


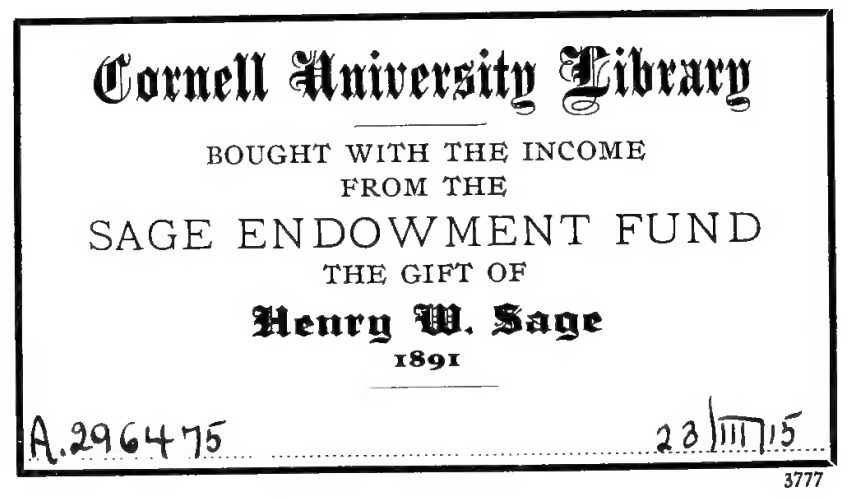




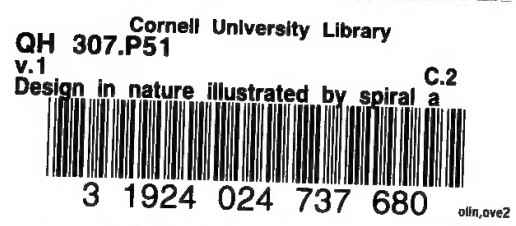


$$
\text { . }
$$ 
DESIGN IN NATURE 


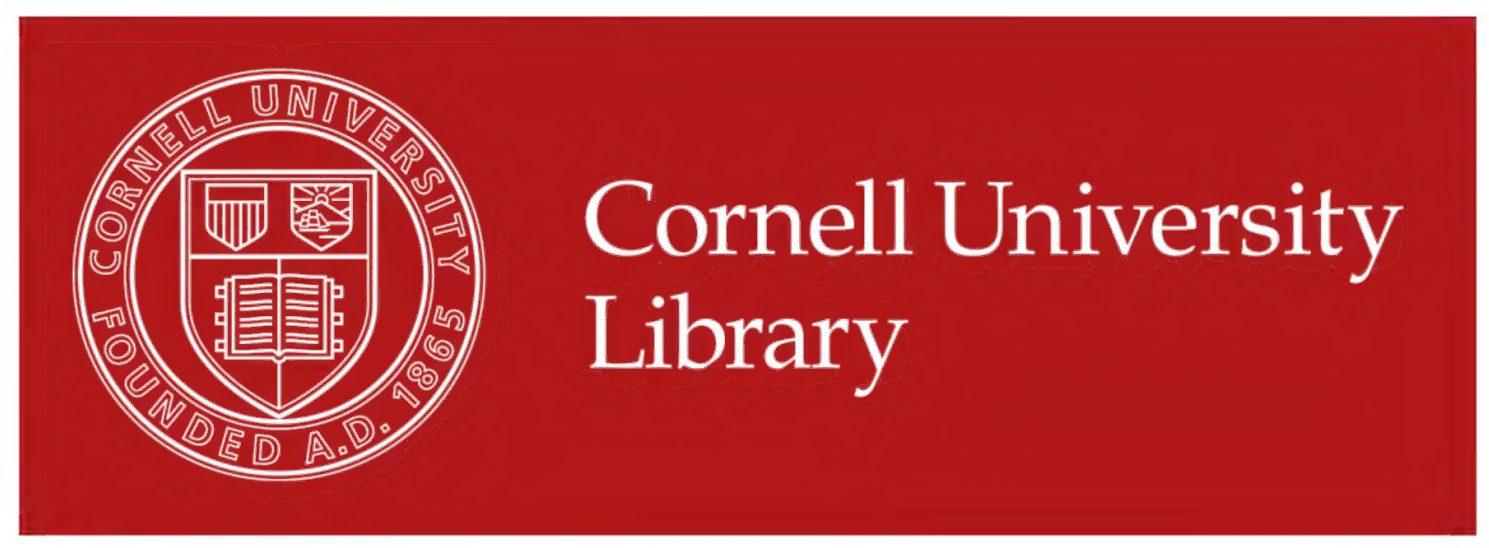

\section{The original of this book is in the Cornell University Library.}

There are no known copyright restrictions in the United States on the use of the text. 


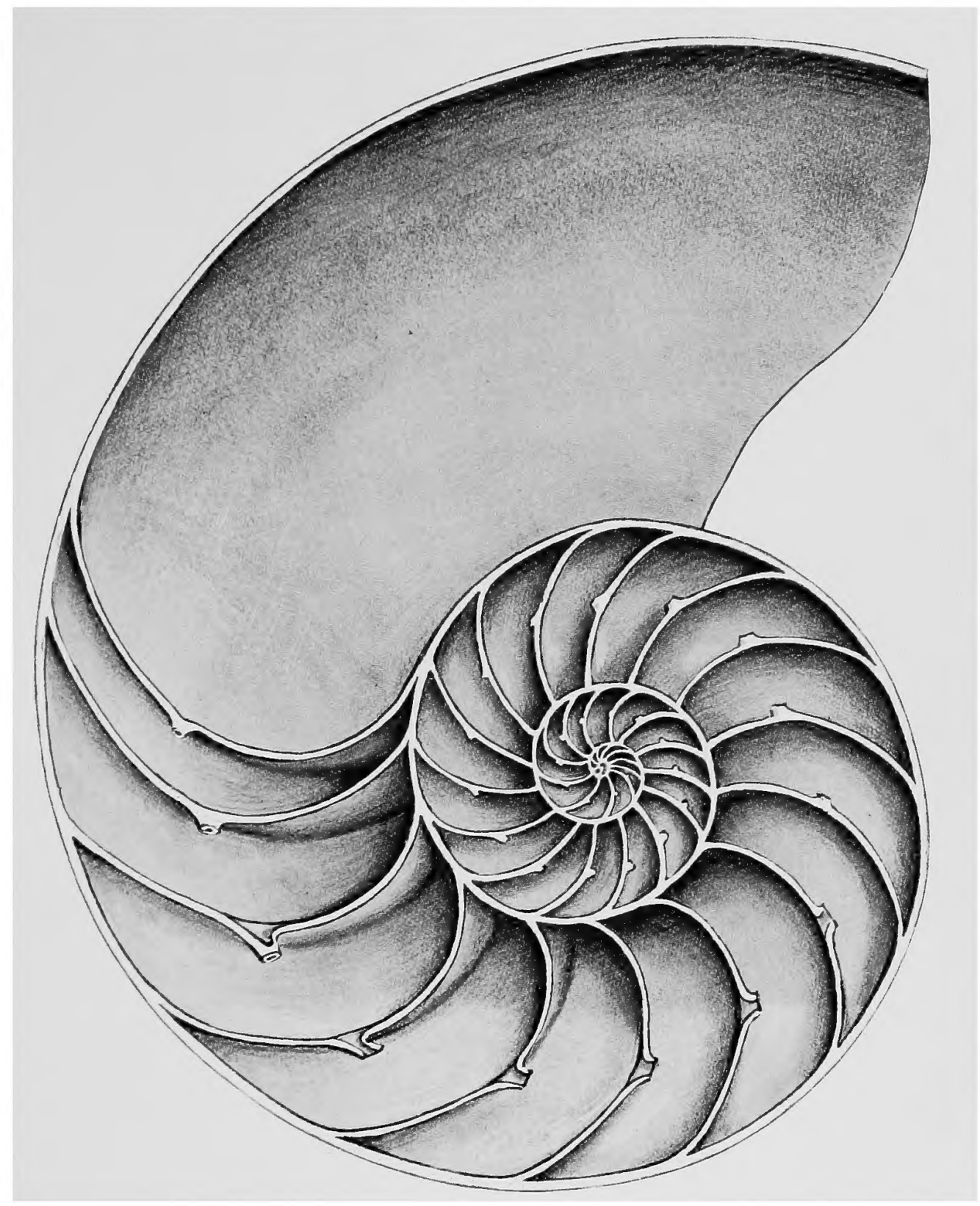

Tracing from nature of a mesial line section of the shell of the Nautilus ( $N$. pompitius), by the Author. Shows beautiful curves and one of the finest sjirals known. 




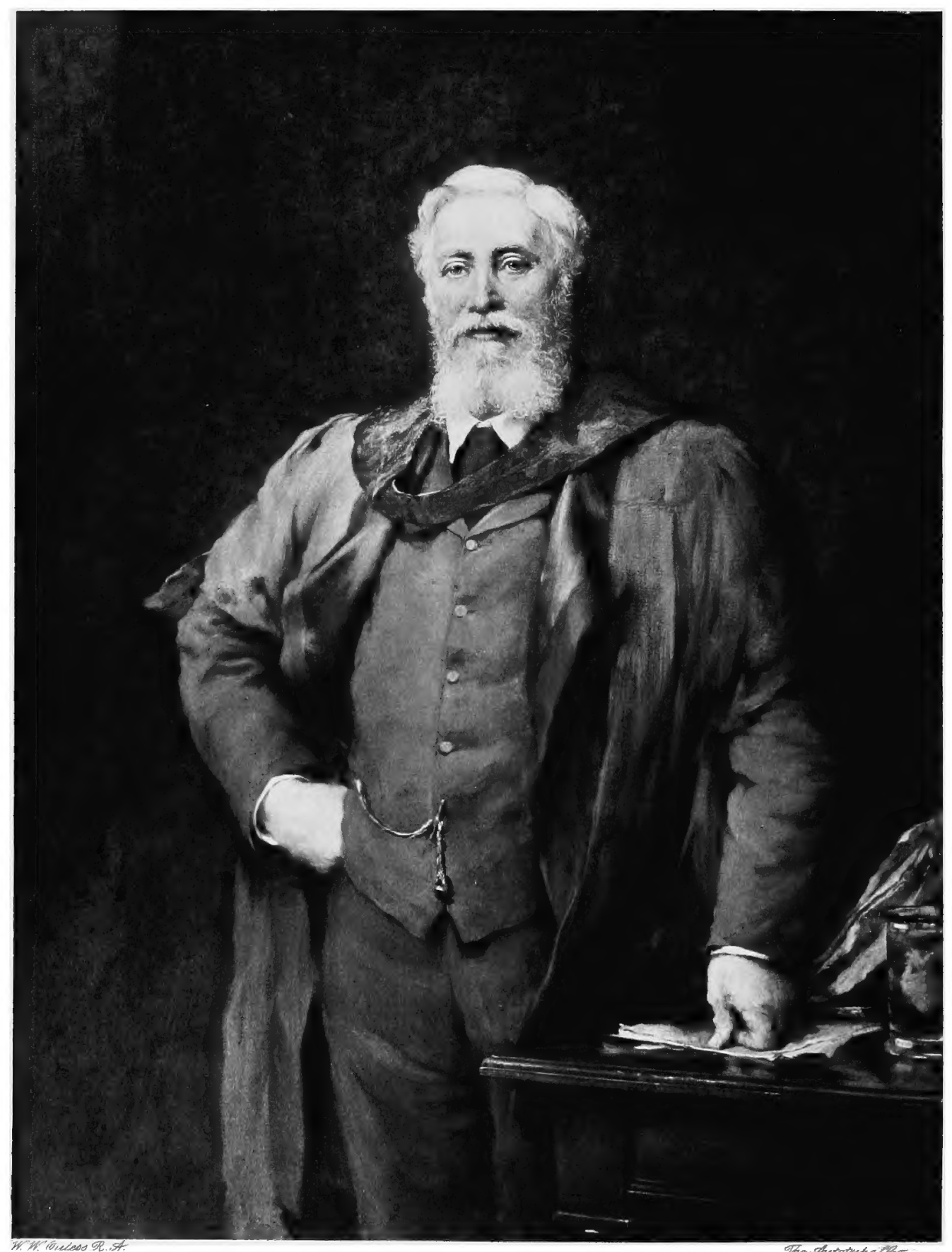

Tho chotatype tho

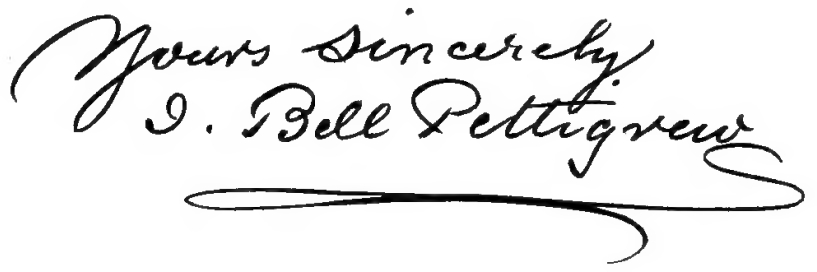






\title{
DESIGN IN NATURE
}

Illustrated by Spiral and other Arrangements in the Inorganic and Organic Kingdoms as exemplified in Matter, Force, Life, Growth, Rhythms, \&c., especially in Crystals, Plants, and Animals. With Examples selected from the Reproductive, Alimentary, Respiratory, Circulatory, Nervous, Muscular, Osseous, Locomotory, and other Systems of Animals

BY

J. BELL PETTIGREW

M.D., LL.D., F.R.S., F.R.C.P.

\begin{abstract}
LAUREATE OF THE INSTITUTE OF FRANCE; CHANDOS PROFESSOR OF ANATOMY AND MEDICINE IN THE UNIVERSITY,
ST. ANDREWS; FELLOW OF THE ROYAL, BOTANICAL, MEDICO-CHIRURGICAL, ROYAL MEIICAL, LITERARY AND PHILOSOPHICAL, HARVEIAN, AND OTHER SOCIETIES: FORMERLY DEAN OF THE MEDICAL FACULTY,

UNIVERSITY, ST. ANDREWS; PRESIDENT OF THE ROYAL MEDICAL AND HARVEIAN SOCIETIES;

MEMBER OF THE GENERAL COUNCIL OF MEDICAL EDUCATION AND REGISTTRATION

OF THE UNITED KINGDOM; EXAMINER IN ANATOMY AT THE UNIVERSITY,

GLASGOW, AND IN PHYSIOLOGY AT THE ROYAL COLLEGES OF PHYSICIANS AND SURGEONS, EDINBURGH, ETC.
\end{abstract}

Illustrated by nearly Two Thousand Figures, largely Original

and from Nature

IN THREE VOLUMES

VOLUME ONE

L. ONGMA N S, GREEN, A N D C O.

39 PATERNOSTER ROW, LONDON

NEW YORK, BOMBAY, AND CALCUTTA

1908 



\section{TABLE OF CONTENTS}

\section{VOLUME ONE}

\section{PAOM

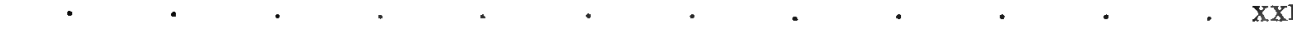 \\ INTRODUCTION . . . . . . . . . . . . . . . . . . . . . . . . . . . . . .}

INORGANIC AND ORGANIC MATTER DISTRIBUTED ACCORDING TO THE SAME

GENERAL LAWS: GLOBULAR, CONCENTRIC, CURVED, SPIRAL, RADIATING,

BRANCHED, AND SEGMENTED ARRANGEMENTS OF MATTER. MOVEMENTS

AND RHYTHMS IN THE INORGANIC AND ORGANIC KINGDOMS-A FIRST

CAUSE NECESSARY

$\S 1$ 1. Atoms and Molecules under Guidance . . . . . . . . . . . . . . . . $\quad 1$

$\S 2$. Straight-Line, Radiating, Concentric, and Spiral Formations with Traces of Segmentation . . . 2

§ 3. Radiating, Dendritic, Segmented, Concentric, and Spiral Arrangements common to Crystals, Plants, and Animals . . . . . . . . . . . . . . . . . . . . 5

PREVALENCE OF SPIRAL ARRANGEMENTS ON A GRAND SCALE IN THE PHYSICAL UNIVERSE

$\$$ 4-7. Origin of Spiral Structures-Spiral Arrangements in Crystals, Plants, and Animals

$19-26$

$\S$ 8. Examples of Radiating and Concentric Arrangements in Plants and Animals as illustrating Symmetry of

Form

§9. Dendritic or Branching Movement in Electric Fluid-Dendritic Formations in Crystals, Plants,

Animals, \&c.

9. Dendritic or Branching Movement in Electric Fluid - Dendritic Formations in Crystals,

\section{HEXAGONAL STRUCTURES}

$\S 10$. Recapitulation

MATTER DIVISIBLE INTO SOLIDS, LIQUIDS, AND GASES-DISTRIBUTION OF MATTER-ORDER OF CREATION, \&c.

$\S 11$. Atoms and Molecules Fundamental in the Inorganic and Organic Kingdoms _ . . . $\quad . \quad 93$

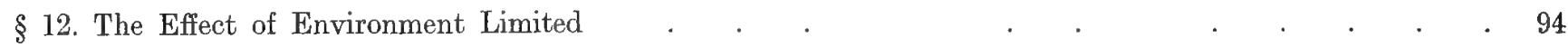

§ 13. The Organic and Inorganic Kingdoms reciprocate . . . . . . . . . 94

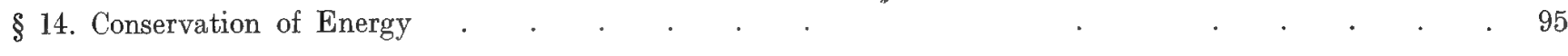

§ 15. Protoplasm: Its Nature and Function . . . . . . . . . . . . . . $\quad$. 95

THE REPRODUCTIVE ELEMENTS OF PLANTS AND ANIMALS ESSENTIALLY AND FUNDAMENTALLY DIFFER FROM THE BEGINNING

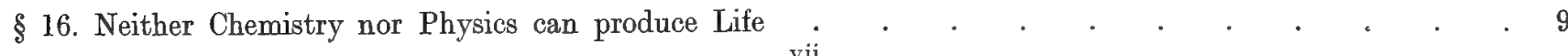
98 
$\$ 18$. Growth in Straight Lines and in Spirals-Longitudinal and Transverse

Wings, \&c

$\S$ 19. Atoms and Molecules the same in Dead and Living Matter..

$\S 20$. Resemblances between Crystals, Plants, and Animals: Anomalous Resemblances . • $\quad . \quad 102$

21. Unity of Plan in Nature as regards Form and Colour: so-called Mimicry...

LINES OF FORCE IN CONNECTION WITH MAGNETISM, ELECTRICITY, OPTICAL PHENOMENA, \&c., AS BEARING UPON STRAIGHT-LINE, CURVED, AND SPIRAL FORMATIONS IN PLANTS AND ANIMALS

§ 22-29. The Lodestone; the Bar-Magnet; Compound Magnet; Horse-Shoe Magnet; Electro-Magnet; Magnets with one degree of Freedom; the Compass; Magnetism, Electricity, Light, Heat, and Motion Correlated

\title{
ATMOSPHERIC AND OTHER ELECTRICITY
}

\section{ANIMAL MAGNETISM}

$\S 30$. Lines of Communication and Force, Nervous and Otherwise.

\begin{abstract}
ATOMS, MOLECULES, AND CELLS, AS FACTORS IN ORGANIC STRUCTURE AND FUNCTION
\end{abstract}

\section{EVIDENCES OF DESIGN IN THE REPRODUCTIVE ELEMENTS OF PLANTS AND ANIMALS}

The Globular, Concentric, Radiating, Branched, Segmented, Curved, and Spiral Arrangements make their Appearance at the very Threshold of Life

PROGRESSIVE ADVANCE IN LOWER PLANT AND ANIMAL FORMS

THE VISIBLE AND INVISIBLE WORLD - Views of Stewart, Tait, Thomson, Helmholtz, Stokes, Young, Crookes, \&c. . . . . . . . . . . . . . . . . . . . 180

$\S 31$. New Theory of Matter . . . . . . . . . . . . . . . . . . . $\quad$. 184

$\S 32$. Inter-action between the Mental and the Material Aspects of Things . . . . . . . . 191

MATTER AND FORCE IN THE INORGANIC AND ORGANIC KINGDOMS: THEIR RELATION TO LIFE, AND TO PLANTS AND ANIMALS-Author's Views regarding Creation, Evolution, Natural Selection, Type, Locomotion, Environment, Geology, \&c. . 193

The Elements and their Combining Weights . . . . . . 194

§33. Professor Haeckel's Belief in the Omnipotence of Matter $\quad . \quad$. . . . . . . 196

§ 34. Mechanical Views of Kant and Laplace as interpreted by Haeckel . _ . . . . . . . . 201

$\S 35$. Professor Huxley's Views on Evolution, especially in Connection with the Reptile, the Bird, and the Horse 


\section{THE TRAVELLING ORGANS OF ANIMALS TO BE REGARDED AS ORIGINAL STRUCTURES}

$\S 36$. The Travelling Organs in Relation to Environment .

\section{CREATION A PROGRESSIVE WORK}

$\S \S 38-39$. Scriptural Account of Creation-Geology as Bearing on Creation .

\section{ORDER IN WHICH PLANTS AND ANIMALS APPEARED ON THE EARTH}

$\S 41$. Plants and Animals Improvable up to a Point

\section{NATURAL AND ARTIFICIAL SELECTION CONTRASTED AND CONSIDERED}

$\S 42$. Everything Controlled and under Supervision

$\S 43$. Plants and Animals subject to Disease.

\section{THE UNIVERSE AS A WORKING SYSTEM}

$\S \S 4-48$. Consideration of the Terms Irritability, Stimulation, Environment, and Instinct

. $239-243$

Instinct and Intelligence

§ 49. Effect of Cosmic Changes on Plants and Animals . . . . . . 246

$\S 50$. Rhythmic Movements in Plants and Animals are Repetitions of Rhythms occurring in External Nature . 249

Rhythms and Reflexes in Plants and Animals: their Nature and Uses . $\quad . .252$

§ 51. Muscular Movements Inherent, Spontaneous, and Independent-Not caused by Nerve Action . . 253

§ 52. Nerve Reflexes in Animals-Definitions of Reflex Acts-Subjects Connected with Reflex Manifestations, \&c. . . . . . . . . . . . . . . . . . . 258

§ 53. Rhythmic Muscles-Rhythms not Confined to Involuntary Muscles 269

54. Respiratory Rhythmic Movements in Animals-New Explanation of these Movements . 272

§ 55. The Respiratory Organs in Animals and in Man Structurally Considered . . $\quad 274$

$\S 56$. The Respiratory Movements, especially in Man-New View of the Mechanism of Respiration-The Muscles

of the Chest, Abdomen, and Diaphragm all Involved 277

§ 57. The Mycetozoa . . . . . . . . . . . 299

§ 58. Protoplasmic, Amobic, Muscular, and other Movements $\quad$. . . . 312

§ 59. Muscular Action (Voluntary and Involuntary), as bearing on Locomotion, Respiration, Circulation, Alimentation, Urination, Defecation, and Parturition . . . . . . 327

\section{RUDIMENTARY FORMS IN RELATION TO MOVEMENT, REPRODUCTION, AND LIFE}

$\S \S 60-65$. Movements, \&c., of the Amœba, Paramecium, Gromia, Mycetozoa, Zooid, Monad, Vorticella, \&c. $332-340$

\$66. Animals specially constructed as Air-breathers, and Water-breathers, and for Land, Water, and Air

Transit . . . . 342

§67. A Creator, Designer, and Upholder necessary to the Universe as we know it . . 347 VOL. $\mathrm{Y}$. 


\section{DEVELOPMENT AND DIVISION OF LABOUR IN RELATION TO DESIGN}

§ 68-69. Design a Prominent Factor in Nature-Design as seen in the Gradation of Plants and Animals and

in the Arrangements for Walking, Swimming, and Flying . . . . . . 359-364

$\S \S 70-71$. Design as Manifested in the Growth and Reproduction of Plants and Animals, especially the Latter

$\S \S 72-77$. Parts Entering into the Composition of the Human Ovum-Ripening of the Ovum : Formation of Polar Globules-Fertilisation of the Ovum-Reproduction in the Bird-Division of the Impregnated Ovum--The Development of Membranes and Layers from which the Future Being Proceeds

$\S \S 78-80$. Subjects Connected with the Development of the Human Embryo and Fœetus-Career of the Unimpregnated and Impregnated Ovum-Development of the Brain and Vessels of the Body-Placental and Fœtal Circulation-Successive Changes Witnessed in the Growing Parts

§§ 81-82. Transition Links in Relation to Types-Changes in, and Peculiarities of, the Human Fœetus 401-405 § 83. The Development of Blood, Blood-vessels, Nerves, Muscles, Bone, Lungs, Glands, Sense Organs, \&c., in Man and Mammals.

$\$ \$$ 84-85. Design as Witnessed in the Migration of Birds and Other Animals-Design as seen in the Production and Distribution of Seeds

\section{VOLUME TWO}

\section{THE CIRCULATION IN PLANTS, IN THE LOWER ANIMALS, AND IN MAN}

$\S \S 86-87$. Epitome of the History of the Circulation-Definition of the Circulation.

$428-429$

\section{THE CIRCULATION IN PLANTS}

$\S \S$ 88-90. Two Different Systems in Plants-Two Principal Sap Currents in Plants--Proof that the Saps of Plants Ascend and Descend

$\S \S$ 91-93. Endosmose and Exosmose as Adjuncts of the Circulation-The Vessels of Plants: their Function

-Points of Resemblance between the Vessels of Plants and Animals . . $433-435$

$\S \S 94-97$. Respiration in Plants and Animals-Cells of Plants : their Nature and Function-The Intra-Cellular Circulation in Plants-The Lactiferous Circulation in Plants-The Forces which Produce the Circulation in Plants.

$436-444$

\$ 98-99. Original Experiments to Show that the Vessels of Plants in Summer form Syphons-Experiments bearing on the Intra-Cellular Movements in Plants

$\S \S$ 100-102. Absorbents of Plants-Analogy between the Leaves and Roots of Plants_-The Effects produced on the Circulation by the Swaying of Plants in the Wind: Mr. Herbert Spencer's Views $449-451$ $\S \S 103-106$. Epitome of the Forces engaged in the Circulation in Plants_Organic Forces a Modification of Inorganic Forces-Motion a Condition of Matter-Circulation in Metals.

$453-459$

\section{THE CIRCULATION IN ANIMALS (Invertebrata)}

$\S 107$. Symmetry of Form in the Organs of the Circulation and in the Body generally 
$\S \S 110-113$. Circulation in the Leech contrasted with that in Plants-How the Circulation connects the Organic and Inorganic Kingdoms-The Digestion and the Circulation-Cilia, their Form and Function . 463-465

$\S \S$ 114. Undefined Forces of the Circulation-Slowing and Quickening of the Circulation

$\$$ \$15-121. Circulation in the Star-Fish, Spider, and Insect-Functions performed by the Valves of the Vessels in Insects--Circulation in the Lobster-Position of the Respiratory Apparatus in the Lobster, Fish, \&c.

-Circulation in the Brachiopoda, Gastropoda, and Cuttle-fish

. $469-472$

\section{THE CIRCULATION IN ANIMALS (Vertebrata)}

$\S$ 122-126. Circulation in the Fish, Batrachia, Frog, Isacerta ocellata, Python, Crocodile, \&c.-Presence of Septum Ventriculorum

. $473-477$

\section{THE FETAL CIRCULATION}

$\S \S 127-129$. Points of Analogy between the Circulation in Reptiles and that in the Human Fotus-Circulation in the Body of the Foetus-Circulation in the Placenta

$\S 130$. Distribution of the Great Vessels in Reptiles, Birds, and Mammals

$\S 131$. Valves in the Heart of the Bird . . . . . . . . . . . 493

$\S$ 132-133. The Respiration and Circulation in Birds-The Air-Sacs of Birds, \&c. . . . 494

\section{THE CIRCULATION IN THE MAMMAL}

$\S 134$. The Auricles, Ventricles, Valves, Septa, \&c., of the Heart of the Maminal

$\S$ 135-138. The Right Heart of the Mammal-The Blood urged on by a Wave-Movement: the Heart and Vessels Open and Close in Parts—The Valves of the Right Heart of the Mammal—Safety-Valve Action of the Tricuspid

$497-500$

$\S \S 139-144$. The Left Heart and Valves of the Mammal. The Circulation in the Head, Liver, and Erectile Tissues-The Lymphatic and Capillary Vessels of Animals-Structure of the Capillaries and Small Arteries and Veins: Vessels Close and Elongate, and Open and Shorten-Elastic and Vital Properties of Vessels-Structure of the Large Arteries and Veins

$501-504$

\section{STRUCTURE OF THE HEART OF THE MAMMAL}

$\S 145$. The Arrangement of the Muscular Fibres in the Auricles and Ventricles, especially in the Ventricles

\section{ARRANGEMENT OF THE MUSCULAR FIBRES IN THE HOLLOW VISCERA GENERALLY}

$\S 146$. Arrangement of the Muscular Fibres in the Bladder and Prostate . . . 519

$\S 147$. The Distribution of the Fibres in the Muscular Coats of the Stomach of Man and Other Mammalia 522

§ 148. Esophageal or Cardiac Sphincter not hitherto described . . . . . . . . 525

§ 149. The Pyloric Sphincter-its Ultimate Structure . . . . . . . 525

Movements of the Human Stomach and its Sphincters . . . . . . . . . . . . 527

$\S 150$. Analogy between the Muscular Arrangements and Movements of the Hollow Viscera (Heart, Stomach, Bladder, Uterus) and those of the Trunk and Extremities of Vertebrates . . . . . . . 531 


\section{MUSCLES ENDOWED WITH CENTRIPETAL AND CENTRIFUGAL MOVEMENTS- SARCOUS ELEMENTS OF MUSCLE-THEIR PECULIAR ACTION}

$\S \S 154-155$. Muscular Motion as Bearing on the Functions performed by the Heart, Blood-vessels, Thorax, Extremities, \&c.-How the Muscular Fibres Open and Close the Blood-vessels and the Several Compartments of the Heart-also how the Stomach, Bladder, Thorax, and Abdomen Open and Close as Wholes or in Parts

$\S$ 156. Analogy between the Movements of the Thorax, Abdomen, and Heart

$\S 157$. The Movements of the Mammalian Heart, Interrupted and yet Continuous. How the Heart Rests

$\S 158$. Size of the Cavities of the Heart (Mammal) .

$\S 159$. Impulse of the Heart (Mammal)

\section{THE VALVES OF THE VASCULAR SYSTEM, ESPECIALLY IN MAN}

$\S \S 160-164$. The Venous Valves, their Structure, \&c.-The Venous Valves in Action--The Arterial or Semilunar Valves, their Structure, \&c.-The Arterial or Semilunar Valves in Action-The Semilunar and Other Valves of the Fish, Reptile, Bird, \&c.

$\S 165$. Intricate Structure of the Bicuspid and Tricuspid Valves in Mammalia-CRelations of the Chordæ Tendineæ to these Valves and to the Musculi Papillares

$\S \S 166-168$. The Bicuspid and Tricuspid Valves of the Mammal in Action-The Mechanical and Vital Theories of the Action of the Bicuspid and Tricuspid Valves considered-The Sounds of the Heart : to what Owing

THE GANGLIA AND NERVES OF THE HEART, AND THEIR CONNECTION WITH THE CEREBRO-SPINAL AND SYMPATHETIC SYSTEMS IN MAMMALIA

$\$$ 169-175. The Cervical Cardiac Branches-The Thoracic Cardiac Branches-The Superior Cervical Ganglia - The Middle and Inferior Cervical (anglia-The Upper Cardiac Nerve-The Middle Cardiac NerveThe Inferior Cardiac Nerve

$\S \S 176-177$. Nerve Plexuses formed on the Roots of the Pulmonary Artery and Aorta in the Calf-Nerve Plexus formed on the Coronary Sinus of the Calf .

$\S \S 178-180$. Distribution of the Nerves on the Surface and in the Substance of the Auricles of the Heart of the Calf-Distribution of the Nerves on the Surface and in the Substance of the Ventricles of the Heart of the Horse and Calf-Distribution of the Nerves on the Right and Left Ventricles $573-575$

$\S$ 181-182. Distribution of the Nerves in the Human Heart-Nerves and Ganglia of the Human Heart 576-577

\$ 183. Microscopic Appearances presented by the Ganglia found on the Coronary Sinus and Cardiac Vessels

$\S 184$. Why does the Heart act Rhythmically?

§ 185-186. Proofs that the Heart may Act independently of the Nerves-Proofs that the Movements of the Heart are Regulated and Co-ordinated by the Nerves - $580-581$ 
THE MOVEMENTS AND FUNCTIONS OF SENSITIVE, INSECTIVOROUS, CLIMBING, AND OTHER PLANTS

$\S \S 188-189$. The Sensitive Plants and Pitcher Plants

The Droseraceæ or Insectivorous Plants Proper

$\$ \$$ 190-195. Aldrovande vesiculosa-Drosophyllum lnsitanicum-Roridula-Byblis gigantea-Dionipa muscipula or Venus's Fly-trap-The Sun-Dew (Droserce rolundifolia)

Sensitive Moving Plants-Spiral Climbing Plants-Revolving and Twisting Stems, Tendrils, Leaves, \&c.

$\S \S 196-200$. Stem and Tendril Climbing Plants-Spirals Formed by the Tendrils of the Passion-flower, Sweetpea, Cucumber, and Vegetable Marrow

Design in Climbing, Sensitive, and Insectivorous Plants

$\S$ 201. Growth in Plants and Animals

The Universality of Spirals in Nature as indicated by Stereo-Chemistry, \&c. . $\quad 633$

Spiral Arrangements in the Physical Universe

Spiral Arrangements in the Vegetable Kingdom . . . . . 636

Spiral Arrangements in the Animal Kingdom.

\section{SPIRAL FORMATIONS IN PLANTS}

$\S \S 202-209$. Spirality in Plants the Product of Life and Original Endowment-Spiral Seeds-Spiral and Other Cells in Plants-Spiral Fronds and Flowers-Spiral Hairs-Spiral Distribution of Leaves and BranchesSpiral Stems of Plants and Boles of Trees-Spiral Fossil Stems

Spiral Climbing Plants-Revolving and Twisting of Stems, Tendrils, \&c.

THE GROWTH OF SPIRAL SHELLS, HORNS, BONES, TEETH, FEATHERS, \&c., IN RELATION TO SPIRAL PLANTS, BRANCHES, LEAVES, TENDRILS, FLOWERS, FRUITS, SEEDS, \&c.

$\S$ 212. The Prevalence of Spiral Formations : their Physiological Significance in Relation to Walking, Swimming, and Flying-The double figure-of- 8 Curves and Spirals made by the Wings in Flight not known to Leonardo da Vinci

\section{THE ORIGIN OF SPECIES-VARIOUS VIEWS}

§213. Consideration of the Theory of the Origin of Species by Means of Natural Selection-Objections to the Theory

$\S \S 214-215$. The Origin of Species Undetermined and Indeterminable-Professor Haeckel an Advanced Exponent of the Darwinian Theory

Heredity and Descent-Transmission of Peculiarities Natural and Acquired-Formation, Use, and Disuse of Organs

\$217. A First Cause Necessary to Life-Life Transmissible-Types of Plants and Animals versus Evolution and Spontaneous Generation 


\section{THE FIRST APPEARANCE OF A NERVOUS SYSTEM-THE NERVOUS SYSTEM GENERALLY}

§ 218. The Nature and Peculiarities of the Nervous System

Proofs that the Brain is the Organ, Apparatus, or Laboratory of the Mind

The Nervous System as Bearing on Sensation, Volition, Muscular, and other Action .

$\$ \S 219-227$. The Brain - Olfactory Ganglia_Cerebral Ganglia or Hemispheres_Corpora Striata_Optic Thalami-

Tubercula Quadrigemina-Cerebellum_Tuber Annulare and Medulla Oblongata

$777-780$

Reflex Action

$\$$ 228-231. Unconscious Cerebration-Phrenology in Relation to Cortical Brain Areas-Nerve Endings in the Brain, Skin, and other Structures-The Relations Existing between Brain and Muscle

\section{CONSCIOUSNESS AND MEMORY}

The Sensory Organs developed from Within and not from Without . . . 814

§ 232. Environment-The Production of the Eye

$\S 233$. Consideration of the Argument for Design

The Sense-Organs as Bearing on Adaptation and Design

$\S \S 234-241$. The Sense of Touch-The Sense of Taste-Conditions under which Taste is Experienced-The Sense of Smell-Conditions under which smell is Produced-The Sense of Hearing-Internal Ear or Labyrinth—The Sense of Sight—The Structure of the Eye, \&c.

$8: 36-8 \pm 7$

\section{THE PHONOGRAPH AND TELEPHONE IN THEIR RELATIONS TO THE HUMAN} VOICE AND EAR, AND AS ILLUSTRATING THE INTIMATE CONNECTIONS SUBSISTING BETWEEN THE INORGANIC AND ORGANIC KINGDOMS

§ 242. The Structure and Working of the Phonograph

$\S \S 243-244$. Mechanism of the Human Voice-Mechanism of the Human Ear

$\S \S 245-247$. Structure and Working of the Telephone-The Reiss (musical) Telephone-Professor A. Graham

Bell's Telephone

Reflex Action, Instinct, and Reason

$\S 248$. The Alternate Propelling and Retaining Muscular Structures of the Body (Heart, Stomach, Bladder,

Uterus, \&c.) in Relation to so-called Reflex Action

Movement in Relation to Intelligence-Development of Intelligence, \&c.

$\S \S 249-257$. Voluntary Movements in the Protozoa-The Coelenterata-The Annelida-The Larvæ of Insects-

The Mollusca-The Spiders-The Ants-The Sense Organs of Ants-The Termites . 890-909

$\$ 258-259$. Bees and Wasps-Intelligence of the Bees, \&c.

\section{CONSTRUCTION OF THE DOUBLE HONEY-COMB OF THE DOMESTIC BEE (Apis mellifica)}

\section{Evidence of Design in said Construction}




\section{INTELLIGENCE OF MAMMALS}

$\S$ 264-268. Intelligence of Sea Mammals (Porpoise, Whale, Sirenidæ, Walrus, Seal, and Sea-Tion)

Intelligence of the Land Mammals in the Wild and Domesticated State .

\$ 269-287. Rabbit, Hare, Wild Pig, Buffalo, Bison, Beaver, Lion, Tiger, Bear, Otter, Wolf, Jackal, Fox, Weasel, Ferret, Polecat, Wolverine, Rat, Ox, Sheep, Goat, Horse, Cat, Dog, Elephant, Monkey, \&c.) $966-1002$

$\S 288$. Man as a Separate Creation

\section{THE MUSCULAR AND OSSEOUS ARRANGEMENTS OF MAN AS BEARING ON EXPRESSION, RESPIRATION, LOCOMOTION, THE ERECT POSITION, \&c.}

$\$$ 289-294. The Muscular and Osseous Systems Interdependent and Complemental_-The Muscular Arrangements of Man as a Whole-The Muscles of the Head, Face, and Neck (Human)_The Muscles and Bones of the Trunk, Thorax, and Abdomen (Human)-Anterior View of the Bones and Cartilages of the Chest (Human)-Lateral Vien of the Bones of the Chest (Humun).

1026-1035

$\S 295$. Bones of the Chest and Shoulder Girdles which take part in the Swinging Pendulum-Movements of the Arms in Locomotion in Man

$\S 296$. Section of the Bones of the Human Pelvis showing the Pelvic Arch, the Lower Portion of the Vertebral Column, and the Upper Parts of the Femurs or Thigh Bones with their Ball and Socket or Universal Joints engaged in the Pendulum-Movements of Walking, Running, \&c.

\$ 297-298. The Muscular Arrangements on the Anterior, Posterior, and Lateral Aspects in Man-The Shoulder, Hip, and other Muscles connected with Locomotion, the Erect Position, \&c. 1036-1037

$\S 299$. Lateral View of the Superficial Muscles of the Human Chest and Abdomen connected with Respiration, Abdominal Expulsive Efforts, \&c.

$\$ \$ 300-302$. Lateral View of the Deeper Muscles (more especially of the Abdomen) connected with Respiration, Abdominal Expulsive Efforts, \&c. (Human)-Lateral View of the Muscles and Ribs of the Left Side of the Body connected with Respiration, \&c. (Human)-Transverse Section of the Abdominal Cavity in the Lumbar Region (Human).

Muscular Arrangements and Position of the Diaphragm or Partition which Separates the Thorax from the Abdomen

§ 303. The Diaphragm as seen from Above and from Beneath (Human) .

$\S \S$ 304-305. Superficial Muscles of the Left Chest and Front of the Arm (Human)-Deep Muscles of the Chest and Superficial Muscles of the Front of the Arm (Human)

$1044-1045$

§ 306-311. Muscles of the Human Arm, Forearm, and Hand-Muscles occurring on the Dorsum of the Scapula, and the Back of the Arm (Human)-Anterior View of the Superficial Muscles of the Forearm (Human) -Anterior View of the Deep Muscles of the Forearm (Human)-Posterior Surface of the Forearm : Its Superficial Muscles (Human)_Posterior Surface of the Forearm : Its Deep Muscles (Human) . 1045-1047

\section{THE BONES OF THE WRIST AND RIGHT HAND-DORSAL SURFACE (Human)}

$\S \S 312-314$. Muscles of the Hand-Dorsal Surface (Human)-Muscles, Tendons, \&c., of the Left HandPalmar Surface (Human) - Lumbrical and Interosseous Muscles of the Hand (Human) . . 1049-1050 
$\$ \$ 316-320$. Muscles of the Tliac and Anterior Femoral Regions (IIuman)-Muscles of the Inner Aspect of the

Thigh (Human)-Muscles of the Front of the Leg; Anterior Tibio-fibular Region (Human)-Superficial and Certain of the Deep Muscles of the Lower Leg as seen from Behind (Human)-Muscles of the Back of the Leg, Deep Layer (Iruman)

\section{BONES OF THE RIGHT FOOT SEEN FROM THE INSIDE (Human)}

§321. Longitudinal Section of the Soft and Hard Parts of the Foot (Human)

$\$ \S 322-326$. Muscles of the Sole of the Foot, Superficial Layer (Human)-Muscles of the Sole of the Foot, Second Layer (Human)--Muscles of the Sole of the Foot, Third or Deepest Layer (Human)-The Dorsal and Plantar Interossei Muscles of the Foot (Human) - The Foot of Man adapted to the Erect Position . 1058-1060

DESIGN AS MANIFESTED IN THE HUMAN FOOT, HAND, AND ORGANS OF LOCOMOTION

$\S 327$. The Human Foot and Hand in Relation to the Foot of the Horse and the Wing of the Bird, Bat, and Pterodactyl

Design as Displayed in the Muscular System generally

\section{VOLUME THREE}

\section{ANIMAL LOCOMOTION}

§ 328. Extended General Statement and Review of the whole Subject .

$\$$ 329-331. Fundamental Axioms-Of Uniform Motion-Motion Uniformly Varied .

\section{PASSIVE ORGANS OF LOCOMOTION}

§ 336. Bones-Joints-Ligaments

\section{ACTIVE ORGANS OF LOCOMOTION}

§ 338. Muscles, their Properties, Arrangement, Mode of Action, \&c. .

\section{MOVEMENTS IN RUDIMENTARY LIVING MATTER}

\$341. Instantaneous Photography a Valuable Aid in Determining Animal Movements

$\$ 342-345$. The Creeping of the Serpent-The Locomotion of Birds (the Ostrich, Adjutant, \&c.)-Locomotion in Man-Locomotion of the Horse 
THE OSSEOUS AND MUSCULAR SYSTEMS OF THE HORSE

$\S 346-352$. The Walk, Trot, Gallop, Canter, Amble, Rack, and Ricochet of the Horse . . 1137-1I4:3

$\$ 353-355$. Locomotion of the Giraffe, Elephant, and Dog

$1144-1146$

\section{PROGRESSION ON AND IN THE WATER}

$\S \S 356-361$. The Swimming of the Jelly-fish, Scallop, Salpa cristata, Octopus, Squid, Winged Medusa, and Sea-Butterfly

$1149-1155$

$\$ \S 362-366$. The Swimming of the Lobster, Fresh-water Tortoise, Turtle, and Triton, and of the Swan and other

Birds

$1156-1160$

$\S 367-368$. Analysis of the Swimming of the Fish-Consideration of the Osseous and Muscular Systems of the

Fish, \&c. .

$1162-1171$

$\$ \$ 369$. Analysis of the Movements of the Swimming of the Whale, Porpoise, Halitherium, Rhytina, Dugong,

Manatee, \&c.

$\S 370$. Analysis of the Swimming Movements of the Walrus, Seal, and Sea-Lion

$\S \S 371-372$. The Swimming of the Penguin-Flight under Water-Difference between Sub-Aqueous and Ä̈rial Flight

$1185-1190$

§ 373. The Flight of the Flying-fish-The Kite-like Action of its Wings

DESIGN AS WITNESSED IN THE SWIMMING APPLIANCES OF ANIMALS - Fins, Flippers, and Wings resemble each other Structurally and Functionally-The Swimming Organs form the Transition Links between the Walking and Running Organs, and the Volant or Flying Organs

$\S 374$. General Statement regarding the Nature and Extent of the Surfaces employed in Walking, Swimming, and Flying

\section{PROGRESSION IN OR THROUGH THE AIR}

§ 375. The Wing can Produce and Utilise Artificial Air Currents

$\$ 376-378$. Weight necessary to Flight-Weight contributes to Horizontal Flight-Weight, Momentum, and

Power as Factors in Flight

1203-1204

§ 379. Air-Cells in Insects, Birds, and Bats not Necessary to Flight

$\S \S 380-382$. How Balancing is Effected in Flight_-The Sound produced by the Wing-Rapidity of Wing Move-

ments partly accounted for-The Wing Area Variable and in Excess . .

$1206-1208$

SERIES OF EXPERIMENTS BY THE AUTHOR SHOWING EXCESS OF WING AREA

IN THE FLY, DRAGON-FLY, BUTTERFLY, HOUSE SPARROW, d.

$\S 383$. The Wing Area decreases as the Size and Weight of the Volant Animal increases

All Wings constructed on a Common Principle, namely, that of the Helix or Screw

\section{THE WINGS OF INSECTS}

\$384-385. Anterior Wings (Elytra or Wing-Cases), their Shape and Uses-The Posterior Wings, their Nervures,

Form, Function, \&c.

$\S 386$. Threefold Action of the Wing in Insects

§ 387. Flight Essentially a Spiral, Gliding Movement

$\S 388$. Points wherein the Screws formed by the Wings differ from the Screw Propellers in Use in Steamships 
THE WING A TWISTED LEVER OR HELIX-ITS MODE OF ACTION IN THE INSECT, \&C. $\S$ 389-391. Arrangement for Moving the Wings of Insects-Speed Attained by Insects-The Centre of Gravity in Insects-Articulation of the Wing to the Body of the Insect _ . . . . . 1225

When the Body of the Volant Animal is Fixed and its Wings are made to Vibrate they Describe the Figure-of-8 Trajectories in the Air . . . . . . . . 1227

When the Body of the Volant Animal is Advancing and its Wings are made to Vibrate they describe first Looped and then Waved Tracks in Space . 1228

$\S 392$. Mode of Investigation pursued by the Author

$\S 393$. The Muscles and Joints of the Wing

$\$ \S 394-395$. Mechanical Theory of the Action of the Insect's Wing as Stated by Chabrier-Objections to the Mechanical Theory of Wing Movements

$\S 396$. Method of Demonstrating the Accuracy of the Figure-of-8 Movements made by all Wings .

$\S \S 397-398$. Flight of the Common House-fly as seen in the Living Insect-Flight of the Crane-fly under similar Conditions

$1230-1231$

\section{THE WINGS OF BIRDS}

$\S \S 399-401$. Structure and General Appearance presented by the Wing of the Bird-Movements of the Wing of the Bird-Flexion and Extension of the Wing-Valvular Action of the Primary and Secondary Feathers of the Wing-Muscles, Bones, Joints, Elastic Structures, \&.c., of the Wing

$\S$ 402. Dimensions and Weights of Important Parts of the Pigeon (Columba livia)

$\S 403$. Consideration of the Forces which propel the Wings of Birds and Bats

$\S \S$ 404-406. Lax Condition of the Shoulder-Joint in Birds and Bats-The Wing Flexed and partly Elevated by the Action of Elastic Ligaments-The Nature and Position of such Ligaments in the Pheasant, Snipe, Crested-Crane, Swan, \&c.--The Elastic Ligaments more highly Differentiated in Wings which are made to Vibrate rapidly

$1251-1252$

$\S 407$. Power of the Wing, to what Owing

$\S \S 408-409$. The Skeleton or Osseous System of the Bird (Golden Eagle)—The Bones of the Wing of the Bird-

Their Articular Surfaces, Movements, \&c.

1253-1255

THE MOVEMENTS PECULIAR TO THE SHOULDER, ELBOW, WRIST, AND OTHER JOINTS IN THE WING OF THE BIRD

§ 410-416. Shoulder-Joint, Elbow-Joint, Wrist-Joint-The Elbow, Wrist, and Other Joints Alternate and Reciprocate-Elevation and Depression of the Wing, how caused-The Elbow, Wrist, and other Joints always at nearly Right Angles--Lateral Movements in the Elbow, Wrist, and other Joints

$1256-1258$

TRACES OF DESIGN IN THE WING OF THE BIRD-THE ARRANGEMENT OF THE PRIMARY, SECONDARY, AND TERTIARY FEATHERS, \&c.

$\S 417$. Flexion and Extension of the Wing of the Bird in Flight. 1259

The Wing Strikes Upwards and Forwards during the Up Stroke, and Downwards and Forwards during the Down Stroke

§ 418. Reasons why the Effective Stroke should be delivered Downwards and Forwards 
$\S 420$. The Flight of Birds divisible into Four Kinds-Differences to be Noted between Flapping or Rowing Flight, and Sailing or Swimming Flight.

$\S 421$. The Regular and Irregular in Flight

The Wing Acts as a True Kite both during the Down and Up Strokes

$\S \S 422-423$. Analogy between the Wing and a Boy's Kite-Points of Difference to be Noted-The Angles formed by the Wing with the Horizon during its Vibrations

$1272-1273$

$\S \S 424-429$. The Margins of the Wing thrown into Opposite Curves during Extension and Flexion-The Tip of the Bird's and Bat's Wing describes an Ellipse-The Wing capable of Change of Form in all its Parts-The Wing when made to Vibrate produces a Cross Pulsation-Compound Rotation of the Wing-The Wing Oscillates unequally with Reference to a Given Line . . . 1275-1277

$\S 430$. The Flight of the Sea-Gull as witnessed on the West Coast of Scotland . . . . . 1277 $\S \S 431-432$. Analysis of the Movements of Extension and Flexion in the Wing of the Gannet-The Arigles of Inclination which the Under Surfaces of the Gannet's Wing make with the Horizon in Extension and Flexion vary

$1278-1279$

\section{MEASUREMENTS, WEIGHTS, \&c, OF THE GANNET AND HERON}

§ 433. Flight of the Gannet as witnessed at the Bass Rock, Firth of Forth, Scotland

$\S \S 434-435$. A Regulating Power necessary in Flight—The Wings at all Times thoroughly under Control 1281-1282

Analysis of the Down and Up Strokes of the Wing of the Bird in Flapping or Rowing Flight 1283

$\S 436$. The Body is made to Ascend when the Wings Descend and vice vers $t$

$\S 437$. The Natural Wing when Elevated and Depressed must move Forwards

$\S 438$. The Body and Wings move in Opposite Curves .

§ 439. Birds fly by causing their large, powerful Wings to act upon the Air as Kites; the Air furnishing a suitable Fulcrum for supporting and carrying them Upwards and Forwards-The Wings are Propelled by Voluntary well-directed Muscular Movements, aided by certain Elastic Structures which contribute to the continued Vibrations of the Wings-In Flight, Weight and Momentum play an important Part

$\S$ 440. Lifting Capacity of Birds

$\S 441$. Mode of Ascending, Descending, Turning, \&c.

\section{THE WINGS OF BATS}

$\S 442$. Where the Bat's Wing agrees with and differs from that of the Insect and Bird .

$\S 443$. The Bones of the Wing of the Bat-The Spiral Configuration of their Articular Surfaces

$\S 444$. Extension and Flexion of the Wing of the Bat

\section{THE WINGS OF PTERODACTYLS (Extinct fying reptiles)}

$\S 445$. The Wings of Pterodactyls are Original Structures in the Sense that they formed part of the Animals when Created.

$\S 446$. The Wings of Pterodactyls conform to the Requirements of Flight, as witnessed in Insects, Birds, and Bats

$\S 447$. The Wings of Pterodactyls afford Examples of Extreme Modification for a Special Purpose 
$\S 448$. Points wherein the Wings of Pterodactyls and Bats Agree and Disagree-Important Differences to be Noted

$\S 449$. The Wings of Pterodactyls are produced not by Dwarfing or Obliterating original Typical Parts, but by the Excessive Growth and Increase in Size of the Parts more especially connected with Flight . . 1295

$\S 450$. The Wings of Pterodactyls are Triangular in Shape, Elastic, carefully-graduated Organs, formed on a C'ommon Type . . . . . . . . . 1295

$\S$ 451. The Wings of Pterodactyls, Bats, and Insects supply Good Models for Winged Flying-machines 1295

\section{EXTINCT PLANTS AND ANIMALS-THEIR PECULIARITIES-LARGE SIZE-} RELATIONS TO THE YOUNG EARTH, \&c.

§ 45\%. Extinct Plants - their unusual Appearance, \&c., as compared with Modern Plants

\section{THE ORIGIN AND CAREER OH MAN}

$\S 45.5$. Antiquity of Man

$\S 456$. The Human Race in Greek and Roman Times

$\S$ 457. Antiquity of Man from the Geological Standpoint.

\section{APPENDICES}

APPENDIX I.-HISTORY OF ANATOMICAL PREPARATION-MAKING AS DEVISED AND PRACTISED BY THE AUTHOR AT THE UNIVERSITY OF EDINBURGH, AND AT THE HUNTERIAN MUSEUM OF THE ROYAL COLLEGE OF SURGEONS OF ENGLAND (LONDON)

(Reprinted from the Lancet of November 23rd and 30th, 1901.)

APPENDIX II.-AËRIAL LOCOMOTION. PETTIGREW versus MAREY. By PROFESSOR COUGHTRIE

(Reprinted from the Quarterly Journal of Science, April 1875.)

APPENDIX III.-REFUTATION OF THE CLAIM PUT FORWARD BY THE ADMIRERS OF LEONARDO DA VINCI TO BE REGARDED AS THE DISCOVERER OF THE TRUE PRINCIPLES OF FLIGHT . . . . . .

("Spiral Formations in Relation to Walking, Swimming, and Flying." Lancet, January 2nd, 1904.) ${ }^{1}$

\section{INDEX}




\section{PREFACE}

THE present work has attained much greater dimensions than was originally intended or was perhaps desirable. This is due to the large number of subjects treated, and to the fact that they are correlated, and, in many cases, overlap.

It was necessary to deal with Physics, Chemistry, Botany, Zoology, Anatomy, Physiology, Psychology, and Palæontology more or less in detail, as these all hang together and bear an important relation to each other in an inquiry such as the present.

"Design in Nature" has a far-reaching significance when proofs thereof are drawn from the cosmos as a whole, that is, from the Inorganic and Organic Kingdoms. If I had confined my researches to one groove, the task would have been light and the compass of the work small. The result, however, would not have been quite satisfactory, as Design is apparent in every part of the Universe, and in no one part more than in another.

The work deals with many of the more important problems of Philosophy and Life so keenly discussed of late years not only by scientists, but also by the general public. To give breadth to the inquiry, the leading sciences have been laid under contribution, it being pointed out that the animal is indebted to the plant, and the plant to the Inorganic Kingdom - that all things living and dead are correlated and interact according to common laws, which alike govern the Inorganic and Organic Kingdoms. A Creator or First Cause, it is claimed, regulates and upholds everything. Nothing comes by chance.

While it is hoped the work is (as far as possible) scientifically accurate and up to date, it is written in a popular style-all technicalities being avoided or explained.

The great bulk of the text is original : care being taken to quote authorities when I am indebted to the labours of others. In not a few cases I give extracts from the writings of fellow-workers, in order that those interested may be fully informed and enjoy facilities for exercising their own judgment in difficult and disputed points.

I have not hesitated to introduce matter from my own memoirs, papers, lectures, \&c., published in the Philosophical and other Transactions, in Journals, and separately, when this seemed desirable. In these instances I give the references and dates, so that opportunities for consultation may be afforded to those desiring more extended. information on any point treated. The matter so incorporated is not, in any case, to be regarded as superseding the source from which it is drawn.

In order to save the time of the reader, and simplify, as much as possible, the various complicated problems discussed, I have introduced into the work a comparatively very large number of illustrations. I have also taken great pains to make the illustrations accurate both as regards outline and detail : quite a large proportion of them being from photographs and drawings from actual specimens either by my artist, Mr. Charles Berjeau, or by myself. The illustrations when not original are (where practicable) duly acknowledged.

I cannot too highly praise Mr. Berjeau's work. It is as artistic and beautiful as it is truthful and striking. But for his hearty co-operation and ungrudging labour under my own eye for several years, it would not have been possible to achieve the results aimed at.

I have to express my cordial thanks to the Messrs. Butterworth of London for the admirable manner in which they have executed the numerous wood-cuts, engravings, \&c., and to my Publishers, Messrs. Longmans, Green \& Co., of London, New York, and Bombay, for the watchful vigilance and forethought displayed by them in everything pertaining to publication, for arranging important details, and for producing the work in an attractive and desirable form: nor must I omit favourable mention of my Printers, Messrs. Ballantyne, Hanson \& Co., of Edinburgh, for their careful and delicate handling of the text and illustrations in their passage through the press. Lastly, my best thanks are due to numerous friends who have kindly assisted me in correcting the proofs. Of these I have specially to mention the names of Professor John Ferguson, LL.D., University of Glasgow ; H. Warren Jones, M.A., Oxon.; Professor F. Jeffrey Bell, M.A.; John H. Wilson, D.Sc. ; and John C. Bagot, Esq. 
It only remains for me to state that the work has been divided into sections, which are numbered for easy reference. Each section is intended to be, as far as possible, complete in itself. It is, however, hoped that the reader will peruse the sections consecutively, as they are mutually explanatory.

If the account given of the relations subsisting between the Inorganic and Organic Kingdoms serves to direct the attention of the reader to the wonderful adaptations of means to ends which everywhere obtain in the Universe, and which can only be explained by the existence of an intelligent Creator, Designer, and Upholder, the object which I have had in view will be fully attained.

The Swallowgate,

ST. ANDREWs,

SCOTLATD.

\section{NOTE}

THE manuscript of "Design in Nature" was quite completed by the Author, and a considerable portion of the Work was printed under his direction. On his death, which occurred on January 30, 1908, the revision of the proof and the passage of the sheets through the press were entrusted to Dr. John H. Wilson, of St. Andrews University, with the assistance of Mr. H. Warren Jones, M.A., and the others named in the Preface. They have endeavoured faithfully to carry out the task allotted to them, but it is very probable that if the Author had lived to see the whole Work in type, means of improvement would have suggested themselves to him. The Index has been compiled by Dr. Wilson. 


\section{INTRODUCTION}

The present work naturally and necessarily covers a wide field, from the fact that it attempts to trace DesiGN, Order, and Purpose in the inorganic and organic kingdoms, especially the latter.

It concerns itself not only with inorganic matter and physical force, but also with organic matter, vital force, and intellect.

It seeks to explain, so far as that is possible, the combinations and movements of atoms and molecules in dead and living matter, particularly where growth is concerned; growth and force frequently acting on essentially the same lines, as witness the formation of dendrites, frost-pictures, lightning-imprints, the branching of plants, and the division and distribution of blood-vessels, \&c., in animals.

It aims at showing that growth occurs in specific or predetermined directions; that atoms and molecules under the influence of life coalesce to form cells, and that from cells all, or nearly all, the tissues of plants and animals are produced.

It attempts to demonstrate that atoms and molecules obey certain laws, and that they are under control, and arrange and group themselves in straight lines, radiating and otherwise; in curves and circles concentric and otherwise; in spirals single, double, and multiple, and in the several forms common in crystals, plants, and animals.

It strives to refer the formative processes of certain crystals and certain plants and animals to the same or similar laws, to show how inorganic and organic products are built up, and how plants and animals tend to split and divide longitudinally and transversely to produce branching and segmentation. The branching is well seen in the majority of plants, and in the blood-vessels and other parts of animals. The segmentation is witnessed in the horse-tails among plants and in the vertebral column of animals.

It endeavours to explain that in plants and animals there is gradation and advance from lower to higher forms, according to a gradually ascending scale, as apart from evolution or the production of the one from the other by unlimited modifications in unlimited time. It makes for advance, by the improvement of individuals and by the creation of higher types with varieties; a state of matters which gives fixity with a certain amount of fluctuation; the fluctuation being confined within prescribed limits in such a way as never to produce confusion. It is only in this way that the absence of connecting links in the geologic and other records can be explained.

It relegates rudiments and vestiges and embryonic changes in the higher forms to a general plan, thus asserting and manifesting itself at every stage of the life histories of plants and animals. It is felt that the resemblances of the embryos of higher forms to the adults of lower allied forms afford no proof that the higher forms are manufactured from the lower ones in the course of untold ages. If this theory were true, and if, as believed by many, the production of the lower forms was confined to a particular period, a time would inevitably come when no lower forms would be left; the fact being that countless millions of such forms exist and always have existed.

It advocates the doctrine that like produces like in endless sequence, and that each begets only its own kind. It asserts that plants and animals differ, and fundamentally differ, from each other from their first inception, and that there is no such thing as a universal sarcode or protoplasm common to all. The physical conditions of reproduction are to a large extent the same as regards climate, atmosphere, moisture, heat, \&c., and nothing short of fundamental differences in the ultimate embryonic elements themselves can account for the amazing multitude and variety of plants and animals found on the earth.

It proclaims the belief that a nervous system (in the ordinary sense) is not necessary to what are essentially voluntary movements, and that myriads of lower forms act in definite directions and to given ends as apart from it. It further attributes to the lower animals with a nervous system a certain degree of consciousness and the power of reasoning; the reasoning faculty being graduated and culminating in man.

It endeavours to illustrate the several points alluded to above by constant references to structures and movements occurring in the two great kingdoms of nature, namely, the inorganic kingdom, represented by "brut" matter, and the organic kingdom, consisting of plants and animals in endless variety.

It proceeds on the conviction that in the universe there is a store of matter and of force which, humanly speaking, can neither be increased nor diminished; that all the matter which enters into the composition of plants and animals is taken from and ultimately restored to the inorganic kingdom; that a certain proportion even of the 
force which actuates plants and animals is traceable to the same source; and that the inorganic and organic kingdoms are co-extensive, complemental, conditioned, and correlated; the one being made for the other.

It attributes the production and correlation of matter, force, and mind to a Creator or First Cause. It advocates a primal power or force from which, directly or indirectly, everything proceeds. It is not possible to separate force from matter, and mind and intelligence from matter, as we know them. Intelligence in varying degrees is the product of certain forms of living matter. It is the mainspring of action in the universe. To it are to be referred all the creative acts, all the known elements, and all forms of force whether physical, vital, or mental. To it, design, law, order and the constitutions and movements of things in general are traceable. The spherical shapes and well-defined movements of the heavenly bodies, and the symmetrical forms and regulated actions of plants and animals, support this view. The intelligence of animals and of man is to be regarded as a special endowment-an emanation from the divine Intelligence, with this great difference, that in the creature it is finite, but in the Creator infinite. The Creator works in and through matter and mind, and has implanted reason, and consciousness of a kind, in varying degrees in the lower and higher organic forms. Originally, matter, force, mind and consciousness have a common source. This accounts for the interdependence of all created things, and for the harmony which characterises the inorganic and organic kingdoms. It also accounts for the uniformity of vital and mental manifestations. No purely physical or mechanical explanation of the universe can suffice. Living things are not automata, and life is a factor which cannot be overlooked. Neither can the abiding presence of the Deity be ignored when discussing the properties and powers of matter, and the peculiarities of mind and consciousness, whether in their rudimentary or advanced forms.

Deviations from recognised laws are possible with the Creator. The Maker of all things can re-arrange without destroying His work. Aberrations apparent or real (and such do occasionally occur) are not, in a sense, miraculous.

In the present work I endeavour to show that the combinations of matter, force, mind, and consciousness are practically endless. I seek to prove that inorganic and organic matter, and physical, vital, and mental force, are not opposed to each other; that a ('reator, Prime Mover, or First Cause is necessary to produce dead and living matter, and physical, vital, and mental force, and that the same laws, to a large extent, dominate all.

I find everywhere in nature a well-ordered scheme, where everything, living and dead, fits into some other thing specially prepared to receive it.

I observe law and order and specific arrangements and design throughout the entire cosmos.

Nothing, so far as I can make out, is left to chance. There is no room for accident in the great scheme of the universe. The inanimate lingdom gives of its best to the animate, and in due time the animate repays its debt to the inanimate. The inanimate gives its substance and a considerable proportion of its force to the animate. The coming and going and give-and-take movements (essentially rhythmic in character) which obtain in the physical universe, and which are represented by day and night, the seasons, the rise and fall of the tides, \&c., are reproduced in various ways in plants and animals; in respiratory movements and interchanges; in circulatory movements; in the ingress of fond and the egress of effete matter; in endosmotic and exosmotic currents which add to and take from living structures; in secretion and excretion, in pulsating spaces, contractile vesicles, hearts, and so on. The great rhythms of the physical universe are transferred in a multitude of ways to the vegetable and animal kingdoms; a circumstance of the deepest import, as showing that the inorganic kingdom is, in a sense, the parent of the organic kingdom, and that the two kingdoms are in complete accord, and complemental, even in matters of detail. All the movements in the inorganic and organic kingdoms are ordered, correlated, complemental movements. They bespeak a Creator, a Designer, an Intelligent First Cause.

While we cannot penetrate the veil which conceals the beginnings of things, we are permitted and encouraged to reason about things as they are. If we find matter and force and the manifestations of mind everywhere present in the organic and inorganic kingdoms; if the matter and force and mind are regulated by unalterable laws; if traces of design and the most wonderful adaptations of means to ends present themselves on all hands ; we are forced to postulate not only a Creator and Prime Mover but also an Upholder. An intelligent agent of the highest conceivable kind becomes a necessity. It is not possible for a well-balanced mind to imagine that the universe, teeming with life and movement, correlated and interacting in the most extraordinary manner, is the result of accident. We cannot believe that the heavenly bodies marked out their own orbits, that the sea set its own boundaries, and that plants and animals with their wealth of structure and function are the offspring of accident. There is nothing in nature to countenance the doctrine of chance, of natural selection, and of spontaneous generation, which ignores a Creator and proposes to dispense with a First Cause and Design. "Life, wherever it occurs, is transmitted. It is an emanation from the primordial source, in the same sense that the matter in which it is manifested is an emanation. The connection between the matter and force of the universe, and between plants and animals, is of the most intinate character. Iife works in and through matter, which it is continually incorporating and discharging. The matter, and the force inhering in it, are, as a rule, readily available for the purposes of life; the atoms and mole- 
cules being so arranged that they admit of being easily separated and appropriated. Life selects and rejects at discretion both matter and force, and living things are superior to their surroundings. They are not the slaves of environment, and all the changes and modifications which occur in them, at every stage of their histories, primarily originate in themselves according to fixed laws. Environment, while it is indirectly associated with modifications up to a point, cannot be credited with the modifications, any more than it can be credited with the production of structures and organs. As a matter of fact, plants and animals are very little amenable to environment; they are not moved by dead substances acting simply as stimuli, and are not irritable in the modern sense. They, as a rule, feel, but feeling and irritability are essentially different things, the one being natural, the other unnatural, that is, abnormal. Plants and animals are entities, and represent creations in time and space. They have their incomings and outgoings, their rise and fall ; their habitat is provided, their food assured, and quite an extraordinary degree of permanence guaranteed to leading types. Nothing is left to the fates; not only are the essential factors of plants and animals provided for in the great scheme of nature, but trivial details are arranged and boundaries thrown up which restrict modifications and variations to comparatively narrow dimensions. In no case is endless modification permitted. Boundaries and limits are set to the changes and movements which occur in the inanimate and animate kingdoms, and everything that is is amenable to law and order, and virtually to the same law and order. There can be but one Creator, Regulator, and Upholder.

I am wholly opposed to the theory of irritability, and its ally, extraneous stimulation, as applied to plants and animals. All my researches go to prove that plants and animals are masters within their own domain, and that they select, subjugate, and utilise matter in every form, whether that be solid, liquid, or gaseous. It is more reasonable to believe that living things inaugurate and regulate their own movements than that their movements are inaugurated and regulated by dead matter outside of themselves. While living things must be credited with sensitiveness, sensitiveness must not be confounded with irritability. Neither is responsiveness to external stimuli any proof of irritability of constitution in plants and animals. Finally, it does not follow that because plants and animals respond, within limits, to external stimulation, the external stimulation or outside influence is identical with the internal impulse which, under normal conditions, initiates and determines all the movements and functions of plants and animals. It is an error to suppose that plants and animals must, of necessity, be possessed of irritable constitutions, and be jogged into activity by externalities. Such views ignore the powers and potentialities of life, and regard plants and animals as mere automata, which they certainly are not.

Plants and animals never lose their identity, or abrogate their powers. Climate and other external conditions only affect them up to a certain point. That there inheres in plants and animals a power of endurance, a power of resistance, and a power of initiation and adaptation is proved in various ways. Plants and animals of various orders protect themselves by developing structures calculated to ward off inimical influences. Thus plants which in temperate climates, where evaporation is moderate, have smooth stems and thin leaves, develop rough stems and thick fleshy leaves in tropical climates where evaporation is excessive and moisture has to be conserved and water stored. They also, in many cases, alter their shape and position and diminish or altogether dispense with leaves; developing scales, prickles, hairs, \&c., and exuding gums, waxes, and protecting varnishes. They likewise, in not a few instances, develop protecting epidermic cells and ligneous and other tissues.

In the case of invasion by insects and grubs, plants throw up defensive works, as happens in the formation of briar and other galls. They protect themselves from poison wounds by exuding callous substances to prevent absorption of the materies morbi. Similar remarks may be made of animals. The skin of the European is white, thin, and dry; that of the negro dark, thick, and oleaginous, and adapted to high temperatures. Animals in the Arctic region have their skins protected by an abundance of fur. In tropical climates, animals have fine coats of hair or are hairless and thick-skinned, as witness the elephant, rhinoceros, and hippopotamus. The fact that plants and animals adapt themselves to their surroundings for their own protection and comfort lends no countenance to the doctrine that they are a prey to circumstances, and wholly at the mercy of external conditions and environment.

The time has now come when, it appears to me, the subject of environment, irritability, and external stimulation must be reconsidered. Environment undoubtedly exercises a certain influence on the structural peculiarities and movements of plants and animals, but the influence is of an indirect and limited character, and all changes of structure, and all movements resulting therefrom, begin and terminate in the plants and animals themselves. In other words, environment does not act as a cause in the strict and proper acceptation of that term, and it does not, however great the time allowed, alter plants and animals beyond recognition. Mr. Charles Darwin in part realised this fact. In his "Origin of Species by Means of Natural Selection" he says (p. 11): "We clearly see that the nature of the conditions is subordinate in importance in comparison with the nature of the organism in determining each particular form of variation." He, however, modifies and virtually alters his opinion in other passages, for he adds (p. 46) : "Changed conditions of life are of the highest importance in causing variability, both by acting directly on the organisation, and indirectly by affecting the reproductive system. . . . Variations of all kinds

VOL. I. 
and degrees are directly or indirectly caused by the conditions of life to which each being, and more especially its ancestors, have been exposed."

Dr. St. George Mivart states the case as follows: "It seems, then, to be undeniable that the characters and the variation of species are due to the combined action of internal and external agencies acting in a direct, positive and constructive manner." I It will be seen that Dr. Mivart, while attributing the characters and the variation of species to the operation of internal and external agencies, properly assigns the internal agency the first place.

It has been customary of late years, especially on the Continent, to ignore a First Cause and the Design which a First Cause implies, and to attribute the universe and everything it contains to a chance assemblage of material particles ; to matter fashioning itself ; to matter assuming movements and life, and, in the fulness of time, evolving from a monad to a man.

Thus Professor Ernst Haeckel says: "The homogeneous, viscid, plasma substance, which singly and alone formed the bodies of the first organisms, and even at this day quite alone forms them in the case of the monera, or simplest amoebic forms, is analogous to the tenacious and viscid planetary substance which contains the elements and substance of the young earth, as well as the other glowing world bodies. In both cases the form of the creation happened, not through the capricious interference of a personal Creator, but through the original power of matter fashioning itself. Attraction and repulsion, centripetal force and centrifugal force, condensation and rarefaction of the material particles, are the only creative powers, which at this point lay the foundations of the complicated structure of creation." ?

This is the position taken up by extreme evolutionists. Nothing, however, is gained by accepting such an exacting hypothesis, which seeks to set aside a Creator, Design, and Law and Order in the old sense, and to substitute what is practically a stone for bread. Ex nihilo nithil fit.

Matter cannot possibly create itself, and cause and effect obtain in the Universe as we know it. It is more rational to believe in a First Cause and Design than to leave everything to a fortuitous concatenation of circumstances. In the one case there is law and order and the adaptation of means to ends from the beginning; in the other there is uncertainty, confusion, and marked disorder. The gulf between life and death is wide and deep, but evolutionists, with an assurance worthy of a better cause, perseveringly ask us to take a stupendous leap in the dark without, in a sense, looking before or behind. They say, in so many words, that inanimate or dead matter can create itself, can usurp life, can develop intellect, and can control and shape the destinies of men and nations.

Every intelligent being, however, who is capable of thinking and reasoning, has in himself the evidence of an absolutely opposite state of things. He knows that he can control and change the shape of inanimate matter. He further knows that he can control and alter the direction of physical force.

Notwithstanding all this, evolutionists calmly and confidently invite us to believe that matter, dead matter, is eternal and omnipotent, and that everything that exists is produced from it, in the lapse of time, by infinite permutations. They assert that, given sufficient time and sufficient modifications, "brut " matter assumes and exercises the prerogative of life and produces rudimentary plants and animals which trend upwards, and ultimately culminate in man. Evolutionists assuredly make large demands upon our credulity, if not upon our reasoning faculties, and in doing so they intentionally or unintentionally take for granted what requires to be proved: theirs is a case of petitio principii pure and simple. Educated, thoughtful men may be pardoned if they gravely shake their heads and refuse to accept a theory which virtually asks them to suppress their reasoning powers, and to keep their judgments indefinitely in abeyance.

There are serious objections to the evolutionist view in its extreme form. Thus there are breaches of continuity and gaps in the geological record which apparently cannot be bridged over. There are, moreover, existing plants and animals on which little or no change has occurred for untold ages. It happens also, that when a race of plants and animals becomes extinct, they are, in not a few instances, replaced by forms not occupying a higher position in the scale of being. The continuous upward trend claimed for plants and animals by evolutionists is not uniform or universal. Certain plants and animals in geological time culminate or attain perfection and then deteriorate or altogether disappear. Parasites, in many cases, afford examples of retrogression.

Egyptian and Chaldean tombs, monuments, temples, and writings conclusively show that man has not changed perceptibly for at least 6000 years. The same is to be said of many plants. Mr. William Carruthers, a high authority in botany, has shown (British Association Proceedings, 1886) that the earliest vegetable specimens described by Dr. Schweinfurth from the Egyptian tombs present no appearance of change. This fact appears also in the leaves and other organs of plants preserved in the nodules of the Pleistocene clays of the Ottawa, and in specimens of similar age found in various places in Britain and the Continent of Europe. One of his illustrations

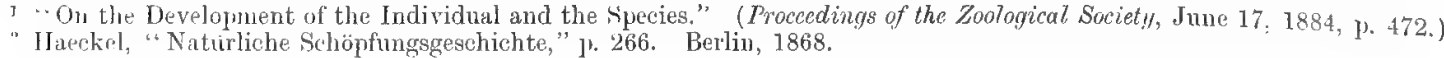


is the ancient willow, Salix polaris, which now lives in the Arctic regions, and is found fossil in the Pleistocene beds at Cromer and Bovey Tracey.

Professor Huxley, in his lecture "On the Hypothesis of Evolution," in discussing the permanency of type, remarks: "The progress of research has supplied far more striking examples of the long duration of specific forms of life than those which are furnished by the mummified ibises and crocodiles of Egypt. A remarkáble case was found in the neighbourhood of the Falls of Niagara. In the immediate vicinity of the whirlpool, and again upon Goat Island, in the superficial deposits which cover the surface of the rocky subsoil in those regions, there are found remains of animals in perfect preservation, and among them shells belonging to exactly the same species as those which at present inhabit the still waters of Lake Erie . . We are fairly justified in concluding that no less a period than 30,000 years har passed since the shell-fish, whose remains are left in the beds to which I have referred, were living creatures.

"But there is still stronger evidence of the long duration of certain types. I have already stated that, as we work our way through the great series of the Tertiary formations, we find many species of animals identical with those which live at the present day-diminishing in numbers, it is true, but still existing, in a certain proportion, in the oldest of the Tertiary rocks. Furthermore, when we examine the rocks of the Cretaceous epoch, we find the remains of some animals which the closest scrutiny cannot show to be, in any important respect, different from those which live at the present time. That is the case with one of the Cretaceous lamp-shells (Terelratula) which has continued to exist unchanged, or with insignificant variations, down to the present day. . . Hence it must be admitted that certain existing species of animals show no distinct sign of modification or transformation in the course of a lapse of time as great as that which carries us back to the Cretaceous period; and which, whatever its absolute measure, is certainly vastly greater than thirty thousand years." 1

Sir J. William Dawson in his "Geological History of Plants" (p. 110) conclusively shows that plants do not always advance as time rolls on, but that they occasionally deteriorate, and even disappear. Thus he states that "the old Cambrian and Silurian seas were tenanted with seaweeds not very dissimilar from those of the present time.

"Ascending from the Erian to the ('arboniferous System ... we are still within the limit of the Palæozoic period and the reign of the gigantic club-mosses, cordaites, and taxine pines. At the close of the Erian there had been over the whole northern hemisphere great changes of level, accompanied by active volcanic phenomena, and under these influences the land flora seems to have much diminished. At length all the old Erian species had become extinct, and their place was supplied by a meagre group of lycopods, ferns, and pines of different species from those of the preceding Erian. This is the flora of the Lower Carboniferous Series. . . But the land again subsided, and the period of the marine limestone of the Lower Carboniferous was introduced. In this the older flora disappeared, and when the land emerged we find it covered with the rich flora of the coal formation proper, in which the great tribes of the lycopods and cordaites attained their maxima, and the ferns were continued as before, though under new generic and specific forms. There is something very striking in the succession of a new plant world without any material advance."

The curious and interesting aquatic plants known as Rhizocarps seem to have reached a climax in the Erian age, since which time they have occupied an inferior position.

The Lycopods "have long ago descended from their pristine eminence to a very humble place in nature." 2 The ferns too have deteriorated, although " in the southern hemisphere at least they retain their arboreal dimensions and ancient dominance." Similar remarks may be made of the Equisetaceæ, which in the older time formed considerable sized trees. In the Erian forests the family of Cordaites flourished, but has long since perished.

A question of considerable importance arises here in connection with the modern theory of the "Origin of Species by Means of Natural Selection." This theory proceeds on the assumption that species are formed by modifications and variations with an upward trend extending over long periods; the modifications and variations conveniently ceasing when a new species is formed-the species which was formed by fluctuation becoming somewhat suddenly fixed and permanent. It may reasonably be urged that it is not possible or allowable to fix limits arbitrarily to modifications and variations in plants and animals at any one stage, if these changes are taken for granted and recognised at all previous stages : logically the changes with an upward trend must go on. In other words, if changes are necessary to form a species, it is illogical to dispense with them when a certain point is reached, the causes which produce the changes still existing. This circumstance of itself goes far to prove the necessity for types, or points of departure for plants and animals; types being central creations to which plants and animals recur or breed back when they have strayed by variation under peculiar circumstances, accidental or otherwise.

There would seem to be, not only types or central forms for plants and animals in the scheme of creation, but also an arrangement whereby types, like individuals, grow, increase, and perfect themselves up to a certain point, after which they cease to grow, diminish, deteriorate, and disappear. The great size and importance attained by

\footnotetext{
1 "Lectures and Essays by Thomas Hemry Huxley" (Macmillan's series). London, 1904 ; 1)ages 22 and $23 . \quad 2$ Op. cit., 1. 78.
} 
the Equisetaceæ in plants, and the Nautilidæ in animals in geologic as compared with modern time, support this view. ${ }^{1}$ The view here advocated would account for the disappearance of races of plants and animals in the prehistoric period, and for otherwise unaccountable gaps in the geologic record. Plants and animals, as already explained, are conditioned. Atmospheric changes, varying climatic states, the rise and subsidence of land, volcanic upheavals, the unequal distribution of mountains, plains, islands, seas, lakes, rivers, \&c., would largely account for the appearance of new plants and animals on the globe, for their continuance thereon in some cases for practically indefinite periods, and for their deterioration and complete disappearance in others. Their appearance, continuation, and disappearance, there can be little doubt, are regulated by design, law, and order, and it seems certain that plants and animals appear, live, and disappear, and keep pace with the changes incident to the physical universe, particularly the earth. Plants and animals, as has been pointed out, are complemental parts of the great scheme of nature; the organic and inorganic kingdoms being conditioned, correlated, and made for each other. Plants and animals are made from and for, but not by, the physical universe.

While the heavenly bodies and movements proclaim Creative Energy, Law, and Order on a grand scale, the prevalence of plants and animals in such profusion and variety on the earth, each of which is cared for even in matters of detail, attests the existence of an Omnipresent, All-ruling Providence. The interdependence of the inorganic and organic kingdoms, and the prevalence of essentially the same law and order in both, further declare that the Great First Cause, Prime Mover, and Upholder is one and indivisible.

The magnitude of the creative acts and the enormous antiquity of the universe conclusively point to the eternal nature of the Being by Whom, and through Whom, everything exists. His presence in matter, force, life, and intellectual manifestations of all kinds can scarcely be gainsaid if the reasoning faculties be allowed free play and the dictates of conscience followed.

As I cannot conceive of the earth with its gradual but stupendous changes of atmosphere, climate, distribution of continents, mountain ranges, islands, seas, lakes, rivers, volcanic upheavals, \&c., as apart from Design, Law, and Order, so I cannot imagine the great races of plants and animals in geologic and modern time as other than fixed, in the sense that their forms and functions, their duration and their distribution, have limits which may not be exceeded. The symmetric nature of plants and animals, their life-histories, their birth and death, their tendency to breed back, their unwillingness to form hybrids, the barrenness of hybrids in the majority of cases, \&c., all point to a well-ordered plan which, amid much apparent fluctuation and mutability, nevertheless remains immutable.

The remarkable resemblances between plants and animals in many cases, to say nothing of the likeness of both to crystals, dendrites, spiral, and other inorganic forms and combinations, conduct to similar conclusions.

It is an astonishing fact, and one deserving of very special attention, that the crystallic and dendritic arrangements seen in frost-pictures on window-panes and pavements in winter, in certain minerals and metals, in the lightning flash obtained by instantaneous photography, or as it impresses itself on the human skin, are reproduced in infinite variety in plants and animals. Nor less astounding is the circumstance that the spiral arrangements which everywhere obtain in plant and animal structures and movement have their prototypes and counterparts in the physical universe; in the spiral arrangements of nebulæ; in spiral waterspouts and sand-storms; in whirlpools of air and water, \&c. These cannot be chance coincidences. The same laws evidently apply to the organic and inorganic kingdoms. There is, it appears to me, no possibility of getting away from a First Cause; Design, Law, and Order when the facts are squarely looked at.

The modern arguments in favour of evolution, instead of destroying the arguments for a First Cause and Design as factors in creation, do not, it appears to me, raise serious objections, and scarcely touch the hem of the subject.

If evolutionists have nothing to offer. but crude conjecture and wild speculation, they are not entitled to sap and destroy the foundations and superstructures of a former, and on the whole satisfying, belief. Better to leave well alone than to attempt to ground our faith upon what is virtually an ignis fatuus. A doctrine is not necessarily scientific and profound because it is destructive and unintelligible.

I may not succeed in marshalling and stating the evidence in support of a First Cause and Design with all the skill or force desirable, but holding the views expressed above, I feel it incumbent upon me to make at least the attempt.

While the spiral and other arrangements discussed in the present work exist in the inorganic and organic kingdoms alike, I hope to be able to show that in the latter, as seen in plants and animals, they are due to design and to the operation of life and of growth, and very little, if at all, to external stimulation. Plants, in many cases, twine and revolve, and bones, muscles, horns, and shells twist and coil spontaneously because of inherent bias, and as apart from irritability and artificial stimuli of all kinds. Similar remarks are to be made of the spiral arrange-

1 "In the Palrenoic age the club-mosses, lerns, and horse-tails engrossed the world and grew to sizes and attained degrees of conplexity of structure not known in modern times." The cuttle-fishes in the olden time were greatly in excess of those existing in the present day. They
were not mily more numerous aud represented more species, hut they were also nore lighly developed and complieated as regards structure. 
ments met with in the stems and vessels of plants, leaves, hairs, fruits, \&c.; in the nerves, the umbilical cord, the cochlea of the ear, the ventricles of the mammalian heart, the stomach, bladder, uterus, \&c. All these structures are spiral because of original endowment and bias.

An outstanding argument in favour of Design and Original Purpose is to be found in the give-and-take movements or rhythms common alike to the inorganic and organic kingdoms. The physical universe gives to and takes from plants and animals light, heat, moisture, \&c., during the day and night, and the seasons. The importance of day and night and the seasons to plants and animals cannot be over-estimated. These changes ensure periods of activity and periods of comparative repose; periods for feeding and growing; periods for reproducing, \&c. If there were no alternations of day and night and of the seasons, the whole economy of plants and animals would be changed. They would cease to be healthy, and would probably cease to exist.

Day and night and the seasons are due to well-known cosmic changes; namely, to the rotation of the earth on its axis every twenty-four hours, and to the earth revolving round the sun once in 365 days. The sun and light and the earth, and the earth's atmosphere, were necessarily created before plants and animals. The latter are parts of a designed whole.

The unfailing recurrence of day and night and the seasons were all considered before plants and animals were formed. The inorganic and organic kingdoms were clearly made for each other, and form complemental parts of one great scheme.

"Oldest of all the formations known to geologists, and representing perhaps the earliest rocks produced after our earth had ceased to be a molten mass, are the hard, crystalline, and much contorted rocks, named by the late Sir W. E. Logan Laurentian, and which are largely developed in the northern parts of North America and Europe, and in many other regions. .. . In the lower part of this great system of rocks which, in some places at least, is thirty thousand feet in thickness, we find no traces of the existence of any living thing on the earth. But in the middle portion of the Laurentian, rocks are found which indicate that there were already land and water, and that the waters and possibly the land were already tenanted by living beings. The great beds of limestone which exist in this part of the system furnish one indication of this. . . The climate and atmosphere of the Laurentian may have been well adapted for the sustenance of vegetable life. We can scarcely doubt that the internal heat of the earth still warmed the waters of the sea, and these warm waters must have diffused great quantities of mists and vapours over the land, giving a moist and equable if not a very clear atmosphere. The vast quantities of carbon dioxide afterwards sealed up in limestones and carbonaceous beds must also have still floated in the atmosphere and must have supplied abundance of the carbon, which constitutes the largest ingredient in vegetable tissues. Under these circumstances the whole world must have resembled a damp, warm greenhouse, and plants loving such an atmosphere could have grown luxuriantly. In these circumstances the lower forms of aquatic vegetation and those that love damp, warm air and wet soil would have been at home.

"It may fairly be assumed that in the present world, and in those geological periods with whose organic remains we are more familiar than with those of the Laurentian, there is no other source of unoxidised carbon in rocks than that furnished by organic matter, and that this has obtained its carbon in all cases, in the first instance, from the deoxidation of carbonic acid by living plants. . .

"In the later geological formations the limestones are mostly organic-that is, they consist of accumulated remains of shells, corals, and other hard parts of marine animals."

Sir William Dawson, to whom I am indebted for the foregoing extracts, furnishes the annexed table indicating the order in which plants and animals appeared on the earth. ${ }^{1}$

It will be seen from the table on p. xxx that plants and animals become more complex with the advance of time, there being what may be regarded as types of plants and animals, and an ascending series in both. Thus the Eozoic age furnished Protogens and Algæ in plants, and Protozoa in animals; the Palrozoic age, Acrogens and Gymnosperms in plants, and Invertebrates, Amphibians, and Fishes in animals; the Mesozoic age, Cycads and Pines in plants, and Reptiles in animals; the Kainozoic age, Angiosperms, Palms, \&c., in plants, and Mammals and Man in animals.

It also shows that creation, considered from the geologic standpoint, is a progressive work, that is, a work which consists of stages and has been accomplished at different times. This was it priori to be expected. The earth and its climate had to be prepared for the advent of plants and animals, and the plants and animals were created in succession, and varied according to the condition of the earth and climate at particular periods. This accounts for the prevalence of rank vegetation and huge animals at one period of the earth's history, and for a less luxuriant vegetation and a more refined fauna at another. It also accounts for changes in the flora and fauna of different regions of the earth at different times, due to vicissitudes of climate; the remains of temperate and tropical plants and animals being not unfrequently found in the same district.

1 "The Geological History of Plants" (International sientific Series, London, 1888, 11. 4 and 8). 
If (and I think the point must be conceded) creation is a progressive work, a strong argument is furnished for separate creative acts. If, moreover, separate creative acts follow changes in the earth's crust and climate, the inference is that the creation of plants and animals, and modifications thereof, go on in the present day as in past ages, all which means that the Creator or First Cause is at work now as $\mathrm{He}$ has always been and will continue to be while the world lasts. The Creator, in this sense, is to be regarded not only as the Framer but also as the Upholder, Regulator, and Sustainer of the Universe. This, on the whole, is the most comforting and sensible view to take of creation, as it guarantees to plants and animals a home, food, and constant supervision; and to man security both as regards the here and the hereafter. Creation as a progressive work differs from evolution in the sense that the different types of plants and animals which prevail now and which prevailed in the past are produced separately, and are not manufactured the one from the other in endless sequence by infinite modifications in infinite time.

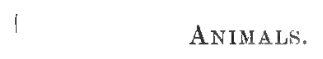

Age of Man and Mammialia

Age of Reptiles Age of Amphibians and Fishes
A ge of Invertebrates
Palreozoic

Mesozoic Pate

Euzoic.
Sistems of Formations.

$\left.\begin{array}{l}\left(\begin{array}{l}\text { Modern } \\ \text { Pleistocene } \\ \text { Pliocene } \\ \text { Miocene } \\ \text { Eocene }\end{array}\right) \\ \left\{\begin{array}{l}\text { Cretaceous } \\ \text { Jurassic } \\ \text { Triassic }\end{array}\right) \\ \left(\begin{array}{l}\text { Pernian } \\ \text { Carboniferous } \\ \text { Erian } \\ \text { Silurian } \\ \text { Ordovician } \\ \text { Cambrian } \\ \text { Huronian (Upper) }\end{array}\right) \\ \text { Huronian (Lower) } \\ \text { Upper Laurentian } \\ \text { Middle Laurentian } \\ \text { Lower Laurentian }\end{array}\right)$

Plants.

$$
\begin{aligned}
& \text { Angiosperms and Palms domi- } \\
& \text { nant. }
\end{aligned}
$$

Cycads and Pines dominant.

Acrogens and Gymnosperms
dominant.

Protogens and Algre.

Dana estimates "that the time-ratios for the first three great ages may be as one for the Kainozoic to three for the Mesozoic and twelve for the Palæozoic, with as much for the Eozoic as for the Palæozoic. . It is further held that the modern period is much shorter than the other periods of the Kainozoic, so that our geological table may have to be measured by millions of years instead of by thousands." Geological time, there can be little doubt, "has been vastly long in comparison to that covered by human history." One of the most astonishing circumstances connected with the Laurentian period is the appearance of protoplasm, chlorophyll, and cells as pointed out by Sir William Dawson. He writes, "In any case we have here presented to us the strange and startling fact that the remarkable arrangement of protoplasmic matter and chlorophyll, which enables the vegetable cell to perform, with the aid of solar light, the miracle of decomposing carbon dioxide and water, and forming with them woody and corky tissues, had already been introduced upon the earth. It has been well said that no amount of study of inorganic nature would ever have enabled any one to anticipate the possibility of the construction of an apparatus having the chemical powers of the living vegetable cell. Yet this most marvellous structure seems to have been introduced in the full plenitude of its powers in the Laurentian age."

As indicating the very close connection which obtains between the organic and inorganic kingdoms it need only be stated that plants and animals require light and darkness, heat and cold, moisture and drought, and a great variety of cosmic conditions of a give-and-take, rhythmic character which physics, and the movements of the heavenly bodies, can alone supply. But (and this is the remarkable circumstance) plants and animals, as indicated, exhibit give-and-take, rhythmic movements of their own. The cosnic rhythms are repeated, and, as it were, perpetuated in plants and animals. Without them plants and animals could not possibly exist. Plants and animals of necessity take in pabulum and give off waste products at intervals. The intake and output movements are essentially interrupted rhythmic movements. Plants and animals feed and evacuate at intervals, they work and rest at intervals, they reproduce themselves at intervals. Everything about them is of the give-and-take order. Even in plants and animals consisting of single cells the give-and-take movements occur. In these rudimentary 
organisms there is an ingoing endosmotic or nutritive current and the outgoing exosmotic or waste product current. These currents vary in activity at intervals. The ingoing and outgoing currents are largely determined by the osmotic nature of the wall bounding the cell, and by the varying densities of the fluids within and without the cell. The ingoing and outgoing currents, essentially osmotic in their nature, occur in individual cells, and are to be regarded as primary, fundamental currents. Given living cells, cell contents, cellular plants and animals, plus moisture, ingoing and outgoing currents become a necessity. These currents are indispensable alike to the lowest and highest plants and animals. Plants and animals can have no health as apart from the more or less constant inflow and outflow of gases, fluids, and semi-solids into and out of their bodies. The ingoing and outgoing currents are required to change at shorter or longer intervals all the particles entering into the formation of plants and animals, and plants and animals are healthy in proportion as the currents are vigorous, well maintained, and duly balanced. If the ingoing currents are in excess there is turgescence, œedema, and swelling; if defective, there is shrinkage and diminution of bulk. From the foregoing, it will be evident that plants and animals, and all parts thereof, are constantly changing, and that there is no such thing as stability, in the literal acceptation of that term, in the organic kingdom. The same is to be said of the inorganic kingdom. The want of stability or the ceaseless ebb and flow of matter without and within living plants and animals make the examination of atoms and molecules imperative in the present work. This follows for four reasons :-

(a) Plants and animals are primarily and fundamentally composed of atoms and molecules, and these, in turn, form cells and cell structures.

(b) Atoms and molecules are continually entering into and leaving plants and animals.

(c) Reproduction and growth and all the changes occurring in plants and animals are dependent on atoms and molecules, and the cells formed by them.

(d) Movements of all kinds, be they great or small, connected with the circulation, respiration, locomotion, \&c., originate in atoms and molecules.

In the present work I propose to deal more especially with life, growth, and movement as indicating design; life determining the amount and direction of growth; growth determining form, particularly radiating, concentric, and spiral form, and life, growth, and form determining movement; notably the centripetal, centrifugal, and spiral movements; the latter occurring in climbing plants, the viscera, the circulation, and in walking, swimming, and flying.

I also deal, incidentally, with the reproductive and nervous systems; a knowledge of both being necessary to a just comprehension of the activities of plants and animals. A nervous system, or its equivalent, there is reason to believe, may be predicated of plants and animals alike. The parts of the work devoted to the viscera, the circulation, and the locomotion of animals were largely written by me in the form of Memoirs communicated to, and published by, various learned societies between the years 1864 and 1873 . Of these and other contributions I append a list :-

"On the Arrangement of the Muscular Fibres in the Ventricles of the Vertehrate Heart, with Physiological Renarks." (Proc. Roy. S'ur. Lond., April 19, 1860, and Phil. Trans., Part II., 1864.)

"On the Relations, Structure, and Functions of the Talves of the Vascular System in Vertebrata." (Trans. Roy. Soc. Edin., vol. xxiii., 1864.)

"On the Ganglia and Nerves of the Heart and their connection with the Cerebro-syinal and Sympathetic Systems in Mamnalia." (Proc. Roy. Soc. Eilin., 1865.)

"On the Muscular Tunics of the Stomach in Man and other Mammalia." (Proc. Roy. Soc. Limil., 1867.)

"On the Muscular Arrangements of the Bladder and Prostate, and the Manner in which the Ureters and Urethr"d are Closed." (Phil. Trans., Part I., 1867.)

"On the various Modes of Flight in Relation to Aeronautics." (Proc. Roy. Instit. of Great Brituin. March 22, 1867.)

"On the Mechanical Appliances by which Flight is attained in the Animal Kingdon." (Trans. Linn. Soc., vol. xxvi., read June 6 and 20,1867 .

"On the Physiology of Wings: being an Allalysis of the Movements by which Flight is Produced in the Insect, Bird, and Bat." (Trans. Roy, Soc. Elin., vol. xxvi., 1871. Rirrived by the Society, August 2, 1870 ; rearl January 16. 1871.)

"On the Relation of Plants and Animals to Inorganic Matter, and on the Interaction of the Vital and Illysical Forces." (Lancet, November $5,1873$.

"Animal Locomotion, or Walking, Swimming, and klying." (Anglo-Anerican Series, London, 1873.)

"The Physiology of the Circulation in Plants, in the Lower Animals, and in Man." (Edin. Mt'l. Journ., 1872: Macmillan \& Co., Lond., 1874.)

"Man in his Anatonical, Physical, and Physiological Aspects." (Laneet, Novemlxr 1875.)

"Flight and Flying Machines." (Ency. Britamirt, 9th ed., vol. ix., Edin., 1879.)

"The Phonograph or Speech Recorder in its Relation to the Human Voice and Ear." (Modern Thought, February 1882.)

"Creation-Man's place in Creation-his Development and Education from a Science Point of View." (Brit. Mucl. Journ., November 1882 ; and Educational Times, December 1882.)

"Civilisation a Result of Intellectual Progress.". (Not yet published.)

"The Brain and Nervous System in their Relation to Mind, or the Correlation of the Physical and Psychical Forces." (Not yet published.)

"On the Formation of Crystals, Dendrites, Spiral and other Structures in Relation to Growtl and Movement, especially Rhythmic Movement." (Edin. Med. Journ., March and April 1901.) 


\section{E R R A T A}

\section{VOI. I}

P. 39, line 2 from bottom, for echinoide reirl erhinus.

P. 52, line 8 from hottom, for ratiatiny dendrites on mineral read stellate actinolite.

P. 76, line 14, for Scopognathus read Scaphognathus.

P. 170, line 21, for substallate read substellate.

\section{VOL. II}

P. 433, for Figs. 97, 98, 99 read 99, 97, 98 .

P. 435, Fig. 102, for duckwood lead ductewerd.

P. 597, line 7 from bottom, for Amlrovanda read Aldrovanda.

P. 612, line 3 from bottom, for Camifolim read Periclymenum.

P. 616, line 11, for jucunde read jucunda.

VOL. III

P. 1152, line 20, for Prrson's read Parsons'.

P. 1263, line 30, for Humphrey read Humphry.

P. 1307, line 11 from bottom, for Archropterix read A reharpterys. 


\section{DESIGN IN NATURE}

\section{INORGANIC AND ORGANIC MATTER DISTRIBUTED ACCORDING TO THE SAME GENERAL LAWS: GLOBULAR, CONCENTRIC, CURVED, SPIRAL, RADIATING, BRANCHED, AND SEGMENTED ARRANGEMENTS OF MATTER. MOVE- MENTS AND RHYTHMS IN THE INORGANIC AND ORGANIC KINGDOMS -A FIRST CAUSE NECESSARY ${ }^{1}$}

\section{$\S \mathrm{I}$. Atoms and Molecules under Guidance.}

The distribution and movements of atoms and molecules in the universe, especially that part of it forming the organic kingdom, is of the utmost importance in biology and physiology. No one, so far as I know, has succeeded in giving a complete explanation of them.

Atoms and molecules, there can be little doubt, move and are moved, and arrange themselves under the operation of a First Cause, as represented by life and physical force-gravitation, attraction, repulzion, changes of temperature, condensation, rarefaction, osmose, \&c.

Newton and Swedenborg held strongly to a First Cause.

Newton, when speaking of the formation of the sun and fixed stars, says: "I do not think (this) explicable by mere natural causes, but am forced to ascribe it to the counsel and contrivance of a voluntary agent."

In like manner, Swedenborg remarks "that nothing can be truly known of the visible world without a know. ledge of the invisible, for the visible is a world only of effects, while the invisible, or spiritual, is a world of causes."

Haeckel and Tyndall reject a First Cause. They attribute everything to a power inhering in matter as matter, in virtue of which it assumes shape and movement, as apart from a Creator and as apart from life.

Kant, Herschel, Laplace and others endeavoured to explain the existence and movements of the heavenly bodies by what is known as the nebular hypothesis. Laplace was of opinion that the matter of the solar system " existed originally in the form of a vast, diffused, revolving nebula, which, gradually cooling and contracting, threw off, in obedience to mechanical and physical laws, successive rings of matter, from which subsequently, by the same laws, were produced the several planets, satellites, and other bodies of the system." 2

Descartes attempted to account for the formation of the universe, and the movements of the bodies composing it, by a theory of vortices.

It will be observed that all the philosophers referred to assume the existence of matter. It is the distribution and movements of matter, not only in the physical universe, but also in the vegetable and animal kingdoms, which is still sub judice.

1 What is written under this heading first appeared in an article, entitled "On the Formation of Crystals, Dendrites, $\mathbf{S}$,iral, and other" Structures, in Relation to Growth and Movement, esprecially Rliythmic Movement," by the author, in the Edinburgh Merlical Journal under dates March and April 1901. The article is now reproduced with its original illustrations, which were too numerous and costly for any journal to mondertake. It has been re-cast, and short comnecting passages ardded to introduce the illnstrations, but with these exceptions it is as when first published: the form but not the sulustance has been slightly altered.

${ }^{2}$ An alternative to Laplace's theory of the formation of planetary systems is suggested in an article by Mr. F. R. Monlton, of Chicago University, in the Astrophysical Joumat, Oetober 10, 1905. In 1900 this writer and Prof. T. O. Chamberlin examined the older hylothesis from the dynamical standpoint, and discovered, as they believed, certain contradictions which induced then to frame a new theory.

The theory now suggested supposes that the planets and their satellites have been formed around prinitive nuclei of considerable dimen sions existing in a spiral nebula probably similar to thrise which Prof. Keeler showed to he many tines more numerous than all the nelsulre of other types.

The growth of each nuclens, according to them, is caused by the gradual accretion of smaller masses, and the method of this growtl, it is suggested, accounts for all the different types of bodies now found in the solar system, and for their present motions and velocities, on dynamical principles.

The original spiral nebula is supposed to have heen formed ly the near apuroach of another star to the body which is now our sun. This exterior attraction, it is assumed, set up tides in the solar matter, and, heing continued, caused immense masses to be ejected und drawn out into the spiral form. On this assumption the spiral would emerge from the central nuclens in two directions on opposite sides, and this is the form generally shown in photographs of such nebule, (Short notice in Notu' of November" 23, 1905, headed "The Evolution of the Solar" System.")

VOL, I, 
Atoms and molecules for the most part display a tendency to assume symmetric forms and to dispose themselves in straight lines or in curves; the curves forming spheres, circles, and spirals, especially the latter. As a consequence, increase, growth, and development in the inorganic and organic kingdoms proceed in one or other of the directions indicated. ${ }^{1}$

\section{§2. Straight-Line, Radiating, Concentric and Spiral Formations with Traces of Segmentation.}

What I designate straight-line formations produce bodies bounded by plane surfaces; the curved formations producing spherical, circular, and spiral structures and modifications thereof. The straight-line formations are represented by crystals of every form and variety, crystallites, and dendrites; the latter branching and assuming

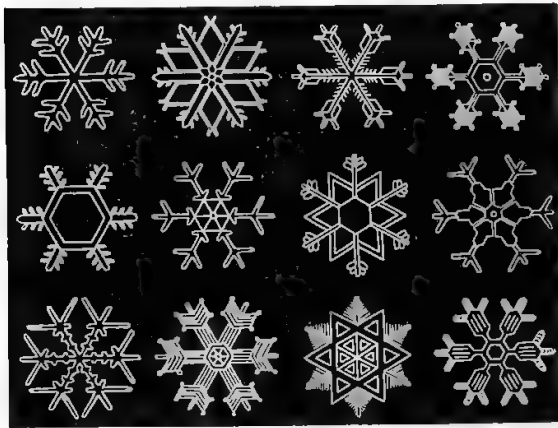

FIr: 1.- Exquisite crystals of snow as figured by Scoresby. Illustrate straight-line formations. a characteristic tree-like shape, with, in some cases, a certain amount of segmentation. Crystals are formed by aggregations of atoms and molecules from without; the additions, for the most part, being made in straight lines, and giving rise to plane surfaces bounded by characteristic angles. Not unfrequently crystals display dendritic, radiating, concentric, and spiral arrangements. ${ }^{2}$

The curved formations are represented by spheres and modifications of spheres, where the atoms and molecules combine to form bodies having concentric and spiral arrangements; the additions being made in successive curved layers.

Beautiful examples of straight-line formations are seen in the crystals of snow, and of the straight-line, radiating, and curved formations in the crystals and conglomerations of hail. In the latter the straight-line, radiating, and concentric arrangements are all present. Perhaps no better

illustration of the extraordinary plasticity and power of nature to assume different shapes and conditions, under slightly altered circumstances, can be given than are afforded by the structure of snow and hail respectively (Figs. 1 and 2).

Examples of crystals are met with in the organic as well as in the inorganic kingdom. Crystals, as a rule, are symmetrical, and characterised by great beauty of outline. They are endless as regards form, and have for the most part an unvarying chemical composition. They occur in the soft snow and in the hardest rocks and metals. They are deposited in the solids of certain plants, and in the fluids of plants and animals, as witness the crystals of sugar, blood, bile, urine, \&c.

Typical examples of crystals displaying straight-line, dendritic, radiating, concentric and spiral arrangements, with traces of segmentation, are given at Plates i. and ii. From a careful examination of the figures in the plates in question it will be seen that crystals assume a very great variety of form; the peculiar shape depending, in many cases, on the condition, for the time being, of the mother liquid as regards temperature, degree of viscosity, and what may be regarded as adventitious circumstances. In other words, crystals, while having a definite chemical composition, and, as a rule, a distinctive form, nevertheless lend themselves to constructive processes, and admit of modification in accordance with certain
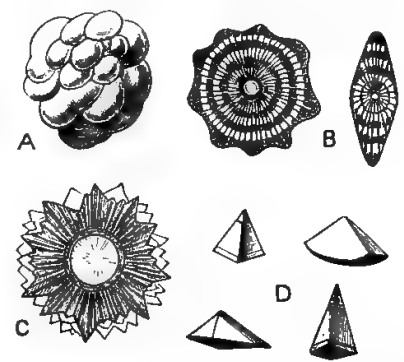

FIr. 2.-Tarious forms of hail as figured by Whitney. A, hailstone which fell at Bonn in 1822, having a diameter of an inch and a half, and weighing 300 grains. B, sections of differently shaped hailstones, showing a radiating nuclens and concentric layers. $\quad C$, section of hailstone with minute crystallic pyramids on its surface displaying a radiating arrange. nient. D, the crystallic pyramids detacheit.

laws. When so modified they bear the most extraordinary resemblances to certain plants and animals and parts thereof (compare Plates i. and ii. with Plates iii., iv., v.), and support the belief that the law of increase and growth applies equally to crystals and to plants and animals; and that one design runs through the in-

1 I desire to point ont that I employ the terms atom and molecule in their generally accepted sense, without prejudice, and with the knowledge that some advanced physicists of late years regard the atom as highly divisible. The divisibility of the atom does not affect my argument as developed in the present work, and I keep an open mind on the subject. It only pushes the division of matter to a further point. It does not jeopardise the existence of natter or the forces which inhere in matter as such : matter and force to the physicist and physiologist are still realities. They are, as hitherto, indestructible and fixed quantities in the universe. The more minute division of natter is one of detail rather than of principle, and is discussed further on (page 180: The visible and invisible worlds; new theory of matter, \&e.).

${ }^{2}$ In making these general statements I am aware that crystallisation is a complicated process, and results from varions and diverse conditions. Herr O. Lehman (Zeitschrift fur Krystallographie und Mineralogie, von P. Groth, vol. i., 1877) traces crystallisation (1) to the evaporation of a solution; (2) to the action of chemical re-agents ; (3) to the solílification of melted masses ; (4) to the condensation of vilpours ; (5) to change of fixed, physical, isometric moditication; and (6) to separation by electrolysis.

Crystals have been divided by Webster into (1) the isometric, which have the axes all equal, as in the cnbe, octahedron, \&c. ; (2) the tetragonal, which have a varying vertical axis, while the lateral are equal, as in the right square prism; (3) the orthorhombic, which have the three axes unequal, as in the rectangular and rhombic prisms; (4) the monoclinic, which have one of the intersections oblique, us in the oblique rhombic

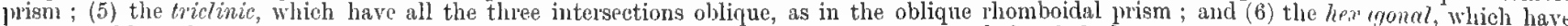
three equal lateral axes, and a vertionl axis of variable length, as in the hexagonal prism and rhombohedron. 
PLATE I
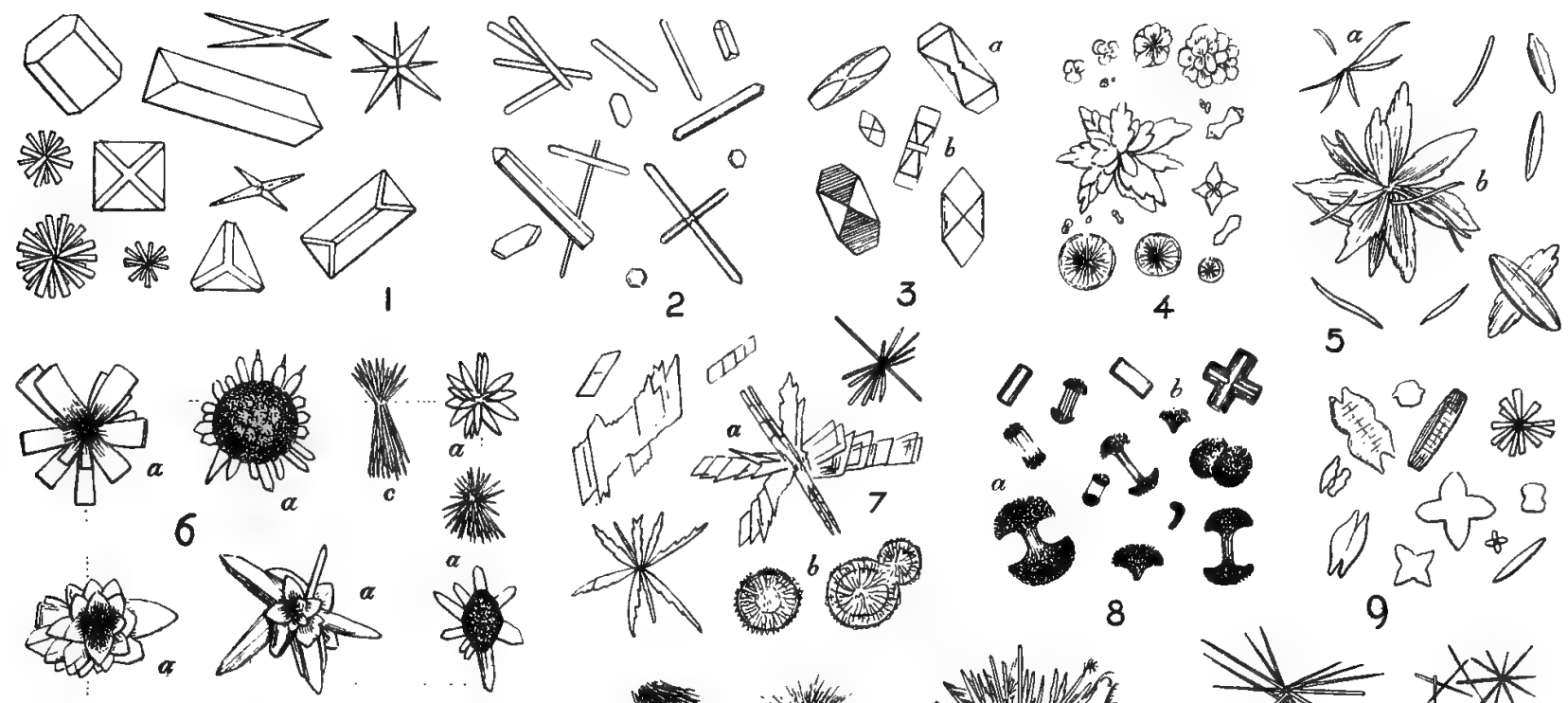

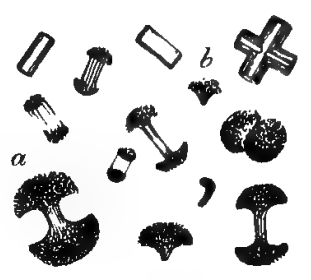

8

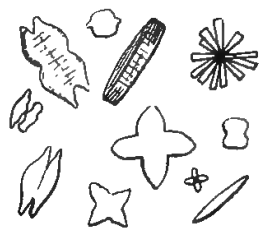

9
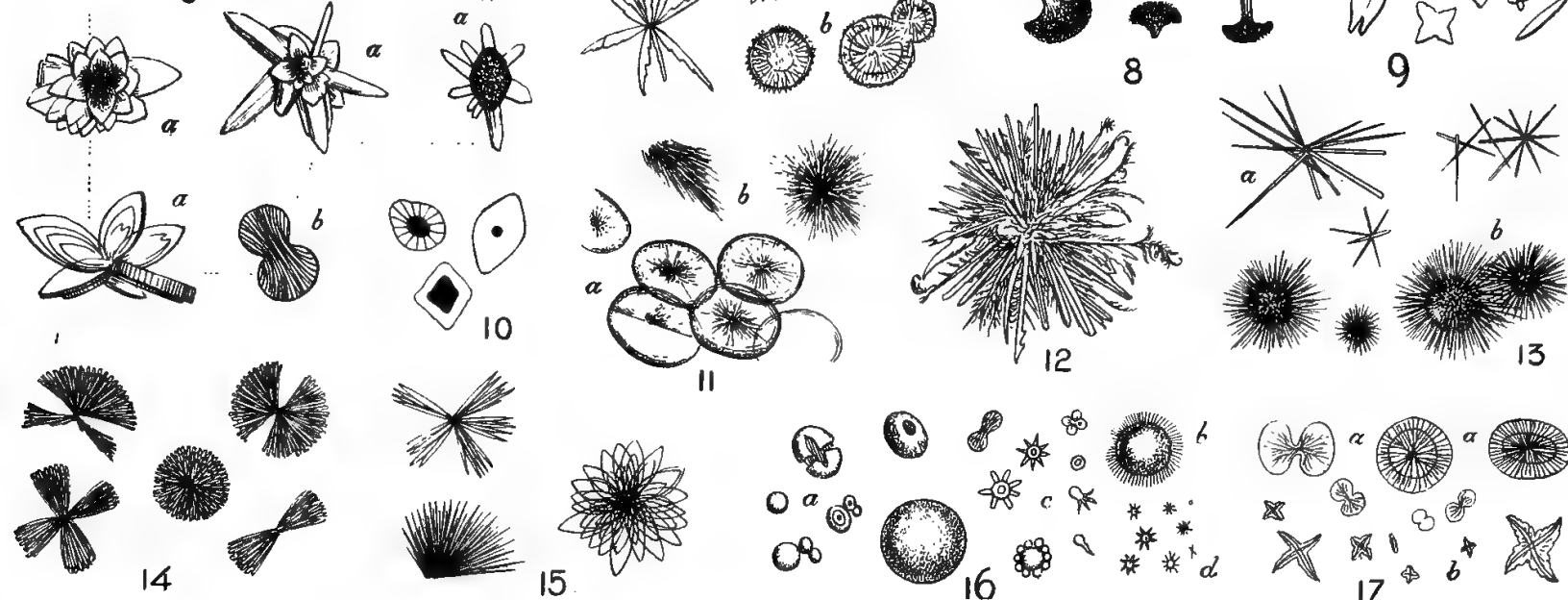

13

15
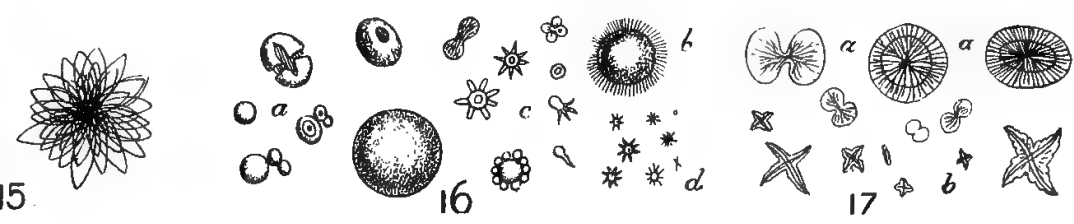

Selection of crystals to illustrate radiating, concentric, and other arrangements frequently met with in plants and animals.

FIG. 1.-Various forms of crystals of cystic oxide. Show star-like, radiating, and prismatic arrangements ; the latter assuming angular, quadrangular, and hexagonal shapes. The stars and rosettes resemlle certain plants and animals; also lines of force (Plates lv. and lvi., 1p. 107 and 109), and embryo cells developing (Plates lxi., lxii., and lxiii., pp. 154, 156, and 158).

Fig. 2.-Crystals of hippuric acid. Elongated prisms or needles (right rhombic prismatic system). Resemble certain low organic forms.

FIG. 3.-Rhombic crystals of uric acid. Human. Natural. ", Front view; b, side view. Show hour-glass markings as in cells aloout to divide.

organic and organic kingdoms; that, in fact, the two kingdoms are complemental, and form parts of each other. This view derives striking confirmation from the fact that, when magnets and electricity are made to act on iron filings and other inanimate substances, they arrange the substances in straight lines, in curves, and in spirals; the straight lines radiating, the curves forming ellipses and circles which are concentric, and the spirals being single or double, and, in some cases, forming figures-of-8, as shown at Plates lv. and lvi., pp. 107 and 109.

The view is further confirmed by an examination of organic cells and the cellular sexual elements after impregnation and during development. Here again, and at the very threshold of life in plants and animals, the radiating, globular, concentric, curved, spiral, branching, and segmented arrangements make their appearance, as shown at Plates lxi., lxii., lxiii., and lxiv., pp. 154, 156, 158, and 160.

These, to many, will appear startling statements, but they are borne out in the fullest manner by the histories of crystals, plants, and animals respectively (vide Plates i., ii., iii., iv., and v. which follow).

As the illustrations in the work are necessarily numerous, the descriptions given of them are as brief as possible, consistent with clearness. The figures, fortunately, for the most part explain themselves. It will suffice, in the majority of cases, if the reader simply glances at the figures, and at the explanatory headings affixed to the descriptions of the plates. 
FIG, 4.-Carbonate of callium from urine of horse. Natural. Shows radiating and rosette arrangements seen in plants, flowers, animals, and parts thereof: also in lines of force (Plates lv. anrl lvi., pp. 107 and 109), and in developing embryo culls (Plates lxi., lxii., and lxiii., l'p. 154, 156, and 158).

FIG. 5.- Crystals of margarine and stearine. Margarine needles $(a)$ and stearine flower-like aggregations of lanceolate plates $(b)$.

FIG. 6.-Uric acid crystals. Human. Natural. a, Various forns of aigrettes, radiating and flower-like; b, duml-bell crystal, resembles cell dividing; i, sheaf-shaped crystal not unlike palm-leaf.

FIG. 7.-Phosphate of calcium erystals; mostly thin, rhombic, radiating plates ( $a$ ); some displaying a radiating and concentric (b) arrangement, as seen in the sections of the stems of plants, and in the bones of animals (b) (Plate v., p. 9).

FIG. 8.-Dumb-bell crystals $(a)$ and rectangular plates $(b)$ of uric acid.

FIG. 9.-Uric acid precipitated from solution in sulphuric acid by water. Shows radiating, star-like, and other crystals, having shapes similar to those seen in rudimentary plants and animals, especially diatoms (Plate iv.).

FIG. 10. - Uric acid rhombs slightly acted $u$ on by potash, showing nuclei.

FIG. 11.-Margarine. ", Fat cells containing star-shaped masses of needles; $b$, star-like clusters of needles. These resemble growing flat bone (parietal lone, for example).

FIG. 12.- Crystals of sugar of milk, displaying radiating and feathery, plant-like arraugements.

Fig. 13. - Crystals of urate of calcium. Show spicular radiating $(a)$ and spicular spherical $(b)$ formations. Resemble palms in plants, and sea-urchins in animals.

FIG. 14.-Crystals of urate of magnesium. Afford examples of radiating bundles of spicules, assuming fan-like, hour-glass, and Maltese cross shapes, common in plants and animals,

FIG. 15. - Stearine crystals. Show suft nacreous lamince or needles presenting radiating, flower-like arrangements.

FIG. 16. - Crystals of urate of sodium and ammonium. Show spheres with nuclei and concentric rings $(a)$; one covered with radiating needles $(b)$, as with cilia. Natural radiating stellate forms, is in flowers and star-fish, are seen at $c$ and $d$. All these crystals represent plant and animal types.

FIt. 17. - Crystals of oxalate of calcium, prepared with acid and showing dumb-bell and concentric and radiating arrangements $(a, a)$. The same salt modified by domble decomposition showing octaliedrons $(b)$. The concentric and radiating arrangements here seen occur in great numbers in plants and animals and parts thereof, especially in developing embryonic cells (Plates lxi., lxii., and lxiii., pp. 154, 156, and 158); in transverse sections of the stems of plants and of animal structures (Plate v., p. 9). The concentric and radiating arrangements are also seen in the lines of force obtained by the action of nuagnets on iron filings (Plates lv. and lvi., pp. 107 and 109). The dumb-bell and stellate crystals resemble cells dividing, and forms common in flowers and animals.

\section{PLATE II}

Selection of crystals to illustrate radiating, branched, segmented, and spiral arrangements, very common in plants and animals. Some of these crystals are beautifully symmetrical; symmetry of form being also a characteristic of plants and animals.

F1G. 1.-Crystals of sulphocyanate of brucine, showing rudiating arrangenent of needles.

FIG. 2. -Margarine, slowing radiating, starlike, and branched dendritic arrangements.

Fro. 3. -Radiating crystals of sulphate of calcium.

FIt. 4.--Radiating spicules of brucine, presentiug a tower-like appearance.

FIG. 5.- Spicules of nurcotine exhibiting radiating, dendritic, flower-like forms. Figs. 1, 2, 3, 4, and 5 exhibit the radiating arrangement to perfection (see Plate lv., p. 107, and l'lates lxii., lxiii., jp. 156 and 158).

Fig. 6.-Bilifulvine. Natural. Human. Shows rhombs $(a)$ and elegantly curved dendritic bundles of needles $(b)$, essentially plant-like in alpearance.

FIG. 7.-Feathery or penniform crystals of magnesium ammoniun phosphate, dendritic in cliaracter.

FIG. 8.-Crystals of ammonium chloride, showing well-marked dendritic, branching arrangements similar to what are seen in plants, in minerals and metals, and on window-panes during frost.

FIG. 9.-Crystals of uric acid of boa, artificially precipitated from solution in potash by hydrochloric acid. Show dendritic formations similar to those seen at Fig. 8.

F1G. 10.-Symmetrical, six-rayed spicule, often seen in sponges.

FIG. 11. - Stellate and leaf-like crystals of mamesium ammonium phosphate. Resemble star-fishes and certain flowers.

FIG. 12. - Crystals obtained from the cooling of a warm solution of ammonia. The more perfect crystals present a remarkaljly regular, segmented apjearance, not unlike a vertebral column.

FIG. 13.- a, Crystals of gypsum, showing well-marked dendritic or loranching arrangements similal to what are witnessed on window-panes during frost, and in plants; $b$, crystals of chloride of barium produced by adding gum to a solution of this substance as a thickening medium. The crystils might well be mistaken for plants.

FIG. 14.--Dendritic, branched crystals of nitrate of barium, obtained by adding gum to a solution of this substance, and rapidly evaporating in thin layers.

Fra. 15.-Crystals of phthalia anhydride, displaying a well-marked tendency to curve and branch as in plants.

FIG. 16.-Feather-shaped structure greatly resembling moss, and consisting of small rhombic crystals obtained from a watery solution of $\mathrm{C}_{6} \mathrm{H}_{2}\left(\mathrm{CH}_{3}\right)_{2} \mathrm{NO}_{2} \mathrm{SO}_{3} \mathrm{~K}+{ }_{2}^{1} \mathrm{H}_{2} \mathrm{O}$ (2 nitro 1-3 xylene 4 sulphonate of potassium).

FIG. 17. - Plant-like structure consisting of individual crystals arranged on stalks and radiating in a non-symmetrical manner. Obtained from an alcoholic solution of $\left(\mathrm{C}_{6} \mathrm{H}_{2}\left(\mathrm{CH}_{3}\right)_{2} \mathrm{NO}_{2} \mathrm{SO}_{3}\right)_{2} \mathrm{Zn}+5 \frac{1}{2} \mathrm{H}_{2} \mathrm{O}$ (2 nitro 1-3 xylene 4 sulphonate of zinc).

FIG. 18.-Group of curling and branching crystals of permanganate of potassium. Magnified 300 diameters. They resenble branches of trees in winter, and branching blood-vessels, bronchial tubes, \&c. Fig. 19.- Symmetrical, rosette-like, branching crystals, obtained by dissolving cam] hor in alcohol or benzol and then evaporating.
Resemble flowers and star-fishes.

FIG. 20. - Rosette crystal of strontianite in which the individual crystals radiate from a common centre. Resembles deepsea coral (Bathyactis symmetrica); growing flat bones (parietal bone, for example); the ends of vertebræ, \&e.

FIG. 21. - $a$, Aggregation of curling, hair-like crystals of dinitroparacresol, showing incipient spirals $\left(\mathrm{C}_{0} \mathrm{H}_{2}\left(\mathrm{NO}_{2}\right)_{2} \mathrm{CH}_{3}(\mathrm{OH})\right.$; $b$, spiral, radiating, leaf-like crystals of styl hnic acid $\left(\mathrm{C}_{6} \mathrm{H}\left(\mathrm{NO}_{2}\right)_{3}(\mathrm{OH})_{2}\right)(2-4-6$ trinitroresorcin). The leaflets are rectangular or oblique bordered, and are always curled and much branched if the viscosity of the solution is diminishen by the addition of alcohol.

FIG. 22. - a, Beautiful symmetrical, segmented crystal of chloride of copper and chlorate of ammonia; $b$, c, graceful starlike crystals of nitrate of sodium and rhombohedral nitrate of potassium; $d$, club-shaped, stellate crystal similar to those obtained from the solidification of saltpetre. 
PLATE II
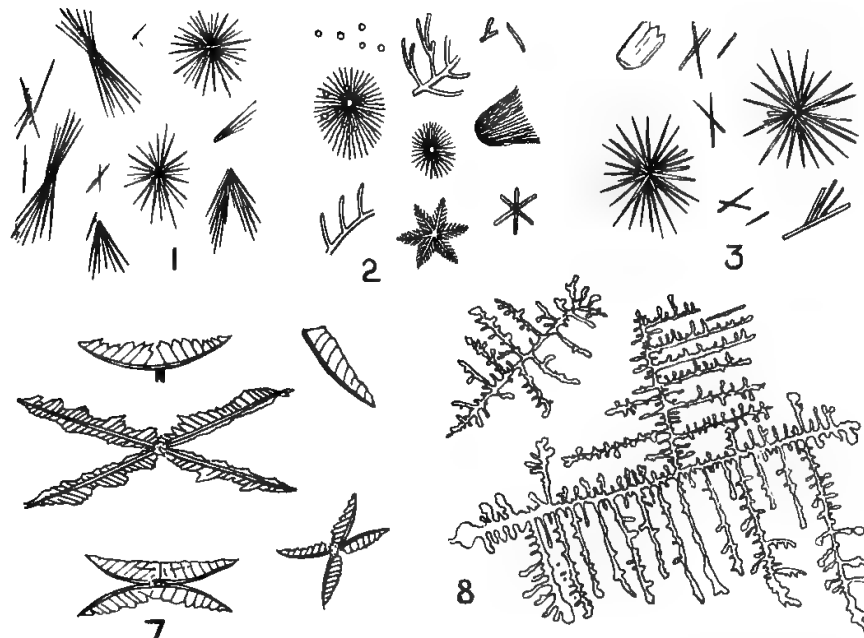

7

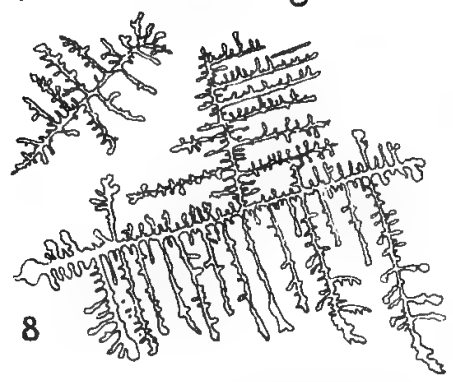

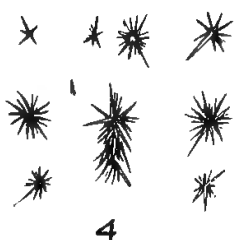
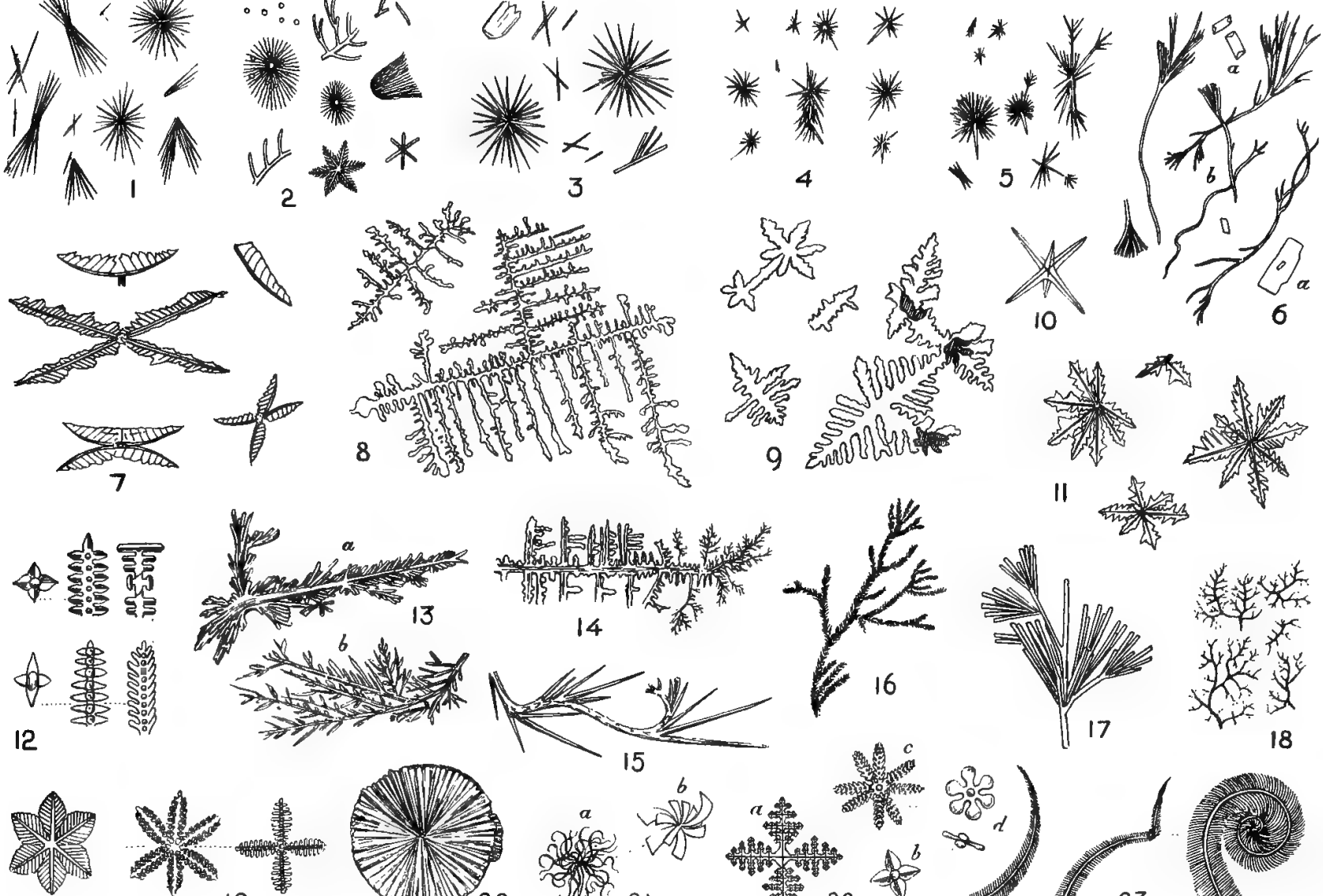

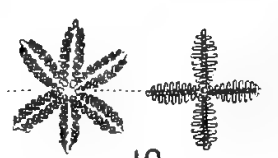

19
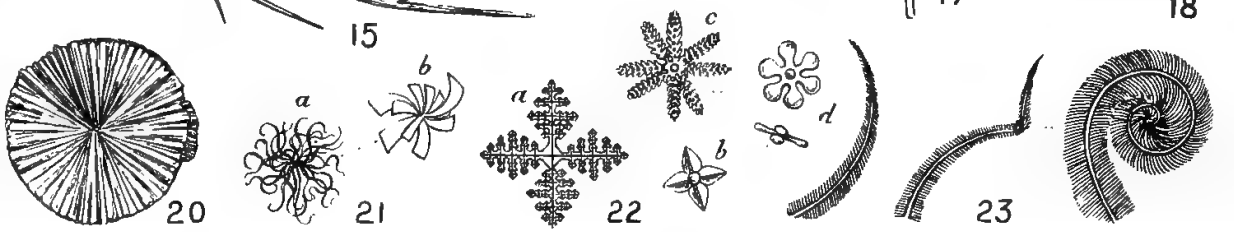

FIG. 23. Remarkable spiral crystals of sulphur obtained by cooling quickly on a microscope slide. These crystals resemble the fronds of ferns and the horns of animals. A still more remarkable example of a spiral crystal is furnished by prochlorite as shown at p. 19, Fig. 7.

\section{§3. The Radiating, Dendritic, Segmented, Concentric and Spiral Arrangements common to Crystals, Plants, and Animals.}

That the radiating, dendritic, concentric, spiral, and segmented arrangements witnessed in crystallic combinations reappear in plants is abundantly shown by an examination of the figures in Plate iii.; and that the same holds true of animals is made equally evident by consulting the figures of Plates iv. and v., especially the latter.

PLATE III

This plate shows a well-marked tendency in living things to aggregation, division, segmentation, and repetition of parts ; to branching, to radiation, and to concentric and spiral distribution of their substance. The plants here figured resemble the crystals shown in Plates $i$. and ii. There is a common plan for crystals, plants, and animals.

FIG. 1.-Developinent of bacteria and vilorios on the surface of an organic infusion (J. H. Bennett). " M, Molecular mass; $b$, short bacteria; ', larger bacteria; $d$, the same more separated; $c$, aggregation of vilorios; $f$, chain-like molecular filaments (Leptothrix).

FIG. 2.-Conferva floccosa. ", Fragment of growing filament; $b$, filament breaking up; $c$, zoospore capable of independent movement. Shows segmentation (compare with $e$ of Fig. 1).

FIG. 3.-Fidogonium vesicatum. ", Fragment of a filament; b, ditto, breaking up and elnitting a zoospore; ", zoospore with a crown of cilia. The roospore is free to move. Shows division, segmentation, and repetitiun of parts, as in the Articulatit and Vertebrata.

FIG. 4.-Stigeoclonium protensum. $\quad a$, Fragment of branched filaments ; $b$, gerninating zoospore. Shows segmentation of filaments and zoospore as in Fig. 3.

FIG. 5.-Brachycladium ponicillatum. An erest filament with fertile branches. Magnified 200 diameters. Shows segmentation and branching in a marked degree.

FIG. 6.-Helicosporium pulvinatum, magnified 200 diameters. a, Branched filament; $b$, sporidia forming lie:ıtiful spirals. $a$ and $b$ afford typical examples of segmentation. 
PLATE III

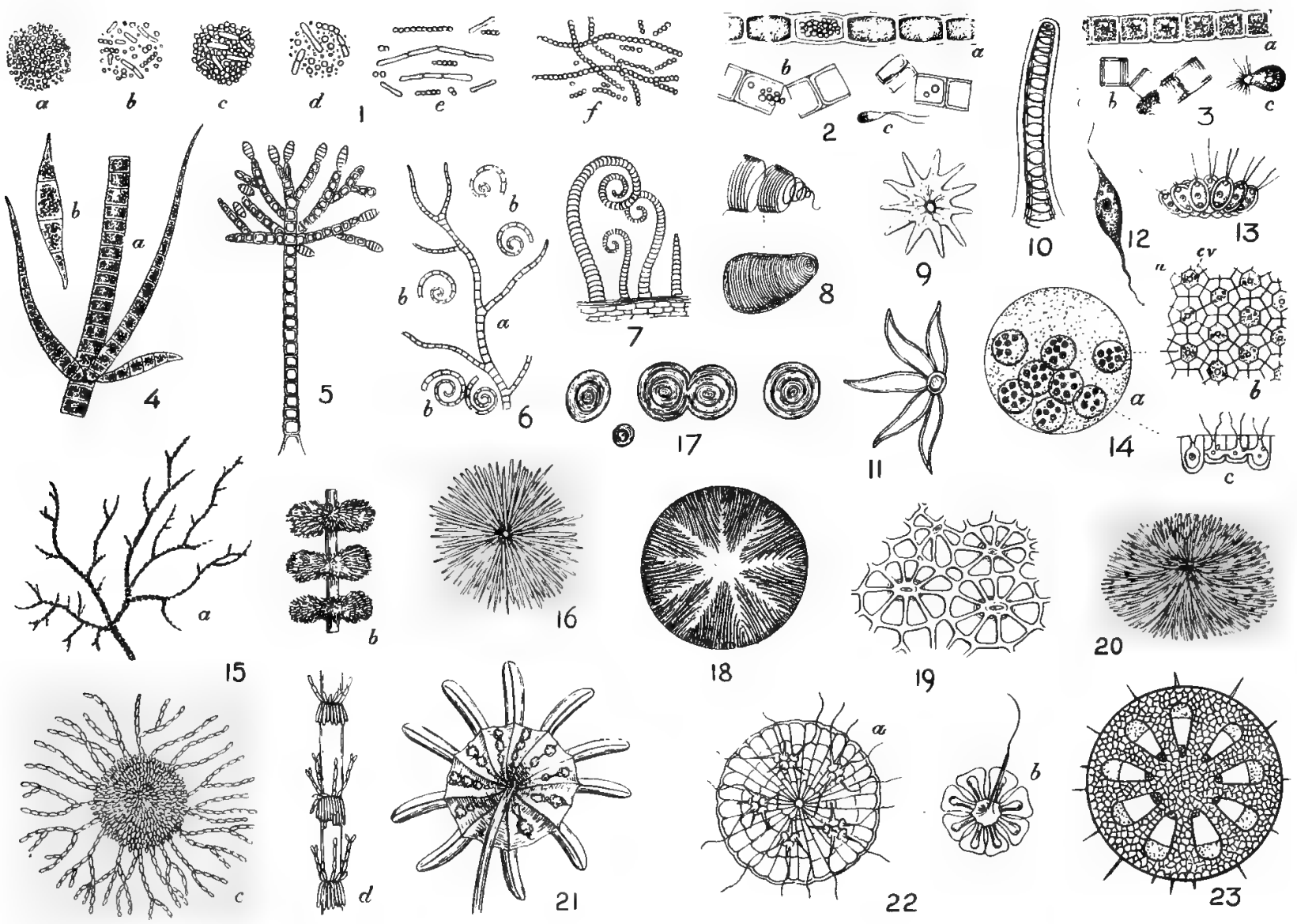

FIG. 7.-Gyrocenus ammonis, magnilied 150 dimeters. Shows expuisite spirals resembling those of the fronds of ferns: also segmentation. Comprare witl Fig. 12, p. 21, Plate xxxvi., Fig. 4, 13. 5\%, and Plate xxi., Fig. 3, 11. 37.

FiG. 8. - Scale-like hairs from the seed of Cobre scandens, magnified 35 diameters, rolled up in graceful conical spirals; the hair being uncoiled in the upper part of the figure. Thuse hairs resemble, in a general way, the sliral tendrils of vines, passion flowers, and climbing plants: also many animal structures.

Fra. 9. - Stellate hairs from the epidermis of Dentzia scubru, magnified 125 diameters. Similar stellate forms occur in crystals, in other plants, and in animals (star-fish, \&c.).

Fig. 10.- Hair from the seed of Salvia, magnified 55 diameters. Displays a leautiful open spiral, similar to that seen in the stem of Vorticella, \&c.

Fig. 11.- Stellate hair of the ivy leaf, magnified 100 diameters. Resembles flower of plant and star-fisl..

Fict. 12. - Cercumonas crassicauda. A one-celled flagellate, magnified 200 dianteters. Resembles spindle-shajned cells in involuntary nuscle.

FIG. 13.-Gonium pectoml, magnified 17.5 diameturs. Composed of cells with double Hagella. The cells exhibit rhythmic movements in the absence of, and as apart from, muscular filures.

Fra. 14. - a, Volvox globator, with daughter and grand-dauglıter colonies, magnified 25 diameters (after Carter); $b$, surface view of volvox, showing cells in hexagonal spaces in common jelly. Compare with epithelial, liver, and pigment cells, and also hexagonal cells of honeycomb. Each cell reveals nuclei $(n)$, two flagella, and a contructile vacuole $(r c)$. $c$, Vertical section of wall of volvox, matrified 400 diameters (after Biitschli).

FIG. 15.-Batrarhospermum momiliformr, natural size. Shows aggregation, branching, and segmentation. ", Segmented axis with branches; $b$, a portion of an axis with whorls of branches magnitied; $c$, a tuft of branches with spores in the midst more magnified; $d$, highly magnified view of a few cells. Shows segmentation and branching.

FIG. 16. - Scale of the epidermis of Hippophuë rhamnoides, magnified 50 diameters. Shows stellate, rarliating arrangement from central point, as in many crystals, plant, and animal structures.

Fig. 17.-Cnpora amylacen from the human ependyma, magnified 350 diameter's. Shows well-narked concentric arrangement, as seen in certain crystals, plants, and animal structures.

FIG. 18. - Anterior view of human crystalline lens (adult), magnified $2 \frac{1}{2}$ diameters. Slıws stellate, radiating arrangement of filures.

FIs. 19. - Funaria hygrometrica, magnified 150 diameters. Stomata of the epidernis of the column. Show concentric and radiating arrangements, resembling the Haversian systems of bone on transverse section.

FIG. 20.-Actinothyrium graminis, hiohly magnified. Shows radiating arrangement. Conuare with Fig. 16.

FIG. 21. Marchantia polymorpha, magnified 5 diameters. A sporangial receptacle seen from below. Shows concentric and radiating arrangement of parts. Compare with Figs. 9, 15c, 18, and 20.

FIG. 22.-Coleochete scutata. a, Perfect plant, magnified 25 diameters; $b$, commencement of the development of a frond, magnified 10 diameters. Show concentric and stellate arrangement of parts.

FIG. 23. - Horizontal section of a yearling shoot of a Dicotyledon, magnified 25 diameters. Shows well-marked concentric and radiating arrangements; similar arrangements are seen in crystals ancl in niany parts of plants and animals. 


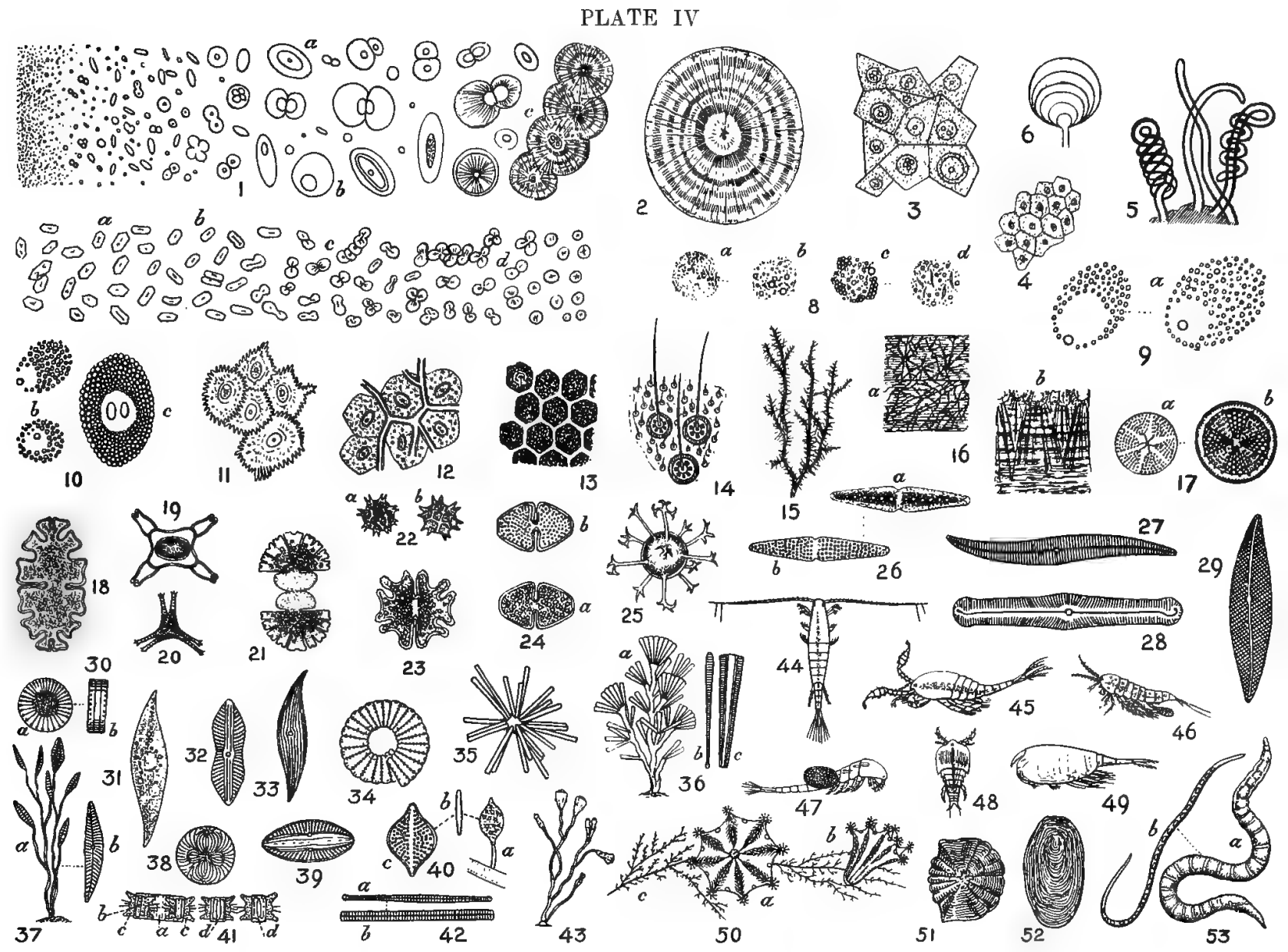

This plate shows how certain crystals formed in viscous fluids present a radiating, concentric arrangement; how other crystals display pentagonal and hexagonal shapes; how protagon develops spiral forms; how molecules tend to aggregate and form cells with nuclei; how plants and animals display various markings which result in radiating or concentric arrangements or both; how plants and animals exhibit for the most part some kind of division or segmentation by which they can build themselves up and divide and separate part of their substance in the process of reproduction. Lastly, how growth and pressure in constricted areas modify form.

Fra. 1.-Precipitation of carbonate of calcium from a viscous solution on a slide of glass, showing inolecular, nuclear, and cell forms in various stages of development. $a$, Oval body containing nucleus; $b$, round body containing nucleus; $c$, glolsular crystals of carbonate of lime, showing concentric and radiating arrangements (J. H. Bennett).

FIG. 2.-A perfectly formed globular erystal of carbonate of calcium with concentric and radiating lines (J. H. Bennett). The concentric and radiating arrangements so well seen here are frequently met with in the transverse sections of the stems of plants and of bones and other tissues of animals, as shown more especially in Plate v., p. 9; and in Plates lxii. and lxiii., pp. 156 and 158.

FIg. 3.-Flattened crystals of carbonate of calcium adhering at their edges, on the surfice of lime water, resembling epithelium in form (Fig. 4). The crystals are pentagonal in shape, and resemble the pentagonal and lexagonal liver cells (Fig. 12), and the pigment cells of the choroid coat of the eye (Fig. 13). Compare with hexagonal cells of honeycomb; also witlı transverse sections of basaltic columns of Staffa in Scotland, and the Giant's C'auseway in Ireland (Plate xl., Figs. 1 and 2, p. 63) (the Author).

Fig. 4.-Pavement epithelium from human skin, showing jentagonal and hexagonal forms, chiefly the latter. ('ompare with Figs. 3, 12, and 13 (the Author).

Fig. 5.-Shows straight, curved and spiral processes shooting out from a mass of protagon on the addition of water, artificial formation (J. H. Bennett). The spiral is a fundamental formation in nature (the Author).

FIG. 6.-Concentric layers occasionally formed at the extremities of free ends of the protigon (J. H. Bennett).

Fig. 7.- This figure comes under Fig. 1. Shows crystalline forms assuming the cell shape. "Crystalline forms; b, angles of crystals rounded off ; $c$, ovoid cell forms; $d$, cell forms aggregated together (J. H. Bennett).

Frg. 8. - Various arrangements of molecules. a, Finely molecular mass; $b$, molecules varying in sizu as seen in milk ; $r$, molecules aggregated in groups ; $d$, histolitic molecules houn broken-down fibrous tissue (J. H. Bennett).

FIGs. 9 and 10.-Fatty molecules precipitated from an alcoholic solution arranging themselves to resemble cells with a nucleus $(a, b)$ and nucleoli (c) (J. H. Bennett).

FIG. 11. - Spinous cells from the Malpighian layer of the human epidermis (after Schultze). Show concentric and radiating arrangements with a certain amount of angularity. 
Fra. 12.-Hepatic cells with biliary duct between. Show pentagonal and hexagonal forms, chiefly the latter. Compare with Figs. 3,4 , and 13 .

Fra. 13.- Hexagonal pigment cells from the choroid coat of the eye. Compare with Figs. 3, 4, and 12.

Fig. 14.-Pygidium of a flea with bristles. Shows concentric and radiating arrangement of the areolæ (the Author).

FIG. 15.-.Portion of sponge with spicules projecting from the surface. Shows branching, plant-like form (the Author).

FIG. 16.- Portions of shell of oyster (a), and egg of ostrich $(b)$. Show radiating and concentric arrangements (the Author).

FIG. 17. - a, Asterolampra marylandica, side view; b, Heliopelta Leeuwenhackir, side view. Show heautiful concentric and

radiating arrangements (the Author).

FIG. 18.-Euastrun oblongum. Shows rudimentary segmentation (the Author).

FIG. 19.-Tetmemorus lævis (in conjugation). Shows symmetrical form and angmentation.

FIG. 20.--Staurastrum gracile. Shows triangular radiation.

FIG. 21.-Micrasterias denticulata. Cell dividing transversely.

FIG. 22.--a, Pediastrum boryanum. Shows stellate radiation and reticulated surface, b, Pediastrum granulatum. Enpty cell.

FIG. 23. - Euastrum verrucosum. Shows symmetric radiation and tendency to segmentation.

FIG. 24. - a, Cosmarium pyramidatum. Shows tendency to segmentation or division. b, Empty shell of sance.

FIG, 25.--Micrasterias denticulata (sporangium). Slıws concentric and radiating branching arrangement (the Anthor).

FiG. 26. - a Tetmemorus granulatus; $b$, ditto, empty cell. Show symmetrical markings and tendency to segmentation.

FIG. 27.-Gyrosigma strigile (side view). Shows central point and longitudinal and transverse markings.

FIG. 28.- Pimularia nobilis (side view). Shows central point and longitudinal, radiating, and transverse markings.

FIr. 29. - Fyrosigma transversale. Show's central point and longitudinal and oblique markings which cross each other.

FIG. 30.-Cyclotella operculata. a, Side view ; b, front view. Show concentric and beatiful radiating arrangement.

FIG. 31,-Gyrosigma angulatum (side view with endochrome). Shows longitudinal and central circular markings.

FIg. 32. - Navicula didyma (side view). Shows central point and longitudinal and radiating markings.

FIi. 33.--Gyrosigma littorale (side view). Shows central point and longitudinal striation.

FIG. 34.-Meridion circulare. Shows frustules united into a spiral coil; front view.

Fig. 35.--Synedra splendens. Shows frustules attached at one end and radiating.

FIG. 36. - Licmophora splendida. a, Frustules arranged in a radiating, branched, plant-like form; $b$, side view of single frustule ; $c$, front view, ditto.

FIg. 37.-Cocconema lanceolatum, presenting: $a$, a plant-like appearance; $b$, single valve side view.

FIr. 38.-Eupodiscus sculptus (side view). Shows central point with four sets of pear-shaped radiating markings, arranged at right angles to each other.

FIG. 39.-Amphora ovalis (front view). Shows longitudinal and radiating markings.

FIG. 40.-Doryphora amphiceros. $a$, Side view of frustule with endochrome; $b$, front view, ditto ; $c$, prepared single valve.

FI(. 41.-Biddulphia aurita and frustules undergoing division $(a, b, c, d)$.

FIG. 42. - Synedra splendens. a, Side view of prepared frustule; $b$, front view of ditto. Show longitudinal and transverse markings,

FIG. 43.--Gomphonema acuminatum. Showing plant-like arrangement of parts.

FIG. 44.-Cetocholus septentrionalis (dorsal view). Shows segmentation of body, antenne, \&:.

Frg. 45.-Cyclops quadicornis (male). Shows segmentation in body, antennæ, and legs.

FIt. 46.-Canthocamptus minutus. Shows segmentation in body, anteniæ, and legs.

FIG. 47.-Notodelphys ascidicola (female). Shows segmentation of borly, \&c.

Fir. 48. - Alteutha depressa. Shows segmentation of body, antennæ, and legs.

Fig. 49.-Nebnlia bipes. Shows segmentation of body, \&c.

FIG. 50.--Haliclystus octoradiatw. A Lucernarian medusa $(a, b)$; on sea-weed (c). Shows symmetrical madiation from centre, as in star-fish and many crystals and plants.

FIG. 51. - Scale of dace (Leuciscus vulgaris). Shows concentric and radiating markings.

FIG. 52. - Scale of jack or pike (Esox lucius). Shows concentric markings. Compare with markings on starch corpuscle.

FIG. 53.-Anguillula tritici. a, Mature worm; $b$, young or immature worm. Show tendency to segmentation of bodies.

\section{PLATE V}

In this plate accurate drawings of an animal cell, egg, hair follicle, muscle, nerve, Pacinian body, coral, horn, bone, tooth, \&c., are given. The radiating and concentric arrangements occurring in crystals and plants reappear in a striking form in animals, and are to be regarded as fundamental. Compare with Plates i., ii., iii., and iv.; also with Plates lxi., lxii., and lxiii., pp. 154, 156, and 158, also Plates lv. and lvi., pp. 107 and 109, which deal with embryonical development and lines of force.

Fra. A. Cell with radially disposed reticulum from the intestinal epithelium of a worm (after Carnoy).

Fis. B. Ovum or egg cell of a worm (after Strasburger), Shows radiation from a central area.

FIr. (.. Transverse section of muscular fibre of leg of wasp, showing a ridial disposition of the sarcoplasm (afler Schaefer).

Fir: D. Transrerse section of a Pacinian body (Luch corpuscle), showing concentric arrangement (the Anthor).

FIG. E. Transverse section of two merves: the nerve fibres occupy the central portions of each section, and rod-like structures pass radially between the axis cylinder and the primitive slieath (alter Schaefer).

FIG. F. Transverse section of human molar tooth. Shows radiating and concentric arrangement of dentine and enamel (the Anthor).

FIG. G. Growing parietal bone of a foetus in the pussession of the author. The bony substance is arranged in radiating lines; growth proceeding from the central portion of the bone (the Author).

FIG. H. Parietal bone of an enbryo sheep. Shows the same radiating arrangement as in Fig. G (after Scliaefer).

FIg. I. Vertical section of human os calcis. Shows radiating, curved, cross arrangement of the cancellons tissue of the bone, giving the greatest amount of strength with the least possible material. The os calcis in walking receives the weight of the loody the instant the heel tonches the ground (the Author).

FIr: J. Transverse section of growing horn of stag. Sliows radiating and concentric arrangements (the Author).

Fri. K. Section of hair-follirle (after Piesiadecki). Shows concentric and radiating arrangements.

FIt. L. Trausverse section of human vertebra. Shows radiating arrangement in the body of the vertebra (the Anthor). 
PLATE V
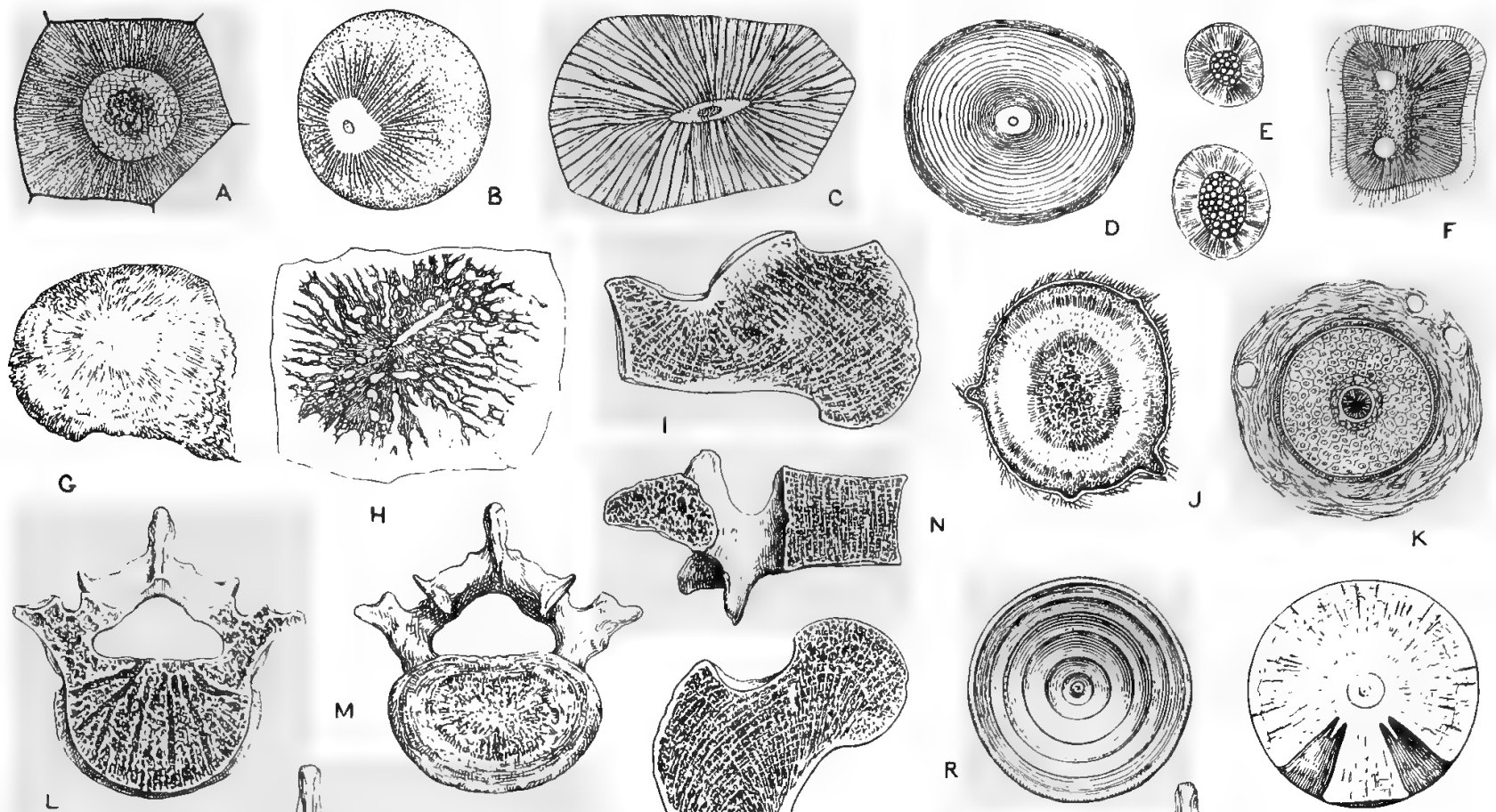

M

M
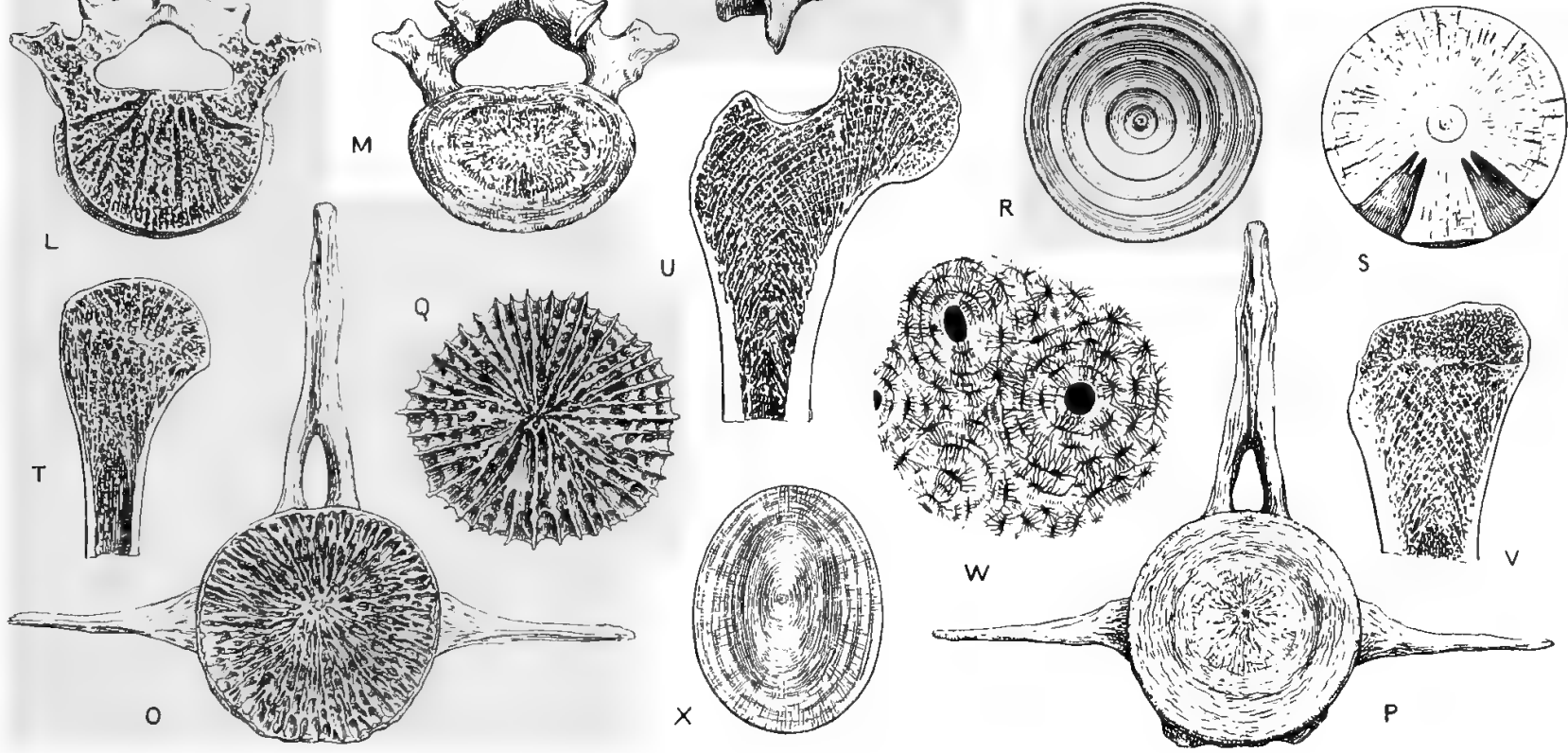

FIf. M. Articular surface of human vertehra, showing radiating and concentric arrangements (the Anthor).

FIG. N. Vertical section of human vertebra. Shows vertical and transverse distribution of the bony tissues-the vertical predominating. This arrangement combines great lightness with great strength, especially vertical strength (the Author).

FIG. O. Epiphysis of vertelora of young whale (proximal or distal surface). Show's well-marked radiating and concentric arrangements. Compare with Figs. J, M, S, Q, W, X, and P.

FIG. P. Articular surface of vertebra of young whale. Shows radiating and concentric arrangements, the latter being most marked (the Author).

Fra. Q. Deep-sea coral (Bathyactis symmetrica), from original drawing by C. Berjeau of specimen in British Museum. Sliows wellmarked radiating and concentric arrangements, the former predominating. Compare especially with Fig. $\mathrm{O}$.

FIG. R. Articular surface of vertebra of shark. Show's leautiful concentric rings like the rings of growth in the stems of trees, Compare with Figs. D and K (the Author).

FrG. S. Transverse section of vertebra of shark. Shows concentric and radiating arrangements. The two spiculæe resemble rudimentary vertebral processes (the Author).

Fig. T. Vertical section of head of human humerus. Shows vertical, transverse, and ollique distribution of the lony tissue, which secures great strength and lightness, letter seen in Fig. U (the Author).

Fig. U. Tertical section of lead, neck, and upper part of human femur, showing the hony tissue radiating and disposing itself in beautiful curved arches which interweave and produce an extraordinary degree of strength combined with ixtreme lightness. As is well known, these parts transmit the weight of the body to the inferior limbs. They furnish i very good example of design (the Author).

FIt. V. Vertical section of upper portion of tibia. Presents an arrangement similar to that seen at Fig. U (the Anthor),

FIG. W. Transverse section of shaft of human femur, showing concentric and radiating arrangements similar to those representing rings of growth in trees (the Author).

FIG. $X$. Transverse section of tooth of Cachalot whale. Shows beautiful concentric and radiating arrangements. Compare with Figs. J, M, S, O, P, and:W (the Author).

VOL. I. 


\section{PREVALENCE OF SPIRAL ARRANGEMENTS ON A GRAND SCALE IN THE PHYSICAL UNIVERSE}

The spiral formations of the physical universe are seen in whirlwinds and spiral sandstorms, in whirlpools and spiral waterspouts, in spiral nebular arrangements, \&c. In all these cases, the atoms, molecules, and bodies concerned are arranged spirally. Winds occasionally blow in straight lines, but for the most part in curves, circles, and spirals. The cyclone, one of the most powerful and violent of winds, pursues a circular path. When, however, it advances rapidly it follows a rotatory, spiral course.

"It appears from recent investigations, that hurricanes, tornadoes, and typhoons are to be regarded as great whirlwinds in motion, the meeting of two opposing winds producing the whirlwind. According to M. Dove, the rotatory motion of the air is from right to left in the northern hemisphere, or in the direction opposite to the hands of a watch; from left to right in the southern hemisphere, or with the hands of a watch; gusts and lulls occurring in the vortices. It is further ascertained that the northern hurricanes travel in an oblique direction from the equator towards the north pole, while the southern hurricanes travel more obliquely from the equator towards the south pole. We frequently observe in serene hot weather the whirling motion of the air, betrayed by sand and dust ascending in spiral columns. Larger and stronger whirls carry up leaves and straws, and even buildings lying in their course. These whirlwinds are generally caused by the struggle of two winds meeting at an angle, in the same manner that eddies and whirlpools are formed in water, by two currents being obliquely impelled against each other."

There is reason to believe that whirlpools in water, and also in air, may take place without the intervention of two opposing currents meeting each other obliquely. An artificial whirlpool is readily produced by placing water in a circular hand basin provided with a circular aperture and plug at the bottom. If the plug be withdrawn, the water, in its attempt to escape, invariably forms a tiny eddy or whirlpool. Circumscribed empty spaces favour the formations of vortices and whirlpools.

The peculiar circular and spiral movements of the wind are well seen in certain sand-storms. In various regions of the earth, more especially in warm climates, the sand is raised by the action of the wind in spiral eddies, which take an upward direction, and form a more or less dense column of sand, having a vertical measurement of anything up to a hundred or more feet. The column is distinctly spiral in its configuration, and spreads out at its summit, when the sand-storm ceases. Occasionally a large number of spiral columns may be seen at the same time, arranged in a circle.

In such cases each column rotates, so to speak, on its own axis : the whole careering round in a circle or spirally as indicated by the arrows in Fig. 3. The movements somewhat resemble those of the earth round the sun.

Vortices occur on sea, on land, and in the heavens. At sea, and in great fresh-water lakes and rivers, they take the form of eddies, maelstroms, and spiral water-spouts ; on land they are seen as whirlwinds of sand, leaves, straws, and other light substances. In the heavens the nebulæ are, in certain cases, arranged in great whirls composed of right and left-handed spirals.

The water-spout provides one of the most wonderful displays of spiral molecular action in nature. The spiral is generally single. The water-spout consists of a huge, vertical, spiral mass of watery vapour, sometimes stationary, and sometimes spirally moving along the surface of the water (sea, lake, or river). It descends from the clouds, or rises from the water, in gigantic spiral wreaths, and forms a vertical spiral column from 800 to 2500 feet high; the column is also of a sand-glass shape, that is, it spreads out above and below, and is comparatively narrow in the middle. The effect is to draw up immense masses of water into the clouds, the clouds driven by winds carrying the moisture which they contain to large areas of the earth's surface, on which it is ultimately deposited as rain, sleet, hail, or snow.

The surcharged clouds are the great irrigators of the land, and wherever rains are plentiful, plants and foliage are abundant and luxuriant. The abundance of plant life in turn secures a plethora of animal life; plants, in the great majority of cases, supplying the food of animals; and animals thriving and multiplying in proportion as forage, succulent grasses, leaves, vegetables, and fruits abound.

The water-spout sometimes breaks out with terrific violence far inland. It is variously described. According to one authority ("Descriptive Atlas," by Milner), "whirlwinds usually form a double cone; the upper cone with the vortex inclining downwards, while the point of the lower cone is directed upwards. In passing over the sea, lakes, and rivers, the water is raised by the lower cone in a spiral column, often to the height of several hundred feet, while the clouds are brought down by the upper cone, and a water-spout is formed. Two cones have frequently been observed to separate and re-unite; and in the Mediterranean as many as sixteen water-spouts have been seen 


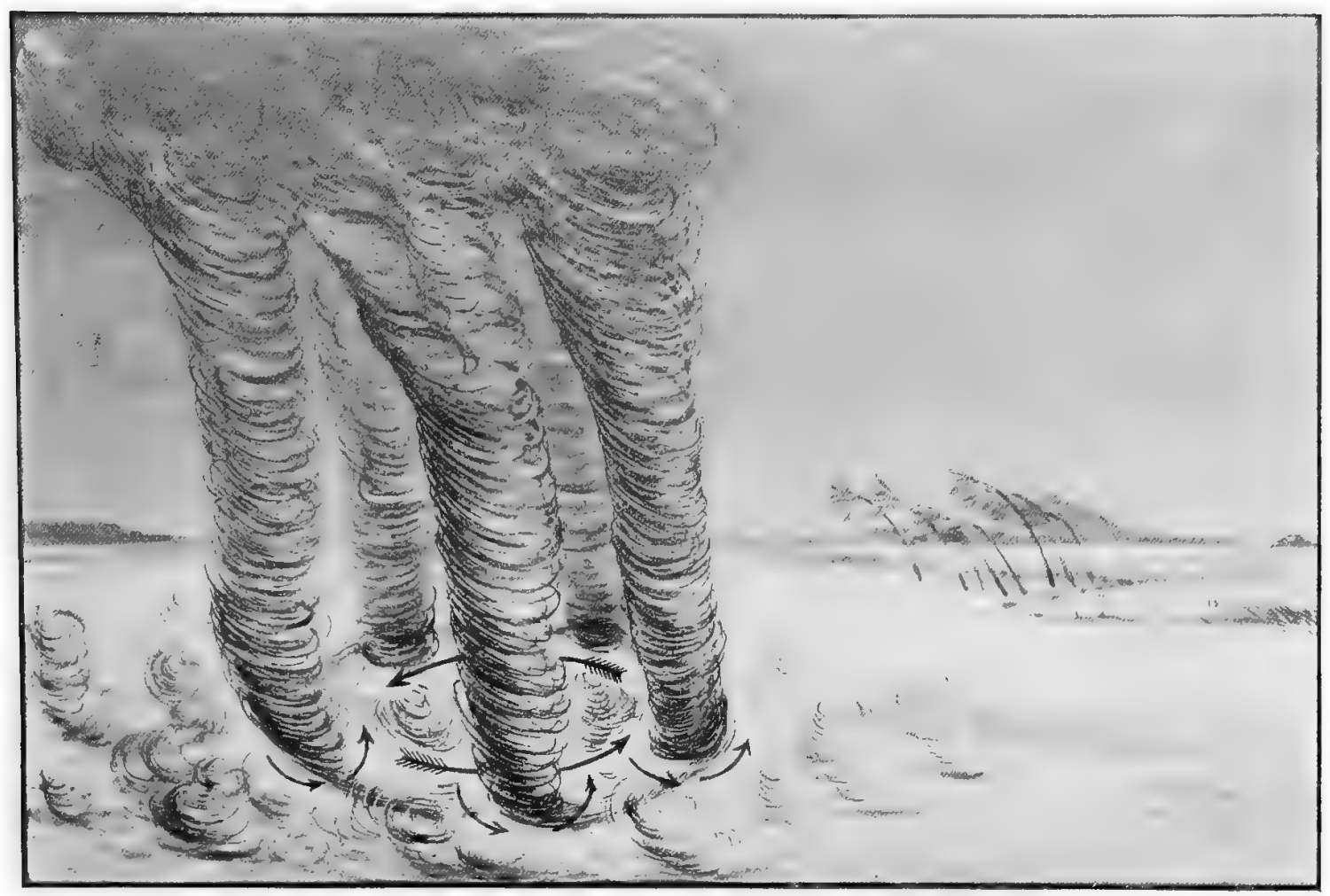

FIr. 3. --Study of a sliral sand-storm by the Author. Drawn'by C. Berjeau.

at the same time. In water-spouts on land the upper cone consists of a mass of clouds, and the lower cone of sand, dust, and other bodies found on land. Deluges of rain accompany their disruption."

According to another authority ("Century Dictionary," edited by Dr. W. Dwight Whitney), "The water-spout is a whirlwind over a body of water, which produces the appearance of a solid column of water, extending from the surface to the clouds. In reality, however, the phenomenon that is seen is the cloud brought down to the earth's surface by the rapid gyratory motion of a vertical whirl, and it consists simply of fine mist surrounding a central axis of rarefaction. At first the cloud has the form of a tapering funnel ; then, descending to near the water's surface, it draws up the water for a distance into its vortex, and imparts to it its whirling motion. The spout is then complete, and appears as an immense column connecting sea and cloud, light in colour near the centre, but dark along its sides. Like other whirlwinds, the water-spout has a progressive as well as a rotatory motion, its axis sometimes being inclined forward in the direction of advance. . . . It is common for a number of water-spouts to be seen simultaneously or successively" (Plate vi., Figs. 1 and 2).

Before leaving the subject of fluid spiral formations it may be interesting to direct attention to the spiral made by the escape of ether in water placed in a red-hot silver basin (Fig. 4), and to the vortex ring made by smoke in its first stage (Fig. 5), as delineated by Mr. F. Hovenden. ${ }^{1}$

The vortices formed by nebulæ are, in some respects, the most wonderful of all. So far as I can make out they originate in three ways :-

(a) By a nebular mass or nucleus assuming a rotatory or spiral motion in space and drawing towards it, in well-marked spiral lines, smaller nebular masses; $(b)$ by a converse process, whereby the smaller nebular masses assume spiral movements and coalesce to form a spherical or spiral nebular nucleus; and $(c)$ by a resolving or disintegrating process, whereby the nucleus or central core throws off its substance in spiral streams, usually two in number-these forming right or left-handed spirals. There is what is practically a spiral infolding and evolving process at work; a spiral overlapping in one direction, and a spiral unlapping in another and opposite direction.

There are good grounds for believing that spiral nebulous matter is not in every instance formative matter, that is, spiral matter aggregating and building up stellar and other bodies. On the contrary, it may very well be the spiral detritus of stars and other heavenly bodies breaking up and disintegrating. If this threefold view 1 "What is Heat and what is Electricity?" by F. Hovenden, F.L.S., F.G.S., F R.M.S. London, 1900. 
be adopted the cosmogony will consist of spiral and other matter in a perpetual state of flux; the matter circulating in time and space and being free to combine and resolve itself in obedience to natural laws. The view here propounded is in harmony with creation as a progressive work, and also in accordance with the ceaseless changes witnessed in the inorganic and organic kingdoms. It further secures to all things a beginning, a career, and an end, and opportunities of renewal. ${ }^{1}$

The nebular spiral arrangements were first figured by Lord Rosse. They were subsequently very successfully photographed by Dr. Isaac Roberts in his interesting work, "Stars, Star-clusters, and Nebulæ."

The origin of the spiral movements in inorganic matter is involved in great obscurity. In whirlwinds, in

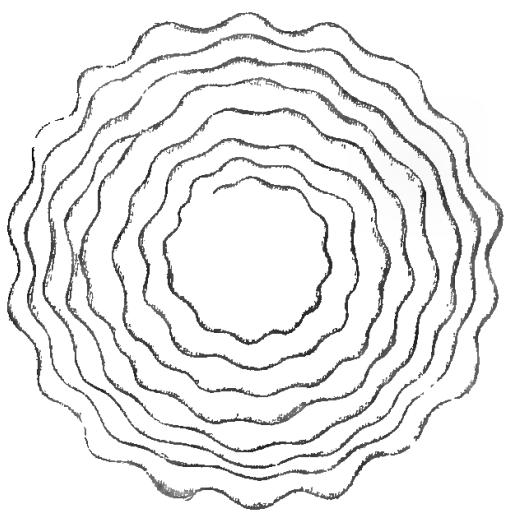

FI: 1. - In Fig. 4 the liquid is seen in plane, and the corrugated edges show where the free ether is "rippling" or" flowing up from below and through the fluid (Hovenden).
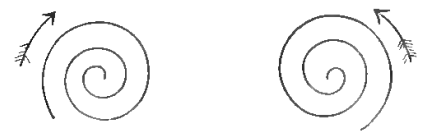

FIt: 5,-Diagram showing section of a vortex ring in its first stage. The arrows indicate the direction of the motion of the molecules (Hovenden). whirlpools, and in nebulæ it is usually attributed to two currents meeting at a certain angle and clashing, with the result that the colliding matter is obliquely deflected and made to assume a spiral course. Such colliding is, however, not necessary. It suffices if matter be allowed to flow into empty spaces, or if it be subjected to varying pressure, or exposed to attractions from nearly, but not quite, opposite points.

The colliding, clashing theory of the origin of spiral movements is negatived by the spiral movements occurring in organic matter, to wit, in the fluids and solids of plants and animals.

The spiral movements occurring in the organic kingdom, as I show further on, are essentially identical with those occurring in the inorganic kingdom. The spiral movements in plants and animals are not, however, exposed to collisions of any kind. On the contrary, they manifest themselves without the least commotion, and under circumstances which preclude the possibility of collisions. Thus, they make their appearance in the growing stems, leaves, flowers, fruit, and tendrils of plants; in the developing reproductive cells of certain plants and animals; in growing shells, horns, and teeth; in the movements of the heart of the bird and mammal in the circulation; and in the movements of the muscles, bones, and joints of the higher animals in the several kinds of locomotion.

The origin of spiral movements in the inorganic and organic kingdoms by no means lies on the surface.

From a wide survey of the subject I am disposed to regard them as predetermined, inherent, fundamental movements. This belief is favoured by the new electric theory of matter, according to which the atom is divisible into innumerable sub-atoms, corpuscles, or electrons; these sub-atoms or electrons forming a system of bodies not unlike the sun and the planets, there being according to the most recondite physicists a kind of "planetary theory of the atom."

According to the electrical theory of matter, the sub-atoms, corpuscles, or electrons are subject to various kinds of strain, are in a constant state of excitement, and in a perpetual whirl of movement, spiral or otherwise; the movements being as important as the matter moving.

The electric theory of matter, it will be observed, puts spiral and other movements in the forefront of cosmic arrangements.

As originally pointed out by Professor Faraday, all things are electric. He divided the several substances, inanimate and animate, into two great classes, namely, the paramagnetic and the diamagnetic, according as they arrange themselves in a line with or across the poles of a horseshoe magnet, as indicated by the magnetic needle.

1 Since writing the foregoing, I find Professor Ernst Haeckel has exjressed a somewhat similar belief as regards the making and the ummaking of the heavenly hodies. He says: "The universe or the Cosmos is eternal, infinite, and illimitable. Its substance, with its two attributes (nuatter and pnergy), fills infinite space, and is in eternal motion. This motion ruus on throngh infinite time, as an unbroken development, with a periodic ehange fron life to death, from evolution to devolution. . . . While the rotating masses slowly move towards their destruction or dissolution in one part of slace, others are springing into new life and development in other quarters of the Universe " "Riddle of the Universe," English edition, 1902, p. 5).

Dr. Isaac Roherts, F.R.S., in 1899 enunciated practically the same views. Leaving out of consideration the origin of matter itself, and leginning at the epoch of re-construction, he summarises the order of stellar evolution as under :-

1. Dark or light aggregations of matter in globular, conletic, meteoritic, or dust-like form, and in gaseous clouds scattered abont, isolated, in spatice.

2. Collisions hetween any two or more of such bodies or argregations

3. Re-conibisation of the naterials after collision into webule, mostly of the spiral type, and then into stars.

4. Arrival again at the epoch of maturity.

5. Decay, and then retum to the epoch of quiescence preparatory to undergoing another cycle of wreckage and of re-constitution ("Stars, Star-clusters, and Nelunle," vol. ii., p. 178). 
PLATE VI

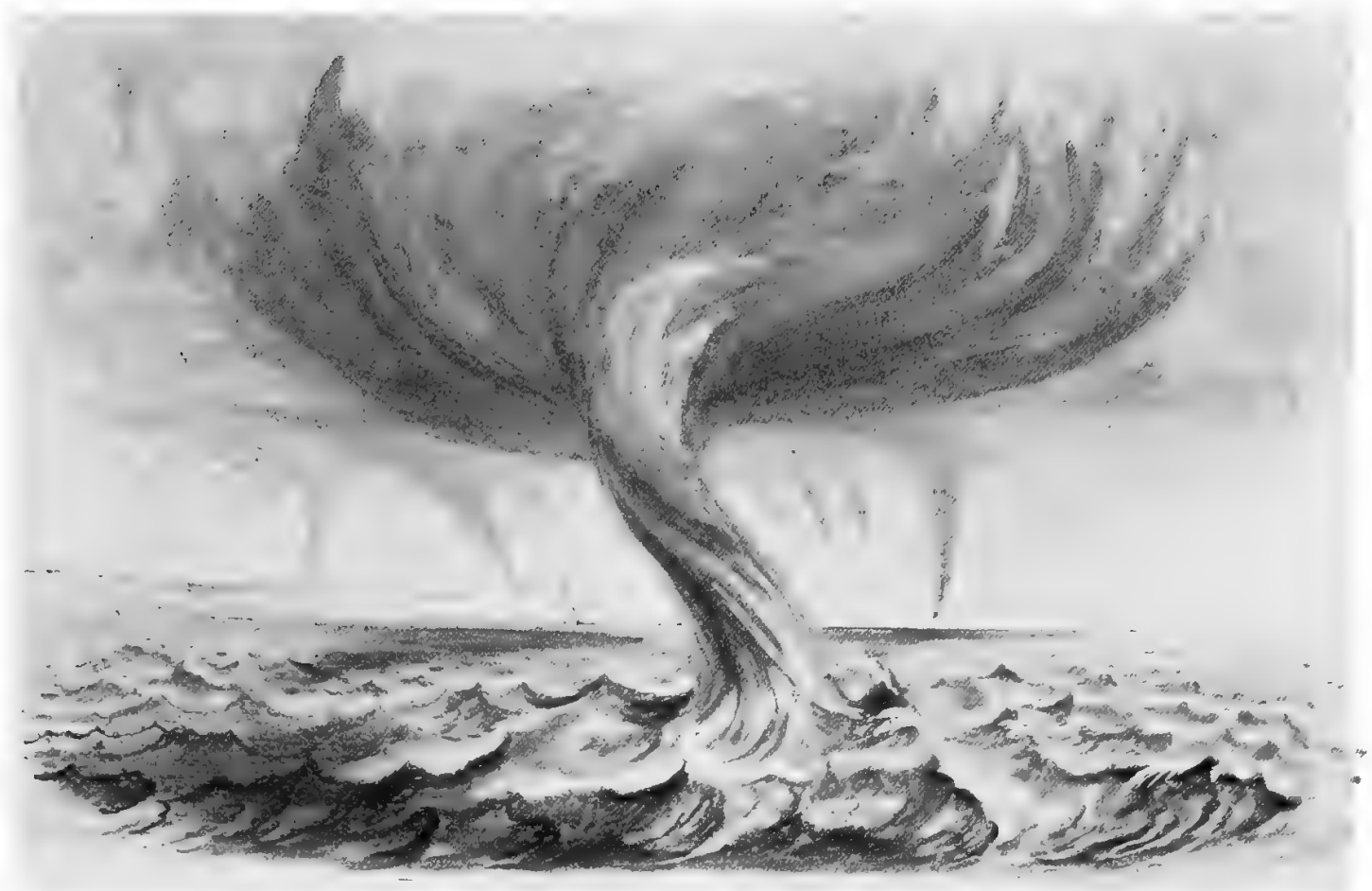

FIr: 1.-Study of a spiral water-spont by the Author. Drawn by C. Berjean.

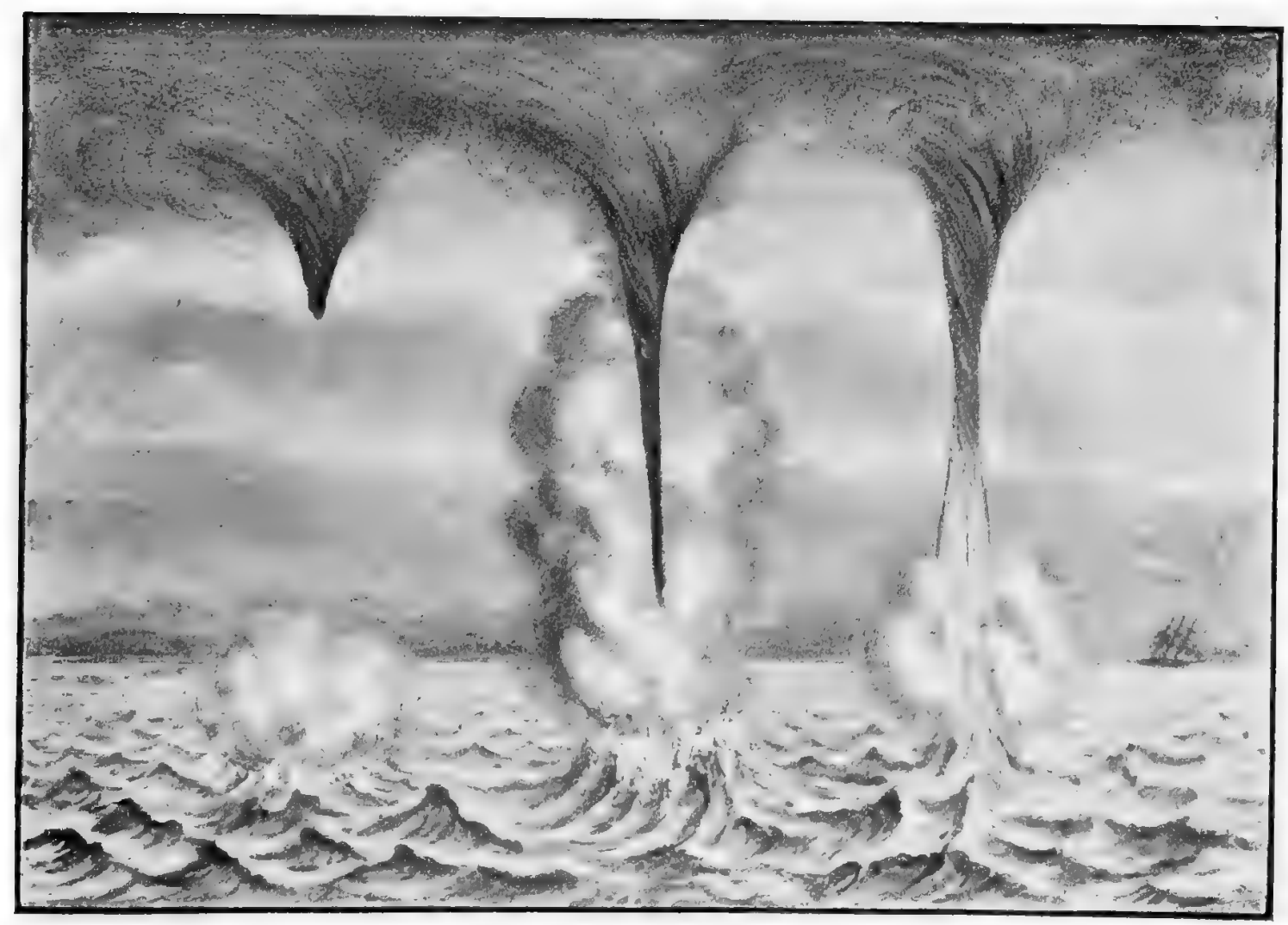

Fia. 2.-Study of spiral water-spouts by the Author. Drawn by C. Berjeau. 
The presence everywhere of electricity and electrical strain invests the new theory of matter with quite a remarkable interest.

I know nothing in the whole range of physics more wonderful than the so-called spiral nebulæ. They are now known to occur in very large numbers, and to form right and left-handed symmetrical spirals, being composed of two parts which wind within each other round a centre or nucleus, which consists of one or more stars with a nebulous matrix. How stars and nebulous matter, in unlimited space, should arrange themselves in spiral wreaths baffles comprehension They can only do so in accordance with certain laws which are the outcome of design. When it is stated, as has been done, that similar spiral arrangements can be traced in large numbers in plants and in animals, the mind is overwhelmed, and is, at first, inclined to withhold belief. ${ }^{1}$

The resemblance between the inorganic nebular spirals and the organic plant and animal spirals is in no sense fanciful but founded upon the most convincing evidence. In proof of what is here stated I invite the reader to compare the spiral nebula (Fig. 4 of Plate vii.) with the spiral flower (Fig. 13, p. 21); the spiral seed (Fig. 11, p. 20); the spiral fruit (Plate xi., Fig. 1, p. 25); the spiral spermatozoon (Plate xii., Fig. 1, E, p. 27); the two spiral shells (Plate xiii., Fig. 1, F and G, p. 28); and the spiral apex of the mammalian heart (Plate xvii., Fig. 3a, p. 32).

If proof were wanting of the intimate relations subsisting between the inorganic and organic kingdoms, and of the unity of plan which pervades all nature, these remarkable coincidences in spiral formations are well calculated to supply it.

The inorganic and organic spirals cannot, everything considered, be regarded as chance productions. They undoubtedly owe their origin to the operation of a common law, and afford a striking proof of a First Cause.

The inorganic and organic kingdoms are constructed on similar lines. They are not opposed to each other. On the contrary, they are interdependent, complemental, co-ordinated, and conditioned. They are made for each other. No marvel, then, if plants and animals assume shapes and movements which are common in the heavenly bodies. Endless examples of spiral structures and spiral movements are given further on.

I append photographs of the extraordinary nebular arrangements (Plates vii. and viii.). They transcend in grandeur and beauty everything of the kind which has hitherto appeared. These arrangements, moreover, are extremely suggestive in their bearing on movements in general, and on the movements in plants and animals in particular.

\section{PLATE VII}

Plate vii. shows actual photographs of nebulæ and star clusters; the upper two figures of the plate displaying curves and spiral arrangements - the lower two figures astral nuclei with nebulous envelopes.

FIG. 1.-Photograph of star-cluster M. 13, Herculis, by Dr. Isaac Roberts, F.R.S. Dr. Roherts savs, "The photograph shows the star's in the central part of the cluster to be involved either in faint nebulusity or in almospheric glare cansed by the light of the stars, and some of them appear to be deformed in outline lecatse of the overlapping of two or more star inages. The general configuration of the stars is suggestive of their development from a spiral nebula. The forms of the convolutions still remain visible in the arrangement of the stars, whilst the nebulnus matter appears to have been absorbed, and the nuclear condensations at their centres account for the dense aggregations of stars."

Very beantiful spiral nebulæ are figured in Plate viii.

FIG. 2.-Photograph of Nebula HI I., 143, Virginis, loy Dr. Isaac Roberts, F.R.S. The photograph shows the nebula to resemble somewhat the letter 1$)$ with the curve in the n.p. direction, and a star of about the 15th magnitude in the centre, the interior being filled with nebulosity of different densitirs, within which are five or six star-like coudensations. Dr. Roberts remarks that the nebulous condensations resemble those invariably seen in spiral nebula. (Photographs of Stars, Star-clusters, and Nehulæ.)

Fig. 3.-Photograph of Annular Nebula M. 57, Lyræ, by Dr. Isaac Roberts, F.R.S. Sir J. Herschel describes this nebula as a magnificent object; annular, bright, and considerably extended. There is no appearance of stars involved in the ring, but the s.f. and n.p. sides are denser than the s.p. and n.f. ones. There is, as explained by Lurd Rosse, an extension of faint nebulosity beyond the margins at each end of the major axis. 'This nebula resembles, in a general way, the cell of a plant or unimal, which consists of a nucleus or central core, cell contents (protoplasm), and a cell wall or enveloue. (Photographs of Stars, Star-clusters, and Nebula.)

FIG. 4.-Photograph of Nebula M. 97, Urse Majoris, by Dr. Isaac Roberts, F.R.S. Lurd Rosse compared this nebula to the face of an owl, and was inclined to assign to it a spiral form. It lias a star for its centre, ant the nelulous envelope is concentrated at two points and projects inwards as if preparatury to assuming the spiral shape. A two-fold spiral grouping is very well seen in Fig. 1 of Plate viii. (Photographs of Stars, star-clusters, and Nebula.)

1 Some will no doubt endeavour to explain the marvellous spiral arrangements witnessed in nebule to attractions and repulsions of various kinds; they will regard the phenomenon as of purely physical origin. Such an explanation, however, wonld, as I have endeavoured to show, be quite inadequate to explain similar spiral arrangements occurring in plants and animals where no such attractions and repulsions can possibly be present. 


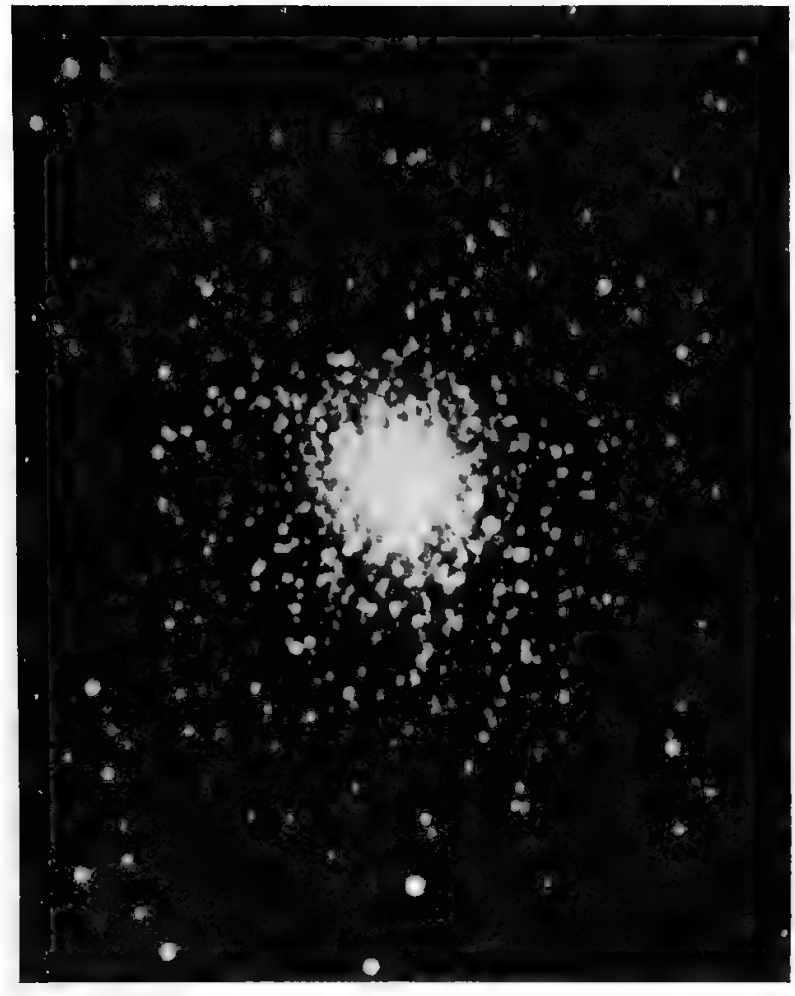

FIG. 1.

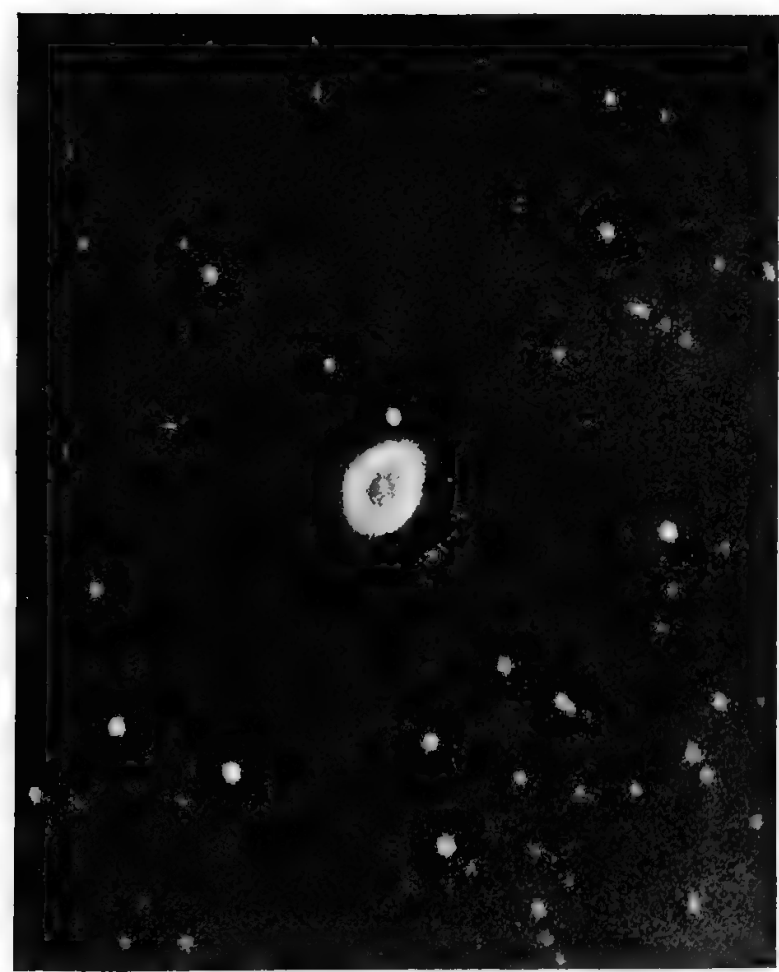

FIG. 3 .

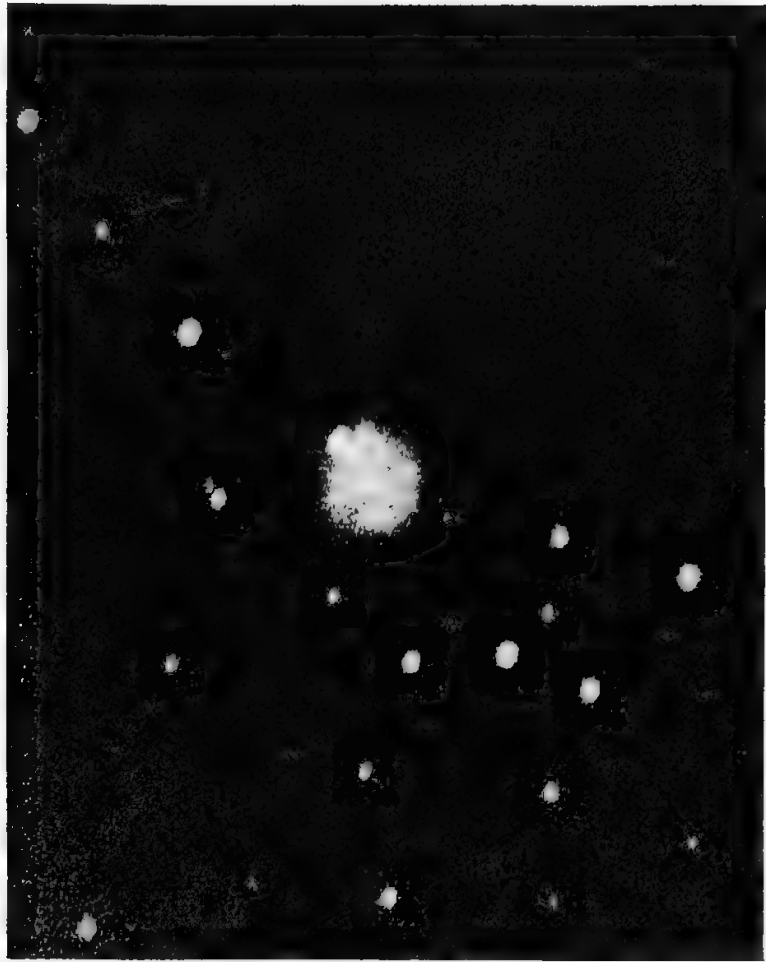

FIG, 2.

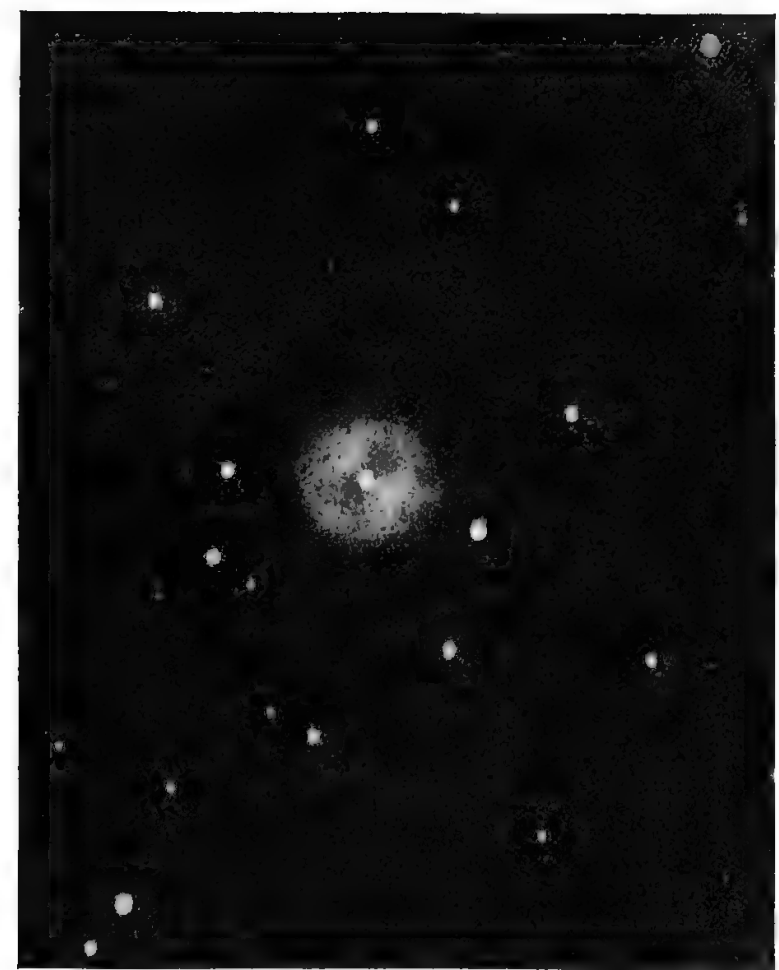

FIG, 4, 


\section{PLATE VIII}

Plate viii. shows photographs of transcendently fine spiral nebulæ. The first three figures of the plate reveal left-handed spiral nebulæ; the fourth figure displaying a right-handed spiral nebula. The nebulæ have an astral centre or core, and are symmetrical, each nebula being composed of two portions which wind into each other from opposite points, either from above and below, or from the sides. These spiral arrangements in the heavens are very striking, and demand the most careful consideration. Dr. Isaac Roberts, who has devoted much attention to the subject, says of them: "The records of these features are now so numerous and accordant that they cannot be attributed to accidental or fortuitous circumstances" ("Stars, Star-clusters, and Nebulx," vol. ii. p. 67).

Fra. 1.-Photograph of Spiral Nehula H IV., Ur'se Majoris, by Dr. Isaac Roberts, F.R.S. Lord Rosse observed this nebula between 1851 and 1862, and detected its spiral nature. He says that light, mottled, faint nebulosity fills mp the space between the arms. The photograph shows the nebula to be a left-handed sliral with bright stellar uncleus, and with both stellar and elongated condensations in the convolutions. (Photograjhs of Stars, Star-clusters, and Nebulæ.)

FIG. 2.-Photograph of Spiral Nebula M. 100, Comæ Berenicis, by Dr. Isaac Roberts, F.R.S. This nehmla forms a most exquisite left-handed spiral, with the nucleus very sharply stellar in the midst of faint netulosity. The convolutions, according to Dr. Roberts, are strikingly perfert, and have several aggregations of nelulosity in them; three or four faint stars are also involved. (Photographs of Stars, Star-clusters, and Nebulæ.)

FIG. 3.-Photograph of Spiral Nebula M. 51, Canum Venaticorum, hy Dr. Isaac Rolierts, F.R.S. Lord Rosse describes this nebula, and figures it as a strong, left-handed, spiral structure. According to 1\%. Roberts the nucleus of the large nebula consists of a small bright star in the midst of a patch of very dense nebulnsity, from which the convolutions radiate in approxinately symmetrical forms. The convolutions are broken up into numerons stars and star-like condensations, and there are wisps of nebulosity, with a star involved in each of them. One of these appears to have been deformed, probably by the action of the second utuclens, as indicated by the disarrangement of symmetry. (Pliotographs of Stars, Star-clusters, and Nebule.)

Fir. 4.-Photograph of Spiral Nebula M. 74, Piscium, by Dr. Isaac Roljerts, F.R.S. This nebula forms a beautiful right-handed spiral. It is the reverse of the spirals seen at Figs, 1, 2, and 3; these, as stated, being left-handed spirals. Lord Rosse describes it as a spiral nebula with the centre formed of stars, and several stars visilble through the nebula. According to Dr. Roberts the convolutions are studded with many stars and star-like condensations-the nebulous matter forming the convolutions being broken up into stars and star-like loci which vary in brightness. Dr. Ruberts remarks that every spiral nebula photographed hy him has a stellar nucleus surrounded by dense nebulosity in the centre of revolution. (Photographs of Stars. Star-clusters, and Nebulæ.)

I add a striking photograph of the Great Nebula in the constellation of Andromeda, which, according to recent astronomical authorities, shows a world in process of formation. For vastness and grandeur it transcends everything yet discovered. The photograph reproduces the outstanding features and details of the nebula, and has been faithfully rendered at Fig. 6.

In the photograph, the Great Nebula is seen more or less sideways, and presents an elliptical form. Viewed from before it would be nearly circular. The new sun, all but completed, occupies the centre of the system, and is distinguished by its greater luminosity. The vast circular rings and spiral folds which invest it are believed to be undergoing a process of condensation at their free margins, and preparing matter for the formation of future planets. They present an arrangement similar to that witnessed in the Milky Way. Already the sites of various heavenly bodies differing considerably in magnitude are indicated - some of them being in position. This is especially the case in the outer coils of the Nebula, where planets formed and in process of formation are seen. The whole system is evidently in a state of flux, and if it could be examined as a near object a scene of unparalleled activity would doubtless present itself-matter moving in all directions in apparent confusion, but in reality in obedience to law and order, and according to a well-considered, preconceived, predetermined plan.

Some parts of the Nebula are semi-transparent, and stars are seen through and beyond it. The spectroscope, however, indicates that the gaseous period has been passed and, in all probability, the fluid stage reached. Changes of a far-reaching nature are evidently occurring slowly but surely in strict sequence, and according to unalterable laws.

The distance of the Nebula from the earth is inconceivably great, and its size so enormous that it completely dwarfs our solar system. An approximate idea of its vast dimensions may be formed from the following calculation. "Light-which travels at the rate of 185,000 miles a second, and could cross our solar system in a little over four hours-would require more than five years to pass from one end of it to the other."

The following graphic account of the Great Nebula is from the pen of Mr. Norman Lattey: "Far away, floating in the immeasurable depths of infinite space, gleams a tiny patch of luminosity. To the naked eye it looks like a hazy star, and is visible in this country from September to January on clear nights. Astronomers call it the Great Nebula in Andromeda. It is really the immense spirit of a yet unborn universe, whose story of creation has only reached the first chapter, and cannot be concluded for countless centuries. There are, of course, other nebulæ in the sky in various stages of evolution; but they are mostly mere tangled masses of glowing gas, shape. less and inert, as yet untouched by the vitalising power of motion which is essential in order that their dead matter may be converted into living worlds 


\section{PLATE VIII}

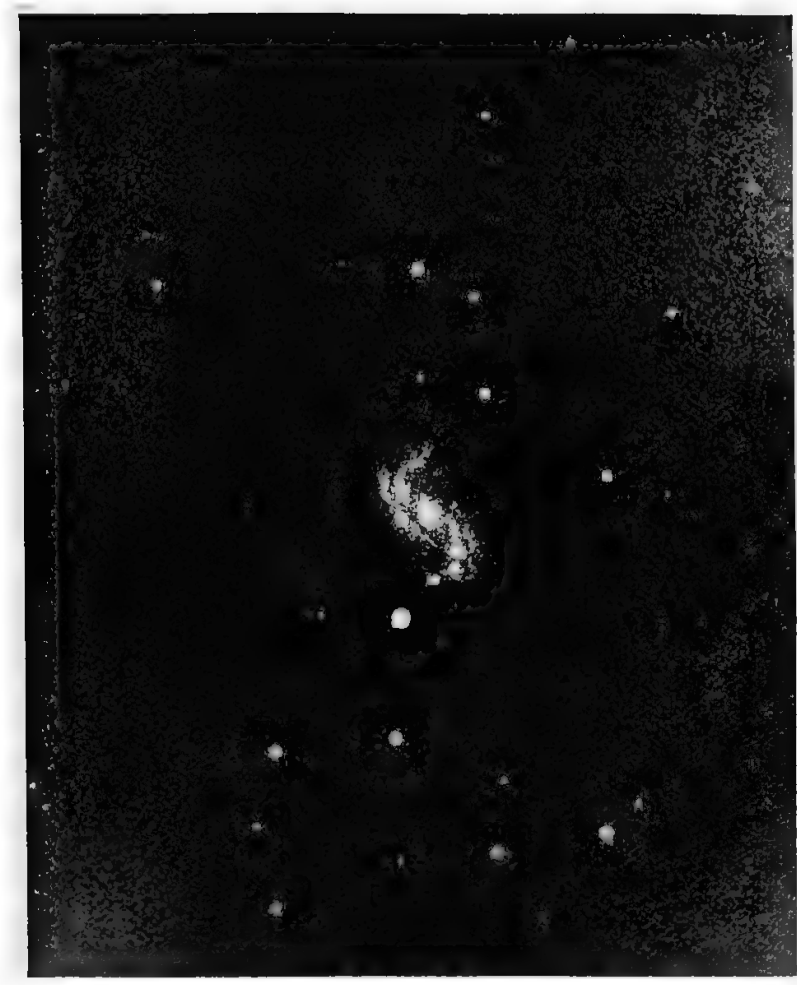

FIG, 1.

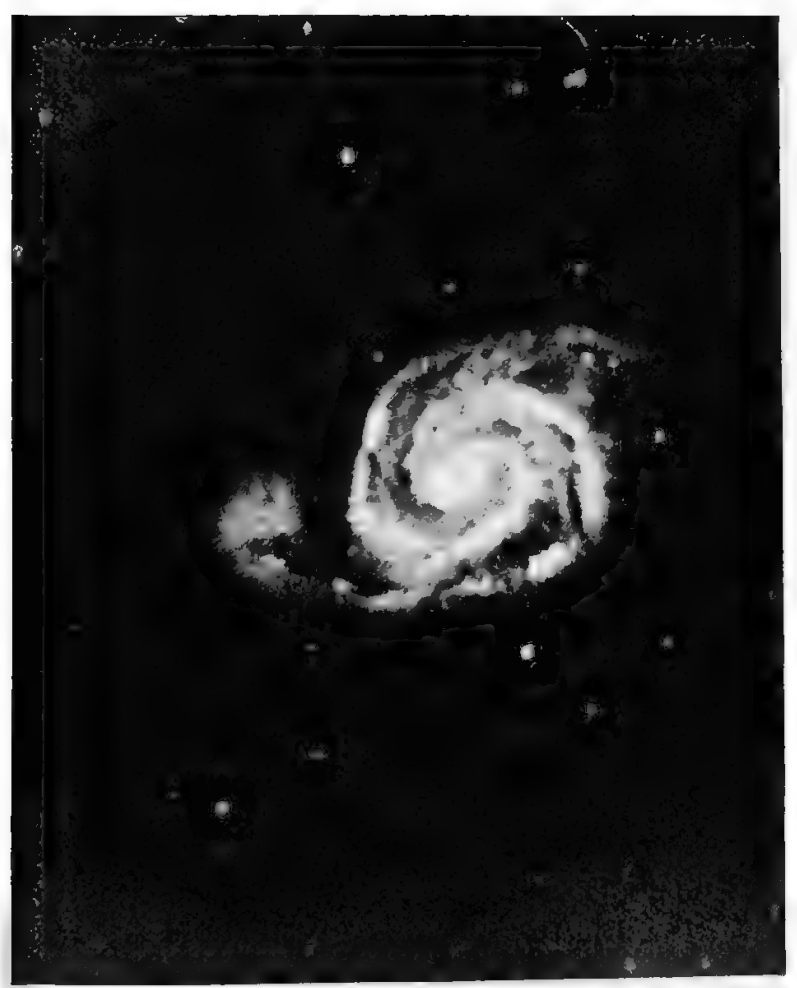

VOL. I.

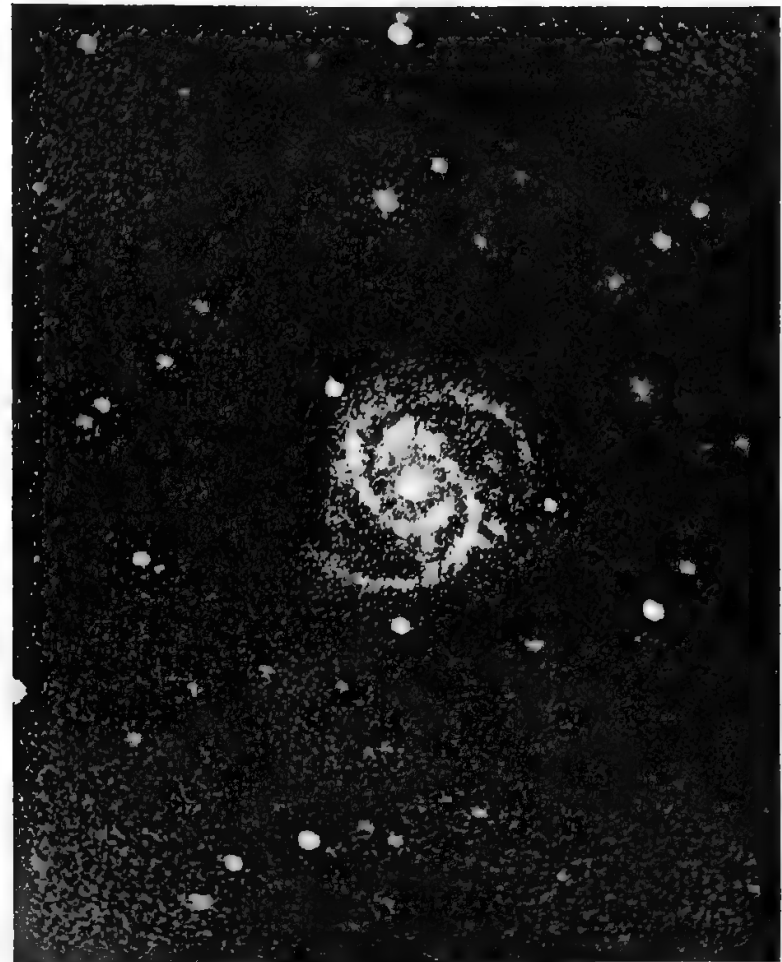

FIG. 2.

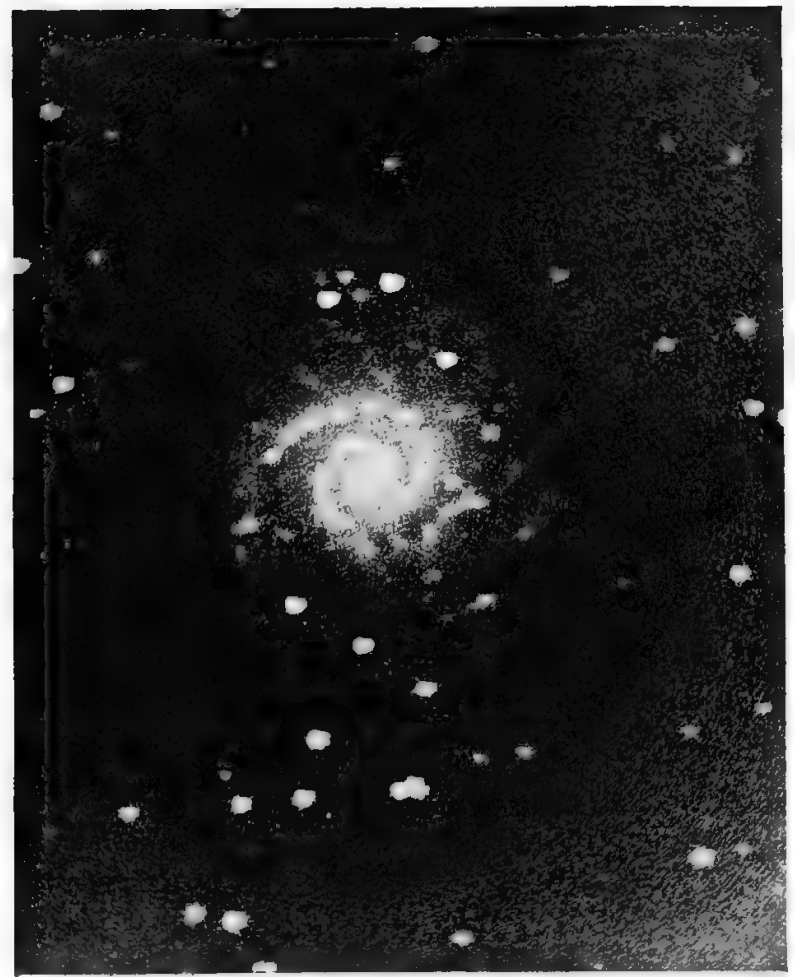

FIG, 4. 
"Immanuel Kant, the great German philosopher, was the first to guess the wonderful process that preceded the foundation of solar systems; but it was reserved for the celebrated French mathematician and astronomer, Laplace, to formulate definitely Kant's now famous nebular hypothesis. This supposed that the sun and all the planets, with their retinues of satellites, originally existed as a huge globe of fiery vapour, which, having acquired a slow rotary motion, gradually assumed the form of a gigantic whirlpool. As time went on, the vast rings of tenuous

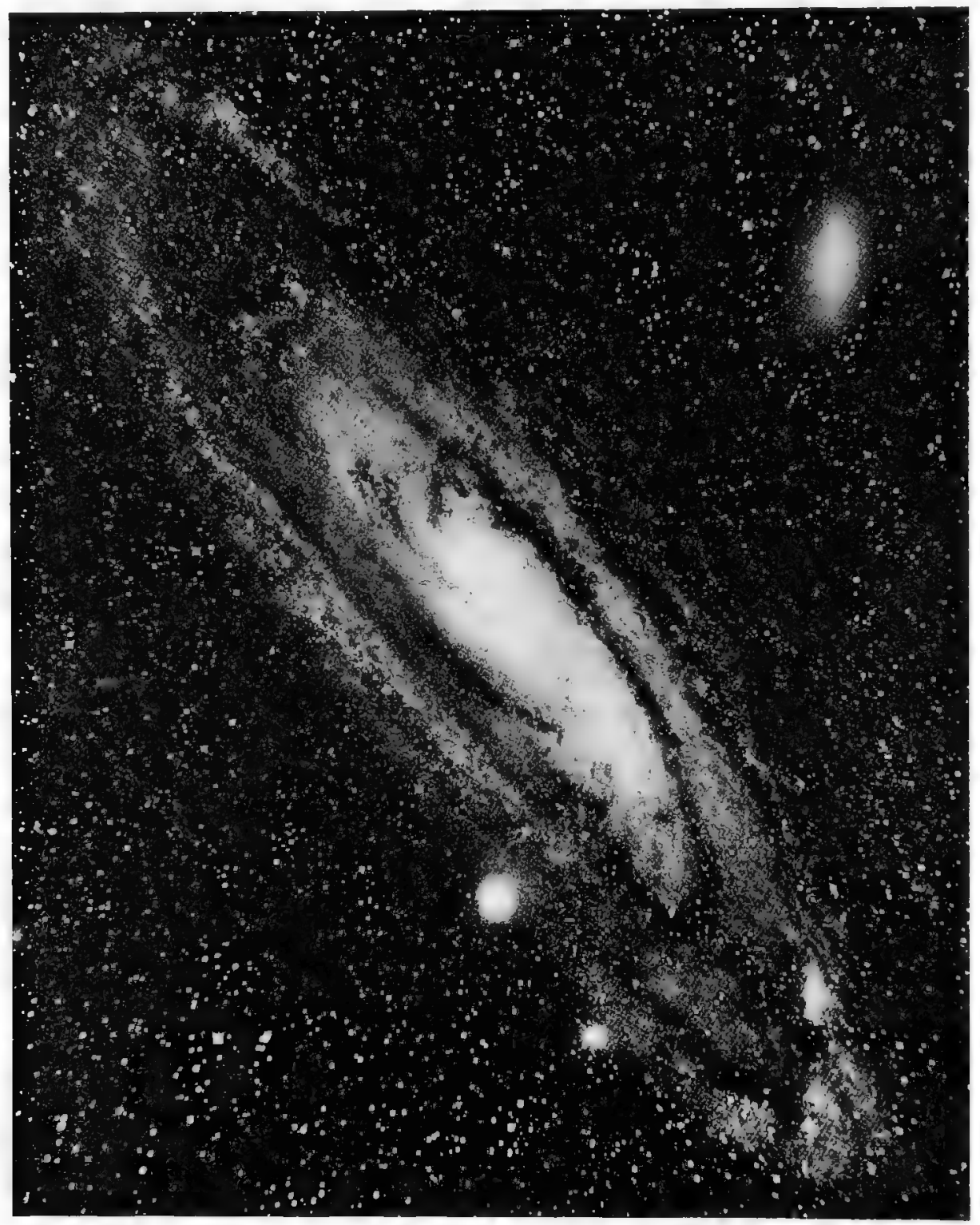

FIr. 6.-Photograph of the Great Nebula in the constellation of Andromedn, by Dr. Isaae Roberts, F.R.S. He describes it as Spiral Tebula M. 31, Androneda. The photograph was taken with a 20 -inch reflector with an exposure of ninety minutes on October 17, 1895, and covers the region between R. A. $0 \mathrm{~h}$. $34 \mathrm{~m} .0 \mathrm{~s}$. and R. A. 0h. 40m. 41s. The nebula, Dr. Roberts explains, fornis a left-handed spiral, and is better seen in the negative than in the photographic print.

matter so created gradually condensed unevenly here and there, each patch becoming a centre of attraction, drawing more and more material around it, the largest, of course, remaining approximately in the middle. In this way the central mass became the sun, and the smaller condensations resolved themselves into the planets. Visual demonstration of this daring theory was not long delayed. About the middle of last century Lord Rosse, with his huge telescope at Parsonstown in Ireland, discovered the first of the so-called spiral nebulx, and soon afterwards Sir William Huggins announced their gaseous character. Even then, however, their extraordinary nature was not fully realised, and it was only a short time ago that photography, supporting the joint testimony of the telescope 
and spectroscope, conclusively proved the correctness of Laplace's hypothesis." (For additional information on this subject, see the beginning of the present section-" Prevalence of spiral arrangements on a grand scale in the physical universe," p. 10.)

While the vast systems of spiral nebulæ, spiral cyclones, spiral sand-storms, spiral water-spouts, whirlpools, \&c., are on a scale of unexampled magnificence and splendour, there yet exist microscopic spiral arrangements in great plenty which are quite as interesting in a way to the chemist and physiologist as the others are to the astronomer and physicist. Examples of the minute spirals are to be found in crystals, plants, and animals; especially the two latter.

\section{§4. Origin of Spiral Structures.}

Stereo-chemistry has shown that "optically active substances may be divided into two classes. Some, like quartz, sodium chlorate, and benzil, produce rotation only when in the crystalline states; the dissolved (or fused) substances are inactive. Others, like oil of turpentine, camphor, and sugar, are optically active when in the liquid state or in solution. In the former case the molecules of the substance have no twisted structure, but they unite to form crystals having such a structure. As M. Pasteur expressed it, we may build up a spiral staircase-an asymmetric figure-from symmetric bricks; when the staircase is again resolved into its component bricks, the asymmetry disappears. In the case of compounds which are optically active in the liquid state, the twisted structure must be predicated of the molecules themselves, that is, there must be a twisted arrangement of the atoms which form these molecules."

Pasteur, in discussing the molecular constitution of tartaric acids, says that "the molecular structures of the two tartaric acids are asymmetric, and, on the other hand, that they are rigorously the same, with the sole difference of showing asymmetry in

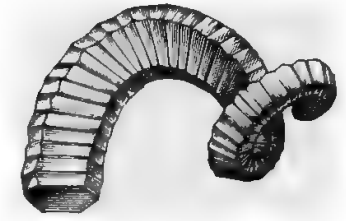

Fir.7.-.-Prouhlorite. Shows elegant spiral with transverse markings. (Fron "System of Mineralogy," by D. T. Dana, 1892.)

opposite senses. Are the atoms of the right acid grouped on the spirals of a right-handed helix, or placed on the solid angles of an irregular tetrahedron, or disposed according to some particular asymmetric grouping or other? We cannot answer these questions. But it cannot be a subject of doubt that there exists an arrangement of the atoms in an asymmetric order having a nonsuperposable image. It is not less certain that the atoms of the left acid realise precisely the asymmetric grouping which is the inverse of this." Pasteur regarded the formation of asymmetric organic compounds as the special prerogative of the living organism. "Most of the substances of which the animal and vegetable tissues are built up-the proteids, cellulose-are asymmetric organic compounds, displaying optical activity. . . Mesotartaric acid contains two equal and opposite asymmetric groups of atoms within its molecule." Pasteur was of opinion that compounds exhibiting optical activity were never obtained without the intervention of life. He also says: "Artificial products have no molecular asymmetry; and I could not point out the existence of any more profound distinction between the products formed under the influence of life, and all others." And, again, he refers to the molecular asymmetry of natural organic products as the great characteristic which establishes, perhaps, the only well-marked line of demarcation that can at present be drawn between the chemistry of dead matter and the chemistry of
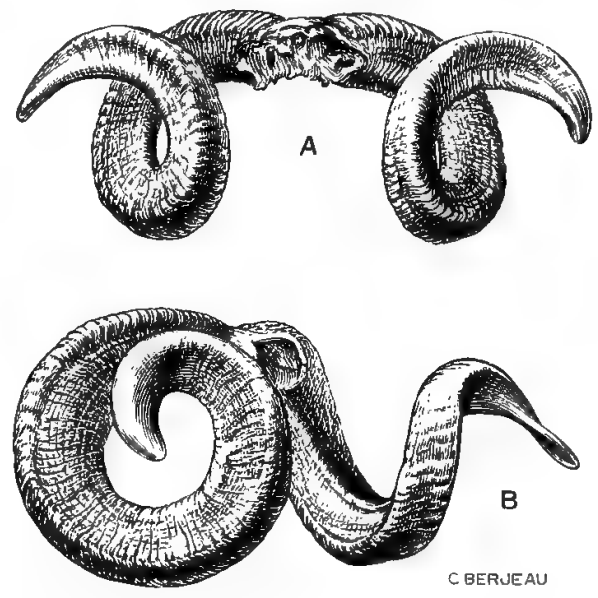

Fis. 8.-Photographs of rani's holns by the Author ; drawn on wood hy C. Berjean. A, front view; $B$, three quarter-view. Show hine right and lelt-handed spirals with transverse markings.

living matter. "Non-living, symmetric forces, therefore, acting on symmetric atoms or molecules, cannot produce asymmetry, since the simultaneous production of two opposite asymmetric halves is equivalent to the production of a symmetric whole, whether the two asymmetric halves be actually united in the same molecule, as in the case of meso-tartaric acid, or whether they exist as separate molecules, as in the left and right constituents of racemic acid. In any case, the symmetry of the whole is proved by its optical inactivity." 1

\section{§5. Examples of Spiral Arrangements in Crystals.}

Subjoined the reader will find a striking example of a spiral crystalline formation (Fig. 7) which greatly resembles a ram's horn (Fig. 8). Other examples of spiral crystals will be found at Plate ii., Fig. 23, p. 5.

1 "Stereo-Chemistry and Vitalism," by Professor F. R. Japp, as given in the "Report of the British Association for the Advancement of Science," 1898. 
It may be useful to give here a few illustrative examples of spiral formations in plants and animals.

As spiral plant and animal structures occur in great numbers throughout the work, it is only necessary, at this stage, to emphasise the fact that spiral arrangements are fundamental, and that they make their appearance at the very dawn of life, being found in seeds, seed-cases, flowers, fruit, adult plants and parts thereof; in the ova and spermatozoids of animals, in young and adult animals, and in many parts of animals, especially in the more complex and highly differentiated animals. Spiral movements are equally abundant. These make their appearance in the spores and seeds of plants which, in many cases, move and swim freely about until they find a suitable habitat, when they fix themselves permanently.

Fig. 9 gives an accurate representation of the spiral forms and movements of certain Bacteria.

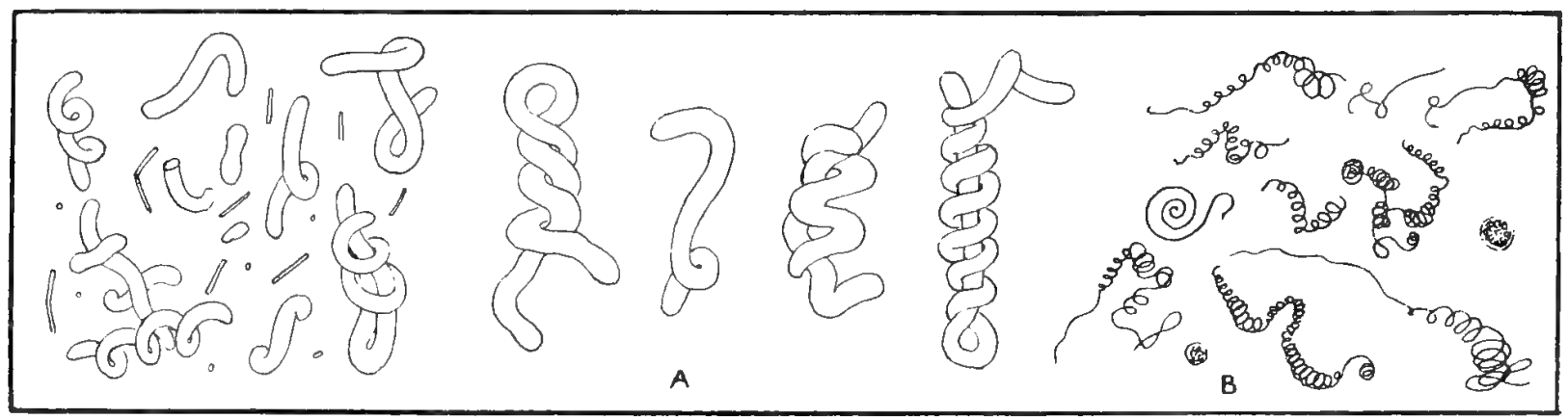

Fri, 9.--A, Butcrium vermiforme. B, Bacteria from the river Thames (Protchs type)-young colony from plate culture, greatly enlarged (Marshall Ward). At Figr. A, the spiral contortions and movements are very marked. At Fig. B, the spiral contortions pursue two directions and reverse, as in the tendrils of regetable marrow, the passion-flower, the eggr-purses of sharks, \&c: ; one part folming a right-lianded, the other a left-lianded spiral (the Anthor').

The spiral movements are also seen in Infusoria and low animal forms generally. They can be traced without difficulty from the lowest to the highest animals, and, incredible as it may appear, fishes swim, birds fly, and quadrupeds and bipeds walk, by double spiral or figure-of- 8 movements, as was demonstrated by me somewhat exhaustively in the years $1867,1868,1870$, and $1873 .^{1}$

\section{§6. Spiral Arrangements in Plants.}

The spiral arrangements in plants are at once striking and graceful, as the annexed illustrations show.
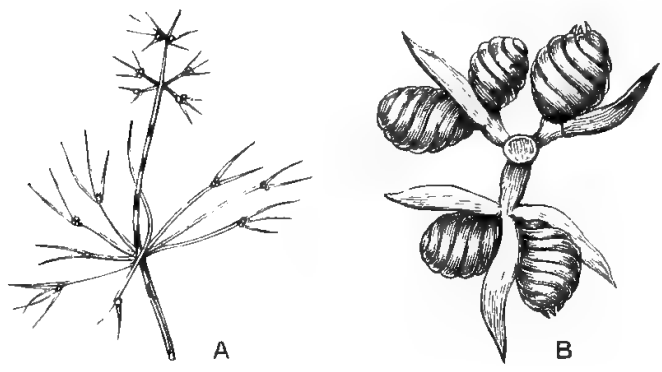

F11;. 10.

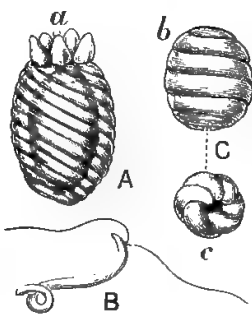

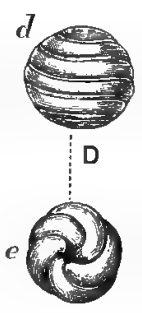

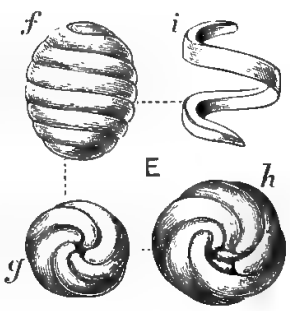

FI 1 . 11.

Fiu. 10. Chara elastica: recent. Italy.-

A. Sessile oogonia between the divisions of the leaves of the female plant.

B. Magnified transverse section of a branch, with five oogonia, seen from below (after Lyell).

FIC. 11.-

A. Oogonium of Chara entire, composed of five cells wound round a large central cell in a spiral manner, with corona (a).

b. Spiral spermatozoid of Chara separated from a cell. Shows two vibratile cilia (after J. H. Balfour).

C. Fossil carpogonia of Chara. b, Side view of Ghara Lemani, $\times 10$; c, under vjew, showing spiral arrangement of cells.

1). Clara midicagiumla, $\times 10$. $d$, Side view ; ', under view, showing spixul arrangement of cells.

E. Chara helicteris, $\times 10$. $f$, Side view; $g$, under view; and $h$, top view, showing spiral armengements of cells; $i$, one of the spiral cells detached (after Cuvier). The spirals shown at $\mathrm{D}$ and $\mathrm{E}$ of this figure resemble the spirals seen in nebulae (Plate viii.), certain shells (Plate xiii., Fig. 1 ,
$f, g$ ), certain cones (Plate xi, Fig. I, bottom row), and the apex of the heart (Plate xvii., Fig. 3, A). 1 Procedingris Royal Institution of Great Britain, 1867; Transactions Linnean Society, 1868; Transactions Royal Society, Edinburgh, I870;
Anglo-American Sricuce Series, 1873. 


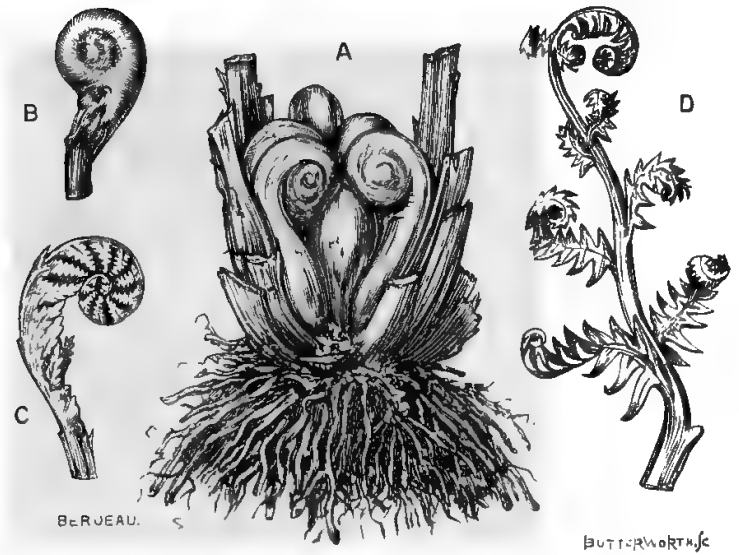

FIs. 12.

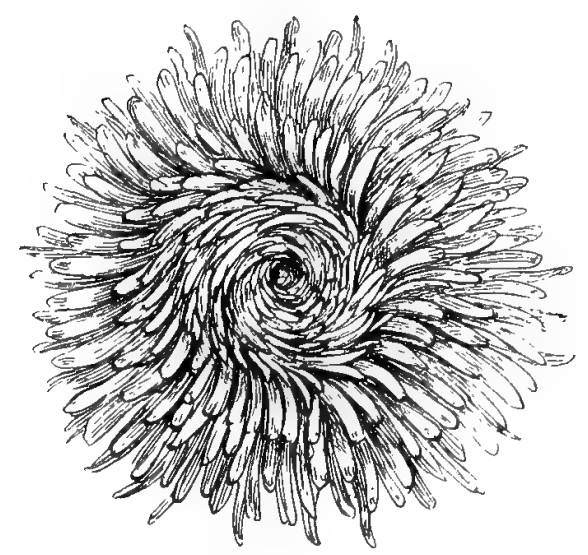

FIG. 13.

FIG. 12.-A, B, C, D. Spiral fronds of an actively growing fern. Drawn natural size by C. Berjeau from a fresh specimen collected by the Author. Shows right and left-handed spirals.

Fig. 13.-A spiral Chrysanthemum. Drawn from nature for the present work ljy C. Berjeau. Displays a left-handed spiral arrangement as seen in spiral nebulæ (Plate viii.), spiral seeds (Fig. 11, D, E), spiral spermatozoon (Plate xii., Fig. 1, E), spiral shell (Plate xiii., F, G), and the spiral apex of the heart (Plate xvii., Fig. 3, A).
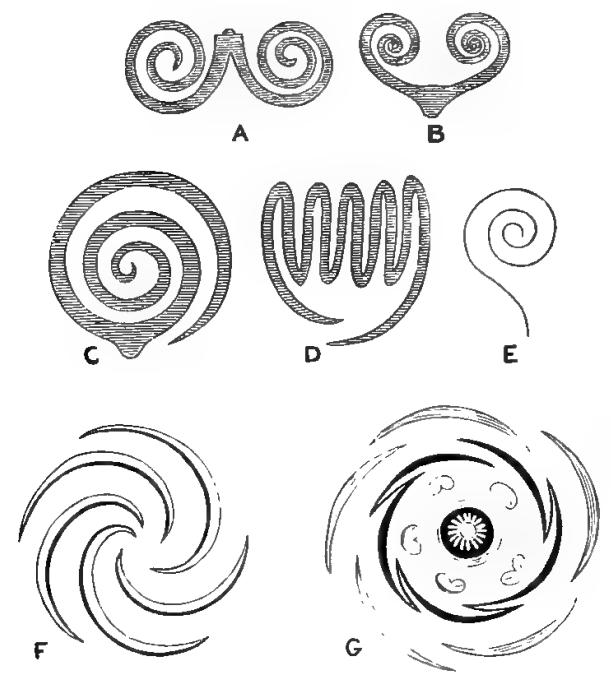

FIG. 14.
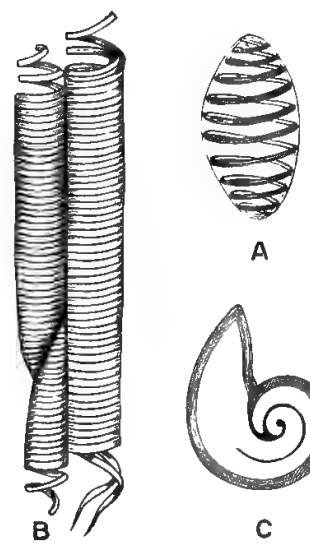

A

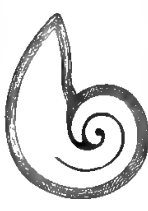

C

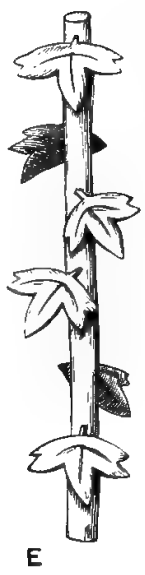

FIG. 15.

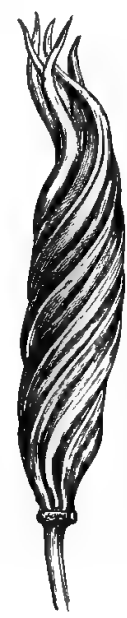

Fig. 16.

Figs. 14, 15, and 16 illustrate the spiral arrangements in leaves, flowers, and fruit; in cells and seeds; in vessels, climbing plants, \&c.

FIG. 14.-A. "Transverse section of a revolute leaf. The two edges are rolled outwards or away from the axis.

B. "Transverse section of an iuvolute leaf. The two elges are rolled inwards or towards the axis.

C. "Transverse section of a convolute leaf. The leaf is rolled upon itself so as to form a continuous coil.

D. "Transverse section of a plicate or plaited leaf. The parts of the leaf are folded together like a fan.

E. "Circinate vernation, in which the leaf is rolled up from apex to base, like a crosier.

F. "Diagrarn to illustrate contorted or twisted astivation, in which the parts of the whorl are overlapped by each other in turn, and are twisted on their axes as in the mallows.

G. "Diagram of the flower of the Sowbread (Cyclamen) showing the five sepals overlapping each other, and five petals arranged in a contortive manner, five stamens, and the pistil in the centre" (Professor J. Hutton Balfour).

Fig. 15.-A. "Spiral cell, or cell with a spiral fibre inside, from an orchid.

B. "Spiral vessels taken from the melon, showing the elastic filres uncoiled.

C. "Vertical section of the seed of Bunias, with its spiral embryo. The cotyledons are rolled upon the radicle in a spiral manner, hence the name spirolobex.

D. "Exogenous stem, surrounded by a woorly climbing plant (Bush rope) which causes contractions ind swellings of the stem.

E. "A stem with alternate spiral leaves arranged in a '[uincuncial manner. The sixth leaf is directly above the first, and commences the second spiral cycle" (Professor J. Hutton Balfour).

FIG. 16.-Spiral fruit of Helicteres Isora. Forms right-handed spiral (after Baillon). 
PLATE IX

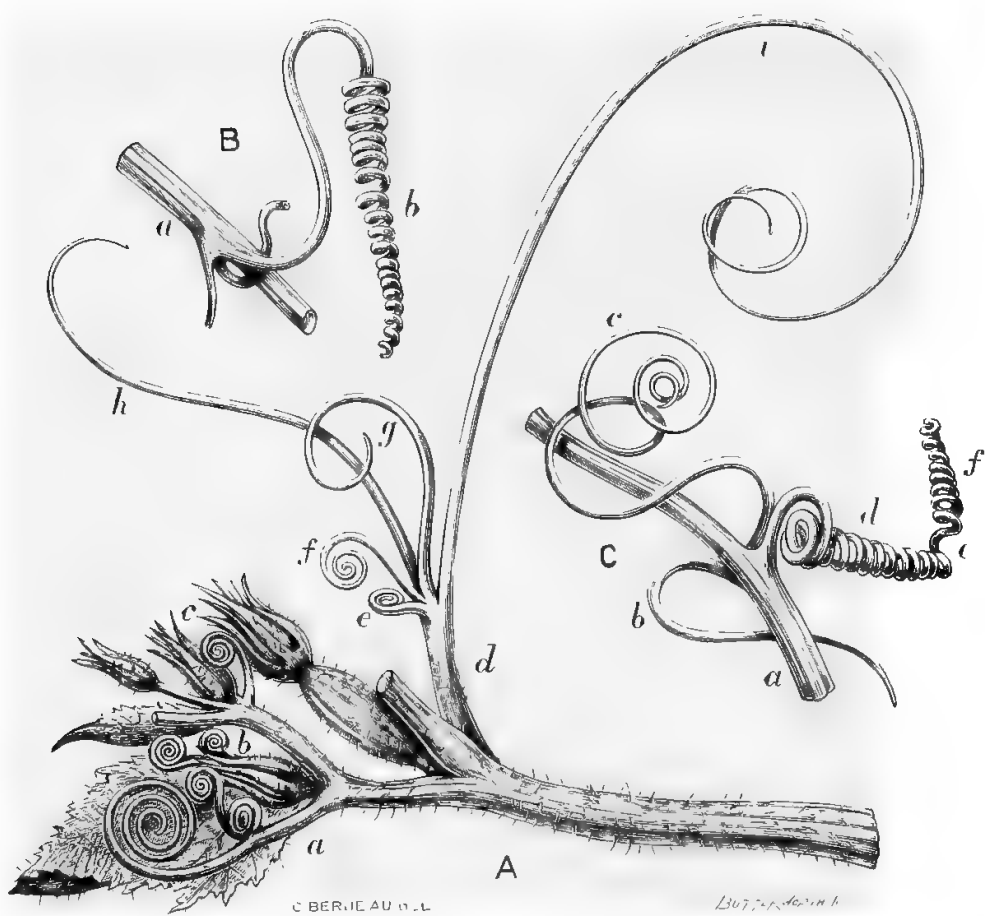

FII: 1.

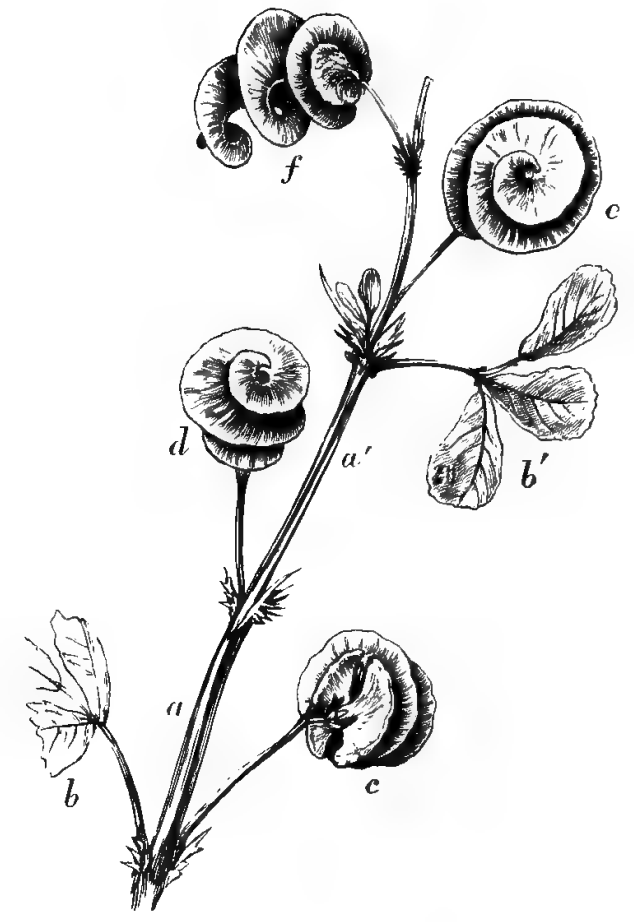

FII i : 2.
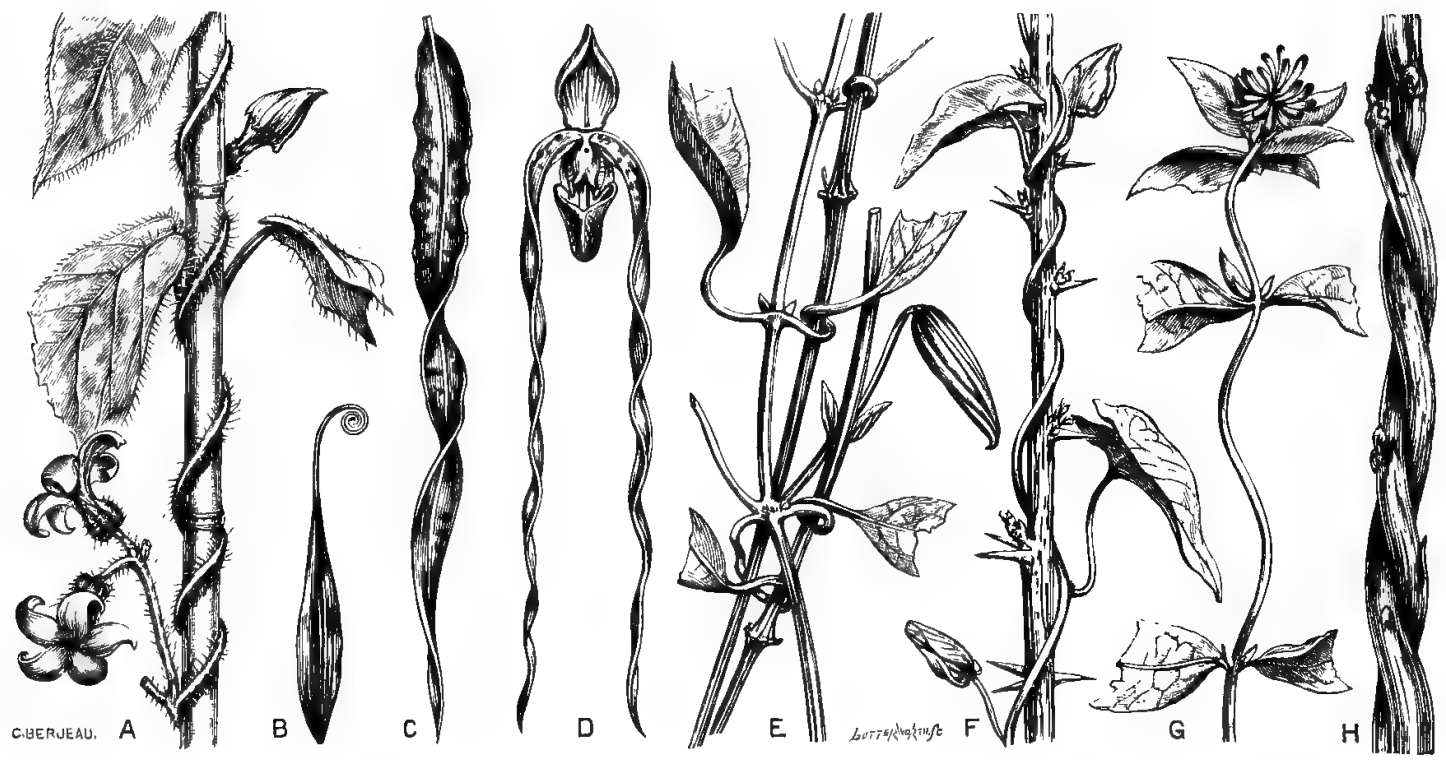

Fur. 3.

Plate ix. illustrates spiral formations in plants.

Fig. 1. - Terminal stem and shoot of vegetable marrow with tendrils in various stages of derelopment, and furming single right and left-handed spirals, and one double or reversing spiral.

A. $a, b, c$, three sets of young tendrils closely coiled up and forming right and left-handed spirals; $d$, peduncle bearing five tendrils in varisus stiges of development; $e$, very young tendril closely coiled and forming a left-handed spiral ; $f$, young tendril uncoiling and forning a right-handed spiral; $g$, young tendril uncoiling and forming a right-handed spiral; $h$, young tendril uncoiled and leginning to reverse its spiral; $i$, mature tendril re-coiling and forming a lelt-handed spiral. The tendrils $e, f, g, h, i$ have not tonched or caught hold of anything, and are therefore natural spiral formations. 


\section{SPIRAL ARRANGEMENTS IN PLANTS}

\section{PLATE IX (continued)}

B. Stem, $a$, of vegetable marrow with three tendrils; $b$, mature tendril growing freely in slace whith has coiled upon itself to form a single cone-shaped left-handed spiral.

C. Stem, $a$, of vegetable marrow having three tendrils, $b, c, d ; b$, young tendril which has uncoiled and straightened itself; $c$, mature tendril in the act of re-coiling and forming a left-handed spiral ; $d, e, f$, mature tendril which has re-coiled and forms a double or reversing spiral: at $d$, the spiral is left-handed; at $e$, the spiral is reversing, and at $f$, it is right-handed. The power possessed by plants of forming right and left-handed and double or reversing spirals is a feature of extrundinary interest, as proving original endowment. In Fig. C, as in Figs. A and B, the tendrils are growing freely in space: the single right and left-handed and double reversing spirals are therefore natural formations, and are not aue to inherent irritability or stimulation caused hy coming in eontact with anything living or dead. Similar remirrss are to be made of animal tissues : nusciles, nerves, lones, horns, claws, teeth, shells, \&c., all assume spiral forms quite apart from either irritition or stimulation.

Drawn three-fourths natural size, by C. Berjean from fresh specimens collected by the Author.

FIr. 2.--Species of Medicago. Shows flattened spiral pods (legume); at', spiral sten, $b h^{\prime}$, leaves; $c$, back view of spiral porl $d$, side view of pod; $e$, frout view of lod; $f$, pod opened out, seen from behind and laterally. Here thute can be no question as to inhereut spiral endowment, as the pods are not, and never have been, in contact with anything hut air. Irritability ind contact with foreign bodies take no part in the spiral formations. Drawn natural size, hy C. Berjeau from fresh specinen collected by the Author at Ronda, Spain.

FIG. 3.-Shows striking examples of spiral formation in plants.

A. Schuhertia (I'hysianthus). Stem forming right-handed spiral.

B. Cloriosa superba. Leaf terminating in spiral tendril.

C. Croton ('odizum). Leaf forming right-handed spiral.

D. Orchid (Cypripedium). Right-handed spiral.

E. Clematis (C. inontana). Leaf stalks form right and left spirmls round alien stems.

F. Convolvulus (C. arrnsis), forming right-handed spiral round gooseberry twig.

G. Honeysuckle (Lonicere Prirlymentum), winding from left to right.

H. Two honeysuckle stems entwined and forming left-handed spiral as in the human umbilical cord (Plate xii., Fig. 3, 1. 27 ), and spiral intestine (Plate xii., Fig, 4).

Drawn by C. Berjeau from fresh specimens collected by the Anthor.

\section{PLATE $\mathrm{X}$}

Plate $\mathrm{x}$. illustrates spiral formations in the stems, tendrils, and fruit of plants.

FIG. 1.-Spiral stems and tendrils of the passion-flower (Passiflora alata). Shows right and left-handed spirals, double reversing spirals, and spiral tangles.

A. $a$, Stem forming right-handed spiral; $b$, revolving summit of passion-flower with young coiling tendrils; $c, i$, $c$, mature tendrils forming single and double reversing spirals.

B. $d, e$, Stem of passion-flower twisting from left to right and forming right-lianded spiral; $f, f$, donble reversing spiral and spiral tangle; $g, h$, typical example of double reversing spiral tendril.

C. $i, j$, Right-handed spiral formed by stem of passion-flower $; k, l, m, n, n, p$, well-marked examples of donlule reversing spiral tendrils. The stems and tendrils of the passion-flower are natural spiral formations. The nature and degree of spirality exhibited by them are due to inherent endowment and not to irritability and external stimulation. This follows because the stems and tendrils curve and twist when not in contact with anything save the air. The tendrils even reverse their spirals when similarly circumstanced.

Drawn by C. Berjean from fresh specimens collected by the Author.

FIG. 2.-Specimens of fresh hops (Humilus Lupullus) sent to the Author from Kent. Drawn by ('. Berjeau.

A. Spiral bundle of hop stems. The stems twine into each other and form left-handed spirals.

B. Single hop stem forming a left-handed spiral.

C. Another portion of the same stem forming a right-lianded spiral. It is not possible that any form of extraneons stimulation could produce right and left-handed spirals in the same stem. This can only be referred to design and original endownent.

FIG. 3.-A. Spiral stems of Tecoma (Bignonia) twisting and forming a right-handed spiral.

B. Sprig of Sweet Pea (Lathyrus odoratus) with tendrils forming right and left-handed spirals.

C. Stems of Asparagus (Asparagus plumosus) twisting spirally into each other. The twist is in an olposite direction to that figured at $\mathbf{A}$. They form a left-handed spiral.

Drawn hy C. Berjeau from fresh specimens collected by the Autlor.

FIG. 4.-A, B. Diagrams illustrating the spiral arrangements of the leaves and fruit of a plant. At A the divergence between every

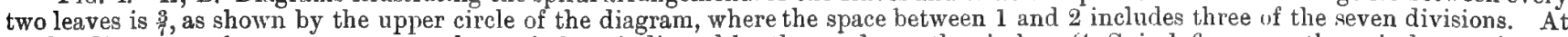
B, the divergence between every two leaves is $\frac{1}{t}$, as indicated by the marks on the circle. (c. Spirnl fir cone-the spirals running in two principal directions, as shown by the darts; the one spiral being more vertical than the other. The secondary spirals indicate the difference between each scale of the cone in a single spiral. The cone is composed of numerous scales, which are metamorphosed leaves arranged on a common axis and covering the seeds (after J. H. Balfour). 
PLATE X
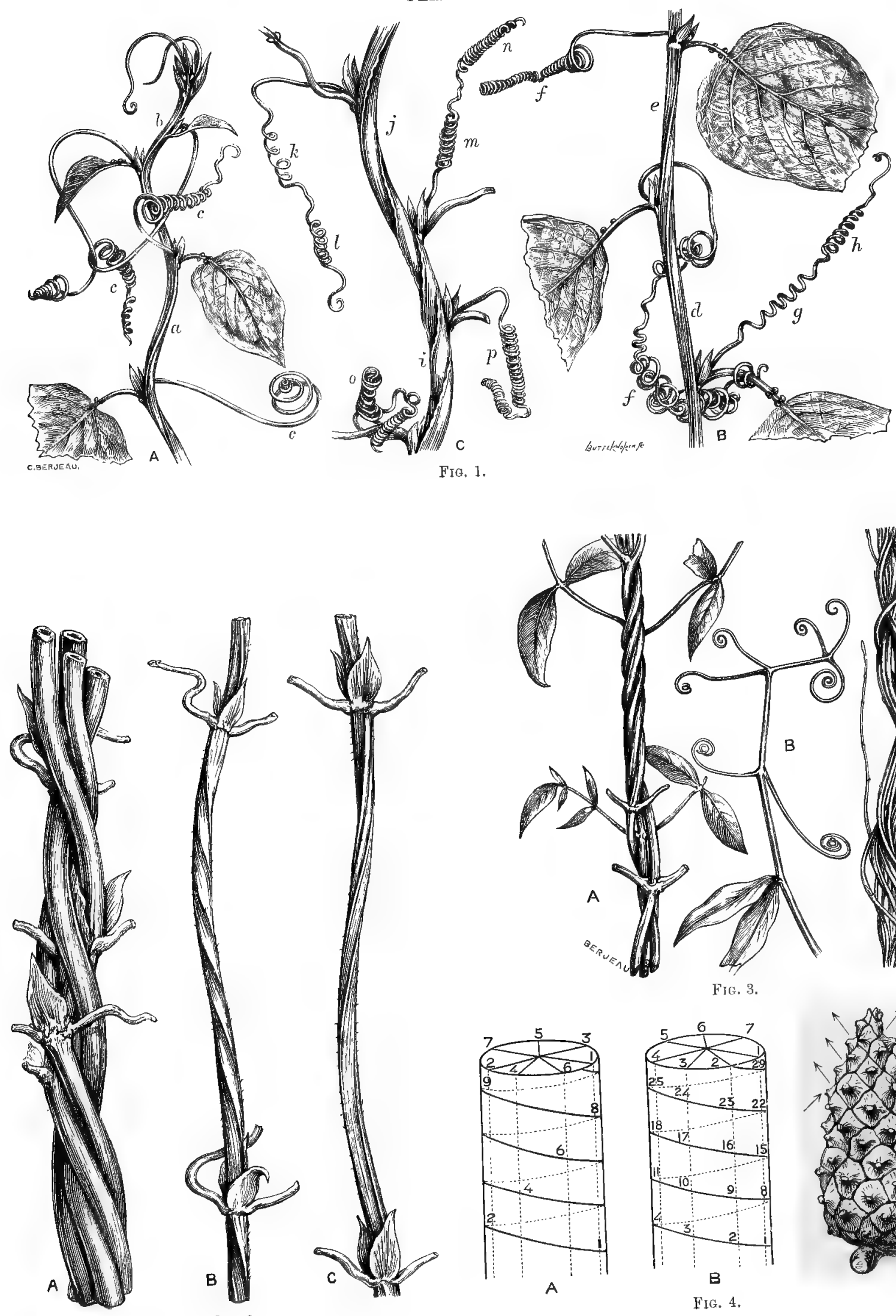

Fig. 2.
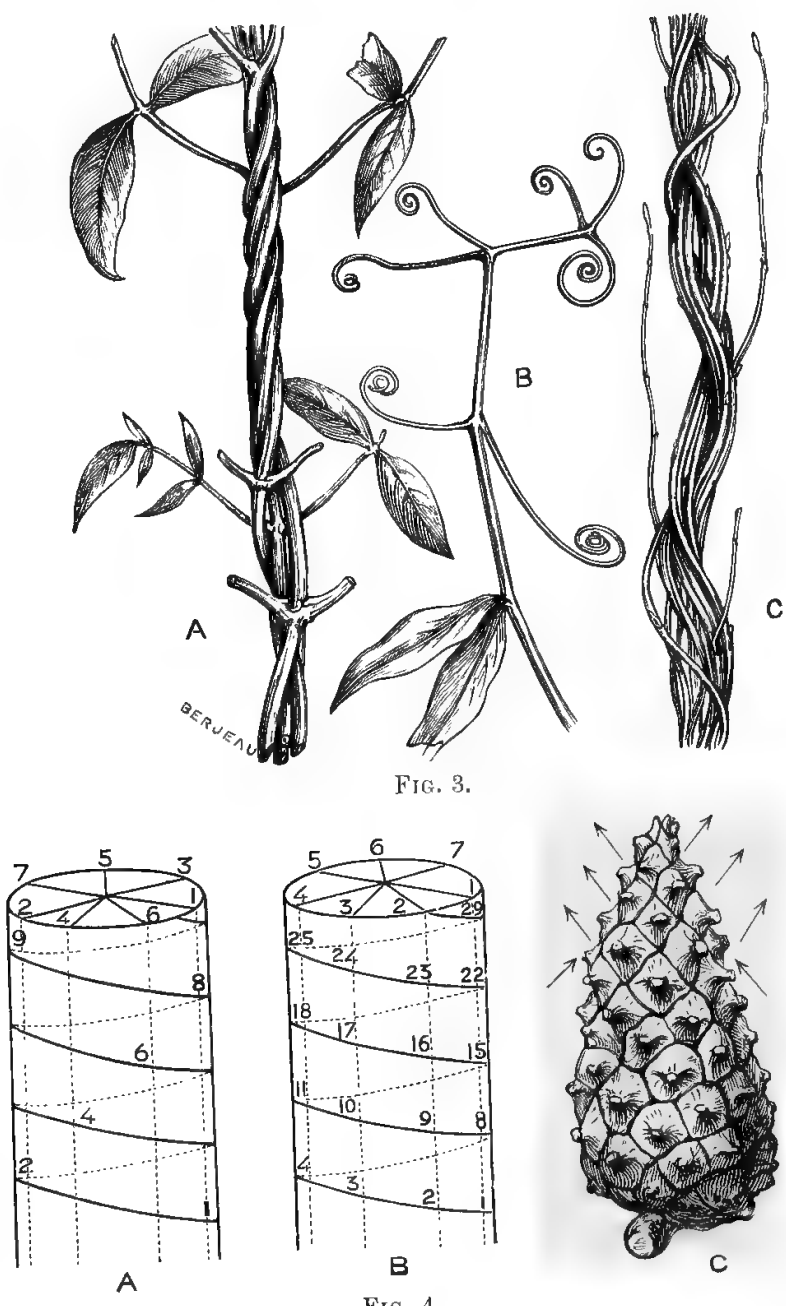

FIG. 4. 

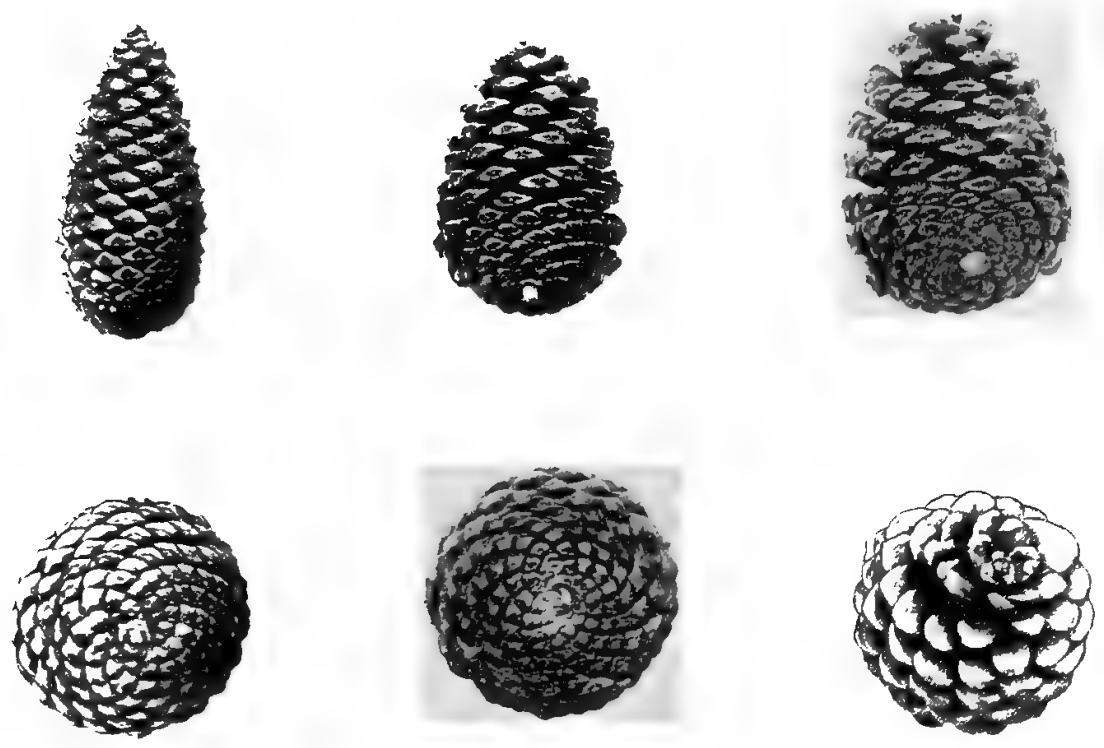

Fis. 1.

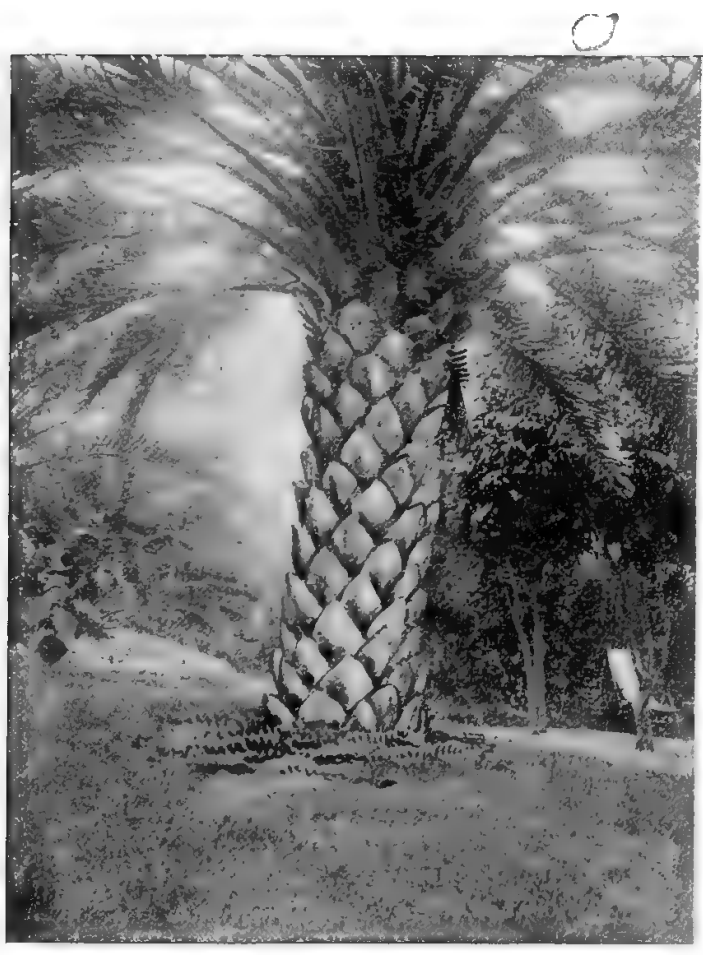

FIs: 2.

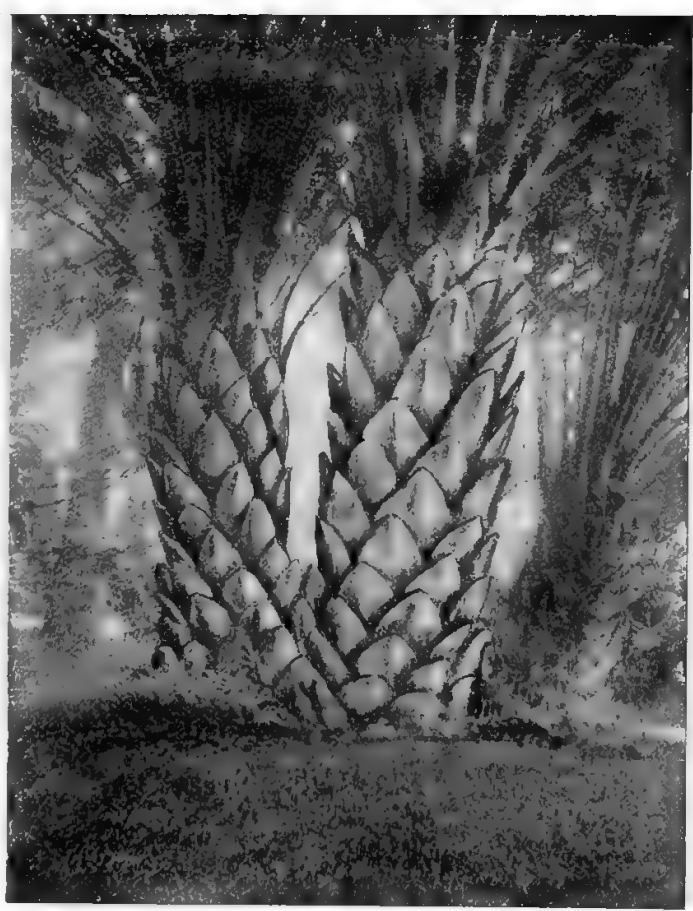

$F_{I T} \div 3$.

Plate xi. illustrates spiral formations in fir cones and palm trees.

FIg. 1. - Cones of Pinus maritina, collected by the Author at San Romolo, Italy. The upper left cone (lateral view) shows double spiral arrangement of scales The upper central cone shows a beautiful right-handed spiral, the upper right cone showing a beantiful left-handed spiral. These two cones are weathered and the scales opened out by exposure. The lower left and central cones (basal end views) show the right and left-handed spirals emerging from the axis of stalk of the cone and producing perfect symmetry (compare

VOL. I. 
PLATE XI (continued)

with $\mathrm{D}, \mathrm{E}$ of Fig. 11, 1, 20). The lower right cone (npicial view) shows the double spiral arrangement in a weathered specimen, where the scales of the cone are opened ont or separated. From photographs taken for the Author by his nephew, J. Bell Pettigrew.

FIG. 2.-Date paln growing at San Remo, Italy, photographed for the Author by John A. McMordie. Shows double spiral stem, the right spiral being the more vertical and more pronounced.

FIG. 3.-Date palms growing near that represented at Fig. 2, and photographed for the Author by John A. McMordie, at the same time. In the palm to the left, the left spiral is the more vertical and more strongly marked; in the right, the right spiral is the more vertical and more strongly pronounced. Petter examples of right and left-handed leading spirals caunot be inagined.

\section{§7. Spiral Arrangements in Animals.}

The spiral arrangements in animals are, if possible, more remarkable than those witnessed in plants. In animals the hardest as well as the softest tissues assume the spiral form. The spermatozoa, the ova, the muscles, nerves, feathers, shells, horns, bones, and teeth all attest the prevalence of the spiral as a factor in organisation. Nothing short of design and pre-arrangement and fundamental bias can account for the occurrence of spiral arrangements in such a great variety of substances. The presence of spirals in great numbers in plants where there is comparatively little differentiation would have been sufficiently striking, but when we find them in great abundance in the tissues of all animals, even the highest and most complex, we are forced to look for a cause, and to remove them from the category of chance formations. We are obliged to fall back upon Design and Law and Order. The necessity for this procedure becomes imperative when it is remembered that spiral arrangements are not confined to plants and animals, but are also found in molecules, crystals, nebulæ, cyclones, sand-storms, water-spouts, whirlpools, \&c.

The spiral formations in animals are not only outstanding and typical but they are bewildering as regards variety and detail. It would be easy to adduce any number in corroboration, but it will suffice for the present if I give sufficient to illustrate the general principle, which I do in the following ten plates (xii. to xxi. inclusive). Further illustrations of an important kind will be found in the body of the work.

\section{PLATE XII}

Plate xii. illustrates spiral formations and structures in spermatozoids, umbilical cord, intestine, and nerve cells.

FIG. 1. Spiral spermatozoon of Crayfish (Astacus fluviatilis) (after Huxley), $\times 850$.

A, B, C, D. Different stages in the development of a spermato\%orn from a seminal cell.

E. A mature spermatoznon-front view. The mature spermatozon (E) consists of a right and left set of radiating spiral elements seen in the uncoiled state at $\mathrm{C}$. The spermatozoon is bi-laterally symmetrical, and bears a remarkable resemblance to the two sets of spiral nebule seen at Plate viii., p. 17; to the two spiral shells seen at Plate xiii., Fig. 1, D, E; and to the spiral apex of the heart, seen at Plate xvii, Fig. 3, A. The spermatozoon is an embodinent of evolving spiral force (the Author).

FIG. 2. Spiral spermatozoa of various kinds.

A. Spermatozoon of Triton (Triton cristatus), $\times 450$.

B. Spermatozoa of labbit (Lepres cuniculus), $\times 450$.

C. Spermatozoon of field mouse (Avicola crverlis), $\times 450$.

D. Spermatozoon of wood shrike (Lanizes rufus), $\times 450$.

E. Spermatozoon of goldfinch (Frimilla elegans), $\times 450$.

K. Spermatic cyst of common creeper bird (Certhia familiaris), containing a bundle of spermatozoa, $\times 500$.

L. Slermatic cyst of rahbit. "The globules, each of which contrins a spermato\%oon, $\times 350 ; b$, separate globuli, $\times 500$ (after Griffith and Henfrey).

FIG. 3.-Human umbilical cord, composed of one vein $(a)$ and two arteries $(b)$, intertwining to form a symmetrical left-handed spiral. Resembles spiral water-spont (Fig. 5), and the twining stems of the hop (Plate x., Fig. 2, A). Two spirals at least are necessary to [roduce symmetry, structures composed of one spiral being incomplete or lop-sided. Drawn from injected specimen in the possession of the Author by C. Berjeau.

FIG. 4.-Sipmulus nudus laid open from the side, showing left-handed spiral intestine (a); $b$, anus (after W. Keferstein). Resemhles spiral umbilical cord (Fig. 3 of this Plate), and spiral hop stems (Plate x., Fig. 2, A, B).

Fra. 5.- A. Ganglion cell of a frog, with right-handed spiral nerve fibres, magnified. $a$, $a$, Straight fibre; $b$, large coiling fibre; $r$, small coiling fibre (after Lione] S. Beale). Resembles twining plants (Plate $x$, Fig. 3, A).

B. Ganglion cell from the sympathetic system of the frog, with left-handed spiral nerve filıe magnifier. a, Straight fibre; $b$, coiling fibre, arising by a superficial netting connected with the nucleolus of the cell; $c, c$, capsule with nuclei (after J. Arnold).
Resembles twining plants (Plate ix., Fig. 3, A and F, p. 22). 
PLATE XIL

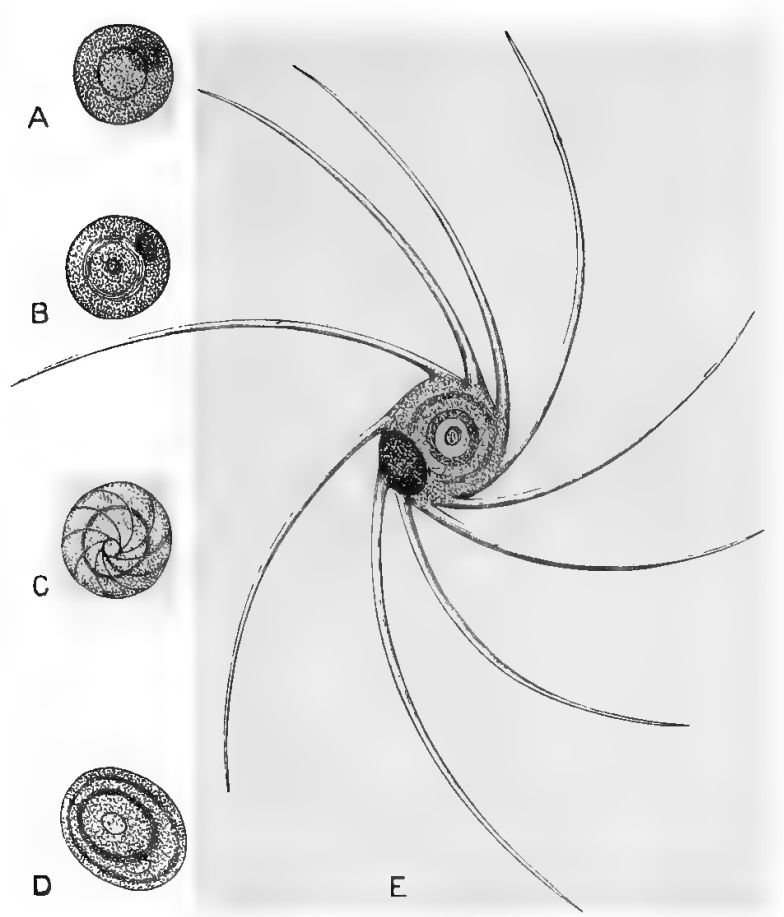

FIG. 1.

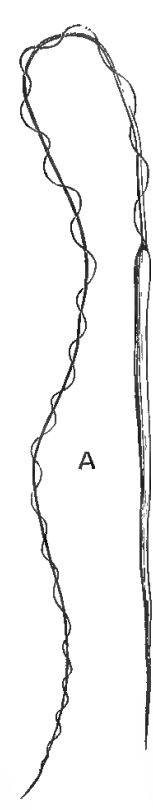

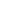

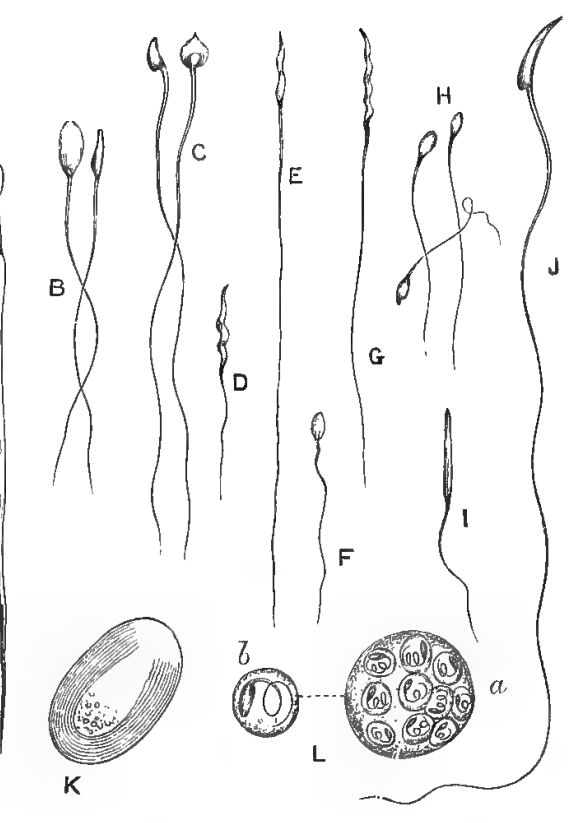

FIG. 2.

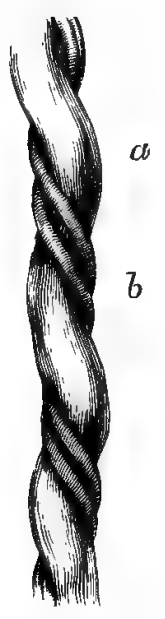

FIG. 3.

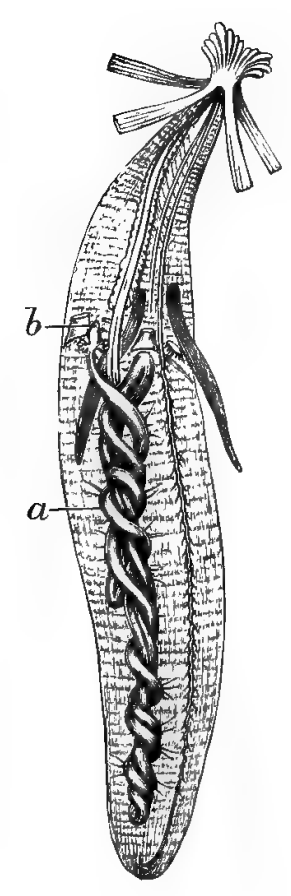

FIG. 4.

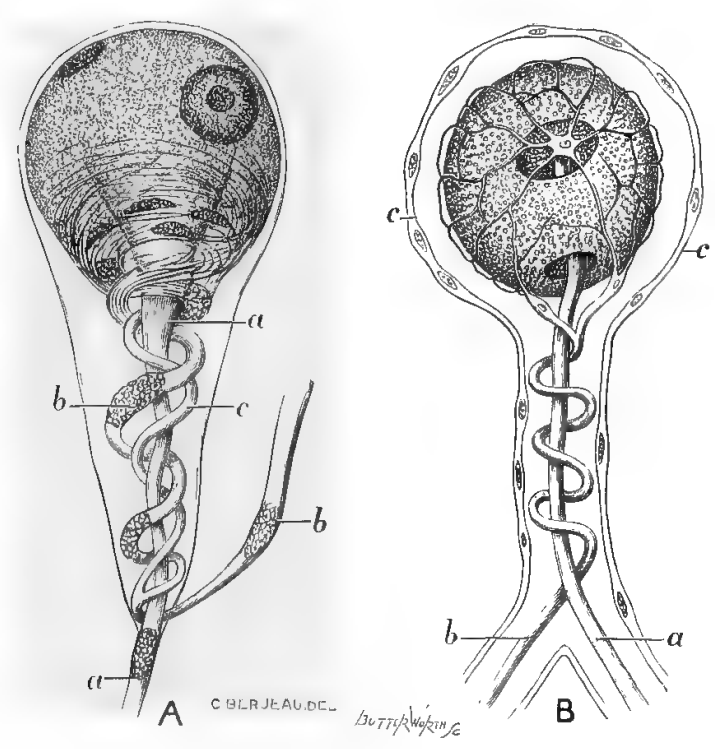

Fit. 5. 
PLATE XIII

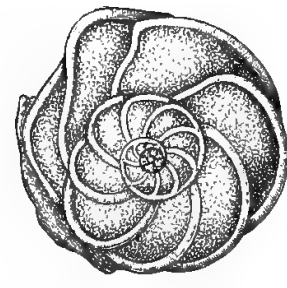

A

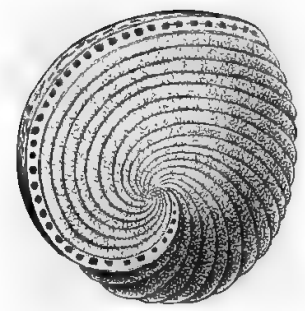

C.Berueaul E

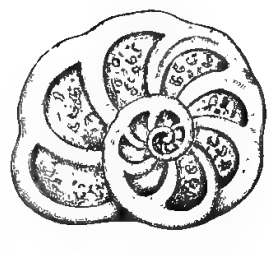

B

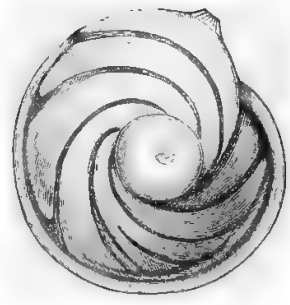

F
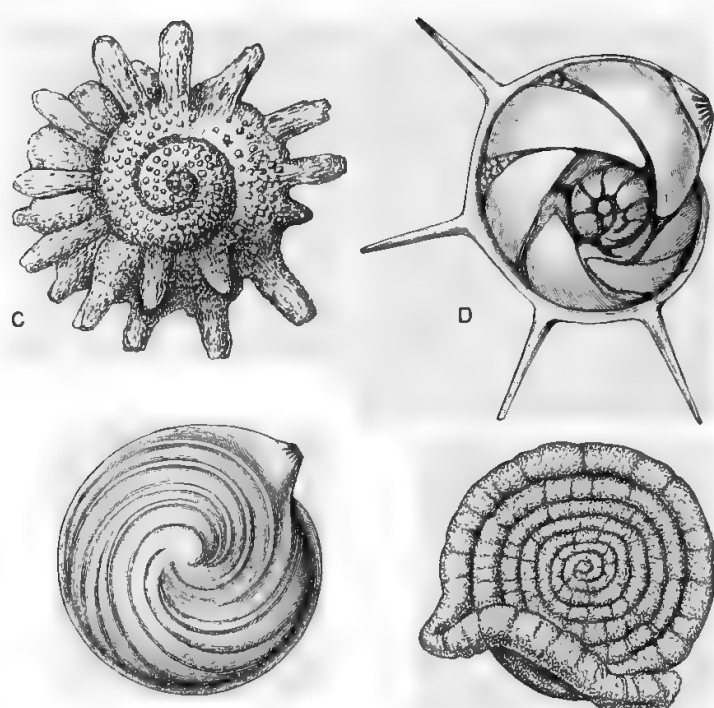

G

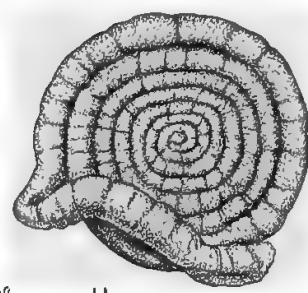

$\mathrm{H}$

Fra. 1.
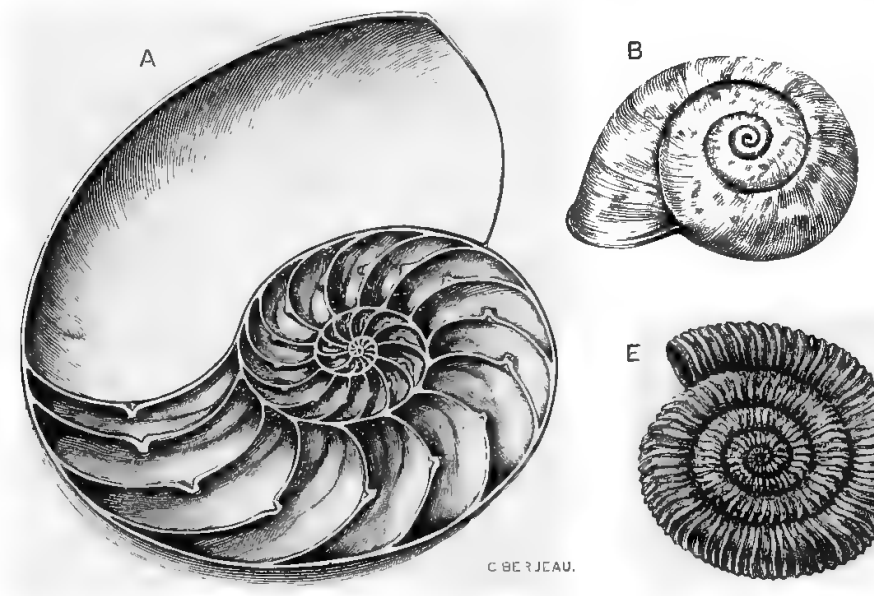

C

D
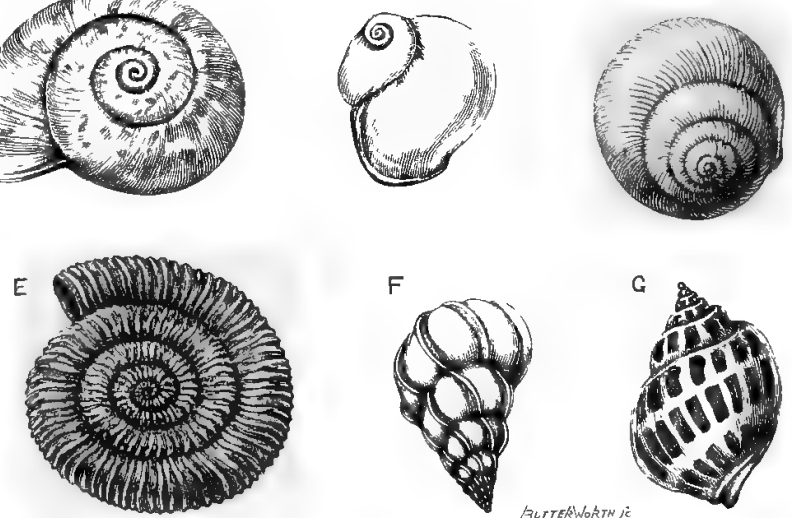

F
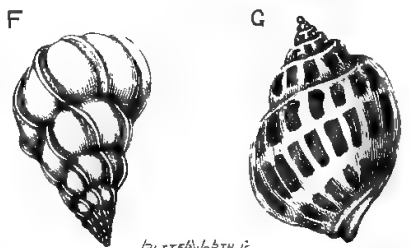

FiG. 2.

Plate xiii. illustrates spiral formations in shells.

FIG. 1. - Spiral Foraminilela after Brady, challenyer Reports, vol. ix. In estimating the direction of the spirals in these shells, the spirals are traced from without inwards, ol from base to a] iex (the Author).

A. Discorbina eximia, $\times 35$. Chamlord right-handed spiral shells.

C. Calcavina defrancii, $\times 20$. Spiculated left-handed spiral shell.

D. Cristelleria calcar, $\times 3$. . Curious plicated left-handed spiral shell.

E. Orbiculina aduma, $\times 20$. Syumetrical shell with left-handed spiral whorl at apex. Resembles spiral nebulæ (Plate viii.), certain spiral cones (PJate xi., Fig. 1, lower low), and spiral cast of heart (Plate xvii., Fig. 3, B).

F. Cristelleria orbicularis, $\times 20$.

G. Cristellaria vorter, $\times 20 . \quad F$ and $G$ are symmetrical shells with right-handed spiral whorls at apex. They resemble nebula (Plate viii., Fig. 4); spiral seeds (Fig. 11, D, E); spiral spermatozoon (Plate xii., Fig. 1, E); thd the spiral apex of the heart (Plate xvii., Fig. 3, A).

H. Ammoliscus tenuis, $\times 12$. ('renated right-landed spirnl shell with many and fune convolutions.

FIG. 2.-A. Mesial line section of shell of Nautilus (Nantilus pompitius). Forms an expuisite left-handed spiral, with beautiful curved partitions becoming smaller as the apex of the shell is approached. Nothing cun exceed the grace and betuty of the section here exhibited.

B. Rhyssota brookei. Forms right-lianded spiral.

C. Acavus phunix (white variety). Forms left-handed spiral.

D. Strophocheilus (Borus) oblongus. Forms left-handed spiral.

E. Colocerce (Ammonites) communis. Fossil, Upper Lias formation. J. Scoresby. Forns a striking, closely-coiled, right-handed spiral.

F. Scalaria scalaris. Forms a beautiful pyramidal, spirally-oruamented, left-handerd spiral.

G. Eburna spirate. Forms a finely-marked left-handed spiral.

Drawn by C. Berjean from lihotographs lyy the Author. 


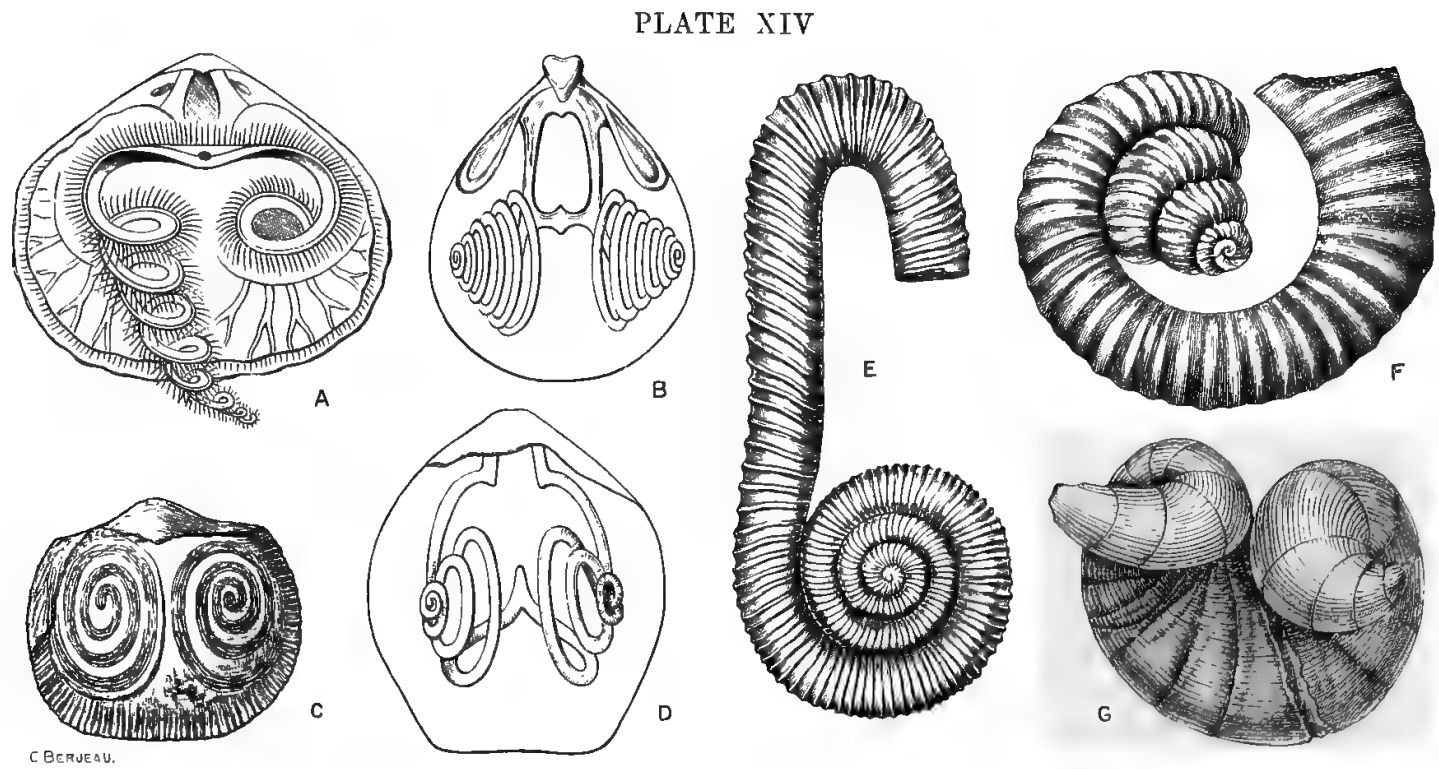

FIG. 1.
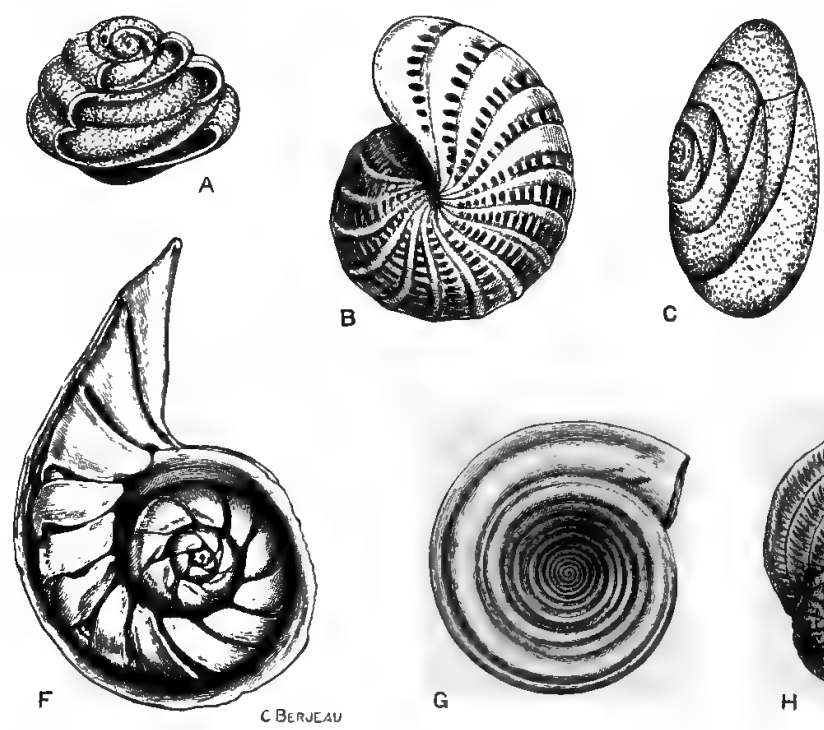

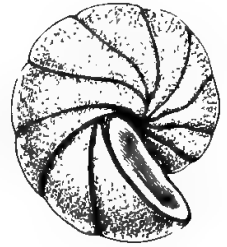

D

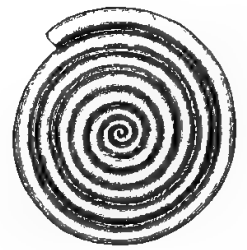

E
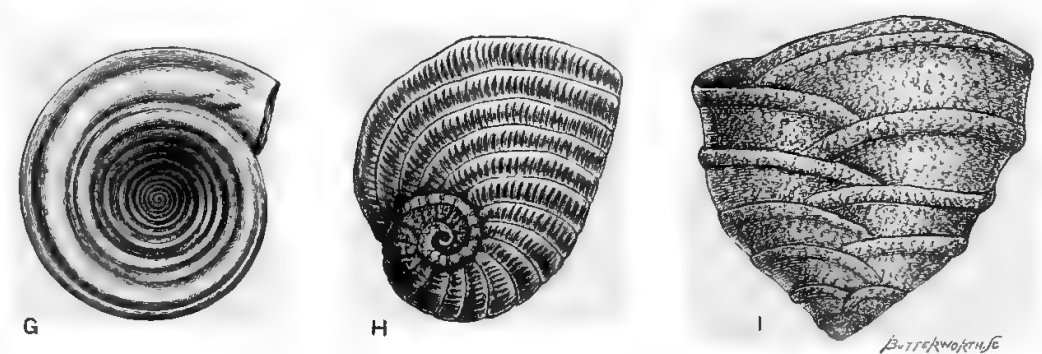

Fis: : 2.

Plate xiv. illustrates spiral configuration of shells.

Fig. 1.-A. Rhynchonella puttucen. Shows interior of dorsal valve. The spirml labial alpendage at the right of the figure is coiled and occupies its normal position : the spiral labial appendage at the left side is uncoiled and displaced (after Javidson).

B. Restored interior of dorsal valve of Uncites gryphus from the Middle Devonian, showing berutitul right and left-handed spirals (after Davidson).

C. Koninckina leonhardi from the Trias of St. Cassian, enlargerd. Displays right and left-handed brachial processes (after Zittel).

D. Interior of the dorsal valve of Dayia nuvicula, Silurian, enlarged (after Davidson). Repeats the right and left-handed spiral arrangements seen in $\mathrm{A}, \mathrm{B}$, and $\mathrm{C}$.

E. Macroscaphites ivanii. Cretaceous (Neocomian).

F. Heteroceras emerici. Cretaceous.

G. Diceras arietina. Upper Jurassic.

Figs. E, F, and $G$ display unusual spiral forms, $E$ forming a left and $F$ a right-handed spiral, and $G$ a right and left-handed spiral united. The number and variety of spirals in shells is incredibly great.

FIG. 2.-Spiral Foraminifera after Brady. Challenger Reports. Zoology, vol. ix. These shells supply beautiful examples of right and left-handed spirals : also plication and symmetric markings.
A. Ammodiscus charoides, $\times 70$.
D. Cyclammina orlicularis, $\times 15$.
B. Polystomella macella, $\times 35$.
E. Spirillina limbata, $\times 60$.
C. Chilostomella ovoidea, $\times 70$.
F. Cristellaria variabilis, $\times 50$.
G. Cornuspira carinata, $\times 30$.
H. Peneroplis pertusus, $\times 35$
1. Textularia trochus, $\times 25$. 

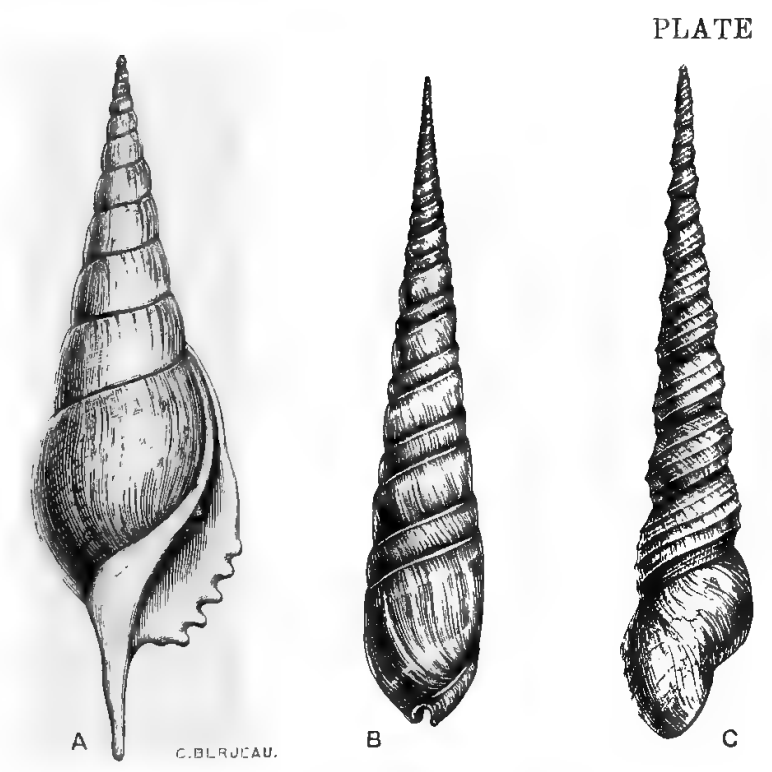

Fig. 1.
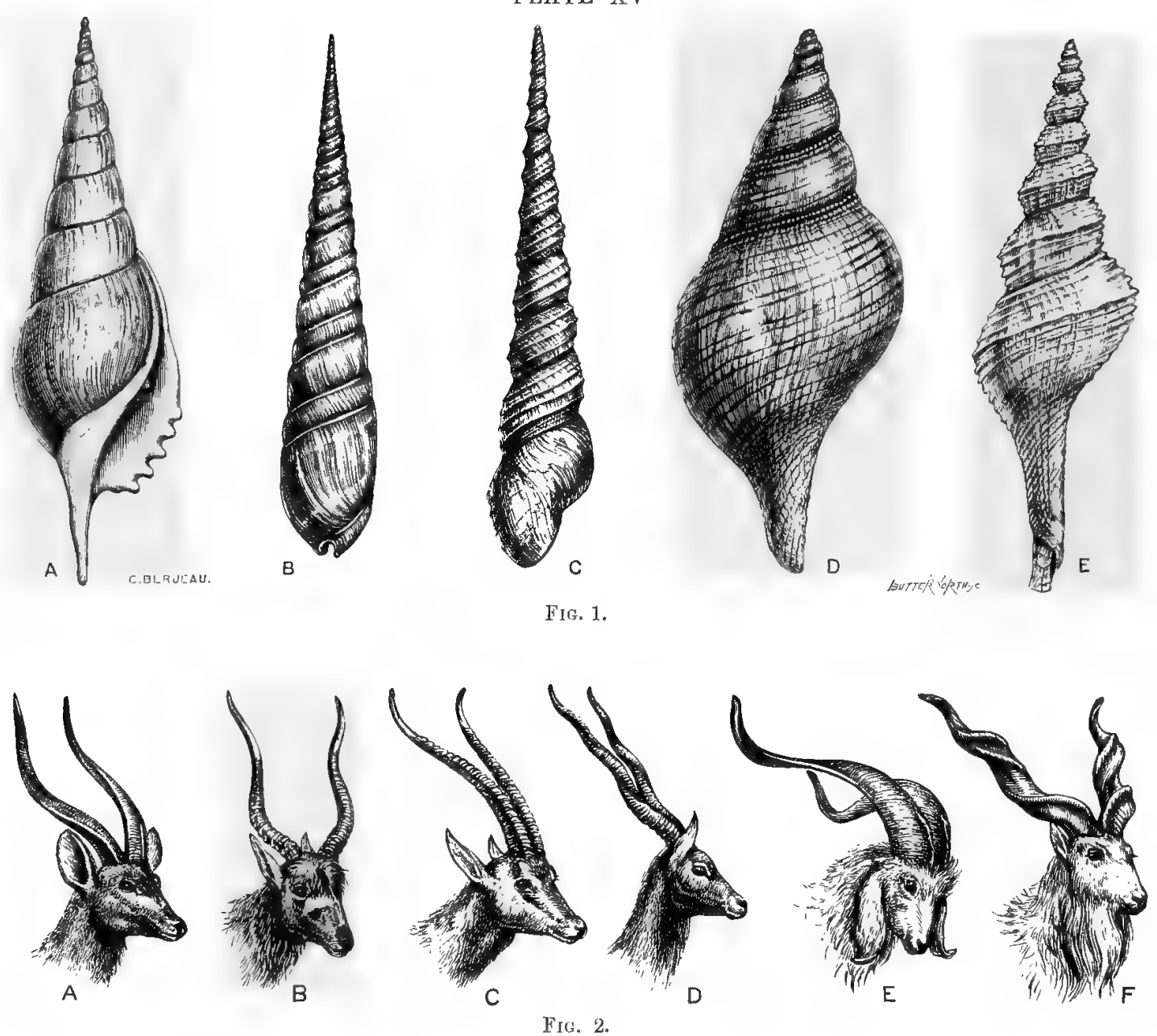

Plate xv. illustrates spiral formations in shells and horns.

Fic. 1.- Hhows typical pyramidal-shaped, right-lianded shells which bear a narked resumblance to many houns.
A. Rostellaria curvirostris:
D. Fuscioluria tulipa.
B. Terebra dimiliate.
E. Fusus delpetit-thoucursie.
C. Turritella (spr.).

J)awn hy C. Berjeau from photographs by the Author.

Fic. 2. Shows typical right and left-landed spiral horns. These horns, especially those figured at F, bear a striking reseml, lance to the spiral shells (Figs. 1 of this Plate).

A. Head and spiral horns of West African hannessed antelope (Tragelephus gratus).

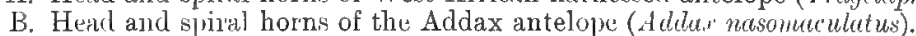

C. Head and spiral horns of Grant's gazelle (Cranella yranti).

1). Hearl and spiral horns of the liack luck (Antilope cervicupra).

E. Head and spiral horns of the Angora goat (Capra hircus).

Diawn by C. Berjeau for the Author.

F. Heal and spiral homs of the Markhor goal (Cupra falconeri).

\section{PLATE XVI}

Plate xvi. illustrates spiral structures as seen in shells, horns, tusks, teeth, feathers, proboscides, tentacles, \&c. spirals.

Frc. 1. - Skull and spiral horns of the Pamir sheep (Ovis poli) (after Sir V. Brooke). Shows beautiful right and left-lianded

FIG. 2. -A. Head and spiral tusks of mammoth (Ellephas primigenius) (after Tilsius). The great tusks of the manmoth furnish striking examples of spiral formations in the animal kingdom.

B. Head, tusks, and spiral trunk of the African elephant (E'lephus africanus). Thle trunk of the elephant sujplies in example of a soft, yielding, temporary spiral, similar to that seen in the tail of the spider monkey, the two-loed ant-eater, Merian's opossum, and 
PLATE XVI

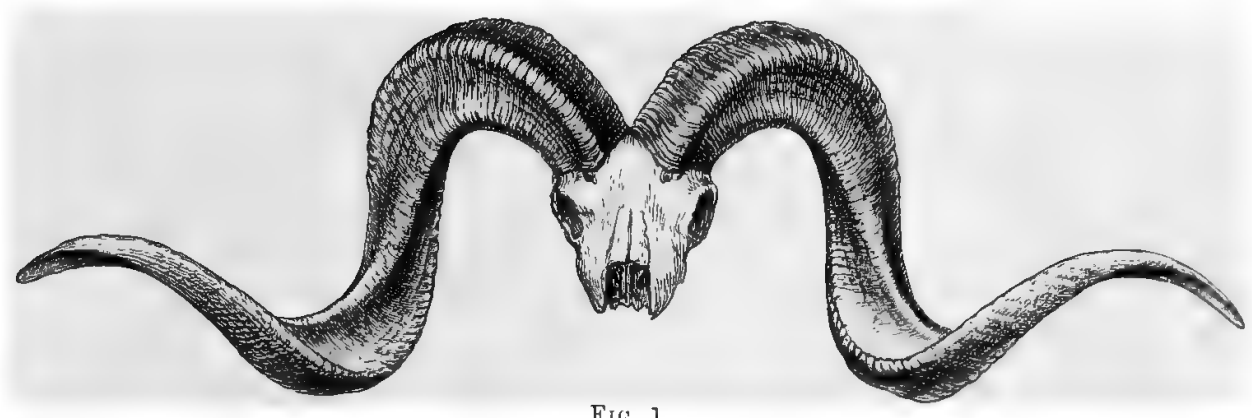

Firi. 1.
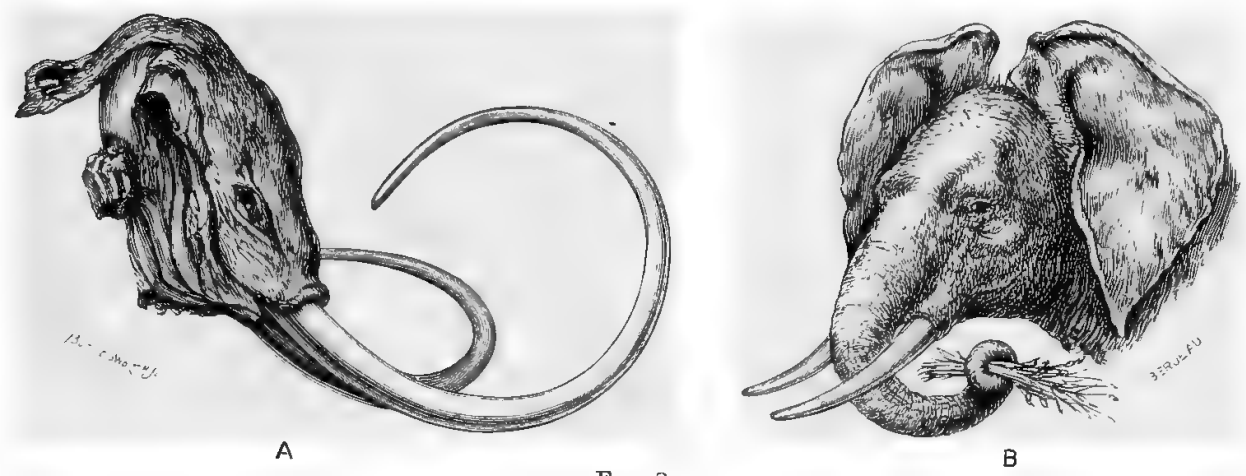

FIG. 2.

A

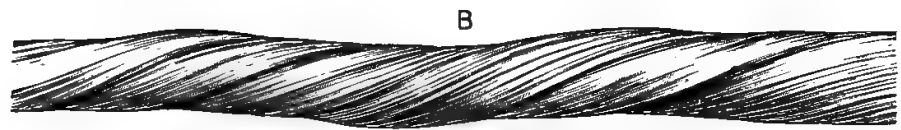

Fir. 4.
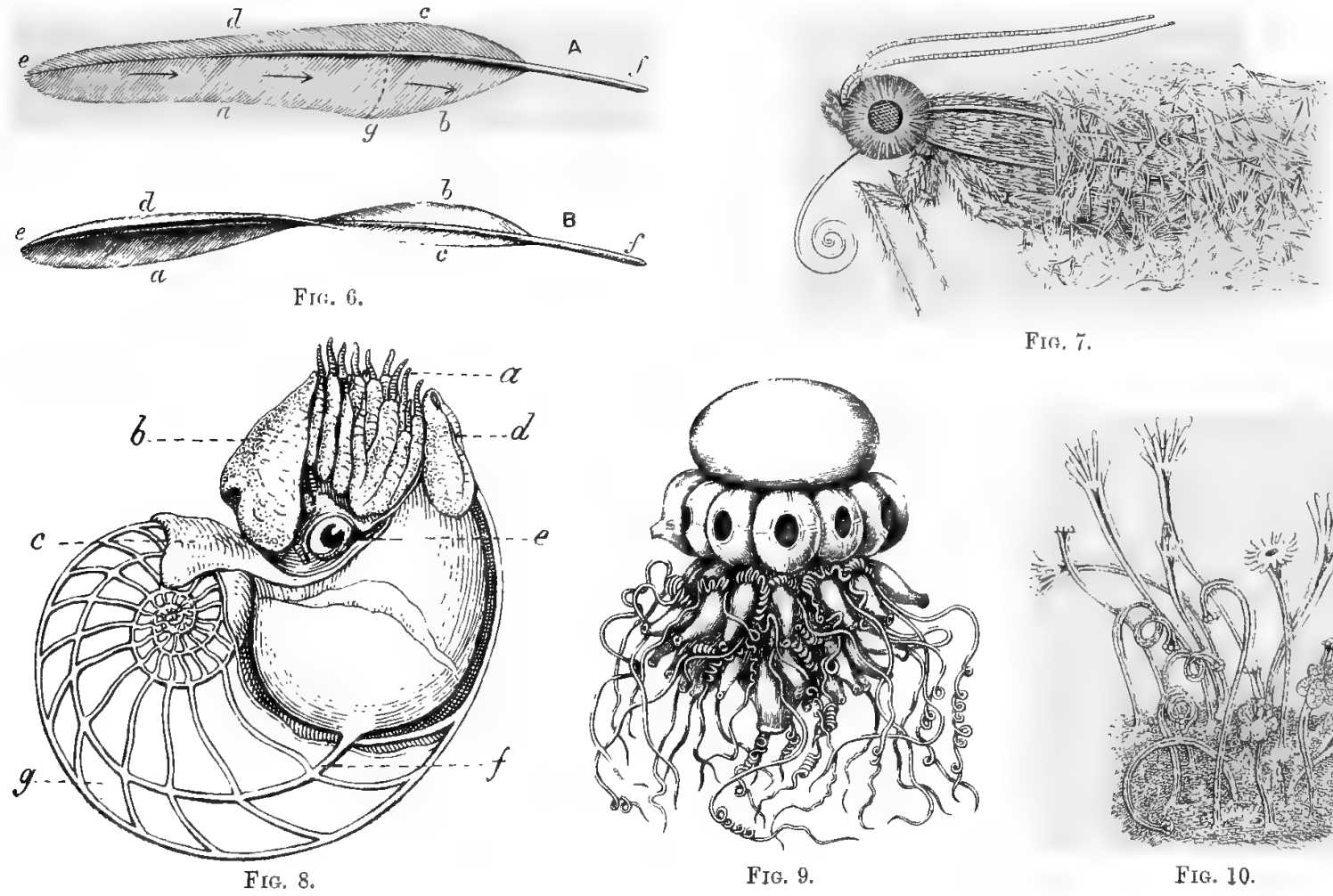

FIn. 7 .
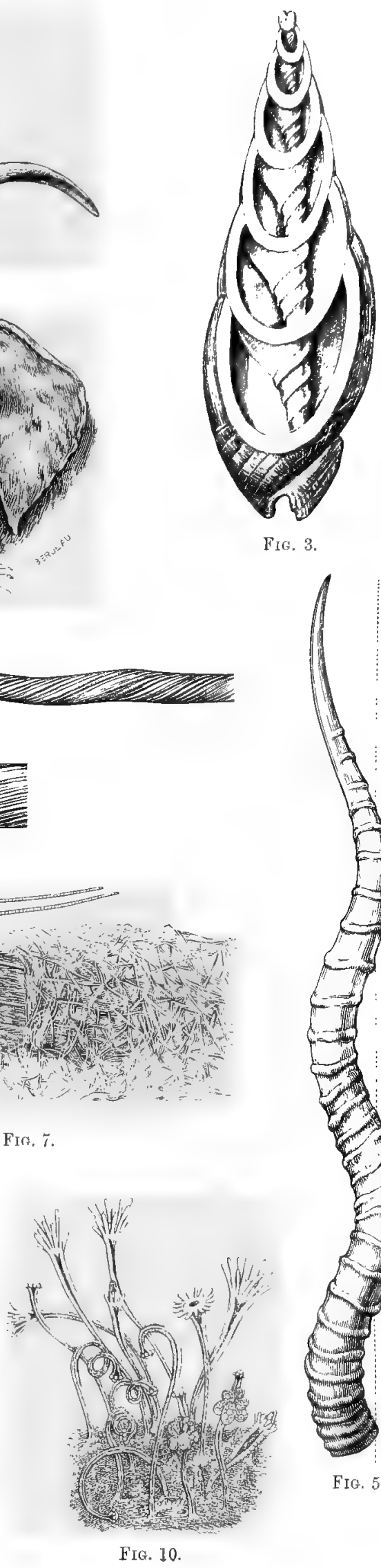

FIG. 3.

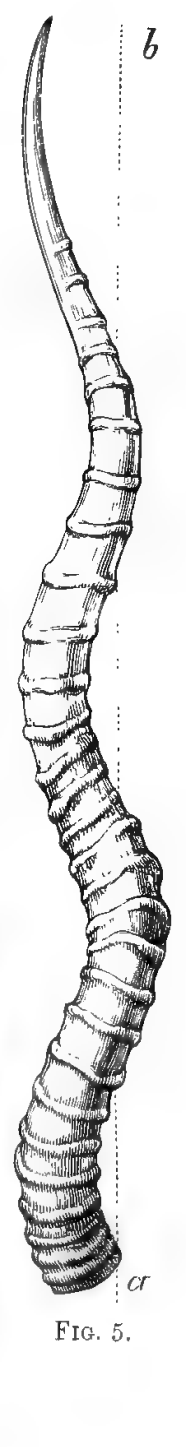


PLATE XVII

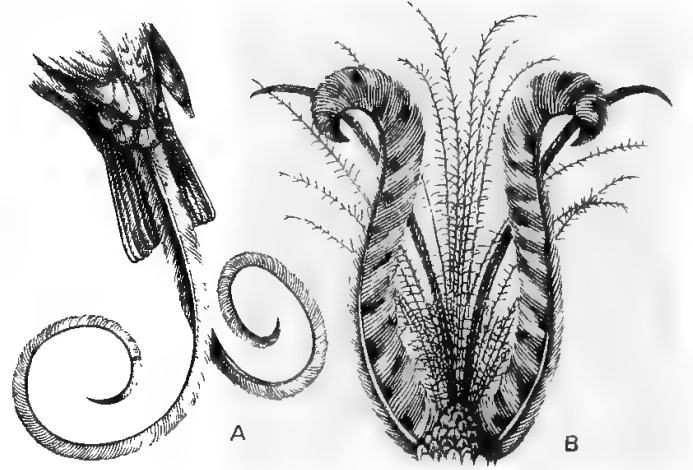

FIG. 1.

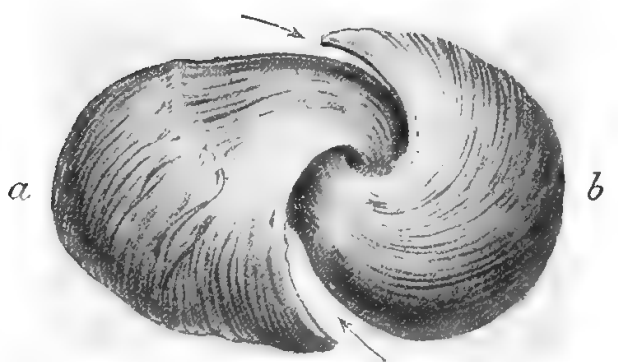

A

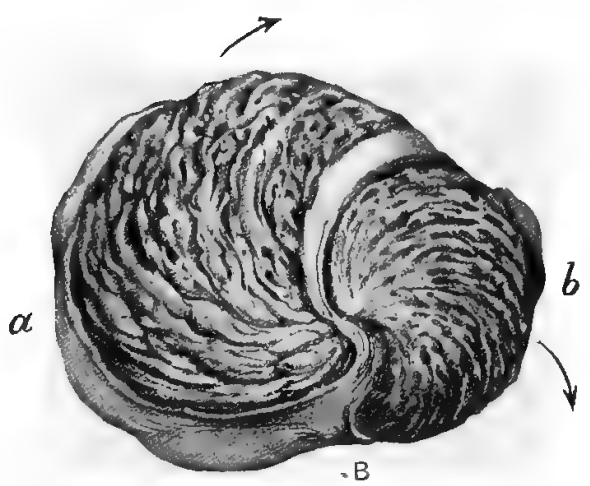

Fili, 3.

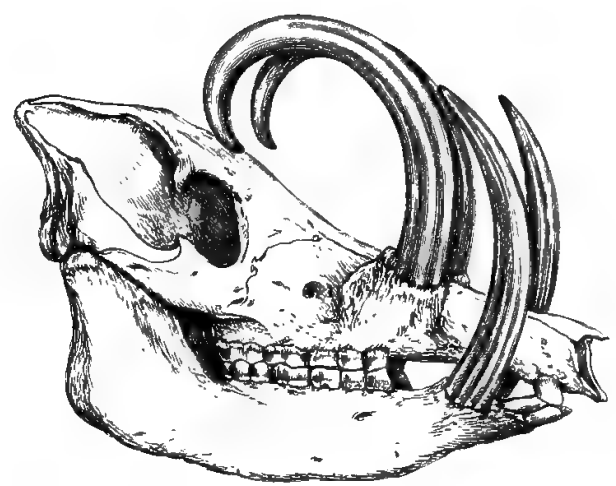

FIG.2.

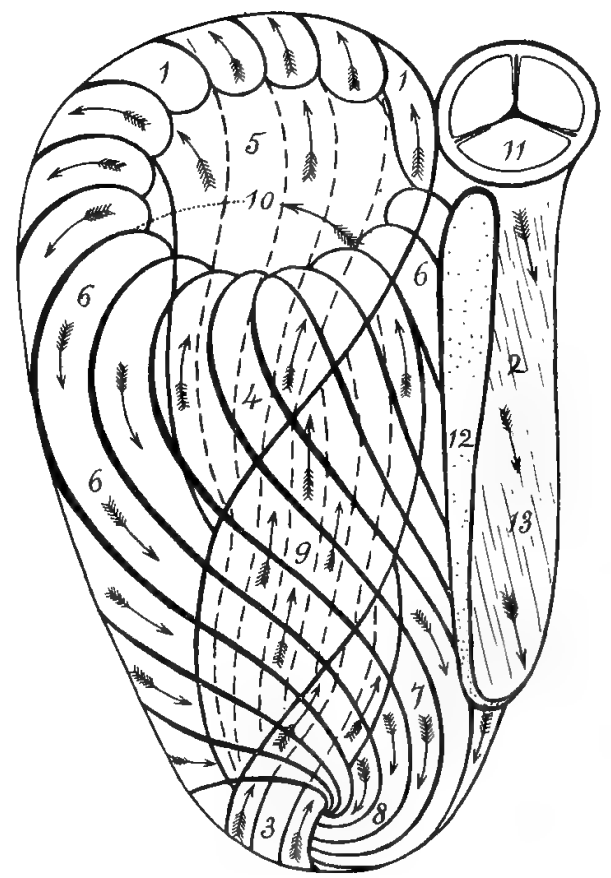

FIG. 4.

PLATE XVI (continued)

the tendrils of certain climbing plints; the tusks of the elephant and mammoth supplying examples of permanent spirals scen also in teeth, horns, bones, \&c. Drawil by C. Berjeau for the Author.

Fir. 3.- riew of spiral shell (Mitra episcopalis), sawn through longitndinally, showing colmmella with right-handed spiral folds (after Zittel). This affords the type of many spiral staircases.

FIG. 4.-A. Siriml tusk of narwhal (Monodon monoceros). The tusk forms a left-handed spiral composed of several strands, and producing a symmetrical combination. This is one of the largest examples of a spiral tooth. B. A portion of the same on a larger scale.

FIG. 5.-Spiral annulated horn of the addax (Addax nasomaculatus). The line $a, b$ shows the amount of spirality.

FIr. 6.-Primary feather of the wing of the swin, seen from above and edgeways, to show the spiral nature of the feathers on which flight mainly depends.

A. Primary feather seen from alove; curves in every direction. $a, b$, Posterior margins; $c, d$, anterior margins; $e, f$, vane or mid-ril, ; $c, g$, double curve formed by the margins.

B. The same feather, seen edge-ways. Slows spiral nature of feather. $a, b$, Posterior margin forming doulle curve arranged on a different plane from anterior nargin $(c, d)$, which also forms a double curve. The two margins cross each other fignrt-of-8 fashion; $e, f$, vane of feather. A primary feather is, in a way, a diminutive and perfect wing. Drawn from nature by the Author.

FIt*. 7.-The cloth moth with spiral proboseis. Drawn by C. Berjeau for the Author.

FIG. 8. - Lateral view of the nautilus in its shell (after Owen). Shows exquisite right-handed spiral. $a$, Tentacles; $b$, hood; $c$, mantle; $d$, funnel ; $e$, eye ; $f$, siphuncle; $g$, compartment of shell. 


\section{PLATE XVI (continued)}

Fra. 9.-Stephalia Corona (after Haeckel). Shows extraordinary spirnl tentacles greatly resembling the spiral tendrils of many plants. The tentacles make right and left-handed spirals spontaneously, and as apart from stinulatiom or inritation of any kind.

Fıa. 10,-Hydratenia elinata. Shows beatiful curved spiral stems, which can he extenderd and withdrawn at pleasure.

\section{PLATE XVII}

Plate xvii. illustrates spiral formations in feathers and teeth, in the muscular arrangements of the heart, and in the cast of the ventricular cavities of the heart.

FIr. 1.-A. Exquisite spiral tail feathers of Wilson's bird of paradise (Jiphyllonles wilsoni) (afier. Guillemard).

B. Spiral tail feathers of lyle bird, showing beautiful double curves ("Royal Natural History").

Ficr. 2.--Spiral teeth of Balirusu alfurus (after Guillemard). Resemble the tusks of the mammoth and elephant in general shape (see Plate xri., Fig. 2, A and B).

FIs. 3.-A. Dissection and photograph by the Author (1860) of the apex rif the left ventricle of the lueart of the sleep. Preserved in the Anatonical Museum of the University of Edinburgh. Shows beantiful right-handed symmetrical spiral whorl conposed of two sets of museular fibres $(a, b)$ which wind into the interior of the ventricle as indicated by the darts (compare with Plate xiii, Fig. 1, G).

B. Plaster of Paris anst hy the Anthor (1876) of the right and left rentricular cavities of the human heart. Preserved in the Museum of the Royal College of Surgeons of England. Shows fine left-handed symnetrical spiral composed of two parts (a, b). In this case the spiral runs from the apex to the base of the ventricles (vide darts), which is the opposite of A, where the spiral runs from the base to the apex. Compare with spiral nehulæ (Plate viii.); spiral flowers (Fig. 13); spiral seeds (Fig. 11, D, E); and spiral shell (Plate xiii., Fig. 1, E).

Frg. 4.-Diagram by the Author (1859), showing that external and internal muscular fibres of the left ventricle of the heart are arranged spirally, and furm two sets of left and two sets of right-handed spirals: that the two sets of external filures which constitute the left-handed spirals involute and enter the apex and become continuous with the two sets of internal fibres constituting the righthanded spirals, which latter in turn evolute at the base and become continuous with the two sets of spiral external fibres as indicated by the arrows and the numerals $1,2,3,4,5,6,7,8,9,10$.

\section{PLATE XVIII}

Plate xviii. illustrates spiral formations in the egg-purses of the shark and dog-fish, horns, and sea fan.

FIG. 1.-A. Spiral egg-purse of Port Jackson shark (Heterodontus philippi).

B. Ditto. Half natural size.

C. Egg-purse of dog-fish (Śqualus acunthias). These egg-purses are provided with spinal filaments resembling the tendrils of plants, by which they can fix themselves to objects in their vicinity. Like tendrils, they form double reversing spirals.

FIs, 2.-A. Horns of eland (Oreas canna), forming typical right and left-handed spirals. These horns furnish examples of close spirals, and resemble screw nails (compare with Plate cxi., Fig. 1, $c$ ).

B. Head and horns of koodoo (Strepsicerns $K u$ ulli). The horns in this case form beautiful opren right and left-handed spirals similar to those seen in climbing plants.

C, D. Skulls and spiral horns of small antelopes. Drawn ly C. Beriean from photographs by the Author.

FIG. 3. Corkscrew sea fan (Streptocaulus pulcherrimus). Forms elegant right-handed spiral $(a, b, e, d, e, f, g, h)$.

\section{PLATE XIX}

Plate xix. illustrates spiral shell formations and their resemblance to similar spiral formations in the bony portions of the inner ear (human).

FIG. 1.-Examples of various shells forming elegant right and left-handerl close and open spirali.

A. Eumphalus pentanfulictus (Woodward). Forms left-handed, close, flat spiral.

B. Crioceras emerivi. Forms left-handed, open, Hat spiral.

C. Ecruliomphalus distans. Forms riglit-handed, open, flat spiral.

D. Siliquaric anguina. Forms left-handed, semi-open, conical spiral,

E. Shell ("phragmacone") of S'pirula fragilis. Forms left-hander, open, Hat spiral.

F. Stephanoceras (A mmonites) humphresianum. Forms right-handed, close, flat spiral.

G. Axis of Archimedes wortheri. Forms left-handed, elongated, close spiral. Fron Niclulson's and Lydekker's "Palrentolngy."

FIG. 2,-Remarkable examples of right and left-lınderl spirals occuring in the inner prition of the human ear.

A. Cochlea of ear laid open. $a, a^{\prime}$, Osseous wall; $b, b^{\prime}$, lanina spiralis; $c$, strands of cochlear nerve folding over at $d, d^{\prime}$.

B. Osseons labyrinth of left internal ear seen from without. ", Cuchlea; b, senicircular canals (after Hirsehfeld and Leveillé).

Frf. 3.-A. Lamina of cochlea of internal ear exposed (after Riidinger).

Fig. 4.-Bony labyrinth of right internal ear of child, $a$, Cochlea. The semicircular canals are seen at the left of the figure (after Ruidinger),

VOL. I. 
PLATE XVIII
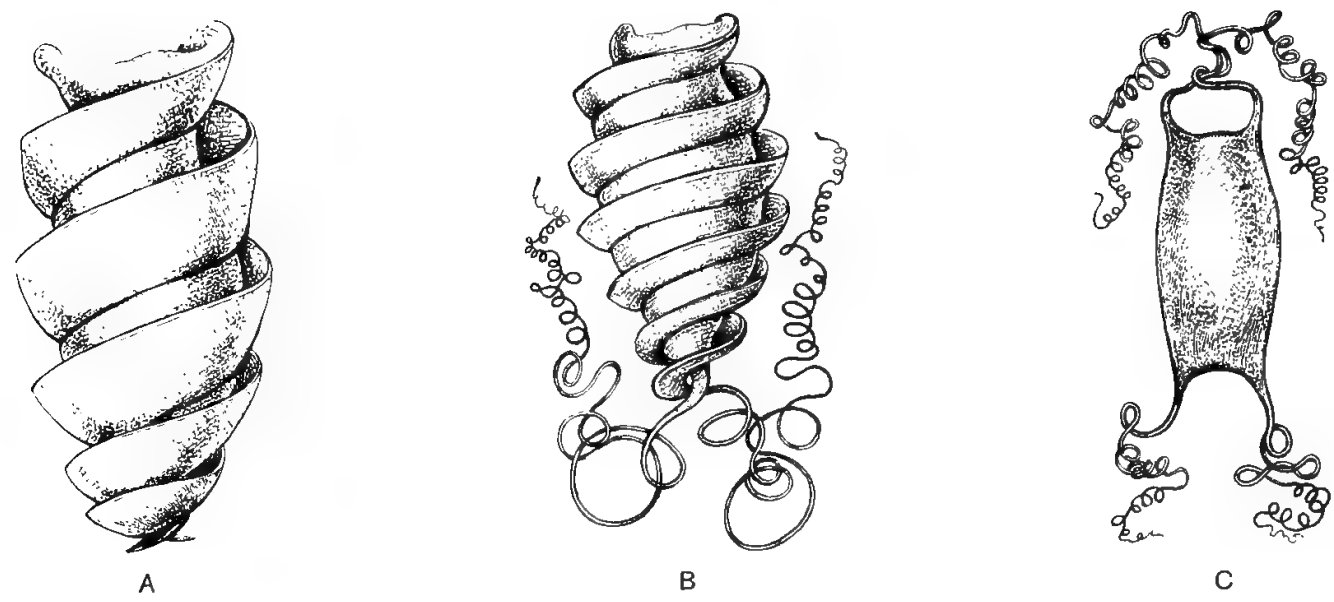

C

FI $\because \div, 1$.

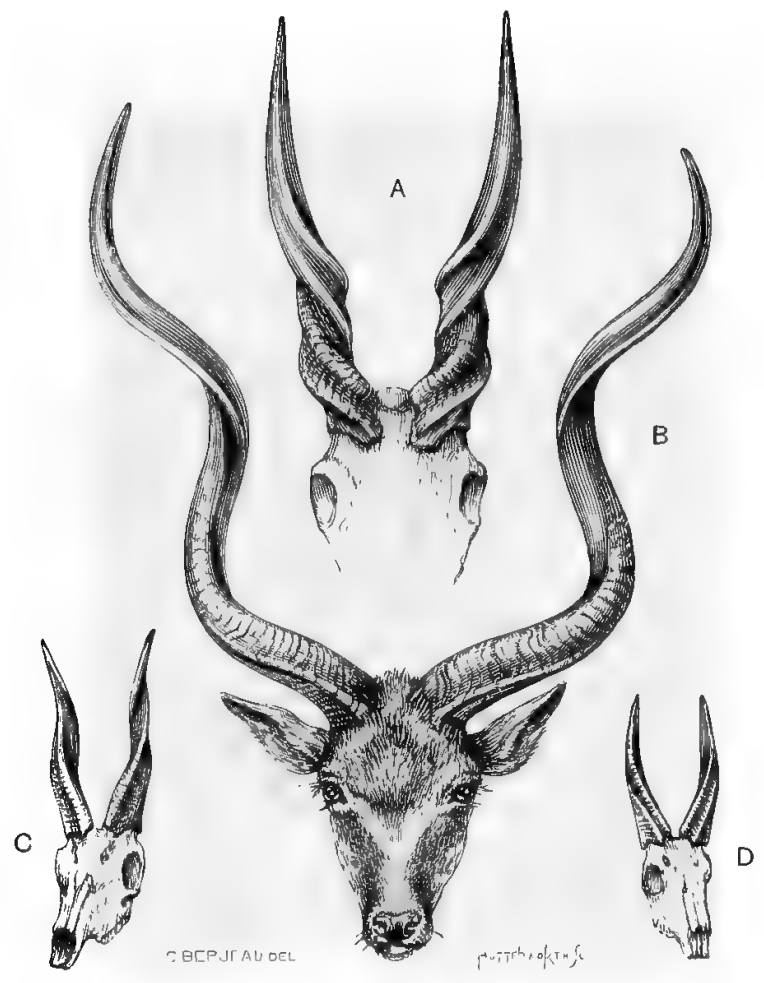

FIri, 2.

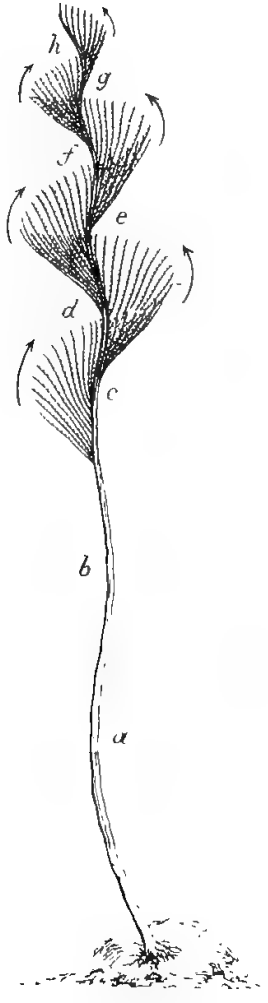

Fir: 3.

PLATE IX

Plate xx. illustrates temporary and permanent spiral formations in star-fishes and in the limbs, ribs, and turbinated bones of vertebrates.

FIr. 1. - Striking example of spirals formed by the coiling of the limbs of a brittlestar (Astrunyx lovemi). The spirals resemble those made by the tendrils of certain plants, for example the vegetable narrow (Plate ix., Fig. 1, B, C) and passion Hower (Plate $\mathrm{x}$., Fig. $1, f f, g h, k l, m n, o, p)$. Drawn by C. Berjeall from a photograpli taken for the Author.

FIt. 2.-Humau ribs twisted upon themselves and forming thus spirals. The twist is indicated by the darts and letters $a, b$, and $c$, d. Draw by C. Berjeau from specimens in the Author's museun.

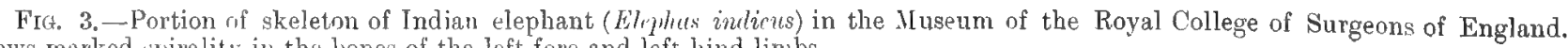
Shows marked spirality in the hones of the left fore and left hind limbs, 
PLATE XIX
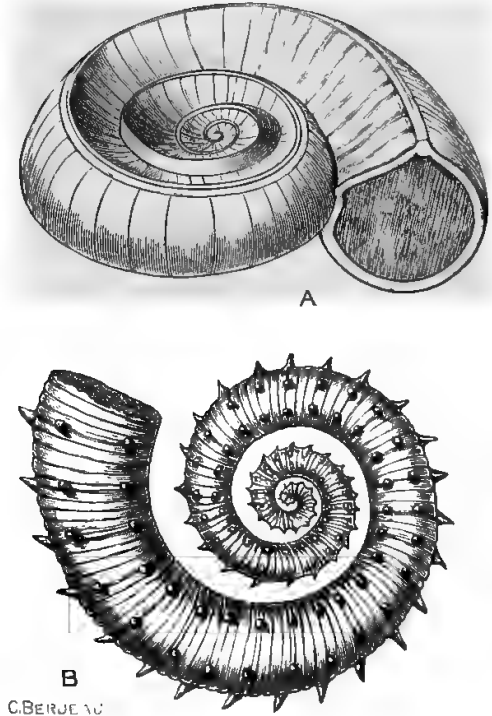

C.BEITUE

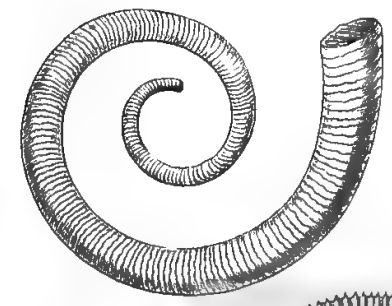

c

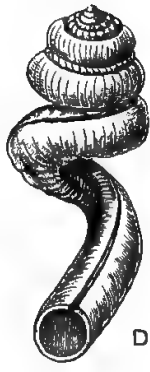

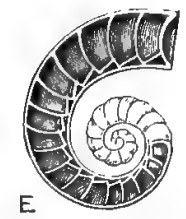

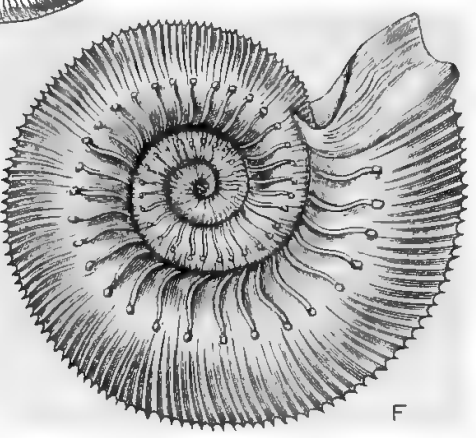

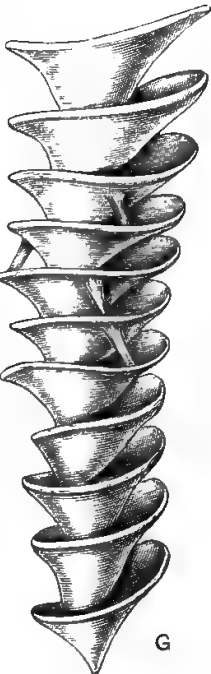

BuTrLA AWOATH:S

K'LG. 1.

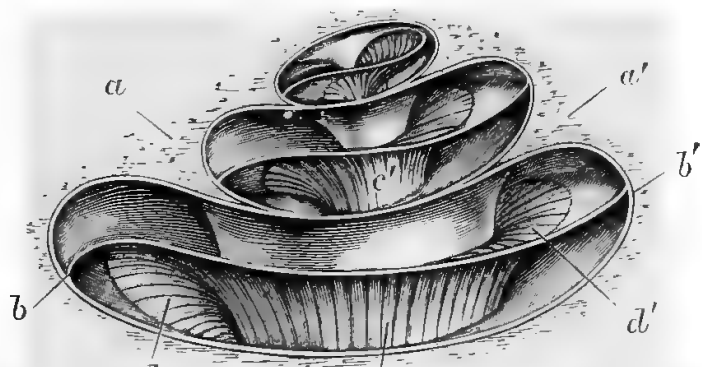

A

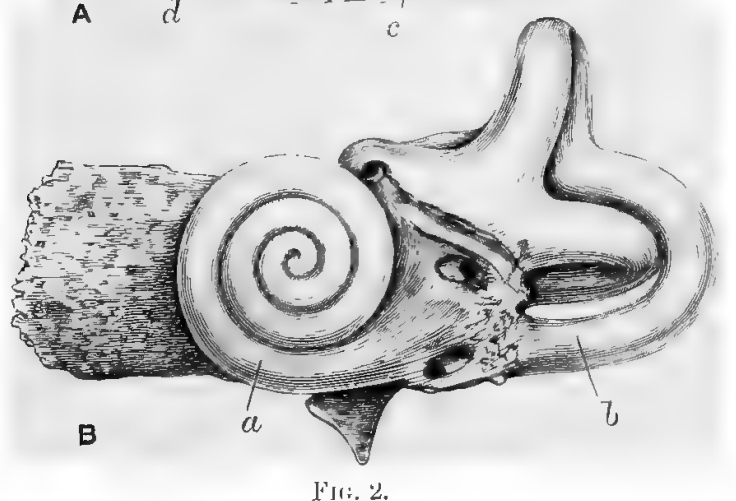

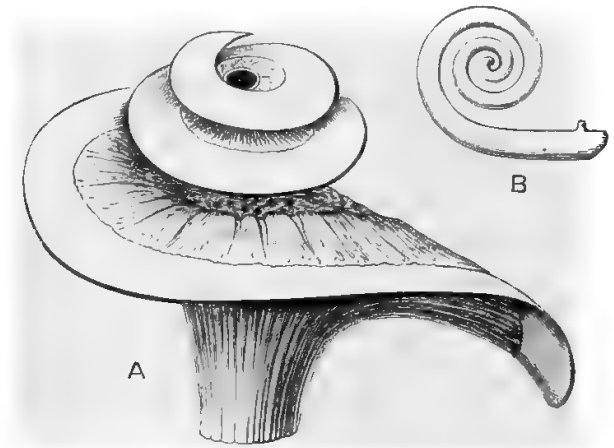

FI 6.3.

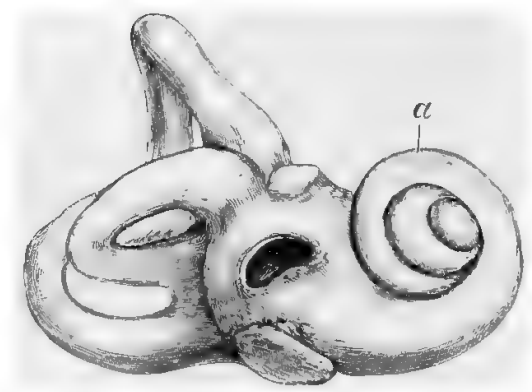

FIG. 4.

PLATE XX (continued)

A. Vertebral columu; $b$, sternum; $c$, rilis; $d$, scapula; $e$, liumerus; $f$, ludius and ulna; $g$, hones of foot; $h$, pelvis; $i$, femur; $j$, tiljia and filula; $k$, bones of foot. The bones of the limbs are not only twisteu upon themselves lut, in the case of the radius and ulna, and the tilia and fibula, they twist round each other. The spirality resembles that seen in the lrones of the extremities of man (Plate xxi., Fig. 1); the wing of the bird (Fig. 185); the cast of the left ventricle of the heart (Plate xvii., Fig. 3); and certain trees (Plate xi., Figs. 2 and 3 ; Plate xlii., Fig. 1); fruit (Fig. 16), and horns (Plate xv., Fig. 2, D, E).

Drawn by C. Berjeau from photograph specially taken for the Author.

Fig. 4. - Transverse section of turbinated or scroll bones of human skull (after Hirschfeld and Leveillé). The middle $(a, b)$ and inferior $(c, d)$ turbinated bones are distinctly spiral in their nature; the right ones $(a, c)$ forming right-handed and the left ones lefthanded spirals. 
PLATE XX

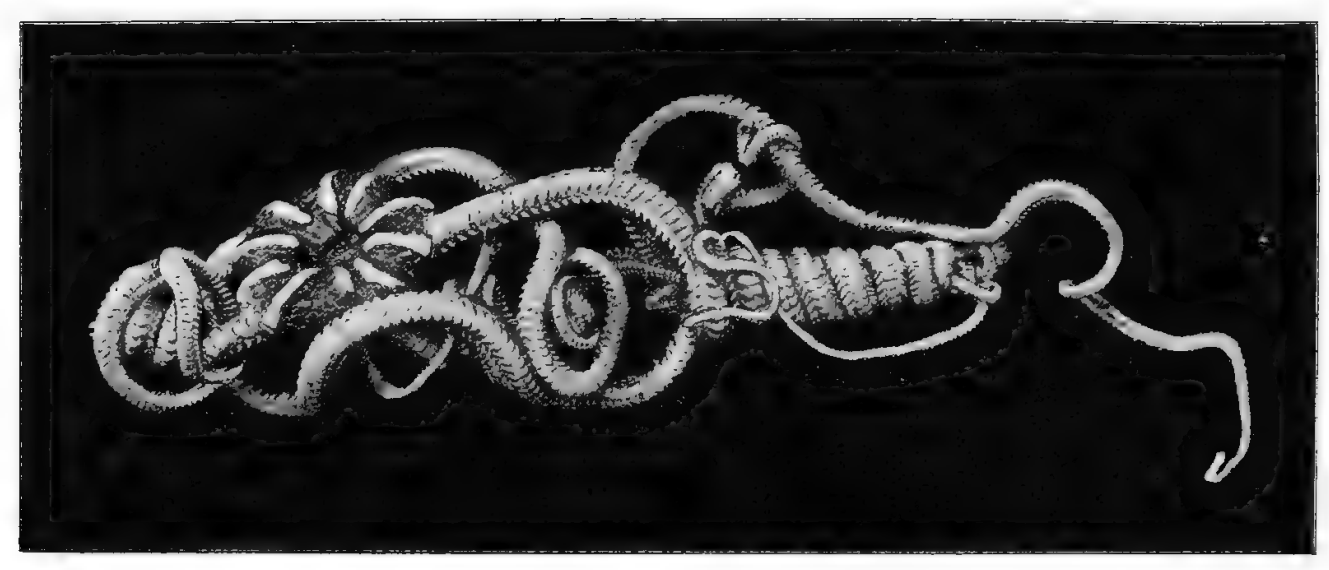

FIx, I.
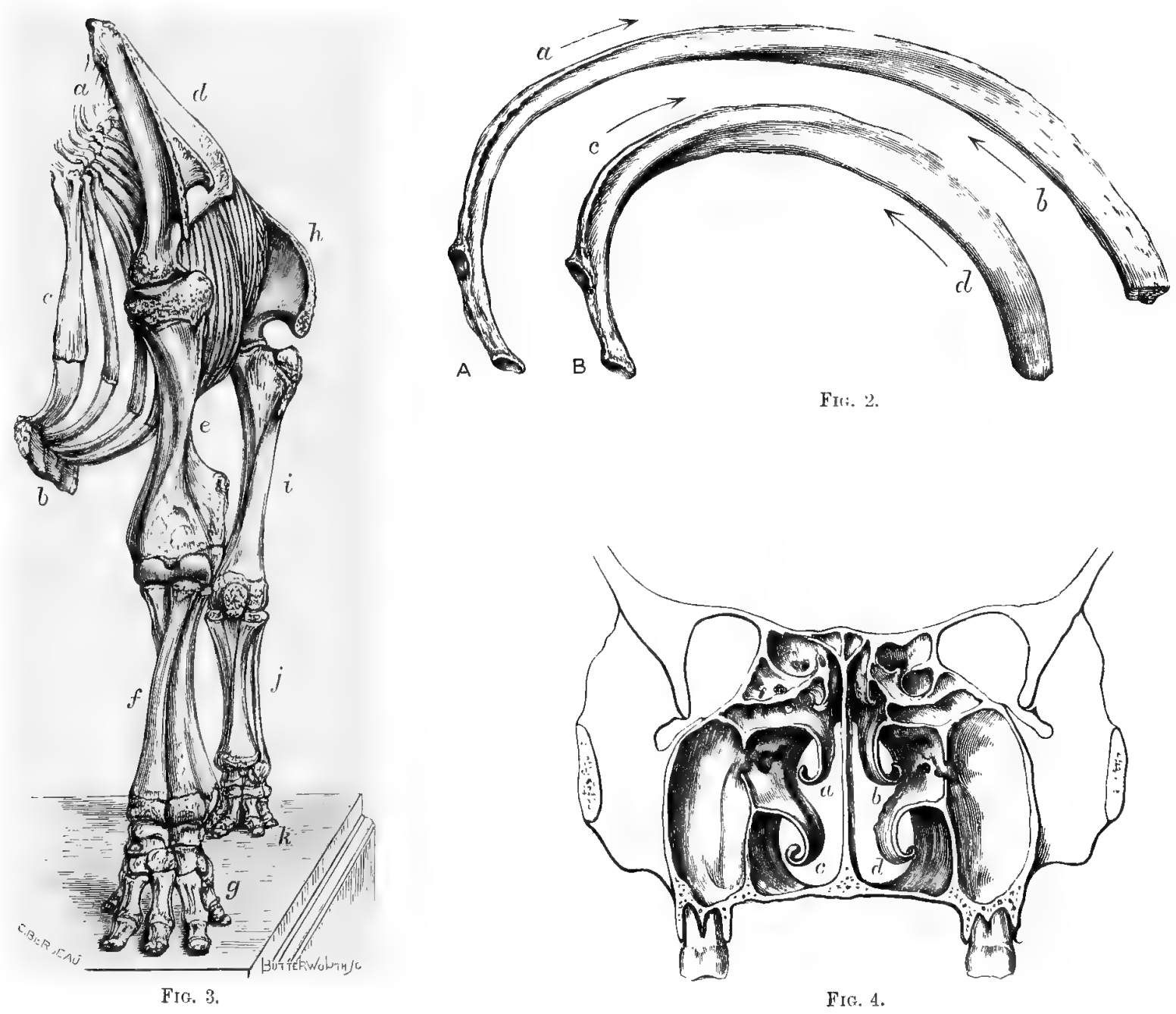

FIt: 2.

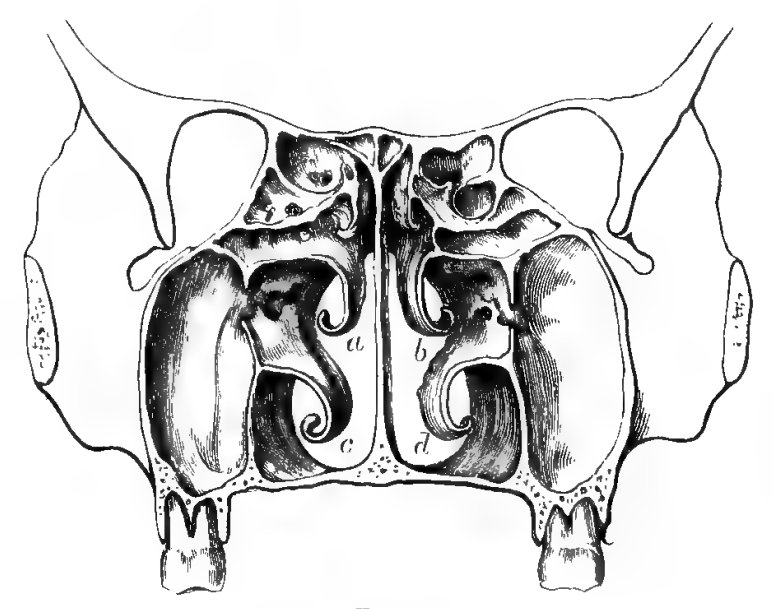

FIf. 4. 
PLATE XXI

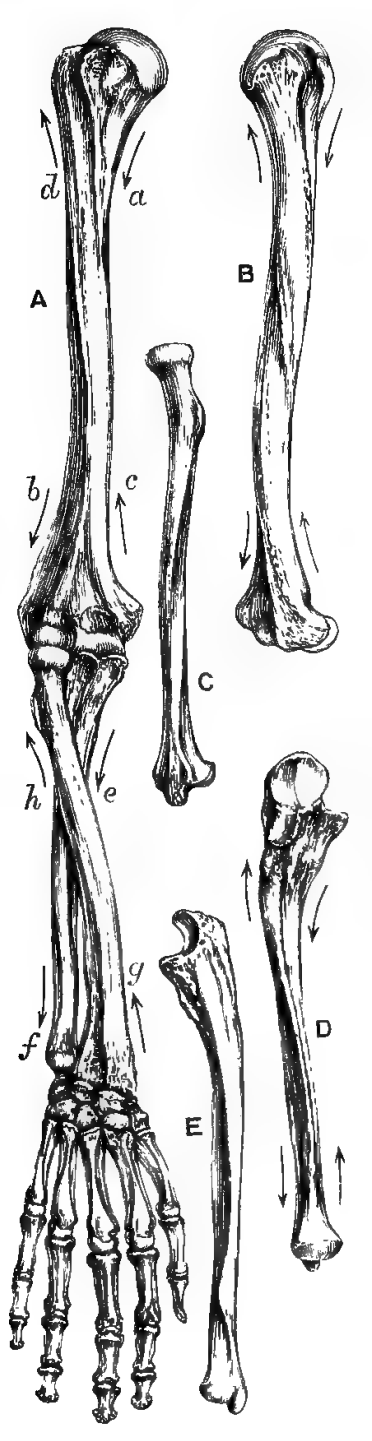

FIG. 1.

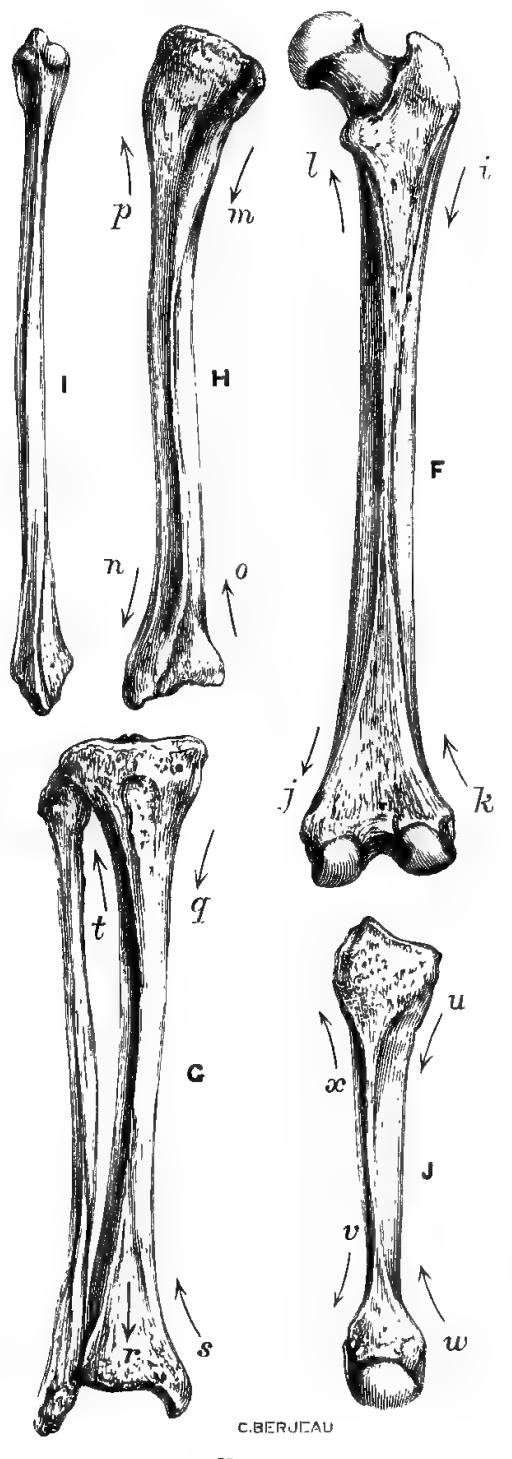

FIG. 2 .

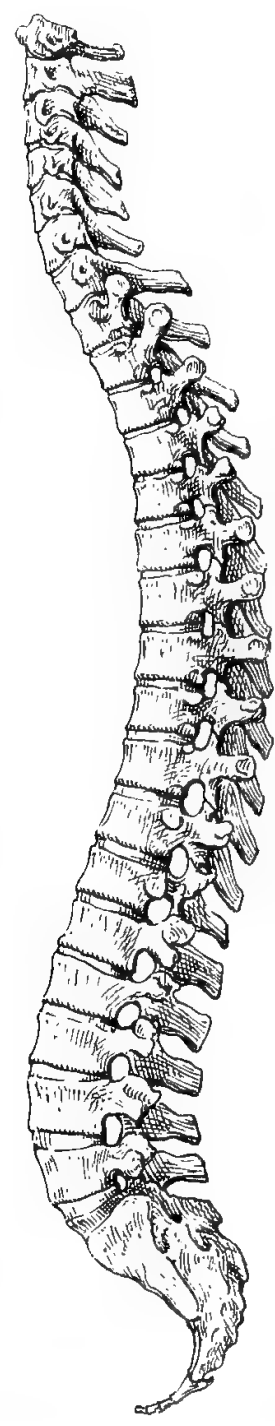

FIs. 3.

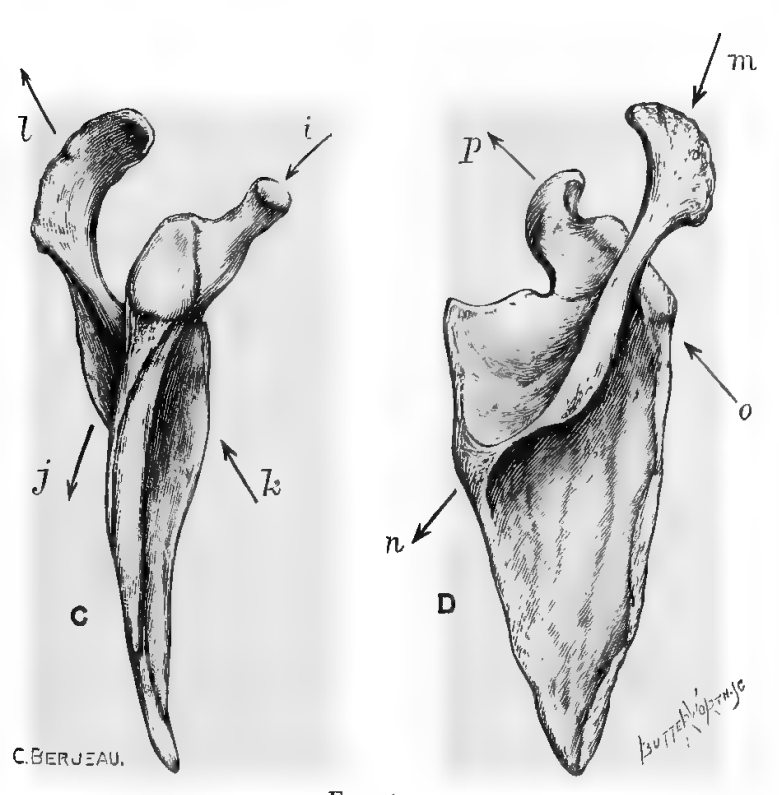

Fli, 4,
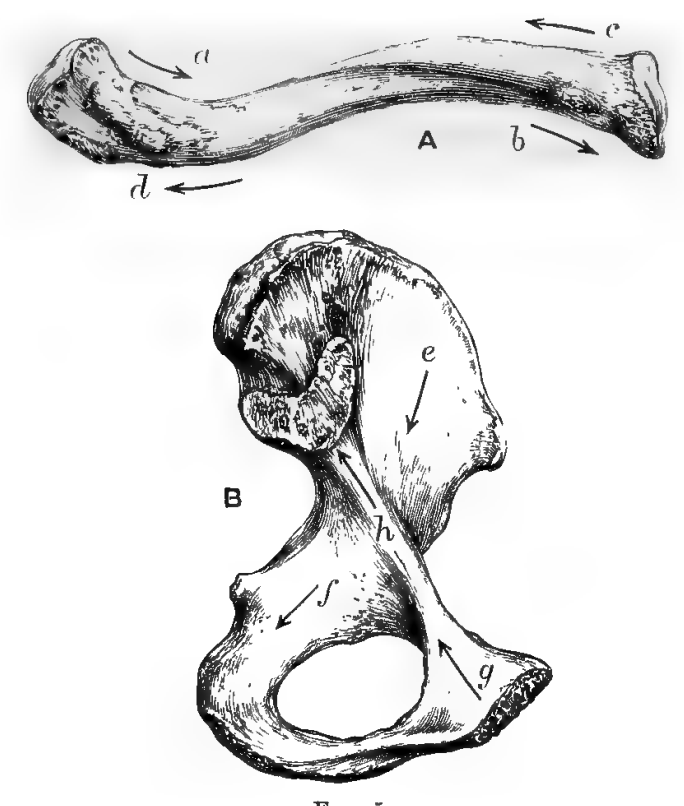

FIG. 5. 


\section{PLATE XXI}

Plate xxi. illustrates the spiral formation of human bones as seen in the arm, leg, vertebral column, clavicle, scapula, and pelvis.

FIG. 1.-Bones of right upper extremity of man with hand pronated: also suparate bunes. All these bones present fine examples of spiral strurtures; pronation of the hand being performed by the radius twisting round the ulna.

A. The spirality or twist in the humerus or an'm bone is indicated by the darts $a$, $b$, and $c$, $d$, and in the radius and ulna by the darts $e, f$, and $y, h$.

B. Another view of the humerus, the darts, as before, indicating the spirality, which is very marked.

C. Radius (one of the bones of the fore-arm), twisted upon itself.

D. Lina (bone of fure-arm), tiwisted upon itself (see darts).

E. Different view of una. The lones of the lower extremity (fenur, tibia, and fibula) are also twisted, and so resemble the upper ones. Compare with the fore limb of the elephant (Plate xx., Fig. 3) and the structures mentioned in connection therewith. Drawn to scale by C. Berjeau from specimens in the Author's museum.

FIG. 2.-Bones of right lower extremity of man, with metatarsal bone of foot. All these bones are spiral in their nature; the spiral leing particularly well seen on the posterior surface of the femur or thigh lione.

F. Femur or thigh bone seen posteriorly. The darts $i, j$, and $k, l$, indicate the direction of the spirality and how the ridges of the lone tend to uross each other.

G. 'lilid and fibula. In this figure the direction of spirality is indicated hy the darts $q$, $r$, and $s, t$.

$\mathrm{H}$. Another view of the tilia; the spirality being indicated by the darts $m$, $"$, and $o, p$.

I. A nother view of the tilsula in which the spirality is well narked.

Drawn to scale by C. Berjeau from specimens in the Authol's museum.

Fig. 3.-The luman vertelral column or backbone. It consists of a cliain of small sfuare-shapred bones with spines and transverse processes for the attrchment of muscles; the bones heing held tugether by liganents and inter-vertebral cartilages, which act as buffer's and diffuse shock. The colum displays four antero-pusterior curves; the convexities of two of which are directed anteriorly and two prsteriorly. There is also as certain amount of lateral curring which enables the column to move practically in every direction, namely, fron before backwards, from side to side, and spirally round its long axis. The column is at once one of the strongest min most mobile parts of the body; it is also the most elastic and springy part. It furnishes in perfect structure for neutralising shock, especially vertical shock.

FIGS, 4 and 5.-Illustrate the spiral twisting of the lyones in the human elavicle, scajnlla, and pelvis. These bones, like those of the upper and lower extremities, the rilos, s., are all distinctly twisted and spiral in their nature.

A. Hunan clavicle spirally twisted and resemhling the letter $f$, the true line of beaty and of strength (see darts $" l, l$, and $c^{\prime}, d$ ).

B. Human pelvis, on the whole the strongest combination of bones in the body. It several parts are distinctly spiral, as indicated by the darts $c, f$, and $g, h$.

C. Human scapula seen anteriorly. This bone is strengthened by strong ridges and processes, which distinctly tend to cross earli other spiral-fashion (see darts $i, j$, and $k, l$ ).

D. Human scapula seen pusteriorly. The same spiral twisted arrangement seen in C is lepeated (see darts $m$, $\mu$, and $u, p$ ). Drawn to scale by C. Berjeau from specimens in the Author's muscum.

\section{$\S$ 8. Examples of Radiating and Concentric Arrangements in Plants and Animals, as illustrating Symmetry of Form.}

I have directed attention to the radiating and concentric arrangenents which olbtain in crystals, in plants, and in animals, and the subject, it appears to me, is so important, from a fundamental point of view, that I will be excused if I devote a little more time to its elucidation and illustration. On the radiating and concentric arrangements very largely depend the symmetry of certain crystals and that of the majority of planis and animals. symmetry, where radiating and concentric arrangements prevail, is more or less assured. It is otherwise in the case of spirals. Single spiral structures are always unsymmetrical, and in order to secure symmetry the spiral must be divided into two (or more) portions; the portions being made to start from corresponding opposite points above and below or from the sides, and made to wind into each other. While spiral symmetrical structures must consist of at least two parts, they may consist of four, eight, or multiples of these. Symmetry is also produced when right and left-handed spirals are superimposed and made to cross each other.

The radiating and concentric arrangements receive their most striking illustrations in transverse sections of plants and trees, but they are seen everywhere; in crystals, and in plants and animals, and parts thereof. The sections of hail seen at Fig. 2, p. 2, and the sections of animal structures depicted at Plate v., p. 9, are very illustrative. In order, however, to save the reader the trouble of comparison I append additional examples of these arrangements (vide Plates xxii. to lii. inclusive).

The radiating and concentric arrangements are seen in sections of the stems, branches, and roots of plants and trees; in the arrangements of flowers and fruit; in the arrangements of spores, zooids, and corals; in the arrangements of shells, scales of fishes, horns, hoofs, and claws; in the arrangements of muscles, nerves, bones, teeth, \&c. In plants, trees, shells, scales, \&c., the concentric arrangements, for the most part, indicate layers of growth.

The following illustrations (Plates xxii. to xxviii. inclusive) will explain themselves. 


\section{RADIATING AND CONCENTRIC ARRANGEMENTS}

\section{PLATE XXII}

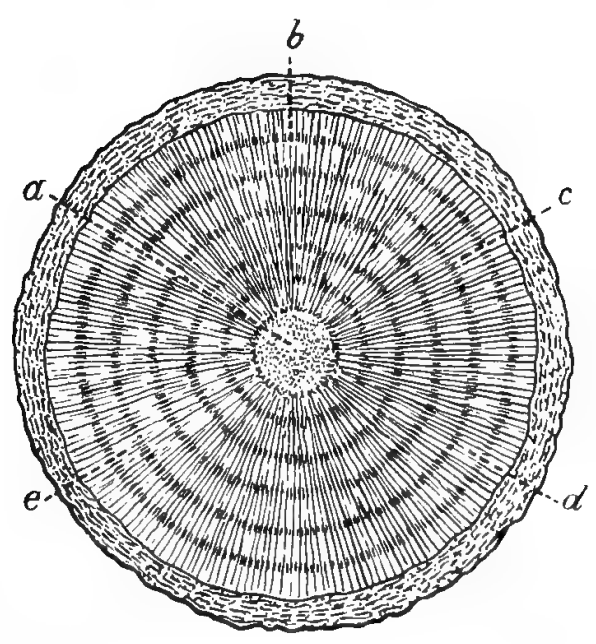

A

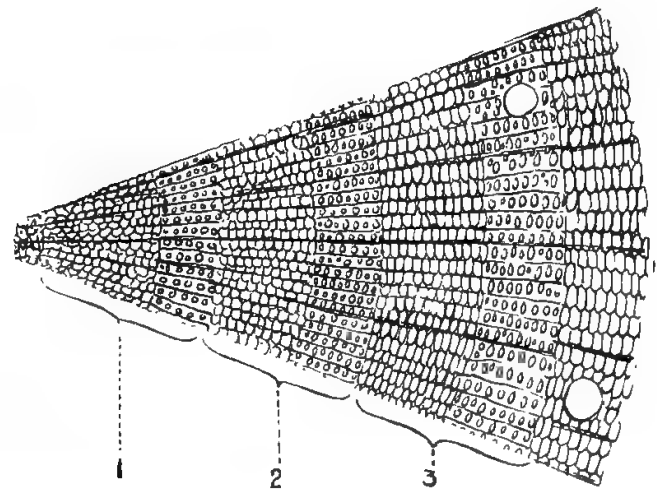

B

FIr, 1.

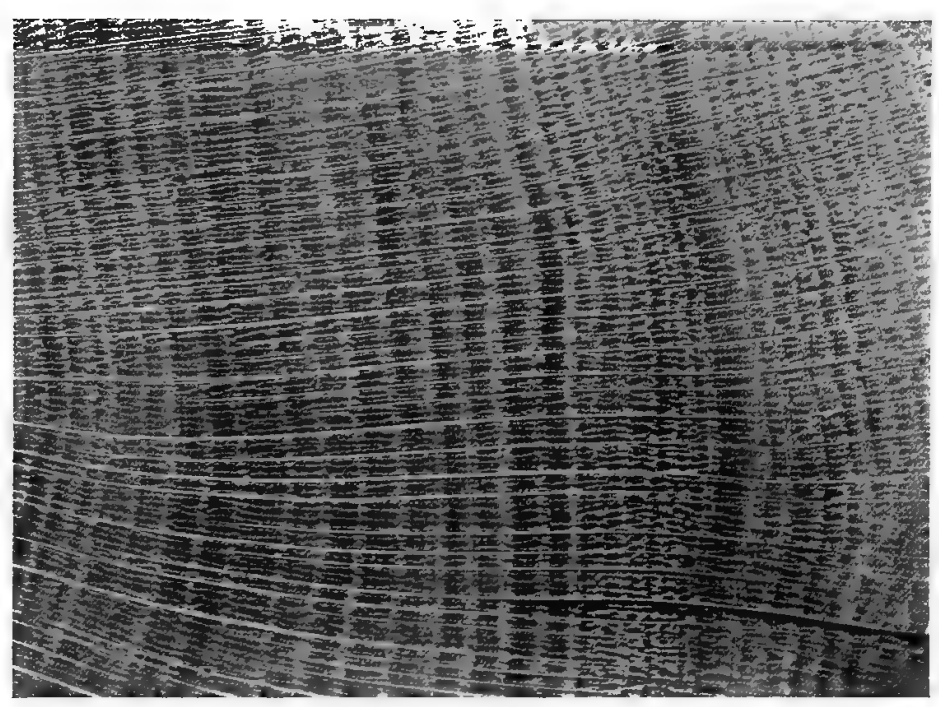

Firi.12.

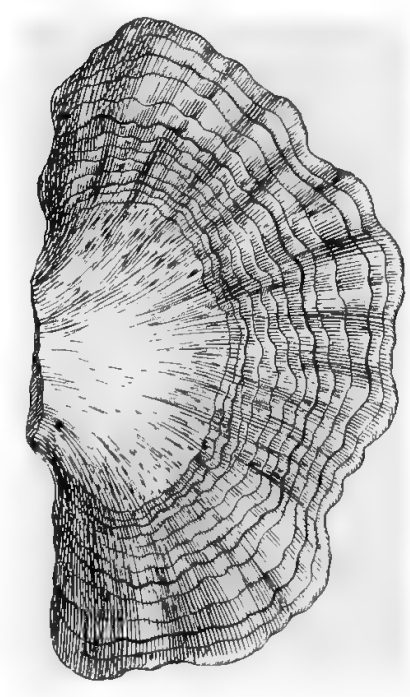

FIr.:

Plate xxii. illustrates radiating and concentric arrangements in the oak, fir tree, and fungus.

FI $(x .1$. A. Transverse section of stem of oak, showing radiation and concentric rings of growtls. a, Pith nr wentral portion of stem ; $b, c, d, e$, concentric wrody formations indicating that the olak is six years old. The bark also consists of six layers.

B. Portion of transverse section of stem of fir tree, illustrating radiation and rings of growth. In this case the fir is three years old, as indicated by the numernls 1, 2, 3 (after J. H. Balfour).

FIG. 2.-Portion of transverse section of old rak, showing radiation and concentric rings of srowth. From fine photograph presented to the Author ly Mr. R. A. Rohertson.

FIr. 3.-Fungus (Hexrngonia glabra), displaying radiation and coneentrie rings of growth (Miern-1)intionary).

\section{PLATE XXIII}

Plate xxiii. illustrates radiating and concentric arrangements in the pine-apple, echinus, radiolarian, diatom, haliomma, and xiphacantha.

FIG. 1,- Transverse section of fresh pine-ipple, showing radialing and concentric arrangements. a, ('ore or central part of apple, concentric in its nature; $b$, concentric part ontside core; $r, d, e$, radiating portions of pine-aplle. Drawn from fresh specimen by C. Berjean for the Author.

FIG. 2.--Transverse section of sjine of echinoide, revealing a radiating and concentric arrangement. ", Core or central portion; $b$, concentric rings ; $c, d, e$, radiating parts of spine (after Griffith and Henfrey). Greatly resembles transver'se section of stem of tree 
PIATE XXIII

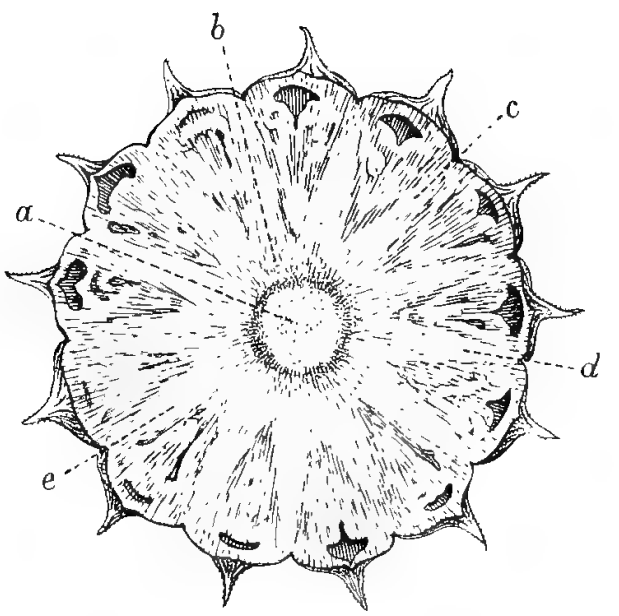

Fif, 1.

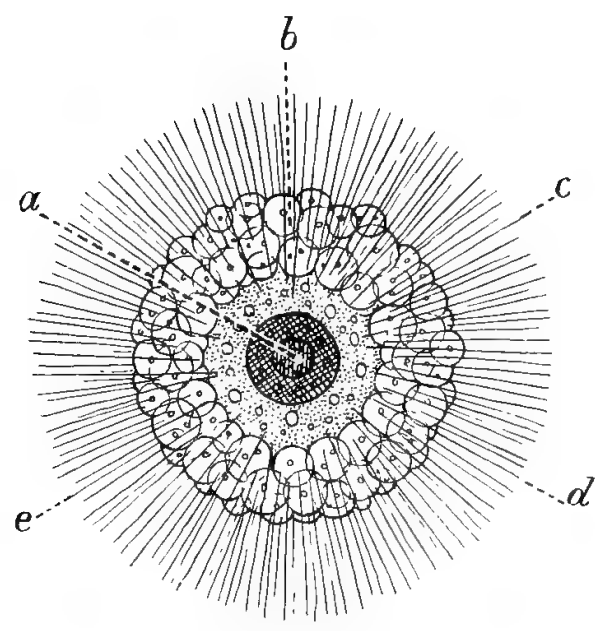

FIf. 3.

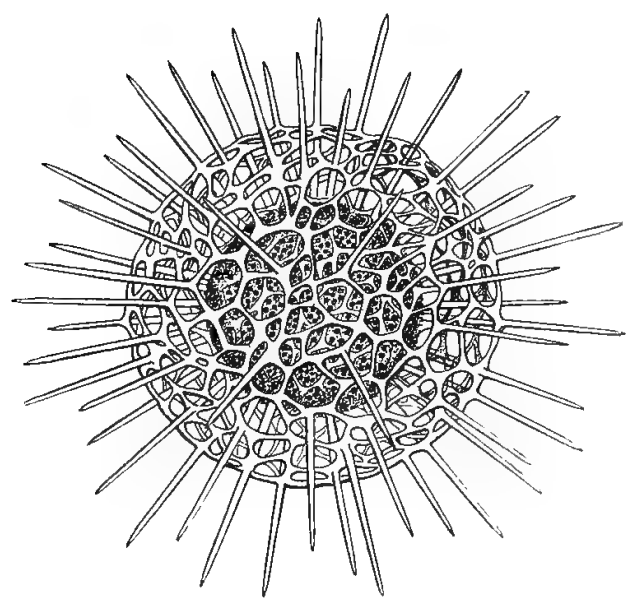

Fig. 5.

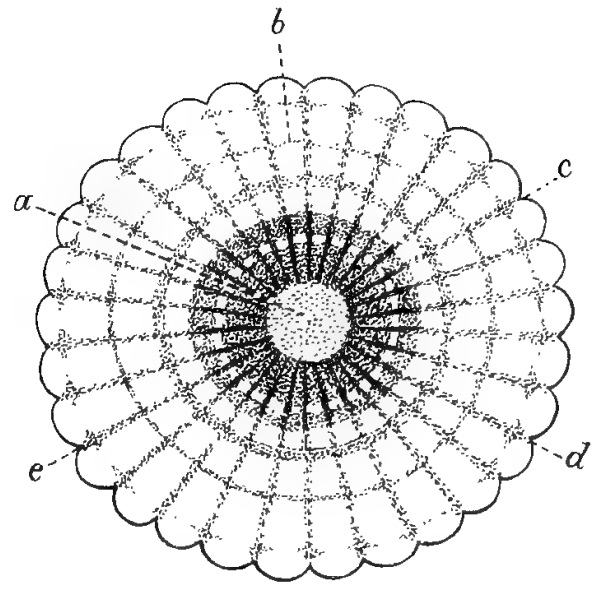

FIf 2.
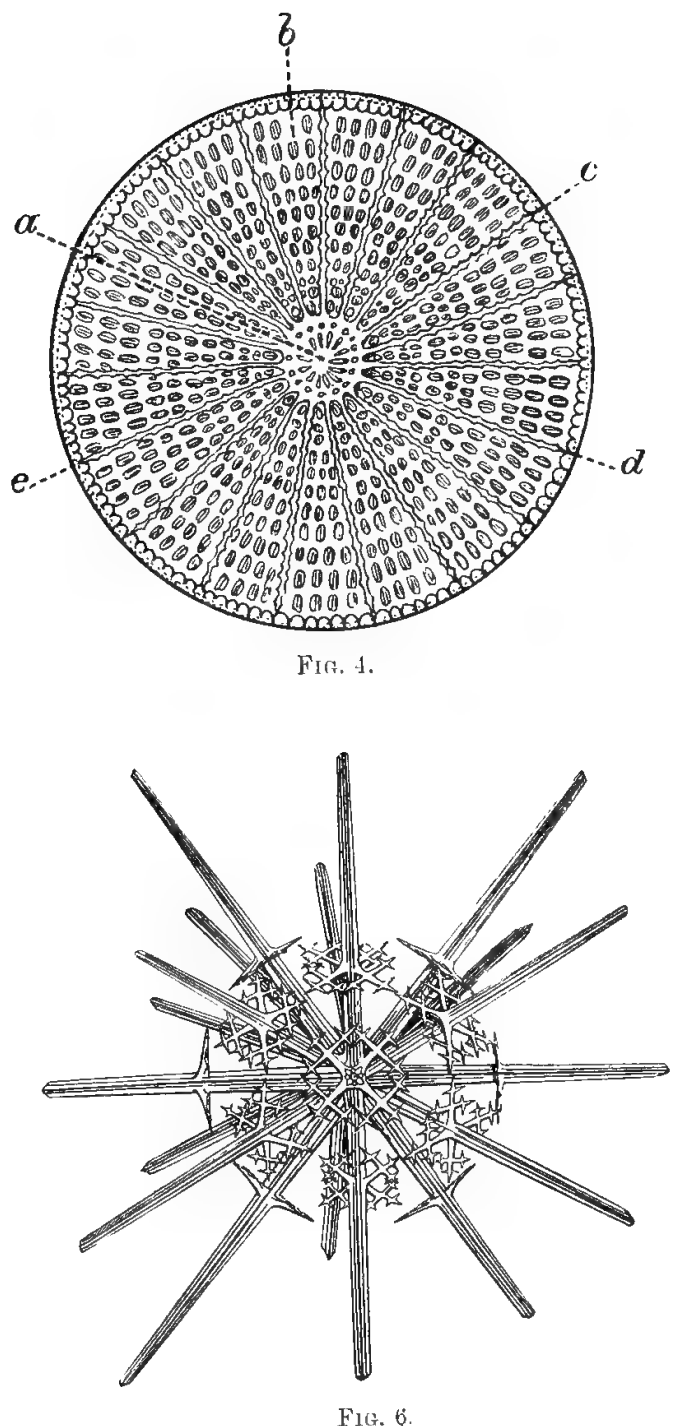


\section{RADIATING AND CONCENTRIC ARRANGEMENTS}

PLATE XXIV

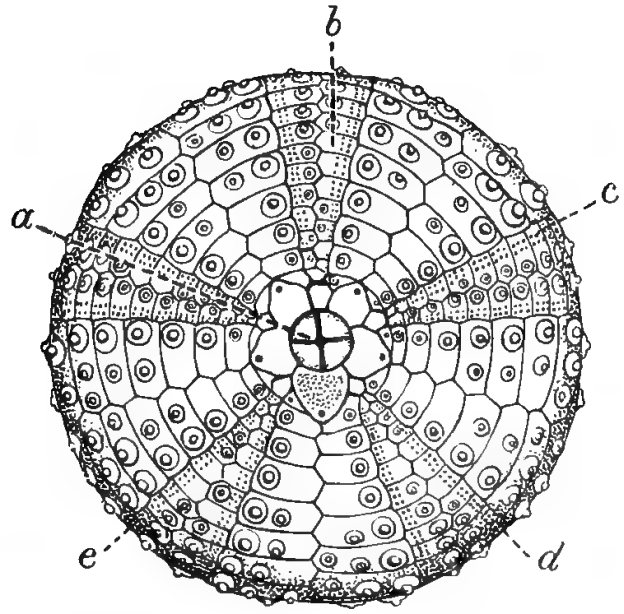

FIG. 1.

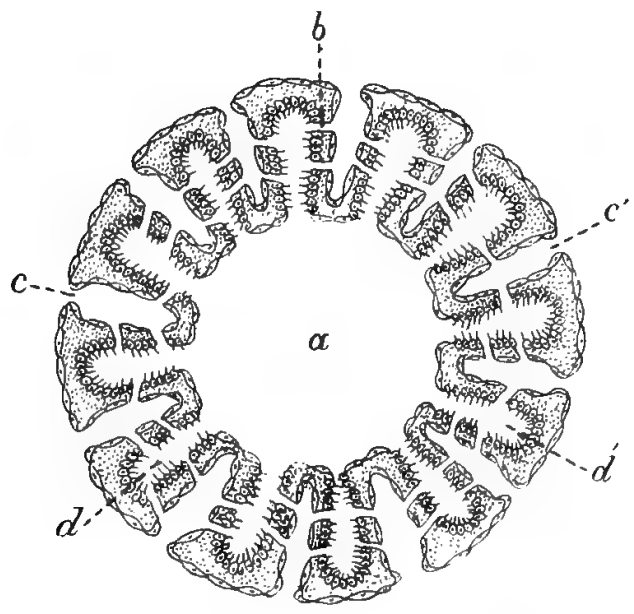

FIG, 2.

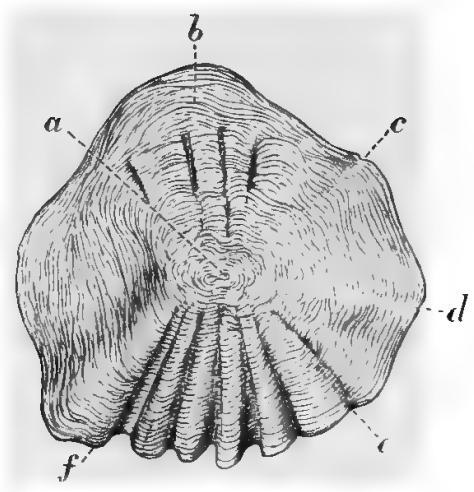

A

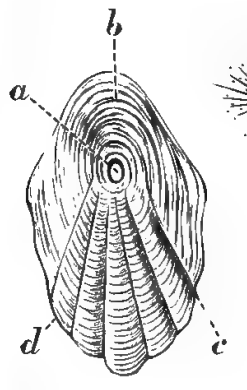

c

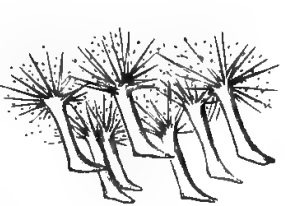

D

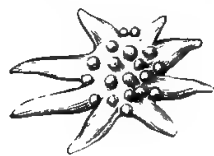

F

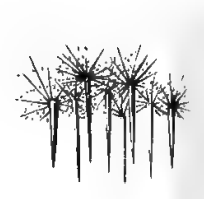

E

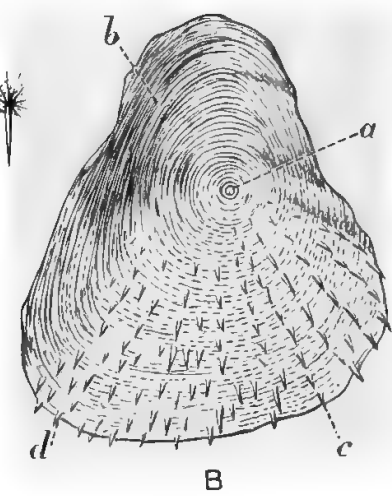

B

FIs. 3.

PLATE XXIII (continued)

(Plate xxii., Fig 1, A); radiolarian (Fig. 3 of this Plate); diatom (Fig. 4 of this Plate); sea-urchin (Plate xxiv., Fig. 1); the scales of fishes (Plate xxiv., Fig. 3); sections of hail (Fig. 2, B and C); various crystals as figured at Plates i., ii., iii., and iv.; and various structures delineated in Plate $v$. The radiating and concentriv arrangements obtain in certain crystals and in all parts of plants and animals.

FIG. 3.-A radiolarian (Thalassicola pelarira) (after Haeckel). Shows radiating and concentric arrangements. a, Central portion; $b, c$, concentric parts; $d, e$, radiating parts.

FIG, 4.-A diatom (Avachnodiscus ehrenbergii), illustrating the radiating and concentric arrangements. ", Central portion of diatom; $b$, concentric portions ; $c, d, e$, radiating parts.

FIG. 5.-Haliomma wyvillei (after Wyville Thomson). Shows radiating, concentric, and network arrangement of parts.

FiG. 6. - Skeleton of Niphacantha murrayana (Guide-Book Coral Gallery, British Museum). Reveals radiating and concentric arrangement in hard parts of animals. Resembles the arrangentent met with in the spicules of glass sponges (Plate xliv., Fig. 7 ); corals (Plate xxv., Figs. 5 and 6); blood-vessels of lohule of liver (Plate xxxiii., Fig. 1) ; ganglion nerve cell (Plate xxxviii., Fig. 4); various flowers and trees (Plate xxvi., Fig. 1; Plate xxviii., Figs. 3 and 5); star-fishes (Plate xxviii., Figs. 2 and 4); sea anemones (Plate xxvii., Fig. 4); crystals and dendrites (Plate xxxiv., Figs. 3 and 4; Plate xxxv., Fig. 4) ; electric spark (Plate xxxi., Fig. 2), \&c. The radiating and concentric arrangements are fundamental and universal in the inorganic and organic kingdoms (the Author).

\section{PLATE XXIV}

Plate xxiv. illustrates radiating and concentric arrangements in the sea-urchin, sponge, and scales of fishes.

F1G. 1.-Aboral surface of sea-urchin (Arbacia). Shows radiating and concentric arrangements. $a$, Central portion of case or shell; $b$, hexagonal plates of same arranged in concentric rings; $c, d$, $e$, hexagonal plates arranged in wedges, and radiating. Drawn by C. Berjeau for the Author.

FIG. 2.-Transverse section of sponge (Sycandra) (after Masterman). Shows modification of radiating and concentric arrangements. $a$, Central cavity ; $b$, prosopyle ; $c$, $c^{\prime}$, imhalent canals ; $d$, $l^{\prime}$, exhalent canals,

VOL. I. 
PLATE XXV

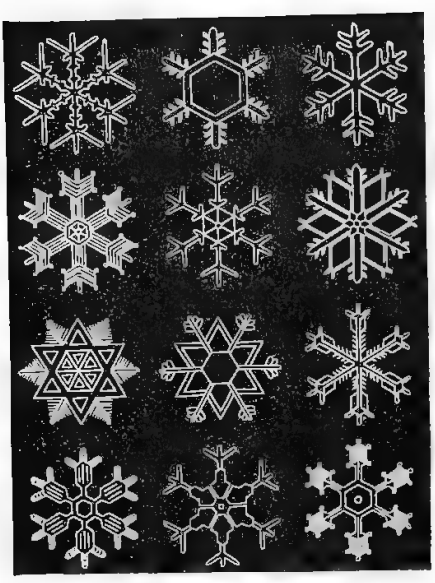

FIf: I.
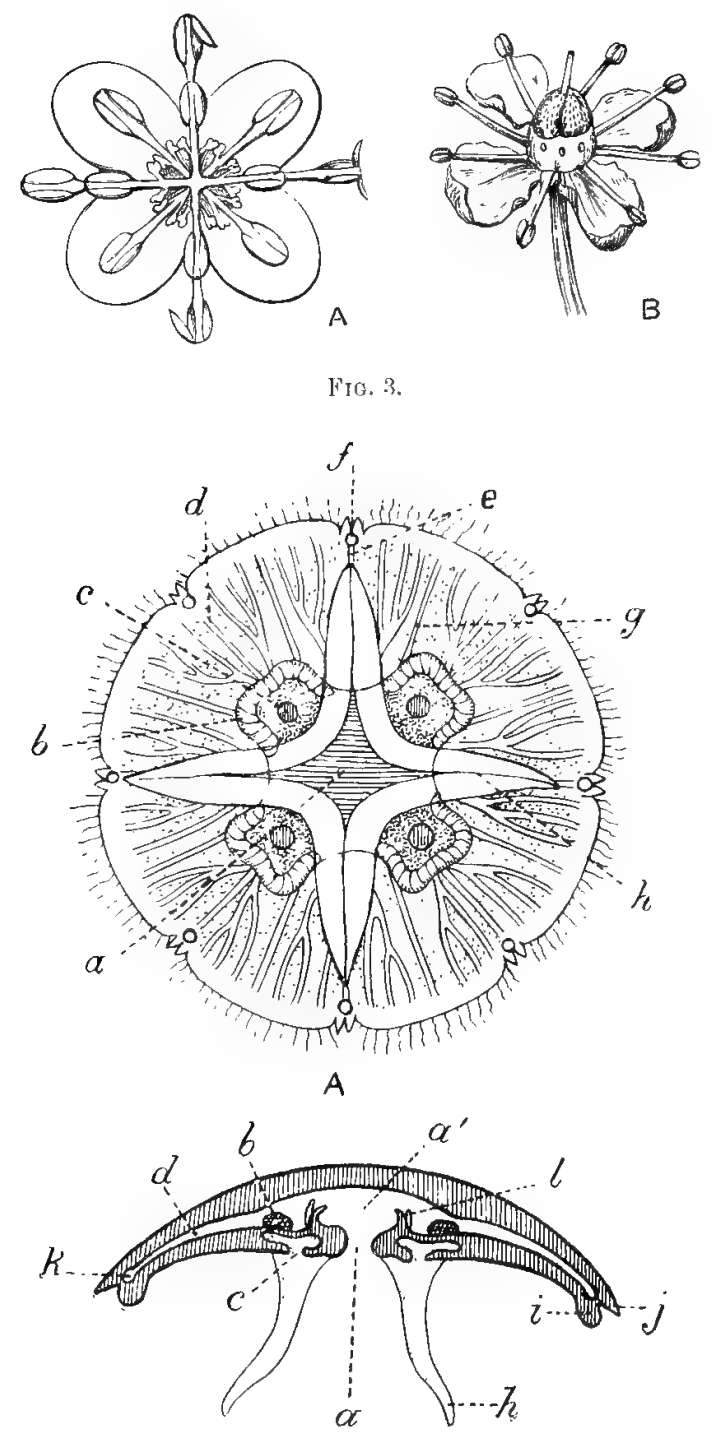

B

$F[1 \div 7$

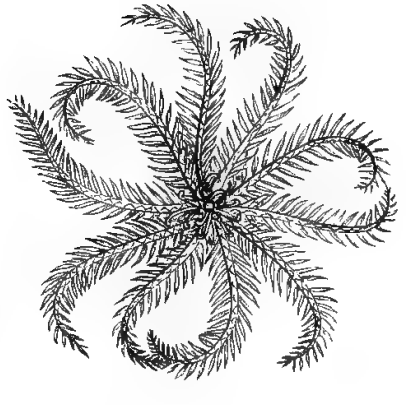

FIn. 2.
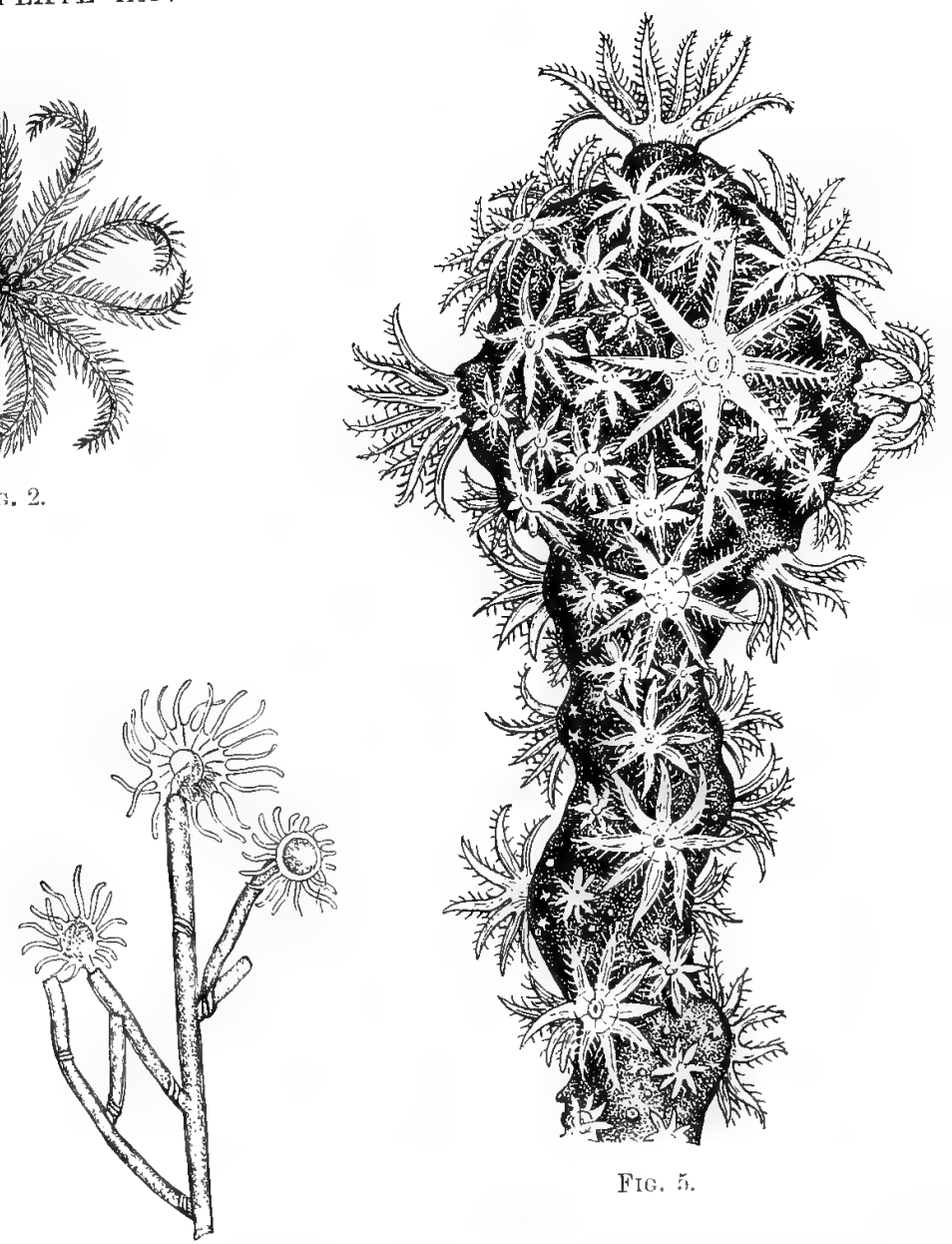

FIG, 4.

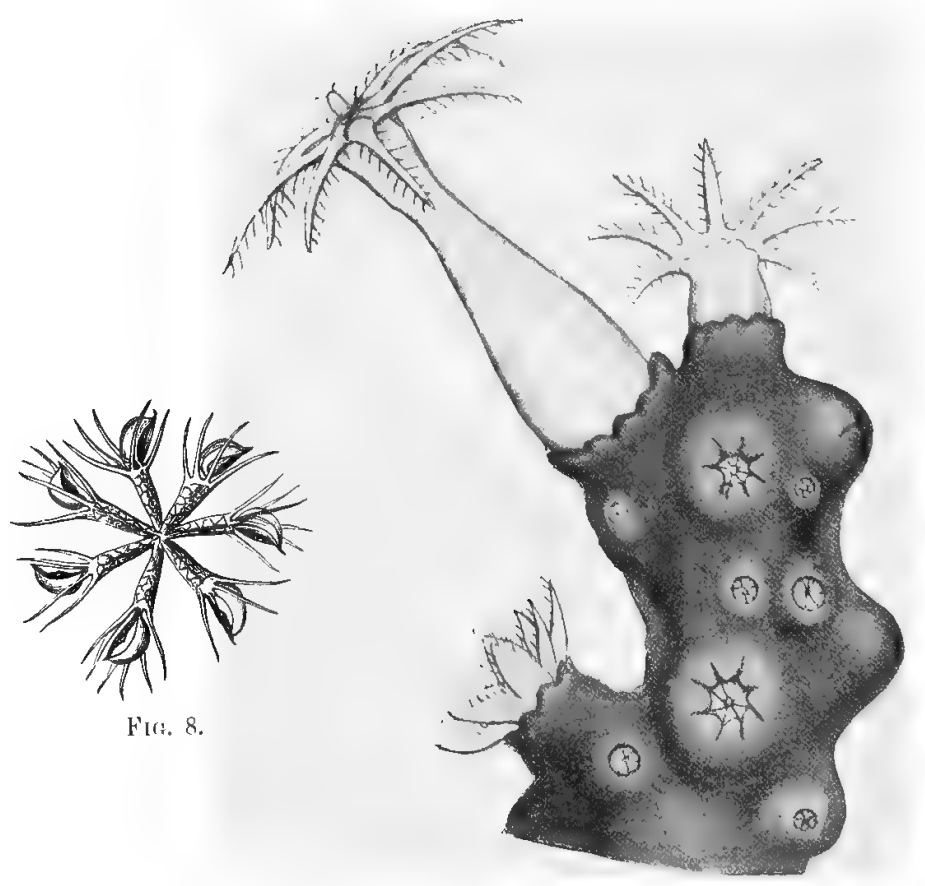

FIG. 6 , 


\section{RADIATING AND CONCENTRIC ARRANGEMENTS}

PLATF XXVI

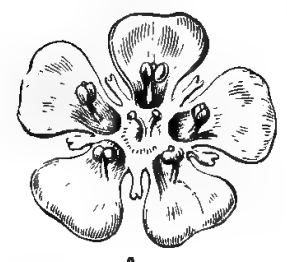

A

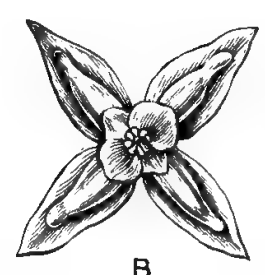

B

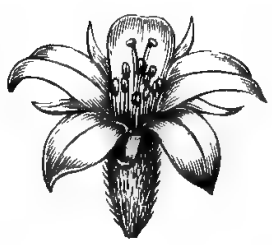

C
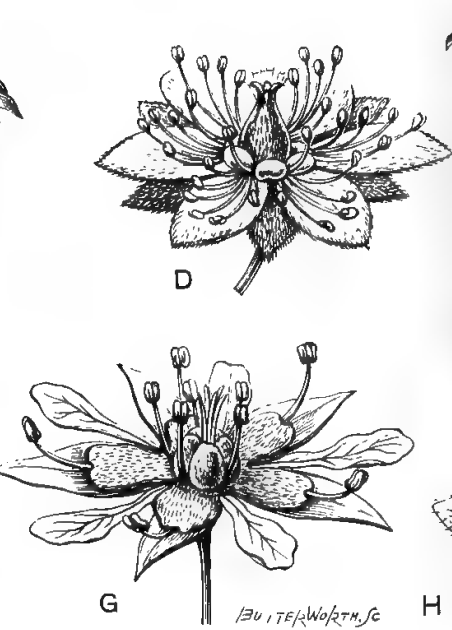

(1)

$\mathrm{H}$

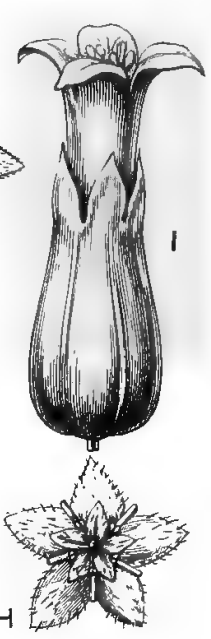

FIG. 1 .

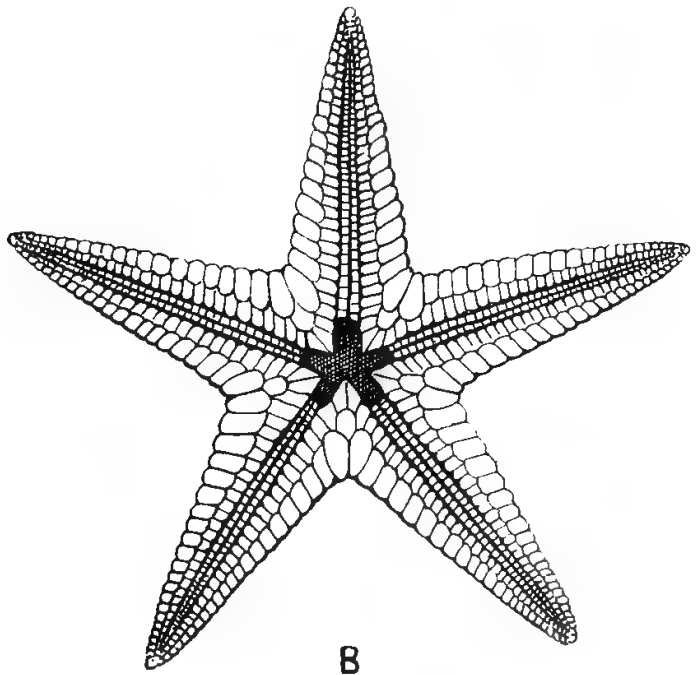

B

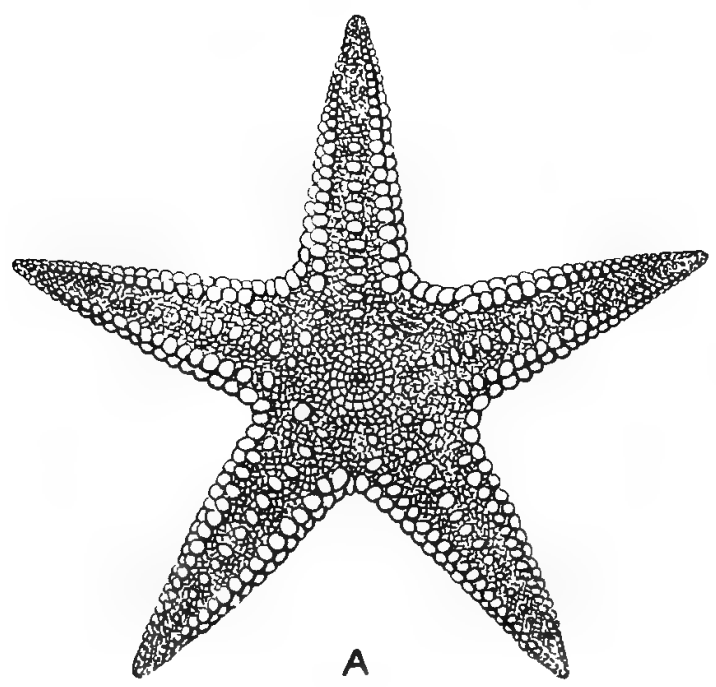

FIG. 2.

\section{PLATE XXIV (contimued)}

Fici. 3. - Scales of fishes (after Giinther). Reveal radiating and concentric arrangement of parts. The concentric rings, as in trees, represent layer's of growth.

A. $u$, Centre of system ; $b$, cuncentric rings ; $d, e, f$, radiating portions of scale.

$\mathbf{B}$ and $\mathbf{C}$. Similar arrangements and similar letters as in $\mathbf{A}$.

D, E, F. Slightly modified scales, the radiating or stellate arrangement prevailing. The arrangements seen at $\mathrm{A}, \mathrm{B}$, and $\mathrm{C}$ resemble those occurring in transverse section of trees (Plate xxii., Figs. 1 and 2); certail fungi (Plate xxii., Fig. 3); crystals (Plate xxv., Fig. 1); Howers (Plate xxv., Fig. 3); corals (Plate xxv., Figs. 5 and 6); sea anemones (Plate xxvii., Fig. 4); limpet and scallop shells (Plate xxvii., Figs. 1 and 2); vertical section of human brain (Plate xxvii., Fig. 3), \& .

\section{PLATE XXV}

Plate xxv. illustrates radiating and concentric arrangements in crystals, flowers, polypes, \&c.

FIG. 1.-Snow crystals as figured by Scoresby. These reveal wonderful symmetry and beauty of form. They display fundamental radiating and concentric arraugements, and may be regarded as starting-points in organisation. Curiously enough, the divergency or' radiating portions of the crystals are six in number, aud hexagonal forms are not uncommon in igneous I'ocks (Plate xl., Figs. I and 2). Hexagonal shapes occur in the vessels and other parts of plants, in the epidermis and pigment cells of the choroid coat of the eye, and in the comb of the honey bee (Plate iv., Figs. 4 and 13), \&c.

FIG. 2.-Comatula or feather star (after Dallas). In its young state it is furnished with a stem, and greatly resembles a plant. Shows radiating arrangement to perfection.

FIG. 3.-Symmetrical flowers (after J. H. Balfour), showing radiating and concentric arrangements.

A. Laurus nobitis. B, Ruta graveolens.

FIG. 4.-Polype (Eudendriun ramosum), magnified (after Dallas). Much resembles a plant. Shows radiating and concentric arrangements. Compare with Figs. $3,5,6$ and 8 of this Plate. 


\section{l'LATE XXV (continued)}

FIG. 5.-Red coral (Corllium rubrum) (after Lactze-Duthiers). Shows (xxtuisite iadiatiıg, concentrie, flower-like arrangements. For other view see Fig. 6.

FIG. 6.-The same, with polype extended.

FIG. 7. -Jelly-tish. (A urelicu aurite) (after Masterman).

A. Oral view. Shows radiating and concentric symmetrical arrangements. $u$, Mouth; $b$, gonad; $c$, aperture of sul)-genital jit ; $d$, inter-radial canal ; $e$, pro-radial cmal ; $f$, tentaculocyst; $g$, ad-radial canal ; $h$, oral tentacles.

B. Median longitudinal section through inter-radial plane. Shows mushnoon-shaped disc of jelly-tish (A ureliu aurita). " Mouth ; $a$, stomach ; $b$, gonad ; $c$, sub-genital pit ; $d$, inter-radial canal; $h$, oral tentacle; $i$, tentaculocyst; $j$, lappet; $k$, ring-canal ; $l$, gastric filaments.

FIG. 8.- Whorl of leaves of insectivorous plant (Allrovenda vesiculosa) (after Darwin). Slıow radiating, star-like arrangement.

\section{PLATE XXVI}

Plate xxvi. illustrates radiating and concentric arrangements and symmetry in flowers and star-fishes.

FIG. 1.--Examples of symmetrical flowers (alter Baillion). Show radiating and concentric arrangements. Flower's in many cases resemble crystals, as shown in Plates i., ii. and iil., and in Plate xxv., Fig. 1. They also resemble many auinals, especially infusoria, (Plate xxxvii.); polypes and corals (Plate xxv., Figs. 4, 5, and 6); sea anemones (Plate xxvii., Fig. 4); and star-fishes (Fig. 2 of this Plate, and Figs. 2 and 4 of Plate xxviii.).
A. Mitella nuda.
B. Epinedium alpinum.
D. Homalium racenosum.
E. Tarira volyantha.
G. Quillaja saponaria.
(.) Raphiolepis rubra.
F. Alchemilla vulyaris.
H. Buettueria salicifoliu.
I. Bryophyllum calycinum.

FIG. 2.-Typical star-fish (I'uletester cuchuris, Devonian) (after Hall). Shuws raliating and concentric arrangements, and resembles nany crystals (Plates i., ii., and iii., and Plate xxv., Fig. 1); and many plants (Plate xxv., Fig. 3 ; l'late xxviii., Figs. 1 and 5 ).

A. Upper surface of star-fisl. B. Under surface of star-tish.

\section{PLATE XXVII}

Plate xxvii. illustrates radiating and concentric arrangements in the limpet, scallop, human brain, and sea anemones.

FIo. 1.-Limpet (Fissurelle nimbusa). Shows radiating and concentric arrangements to perfection. Resembles many erystals (Fig. 2, B ; Plate iv., Figr. 2); sone fungi (Plate xxii., Fig. 3); the transverse sections of trees (Plate xxii., Fig. 1); lones, teeth, (ce. (Plate v.). Drawn by C. Berjeau from specimen in the Author's imseum.

FIG. 2.-Clam or scallop shell (Pecten redians), furnishing a striking example of radiating and concentric arrangements. Resenbles wedge-shaped portion of transverse section of tree (Plate xxii., Fig. 1), also fungus (Plate xxii., Fig. 3), \&c. ", Starting-point of shell ; $b$, concentric rings corresponding with rines of growth; $c, d, e$, radiating portions of shell. Drawn by C. Berjeau fronı specimen in the Author's museum.

F'ri. 3.-Vertical mesial line section of human brain (after Dalton). Shows radialing and concentric arrangements in a marked degree. Resembles certain plants (llate xxii., Fig, 3).

A. Olfactory ganglion.

B. Ridiating tibre of cerehrun enveloped by concentric convolutions.

C. Corpus striatum.

E. Tubercula quadrigemina.

F. Cerebellum.

(i. Canglion of tuber anuulare.

D. Optic thalamus.

H. Ganglion of medulla oblongata.

Fıc. 4. - Sea anemones, frum water-colour drawing in the Natural History Department of the British Musenm by C. Berjeau. Display radiating and concentric arrangenents in a striking manner. Resemble certain crystals (Plate i., Fig. 13) and many fluwers (Plate xxvi., Fig. 1) and trees (Plate xxviii., Fig. 3).

\section{PLATE XXVIII}

Plate xxviii. illustrates radiating, concentric, and spiral symmetrical arrangements in plants and star-fishes.

FIG. 1.-Alstromeria, displaying radiating whorl of spiral leaves. $a$, Leaf stem ; $b$, c, $d$, c, leaf twisted upon itself, the margins of the leaf being disposed in different planes and revealing double or figure-of-8 curves. Drawn by C. Berjeau from specimen collected by the Author ; half natural size.

FIG. 2.-Silurian star-fishes (after Salter). Show primitive radiation of a pronounced type. Resemble many crystals (Plates i. and ii.; Plate xxv., Fig. 1); plants (Plate xxvi., Fig. 1, and Figs. 1 and 5 of this Plate); they also resemble modern star-fishes (Fig. 4 of this Plate).
A. Plasterina primæru.
B. Palsaster rutheni.
C. Palaocoma Colvini.

FIG. 3. --Palm tree. Shows branches radiating from a central point. Resembles many crystals (Plates i. and ii.), and animals (Plate xxv., Figs. 4, 5, 6, and $\zeta$; Plate xxvi., Fig. 2 ; and Figs. 2 and 4 of this Plate), and parts of animals (Plate $v$.). Drawn by C. Berjeau.

FIG. 4.-Modern star-fish (after Wyville Thomson). Shows typical radiation, and resembles many crystals and plants, as explained nnder Fig. 2.

F1G. 5.-Summit branches of Norfolk Island pine (Arancaria excelsa), showing characteristic radiation. The summit branches vary from four to seven in number, and present a dainty, symmetrical, feathery appearance. The arrangement resenbles that found in crystals, animals, and parts thereof, as stated under Fig. 3. Drawn by C. Berjeau, from growing specimen in the possession of the Author, 


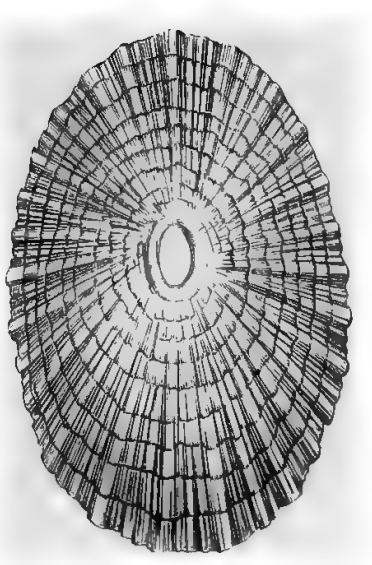

FIt. 1 .

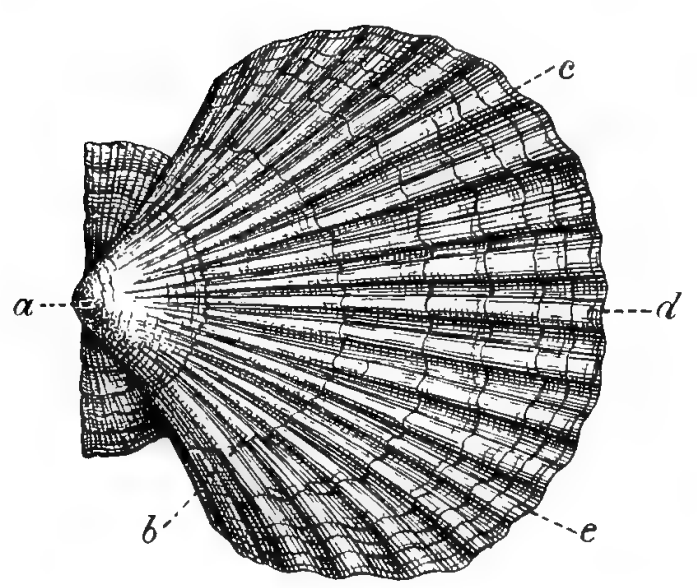

FIC: 2.

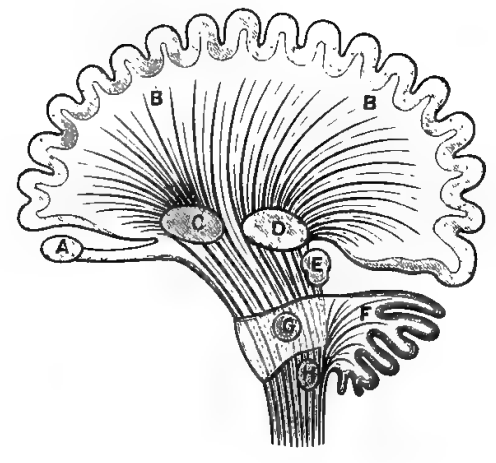

FIG. 3..

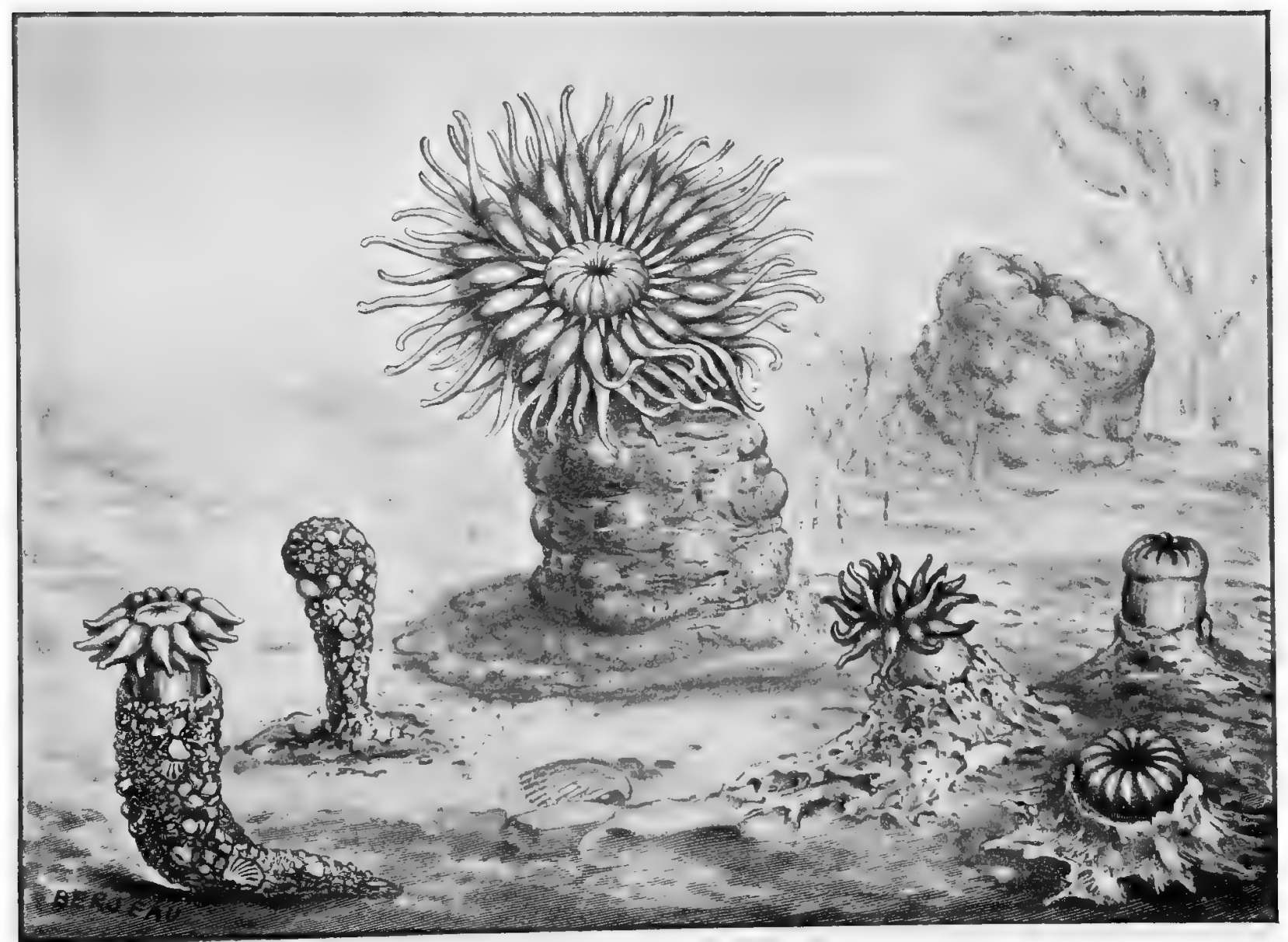

FIG. 4, 
PLATE XXVIII
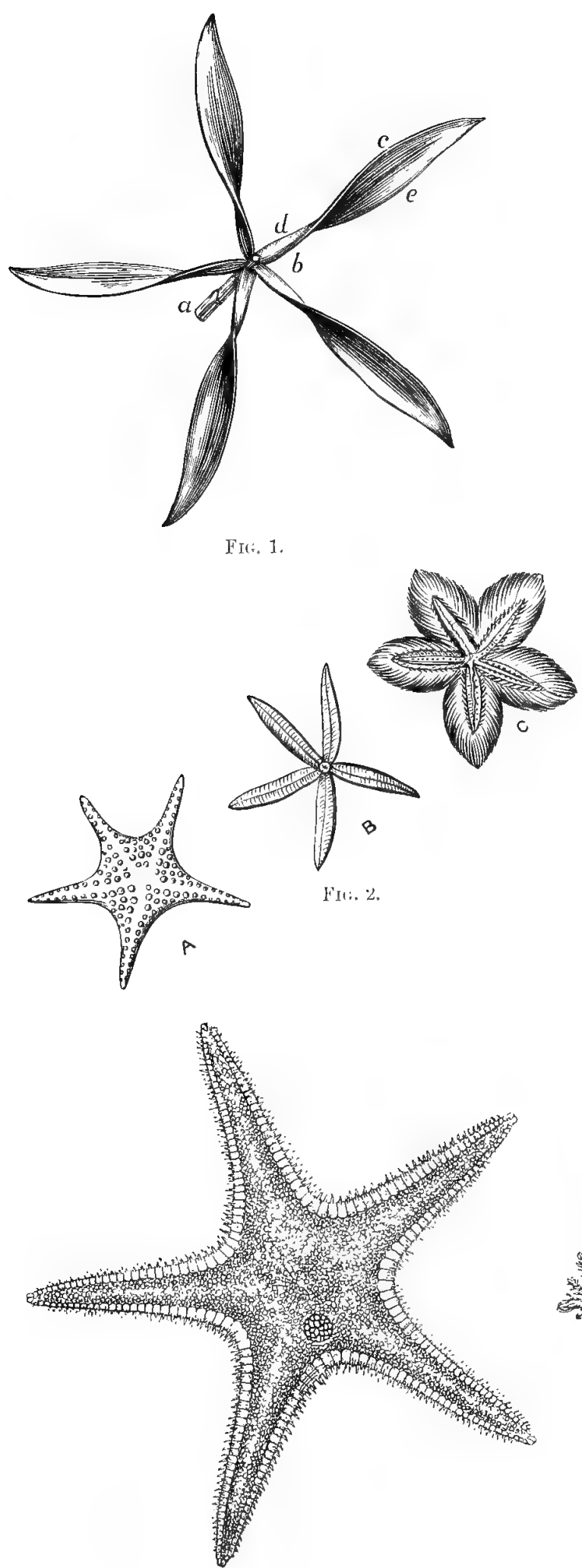

FIG. 4,
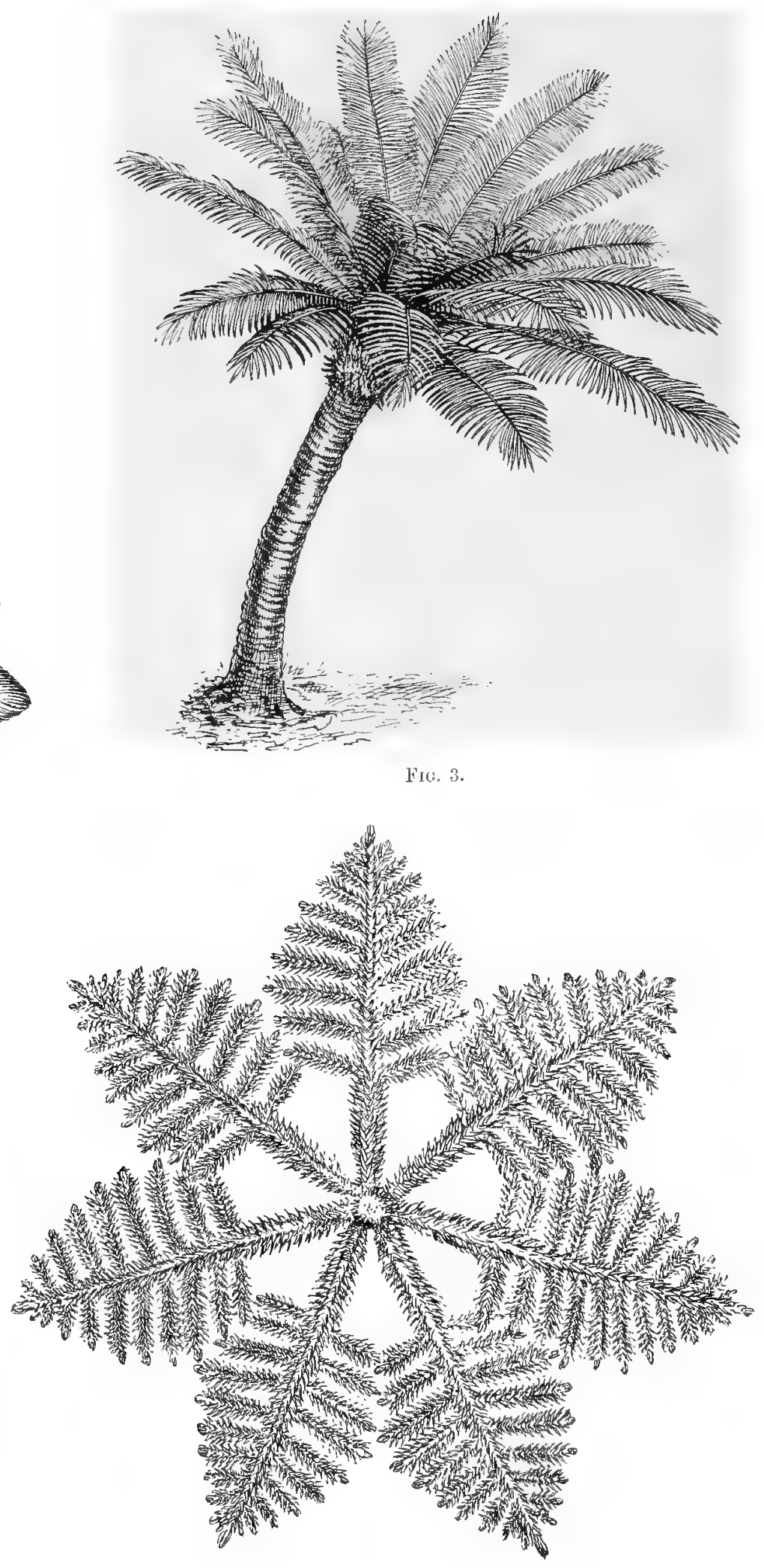

FIG, 5. 


\section{DENDRITIC OR BRANCHING ARRANGEMENTS}

\section{§ 9. Dendritic or Branching Movement in Electrical Discharge-Dendritic Formations in Crystals, Plants, Animals, \&c.}

Having shown how very intimate the connection is as between crystals, plants, and animals in the matter of radiating, concentric, and spiral arrangements, and having further shown that spiral arrangements exist on a grand scale in the physical universe, I now proceed to demonstrate that dendritic or branching arrangements also exist in crystals, in plants, and animals, and in the physical universe. My contention here is that there is an inherent tendency in physical and vital forces to split up the matter (dead and living) on which they act; the amount of splitting increasing in proportion to the amount of resistance encountered, there being less resistance in ether than in common air, less in fluids than in solids, and so on. A river when it reaches the flat sands and mud of the estuary usually divides into two or more channels; water forced through a hose on a dry pavement spreads. and so does water spilt upon the ground. In like manner the resistance experienced by the fluids permeating the body causes the fluids to split up, divide, and bifurcate, and this, it appears to me, has much to do with the dendritic or branching appearance presented by the blood-vessels, the coats or coverings of which are laid down where the nutritive and formative fluid first meanders. Force takes the line of least resistance in this as in other cases, and, as I have explained elsewhere, the vital and physical forces are, as a rule, not opposed to each other but work in unison. What is true of the blood-vessels is true of the lymphatics, nerves, and all structures where division and branching occurs. The division is, for the most part, longitudinal, but not unfrequently it is transverse, when it is known as segmentation. Division may, of course, take place as apart from obstruction in growing structures. When a formative agency is at work, as in growth and development, the direction and extent of the division or bifurcation is predetermined, and limits set. That a certain amount of resistance is experienced in many cases during development is shown by this, that at the points of bifurcation in growing structures there is generally a little increase in substance in solids and a slight dilatation of the channel in tubes. The thickening and increase in question are seen to advantage in the venation of leaves, and the branching of blood-vessels (Plate xxxiii., Figs. 1 and 3); the increase referred to is seen in the physical universe in electric sparks (Plate xxxi., Figs. 2 and 3, and Plate xxxii., Fig. 2); lightning flashes (Plate xxix., Figs. 1 and 2); and in branching structures generally (Plates xxxviii. and xxxix.).

The vital force during development presses forward, as is well shown in the streaming or river-like advance of plasmodium when feeding (Fig. 59, p. 302). In this case also there is increase where the living stream divides.

The dendritic or branching arrangements in the inorganic and organic kingdoms are illustrated by twenty-five plates, namely, Plates xxix. to liv. inclusive.

\section{PLATE XXIX}

Plate xxix. illustrates radiating, branched, waved arrangement in lightning flashes.

Fra. 1,-Ramified, waved lightning, photographed by J. Cruik. Shows radiating, dendritic, tree-like shapes wholly unlike the conventional zigzag lightning pourtrayed by artists. Resembles electric sparks (Plate xxxi., Figs. 1, 2 and 3 ; Plate xxx., Fig. 3; Plate xxxii., Fig. 2); various crvstals (Plate ii.); dendrites (Plate xxxiv., Figs. 1 and 2 ; Plate xxxv., Figs. 1 and 4 ; Plate xxxvi., Fig. 2); leaves and trees generally (Plate xxxiii., Fig. 3 ; Plate xlv., Figs. 2 and 3) ; corals (Plate xxxv., Fig. 5 ; Plate xxxvi., Fig. 3); and parts of animals such as the bronchial tubes, blood-vessels, and other liranching structures (Plate xxxix., Figs. 1 to 9 inclusive).

Fig. 2.-Ramified, waved lightning as seen at Sydney, New South Wales, photographed by H. C. Russell. Shorvs radiating, branching, dendritic, tree-like forms similar to those represented at Fig. 1 of this Plate. The description given under Fig. 1 applies to this figure.

FIG. 3.-Photograph of electric spark where the terminals or discharging poles (positive and negative) are separated four inches. Similar photngraphs are seen at Plate xxx., Figs. 1, 2, and 3. The descriptions given of these figures apply to this. Pliotographed for the Author hy Mr. T. C. Gamlen.

\section{PJATE XXX}

Plate xxx. illustrates lines of force, motion, and light as revealed by electric sparks.

FIGs, 1 and 2.-Electric sparks specially photographed for the Author by Mr. T. G. Gamlen of West Hartlepool. The sliarks were the aid of a 10 -inch induction coil with 12 volts on coil. Exposure a pratte of a second. 'The terminal taken in a dark room by the aid of a 10-inch ine separated 2, 3, and 9 inches respectively. 'J'he photographs have been reduced to or discharging poles (positive and negating lines of force, motion, and light similar in many respects to the lightning fashes representer suit the size of the page. Show branching lines of force, motion, and the

FIG. 3.-Branching lines of force, motion, and light obtained as in Figs. 1 and 2 ; the terminals in this case being separated 9 inches. These resemble in a striking nanner the branched lightning flashes represented at Plite xxix., Figs, 1 and 2 , especially the latter. Photographed for the Author by Mr. T, G. Gamlen. 
PLATE XXIX

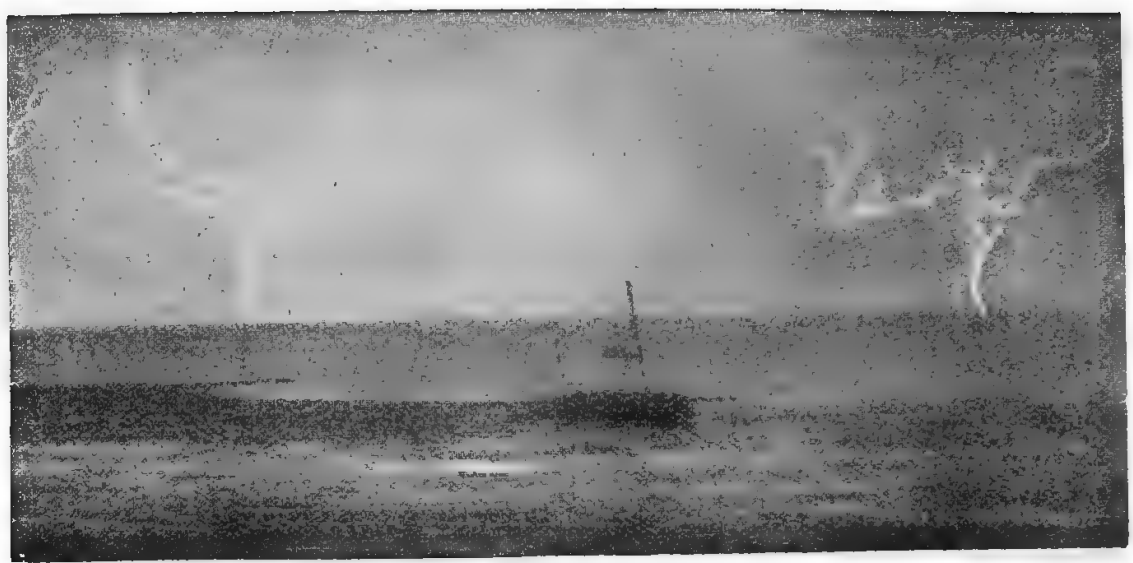

FIG, I.

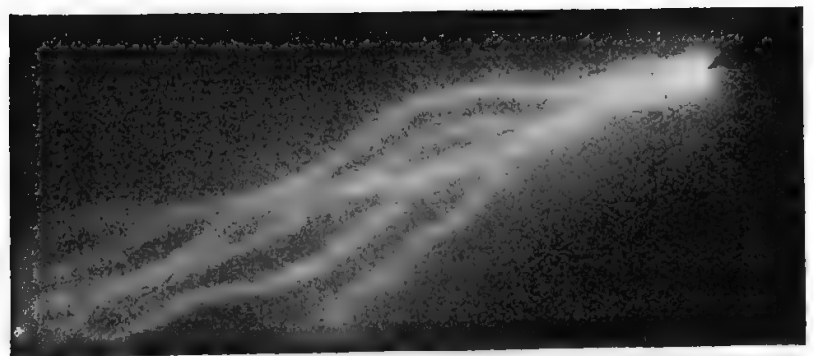

Fi: 3: 3.

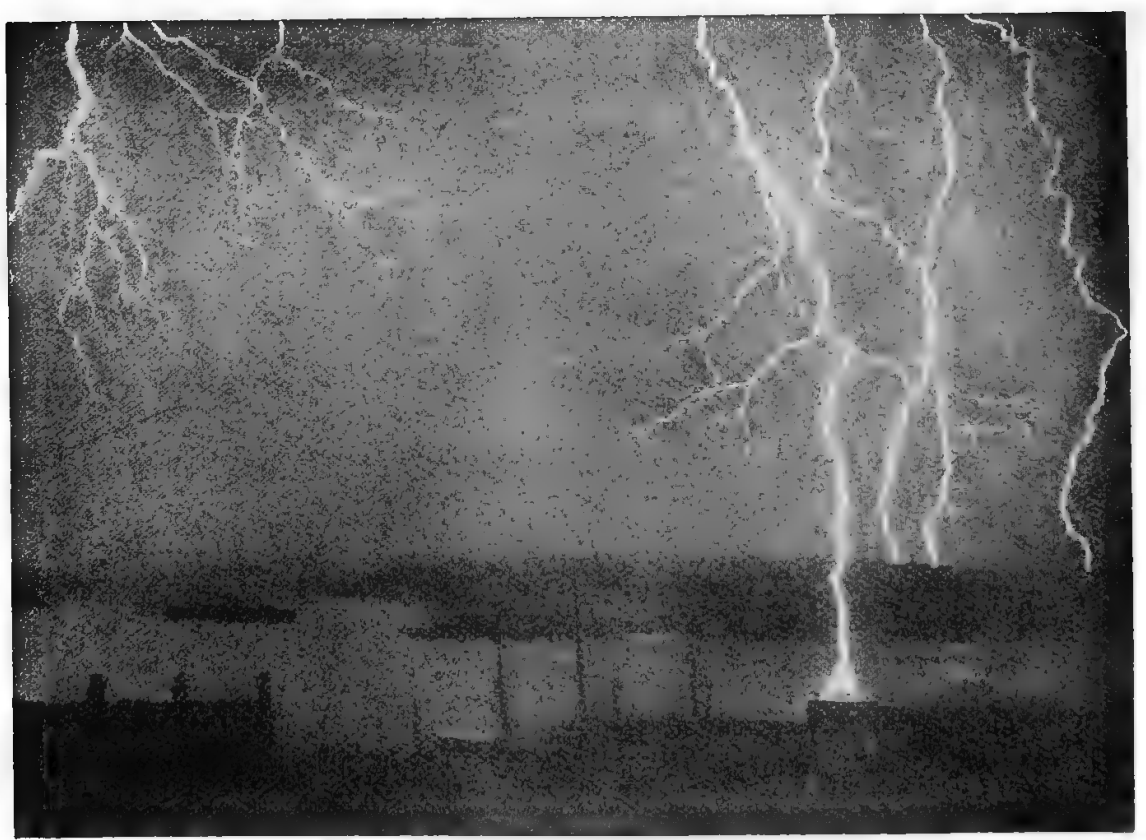

Fis: 2 . 
PIATE $\mathrm{XXX}$
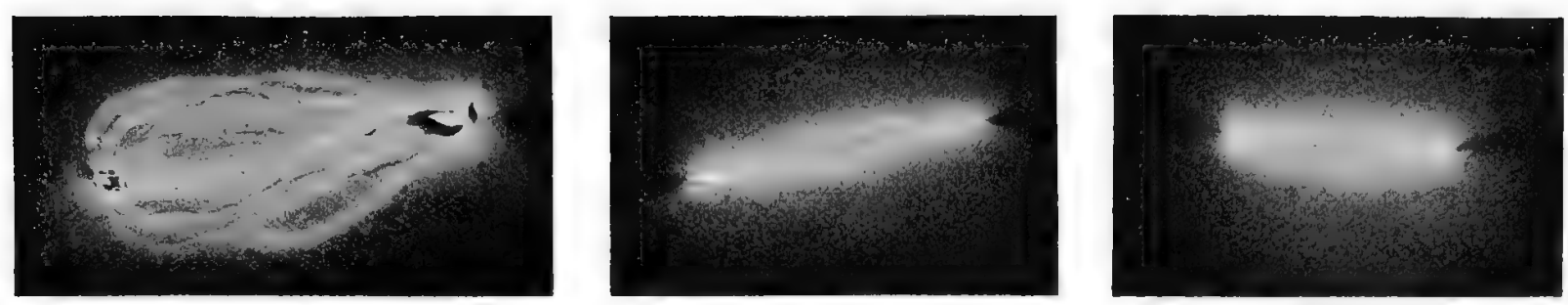

Fir. 1.
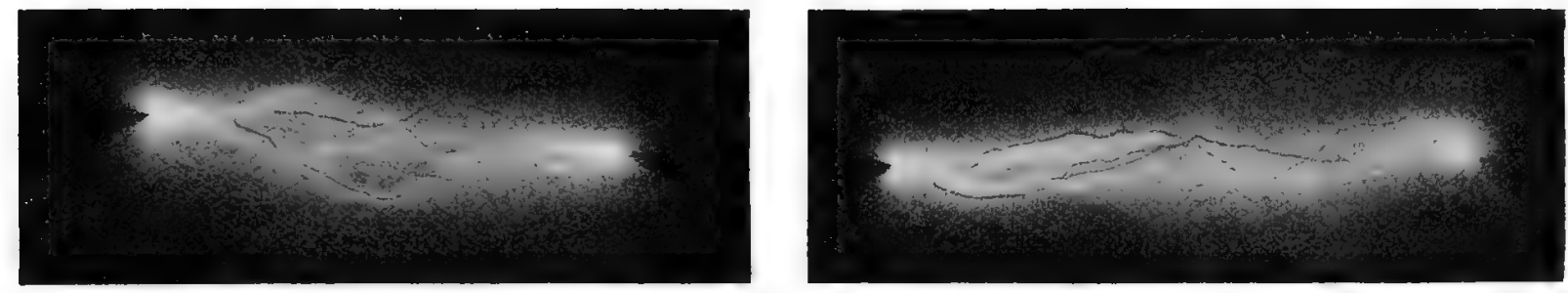

FIG. 2.

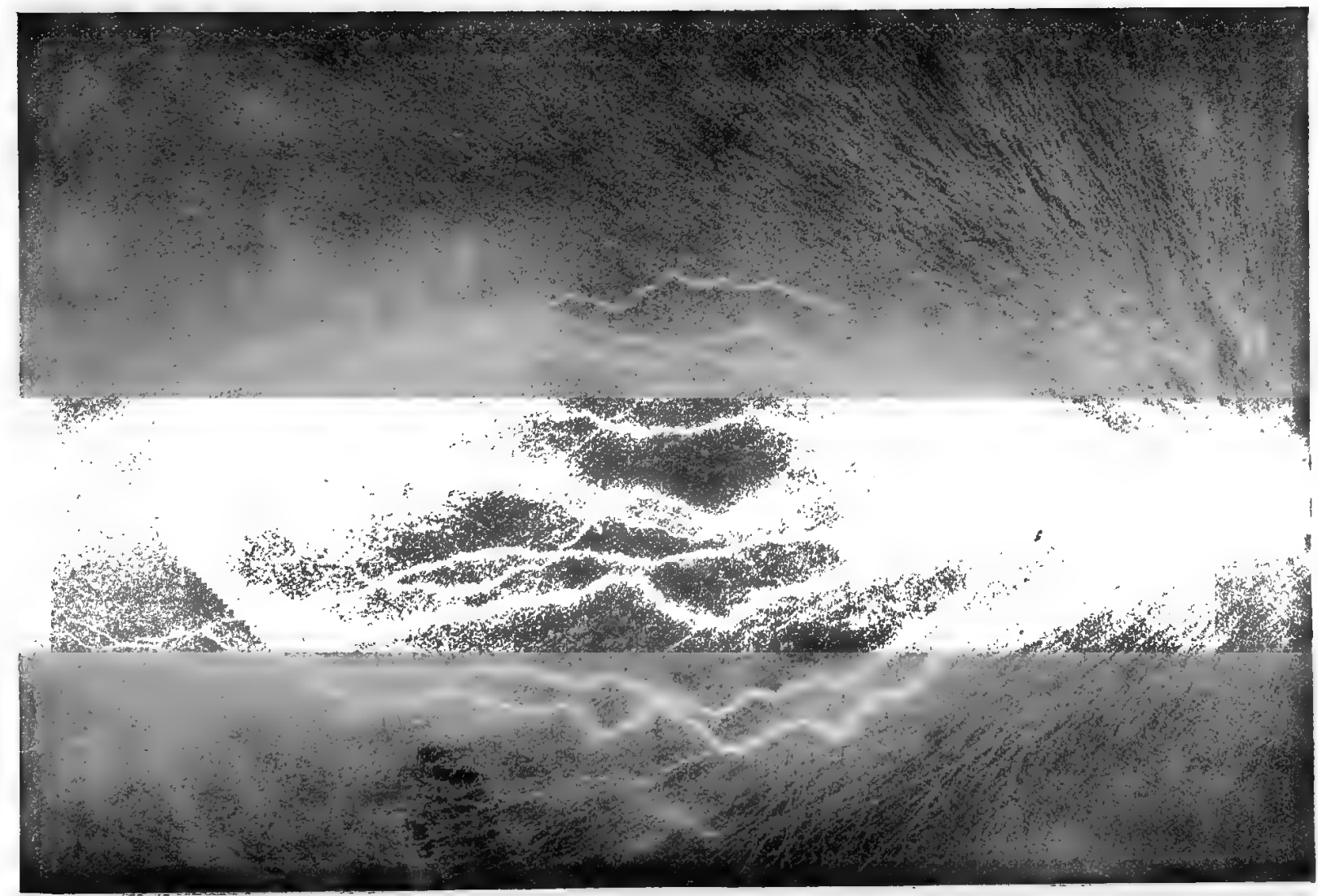

FiG. 3,

VOL. I, 


\section{PLATE XXXJ}

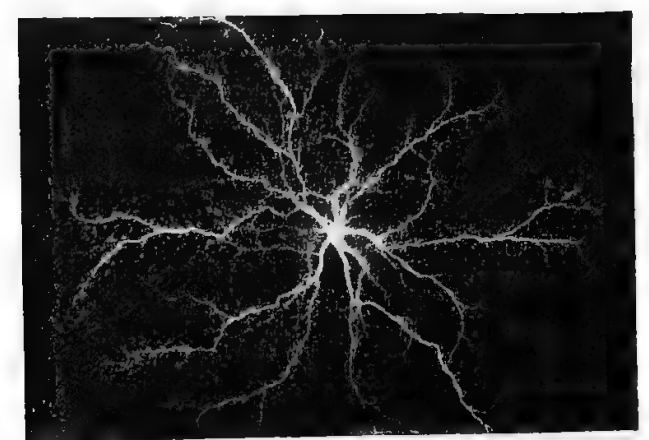

FII 1.

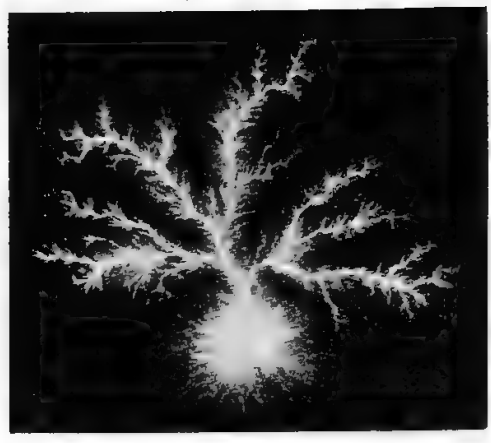

ENa, 2.

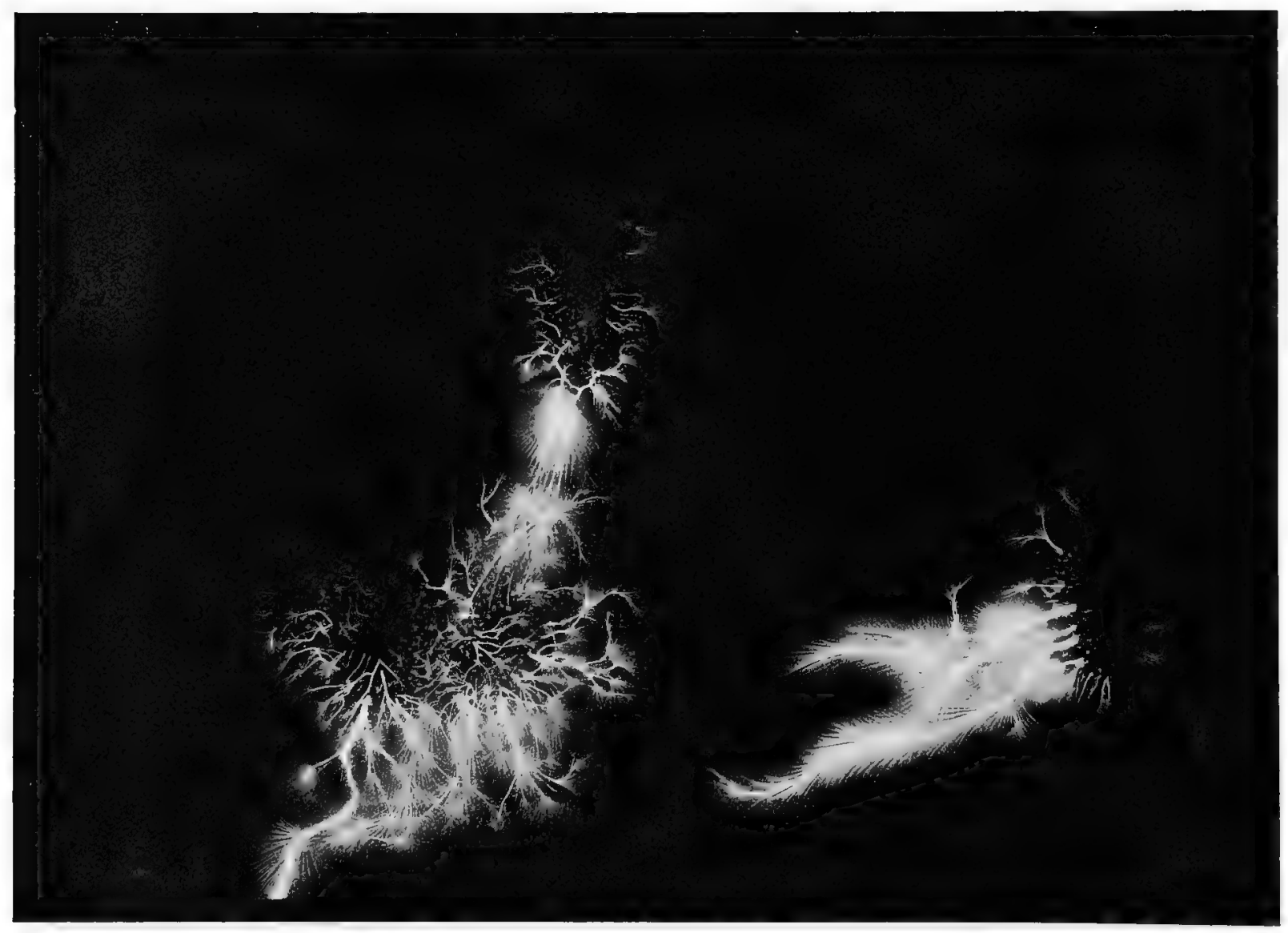

Fis: 3.

Plate xxxi. illustrates lines of force, motion, and light as seen in electric sparks.

Frg. 1.-Photograph of a typical electric spark by A. Thurhurn. Shows radiating, branched appearance presented by the electrical discharge when acting on a highly sensitive photographic plate.

FIG. 2.-A similar photograph by A. Thurburn, where the electric spark assumes a radiating and still more lyranched and tree-like appearance.

FIG. 3.--A somewhat similar photograph by D1. Adans, where the electric sparks have assumed a radiating, branched, and feathery appearance; Röntgen ray pictures of the hands being seen in the background. Pictures of this class are most conveniently obtained by placing two photographic plates back to back and inserting between them the points of a discharger connected to an induction coil ; the contact being broken by hand to prevent more than one discharge falling on the plates. Fig. 2 of this plate represents a photograph taken from the plate next the negative terminal; Fig. 3 , that taken from the plate next the jositive terminal. 
PLATE XXXII

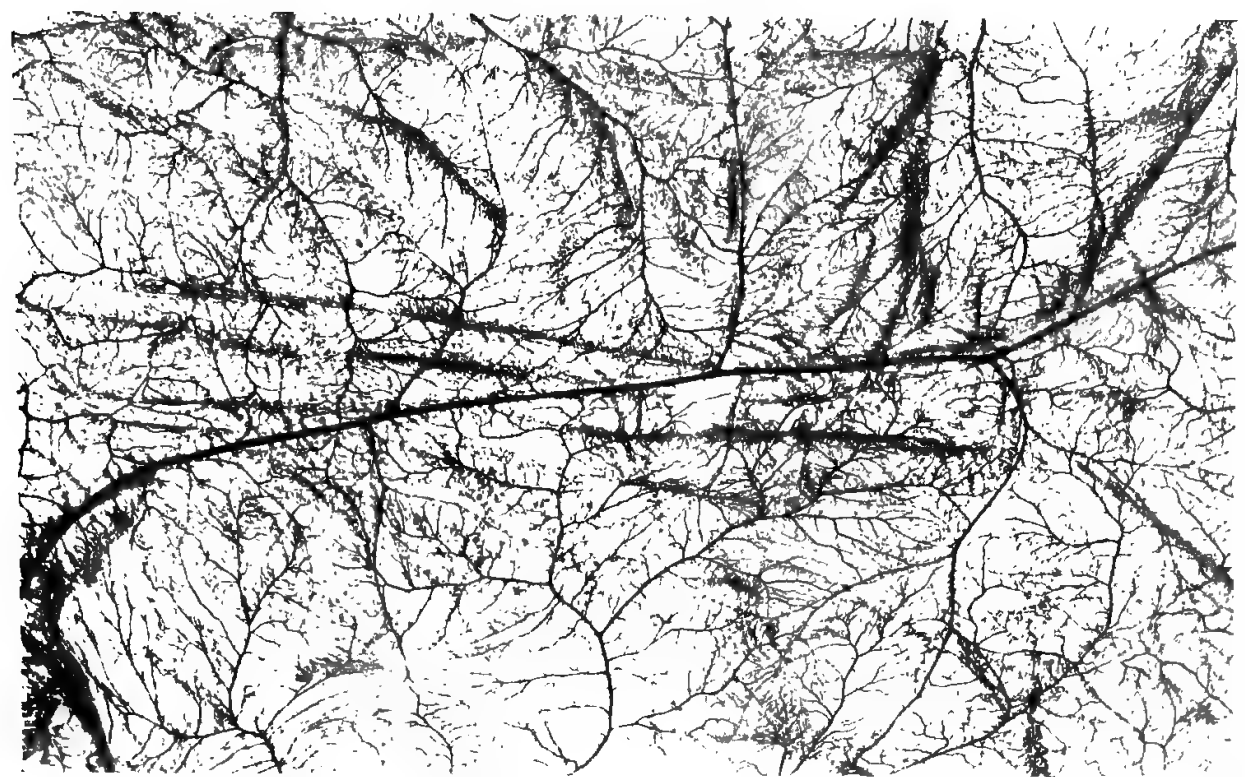

Fili, 1.

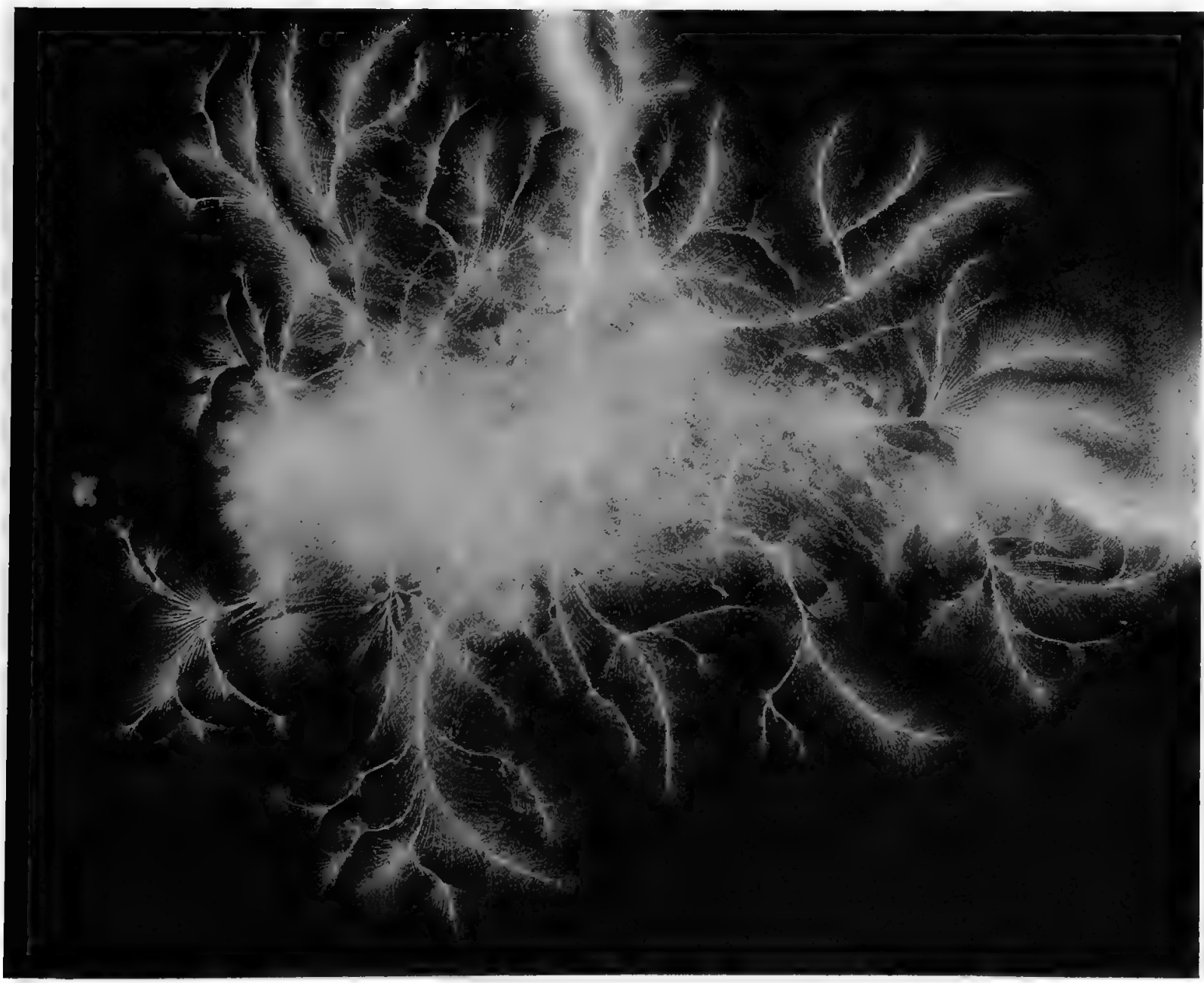

Fis.2. 


\section{PLATE XXXII}

Plate xxxii. illustrates lines of force and movement as indicated by blood-vessels and electric sparks.

FIG. 1.- Portion of the chorion of a mare injected and prepared by the Author in 1863: preserved in the Hunterian Museun of the Royal College of Surgeons of England. Shows the extraordinary branching arrangements of the blood-vessels, resulting in an exquisite network or tracery resembling that formed by the stems, branches, and twigs of trees in winter, and by the venation of leaves in sumner (Plate xxxiii., Fig. 3). It also resembles that seen in crystals (Plate ii.); dendrites (Plate xxxiv., Figs. 1 and 2 ); polypes (Plate xxxvi., Figs. 1 and 3 ); growing lone (Plate xxxviii., Fig. 3); nerve ganglia (Plate xxxix., Fig. 2); and especially the ramificatiuns of electric sparks and Rüntgen ray pictures (Plate xxx., Figs, 1, 2, and 3; Plate xxxi., Figs. 1, 2, and 3); and certain forms of lightning (Plate xxix., Figs. 1 and 2). Front photograph by the Author.

Fig. 2.-Remarkable photograph presented to the Author by Count Jodko, Shows electric sparks branching and terminating in delicate feathery forms. The photograph makes visible the lines of force, motion, and light; the bifurcations or branching being characterised by enlargements which represent minor electrical discharges or explosions-the main discharges or explosions being seen in the more luminous left portion of the photograph. It specially resembles the branching blood-vessels seen in Fig. I of this Plate, and the several structures and phenomena referred to in the description thereof.

\section{PLATE XXXIII}

Plate xxxiii. illustrates branching arrangements in the blood-vessels of the liver, in a lightning flash, and in a leaf.

FIt. 1,- Blood-vessels as seen in a transver'se section of a lobule of the human liver injected (after. Sappy). Show branching, radiating, and concentric arrangement, producing a marvellous network of capillinies greatly resembling that seen in many crystals (Plates i. and ii.); dendrites (Plate xxxiv., Fige, 3 and 4); polypes (Plates xxxv., Figs. 3 and 5 ); the venation of leaves (Fig. 3 of this figure); and various animal stmctures (Plate $V_{0}$ ). a, Intrit-loljular or hepatic vein radjating and lranching to form minute capillary plexuses; $b$, intra-lobular or portal veins breakiner up and inosculating witl the intra-lobular ones.

FlG. 2.-Photograph of dendritic markings produced ly a lightning flash on the human arm. Resembles crystals (Plate ii.); electric sparks, \&. (Plates xxx., xxxi., and xxxii.); dendrites (Plate xxxiv., Fiss. 1 and 2 ; Plate xxxvi., Figs. 1 and 4); branches and leaves of plants (Fig. 3 of this Plate), and branching structures in animals (Plate xxxvi., Figs. 1 and 3 ; l'late xxxviii., Fig. 4 ; Plate xxxix., Figs. 1, 2, 4, 6, and 9).

ligu. 3.-Plotograpl of a leaf by ('ount Jodko showing typical, radiating, branching venation similar to what oceurs in crystals (Plate ii.); electric sparks (Plate xxxi., Figs. 2 and 3 ; Plate xxxii., Fig. 2); dendrites (l'late xxxiv., Figs. 1, 2, 3, and 4 ; Pliate xxxv., Figs. 1 and 4 ; Plate xxxvi., Fig. 2); polypes (Plate xxxvi., Fig. 1); and the lnancling structur. s in animals enumerated under Fig. 2 of this Plate.

\section{PLATE XXXIV}

Plate xxxiv. illustrates radiating and branching crystallisation as seen in window-panes of houses during frost and in rocks.

Figs, 1 and 2.--Honr-frost crystals forming branching, dendrilic arrangements on window-panes ant pavements in winter (from photograjhs by Mr. J. Leadbeater). Sinilar dendrites are formed on stones (Plate xxxv., Fig. 1 ; Plate xxxvi., Fig. 2); on metals and on ice (Plate xxxv., Fig. 4). They resemble electric sparks (Plate xxxi., Figs. 2 and 3); lightning flashes (Plate xxix., Figs. 1 and 2 ; Plate xxxiii., Fig. 2); crystals (Plate ii.); plants (Plate xliii., Fig. 7; Plate xlv., Figs, 1, 2, and 3); polypes (Plate xxxv., Figs. 2, 3, and 5 ; Plate xxxvi., Fig. 1); and branching structures generally.

F1a. 3.-Photograph by Valentine of the famous "Rock and Spindle" in the vicinity of St. Andrews, Fifeshire, Scotland. Displays a remarkable radiating crystallic arrangenent J'esembling a huge wheel with prominent spokes. The Rock and Spindle belongs to the Plutonic system of rocks, and is situated on the seashore; its base being immersed at each rise of the tide. Resembles many crystals (Plates i. and ii.; Plate xxv., Fig. 1) ; electric sparks (Plate xxxi., Figs. 2 and 3); dendrites (Plate xxxv., Figs. 1 and 4, and Fig. 4 of this Plate); flowers (Plate xxv., Figs. 3 and 8 ; Plate xxvi, Fis. 1); trees (Plate xxviii., Figs. 3 and 5); polypes (Plate xxxv., Figs. 2 , 4, 5, anı 6; Plate xxxy., Fig. 5); sea anemones (Plate xxvii., Fig. 4); stal-fishes (Plittexxvi., Fig. 2 ; Plite xxviii., Figs. 2 and 4 ); and the several parts of animals (Plate v., B, C, F, H, L, S, Q, W, and O).

FIG. 4.-Photograpl ly Mr. Alexander Thoms of beautiful radiating dendrites on a mineral in his pussession. All that is said under Fig. 3 applies to this figure.

\section{l'LATE XXXV}

Plate xxxv. illustrates radiating, branching, concentric, and segmented arrangements in dendrites, in polypes, \&c.

FIG. 1.-Photograph of dendritic manganese on lithographic limestone. Shows radiating, branching arrangement so common in electric sparks, lightning flashes, hoar-frost pictures, crystals, plants and trees of all kinds, and animals and parts of animals, where there is division and branching of parts. From specimen in the Collection of Scottish Minerals in the Musetum of Science and Art,
Edinburgh. Photographed for the Author by George Rodger. 


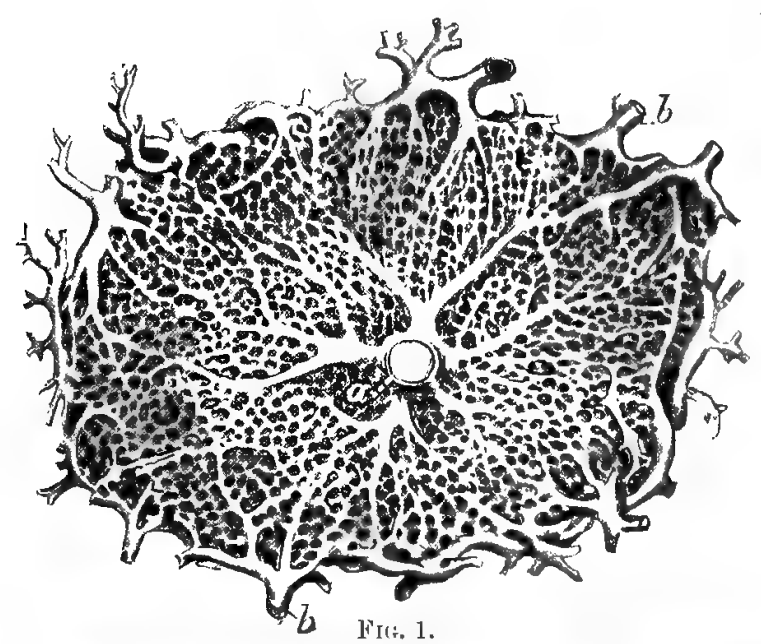

PLATE XXXII

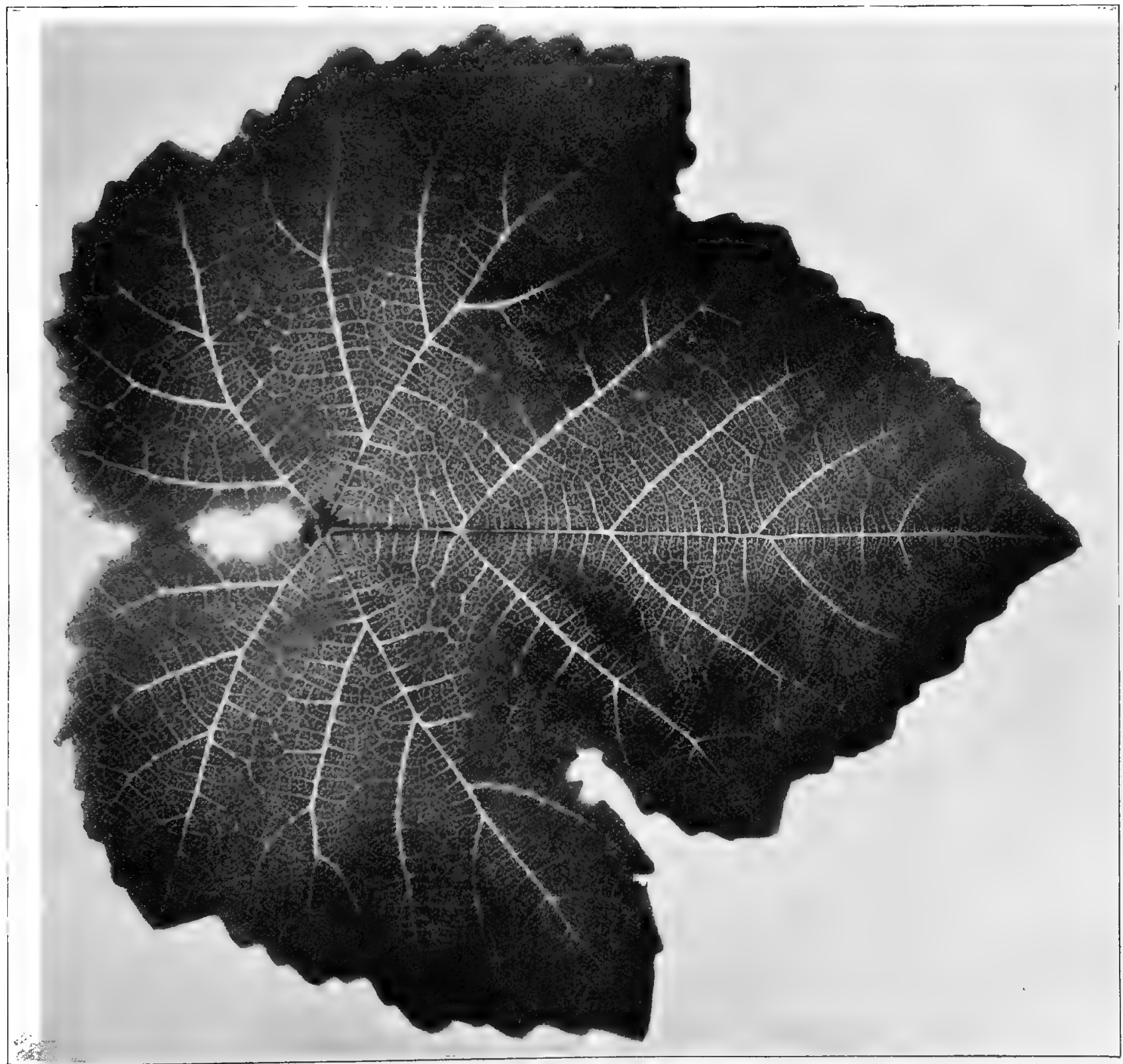

His. 3. 
PLATE XXXIV

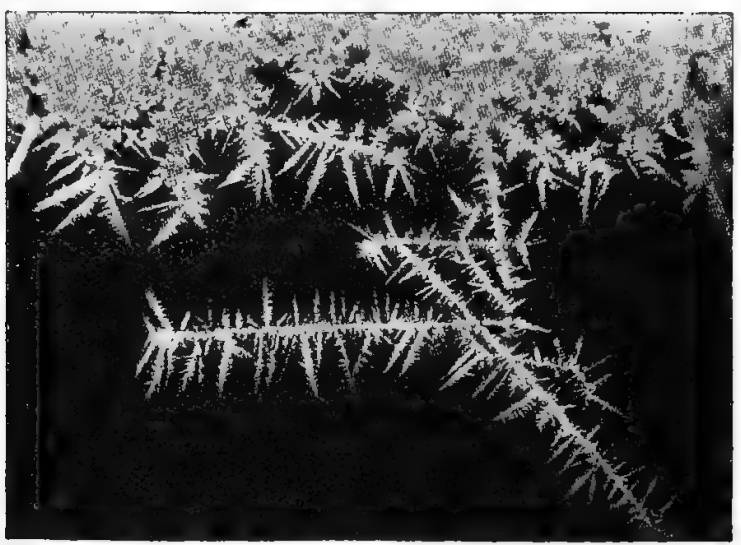

FIs. I.

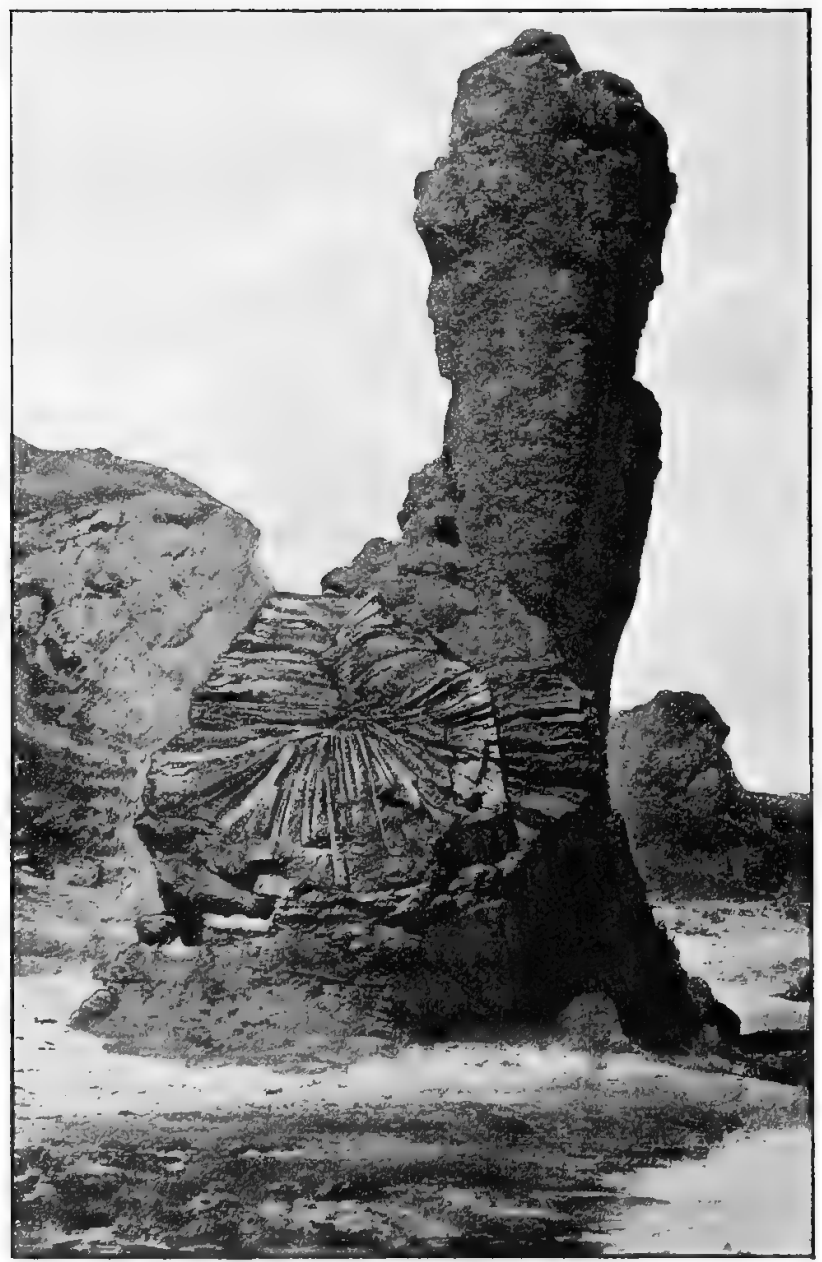

FI: 3

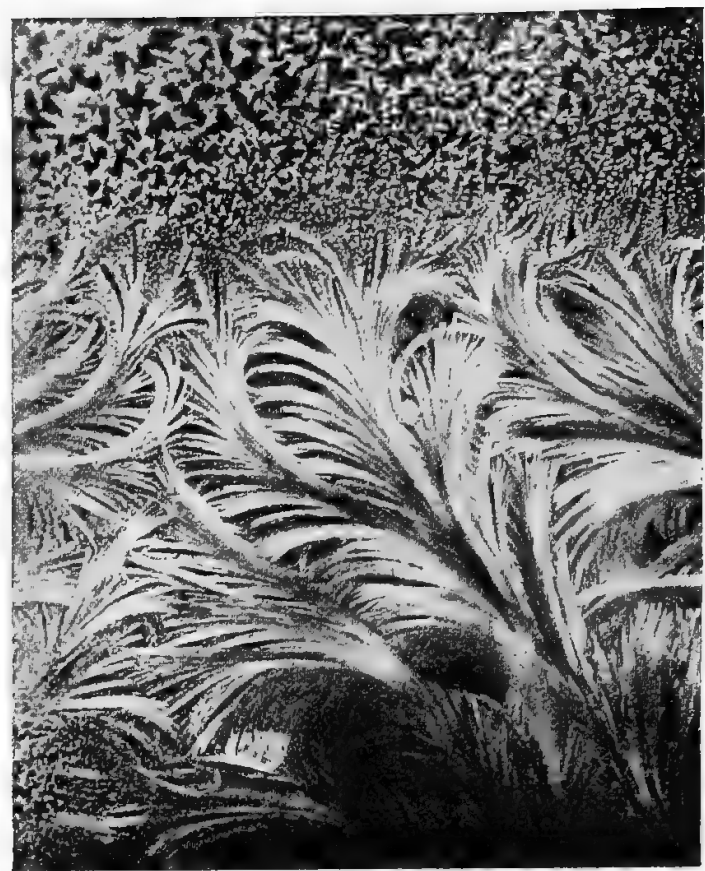

Fis. 2.

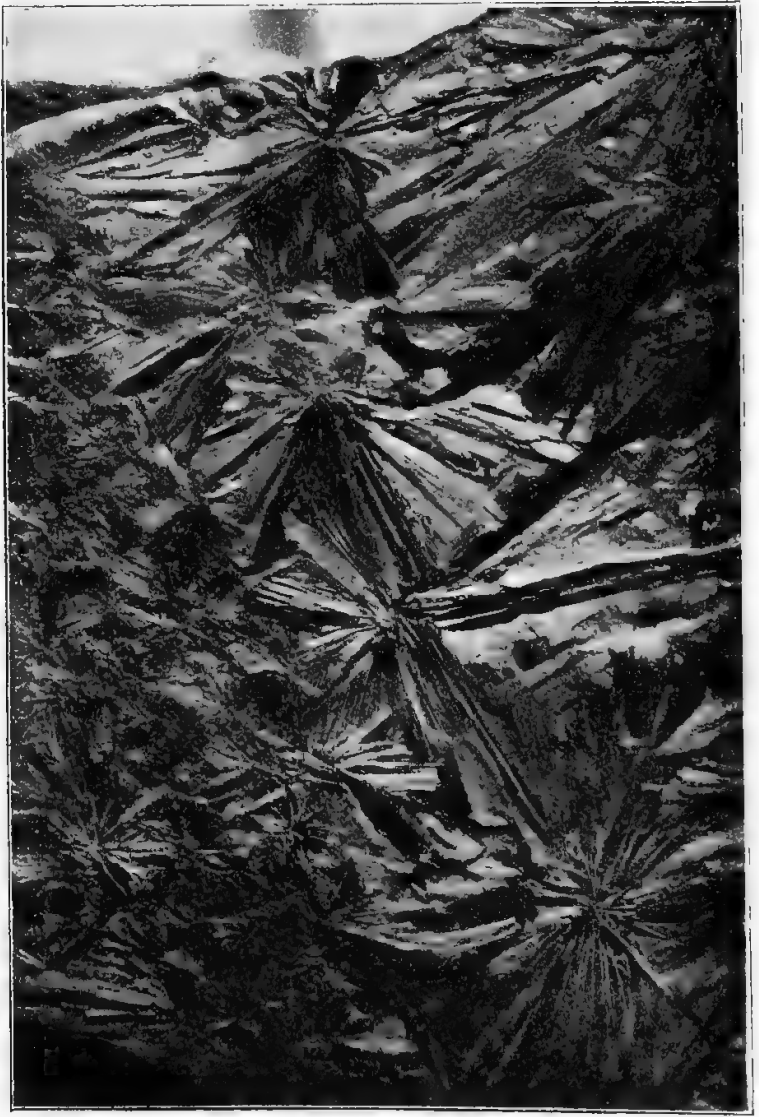

Fili. 4 . 


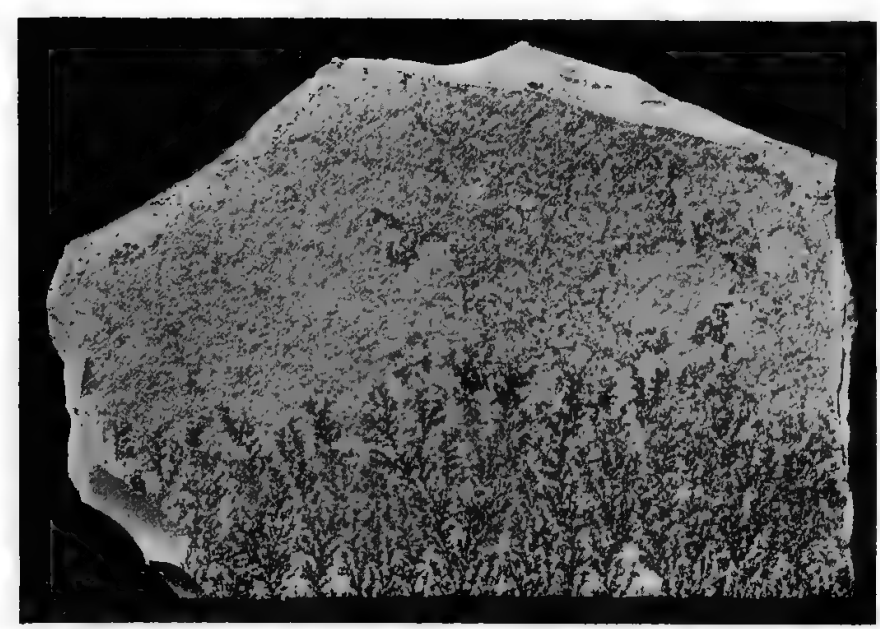

Fis. 1.

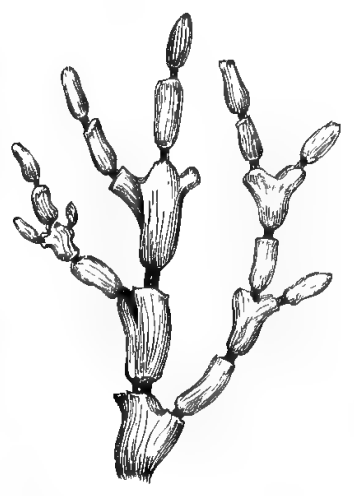

FIf: 2.

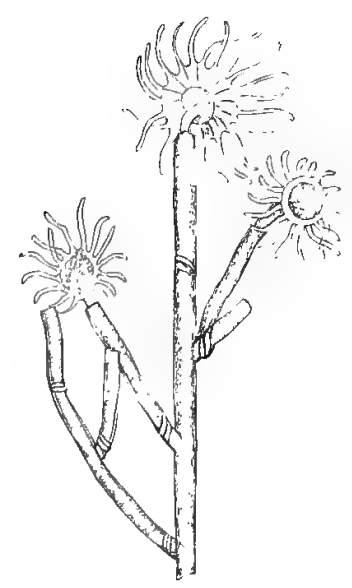

Fin. 3.
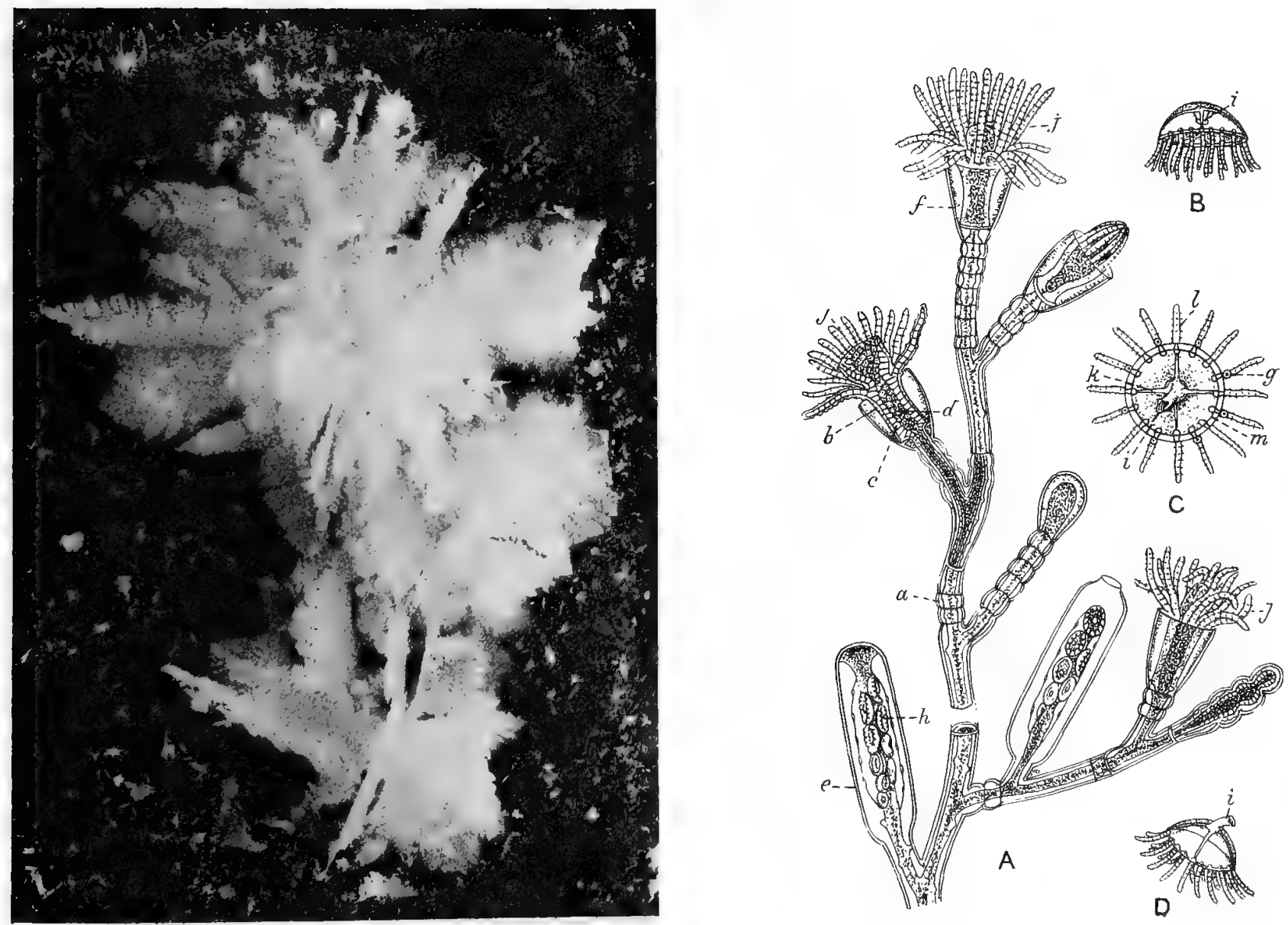

Fitr. 4. 


\section{PLATE XXXV (contimud)}

Frf. 2.-Sertularia filicula (alter Dallas). A jolype showing a radiating branching of parts and segmentation common in plants and a large number of animals.

FIG. 3.-Eudendrium ramosum (after Dallas), magnified. A polype presenting a flower-like appearance and segmentation of stem.

FIG. 4.-Photograph of frost flower obtained from the frozen surface of the lake at Davos Platz in winter by Colonel Hume. Shows striking resenillance to many crystals, to numerous plants and flowers, to various polypes, and to many radiating structures in animals.

FIG. 5. Obelia (after Parker and Haswell). A polype showing radiating, branched, and segmented arrangements common in crystals (Plate ii.), electric sparks (Plate xxxi., Figs. 2 and 3 ); dendrites (Figs. 1 and 4 of this Plate); and plants and animals.

A. Obelia as a whole. B, C. Medusæ of obelia. D. The same inverted. $a$, Common body tissue of obelia; $b$, ectoderm; $c$, endoderm ; $d$, stonarh; $r$, generative capsules; $f$, horny cusps; $g(C)$, lithocyst; $h(A)$, medusa bud; $i$ (B, C, D), nanubrium; $j(\mathrm{~A})$, three feeding polypes; $k(\mathrm{C})$, radial canal ; $l(\mathrm{C})$, tentacle; $m(\mathrm{C})$, velum.

\section{PLATE XXY Y}

Plate xxxvi. illustrates radiating, branching, segmented, and spiral arrangements as seen in dendrites, polypes, \&c.

Fig. 1.- Photographs of three beautiful poly pes.

A. Fern coralline (Sertularia filicula). B. Sea tamarisk (Diphasia tamarisca). C. Lobster horn coralline (Antennularia ramosa).

These greatly resemble plants, display radiating, branching arrangements seen also in crystals (Plate ii.); dendrites (Plate xxxiv., Figs. 1 and 4, and Fig. 2 of this Plate); nerve cells (Plate xxxviii., Fig. 4; Plate xxxix., Fig. 2); and other structures, such as bronchial tulbes, blood-vessels, lymphatics, \&c. (Plate xxxix., Fig. 4). Photographed for the Author by George Rodger.

Fig. 2.-Photograph of dendrite of manganese oxide. Shows radiating, branching arrangement seen in electric sparks, hoarfrost pictures, crystals, plants and animals, and parts thereof. From specimen in the Collection of Scottish Minerals in the Museum of Science and Art, Edinburgh. Compare with Plate xxxv., Fig. 1. Photographed for the Author by George Rorger.

Fig. 3.-Red coral (Corallizin rubrum), (after Dallas). Shows branching, tree-like form observable in lightning flashes (Plate xxix., Figs. 1 and 2 ); in dendrites (Plate xxxiv., Figs. 1 and 2 ; Plate xxxv., Figs. 1 and 4 , and Fig. 2 of this Plate); in crystals (Plate ii.); in plants (Plate xlv., Figs. 1, 2, and 3); and many branching animal structures, notably bronchial tubes and blood-vessels.

FIG. 4.-Pentacrinoid larva of the rosy feather star (Antedon bifida), (after Herbert Carpenter). Shows radiating, branching, spiral, segmented arrangemeuts. The rosy feather star presents a graceful, flower-like appearance which cannot fail to attract attention.

\section{PLATE XXXYI}

Plate xxxvii. illustrates binary and other forms of division and branching in Infusoria, as figured by W. Saville Kent. It also shows how colonies of zooids strikingly resemble plants and flowers in general appearance.

FıG. 1.-Adult umbellate colony-stock or zoodendrium (Codosiga allioides), bearing numerous terminal clusters of associated collared zooids, $\times 650$. This colony-stock presents an exceedingly elegant branched appearance, and might readily be mistaken for a plant in flower. The arrangement of parts points to a common law of development in plants and animnls. forms.

FIG. 2. -Two colony-stocks or polythecia (Polyace dichotoma), $\times 1000$. Illustrates binary division or branching in low animal

FIG. 3.-Adult zooid (Monosiga angustata), $\times 2500$, with nucleus, contracting vesicle, collar, flagellum, \&c.

FIG. 4.-Adult zooid (Salpingoca ampulla) with fully-ileveloped protecting covering or lorica, $\times 1250$.

FIG. 5.-A more advanced condition of the same.

FIG. 6.-An Infusorian (Ślpingoeca minuta) with sarcode protruding. This figure illustrates the extraordinary power of changing shape possessed by the lower animal forms. The collar has been retracted by a centripetal movement, and the animal has rirtually assumed a new shape.

FIG. 7.- Single form dividing by longitudinal fission. Here the bifurcation or division which is so conmon in plants and animals, and parts thereof, is very well seen.

FIG. 8.-Monosiga consociata with collar and flagellum in the extended (a) and retracted condition (b). In the latter case the zooid is entering on the encysted state, $\times 1500$. The retraction of the collar and flagellum by centripetal movements completely alter the appearance of the zooid.

Fıg. 9.-Spheroidal cluster of associated zooids (Codosiga botrytis) with one zooid detnched, $\times 650$ (Stein). Shows exquisite symmetrical, flower-like arrangement of parts.

Fig. 10.-Colony of three Codosiga grossularia with their collars conically contracted and with protruding psendopodia, $\times 1000$. Illustrates the centripetal and centrifugal power possessed by zooids of changing shape. The sarcous elements of nuscle posses, similar powers.

FId. 11.-Colony-stock of Codosiga botryt rs, revealing first traces of membranous collars, $\times 1000$ (Frensenius). 


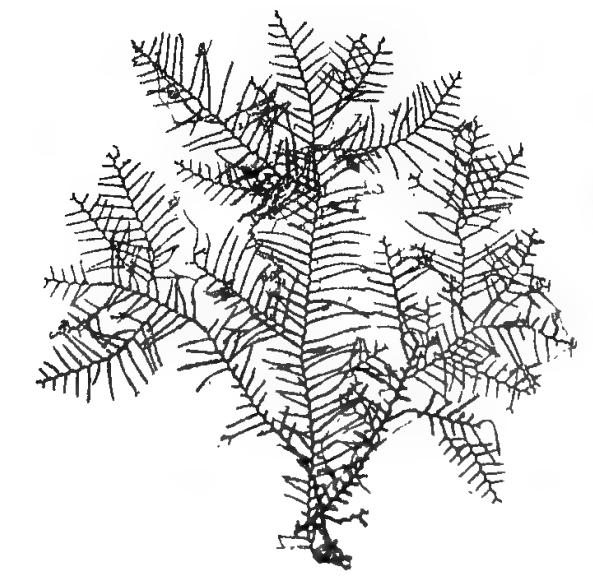

A
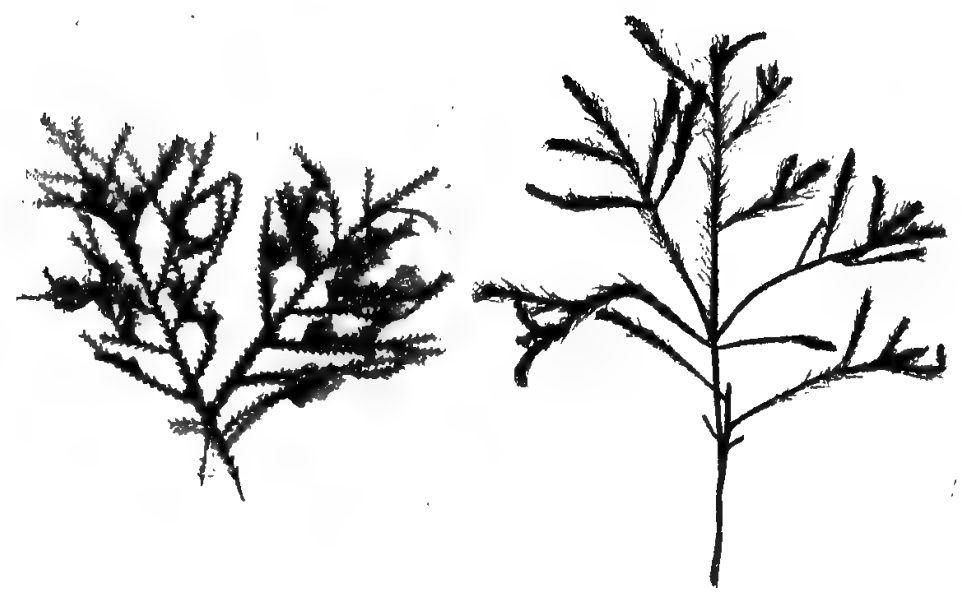

FIG. 1.

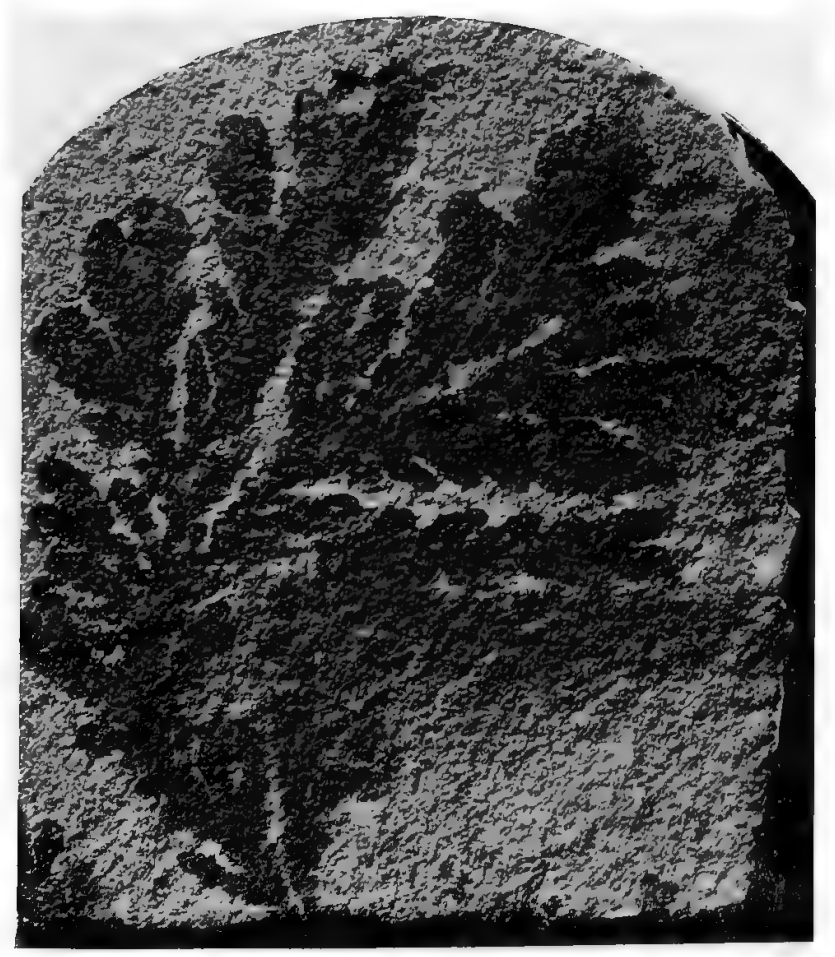

FIc. 2.

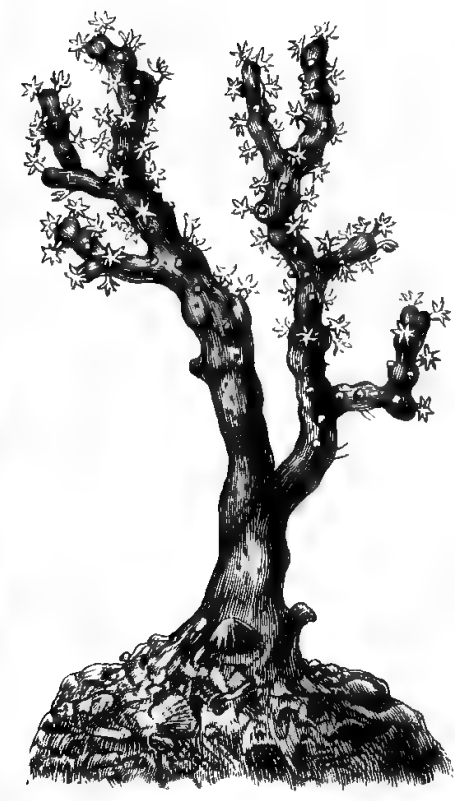

Fis: 3.

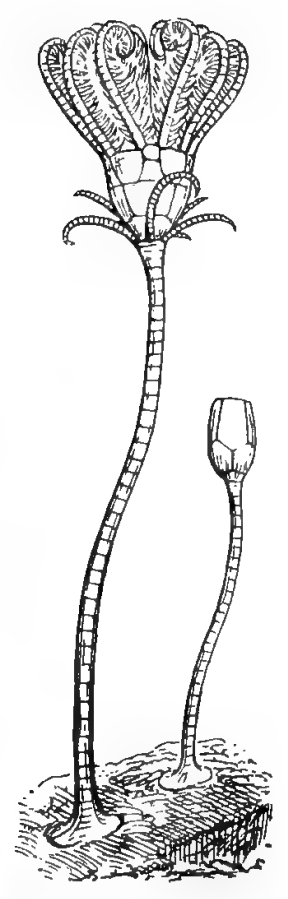

FIG. 4. 
PLATE XXXVII

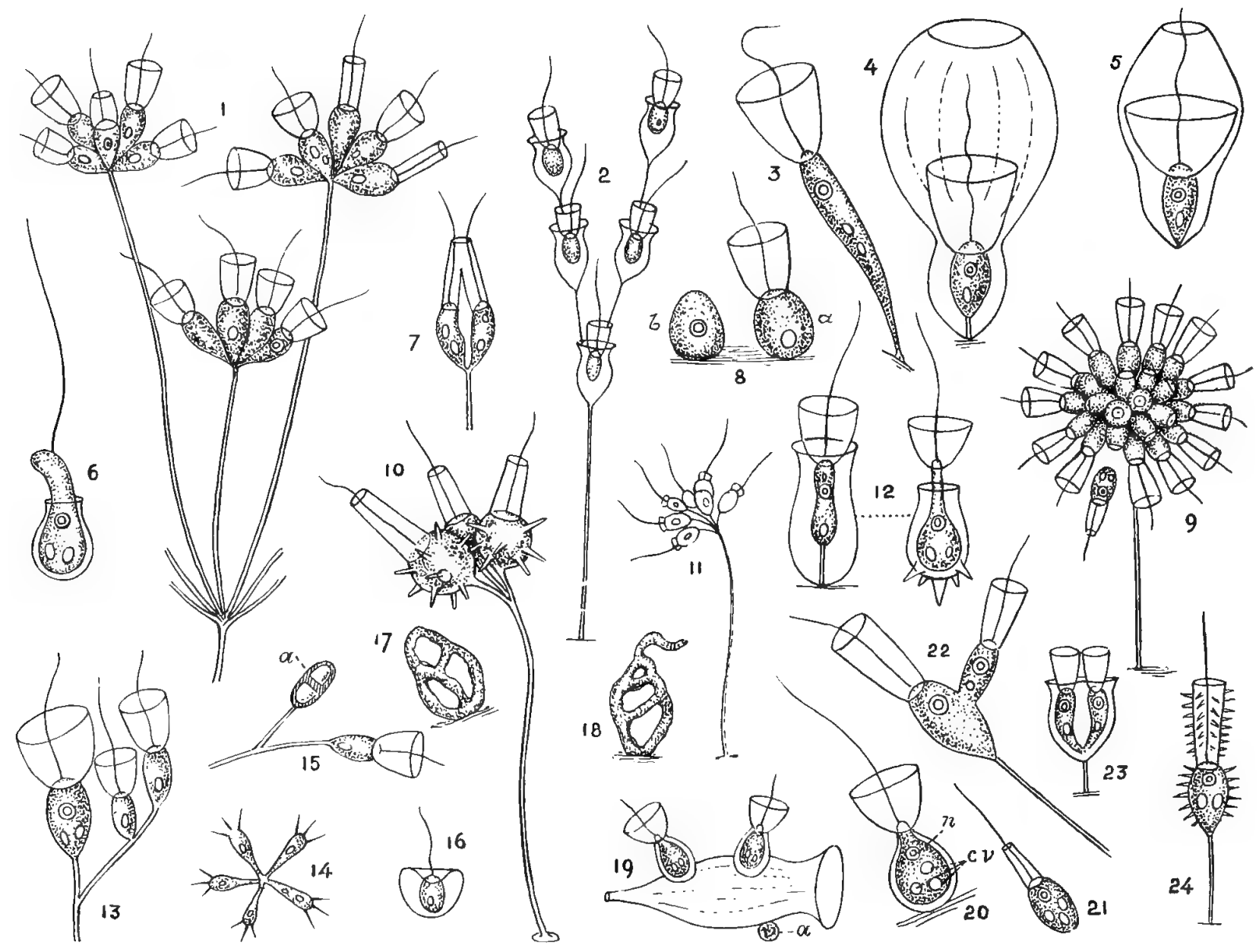

I'LA'T'E XXXVII (rontinued)

Frg. 12.-Two collar-bearing, loricate mon uds (Salpingseca petiolata and Lagenaca cuspidata), x 1250 and 1500.

FIG. 13. - Branchlet with three zooirls of fully-developed colony-stock (Codosiga cymosa), $\times 1250$.

FIs. 14.-Free-floating colony of zooids with collars and flagella imperfectly indicated, $\times 600$ (De Fromentel). Shows radiating, stellate arrangement.

Fir. 15.--Branchlet with two zooirls (Codosiga cymosa), the one to the left $(a)$ encysted and separater by segmentation, $\times 1250$. In this ease the segmentation and division is transverse, while in Fig. 7 it is longitudinal. The longitudinal and transverse division of zooids illustrates a general principle, namely, the power possessed hy living things of splitting and subdividing in various directions. Loncritudinal division is illustrited in the branching of plants, and in the branching of parts of animals, as in blond-vessels, bronchial tubes, \&c. The transverse division is seen in many plants, and obtains in articulate and vertebrate animals.

FIG. 16.-Germ of zooid (Salpingaca ampulla) attached and developing its collar and protective lorica, $\times 1200$.

FIGS. 17 and 18.-Zooids (Monosiga ronsociata) with collars and flagella retrncted and assuming vacnolar amoboid phase, $\times 1500$. Here the extraordinary power possessed hy simple animals of reverting to a simple or primitive condition is strikingly manifested.

F'Ix. 19.-Two adult zooids (Salpingarca minuta) and single undeveloped germ (a), attached to an empty lorica, $\times 1000$.

FIG. 20.-A single attached zooid (Salpingoea minuta) showing nucleus $(n)$ and contractile vericles $(c v), \times 1250$.

FIG. 21, - Free swimming zooid (Codosiga botrytis) detached from sedentary colony, with collar contracted, $\times 650$.

FIG. 22. - Similar zooid coalescing or conjugating with a sedentary zooid (Stein). Illustrates how zooids merge and fuse into each other.

FIA. 23. A zooid (Salpingoeca convallaria) dividing ly longitudinal fission, $\times 650$. Illustrates the dichotomous division of zooids. Comprare with Figs. 7 and $15 \alpha$.

FIG. 24. - A zovid enitting minute pseudopodic processes, $\times 650$ (Stein). These processes are produced by a voluntary pushing ontwards or centrifugal action of the body sarcode. They are retracted and merged in the body sarcode by an opposite or centripetal action. Similar powers, as indicated, are possessed by the sarcous elements of muscles, in virtue of which muscles are voluntarily elongated or shortened, 
PLATE XXXVIII

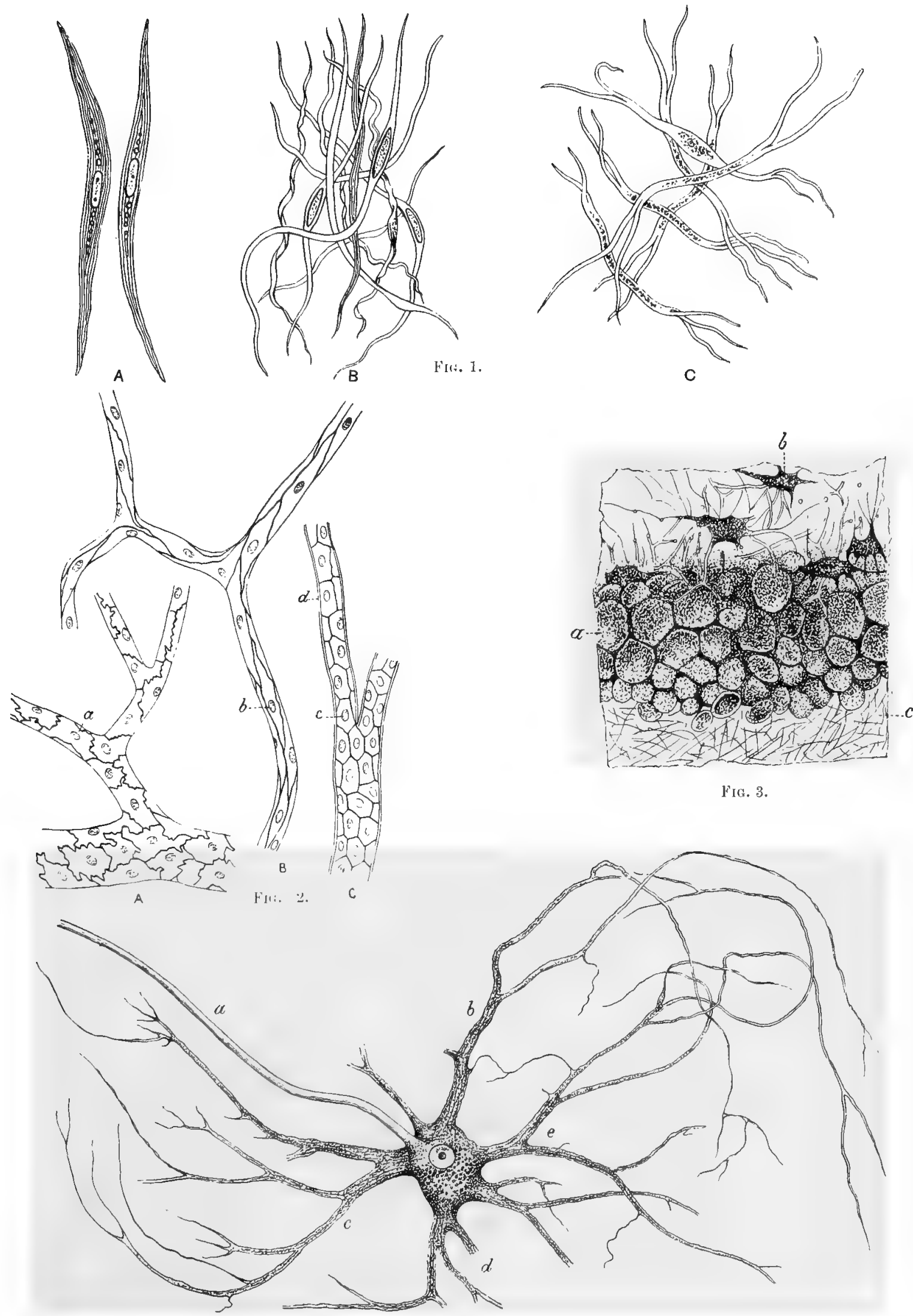

FIt. 4 . 


\section{PLATE XXXVIII}

Plate xxxviii. illustrates branching and radiating arrangements in muscle, blood-vessels, bone, and nerve ganglion.

FIG. 1.- Stindle-shaped nucleated cells occurring in involuntary muscular fibres (after Arnold).

A. Muscular fibre treated with serum.

B. Muscular filres from the intestine isolated by means of nitric acid.

C. Dichotomously divided muscular fibres from a pleuritic meml,rane.

FIG. 2.-Branching capillary blood-vessels (after Stricker).

A. Capillaries from intestine of snail, irregularly lobed cells (a).

B. Small capillaries with futifurm cells (b) from mesentery of Leuciscus.

U. Capillaries of the pecten of the eye of the bird, exhibiting polygonal cells $(c)$; the lyyaloid membrane covering the capillaries is given at $d$.

FIG. 3.-Bone trabeculæe with osteoblastic layer, from the parietal bone of a human embyro it the fifth month (after Rollet). Shows radiating arrangement. a, Cells (osteoblets) erowded and undergoing ossification, more advanced at $b$, where radiating and branching occurs; c, trabecular matrix in which cells occur.

FIG. 4.--Multipolar ganglion (nerve) cell from the anterior hom of the spinal cord of an ox (after Stricker). Shows radiating, branching, and concentric arrangements. The ganglion contains a nucleus and nucleolus. This form of nerve centre virtually discharges brain functions, and may not inaptly be designated a brainlet. $a$, Axis cylinder process; $b, c, d, e, b r a n c h e d$ processes, $\times 300$.

\section{PLATE XXXIX}

Plate xxxix. illustrates radiating, branching arrangements in the human skin, ganglia, and nerve baskets, the mesentery, and papillæ of tongue.

FIG. 1.-Section of the skin of the hand marnified twenty times (modified from Hirschfeld and Leveillé). Shows radiating, lirunching, and spiral structures.

A. $a$, Horny, and b, mucoun layers of the epidermis; $c$, coriun; $d$, panniculus adiposus; e, fat cells; $f$, spiral sweat glands opening on surface at $g, g, g ; h$, nerve ending in Pacinian bodies $(i) ; j$, nerves ending in loops; $k$, nerves endin in f $l$, capiliary plexus of vessels.

B. a, l'wo collateral nerve lranches from the palmar surface of the index linger; b, Pacinian or touch corpuscles attached t" said nerves, natural size.

C. Pacinian corpuscles magnitien 100 times. $a$, Nirve with sheath $(b)$; $c$, free end of nerve surrounded by fluid; $d$, calsule.

Fitr. 2.-Nerye baskets from spinal cord of ox (after Gerlach). Show ladiating and concentric arrangements. a, Nerve filı'e dividing into two branchus $(h, b)$ which communicate with the plexuses of nerve fibres $(c, c)$ in connection with the two nerve cells $(d, d)$. Prepared with carmine and ammonia, from the spinal cord of an ox, $\times 150$.

FIG. 3.-Miliola (Proto:oa). Shows radiation and branching of sarcode (Chapman).

FIG. 4.- Loop of human intestine with mesentery. Shows radiating and branching arrangements in the blood-vessels and lymphatics. $a$, Artery; $b$, vein; $c$, lymphatic gland; $d$, lymphatic vessels. Drawn by C. Berjeau for the Author.

Fra. 5.-Granite veins traversing gneiss, Cape Wrath (Lyell). Shows radiation and branching as in 1yloorl-vessels.

FIG. 6.-Fossil crinoid (cyuthocrinus planus, Niller). Body and arms. Mountain limestone. Shows radiation and lorinuhing as in trees and blood-vessels.

FIG. 7.-Fossil corals.

A. (Lonsdaleic floriformis, M. Edwards). Young speciunen, with buds or corallites on the disc.

B. (Lithostrotion floriforme, Fleming). Part of a full-grown compound mass. Shows radiation and bulding, and how the buds or corallites become hexagonal-shaped from growth and pressure as in epithelium pigment of liver cells, \&c. (after Lyell).

FiG. 8.-Gromia terricola (after Leidy). A rudinentary animal which has the power of projecting and withdrawing its body sulistance (sarcode) by voluntary centrifugal and centripetal movements. Similar movements occur in the sarcous elements of voluntary muscle. The projected sarcode forms a radiating network, and represents lines of movement and force analogous to those seen in electric sparks (Plate xxxi., Figs. 2 and 3). The animal as a whole affords a good example of radiating, branching, concentric ar'rungement,s.

FrG. 9.-A. Section of circumvallate papillæe of human tongue, $\times 150$. a, Epithelium ; b, tiste bud; ", injected l,loud-versels; ", colium ; e, gland with duct (after Heitmmann).

B. Section of fungiform papille of tongue with blood-vessels injected. ", Epithelium; $b$, coriun ; $c$, blood-vessels; $d$, lymphoid 1issue; $e$, muscular fibre (after Heitzmann).

C. Section of two filiform prapilla of tongue. Lettering same as in B (after Hartmann).

D. Two filiform papilla ; the one with the epithelium and the blood-yessels iujected, the other without. "L, Substance of papiilic divider at their upper extremities into secondary papilla; b, "pithelial rovering, laminated portion, between the japillre, but exiended into hair-like j,rocesses over the secondary papillie $(c) ; c, c$, artery and rein. Show radiating, branched arrangements. 
PLATE XXXIX
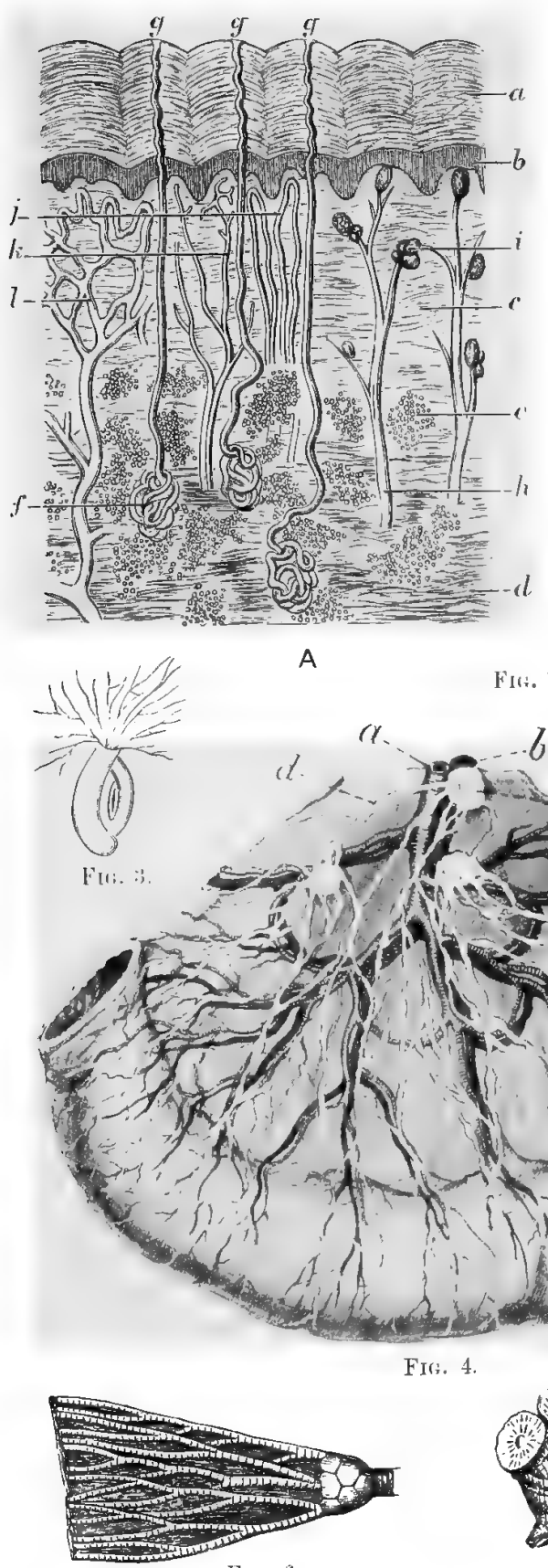

FIr. 6 .

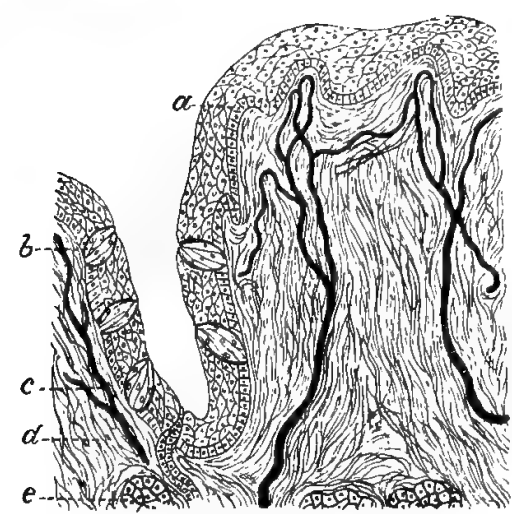

A

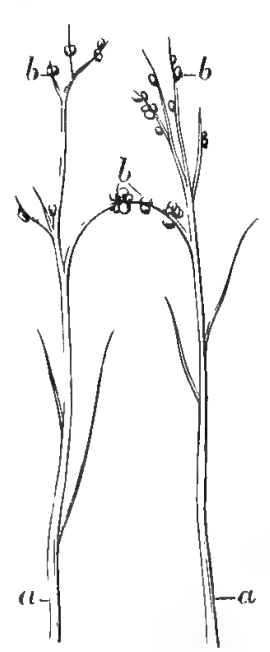

B
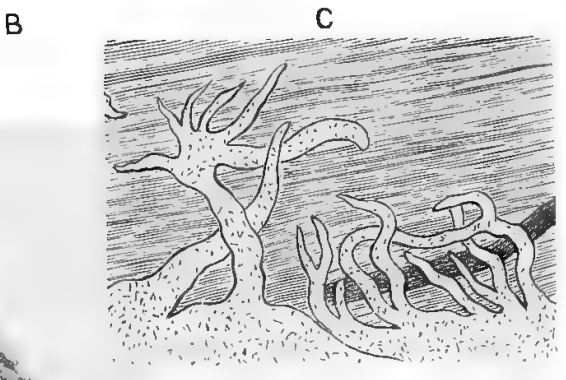

FIt. 5.
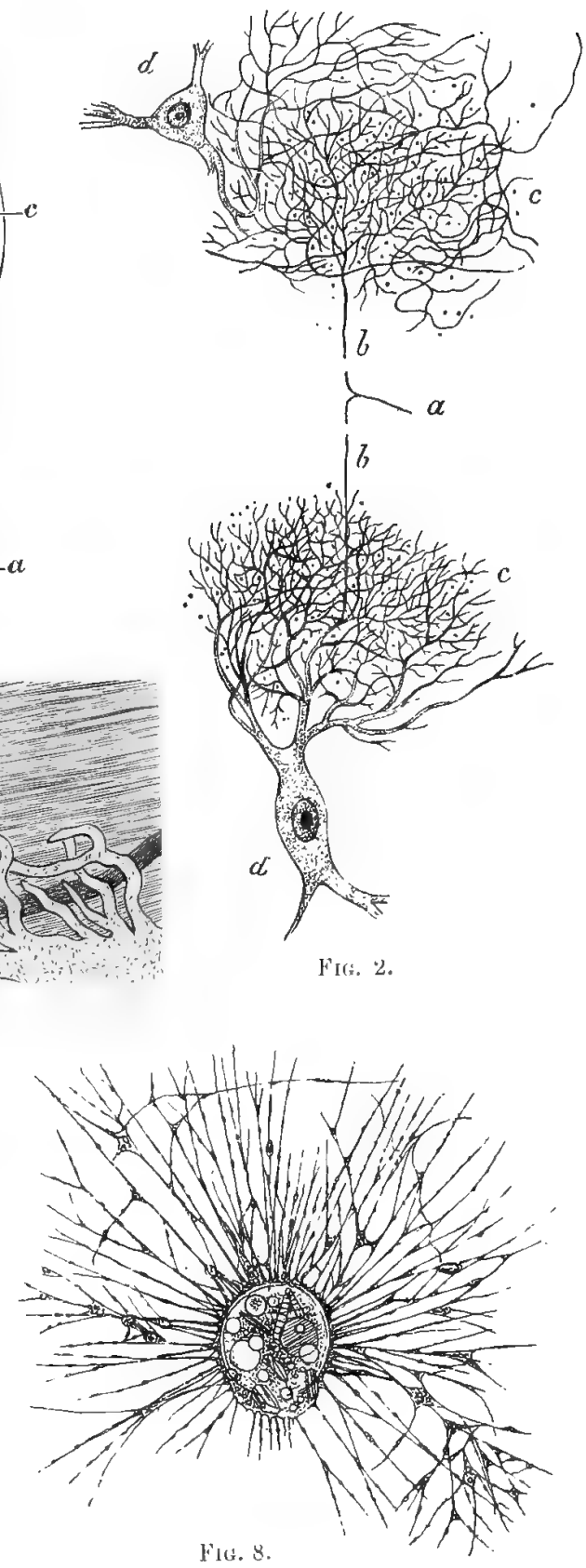

F11. 7 , 手

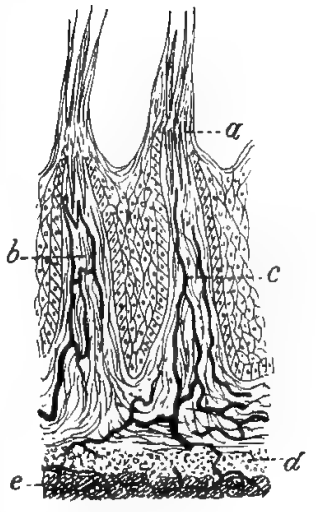

C

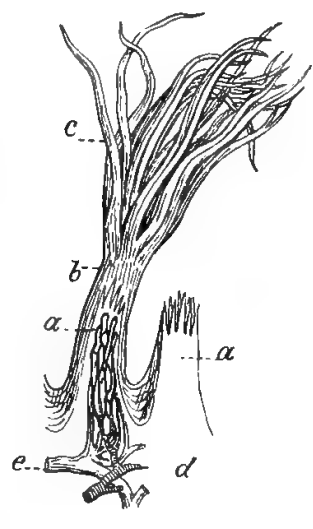

D 
Plate xl. illustrates in a striking manner well-marked resemblances in inorganic and organic structures as regards longitudinal cleavage and transverse cleavage or segmentation.

FIg. 1.- Photograph of Fingal's Cave, Staffa, in the west of Scotland. Consists of vertical bisaltic columns largely hexagonal in shape. They are seprated transversely at short intervals as in a vertebral column.

FIG. 2,-Photugraph of portion of the Giant's Causeway in the north of Ireland. The coluuns resemble those found in Fingal's Cave (Fig. 1 of this Plate). Figs. 1 and 2 of this Plate show the longitudinal and transverse cleavages in Plutonic rocks to perfection. Similar cleavages are seen in hydrated starch (Fig. 3 of this Plate). They greatly resemble the cleavages observed in plants and animals, especially the latter (Figs, 5, 6, 7, and 8 of this Plate), and point to a law of development or differentiation common to the inorganic and organic kingdoms (the Author).

FIG. 3.-Pentagonal and hexagonal prismatic columns resembling those found in Fingal's Cave and the Giant's Causeway, produced artificially hy evaporating and desiceating a fluid mixture of starch (the Author).

FIG. 4.-Fossil coral (Lithostrotion hasaltiforme) found in England, Ireland, Russia, Iova, and westward of the Mississippi (D. D. Owen). Shows heautiful prismatic, lexagonal columns, wonderfully resembling the lessaltic rock columns foutud in Fingal's Cave anil Giant's Cantseway (Figs. 1 and 2 of this Plate), which I have shown can be produced artificially (Fig. 3 of this Plate).

FiG. 5.-Fin or wing of the sturgeon (Acipenser), (after Ginther). Displays well-marked longitudinal cleavage with a certain amount of transverse cleavage. $c$, $e, d$, Root and thick, tapering semi-rigid murgin of fin; $f$, thin, elastic margin of fin. The triangular shape and graduated structure of the fin furnish the type for all propelling fins and wings-fins and wings having nuch in common, the latter being larger.

FIG. 6.-Fore-limb of Ceratodus, a member of a family of vely ancient fishes, dating back to the Triassic and Jurassic eras, and still surviving in Australian waters (after Giinther). Shows typical radiating, branching, segmented arrangements. The fore-limb forns a parddle rather than a fin or wing. It is not graduated, as in Fig, 5, to form in jropelling organ. ",$b$, Root of fore-linb; $c$, broad basal cartilage sulporting tapering segmented axis and diverging rays; $d$, lip of fore-limb; $e$, anterior, and $f$, posterior margins of same.

Fis. 7.- Trilolite (Homalonotus delphinocephalus), (after Nicholson). Shows transverse cleavage or segmentation.

Fig. 8.--Human verfehral column or hackbone (after Holden). Shows branching and typical transverse (leavage or segmentation. Compare with Fig. 7 of this Plate, and with the figures of Plate xxxix. The alterniting curves presented by the backbone form a veritahle line of beauty.

FIt. 9.-A young coral (Lonstaleiu fturiformis) with buds or corallites on the disc, illustrating calycular gemmation (M. Edwards).

\section{HEXAGONAL STRUCTURES}

In the United Kingdom and in almost every country in Europe igneous rocks occur. They do not necessarily form hills with cones and craters indicating their origin. Thus the rocks of Staffa in Scotland and the Giant's Causeway in Ireland are called basalt, and are known to be volcanic, as they agree in their columnar prismatic structure and mineral composition with the lava which has flowed from the craters of volcanoes. The remarkable structure of these rocks is supposed to be due to shrinkage and consolidation on cooling. Similar columnar prismatic structures are produced when a mixture of hydrated starch is slowly dried off in the sun or in an oven. In both cases the columns, generally pentagonal or hexagonal in shape, are divided transversely at more or less regular intervals. ${ }^{1}$

I direct attention to the basaltic prismatic columns as the longitudinal and transverse cleavages and hexagonal outline which they reveal re-appear in many living plant and animal structures. The longitudinal and transverse cleavages are seen in the Equisetacer, in cones and branches among plants, and in the segmented animals with limbs, bronchial tubes, \&c. The hexagonal formations are witnessed in epithelial and liver cells, in scales and coverings, in transverse sections of fasciculi of muscle, in the enamel of teeth, in the honeycomb, in old-world corals, \&c. A familar and striking example (Eunomia radiata) occurs in the coralline limestones. This is found in the Great Oilite, near Bath, where it is very conspicuous; single individuals forming masses several feet in diameter, and which probably required centuries for their formation. Another good example (Favosites cervicornis) is met with in large numbers in the Devonian and Silurian groups, in the great limestones of Plymouth and Torbay. An

1 The lollowing is the accont given of these curions and interesting structures by sir charles Lyell: "One of the characteristic forms of" voleanic rocks, especially of basalt, is the colunmar, where large masses are divided into regular misnis, sonetimes easily separ'alule, lut in other" cases adhering firmly together. The columns vary in the number of angles, from three to twelve; liut they have most comnonly from five to seven sides. They ire often divided transversely, at nearly equal distances, like the joints in a vertebral column, as in the (riant's Causeway in Ireland. They valy axcendingly in respeet to length and dianeter. Dr. Macculloch mentions some in Skye which are about 400 feet long; others, in Morven, not rxceding an inch. In regard to dianeter, those of Ailsa measure 9 feet, and those of Morven an inch or less. They are usually straight, but sometimes curved; and eximples of loth these occur in the island of Statfa. In a horizontal bed or shecet of trap the columns are vertical ; in a verticul dike they are liorizontal." 
PLATE XL
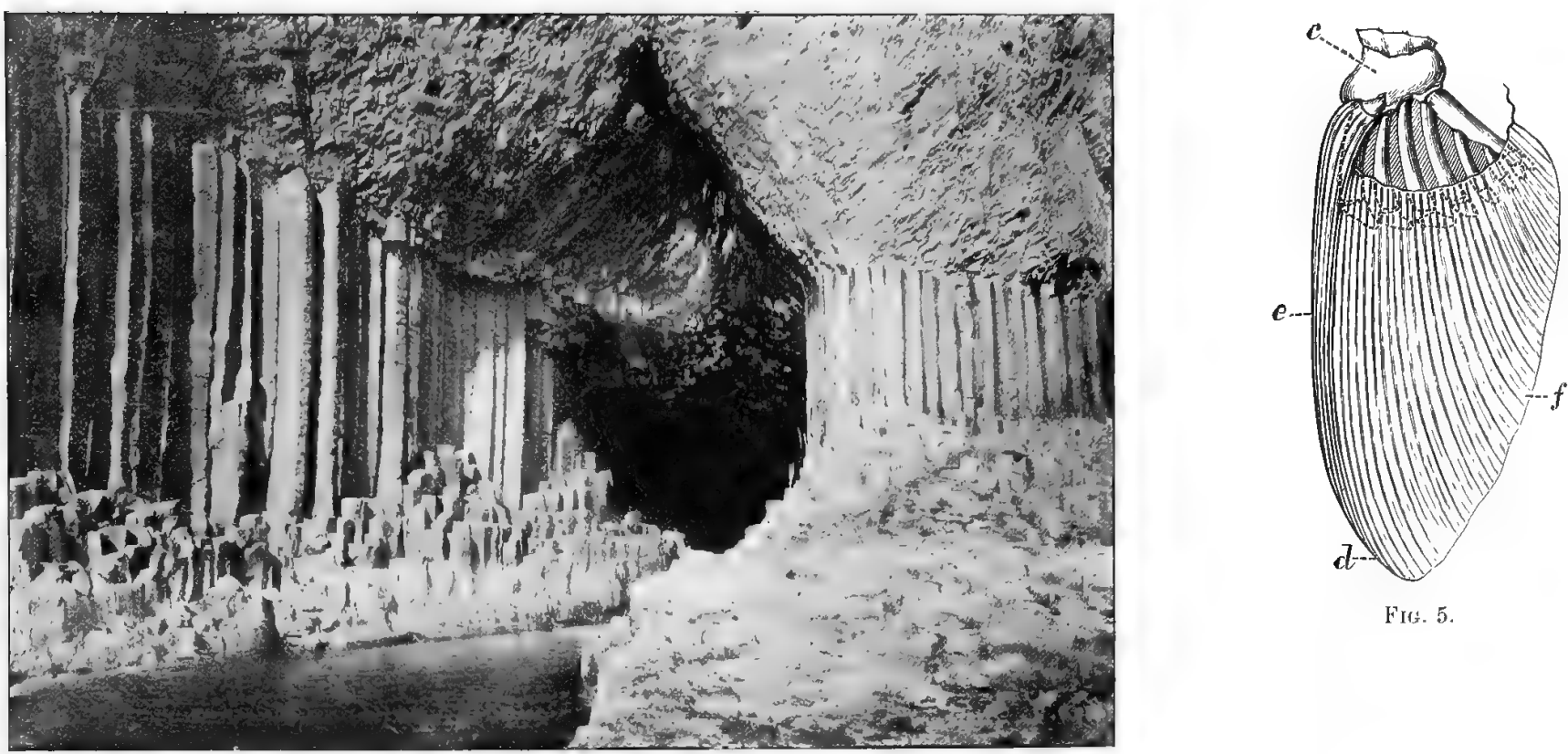

FIG. 1 .
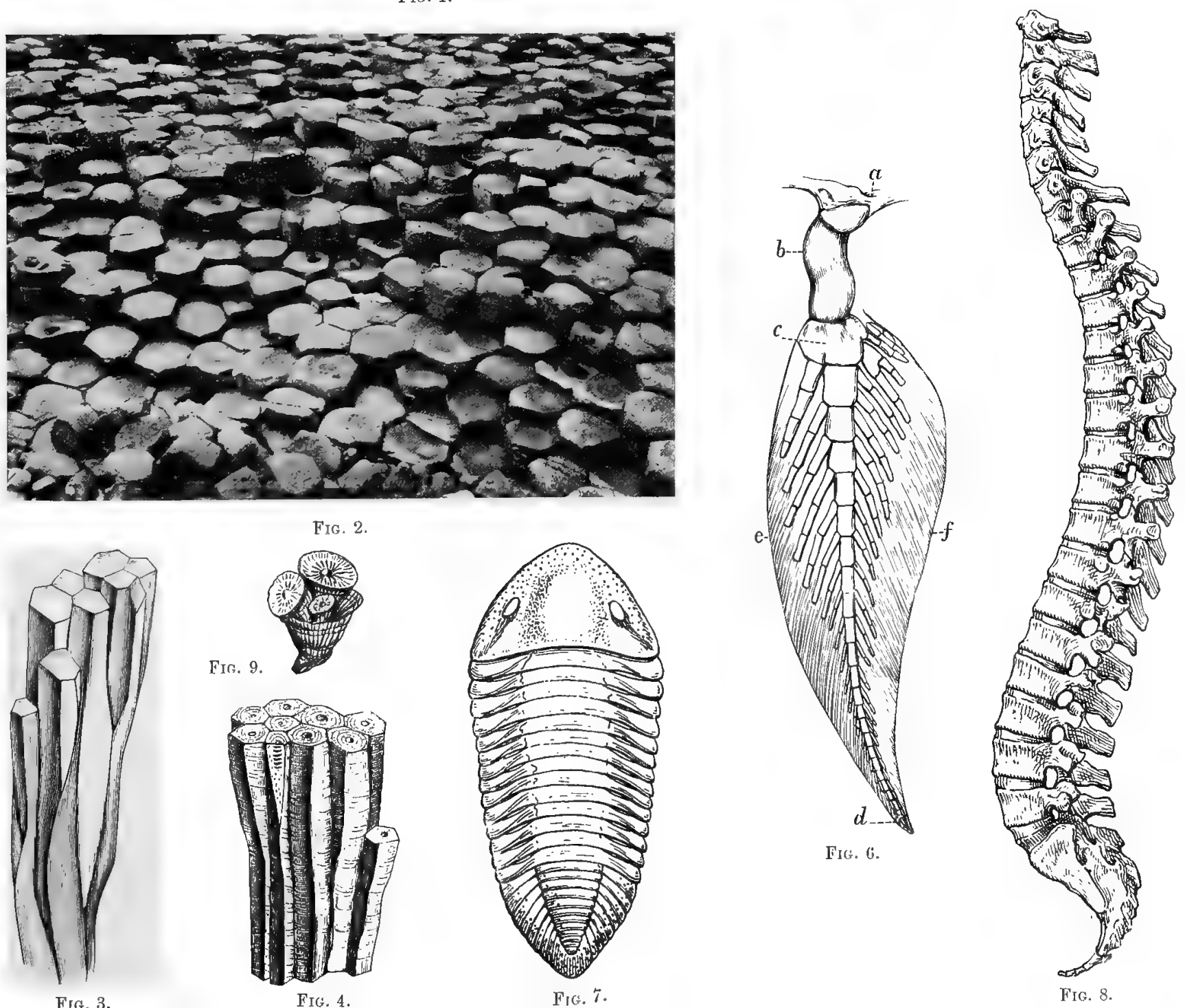
outstanding and very illustrative example is found in Isastra oblonga. In this the hexagonal and radiating arrangements are beautifully preserved. The hexagonal arrangement also occurs in the fossil fruit of Podocarya Bucklandi and in the shell of the fossil Echinoderm (Ananchytes ovatus).

Two other fossil corals may be cited in this connection, namely lithostrotion basaltiforme and Lonsdaleia floriformis. These fossil corals are met with in the mountain limestone, and have a very wide range; extending as they do from the eastern borders of Russia to the British Isles, and being found everywhere in both countries. They show a columnar hexagonal arrangement, and also a radiating and concentric arrangement frequently met with in inorganic masses and in crystals.

The radiating and concentric arrangement is seen to advantage in transverse sections of the Palreozoic and Ncozoic types of lamelliferous cup-shaped corals and in transverse section of the fossil tooth of the iguanodon (Iguanodon mantellii). The columnar hexagonal arrangement is also seen in Favosites gothlandica, where the partitions in the tubes greatly resemble the walls of the honeycomb of the bee.

The columnar, radiating, concentric, segmented, and branching arrangements in corals and echinoderms are seen to advantage in the annexed Figs. (17 to 22 inclusive).

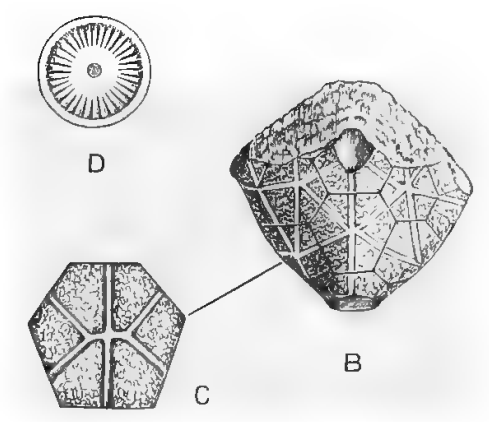

Fis: 17 .

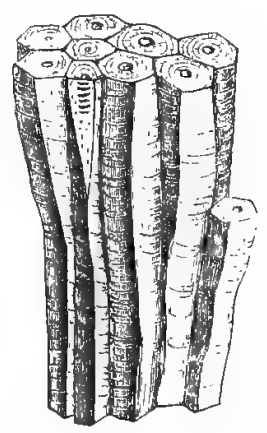

A

FIG. 17.-A. Coral known as Lithostrotion basultiforme (Lithostrotion striutum, Fleming). Occurs in England, Ireland, Russi:, Lowa, and westward of the Mississippi, United States. Shows columnar hexagonal arrangement of tuliules of coral resembling the cells if the loneycomb and the basaltic columns met with at Staffa in Scotland and the Giant's Causeway, Ireland. Compare with Fig. 19 (after D. D. Owen-interpreted by the Author).

B. Cyathocrinus caryocrinoides. Pelvis or body of crinoid ; called also calyx or cup.

C. One of the pelvic plates.

D. Surface of one of the joints of the stem (after McCoy).

F'G. 18.-Other Corals. Farosites gothlandira, Lam. (Dudley).

A. Portion of a large mass of coral; less than natural size.

B. Magnified portion of A, to show the pores and the partitions in the tubes. Illustrates lexagonal and segmented arrangements. Compare with Fig. 3.

C. Omphyme turbinatum, Linn. sil. (Cyathophyllam, Goldf.). Wenlock limestone, Shropshire. Shows longitudinal and transverse markings with radiation and branching (after Lyell---interpreted by the Author).

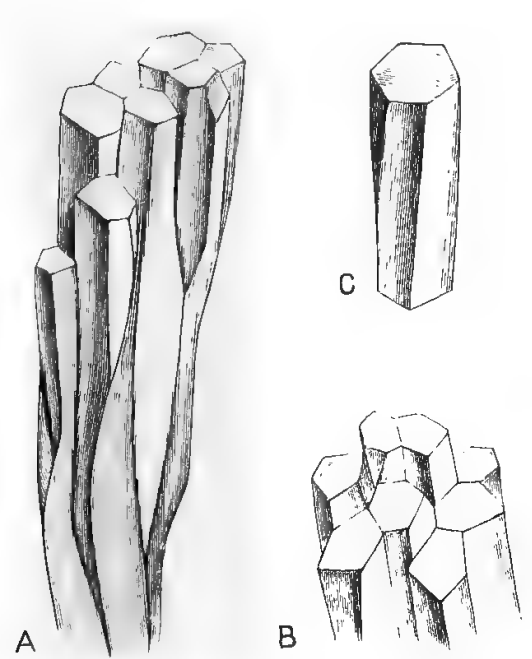

Fił. 19.

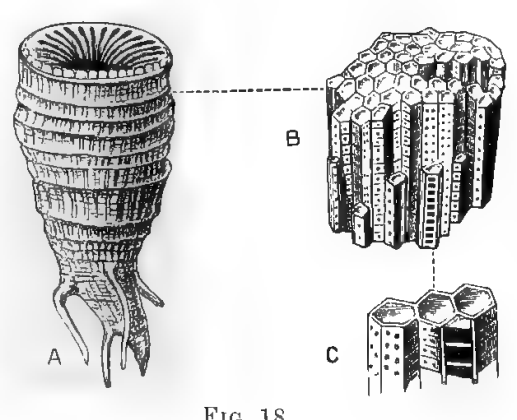

FIG. 18 .

Fig. 19.--Colunmar columns artificially produced by the evaporation and drying of liydrated starch. These columns consist of five and six-sided pisms resembling sinilar columns of igneous origin occurring at Staffa, Scotland, and the Giant's Causeway, Ireland. Their general outline corresponds with that of the tubes of many corals, and with the cells of the honeyconl, \&c.

A. Mass of starch columns, for the most part six-sided.

B. Mins of starch columns with five sides.

C. Very perfect hexagonal column slightly twisted upon itself.

The hexagonal columus formed by the action of fire and by evaporation and shrinkage are solid; those formed by the coril and the bee are lollow (the Author). 
FIG. 20.-Palæozoic type of lamelliferous cup-shaped coral. Order Zoantharia rugosu (Milne Edwards).

A. Vertical section of Campophyllum flexuosum, half natural size, from the Devonian of the Eifel. The lamellø are arranged around the inside of the cup; the walls consist of cellular tissue, and large transverse plates, called tubulde, divide the interior into chambers.

B. Arrangement of the lamellæo in Polycelia profunda, natural si\%e, from the magnesian limestone, Durham. Shows quadripartite arrangennent of primary septa, there being four principal and eight intermediate lamellæ-multiples of four.

C. Stantria astreiformis (Milne Edwards). Young group, nutural si\%e. Ulper Silurian, Gothland. The lamellæ in each cup are divided by four prominent ridges into four groups. These corals illustrate radiating, concentric, and segmented arrangements (M. Edwards-interpreted by the Author).

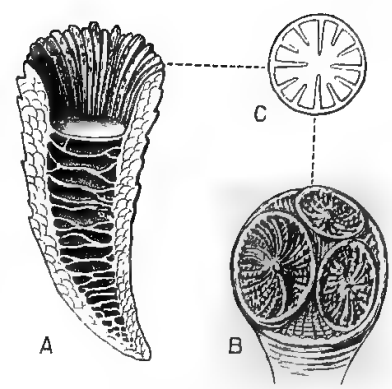

FIS. 20.

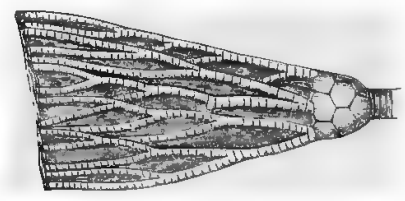

A

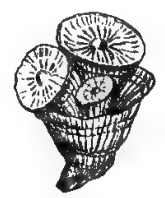

B

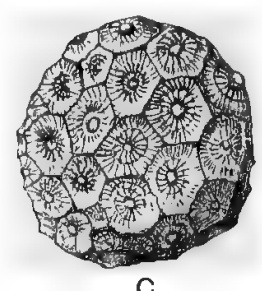

C

Firt. 21.-A. Body and arms of crinoid (Cyatlocrinus planus, Miller). Occurs in mountain limestone. Shows hexagonal arrangement at base and branching arrangement at body and free extremity. Naturally occupies vertical position.

B. Young specimen of coral (Lonsdrleia floriformis, M. Edwards), with buds or corallites on the disc illustrating calycular gemmation.

(1. Lithestrotion florifonme (Fleming). Part of a full-grown compound mass. Shows hexagonal outline of tubules and concentric radiating arrangements of interior. Bristol, \&c., Russia (after Lyell).

FiG. 22.- Neozoic tylue of lamelliferous eup-shaped coral. Order Zoanthario aporosa (Milne Edwards).

A. Parasmilia centralis (Mantell). Vertical section, natural size. Upper chalk, Gravesend. In this type the lamellæare massive and extend to the axis or columella, composed of loose cellular tissue without any transverse plates.

B. Cyathina boverbenkii (II. Edwards). Transverse section enlarged. Gault, Folkestone. In this coral the primary septa are a multipl of six. There are twelve principal plates, and between each pair three secondaries--in all forty-eight.

C. Fungia patelleris (Lamk). Recent; very young state. Magnified. Shows six primary and six secondary septa. Illustrates radiating arrangentent (after Lyell-interpreted by the Author).

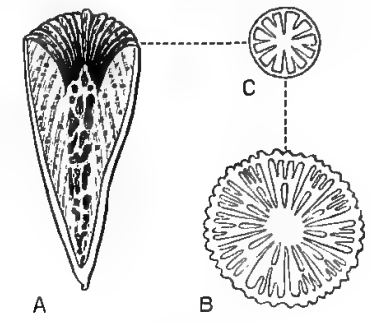

Fir: 22.

The columnar, hexagonal, prismatic, radiating, and concentric arrangements met with in inorganic and organic structures are, it appears to me, governed by laws not at present understood. Neither physical pressure, contraction and shrinkage, growth and expansion, nor the action of heat and cold of themselves fully account for them. The hexagonal, prismatic columns of basalt and starch are solid; the hexagonal columns of corals and the honeycomb of the bee are hollow. The basaltic columns are produced from a hot fluid mixture, those of starch from a cold fluid mixture. The columns divide transversely as well as longitudinally. The more or less shapeless living sarcode of the coral cannot possibly supply the model for the hexagonal symmetrical tubes which these interesting creatures produce singly and collectively; neither can the cylindrical body of the bee furnish the model for the hexagonal cells of the honeycomb; a cylinder cannot be the pattern for a hexagon, the cylinder being round, the hexagon angular. Neither can shrinkage and expansion, taken separately, wholly explain the phenomena. The basalt and starch assume the hexagonal prismatic form presumably on contracting; the epithelial cells of the sikin, the liver cells, and the pigment cells of the choroid of the eye presumably on expanding and indenting each other. The same results are obtained by apparently diametrically opposite methods and, what is remarkable, in inorganic or dead, and in organic or living matter equally. Similar observations have to be made regarding the radiating and concentric arrangements. These, curiously enough, occur in crystals and in plants and animals. Here again the boundary between the dead and living disappears. The more this subject is looked into, the more it will appear that a hard and fast line cannot be drawn between inorganic and organic matter, and between physical and vital force. This circumstance, singly and alone, goes far to prove the presence of a Creator and the existence of laws which apply equally to the rocks, crystals, \&c., and to plants and animals. The atoms and molecules which enter into the composition of all known substances, dead and living, behave similarly in the inorganic and organic kingdoms. Of this I am fully convinced.

The two great kingdoms of nature are correlated, and interact to an extent at present undreamt of. They are not opposed to each other, as some hastily conclude. The organic is taken from and returns to the inorganic, and the inorganic, or part of it, becomes animated from time to time. Both kingdoms form one great whole, and they are created, regulated, and supervised by the same master-intellect. It is not a case of a house divided against itself, but one of complete harmony, where everything works to a given end, and for good,

VOL. I. 


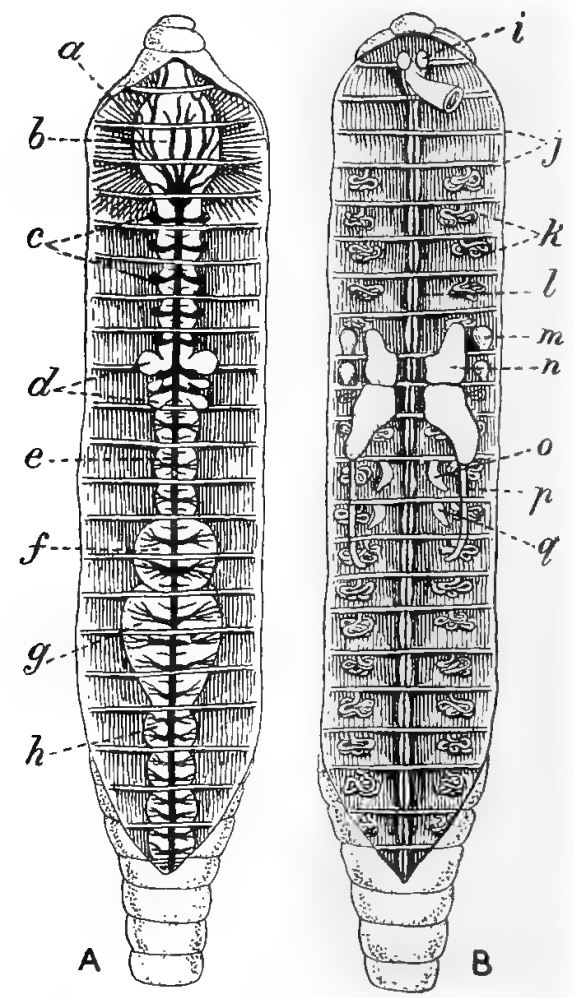

PLATE XLI
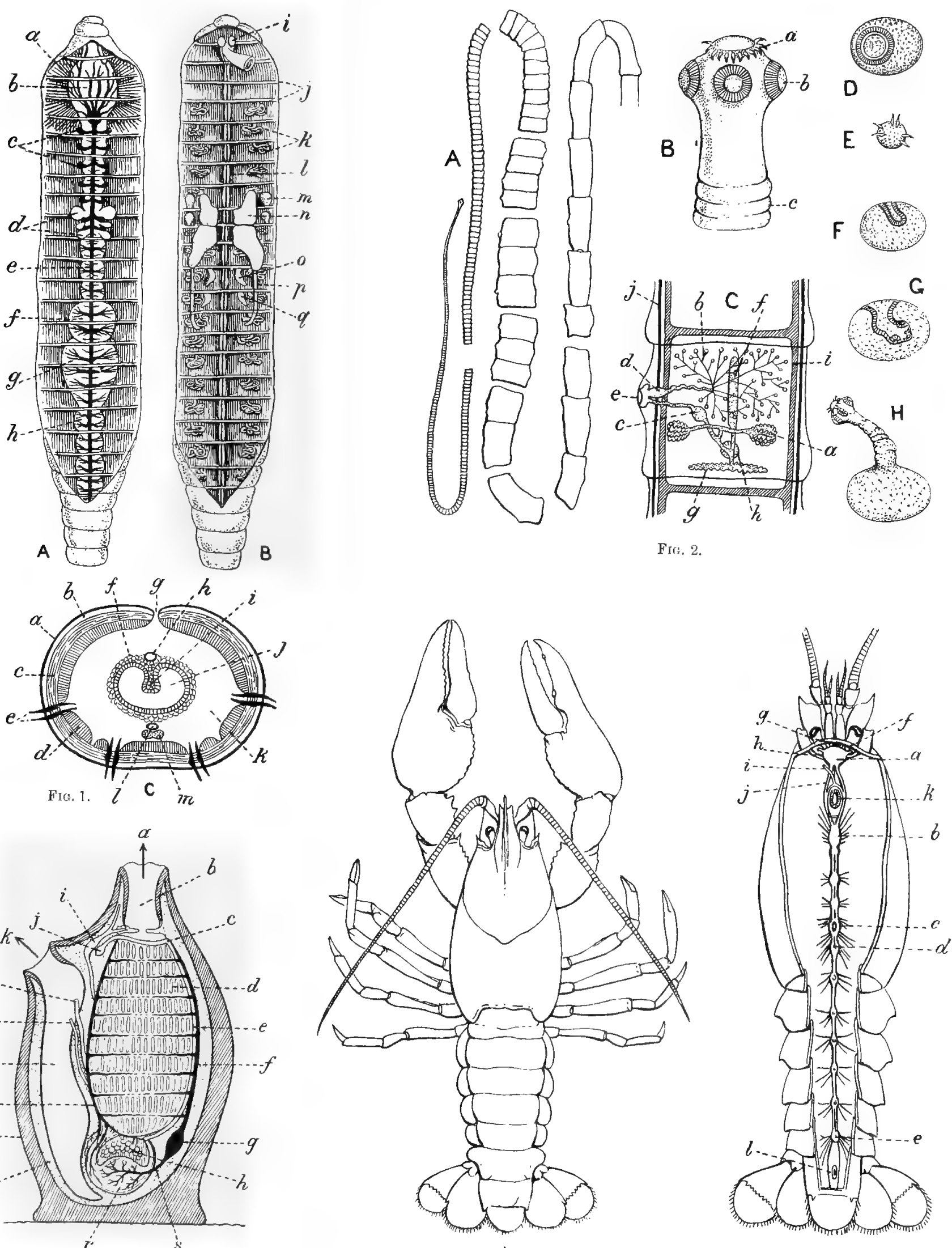

Fus.

A

Fig. 4. 
PLATE XLI

Plate xli. illustrates cleavage or segmentation in animals; also, in a minor degree, radiating and concentric arrangements.

FIg. 1.-Dissection of earthworm, by Masterman-dorsal view. Shows transverse divisions and septa.

A. Superficial dissection. $a$, Pharyngeal muscles; $b$, pharynx ; $c$, lateral hearts ; $d$, cesophageal glands; $e$, dorsal blood-vessel; $f$, crop ; $g$, gizzard ; $h$, intestine.

B. Deep dissection with alimentary canal, \&c., removed. $i$, Brain; $j$, septa; $l$, nephridia; $l$, nerve cord; $m$, spernatheca; $n$, seminal vesicle; $o$, ovary; $p$, vas deferens ; $q$, oviduct.

C. Transverse section of earthworm in intestinal region. Shows radiating and concentric arrangenents. $a$; Cuticle ; $b$, ectoderm; $c$, circular muscles; $d$, longitudinal muscles; $f$, yellow cells ; $g$, dorsal pore; $h$, dorsal blood-vessel ; $i$, typhlosole; $j$, intestine ; $k$, peri-visceral cavity ; $l$, nerve cord ; $m$, ventral blood-vessel.

FIG, 2,-Views of adult and young tapeworm (Tænia solium), (after Lenckart). Show segnentation, radiating, branched, and concentric arrangements,

A. Body of adult Tænia segmented transversely (Tania saginata).

B. Head of Tænia with circlet of hooks $(a)$, four suckers $(b)$, and primary segments $(c)$.

C. Semi-diagrammatic view of a single proglottis of Trenia. $a$, Ovary; $b$, testis ; $c$, receptacle; $d$, seminal vesicle; $c$, common sexual aperture ; $f$, uterus; $g$, yolk-gland; $h$, shell-gland ; $i$, excretory canal ; $j$, nerve cord.

D, E, F, G, H. Development of Tænia solium.

FIG. 3.-Diagrammatic, median, longitudinal section of an ascidian by Masterman. Shows longitudinal and transverse division. $a$, Mouth; $b$, buccal cavity; $c$, peri-pharyngeal groove; $d$, pharyngeal clefts ; $e$, endostyle; $f$, pharyngeal part of ventral vessel ; $g$, heart ; $h$, gonad; $i$, nerve ganglion ; $j$, subneural gland; $k$, atriopore; $l$, intestine ; $m$, genital duct ; $n$, atrium ; $v$, dorsal bloodvessel ; $p$, test (cuticle); $q$, mantle (ectoderm); $r$, stomach; $s$, intestinal part of ventral vessel.

FIG. 4.-A. The common crayfish (Astacus fuviatilis, male), (after Huxley). Natural size. Shows radiation, brancling, and segmentation in the limbs, claws, \&c., and transverse division and segmentation in the body.

B. Dissection of the same from above, to show the central nervous system, \&c. a, Supra-cesophageal ganglion; $b$, infra-cesophageal ganglion; $c$, fifth thoracic ganglion; $d$, last thoracic ganglion; $e$, last abdominal ganglion ; $f$, optic nerve ; $g$, antennulary nerve ; $h$, antennary nerve; $i$, stomato-gastric nerve $; j$, circum-cesophageal commissures; $k$, cesophagus in cross section ; $l$, vent.

\section{PLATE XLII} thereof.

Plate xlii. illustrates spiral, longitudinal, radiating, and transverse division in plants and animals, and parts

FIG. 1.-Plotograph of plane lree (Acer pseudoplatanus) growing at Deans Court, St. Andrews, N.B. Shows spiral, longitudinal division of stem or bole. This is a rather common arrangement in the trunks of trees generally. The spirals may be right or lefthanded. Similar spiral arrangements are scen in water-spouts (Plate vi., p. 13); the stems of many plants (Plate x., Figs. 1, 2, and 3); various shells (Plate xv., Fig. 1); horns (Plate xv., Fig. 2 ; Plate xviii., Fig. 2); tusks (Plate xvi., Fig. 2 ); the bones of animals, especially the bones of the extremities (Plate xx., Fig. 3 ; Plate xxi., Fig. 1); the umbilical cord, and certain intestines (Plate xii., Figs. 3 and 4 ); the muscles of the left ventricle of the heart (Plate xvii., Fig. 4), \&c. The spiral, it will be seen, is no chance product in nature (the Author).

FIG. 2.-General views of the layers of the retina of the human eye (after Max Schultze). Show longitudinul and transverse divisiou.

A. The layers magnified 400 diameters. $a$, Membrana limitans interna ; $b$, optic fibre layer; $i$, ganglion-cell layer; $d$, internal granulated (molecular) layer; e, internal granule layer ; $f$, external gruulated (intergranule) layer ; $g$, external granule layer, including the external fibre layer; $h$, membrana limitans externa; $i$, layer of rods and cones; $j$, pigment layer:

B. The posterior part of the fundus of the human retina magnified 800 diameters. $f$, External granulated layer; $f$, external granule layer; $h$, limitans externa; $i$, rods and cones; the supporting connective tissue is onitted in this figure.

C. Cone and cone fibre, the latter presenting varicosities: from the vicinity of the yellow spot of the retina of the human eye. Magnified 500 diameters.

FIG. 3.-.Cuttlefish (Sepiu officinalis). Shows longitudinal, slightly radiating division and transverse markings.
A. Dorsal view of cuttlefish.
B. Ventral view of cuttlefish.

FIGs. 4.-Vertical section of sea anemone, copied from a water-colour drawing by C. Berjeau in the Zoological Department of the British Museum. Shows radiating, branched arrangement. a, Non-pinnate tentacles, the cavities of which communicate with the general cavity $(b)$, which is divided into compartments by septa $(c)$, on the walls of which the gonads $(d)$ are developed. The axis is occupied by the stomach (e), which communicates below with the general cavity $(b)$ and opens above by a mouth narked by a special slit $(f)$. $g$, Marks the point at which a chamber is in communication with its neighbour, and $h$ is the lower surface of the disc.

FIG. 5. - Filbrils of voluntary striped muscle in the uncuntracted and contracted states, as figured by Professor Rutherford. Show longitudinal and transverse cleavage.

A. Fibrils of extensor muscle of crab's leg, uncontracted and fully extended.

B. Fibrils of muscle of $\mathrm{crab}^{2} \mathrm{~s}$ leg, uncontracted and unextended.

C. Bundle of semi-contracted fibrils from muscle of crab's leg.

D. A further stage of contraction of muscle of crab's leg.

E. Fully cuntracted muscle of crab's leg, showing complete reversal of stripes.

$\mathrm{F}$. Contracted muscle from same preparation as that figured at $\mathrm{E}$.

G. A bundle of contracted fibrils.
The sarcous elements of the fibrils, as the author explained in 1872-3 (Edin. Med. Jour.), are endowed with a centripetal and centrifugal power, whereby they can elongate in the direction of the length or breadth of the fibril, according as the fibril is to be lengthened or shortened in the so-called relaxation and contraction of muscle. The original diagrams illustrating this point are given at Plate lxxxiii., p. 320 . 


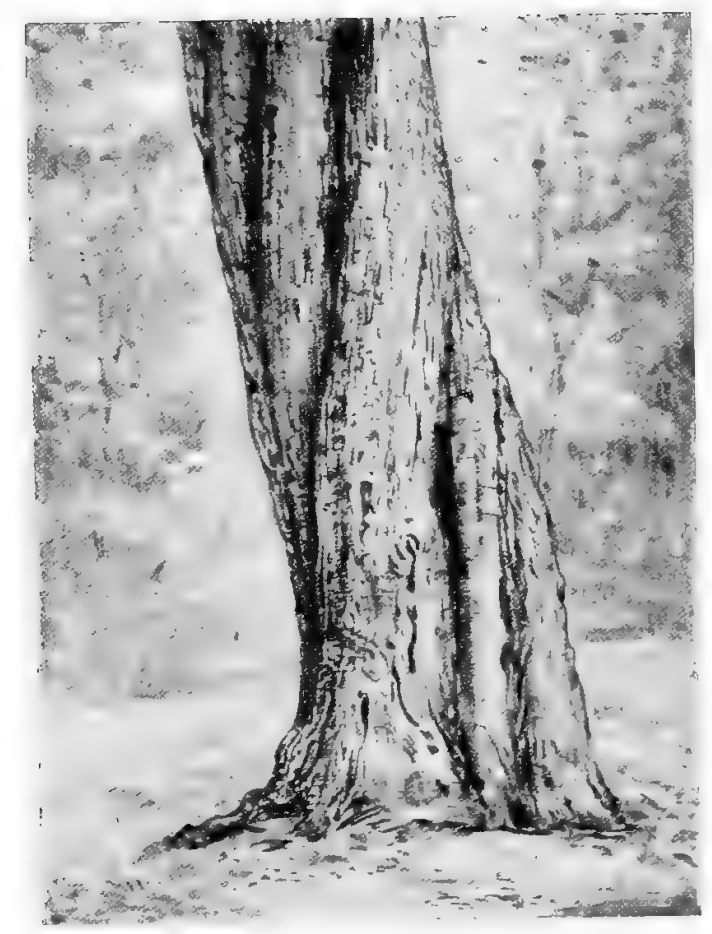

Fit. 1 .

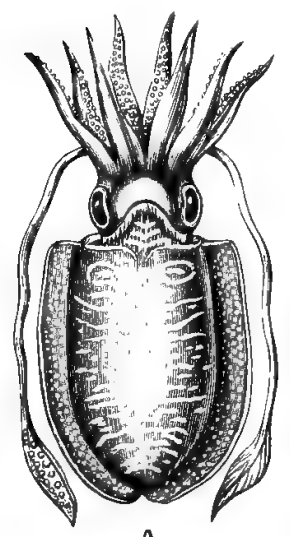

A

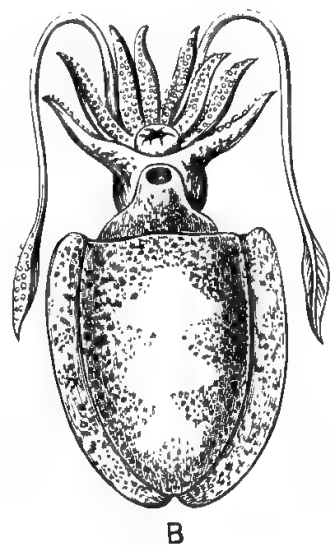

Fir. 3.

PLATE ILII
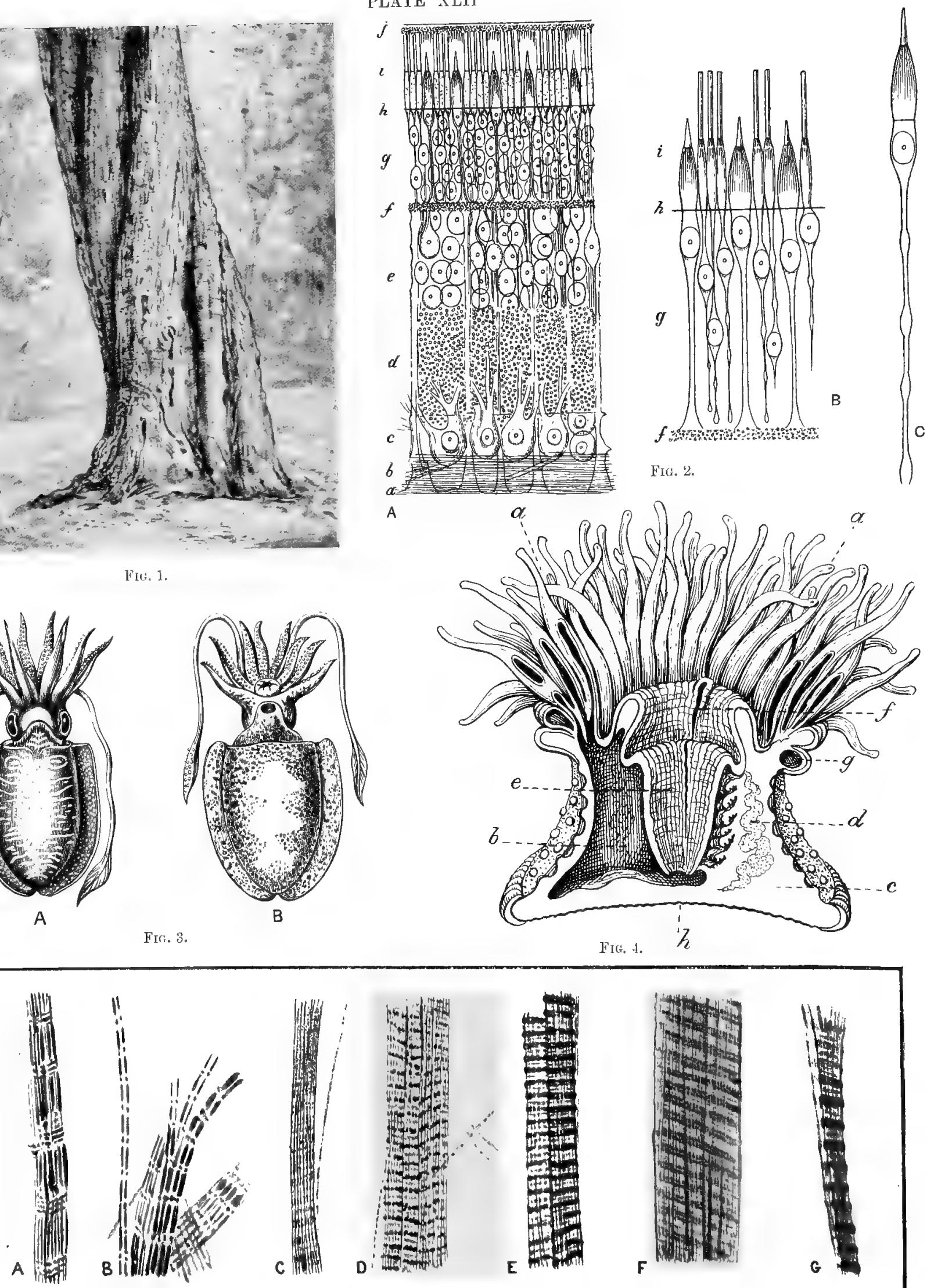

FIG. 5. 


\section{LONGITUDINAL AND TRANSVERSE CLEAVAGE}

PLATE XLIII

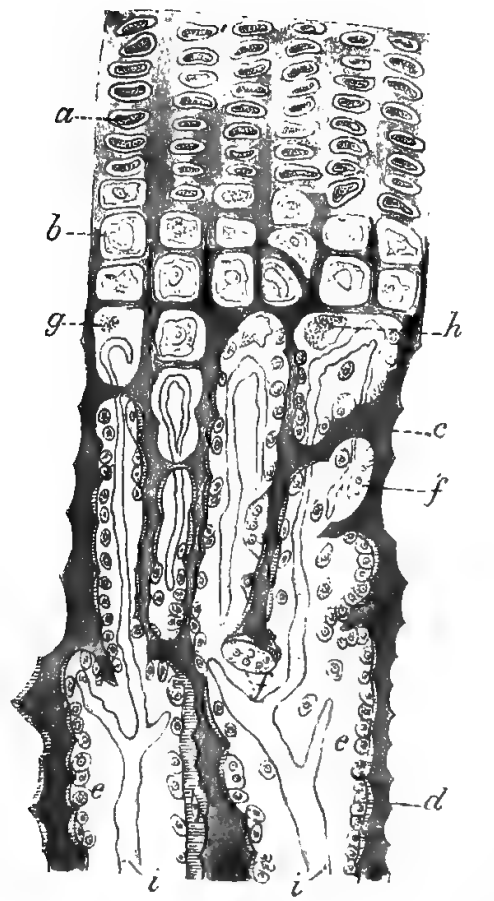

Fig. 1.

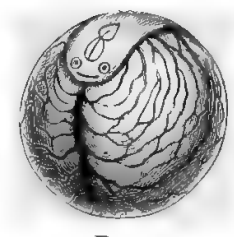

FIG. 4.

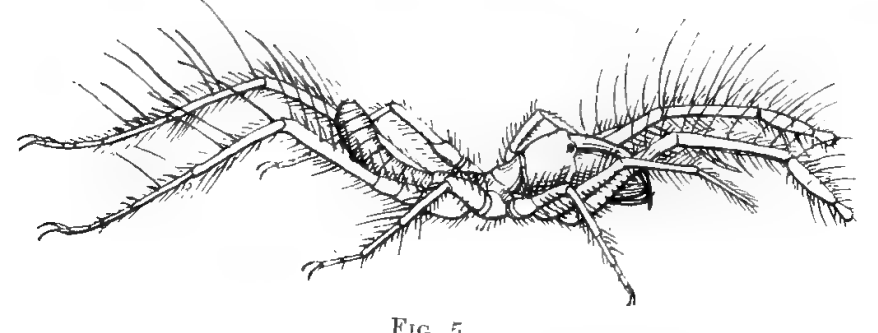

FIG. 5.

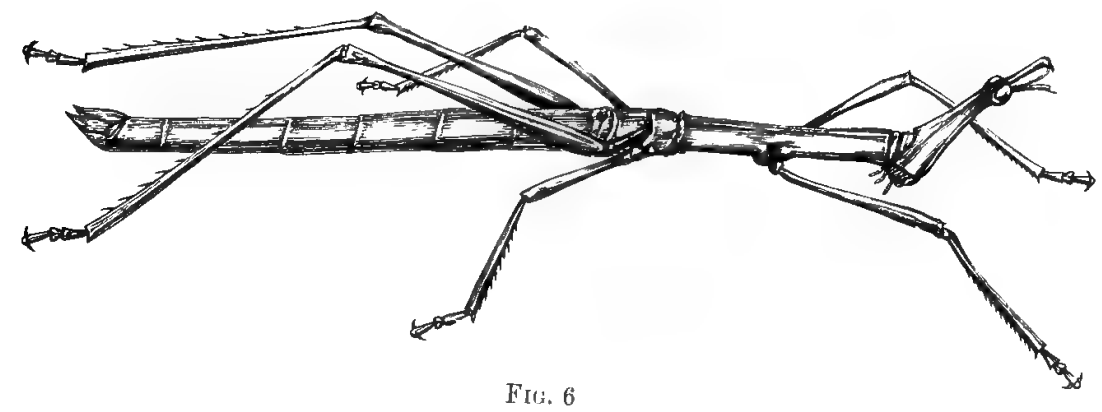

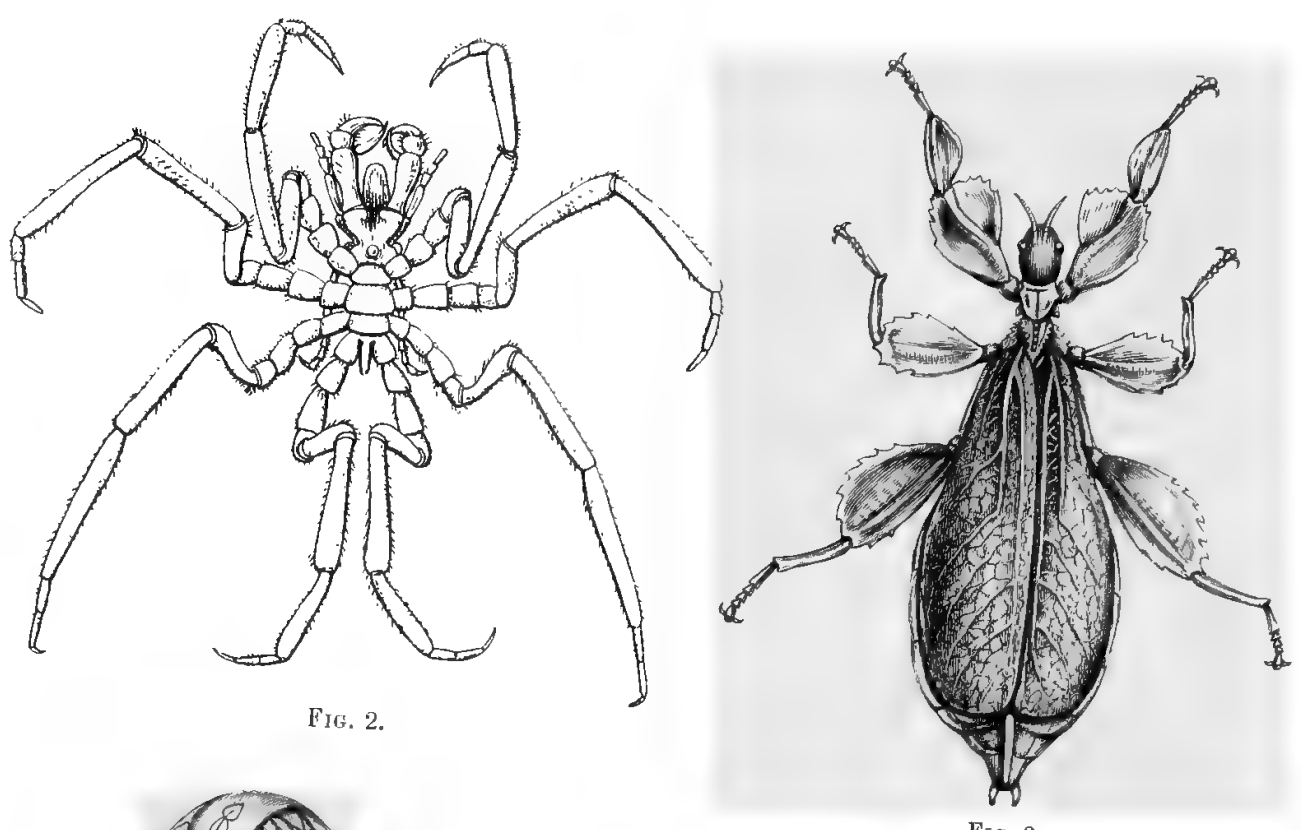

FIG. 3.
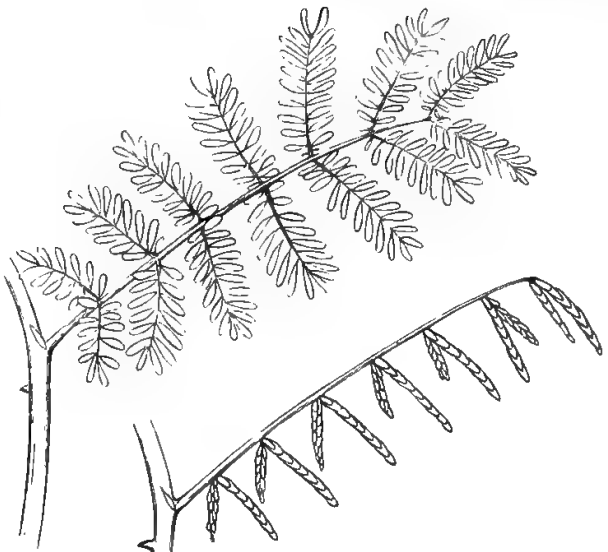

A $\begin{cases}\text { B } \\ B\end{cases}$

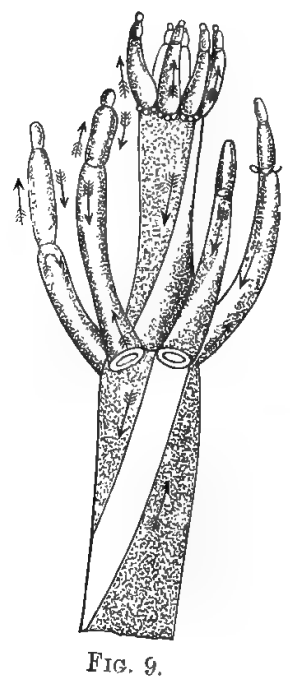




\section{PLATE XLIII}

Plate xliii. illustrates longitudinal and transverse cleavage and branching generally in plants, animals, and parts thereof.

FIG, 1.-Part of longitudinal section of the developing femur of the rabbit, magnified 350 diameters (after Klein and Noble Smith). Shows longitudinal and transverse cleavage very distinctly. $a$, Rows of flattened cartilage cells; $b$, greatly enlarged cartilage cells close to advancing bone, the matrix between them being partly calcified; $c, d$, bone already formed, the osseous trabeculæ being covered with osteoblasts $(e, e)$, except here and there, where a giant cell or osteoclast $(f, f)$ is seen eroding parts of the trabeculæ; $g, h$, shrunken irregular cartilage cells; $i$, $i$, vascular loops. The lower half of the figure shows the disappearance of the cartilage cells, the formation of calcified cartilage matrix, and the deposition by the osteoblasts of secondary osseous substance.

FIG. 2,-Nymphon abyssorum (after Cuvier). Shows an extreme example of segmentation in body and linibs of deep-sea crab (after Wyville Thomson).

FIG. 3.-._"Dead leaf" insect (Phyllium siccifolium, fenale). Shows resemblance to the venation and branching of leaves in its flattened body and legs. The peculiarity in form in this insect and in the stick insect (Fig. 6 of this Plate) cannot be explained by nimicry. Animals have no power to imitate or grow like anything but thenselves. Like can only beget like.

FIG. 4. - Egg of fish (Jarrabatur), showing the branching of the vessels in the vitelline circulation (after Dalton).

FIG. 5.-Fralcodes araneoides, a spider-like animal (family Solpugidx). Shows segmentation of body and great elongation and lranching of the limbs. The limbs might readily be mistaken for small dead branches.

FIG. 6.-The stick insect (Proscopia nodula). So named from its marked resemblance to small dead branches (after Cuvier). Shows segmentation and branching of body and legs. The peculiar form of this insect cannot be referred to minicry. It can only be explained by a common law of development.

FIG. T.-Acacia farnesiana (after Darwin). Affords a good example of branching.
A. Shows the leaves expanded during the day.
B. The same folded at night.

Fru. 8.-Portion of leaf of insectivorous plant (Drosophyllum lusitanicum), (after Darwin). Shows peculiar mushroom-shaped glands.

FIG. 9. Chara, a plant allied to the Alga (after J. H. Balfour). Shows lranching and spiral formation. The arrows indicate the direction of the spiral intra-cellular circulation.

\section{PLATE XLIV}

Plate xliv. illustrates striking resemblances between the convolutions of the human brain and the hard parts of certain corals; also between the hard parts of Venus's flower-basket and the spiral muscular fibres of the left ventricle of the heart of the bird and mammal ; also between the spicules of sponges and certain crystals.

FIt. 1.-External convolutions of tlıe human brain. Shows folded plicate arrangement.

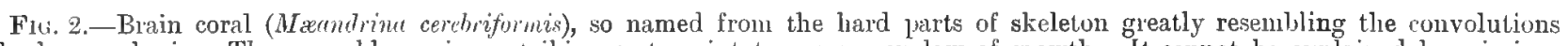
of the human brain. The resemblance is so triking as to point to a common law of growth. It cannot be explained hy mimicry (photographed by the Author).

FIG. 3.--Transverse section of upper part of tooth of Lalyrinthodon jaegeri, magnified. a, h, Margins of section (after Uwen). Shows a remarkable convoluted arrangement greatly resembling the liuman brain (Fig. 1 of this Plate) and the brain colal (Fig. 2 of this Plate).

Fis. 4.-The same tooth : natural size. The centre is the pulp cavily from which the processes of pulp and dentine radiate. The cumbined radiation and plaiting and folding of the sulistance of the tooth is, in a way, unique. The letters $a, b$, indicate the position from which the section shown in Fig. 3 was taken.

FIG. 5.-Snow crystals as figured by Scoresby. These are conpound branched crystals, the branches being six in mumber. Curiously enough the sponge spicules have also, in many cases, six branches or rays. The radiating arrangenent is well marked in both. Here again there seems to he a coumon law of development.

FIG. 6. - Fertilisation of the ovum of an echinoderm (after Selenka). "Z, Zona pellucida; $b$, entrance of the spermatozoon into the protoplasm of the vitellus $(c)$, with radiating and concentric arrangement of its grauules. Shows the fundamental nature of the radiation and concentric arrangements. Here they present themselves at the very beginning of life. They are also found in all kinds of adult animal structures (vide Plate v.), and in crystals and plant structures (Plates i., ii., iii., and iv.).

FIG. 7.- Spicules of glass sponges (milgnified). In the centre is a node of the dictyonic network of a ventriculite (Guide to Coral Gallery, British Museum). Show leautiful symmetrical forms greatly resembling crystals. Compare with the snow crystals delineated at Fig, 5 of this Plate.

FIG. 8.-Venus's Hower-basket or glass sponge (Euplectella cespergillum). Shows the hard parts of skeleton. These resemble spun glass, and form an intricate and exquisitely beautiful network of longitudinal, transverse, and oblique spiral fibres, which may not inaptly be likened to the spiral muscular fibres forming the left ventricle of the heart. The arrangement in both cases consists of layers, the spiral elements of which cross each other at various angles to form structures remarkable for their lightness and strength. (Compure with Figs. f and 7 of Plate xevii.; and the first eight Figs. of Plate xeviii., where dissections and models of the heart are given.) a, Longitudinal portions of skeleton; l, i, obligne spiral portions; $\lambda$, transverse portions (photographed by the Author). 

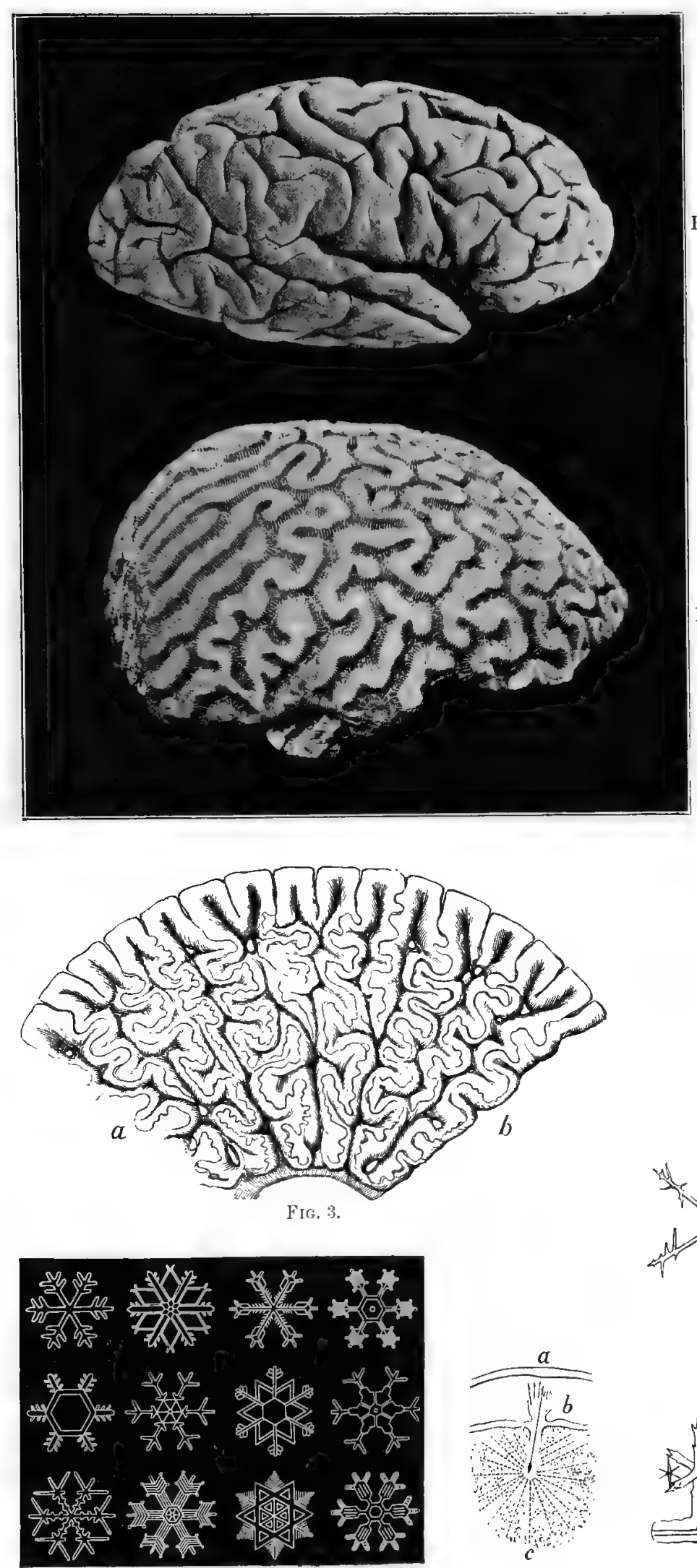

FIG. 5.

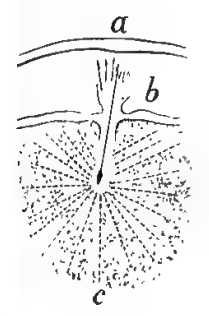

FIG. 6 .

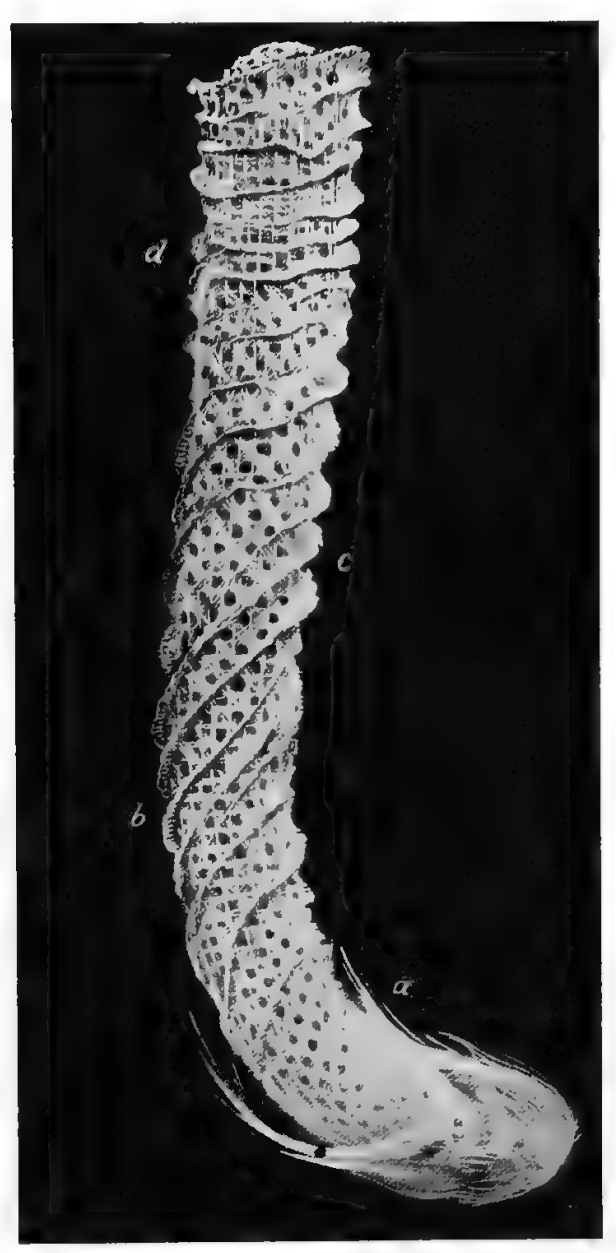

Fir. 8.
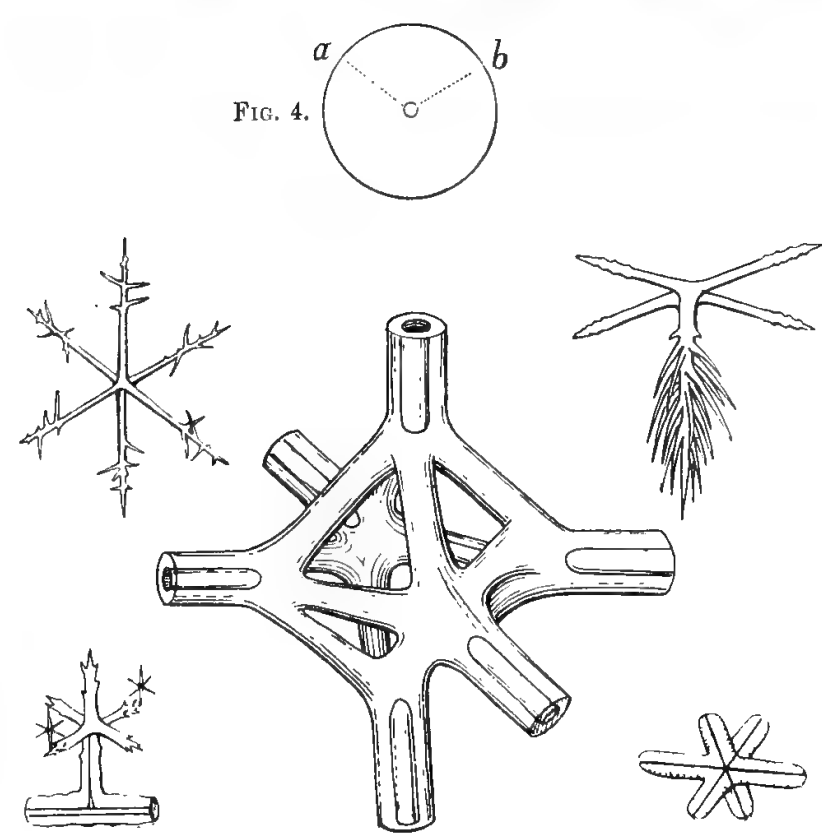

FIG. 7 . 
PLATE XLV

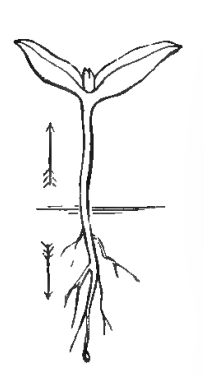

Fin. ].

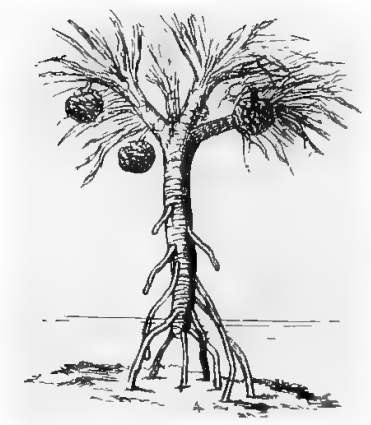

FIG. :.

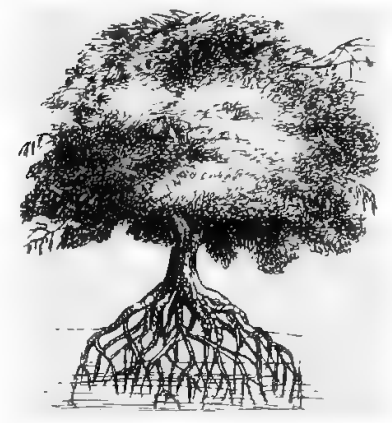

Fint. :3.

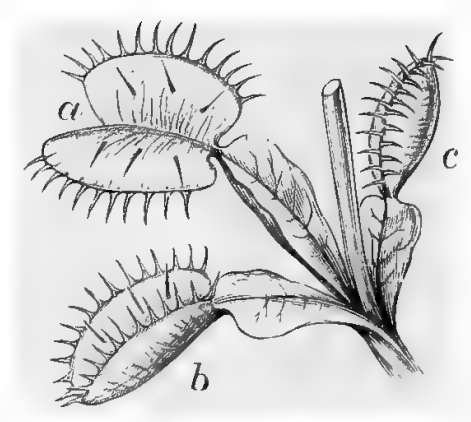

$\mathrm{F}[1 \div .4$.

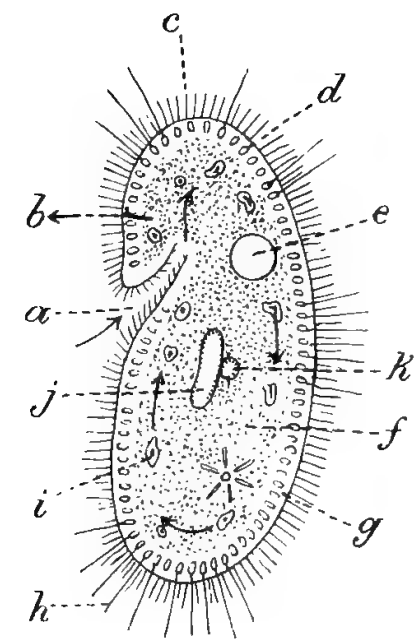

FIF. 5.

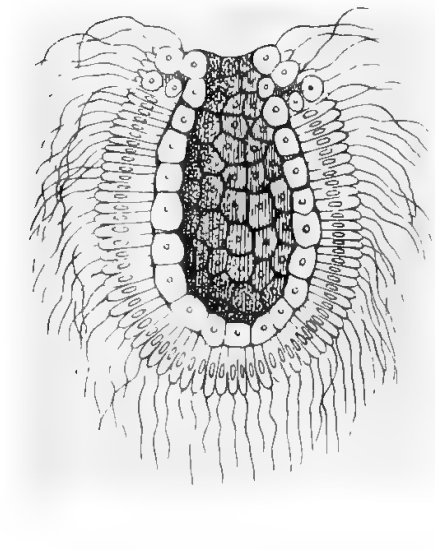

Fis: 6.

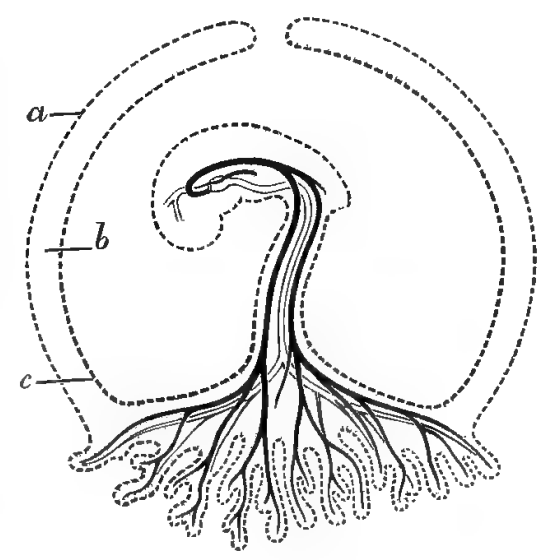

FIr. 7 .

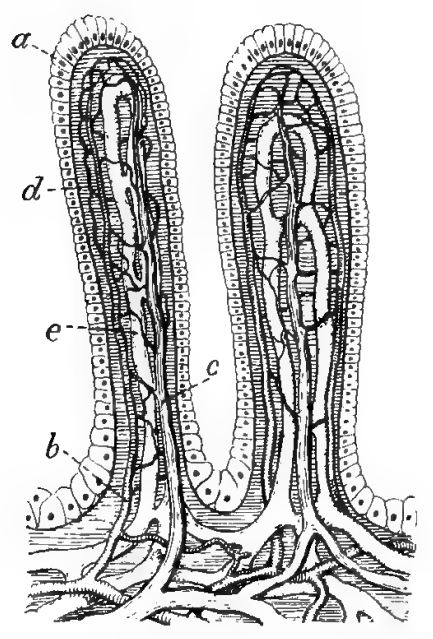

A

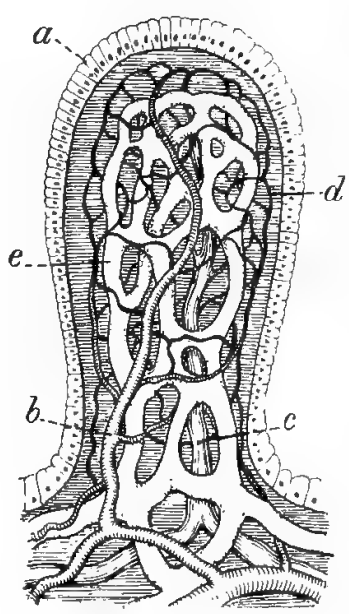

B

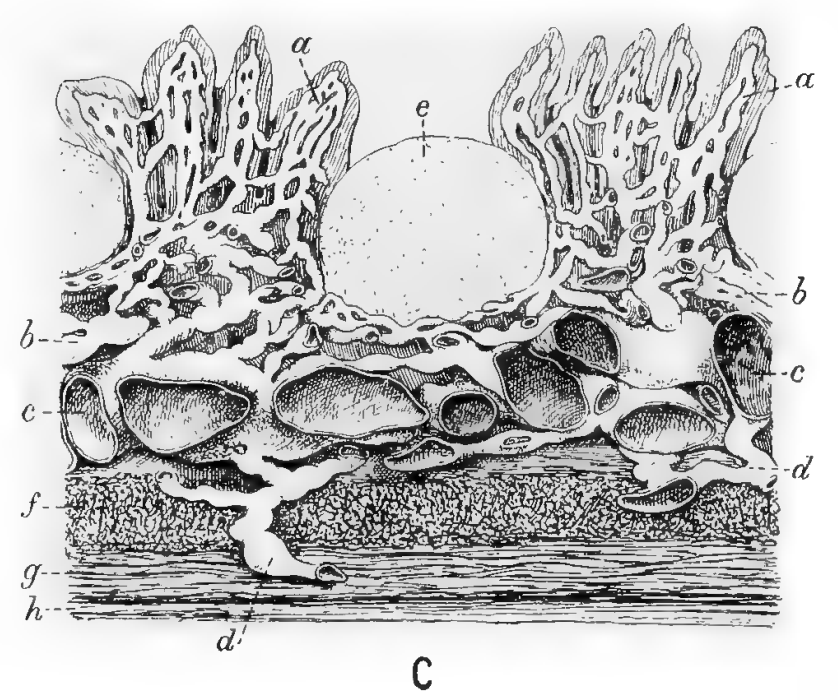

Fig. 8. 
PLATE XLV

Plate xlv. illustrates radiating and branching in plants and animals and parts thereof.

FIG. 1.-A dicotyledonons seedling with an ascending and descending axis (Henfrey). Shows branching above and below ground.

FIG. 2.-The screw pine (Pandanus odoratissimus) with adventitious roots supporting the truuk (Henfrey). Shows radiation and branching at either end of trunk.

Fig. 3.-The mangrove tree (Rhizophora), supported as it were upon piles; the roots ruising up the trunk (J. H. Balfour). Shows radiation and branching in two directions.

Fira. 4.-Venus's fly-trap (Dionæe muscipula), a sensitive, insectivorous plant. Shows radiation and division in the leaves. $a$, Leaf fully expanded; $b$, ditto partly closed ; $c$, ditto closed (J. H. Balfour).

Fra. 5.-Paramecium (after Masterman). Shows radiation and division of surface. $a$, Month; $b$, anus ; $c$, cilia; $d$, trichocyst; $e$, contractile vesicle; $f$, medulla ; $g$, cortex $; h$, thread of trichocyst ; $i$, food-vacuole $;$, micro-nuclens ; $l$, macro-nucleus.

FTG. 6. -Larva of calcareous sponge at the Gastrula stage (Haeekel). Shows radiating and branching in a low form at a very early period of development. The larva consists of an outer and inner cell layer, and forms a pouch with a mouth.

FIG. 7.-Diagram of human ovum and placenta (after Fothergill). Shows well-marked radiating and branched arrangement. $a$, Epithelium or chorion; $b$, connective tissue ; $c$, epithelium or amnion.

Fig. 8.-Villi of intestine of mare and sheep (after Carpenter). Show branching of villi and of the blood-vessels and lymphatics of villi. The branching is minute and complicated.

A. Villi of mare. $a$, Epithelium ; $b$, artery ; $c$, vein ; $d$, capillaries ; $e$, lacteals.

B. Villus of sheep. Same lettering as in A.

C. Vertical section through one of Peyer's patches in the lower part of the ileum of the sheep. $a, a$, Lacteal vessels in the villi; $b, b$, superficial layer of the lacteal vessels $; c, c$, deep layer of the lacteal vessels ; $d, d$, afferent lacteals provided with valves ; $e$, Peyer's gland ; $f$, circular muscular layer of the wall of the intestine; $g$, longitudinal muscular layer of ditto; $h$, peritoneal layer.

\section{PLATE XLVI}

Plate xlvi. illustrates the first appearance and mode of formation of limbs. This is a form of budding, division, and branching. The longitudinal division is seen in the fins and tails of fishes, the several kinds of flippers, and the fore-arms, legs, and digits of bipeds and quadrupeds : the transverse division is seen in the vertebral column or backbone.

FIG. 1.-Lepidosiren annectens (Owen); Protopterus annectens (Günther). Show branching filamentous appendages. a, Filamentous anterior extremities or pectoral fins; $b$, filamentous posterior extremities or ventral fins; $c$, scapulo-coracoid bone with jointed cartilaginous ray of pectoral fin ; $d$, pelvic cartilage and jointed cartilaginous ray of ventral fin (after Owen and Günther).

FIG. 2.-Siren (Siren lacertina), Amphiuma (Amphiumu tridactylum), and Menobranchus (Menobranchus lateralis); revealing rudimentary limbs, and branching thereof.

A. Siren. Shows rudimentary anterior extremities with imperfect digits.

B. Amphiuma. Shows rudimentary anterior and posterior extremities, each with three digits. nature).

C. Menobranchus. Shows more highly developed anterior and posterior extremities with four digits (after Cuvier and from

FIG. 3.-Development of the human embryo from the third to the seventh week. Shows division and segmentation of the body (visceral arches and spinal column), the growth of the head and body, and the growth and budding of the limbs. The lettering is the same in all the figures.

A. Three and a half weeks embryo. a, Rudimentary vertical colunn terminating in a caudal process or tail; $b$, budding, unformed, superior limb; $c$, ditto, inferior limb; $d$, branchial arches; $e$, rudimentary umbilical or nourishing cord; $f$, head beginning to assurne shape.

B. Four weeks embryo. In this the spinal column $(a)$ is a little more pronounced than at A, especially the caudal portion. The branchial arches $(d)$ are also more defined, and the upper $(b)$ and lower $(c)$ extremities are increased in size.

C. Four and a half weeks embryo. In this the parts are becoming more defined.

D. Five weeks embryo. In this the parts referred to under $A$ are altering slightly in appearance, the left superior extremity $(b)$ developing a rudimentary hand and five digits or fingers, the head $(f)$ being increased in volume, and the spinal column $(a)$ being less prominent from being partly covered in. a foot.

E. Five and a half weeks embryo. The changes referred to under D are more pronounced, the posterior extremity $(c)$ developing

F. Six weeks embryo. In this the head and body have increased in size, and the segmentation of the spinal column has disappeared from being covered in. 'l'he left superior $(b)$ and left inferior $(c)$ extremities have grown, and the hands and feet, fingers and toes, are becoming more distinct. The digits on the feet, as on the hands, are five in number.

G. Seven weeks embryo. In this the head and body are growing and assuming shape, the loranchial arches $(d)$ and the spinal column $(a)$ being less obvious. The left superior $(b)$ and left inferior $(c)$ extremities are elongated and gradually assuming shape. The changes begun in the embryo, it need scarcely lie stated, are completed in the foetus at full term, but need not be further described. Drawn for the present work by C. Berjeau,

YOL. I. 


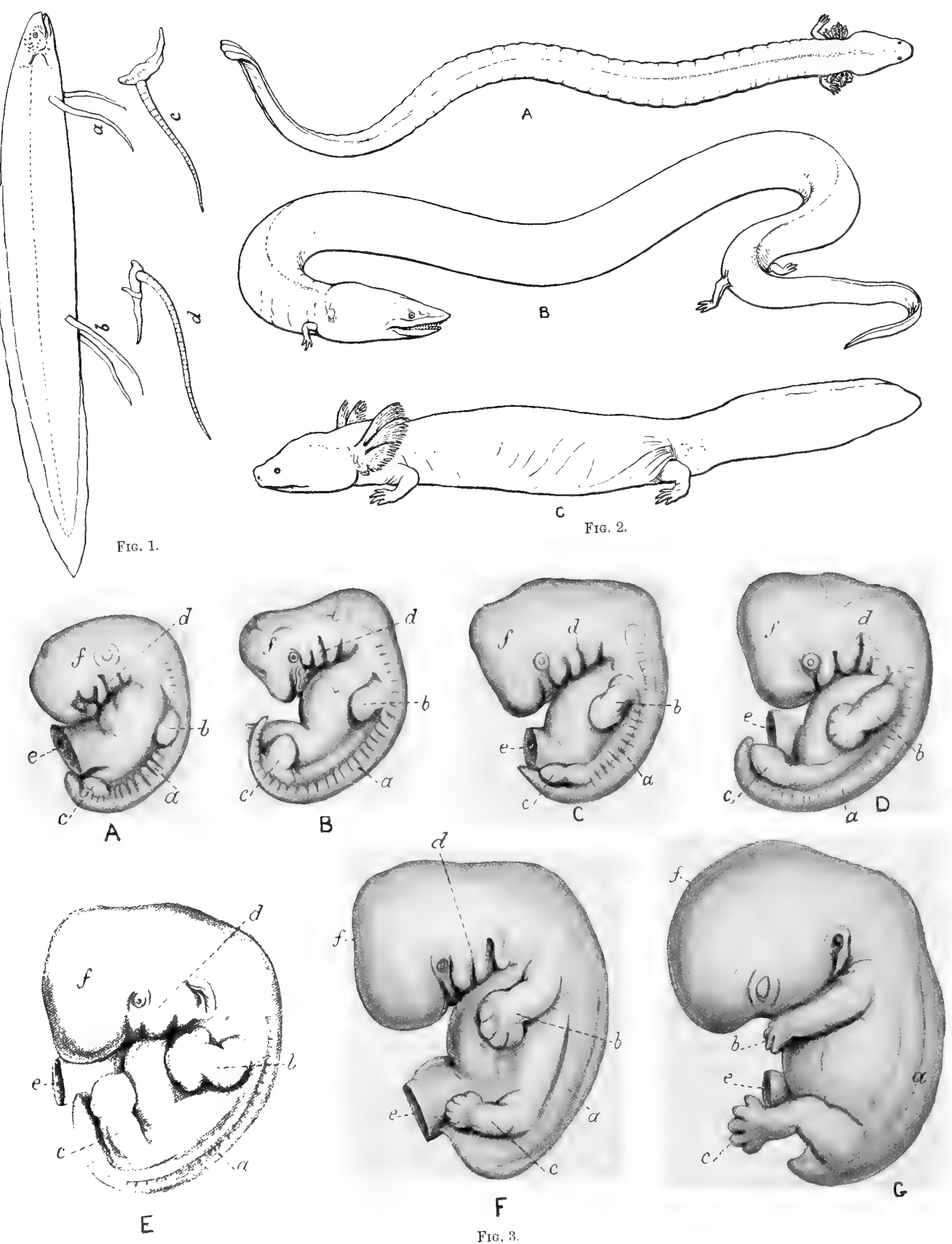

PLATE XLVI 

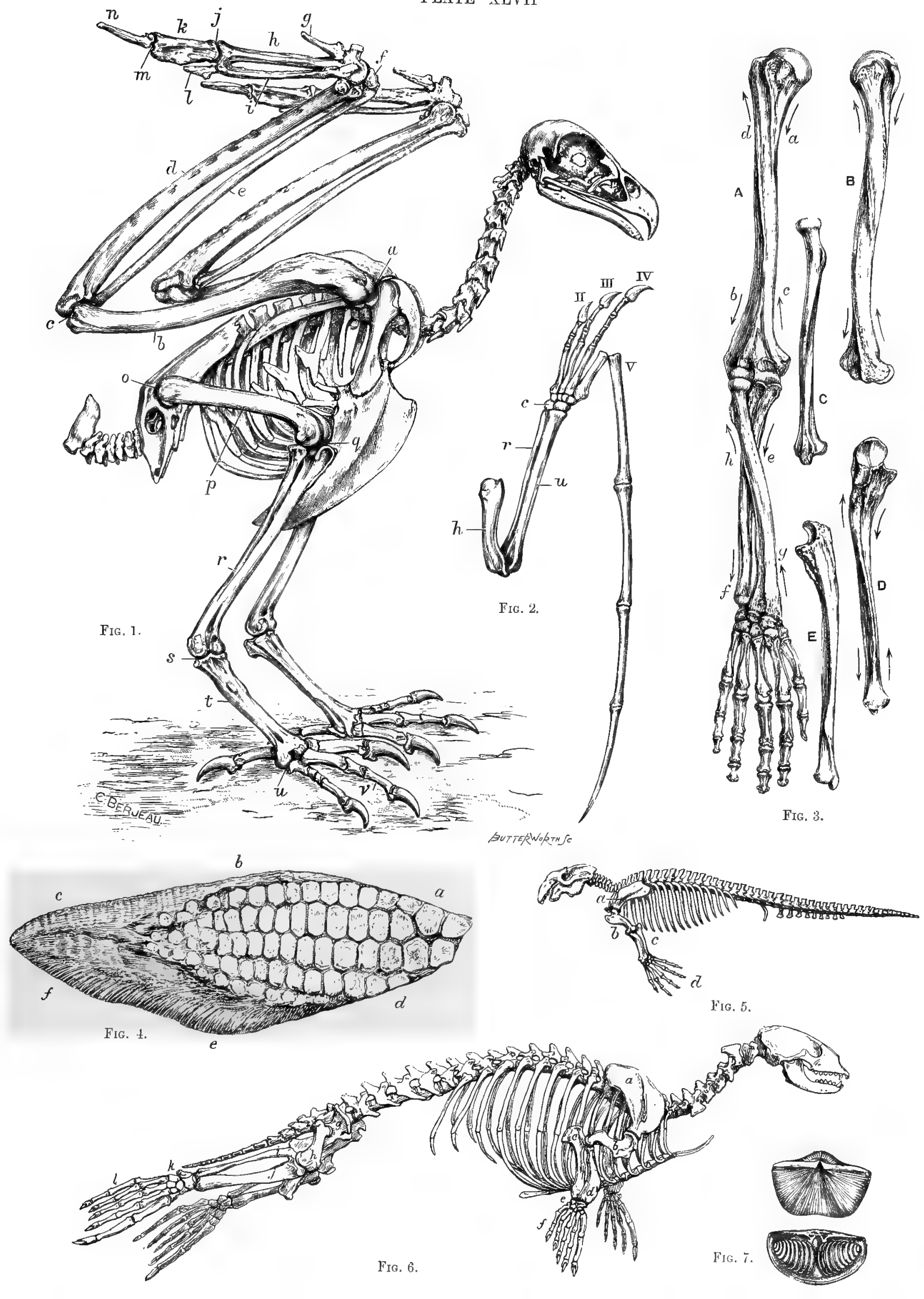


\section{PLATE XLVII}

Plate xlvii. illustrates the longitudinal and transverse cleavages or segmentation of the body and limbs in vertebrates. The longitudinal, with a certain amount of transverse, cleavage is well seen in the limbs; the transverse cleavage being marked in the vertebral column and ribs. This plate also illustrates division and radiation, seen more particularly in the hands and feet or their representatives, namely the fins, flippers, and swimming tails of fishes and sea mammals, and the diving, swimming, and flying wings of birds. It further shows that the extremities and other parts of the body are spirally constructed. (Compare with Plates xlv., xlvi., xlviii., and xlix.)

FIG. 1. - Skeleton of golden eagle (Aquila chrysaëtos), Scotland. Specially drawn for the present work from specimen in the British Museum, by C. Berjeau. a, Shoulder joint; $b$, humerus ; $c$, elbow joint ; $d$, e, bones of fore-arm ; $f$, carpus and wrist joint; $g$, thumb or false wing; $h, i, j, k, l, m$, n, bones of the hand more or less fused together. This part of the wing bears the primary or Hying feathers, where division and radiation are carried to an extreme. $o$, Hip joint; $p$, femur; $q$, knee joint; $r$, bones of leg; $s$, ankle joint; $t, u$, tarsus; $v$, foot. It will be observed that the wings are powerful structures as compared with the legs and other parts of the body. This is necessary for the purposes of flight. The modifications of the hands are at once striking and renıarkable. Compare with the hand of extinct flying reptile (Scopognathus crassirostris), Fig. 2, and the hand of man (Homo sapiens), Fig. 3 of this Plate.

FIG. 2.-Skeletou of arm, fore-arm, and hand of extinct flying reptile (Scopognathus crassirostris), Nicholson and Lydeker. Shows longitudinal and transverse cleavage and extreme modification of the fifth digit (v.) to carrying a flying membrane. $h$, Humerus; $r$, $u$, radius and ulna (bones of fore-arm); $c$, carpus or wrist joint; ii., iii., iv., v., bones of hand; the fifth digit (v.) being very greatly enlarged for the purposes of flight.

Fig. 3.-Bones of human arm, fore-arm and hand. Shows longitudinal and transverse cleavage, and how all the bones are twisted upon themselves and form spiral structures.

A. Entire arm. The humerus is twisted upon itself, as indicated by the darts $a, b, c, d$. The same is true of the radius and ulna (vide darts $e f, g h$ ), and the bones of the hand.

B. Another view of humertus, showing marked spirality.

C. Shows spiral structure of radius.

D. Shows spiral structure of uIna.

The bones of the wing of the bird and even the primary feathers are twisted upon themselves to form spiral structures. The bones of the human leg and those of birds and quadrupeds are also spirally formed. Compare with extremities of elephant (Plate xx., Fig. 3). Drawn from specimens in the Author's museum, by C. Berjeau.

Fig. 4.- - Hind flipper of extinct Ichthyosaurus or fish reptile (Ichthyosaurus tenuirostris), (after Owen), with portion of skin and soft parts adhering. Shows longitudinal and transverse division and segmentation of a marked kind. $u, b, c$, Anterior or thick margin of flipper; $d, e, f$, posterior or thin flexible margin of flipper. The flipper is triangular in shape and graduated, and is a true swimming organ. It is movable in all its parts, aud resembles a flying wing. The flipper is essentially a modified hand. It is seen in position at Plate xlviii., Fig. 3. Compare with the flippers of the extinct Plesiosaurus in same Plate, Fig. 2, and with the modified hand of the bird's wing and extinct flying reptile (Figs. 1 and 2 of this Plate).

Fin. 5.- Skeleton of the dugong (Halicore dugong), one of the fish-like, swimming nammals (after Dallas). Shows well-marked longitudinal and transverse cleavage of body and limbs. The flipper or swimming arm in this case consists of a scapula $(a)$; an arm $(b)$; a fore-arm $(c)$; and a hand with five digits $(d)$. The bones are all twisted, and the Hipper is moved spirally so as alternately to seize
and evade the water.

FIG. 6.- Skeleton of common seal (Phoca vitulina), (after Cuvier). Shows longitudinal and transverse cleavage in body and limbs, especially in the latter. The fore limb consists of a scapula $(a)$; a humerus $(b)$; radius and ulna $(c, d)$; wrist $(e)$; and hand with five digits. It closely resembles that of the dugong (Fig. 5 of this Plate). The hind limb consists of femur ( $h$ ), attached to pelvis $(g)$; radius and ulna $(i, j)$; ankle joint $(k)$; and foot with five toes $(l)$. The movements of the fore limbs are similar to those occurring in the dugong. The novernents of the hind limbs resemble in all respects those made hy the lower part of the body and tail of the tish. 'The hind limbs are held together and made to lash from side to side in swimming, the toes of the feet and the membrane supported by them being alternately spread out and contracted during the swimming movement, as happens also in the caudal fins of fishes. The contour of the seal, like other sea mammals, is fish-like.

FIG. 7.-Spirifer striatus. Shows radiating and right and left-lıanded spiral arrangements. Compare with Plate xiv., Fig. 1, $\mathrm{A}, \mathrm{B}, \mathrm{C}, \mathrm{D}$.

\section{PLATE XLVIII}

Plate xlviii. illustrates longitudinal, radiating, and transverse cleavage and segmentation in the fins, tail, and body of the fish; in the flippers and bodies of the extinct bird-like and fish-like reptiles (Plesiosaurus and Ichthyosaurus); and in the arms and tentacles of modern and extinct cuttle-fishes.

FIG. 1. Skeleton of perch (Perca), (after Cuvier and Valanciennes). Shows elliptical-shaped, flattened body, with pointed backbone furnished with spines and ribs, specially arranged to admit of the sinuous lateral movements necessary in swimming. In these movements the radiating, branching parts of the tail play a conspicuous part, the radiating, hranching fins being engaged in a minor degree. $a, a^{\prime}$, Dorsal fins ; $b$, pectoral fin ; $c$, ventral fin ; $d$, anal fin; $e$, $e^{\prime}$, caudal fin. The pectoral and anal fins occupy the positions of the anterior and posterior extremities of the seal and other sea mammals.

FIG. 2.-Skeleton of the great extinct bird-like reptile, Plesiosaurus (Plesiosaurus dolichodeirus, Upper Triassic and Liassic), (after Cuvier). Shows typical longitudinal and transverse segmentation in the four flippers, and pronounced transverse segnentation in the long, graceful, spinal column with its spinous processes and ribs. While the spinal column in this extinct form was flexible as in the fish, there can be no doubt that locomotion was effected almost exclusively by the flippers, which are specially modified to act as 
PLATE XLVIII
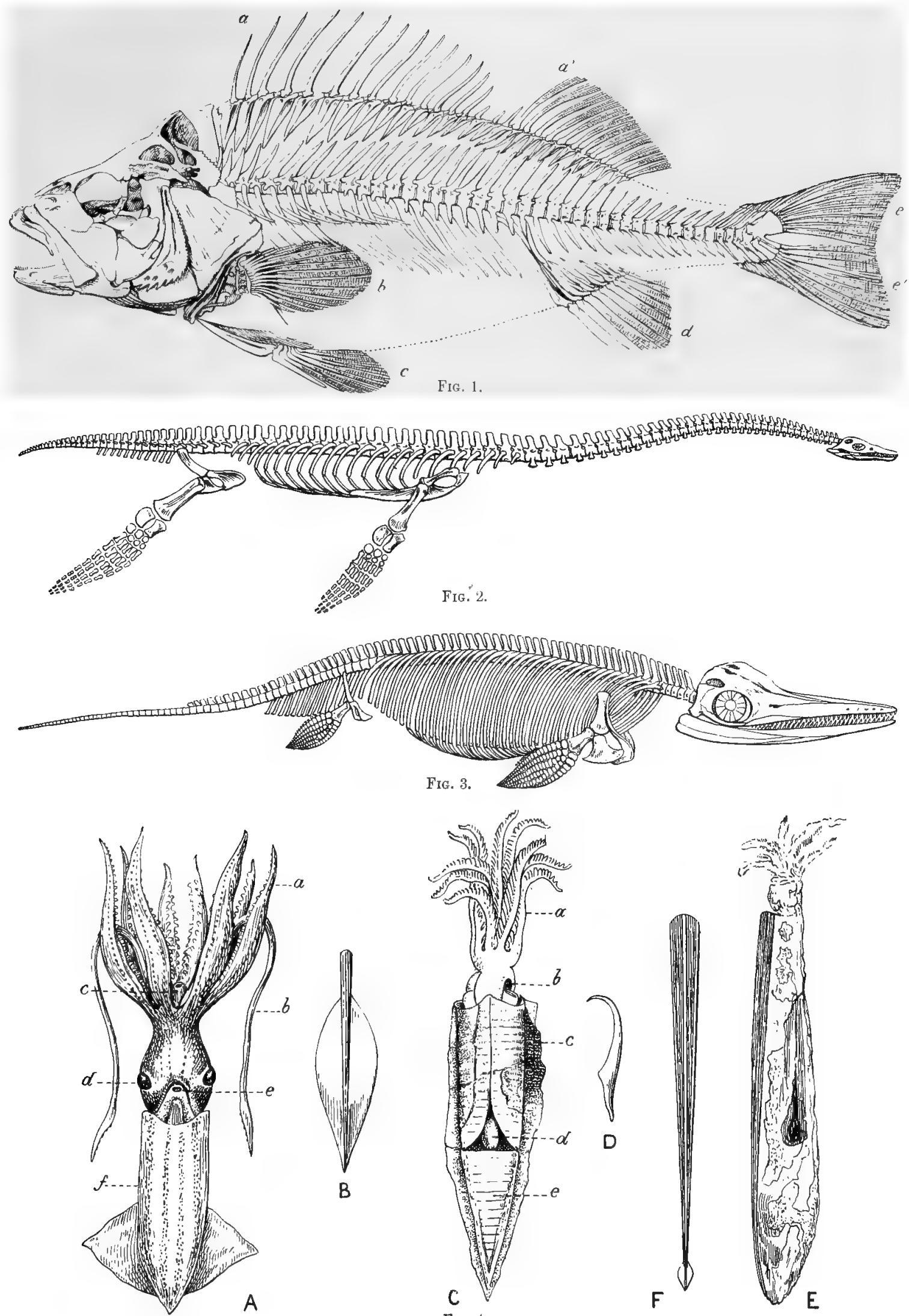

FIG, 4. 


\section{PLATE XLVIII (continued)}

propellers. These are constructed on the principles of true wings, being triangular in shape and graduated; the root and anterior margins of each flipper being thick and semi-rigid, while the tip and posterior margins are thin, flexible, and elastic. This peculiar structure, when the flippers were made to vihrate, would produce a rapid forward motion akin to flying, and the speed of the Plesiosaurus must have been as phenomenal mmong animals as that of the dragon-fly among insects. The flippers represent modified arms and legs, the fingers and toes consisting of five tajering rows of small, slightly elongated, square-shaped boues. As the flippers are loosely jointed at the shoulder and pelvis by universal joints, and all the hones composing them are free to move, the range and variety of motion must have been very great. The movements, there is reason to believe, consisted of vertical vibration with a certain anount of rotation along the anterior margins of the flippers, accompanied by triangular twisting of the flippers, as happens in wings. The flippers somewhat resemble those of the sea hear (Otaria jubata). (Compare with Plate lii., Fig. ].

FIG. 3. - Skeleton of the great old-world fish-reptile (1chthyosaurus tenuirostris), (after Cuvier). Shows the same features as in the Plesiosaurus just described, with the following differences. The four fippers are shorter, broader, and less highly developed as propelling organs. To remedy this, the animal was provided with a powerful swimming tail, believed at one time to have had no caudal fin. From a specimen, however, discovered in the Lias of Wurtemburg, where the contour of the soft or fleshy parts could be made ont, a caudal fin was present, the terminal portion of the spinal column occupying its lower lobe, while in the sharks it occupies the upper lobe. Professor R. Owen, with remarkable sagacity, predicted the presence of a caudal fin in the Ichthyosaurus. The tail of the Ichthyosaurus, there is reason to believe, was made to vibrate vertically in swimming, as in the dolphin, whale, dugong, manatee, \&c., and not laterally as in the fish. This is almost certain from the uumber, position, and configuration of the ribs, and the nature of the breathing, which was aërial.

FIG. 4.-Recent and extinct cuttle-fishes (after Zittel and Mantell). Show longitudinal, radiating cleavage and transverse markings.
A. Modern cuttle-fish from Pacific ocean (Enoplotenthis leptura). $u$, arms ; $b$, tentacles; $c$, mouth ; $d$, eyes ; $e$, funnel; $f$, mantle.
B. Internal shell of ditto.
C. Fossil cuttle-fish partly restored (Belemnoteuthis antiqua). a, arms; $b$, eyes; $c$, nantle; $d$, ink-bag ; e, phragmacone.
D. Ink-bag of ditto.
E. Fossil cuttle-fish (Plesioteuthis prisca).
F. Shell of ditto.

\section{PLATE XLIX}

Plate xlix. illustrates longitudinal, radiating, and transverse cleavage and expansion in relation to the organs of locomotion.

FIG, 1. - Skeleton and outline of the deer (Cervus eluphes), (after Pander and D'Alton). Slows longitudinal and transverse cleavage of limbs and the small feet adapted for land transit. In this fleetest of animals the angles made by the bones of the limbs with each other and with the shoulder (scapula) and hip (pelvis) are comparatively acute, and afford facilities to the muscles for suddenly shortening and elongating the limbs in rapid progression. a, Angle made by the femur with the innominate bone (pelvis); $b$, angle made by the tibia and fibula with the fenur; c, angle made by the cannon bone with the tibia and fibula; $d$, angle made by the phalanges with the cannon bone; $e$, angle formed by the humerus with the scapula; $f$, angle made by the radius and ulna with the humerus; $y$, angle made by the camnon bone with the radius and ulna; $h$, angle made by the plialanges with the cannon bone.

FIG. 2.--Thresher or fox shark (Curcharias vulpes). Shows large pectoral and candal fins, indicating great speed in swimming. The pectoral fins are true wings as regards construction; that is, they are triangular in shape, thick and semi-rigid at the root and along the anterior margins, and thin and elastic at the tips and along the posterior margins. When made to vibrate in a vertical direction they act as powerful propellers. The huge tail, which is the principal swimning organ, is sinilarly constructed. Photographed for the Author by E. Millar.

Fig. 3.-Feet of swan and grelse. Show longitudinal cleavage and radiation in the bones of the feet.

A. Legs and feet of the swan (Cygnus), with the latter closed and spread out as when making the non-effective (a) and effective stroke $(b)$ in swimming. The closing and opening of the feet enable the bird alternately to evade and seize the water very effectually. Drawn from nature for the Autlior by C. Berjean.

B. Foot of the grehe (Podiceps), (after Dallas). In this foot each toe is provided with a swimming membrane, the membrane being closed when the loot is flexed and making the non-effective stroke, and expanded when the foot is extended and making the effective stroke.

FrG. 4.- Hind extremities or posterior flipper of the elephant seal (Macrorhinus leoninus) in the closed and expanded condition. (Chullenger Reports, vol. xxvi.) Show cleavage and radiation of bones of feet. The Hippers in swimming are alternately opened and closed, as also happens in the tail of the fish when making the non-effective and effective strokes. Each flipper consists of five toes supporting a flexible swimming membrane. Compare with A and B of Fig. 3 of this Plate.

Fri. 5.- Heterocercal or unsymmetrical tail of the sturgeon (Acipenser sturio), (after Giinther). Shows division and radiation resulting in a leautifully-graduated swimming organ. The terminal, tapering portion of the spinal column occupies the ulyer lobe of the tail, and the tail is thick at the root and thin at the free margin $(f, g, h)$. It is also thicker and stronger aloove $(a, b, c)$, and thinner and more flexible below $(d, e)$. It bears a considerable resemblance to a wing. In reality, candal and other fins, flippers, and wings are constructed on a common type, and discharge essentially similar functions.

FIG. 6.-Skeleton and outline of the ostrich (Struthio camelus), (after Dallas), Show longitudinal and transverse cleavage in legs, wings, vertelral column, and rilss. Remarkable for its large, powerful legs, small feet, and rudinentary wings-conditions necessary for rapid land transit. What is said of the limbs of the deer (Fig. 1 of this Plate) applies to the ostrich. $a$, Angle made by the femur with the pelvis; $b$, angle made by the tibia and fibula with femur; $c$, angle made by the tarso-metatarsal bone with the tibia and fibula; $d$, angle made by the lones of the feet with the tarso-metatarsal bone; $e, f$, bones of aborted wing making nearly right angles with each other. 
PLATE XLIN
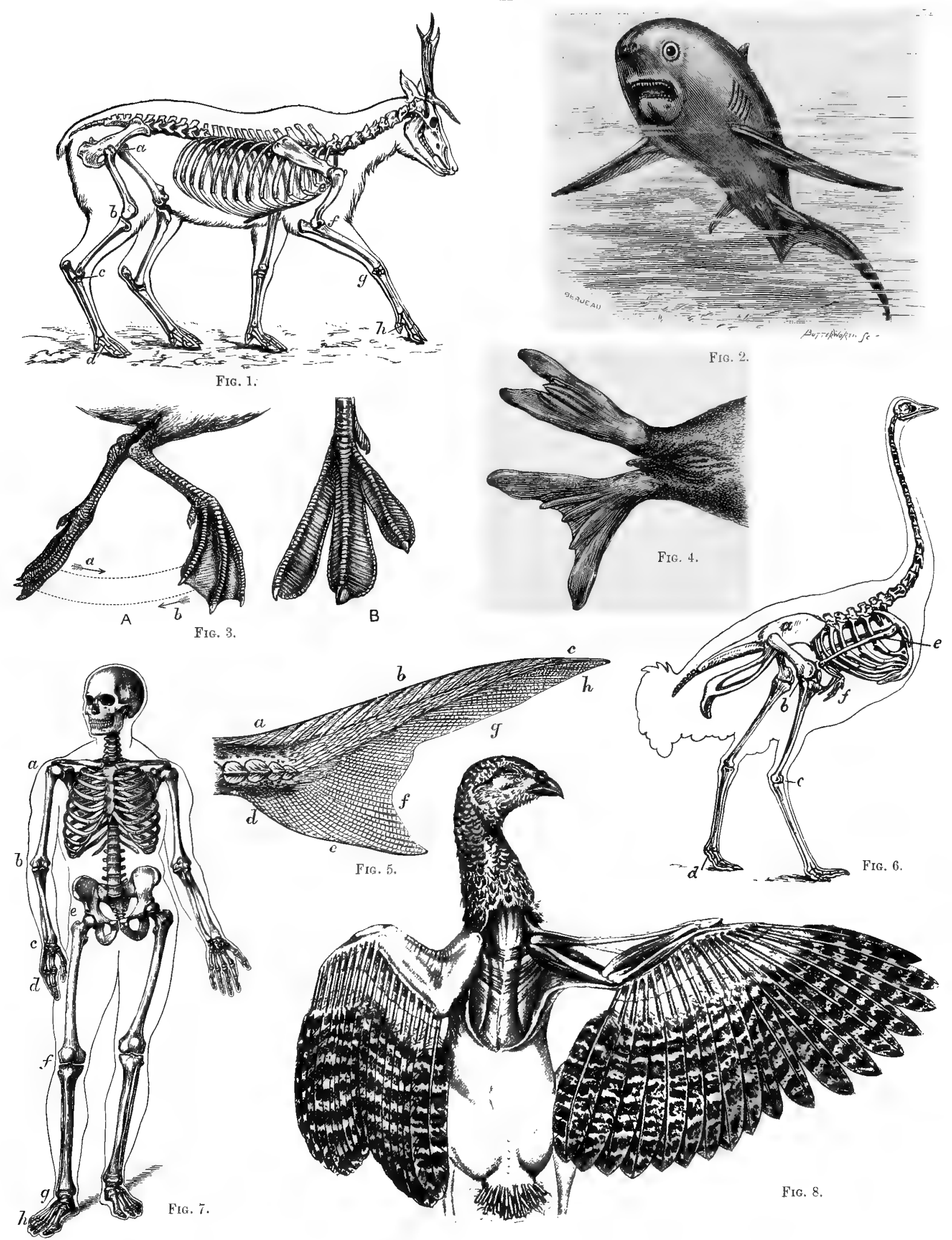

FIG. 8. 


\section{PLATE XLIX (continued)}

FIG. 7.--Skeleton and outline of man (Homo sapiens). Shows longitudinal and transverse cleavage in arms and legs; radiating cleavage in the hands and feet; and transverse cleavage in the vertebral column and ribs. $a$, Shoulder joint (universal); $b$, elluow joint (spiral and hinge); $c$, wrist joint (universal in a way); $d$, hand joiıts (spiral and hinge); $a, b$, humerus; $b, c$, radius and ulna plaited and showing spirality $; c, d$, hones of wrist and hand; $e$, hip joint (universal); $f$, knee joint (spiral and hinge); $g$, ankle joint (universal in a way); $h$, foot joints (spiral and hinge); $e, f$, femur; $f, g$, tibia and fibula; $g$, $h$, bones of foot. The spiral configuration of the bones forming the human skeleton is given at Plate xix., Figs. 3 and 4 ; Plate xx., Figs. 2 and 4 ; and Plate xxi., Figs. 1 and 2. The spiral configuration of the bones of the elephant is seen in Plate xx., Fig. 3.

Frg. 8.-Dissection and drawing by the Author of the wings of the pheasant (Pluasianus colchicus). Shows extreme cleavage and radiation and large superficial area of the wings. Compare with Figs. I and 2 of this Plate. The right wing is extended, and shows the muscles of the shoulder, the muscles and elastic structures of the wing, and the arrangement of the primary and secondary feathers in the wing. The left wing is flexed, and displays the skin and elastic structures which assist in folding the wing as corrugated and puckered. For details of the anatomy of the wing see further on.

\section{PLATE L}

Plate 1. illustrates longitudinal cleavage and radiation in the travelling organs of animals (caudal and other fins, flippers, feet, wings, \&c.), and how intimately these fundamental cleavage arrangements are associated with locomotion in all its forms.

FIG. 1.- Shows a series of original drawings made for the Author by C. Berjeau in 1867 (vide Trans. Linn. Soc. vol. xxvi.). These drawings, taken from nature, reveal the important fact that the travelling organs of animals increase in size according as the medium traversed becomes more and more tenuous and affords less and less support. Thus the feet of the deer, adapted for land transit, are smaller than the feet of the bird, otter, frog, platypus, seal, and turtle adapted for swimming; these, again, being smaller than the pseudo-wings of the flying fish, flying lizard, and flying lemur ; the latter being, in turn, smaller than the wings of insects, birds, and bats adapted for flight. The subjects are arranged with a view to comparison and to facilitate reference.

A. Small foot of deer adapted to land transit.

B. Webbed foot of otter slightly expanded. Adapted for land and water transit.

C. Webbed foot of frog, considerably expanded. Adapted for land and water ; chiefly water.

D. Webbed foot of platypus (duck mole), greatly expanded. Adapted chiefly for water.

E. Webbed hind foot of seal, greatly expanded. Adapted mainly for water.

F. Wings of swallow, enormously expauded. Adapted solely for aërial transit. Compare with foot of deer (A), adapted solely for land trausit.

G. Flippers of turtle, greatly expanded. Adapted almost exclusively for water. $a, b$, Anterior thick margin; $c$, posterior thin margin of fore flipper ; $d, e$, posterior thin margin of hind flipper.

H. Tail and caudal fin of fish, largely expanded. Adapted wholly for water transit. The fish moves its tail laterally and with a spiral figure-of-8 movement; the vibration occurring on either side of the mesial plane $(a)$.

I. Tail and caudal tin of manatee (sea cow), greatly expanded as in fish. Adapted wholly for water transit. The tail moves vertically on either side of the mesial line $(a)$, and makes a figure-of-8 track on the water, as happens in the fish.

J. Portion of body and swimming wing and foot of penguin. The swimming wing is much smaller and stiffer than the flying wing. It twists and untwists in action and forms a beautiful screw $(a, b, c)$. The swimming foot $(d)$ is webbed, and larger than the unwebbed terrestrial bird's foot.

K. Flying-fish with greatly enlarged pectoral fin, which forms a psendo-wing. It makes short flights, and connects the travelling organs adapted for the water and the air.

L. Flying lizard with flying membrane (pseudo-wings) supported by its ribs, It glides from heights over considerable distances.

M. The bat with its wings fully extended: the increase in the size of the travelling orgins (wings) is excessive. Compare with the foot of the deer $(A)$, the tail of the fish $(\mathrm{H})$, the flipper of the turtle $(\mathrm{G})$, and the flipper of the seal (E). The bat is adapted exclusively for aërial transit.

N. Flying lemur with fying membrane supported by boly, extremities and tail. The flying lizard (L) and flying lemur (N) connect the travelling organs adapted for the land and air. The increase in the extent of the surfaces engaged in transit is very con-
siderable.

FIr: 2.-The tail of the herring (Clupea harengus), drawn hy C. Berjeau for the Author, in the closed, semi-closed, and fully expanded condition. Shows longiturlinal cleavage and radiation. The tail is the chief swimming organ, and no better eximple can be given of the utility of cleavage and radiation for the purposes of progression. The tail divaricates when making the figure-of- 8 movements in swimming, and so alternately seizes and lets go the water on which the progress of the fish depends. The tail is a finelygraduated, flexible, elastic structure.

FIG. 3.- The tail of the mackerel (Scomber scomber), drawn by C. Berjeau for the Author, in the closed and expanded condition. Displays the same characteristics as the tail of the herring (Fig. 2).

Fic. 4. - The heterocercal tail of a shark with the caudal portion of the vertebral column terminating in the upper or major lobe. Shows longitudinal and radiating cleavage. The organ, as in the fish, is finely graduated, flexible, and elastic. It tapers from the root in every direction. Drawn by C. Berjeau from a specimen in the museum of the Author. 


\section{LONGITUDINAL AND RADIATING CLEAVAGE}

PLATE L
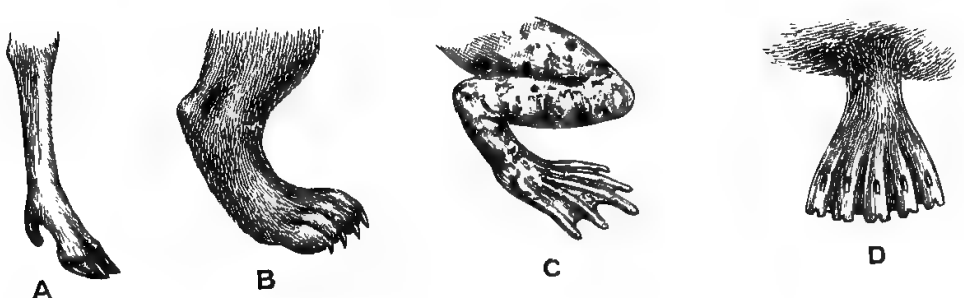

c

D
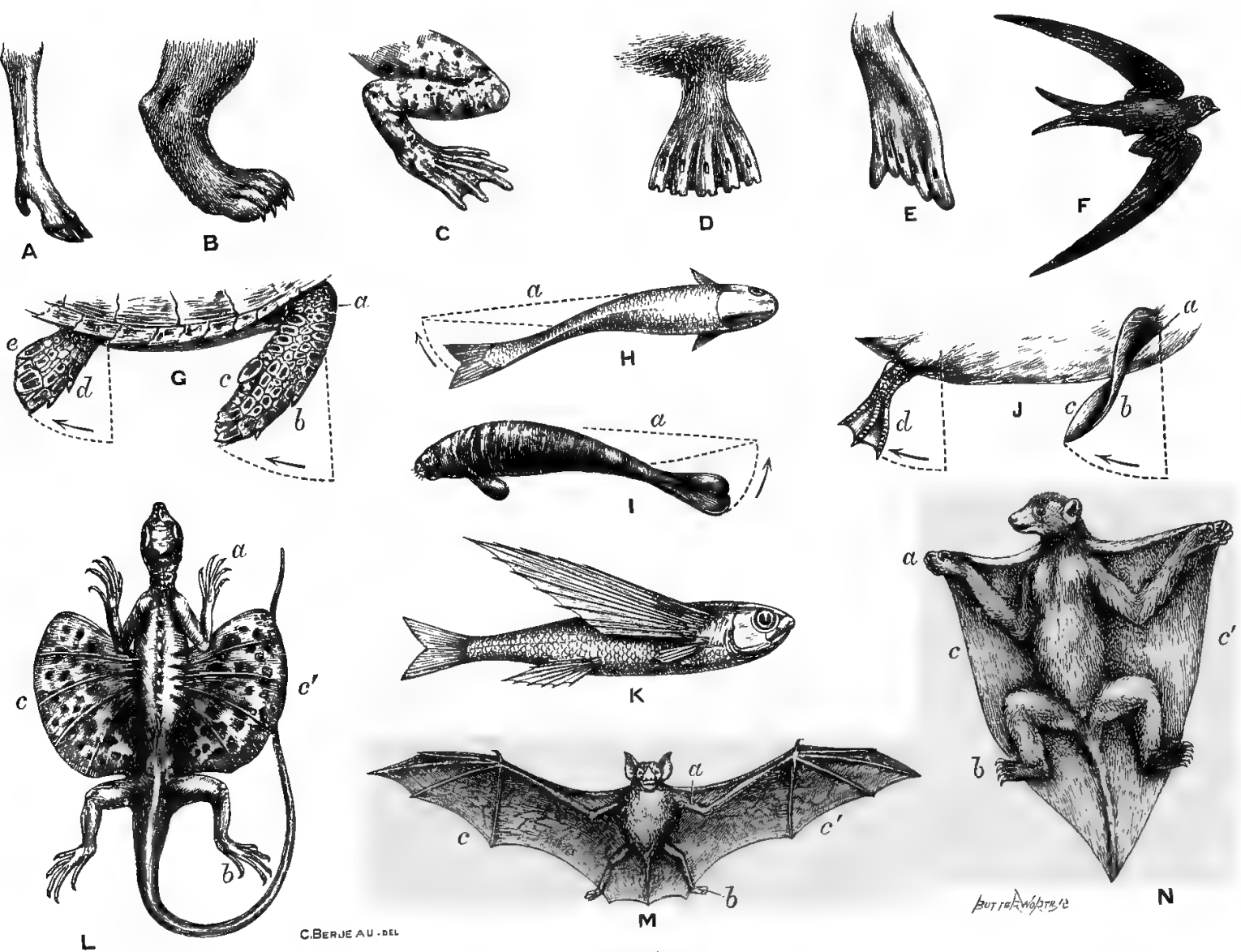

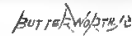

N

Fig. 1.
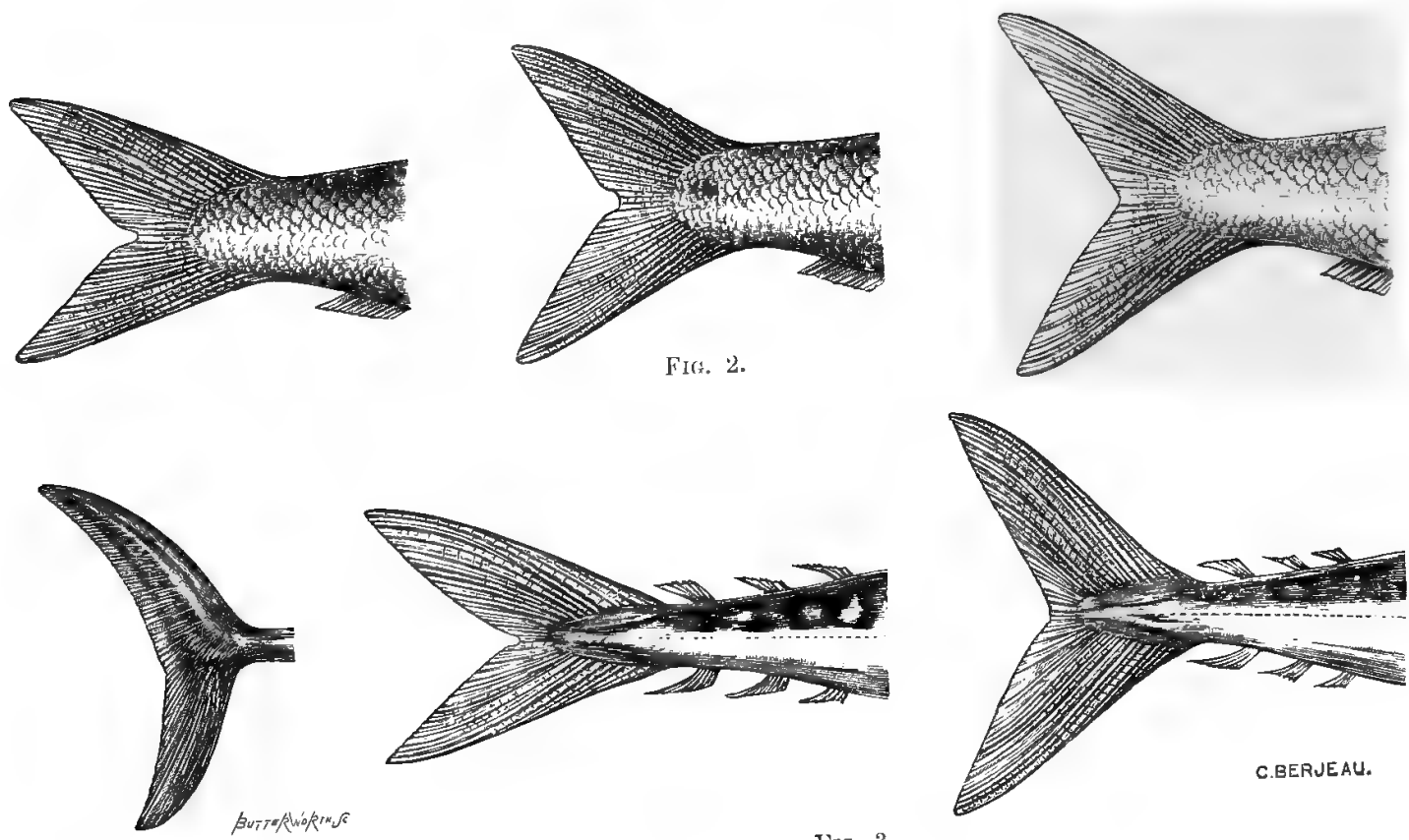

FIG. 4.

FIG. 3 ,

VOL. I. 
Plate li. illustrates longitudinal, radiating, and transverse cleavage as seen in the tail of the manatee and dog-fish, in the wing and body of the insect, and in the wing of the bird and bat. These cleavages and radiating expansions are necessary for the purposes of locomotion in the water and the air.

FIG. 1.-Various views of the swimming tail of the manatee or sea-cow (Manatus americanus) as drawn by C. Berjeau, for Dr. James Murie, from a fresh specimen. The tail of the manatee is symmetrical, and more Heslyy than that of the fish. In swimming it is made to vibrate vertically, as in the whale and other sea mammals, such as the rhytina, dugong, porpoise, \&c. The vertical movements enable the air-breathing sea mammals readily to reach the surface, which is a necessity of their being. The arrangenent is not accidental, and affords a proof of design. The movement differs from that of the tail of the fish, which is made to vibrate horizontally or laterally.

A. The tail of young male as seen from the side and partly dissected. $a$, Skin; $b$, vertebral columu, showing transverse segmentation; $c$, right lobe of tail with skin in situ; $d$, left lobe of tail with skin removed, showing radiating fibrous expansion of tail; $e$, sacro-coccygeus and infra-coccygens muscles, divided obliquely.

B. Lateral view of tail. Shows symmetrical nature of the organ, the lower part of the body tapering to a point and terminating in the tail. The organ is flexible and elastic, as in the fish, and is a powerful propeller.

C. Under surface of the tail. It lacks the conical ridge seen on the upper surface (B).

FIG. 2.-Heterocercal or non-symmetrical tail of the large, male dog-fish as drawn by the Author from specimen in his possession. This form of tail has an eccentric movenent which differs from that of symmetrical tails. Shows longitudinal and radiating cleavage in caudal fin and transverse cleavage in terminal portion of vertebral column; this occupying the superior or major lube. The tail is fimely graduated in all directions and a powerful propeller, the dog-fish and shark families being the greyhounds of the ocean. The non-symmetrical and eccentric action of the tail increases the degree of rotation in the body in swimming, and enables these voracious creatures, whose mouths are situated in the ventral surface, to turn readily on their side or back when seizing their prey. This affords a good example of design. $r$, Tapering terminal portion of spinal column; $a, b, c$, superior utargin of tail; $d$, $e$, inferior margin; $f, g, h$, free margin.

F1G. 3.- Right wing of male pheasant (Phasiamus colchicus), seen from above. Drawn by C. Berjeau from a specimen in the Author's museun. Shows radiating cleavage to perfection. The wing, like all others, is triangular in shape, and is flexible and elastic. It tapers from the root towards the tip and from the anterior $(a, b, c)$ towards the posterior $(d, e, f)$ maruin. It is a carefully graduated structure, the root being stronger than the tip and the anterior than the posterior margin. It forms the most complicated and perfect travelling organ known, and is a marvel of design. Each feather is a masterpiece in itself. 'The feathers are divided into three chief sets-the primary or rowing feathers, nine in number $(d)$, the secondary feathers $(e)$, and the tertiaries $(f)$. The main covering feathers of the wing are seen between $a, b, g$. The wing of all the travelling organs is the largest as compared with the body. It has literally to tread the air, this affording very little support when contrasted with the water and the earth. It has, moreover, to support as well as propel. Viewed from every point it is one of nature's triumphs.

FIG. 4.-Body and right wing of bat (Vespertilio murinus), seen from above. Drawn by C. Berjeau from a specimen in the Author's museum. The bat is the only mammal which flies. Its wings present extraordinary modifications of the anterior extremities. They are outstanding examples of adaptation of means to ends, and afford a unique example of design. Shows division and radiation in the anterior and posterior extrenities, body, and tail, which support the flying membrane. This, as in the insect (Fig. 5 of this Plate), is not broken up, but continuous. In the bird (Fig. 3 of this Plate) the fiying membrane is conposed of feathers which can be separated. The wing of the bat, as in the bird, is flexible, elastic, and finely graduated. It forms a perfect organ of fight. ", Anterior, thick, semi-rigid maryin of wing; $b$, posterior, thin, highly elastic margin.

FIG. 5.-Body, right wing, and wing case of large beetle (Goliathus micans), seen from above. J)rawn by C. Berjeau from specimen in the Author's museum. Shows radiating and transverse division in the wing and body. The wing greatly resembles that of the bat in general structure, consisting as it does of a continuous menbrane supported by a radiating framework of elastic materials (vanes). The wing is beautifully graduated, being thicker at the root and along the anterior margin, where it is jointed (a), than at the tip and along the posterior margin $(b)$. It is Hexible and elastic in all its parts, as in other wings. It can be folded on the back of the insect when not in use, and protected by the wing cover or elytron $(c)$.

FIG, 6.-Right wing of the albatross (Diomedea exulans), seen from above. Drawn by the Author from a specimen in his possession. This forms the longest and narrowest of all wings. The wing here represented is 6 feet long hy 9 inches wide. It resembles that of other birds if allowance be made for its greater length and narrowness. $a, b, c$, Anterior margin of wing; $d$, $c, f$, posterior margin of wing. The primary or rowing feathers, nine in number, are seen at $d$; the secondary feathers at $e$; and the tertiary feathers at $f$. While all wings are triangular in shape and carefully graduated, they are shorter or longer according to requirement. Compase the wing of the pheasant (Fig. 3 of this Plate) and that of the albatross (Fig. 6 of this Plate), and both with that of the bat (Fig. 4 of this Plate) and that of the insect (Fig. 5 of this Plate). The long wings are adapted for slow movements, the short ones for quick movements.

\section{PLATE LII}

Plate lii. illustrates the manner in which the travelling organs, originally formed by budding and by longitudinal, radiating, and transverse cleavages, are modified in animals widely divergent, to meet the requirements of transit in water and air. All the swimming animals which live in the water conform, as a rule, to the fish shape; the swimming organs, whether fins, flippers, wings or feet, being similarly constructed, and acting on a common principle.

Fig. 1.-The sea-bear (Otaria hookeri). Drawn by O. Berjean from life. Shows greatly modified anterior and posterior extrenities; the former being converted into thippers which greatly resemble wings, the latter being expanded like fish tails. The animal can fly through the water at a great speed with its Hippers, or it can employ its expanded, webbed hind extremities as fish tails, and swim after the manner of the fish. The Author has frequently witnessed both kinds of progression. The specially modified limbs are
plainly the outcome of design.

FIa. 2.- The seal (Phoca fotida). Drawn by C. Berjedu from life. The extremities are smaller and still more modified than in the sea-bear. The general shape of the body, moreover, is more fish-like, In both the sea-bear and seal the anterior extremities 
LONGITUDINAL, RADIATING, AND TRANSVERSE CLEAVAGE ${ }_{3}$

PLATE LI
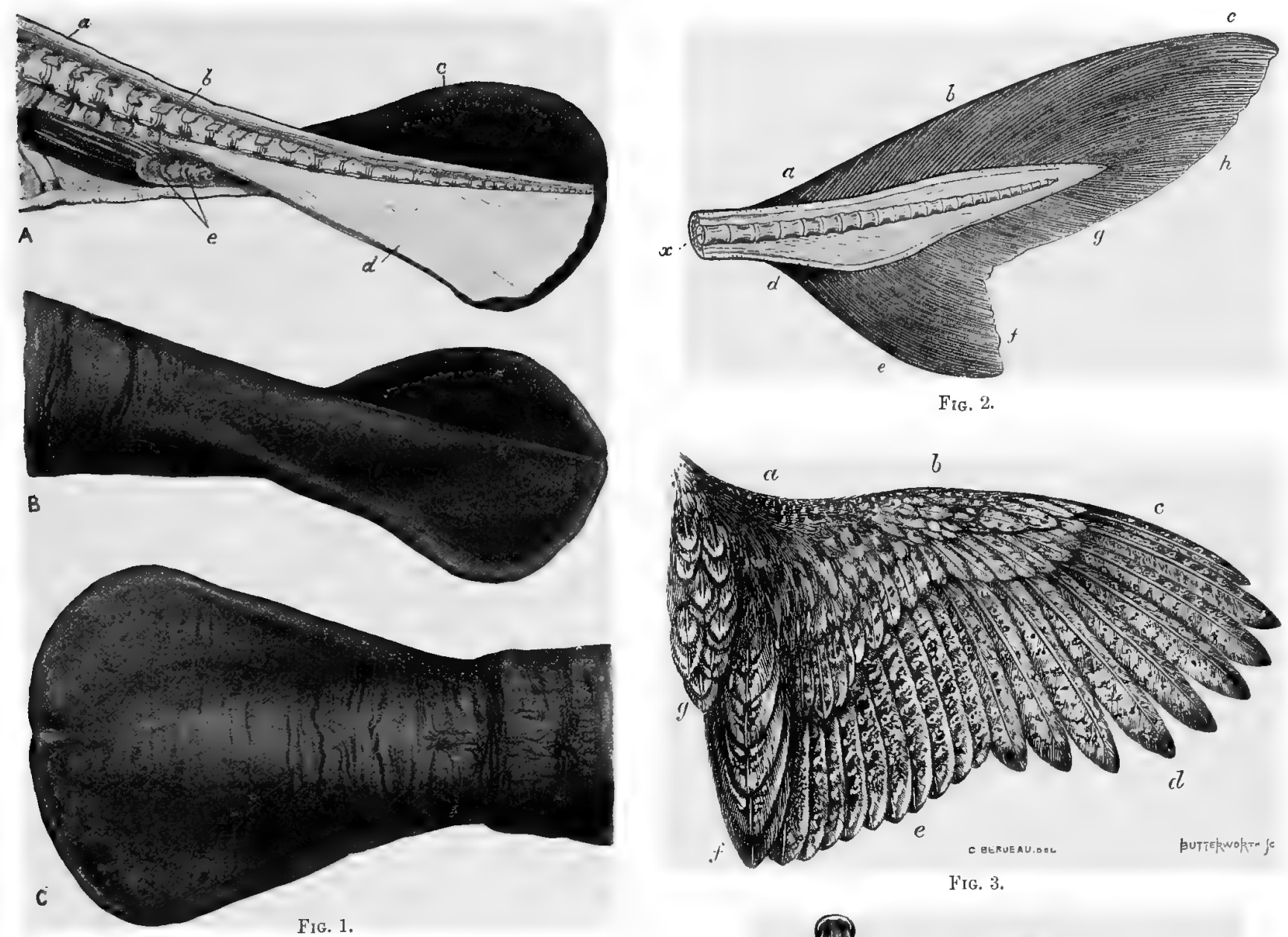

Fig. 2.
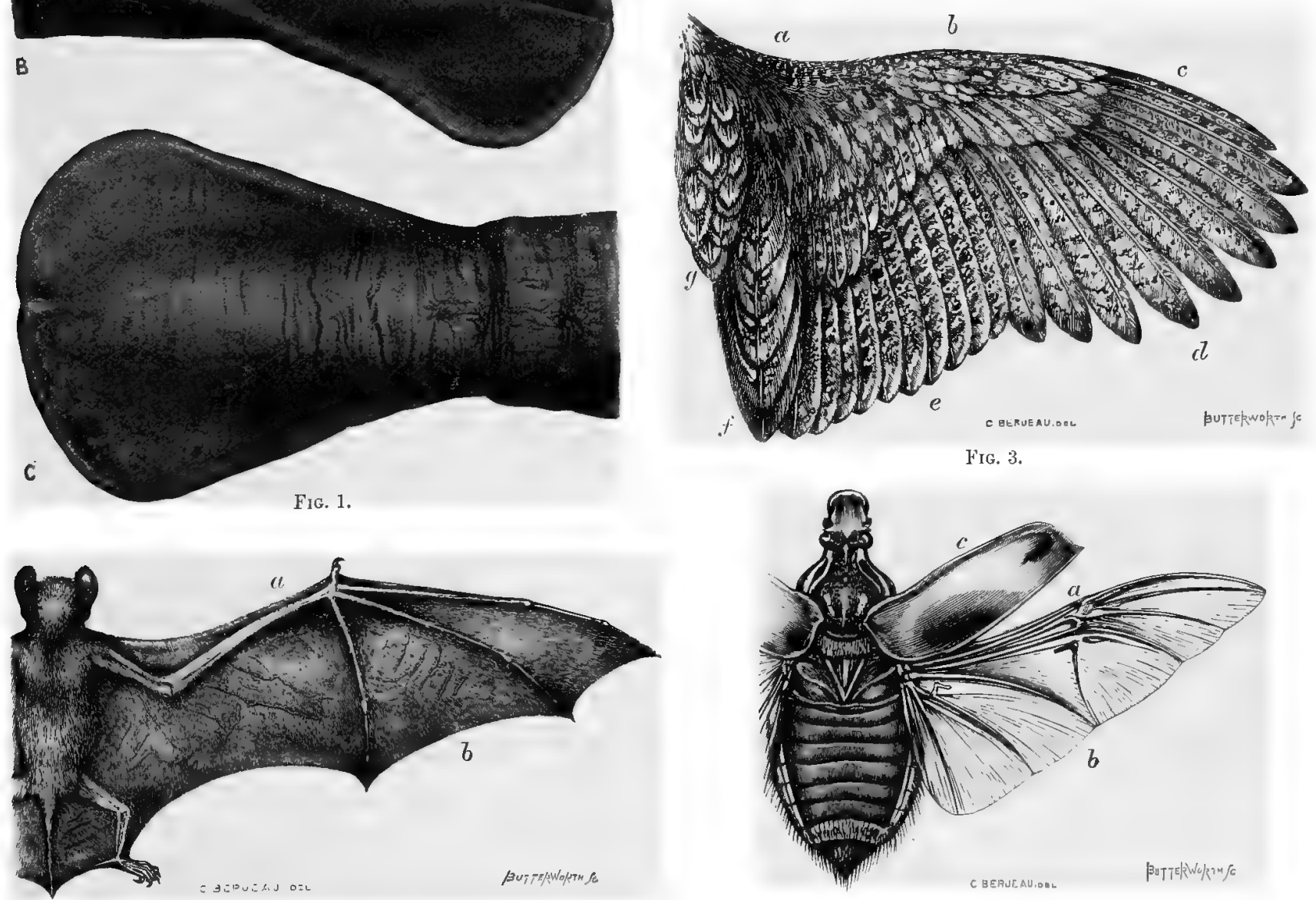

FIG. 4.

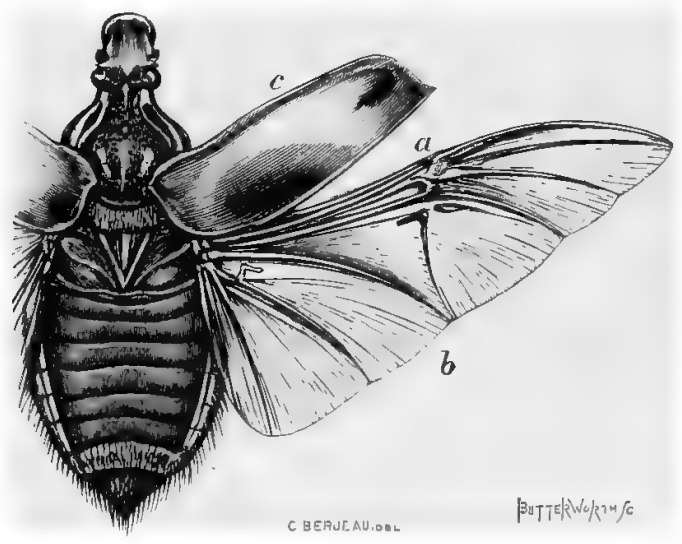

FIG. 5.

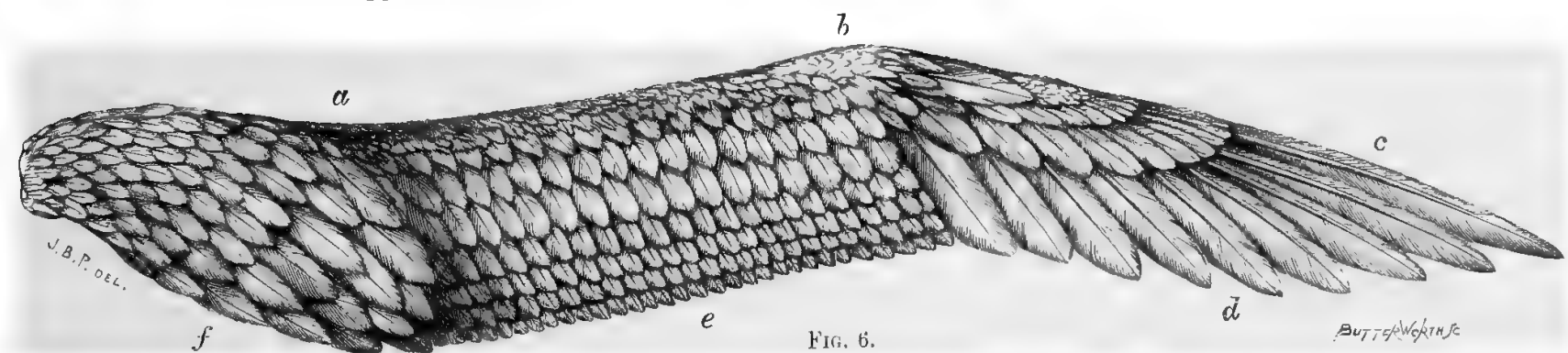




\section{PLATE LII (continued)}

exhilit five digits, and the posterior extremities five toes, with swimming membranes between. The seal swims almost exclusively by means of its expanded, webbed, hind extremities or flippers, which it lashes vigorously from side to side as a fish does its tail. The hind flippers open and close during the effective and non-effective strokes, and confer on the trunk in swimming aslight degree of rotation. The anterior extremities or flippers are employed chiefly in balancing and turning, and in seeking the surface of the water for breathing purposes.

Fig. 3. The manatee or sea-cow (Mamatus americanus). Drawn from a fresh specimen by C. Berjeau. The anterior extremities or flippers alone persist; the posterior ones leing replaced by a broadly expanded tail. The tail resembles that of the fish, but in swimining it is made to vibrate from above downwards or vertically, instead of laterally. This arrangement enales the animal to reach the surface of the water quickly for breathing purposes and to disappear suddenly if in danger. The anterior extremities or flippers assist in these movements and in turning. The manatee is more fish-like in appearance than either the seal or sea-Tyear. Design and adaptation can alone account for the slape and movements of the manatee.

FIG. 4.-The porpoise (Phocæna communis). Drawn from a fresh specimen by C. Berjeau. This sea mammal altogether resembles fish in general appearance. The anterior thippers can no longer be recognised as arms. They are so diminished in size and so altered in shape as to resemble the pectoral fins of fishes. In like manner the posterior flippers are supplanted by a delicately-outlined, powerful swimming tail. The porpoise is one of the best swimmers known. The tail is made to vibrate vertically, and makes figure-of- 8 movements. The same is true of the manatee, though to a less extent. In both cases the tail in swimming twists and untwists after the manner of a screw. The swimming adaptations observable in the sea-bear, seal, and manatee culminate in the porpoise. Design has produced a perfect swimming form.

FIG. 5. - The salmon trout (Salno trutta). Drawn from a fresh specimen by C. Berjeall. This may be taken as a typical fish. It has pectoral, dorsal, ventral, and anal fins, and a finely-formed tail. The fins and tail are carefully-graduated structures, and supply good examples of longitudinal and radiating cleavage. The body is specially designed to diminish resistance when it is forced through the water, and the tail and lower third of the body combined form a powerful propeller. The stroke is delivered laterally, and the movements made by the tail are figure-of-8, slightly rotatory screw movements. In the fish, the anterior and posterior extrenities may be sajd to have entirely disappeared, their places leing taken by the pectoral and ventral fins. The fins in fishes assist in balancing and turning, but, in the majority of cases, take little or no part in propulsion.

FIs. 6-The flying-fish (Exocotus robustus). Drawn from a recent specimen by C. Berjeau. The flying-fish forms a connecting link between the water and the air: it swims well, and takes considerable flights. It is very amply supplied with fins. The pectoral fins are as large relatively to the size and weight of the body as the wings of many insects and birds. They are true wings as regards shape and structure. They and the other fins furnish a striking example of longitudinal and radiating cleavage. The flying-fish conclusively proves that in order both to swim and fly special arrangements must be made; what are virtually true wings must be added to the swimming tail. Here again design and aclaptation come in. Neither the wings or tail are chance products. Similar remarks apply to all organs of locomotion, however fashioned and however applied. They are in every case specially designed structures.

Fig. 7.-The little auk (Aptenodytes minor, Linn.). Drawn from living specimen by C. Berjeau. In this bird the flying wings are dwarfed and employed as swimming and diving crgans, with or without the feet. They are triangular in shape and graduated like other wings, their small size and want of feathers rendering them useless for flying in the air. They are flexible and elastic, but stiffer than ordinary wings; a modification necessitated by the greater densily of water as compared with air. They are made to vibrate like other wings and twist and untwist during their action and make figure-of- 8 screw novements. The flight of the bird under water is astonishingly rapid and graceful, as the Author can testify from careful olservation. The modified wings of the little auk furnish another example of design.

FIG. 8. - The triton (Triton cristatus). Drawn from the life by C. Berjeau. This curious creature is adapted for land and water transit, and is accordingly furnished with travelling extremities and a large, powerful, swimming tail. Similar arrangements obtain in the crocodile. The triton connects the land and water as the flying-fish connects the water and the air in the matter of transit. In both cases special modifications are required in the locomotory organs. The means and the ends are alike proofs of design.

\section{PLATE LIII}

Plate liii. illustrates the variety in shape and increase in size of the travelling organs required for land, water, and air transit respectively: how fishes in swimming, birds in flying, and quadrupeds and bipeds in walling, make double or figure-of-8 curves as explained by the Author in 1867, 1868, and 1870. ${ }^{1}$

FIG, 1.--Shows the extrene modifications in the travelling organs required for land, water, and air.

A. Chillingham bull (Bos scoticus). Reveals ponflerous body and the small extremities and feet adapted for land transit; $t$, indicate the doulle curves made by the two anterior extremities, and $r, s$, those made by the posterior extremities in walking. As the right fore and left hind legs move together to form one step, and the left fore and right hind legs move together to form a secomr step, the donble curves form a figure-of- 8 .

B. 'The hawk's-bill turtle (Eretmochelys imbricuta). Displays large modified anterior and posterior extremities, adapted for' water transit. Both the anterior and posterior extremities, especially the former, are constructed on the wing model, being triangnlar in shape, and thick and semi-rigid at the roots and along the anterior margins, and thin and elastic at the tips and along the posterior margins. 'They act as wings in swinming. 'The travelling organs of the turtle are relatively much larger than those of the Chillinghan bull (A)

C. The bat (Phyllorhina gracilis, Peters). This anoulous and yuaint creature exhibits a still greater degree of modification in the anterior and posterior extremities to meet the exigencies of aërial transit. Here the body is dwarfed and the anterior extremities enormously enlarged to produce the necessary framework to support, in conjunction with the posterior extremities and tail, the widely expanded wings. It would be difficult to adince more perfect examples of design than are furnished by $\mathrm{A}$, $\mathrm{B}$, and $\mathrm{C}$, respectively.

1 "On the Various Modes of Flight in Rulation to Aeronautics" (Proc. Roy. Inst. Gt. Britain); "On the Mechanical Appliances by which

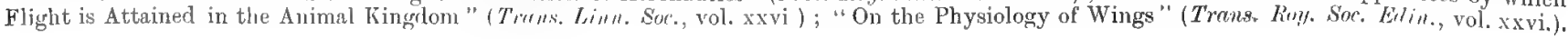


PLATE LII

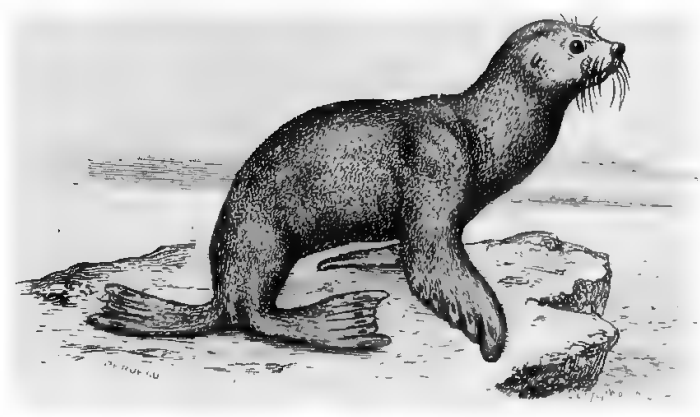

Fig. 1.

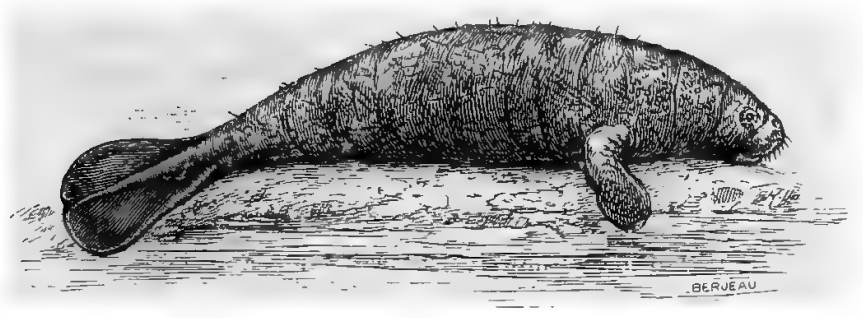

FIG. 3.

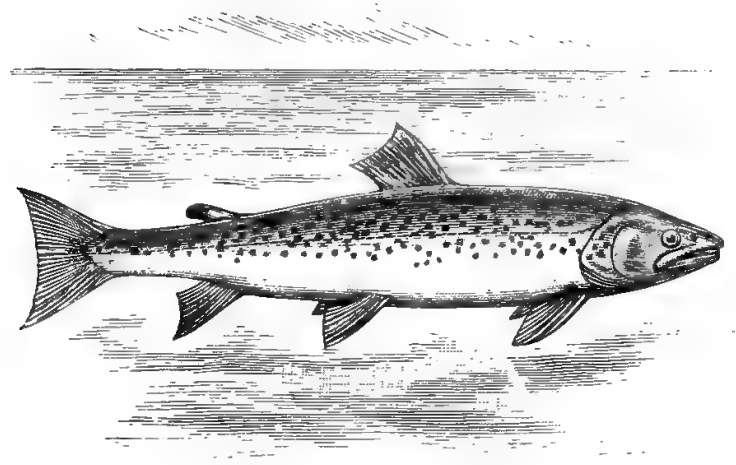

FIG. 5.

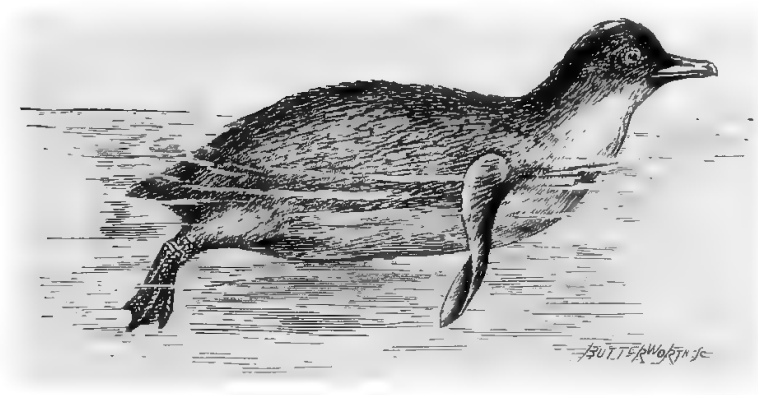

Fig. 7.

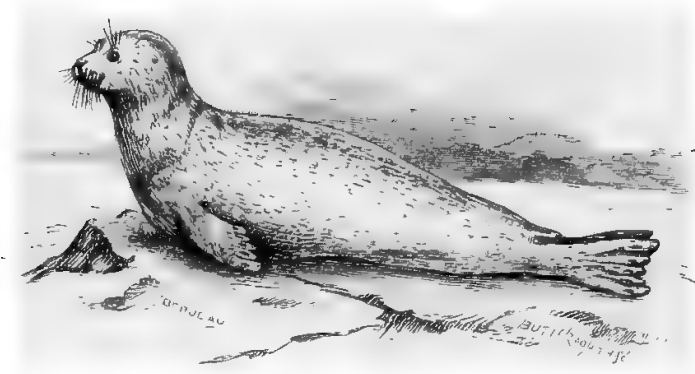

FIG. 2.

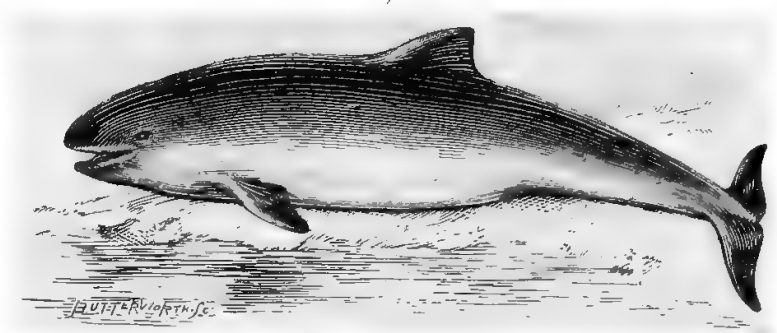

F1G. 4 .

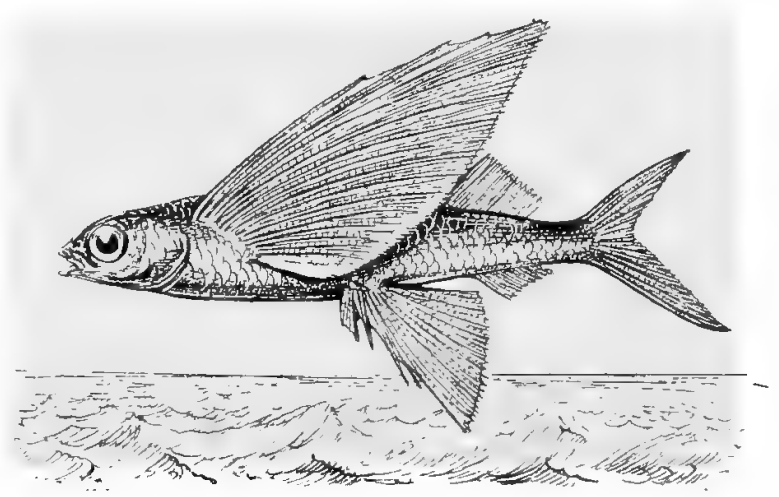

Fig. 6.

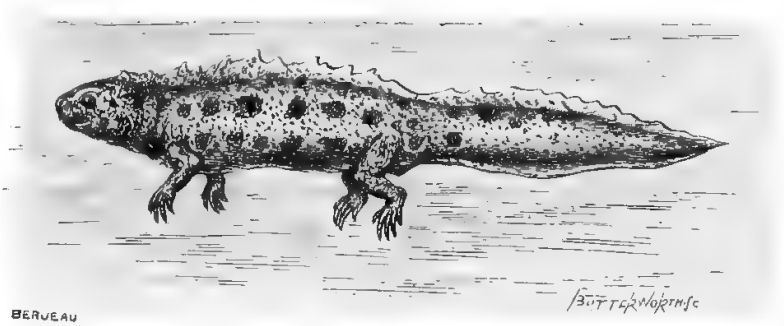

FIG. 8. 


\section{PLATE LIII (continued)}

Small feet suffice for support and propulsion when the surface trod, which supplies the fulcrum for the travelling organs, is unyielding. Larger travelling organs are recuined for the water, which furuishes a yielding fulcrum; still larger travelling surfaces being required for the air, where the fulcrum is still more mobile and treacherous (the Author).

FiG. 2.-Photographs of men walking at a brisk pace, seen from before and behind. Shuw the double figure-of- 8 curves made by the superior and inferior extremities, and the double twisting movements which oceur at the shoulders and hips in walking. The left arm (a) and right leg (b) advance together in curves (see darts) to make one step; the right arm (c) and left leg (d) advancing together in curves (see darts) to make a second step. The same movements are seen at Fig. 4 of this Plate, where the interrupted line represents the curves made ly the arms in walking; the continuous line representing those made by the legs. The twisting movements which oceur at the shoulders and hips in walking are seen at $k, l$, and $m, n$ (see darts); those made by the legs and arms at $h, i$, and $j, g$. In the central figures the extremities are in the act of reversing (the Author).

Frg. 3.--The swimming of the sturgeon (Acipenser sturio), as depicted by the Author in 1867. Shows the double figure-of-8 curves macle by the body and tail of the fish as it progresses through the water. The tail makes similar curves. "i, $b$, Line of progress; $c$, candal curve; $d$, cephalic curve. The candal and cephalic curves are complemental and afford fulcra for each other when the effective strokes are made.

FIG. 4.-Double or figure-of-8 curves made by the arms and legs in walking, as depicted by the Author in 1867. The interrupted line represents the curves made by the arms; the continuous line the complemental curves made by the legs. The right leg and left arm advance together to make one step; the left leg and right arm advancing together to make a second step. The legs and arms move diagonally, and the curves made by them eross and produce figures-of-8. The dingonal movements of the limis necessitate twisting movements at the hips and shoulders.

Frg. 5.-Blow-fly with its wings at rest and in motion, as delineated by the Author in 1867.

A. Blow-fly at rest.

13. Blow-fly with its body fixed and its wings in motion (captive flight). In captive flight the wings twist and untwist as they hurry to and $f r o$ and make double or figure-of-8 curves. The tips of the wings describe a tigure-of- 8 trajectory in space. In free flight the figure-of- 8 is opened out to form first a spiral and then a waved track, as shown at Figs. 6 and 9 of this Plate.

FI ${ }^{\prime}$. 6. - Spiril and waved track made by the wings of the insect in free flight. The track in question is indicated by the arrows marked $a, b, c, d, e, f, g, h, i, j, k, l, m, n, o, p, q, r, s, t ;$ the recoil obtained from the air is indicated by the same letters with a dash prefixed (the Author', 1867 and 1870).

Fig. 7. - The flight of the heron (Avdea cinerea), from a photograph by the Author. Shows very large wings and snall boà, as in the bat (Fig 1, C, of this Plate). The wings of the heron and all other birds make figure-of-8, spiral, and waved tracks in Hight, as shown at Figs. 6, 9, and 10 of this Plate.

Fig. 8. - Double spiral curves made by the fect of a bird in swimming, as figured by the Author in 1867 . The arrow a, represents the curve made by the right foot during jts back or effective stroke; the arrow b, the curve made at the same time by the left foot during its forward or non-effective stroke. The right and left legs and feet move alternately, and the feet are expanded during the effective strokes and closed during the non-effective strokes. The swan occasionally moves both feet together.

Fic. 9 - Spiral and waved track made by the wing of the bird in free flight, as first explained by the Author. The spiral and waved track is indicated by the arrows marked $a, b, r, d, e, f, g, h, i, j, k, l, m, n, o, p, q, i, s, t$ (the Author, 1867 and $i 870$ ).

FIG. 10.-Waved track made by the wing of the bird in free flight, as figured by the Author in 1867. $a, b$, Represent the line of flight. The letter's from $c$ to $m$ inclusive, represent the angles nade by the wing with the horizon during the down and up strokes. This wing flies forward as a true kite during both the down and up strokes (aide arrows).

\section{PLATE LIV}

Plate liv. illustrates the double, complemental, figure-of- 8 curves made by the extremities of quadrupeds and bipeds in walking; how similar curves are made by the wing in flying; and how there are good grounds for believing that these curves are fundamental, and first make their appearance in the sinuous movements of spermatozoa, the serpent, and other creeping things.

F1f. 1.- Trotting horse drawn by the Author to show the double figure-of- 8 curves made by the limls. $t, r$, Donble curves made by the right fore and left hind legs; $u, s$, double and complementary curves made by the left fore and right hind legs. The double complementary curves when superposed produce an elongated figure-of- 8 . 'The double curves are caused by diagonal movements of the fore and hind limbs of opposite sides, and by twisting movements occurring at the shoulders and hips of the horse.

FIt. 2.-The serpent as seen crawling, incorrectly and correctly pourtrayed (after Günther).

A. The serpent in this figure is wrongly represented as making simnous vertical movements; the body being supported at three points only.

$B$. The serpent in this tigure is rightly represented as making sinuous lateral movements; the body being more or less in contact with the ground in its entire length. At $", b$, the serpent is glining forward in double, complemental, figure-of-8 reversing curves ; at $c, d$, it is stretched out and at rest. In 1867 the Author explained that the travelling organs and extremities in the higher animals, whether fins, flippers, wings, or feet, were outgrowths from the trunk and vertel,ral column, and deriver their sintous figure-of-8 movements therefrom. The following were the words employed at the date in question: "That the movements of the extremities primarily emanate frum the spine is rendered probable by the remarkable powers possessed by serpents. . . The peculiar endowments, therefore, which accompany the possession of extremities, would seem to present themselves in an undeveloped or latent form
in the trunk of the reptile."

FIt. 3.-Photographs of nude female walking, as seen before and behind (after E. Muyloridge). The darts are added by the Author, and he is responsible for the description given of this and other photographs by Muybridge employed in the work. The darts show 


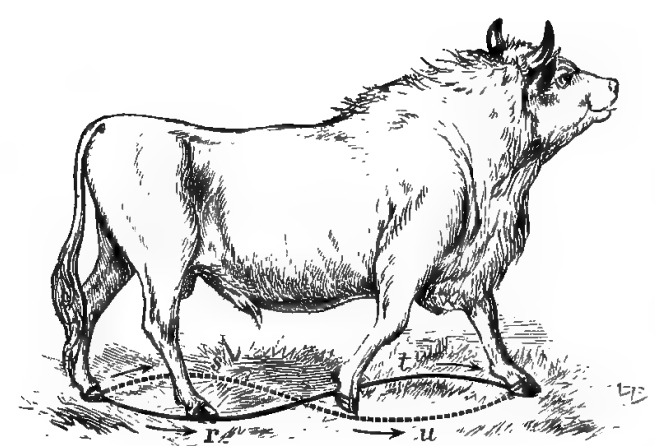

A
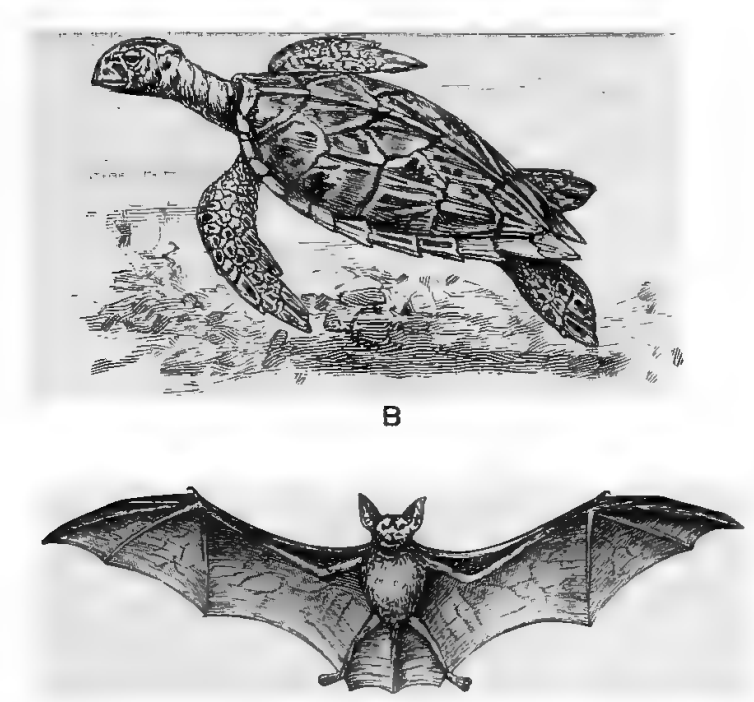

c

Fig. 1.

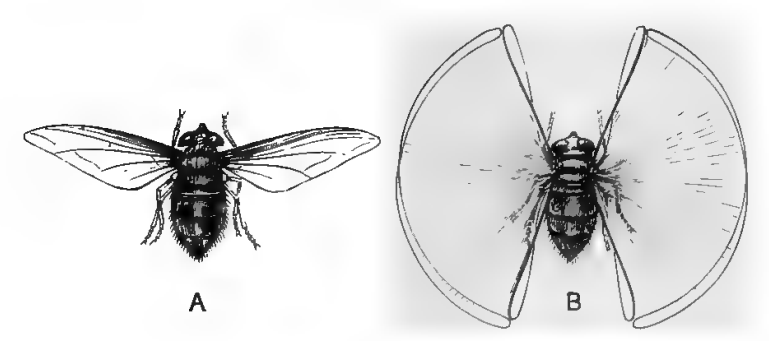

FIG, 5.

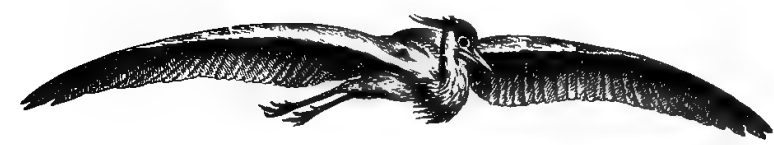

Fig. 7 .

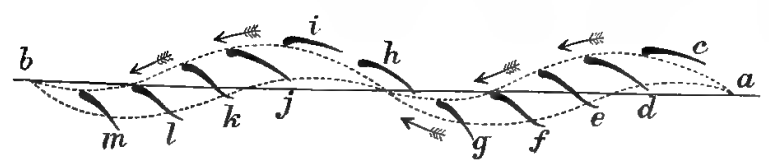

Fig. 10 ,
PLATE LIII
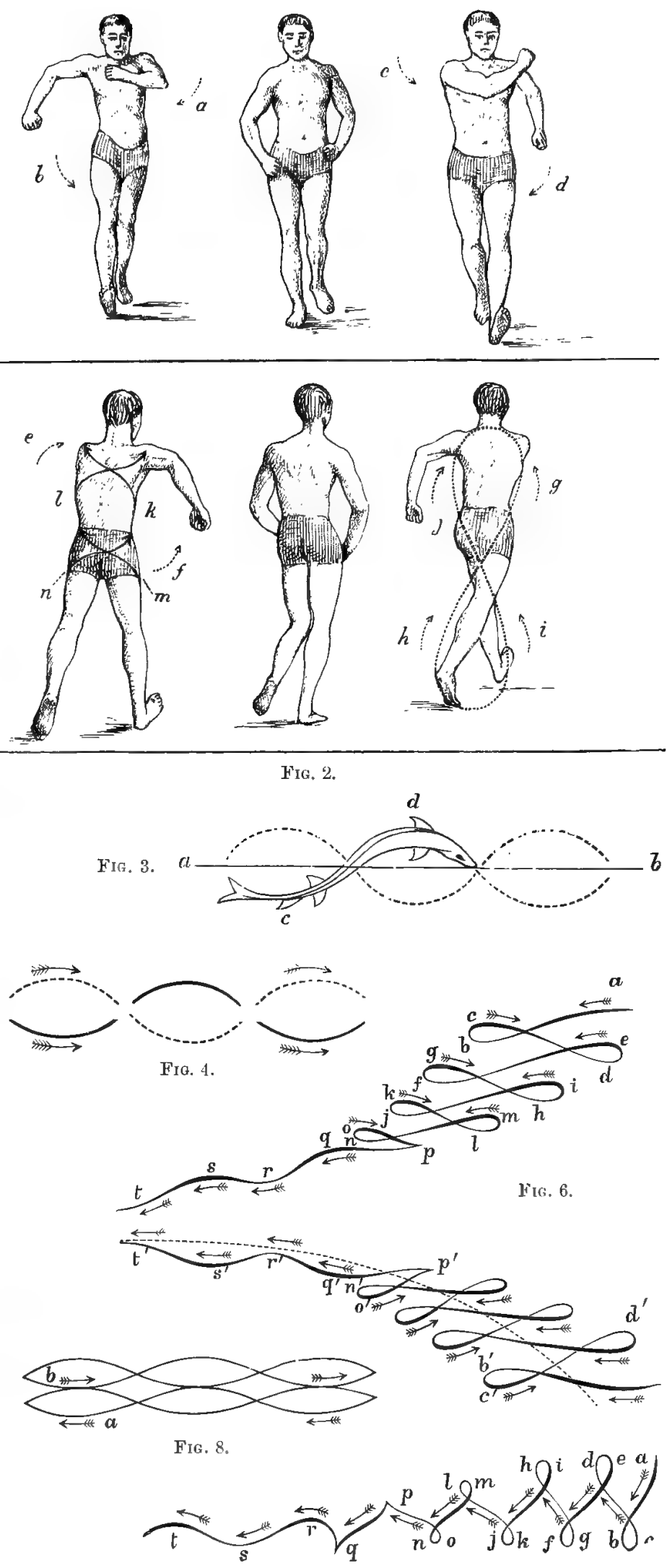
FIG. 9. 


\section{PLA'TE LIV (continued)}

that the lower limbs plait and overlap slightly in walking: they in fact form complemental and opposite curves in each successive step, as shown at Plate liii., Figs. 2 and 4. A similar plaiting of the limbs occurs in the walking of the bird (Figs. 7 and 10 of this Plate), and in the walking and running of quadrupeds, as for example the horse, \&c. (Fig. 1 of this Plate).

FIG. 4.-Double, complemental, figure-of-8 spirals made by the wing of the bird in extension and flexion, as drawn by the Author in 1867. The solid line and arrows indicate the double curve made by the wing in extension; the interrupted line and arrows the double curve made by the wing in flexion.

FIG. 5. - Shows the figure-of- 8 movements made by the wing of the wasp in active flight, and also the angles made by the wing with the horizon at every part of each stroke, as figured by the Author in 1867 and 1870 . In the insect the direction of the stroke is more horizontal than in the bird and bat. The forward and backward stroke and figure-of- 8 made by the tip of the left wing is seen in the two upper figures at $a, b, e, d, e, f, g$, and at $g, h, i, j, k, l$. The various angles made by the left wing with the horizon at successive stages of the forward and lackward stroke and when reversing are given in the two lower figures. The same letters in the upper and lower figures represent the wing in the several phases through which it passes during the forward and backward stroke.

FIG. 6. - The cicala (C. septemdecim). Shows four typical wings. As the wings are transparent the venation and structure can be seen to great advantage. The two riglit and the two left wings, when taken together, present a triangular outline; the roots of the wings being thick and semi-rigid, the tips thin and elastic. The anterior margins, moreover, are thicker than the posterior ones, The wings taper in two directions, namely, from the roots to the tips, and from the anterior to the posterior margins. They are carefully graduated structures and, as such, perfect organs for aérial locomotion. Wings so constructed inevitahly fly forward during both the down and up strokes when made to vibrate. Drawn by C. Berjeau from specimen in the Author's museum.

FIG. 7.-Photograph of the ostrich (Struthio camelus), as seen from behind, running. Shows how the right leg (a) and left wing and shoulder $(b)$ move together to form a double diagonal curve (see darts) and one step; and how the left leg (c) and right wing and shoulder (d) move together to form a second and oplosite douljle diagonal curve (see dart) and a second step. These movements are repeated so long as the bird continues to run (the Author).

FIG. 8.-Diagram constructed by the Author in 1867 to show that the wing of the bird, contıary to prevailing belief and preconceived notions, strikes downwards and forwards during the down stroke. Prior to 1867 the wing was supposed to strike vertically downwards or downwards and backwards. The Author's view has been confirmed by instantaneous photography, and is now generally accepted. $x$, Axis of body of bird ; $d$, root of wing ; $a$, $a^{\prime}$, double forward curve made by tip and posterior margin of wing during their descent; $f, f^{\prime}$, similar but opposite curve made by root and anterior margin of wing. The two margins make double and opposite diagonal curves when the wing is in action. The wing, as a matter of fact, twists and untwists diagonally during the down and up strolies, and makes figure-of- 8 movements similar to those made by the tails of fishes, the flippers of sea mammals, and the extremities of quarirupeds and bipeds.

FrG. 9.-Photograph of vulture (Vultur monachus), flying with the wings flexed as seen during the up stroke, and fully extended as seen during the down stroke and in sailing flight. During the up stroke the wings are not only flexed or folded, but all the feathers are separated so as to diminish to the utmost the resistance experienced from the superimposed air. During the down stroke the wing is fully opened out and extended, and the feathers, for the most part, tightly closed to seize and prevent the escape of the nether air. The right wing of the lower figure is twisted upon itself, and forms double figure-of-8 curves (the Author).

FIG. 10.--Photograph of the adjutant (Ciconin alba) walking. This quaint birl walks precisely as a man does : thus it begins its step by extending the toes of the right foot (1st figure, left side); it then folds and shortens the right leg ( 2 nd figure); it then advances the right leg and folds the right foot (3rd figure); it then straightens the right foot and somewhat straightens and advances the right leg (4th figure); it then further straightens and advances the right leg and straightens and points the right foot downwards (5th figure); it then still further straightens and advances the right leg and spreads ont the toes of the right foot preparatory to placing the right foot on the ground (6th figure). This completes one step. During the step the right leg and foot make a curve the convexity of which is directed outwards or towards the spectator (see darts). The left leg and foot perform precisely similar movements in making a second step, and make an opposite or complemental curve (the Author).

\section{§ I0. Recapitulation.}

The points which I have endeavoured to establish by the aid of the foregoing illustrations are :-

1. That many crystals bear a striking resemblance to plants and animals and parts thereof.

2. That crystals in many cases present radiating, branching, segmented, and concentric arrangements also found in plants and animals.

3. That crystals form frost pictures and dendrites which resemble plants and animals and also electric sparks and lightning flashes.

4. That crystals in some cases form spirals which resemble the fronds, tendrils, and other parts of plants, and the bones, horns, teeth, and other parts of animals.

5. That plants and animals are composed, for the most part, of the same elements and have much in common; their constituent parts being, in the majority of cases, arranged in radiating, branching, and concentric lines, with longitudinal and transverse cleavages in a more or less marked form.

6. That these cleavages also occur in inorganic matter, as in basaltic rock formations.

7. That the basaltic columns form pentagonal, hexagonal, and other prisms, which find their counterparts in certain corals; these again resembling the prisms found in the enamel of teeth, the fasciculi of muscles, and, as far as general outline is concerned, the cells of the honeycomb, epidermic cells, pigment cells, \&c.

8. That organic structures which are widely separated and which apparently have nothing in common do never- 
PLATE LIV
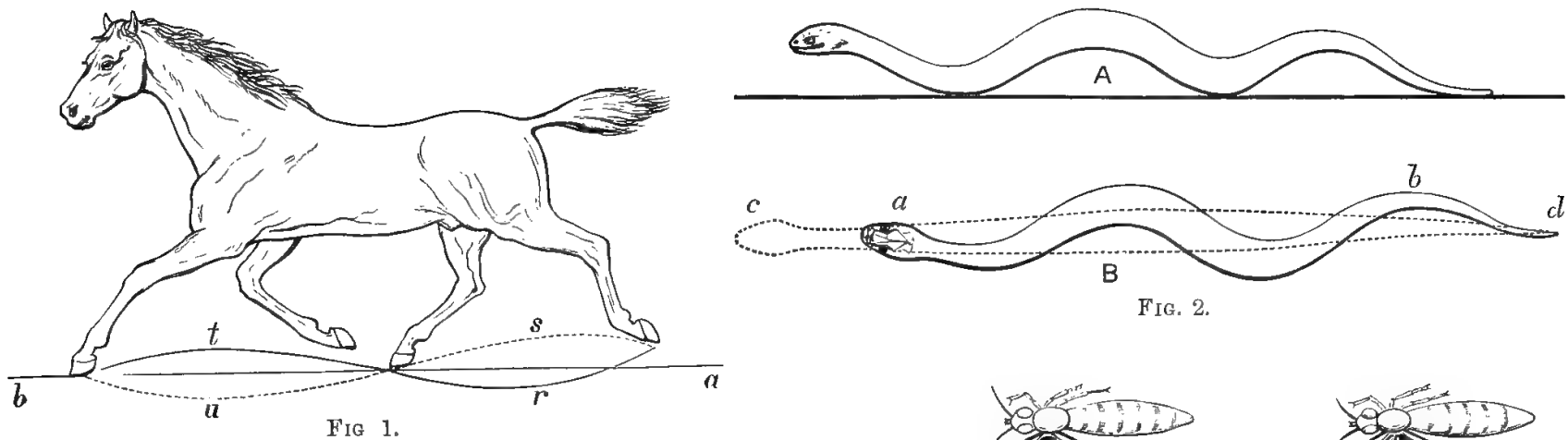

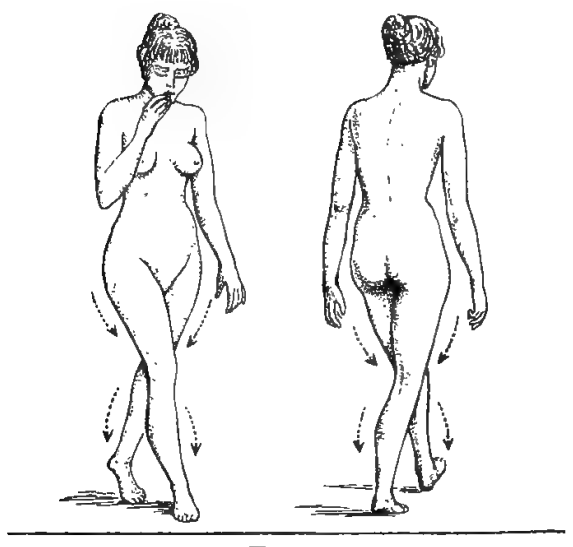

FIG. 3.

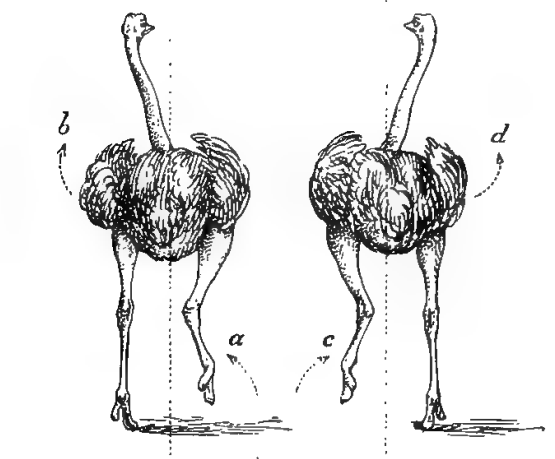

FIG. 7

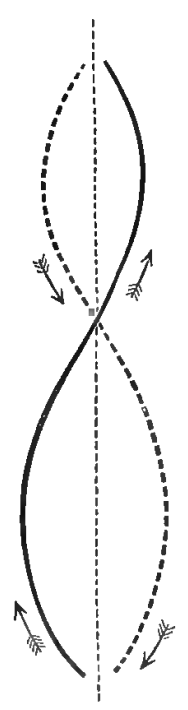

FIg. 4.

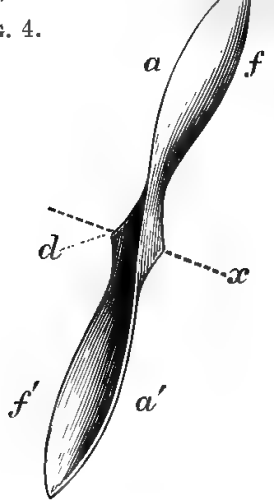

FIG. 8.

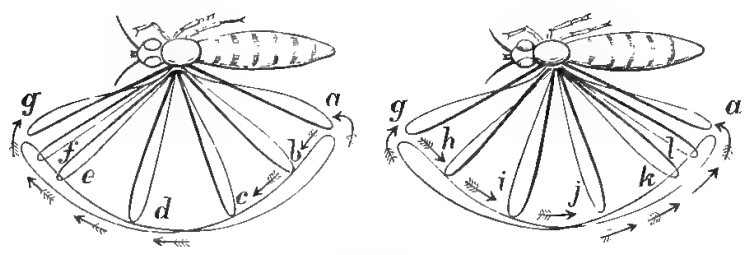

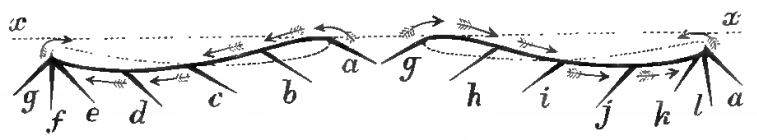

FIG. 5.

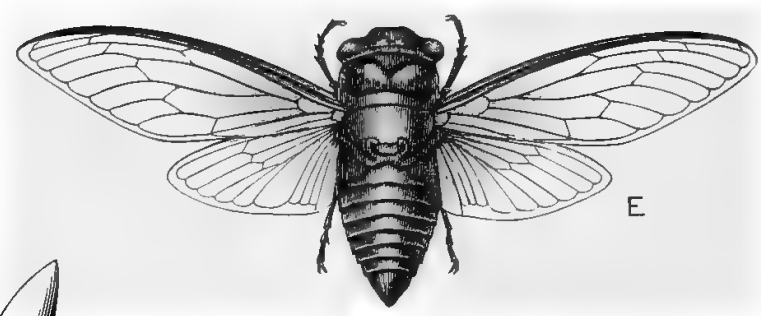

FIG. 6.

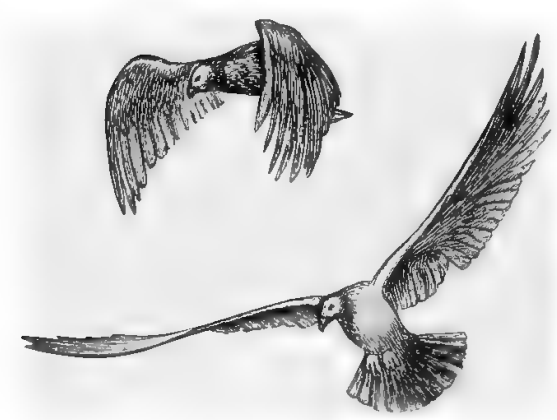

FIG. 9.

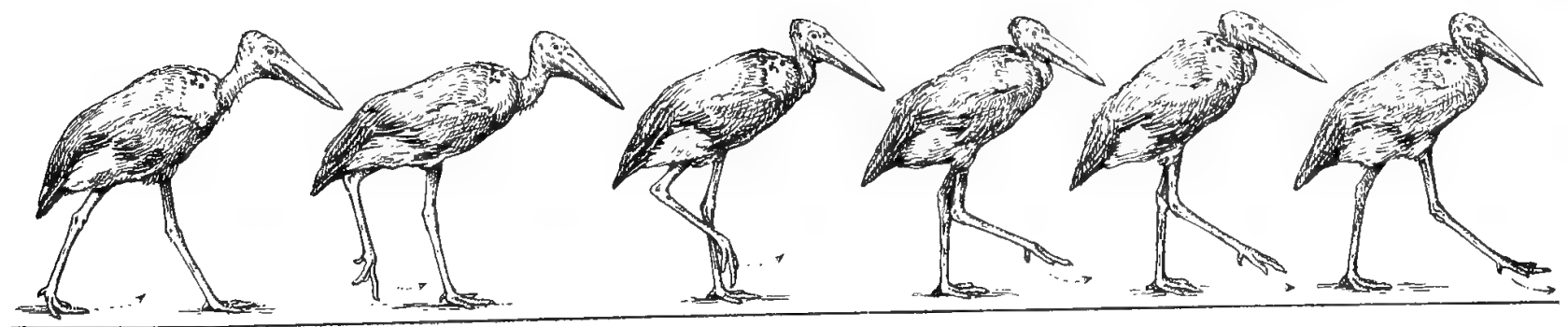

FỊG. 10.

VOL. I. 
theless greatly resemble each other, as witness the tooth of the labyrinthodon, brain coral, and human brain, all of which are curiously convoluted; also Venus's flower-basket (Euplectella), which presents an intricate arrangement of interlacing spiral fibres not unlike that found in the ventricles of the heart of the bird and mammal.

9. That crystals, plants, and animals are, for the most part, symmetrical; the symmetry being due to the radiating, branching, and concentric arrangements of the atoms and molecules, which act in specific directions according to fixed laws.

10. That the atoms combine to form structures with plane, curved, and spiral surfaces, and that these are found in great plenty in the inorganic and organic kingdoms, as witness angular crystals, dendrites, spheres of all kinds, twining plants, spiral shells, spiral nerves, spiral bones, spiral horns, spiral teeth, \&c.

11. That the spiral formations seen in plants and animals are accompanied in many cases by spiral movements, and that these movements have their analogues in nebular eddies, cyclones, spiral sand-storms, spiral water-spouts, whirlpools, \&c.

12. That the spiral movements in the higher animals play a prominent part in walking, swimming, and flying.

13. That the spiral movements are fundamental, and intimately associated with the distribution of matter, as seen in the formation of nebular, air, and water vortices; in the formation of cells and spiral structures in plants ; and in the formation of spiral horns, teeth, bones, muscles, and other parts of animals.

$13(a)$. That the spiral arrangements make their appearance at the very threshold of existence in plants and animals.

14. That force and growth act in the same direction, on parallel lines, and to given ends; the inorganic and organic kingdoms being complemental and interacting.

15. That no part of nature is at war with any other part; all plants and animals coming from and returning to the inorganic kingdom; and all force, physical, vital, and mental, being under divine guidance, and working harmoniously to produce the existing state of things.

16. That everything, objective and subjective, points to design, law, and order, and supreme intelligence ; intelligence being always present and adapting the means to the ends.

17. That the intelligence is, in every instance, either in the thing acting or behind it, and that nothing occurs by accident or chance.

18. That there is no such thing as spontaneous generation or life de novo, and that living plants and animals are created things and only beget themselves, each after its kind.

The points here indicated have now to be considered somewhat in detail.

As explained in the opening sentences of the work, atoms and molecules for the most part display a tendency to dispose themselves in straight lines or in curves, the curves forming circles and spirals, especially the latter. As a consequence, growth and development in the organic and inorganic kingdoms proceed in one or other of the directions indicated.

The straight-line formations produce bodies bounded by plane surfaces; the curve formations producing spheres and spiral structures and modifications thereof. The straight-line formations are represented by crystals of every form and variety, crystallites, and dendrites; the latter branching and assuming a characteristic tree shape. Crystals are formed by aggregations of atoms and molecules; the additions, as a rule, being made in straight lines, and giving rise to plane surfaces, which, in many cases, result in the most exquisite symmetric forms. Very frequently they occasion radiating or stellate arrangements.

The curve formations, as stated, are represented by spheres and modifications of spheres, where the atoms and molecules combine to form bodies having concentric arrangements; the additions being made in successive curved layers. Beautiful examples of straight-line formations are seen in the crystals of snow, and of associated straight-line and curve formations in the crystals and conglomerations of hail.

The spiral formations of the physical universe are seen in spiral nebular arrangements, in whirlwinds and spiral sand-storms, in whirlpools and spiral water-spouts, \&c. In all these cases, the atoms, molecules, and bodies concerned are arranged spirally. The straight-line, curved, circular, and spiral formations obtain also in plants and animals. The globular, rod-like, and spiral structures are seen in the very beginnings of life, in the reproductive elements of plants and animals, and in the very lowest living forms, for example, cocci, bacilli, and spirilla, measuring from the $\frac{1}{100 \sigma}$ to the $\frac{1}{500}$ of an inch in diameter. Examples of the straight-line arrangements are met with in the stems, roots, branches, and other parts of plants. Examples of curve formations are to be seen in the concentric arrangements (rings of growth) in the stems of plants and trees; and examples of spiral arrangements occur in the twisted stems of climbing plants, tendrils, \&c. Twisted stems are not uncommon also in forest trees. Trees and plants split up and branch after the manner of dendrites. They thus combine, in their structure, the straightline and curve formations to which reference has been made. 
The same thing, within limits, happens in animals. Animals are symmetric and asymmetric. A good example of a symmetric animal is the five-rayed starfish. In general configuration it greatly resembles a crystal. The body, as a whole, displays the straight-line and curve formations in combination; the diverging rays affording an illustration of the former, and the curved surfaces of the rays, of the latter.

The Aplysia, one of the molluscs, furnishes an example of an asymmetric animal.

The symmetric animals are in the ascendant. In matters of symmetry they, in not a few cases, resemble crystals. They also resemble dendrites from the fact that, in a great many instances, they branch out in more or less straight lines, as witness the integumentary appendages, and the extremities and travelling organs of animals.

Animals reveal spiral formations in their bodies, as a whole, and in certain parts of their bodies. The animals inhabiting shells are almost all spiral, and quadrupeds and bipeds have spiral extremities; these being composed of spiral bones, spiral joints, spiral muscles, \&c. The quadrupeds have, in many cases, spiral horns. Both quadrupeds and bipeds have, as a rule, spiral hearts.

The general configuration of animals is rounded or convex, while the internal arrangements, in the majority of cases, are dendritic, that is, they branch out in straight lines after the manner of trees. This holds true of the blood-vessels, lymphatics, bronchial tubes, nerve cells, \&c., in the higher animals. It also holds true of the respiratory, alimentary, and other systems in the lower animals.

Animals, like plants, are the products of straight-line, curve, circular and spiral formations. The movements in the organic and inorganic kingdoms are also in straight lines, curves, ellipses, circles, and spirals.

In the physical universe we have examples of straight-line movements when a body flies off at a tangent into space. We have examples of elliptical and rotatory movements in the earth travelling round the sun, and turning upon its axis while so engaged. The spiral movements are seen to advantage in the whirlwind, the water-spout, and the vortices formed by nebulæ.

In plants and animals, the straight, circular, and spiral movements reappear. In plants, straight-line movements are seen in the general circulation, in the upward and downward progress of branches and roots in the process of growth; in the bifurcation and growth of the stem, branches, leaves, \&c. The circular movements are seen in the free ends of plants whose summits revolve; and the spiral movements in climbing plants which wind round supports, and which have, for the most part, twisted or spiral stems. In animals, straight-line movements are witnessed in the pseudopodia of the Protozoa, which supply the most plastic and direct form of movement, also in the forward movements of worms, slugs, \&c. The circular movements are seen in the Rotifera; the curve movements in fishes and snakes; and the curve and spiral movements in the walking of quadrupeds and bipeds, in the swimming of aquatic birds, and in the flight of insects, birds, and bats.

In the higher animals, the travelling organs are spiral, both as regards form and function. The same remarks may be made of the viscera of several of them, namely, the ventricles of the heart, the stomach, bladder, uterus, \&c.

As regards both form and movement, plants and animals find their analogues in the physical universe; the organic kingdom is not separated from the inorganic one, unless in the matter of life. Nor will this, on reflection, occasion surprise. All the elements entering into the formation of living plants and animals come directly or indirectly from the physical universe. They also return to it at death. There is in the universe a store of matter and a store of force, which, humanly speaking, admits neither of increase nor diminution. The organic and inorganic kingdoms are inseparably united, and there are good grounds for believing that the life, in building up plants and animals, not only appropriates all the materials employed in the constructive process from the physical universe, but also a considerable proportion of the force which inheres in those materials, and which, strictly speaking, cannot be separated from them.

If the views now put forth be adopted, most, if not all, the structures and movements with which we are familiar in the organic and inorganic kingdoms fall into line. They go far to explain how the heavenly bodies are built up, and how they move and wheel in space-in tangents, ellipses, circles, spirals, \&c.: how the nebulæ, in many instances, form vortices; how there are eddies and whirlpools in the water; cyclones or circular storms in the air; spiral water-spouts and spiral sand-storms on sea and land, \&c. They also go far to show how crystals and dendrites are formed in the inorganic kingdom; how structures, bounded by plane and curved surfaces, are produced in the organic kingdom; and how straight, curve, circular, and spiral movements in plants and animals, and parts thereof, are produced.

Movement at once precedes and follows structure, and the direction of movement in living things is, in every instance, determined by the composition and configuration of the moving parts.

There are good reasons why atoms and molecules should arrange themselves, and move in straight lines and in spirals. Straight lines and spirals do not return upon themselves and admit of indefinite extensions, that is, 
matter can be added in straight lines and in spirals to any amount, and movement in either direction has practically no limit. Those peculiarities of straight-line and spiral formations and movements are of the utmost consequence in growth and progression, especially in the locomotion of animals.

\section{MATTER DIVISIBLE INTO SOLIDS, LIQUIDS, AND GASES-DISTRIBUTION OF MATTER-ORDER OF CREATION, \&c.}

The straight-line formations are crystalline and dendritic in character, that is, they form structures bounded by straight lines and plane surfaces, as in crystals; the dendrites of minerals and metals; the frost pictures seen on window-panes and pavements in winter; the lightning flash, \&c.

Straight-line formations are also witnessed in the arborescent arrangements of the roots, branches, leaves, and other parts of plants, and in the branching of nerve cells, blood-vessels, lymphatics, bronchial tubes, and other parts of animals.

The spiral formations and movements everywhere abound in the vegetable and animal kingdoms; in spiral growth and development; in the spiral cells, hairs, and vessels of plants; in the spiral stems and tendrils of climbing plants; in the revolving movements of plants ; in the spiral distribution of branches, leaves, fruits, \&c.; in the spiral configuration of shells, horns, and teeth; in the spiral structure and distribution of the bones and joints of the vertebrate skeleton; in the spiral formation and movements of the soft parts, especially of the voluntary and involuntary muscles, as seen in the limbs, the ventricles of the heart, stomach, bladder, uterus, \&c.

In suggesting possible explanations of the distribution and movements of atoms and molecules in living plants and animals, I recognise most fully the existence of a great First Cause, as regards the creation, disposal, and supervision of matter, both in the organic and inorganic kingdoms.

The First Cause may operate in two different ways: $(a)$ In creating matter (inorganic or organic) and in giving it a certain rôle to perform as apart from supervision. (b) In creating matter, and in supervising every change which occurs in it.

In the former case, creation is as it were a completed work; everything infallibly working out its own destiny, according to law and order. In the latter case, creation is only in part completed-in other words, is progressive, and requires constant supervision and guidance. The first view is that adopted by many evolutionists; the second, that favoured by those who believe in separate creations.

Nothing short of a First ('ause can, it appears to me, explain many of the phenomena with which biologists and physiologists have to deal.

The order of creation is briefly as follows: The physical universe was first formed, then plants, then animals. Plants and animals could not exist as apart from the physical universe. The materials and forces of the physical universe not only enter into the composition of plants and animals, they also provide them with food. "Brut" matter forms the food of plants; and plants, for the most part, that of animals. The Carnivora feed upon animals, and the Omnivora upon animals and plants. The physical universe is, in a sense, the great primeval parent, and provides a beautiful table and a home, as well as a grave, for plants and animals.

All living things come from the physical universe and return to it. The subtle element of life borrows from the physical universe, for a longer or shorter period, the materials which it converts into plants and animals; but sooner or later it returns them, in their original or in a modified form, and in undiminished quantity.

The restoration or day of reckoning occurs at death; and I can conceive no more striking picture, no more convincing illustration of the tremendous gulf between living and dead matter, than is furnished by living and dead plants and animals respectively.

The stillness of death, as contrasted with the ceaseless activities of life, produces a profound impression on even the most careless and thoughtless observer. The living and dead thing are as the poles asunder. Dead once, dead always. No power on earth can resuscitate a dead plant or a dead animal. Similarly, only a living thing can beget a living thing. No fortuitous assemblage of dead particles can, under any possible circumstances, assume life de novo. Spontaneous generation, even more than the philosopher's stone, is the illusory figment of the imagination.

There is no proof that plants and animals were ever produced otherwise than they are to-day.

I am not called upon here to discuss the popular modern theory of evolution. That is more conveniently done further on. It is enough for my present purpose to be able to state that plants and animals, in endless succession, have come down from the earliest pre-historic times, and are, so far as we can make out, very little changed at the present time. Man himself has undergone no radical modification for 6000 years at least, and 
some anthropologists and geologists refer his appearance on the earth to an inconceivably early period-a period which the mind has difficulty in grasping.

That modifications have taken place in the organic and inorganic kingdoms, in the fulness of time, no one will doubt; but the grand fact remains, that living plants and animals are the direct products of ancestors, that is, of living plants and living animals; and that the offspring, in every instance, resemble their parents and prototypes.

No one has ever seen the birth of a new plant or animal, and it goes without saying that if thousands of years are required to manufacture a new genus, and, it may be, even a new species, the proofs of the said manufacture are necessarily absent. The subject is straightway removed from the category of things about which the human mind can reason. The matter logically becomes a petitio principii. Endless modifications through endless time are required and claimed for evolution, but these conditions, as indicated, remove it from human experience, and, to a large extent, even from human history.

The modifications in the organic and inorganic lingdoms are no doubt very slow, gentle, and minute, but they are not such as to break down the great and well-defined boundaries between the several orders of plants and the several orders of animals. Cultivated plants and animals, if left to themselves, revert to their original types, and the progeny of allied animals, crossed in breeding, become, as a rule, barren.

The modifications in question, moreover, occur simultaneously in the inorganic and organic kingdoms, so that there is no dislocation of the great scheme of the universe, whereby, as I have endeavoured to show, the inorganic kingdom becomes at once the foster-parent, the storehouse, and the grave of everything that lives-plant and animal alike.

\section{$\S$ II. Atoms and Molecules Fundamental in the Inorganic and Organic Kingdoms.}

The atoms and molecules of the physical universe have always been, and still are, the atoms and molecules from which plants and animals are built up, with the addition of that far-reaching and mysterious factor called life. The atoms and molecules aggregate and are marshalled by life into the symmetric and asymmetric forms (plant and animal) with which all biologists and physiologists are familiar. The atoms and molecules, as already explained, arrange themselves in straight lines and in spirals, and combine to form tissues bounded by straight lines and plane surfaces, or by curves and spirals. The straight-line and spiral formations are met with in the simplest plants and animals, as well as in the most complex. They are seen even in the spores, seeds, and eggs. Rod-like structures are not uncommon in the beginnings of life, and spiral seeds and ova are of frequent occurrence in plants and animals. The carpogonia of Chara helicteris, and the spermatozoa of the cray-fish (Astacus fuviatilis), provide the necessary illustration. The egg-purses of sharks and dog-fishes also reveal beautiful spirals. The rod-like and spiral formations are not chance structures. They are the results of reproduction, growth, and development, and have been going on throughout the ages. No one can, of course, say why the atoms and molecules arrange themselves in the manner indicated. We must accept the arrangements as we find them in both the inorganic and organic kingdoms. The arrangements are to be regarded as ultimate facts in physics, biology, and physiology. There is no getting behind or beyond them. It is not possible even to inagine that the endless examples of crystals, dendrites, and spiral formations and movements which we behold in the universe, and in plants and animals, are the result of accident.

The fact that crystals, dendrites, spirals, \&c., are formed in unvarying sequence, and that plants and animals reproduce themselves, and have done so for countless ages, shows that they are, and have always been, under law and order. That they will so continue, while the present order of things lasts, can scarcely be doubted.

There is no need to assume that the plants and animals of the present day materially or fundamentally differ from the plants and animals of pre-historic times. That species, and even races of plants and animals, may have died out, and new forms come into existence, does not destroy the argument that the atoms and molecules forming the plants and animals of the present day act in precisely the same directions as they have always done. Neither does it disturb the relations which exist as between the inorganic and organic atoms and molecules as a whole. The mere form or external configuration of plants and animals does not destroy the fundamental relations which are known to exist as between the inorganic and organic kingdoms. Great physical upheavals have, no doubt, occasionally dislocated, for the time being, those fundamental relations, but out of the wreck, so to speak, new races of plants and animals have arisen to supplement those which have perished; and the new races, like the old ones, have been linked to the physical universe by atomic and molecular action quite as closely as the primeval plants and animals were linked to a pre-existing state of things.

The relations between the inorganic and organic kingdoms are now, and always have been, of the most intimate description; a state of matters which compels us, when speaking of the origin, distribution, and lives of plants and animals, to consider the universe as we find it. 


\section{§ I2. The Effect of Environment Limited.}

The question of environment is in all cases an important one, but it must never be forgotten that environment, while it slightly modifies plants and animals, never alters their constitution; it never makes a plant or an animal other than what it is by original endowment.

The life, and not the environment, makes plants and animals what they are. Environment never produces a new organ in any organism; neither can environment, as apart from life, goad plants and animals into activity.

Plants and animals, once created and endowed with life, work out their own destinies on the lines laid down for them. They defend themselves against environment when that is inimical, but the initiative, in every instance, comes from themselves. Environment is a dead thing. Plants and animals are living things, and are therefore superior to environment. It is a mistake to exalt environment over life, as is very frequently done. 'This necessitates the assumption and aid of a set of artificial conditions which, I venture to assert, do not exist. It requires us to take for granted that all the tissues of plants and animals, and plants and animals themselves, are irritable, and that they can only act in response to outside stimulation. This view robs life of its characteristic power, namely, the power of independent initiative.

\section{I3 The Organic and Inorganic Kingdoms Reciprocate.}

In order fully to explain some of the more tangible, outstanding, and salient features which obtain between the inorganic and organic kingdoms, and which they have in common, it is necessary to refer very briefly to the sun, solar heat, light, the atmosphere, soil, moisture, winds, \&c., in their relations to growth, development, and organic movement.

The sun is the great giver of heat and light, and on its presence the life of plants and animals practically depends. ${ }^{1}$ The sun, as far as our planet is concerned, is fixed, but as the earth rotates upon its axis every 24 hours, and revolves round the sun every 365 days, it follows that the alternations of day and night, and the seasonsspring, summer, autumn, and winter-so indispensable to the health and well-being of plants and animals-are infallibly secured. The sun is directly connected with the production of winds, as these largely depend on changes of temperature in certain localities, on the rarefaction and condensation of the air, irregular atmospheric pressure, vacua or partial vacua, the presence or absence of moisture, \&c.

Winds are useful in carrying and diffusing heat and moisture, and in purifying the air which plants and animals breathe. The organic and inorganic kingdoms are mutually interdependent.

The organic substances are, so to speak, manufactured from the inorganic by plarits and animals. A plant to live must be supplied with soil, with water, and air. The soil contains saline matters, to which are generally added decomposing substances, which furnish carbonic acid and ammonia. The water furnishes oxygen and hydrogen in chemical combination; and the air oxygen and nitrogen, and small but important quantities of carbonic acid and ammonia. The plant is thus supplied with water, salts, carbonic acid, and ammonia. But the plant can disintegrate the carbonic acid, and appropriate its carbon, which it can subsequently build up with oxvgen and hydrogen into sugar, oil, and starch; or it can combine carbon, hydrogen, oxygen, and nitrogen to form those peculiar nitrogenous substances recently known as protoplasm. The sun lends its aid to the plant in these transformations; in fact, the heat of the sun enables the plant to separate the oxygen from the carbon and the nitrogen from the hydrogen in the formation of sugar, oil, and starch. The heat of the sun is, marvellous to relate, not lost in the process. It is stored up in the newly-formed sugar, oil, and starch; and may be reconverted into heat either by burning in the animal body or in an open fire. The heat thus obtained can, when collected, be made to perform a definite amount of mechanical work. The physical and vital forces are correlated and interact. Sugar, oil, and starch are manufactured by a vital chemistry, and, when once produced, are appropriated, with other substances, as food by the Herbivora, and subsequently by the Carnivora and Omnivora. By vital, chemical, physical, and other forces the inorganic material of the outer world is incorporated in the organic or inner world of plants and animals.

The animal, built up, as it were, indirectly from the inorganic and directly from the organic kingdoms by means of the plant, reverses the operations of the latter. It returns to the inorganic world the substances abstracted from it by the plant. Thus the animal takes the complex bodies produced by the plant, and oxidises or burns them. It restores the carbon of these bodies to the atmosphere chiefly in the form of carbonic acid, the hydrogen as water, and the nitrogen, with the remainder of the carbon, as urea, to the soil.

While these transformations are going on in the animal body, the tissues are being built up and conserved.

1 "The life of men, animals, and plants could not continue if the sun had lost its high tenuperature, and with it its light" (Popular scientific lectures by HeInuholtz, 1873.) 


\section{CONSERVATION OF ENERGY}

secretions formed, and work done. This work may be either mechanical or mental, or both. The animate and inanimate kingdoms plainly interact. The plant lays the inorganic world under contribution, and the animal lays the vegetable world under contribution; but the animal in due time restores to the inorganic or mineral kingdom, in undiminished quantity, the substances abstracted from it by the plant. In like manner, the energy displayed by the animal is to be regarded as the sum of the potential energy stored up in chemical compounds; and this in turn is restored to the outer world as heat, which, like the matter, is undiminished in quantity. There is, therefore, a cycle of force and a cycle of matter in living beings. "Animals, like machines, can only move and accomplish work by being continuously supplied with fuel (that is to say, food) and air containing oxygen; both give off, again, this material in a burnt state, and at the same time produce heat and work. All investigation, thus far, respecting the amount of heat which an animal produces when at rest is in no way at variance with the assumption that this heat exactly corresponds to the equivalent, expressed as work, of the forces of chemical affinity then in action. As regards the work done by plants, a source of power in every way sufficient exists in the solar rays which they require for the increase of the organic matter of their structures . . . : a certain portion of force disappears from the sunlight, while combustible substances are generated and accumulated in plants, and we can assume it as very probable that the former is the cause of the latter. . . The immense wealth of ever-changing meteorological, climatic, geological, and organic processes of our earth are almost wholly preserved in action by the light and heat-giving rays of the sun. . . . In the series of natural processes there is no circuit to be found by which mechanical force can be gained without a corresponding consumption." 1

\section{$\$$ I4. Conservation of Energy.}

It is necessary to say a word here regarding the great physical law of the conservation of energy. This law resolves itself into three parts, namely, the conservation of energy, the transformation of energy, and the dissipation of energy. We have examples of the conservation of energy in physiology wherever we have mechanical or physical adaptations which produce a maximum of work with a minimum of power. We have examples of the transformation of energy in the muscles moving the bones, or the heart the blood; and we have examples of the dissipation of energy in the friction accompanying the movements of both bones and blood. In all the vital, physical, and chemical actions and reactions the law plays a prominent part.

The interaction of the physical, chemical, and vital forces is thus expressed by Professor Helmholtz: "If a certain quantity of mechanical work is lost, there is obtained an equivalent quantity of heat, or, instead of this, of chemical force; and, conversely, when heat is lost, we gain an equivalent quantity of chemical or mechanical force; and again, when chemical force disappears, an equivalent of heat or work; so that in all these interchanges between various inorganic forces, working force may indeed disappear in one form, but then it reappears in exactly equivalent quantity in some other form. . . The universe possesses, once for all, a store of force which is not altered by any change of phenomena, can neither be increased nor diminished, and which maintains any change which takes place on it. . . The force of falling water can only flow down from the hills when rain and snow bring it to them. To furnish these we must have aqueous vapour in the atmosphere, which can only be effected by the aid of heat, and this heat comes from the sun. The steam-engine needs the fuel which the vegetable life yields, whether it be the still life of the surrounding vegetation, or the extinct life which has produced the immense coaldeposits in the depths of the earth. The forces of man and animals must be restored by nourishment; all nourishment comes ultimately from the vegetable kingdom, and leads us back to the same source. . . We are thrown back upon the meteorological processes in the earth's atmosphere, on the life of plants in general, and on the sun." 2 It would appear from this that living force can generate the same amount of work as that expended in its production.

\section{§ I5. Protoplasm: its Nature and Function.}

The link which binds the organic to the inorganic, and the living to the non-living, is protoplasm in one form or other. This mysterious life-stuff apparently provides the bridge which connects the inorganic and organic kingdoms. The approaches to this bridge are numerous, but, unfortunately, not altogether satisfactory or safe. Professor Haeckel says: "The homogeneous, viscid, plasma substance which singly and alone formed the bodies of the first organisms, and even at this day quite alone forms them in the case of the monera, or simplest amœbic forms, is analogous to the tenacious and viscid planetary substance which contains the elements and substance of the young earth, as well as of the other glowing world bodies." Mr. Herbert Spencer, who was one of the advanced

$$
\text { Op. cit. }
$$$$
2 \text { Op. cit. }
$$ 
leaders of modern science, ave red "that the chasm between the inorganic and organic is being filled up, and that organisms are highly differentiated portions of the matter forming the earth's crust and its gaseous envelope." In like manner, Professor Huxley, also in the vanguard of science, stated " that protoplasm can originate only in that into which it dies-the elements-the carbon, hydrogen, oxygen, and nitrogen of which it is found to consist. Hydrogen, with oxygen, forms water; carbon, with oxygen, carbonic acid; and hydrogen, with nitrogen, ammonia. Similarly water, carbonic acid, and ammonia form, in union, protoplasm. . . . Protoplasm, then, is but an aggregate of physical materials, exhibiting in combination-only as was to be expected-new properties. . . . All vital action whatever, intellectual included, is but the result of the molecular forces of the protoplasm which displays it." 1 Protoplasm, according to Huxley, is the formal basis of life. "It is the clay of the potter, which, bake and paint it as he will, remains clay, separated by artifice, and not by nature, from the commonest brick or sun-dried clod. Thus it becomes clear that all living powers are cognate, and that all living forms are fundamentally of one character." Huxley regards protoplasm as identical in composition and uniformly diffused in plants and animals ; that is, not contained in cells. In this he differs from the majority of German histologists, who still regard the cell as the precursor and parent of protoplasm. With them "there is as yet no matter of life; there are still cells of life." Huxley claims for protoplasm a threefold unity-a unity of faculty, a unity of form, and a unity of substance. Each of these positions has been disputed, and properly ; for how, say Huxley's opponents, can there be unity of substance if the elements, carbon, oxygen, hydrogen, and nitrogen, which constitute the protoplasm, are combined in varying quantity in different kinds of protoplasm? If there is not unity of substance there cannot be unity of form, and if there is neither unity of substance nor of form there cannot possibly be unity of function. The unity of substance of protoplasm depends for its proof mainly upon ultimate chemical analysis. Ultimate chemical analysis, however, teaches next to nothing in such cases. "Ozone is not antozone, nor is oxygen either, though in chemical constitution all are alike." Further, some protoplasm, in addition to carbon, oxygen, hydrogen, and nitrogen, contains a certain proportion of sulphur; and the Germans have shown that the cells which produce protoplasm contain in some cases glycogen, in others cholesterine, in others protagon, and in others myosin. According to Professor Stricker protoplasm varies almost indefinitely in consistence, in shape, in structure, and in function. In some cases it is fluid, in others semi-fluid, in others firm and resisting. Occasionally it is club-shaped, bottle-shaped, spindle-shaped, branched, prismatic, polyhedral, \&c. One kind produces fat, another pepsine, another pigment. There is a protoplasm for each of the tissues-nerve, brain, bone, muscle, \&c. There is, further, a protoplasm for the several kinds of plants and animals, each producing its own kind.

Analogous in many respects to the protoplasm of Huxley is the bioplasm of Professor Lionel S. Beale. This, as its name implies, is also an elementary life-stuff. It is undifferentiated, and in this respect is identical with Huxley's protoplasm. Beale and Huxley differ as to the mode of production of their elementary life-stuffs. Beale affirms that they are the product of the vital forces per se; Huxley of the physical ones. Beale attributes all organisation to life, as apart from chemical, physical, and other forces. According to him, the bioplasm or germinal matter is the same always and everywhere, and consists of small masses of a structureless, colourless, and transparent viscid substance. Beale claims for his bioplasm or life-matter similar properties to that claimed by Huxley for his protoplasm. To both the same objections apply. Beale grounds the homogeneity and identity of his bioplasm on microscopical research, and Huxley, as explained, upon ultimate chemical analysis. Neither, however, is trustworthy. Experience teaches us that the microscope is limited in its powers, and that chemical analysis, instead of simply disintegrating and breaking up a body into its ultimate elements, not unfrequently produces new combinations, and consequently new substances. On carefully considering this matter, I am still of the opinion which I expressed in 1873, that both Huxley and Beale are in error, and that protoplasm is not the product of either the physical or the vital forces per se, but of both combined. Beale thus expresses himself: "Force (that is, physical force) is actually opposed to construction; and before arything is built up the tendencies of force must be overcome by formative agency or power. . . The vital power transcends altogether physical forces ; for it controls, guides, directs, arranges ; while the latter are controlled, are guided, are directed, \&c." 2 In proof of the foregoing, Beale states that a tree grows against gravitation. This is quite true; but it is equally true that in such cases the physical forces of capillarity, osmosis, chemical affinity, \&c., as I endeavoured to show in my "Lectures on the Physiology of the Circulation in Plants, in the Lower Animals, and in Man " (Edin. Med. Journ., 1872), are largely employed. The vital forces may override or bridle certain physical forces while they act in conjunction with others. In like manner nature may override or bridle one or more of the physical forces by employing stronger physical ones; but the physical forces subdued or inoperative for the time being are not on this account destroyed. They are ready for use when the proper time arrives. Capillarity and osmosis (purely

2 Huxley, as "pitonised by I. Hutchison Stirling, LL.D., to whose able critique on "Protoplasm" the reader is referred. 2 "Life Theories and Religious Thought," 1871 , pp. 6 and 78. 


\section{THE REPRODUCTIVE ELEMENTS OF PLANTS AND ANIMALS}

physical forces) in certain cases overcome gravitation equally with the vital forces, but it does not follow from this that gravitation as a power is extinguished. When gravitation (and this remark applies to all the physical forces) is useful in building up the organism, it is employed by nature in conjunction with the vital forces; and when capillarity, osmosis, \&c., are more convenient, they are preferred. The vital and physical forces must be regarded in their totality, nor must the object in view be lost sight of. Natural laws and forces (vital, chemical, and physical) act and react upon each other ; and though at times some are inoperative, they are not annihilated, neither are they opposed when the final effect to be produced is taken into account. Organised structures are built up in accordance with natural laws. Thus, their parts and particles consist of substances which naturally have a chemical affinity for each other; those substances absorb and respire-that is, take in and give off fluids; they exhibit, for the most part, capillary spaces and interspaces in their interior; they have weight, porosity, elasticity, and various other purely physical properties. In this way chemical affinity, osmosis, capillarity, gravitation, \&c., have free play, and each in turn assists not only in forming and maintaining the body, but also in disintegrating it. The vital forces, if I may be allowed the expression, use and abuse the physical ones. They do not, they cannot, annihilate them. As well may we say the vital forces annihilate matter.

\section{THE REPRODUCTIVE ELEMENTS OF PLANTS AND ANIMALS ESSENTIALLY AND FUNDAMENTALLY DIFFER FROM THE BEGINNING}

Whatever may be said regarding the ultimate composition of protoplasm, the grand fact remains, that the germs and seeds of plants and the eggs of animals, apparently homogeneous and identical, invariably produce not only their own kind, but, during the process of development, substances which are altogether dissimilar. Even man, with his wealth of imagination and thought, is the product of an egg, which to the eye of the microscopist and chemist is, as to its several parts, homogeneous and identical. Yet this egg, with its several parts placed in exactly the same conditions, produces the tender pulp of the brain, the flaccid muscles, the tough tendons, the elastic cartilages, the hard bones, the still harder teeth, the enamel, a substance nearly as brittle as glass, and in due time the several secretions, the major portion of which is composed of water. To these are to be added the external skin, with its hair, nails, \&c.; the internal skin or mucous membrane, with its numerous glands; the secreting and excreting organs; the nerves; the lymphatics; and the blood-vessels, with their contained blood, so rich, so complex, so marvellous in composition. These various products, so diverse in form, composition, and function, are, we know, the product of an egg. Shall we be told, or, if told, shall we believe, that this egg is homogeneous and identical in all its parts and particles? Identical these parts may be to the eye of sense, but to the eye of reason they are not so. Matter similarly constituted, similarly disposed, and similarly conditioned-that is, placed under precisely similar circumstances-behaves in precisely the same manner; in other words, all the parts are influenced alike. If life had to operate on absolutely identical matter, the living mass would be simply homogeneousthat is, undifferentiated; and if undifferentiated, it would have no power to build up either a plant or an animal. It is impossible to produce muscle, bone, brain, \&c., from matter identical in ultimate composition. In the germ, the seed, the egg, even from the first, there is differentiation, actual or potential. ${ }^{1}$ The first embryonic changes are the result of this differentiation. It is this which enables the different parts of the embryo to attract and assimilate different substances and to build up different tissues. One embryonic change involves another, and gradually the individual is developed, each according to its kind. The weaver may not produce a web dissimilar in texture and pattern in the absence of dissimilar elements. He must be supplied with the warp, the woof, and the varied pigment. A queen-bee may be formed from an ordinary bee grub by changing the quantity and quality of its food, but no amount of change of diet can produce from the same grub a dragon-fly or a locust.

In making these statements regarding the non-identity of protoplasm, bioplasm, and the several parts of

1 Mr. Herbert Spencer, in his "Principles of Biology," expressed the belief that in reproduction the cell must be regarded as the morphological unit, but that in addition there must be a chemical unit consisting of the simplest combination of molecules capable of displaying life. Others, who regarded the nucleus as the most important part of the cell, discovered that by the employment of suitable dyes one portion of the mucleus could be distinguished from all the other narts. This they designated the chromatin. The chromatin, it was found, could be broken up into a number of rod-like bodies, which were designated chromosomes. The chromosomes, when stained, were found in turn to be compound and divisible into microsomes. This much could be denonstrated by the aid of the microscope with stained muclei : when its powers failed, Professor Weismann, by a process of reasoning, inferred that each of the microsomes consisted of a number of still smaller bodies, which he called determinants. The determinants Weismann regarded as the structures forming the germ-plasm which decided the nature and character of the adult organism. Weismann went further, and assumed that not even the determinants constituted the ultimate living elements. The determinants, he believed, were further resolvable into biophores. Weismann, who has done much to expiscate this most involved and difficult subject, was by no means the first to deal with it in a more or less exhaustive manner. It has been a veritable bone of contention for over thirty years-each author employing his own nomenclature.

From the foregoing it will be seen that the reprodnctive cell is by no means a simple structure, and that it is seen to be more complex in proportion as our opportunities of investigating it increase,

VOL. I. 
the germ, seed, or egg, I do not mean to affirm that all the tissues enumerated are contained in the original germ, seed, or egg. Only this: that the original germ, seed, or egg is differentiated sufficiently to inaugurate the first series of changes, and that these inaugurate others in constant and rapid succession, until the plant or animal is completed. The first series of changes provides the materials and forces for the second, the second for the third, and so on, according to the degree of differentiation required in each particular organism. It is initial force and matter that are primarily required. Granted these, development in specific directions proceeds as a matter of course. ${ }^{1}$

\section{§6. Neither Chemistry nor Physics can Produce Life.}

Chemistry and physics are not the whole of physiology. These sciences can conjure up an automaton, but are absolutely powerless when an ovum is desired. Dumas, a leading authority in modern chemistry, thus limits its province: "The chemist has never manufactured anything which, near or distant, was susceptible even of the appearance of life. Everything he has made in his laboratory belongs to 'brut' matter; as soon as he approaches life and organisation, he is powerless. . . Organised matter, not capable of being crystallised, but destructible by heat, the only matter which lives or has ever lived-this matter, a subordinating agent of the vegetating power in plants, of the motion and sensation of animals, cannot be produced by chemistry; heat does not give birth to it; light continues to engender it under the influence of living bodies." "

The pretensions of the physicist may be disposed of even more cavalierly.

No machine hitherto devised by human ingenuity at all resembles or can compare in efficiency with a living organism, when the consumption of material and the amount of work done are taken as the standard of comparison. A man is as far in advance of a steam-engine in this respect as day and light are of night and darkness. ${ }^{3}$ If, then, neither the chemist nor physicist, nor both combined, can produce a living organism or anything even remotely resembling it, we are forced to fall back upon other than the chemical and physical forces, and the only ones we can under the circumstances fall back upon are the vital.

\section{INORGANIC AND ORGANIC RHYTHMS}

The scheme of creation hangs together in a most extraordinary manner. The phenomena of day and night, and of the seasons, which exert such a beneficial influence on plants and animals, are directly due to cosmic movements now well understood. They are of the give-and-take order. They ensure periods of activity and repose, or, more strictly speaking, periods of comparative activity and comparative repose, to plants and animals; this alternating activity and repose being essential to their health and well-being. Day and night and the seasons come and go at regular and calculable intervals. Day and night give and take light. The seasons give and take heat, moisture, \&c. Day and night and the seasons provide, for plants and animals, what is virtually a series of rhythmic movements. These movements are of primary importance in the organic kingdom, as they regulate, within limits, the time of feeding, building up, assimilating, secreting, excreting, \&c., and the resting and hibernating of plants and animals.

The give-and-take, rhythmic movements of the physical universe assume a great variety of forms. They appear in the alternations of day and night and the seasons. They are seen in the rise and fall of the tides, in cycles of drought and moisture, in wave movements of all kinds, such as those of light, heat, sound, \&c.; but-and this is the marvel-they reappear in the rhythmic movements of plants and animals; for example, in the spontaneous to-and-fro movements of certain leaves; the time-regulated opening and closing of the vacuoles of various water plants; contracting vesicles of all kinds; and the respiratory and circulatory movements in animals, as witness the opening and closing movements of the chest, heart, stomach, bladder, uterus, \&c.

The physical universe, under the influence of a First Cause and of life, inaugurates the beginnings and the building up of plants and animals; it also, under the same influences, takes the initiative in everything that pertains to their daily economy. Plants and animals are to be fed and rested; give-and-take movements on the part of the physical universe are consequently an absolute necessity. Plants breathe. During the day they take carbonic acid from the air and give oxygen to it. Animals also breathe. They take oxygen from and return carbonic acid to the air. Plants and animals in the matter of respiration reciprocate; the one supplying what

1 These views were first enunciated by me in an Introductory Lecture "On the Relation of Plants and Animals to Inorganic Matter, and on the Interaction of the Vital and Physical Forces," published in the Lancet of November 15, 1873.

? Faraday Lecture.

${ }^{3}$ Count Rumford long ago showed how a much greater quantity of work could be performed by a horse than could be performed l,y its food, when employed as fuel in a steam-engine, 


\section{SPIRAL STRUCTURES AND MOVEMENTS IN NATURE}

the other requires. The respiratory movements in plants and animals are give-and-take, interrupted movements, occurring at stated intervals, and therefore rhythmic in character.

Plants and animals, in addition to breathing, feed, and circulate their nutritious juices. The feeding and circulatory movements, like the respiratory ones, are give-and-take movements, that is, they occur in a certain order, and at stated intervals. Plants and animals not only take in, assimilate, and circulate extraneous substances rhythmically; they also give out the detritus or waste products rhythmically. The give-and-take, rhythmic movements referred to have to do with every change which occurs in plants and animals; with the respiratory act, with the circulation of gases and fluids, with the absorption and assimilation of food, with the extrusion of waste products, with secretion and excretion, metabolism, \&c. The give-and-take, rhythmic movements are provided for in the very beginnings of life. The cells of plants and animals afford the necessary conditions. Vegetable and animal cells are composed of a cell wall, a nucleus, and protoplasmic cell contents. The cell wall is porous, and provides an osmotic medium. When the cells are exposed to moisture, gases, heat, \&c., two opposite or give-and-take virtually rhythmic currents are at once established; there is the comparatively rapid ingoing or endosmotic nourishing current, and the slower outgoing or exosmotic waste product current. This explains how a plant or an animal composed of a single cell can live, grow, attain maturity, and reproduce itself. Living matter, lower than cells, possesses the same powers and exercises the same privileges. What is true of a single cell is true of every congeries of cells, and of all the tissues and parts formed by cells, in the simplest and most complex plant and animal organisms. Giveand-take movements are necessary to all.

\section{§ I7. Spiral Structures and Movements Universal in Nature.}

The next arrangement and order of movement to be considered is the spiral. As already stated, the spiral formations and movements reveal themselves in the physical universe in a variety of forms; in the spiral distribution of nebulæ, in the spiral water-spout, the spiral sand-storm, \&c.

Has this spiral distribution and spiral movement of the atoms and molecules of matter in space any counterpart in plants and animals? Most assuredly it has. The seeds of certain plants, and the ova and embryos of certain animals, exhibit distinct spiral formations and movements.

While we have spiral formations and movement at the very beginnings of plant and animal life, these are multiplied and emphasised as growth and development proceed; the highest representatives of the organic kingdoms providing the most numerous and striking examples. Thus in plants we find spiral cells, spiral hairs, spiral vessels, spiral stems, spiral branches, spiral leaves, spiral flowers, spiral fruits, \&c. We also find spiral movements in climbing and other plants.

Similar remarks are to be made of animals. In these, the muscles, bones, and joints are spirally constructed and arranged; the heart, stomach, bladder, uterus, \&c., also display spiral structure. All exhibit spiral movements. The movements of walking, swimming, and flying are, in every instance, spiral in their nature.

Locomotion for the most part consists of spiral, sinuous, double-curve, figure-of- 8 movements. These movements make their appearance in bacteria and the lower plant and animal forms. They occur in the cilia of infusoria and other organisms, where they take part in tactile, feeding, and swimming operations. The cilia and sinuous movements are not unfrequently met with on mucous and other surfaces, where they produce currents in given directions. The double-curve movements can be traced through the whole series of creeping things. They are seen to advantage in the wave movements of the caterpillar, and produce the characteristic wriggling of the worm: they appear in spermatozoa and in quite a large number of soft-bodied animals : they reappear in skeletal animals, and are witnessed in the swimming of the fish and in the creeping of the serpent. They are likewise the chief factors in the walking of quadrupeds and bipeds, and in the flying of insects, birds, and bats.

The spiral structures and movements, as already stated, are fundamental- that is, they are not dependent on fortuitous circumstances, or any form of stimulation or irritation.

Examples of spirals (single and double) are found in large numbers in plants and in animals. Spiral formations are symmetrical when two or four opposite spirals are employed. They are non-symmetrical.or lop-sided when only one spiral is employed. Complementary spirals are by no means infrequent.

The unaccountable thing is that crystalline, dendritic, and spiral formations and movements occur both in inorganic dead matter and in organic living matter.

The subject of organic growth, development, and movement has many side-lights thrown upon it by a consideration of what may be regarded as inorganic growth, development, and movement-namely, the arborescent frost pictures, as they appear on window-panes and pavements in winter, the branching dendrites formed in minerals and metals, and similar dendritic displays made by lightning (revealed by instantaneous photography), and when it strikes and scorches the human skin. 
A hasty generalisation would naturally lead to the conclusion that there is in the universe only one kind of matter and only one kind of force. It might even be inferred that the living or organic kingdorn is an outgrowth or product of the inorganic kingdom, and that vital force is a mere variety and product of physical force.

As, however, it has been satisfactorily proved that there is no such thing as spontaneous generation, a line of demarcation must be drawn as between dead and living matter, and between physical and vital force.

The question to be finally put is, How are the spiral structures and movements produced? They are, so far as I can make out, the result of independent growth and inherent endowment. The atoms and molecules of the growing and moving parts are distributed in spirals and move in spiral directions. This they do under the influence and guidance of a First Cause and of life.

The spiral is a fundamental factor in structure and movement, behind which we cannot get. The spiral formation of soft and hard parts, seeds and ova, muscles, bones, joints, shells, horns, \&c., are all, in my opinion, directly traceable to growth and original endowment; and to the spiral distribution of the atoms and molecules which constitute each particular form of growth and each particular kind of movement.

The spiral distribution and movements of atoms and molecules, in the organic and inorganic kingdoms, are not only under law and order, but they are under the same law and order. There is not one law for the spiral inorganic, and another for the spiral organic formations and movements.

The spiral distribution and movements of the atoms and molecules in the two kingdoms are never accidental or haphazard. If this were so, the stability of the two kingdoms would suddenly cease and determine.

\section{$\S$ I8. Growth in Straight Lines and in Spirals; Longitudinal and Transverse Cleavage: Limbs, Fins, Wings, \&c.}

Growth and development, in order to meet the requirements of plants and animals, of necessity proceed in two principal directions, namely, in straight lines and in spirals. Plants and animals modelled on these lines are readily constructed, because they can grow or extend themselves into space without let or hindrance. They can also move with perfect freedom, as their moving parts cannot, under any circumstances, over-reach or interlock.

Many examples might be adduced to prove that inorganic and organic structures and movements very closely resemble each other.

The dendrites of minerals and metals, the frost pictures on window-panes and pavements in winter, the lightning flash, \&c., are amazingly like the arborescent forms seen in the branches and leaves of plants and trees, the division and subdivision of blood-vessels and lymphatics, the branching of nerve cells, bronchial tubes, \&c., to say nothing of the body itself, which breaks up into extremities; the extremities in turn dividing into digits, and their expansions as seen in the fins and tails of fishes, the flippers of sea mammals, and the wings of insects, birds, and bats.

I am not aware that attention has ever been specially drawn to the manner in which the trunks of animals, as it were, proliferate by a process of budding; the limbs, even in the highest animals, appearing as fleshy projections or buds in the developing embryo. The limbs, while growing, also bud at their free extremities into digits, and the digits, in many cases, as stated, divide and split up to produce rays and membranes, as witness the pectoral, ventral, and caudal fins of fishes, the nervures of the wings of insects, the flying membranes of the bat, the primary, secondary, and tertiary feathers of the wings of birds, \&c. A very large percentage of all known animals assume the dendritic form. The dendrites in animals may be symmetric or asymmetric, that is, they may display perfect symmetry as in certain star-fishes, or asymmetry as in certain molluscs. The symmetrical animals bear a recognisable resemblance to crystals and symmetric plants. The non-symmetric animals are comparatively few in number, and may be regarded as exceptions to prove a rule. The dendrites in animals, in many instances, take the form of appendages-antennæ, hairs, cilia, suckers, probosces, \&c.; they also, as explained, figure as arms, legs, wings, \&c. The dendrites or outgrowths are functionally of great importance as sense-organs, as vehicles for securing food, and as instruments of locomotion. Even pseudopodia take on the dendritic type and act in straight lines.

In the lower animals, we have examples of the dendritic arrangement in the Vorticella, Hydra, Sertularia, Red Coral, Sea-pen, Actinia, Medusa, Comatula, Serpula, Rotifer, Sand-hopper, Beetle, Galeodes, Spiny Lobster, Crab, Spider, the Leaf Insect, Cuttle-fish, \&c.

In the higher animals, the dendritic arrangements appear, partly as tegumentary appendages (scales, hairs, feathers, horns, hoofs), and partly as travelling organs--fins, flippers, arms, legs, wings, \&c.

The serpent-a typical vertebrate animal - if the scales and ventral plates be excepted, is destitute of dendritic outgrowths. In the Lepidosiren, four small tapering processes project from the under surface of the body, and 
prefigure limbs. In the siren, two very tiny rudimentary anterior limbs, with imperfect digits, appear. In the Amphiuma, these are increased to four (two anterior and two posterior). In the Menobranchus, the same arrangement obtains; the limbs, though still very rudimentary, being a little larger. The limbs increase in size, and become more differentiated in the triton and crocodile, and are perfected in quadrupeds and bipeds.

The tendency of the body to proliferate into extremities, digits, rays, \&c., proves clearly enough that the dendritic, or branching type, is common both in the organic and inorganic kingdoms. In fact, the branching formations which occur in such great numbers in plants and animals are the analogues of the dendrites seen in minerals and metals; in the arborescent frost pictures; in the branching lightning, \&c. The spiral formations so common in plants and animals have also their representatives in the physical universe. These, as already indicated, appear as physical vortices, in spiral sand-storms, spiral water-spouts, and spirally-arranged nebulæ. The physical spiral arrangements may not inaptly be regarded as the forerunners or heralds of the twisted stems and the revolving movements of climbing plants; and of the twisted bones and extremities of quadrupeds and bipeds, with their spiral joints and complement of spiral muscles and spiral movements.

No doubt many will fail to perceive any possible connection between spiral sand-storms, spiral water-spouts, spiral nebulæ, \&c., and the spiral stems and revolving movements of plants, and the twisted limbs and spiral movements of animals, as seen in walking, swimming, and flying; and if so, no one will be disposed to cavil or impute blame; still the resemblances are so striking, when carefully looked into, that no scientific inquirer can afford to pass them over. They are especially striking, when it is felt that they are the results of the spiral distribution and spiral movements of the atoms and molecules common alike to the inorganic and organic kingdoms. They, in fact, point to the existence of a great law of growth, development, and movement which reigns supreme in both kingdoms, whereby atoms and molecules coalesce to form crystals and dendrites in the physical universe, and plants and animals in the mysterious universe of life. The law indicated, there is reason to believe, governs not only the major operations which produce movement and form in crystals, dendrites, plants, and animals, but also the minor operations which determine the arrangements and movements of the atoms which form molecules, of the molecules which form cells, and of the cells which form tissues.

\section{§ I9. Atoms and Molecules the same in Dead and Living Matter.}

There are good grounds for believing that the distribution and movements of atoms and molecules in the organic and inorganic kingdoms are not essentially different, what difference there is being traceable to the presence of life in the organic kingdom; life controlling, within limits, the elements of the inorganic kingdom, but working virtually on the same lines as obtain in that kingdom.

The ultimate distribution and movements of matter in the organic and inorganic kingdoms, when one comes to think of it, should not materially differ. The one is part of the other. The organic comes from and returns to the inorganic; the elements in both are the same. The plant and animal borrow all the materials forming their bodies, and much of the force which energises them, from the inorganic kingdom. There is therefore no room for antagonism between the organic and inorganic kingdoms, in so far as the dendritic and spiral formations and movements which prevail in both are concerned.

The oneness, the practical identity, structurally and functionally, which obtains in the organic and inorganic kingdoms, affords perhaps the strongest argument for design yet put forward. It shows that the great First Cause is the Author of the organic and inorganic kingdoms alike; that $\mathrm{He}$ works continually, and on the same lines in both; that the two kingdoms are complemental, in fact, working parts of each other; that the organic kingdom, while an elaboration and differentiation of the inorganic kingdom, is in no sense an inimical elaboration.

This accounts for the reciprocation which is known to exist as between plants and animals, and as between both and the physical universe; for the reciprocation which is known to take place as regards the intake and output of extraneous matters (solid, fluid, and gaseous) during the lives of plants and animals; for the reciprocation of day and night, and the seasons, in their bearing on the growth, development, and rhythmic movements of plants and animals; for the continued prevalence of law and order in the physical universe, and in the world of plants and animals, whereby the latter, and all parts of the latter, are duly provided for as regards locality, environment, food, \&c.

As, on the one hand, it is impossible to conceive of the physical universe as a chance combination of heterogeneous substances and movements, so, on the other hand, it is impossible to imagine the great kingdom of plants and animals as the product of spontaneous generation and of accident. The controlling power of the Creator alone can explain the glories of the heavens and the wonders of animated nature. The same Master-Mind regulates the outgoings and incomings of both. It is unphilosophical to assume that law and order prevail in every depart- 
ment of the physical universe, while no care, or very little, is bestowed on the members of the vegetable and animal kingdoms; these being, as it were, left to shift for themselves, and to fight their own battles, or struggle for existence. Iogically, we are bound to conclude that the myriads of tiny rudimentary plants and animals, as well as the multitude of differentiated, complex organisms, and all the organs and parts thereof, are directly under control and supervision. This alone will explain the mysterious powers exhibited by untold millions of almost invisible low plant and animal forms, and the vegetative functions in the higher animals and in man himself.

The resemblances between inorganic dendrites, if I may be allowed the expression, as revealed in minerals, in metals, and in frost and lightning pictures, and organic dendrites, as seen in the branchings of plant and animal structures, are so very striking and remarkable that I have deemed them worthy of special, and even profuse, illustration. A careful examination and study of the photographs and drawings submitted compel me to believe that the lines of force represented in dendrites and in frost and lightning pictures correspond in the main with the lines of growth in plants and animals where subdivision and bifurcation take place. One law obviously controls and shapes inorganic and organic matter, and physical and vital forces. The living organic forms resemble the dead inorganic forms to such an extent as to leave no doubt in my mind that both are traceable to the operations of one and the same First Cause.

The dendrites of minerals and metals, and the frost pictures, so closely resemble plants, and the venation of the leaves of plants, that they might readily be mistaken for them. The dendrites formed by lightning and electric sparks in space and when they touch the human skin, are marvellously tree-like in character, and reveal the most exquisite arborescent details and tracery. They bear an extraordinary resemblance not only to the branching of plants but also to the branching of blood-vessels, lymphatics, bronchial tubes, nerve cells, \&c. in animals. They are in both cases essentially straight-line formations. The lightning and electric spark very specially resemble the branching of the neurons and ganglia in the human brain and spinal cord, and display at their bifurcations little subsidiary angular swellings representing discharges or explosions of force which strongly suggests similar discharges on the part of nerve centres in the several parts of the body, as witnessed in normal nerve action and as emphasised in abnormal nerve action, in certain forms of epilepsy. That there are such discharges of nerve force all over the body wherever there are aggregations of nerve-cells, ganglia, and neurons is to me a matter of certainty。

\section{$\S 20$. Resemblances between Crystals, Plants, and Animals: Anomalous Resemblances.}

I have submitted what some will regard as a plethora of illustrations to show that many plants resemble crystals and that many animals resemble both plants and crystals. Other remarkable similarities are to be noted. A large number of fossil corals bear a striking likeness to basaltic columns as found at Staffa in Scotland, and the Giant's Causeway in Ireland; they also resemble, in their general outline, the prisms found in the enamel of teeth; the fasciculi of voluntary muscles; the hexagonal cells of the honeycomb; the pigment cells of the choroid coat of the eye, \&c. Other corals— the brain coral (Mrandrina cerebriformis), for example, which is a hard skeletal mass-wonderfully resemble in general appearance the convolutions of the semi-fluid human brain. The fossil tooth of Labyrinthodon Jaegeri does the same. In this tooth, as a transverse section shows, are concealed not only the general characteristics of the convolutions of the human brain, but many of the details; the external and internal convolutions and the peculiar markings of the latter can be readily made out. Similarly, the hard skeleton of the sponge known as Venus's flower-basket (Euplectella asperyillum) reproduces very accurately the intricate spiral arrangements which obtain in the muscular fibres of the ventricles of the heart of the bird and mammal. These can scarcely be regarded as chance resemblances.

It is difficult to account for the extraordinary similitude of the brain coral and the transverse section of the tooth of the labyrinthodon to the convolutions of the human brain. In the case of the brain the convolutions occur in a soft pulpy mass : in the case of the brain coral, in a combined soft and hard mass : in the case of the tooth, in a substance harder than bone. It is not a question of the mere stowing away of material to occupy the least possible space. If the stowing away principle afforded an explanation of the convolutions of the brain within a bony case, it would not explain the formation of the coral free to grow in any direction; or of the tooth free to increase in length and breadth. Moreover, all brains confined within bony cases are not convoluted. There are many intelligent animals with no convolutions in their brains. A somewhat similar convoluted arrangement to that witnessed in the brain, brain coral, and tooth is seen in the kidneys, lungs, blood-vessels (rete mirabile), glands, and placenta of certain animals. The folded, convoluted arrangement is common to many structures, and is, in a sense, fundamental. Primarily it increases the available surface of any particular part, organ, or organism : secondly it increases the strength and heightens the function of any particular part, organ, or organism. The 
arrangement acquires importance from its frequency, and is referable to a law in anatomy and physiology based upon reduplication and repetition. Nature, like history, repeats itself, and the same forms and conditions reappear under various guises and sometimes in the most unlooked-for quarters-hence the ubiquitous and perplexing vestiges or remnants.

The community of structure observable in Venus's flower-basket and the ventricles of the heart affords another example of repetition in things widely separated. Nor does the matter rest here. The radiating, branching, concentric and spiral arrangements; the longitudinal and transverse cleavage; the distribution of matter in prismatic columns, \&c.; are found in inorganic and organic substances alike. The arrangements in question obtain in plants and animals, and also in the matter from which they are originally formed. This bespeaks a common origin, and the operation of similar laws in the inorganic and organic kingdoms. It testifies to the oneness of the universe as regards its dead or "brut" matter, and its living or vitalised matter.

\section{$\S 2$. Unity of Plan in Nature as regards Form and Colour: so-called Mimicry.}

It follows that there is, within limits, a unity of plan as between the productions of the inorganic and organic kingdoms. The unity of plan extends not only to the objects found in the inorganic and organic kingdoms but also to the colours of the objects forming these kingdoms; and many, if not all, of the colours of plants and animals attributed to mimicry may, it appears to me, be referred to the unity of plan indicated. The prevalence of formtypes and colour-types in nature all points to harmony and design.

Certainly the infinite variety of tints, and the gorgeous display of colour witnessed in the heavens, in flowers, feathers, hairs, shells, minerals, \&c., cannot be accounted for by any theory of mimicry or imitation. Harmony of form and colour in the inorganic and organic kingdoms is a proof of unity and unity only. The harmony in question can scarcely be regarded as capricious or accidental, or even as the result of effort on the part of the things constituting the harmony.

Animals have been said to imitate their surroundings in order to avoid detection and so save themselves from their enemies. The leaf-insect is stated to assume the characteristics of a leaf, and the stick-insect of dead branches. This reasoning could not apply to the brain-coral, and the transverse section of the tooth of the labyrinthodon, both of which are literal transcripts of the convolutions of the human brain. Neither could it account for Venus's flower-basket structurally resembling the ventricles of the heart, or the spicules of sponges resembling certain crystals, or crystals and plants resembling each other. Still less could it explain how corals, which are the skeletons of living things, resemble basaltic rock formations, and how the latter resemble the prisms found in the enamel of teeth, in the fasciculi of voluntary muscles, in the honeycomb, and other cell structures.

All these resemblances are to be referred to a law of development, and a general plan, which applies both to the inorganic and organic kingdoms. Neither plants nor animals have the power of imitating or growing like anything but themselves. Such power implies design and creative agency outside both. The living thing cannot fashion itself or assume fantastic shapes and colours as apart from a Creator and a general scheme of form and colour. The power of mimicry for protective purposes is attributed to a comparatively few plants and animals. What is to be said regarding the majority of plants and animals not so protected? The question naturally arises, Why should the few enjoy powers and privileges not possessed by the many? If the colour and spots of certain flat fishes resemble the sand and gravel on which they rest, and the plumage of the grouse and ptarmigan resemble the heather and the snow, it is because there is a scheme of colour in creation as there is a scheme of form. The presence of the most exquisite colours in the skies, and in minerals buried deep in the earth, as well as in plants and animals and their tissues, attests the accuracy of this observation. If the chameleon changes colour in a living, natural condition, the dolphin does the same when dying. Mimicry affords no explanation. Moreover, there are myriads of plants and animals with bright colours, which, while they are effective and appropriate objects in the landscape, and contribute to the general harmony, would mark them only as objects to be destroyed by their natural enemies The explanation lies deeper than mere mimicry. The power to assume what are virtually new shapes and colours, as explained, does not inhere in plants and animals, but is conferred on them by the Creator. The same is to be said of developing plants and animals. Every differentiation, every change and modification, of furm and colour, is predetermined. Like can only beget like. The leopard cannot change its spots, neither can the zebra change its stripes. 


\section{LINES OF FORCE IN CONNECTION WITH MAGNETISM, ELECTRICITY, OPTICAL PHEN()- MENA, \&c., AS BEARING UPON STRAIGHT-LINE, CURVED, AND SPIRAL FORMATIONS IN PLANTS AND ANIMALS}

The peculiar straight-line, curved, and spiral formations due to radiating, concentric, and other arrangements witnessed in crystals, plants, and animals, which are discussed and illustrated in the early sections of this work, have their representatives in the physical universe. They are seen to advantage in the action of magnets, electricity, \&c., upon iron-filings and other substances. Generally speaking, they correspond to the "lines of force" produced by these agencies, and are physically and physiologically of the greatest possible importance, from the fact that they are fundamental and, within limits, common to animate and inanimate bodies. The distribution of matter in the organic and inorganic kingdoms opens up a wide subject, but it has such an obvious bearing on a First Cause and Design that it is necessary to treat it more or less exhaustively. It is now known that almost all substances are magnetic, and the relation between magnetism and electricity is of the closest possible kind; indeed the majority of physicists believe that they are different forms of one and the same thing. It is necessary to take up these subjects more or less in detail. ${ }^{1}$

To Franklin, Beccaria, Galvani, Oersted, Davy, and Faraday primarily, and to Maxwell, Kelvin, Helmholtz, and Henry secondarily, is due most of our knowledge regarding magnetism and electricity.

The researches of Heinrich Hertz have confirmed the modern conceptions of these subjects. Professor H. Ebert in a recent work ${ }^{2}$ has further simplified matters by utilising the conception of lines of force in the systematic exposition of the phenomena concerned. He lays special emphasis on three fundamental ideas, namely, $(a)$ that of lines of force; (b) that of energy in a magnetic or electro-magnetic field; and $(c)$ that of the symmetry of a medium which is the seat of magnetic force. He points out that magnetic attraction is not due to fluids concentrated at certain points, but to pressures and tensions in the field surrounding the attracting bodies. According to him, "When an electrical current is flowing, the seat of the phenomenon is not the conductor merely : the most important part of the whole phenomenon takes place in the field of magnetic force surrounding the conductor. It is accordingly to these fields, and the forces continuously propagated through them from point to point, that the highest interest is attached."

\section{\$22. The Lodestone.}

In considering the subject of magnetism and electricity and lines of magnetic force it is necessary to say a few words regarding natural and artificial magnets.

The natural magnet, or lodestone, which is a certain native oxide of iron, termed magnetic iron ore, possesses the remarkable property of attracting iron-filings.

When or by whom this property was originally discovered is not known, but the Greeks called such iron ore $\mu a \gamma \nu \bar{\eta} \tau \iota s$, from the name, as it is supposed, of a shepherd who first observed its property.

Pliny describes it under the name of " magnes," a term derived from Magnesia, a province of Lydia in Asia Minor.

This iron ore consists chiefly of the two oxides of that metal $\left(\mathrm{FeO}, \mathrm{Fe}_{2} \mathrm{O}_{3}\right)$ or $\mathrm{Fe}_{3} \mathrm{O}_{4}$.

The term lodestone has been derived from leading stone, i.e. "lode," a way, for the directive properties of the magnet were for many years utilised for navigation purposes before its other properties were understood. Many of these other properties, but to a far less extent, are exhibited by the ore of nickel, cobalt, and a few other metals.

The powers possessed by the natural magnet are extraordinary and peculiar. Thus it is found that if a natural magnet be dipped in iron-filings, tufts and strings of the iron-filings adhere to it, especially at its corners and edges. The magnet exerts a special influence on the filings, and the tufts and strings of filings increase in length; the particles attached acquiring the properties of the lodestone and attracting others. The force exerted is greatest nearest the magnet. It is diminished as the magnet is receded from; a point being reached when the attracting

1 The foregoing was written by me in the summer of 1902 . Early in November 1905 I received in due course my copy of the "Proceedings of the Royal Society," in which I found a paper with three plates by Marcus Hartog, M.A., D.Sc., entitled "The Dual Force of the Dividing Cell " (Part I. . The Achromatic Spindle Figure Illustrated by Magnetic Chains of Force). As this paper traverses much of the ground occupied by me more than two years before Mr. Hartog's paper appeared, and as his plates and figures, in some cases, greatly resemble my own, I deem it important to mention the fact. There is this difference between us: Mr. Hartog confines his observations and illustrations to the "dividing cell" as seen in reproduction. I, on the other hand, endeavour to establish a parallel between vital and physical lines of force as witnessed not only in reproduction, but also in growth generally, as seen in plants and animals at every period of their life histories. In other words, I endeavour to show that every part of plants and animals has its analogue in the inorganic kingdom. I give a wider scope to the inquiry, and point out that the general shape of plants and animals, and the particular form of all their parts, have their prototypes in the inorganic kingdom. I do not, however, attempt to identify electricity and life, or to substitute physical force for vital force. These I regard as separate entities.

2 "Magnetic Fields of Force-An Exposition of the Phenomena of Magnetism, Electro-magnetism, and Induction based on the Conception of Lines of Force." Translated by C. V. Burton, D.Sc. London, 1897. 
force is so feeble that the chain of filings breaks. "The adherent tufts of iron-filings are apparently not only subject to the influence of gravity, but are themselves the seat of some special kind of influence. Between the separate particles some modifying cause is evidently at work; they possess a certain rigidity. If we try to separate them from the lodestone they become drawn out into chains, the filings adhering one to another in succession: the magnetic influence is taken up by the chains of particles, and transmitted from one to another." In fact each particle of iron becomes a separate magnet.

The power exerted by the lodestone differs from that exerted by other bodies. If the finger or other substance be presented to the filings, some may adhere, but they do so in quite a different way, and in a smaller degree. If the lodestone be dipped in sand, or in filings of copper or brass, there is no special action of the lodestone in these cases, but only that form of cohesion which exists between all bodies in contact. It is not to be inferred from the last experiment that sand, copper, and brass cannot be brought under the magnetic influence : only this, that they are feebly magnetic when compared with the iron-filings, which are strongly magnetic, and are consequently the most convenient for illustration and the construction of magnetic diagrams and pictures.

Faraday pointed out that all bodies (even gases) are magnetic, and he divided them into dia-magnetic and paramagnetic, according as they arranged themselves across the poles or parallel with the poles of a horse-shoe magnet.

The lodestone or natural magnet can convey its properties to other substances. Thus, if a piece of steel, say a sewing or knitting needle, or the blade of a knife, be stroked in a given direction and for a certain time by a magnet, the needle and the knife become magnetised. The magnetic properties of the lodestone are conveyed to the needle and knife, and they become artificial magnets. Similarly, in the case of frictional electricity, if a stick of sealing-wax or a piece of amber be vigorously rubbed with a piece of silk, the sealing-wax and amber attract light substances, such as hairs, feathers, and cork, to them. They acquire properties not previously possessed by them. It is thus possible to produce artificial magnets. The most common forms of artificial magnets are those known as bar-magnets and horse-shoe magnets : the former consisting of a straight bar of steel with a north pole at one end or extremity, and a south pole at the other end or extremity; the latter consisting of a bar of steel bent like a horse-shoe with its poles approximated. The horse-shoe magnet is the more powerful. In both forms of magnet the lines of force pass from north to south pole and consequently the north pole attracts the south pole and vice versa. To the bar and horse-shoe magnets, the important electro-magnet is to be added.

By means of iron-filings the influence exerted by the different parts of a magnet and the lines of force generated in each particular locality can be traced.

Magnetic and electric currents are produced in various other ways---by the action of acids on different metals united by a wire, by the application of heat and cold to different metals joined at two points, by friction, by induction.

Magnetism, electricity, heat, and light are now regarded as forms of motion, and are closely allied to each other. They are all dependent for their manifestation on matter in a highly minute state of division; the particles and forces inhering in them acting and reacting on each other in rapid and constant succession. ${ }^{1}$

In estimating the lines of force it is of primary importance to eliminate, as far as possible, the effect of gravity in the particles, and this is most readily done by scattering the iron-filings over a sheet of white paper placed on a horizontal support in the region of magnetic influence. The filings are best distributed from a sieve held above the paper, as this ensures a greater or less degree of uniformity. When the magnet is placed on the paper with the iron-filings, and the paper gently tapped, the filings arrange themselves in beautiful curved lines known as Faraday's lines of force.

The pictures produced by the action of magnets on iron-filings had been observed prior to Faraday's time, but he was the first to determine their true nature and value.

These pictures supply, as it were, a chart of the lines of force generated by the magnet in the first instance, and by the particles of iron in the second instance.

Very satisfactory pictures of iron-filings can be obtained by employing ferrum pulveratum or ferrum redactum shaken from a small linen bag on a horizontal sheet of smooth white cartridge paper. When the lodestone is applied and the picture completed, the iron particles indicating the lines of force can be fixed by spraying the paper with a thin solution of white shellac varnish. The picture so produced and fixed can be photographed and preserved for future study and reference.

1 "When a Leyden jar is discharged the knobs of the jar become alternately positive and negative. The medium around the jar is therefore polarised alternately in opposite directions. This polarisation starts from the knob and spreads through space, at each point of which there are to-and-fro motions, and waves of opposite polarisations are sent through the mediun, carrying the energy which had been stored up in the Leyden jar. There is a periodic or to-and-fro movement in the ether, and if we could make a Leyden jar of molecular dimensions, charge it, and discharge it, we could produce a periodic movement in the ether which is analogous to that which occurs in the propagation of light. Maxwell's electromagnetic theory of light supposes that the periodic motions which constitute light are of the same nature as those which arise when the positive and negative conditions of the ether are rapidly alternated in the case of the discharge of a Leyden jar. Light, heat, and electricity are therefore manifestations of electro-magnetic waves which come to us from the sun." ("What is Electricity?" By John Trowhidge, Rumford Professor, Harvard University. International Science Series, 1897, pp. 265, 266.)

VOL. I. 
Another plan is to cover a sheet of paper with a thin layer of paraffin-wax, form the line of force picture, and fix it by gentle heat. The pictures of iron-filings may also be photographed directly by sprinkling the finest iron dust over the gelatine film of a bromide of silver dry plate in a dark room, by placing the magnet which produces the lines of force on the dry plate, and by tapping the edges of the plate gently with a glass rod. When the picture is formed the lodestone is removed, the plate exposed, and the image fixed in the usual way.

I append two plates (Plates lv. and lvi.) containing lines of force representations of iron-filings produced by magnetic action; my object in the present instance being to direct attention to the existence of "lines of force" in the inorganic kingdom in relation to similar lines of force which I believe exist in the organic kingdom, as indicated by the movements, growth, and structures in plants and animals. The inorganic lines of force can also be traced in electric and analogous discharges (lightning flashes, electric sparks), in the formation of crystals, frost pictures, dendrites in minerals and metals, \&c., as seen in Plates xxix. to xxxvi. inclusive.

\section{"LINES OF FORCE" PLATES (Plates LV. and LVI.)}

These plates contain representations of "lines of force figures" obtained by experiments with natural and artificial magnets on iron-filings and other substances. The lines of force figures remarkably resemble in their general arrangement, and even in their details, similar figures met with in crystals and in the several parts of plants and animals. They point to general laws which govern equally the inorganic and organic kingdoms. They reveal radiating or stellate, curve, concentric, circular, and spiral arrangements, all of which are found in dead and living matter alike.

All substances are magnetic (dia-magnetic and para-magnetic), and it is difficult to say what precise part magnetism and electricity play in the disposal, arrangement, and movements of the ultimate atoms and molecules of animate and inanimate bodies.

That the rôle performed by magnetism and electricity in the arrangements and movements of ultimate matter is a leading and important one cannot be doubted. This becomes more apparent as the circle of the sciences widens, and the relation of inorganic to organic matter becomes better understood.

The "lines of force figures" are from Professor H. Ebert's work "Magnetic Fields of Force," translated by C. V. Burton, D.Sc. (London and New York, 1897), but the inferences drawn from the figures are those of the Author only.

\section{PLATE LV}

FIG. 1.-The dark, radiating, nearly straight and curved lines in this figure are dne to the influence exerted by the lodestone or natural magnet on iron-filings uniformly distributed over a horizontal sheet of white paper. They represent the lines of force; in other words, the arrangement of the iron particles by the lodestone. The white patches $a, b, c, d$ indicate the absence of iron-filings, these having adhered to the lodestone when it was removed after the formation of the lines of force picture.

FIG. 2.-Lines of force figure produced by the action of an artificial horse-shoe magnet on iron-filings. $s, n$, The poles of the magnet; $i$, region of least attraction or indifferent zone of the magnetic field. The lines of force are most marked at the poles. Here they radiate in nearly straight and in graceful curved lines: the nearly straight lines proceeding from the outer corners of the polesthe curved ones from the upper ends of the poles. At the central portions of the magnet the lines are nearly straight and transverse. At the bend of the magnet the lines curve round and follow generally the outline of the bend. The lines in this region are less under the influence of the magnet, and form its indifferent zone (i).

FIG. 3.-Lines of force figure produced by the action of a lodestone or natural magnet on iron-filings: the poles of the magnet being armeri with pole pieces and rods of soft iron to concentrate and increase their power. $s, n$, The poles of the magnet. In this figure, the radiating and curved arrangements are seen to perfection. The curves are best seen between the poles. They resemble centres of development in crystals and of growth in plants and animals. Compare with Plates i., ii., iii. and iv.

FIG. 4.-Lines of force figure produced by the action of an artificial round bar-magnet on iron-filings when the bar-magnet is held in a vertical position. Shows concentric and radiating arrangements of the iron-filings similar to those met with in crystals, in the stems, branches, and other parts of plants, and in the bones, teeth, and other parts of animals in cross section. Compare with figures in Plates i. to v. inclusive.

FIr. 5,- Lines of force figure of the earth considered as a natural magnet. $x$, Centre of the earth. The axis of the earth is indicated by the short oblique lines proceeding inward from the periphery of the figure. $n, s$, North and south poles of the earth, where the lines of force are most numerous and intense: they radiate and curve round, those from the north pole curving round and re-entering the earth at the south pole. The lines of force are feeblest at $a, a$; those regions representing the indifferent zones of the magnetic field.

FIG. 6.-Lines of force figure prodnced by an artificial bar-magnet acting on iron-filings as seen in a horizontal plane parallel to the axis of the magnet. $\varepsilon, n$, The polar regions, or centres of greatest magnetic intensity; $j$, the centre of least magnetic intensity (indifferent zonc). Shows the radiating, nearly straight, and curved arrangemeuts in a striking manner. The curves are most marked letween the poles, where they form incipient concentric rings; they are least marked and straightest at the outer edges of the poles. The arrangement is that seen in the formation of many crystals and at growing centres in plants and animals.

FiG. 7. - Lines of force figure obtained by two conductors passing perpendicularly through a plane of paper and iron-filings, with a little bar-magnet or needle placed near, whose axis points directly towards the axis of the conductors. $c_{1}$, $c_{2}$, Spots at which the conductors pass perpendicularly through the paper and iron-filings. At $c_{1}$ the current passes from below upwards and eninbraces the conductor in a direction the reverse of that in which the hands of a clock move. At $c_{2}$ the current passes from above downwards and 
PLATE IV

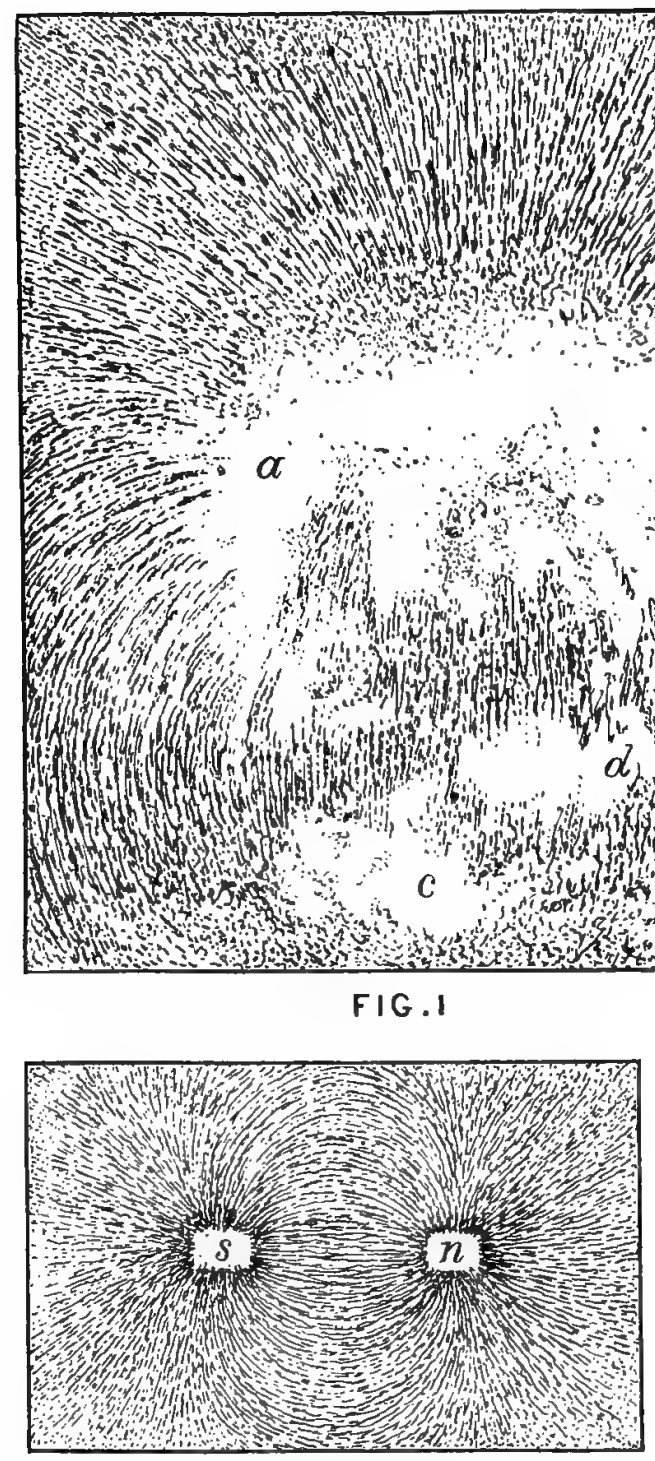

FIG.3

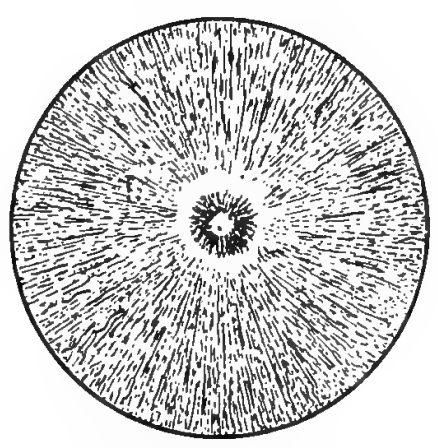

FIG. 4

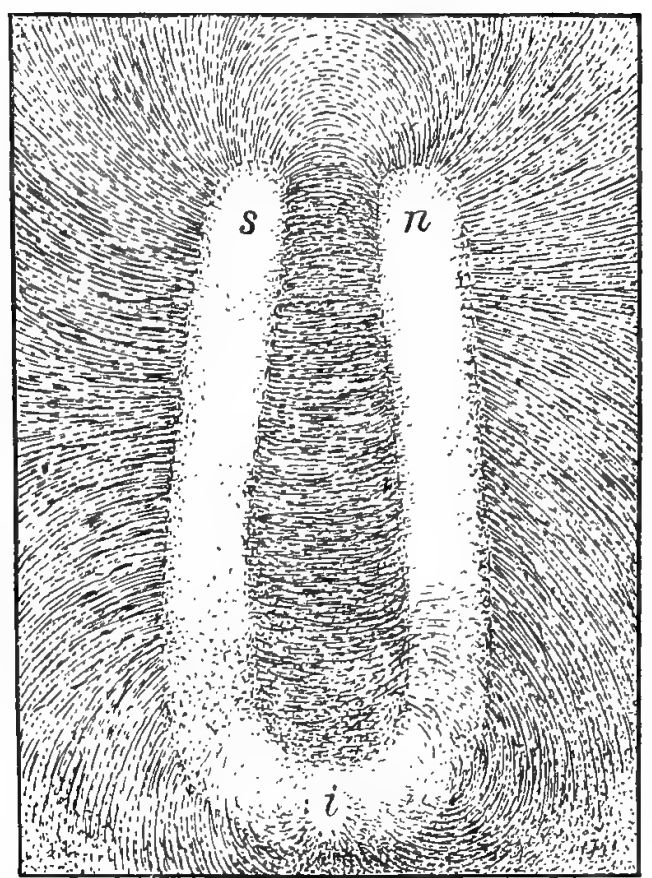

FIG . 2

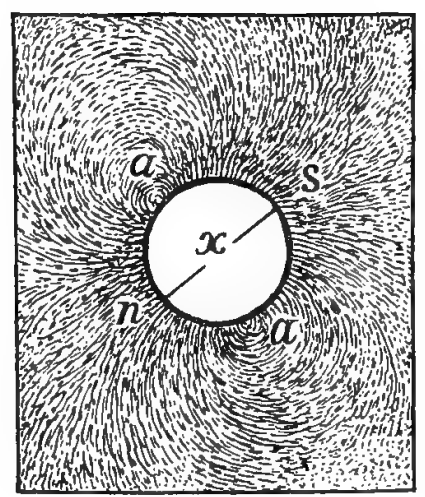

FIG. 5

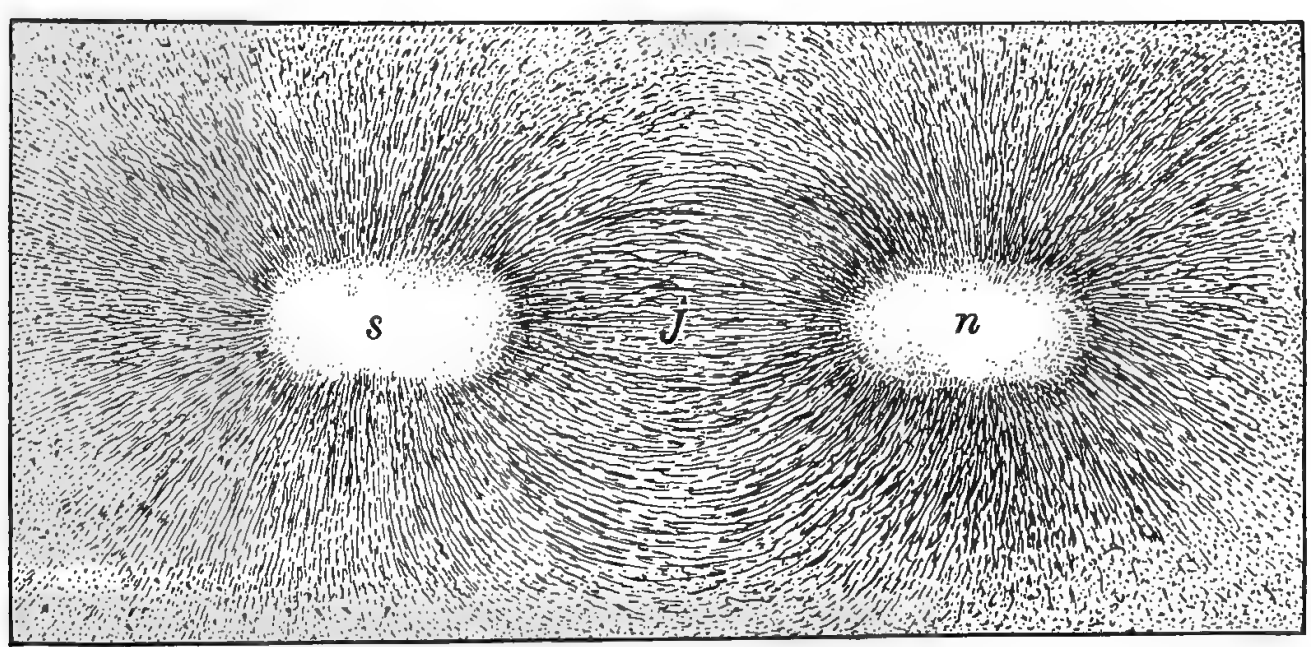

FIG. 6

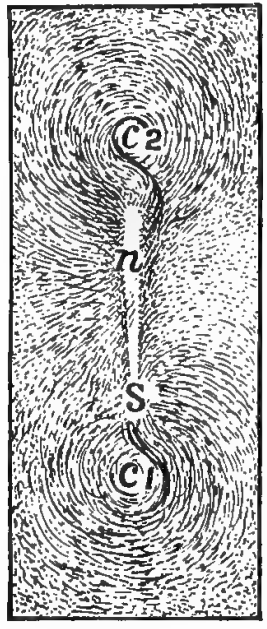

FIG.7 


\section{PLATE LV (continued)}

embraces the conductor in an opposite direction. Two systems of opposite concentric rings are produced. Between the points $c_{1}$ and $c_{2}$ lies the needle $n, s$, its position being indicated by the disturbance of the lines of force. Two bundles of lines of force are attached to the needle, due to the current passing in opposite directions. These meet in the middle of the magnet and form an indifferent zone. The tension along the lines of force tends to deflect the needle out of the plane of the current, causing the north pole $(n)$ to move downwards and the south pole $(s)$ upwards.

This figure shows that lines of force are influenced and modified by the presence of foreign bodies. Bodies act and react on each other, and so of movements.

The figure illustrates the spiral, figure-of-8, and circular arrangements frequently met with in the tissues and organs of plants and animals, and in the movements of walking, swimming, and flying.

\section{PLATE LVI}

FIG. 1.-Lines of force figure produced by the action on iron-filings of two magnets with their north poles turned towards each other. In this case the magnets $\left(M_{1}, M_{2}\right)$ and lines of force repel each other, as indicated by the darts $\left(i_{1}, i_{2}\right)$. n $n_{1}, n_{2}$, The north poles of the magnets ; $s_{1}, s_{2}$, the south poles of the magnets $; \dot{j}$, indifferent or zero point caused by mutual and equal repulsion. At this point a short magnetic needle is not influenced or exposed to any directive agency. This figure shows that the lines of force proceeding from each north pole $\left(n_{1}, n_{2}\right)$ are bent back on encountering each other, and curve sharply round, and at points become more or less parallel.

FIG. 2.-This figure is the opposite of Fig. 1. In this case the north and south poles of two magnets $\left(M_{1}, M_{2}\right)$ are directed towards each other, with the result that the lines of force are attracted or drawn towards each other as indicated by the darts $\left(i_{1}\right.$, $\left.i_{2}\right)$. $n_{1}$, $n_{2}$, the north poles of the magnets ; $s_{1}, s_{n}$, the south poles of the magnets. This figmre shows that the lines of force proceeding from the pole $n_{1}$ of the one magnet $\left(M_{1}\right)$, bend round and unite with the lines which terminate in the pole $s_{2}$ of the other magnet $\left(M_{2}\right)$. In the interspace between the anlike poles of the two magnets $\left(n_{1}, s_{2}\right)$, the lines of force are crowded together and very Jittle bent or curved (j). In the more outlying parts of the field they are more spread out, and pass in wide curves from one pole to the other. The lines of force are less spread out and curved than in Fig. 6, Plate lv.; a circumstance due to tension along the lines of force and to cross pressure.

Figs. 3 and 4 are produced by two bar-magnets of equal power fixed in a vertical position at different distances from one another; the poles being like or unlike according to arrangement. In Fig. 3 the poles are unlike $(n, s)$, whereas in Fig. 4 they are like ( $n$, $n$ ). In Fig. 3 the iron-filings radiate from the unlike poles $(n, s)$, and bend towards each other in graceful curves between the poles; the curves becoming larger as the mesial line between the poles is receded from. In Fig. 4 the iron-filings radiate, but the curves formed by them in the interspace $(j)$ between the poles $(n, n)$ are absent; a circumstance due to like poles and the lines of force proceeding therefrom repelling each other. The rule in such cases is, "Like poles repel and unlike poles attract." The interspace (j) forms an indifferent zone. In Figs. 3 and 4 the action and reaction of the poles on each other is very marked. The figures illustrate the effects produced by attraction and repulsion respectively, and how plant and animal structures and movements may be modified.

FIG. 5.-Lines of force figure produced by the action of the unlike poles of two bar-magnets adjacent to one another on iron-filings. The lines of force proceeding from the poles $\left(n_{1}, s_{1}\right)$ of the one magnet bend round until they reach the poles $\left(n_{2}, s_{2}\right)$ of the other magnet. They unite with the lines proceeding from the latter poles, and establish a connection between the two bars-the lines of force near the poles being almost straight. In the space outside the nagnet the lines of force are bent, and form larger and larger curves, because of pressure perpendicular to their direction. $i_{1}, i_{2}$, The different zones of the magnetic field; $j$, region where the lines of force are absent.

In the case of two bar magnets with like poles adjacent to one another the lines of force which proceed from the poles $\left(n_{1}\right.$, $\left.s_{1}\right)$ of the one magnet curve back on meeting the poles $\left(n_{2}, s_{2}\right)$ of the other magnet, and in so doing largely avoid the space between the two magnets and run parallel.

The lines of force in Fig. 5 very closely resemble similar lines seen in centres of development in crystals, and lines of growth in plants and animals.

F1G. 6.- Lines of force figure obtained in a plane perpendicular to a rectilinear conductor. In the middle of the picture is seen the circular section $(c)$ of the cylindrical wire; the iron-filings having arranged themselves in complete rings embracing it concentrically. There are no points at which the lines of force originate or terminate; in other words, there are no sources or sinks in the field. The force exerted is greatest in the vicinity of the wire, where the iron-filings are aggregated and form a dark ring: the force decreases as the wire is receded from, the rings of iron-filings becoming larger and more faint in a direct ratio to the distance. "All the lines of force due to a current in a straight wire lie upon cylindrical surfaces whose axis coincides with that of the wire." This figure has an olvious bearing on the formation and growth of all concentric structures, as witness the cross sections of many crystals, the stems, branches, and other parts of plants, and the bones, teeth, and other parts of animals.

FIG. 7.- Shows a lines of force diagram with a current flowing round a ring. The arrangement consists of a horizontal sheet of paper with iron-filings, through which a thick copper circular wire, the ends of which are not quite in contact, runs in a perpendicular direction. When a current is sent round the wire in the direction $(+$ to -$)$ indicated by the darts, the concentric lines of force seen within and without the circular copper wire are obtained : they are more crowded within than on the outside of the wire. The actual lines made by the iron-filings are given at Fig. 8 .

FIG. 8.--Lines of force figure produced by a current sent along an open copper ring runuing perpendicularly through a horizontal sheet of paper with iron-filings. According to Professor Ebert, "The direction in which the lines of force are to be positively reckoned may be easily deduced from the positive direction along the conductor (axis of force). If this latter direction is considered clock-wise from our point of view, the lines of force will encircle the left limb of the wire clock-wise as seen from above. These lines, then, as they spread through the ring are directed towards the observer, the same being true of the lines which encircle the right limb of the wire, and, in fact, all the lines of the system. Thus, a translation in the direction of the magnetic force, combined with a rotation in the sense of the current, would constitute a right-handed screw motion. We may say that a current and the lines of induction which it produces enbrace one another right-handedly" (Ebert, Part I. p. 193).

This lines of force figure bears a general resemblance to cross sections of various parts of plants and animals.

FIG. 9.-Lines of force figure produced by the action of a conducting wire and the south pole of a bar-magnet on iron-filings. The presence of the sonth pole $(s)$ of the magnet deforms or disarranges the field of concentric magnetic lines of force produced by the action of the conductor $(c)$, and gives rise to a spiral arrangement of the iron-filings. Contrast with Fig. 6, where the magnetic field is undisturbed. The current passes through the plane of the picture from below upwards. "Of the lines of force which densely encircle the cross section of the conductor' $(c)$ a considerable number end at the 'sink' $s$ (south pole of bar magnet). Here they leave the open 
PLATE LVI

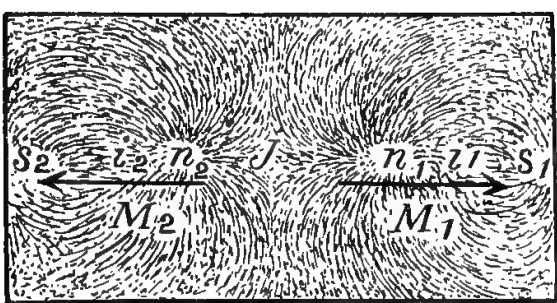

FIG.I

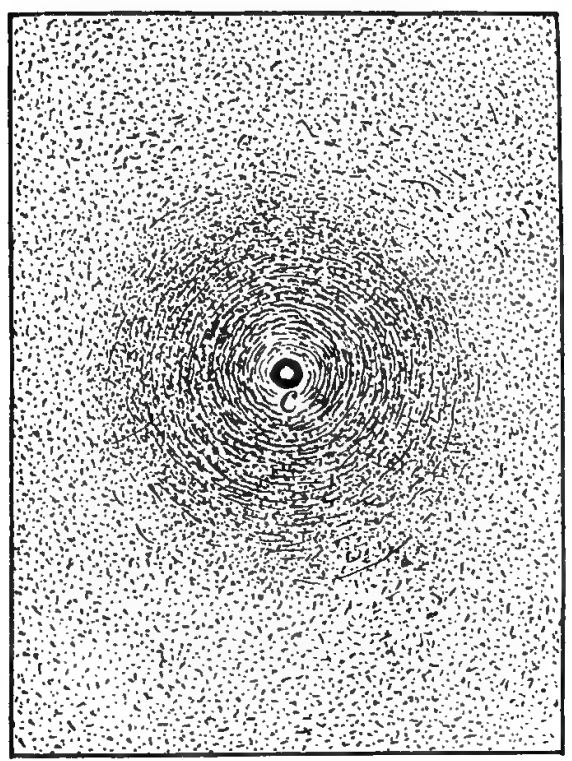

FIG. 6

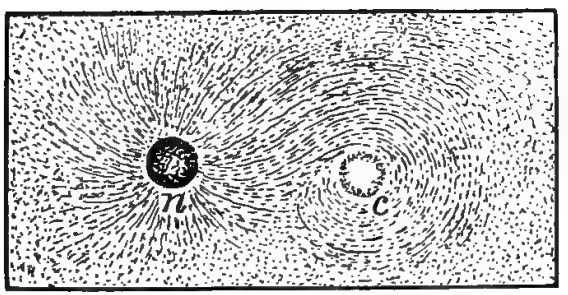

FIG. IO

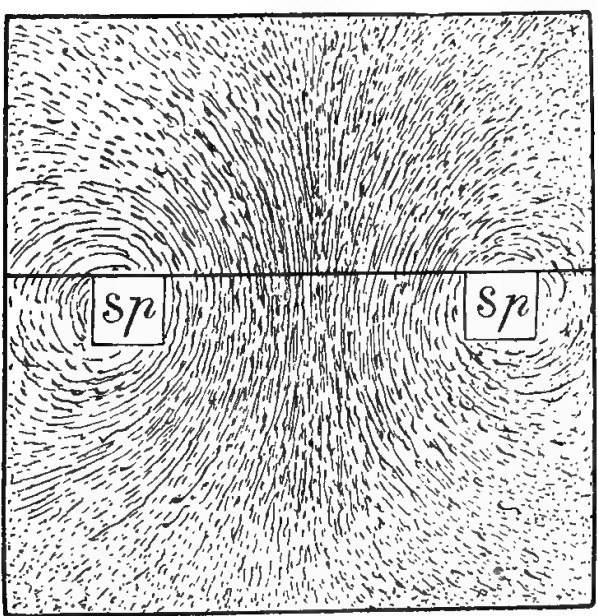

FIG. 14

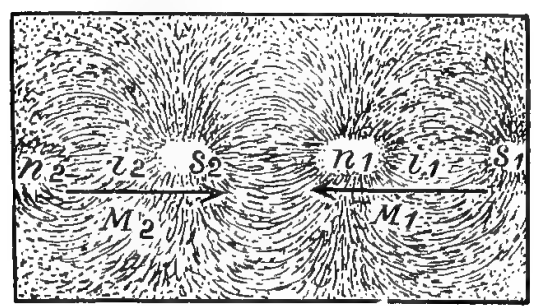

FIG. 2
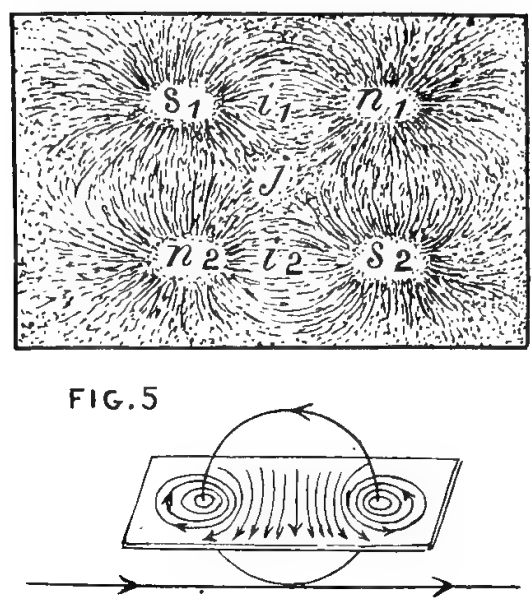

FIG. 7

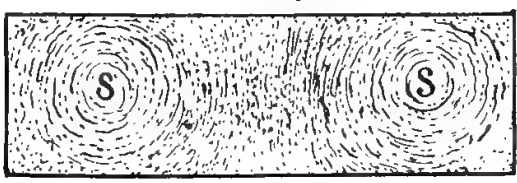

FIG 8

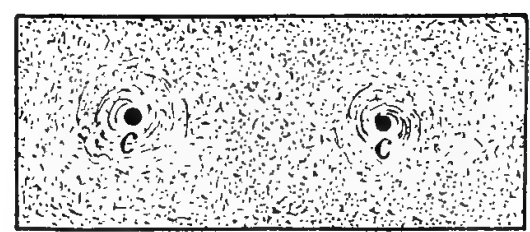

FIG.II

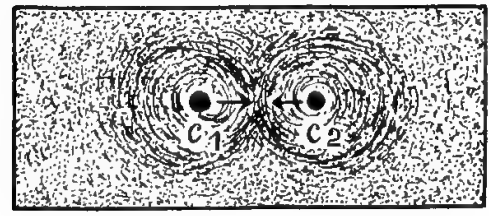

FIG. I2

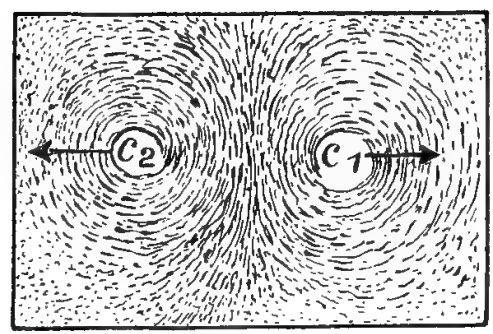

FIG.15

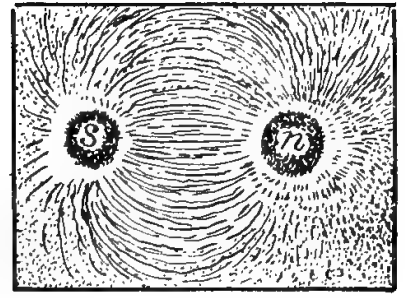

FIG.3

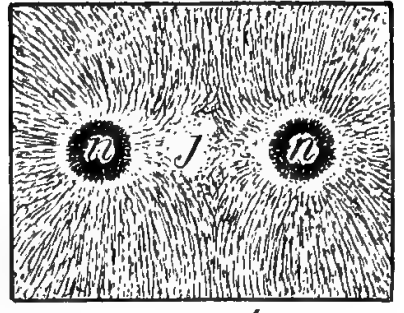

FIG. 4

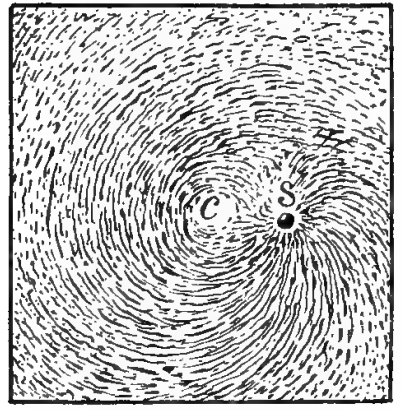

FIG. 9

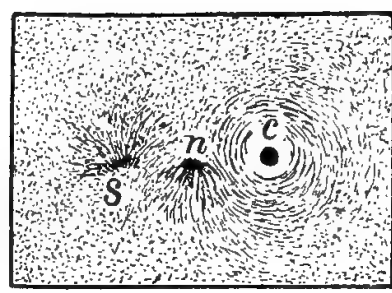

$F \mid G .13$

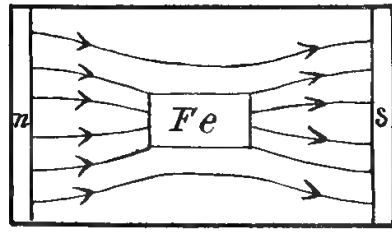

FIG.16

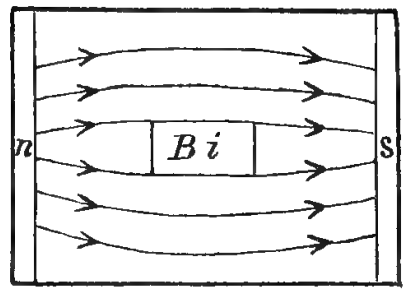

FIG.17 


\section{PLATE LVI (continued)}

field and pass into the substance of the magnetised steel bar; a place of no magnetic force making its appearance just above $s$. Beyond the range of the sink $s$, the lines of force bend round in wide curves, passing through the undisturbed side of the field and completely encircling the conductor" (Ebert, p. 208).

This picture shows how concentric lines of force can be directed and converted into spiral lines by the presence of a foreign body. Spiral arrangements occasionally occur in crystals, and are very common in plants and animals. Spiral structures and spiral movements are everywhere present in the inorganic and organic kingdoms, as witness the whirlpool, sand-storm, cyclone, and the movements in walking, swimming, and flying.

Fig. 10.-Lines of force figure produced by a conductor and the north pole of a bar-magnet acting on iron-filings. In this case the concentric lines of force investing the conductor $(c)$ become mixed up with a certain number of the lines of force proceeding from the north pole $(n)$ of the bar-magnet. At $c$ there are concentric lines of force, and at $n$ radiating lines of force. In the interspace between $c$ and $n$, the two systems of lines become mixed and blend together. In the lower part of the picture there is a space nearly free fron lines of force. The current passes in a downward direction clock-wise round $c$. This tigure shows a transition as between radiating and concentric lines of force which results in a certain degree of spirality.

FIG. II.-Lines of force figure produced lyy the action of two current-conductors (placed widely apart) on iron-filings. When the conductors are separated by considerable intervals they do not disturb each other's magnetic fields. 'Thus in cross sections the lines of force investing each conductor are disposed in concentric circular lines; each system of lines being complete in itself, and not being influenced or modified by the other system. It is otherwise when the conductors are placed near each other, as seen at Fig. 12, which see. This figure resembles the arrangement found in cross sections of the vascular bundles of plants and the blood-vessels, \&c., of animals.

FIG. 12.--Lines of force figure produced by the action of two current-conductors (placednear each other) on iron-filings. In this case each of the two axial fields extends into the range of the other, with the result that the two fields are disturbed and considerably modified. Provided the currents of the two conductors flow downwards their lines of force run clock-wise around $c_{1}$ and $c_{2}$. Between the conductors their annular lines of force run in contrary directions, and tend to cross each other figure-of-8 fashion. Exactly in the middle, between $c_{1}$ and $c_{2}$, there is a point where the two halves of the figure-of- 8 curves meet. Within the two halves the lines of force are in the form of rounded ovals, with elongated ends turned towards each other. Outside the two sets of ovals with the elongated ends the lines of force pursue a common, elongated, oval course.

This figure is important, as showing that figures-of- 8 may be produced from rotatory movements in opposite directions, and lyy the juxtaposition of the current conductors. Figure-of- 8 structures and movements are very common in animals, especially in the hollow viscera and organs of locomotion.

Fra. 13.-Lines of force figure produced by a conductor and a bar-magnet actingr on iron-filings. The lines of force of the current produce a concentric system of rings seen at the cross section of the conductor $(c)$. At the side where the magnet is, with its source $n$ and $\operatorname{sink} s$, the two fields are superposed. 'The current flows from below upwards, through the plane of the figure, the lines of force, viewed from above, encircling the conductor-currents counter-clock-wise, as nay be seen from their behaviour on encountering the poles $(n, s)$ of the magnet. If the magnet is free to rotate about its middle point it is deflected. This figure shows conclusively how conductor's and magnets mutually influence each other.

FIG. 14. - "Shows a lines of force diagram for a ring-shaped coil, in a plane passing throngh the axis of symmetry. , hi", Sp are the places where the coil intersects the plane of the diagram, on either side of which it projects semicircularly. The lines of force surrounding all the separate turns follow approximately the same course, and unite to form continuous curves, those which niost closely embrace the cross section of the coil being nearly concentric with it. On the other hand the more remote lines, owing to the pressure of the inner ones, are bowed out excentrically into widely spreading curves. In the neighbourhood of the axis of the coil the field is approximately uniform" (Ebert, pp. 241-2).

Fig. 15.- Lines of force figure produced by two oppositely-directed parallel currents and their electrodynamic action on one another (repulsion). i" 1 , , Cross sections of the two conductors. Provided with current along $c_{1}$ from above downward through the plane of the figure, its lines of force follow the hands of the clock. The current along $c_{2}$ in this case flows from below upwards, and in a direction opposed to the hands of the clock. In the mesial line between the conductors $\left(c_{2}, c_{1}\right)$ the lines of force which run side by side have the same direction. Their tensions and pressures are added together. The annular rings of force because of mutual pressure are crowded together in the direction of the mesial line, and bulged out in a contrary direction, and cease to be strictly concentric, as happens in Fig. 6, Plate 1v. The pressure across the lines of force tends to drive the two current conductors apart, as indicated by

FIGS. 16 and 17,-Show the disposition of the lines of force in para-magnetic and dia-magnetic bodies. Thus in iron ( $F e)$, which is a para-magnetic substance, the lines of force from the north pole $(n)$ of a magnet converge hefore the iron is reached, and diverge after passing through it. The ends of the iron facing the north and south poles become a source and a sink. In bismuth (Bi), which is a dia-magnetic substance, the lines of force behave quite differently: they diverge on reaching the bismuth and converge after liaving passed it. They pass for the most part throngh the surrounding medium. In the case of the iron there is axial or polar setting: in the case of bismuth equatorial setting. Different substances are differently affected by magnetism, and the substances in turn react upon the currents: the lines of force in the case of para-magnetic bodies are made to converge and diverge; whereas in dia-nagnetic bodies they are made to diverge and converge. These opposite effects are of the utmost consequence in the distribution of the ultimate atoms and movements of inorganic and organic matter. They prefigure the opening or expanding centrifugal structures and movements, and the closing or contracting centripetal structures and movements, evervwhere observable in plants and animals.

The iron-filing pictures afford indications of the direction and magnitude of the magnetic force.

There are in the iron-filing pictures what are virtually outgoing and returning lines of force. These are due to opposite properties at different points of the surface of the magnet, whereby the influences which proceed from certain regions of the magnet re-enter it at other regions. These regions are designated polar regions or poles, and are similar to the poles of the earth, "from one of which magnetic lines of force proceed outwards into space, while at the other the lines pass back into the interior of the earth."

It is convenient to speak of the points from which the lines of force issue and re-enter the magnet in greatest 
number as polar regions or poles. They are, so to speak, points of distribution and convergence. They are also foci or points of intensity.

In studying Fig. 1, Plate lv., which represents the effect produced by the contact of a lodestone or natural magnet with iron-filings equally distributed on a horizontal sheet of white paper, it is important to note the general appearance of radiation so well seen in cross sections of the stems and other parts of plants, and in cross sections of the muscles, bones, teeth, horns, and other parts of animals. It is also important to observe the first traces of curve, and circular formations, in the several parts of plants and animals. Compare with Plates xxii., xxiv., and xxvii.

The idea presented by the contact of the lodestone with the iron-filings is that of a power radiating its energy in more or less straight lines, stellate-fashion, and in more or less curved lines, concentric-fashion. Both the radiating and concentric forms of energy occur in plants and animals, and the two kinds of energy are to be credited with the radiating and concentric arrangements so common in plants and animals, and to which plants and animals largely owe their synmetry and their strength. The radiating and concentric arrangements are also seen in crystals (compare Plates i. and ii.). The radiating and concentric arrangements in question point to a common law of construction in crystals, plants, and animals.

From what has been stated it will be apparent that the iron-filings equally distributed on a horizontal sheet of white paper are acted upon and influenced by the lodestone or natural magnet. The filings form the so-called "magnetic field"; this consisting of the space to which the influences of the magnet extend. As the external surface of the magnet and the magnetic influences exerted thereby only have to be considered, it is permissible to speak of the magnetic field as confined to one or more planes. As, however, the interior portions of the magnets and the magnetic influence exerted by them are also to be considered, it follows that the magnetic field must be enlarged, the field of the magnet being extended in three directions. As a matter of fact, the magnetic field in the extended or ultimate sense surrounds or invests the magnet as a whole. It is therefore best represented as a spherical space with the magnet in the centre; the lines of force emanating from the magnet radiating into space to a practically unlimited extent.

As the polar regions or poles of the lodestone or natural magnet are unequally distributed, from irregularity of shape and other circumstances, it is necessary to direct and concentrate the magnetic influences within limited areas. This is best done by the employment of soft iron shields and rods which are readily permeable to magnetism. The iron shields and rods are applied to the polar regions of the lodestone; the polarity being transmitted to the exterior of the so-called "pole-pieces" in an intensified form.

When the pole-pieces are applied to iron-filings evenly distributed on a horizontal sheet of white paper a lines of force picture is produced, as shown at Fig. 2 of Plate lv. The lines of force picture is remarkable for its symmetry, and for the localisation of the poles. There is radiation of the iron particles round each pole as a centre, and between the poles the iron particles are disposed in what are virtually concentric lines. The radiating and concentric arrangements indicated in Fig. 1, Plate lv., reappear in a slightly modified form, and produce arrangements met with in crystals, plants, and animals.

The pole-pieces of the lodestone may be connected and the magnetic circle closed by applying a band or bridge of soft iron, known as the keeper, to them. In this case the magnetic properties of the lodestone are conserved. The keeper aggregates and transmits through it the lines of force which pass from pole to pole. It adheres to the pole-pieces and so overcomes gravity.

As will be seen, the magnetic properties of the lodestone may be intensified, directed, and conserved by the aid of the pole-pieces and keeper. Whatever the nature of the magnetic power, it is evidently non-material. This follows because the finest and most sensitive scales or balances fail to detect any increase in weight of substances after magnetisation. In other words, the substance magnetised is of exactly the same weight as the substance unmagnetised. Magnetisation can only consist of a "rearrangement of the matter already existing in the substance, or in giving to the particles of the substance, and those surrounding it, some kind of motion, most probably of a cyclical or rotatory nature; the motion not affecting the magnet as a whole, but only its component parts.

\section{§23. The Bar-Magnet.}

The production of lines of force pictures is facilitated by the employment of artificial magnets, as these can be made of suitable sizes and of convenient shapes. The simplest is the bar-magnet. This consists of an elongated bar of steel magnetised by stroking it with the pole-piece of a lodestone in the same direction forty or fifty times. Its magnetic properties are most marked at, and indeed almost exclusively confined to, its free ends; these constituting its poles. The bar-magnet, when applied to iron-filings evenly distributed on a horizontal sheet of white paper, as 
explained, produces the striking, well-defined lines of force picture seen at Fig. 3 of Plate lv. This picture resembles in its general features that seen at Fig. 2, Plate Iv., and need not be further described.

If a round bar-magnet be supported vertically on a horizontal sheet of white paper, over which iron-filings are evenly spread, a lines of force picture in a horizontal plane is obtained, as seen at Fig. 4 of Plate lv.

This figure is very remarkable, as showing a radiating and concentric arrangement of the iron-filings such as is frequently witnessed in crystals, plants, and animals, and which, as already explained, makes for symmetry and strength.

In the case of a very long, thin, bar-magnet the iron-filings attach themselves almost exclusively to its extremities, and assume the form of nearly spherical tufts : the filings between the extremities, which constitute its "indifferent zone," being scarcely at all influenced. The extremities or poles act, in a great measure, independently. The field at either end of a long bar-magnet is said to be "unipolar." In the cross sections of such a magnet the lines of force proceed radially. As many of the lines of force originate at the circumference of the end of the magnet, the iron-filings aggregate and form a black ring in the middle; the portions on either side of the black ring being denuded of filings and appearing white, as shown at Fig. 4, Plate lv.

\section{§ 24. The Compound Magnet.}

As already stated, the power of a magnet depends on the number of lines of force issuing from it; the greater the number of lines of force proceeding from a magnetically active plane, the greater their effect. The power of a bar-magnet is increased by combining it with other bar-magnets, care being taken to arrange the ends of the bars having the same kind of magnetism together; the lines of force all diverging from one end and converging again at the other end. In this way a compound magnet is formed.

There are various special magnets to which it is only necessary to allude in passing. Very thin magnets are manufactured from clock-springs by cutting a long, thin strip of steel in the form of a very elongated rhornbus and magnetising the cut portion by stroking it with another magnet, so that the poles are at the acute corners. Such a magnet forms the so-called magnetic needle so useful in navigation and in electric experiments generally.

If a short, thick bar of steel be magnetised perpendicularly to its axis, "the transversely magnetised body so obtained has polar regions on the two sides of its bounding surfaces." If the length of the bar be excessively small, a plate with poles at opposite points of its circumference is produced. A steel ring can be magnetised to have poles at opposite ends of a diameter. If a rod be strongly magnetic at both ends, and the length of the rod in the direction of the axis sufficiently reduced, a plate which has a distribution of magnetism on each face is produced. This gives rise to the "magnetic shell." In this case the lines of force proceeding from one face bend round, embrace the rim of the plate, and reach the other face, where they terminate. A long bar-magnet may be regarded as consisting of a succession of magnetic shells placed face to face, all the faces of one kind being turned in the same direction.

\section{§ 25. The Horse-shoe Magnet.}

Seeing the effects produced by bar-magnets are most marked at the ends where the lines of force are most numerous, stronger fields are secured by bending the bars into the form of horse-shoes, and so approximating the ends. In the horse-shoe magnet the ends are filed to make their terminal faces lie in one plane; the bar being magnetised along its whole length. The power of the horse-shoe magnet is increased if the magnet is made of thin steel plates separately magnetised.

If such a magnet be supported vertically with its ends upwards and a lines of force picture formed on a sheet of paper with iron-filings held immediately above it, a figure similar to that seen at Fig. 2, Plate lv., will be obtained.

The lines of force picture produced by the horse-shoe magnet is given at Fig. 2, Plate lv.

In the median plane of the magnet the lines of force proceeding from the ends $n$ and $s$ of the horse-shoe are closely crowded together, while in the space between the limbs of the horse-shoe they pass by nearly the straightest and shortest paths. Beyond this space the lines are bent out into wider and wider arcs; those proceeding from the outer portions of the ends extending to the more remote parts of the field. As the bend of the horse-shoe is reached, the lines of force proceeding from the body of the magnet are diminished in number. Finally, the lines run parallel to the bend, where very little force is exerted upon them. The bend thus forms the "indifferent zone" of the horse-shoe magnet.

In the case of compound magnets, where the magnetic forces are strong, the lines of force can be traced not only in a plane, but in three dimensions. The lines of force can also be fixed by stirring iron-filings into a warm solution 
of gelatine and placing the poles of the horse-shoe magnet in the solution. When the gelatine cools and hardens a transparent lines of force model is produced.

\section{§26. The Electro-Magnet.}

This particular form of magnet consists of a bar of soft iron (frequently bent horse-shoe fashion), placed within an extensive coil of insulated copper wire through which an electric current is passing. The combination confers greatly increased strength on the magnet; the powers being augmented in proportion to the length of wire in the coil. The electro-magnet is especially useful where interrupted or alternating currents are a desideratum, as it affords facilities for rapidly inaugurating and breaking the electric circuit. It is employed in the telephone, the telegraph, in wireless telegraphy, and in the arts generally.

\section{§27. Magnets with One Degree of Freedom.}

Hitherto the magnets producing the lines of force pictures have been fixed. It is now necessary to consider cases where the axis joining the poles of the magnet is free to rotate in a horizontal plane about its middle point, as in the magnetic needle, where one degree of freedom is permitted. Naturally, the axis of a magnet can occupy an infinitely great number of positions.

In the case of the horse-shoe magnet " one degree of freedom " is given by suspending the magnet by a thread (previously untwisted) attached to the centre of its curved portion or bend. In the bar magnet (say the magnetic needle) the same result is obtained by suspending it at its middle portion by a single untwisted fibre of silk, or setting it centrally on an agate point, which enables it to swing freely in a horizontal plane. The bar-magnet so poised, and free to move in a horizontal plane, points, when left to itself, north and south with its poles. When a magnetic needle is deflected or disturbed it makes smaller and smaller vibrations until it attains its position of rest; the one pole pointing to the north, the other to the south. The end of the needle rubbed with the north pole of an existing magnet becomes a south pole and vice versa.

"The lines of force have, at every point, a determinate direction. The north pole (N) of a fixed magnet always repels the north pole $(n)$ of a movable magnet along the lines of force proceeding from N. The south pole (S) of a fixed magnet always attracts the north pole $(n)$ of a movable magnet along the lines of force proceeding towards S."

Contrariety of direction is conveniently indicated by a plus sign $(+)$ for the positive direction, and a minus sign ( - ) for the opposite or negative direction.

If we inhabit the northern hemisphere of the earth it will be necessary to reckon positively the force exerted upon a north pole placed in the magnetic field. "In all magnets the lines of force issue from the north pole and pass through the field to the south pole, where they re-enter the magnet."

The so-called compass-needle is free to move in a horizontal plane, and also to dip vertically at either end. The horizontal or declination movements indicate the position with regard to the poles of the earth; the dip, the position with regard to the equator or central region of the earth. The magnetic needle is the essential portion of the mariner's compass, and enables the mariner to ascertain his position on the earth's surface.

"Generally speaking, the further we go north or south from the equator the more markedly does the magnetic needle point downwards, the dip increasing with the latitude. In the northern hemisphere it is the north-seeking pole that points downwards; in the southern hemisphere it is the south-seeking pole. In the neighbourhood of the equator the earth is encircled by a line, at each point of which the needle sets itself horizontally, so that the angle of dip is zero. This line is called the 'magnetic equator,' and is not coincident with the geographical equator. There are also points, one in the northern and one in the southern hemisphere, where the direction of the dipping needle is exactly vertical. From the analogy to artificial magnets, these are called the 'magnetic poles of the earth.' They do not coincide with the geographical poles. The north magnetic pole is in $70.5^{\circ}$ north latitude and $98.5^{\circ}$ west longitude from Greenwich, a point in the North American Archipelago; the south magnetic pole is about $74^{\circ}$ south latitude and $148^{\circ}$ east longitude. The line joining these poles does not pass exactly through the centre of the earth. . . The magnetic equator is continuously shifting from east to west, and with it the whole system of isoclinic lines, the angle of dip throughout Europe gradually decreasing. The total magnetic force or intensity at any given place is also changing; at some places it is becoming greater, at others it is becoming less."

\section{§28. The Compass.}

"If the declination is known for any given place, we may conversely use the magnetic needle to determine the direction of the geographical meridian, and hence the 'four points of the compass.' A form of declinationneedle convenient for this purpose is the so-called compass-needle. The needle moves over a circle divided into VOL. I. 
360 degrees or (for miners) into twenty-four hours or (for seamen) into thirty-two points. The points corresponding to north, south, east, and west are generally marked more conspicuously than the rest. The instrument is enclosed in a case, and is furnished with a contrivance, called an arrestment, which serves to lift the needle off the point when not in use.

"To the Italian, Giovanni Gioja, is due the device of attaching the needle to a divided circle, or a disc marked with the thirty-two points, thus producing the compass so indispensable at sea. The needle (or system of needles), with the disc attached, turns upon a fixed point within a case, which is closed by a glass plate. A fixed mark within the case gives the direction of the ship's head. Since the graduated dise is always maintained in the proper orientation by the needle attached to it, the steersman can read off immediately on the graduations the direction of the ship's head, that is, the direction in which he is steering. The compass usually hangs in a system of movable rings (Cardani's suspension), and its graduated disc floats upon alcohol, so as to be as little influenced as possible by the oscillations of the ship."

The earth itself is a great magnet, with north and south poles, in which the lines of force are aggregated. It has also its magnetic field and indifferent zone.

"The intensity of the earth's field is least in the neighbourhood of the equator, and becomes greater towards the poles, though not according to any regular law. The places where the magnetic force is a maximum are not situated at the poles, but at some distance from them. The earth is thus seen to be an irregularly magnetised body. The variations of intensity or 'total force' may be indicated by means of curves such that for all points on any one curve the 'total force' is the same. These are called isodynamic lines. . . We must picture the lines of force as emerging with considerable density in Victoria, South Australia. At greater distances from the south pole (magnetic north-seeking pole), lines of force also emerge from the earth, but less thickly, and with a component of direction towards the north. Still further northwards we come to the indifferent zone of the terrestrial magnet, which is a somewhat broad band running equatorially round the earth. After this there are regions where the lines of force from without re-enter the earth, somewhat few at first, and nearly horizontal in direction, but always denser and more nearly vertical as we approach the north magnetic pole, which has the pelarity we have called south-seeking. Finally, when we reach this point, in the North American Archipelago, the lines of force have something like their greatest density of distribution, and run vertically downwards into the body of the earth" (Plate lv., Fig. 5).

The magnetic field of the earth comprises all the space surrounding our planet, so far as we can detect in it any magnetic influence. The portion of the field best known to us is the earth's crust and its atmosphere. The earth affects neighbouring heavenly bodies and ordinary magnets, and is affected by them. There is reciprocity as between the heavenly bodies and our planet. Matter and force are continually acting and reacting on each other directly or indirectly at near or remote distances. The magnetic field of the earth is not constant, but is subject to periodic variations. It is convulsed by sudden changes of condition, and is slowly and progressively altering its distribution. In the variations of the field we can recognise the influence of astronomical events, so that there would seem to be magnetic lines of force connecting our planet with other worlds. The knowledge of this fact gives to magnetic forces a universal interest and significance.

"Very closely connected with the earth's system of magnetic lines of force are the luminous phenomena which at certain times produce an illumination of the upper layers of the atmosphere in both polar regions, often on a very magnificents scale. They are a direct visible expression of the magnetic condition of the earth, and are so especially related to the variations of this condition that they were justly called by Humboldt 'magnetic tempests' (magnetische Ungewitter). When a magnetic storm passes over the earth, and the magnetic needles are set trembling, the lights at the northern and southern poles, at no time quite extinguished, become especially brilliant. . . . The best known are the 'northern lights' or 'southern lights' (aurora borealis or australis), and consist of luminous streamers which flicker unsteadily to and fro, passing downwards from the higher and more rarefied regions of the atmosphere, and vice versa." 1

It is not necessary to pursue the subject of inorganic and organic electricity further; suffice it to say that, as the circle of the sciences widens, it becomes more and more apparent that it is not possible absolutely to separate the inorganic and organic kingdoms either as regards their matter or their force. They merge and blend at innumerable points, and practically the same laws control both. The organic kingdom is indebted for its substance and not a little of its force to the inorganic, and virtually the same constructions and movements prevail in both. These for the most part consist of radiating, concentric, curve, and spiral arrangements and movements seen in plants and animals and parts thereof. 1 The above quotations are fronı Professor H. Ebert's recent work, "Mlagnetic Fields of Force," trauslated by C. V. Burton, D. Sc.
London and New York, 1897 . 
It follows that dead and living matter exhibit, for the most part, a community of structure and movement. There is an obvious sympathy as between the inorganic and organic kingdoms. The subjective organic finds its correlate in the objective inorganic.

The inorganic and organic lingdoms are adapted to each other. The eye and the light are made for and are physiologically the counterparts of each other. The same is true of all the sense organs, and the objects on which they act. There would be no need for eyes, ears, taste, smelling, and touch bodies in animals if there was no light, and no sounding, smelling, sapid, and extraneous substances outside the animals. Similarly, the inorganic and organic movements, if not co-ordinated, would clash, oppose, and nullify each other. In nature the law of "least action" is always at work, and neither in the inorganic nor organic kingdoms is matter or energy ever wasted. The phenomena of heat, light, magnetism, electricity, \&c., are not confined to the inorganic kingdom. Plants and animals both generate heat, and animals also generate electricity.

It thus happens that the living plant and animal are in sympathy with everything around them. The inorganic and organic kingdoms and physical and vital force reciprocate to quite an extraordinary extent, and prove very conclusively that the universe is to be considered as a whole. The science of optics supplies us with the microscope and telescope, and these in turn enable us to perceive and study infinitely minute near objects, and infinitely vast distant objects: the electric telegraph not only connects all parts of the earth physically-it also connects nations with common aims mentally, and, in a sense, enables them to annihilate time and space: the telephone and wireless telegraphy permit them practically to speak into space and be heard and understood thousands of miles away; and the phonograph literally enables them to embalm and preserve for long ages and future generations the human voice even to its most subtle inflections. In this way even the dead speaketh. The reciprocation here alluded to is traceable to an intelligent Creator or First Cause, Who correlates and co-adapts everything, living and dead. The palpable design everywhere apparent in the universe negatives the idea that anything organic or inorganic exists by chance.

Everything had to be created according to a preconceived plan, and all the parts of the inorganic and organic kingdoms had to be adapted to each other. The inorganic came first, then plants, then animals, and lastly man. An atmosphere and soil had to be prepared for plants; and an atmosphere, soil, and plants for animals. An atmosphere and soil were required for the leaves and roots of plants, plants had to be produced for the food of animals, and plants and animals were both necessary for the sustenance of man. A habitation and pabulum were required for plants and animals, and special plants and animals had to be formed, and adapted to meet the exigencies of certain external conditions, at particular periods.

This necessitated the gradual cooling of the incandescent young earth, the preparation of various subtle gases and fluids, the slow formation of soil and other solids, \&c. The more simple plants and animals were necessary to the existence of the more complex ones, and physical and vital changes followed each other in an endless but wellordered sequence until the universe, as we know it, was evolved. At no point in the great evolutionary process has the Master-Builder's plan broken down. That, notwithstanding all that has been said of late years to the contrary, is sound in warp and woof. It is impossible, even by a stretch of the imagination, to regard the universe as a chance product.

\section{§29. Magnetism, Electricity, Light, Heat, and Motion Correlated.}

The universality of magnetism, electricity, light, heat, and motion in the world necessitates a brief consideration of these subjects. They all are the outcome of law and order, and, as such, afford important testimony of Design and of the existence of a Creator or First Cause. If it can be shown that the subjects referred to all hang together and are inorganic in their nature, and if, further, it can be demonstrated that they occur also in the organic kingdom, and that the inorganic and organic kingdoms have much in common and are correlated, it goes without saying that the great fabric of the universe is a designed whole, and the product, not of chance, but of an Intelligent Designer. When in every direction in the inorganic and organic kingdoms law and order, and only law and order, can be traced, and when it is seen that everything inorganic is carefully adapted to everything organic and vice vers $\hat{a}$; the most perfect correlation and co-adaptation being, in every instance, scrupulously carried out; it is next to impossible to ignore the manifestations of Intelligence which everywhere present themselves. This condition of things acquires additional significance when it is pointed out that the lines of force produced by the action of natural and artificial magnets and electricity on iron-filings resemble in their general arrangement, and ever. in their details, similar lines of force employed in the formation of crystals, plants, and animals; the lines of force explaining in large measure not only the structure of crystals, plants, and animals, but also the chief movements of the two latter. If law and order were confined to the inorganic and organic kingdoms respectively, there would, 
even then, be cumulative proof that a Creator produced both kingdoms; but when it is found that the two kingdoms dovetail into each other and interact at innumerable points, and are, in the widest and fullest sense, complemental as regards their matter and much of their force, then the proofs of a First Cause, a Sustainer, and Regulator are, it appears to me, indefinitely strengthened.

In considering magnetism, electricity, heat, and light we have to deal with the movements of the atoms and molecules of matter, and of the ether which pervades space. Magnetism, electricity, heat, and light may be said to merge into each other. They are all forms of motion, and are best treated together. A knowledge of electricity is necessary to a comprehension of the others, and forms, in a sense, a preliminary inquiry.

The precise nature of electricity is unfortunately not yet quite understood. Recent researches, however, make it all but certain that the old idea of an electric fluid must be abandoned. Electricity, there can be little doubt, does not flow as water and other liquids do. When an electric current passes along a wire, the transference is effected by continuous molecular action propagated within and outside the wire: the action extending to the atmosphere. There is a movement of the molecules of the wire, and of the molecules of the ether between and outside those of the wire in the atmosphere; the latter constituting the so-called magnetic field. ${ }^{1}$ Both sets of movements take the form of waves, and the molecules rotate as well as vibrate or pulsate: there are longitudinal wave and rotatory transverse movements. The molecular action is primarily between neighbouring inolecules; the remote molecules being affected secondarily. The movements are not those of fluids streaming steadily in a particular direction between the molecules, but vibratory wave movements of the molecules themselves in several directions; the advance or transference being due to what is practically a system of percussion, one molecule acting on another in rapid succession not only in the wire but throughout space. The molecules have a vibratory progressive wave movement in the aggregate. They have also transverse, rotatory, and, some think, spiral movements individually. Each molecule has an environment to which it is confined, but the molecules as a whole have movements apparently co-extensive with space.

Professor Trowbridge of Harvard University, in his work, "What is Electricity?" " points out that when fluids or compressed air are made to flow through tubes there is no disturbance outside the tubes in the surrounding medium ; a magnetic needle placed outside the tubes not being affected. It is otherwise when a current of electricity is passed along a copper wire. In this case, when a magnetic needle is placed in the vicinity of the wire it is disturbed or deflected, showing that the atmosphere outside the wire is involved. The conducting wire is surrounded by an atmospheric magnetic field, which influences substances placed in it. While it is convenient in popular parlance to speak of the flow of electricity, and of electrical currents, it is necessary to make a distinction between the passage of fluids in the ordinary sense and that of electricity. In electrical transferences it is the ether principally which is concerned. That ether exists is all but proved by the transference of energy in magnetic induction. When an electric current is sent through one circuit it begets a current in a neighbouring circuit. The energy which disappears in the exciting current reappears in the induction current, and must have existed in the space separating the currents during the transference. A medium of some kind (believed to be ether) is necessary to convey the energy through the intervening space in question. The phenomena of light also involves the existence of a medium.

"The old fluid theories implied that when a body was electrified it had something upon it which was called electricity. According to the modern views, we regard the ether around the body as charged with energy which is the result of the work we have done in charging the body. This energy in the ether is the energy of motion. There is asstate of strain in the ether which we term a polarised condition. Around a positively charged body this polarisation has a certain direction and a certain amount. With a negatively charged body this polarisation is in an opposite direction. It is suggested that these polarisations may be like right-handed and left-handed rotations or twists. When we electrify a conductor we store up energy around the conductor in the ether. The work we do is spent in changing the state of the medium. When a body is discharged, the medium returns to its original state, and the energy is dissipated as heat in the electric spark or as heat in the conductor. The electric current is therefore the manifestation of energy in the ether along the wire through which the current appears to flow. The consideration of the rotation of the plane of polarisation of light by magnetic force led Maxwell to a theory of magnetism which is called 'the hypothesis of molecular vortices.' Since there is good evidence for the belief that there is some kind of rotation going on in the magnetic field, Maxwell investigated the condition of motion which exists when a great number of very small portions of matter rotate on their own axes, these axes being parallel to the direction of the magnetic force. The motion of these vortices does not sensibly affect the visible motions of large bodies, but it can be supposed to affect the periodic motion of the medium which constitutes the phenomena

\footnotetext{
Sellmeyer in 1872 expressed his belief that the ponderable atoms vilorate, but with much smaller amplitude than the ether particles.
2 International Scientific Serics. London, 1897 .
} 


\section{MAGNETISM, ELECTRICITY, LIGHT, HEAT, AND MOTION}

we call light. According to this theory, the displacements of the ether will produce a disturbance of the vortices, and this disturbance of the vortices can be supposed to react on the ether, and in this way can affect the propagation of light." 1

If a Leyden jar be discharged through a great circle of wire placed in one room, electro-magnetic waves can be sent through brick walls and detected in another room by the sparks excited in a similar circle of wire connected with a second Leyden jar. The electro-magnetic waves undulate in the direction of their length; they also undulate athwart the path of the wave's advance. "The ether, though homogeneous and continuous, may be, as regards its density, rendered heterogeneous by motion ('Hypotheses of Vortex Molecules,' by Lord Kelvin). Magnetic influence on light indicates a rotational motion of the media when magnetised. This motion does not imply a dissipation of energy." No substance cuts off the lines of magnetic force: they pass through wood, stone walls, copper, and all metals. Ether transmits transverse vibrations to very great distances without sensible loss of energy. Ordinary atmospheric air can transmit waves of sound which move to and fro in the direction in which they are propagated. It cannot, however, transmit waves of heat, light, and electricity, which require transverse vibrations and the presence of ether. A good illustration of heat and light waves is obtained by throwing a stone into a placid pool of water. The waves spread in ever-widening circles from the centre of disturbance, and indicate the direction of progress. They have also a vertical movement at right angles (transverse in character) in addition to the spreading movement.

As there is no limit to space, and ether (or its equivalent) occupies all space, electrical and other movements are propagated to all parts of the universe, and hence the possibility of wireless telegraphy, which is mainly due to molecular vibrations and pulsations of the ether in space. The same is true of the telephone, where the vibrations are originally produced by the vocal chords (human voice) or other sounding bodies. The vibrations are, in the first instance, conveyed to a thin vibrating disc, which, having to-andfro movements communicated to it, causes the molecules in space to react upon each other in waves to a practically unlimited extent. The vibrations generated in one locality can be collected by a duplicate vibrating disc in another, with the result that messages can be trans-

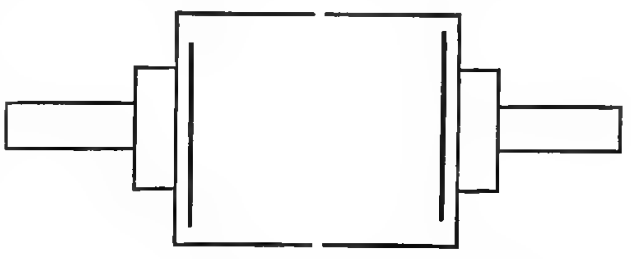

Fig. 23.-Diagram of telephone (after Trowbridge).

mitted incredibly long distances. ${ }^{2}$ This extraordinary achievement is only possible when there is unlimited matter and motion: any breach of continuity either in the matter or the motion would be fatal to the principle and practice of wireless telegraphy and telephony. The telephone, a sketch plan of which is appended (Fig. 23), is an instrument based entirely on Faraday's discovery of magnetic induction. It was discovered by Professor Graham Bell, who has acquired a great reputation as an authority in the physics of sound.

The electric telegraph (discovered before the telephone by Morse and others) acts on essentially the same principle as the telephone. Both transmit messages by means of electro-magnets and insulated wires; the earth in certain cases acting as a return wire. Dynamo machines, electro-magnets, and insulated wires perform the bulk of electric work. Electricity, like heat and light, as already indicated, is regarded as a form of motion; the only difference between electricity, heat, and light consisting in the length of the waves. The waves of heat, for example, approximate to those of electricity, and are longer than those of light. "All space is filled with a medium which transmits electro-magnetic waves to us from the sun." The sun, which is the source of the electro-magnetic waves, has been compared to an enormous electrical furnace, consisting of a mass of molten metals where carbon is freed from its impurities, and the metals driven off in vapour. The presence of oxygen in the sun seems necessary to account for the exceeding splendour and brilliancy of that magnificent orb. Faraday and Dewar have shown that oxygen is strongly magnetic. ${ }^{3}$

"We have already strong grounds for believing that we live in a medium which conveys to-and-fro or periodic movements to us from the sun, that these movements are electro-magnetic, and that all the transformations of light

1 Trowbridge, op. cit. pp. 264, 265, 272 and 273.

2 As to the distance which may be covered by wireless telegraphy the annexed report by Signor Marconi's assistant in the Scofsman of March 12, 1907, is at once instructive and impressive:- "Nine years have passed since the writer became assistant to M. Marconi at the first modern wireless telegraph station. The distance over which messages were transmitted was not great, only about 14 miles, but the tiansmission was regular, if slow, and suffered but little from interruptions. Previous wireless systems, such as those of Morse, Lindsay, Willoughby-Smith, Stevenson, and Preece, had been based on different principles, and had never attained to distances above a very few miles. Great distance is now a matter of so little notice that few people are even aware that there are at least a dozen wireless stations in different parts of the world which maintain regular telegraphic conmunication with others which are over a thousand niles off. The Atlantic Ocean is 3000 miles wide, but the great Cunarders are never ont of direct touch with either England or America. Panama is connected directly with Manlattan Beach, 2170 miles distant, and recent results show that Boston, though 3000 miles off, will be very shortly in jermanent conmmuication with Machrihanish, in Kintyre."

${ }^{3}$ Oxygen is said to constitute the largest portion of the solid and liquid substances of our planet so far as we know it; and nitrogen is by far the predominant constituent of our atmosphere. 
and heat, and indeed the phenomena of life, are due to the electrical energy which comes to us across the vacuum which exists between us and the sun-a vacuum which is pervaded by the ether, and which is a fit medium for the transition of the electro-magnetic waves."

Clerk Maxwell propounded a great generalisation known as "the electro-magnetic theory of light." According to him all the phenomena of electricity, heat, and light are manifestations of electrical energy. Electricity under certain conditions produces heat and light, and motion in turn produces all three. Electricity, heat, and light can be produced by friction or the rubbing of certain substances together. The trituration of even two pieces of ice, as Tyndall showed, generates enough heat to partly melt the ice. Count Rumford boiled water by rotatory and other movements as apart from fire, and Faraday demonstrated that a soft iron bar could be magnetised if placed within a coil of insulated copper wire through which an electric current was flowing. In such a case, the degree of magnetisation is increased by lengthening the coil of wire.

These various transformations afford examples of the conservation of energy; the direction of the energy being altered, while the energy itself is not dissipated or destroyed. One of the best examples of this great law is the formation of coal and the burning of it in the production of steam ; steam being, until lately, the chief generator of electricity as employed in the arts for propelling machinery, tram-cars, \&c., and for producing artificial lighting. The sun's rays (electro-magnetic in character) were required to grow the great tree ferns and other rank vegetation in the carboniferons era. The great tree ferns and their congeners lived, died, and were buried for long ages in the bowels of the earth. They were gradually transformed into coal, but the sun's heat and light, which originally assisted in the formation of the coal, though temporarily eclipsed, were not destroyed. The coal, when exhumed and burned, restores the heat and the light originally obtained from the sun, and can be employed in raising steam. Steam can be converted into motion, and motion into electricity, heat, and light. There is a cycle of apparently different results produced by one and the same cause. Flectricity, heat, and light, as a matter of fact, are varieties of motion.

Professor Faraday endeavoured to identify gravitation with electricity, heat, and light, but did not succeed, and at present we are wholly ignorant of the relations (if any) subsisting between gravitation and the others.

There are those who endeavour to explain gravitation by movements occurring in the ether. They regard the ether as a vast ocean, with a definite tide, moving throughout space; the ether passing through the sun and the planets with more or less difficulty; its motion forcing the particles of matter together. The ether, as already stated, is believed, and not unnaturally, to be the means by which the waves of electricity, heat, and light are conveyed to us by the sun.

While Faraday failed to explain the nature of gravitation, he conferred untold advantages on what he regarded as cognate subjects by discovering induction and the conversion of motion into electricity. Whereas magnetism originally led the way to a knowledge of electricity, electricity, in turn, has thrown much light on magnetism. Weber and others have sought to explain the nature of magnetism by the so-called molecular theory. They suppose that the molecules of a bar of iron form small magnets which, when the bar is unmagnetised, point indiscriminately in all directions, but when magnetised certain of them point in a definite direction. The theory holds good up to a point, but does not quite explain the action of magnetism on light, which compels us to assume a medium between the molecules, and to attribute rotatory or vortex movements to it. "There are not only attractive forces between the molecules of the iron, but also rotatory motion in the medium within and around the magnet."

Since the discoveries of Professor Oersted in 1820, the chief interest has centred in the magnetic field or medium in which the attracting bodies are placed. Prior to this, no notice was taken of the surrounding medium, "and one magnetic pole was considered to act upon another as if it were an action at a distance and not from point to point in the medium between the attracting poles." Investigators did not recognise the lines of force in the magnetic field, or the disturbance occasioned by them to a magnetic pole introduced into the field. They did not realise that the space around the earth is filled with lines of force which emerge and radiate from the north pole, and curve round, converge, and re-enter the south pole. The magnetic field or medium, and the lines of force pervading it, are now subjects for close scrutiny. "Faraday's great achievement was in his conception of the lines of force which emanate from a magnetic pole and stretch through the ether of space; in his pointing out that the medium surrounding the wires carrying electric currents, and the medium in which magnets are situated, is in a state of strain; that there is what he called an electro-tonic state of this medium. It was like a mass of quivering jelly-any movement at one point producing a quiver in all neighbouring points."

Maxwell, in his great work on electricity, thus speaks of Faraday's conception: "Faraday saw lines of force traversing all space where the mathematicians saw centres of force attracting at a distance. Faraday sought the 
seat of the phenomena in real actions going on in the medium; they were satisfied that they had found it in a power of action at a distance impressed on the electric fluids." The theory of centres of force attracting at a distance was no doubt founded on the effects known to be produced by the heavenly bodies on each other. It, however, does not apply to molecular action.

For some fifty years after Faraday's brilliant discovery of magneto-induction, attention was almost exclusively directed to the production of electric currents in one direction instead of interrupted or to-and-fro currents, such as are obtained by the rapid alternate thrusting and withdrawing of the north pole of a magnet into a spool of insulated wire. The interrupted currents are, however, the more varied and powerful, especially when the lines of magnetic force are made to quiver at high speeds. They can by means of a transformer convert a comparatively low electric pressure or voltage into an enormously high one. They can produce electric light, heat wires, and accomplish most, if not all, the work performed by the constant currents. The interrupted currents are daily becoming more important, from the great variety of work they are capable of performing.

In order to convert interrupted or to-and-fro electric currents into constant currents flowing in one direction, a commutator is necessary. This is to the electric circuit what the eccentric is to the valves of the steam-engine when it lets on and cuts off the steam which gives continuity of movement to the piston and fly-wheel. The commutator is briefly a mechanical arrangement by the aid of which the free ends or terminals (electrodes) of wires conveying interrupted or alternating currents of electricity are transposed or crossed, with the result that the interrupted currents are converted into constant currents.

Electric currents are peculiar in this : if the currents in two neighbouring wires are running in the same direction, the wires attract each other; if in opposite directions, they repel each other. In the case of magnetic poles, like poles repel and unlike poles attract.

The production of electricity is not less interesting than its mysterious manifestations. It occurs as a natural product in the lodestone, the lightning, and in certain fishes; notably the electric eel of tropical America, the electric rays, and the electric cat or sheath-fish.

In the fishes, electricity is manufactured by special glands, and discharged in self-defence or attack. The discharge results in a shock like that obtained from ordinary electricity or from lightning. ${ }^{1}$ In the case of the electric eel, according to Humboldt, the discharge is sufficiently strong to destroy a horse or other large animal.

\section{ATMOSPHERIC AND OTHER ELECTRICITY}

The mode of production of atmospheric electricity is still sub judice. It has been ascertained not to be due, as was supposed, to the evaporation of water, although the friction of the particles of water against material substances can produce it. Thus, in Armstrong's electrical machine, where jets of spray are forced through nozzles, a high state of electrification is obtained. The friction of dust particles is also a potent cause of electrification; the tops of the Egyptian pyramids during sand-storms being strongly charged.

Electricity is produced artificially in many ways - for example, by the action of certain fluids on different metals, as in galvanic batteries-that is, when two dissimilar metals are connected by a wire and immersed in a liquid that acts upon one of them; by heat, when two dissimilar metals are mechanically joined and heat is applied to the junction; by friction, when a warm glass rod is rubbed with dry silk. The most common form of artificial electricity is that produced by the aid of the dynamo machine, which is driven by steam-engines, petrol-engines, or water running at high velocities. The modern dynamo consists of a number of wire coils arranged on a rapidly revolving shaft surrounded by fixed pieces of iron, around which the currents formed in the revolving coils are made to circulate. The speed of the shaft, in some cases, exceeds one thousand revolutions per minute. "Currents of electricity are excited in a coil of wire passing rapidly near the poles of a magnet; a current being excited in the coil in one direction by movement near a south pole, and in the opposite direction by movement near a north pole; a commutator can be made to direct these opposite currents in the same direction through another coil." The electricity generated by the dynamo is carried away by wires, and used up or stored as desired. The electricity flows so long as the dynamo is at work, and is delivered at the extremities or electrodes of the wire.

1 According to Trowbridge the bolt of lightning is not one continuous discharge, but is an interrupted, alternating current which pulsates to and fro ten or twelve times, or even more, in a nillionth of a second. Photographs of powerful electric sparks lead one to conclude that a discharge of lightning makes way for its oscillations by first breaking down the resistance of the air by means of a disiuptive pilot spark; through the hole thus made in the air the subsequent surgings or oscillations take place. In lightning discharges high electro-motive force and great quantity are frequently combined in a very short interval of time. The study of the disruptive or oscillatory discharge of lightning is closely related to that of the brush discharge and the phenomenon of the aurora borealis. 
The dynamo can, in some cases, produce sparks five feet long, and these are believed to be identical with discharges of lightning. In some cases the time occupied by the oscillations of electric sparks is less than one ten-millionth of a second. "In lightning discharges high electro-motive force and great quantity are frequently combined in a very short interval of time." A diagram of a series dynamo is given at Fig. 24.

Electricity, when made, can be stored by conducting it to a storage battery. "The storage battery or accumulator in its commonest form consists, before it is charged, of red oxide of lead with electrodes of lead. The oxide and the electrodes are suitably immersed in dilute sulphuric acid, and a strong current of electricity is sent from one electrode to the other. The oxygen resulting from the electrolysis of the water converts the red oxide of lead at the future positive pole of the battery into peroxide of lead and into metallic lead at the future negative pole of the cell. When the charging current is removed and the peroxide of lead pole is connected with the porous metallic lead pole, a current of electricity is produced, and the peroxide goes back to a lower oxide." 1 There are other batteries where peroxide of lead and zinc are employed, and from which more powerful currents can be obtained. These have been advocated by Professor Main, and are known as zinc-lead storage batteries.

The presence of electricity is detected by introducing a small bar-magnet (magnetic needle) or a galvanometer into the magnetic field. The magnetic needle is composed of a slender bar of magnetised steel suspended at its middle by a single fibre of untwisted silk, or set on an agate point, which leaves it free to move in a horizontal

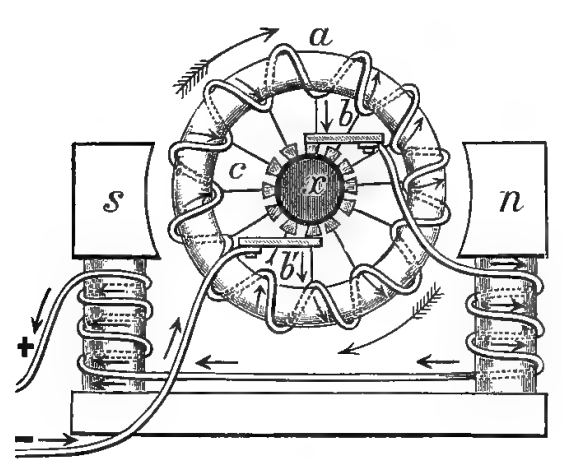

FIG. 24. - Series dynanio, $x$, Revolving commutator with segments $(c) ; b, b^{\prime}$, brushes for taking off the electric current; $a$, iron ring with coils of wire connected with segments $(c)$ of commutator $(x) ; n, s$, field magnets with north and south poles. Positive electricity $(+)$ is obtained at $b$, and negative electricity $(-)$ at $b^{\prime}$. The current is made to flow round the field magnets $(n, s)$, so as to make north and south poles. direction and also to dip at either end. This needle exhibits a north and south pole, and arranges itself in the direction of the poles of the earth. It sets or points towards a wire through which electricity is flowing, and is thrown into vibration by any electrical disturbances in its vicinity. The galvanometer is a still more delicate and sympathetic instrument for detecting electrical currents. "It consists essentially of a coil or bobbin of wire like a spool of thread, with a tiny magnet hung by a spider thread near one end of the spool." It shows that "electrical actions pervade all matter, and that there are electric waves in the ether of space." It is in physics what the microscope is in anatomy and physiology. By its use the faintest electrical currents are brought into prominence, and their strength measured. The galvanometer is provided with the lightest possible bar-magnet or needle with hair pointers at either end, a graduated scale, and two spools of thin insulated copper wire; one end of the one spool being connected with one end of the other, i.e. they are connected in series. If one spool be placed on the stage of a microscope bearing iron-filings on white paper, and the other spool be placed on a third spool made of coarse wire, the ends of which are connected with a battery, a glance at the microscope shows that when the battery current is suddenly made or broken the iron particles vibrate, proving that currents of electricity are passing in the circuit of wire on the two connected spools. The current set up by the battery can at once be detected, and also in the wires and in the atmosphere investing the wires. "Any change in an electric current on a wire, any pulsation, causes instantly a similar pulsation in any neighbouring wire not connected with the first wire and placed parallel to it."

The important part which electricity plays in the inorganic kingdom extends, as indicated, to the organic. Siemens exposed certain plants to the rays of electric light for long periods, with the result that he greatly facilitated their growth. The effects of electricity as a curative agent, especially in nerve and muscular diseases, are well known. Electricity, there is reason to believe, plays a leading rôle not only in structural development, but also in functional processes and movements.

Closely connected with electricity and light are the mysterious cathode, Röntgen, and other rays. These rays have marvellous penetrating powers; photographs being taken by their aid through various solid substances, such as wood, metal, the tissues of the body, \&c.

The waves of electricity travel with the velocity of light; light attaining to the incredible speed of 185,000 miles per second.

The discharges from a Leyden jar also travel at enormous velocities. Professor Joseph Henry has shown that the discharge of a Leyden jar is oscillatory, and that induction effects are produced by remarkably small quantities of electricity. 
The cathode rays possess peculiar interest in relation to ether, electricity, light, phosphorescence, \&c. The term cathode, which was introduced into electrical nomenclature by Faraday, is derived from the Greek кará (down), and ódós (a way), and originated in the early belief that electricity flowed in a downward direction from the positive to the negative pole. The term is applied to the negative terminal of a battery or of a Ruhmkorff coil or transformer. The counter term anode is applied to the positive terminal. If the wires connecting the cathode and anode of a Ruhmkorff coil be imbedded in a small glass vessel, and the air in the vessel exhausted, it is found that when the coil is excited, and a high state of exhaustion reached, the cathode rays stream from the cathode in straight lines, and the vessel is filled with luminosity. There is apparently no connection between the cathode and anode, the rays from the cathode not seeking the anode. This seems proved by placing the cathode and anode above each other at the end of an exhausted tube. In such circumstances, the cathode rays hold on in straight lines to the opposite end of the tube, and do not bend round to the anode. It is not quite certain whether the luminosity produced is wholly electrical, or, as Sir William Crookes suggests, partly due to the impact of molecules of the gas on phosphorescent substances which emit light, which results in bombardments of the electrodes on the walls of the containing vessel. In the extraordinary light effects produced by currents of high frequency "we have to do with an increased activity of the molecules of a rarefied gas, which is produced by the electrical energy stored up in the medium near the electrodes. The cathode rays may be considered as a radiation of electric energy, which is made visible in rarefied media, and which can also be detected outside such media. The visible transformation of electric energy from the electrode, termed the cathode, can be made to pass through metal walls, and can be seen outside those metal walls. The cathode rays can be made to pass through substances which are entirely opaque to ordinary light. Thus, they can be made to pass through sheets of aluminium, gold, silver, and many other opaque materials which entirely cut off ordinary light. The cathode rays spread in all directions. They excite phosphorescent bodies, such as uranium glass, to a brilliant glow, and blacken photographic plates. No heating effect of the cathode rays has been detected." 1

According to I-enard, ether is the medium by which the cathode rays travel and in which they manifest their peculiar phenomena. This follows, because in tubes where the exhaustion is pushed to an extreme, and where the vacuum is nearly perfect, the cathode rays cannot be produced, and all electrical manifestations visible as light disappear.

Scarcely less interesting than the cathode rays are the Röntgen or X-rays discovered by Professor Röntgen. These rays will pass through several inches of wood and thin layers of aluminium. They are, however, almost entirely intercepted or cut off by layers of glass or silver.

Röntgen, by the aid of the X-rays and by employing ordinary dry plates, has been able to take photographs through wood and the soft tissues of the body; the rays, because of specific absorbing powers, distinguishing between the soft and hard parts and revealing the skeleton. He is of opinion that the $\mathrm{X}$-rays are excited either in the glass walls of a Crookes tube, or in the media outside the tube by means of the cathode rays.

The Röntgen rays are rendering immense service to surgery, as they enable the surgeon to photograph not only the bones of the body, but also foreign opaque substances, such as bullets, needles, pins, portions of glass, \&c., accidentally lodged in the soft tissues. Already the bones of the arms and hands, the legs and feet, the chest, pelvis, and backbone have been photographed. Even the heart has been shadowed forth, and some are sanguine enough to believe that by and by the whole internal economy of the living body will be revealed by Röntgen ray and similar photographic processes. The late Professor Sir J. Y. Simpson, of Edinburgh, expressed his confident belief that the day would come when the whole living human body would, by means of electrical or other light, be rendered diaphanous or transparent, and so exposed to the gaze of the anatomist, physiologist, physician, and surgeon. This would be a notable advance in another, though not wholly different, direction.

The Röntgen rays pass more readily through the flesh than through the bones and denser parts, and the hope is entertained that the rays may ultimately be made to discriminate between the softer and harder tissues, and provide graduated photographs.

It is difficult to say whether the Röntgen rays are due to radiant matter streaming from the negative pole (cathode) or to longitudinal waves of electricity.

According to Maxwell's electro-magnetic theory of light, only transverse waves are set up in the ether; longitudinal waves being absent. According to the electro-magnetic theory of Helmholtz, both transverse and longitudinal waves are present. The Röntgen rays are probably identical with the longitudinal rays postulated by Helmholtz, and which travel with incredible velocity. Röntgen himself inclines to the longitudinal wave theory. D. A. Goldhammer ${ }^{2}$ supports the hypothesis that the X-rays are ordinary transverse vibrations of the ether analogous

\footnotetext{
1 Trowbridge, op. cit. pp. 281, 282, 283.

2 Annalen der Physik und Chemie, No. 4, 1896,
}

VOL. I. 
to, or identical with, ordinary ultra-violet light, with the difference that the wave lengths of the $\mathrm{X}$-rays are very greatly reduced as compared with those of any ultra-violet rays hitherto observed. It is just possible that the Röntgen rays are cathode rays modified.

In order to obtain good Röntgen ray photographs, a high electro-motive force is necessary, and the exhaustion of the air must also be carried to a high pitch. It is all but certain that the radiations which characterise the Röntgen rays proceed from the solid body upon which the cathode rays impinge. "One of the most successful forms of Crookes tubes for producing the Röntgen photographs is the so-called focus-tube. The method of studying the effects of the X-rays by means of fluorescent screens is more expeditious than that of photography. A fluorescent screen is simply a sheet of pasteboard covered with a fluorescent substance. Edison has discovered that crystallised tungstate of calcium is highly fluorescent. A pasteboard covered with this substance forms the closed end of a box into which one looks, the hand or limb being pressed against the outside of the pasteboard screen, the fluorescent substance being on the side at which one looks-that is, inside the box. By means of such a fluoroscope one can see the shadow of one's hand after the X-rays have passed through several doors, and at a distance of at least fifteen feet from the Crookes tube. Sensitive photographic plates are fogged through brick walls a foot thick."

The cathode and Röntgen $(\mathrm{X})$ rays, mysterious and inexplicable as they are in many respects, find a parallel in the extraordinary N-rays, as described in 1904 by M. Jean Becquerel to the French Academy of Sciences. The $\mathrm{N}$-rays are emitted by human and other living bodies, and also by certain inanimate substances, in particular by metals. Animals put under chloroform, it has been ascertained, cease altogether to emit the rays- the emanation recurs when the effect of the anæsthetic has gone off. Death causes final cessation of the emission of the N-rays. Flowers, from which also the rays emanate usually, have been subjected to chloroform and likewise went to sleep, the emission ceasing, but recommencing after a time, presumably when the influence of the anæsthetic had disappeared. This is a sufficiently remarkable phenomenon, but it is nothing to what follows. Similar experiments have been made with the inanimate substances which possess the property of giving out the $\mathrm{N}$-rays, and it is averred that those substances behave exactly as do animate beings under the same conditions. That is to say, a metal emitting the rays in a normal state ceases to emit them when subjected to chloroform vapours. In short, anæsthetics will apparently cause metals as well as flowers and animals to sleep. It has yet to be determined whether metals (to speak figuratively) can be killed permanently by the continued administration for a given time of anæsthetics and other poisons in the same way that plants and animals can. If M. Becquerel's statements be confirmed, justification in part will be found for them in the fact that quite a large percentage of all the known elements (particularly the metals) is found in the bodies of plants and animals.

The new theory involves questions of very considerable magnitude, namely, the nature and range of life. It will naturally be asked in the near future, Can the term life be confined exclusively to plants and animals, or must it be expanded to include certain so-called inanimate substances? Hitherto, and by common consent, life has been regarded as forming the distinguishing feature between the organic and inorganic kingdoms; the members of the organic kingdom living, feeling, and moving, those of the inorganic kingdom moving, but neither living nor feeling. While it will require much additional observation and experiment to establish the new doctrine, it would be premature at present to affirm that it is a wholly impossible doctrine. Like other new doctrines, this latest physico-physiological departure is on its trial, and will have to be corroborated by independent workers in kindred fields, and sufficient time allowed to test the accuracy of the theory in relation to known phenomena.

Striking specimens of lightning, electric sparks, and cognate subjects are given in Plates xxix. to xxxiii.

\section{ANIMAL MAGNETISM}

The electric fishes are endowed with extraordinary powers, and deserve more than a passing notice.

They are mostly found in tropical and sub-tropical regions, and although not numerous, are not confined to any one family of fishes. They are, moreover, not the exclusive product of modern times; their fossil remains proclaiming them old-world forms. They are smooth-skinned-that is, they are not covered with scales as other fishes. The electricity which they generate in their bodies in every respect resembles ordinary electricity, and is completely under their control. They can discharge it in small or large quantity, and at pleasure, either in defence or in attack. In certain cases, and under provocation and excitement, the shock transmitted is sufficient to disable or even kill a man. The electrical organs are elaborate structures, and have a remarkably bountiful nerve supply. They have also an ample blood supply. The very numerous and large nerves extending between 
the electric organs and the spinal cord and brain sufficiently account for the control the fish has over them, and the rich blood supply accounts for their high organisation and great activity. They are as completely under control as ordinary voluntary muscles, and many are of opinion that the electric and muscular systems have many points in common.

The resemblance is traced in several directions: $(a)$ in development; from researches into the development of the electric organ of Torpedo it is supposed that the organ in question is developed from muscular substance; (b) peculiar muscular organs, the functions of which have not been discovered, are found in the rays, Mormyrus and Gymnarchus; $(c)$ the electric organ and muscle behave similarly under the same circumstances. Thus, if the electric organ and muscle be exhausted, from whatever cause, they require rest and nourishment to restore them to their normal state. If strychnia be administered, a rapid succession of involuntary electric discharges is induced in the one, and tetanic convulsions in the other. If the motor nerves distributed to the electric organ and muscle be divided, the brain loses its initiating and controlling power: under such circumstances, artificial activity may be induced by irritating the peripheral cut ends of the nerves going to the electric organ and muscle respectively. The more healthy and vigorous the electric organ and muscle and their possessors, the more severe the shock given, and the greater the muscular force evolved.

"All muscles evolve a constant stream of electricity, which may be shown by a multiplying galvanometer to pass from the long external surface, which is positive, to the transversely cut section, which is negative. . . . Du Bois-Reymond discovered that, like muscles, nerves possess an electric current, but much weaker, running from the longitudinal external surface, which is positive, to the transverse internal one, which is negative." 1

The brain, as indicated, exercises supreme control over the electric organ and muscle alike.

The structure of the electric organ varies somewhat in the several electric fishes.

The electric fishes possessing fully formed electric organs, and which can gradually make, store, and give electric shocks of greater or less intensity at will, are the electric rays (Torpedinidx), the electric cat or sheath-fish of tropical Africa (Malapterurus), and the electric eel of tropical America (Gymnotus).

The electric rays have been carefully described by Dr. Günther as follows: "The electric organs with which these fishes are armed are large, flat, uniform bodies, lying one on each side of the head, bounded behind by the scapular arch, and laterally by the anterior crescentic tips of the pectoral fins. They consist of an assemblage of vertical hexagonal prisms, whose ends are in contact with the integuments above and below; and each prism is subdivided by delicate transverse septa, forming cells, filled with a clear, trembling, jelly-like fluid, and lined within by an epithelium of nucleated corpuscles. Between this epithelium and the transverse septa and walls of the prism there is a layer of tissue on which the terminations of the nerves and vessels ramify. Hunter counted 470 prisms in each battery of Torpedo marmorata, and demonstrated the enormous supply of nervous matter which they receive. Each organ receives one branch of the trigeminal nerve and four branches of the vagus-the former, and the three anterior branches of the latter, being each as thick as the spinal cord (electric lobes). The fish gives the electric shock voluntarily, when it is excited to do so in self-defence or intends to stun or to kill its prey; but to receive the shock the object must complete the galvanic circuit by communicating with the fish at two distinct points, either directly or through the medium of some conducting body. If an insulated frog's leg touches the fish by the end of the nerve only, no muscular contractions ensue on the discharge of the battery, but a second point of contact immediately produces them. It is said that a painful sensation may be produced by a discharge conveyed through the medium of a stream of water. The electric currents created in these fishes exercise all the other known powers of electricity; they render the needle magnetic, decompose chemical compounds, and emit the spark. The dorsal surface of the electric organ is positive, the ventral surface negative.

"Of the genus Torpedo six species are known, distributed over the Atlantic and Indian Oceans; three of them are rather common in the Mediterranean, and one ( $T$. hebetans) reaches the south coast of England. They attain to a width of from two to three feet, and specimens of that size are able to disable by a single discharge a full-grown man, and, therefore, may prove dangerous to persons bathing. Other genera, differing from the Torpedo in the position and structure of some of the fins, are found in other tropical and sub-tropical seas, namely, Narcine, Hypnos, Discopyge (Peru), Astrape, and Temera. All, like electric fishes generally, have a naked body.

"A large fish, of the general appearance of a torpedo, has been found at Monte Bolca; and Cyclobatis, from the upper cretaceous limestone of Lebanon, is probably another extinct representative of this family."

What is said of the electric rays is, for the most part, true of the electric cat or sheath-fishes, and the electric eels.

The electric sheath-fishes occur not unfrequently in the fresh waters of tropical Africa, and of these three

1 "Physiology, General, Special, and Practical," hy John Hughes Bentett, M.D., F.R.S. E., \&c., Professor of the Institutes of Medicine, University of Edinburgh. 
species are known - one of which (M. electricus) is met with on the Nile. They grow to four feet or thereby. I had an opportunity of studying some fine specimens of the electric fish of the Nile at the Gezireh Aquarium, Cairo (1904-5), and, on one occasion, very many years ago, I received, at Edinburgh University, an electric shock from an electric fish from Old Calabar. It sufficed to excite the Calabar specimen slightly, and to put one's hand into the water in which it was swimming, whereupon one or more very distinct shocks would be felt, and, once felt, could never be forgotten.

In the electric sheath-fish, the electric organ is not confined to one particular part, but extends all over the body, being most developed in the abdomen. It is contained between two aponeurotic membranes beneath the skin, and is composed of rhomboidal cells which contain a gelatinous substance of rather firm consistence. The nerve distributed to the organ forms an outstanding feature of the arrangement. It has its origin in the spinal cord, has no connection with nerve ganglia, and consists of a single enormously developed nerve trunk. In this it differs materially from the arrangement met with in the electric eel, where more than 200 separate nerves are distributed to the electric organ.

The greatest of the electric fishes is the electric eel (Gymnotus electricus) of tropical America. It attains to as much as six feet in length, and, according to the famous traveller, Humboldt, is dangerous to man and beast. It occurs in great plenty in Brazil and the Guayanas.

The astounding feature in the electric organs of the gymnotus is their enormous nerve supply. More than 200 nerves are distributed to the organs, and the nerves are very much larger than the sensory and motor nerves going to other parts of the body. As the electrical organs are special, so, in a large measure, are the nerves distributed to them. The nerve supply consists of continuations of the anterior branches of the spinal nerves. They furnish branches not only to the electric organs but also to the skin and muscles of the back of the gymnotus.

The electric organs take the form of two pairs of longitudinal bodies; the one pair situated between the skin and muscles on the back of the tail, the other pair between the skin and muscles along the anal fin. They have an involved and intricate structure, consisting as they do of a numerous series of perpendicular and transverse septa, in the interstices of which are imbedded prismatic cells containing a gelatinous substance. The septa are separated from each other by about the thirtieth of an inch, measure an inch in length, and contain some 240 cells, which secure for the electric organs a simply enormous surface.

It is difficult to realise how intimately related the electric organs of fishes are to those of ordinary electric batteries, and how certain living creatures should have been provided with an apparatus for manufacturing, storing, and discharging electricity, giving them a power over their victims, or mayhap enemies, not possessed by eren man himself.

The electric organs of fishes can only be regarded as special creations, as the sense organs are. It is inconceivable that they could have been evolved from any living structure in the remote past, or that they could have been produced by natural selection, into which the elements of chance and utility enter. The electric organs are massive and heavy, and, while they are eminently serviceable, when fully developed, to the electric fishes possessing them, no fish or series of fishes throughout the ages could have been burdened by them while undergoing a slow process of evolution, and while they were totally unfit for work of any kind. Mr. Darwin was wholly unable to explain their existence, and keenly felt the strain they put on his theory of "natural selection." Indeed he is said to have experienced a cold shiver whenever confronted with what, to him, was an insuperable difficulty.

\section{$\$ 30$. Lines of Communication and Force, Nervous and Otherwise.}

Every one who has studied vital manifestations must have been struck with the fact that in plants and animals--the simplest as well as the most complex-there are means of communication as between their several parts and particles, and as between each individual organism and the physical universe. In other words, there is a means of communication between the atoms and molecules of the cells and tissues of plants and animals on the one hand, and between the individuals themselves and their surroundings or environment on the other.

The lines of communication in the lowest plants and animals are by no means well defined. Thus in the cell plants and animals - the yeast plant and amoba, for example--where there is little differentiation, and where there is, so to speak, a common structure and a common function, every part of the individual seems capable of transmitting impulses either from within or from without. In these cases, the living mass is endowed with a power which enables it to grow, divide, reproduce itself, and move. Every part reacts upon every other part, and all are under the influence of life and vital and physical force. Every part of the individual displays a low form of sensitiveness or its equivalent, and this in turn involves a rudimentary form of cognition and consciousness. The sensitiveness connects the several parts of the individual with each other, and the individual as a whole with its surroundings. 
A low form of sensitiveness, cognition, and consciousness is necessary to every independent organism, however rudimentary and minute. Without these the individual could not possibly maintain its place in nature.

If an umœba-which consists of a more or less homogeneous, jelly-looking speck-be examined under the microscope, it will be seen to move in every direction, to feel about for food, and when it finds it to throw its body over the food and convert whatever part of the body the food touches into a temporary stomach. Every part of the arnoba can discharge the aggregate functions of the individual. The movements of the amœeba are independent, deliberate, and purpose-like. They are controlled movements; the control extending to all parts of the amoba, and, within limits, to the matter on or in which the amœba moves.

In the lowest plants and animals, where there is little or no differentiation, and where every part virtually resembles every other part, the lines of communication and force, whatever they are, are of the simplest and most direct. The conditions which obtain are analogous to those which obtain in wireless telegraphy, where messa.ges are despatched and received without the aid of wires or visible means of communication. That the messages in both cases are transmitted through matter in a state of vibration goes without saying. That the messages in both cases are transmitted in waves in more or less straight lines seems equally certain. As in wireless telegraphy obstacles such as buildings, stone walls, \&c., do not prevent the transmission of messages, so in plants and animals, even where differentiation occurs, and where heterogeneous substances are present, messages between the different parts of individuals and the outer world are possible. It would seem that wherever there is continuity of matter (not necessarily homogeneous matter) in the organic and inorganic kingdoms, messages may be sent and received in the absence of separate, independent, visible lines of communication, such as telegraph wires on the one hand and nerves on the other.

There is reason to believe from recent observations that force travels in waves and in nearly straight lines, with a certain amount of similar lateral, branching wave movement. This is seen in the lightning flash, rendered visible by the aid of instantaneous photography. The movements of lightning are not, as was generally believed, simple zigzag, straight-line movements, as depicted by artists, but arborescent or branched wavy movements, where there is the central or main movement in a more or less straight line, and a great many similar branching, subsidiary wave movements. A flash of lightning, as revealed by instantaneous photography, resembles a tree with its branches, or a leaf with its midrib and subsidiary venation.

The instantaneous photograph gives what is practically an analysis of the movements of lightning, and these movements are amongst the most rapid and striking in nature (vide Plates xxix. to xxxi. inclusive).

If we regard lightning as one of the representative forces of nature, it is difficult to escape from the conclusion that all force, whether in the inorganic or organic kingdom, is primarily and essentially of the dendritic type-that is, power acting upon matter in one particular and main direction, and in lateral, branching, subsidiary directions. Such a view would very largely account for the general form of plants and animals, and for the major part of their details. It would also account for the main and subsidiary lines of communication in plants and animals.

The nerves branch from the neurons, ganglia, and nerve cells found in the brain and spinal cord; the arteries branch from the heart; and the blood-and other vessels are laid down and formed in the dendritic tracts mapped out by the saps of plants and animals in the processes of development and growth. Fluids permeating matter tend to separate into streams, and this holds true equally of organic and inorganic fluids. It is also true of organic and inorganic matter.

This community of force and of form in plants and animals, and in the universe, is in keeping with the origin and nature of plants and animals. Plants and animals derive all the materials composing them directly or indirectly from the external universe. They also derive $a$ considerable proportion of the force which actuates them from the same source. If the life or vital force which characterises plants and animals were eliminated, the elements, or such of the elements as are incorporated in plants and animals, would in no way differ from similar elements found in the physical universe. The elements are common to the inorganic and organic lingdoms alike, and the so-called inorganic elements build up at the outset, and continue to circulate through, plants and animals so long as they continue to live.

It is now known that masses of plant and animal protoplasm are connected to each other by the most delicate protoplasmic threads, requiring in many cases very high powers of the microscope to see them. 'These delicate, almost invisible threads, there is reason to believe, reveal the furst traces of lines of communication along which force can travel in living matter. It is difficult to understand how the almost invisible protoplasmic threads referred to are formed, but they can scarcely be a product of mere viscosity, as in that case they would almost inevitably break long before reaching the extreme degree of tenuity which characterises them.

Plants and animals are derived from cells, spores, and eggs, and the lines of communication and of force in many cases radiate from or converge towards a central point; the point in question corresponding with the seat 
of origin of the individual or with a centre developed during the process of differentiation. The ovum is the centre of the developing fœetus-the brain is the centre or terminal of the adult vertebrate animal.

While the shape of the plant and animal is determined to a large extent by the lines of communication and force, the latter in turn are dominated within limits by the former. Thus, in rod-like plants and animals the lines of communication and the direction of force are in straight lines; in globular plants and animals with a central origin they are in straight lines and in curves, whereas in spiral plants and animals they are helical.

In plants and in the lowest animals there is no trace of a nervous system as we know it, but a nervous system or its equivalent may nevertheless exist in both in an undifferentiated form. The fact that some of the lowest animals - the amoba for instance, and certain of the higher plants, such as the sundew - can, and do, move in given directions, at certain times, and to definite ends, goes far to prove not only that there are lines of communication akin to nerve tracts, but that there are also controlling forces which move along these lines, resembling in many respects nerve forces.

To trace the rise and progress of the nervous system in animals to its culmination in man is one of the most difficult problems in physiology. Animals which were long supposed to be devoid of a nervous system (the jellyfish for example) are now known to possess it. The nerves in the jelly-fish are not only extremely delicate, but they are also very soft. The condition of fluidity or softness is characteristic even of the human brain, which is the highest representative of nerve substance. The brain, which transcends all other organs in power, is, strange to say, nine-tenths water. A little excess of softness in the nervous system of the jelly-fish would make the nerves so transparent as to be invisible. It follows from this that a fluid or semi-fluid nervous system in animals, and even in plants, is well within the possibilities. Certainly the movements and simultaneous power of feeling possessed by certain plants, and the majority of even the lowest animals, plainly indicate some such arrangements.

If any one takes the trouble to study under the microscope the countless myriads of animalcules and low animal and vegetable forms which swarm in water and liquid generally, and if, in particular, he watches how in their darting about in search of food they regulate their speed, the direction in which they move, and, in especial, how they avoid colliding with each other, he will be forced to admit that none of the movements he observes are chance movements. If this be conceded, then the obvious corollary is that the movements referred to are voluntary. Voluntary movements, however, as we know them, imply a nervous system, or its equivalent, and this in turn necessitates sensitiveness of a kind. Sensitiveness implies feeling, and feeling cognition, which culminates in the consciousness of the higher animals. The most complex structures are, at their beginnings, developed from comparatively simple and very slightly differentiated protoplasm. In plants and the lowest animals, if nerve substance exists, it must occur in a fluid or semi-fluid, transparent state. The semi-liquid condition of nerve matter in the jelly-fish favours this view. Living things are not chance products. They are, on the contrary, distinct entities. From the lowest to the highest they have independent rôles to perform, and they perform them with remarkable regularity and uniformity. In order to do so, they must be provided with bodies in the form of living matter, and these bodies must be amenable to control as regards the intake and output of food, as regards respiration, circulation, sensation, movement, excretion, \&c.; but control implies directly or indirectly the possession of a nervous system, or its equivalent in an undifferentiated or invisible form. This argument is valid as regards the entire race of animals. It also applies, within limits, to quite a large number of plants.

Founding as we do our ideas of sensation, perception, movement, \&c., on a brain, sensory and motor nerves, muscles, \&c., as we find them in the higher animals, we are apt to deny to the lower and lowest animals, even in a rudimentary form, the structures and attributes which we find in a greater or lesser degree of perfection in the higher and highest animals. Nothing could be more unphilosophical. Brain and nerve substance and muscle, as mere living matter, have not necessarily any advantage over other living matter, and there can scarcely be a doubt that in all living matter, even in protoplasm, there are the potentialities and powers which lend themselves to the formation at once of the simplest and most complex plant and animal organisms. These organisms, the simplest equally with the most complex, are independent beings, capable of managing their own affairs, each in its own way. If the most complex exercise their functions through the instrumentality of a brain, nervous system, and muscles, that is no reason why the more simple should not exercise similar functions by other means and methods, or by similar means and methods in what appears to us (with our limited powers of observation) an undifferentiated form. To take an example. The vacuoles in certain water plants exhibit rhythmic movements in every respect similar to those displayed by the mammalian heart; the vacuoles closing suddenly and opening slowly, as in the several compartments of the heart. But plants have neither muscles nor nerves in the ordinary sense. Again, the heart of the chick, while yet a mass of nucleated cells, and before it even contains blood, displays characteristic rhythmic movements. Here again the muscles and nerves to which we attribute the rhythmic movements of the adult heart are absent. 


\section{LINES OF COMMUNICATION AND FORCE}

Nature can clearly achieve the same results by various means.

It is extremely difficult and exccedingly dangerous to attempt to define the properties and powers of living matter. We certainly cannot dogmatically affirm that all living things devoid of a visible nervous system and muscles are incapable of feeling and moving. The fact that living things devoid of muscles do move, and to definite ends, should go far to prove that a nervous system in a visible form is not necessary to sensation and voluntary motion.

The operations of nature are mysterious and often inscrutable. That she can produce similar results by what
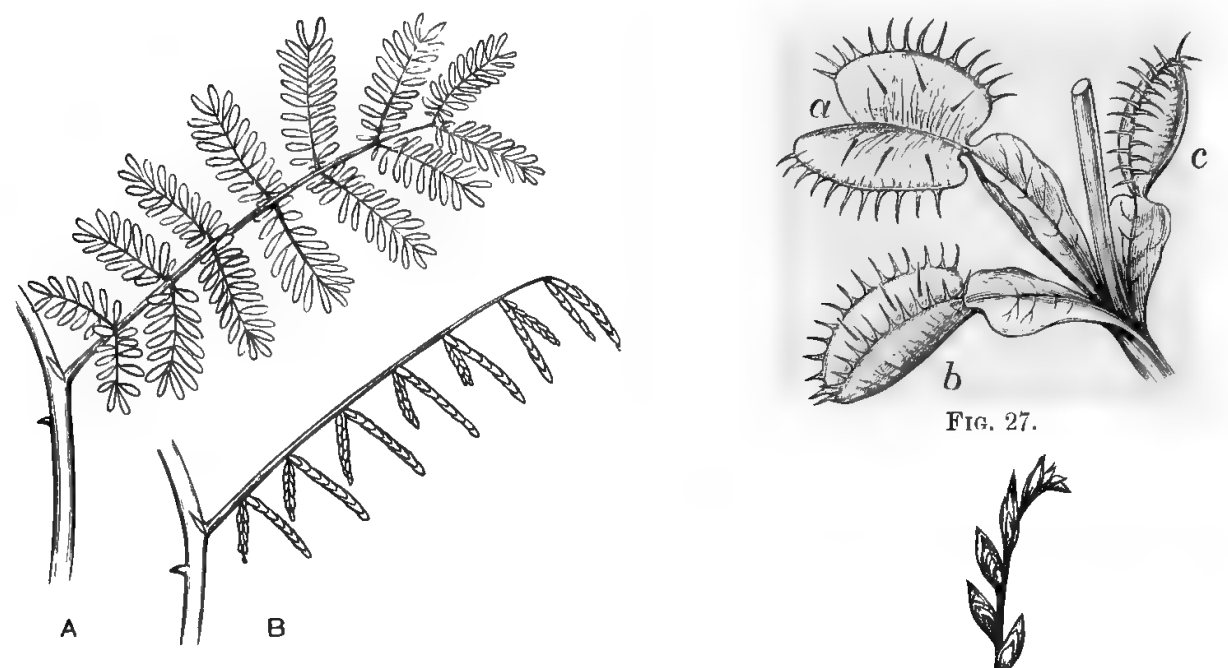

Fr(*, 25.

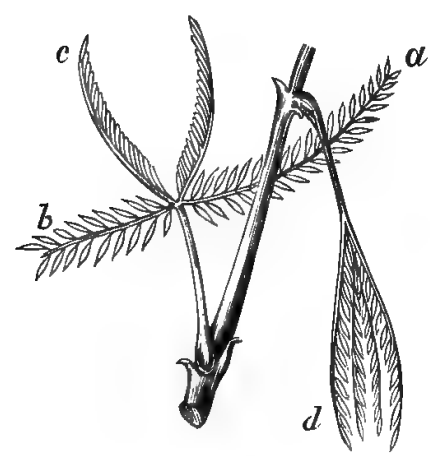

Frs. 26.

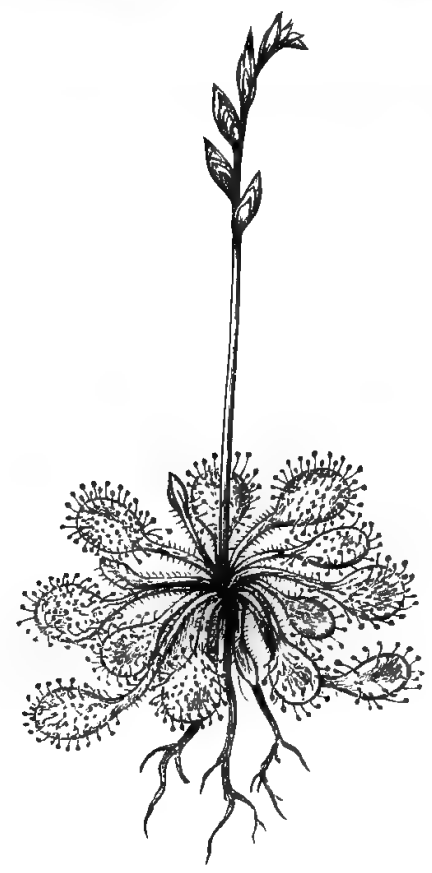

FIG. 28.

FIG. 25.-Acacia farnesiana. A. Leaf during day. B. The same at night (after Darwin).

FIG. 26.-Branch and leaves of sensitive plant (Mimosa pudica), showing the petiole in its erect state (a) and in its depressed state $(d)$; also the leaflets closed $(c)$ and the leaflets expanded $(b)$. During darkness the leaf stalks hang down, and the leaflets are closed, while the reverse is the case during light (after Balfour).

FIG. 27.-Leaves of Dionæa muscipula, called Venus's fly-trap, showing the expanded blade of the leaf $(a)$ with three irritable hairs on each division of the lamina. These hairs, when touched, cause the closure $(b, c)$ of the two halves of the lamina or blade of the leaf (after Balfour).

FIg. 28.-Sundew (Drosera rotundifolia); grows on poor bog land and lives largely on insects caught by its leaves. The leaves are round-shaped, and covered with 200 or more highly sensitive hairs or tentacles. Each hair secretes a pure viscid fluid, which clogs the feet and wings of the insects when they alight on it, the hairs bending down upon their victims and fixing them at or near the centre of the leaf, where they are digested (Century Dictionary).

appear to us dissimilar methods and means is abundantly proved by this. Electricity can be manufactured by various living animals from organised materials, and it can be produced by water or other power from inorganic materials by means of friction, \&c. It is also produced, under certain conditions of the atmosphere, as a natural force. However produced, electricity is one and the same. The electrical cycle, if I may so designate it, brings us 
back to lines of communication and lines of force, to the interdependency of organic and inorganic matter, and to form and movement as the outcome of lines of communication and force in the interdependency of matter as a whole. There is a sequence of events which follow necessarily upon each other, and organic and inorganic matter and vital and physical force are continually acting and reacting upon each other.

It has long been known that the sensitive and insectivorous plants are capable of receiving impressions from without and transmitting impressions from within, and so imitating the so-called reflex nervous actions and movements of animals. Thus, if the sensitive plant (Mimosa pudica) be touched it immediately begins to fold up its leaflets. If the contact be continued and increased, the leaf as a whole becomes depressed, and ultimately the leaf stalk also. These movements in response to an external impact necessarily imply two things : first, that the plant feels the touching body, which means that the impact so produced travels into its substance from without inwards; and second, that the plant, having felt the touch or impact, generates in its substance a counter impulse, which travels in a direction from within outwards, and causes the movements of the leaflets, leaf, and leaf stalk referred to. No other explanation, it appears to me, is possible. The plant, after a longer or shorter time, elevates its leaf stalk, raises its leaf, and opens its leaflets as it were voluntarily.

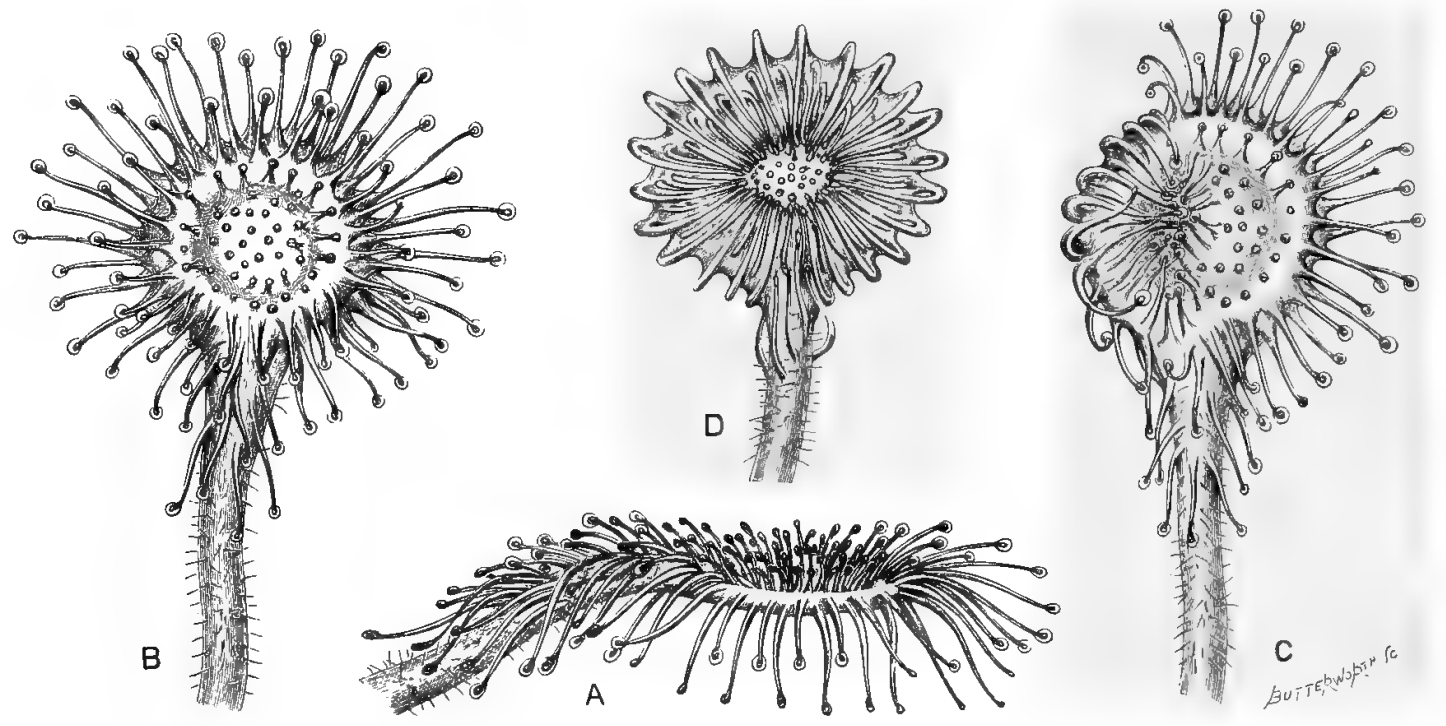

FIG. 29.- Shows the shape of the leaf of the sundew (Drosera rotundifolia) and the appearance of the tentacles when at rest and in action.

A. Leaf of the sundew with its numerous long, tapering, highly sensitive hairs or tentacles, each terminating in a small oval swelling, and tipped with a bleb of clear, viscid secretion very attractive to insects. Magnified four times (seen laterally).

B. Another leaf seen from above. Magnified four times. In A and B the tentacles are not in action.

C. In this figure a tiny speck of meat has been placed on the leaf, and the tentacles to the left of the spectator are seen bending and pressing it in the direction of the centre of the leaf.

D. In this figure all the tentacles are in action, due to the leaf having been immersed in a very weak solution of ammonia (one part to 87,500 of water). The leaf presents the appearance witnessed when an insect is caught and conveyed to its centre by the bending of the tentacles, where it is crushed and held firmly until the digestive secretion is exuded and the act of digestion, absorption, and assimilation completed. The assimilation over, the tentacles gradually unbend and straighten, and prepare to receive new prey (after Darwin).

The sensitiveness and movements of the insectivorous plants are still more extraordinary. In the case of Venus's fly-trap (Diomea muscipula) the plant is provided with an expanded, bi-lobed, hinged leaf, on the surface of which occur six very highly sensitive hairs. When any insect, however minute, alights on or crawls along the leaf, and cones in contact with the sensitive hairs, the leaf immediately folds up, closes, and captures the intruder. Nay more, it crushes the insect, and exudes a secretion akin to gastric juice, with which it actually digests it. Here the double lines of communication and of force become very obvious. An impulse travels inwards from the sensitive hairs to the substance of the plant. This begets a counter or outward impulse, which results in the closing of the bi-lobed leaf of the plant. Nor does the matter rest here : other inward and outward impulses are brought into play, whereby digestion and assimilation are accomplished (Fig. 27).

In Venus's fly-trap there is a high degree of sensitiveness, a low form of cognition, and a power to move in given directions and to definite ends. 
In the sundew (Drosera rotundifolia) the arrangements are, in some senses, even more complex and astounding (Figs. 29 and 30).

In this extraordinary plant, the expanded leaf is studded with a very large number (200 or so) of amazingly sensitive hairs or tentacles, and the tips of these tentacles, marvellous to relate, are each provided with a speck of glistening viscid secretion which is very attractive to insects. The trap in this case is not only set but baited.

When an insect touches one or more of the numerous tentacles, the viscid secretion clogs the wings and feet of the insect, and makes escape difficult, and, in most cases, impossible. This is the first stage of capture. No sooner is the insect entangled than the sensitive hairs or tentacles all around begin to inflect; the inflection is continued until by a progressive rolling movement the insect is carried to the centre of the leaf, where it is firmly pinned down. This is the second stage of capture. The third consists in the crushing of the insect against the surface of the leaf by the infolded tentacles and the exudation of an acid secretion resembling the gastric juice of the human stomach. The insect caught and crushed is finally digested and assimilated. Digestion and assimilation having been accomplished, the sensitive tentacles unbend and become straight as at first, each in due course being provided at its tip with a tiny drop of glistening secretion calculated to entice and ensnare other insects of all kinds, from small beetles to even butterflies and dragon flies. The mechanism employed by the sundew to catch and devour insects is, in some respects, highly complex. It certainly involves lines of communication and of force, but is not a reflex act in the ordinary sense; the latter requiring an elaborate nervous mechanism.
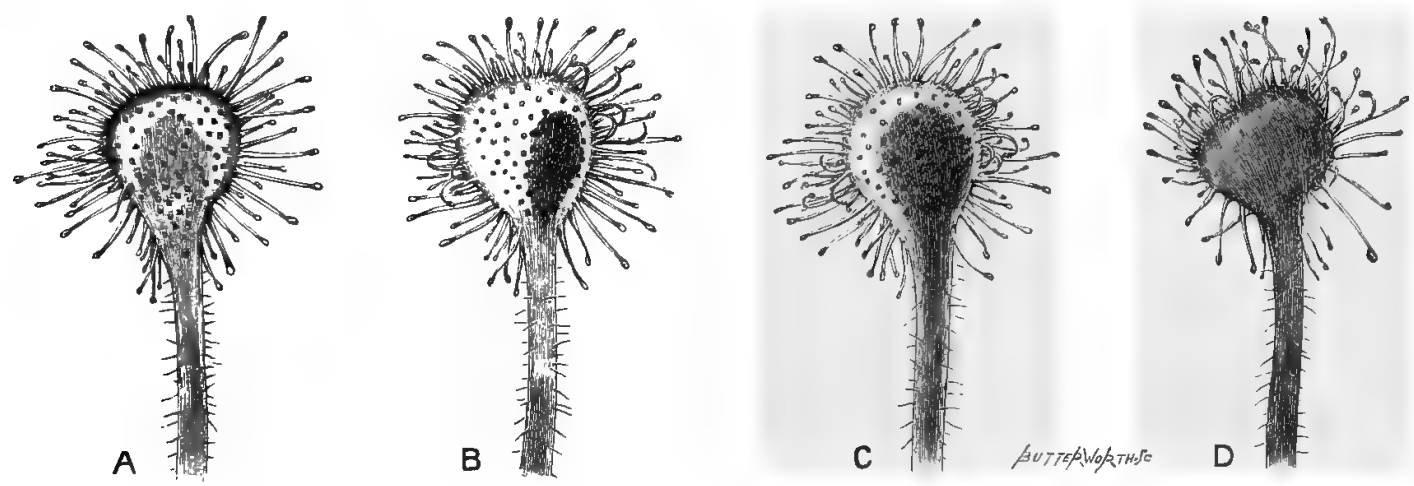

FIG. 30. - Shows the clouding or staining of the leaf of the sundew during digestion (magnified twice).

A. Appearance presented by the leaf before feeding.

B. The same leaf five minutes after a small portion of dry proto-albuminose had been placed on it. The clouding or dark stain represents the position of the proto-albuminuse; some of the tentacles are seen bending towards it.

C. The same leaf twenty-eight miuutes after feeding. The proto-albuminose is partly dissolved, and the clouding or staining occasioned is spreading over the leaf and down the leaf stalk. More of the tentacles are also bent.

D. The same leaf twenty-eight hours after feeding. The proto-albuminose is now largely dissolved, and the area of the clouding or staining of the leaf and leaf stalk greatly enlarged. Absorption is evidently occurring. The number of tentacles bent is also increased (after Gillespie).

First, there is the touch or impact of the insect against the sensitive hairs; second, the inflection or bending of the individual hairs touched; third, a combined inflection and rolling movement of other hairs whereby the insect is carried to the centre of the leaf; fourth, the crushing of the insect on the centre of the leaf; fifth, the exudation of a substance analogous to gastric juice, and the digestion and assimilation of the insect. Finally, there is the unbending or straightening of the tentacles and the supplying afresh of their tips with what is practically a tempting bait with a view to fresh captures of insects. These arrangements plainly necessitate sensitiveness and double lines of communication and of force. The plant feels, and, within limits, knows when the insect touches it. This implies an impulse travelling from without inwards, that is, from the insect into the substance of the plant. The tentacles bend not at their tips but at their base, and digestive secretion is poured forth; which means counter impulses which travel from within outwards. The unbending movement of the tentacles is also occasioned by an impulse travelling from within outwards. This follows, because it is quite evident that the tentacles which are made to bend by one impulse or force cannot be made to unbend by the same impulse or force. They are counter movements, and must be produced by counter impulses or forces and counter lines of communication.

The co-ordinated, purpose-like, semi-intelligent movements and acts of the insectivorous plants can only be explained either by the direct interposition of a First Cause, or by assigning to them powers not dissimilar and in some respects not inferior to those exercised by many animals.

VOL. I. 
If we now turn to Badhamia utricularis we encounter an organism which may fitly be regarded as an intermediate or connecting link between the plant and the animal (Fig. 31). This plant-animal, if I may be allowed the expression, which has no definite shape, advances upon its food in successive waves by means of its plasmodium or sarcode-a form of protoplasm. It is endowed with what are virtually rhythmic movements, in virtue of which it not only advances but retires. It lives upon fungi, and these it devours by any part of its substance. The absorption and assimilation of food are of the simplest description.

The organism is active and quiescent by turns - that is, it rests and feeds at intervals. It reproduces itself by means of spores. Its sensitiveness is of a low type, as are also its powers of digestion and assimilation. It is, however, believed to exercise a rudimentary form of secretion and excretion. Its movements are certainly very remarkable. Altogether Badhamia utricularis must be credited with the double lines of communication and of force

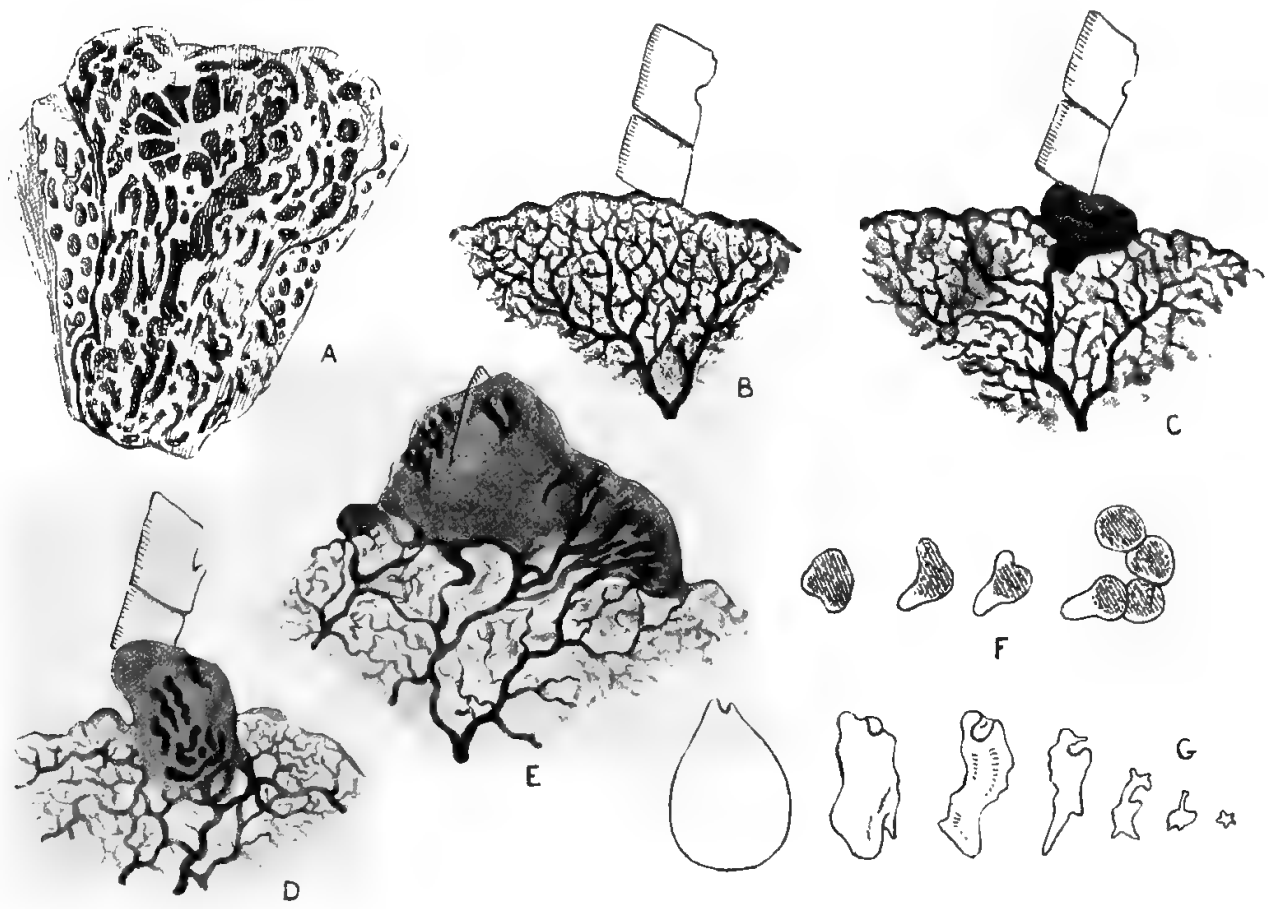

FIG. 31.-Badhamia utricularis in its resting, active, and'feeding states.

A. Sclerotium or dry state of Badhamia, $\times 4$.

B. to E. Plasmodium of Badhamia advancing on section of stenı of Agaricus melleus. B, drawn at 12.25 P.M. ; C, at 12.40 P.M. ; D, at 1 P.M. ; and F, at 2 P.M., $\times 3 \frac{1}{2}$.

F. Young spores of Badhamia, showing amoeboid movements, the lower spore at right hand of figure changing its shape in the course of a few ninutes to that seen at the extreme left of the figure, $\times 565$.

G. Vegetahle substance dissolved by plasmodium of Badhamiu diminishing in size from left to right of figure, taken at intervals of about a quarter of an hour, $\times 250$ (after Lister).

claimed for the sersitive and insectivorous plants. It could not otherwise search for, find, and devour its food and get quit of waste products.

It would be an endless task to trace the double lines of communication and of force throughout the lowest and lower animal series, as with the means of research at our disposal at present it is impossible to discover when the nervous system first makes its appearance. Fnough has been said to show that double lines of communication and of force are a necessity, and exist in one or other form, visible or invisible.

As bearing on the non-necessity of nerve matter as a vehicle of communication and of force it may be stated that even in the highest animals in their early stages of development neither nerves nor brains are present. As a matter of fact, development has proceeded to an advanced stage before either make their appearance. Growth and movement occur independently of nerves. In the anencephalous human fœetus the child is full grown notwithstanding the absence of a brain, which is the central controlling power of the nervous system. The lines of communication 
and of force of the child in utero are twofold, namely, as between the separate parts of the developing foetus, and as between the fœetus as a whole and its parent.

Perhaps the best example of a rudimentary typical nervous system is that supplied by the five-rayed starfish (Fig. 1, Plate lvii.). This consists of a commissural ring of nervous matter surrounding the mouth. In this ring. at the base of each ray or limb, occur nerve ganglia, and in connection with them two sets of nerves-namely, a sensory set, extending between the skin or integumentary system to the ganglia, and a motor set, proceeding from the ganglia to the muscular system. There is as yet no trace of a brain. The nervous system of the starfish lays down, unmistakably, the apparatus by which lines of communication are established, and impulses transmitted. It provides for the transmission of sensory impressions from without and motor impulses from within. The starfish is a sensitive living thing, and can move voluntarily. A brain is not necessary to the more rudimentary and lower forms of voluntary movement. By means of its sensitive nerves the starfish is made aware of matters outside itself. By means of its motor nerves and muscles it can move its body towards anything it fancies. It can also remove its body, or part of it, out of the way of danger. The movements of the starfish are generally spoken of as reflex movements, but they can only be regarded as reflex if the starfish be proved to be an automaton pure and simple, which it cannot possibly be.

The movements of the starfish are not identical with the so-called reflex movements of a decapitated frog, or those of the higher animals where, from a lesion in the upper part of the spinal cord, the brain is virtually detached from the cord and the nervous system generally.

The theory of reflex action utterly breaks down when applied to the starfish in the following particulars: (a) it makes no provision for the performance of voluntary movements on the part of the animal; $(b)$ it assumes that all its tissues are highly irritable and excitable (which is not the case); (c) it necessitates the application of artificial stimulation before the animal, or any part of it, can or will act; $(d)$ it regards the starfish as an automaton, the movements of which are purely mechanical and involuntary, which they certainly are not. The starfish can feel, but there is no proof that the animal in its normal condition is irritable and excitable, and that it must be jogged into activity by outside stimulation. I direct attention at the earliest opportunity to the theory of reflex action as applied to the starfish, because its supporters seek to obscure what they cannot explain, and to set up an artificial boundary as between animals with nerve centres or ganglia plus sensory and motor nerves on the one hand, and animals with all these plus a brain on the other. No such distinction can be drawn. The theory would destroy the common ground (whatever its nature) which exists between the lower animals and the highest plants, and between the lower and higher animals themselves. I discuss this subject at considerable length further on when dealing with reflex action, instinct, and reason; but I desire to warn the reader against rashly accepting a theory which conveys an entirely erroneous impression of the nervous system as a whole.

Dr. John C. Dalton, the distinguished professor of physiology to the College of Physicians and Surgeons, New York, gives expression to the prevailing belief in these words: "When any stimulus or irritation is applied to the integument of one of the arms (of the starfish), it is transmitted by the nerves of the integument to the ganglion situated near the mouth. Arrived here, it is received by the grey matter of the ganglion, and immediately converted into an impulse, which is sent out by other filaments to the muscles of the corresponding limb; and a muscular contraction and movement consequently take place. The muscles therefore contract in consequence of an irritation which has been applied to the skin. It must be recollected that this action does not necessarily indicate any sensation or volition, nor even any consciousness on the part of the animal. The function of the grey matter is simply to receive the impulse conveyed to it, and to reflect or send back another; and this may be accomplished altogether involuntarily, and without the existence of any conscious perception. It is the simplest form of reflex action. Where the irritation applied to the integument is of an ordinary character and not very intense, it is simply reflected, as above described, from the corresponding ganglion back to the same limb. But if it be of a peculiar character, or of a greater intensity than usual, it may be also transmitted by the commissures to the neighbouring ganglia; and so two, three, four, or even all five of the limbs may be set in motion by a stimulus applied to the integument of one of them. According to the character and intensity, therefore, of the original stimulus, it will be followed by a response from one, several, or all of the different parts of the animal frame."

In speaking of the nervous system of the centipede he says, "It consists of a linear series of nearly equal and similar ganglia arranged in pairs, situated upon the median line, along the ventral surface of the alimentary canal. Each pair of ganglia is connected with the integument and muscles of its own articulation by sensitive and motor filaments; and with those which precede and follow by a double cord of longitudinal commissural fibres. In the first articulation, moreover, or the head, the ganglia are larger than elsewhere, and send nerves to the antennæ and to the organs of special sense. This pair is termed the cerebral ganglion, or the 'brain.'

"A reflex action may take place, in these animals, through either one or all of the ganglia composing the nervous 
chain. An impression received by the integument of any part of the body may be transmitted inward to its own ganglion and thence reflected immediately outward, so as to produce a movement of the limbs belonging to that articulation alone; or it may be propagated, through the longitudinal commissures, forward or backward, and produce simultaneous movements in several neighbouring articulations; or, finally, it may be propagated quite up to the anterior pair of ganglia or 'brain,' where its reception will be accompanied with consciousness, and a voluntary movement reflected back upon any or all of the limbs at once. The organs of special sense, also, communicate directly with the cerebral ganglia; and impressions conveyed through them may accordingly give rise to movements in any distant part of the body." 1 Similar reasoning, I would point out, is applied to the nervous system of man, especially to the ganglia or nerve centres of the spinal cord. It is also applied to the nerve centres of the head and other parts in their relation to the sense organs. Figures illustrating the comparative anatomy of the nervous system of the lower and higher animals are given at Plate lvii. which follows.

The starfish is capable of controlling its movements. It can move towards an object which it fancies, or away from an object which it dislikes or fears. The element of voluntariness enters into all its acts. It takes full advantage of the lines of communication which connect it with the outer world, and the lines of force which enable it to move its several parts as a whole (Plate lvii., Fig. 1, A).

\section{PLATE LVII}

This plate shows the beginnings and gradual advance of the nervous system as represented by ganglia and sensory and motor nerves in the lower animals: also the progressive development of the spinal cord and brain in the higher animals. The brain is to be regarded as an expansion of the spinal cord, and consists of essentially the same nerve elements.

FIG. 1.-Illustrates the nerve arrangements in the five-rayed brainless starfish. It consists of a circular commissural nerve ring with five nerve centres or ganglia (one at the foot of each limb). Each ganglion receives and gives off sensory and motor nerves.

A. $a$, Commissural nerve ring surrounding the mouth of the starfish; $b$, oral aperture of starfish; $c$, one of the five triangularshaped ginglionic masses; $d$, motor nerves; $e$, sensory nerves. The starfish in virtue of its nerve arrangements can receive sensory impressions from withont and send out motor impulses from within. 'The animal can feel and move voluntarily, and is in no sense dependent for its movements on inherent irritability, stimulation, or so-called reflex action. The five-rayed starfish and its nervous system are symmetrical (after Daiton).

B. The Aplysia_an unsymmetrical nollusc-with an unsymmetrical nervous system. In the aplysia a rudimentary brain makes its appearance. The cephalic or bran gunglion is composed of two small ganglionic masses fused together and connected with the ganglionic nerve centres in all the other parts of the body by means of commissural nerve fibres. The ganglia receive and give off sensory and motor nerve fibres, and the animal can, like the starfish, feel and move to given ends, irrespective of inherent irritability, artificial stimulation, and so-called reflex action. $u$, Digestive or cesophageal ganglia; $b$, cephalic or cerebral ganglia; $c c$, pedal or locomotory ganglia; $d$, respiratory ganglion. The nervous system of the aplysia is symmetrical in the upper, and asymnetrical in the lower part of the body.

FIG. 2.--Nerve cells from a lumbar sympathetic ganglion without a sheath (A), and with a sheath (B), from the nervous system of an adult man. The cell substance contains pigment of a vivid yellow tint, and is consequently darkly granular (after Max Scliultze).

FIG. 3.--A. Illustrates the nervous system of the centipede (Scolopendra). It consists of a double chain of ganglia connected together by longitudinal and transverse nerve commissures; two ganglia with sensory and motor nerves being provided for each articulation of the animal. The cephalic ganglia are increased in size and united to form a fairly well developed lorain. The brain of the centipede sends nerves to the antennæe and to the organs of special sense. $a$, Brain composed of two symmetrical portions united longitudinally and transversely by nerve commisstures; $c c^{\prime}$, the longitudinal commissural fibres of the left side of the nervous system.

B. Illnstrates the nervous system of $\operatorname{man}(H n m o$ sapiens). The human nervous system is usually divided into a cerebro-spinal and sympathetic portion-the former only being here represented. The cerebro-spinal system closely resembles that of the centipede which furnishes its type. It consists of two symmetrical halves with collections of ganglia corresponding to the segments of the body. The ganglia are provided with sensory and untor nerves, and are united longitudinally and transversely by nerve commissures to secure harmonions working. 'The peculiarity of the cerebro-spinal system in man is the enormons expansion of the cerebro-spinal lobes or hemispheres which grow upwards, forwards, and backwards, and so cover in the great ganglionic nerve centres situated at the base of the brain. $a$, Cerebral lobes or great brain (cerebrum); $b$, ctrehellum; $c$, cervical portion of spinal cord : $c^{\prime}$, dorsal portion of spinal cord : $d$, brachial plexus and nerves going to the arms; $e$, lumbar plexus and nerves going to the legs.

FIG, 4.-Transverse sections of cervical portion of human spinal cord, showing the grey ganglionic nerve centres with their sensory and motor nerves. 'The grey ganglionic matter is arranged in two symmetrical crescentic masses within the cord: this being united longitudinally and transversely lyy nervous commissures. 'The sensory and motor nerves are connected with the free extremities (horns) of the crescentic grey substance and are white in colour. The cord is divided into two portious by anterior and posterior fissures. It is further divided by the grey crescentic matter of the cord and the ingoing and outcoming sensory and motor lierves, so
that it may be said to consist of six longitudinal columns.

Numerals are employed in describing the figures given of the spinal cord, and the same numerals indicate the same parts.

A. Anterior view of spinal cord.

B. View of right side of cord.

C. Upper surface of cord.

D. The nerve roots and ganglia seen from below.

(1), Anterior median fissure of cord; (2), posterior meilian fissure of cord; (3), antero-lateral depression; (4), posterior lateral

1 "A Treatise on Human Physiology," by John C. Dalton, M. D., \&ce, 5th edition, pp. 385-388. 
PLATE LVII

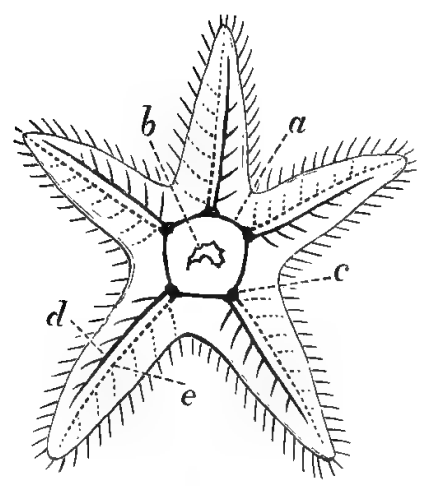

A

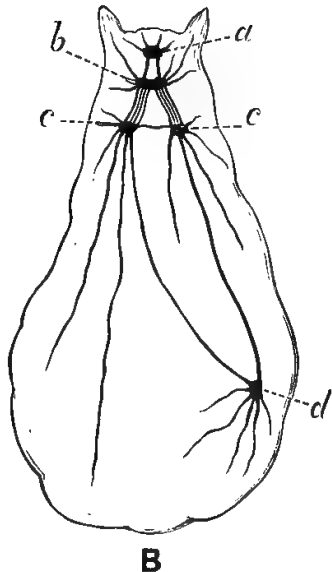

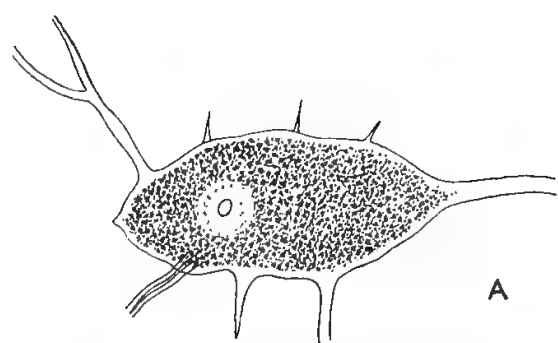

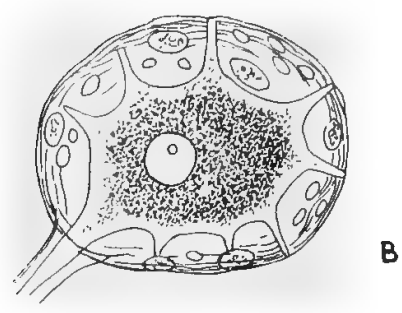

FIs. 2.
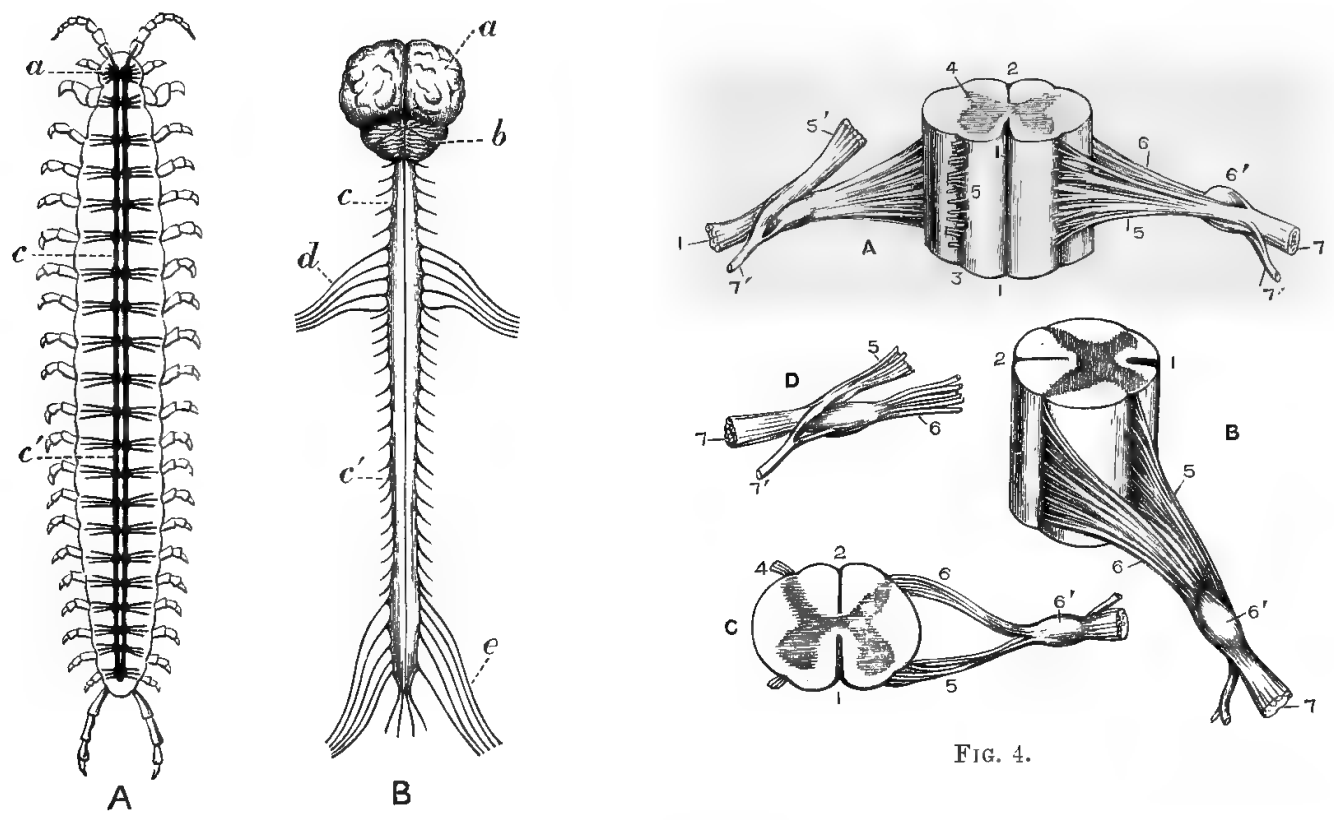

FJG. 4.

FIG. 3.

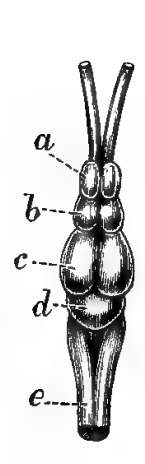

A

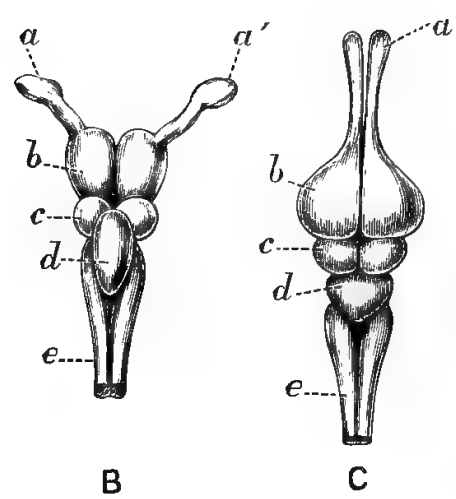

FIG. ธ.

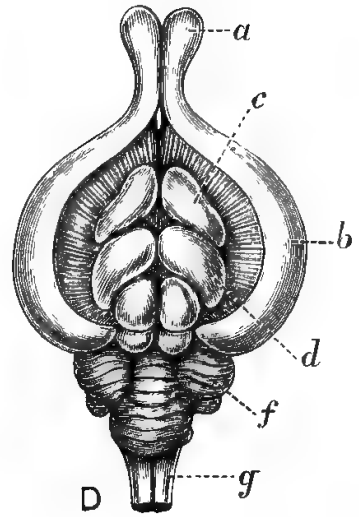

D III.-.

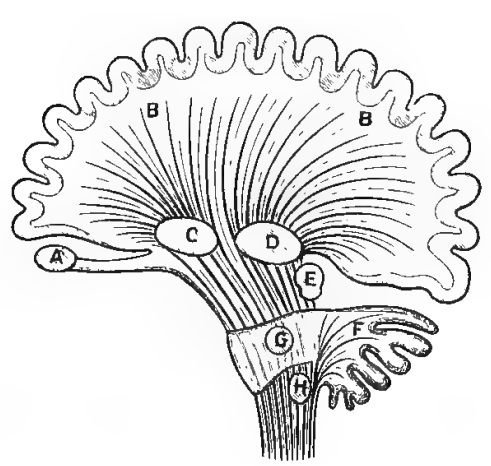

FIG, 6. 


\section{PLATE LVII (continued)}

groove in which the posterior sensory nerve (6) disappears; (5), anterior motor nerve sinking into antero-lateral depression of cord. These fibres display a ganglionic swelling $\left(6^{\prime}\right)$ in their course; $(7)$, the united compound or mixed nerve; $\left(\bar{l}^{\prime}\right)$, the posterior primary branch of same. This nerve is derived partly from the anterior and partly from the posterior roots of the spinal nerves (after Allen Thomson).

FIG. 5. - Illustrates the comparative anatomy of the brain and spinal cord in the fish, reptile, and mammal. The brain and spinal cord are divided longitudinally into two portions, and ars bilaterally symmetrical.

A. The brain and spinal cord of cod-fish. $a$, Olfactory lobes, two in number; $b$, cerebral lobes or hemispheres, also called cerebrum ; $c$, middle brain, giving rise to the optic nerves ; $d$, cerebellum ; $e$, spinal cord, with expansion of cord (medulla oblongata).

B. Brain of hammer-headed shark. The lettering is the same as in A (after Dallas).

C. Brain of alligator. $a$, Olfactory ganglia ; $b$, cerebral ganglia or hemispheres ; $c$, optic tubercles; $d$, cerebellum ; $e$, expansion of the spinal cord into the medulla oblongata.

D. Brain of rabbit. a, Olfactory bulbs or ganglia; $b$, cerebral ganglia or henispheres, separated to show the corpora striata (c), optic thalami $(d)$, and tubercula quadragemina, situated behind the optic thalami $(d) ; f$, cerebellum; $g$, spinal cord expanding into the medulla oblongata (after Dalton).

FIG. 6.-Vertical mesial line section of the human brain and spinal column (semi-diagranmatic), showing the situation of the several great ganglia at the base of the brain and the course of the conducting nerve fibres.

A. Olfactory ganglion; B, cerebral lobes or hemispheres (cerebrum); C, corpus striatum ; D, optic thalamus; E, tubercula quadragemina; $\mathrm{F}$, cerebellum; $\mathrm{G}$, ganglion of the tuber annulare; H, ganglion of the medulla oblongata.

In considering A, B, C, and D of Fig. 5 it will be seen that the great ganglia forming the lrains of the fish, reptile, and mammal are arranged in the same plane as the spinal cord, of which they are mere expansions. In Fig. 6 the great ganglia (the cerebral hemispheres excepted) are bent slightly forwards. These ganglia are situated on a different plane to the spinal cord, of which, as in the lower animals, they are mere expansions. The peculiarity of the human brain is the conparatively very great size of the cerebral lobes (cerelorum) which have grown upwards, forwards, and backwards, so as to cover in and conceal the ganglia at the base of the brain. The brains in Fig. 5 are seen from above. Fig. 6 gives a vertical mesial line section of the human brain (after Dalton).

In the aplysia, one of the molluses, a rudimentary brain makes its appearance. In this case there is a double row of ganglia, with their sensory and motor nerves in the cephalic portion of the animal ; the two ganglia which form the brain being more or less completely fused. The nervous system is symmetrical in the upper part of the body, but unsymmetrical in the lower part, where the respiratory nerve centre occurs (Plate lvii., Fig. 1, B).

In the centipede the nervous system has made a considerable stride. It consists of two longitudinal commissural tracts of nerve matter with a double series of ganglia, each segment of the animal being provided with two ganglia and with sensory and motor nerves extending between the skin and ganglia on the one hand, and between the ganglia and muscles on the other. The ganglia and nerves are united longitudinally and transversely, so that all parts of the body are capable of receiving sensory impressions, and are under control.

The two cephalic or highest ganglia are larger than the others. They are also united to each other, and more or less fused. A fairly well-developed brain can now be detected. The centipede is quick to perceive and flee from danger. Its power of voluntary independent movement is very remarkable. That the centipede feels by its antennæ and other parts cannot be doubted, and that it is aware of matter dead and living outside of itself is equally certain. If, however, the centipede feels and is capable of controlling and directing its own movements, and is aware of matter, however limited, outside of itself, then the question of self and conscious self, in however rudimentary a form, is raised: the question of a low form of cognition and a rudimentary power of reasoning is also raised. This subject will be discussed further on. It is enough to state here that in the nervous system of the centipede the lines of communication for receiving sensory impressions and sending out motor impulses are well marked, there being distinct sensory and motor nerves, and a rudimentary brain (Plate lvii.).

The nervous system of the centipede is the harbinger of similar systems in the vertebrata up to man. In man, the brain is an expansion of the upper part of the spinal cord. The spinal cord itself is composed of two bilateral halves, with longitudinal and transverse nerve commissures and symmetrical groups of ganglia on either side, associated with sensory and motor nerves. The brain, like the cord, is bilaterally symmetrical. It too is connected by longitudinal and transverse commissural and sensory and motor nerves, with their concomitant groups of ganglia and nerve centres. The brain and spinal cord with their groups of ganglia and sensory and motor nerves form an elaborate and complicated whole: the lines of communication and of force are all definitely laid down, and may be readily traced by the trained anatomist and microscopist. The nervous system in the higher animals, and in man, very closely resembles the telegraphic system of a great empire, where the brain corresponds to the capital, and represents the central telegraphic station; the ganglia in the spinal cord corresponding to the towns and villages with their lesser and intermediate stations, and the sensory and motor nerves to the several telegraphic wires along which messages are sent.

The sensory nerves transmit impressions or messages from without, as from the skin and sense organs; the motor nerves transmit messages from within, as from the brain to the muscles. 
So long as the nervous system is intact these messages are possible. If, however, any part of the nervous system be broken down by injury or disease, the messages become unintelligible or altogether cease. The same thing happens when there is a break-down in the telegraphic system.

It will be convenient to discuss this important subject somewhat more fully, especially in relation to the nerve elements themselves.

That the ganglia of the spinal cord with their complement of sensory and motor nerves can act independently of the brain can scarcely be doubted. Such action, however, implies no irritability on the part of the ganglia and nerves of the cord, and no extraneous stimulation. It simply means that the cord with its ganglia and sensory and motor nerves may be regarded as an independent nerve centre to the more or less complete exclusion of the brain. The ganglia or nerve centres of the cord can receive sensory impressions on their own account. They can also convert sensory impulses into motor impulses. This they can do at first hand, and without extraneous intervention. The history of the ganglia or nerve centres, spinal cord, and brain, makes this abundantly clear. The ganglia or nerves centres can act without a spinal cord, and the spinal cord can act without a brain, when the latter is diseased or removed. The object of the nerve centres in every instance is, firstly, to co-ordinate and bring into line the several parts of the body by means of more or less perfect voluntary movements; and secondly, to connect the animal with the outside world. This the nerve centres do by the aid of ganglia and sensory and motor nerves; the former going to, the latter coming from them. No hypersensitiveness or excitability of the nerve centres, and no extraneous stimulation of them is required for their normal action.

In the jelly-fish, which is void of a brain, even the ganglia and sensory and motor nerves are rudimentaryyet the animal is capable of feeling and acting voluntarily. In the starfish, as has been shown (and the same is true of the aplysia), there is still no trace of a brain, but the ganglia or nerve centres and the sensory and motor nerves are more fully developed. The starfish and aplysia can certainly feel and move voluntarily and to given ends as apart from irritability and artificial stimulation. In the centipede the nervous system is arranged symmetrically in two longitudinal lines; each segment of the animal being provided with two ganglia and two sets of sensory and motor nerves: the two cephalic ganglia running together to form a rudimentary brain. The power of the centipede to feel and move voluntarily goes without saying. In the double linear chain of ganglia forming the nervous system of the centipede can be traced the first beginnings of a spinal cord and brain. As a matter of fact, the brain, even in man, is to be regarded as an expansion and elaboration of a bilaterally symmetrical spinal cord. Virtually the same nerve elements enter into the composition of both. Both the brain and spinal cord are provided with ganglia or nerve centres and with nerve commissures and sensory and notor nerves in great profusion. They are also provided with an ample supply of conducting nerve fibres. The nerve commissures connect the ganglia longitudinally and transversely to secure united, harmonious action. The ganglia or nerve centres, the spinal cord, and the brain are furnished with an abundant supply of rich blood, and the cord and brain are provided with fibrous and osseous coverings to protect them from injury.

In the fishes and reptiles, the ganglia which largely form the brain consist of a double chain or series, and these are arranged on the same plane and on a line with the spinal cord itself. It is only in the birds and mammals, especially the latter, that the cerebral lobes or hemispheres (cerebrum) of the brain sprout upwards umbrella-fashion, and cover in more or less completely from above the double chain of ganglia which, as explained, are to be regarded as continuations of the ganglia of the spinal cord itself.

These several points are illustrated at Plates lvii. and lviii.

A subject of peculiar interest in this connection is the nature of the ganglia or nerve centres themselves, whether regarded as isolated, independent entities as they occur in animals, with no spinal cord and no brain; or as they occur in animals, with a spinal cord and a rudimentary brain; or as they are found in animals, with an elaborate spinal cord and with a wonderfully complex and highly differentiated brain. A microscopic examination of the ganglia or nerve centres, and the cells composing them, shows them to be variously shaped; some being round, some oval, some triangular, and some stellate. The star-shaped appearance is, for the most part, due to the entrance and exit of the sensory and motor nerves or conducting nerve substance of some kind; the points at which the sensory nerves enter and the motor nerves leave the nerve centres being known as poles. The ganglia, whether they belong to the cerebro-spinal or sympathetic system of nerves, consist of grey matter, the darker nerve substance. It occupies a central position in the spinal cord, and a peripheral one in the brain. This grey matter discharges very important functions, being accredited with the power of thinking in the brain, and of converting sensory into motor impulses in the spinal cord. The conducting nerves of the spinal cord and brain are composed of white nerve substance. The nerve ganglia are, in many cases, highly elaborated and exceedingly complex (Fig 2, A, B of Plate lvii. and also 1, 2, 3, 4 of Plate lviii.). They consist, like ordinary cells, of a cell wall or envelope, a nucleus, and a nucleolus - the contents of the nerve cell being molecular, granular, and protoplasmic in character. It is in 
the grey protoplasmic mass that the power of the ganglia chiefly resides. It is the grey matter of the ganglia which converts sensory into motor impulses, and which is the active agent in thinking and in voluntary movement.

Some of the ganglia may very well be regarded as independent nerve centres, exercising what is virtually brain power. The more highly differentiated ganglia may not inaptly be designated brainlets or little brains, that is, microscopic collections of grey nerve substance with a measure of latent brain power. They can act directly after the manner of brains, and as apart from irritability, artificial stimulation, and so-called reflex action.

While the brainlet does not represent the brain, it is nevertheless a part of the same whole, and essentially the same in kind.

I append figures of ganglia from the cerebro-spinal system of nerves, with the sensory and motor nerves which enter and leave them. Numerous other examples of ganglia are figured in other parts of the work.

\section{PLATE LVIII}

Plate lviii. shows the general resemblance of the several kinds of ganglia or nerve centres in the different animals and parts thereof. The ganglia are highly complex both as regards their structure and function. This is especially the case in the spinal cord and brain. The brain is a continuation and expansion of the spinal cord, and the nerve centres and elements found in the latter are repeated in the former. It is a question less of kind than of degree, the brain being the more highly developed and differentiated of the two.

FIG. 1.-Multipolar ganglion cell from the anterior horn of the spinal cord of the ox; $a$, axis cylinder process; $b, c, d, e$, branched processes. $\times 300$ (after Dieters).

Fig. 2.-A. Three bipolar ganglion cells from the ganglion Gasserii of the pike. The ganglia are seen at $a, b, c$ (after Bidder).

B. Three bipolar ganglion cells from the auditory nerve of the pike. At $d$, they are invested by the medullary sheath. At $e$, they are partially exposed; and at $f$, wholly exposed. Figure $\mathrm{B}$, shows the ganglion cells to be mere dilatations of the axis cylinder (after Max Schultze).

FIG. 3.-Ganglion cells from the electric lobes of the brain of the torpedo. Medium-sized specimen, $\times 600$. $u$, Axis cylinder process ; $b, b, b, b$, branched processes. Recent. Prepared by short maceration in serum containing a little iodine (after $\lambda$ Iax Schultze).

Fig. 4,-A medium-sized ganglion cell from the anterior horn of the spinal cord of the calf. $a$, Axis cylinder; $b, b, b, b, b$, branched processes abruptly broken off (after Max Schultze).

In discussing the lines of communication and of force in plants and the lower animals, I have alluded incidentally to what may be regarded as rudimentary sensation, perception, and a low form of cognition. I shall have occasion to return to this subject when I come to speak of the nervous system and of the reasoning powers of the higher animals, where consciousness, memory, judgment, and many other attributes of mind have to be predicated.

\section{ATOMS, MOLECULES, AND CELLS AS FACTORS IN ORGANIC STRUCTURE AND FUNCTION}

The growth and movements of plants and animals are very largely, indeed principally, due to changes occurring in the atoms, molecules, and cells, which enter into the composition of their bodies.

An atom is defined as an ultimate, invisible particle of matter. It is the most minute portion of a chemical element which can exist in a compound. Atoms are never free. They combine to form molecules, and, according to Dr. Thudichum, as many as 1895 atoms enter into the formation of a molecule of hæmatocrystallin.

The molecules, in turn, combine to form cells and tissues. The atom and molecule supply the basis of all matter, organic and inorganic. ${ }^{1}$

1 While the foregoing is the account given of the atom in works on chemistry, it is important to point out that of late years an electrical theory of matter has been propounded, which, if it prove correct, will entirely alter our views as to the non-divisibility of the atom.

In a lecture delivered at the Royal Institution of Great Britain (2nd March 1906), Professor J. J. Thomson of the Cavendish Laboratory, Cambridge, is reported to have stated that the old atom of the chemists since Dalton's days has gone, and in its place we now have corpuscles which make up the atom; and instead of its being a single indivisible unit, it is regarded rather as a system of bodies, not unlike the sun and the planets, and there is among the most recondite pliysicists a sort of "planetary theory of the atom." Following up Sir William Crookes's experiments with his famous tube, Professor. Thomson set to work to find the mass or weight of the electric particles that are thrown off when a current is sent through a high vacuum. He found that some of these particles were not more than the one-thousandth part of the mass of a liydrogen atom, and he gave them the name of corpuscles. The particles in question were all charged with negative electricity, and either they were electricity itself or they were the carriers of electricity. On the former view they have been named electrons. One startling result of the Professor's research was that the mass of the so-called corpuscle was always the same, no matter of what material the electrode was made from which the current was passed through the tube; and this seems to mean that these corpuseles, or sub-atoms, or electrons are "the ultimate particles, common to matter of all kinds," the protyle, of which Sir W. Crookes prophesied many years ago. If matter is an electrical manifestation, what then is electricity? Dr. Larmor replies in an abstruse theory. It is a state of intrinsic strain in the universal medium or ether. Our electrical apparatus are machines for producing this strain. It was at first a grave objection to this doctrine that if atoms are made up of electrons or corpuscles they must be liable to break up, and the breaking up of an atom was then unknown. Radium disposed of that difficulty, for radium is visibly breaking up. Professor Thomson explained the processes by which he had measured the corpuscle, proved its electrical character, and
measured its velocity-from 2000 to 60,000 miles per second. 
PLATE LVIII

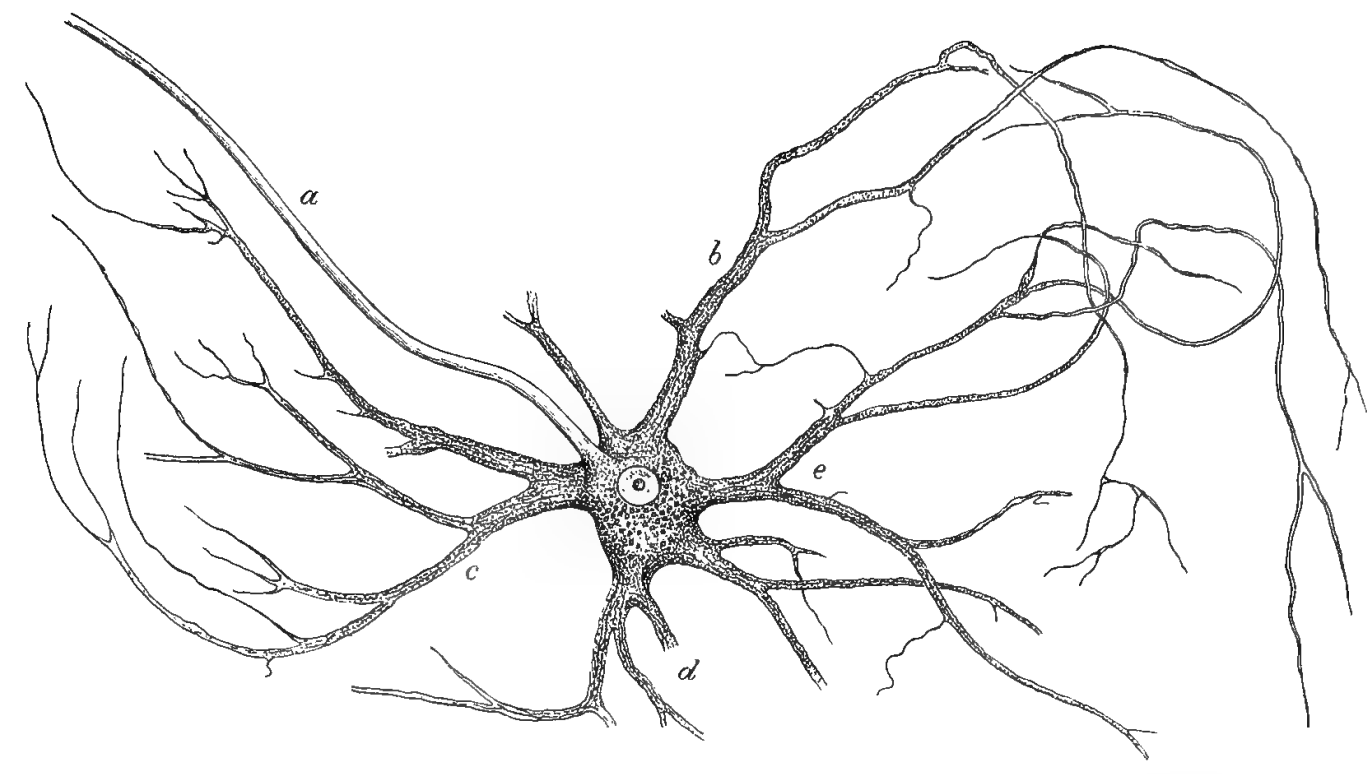

FIG, 1.

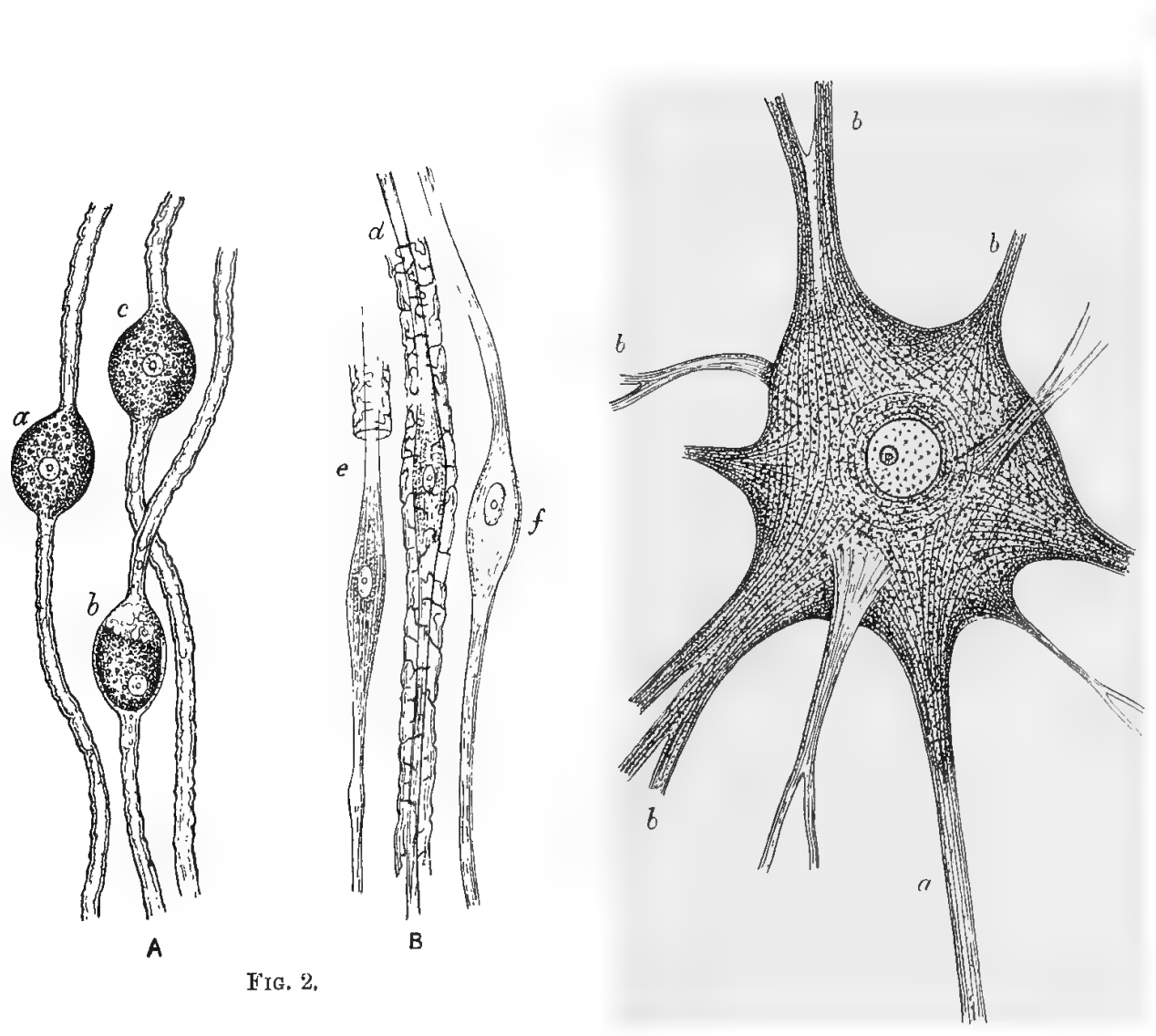

FIG. 3.

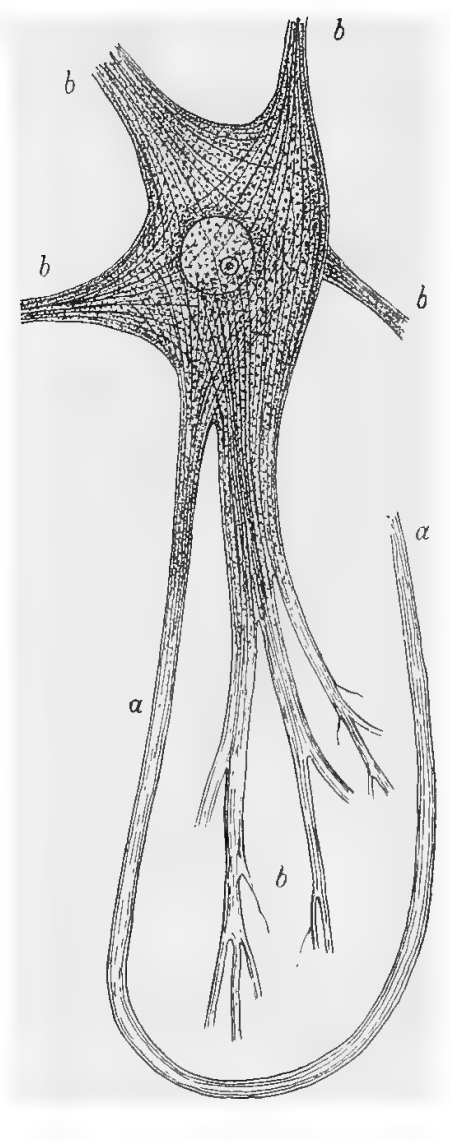

FIG. 4 
Professor J. Hughes Bennett thus describes a molecule: "By a histological molecule is to be understood a minute body, seen under high magnifying power in all organic fluids and textures, varying in size from the fourthousandth of an inch down to a scarcely visible point, which may be calculated at less than the twenty-thousandth of an inch in diameter. The smallest molecule has never been reached, even with the highest magnifying power. In the same manner that the astronomer with his telescope resolves nebulæ into clusters of stars, and sees other nebulæ beyond them, so the histologist with his microscope magnifies molecules into granules, and sees further molecules come into view." 1 The world is infinite in detail as it is vast in its general plan.

According to Bennett molecules are formed in two different ways.

1st. By precipitation in fluids or semi-fluid substances.

2nd. By the disintegration of previously formed tissues.

The former he calls histogenetic, or molecules of formation; the latter histolytic, or molecules of disintegration.

The division of molecules into "molecules of formation" and "molecules of disintegration" is countenanced by the fact that in the embryo the germinal matter breaks up and re-combines.

In the adult, moreover, new matter is being constantly added to the body, and waste or effete products extruded from it.

In the higher animals (man, for example) food before it can be assimilated must be reduced to the molecular condition, when it is known as chyle. Until it becomes chyle it cannot enter the blood and take part in nourishing the tissues.

Molecules lend themselves to endless combinations and transformations, chemical, physical, and otherwise.

The facts which favour the "molecular theory of organisation" are largely embryological in character.

The most essential part of the egg is the vitellus or yolk. From this the body of the embryo is formed, and the organs of the new individual developed. The yolk of the egg, however, is granular, that is, it is composed of molecules. These molecules, there is reason to believe, vary infinitely as regards substance, form, and function.

Molecules form cells and many other structures, such as the vitelline membrane, the sarcolemma, neurilemma, the anterior and posterior layers of the cornea, the capsule of the crystalline lens, \&c. Even the brain is largely molecular.

Molecules are necessary to life and reproduction. The Protozoa consist exclusively of molecules. They nevertheless live, grow, and reproduce themselves perfectly.

It may be stated, broadly, that the primary act of generation both in vegetables and animals is due to the presence of molecules, variously constituted and variously combined. The following is the account given by Professor $J$. Hughes Bennett of the part played by molecules in reproduction :-

"In the higher animals there are male elements, consisting of molecules, generally with, but sometimes destitute of, vibratile filaments ; and female elements, composed of the yolk within the ovum, containing a germinal vesicle or included cell. Both spermatozoid and germinal vesicle are dissolved in the molecules of the yolk, which then, either wholly or in part, by successive divisions and transformations, constitute a germinal mass out of which the embryo is formed. The male and female elements are, moreover, themselves molecular to begin with. Here, as in plants, it is necessary to remember that the spermatozoids, the yolk, and the germinal mass, are all composed of molecules, and that these, combining together, form the nuclei, cells, fibres, and membranes which build up the tissues and organs of the individual. It is not from either the male or female element that the embryo is formed. The essential action is not so much connected, as has hitherto been supposed, with the cell wall or nucleus, as with the molecular elements of the ovum. Many histologists, it is true, employ different expressions in referring to the primitive molecular material from which organisation proceeds : thus it is the 'organised concrete ' of Haller ; the 'solidescible nutritive fluid' of Wolff ; the 'primordial mucous layer' of Burdach; the 'sarcode' of Dujardin; the 'blastema' of Schleiden and Schwann; the 'proligerous pellicle' of Pouchet; the 'germinal matter' of Beale ; the 'protoplasm' of Remak, Von Mohl, and Kiihne; the 'embryo plastic matter ' of Robin; the "primordial protogenes ' of Haeckel, \&c., all which terms express essentially the same thing." 2

Professor J. Hughes Bennett, Professor T. Huxley, Dr. Lionel S. Beale, and others, regard the molecular basis or blastema of the egg as homogeneous and identical in all its parts and particles, but to this view I cannot possibly assent, for how, in reason, can tissues, organs, and organisms, infinitely diverse in ultimate structure and function, be produced from material absolutely identical, seeing the conditions under which development proceeds are, practically, the same? It is not in the nature of things. I "Physiology, General, Special, and Practical," by John Hughes Bemett, M.D., F.R.S.E., Professor of Physiology in the University of
Edinburgh, \&c. 1872, pp. 36, 37.

${ }^{2}$ Professor J. Hughes Bennett, op. cit, ju, 104. 


\section{ATOMS, MOLECULES, AND CELLS AS FACTORS}

The movements of molecules are interesting.

They are seen in the salivary cell, in the cells of various plants, such as Chara and Vallisneria, in the migration of pigment in the skin of the frog, \&c. Movements may also be witnessed in the molecules of many tissues. Indeed the aggregate of molecular movements and chemical changes in a part, constitute the normal function of such part. The movements occurring in glands and in the sarcous elements of muscles furnish examples. Molecules move in relays, in succession, and at stated intervals. This arrangement provides for the due nourishment and rest of every part of the plant and animal. Plants and animals take in food, rest, and work betimes. No plant or animal could be continually feeding, continually working, or continually resting. All this is done in due season, and the molecules are primarily concerned. The human heart, as a whole, is at work from the cradle to the grave; but every part of the heart_-all its sarcous or ultimate elements_have their appointed time for working, resting, and feeding. This goes without saying. Any other arrangement is inconceivable.

Molecules, as a rule, are actuated by two forces; a force of attraction and a force of repulsion-a centripetal and a centrifugal force.

Professor J. Tyndall gives a graphic account of the activity of molecules in his address as President of the British Association (1874). He says : "Everywhere throughout our planet we observe a tendency of the ultimate particles of matter to run into symmetric forms. The very molecules appear inspired with a desire for union and growth, and the question of questions at the present day is-and it is one, I fear, which will not be solved in our day, but will continue to agitate and occupy thinking minds after we have departed-this question of questions is, How far does this wondrous display of molecular force extend? Does it give us the movements of the sap of trees? I reply with confidence, assuredly it does. Does it give us the beating of our own breasts, the warmth of our own bodies, the circulation of our own blood, and all that thereon depends? To this question a similar reply must be given."

As a matter of fact, all movement, of whatever kind, is traceable primarily to atoms and molecules.

Less fundamental, but still most important, is the cell. The cell is a distinct entity, and contains, within itself, all the potentialities of independent existence: thus it has a birth, a period of development, a period of maturity, a period of decay and death. Each cell has a life history of its own. There are, as a matter of fact, cell plants and cell animals, where all the functions of life are adequately and faithfully discharged.

The red-snow plant and oscillatoria consist each of single isolated cells; the cell performing the function of nutrition and reproduction. The fungi and seaweeds-the so-called cellular plants-are composed of numerous cells, arranged according to a definite order, some of the cells discharging the nutritive and others the reproductive functions. The higher or vascular plants have vessels added to the cells; the organs of nutrition and reproduction being more complicated.

In compound animals, where differentiation of structure and function are carried to extremes, and where there is the greatest division of labour, the aggregate life of the cells is the life of the individual. It follows, that life is, in turn, compound, and, as a consequence, there may be particular death, as apart from general death. In other words, parts of plants and animals may and do die; the plants and animals, as a whole, continuing to live. This is the normal condition of living plants and animals in a state of health. The living is inextricably mixed up with the dead. The new matter added to both is dead. The effete or waste products are also dead. The living substance occupies an intermediate position. It exercises the power of selecting, seizing, appropriating, and assimilating new matter, while it discharges the used up or effete products. That living plants and animals may be infinitely minute is abundantly proved by the presence everywhere, in incalculable numbers, of microorganisms the very existence of which was not even suspected until quite recently. These remarks apply not only to plants and animals as wholes, but to living cells, and to every plant and animal structure, whether hard or soft.

The subtle element of life moves mysteriously amongst matter, and is unquestionably superior to it.

Life in plants and animals is, as it were, surrounded by a material atmosphere of death.

The cell is regarded by most authors as the starting-point for all structure and function in plants and animals. This view was strongly urged by Professor Rudolf Virchow.

As already explained, I regard growth and organisation as, primarily, the product of life acting on the atoms and molecules which constitute the cell. So far as I can make out, it is in atomic and molecular protoplasm that all the fundamental changes which result in development and differentiation occur.

This seems satisfactorily proved by the fact that spores, germs, seeds, and eggs, which are apparently homogeneous and identical in all their parts and particles, do, nevertheless, vary infinitely, each producing only its own kind.

The difficulty experienced by investigators in referring the beginning of growth, development, reproduction, 
and all the actions of plants and animals to cells, appears from this, that they are not agreed as to what constitutes a cell.

The value of the cell in the vegetable and animal kingdoms cannot, nevertheless, be over estimated. The cell in biology forms the structural and physiological centre around which life in all its forms may be said to revolve. It exhibits in its multifarious changes well-marked lines of communication and force. The cell has a literature of its own, but this is so extensive as to forbid my dealing with more than a fringe of it. I will therefore confine myself to a consideration of what I regard as the more important parts. I will also limit my observations on the cell itself to its position as a separate living entity, as a centre of reproduction, nourishment, growth, movement, \&c.

A cell may be defined us a microscopic object measuring from the three-thousandth to the four-thousandth of an inch in diameter, which is variously constituted and shaped. Thus it varies in chemical composition : it also varies in shape, being round, oval, caudate, branched, polygonal, cylindrical, stelliform, fusiform, \&c. The cell, as a rule, is round to begin with, but its shape is modified by pressure and various other conditions.

A typical cell consists of three parts, each of which is porous and permeable, namely, the cell wall or envelope, the nucleus contained within the cell wall, and the matter contained between the cell wall and the nucleus. These

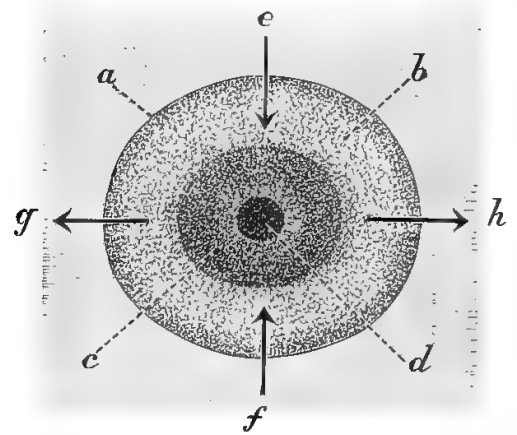

FIG. 32,-Typical cell consisting of a cell wall or envelope, cell contents, a nucleus, and a nucleolus. $a$, Cell wall ; $b$, cell contents ; $c$, nucleus ; $a$, nucleolus. The darts, $r, f$, represent the endosnotic or ingoing nutrient currents, by which the cell is fed; the darts $g, h$, the exosmotic or ontgoing currents, wherelyy the cell rids itself of waste products and injurions substances.

The cell wall, cell contents, nucleus, and nucleolus are osmotic media, and, when the cell is placed in suitable fluids, its vital and mechanical properties are at once evoked (the Author). gives out by the exosmotic currents other substances which are inimical to its well-being and which, if retained, would prove injurious.

That cells can and do discriminate, and that they select and reject within limits, is proved by the behaviour of animal cells as a class. Thus, some cells secrete and others excrete : the secreting and excreting cells are supplied with, and act upon, the same blood. Of the secreting cells some produce saliva, others gastric juice, others bile, and so on. Of the excreting cells some furnish perspiration, some urine and other waste products. The aggregates of cells form plants and animals; they also form the great majority of the vegetable and animal tissues-cellular tissue, woody fibre, muscle, nerve, bone, hairs, feathers, \&c. ; but these several and diverse structures are the product of one and the same vegetable sap, or, in the case of animals, one and the same blood, from which it follows that the several kinds of cells are endowed with special properties to bring about certain predetermined results. In the economy of cells, the division of labour is carried to an extreme. Cells, as structural units, are invested with high powers. They live, grow, and reproduce themselves. They perform the bulk of the work in plants and animals. They are conditioned, and work to given ends, singly and in combination. Their work is duly apportioned: they do nothing in a hap-hazard way. They reproduce, build up, and keep the organism going in all its parts. They, however, do this according to a fixed plan and under supervision. Cells can only work within prescribed limits. They have no power, in the normal or healthy condition, to change either their constitutions or the rôle to be played by them. Least of all can they change or abrogate the function assigned them in the great scheme of organic nature.

The importance of the cell in life and organisation is universally admitted. Indeed the majority of physio- 
logists refer all the changes which occur in reproduction, in the embryo, and in the tissues of plants and animals, directly or indirectly, to the cell. Professor Virchow says, "Omnis cellula e cellula."

Those who advocate this view maintain that all the tissues are developed from cells, and that the first changes in the embryo are inaugurated by cell structures. They assert, consequently, that either the vitelline membrane sends in processes to divide the yolk mechanically, or that the germinal vesicle separates into two ; the two parts attracting the molecules of the yolk around them. Cells, it is claimed, take the lead in reproduction, a bsorption, assimilation, nutrition, growth, secretion, excretion, \&c.

The nature of the cell in cellular plants, the function discharged by it in plants generally, and the manner in which it divides and multiplies to form tissues and new individuals, are shown at Plate lix., Figs. 1 to 4 , and Plate iii. The unicellular animals, and the behaviour of cells in animals, are very fully illustrated in various parts of the work.

\section{PLATE LIX}

Plate lix. deals with cells, cell contents, cellular plants, spores, and the lower plant forms. The cellular animals and lower animal forms are given at Plates lxi. to lxiv. inclusive.

Fig. 1.-A. Cells of the green-snow plant (Protococcus viridis), when fully developed. They contain germs or cellules which when discharged produce new plants. The cells perform nutritive and reproductive functions.

B. Cells of the yeast plant (Torula cerevisia) in different, stages of growth. $a$, Cell in an early state; $b$, cell with nucleus; $c$, cell with nucleoli. This cell plant, like the green-snow plant, performs nutritive and reproductive functions.

C. Cells of the red-snow plant (Protococcus nivalis), in different stages of development, $a$, Cell in the young state; $b$, cell fully formed, containing cellules ready to be discharged, and to form new plants; $c$, cell with contents discharged. Each cell performs the functions of nutrition and reproduction.

D. A species of mould-fungus (Botrytis), consisting of a mycelium, bearing a separate cellular stalk, which branches at the apex; each division bearing a rounded spore.

E. Elongated club-shaped cell of Vaucheria ovoidea, discharging a cellular spore which has been formed in its interior.

F. Spore with numerous cilia surrounding it $(a) ; b$, spore with numerous cilia at one end; , small cell or spore with two vibratile filaments.

G. Two filaments of a cellular plant (Zygnema), united by means of tubes. The plant is formed by a series of cells arranged end to end in a single row. Note-The cells contain S-shaped contents $(a)$; spiral contents $(b)$; and spiral figure-of-8 contents $(c)$.

$\mathrm{H}$. Thallus of the common bladder sea-weed (Fucus vesiculosus), consisting of numerous cells united; some (a, $a$ ) being nutritive, and others $(b, b)$ reproductive.

I. Elaters, or spiral fibres, with spores or reproductive cells $(a, a)$ from liverwort (Marchantia). The fibres are elastic, and serve to scatter the spores (Balfour). The scattering of the spores by elaters is a designed provision of nature.

FIG. 2.-A, B, C, D. Longitudinal section of a young, half-grown cell from the cortical parenclyma. The peduncle of Cephalaria levcantha, treated (B) by a four per cent. solution of potassium nitrate, (C) by a six per cent. solution, and (D) by a ten per cent. solution. $a$, Cell wall ; $b$, protoplasmic lining of cell wall ; $c$, cell nucleus; $d$, chlorophyll-granules; $e$, cell sap. The lettering given for A applies equally to B, C, and D. $f$, Salt solution which has passed through the cell wall (De Vries).

FIG. 3.-Longitudinal section of the middle cell of the hair of a gourd (from the calyx of the young flower-lyud). a, Cell wall; $b$, vacuolated mass enclosing the nucleus of the cell; $c$, branching filaments of protoplasm in active movement and carrying chlorophyll corpuscles (containing starch in their substance); $d$, a crystal being hurried along (after Sachs).

Fig. 4.-A. Tetraphis pellucida, a plant bearing gemmæ (natural size).

B. The same magnified ; at top of figure is the cup in which the gemms collect.

C. Longitudinal section throngh the apex of B, the gemmæ being seen in various stages of development.

$D$. Mature gemma, $\times 550$, consisting at the margin of one, and in the centre of several layers of cells (after Sachs).

E. Development of Pandorinc morum: a swarming family of same.

F. A similar family developed into sixteen daughter-families.

G. A sexual family, the individual cells of which are escaping from their gelatinous investment.

$\mathrm{H}$, I. Conjugation of pairs of swarmers.

J. Zygote which has just been completed.

K. A fully grown zygote.

L. Transformation of the contents of a \%ygote into a large swarm-cell.

M. The same after being set free.

N. A young family developed from the latter (after Pringsheim).

\section{PLATE LX}

Plate $1 \mathrm{x}$. deals with the pollen and cells of plants, and with transverse, longitudinal, and other sections of shrubs and trees. The transverse sections show the concentric arrangements of the cells and woody fibres in certain cases. The radiating arrangements are seen at Plates xxii., xxiii., and xxiv., pp. 39-41. Various kinds of pollen are given at Plate $\mathrm{lxv}$. p. 164.

FIG. 1. A. Funkia cordata. Transverse section of a young pollen sac, before the isolation of the mother cell. a $a^{\prime}$, Mother cells; $b$, epithelium (tapetum) clothing the loculus; $c$, wall of the pollen sac.

B. Loculns after isolation of the mother cells $\left(a a^{\prime}\right) ; b$, remains of tapetum, $\times 550$.

C. Funkia ovata. Development of pollen, $\times 550$. The development proceeds as indicated by the numerals. In No. 7 the 
PLATE LIX

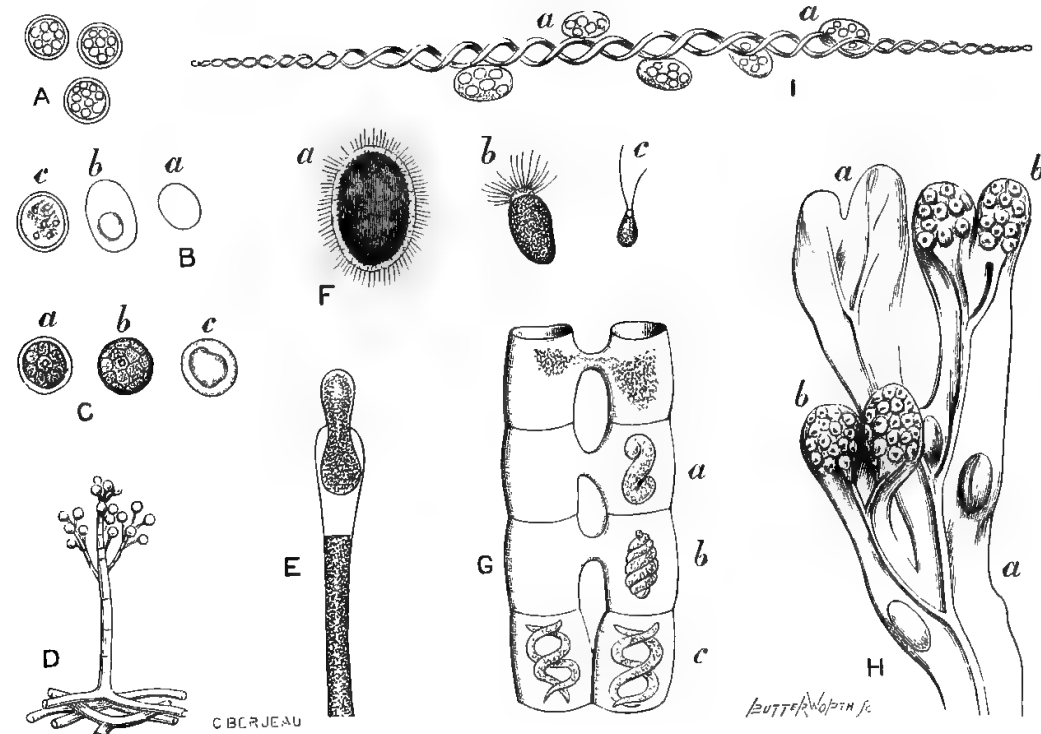

Fig. 1 .

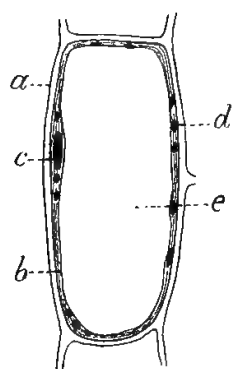

A

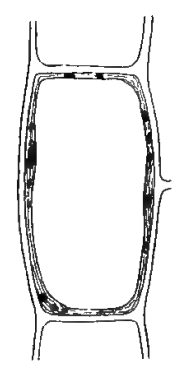

B

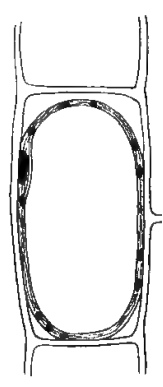

c

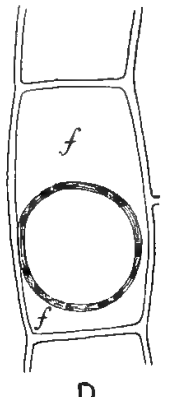

D.
FIG. 2.
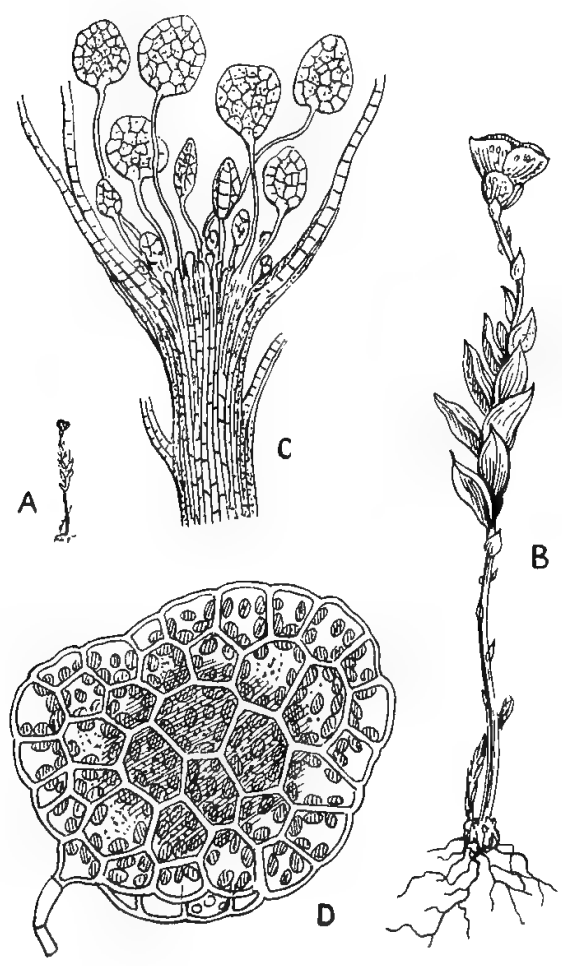

$B$
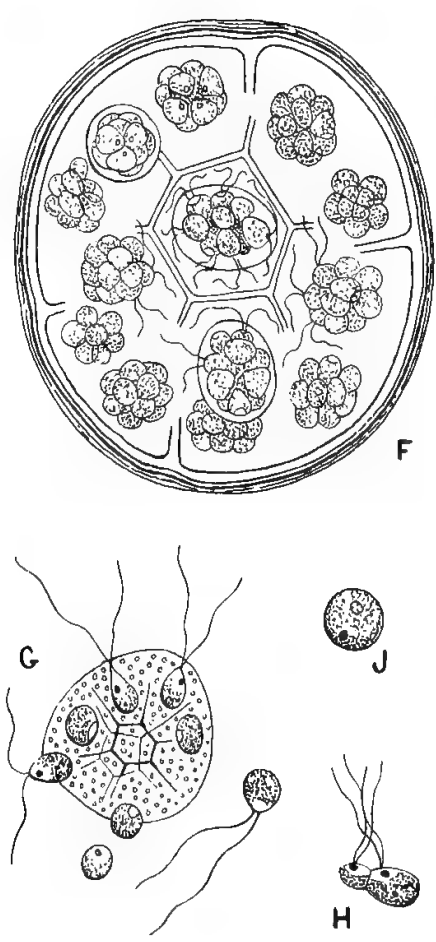

(a)

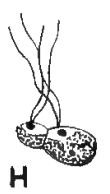

Fig, 4.

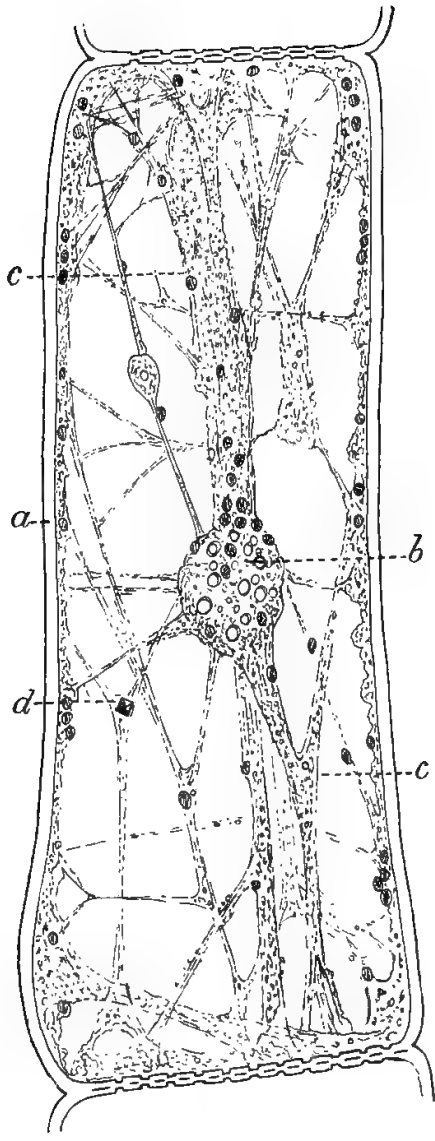

FIG. 3.
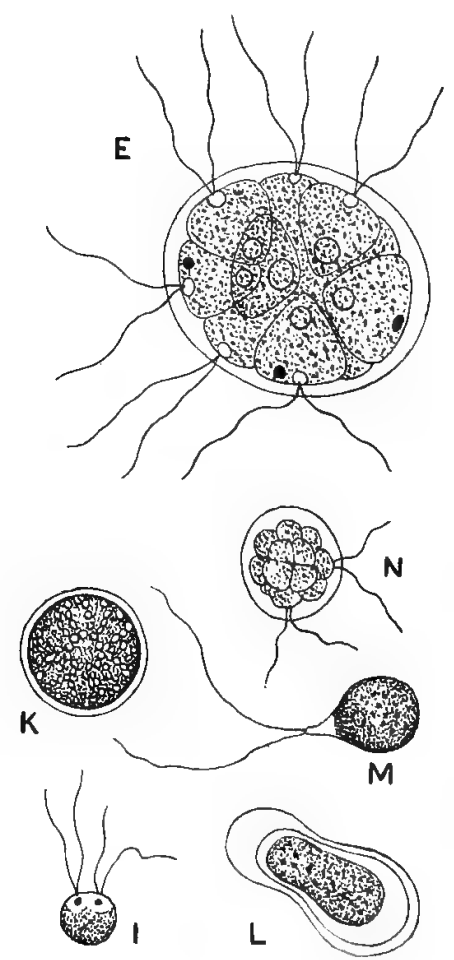
PLATE LX
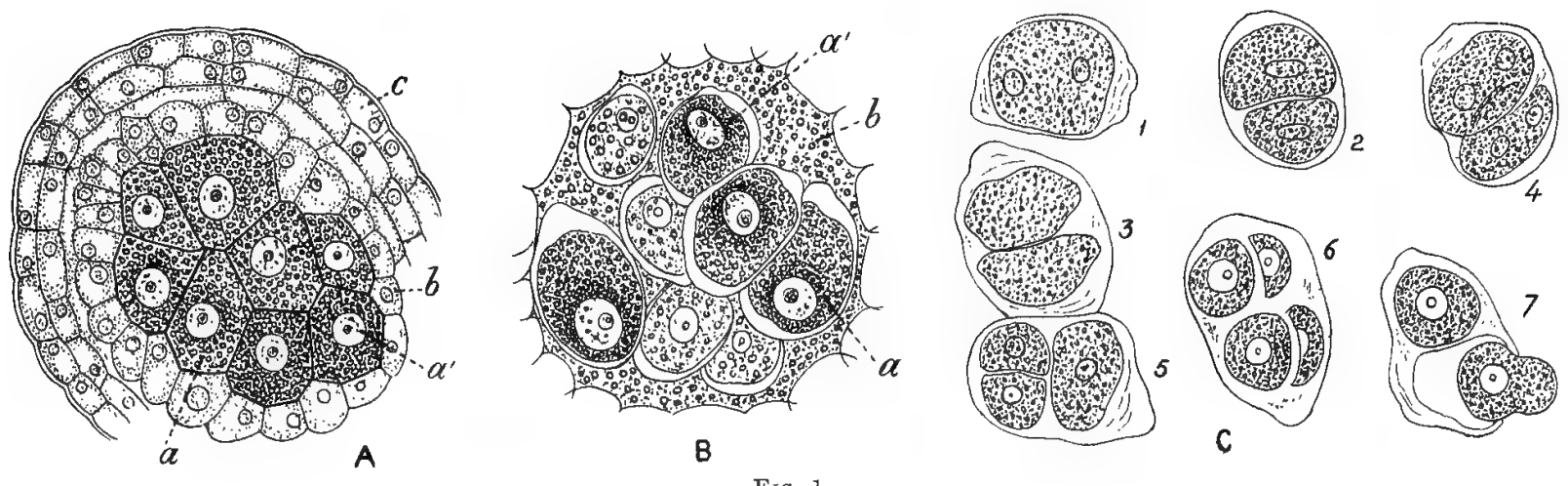

FIG. 1.
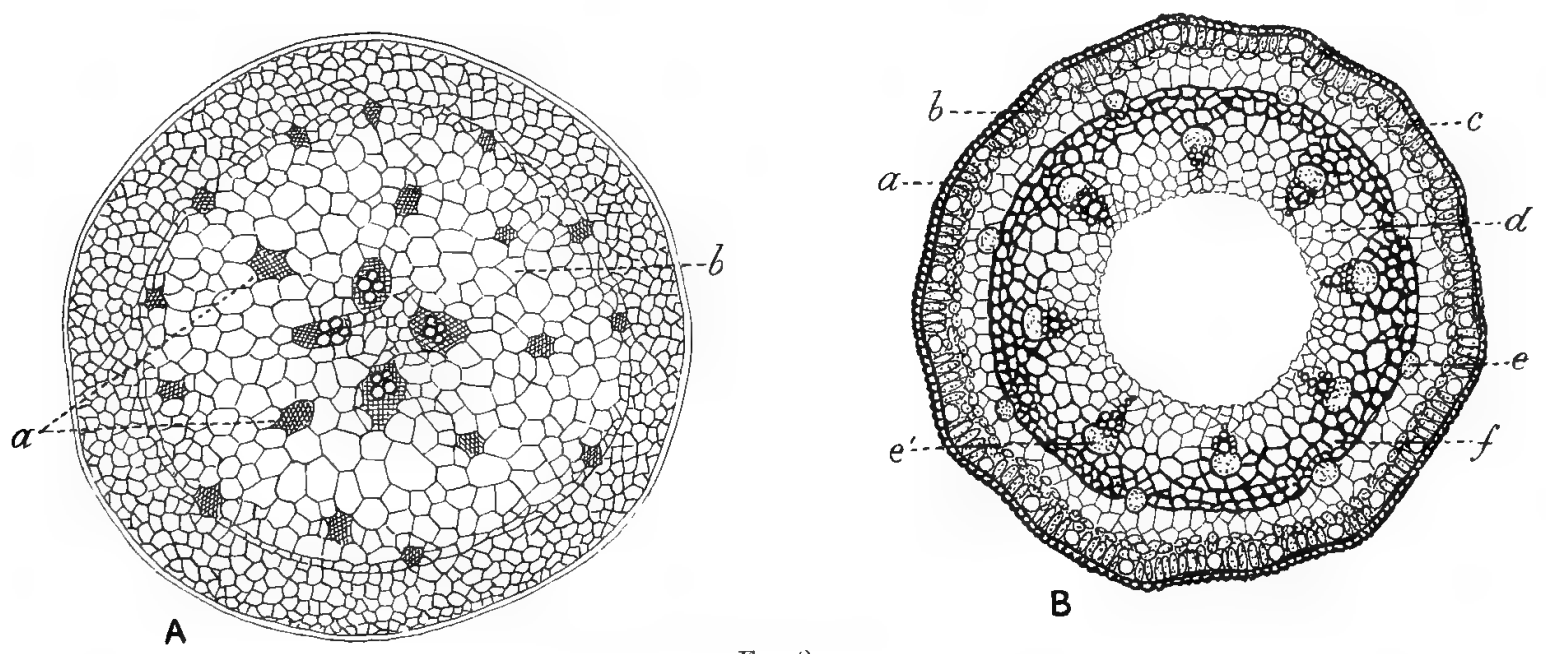

FIG, 2,

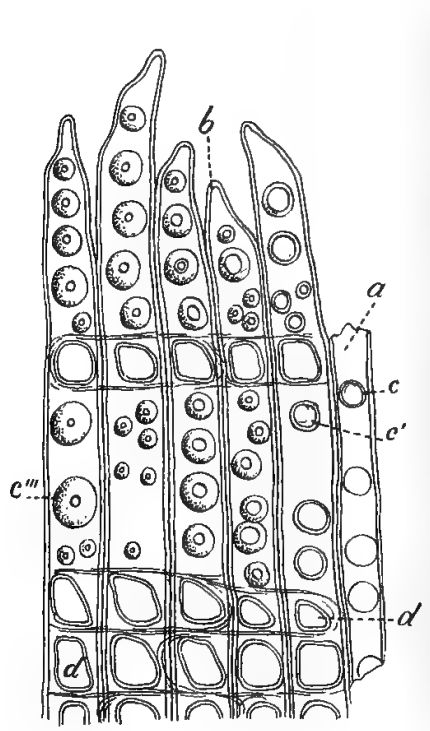

A

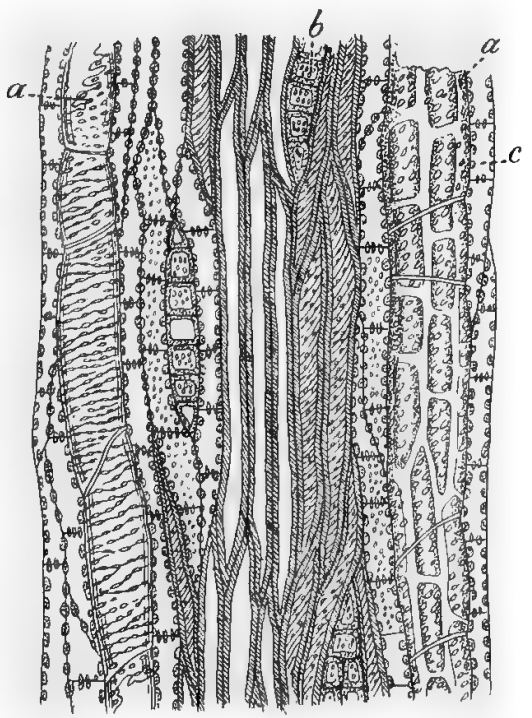

B

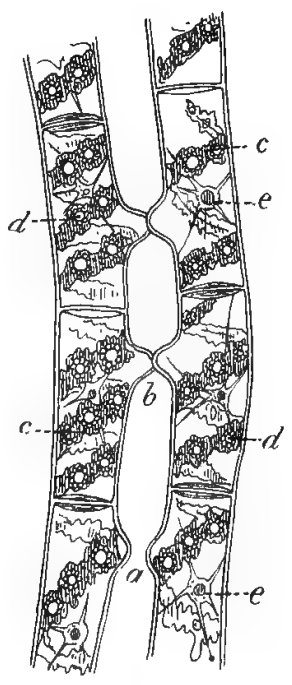

C

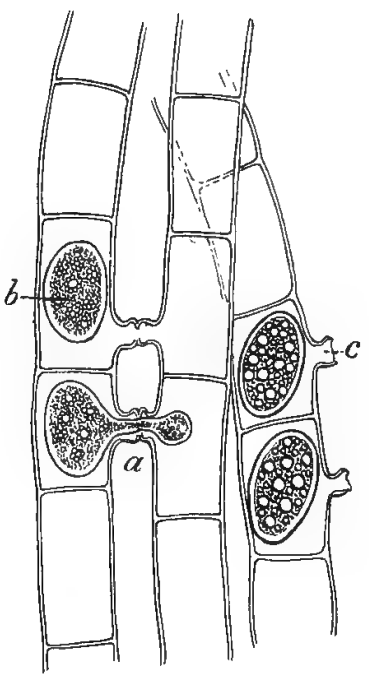

D

FIG.3. 
wall of the daughter-cell has absorbed water and burst; the protoplasmic body having forced itself through the fissure and assumed a rounded contour (after Sachs).

FIG. 2.-A. Transverse section through a young internode of the stem of Tradescantic albiflora. a, Vascular bundles; $b$, large cells forming the parenchyma of the fundamental tissue (after De Bary).

B. Transverse section of the flower-scape of Allium Schonoprasum, xabout 30. $a$, Epidermis ; $b$, chlorophyll cells; $c$, colourless parenchyma of the cortex; $d$, parenchyma of pith; $e e^{\prime}$, vascular bundles; $f$, ring of sclerenchyma (after Sachs).

FrG. 3.-A. Pinus sylvestris. Radical longitudinal section through the wood of a vigorous branch. $a$, Cambial wood-cell; $b$, older wood-cells ; $c, c^{\prime}, c^{\prime \prime \prime}$, bordered pits of the wood-cells in order of age; $d$, $d$, large pits where the cells of the medullary rays are in contact with the wood-cells.

B. Tangential longitudinal section through the wood of Ailanthus glandulosa. $a$, Vessels; $b$, medullary rays cut across; $c$, wood parenchyma.

C. Spirogyra longate. Several cells of two filaments about to conjugate $(a, b)$. Spiral chlorophyll bands $(c)$, with starch grains $(d)$, circularly arranged, and small drops of oil. The nucleus $(e)$ of each cell is surrounded by protoplasm, from which threads go to the cell wall.

D. Cells engaged in conjugation. a, Protoplasm of one cell passing into another cell. At $b$, two protoplasmic masses have already united. At $c$, the young zygotes are provided with a wall (after Sachs).

While the German histologists maintain that everything proceeds from the cell, they differ as to their definition of a cell.

Max Schultze avers that a cell wall or envelope is not necessary to the existence of the cell, and Brücke declares that a nucleus does not necessarily form a part of it. If, however, we eliminate the cell wall and the nucleus, all that remains of the cell is the matter contained between the two, namely, the cell contents or protoplasm, and this is now regarded by Professor Huxley and others as the germinal matter, or physical basis of life.

The history of the cell is instructive.

Schleiden and Schwann (1839), the original promoters of the cell theory, were of opinion that cells were produced from an amorphous mass (cyto-blastema), contained either within or between pre-existent cells. In this mass round, structureless, or minutely granular corpuscles appear.

Around these corpuscles, which constitute the nucleus, a cell wall is formed by molecular disposition, and between the cell wall and its nucleus fluid is stored up. By this process a nucleated cell is produced. From a mass of such cells, according to Schleiden and Schwann, all the textures may be developed. In their opinion "the cause of nutrition and growth resides, not in the organism as a whole, but in the separate elementary parts - the cells."

Professor John Goodsir (1845), to whose writings I refer further on, thus expresses himself: "As the entire organism is formed at first, not by simultaneous formation of its parts, but by the successive development of these from one centre (the germinal spot of the ovum), so the various parts arise each from its own centre, this being the original source of all the centres with which the part is ultimately supplied. From this it follows, not only that the entire organism, as has been stated by the authors of the cellular theory, consists of simple or developed cells, each having a peculiar independent vitality, but that there is in addition a division of the whole into departments each containing a certain number of simple or developed cells, all of which hold certain relations to one central or capital cell, around which they are grouped. It would appear that from this central cell all the other cells of its department derive their origin. It is the mother of all those within its own territory."

Goodsir attached much importance to the nuclei of the cells : these formed his "centres of nutrition." In this matter he proved himself an advanced pioneer. The nucleus is now known to be the seat of the most extraordinary and important changes in reproduction: it is also believed to be the centre of inheritance. "The identification of the cell-nucleus as the vehicle of inheritance, made independently and almost simultaneously in 1884-85 by Oscar Hertwig, Strasburger, Kolliker, and Weismann, must be recognised as the first definite advance towards the internal problems of inheritance through the cell-theory."

Similar in many respects to Goodsir's theory of "centres of nutrition" is Dr. Beale's theory of germinal matter.

Professor Huxley (1853) referred the origin of cells to vacuoles occurring in a homogeneous plasma. These vacuoles contain the cell wall, nucleus, and contents. The cell wall he termed the periplast, the nucleus the endoplast. He attaches no importance to the latter, in which respect he entirely differs from Goodsir. He says, in fact, that not unfrequently the nucleus has disappeared before the really essential changes in the cell begin. Huxley attaches especial importance to the periplast or cell wall and the matter contained within it. In these, according to him, all the really essential, vital, physical, and chemical changes occur. It would be easy to write volumes on the cell theory, but enough has been said to show that authors are not agreed either as to what constitutes a cell, or as to the more important parts of it. Some think that the essential part is the cell wall or envelope, others the nucleus, others the substance cortained between the nucleus and envelope: some again are of opinion that neither cell wall nor nucleus is necessary, and that the contained matter or protoplasm is the only essential element. 
While it is difficult to conceive of a cell minus its cell wall and nucleus, there are good grounds for believing that the intra-cellular substance is really the germinal matter or elementary life-stuff, in which all active growth proceeds, and from which the various tissues are manufactured. Schwann requires a blastema even for the production of cells. The intra-cellular substance is now commonly known as protoplasm (Remak, Von Mohl, and Kühne). It is, however, variously designated. Thus, as already pointed out, it is spoken of as sarcode (Dujardin), blastema (Schleiden and Schwann), solidescible nutritive fluid (Wolff), organised concrete (Haller), germinal matter (Beale), embryo plastic matter (Robin), primordial protogenes (Haeckel), \&c.

A very important part of the cell unquestionably is the cell wall or envelope. This is, strictly speaking, an osmotic membrane, through which, during life, two opposite currents are continually passing: an ingoing or endosmotic current, which carries nutrient materials to the cell, and an outgoing or exosmotic current, which carries away effete matter and waste products.

On the integrity of the cell wall, as an osmotic medium, the health of the cell, and the parts formed by it, largely depends.

If the cell wall in plants and animals becomes thickened, and ceases to be porous, abnormal conditions are at once established. In such cases growth and natural movement are interfered with, and function more or less impaired.

Cells are divided into :-

(a) Permanent healthy cells.

(b) Transition cells.

(c) Unhealthy or morbid cells.

The permanent cells embrace such as do not become tissues: the transition cells include all those converted into tissue : the unhealthy or morbid cells, those which are abnormal.

Cells reproduce themselves in four different ways :--

1. Endogenously or from within, where a cell is generated within a cell.

2. Exogenously or from without, where a young cell formed within the parent cell is extruded.

3. Gemmiparously, where a young cell is produced by a process of budding.

4. Fissiparously, where the original cell splits into two.

The rapidity with which cells multiply is, in some cases, extraordinary. Cell production and cell life is, as a rule, most vigorous at fairly high temperatures. Tropical plants grow more luxuriantly than those in temperate climes. There is, however, a limit as regards temperature. Cell life will not continue below zero, or above $145^{\circ} \mathrm{F}$. Cold beeps back the growth of young plants and animals, while heat brings them forward.

In no department of physiology is the division of labour more thoroughly carried out than in cell structures. This is well seen in the case of plants. In the lowest plants, cells resemble each other and have equal values - that is, they take part equally in the vegetative and reproductive processes. In the higher plants, cells are specialised and have different values as regards their physical and chemical properties; some take part in nutrition, others in secretion, others in reproduction, and so on.

In commori mould, the cells forming the mycelium or spawn absorb nourishment and produce stalks which bear reproductive cells or spores. In vascular plants, certain cells secrete starch, gum, sugar, oils, milky juices, \&c.

The contents of cells are various. Thus, in the grain of the cereals some cells contain nitrogenous compounds, and others starch; in the Equisetacex, and the stems of some grasses, a large proportion of the cells contain silica.

In the case of animals, one set (the blood corpuscles) assist in elaborating the blood; another, in building up the various tissues; another in secreting various products useful to the economy; another in getting rid of effete products ; another, in storing up fat, pigment, \&c.

Cells, there can be no doubt, are endowed originally with special properties, for while they all drink out of the same blastema, some take in and elaborate what others reject. This discriminating power of cells is at the root of all structure and all function.

Cells are highly complex, both as regards their ultimate composition and the nature and variety of the work discharged by them.

They exercise a most important influence in the economy of plants and animals. In the higher animals they contribute to locomotion, sensation, and even mental acts. Through their instrumentality absorption, nutrition, assimilation, growth, development, secretion, excretion, reproduction, \&c., are mainly effected.

Many plants and animals consist of an agglomeration of cells; and whatever plants and animals may be in the mature or adult state, they are, at the outset, composed of cells.

The life of the cell is frequently manifested by movements in the cell wall, in the nucleus, and in the protoplasm or cell contents, These movements are due sometimes to cilia or hair-like processes on the cell wall ; sometimes to independent contractions and expansions in the cell wall ịtself : sometimes to movements confined to the

VOL. İ. 
nucleus, and sometimes to movements occurring in the protoplasm. Spermatozoids have distinct movements, and the heart of the chick, composed entirely of cells, opens and closes with time-regulated beat independently, and before it contains either muscular fibres or blood.

\section{EVIDENCES OF DESIGN IN THE REPRODUCTIVE ELEMEN'TS OF PLANTS AND ANIMALS}

\section{Globular, Concentric, Radiating, Branched, Segmented, Curved, and Spiral Arrangements make their Appearance at the very Threshold of Life.}

I have explained that globular, concentric, radiating, branched, segmented, curved, and spiral arrangements obtain in crystals, plants, and animals (Plates i. to xxvi. and Plates lxxi. to lxxv.), and the reader has only to examine the figures in the latter plates to be convinced that these arrangements make their appearance at the very threshold of life in the reproductive elements themselves. This is an astounding fact, and cannot be too strongly insisted upon. In reality, the structural features of plants and animals are impressed upon them from the first, and are, so to speak, stereotyped. These arrangements have much significance, as showing that plants and animals are, from the outset, built up according to a great general plan, which applies to the inorganic and organic kingdoms alike. Plants and animals are not dissevered from crystals, dendrites, and other physical formations, either in their young or adult conditions; a general plan runs through and regulates all. The globular, concentric, radiating, branched, segmented, curved, and spiral arrangements met with in crystals reappear in plants and animals at their inception, and at every period of their life histories: they are fundamental, and the outcome of design, law, and order. They evince the workings of a great Master Mind, a Supreme Ruler, Director and Upholder - a Controller of events, great and small, in time and space. The only difference between inorganic and organic formations consists in this-that plants and animals live, and are controlled by vital forces; inorganic formations being controlled by physical forces. The vital and physical forces are, however, equally the servants of the Creator, Who makes and sustains everything. This being so, it need occasion no surprise if the male and female sexual elements of plants and animals display in their substance, both before and after impregnation, when they amalgamate, traces of arrangements which pervade matter as a whole. Nor will wonder be excited when it is stated that these arrangements persist, and run through all plants and animals, from the lowest to the highest, in their embryological, young, and adult states; and that plants and animals are created on common types, according to an ascending progressive scheme. This view gives no countenance to the "evolutionary" and "natural selection" theories which maintain that plants and animals are manufactured out of each other by infinite modifications in infinite time, and largely by accident. There is no need, and indeed no room, for such theories. The forces at work in nature are not blind forces-they are controlled: the matter on which they operate is assorted matter-nothing happens by chance.

If the atoms and molecules in the process of crystallisation arrange themselves in a certain order and assume a given form, and if the atoms and molecules of the sexual elements before and after impregnation display characteristic arrangements, shapes, and movements, which can be reckoned upon, it is because the matter and the forces are under law and order, and are controlled to given ends. The variations, of which so much has been made by Mr. Darwin and others, are confined within limits, and these limits mark the boundaries of types which are permanent. If the term "accidental" can truly be applied to "variations," it certainly cannot be applied to types, which are the permanent representatives of law and order as opposed to accident and flux. In the great races of plants and animals the law of reproduction is universal. It is, so to speak, inwoven in their tissues and from the beginning. It dominates the congress of the sexes, their embryological developments, their life histories -everything that pertains to them as individuals, families, and races.

If it can be shown that reproduction is a law of life and fundamental; if further it can be demonstrated that the elements of reproduction exist in plants and animals in young individuals, and long before the adult and reproductive period, when they are required, arrives; if finally, it can be proved that the male and female elements, where they exist, are complemental and adapted to each other, and that when the adult stage is reached they not only seek and meet each other, but that they unite and amalgamate to form new beings, displaying globular, concentric, radiating, branched, segmented, curved, and spiral arrangements, similar to what obtains in crystals, it follows that reproduction is no chance process, but part of a carefully-considered, well-ordered scheme. As, moreover, the process of reproduction, in its entirety, is largely involuntary in character, the only explanation that can be given of it is that it is a designed process-a means to ends-the ends being seen and 
determined from the beginning, but not by the beings who take part in it. It is plain that the reproductive process is not the outcome of any form of "evolution" or "natural selection." Fach plant and animal only reproduces itself, and the reproduction is direct, and not the result of roundabout methods, as claimed by the theories referred to. No plant or animal can deduce itself from another plant or animal; neither can plants and animals vary at discretion, and select or reject structural peculiarities and properties in themselves which, as a matter of fact, have no existence at the period of impregnation. "Natural selection" cannot be applied to a thing before or during its formation. The successive steps in the reproductive process are fixed beforehand, and the structures which are developed, as the result of impregnation, are formed before they are required, and before they are of use to the individual. All this bespeaks design of a high order. It is inconceivable that the things developing can have any idea of the complicated whole they are building up, or of the separate and combined functions they are to perform in the economy of the adult. As already stated, the reproductive act, in its entirety, cannot be regarded as intellectual. It is, in a very large number of cases, quite involuntary. This is especially true where there is only one sex, and in hermaphrodites, where the two sexes are found in one and the same individual. It is also true in the case of plants, especially where the male and female elements occur in separate specimens located at considerable distances from each other, and where the interposition of winds, insects, birds, \&c., becomes a necessity. The latter cannot be regarded as intelligent fructifiers : they can only be considered as the agents of a higher power, which perform work they are predestined to perform as integral parts of the great scheme of creation.

In the lowest plants and animals, where the parts greatly resemble each other, and there is little differentiation, reproduction is largely effected by simple division or by budding (Plates lxi., lxii., lxiii., and lxiv.)

In these cases every part of the individual is capable of the reproductive act. There are no organs of reproduction. In the atoms and molecules of the individual resides the power of reproduction, and this more than any other thing shows the fundamental nature of reproduction. As every living thing can reproduce itself, it follows that reproduction is at once fundamental and universal. As, further, reproduction can be effected in the absence of separate sexual elements, it is clear that the latter, when they exist, are the result of differentiation - the simple thing becoming complex. But (and here comes the crux) the simple cannot of itself become complex. If it does so, the change must be referred to an external controlling power, and that power is the Creator or First Cause. From simple, homo-

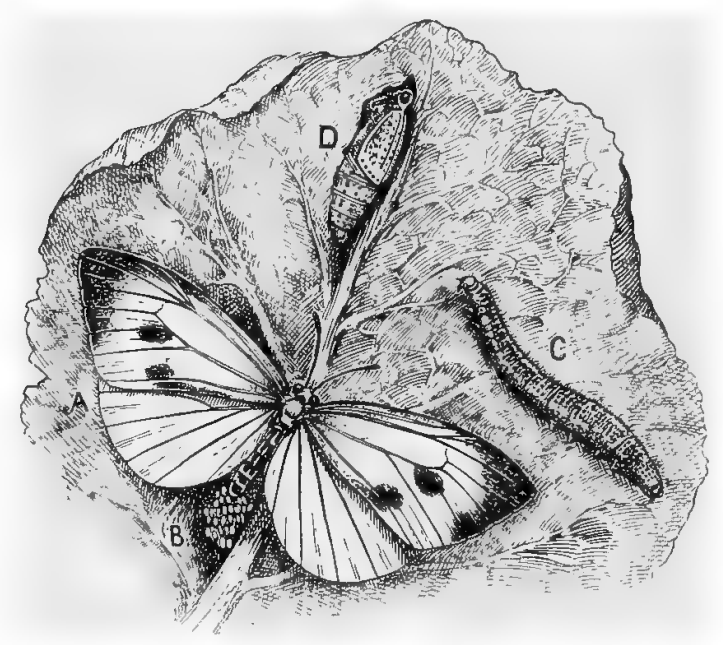

FIG. 33. - Various phases in the development of the white cabbage butterfly (Pieris raps).

A. The white butterfly in the act of laying its eggs (B).

C. The caterpillar stage of the butterfly.

D. The pupa stage of the butterfly (from nature), (the Author).

geneous matter, similarly conditioned and placed, only simple matter can result. The moment a departure is made from the simple to the complex, differentiation (of a kind) is established, and that differentiation involves design and means to ends. The simple plants and animals cannot differentiate of themselves. There is nothing in them to reproduce new structures and to enable them to assume new functions. The power to differentiate must reside in the living things in a potential form, and the act of reproduction proves that plants and animals, be they simple or complex, are the outcome of one or more special substances which have separate existences from the first, and in turn produce others. In no other way can the diversified and countless multitudes of plants and animals be accounted for. Living substances devoted to the construction of plants and animals no more admit of admixture than do the components of crystals. If plants and animals differ markedly from each other, so did the cells and sexual elements from which they were originally derived. It is idle to assert that plants and animals can depart indefinitely from their originals, if only sufficient time be allowed. If this were so, there could be no types or permanent forms, and even the simplest kinds of classification would be impossible. As a corollary, no law and order could anywhere be traced.

In addition to the simple forms of reproduction already referred to there are others which are more complicated, and in which the developing individual seems, for short intervals, to lose its identity. The white cabbage butterfly may be taken as an example. The extraordinary transformations through which the butterfly passes during development are illustrated at Fig. 33.

At $\mathbf{A}$ of Fig. 33 the female butterfly is seen laying her eggs (B), which are living but not moving.

At $\mathrm{C}$, one of the eggs has developed into an active aggressive caterpillar, very destructive to vegetation. 
At $\mathrm{D}$, the dormant pupa stage has been reached, where the caterpillar has gone into winter quarters within ts silent, horny cocoon.

The developmental changes are so unlike each other that it is only the expert who can say that we are dealing all through with one and the same animal.

The changes witnessed in so-called development by "alternate generations" are, if possible, more remarkable. Here the development is, as it were, suppressed at a particular period to be commenced at another period subsequently.

One of the best examples known of development by alternate generations is that furnished by the obelia, one of the smaller medusæ (see Plate xxxv., Fig, 5, A, B, C, D).

Obelia reproduces itself in two ways: $(a)$ as part of a polype, and $(b)$ as free swimming buds which detach themselves from the polype and commence an independent existence. It is hard in either case to trace the development of the animal, and for a long time its possible descent by alternate generations was doubted by even advanced naturalists.

In these cases the offspring in no way resembles the parent.

The animals which reproduce themselves by alternate generations are generally grouped under zoophytes. They belong to the Hydrozoa, a division of the Colenterata, which also includes the fresh-water polypes, and many jelly-fish, mostly small in size. They are a numerous tribe, and are usually found growing on the rocks and seaweeds of the tidal pools. They resemble delicate plants rather than arimals. Their slender, tapering, flowerlike stems and branches keep up the delusion. The colonies of polypes vary in size. Thus, in one of the smaller species (Plumularia cristata) the assemblage varies from 400 to 500 specimens. In some of the larger species (Plumularia falcata, or Sertularia argentea), the colony may contain from 80,000 to 100,000 individuals.

The common tapeworm affords another example of an animal whose developmental changes are interrupted at one point and taken up subsequently at another point. The appearances presented by the various developmental products are, moreover, wholly dissimilar, as a reference to Fig. 34 shows.

In reproduction by "alternate generations" the product does not in the first instance resemble the original parent or parents. In this form of reproduction, the proofs of design are even stronger than in direct reproduction. Certain parasites, the tapeworm for example (Fig. 34), require more than one host or purveyor for their development.

This singular creature has a hermaphrodite parent with a nervous system, but no brain proper. It is produced unconsciously, and blindly launched on the world, but not to perish. It is eaten by a higher animal with other food, and attains its first or cystic stage in the flesh of the unfortunate animal, which involuntarily and unknowingly becomes its host and habitation pro tempore. Nor does the designed chain of events stop here. The host is in turn eaten, in many cases, by man himself. When the tapeworm reaches the stomach and alimentary canal of the second host, it is not killed and digested. On the contrary, it pushes its head out of its cyst and everts itself. It ceases to be cystic, and displays a formidable circle of hooklets which have been gradually forming on the head segment, and with these it securely anchors itself in the mucous lining of the bowel of the second host, imbibes nourishment through its entire substance, and slowly, but surely, becomes an adult tapeworm resembling in all respects its original parent. Neither the tapeworm itself nor its unfortunate hosts display any knowledge of the ultimate object in view. The tapeworm, broadly speaking, is non-intellectual, and the hosts, if intellectual, perform their part of the reproductive process blindly and involuntarily; yet the reproduction of a tapeworm cannot be regarded as an accident. On the contrary, the means to ends are complicated and obvious, and bespeak an intelligent power outside the worm and its unconscious hosts.

What is said of the tapeworm is true of every form of reproduction, even in the higher animals, where there are independent and separate reproductive elements. In the males and females alike, the reproductive elements make their appearance in the young animals very early, and at a period long antecedent to that of puberty, when they are required. This fact alone proves design. How otherwise is it possible to account for the appearance of structures for which there is no use at the time of their formation? This fact saps and destroys the argument that organs are formed by use and wont, by habit, by irritability, by external stimulation, by environment, \&c. In the formation of the reproductive elements none of these causes are at work. The reproductive elements, simple or complex, are original independent entities, and are, in every instance, traceable to their predecessors, whose peculiarities they at once inherit and transmit.

The spermatozoa or male elements, and the ova or female elements, are not produced by the sexes intelligently; yet in healthy animals they are formed in anticipation of the time when they will be required. Not only are they not formed intelligently and voluntarily; they are formed even in spite of the individuals producing them. Even coitus is not necessarily a part of the reproductive process.

The conclusion to be drawn from the foregoing is that the production of the sexual elements and even the sexual desire, is not, strictly speaking, under the control of the individuals concerned. The elements and the 
desire are equally predetermined. The globular, concentric, radiating, branched, segmented, curved, and spiral arrangements of the reproductive cells are also predetermined.

The way in which the male and female reproductive elements in the higher animals deport themselves is worthy of careful study, as further illustrating design. In the human race, the male element or spermatozoon, and the female element, ovum or egg, make their appearance in the boy and girl long anterior to puberty; they can be detected even in the male or female child. The reproductive elements anticipate the reproductive function by a great many years. They are separate creations. The spermatozoa are prepared in incredible numbers in the testes of the male, and the ova or eggs are developed in very large numbers in the ovaries or egg-sacs of the female. The spermatozoa after puberty are always available; the ova or eggs at stated intervals. The spermatozoa and ova in the genus homo (and in animals generally) are living, and have separate existences. Their movements are neither intellectual nor voluntary: yet they seek and find each other with unerring precision. The ovum or ova, as happens, are discharged from the surfaces of the ovaries at the monthly periods, and find their way into the Fallopian tubes of the uterus, where, after the congress of the sexes, they are met by the spermatozoa. The spermatozoa enter the ova, and impregnation, in healthy individuals, follows. The male element penetrates the female element. The male and female elements seek each other as wholes: the essential parts of each element also seek the essential parts of the complementary element and so ensure a perfect amalgamation or blend. The male element even sheds its vibratile flagellum or swimming tail when it is no longer useful. The ovum extrudes a portion of its substance (polar globule or directive corpuscle) to make room for the spermatozoon, and provides an aperture for its entrance. This aperture is, in due course, found and utilised by the spermatozoon. The male and female sexual elements are to be regarded as centres of force, and streams of power and growth radiate from them as light from a star : indeed the term aster, in embryology, is employed to denote certain radiating structures and movements. These extraordinary arrangements can only be accounted for by design and prevision. For changes occurring in the cell, especially in the nucleus,

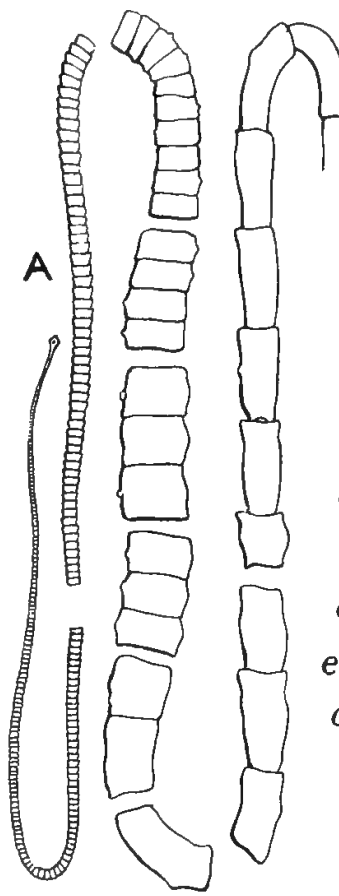

FIg. 34.-A. Tania saginata. B. Head of Truia solium. O. Seni-diagrammatic view of a single proglottis of trenic. $a$, Ovary; $b$, testis; $c$ receptacle; $d$, seminal vesicle; $e$, common sexual aperture; $f$, uterus ; $g$, yolk-gland; $h$, shell-gland ; $i$, excretory canal; $j$, nerve cord. D, E, F, G, H. Development of Trenio solium (after Leuckar't). after impregnation, see Plates lxi., lxii., and lxiii., pp. 154, 156, and 158.

When the male and female sexual elements have met and blended with each other, a still more remarkable series of phenomena present themselves. As a matter of fact, two sets of changes are to be noted, namely, those which occur before impregnation, and those which occur after impregnation.

The series of changes before and after impregnation are involved and complicated, but they form, strictly speaking, different stages or phases of the reproductive process, which is to be regarded as one continuous act. The term evolution, if employed at all in this connection, can only be employed, strictly speaking, in development.

Reproduction consists of a progressive series of co-ordinated transformations and movements in the male and female elements: these elements participating equally, but in different ways. The transformations are of the most remarkable and striking character, and cannot be explained as apart from pre-arrangement, design, and a Designer.

In some animals reproduction results in the most beautiful concentric, radiating, branching, and spiral arrangements, and in a complicated process of weaving as shown at Plates lxi. and lxii. In others it gives rise to bewildering divisions and reunions, and the formation of membranes and other structures as shown in Plates lxi., lxii., and lxiv.

The appearances presented by, and the changes occurring in, the sexual elements of plants are in some respects quite as extraordinary as those witnessed in animals. Plate lxv. shows the appearance presented by the pollen, 
spermatozoon or male element; that presented by the ovule or female element; and by the anthers, elaters, and other parts connected with reproduction. Plants display in a marked degree the globular, concentric, radiating, branched, segmented, and spiral arrangements to which I have so strongly directed attention in the present connection.

The history of the reproductive elements is virtually that of the cell : indeed, to understand the former it is necessary to consider the latter.

A few additional observations regarding the cell from the reproductive point of view will be useful. While the cell may, for practical purposes, be taken as the structural unit for plants and animals, it would be a mistake to regard it as in any way simple. Each cell and each group of cells display peculiarities, and are characterised by features of their own. There is, strictly speaking, no such thing as absolutely simple, identical, homogeneous organic matter.

This seems proved in two ways: $(a)$ by its behaviour while living; and $(b)$ by the appearance presented by it when dead.

Living matter takes in and gives out inorganic matter: it grows, runs a given course, divides, and reproduces itself.

Dead organic matter when subjected to the action of powerful stains, in the majority of cases reveals traces of differentiation and structure.

Even protoplasm presents a reticulated appearance and consists of four or more elements, namely, oxygen, hydrogen, nitrogen, and carbon, with small quantities of sulphur and other ingredients.

Living matter as seen in cells, cell plants and cell animals, the reproductive elements, \&c., presents under high powers of the microscope a great variety of forms--globular, concentric, radiating, branched, segmented, spiral, reticulated, and otherwise.

The cell has a career and history of its own. It cannot be regarded as homogeneous, as it is composed of a cell wall, protoplasm or cell contents, a nucleus, and, generally, one or more nucleoli. In addition to these are to be noted, in many instances, plastids, an attraction-sphere with centrosome, vacuoles, metaplasm, chromatinnetwork, linin-network, net knot (Karyosome), \&c.

The nucleus is, in many cases, a highly complex structure, and runs through many changes which involve differentiation of a kind.

The changes referred to are generally classed under the term Mitosis (from a thread), and include indirect division of the nucleus involving $(a)$ the formation of an amphiaster; $(b)$ conversion of the chromatin into a thread (spirem); (c) segmentation of the thread into chromosomes; $(d)$ splitting of the chromosomes, \&c. (Flemming, 1882.)

The cell plants and animals generally display a nucleus, with or without nucleoli, a contractile vesicle, one or more racuoles, food spaces, \&c.

The reproductive elements-male and female--on which so much depends, are separate and independent entities. They pass through quite a complicated series of phases before they reach maturity, and when they do reach that state and unite and amalgamate in the act of impregnation, they inaugurate, as already stated, a progressive series of extraordinary and even startling changes, characterised by cleavages, reunions, intricate processes of weaving, of tissue forming, of organ forming, of modelling, \&c.

All this, it need scarcely be added, makes for differentiation, division of labour, and heterogeneity.

To Schleiden and Schwann, as already indicated, belongs the great merit of first directing attention to the cell as the fundamental factor in plants and animals. These investigators announced the cell theory in 1838 , and this date marks a new and important era in anatomy and physiology. The cell, as a matter of fact, is itself composed of molecules, and the molecules of atoms; and it is, strictly speaking, in the atoms and molecules that we must search for those properties, peculiarities, and powers which distinguish one cell from another, one plant from another, and one animal from another. To the same source are to be traced the fundamental differences in the sexual elements which determine lineage and transmission by descent. In the atoms and inolecules of the cells and sexual elements are stored up, in a potential form, all the properties, peculiarities, and powers which characterise the parent or parents physically and mentally. When it is stated that the atoms and molecules of cells are, primarily, the parents of plants and animals, it is not meant that the cells to which they give origin contain in miniature all the parts of future plants and animals. They merely contain, in a latent form, the initiatory matter, which, under guidance and hereditary laws, develops in favourable conditions of food, heat, light, moisture, \&c., into plants and animals which in all respects resemble their ancestors.

No provision is made for variations or the perpetuation of variations, which are calculated to introduce confusion, and to subvert or destroy originally created types. When variations do occur, they are, as a rule, speedily eliminated. Neither is provision made for the introduction of acquired physical and mental peculiarities. 


\section{EVIDENCES OF DESIGN IN REPRODUCTIVE ELEMENTS}

other words, parents cannot transmit to their offspring a theological, legal, medical, or scientific training, acquired habits, tricks of trade, handicrafts, \&c., learned and practised by them during their lives. The philosopher cannot transmit his book lore, the watchmaker his deft fingers, or the blacksmith his strong arm. While the arts slightly modify pro tem. those parts of individuals trained in particular directions, they do not alter the atoms and molecules and the cells and sexual elements of the race.

While the cells cannot be fundamentally altered by any form of training, they can, nevertheless, be improved as regards volume and quality. This is the case in nerve cells, especially those occurring in the brain. The brain of educated modern man is larger and of better quality than that of the untutored savage. Education is based on the capacity for improvement of the cerebral nerve centres of the individual up to a point. Mere education, however, can never convert a weak into a strong brain : poets are born, not made. All the transmissible mental peculiarities are the outcome of originally inherited brain cells. The most that can be said in favour of the transmission of acquired characters is that by the continued cultivation for long periods of the cerebral nerve cells in specific directions men and animals occasionally acquire certain tendencies and facilities : the offspring of the bookworm has a penchant for learning; the children of hunting tribes take naturally to the chase; the sheep-dog rounds up sheep, and the sporting dog finds and sets at game. The tendencies and facilities referred to are, however, by no means constant, and cannot consequently be regarded as genuine examples of the transmission of acquired characters. Not unfrequently a strong intellectual father is succeeded by a weak imbecile son.

The advances of, and improvements in, types are traceable to original conformation and to favouring circumstances which individuals, in many cases, cannot control, but of which they freely avail themselves. The over-bred, over-trained racehorse is not unfrequently beaten by a rank outsider, and a city to be healthy physically and mentally must have its old effete blood frequently mixed with fresh young blood from the country.

All biological problems, whether physical or mental, must ultimately be referred to the atoms and molecules of cells, and transmission and heredity are, in a sense, fixed quantities; that is, they are not subject to accidental fluctuations such as are claimed by Mr. Darwin in the production of species. Chance modifications at best influence heredity only for brief intervals-plants and animals tending to breed back to their stereotyped originals.

The cell theory lends itself more to development than to evolution. Evolution implies and carries with it the idea of involution. We cannot take out what is not originally put in. Cells can develop or grow and repeat themselves, but there are no grounds for believing that they can alter themselves and assume new forms and functions, which they would require to do if, in the lapse of time, they produced entirely new plants and animals, as stated by evolutionists. Slight varieties and modifications in plants and animals (which are corrected in time) do not countenance the theory of evolution, which requires the manufacture of plants and animals out of each other by one long, continuous, unbroken process.

It is convenient to regard the cell as the structural unit from which all the tissues of the body, normal and abnormal, proceed.

The cell theory in the hands of Schleiden and Schwann established a common ground as between plants and animals, and enabled Kölliker and Remak to point out similar relations in embryology. It also permitted the great Scottish anatomist, Professor John Goodsir, and the no less celebrated Professor Rudolph Virchow, to demonstrate that "the various functions of the body, in health and disease, are but the outward expression of cell-activities."

Goodsir added much to our knowledge of the cell, and was clearly the pioneer and founder of the "Cellular Pathology" which bulks so largely in modern medicine and surgery. As Goodsir's views, though very important, are comparatively little known, it is necessary to give a brief summary of them in this place. I quote from his "Anatomical and Biographical Memoirs," published in 1868."

"Like all the early observers of 'the cell,' Goodsir met with difficulties. Granted a cell, with its walls, its contents, its nucleus and nucleolus, what then? Did the formation of cells depend on an endogenous or exogenous growth, a fissiparous division, or a gemmiferous thrusting forth of new cells or materials? Theory often ran in advance of observation, and Goodsir, too anxious for a foremost place in the race of competition, went boldly onwards. This mode of procedure could excite no surprise; histology was an almost untrodden field, the explorers of which were enthusiastic and impressionable. Goodsir, no less speculative than scientific, was not the least conspicuous supporter of the new doctrines that bid fair, at one time, to make the cell the whole science of life. Of the lectures delivered (by him) in the theatre of the Royal College of Surgeons of Edinburgh in the summer of 1842 and winter of $1842-43$, a portion was devoted to the consideration of practical subjects-for example, surgical pathology; another portion embraced anatomical and physiological questions of current, or rather special, interest to the younger members of his audience, and were afterwards woven into a work-- Anatomical and Pathological Observations' (vide vol. xi. p. 387).

\footnotetext{
1 "Anatomical Memoirs, with a Biographical Menoir." A. \& C. Black, Edinburgh, 1868.
} 
"The prominent doctrines enunciated by Goodsir in these lectures mainly rested on the existence of centres of force connected with the nutritive and reproductive changes in the normal and pathologica] processes. The term 'centres of nutrition,' or 'germinal centres,' as employed by him, obviously possessed a similar signification to that which at this time (1868) is attached by Dr. Beale to his 'germinal matter,' and by various anatomists of the most modern German school to their masses of nucleated protoplasm. The allocation to these definite 'centres,' not only of the forces engaged in the nutrition of the textures, but in the reproduction of new forms both in normal and pathological processes-a doctrine which has been in its special relations to pathology so systematically pursued by Virchow and his disciples-was unmistakably present in the mind of Goodsir, and also articulately expressed in the pathological papers in the series now referred to. Of the part which the nucleated cell plays in the processes of nutrition, secretion, and reproduction, normal and otherwise, it may perhaps suffice to refer the reader to the paper on 'Centres of Nutrition,' to that on 'Absorption and Ulceration,' to the memoirs on 'Secreting Structures,' 'On Diseased Conditions of the Intestinal Glands,' and the part on the 'Structure and Pathology of the Kidney and Liver.' In these various memoirs and essays the presence of the products of secretion within cells ; the increase which takes place in the size of the cells, and the multiplication of their nuclei when influenced by morbid changes; the rupture of these enlarged proliferating cells, and the discharge of their nucleated contents; all testify to the largeness of his observation of cell-life, both physiologically and pathologically. In the first of these memoirs, not only does he advocate the importance of the cell as a centre of nutrition, but argues that the organism is subdivided into a number of departments, "each containing a certain number of simple or developed cells, all of which hold certain relations to one central or capital cell around which they are grouped.' This idea has since been freely made use of by Professor Virchow, though, it must be admitted, without a due acknowledgment of the quarter in which it was originally stated, and it has obviously influenced many of his physiological and pathological speculations. This reticence is the more strange, as Virchow dedicated his work on 'Cellular Pathology' to the Edinburgh professor in the following complimentary terms: "To John Goodsir, F.R.S., \&c., as one of the earliest and. most acute observers of cell-life, both physiological and pathological, this work on Cellular Pathology is dedicated, as a slight testimony of the deep respect and sincere admiration, by the author.' As Professor Virchow has travelled over much of the ground that had been previously cultivated by Coodsir, it is no less remarkable than disappointing to find in Virchow's volume of 433 pages but one reference to Goodsir, and that in connection with an observation the merit of which might be more fairly ascribed to Dr. Martin Barry. This is scanty civility to a scientific confrire whom he has designated "one of the earliest and most acute observers of cell-life "-one whose labours he has availed himself of, and whose opinions and words he has occasionally adapted." This subject was fully discussed in the British Mcdical Journal (Jan. 12, 1861), in a leading article, "Cellular Pathology, its Present Position," being a review of Virchow's work as translated by Chance. The passages referred to are placed in parallel columns.'

While Germany may be said to have taken the lead in the elaboration of the cell theory, there were able and enthusiastic workers in the field both in London and Edinburgh, foremost among whom may be mentioned Sharpey, Bowman, Carpenter, Gulliver, Busk, Simon, Paget, Martin Barry, Allen Thomson, J. Hughes Bennett, and, as has been shown, John Goodsir, who in some respects was facile princeps of the group.

Various opinions as to the nature of the cell have been hazarded. Thus "Schwann looked upon the vitelline membrane as the outer cell wall, the yolk substance the contents, the germinal vesicle the nucleus, and the macula or maculse the nucleolus or nucleoli. Wagner and Henle regarded the 'germinal vesicle' as the true cell, and the other parts of the ovum as of the nature of superadded structures. Goodsir and Virchow held the cell to be the ultimate morphological element in which there is any manifestation of life, and that the seat of real action must not be transferred to any point beyond the cell."

Latterly, the tendency has been to follow Goodsir's lead, and to identify, more and more closely, the structure and function of the cell. It is now claimed that the cell is not only the unit of structure, but also the unit of function. "It is the cell to which the consideration of every bodily function sooner or later drives us. In the

1 A writer in the Prll Wrill (razette, who seems to have fully realised the many admirable points in Goodsir's cluaracter, says: "Since the days of John Hunter, no greater master of anatomical science, no keener investigator of phenomena, no more comprehensive grasper of generalisations, no clearer or more effective expositor, erer dedicated himself to the great subject of anatomy, human and comparative, than John Goodsir. The only regret will be that he has left so few records of his discoveries and conclusions; that in the kcenness of his pursuit after scientitic truth he left himself so little time to gather up and embody in a lasting form his numerous incidental felicities of investigation and doctrine. But enough, and more than enough, will always remain to prove the brightness of his intelligence, the justness of his reasoning, and the philosophic comprehensiveness of his generalisations. No subject, however remotely connected with his favourite one, but was perfectly known to hin. When in 1854 he suddenly undertook the task of lecturing ou natural history for his deceased friend Edward Forbes, he was found a master, at every loint, in the science which was only accessory to his own. It is indeed impossible to estimate aright the loss which scientific knowledge and academic education sustain through such a death as his. Let us hope that the generous contagion of his teaching and the lustre of his example will arouse in some worthy disciple the masculine enthusiasm, the noble candour, and the chivalrous self-devotion which are buried in 
muscle-cell lies the riddle of the heart-beat, or of muscular contraction; in the gland-cell are the causes of secretion; in the epithelial cell, in the white blood-cell, lies the problem of the absorption of food; and the secrets of the mind are slumbering in the ganglion-cell."

I will conclude this part of the work with one or two short extracts from an instructive volume- "The Cell in Development and Inheritance"--published by Professor Edmond B. Wilson of Columbia University in 1896. The volume referred to is amply illustrated, and I have to acknowledge my indebtedness to this distinguished author for several figures in Plates lix., lx., lxi., and lxii. His allusions to the relations subsisting between the cell and hereditary descent are especially interesting. He observes, "The cell-theory first came into contact with the evolution-theory through researches on the early history of the germ cells and the fertilisation of the ovum. Begun in 1873-74 by Auerbach, Fol, and Biitschli, and eagerly followed up by Oscar Hertwig, van Beneden, Strasburger, and a host of later workers, these investigations raised wholly new questions regarding the mechanism of development and the rôle of the cell in hereditary transmission. The identification of the cell-nucleus as the vehicle of inheritance, made independently and almost simultaneously in 1884-85 by Oscar Hertwig, Strasburger, Kölliker, and Weismann, must be recognised as the first definite advance towards the internal problems of inheritance through the cell-theory; and the discussions to which it gave rise, in which Weismann has taken the foremost place, must be reckoned as the most interesting and significant of the post-Darwinian period. . . By the extreme 'evolutionists' or 'præformationists' the egg was believed to contain an embryo fully formed in miniature, as the bud contains the flower or the chrysalis the butterfly. Development was to them merely the unfolding of that which already existed; inheritance, the handing down from parent to child of an infinitesimal reproduction of its own body. . . Caspar Friedrich Wolff (1759) by precise actual observation showed that the egg does not at first contain any formed embryo whatever; that the structure is wholly different from that of the adult; that development is not a mere process of unfolding, but a progressive process, involving the continual formation, one after another, of new parts, previously non-existent as such. This is somewhat as Harvey, following Aristotle, had conceived it-a process of epigenesis as opposed to evolution. Later researches established this conclusion as the very foundation of embryological science. . . It was reserved for Schwann (1839) and his immediate followers to recognise the fact, conclusively demonstrated by all later researches, that the egg is a cell having the same essential structure as other cells of the body. And thus the wonderful truth became manifest that a single cell may contain within its microscopic compass the sum-total of the heritage of the species. This conclusion, first reached in the case of the female sex, was soon afterwards extended to the male as well. . . Two years after the appearance of Schwann's epoch-making work Kölliker demonstrated (1841) that the spermatozoa arise directly from cells in the testis, and hence cannot be regarded as parasites, but are, like the ovum, derived from the parent-body. Not until 1865, however, was the final proof attained by Schweigger-Seidel and La Valette St. George that the spermatozoön contains not only a nucleus, as Kölliker believed, but also cytoplasm. It was thus shown to be, like the egg, a single cell, peculiarly modified in structure, it is true, and of extraordinary minuteness, yet on the whole morphologically equivalent to other cells. A final step was taken ten years later (1875), when Oscar Hertwig established the all-important fact that fertilisation of the egg is accomplished by its union with one spermatozoön, and one only. In sexual reproduction, therefore, each sex contributes a single cell of its own body to the formation of the offspring, a fact which shows that the sexes play, on the whole, equal though not identical parts in hereditary transmission. The ultimate problems of sex, fertilisation, inheritance, and development were thus shown to be cell-problems."

It only remains for me to direct attention to the figures of Plates lix., lx., lxi., and lxii., where the globular, concentric, radiating, branched, segmented, curved, and spiral arrangements to which I have so frequently referred are delineated. These arrangements are predetermined, and while they can be traced both in the inorganic and organic kingdoms they are especially observable in the cells of plants and animals, particularly in the reproductive or sexual cells. They are fundamental in character, and proclaim their importance by manifesting themselves at the very beginnings of plant and animal life. The early appearance of these arrangements is a startling and unexpected fact, and one which has much significance, as it is calculated to unify and refer to a common standard the ultimate structure of crystals, plants, and animals respectively, and to indicate the presence of law, order, and design in the two great divisions of nature.

Plates lxi., lxii., Ixiii., and lxiv. show that the globular, concentric, radiating, branched, segmented, curved, and spiral arrangements which obtain in crystals, and in plants and animals, make their appearance at the very threshold of life, in cells and in the male and female reproductive elements. This points to general laws in the inorganic and organic kingdoms, and to typical forms which assert themselves in dead and living matter respectively,

VOL. I. 
PLATE LXI

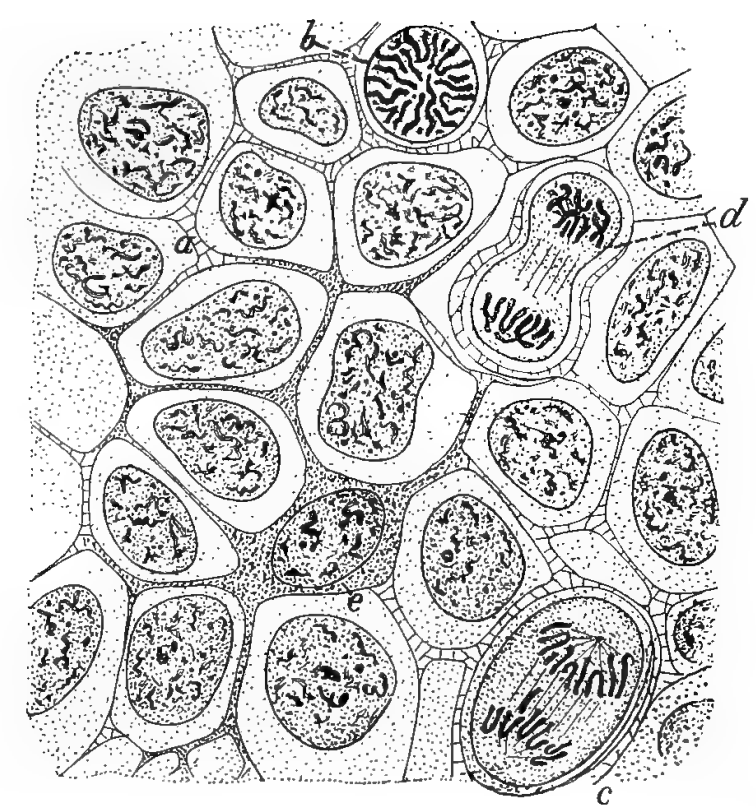

FIG. 1.
(0)

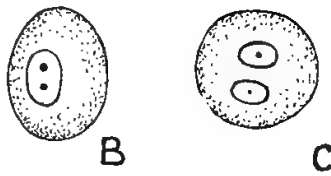

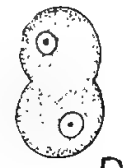

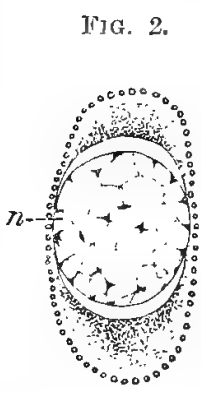

FTล, 3 ,

FIG. 4.
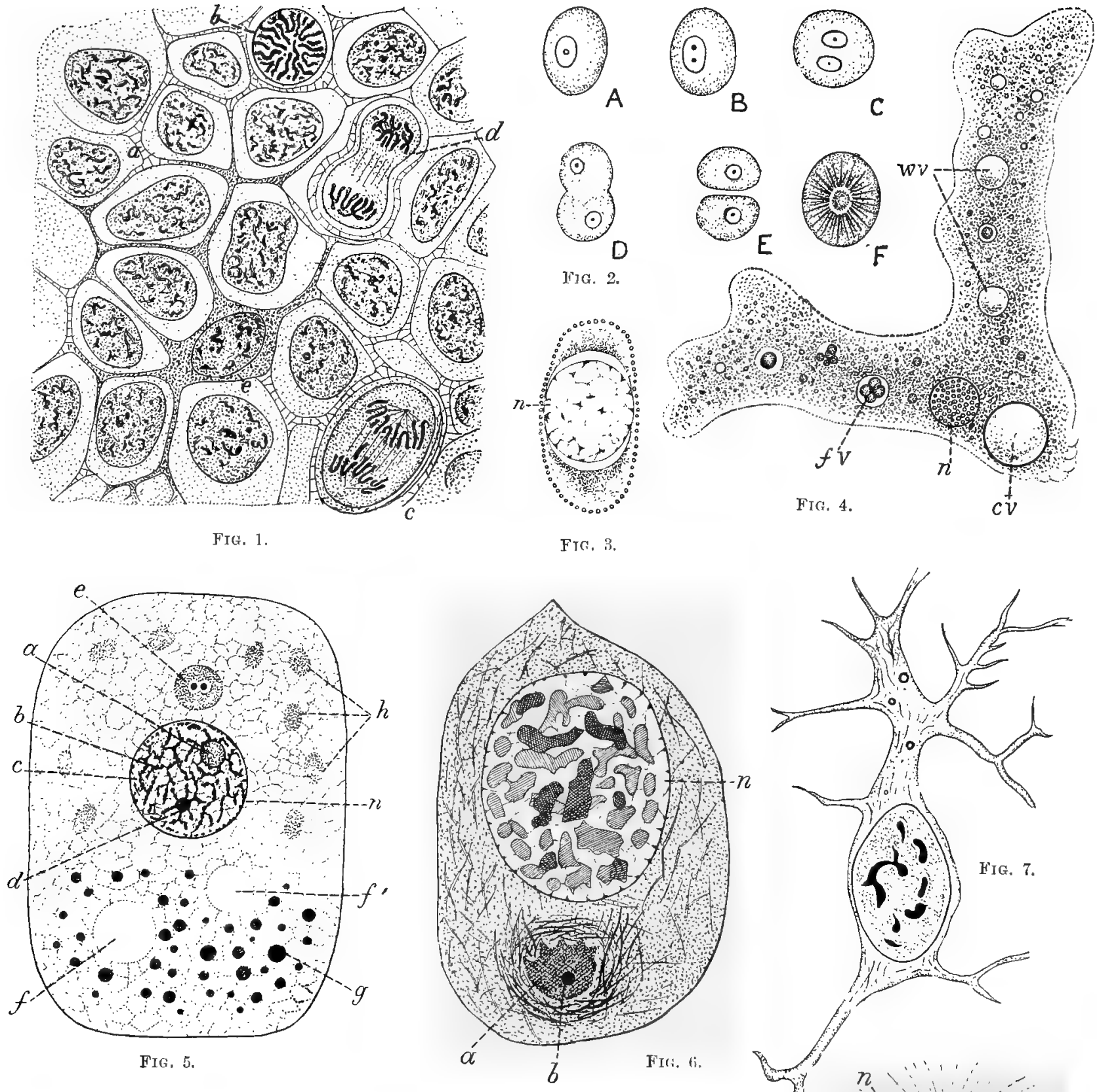

C
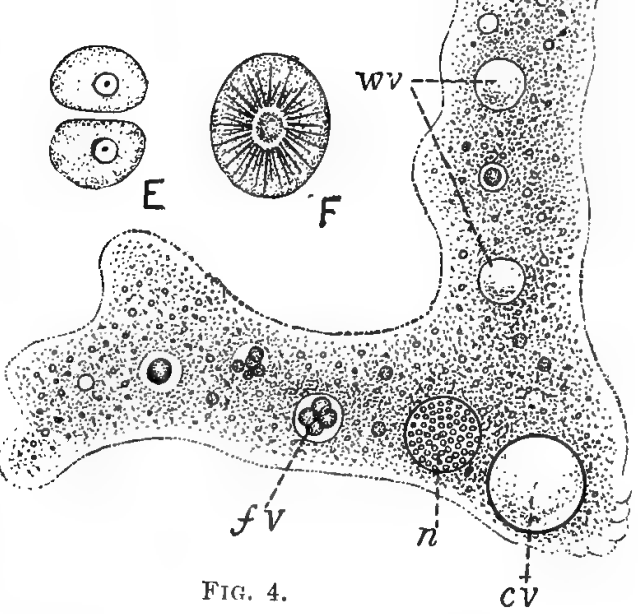

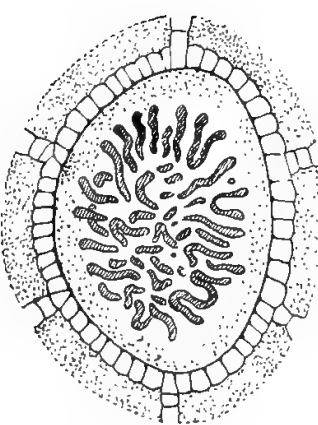

Fir: 8 ,

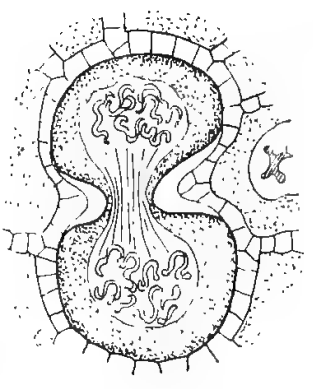

FIG

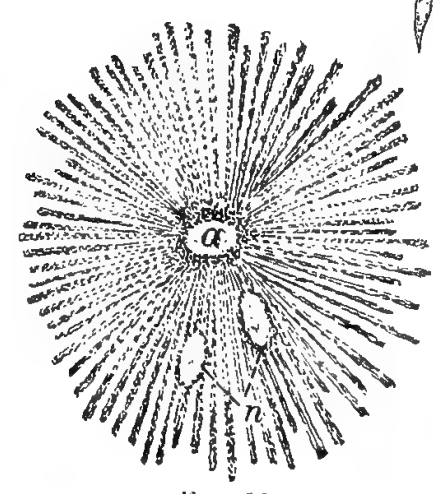

Fit. 10.
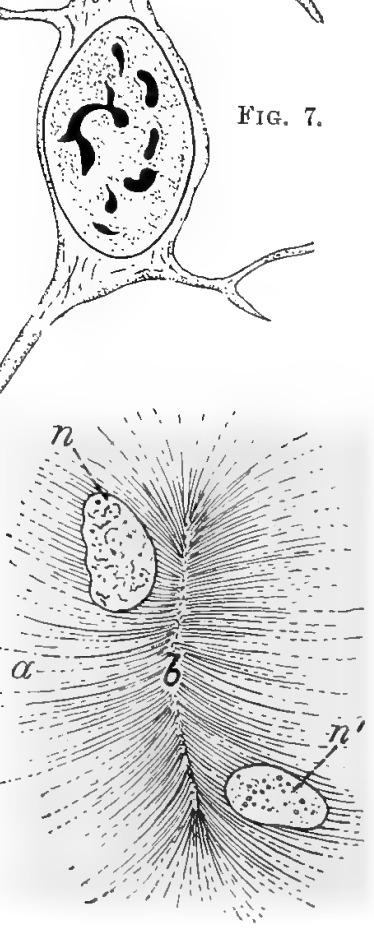

FII 11 . 


\section{EVIDENCES OF DESIGN IN REPRODUCTIVE ELEMENTS}

\section{PLATE LXI}

Plate lxi. illustrates globular, concentric, radiating, branched, segmented, curved, and spiral arrangements.

FIG. 1.-Portion of the epidermis of a salamander in slightly oblique horizontal section, magnified 550 diameters. ('ells rounded and polygonal, connected by delicate bridges of protoplasm and containing large nuclei (Wilson). $a$, Epidermal cells, three of which are undergoing division. 'The earliest stage of division (spirem) is seen at $b ;$ a later stage (mitotic figure in the anaphase), showing chromosomes at $c$; and the final stage (telophase), showing fission of cell-body at $d$. $e$, Branched dark pigment-cell which has crept up from lower layers of skin. This figure illustrates circular, pentagonal, and branched arrangements.

FIG. 2.-Division of the blood-cells in the embryo chick ac'ording to Remak. $a, b, c, d, e$, Successive stages of division ; $f$, cell dividing by mitosis. This figure shows concentric and radiating arrangements.

FIs. 3.-Reticular nucleus $(n)$ of the rhizopod (Actinospharrium) as figured by Brauer. The reticnlation is a form of differentiation-the nucleus possessing a complex organisation.

Fig. 4.-Low aninal form, Amoba protek, consistiug of a single cell. Magnified 280 diameters (Sedgwick and Wilson). $n$, Nucleus; $u v$, water vacuoles; $c v$, contracting vacuole; $f v$, food vacuole. The amoba can reproduce itself and move voluntarily in any direction. It reveals traces of differentiation.

FIG. 5.-Diagram of a cell by Wilson. The basis of the cell consists of a thread-work (mitome or reticulum) composed of minute granules (microsomes), traversing a transparent ground-substance. $n$, Nucleus; $a$, true nucleolus or plasmosome; $h$, cliromatin network ; $c$, linin-network; $d$, net-knot or karyosome: $e$, attraction-sphere containing two centrosomes ; $f, f^{\prime}$, vacuoles ; $g$, lifeless bodies (metaplasm) suspended in cytoplasmic reticulum; $h$, plastids lying in cytoplasm.

This diagrain shows that the cell is not, in any sense, a simple homogeneous boly. On the contrary, it is differentiated to a considerable extent, a circumstance too frequently overlooked. The cell is originally spherical in form, but becomes indented by growth, crowding, and pressure.

FIG. 6.-A resting cell (Spermatogonium) from testis of salamander, showing typical parts (Ravitz). n, Large nucleus bounded by membrane and invested with cytoplasmic thread-work. Contains scattered masses of chromatin and linill-network. $a$, Attraction sphere with centrosome $(b)$. The attraction-sphere is a centre of force, and can act either centripetally or centrifugally.

FIG. 7.-Living branched connective tissue-cell from salamander larva (Fleming). Affords beatiful example of branching arrangement.

FIG. 8.-Living epiderual cell from larva of salamander in early mitosis (segmented spirem), surrounded by protoplasmic bridges (Fleming). Shows concentric, branching, and radiating arranguments.

FtG. 9. -Living dividing cell of salamander larva. The cell when dividing assumes a dumb-bell shape (Fleming).

FIG. 10.-Pigment-cell from epidermis of fish (Blemnius), as given by Zimmerman. a, Central clear space of aster from wlich the pigment-granules diverge; $n$, two nuclei. Shows radiating arrangement to advantage.

FIG. 11.-Pigment-cell of fish (Sargus) showing two nuclei $\left(n n^{\prime}\right)$ with aster $(a)$, and rod-shaped central mass (b), according to Zimmerman. Affords good example of branching, radiating arrangements.

\section{PLATE LXII}

Plate lxii. illustrates globular, concentric, radiating, branched, dendritic, curved, and spindle arrangements in the cell.

FIG. 1.-Leucocyte or wandering cell of the salamander as figured by Heidenhain. The cell contains a large nuclens ( $n$ ) with coarse network of chromatin, two nucleoli $\left(n^{\prime}\right)$, and permanent aster $(g)$, with double centrosome at its centre surrounded by attractionsphere. Illustrates concentric and radiating arrangements.

Fic. 2. - Same as Fig. 1-the cell in this case containing two nuclej.

FItt. 3.-Alveolar or foam-structure of protoplasın in the sea-urchin's egg (Bütgchli). $a$, Aster ; $b$, attraction-sphere ; $c$, centrosome. Shows radiating and concentric arrangements in a marked degree.

FIG. 4.-Branching nucleus from the spinning gland of larva of buttertly (Pieris), as delineated by Korschelt. It would be difficult to find a better example of branching arrangement in a rudimentary structure.

FIGS. 5, 6, 7, and 8.-Assortment of cells by varions investigators showing typical parts.

FIG. 5.-Spinal ganglion-cell of frog as given by Lenhossek. $n$, Nucleus; $a$, attraction-sphere; $b$, centrosome with several centrioles.

Fig. 6.-Spermatogonium of frog (Hermann). ", Attraction-splere (aster), containing a single centrosome (b); $n$, nucleus with single plasmosome $(c)$ and net-knots $(d)$.

FIG. 7.-Peritoneal epithelium from larva of salanander (Fleming). n, Large reticulated nucleus with net-knots (nk); $b$, two centrosomes. 

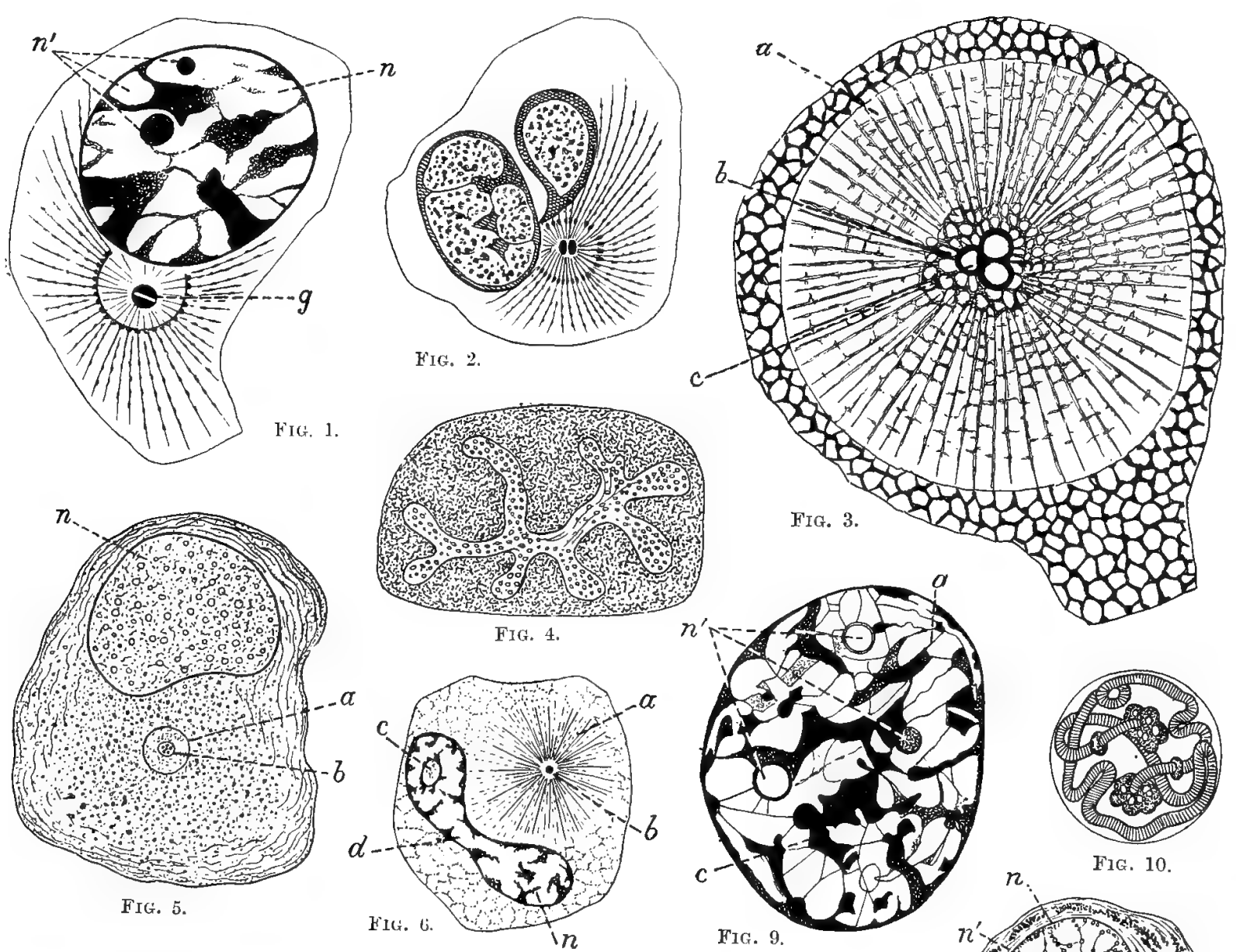

PLATE LIII 


\section{EVIDENCES OF DESIGN IN REPRODUCTIVE ELEMENTS}

\section{PLATE LXII (continued)}

Fig. 8.-Spermatocyte of Proteus (Hermann). ", Nucleus in the spirem or thread stage; ", attraction-sphere with rod-shaped bodies and single centrusome. Figs. 5, 6, 7, and 8 illustrate concentric, radiating, and reticular arrangements.

Fig. 9.-Nucleus from crypt of Lieberkühn in Salamander (Heidenhain). Contains three plasmosomes or true nucleoli ( $\left.n^{\prime}\right)$; also chromatin net-work $(c)$ with some fine linin-threads $(a)$.

FIg. 10.- Remarkable permanent spirem-nucleus from salivary gland of larva of chironomus; chromatin arranged in a single thread with chromatin dises terminating at either end in a true nucleolus or plasmosome (Balbiani). Shows spiral arrangement of a kind.

Fig. 11.-Section of nephridian cell of leech (Clepsine) as drawn by Arnold Gray. $n$, Nucleus invested with chromatin membrane, containing granules and a single nucleolus with vacuoli $(v) ; v^{\prime}, v^{\prime}$, large vacuole in centre of cell filled with watery fluid; $u^{\prime}$, reticulum formed by cytoplasm and containing centrosomes, well marked on peripheral zone.

FıG. 12.-Spinal ganglion-cell of frog (Lenhossek). $n$, Nucleus with intensely chromatic uncleolus $\left(n^{\prime}\right)$ and pale linin-network with chromatin rounded granules (c); $y$, dark cytoplasmic masses (chromophilic granules) with a slightly concentric arrangement; $h$, centrosome, not very distinctly seen ; $i$, two connective tissue cells. The minute anatomy of the nerve cell is by no means simple.

FIGS. 13, 14, and 15.-Metaphase and anaphases of mitosis in cells (spermatocytes) of the salamander (J)rimer).

FIG. 13.-Metaphase. Shows spiudle arrangement of fibres $(s)$; centrosomes $\left(c, c^{\prime}\right)$, asters $\left(a, a^{\prime}\right)$, and two chromosomes $\left(d, d^{\prime}\right)$, attached to contractile mantle-fibres at outside of spindle $(b)$.

FIG. 14.-A Anaphase. Shows divergence of the daughter-chromosomes (d), exposing the central spindle at the inter\%onal fibres $(b)$ with contractile fibres ; centrosomes $\left(c, c^{\prime}\right)$, and asters $\left(a, a^{\prime}\right)$, with concentric markings. This figure illustrates radiating and concentric plus looped arrangements.

FIG. 15.-Later anaphase. Fully exposes central spindle-fibres $(s)$; also the contractile or mantle-fibres $\left(m\right.$, $\left.m^{\prime}\right)$, attached to the chromosomes $\left(d, d^{\prime}\right) ; a, a^{\prime}$, asters ; $c, c^{\prime}$, centrosomes. The cell is now about to divide.

\section{PLATE LXIII}

Plate lxiii. illustrates spherical, concentric, radiating, brawched, and segmented arrangements in the egg.

FIG. 1.-A. Ovarian egg of the sea urchin (Toxopneustes) magnifed 500 diameters (Wilson). gv, Germinal vesicle or nucleus, containing an irregular discontinuous network of chromatin; gs, germinal spot or nucleolus deeply stained with hæunatoxylin; $c h$, naked cell-body composed of a regular network, the threads of which consist of irregular rows of minute granules or microsomes.

B. Spermatozoon or male element, also enlarged 500 diameters.

FIGs. 2 and 3.-Eggs of the annelid Nereis, before and after fertilisation ; magnified 250 diameters (Wilson).

Fig. 2.-Before fertilisation. The large germinal vesicle or nucleus ( $g v$ ) occupies a central position, and contains a network of chromatin in which are five small dark bodies, the quadruple chromosome groups or tetrads, in process of formation: also a double germinal spot $(g s)$ composed of a chromatic and an achromatic sphere. The egg of the Nereis is laden with yolk consisting of clear dentoplasn.spheres $(d)$ and drops of fat $(f)$; no deutoplasm being found in the peri-vitelline layer $(p)$. As reproduction proceeds the tetrads of the germinal vesicle become more pronounced; the network in which they are contained disappearing. This figure illustrates concentric and spherical arrangements.

FIG. 3.-Some time after fertilisation-egg about to divide. The deutoplasm $\left(d, d^{\prime}\right)$ now occupies lower portion of egg, and the peri-vitelline membrane has disappeared. The mitotic figure is also formed and the chromosomes $(c)$ are dividing. $p b$, Polar bodies; $a$, asters. The changes consequent on fertilisation are very marked, and point to progressive development and differentiation. Fig. 3 illustrates radiating and spherical arrangements.

FIGs. 4, 5, 6, and 7.-Young ovarian eggs with yolk-nuclei and deposits of deutoplasm.

Fig. 4.-Myriopod (Geophilus) with one yolk-nucleus (yn), deutoplasm (d), and germinal vesicle (gv) as figured by Balbiani. Shows concentric and spherical arrangements.

FIG. 5.-The same with several yolk-nuclei $(y n, y n)$, germinal vesicle $(g v)$, and attraction-sphere $(s)$.

FIG. 6. Fish (Scorpæna) with deutoplasm $(d)$ forming a ring round the germinal vesicle ( $g v$ ), with irregular mass of “. eliminated chromatin" as delineated by Van Bambeke. Shows well-marked concentric arrangements.

FIG. 7.-Ovarian egg of a young duck, three months old, displaying a yolk-nucleus (yn), a germinal vesicle ( $g v)$, rleutoplasm (d), and an investing capsule $(f)$, as figured by Mertens. Shows concentric and segnented arrangements.

FIGs. 8 and 9.-The middle phases of mitosis in the first cleavage of the Ascaris-egg as represented by Boveri.

Fig. 8.-Closing prophase, the equatorial plate forming. ' ', $c^{\prime}$, Centrosomes; $a, a^{\prime}$, asters. Shows radiatiug, concentric, and spindle arrangements.

FIG. 9.-Metaphase; the equatorial plate (ep) completed-viewed from before, the four chromosomes divided. Centrosomes, asters, and spindle, as in Fig. 8. pb, Polar bodies. It would be difficult to find a finer example of radiating and concentric arrangements than is here furnished. 


\section{PLATE LXIII}
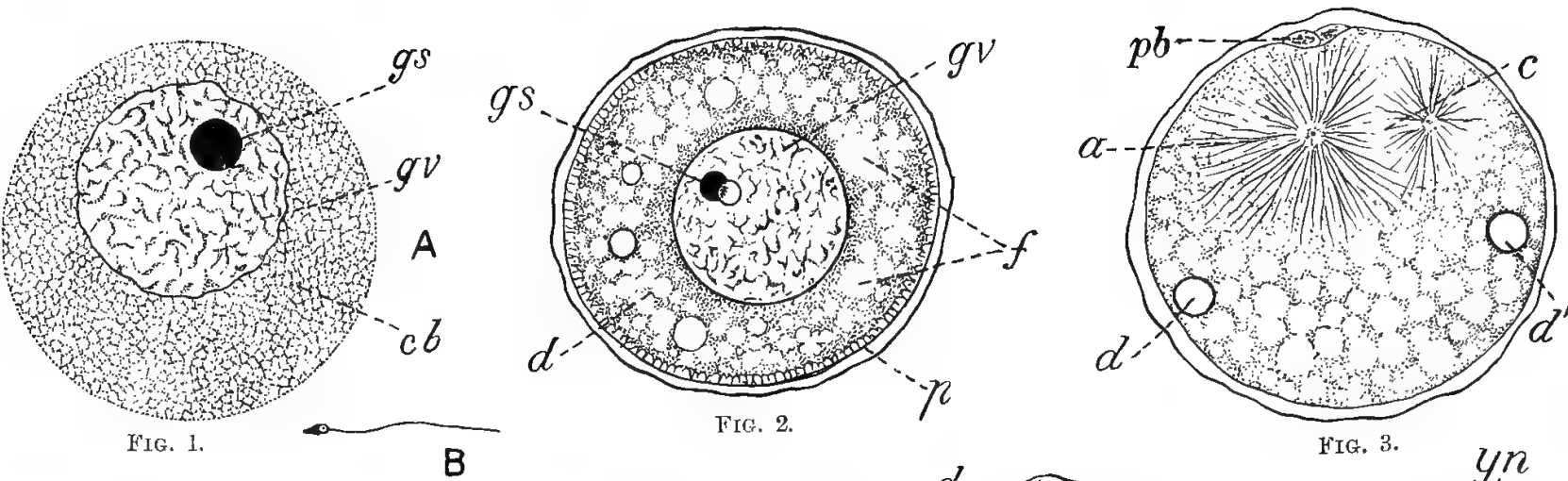

B
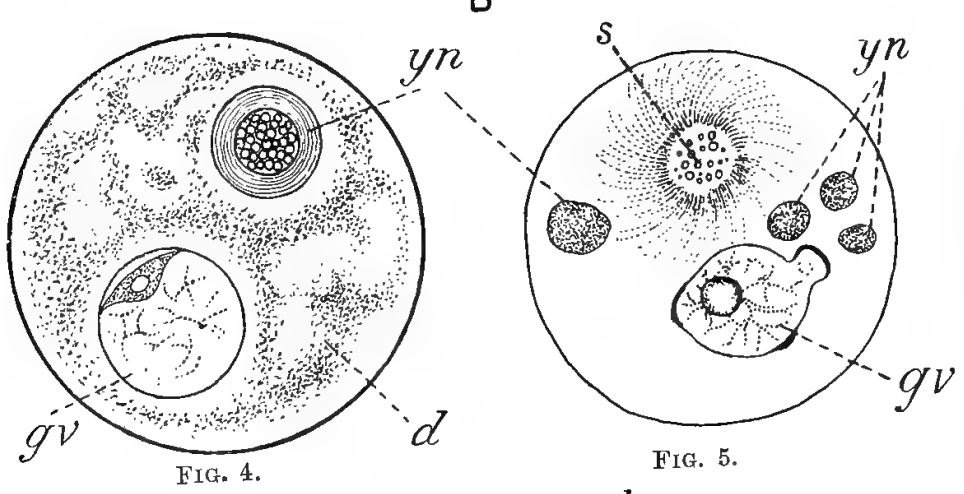

FIG. 5.
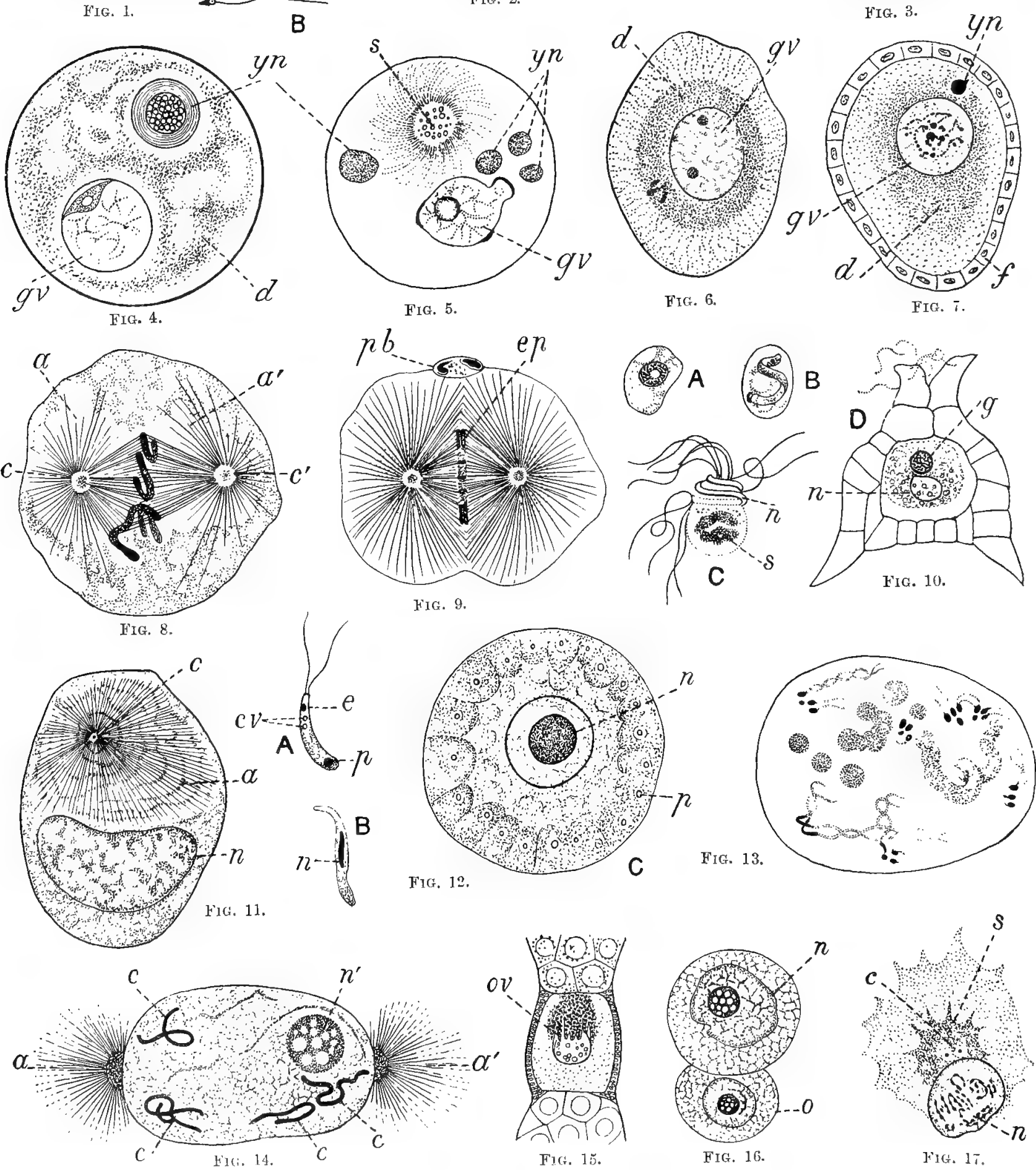

litit. 17 . 


\section{EVIDENCES OF DESIGN IN REPRODUCTIVE ELEMENTS}

\section{PLATE LXIII (continued)}

FIG. 10.- Eertilisation in the plant (Pilularia) as delineated by Campbell.

A, B. Early stages in the formation of the spermatozoid.

C. The mature spermatozoid. The nucleus lies in the spiral turns of the latter at $n$, a cytoplasmic mass containing starch corpuscles being seen at $s$.

D. Archegonium during fertilisation. In the centre, the ovum (o) contains the apposed germ nuclei $(g, n)$. S'lorss spherical, concentric, segmented, and spiral arrangements.

FIG. 11.-Spermatogonium of Salamander as figured by Drüer. $n$, Nucleus; $a$, enormons aster, its rays giving indications of nine concentric circles of microsomes; $\iota$, centrosome, or centre of force, from which the rays of the aster diverge, and round which the circles of microsomes arrange themselves. Aftords an outstanding example of radiating and concentric arringements.

Fig. 12.-Germinal cells of Volvox as figured by Overton.

A. Spermatozoid. $c v$, Contractile vacuoles; $e$, eye spot (chromoplastid) ; $p$, pyrenoid.

B. Spermatozoid stained to show nucleus $(n)$.

C. Ovum (oosphere) with large central nucleus (n), and peripheral layer of chrumatophores. $l^{\prime}$, Pyrenoid. Shows spherical, concentric, and brancling arrangements.

Frgs. 13 and 14.-Germinal vesicles of eggs, showing chromosomes, tetrads, and nucleoli.

FIG. 13.-Cyclops stremun, ilhstrating Häcker's account of the tetrad formation from elongated doulle rods with group of accessory nucleoli (Häcker).

FIG. 14.-Germinal vesicle of an annelid (Ophryotrocha), showing nucleolus $\left(n^{\prime}\right)$, four chromosomes $(c, r, c, c)$, and asters $\left(a, a^{\prime}\right)$, according to Korschelt. Shows spherical, concentric, and radiating arrangements.

FIG. 15.-Living ovarian egg of an insect, water-heetle (Dytiscus), as figured by Korschelt. The egg (ov) lies between two groups of nutritive cells; the germinal vesicle sending amaboid processes into the dark mass of food-granules.

FIg. 16.-Egg and nurse-cell of the annelid (Ophryotroche in the young stage, as depicted by Korschelt. In this condition the nurse-cell $(n)$ is considerably larger than the egg (o). As the ovum grows the egg becomes larger and the nurse-cell snaller-the latter ultimately shrivelling up. The egg practically lives on the nurse-cell so long as it lasts. somes.

Fig. 17.-Mitosis in the flagellate (Noctiluca), early prophase (Ishikawa). $n$, Nucleus ; s, attraction-sphere; $c$, two centro-

\section{PLATE LXTY}

Plate lxiv. illustrates globular, radiating, concentric, branched, segmented, and spiral arrangements, especially the latter (spiral) in the sexual elements.

FIG. 1.-Spermatozoids of plants. A to $\mathrm{D}$ as given by Guignard, and $\mathrm{E}$ to $\mathrm{F}$ as given by Strasburger.

A. Of an alga (Fucus); P. Liverwort (Pellia); C. Moss (Sphrtgnum); D. Fern (Angiopteris); E. Marsilia ; F. Fern (Phegopteris). In $A$, a red chromatophore oceurs at the right of the nucleus, and at $F$, the nuclets is coloured dark. Illustrates spiral, branching, and spherical arrangements.

Frg. 2.-Unusual form of spermatozoa.

A, B, C. Living amoeboid spermatozoa of the Crustacean (Polyphemus), as figured by Zacharias. D, E. Spermatozoa of crab (Dromia); F, of Ethusa; G, of Maja; and H, of Inachus as given by Grobben. I, of Lobster (Homarus)-Herrick; and J, of crab (Porcellana) - Grobben. Illustrates radiating, concentric, and branching arrangements.

FIG. 3.-Diagram of a flagellate spermatozoon. The spermatozoon has been divided to economise space (Wilson). $a$, Apex or apical body; $n$, nucleus; $e k$, end knob (? centrosome); $m p$, middle piece; et, envelope of tail; af, axial filament; $e p$, end piece.

FIG. 4.-Spermatozoa of fishes and amphibia as given by Ballowitz.

A. Sturgeon; B. Pike; C, D. Leuciscus; E. Triton (anterior part); F. Triton (posterior part of flagellum); G. Raja (anterior part). $a$, Apical body; $e$, end piece; $f$, flagellum; $k$, end knob (? centrosome); $m$, niddle piece; n, nucleus. Illustrates spiral and waved arrangements as witnessed in swimming and serpentine movements.

FIg. 5.-Spermatozoa of various animals (after Ballowitz, Von Brunn, and Wilson).

A. Bird (Phyllopneuste) ; B. Bird (Muscicapa); nuclens divided into two parts $\left("{ }^{\prime} x^{2}\right)$, no distinct middle piece; C. Bullfinch with spiral membrane; D. Giant form of spermatozoon of the sheldrake (Tadoma); F. (rill (Lams), with spiral middle piece and apical knob. Illustrates spiral and waved arrangements.

FIG. 6.-Spermatozoa of mammals (after Ballowitz and Wilson).

A. Badger (living); B. The same dead and after staining; C. Bat (Vesperu( ()$)$; D. The same, flagellum and middle piece showing end knobs; E. Opossum (after staining); F. Rat; G. Head of spermatozoon of pig; H. Head of the spermatozoon of the bat (Rhinolophus) showing details; I. Double spermatozoon from the vas deferens of the opossun. he, Head cup (apex); $k$, end knob (? centrosome); $m$, middle piece; $w$, nucleus (in $\mathrm{B}, \mathrm{H}$, and $\mathrm{G}$, consisting of two parts)

FIGs. 4, 5, and 6 illustrate in a striking manner the spiral and waved arrangements which obtain in spermatozoa as a class. 
I 60

DESIGN IN NATURE

PLATE LXIV
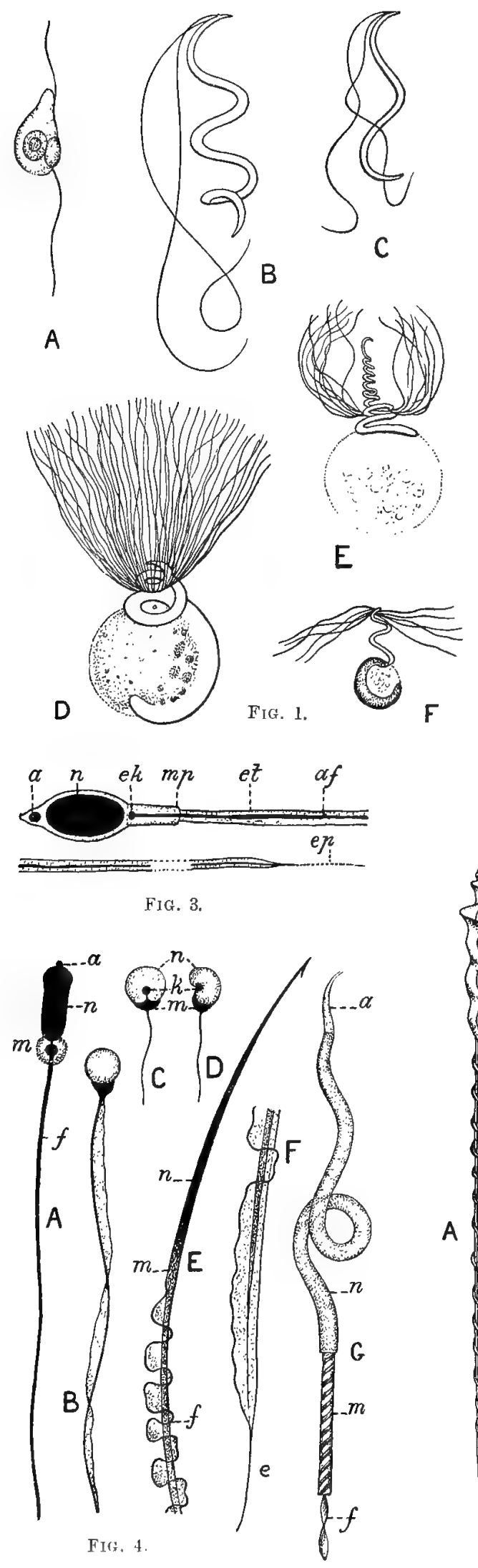

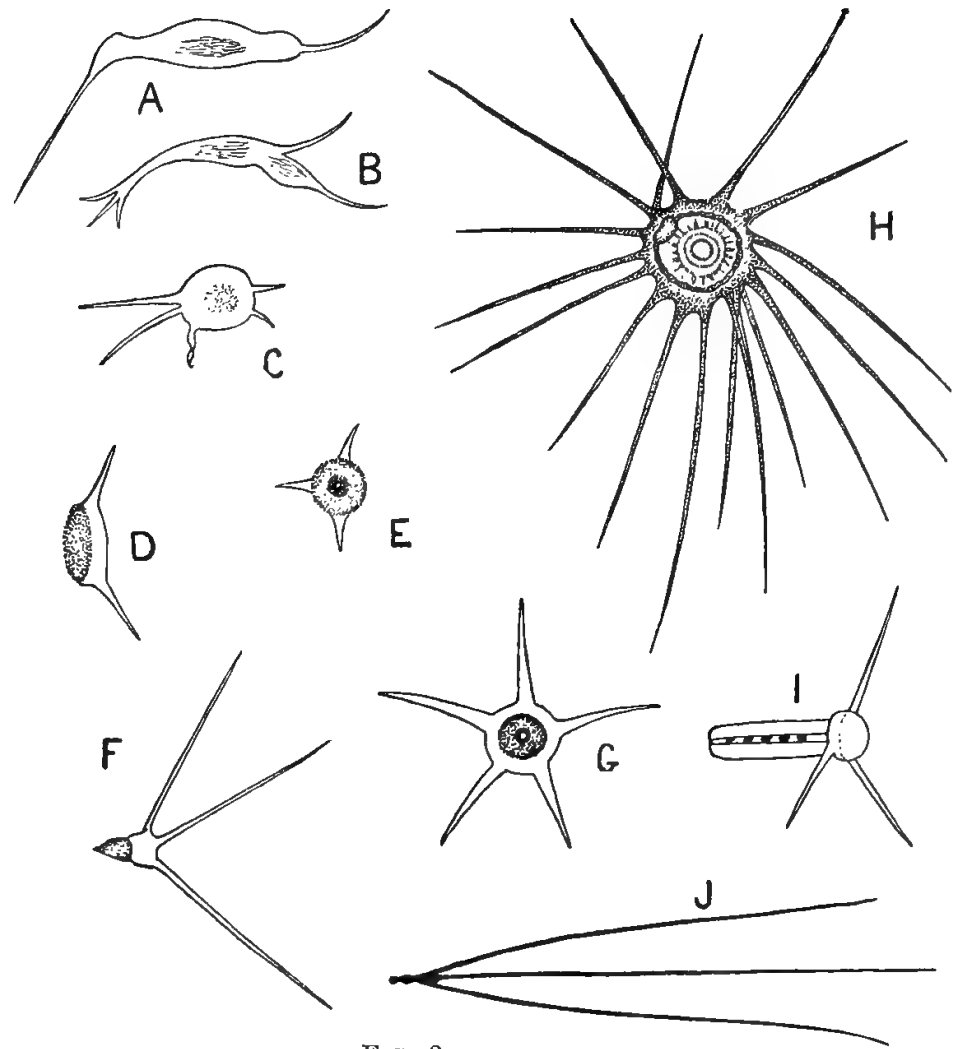

FIG. 2.
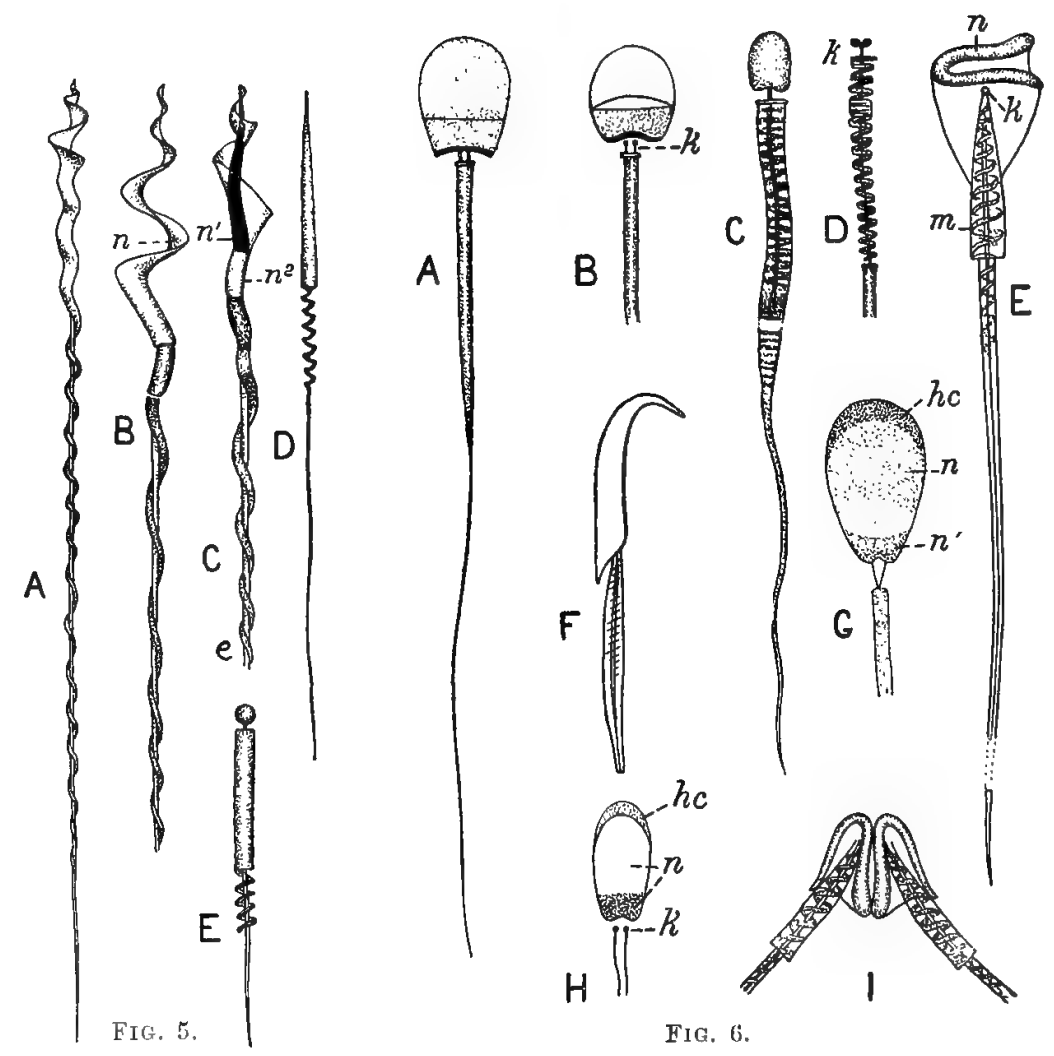


\section{PROGRESSIVE ADVANCE IN LOWER PLANT AND ANIMAL FORMS}

Having shown that crystals, plants, and animals have much in common, and that the substances composing them exhibit globular, concentric, radiating, branched, segmented, curved, and spiral arrangements, I have now to point out that the more rudimentary plants and animals are disposed in an ascending series where improvement in form, structure, and types may be traced.

The rudimentary plants are seen at Plates iii., iv. The rudimentary and other animals are given at Fig. 9, Plate xii., Figs. 1 and 2 ; Plate xvi., Figs 9 and 10 ; Plate xviii., Fig. 3; Plate xx., Fig. 1; Plate xxv., Figs. 2, 4, 5, 6, and 7 ; Plate xxvi., Fig. 2 ; Plate xxvii., Fig. 4 ; Plate xxxv., Figs. 2, 3, and 5; Plate xxxvi., Figs 1, 3, and 4; Plate xxxvii.; Plate xli.; Plate xlii., Figs 3 and 4; Plate xliii., Figs 2, 3, 5, and 6 ; Plate xlvi., Figs. 1 and 2.

The Infusoria, which afford examples of the simplest animals, are alike interesting and instructive, from the fact that they give a clue to the structure of all the higher animals. In these lowly forms, the globular, concentric, radiating, branched, segmented, curver, and spiral arrangements which everywhere obtain in living organisms, and to which attention has been already dirested, form a conspicuous feature. These arrangements, which present themselves at the very threshold of existence, are, it appears to me, so important that I devote no fewer than ten plates to their illustration. In these plates the same lettering is employed in the several figures throughout. Thus $a$, indicates the nucleus or endoplast; $c v$, the contractile vesicle or vesicles; $d$, the oral aperture; an, the anal aperture; $f$, the flagellum; and $c l$, the collar.

In this connection I have to acknowledge my indebtedness to Mr. W. Saville Kent, from whose interesting and erudite work on the "Infusoria" many of the figures are taken."

It is not necessary to describe the several Infusoria in detail. The plates, and the descriptions thereof, will supply the information I desire to convey. The points I wish especially to establish are the following :-

1. The Infusoria display well-marked globular, concentric, radiating, branched, segmented, curved, and spiral arrangements.

2. They show a gradual advance in outline, structure, and function, but no attempt, unless in a general way, has been made at classification.

3. They display symmetry and asymmetry.

4. They exhibit centripetal, centrifugal, spiral, and other movements.

5. They assume a well-marked and distinctive form in the adult condition which, in many cases, they lose during the reproductive process. They advance in the adult state and retrogress and assume the amnebic and shapeless state during transition and the young condition.

6. They reproduce themselves in several ways; $a$, by longitudinal, transverse, and other division; $b$, by budding; $c$, by swarming; and $d$, by reverting to the simple, amorphous, amœbic condition.

7. They move freely about in the young state, and, not unfrequently, become fixed in the adult state.

8. They possess pseudopodia, cilia, flagella, tentacles, sensitive filaments, \&c., which are under control, and which enable them to secure food, and, if need be, to move from place to place.

9. They are provided with a nucleus and one or more contractile vesicles; the latter displaying opening and closing rhythmic movements akin to those occurring in the hearts of vertebrates.

10. They form colonies, being, in some cases, grouped rosette-fashion and presenting a symmetrical flower-like appearance.

11. They, in not a few instances, divide, subdivide, and branch like plants.

12. They frequently display an elegant cup-shaped collar and an exquisite protecting covering or lorica which render them exceedingly beautiful objects.

13. They form, in certain cases, the living substance of sponges.

14. They, on many occasions, reveal longitudinal, transverse, spiral, and other markings.

15. They also afford distinct traces of segmentation.

The globular, concentric, radiating, branched, segmented, curved, spiral, and other arrangements and markings so well seen in the Infusoria are also met with in the pollen, anthers, and other parts of plants connected with reproduction. They are shown at Plate lxv.

In this plate all the peculiarities of structure referred to can readily be studied and appreciated.

Before examining the Infusorial plates it may be useful to describe very briefly one of the typical Zooids, the best for the purpose being the collared monad (Monosiga gracilis).

1 "A Manual of Infusoria, including a Description of all known Flagellate, Ciliate, and Tentaculiferons Protozoa, British and Foreign, and an Account of the Organisation! and Aftinities of the Sponges," by W. Saville Kent, F. I.S., F. Z.S., F. R.M.S., \&c. London, $1880-1882.3$ volumes, VOL. I. 
The collared monad is a minute transparent microscopical creature which lives in fluids and consists of a stem or pedicle by which it is fixed to some foreign object, a pear-shaped body in which can be seen a nucleus, several contractile vesicles, food globules, \&c. Projecting from the upper part of the body is a long, slender, tapering, hair-like process known as the flagellum. Rising from the upper part of the body is the beautifully moulded, delicate collar resembling an egg-cup.

The outstanding features in the collared monad are its small size, transparency, and simple organisation. It possesses no alimentary canal, no breathing apparatus, no circulation, no skeleton, no muscles, no nerves, next

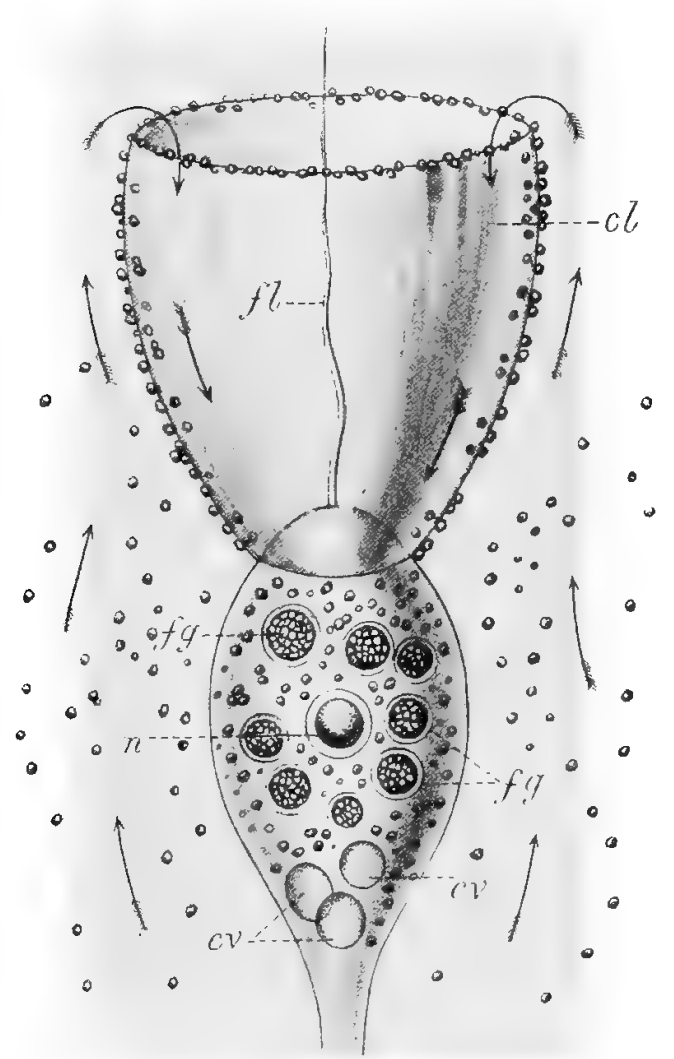

Frg. 35. - Collared monad (Monosiga gracitis) fed on carmine; very greatly magnified. Shows a pear-shaped body surmounted by an egg-cul, collar. The body terminates in a stem or pedicle which is broken off. $c l$, Collar ; fl, flagellum ; $u$, nucleus; $c v$, contractile vesicles ; $f q, f y$, food globules.

The arrows indicate the direction which the food particles, floating in the water, are obliged to take because of the voluntary well-directed movements of the flagellum (II' Saville Kent).

structures which perform such an important rule in the circulation of the higher animals, up to man, may well excite wonder, and that wonder is greatly increased when it is stated that they contain neither muscle nor nerve; the substances to which we usually refer regulated movements in animals. This marvellous arrangement of life is strangely enough not confined to the monad and other rudimentary animal forms. It first makes its appearance in the plant Volvox globator, whose water vacuoles open and close rhythmically with the utmost regularity and precision, the vacuoles opening slowly and closing somewhat suddenly as in the ventricles of our own hearts.

The contractile vesicles of plants and the lowest animals teach a very important lesson, namely, that structure and differentiation, in the ordinary sense, are not necessary to the production of the spontaneous, co-ordinated rhythmic movements on the integrity of which, in ourselves, life depends. They are utterly inexplicable unless we refer them to a First Cause and design.

The function performed by the contractile vesicles in the collared monad is not quite understood. They are supposed to regulate the amount of fluid in the body for the time being, and to assist in the elimination of effete prolurts. They thus seem to be ronnected with ingestion and egestion. 


\section{ADVANCE IN LOWER PLANT AND ANIMAL FORMS}

The food enters the body of the monad as fine, floating particles. Within the body the particles become massed into round cellular-looking bodies known as food globules, which vary in number according to the amount of food ingested at any one time. The carmine-stained food granules shine through the transparent body of the monad and are strongly pronounced.

The collared monad reproduces itself variously: by transverse division, by longitudinal division, by combined transverse and longitudinal division, by swarming, and by assuming an amorphous amobic condition. The last form of reproduction is at once striking, suggestive, and instructive, as it presents the case of a living form which in the adult condition has a well-defined outline and simple structure, both of which wholly disappear during reproduction. In the amobic form of reproduction there is, as it were, a harking back to a simpler condition. The same holds true, within limits, of reproduction in the higher animals. The higher animals, one and all, produce separate sexual elements in which no trace of structure, in the ordinary sense, can be detected. These elements when they come together merge and blend, and form an amorphous mass which divides and subdivides to produce the blastodermic membrane from which, ultimately, all the structures of the body are developed. The higher, as well as the lower animals, return to a humble platform for a fresh start. But, and this is an all-important point, alike in the monad and the man, the potentialities of the future being are present, and can always be relied on to reproduce the new being on the exact pattern of the old.

From what has been stated, it will be evident that in the lowly collared monad the scheme of animal life, as a whole, is not only distinctly foreshadowed, but a working model provided.

The monad is furnished with the means of voluntarily supplying itself with food. It displays various kinds of movements. It can ingest and egest. It can reproduce itself and perform the several functions of life with absolute certainty.

The higher Infusoria will tell their own tale and teach their own lesson in the annexed plates with their accompanying letterpress (vide Plates lxxiii., lxxiv., and lxxv.).

\section{PLATE LXV}

The figures in this plate show a wonderful diversity and differentiation in the pollen, anthers, and other parts of plants connected with reproduction. The shapes, markings, and divisions are very striking, and some beautiful examples of globular, concentric, radiating, branched, curved, and spiral arrangements are to be noted.

FIG. 1.—a, b, Spiral tissue of lining of anther from wall-flower (Cheirunthus Cheiri).

FIg. 2.-Spiral tissue of lining of anther from London Pride (Sauifraye umbrosa).

FIG. 3. - Spiral tisste of lining of antler from Lupinus nanus.

FIG. 4.-Spiral tissue of lining of anther from a cactus (Ctreus speriosis); a, side view; $b$, as seen from above. Show radiation from central point.

FIG. 5.-Spiral tissue of lining of anther from daisy (Bellis perennis).

FIG. 6.-Pollen of Violu orlorata; $e$, side view; $b$, end view with threefold division ; $e$, as moistened by water.

Fig. 7.-Pollen of Periploca grieca. Shows fourfold division of parts.

FIG. 8.-Pollen of A pocynum venetum, showing fourfold division of parts.

FIG. 9.-Pollen of daisy (Bellis perennis) spansely covered with short spines.

FIG. 10.-Pollen of Mesembryanthemum covered with aborted spines.

FIg. 11.-Pollen of Alisme Pluntago with small circular markings.

FIG. 12.-Pollen of Jupinus nuius showing spindle-shaped tesselated patterı.

FIG. 13.-Triangular-shaped pollen of Eililobium montanm with pores at angles.

FIG. 14.-Round indented pollen of garden geranium (Pelargonium specisum). "Front view; $b$, side view.

FIG. 15.-Beautifully marked pollen of passion-Hower (Pussifture carulen); $\iota$, perfect grain; $b$, perfect grain with raised lid and pore opening.

FIG. 16.-Pollen of Scorzonerce hispunicu with syumetrical ridge-prattern.

FIG. 17.-Pollen of Frica multiflore divided into three parts.

FIG. 18.-Pollen of Imputiens Noli-tanyere with jores.

FIG. 19.-Spindle-shaped pollen of Pussiffor atuileyiafolue. a, Side view; $b$, end view; $c$, as muistened ly water. Show rarliating symmetrical patterns.

Fig. 20.-Pollen of Hibiscus Trionum showing conical spines and pores.

FIG. 21.-Pollen of musk-plant (Mimulus moschatus), Displays beantiful spiral arrangement. Compare with spiral oogonium of Chara (Charce elastica), Fig. 10. 


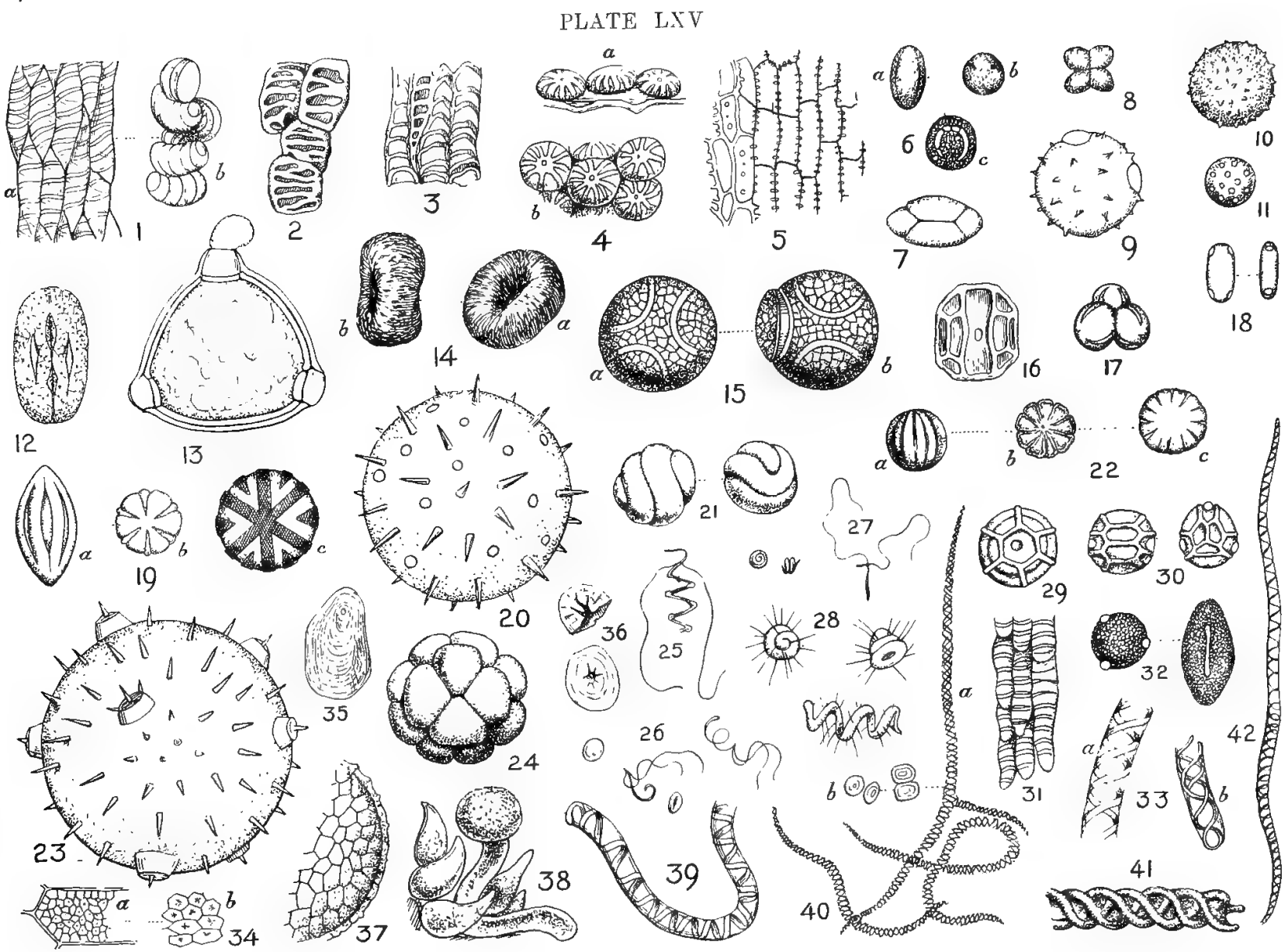

PLATE LXT (continued)

FIg. 22,-Spherical pollen of Sherardia urrensis. a, Side view; $b$, end view; $c$, as moistened hy water. Shows radiating, symmetrical arrangements of parts.

Fig. 23.- Spherical pollen of Cucurbita Prun as seen in water. Silows conical spines and pores.

FIG. 24.--Compond pollen of Acacia lax, with prarts symnetrically grouped.

Fia. 25.- Spermatozoid from the antheridium of Chara fragilis. Shows spiral arrangument. The spermatozoids are capable of independent movements.

FIG. 26.-S Siral spermatozoids from the antheridiun of P'olytrichum commune.

FIG. 27.-Spermatozoids from the antheridium of Marchantia polymorpha, in the coiled and uncoiled state.

FIG. 28.-Beantiful spiral spermatozoids from the intheridium of a fern ('rymnogramma), in the coiled and uncoiled condition.

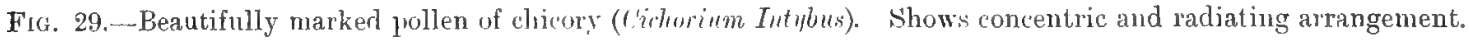

Fig. 30.- Reticulated pollen of Sonchus palustris (side and end views).

Fig. 31.- Spiral fibrous cells of the sporangium of Marhantire polymoryha

FI(x. 32.-Granulated pollen with pores of Statice linifolia (end and side views).

FIG. 33.-Doulle spiral fragments of elater of Marchantia polymor ha, middle (1), and end portions (b).

FIG. 34.- $a$, Portion of a section of a cell of rice, exhibiting very minute starch grains tirmly compacted as in maize; $b$, portion of the same magnified. Reveal hexagonal forms as in liver cells, pignent cells, epithelium cells, \&c.

FIG. 35.--Grain of potato starch showing concentric and radiating arrangement around hilum.

Fig. 36.-.-Statch grains from haricot-bean. Display concentric arrangements.

FIG. 37.--Portion of pullen of Ruellia formosa showing hexagonal arrangement of parts. Compare with Fig. 34.

FIG. 38.--Pollen grain with tube upon the stigmatic papillæe from Lathræa squamaria.

FIG. 39.--Elater of Frullania rlilatata revealing single spiral formation.

FrG. 40.-Elaters (a), and spores (b), of Trichir. The elaters display complicated triple spiral arrangements.

FItx. 41.-Fragment of same elaters greatly maknified showing the remarkable triple spiral arraugement more clearly.

FIG. 42.-Elater of Marchantic polymorphe provided with a beantiful single open spiral. 


\section{PLATE LXVI}
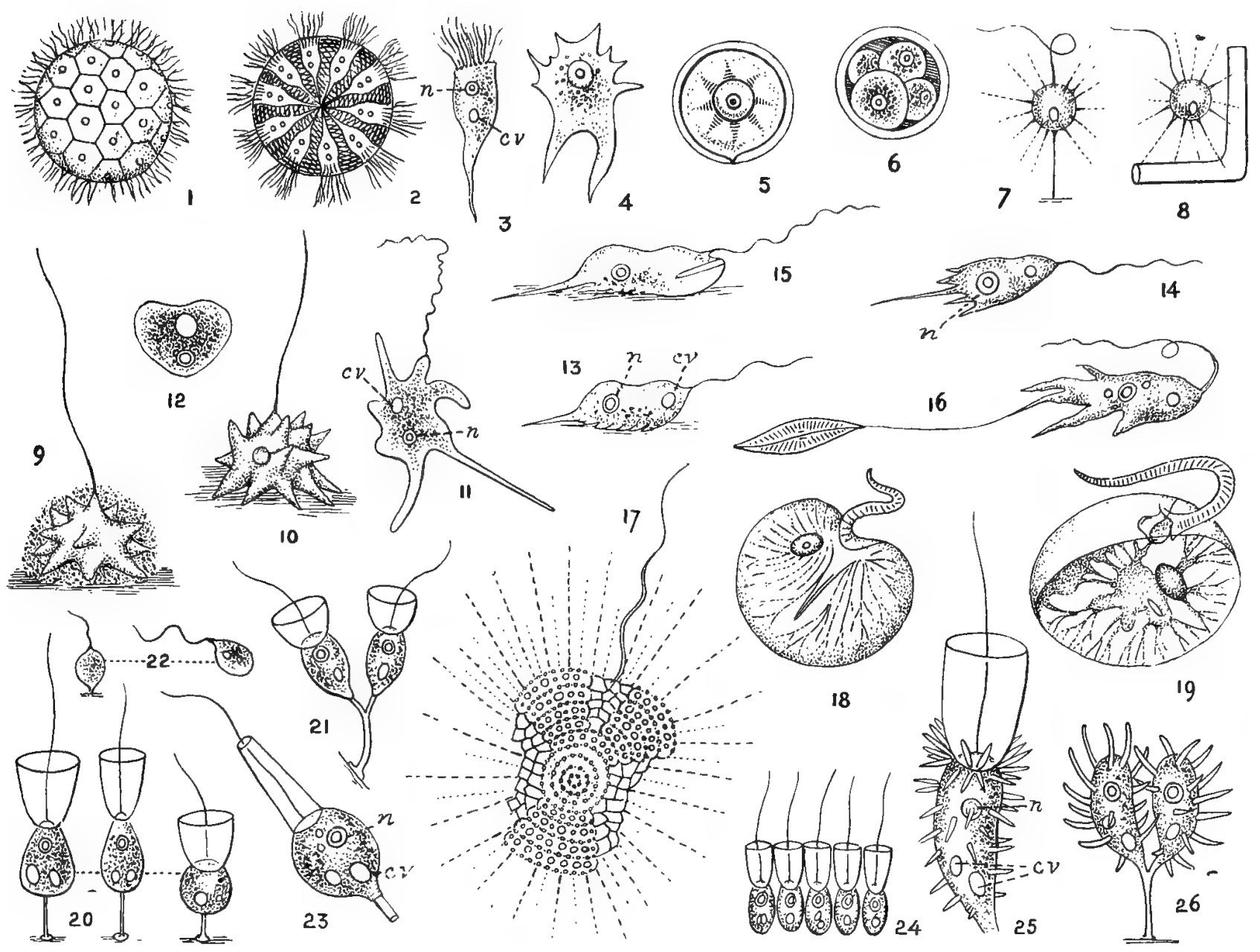

10
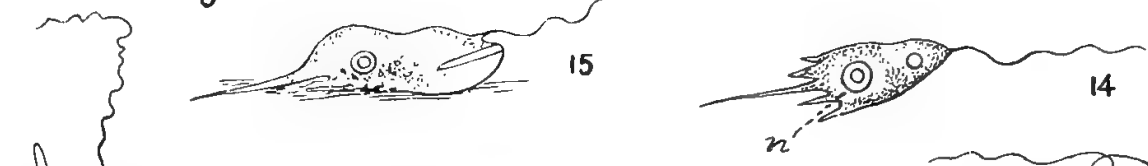

16
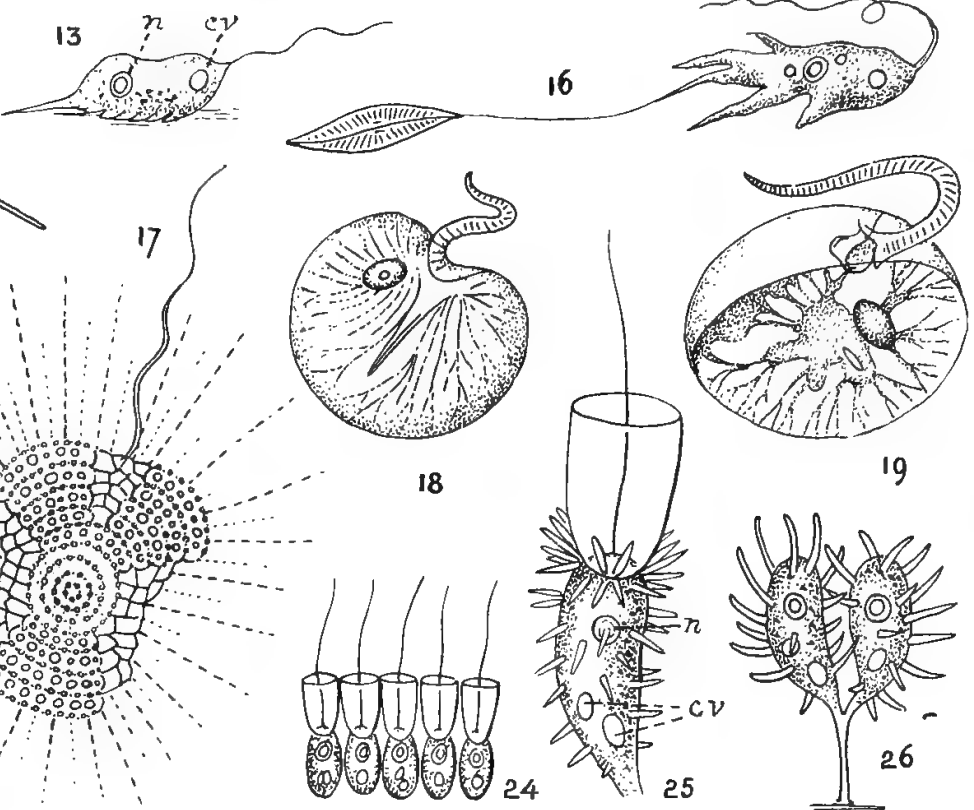

18
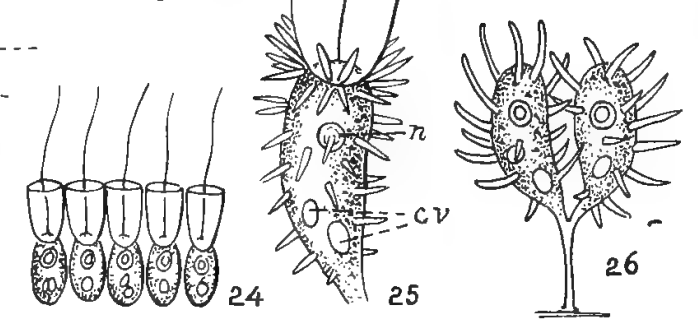

PLATE LXVI

This plate deals with Zooids; with symmetry and asymmetry; with radiating and concentric arrangements; with centripetal and centrifugal movements; with amoeboid forms as the result of retrogression; with growth, force, \&c.

FIG. 1.-Spheroidal colony-stock of adult zooids (Hagospharer planult), $\times 240$ (Haeckel). This figttre presents the general appearance of hexagonal liver cell, pigment cells, epithelial cells, honeycomb cells, \&e.

FIG. 2.-Ideal section of same showing symmetrical cluster of zooids, resembling a flower in appearance.

FIG. 3.-A single isolated zooid from same, composerl of bell-shaped body, with cilia, mucleus $(n)$, and contractile resicle $(c v)$. The cilia are important alike for movement and fur securing food.

FIG. 4.-A similar zooid having assumed an amobihoid phase (Haeckel). Shows retrogression and reversion of zooid to a primitive, simple condition for reproductive purposes analogons to the fusion of the simple male and female elements (ova and spermatozoids) in the higher animals.

Figs. 5 and 6.-Encysted zooids (Haeckel). Fig. 5 slows radiating and concentric arrangements, Fig. 6 has divided by segmentation into four spheroidal sporular bodies. The encysting and clisintegrating or lireaking-up processes in zooids unark important reproductive changes similar to what occur in insects (pupa state) and the segmentation of the volk in vertebrates.

FIG. T.-Zooid (Actinomonas pusilla) attached by single stalk-like filament, $\times 800$ (W. S. Kent).

Frg. 8.-Zooid (Actinomones pusilla) attached by a number of ray-like pseudopodia. Figs. 7 and 8 illustrate the principle of radiation in rudimentary animals. The power possessed by zooids of extruding and withdrawing their sarcode by centrifugal and centripetal movements, as witnessed in psendoporlia, furnishes the key to muscular and other movements in the higher animals. The movements in question are fundamental. They are direct, pushing, or elongating, and pulling or shortening movements. They imply no increase or diminution in bulk, and cannot be referred to elasticity. Botl movements are vital in their nature.

FIG. 9.-Animalcule (Rhizomonas verrucosa) enclosed within granular gelatinous sheath, $\times 750$ (W. S. Kent).

FIG, 10.-Animalcule (Rhisomonas verrucosa) devoid of such sheath. 
PLATE LXVI (continued)

FIG. 11. Animalcule (Polostona filiyerum) with Hagelliferous pseudopodia extended. $w$, Nuclens ; $c v$, contractile vesicle, $\times 250$ (C'lap. and Lach.).

FIG. 12.-Animaleule (Podestomu filigerum) with pseudopodia retracted. Figs. 11 and 12 illustrate the remarkaljle power possessed hy animalcules of voluntarily changing shape in the absence of nerve, nuscle, or other structure. It will be observed that the extended pseudopodia seen in Fig. 12 are conical at their roots and are not produced by constriction or contraction. They are due to a vital extrusion or pushing out of the living sarcode from within (centrifugal movement), : ind ('annot be referred to contraction in any form. The appearance presented by the pseudopodia when drawn in or retracted, as seen in Fig. 12, is due to a vital pulling together of the sarcode (centripetal movement.) Elasticity plays quite an unimportant part in the pseudopodic movements. The centrifugal and centripetal movements here referred to occur in muscle and in all living substances capable of changing shape. They are inherent and fundamental, and are the harbingers of the rhythmic movements by which the respiration and circulation are carried on in the ligher animals.

FLG. 13. - Normal animalcule (keptomonas couduta) ingesting foud by peripheral extension of its body-sarcode. ", Nucleus: c', contractile vesicle, $\times 800$ (W. S. Kent). 'The life of the zooid is devoted almost exclusively to feeding; a rematk which applies to most of the lower animals.

FIG. 14.-Dorsal view of same with short jseurlopodid extensions.

FIt. 15. - Profile view of same.

FIG. 16.-Animalcule (Mustigamobu simplen') with frustule of diatom attached ly sarcode thread, $\times 800$ (II. S. Kent). The sarcode, which can be extruded and withdrawl at pleasure, can be employed either for seizing or ejecting food.

FIG. 17.-Euchitoni Virchowii, $\times 3$ \% $($ Haeckel). Illustrates concentric and radiating arrangements in rudimentary animal forms similar to those found in crystals, scales of fishes, shells, bones, the transverse sections of plants and trees, and different parts of the higher plints and animals. These concentric and radiating arrangements practically represent lines of force and growtl.

FI(t. 18.--Adult animalcule (Noctiluca), dorsal view (Huxley). Shows median groove, stylate rod, and tooth-like process.

Fig. 19. - Adult animalcule (Noitiluca), ventral view. Shows oral cavity with tooth-like process, and contained cilium.

FIt. 20.-Typical zooils, $\times 1200$. These zooids are fixed, and supply themselves with food by the aid of their flagella, which, swirling about, prodnce currents which canse the food particles to enter the cup-shaped collar which forms the ulper part of the body.

FIG. 21. - Colony of two zooids (Codosing furertu) on one pedicle, $\times 1200$ (W. S. Kent).

FIG, 22.-Free swimning and attached condition of monadiform germ (Monosifu globosce); the collar and pedicle being not yet developed, $\times 1200$ (W. S. Kent).

FIs. 23.-A single zooid or animalcule (Codosiga allioisls), with its body spherically, and its collar conjeally contracted, $x 800$ (W. S. Kent). Shows centripetal, and, by implication, centrifugal movements in the body and collar of the \%ooid. The contractile vesicles of the body $(c v)$ execute similar movements. In the zooid three sets of centripetal and centrifugal movements occur ; the one set in the collar, a second in the body, and a third in the contractile vesicles. The voluntary and involuntary movements (fundamentally rhythmic in (haracter) as witnessed in the higher animals are here clearly foreshadowed. $n$, Nucleus or endoplast.

F1li. 24.-A free-floating colony-stock of tive zooids (Desmerella moniliformis) united laterally, $\times 10 n 0$ (W. S. Kent).

Flu. 25.-Zooid sending out slender pseudopodic processes; collar and Hagellum extended. $n, \mathbb{N} u c l e u s ; c r$, contractile vesicle, $\times 2000$.

H'IG. 26. - Two zooids assuming an amueboid condition; collars and Hagella retractecl. Both are sending out psendopodic processes, $\times 2000$ (W. S. Kent). Figs. 25 and 26 illustrate the power possessed by zuoids of changing shape centrifugally and centripetally. This power of changing shape, as explained, is a fundamental endowment in rudimentary animals, and in the tissues (especially the muscular tissues) of the higher animals.

\section{PLATE LIVII}

This plate illustrates the extraordinary powers possessed by zooids and monads of voluntarily changing shape by centrifugal and centripetal movements, and of reverting to simpler amoeboid conditions prior to reproducing themselves.

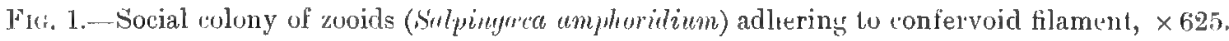

Frc, 2,- One of the same more highly magnified with collar expanded, $\times 1250$. Shows nucleus and contractile vesicles.

Flt. 3.--Another of same with collar contracted within lorica supported on short pedicle. Figs. 2 and 3 afford excellent examples of the power possessed by zooids of opening and closing their collars by centrifugal and centripetal movements.

Fir: 4.-Another of same encysted within lorica, showing retrogression to amcebic primitive condition.

Fic. i.-Another of same with collar retracted, flagellum protruding and thickened at base, body sarcode projecting and lobose. Show how every portion of the sarcode can change shape.

F11, 6.--Anotlier of same with filamentous psendopodia proceeding from top of lorica.

Fiv. 7.-A Another of same where the body sarcode has assumed a loranched, pinnatified appearance (J. Clark).

FI(: 8. - Zooid with lorica flattured below and imperfect collar (Bütschli).

Fir. 9.-Znoid (Salpingeca marina) sending out ray-like psendopodia; the collar and flagellum retracted.

Firis. 10 and 11.- Sarcode ejected from body of zooid assuming stellate form. These figures afford good examples of the power possessed by sarcode of extruding its substance in wedge-shaped masses as apart from contraction and elasticity. 


\section{PLATE IXIII}
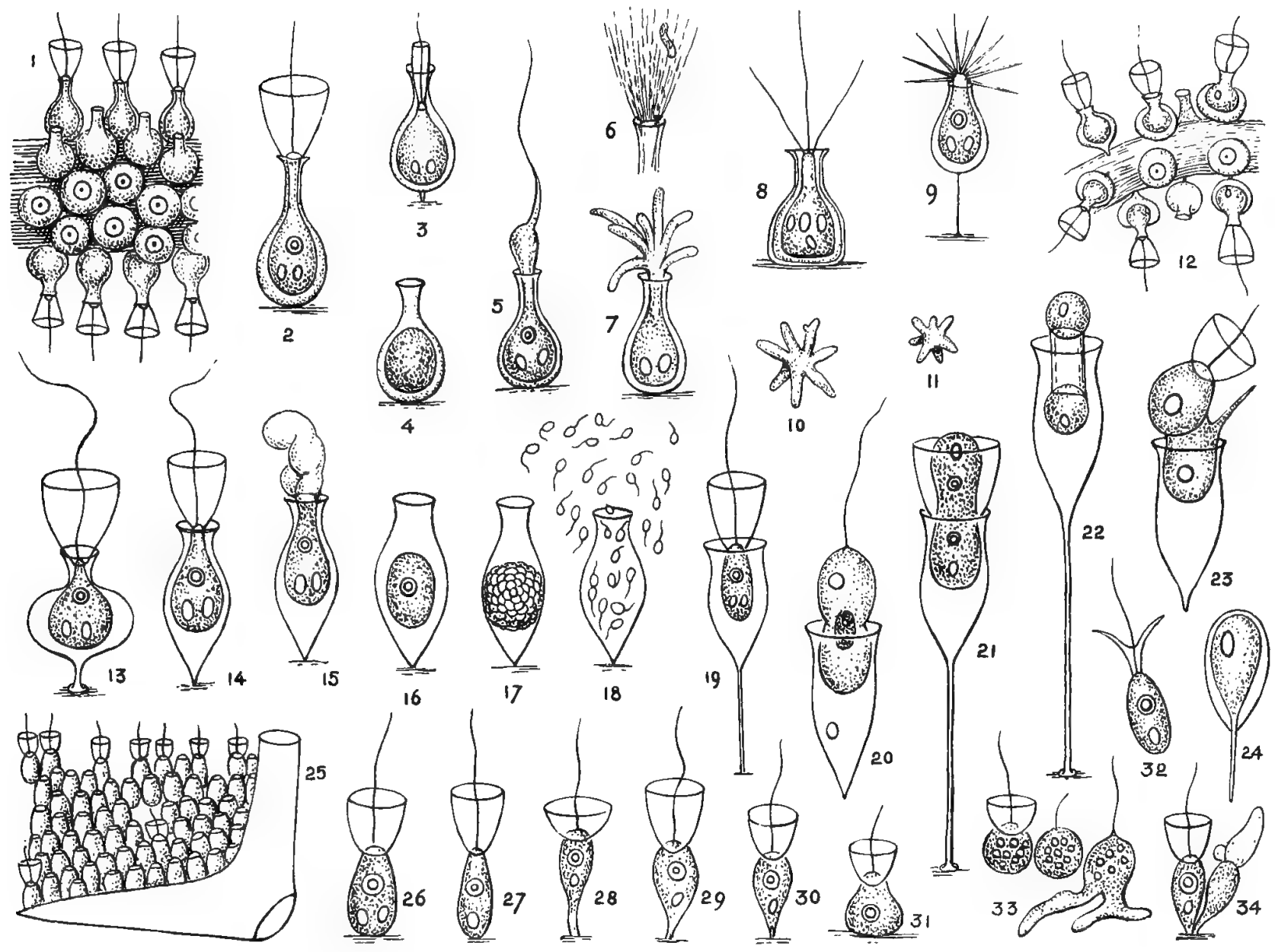

PLATE LXVII (rontimed)

Frr. 12.-A social colony of zooids (Salpinglect napiformis) attached to vegetable fibre, $\times 800$.

FrG. 13.-One of same more highly magnified. This and Figs. 14 and 15 show nucleus and contractile vesicles.

FIti. 14.- Normal fully expanded zooid (Srelpingreca fusiformis), $\times 1500$.

FTtr. 15. Zooid with collar and flagellum retracted assuming amoboid state, $\times 1500$. Zooids have the power of projecting and withdrawing their flagellum and of opening and "losing their collar by centrifugal and centripetal movements (the Author).

FIr. 16.-Encysted zooid, $\times 1500$; the sarcode undergoing change prior to reproduction.

Fir: 17.-Encysted zooid with hody broken up into numerous spore-like substances, $\times 1500$. The changes are analngons to those which occur in the impregnated developing ovum (the Author).

FIIt. 18.-Spore-like substances further developed and escaping from the lorica as monoflagellate germs, $\times 1500$.

FItr. 19.-Zooirl (Salpingceca inquellata) with protecting covering or lorica in its normal fully axtended state, $\times 1250$.

FIfs. 20, 21, 22, and 23.-Zooids in their amoboid form undergoing transverse fission, $x$ 1250. The transverse fission is to be regarded as a form of seginentation (the Author).

FIt, 24.-Attached collarless \%oid secreting its protertive lorica, $\times 1250$.

Fir: 25.--Small calcareons-spicnled sponge ('trontic compresse, Bowerbank) showing pavement arrangement of collared monads with pore apertures, $\times 800$.

FIGs. 26, 27, 28, 29, 30, 31, and 32.-Tarious forms assumed by collared monads of same sponge, $\times 1600$. Show nucleus and contractile vesicles.

FIf. 33.-Isolated monads with bodies filled with ingested carmine particles; one showing collar and flagellum, another Hagellum only, a third witl flagellum and body sarcode projected as pseudopodia, $\times 1600$.

FI $($. 34,-Two collared monarls; the one protruding its flagellnm, the other its body sarcode, $\times \mathbf{I 6 0 0}$. 


\section{PLATE LIVIII}

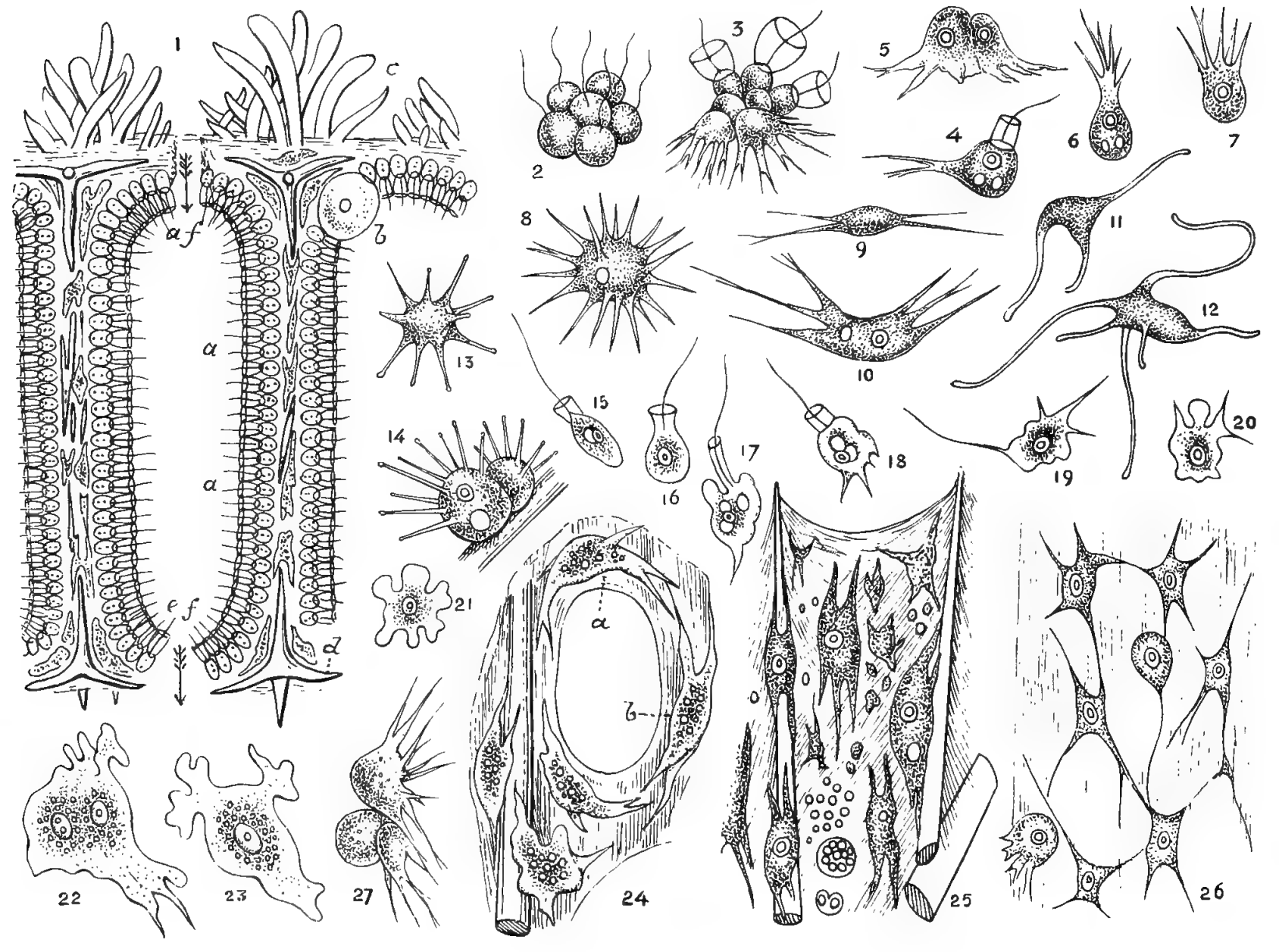

PLATE LXVIII

This plate illustrates the arrangements in a calcareous-spiculed sponge, and the position of the monads and spicula, which latter form a rudimentary skeleton. It also shows the remarkable shapes assumed by monads in their amœboid phases; likewise cytoblasts of various kinds and at different stages.

FIG. 1.-Calcareous-spiculed sponge (Grantia compressa, Bowerbank) shows one entire, and portion of a second monad-lined chamber, $x$ 600. af, Afferent canal for reception of external water; ef, efferent canal leading to central cluacal chamber; a, a, collar-bearing monads; $b$, swarm gemmule in earlier amceboid non-segmented phase of develojment; $c$, external defensive spicula; $d$, internally projecting tri-radiate spicula.

FIGs. 2, 3, 4, 5, 6, 7, and 8.-Polymorphic forms assumed by collared monads (Grantia compressa, Bowerhank), $\times$ 800. It will be observed that the pseudupodic processes on which the variety of form depends are in every instance conical-shaped ; the base of the cones being directed towards the body of the monad. This is important as showing that these processes are the result of a pushing outwards of the body sarcode by a centrifugal action. They are not due to contraction or elasticity of any kind. The same thing hapuens in all amobiform movements. In every case the animal alternately pushes or projects its sarcode out of its body by a centrifugal movement, and retracts or withdraws it into its body by a centripetal movement. Amobiform animals can push out and elongate and retract and shorten portions of their body sarcode at pleasure. Voluntary and iuvoluntary inuscles possess similar properties. But for the double power here clained for living substances and living issues, morements in plants and animals would be improssible (the Author).

FIas. 9, 10, 11, 12, 13, and 14.-Isolated amoboid phises of collared monads of Bread-crumb Sponge (Halichonilria panicea), $\times$ 1000. These monads present similar ajpearances to those seen at Figs. 2 to 8 inclusive. The same remarks apply to all (the Anthor).

FIss. 15, 16, 17, 18, 19, 20, and 21.-Metamorphosed collared monads from calcareous-spiculed sponge (Ascetta primordialis), $\times 700$ (Haeckel). Figs. 17, 18, 19, and 20 display characteristic conical-shaped pseudopodic processes in all respects analogous to those already described (the Author).

Ficis. 22 and 23.-Amceboid zooids from the cytoblastema; the former (Fig. 22) showing two nuclei which may wither represent the conjugation of two zooils or a zooid about to divide hy fission, $\times 700$ (Haeckel).

FTr. 24.-Portions of transparent cytoblastema from Lencosolenia coriacea surrounding a poral aperture and containing amobiform cytrublasts $(a, b)$ enclosing ingested carmine particles, $\times 800$, 


\section{ADVANCE IN LOWER PLANT AND ANIMAL FORMS}

PLATE LXIX

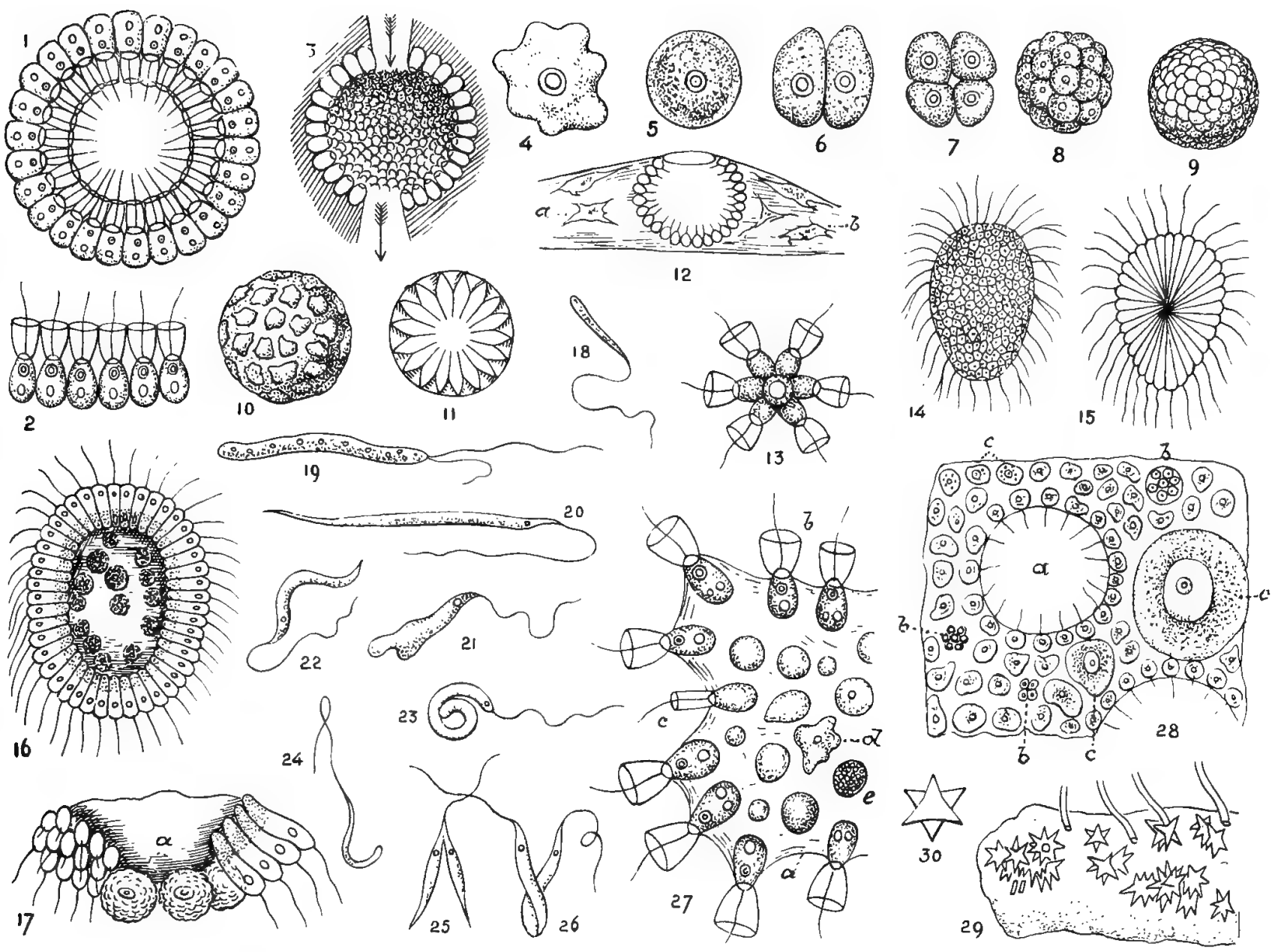

PLATE LXVIII (continued)

FIG. 25.-Portions of cytoblastema of Halichondria panicea containing amobiform cytoblasts in various stages of development; the smaller ones originating from sporular bodies, $\times 1500$.

FIG. 26.-Associated cytoblasts from Aplysilla sulfurea with alternate and interconnecting psendopodia, $\times 400$ (F. E. Schulze). These cytoblasts remarkably resemble in their general appearance the multipolar ganglia found in the spinal cord of mammals (the Author).

FIfr, 27.-A group of metamorphosed collared monads presenting an amoboid aspect.

\section{PLATE LXIX}

This plate illustrates symmetric grouping and symmetric forms; also the various phases through which zooids and monads pass during their life histories.

FIG. 1.-Spheroidal monad chamber or "ampullaccons sac" of sponge (Halisarca Dujardini, Johnston) as seen in optical section without intersecting an afferent or efferent aperture, $\times 800$. The introversion of this monad chamber produces a rosette or ordinary swarm gemmule. Compare with Fig. 13. This figure affords a good example of concentric radiating arrangement (the Author),

FiG. 2.-Six collared monads attached laterally from an "ampullaceous sac" of the same sponge. These when isolated greatly resemble the moniliform colonies of the collared monad (Desmarella moniliformis) seen it Fig. 24, $\times 1000$.

FIG. 3. - "Ampullaceous sac" of same type seen in optical section and intersecting (vide arrows) an afferent and efferent aperture.

FIGs. 4, 5, 6, 7, 8, 9, 10, 11, and 12.-Progressive phases of development of an "ampullaceous sac" by segmentation from a primitive amceboid body, $\times 400$. At Fig. 10 the segmented products resemble amoeboid corpuscles and have no flagellate appendares. At Fig. 12 (profile view of matured ampnllaceous sac) the same elements have developed internally projecting flagella; the characteristic collars being absent. Enclosed cytoblasts are seen at $a, b$ of Fig. 12. Fig. 11 affords a beantiful example of radiating arrangement similar to what occurs in crystals and flowers. The developnent here figured resembles in its main features that witnessed in the segmentation of the impregnated ovum and the formation of the germinal membranes in the higher animals. It begins by a division of the nucleus and the sarcode surrounding it (Dalton).

VOL. I. 


\section{PLATE LXIX (continued)}

FIr. 13.-Spheroidal cell combinations or rosette gemmules of typical collared monads, $\times 800$. The symmetry displayed in this Figure and in Figs. 1 and 11 is very remarkable. The rosette arrangement of Fig. 11 is especially striking (the Author).

FIis. 14 and 15. - Simple planuloid swarm gemmules or ciliated larve of Grantia compressa (Bowerbank) in various stages of development seen superficially and in longitudinal optical section. At Fig. 15 the larva consist of similar closely opposed conical uniflagellate elements, $\times 350$. Fig. 15 reveals a well-marked radiating arrangement. In both figures the cilia form a striking feature. These are useful for causing currents either for securing food or for propulsion.

FIG. 16.-Planuloid swarm gemmule with internally contained cell spherules as seen in optical section in Ascetta primordialis (Oscar Sehmidt).

Frg. 17.-Portion of lateral wall of swarm gemmule of same sponge showing cell spherules (a), derived from the uniflagellate elements which assume an amoboid form and creep into the common central cavity seen in Fig. 16 (Oscar Schmidt).

FIGs. 18, 19, 20, 21, 22, 23, 24, 25, and 26.-Polymorphic phases of adult monads (Herpetomonas muscæ-domestic 2 ), $\times 650$. At Figs. 25 and 26 the monads are dividing by longitudinal fission. The shapes assumed are remarkable and various.

FIG. 27. - Social colony of collared monads (Proterospongia haeckeli) inhabiting a common gelatinous matrix resembling the cytoblastema of an ordinary sponge, $\times 800 . a$, Hyaline gelatinous matrix; $b$, normal zooids with collars expanded; $c$, normal zooids with collars contracted; $d$, zooids with collars and flagella withdrawn and presenting an amcobiform appearance as in the cytoblasts of a sponge body; these divide loy transverse fission; $e$, spore mass (W. S. Kent). Shows centrifugal and centripetal action of collar of zooid, the amceboid and sporular phases of zooid, and its power of changing shape in any direction (the Autlor).

FIr. 28.-Portion of inner wall or lining of sponge (Ascetta primordialis), $\times 350$. a, Pore aperture surrounded by flagelliferous monads; $b, b, b$, spore groups produced from ordinary collared monads believed by Haeckel to be sperm cells; $c$, $c$, large amcboid bodies, the result of metamorphosis and coalescence of collared cells, which later develop into ciliated swarm gemmules (Haeckel).

FIG. 29.--Fragment of peridium or indurated outer wall of sporangium of Didymium farinaceum (Fries), with substallate calcareous spicule, $\times 390$.

FIr. 30.-A spicule of same further enlarged. These spicules present well-marked crystallic shapes.

\section{PLATE LXX}

This plate illustrates how the lower animal forms divide by longitudinal and transverse fission; how they come together and coalesce; how they retrogress and assume the amoboid condition prior to reproducing themselves; how they form sporocysts with spores-the spores being scattered when the sporocysts burst; how, in many instances, they assume plant shapes; how certain of them present a spiral appearance, and how their contractile vesicles open and close as in the diastole and systole of the heart, thus identifying rhythmic movements with the very beginnings of life.

Ficis. 1 and 2.-Progressive plases of transverse fission of adult monad (Cercomonas typica), $\times 1750$.

FI(ss. 3 and 4.-Examples of transverse fission in adult monad (Spumella guttula, Ehr.), $\times 600$. The fusion of smaller with larger monads is seen at Figs. 6 and 7 . The division, segmentation, and blending of rudimentary animal forms have an obvious bearing on
development, differentiation, and division of labour in the higher animals (the Author).

FIGs. 5, 6, and 7. Free-swimning monads (Spumella guttula, Ehr.). u, A perture; $n$, nucleus; cv, contractile vesicle. The monads are coalescing or conjugating in Figs. 6 and 7.

FIix. 8.-Shows a phase of longitudinal fission.

Fra. 9.-An encysted group showing stalked (a) and stalkless (b) cyst, and large cyst with two stalks (c); the latter the result of the conjugation of two zooids, $\times 1500$.

Frr. 10.-Normal attached monad (Śpumella guttula, Ehr.), $\times 600$.

Fitr. 11.- Shows lougitudinal fission of a monarl.

Frss. 12 and 13.-Illustrate the alternating diastole and systole or opening and closing of the two contractile vesicles of a freeswimming monad (Physomonas socialis), $\times 1000$ (W. S. Kent). Similar opening and closing rhythmic movements are witnessed in plants. They have great significance as indicating the inherent fundamental nature of rhythms (the Author).

FIGs. 14 and 15.--Normal adult monads (Heteromita lens, Müll.), $\times 800$. Show nucleus and contractile vesicle.

Fics. 16 and 17.-Irregular-shaped amobiform conditions of same monads.

FI'x. 18 and 19. - Two of same monads about to coalesce.

Fik. 20.-Conjugation or mion proceediug and resulting in a spheroidal sporocyst as seen in Figs. 21, 22, and 23 (same monads). The llending and fusing of nonads into each other and the production of new individuals by spores is analogous to reproduction in
its several phases in certain plants (the Author).

FIr. 24. - Two sporocysts containing eight or sixteen macrospores (same monads).

FIr. 25. -One sporocyst containing four macrospores (same monads).

FIG. 26. - The sporocyst lurst and liberating hiflagellate germs same as in Figs. 14 and 15 , but smaller, $\times 1000$.

FIis. 27, 28, 29, and 30.- Minute uniflagellate monadiform germs developed from macrospores, $\times 2500$.

FIr. 31.-Colony stock or zoodendrium (Cephalothamnium cuneatum), <1250. Presents a very decided plant-like appearance. 


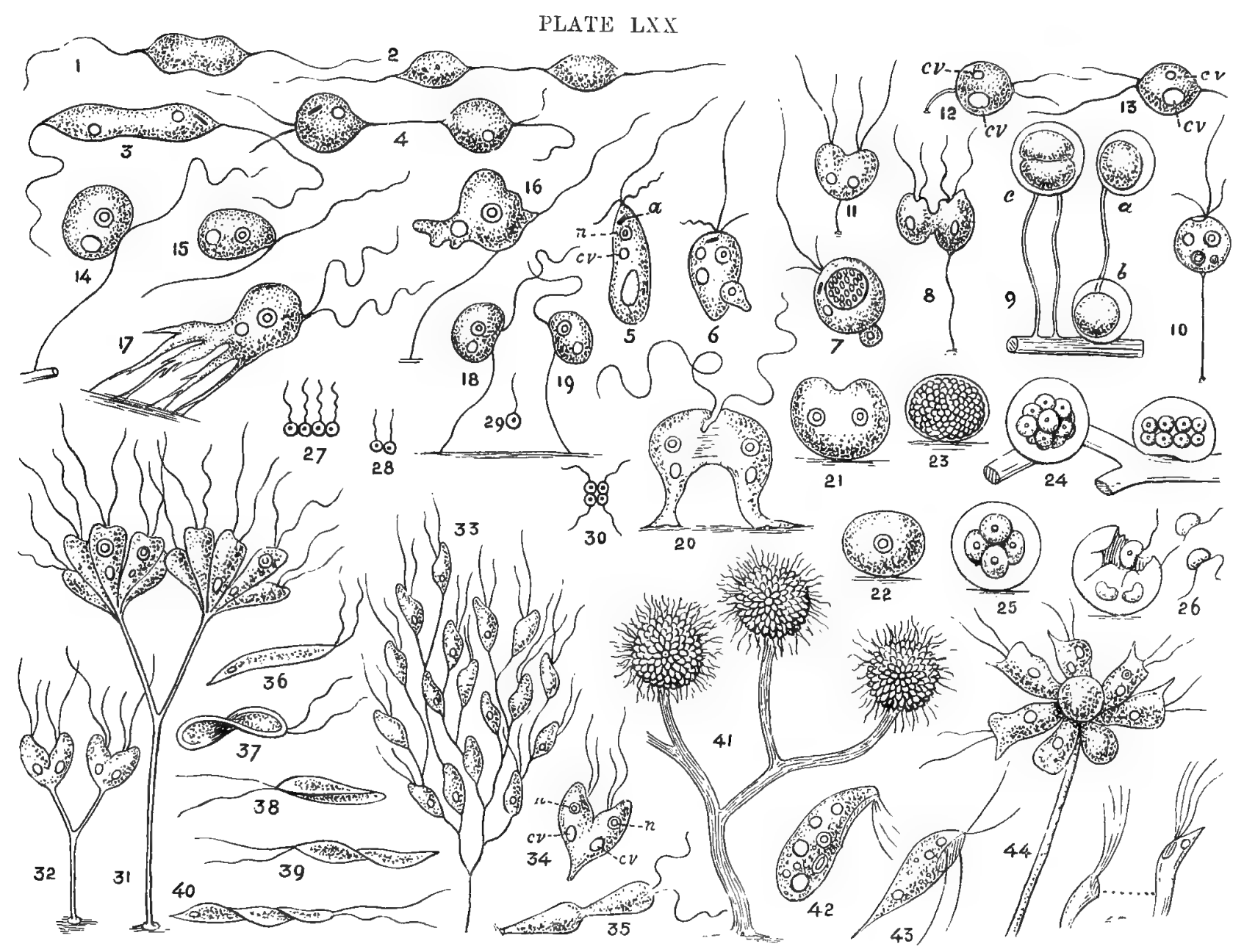

PLATE LXX (continued)

FIG. 32.-Colony of two monads (Dendromonas virgaria, Weisse) dividing by longitudinal fission.

FIG. 33._Adult colony of monads (Cladonema lasmm), $\times 1000$. Reseml,les small shrub in its general asplect.

FIG. 34.-A monad dividing by longitudinal fission, $\times 1500$. $n$, Nurleus; $c v$, contracting vesicle.

FIG. 35.-A monad dividing by transverse fission, $\times 1500$. These cleavages are at the root of development and differentiation (the Author).

FIGS. 36, 37, 38, 39, and 40.-Free-swimming monads (Spiromonces angustata) with their bodies in three cases variously twisted, $\times 1500$. The spiral movements of free-swimming monads mark a new and important departure (the Author).

FIG. 41.-An elect branching colony stock (Anthophysa vegetans, Miill.) with three terminal monad clusters, $\times 400$. The branching appearance presented by this figure resembles that seen at Figs. 31, 32, and 33.

FIGs. 42 and 43.-Two specimens of Tetramitus descissus, $\times 1500$.

Fi(t. 44.- Head of colony stock of zooids (Anthophysa socialis), $\times 1500$ (Buitschli). Presents flower-like rosette.

FIG. 45.-Young animalcules (Tetramitus rostratus), $\times 650$.

\section{PLATE LXXI}

This plate illustrates the power possessed by animalcules of changing shape at pleasure; of dividing longitudinally and transversely; and of assuming spiral shapes structurally and when moving. It also shows advance in organisation; the flagella being increased in number, there being a superior vibratile set employed for catching food, and an inferior set used for fixing or anchoring purposes; hair-like setæe and cilia also make their appearance. Advance in structure and greater functional range proceed pari passu.

Fic's. 1 to 11 inclusive.-Animaleule (Trepomonas agilis) as seen from various points with body straight and twisted, $x 650$. At Figs. 7 and 8 the animalcule is dividing by longitudinal fission. At Fig. 11 the nucleus ( $n$ ), and contractile vesicle (cv), are shown: the arrows in this figure indicate the direction of the circulation of the endoplasm (Stein, Perty, and Bitschli). 'The figures illustrate 
PLATE LXXI

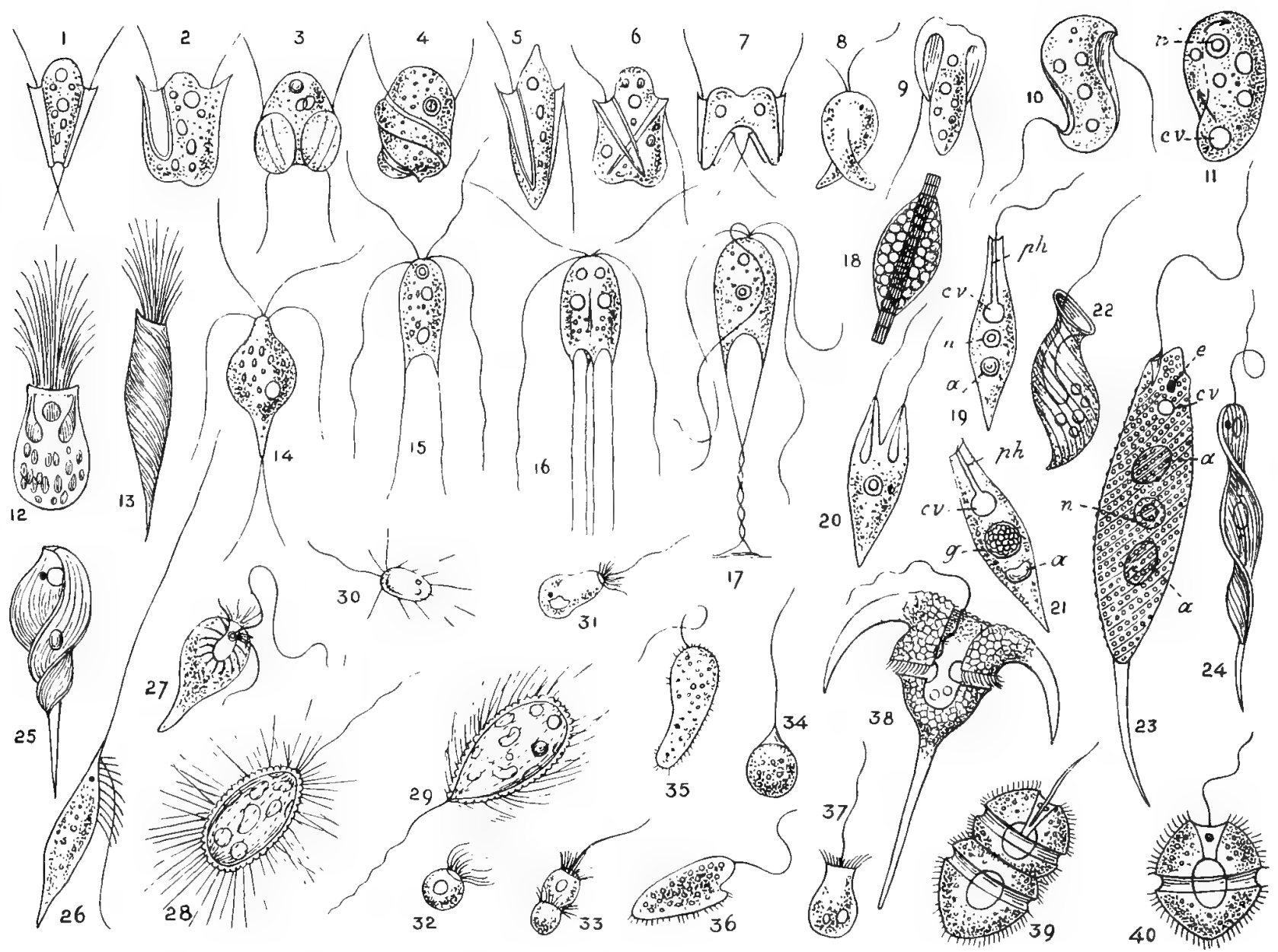

PLATE LXXI (continued)

the power possessed by animalcules of changing slape and of circulating endoplasu. The circulation of living matter in a given direction by a zooid is a new factor and is to be regarded as a fundamental endowment (the Author).

FIGs. 12 and 13.--Lophomonas striala anil Lophomonas blattarum with brush-like flagella-fascicles, $\times 800$ (Buitschli). Fig. 13 shows beautiful spiral formation.

FIG. 14.- Hexamita rostrata with four anterior and two posterior flagella, $\times 650$.

FIGs. 15 and 16.-Aninalcule (Hexamita inflata) dividing by longitudinal fission, $\times 650$.

FIG. 17. - Similar animalcnle gyrating upon its attached posterior flagella and producing a beatiful spiral. The spiral is reversed at intervals, $\times 1000$ (W. S. Kent).

FIs. 18.-Animalcule (Callodictyon triciliutum) enveloping a vegetallle fibre, $\times 400$ (Carter).

FIG. 19.-Animalcule (Atructonema teres). $p h$, Pharynx and vibratile flagellum; rv, contractile vesicle ; n, nucleus ; $a$, amylaceous corpuscles, $\times 640$ (Stein). The direct comnection of the pharyux with the contractile vesicle shown in this figure proclains the advent of a stomach and heart in the higher animals, each endowed with independent rhythmic movements (the Author).

FI6. 20.-The same dividing by longitudinal fission.

FIG. 21.-The same with Hagellum absent and endoplast enlarged and broken up into germiual elements $(g)$. $p h$, Pharynx; $c v$, contractile vesicle; $a$, amylaceous corpuscle.

FIt. 22.-Animalcule (Phialoneme cyclostomum) with spirally ribbed cuticle, $\times 500$ (Ntein). Illustrates spiral structures in low animal forms (the Author).

FIt. 23. --Normal extended animalcule (Euglena spirogyru). aa, Amylaceous corpuscles; "1 nucleus; cv, contractile vesicle; e, eyelike pigment upot (W. S. Keut). Shows oblique spiral markings un surface of hody (the Author).

FIr. 24.-Animalcule (Euglena oxyuris), $\times 200$ (Stein). Shows beautiful spiral arrangement well adapted for swinming. Spiral structures and spiral movements appear very low down in the scale of being (the Author).

FIa, 25. - Spirally twisted non-flagellate animalcule (Ihacus longicaudus), $\times 300$ (Stein). 


\section{PLATE LXXI (continued)}

FIGS. 26 and 27.-Extended and contracted phases of Heteromastix proteiformis, $\times 500$ (J. Clark). These figures show in a striking manner the centrifugal and centripetal power possessed loy animalcules of changing shape and moving all their parts voluntarily and at discretion (the Author).

FIGs. 28 and 29. -Mallomonas plosslii, $\times 800$. Show radiating arjangement of fine hair-like setæe on surface of body.

Fig. 30.-Mallomonas fresenii with long hair-like setæ, $\times 350$.

FIGS. 31, 32, and 33.-Asthmatos cilianis, $\times 400$ (Salisbury). This animalcule presents a flagellum and brush-like fascicle of long flexible cilia. It has a plastic body and is capable of changing shape by centripetal and centrifigal movements. At Fig. 33 the body is dividing transversely to produce two individuals (the Author).

FIG. 34.-Trichonema hirsuta, $\times 400$ (Fromentel).

Figs. 35 and 36.-Mitophora dubia, $\times 350$ (Perty). Body provided with flagellum and peripheral fringe of cilia. The latter can produce food currents or act as organs of locomotion.

FIG. 37.-Stephanomonas locellus, $\times 400$ (Fromentel).

Frg. 38.-Ceratium tripos, Müll. Normal short-armed type, $\times 250$.

Frgs. 39 and 40.-Normal animalcule (Melodinium uberrimum) dividing by transverse fission, $\times 300$ (Allman).

\section{PLATE LXXII}

This plate illustrates transverse and annular markings and divisions; radiating, concentric, and spiral arrangements, and the plant-like distribution of colonies of zooids. Very remarkable and beautiful as regards shape and markings is the lorica or protective covering of zooids. This is, in some respects, as extraordinary as the hexagonal-shaped cell of the comb of the honey-bee. It is produced by a process of secretion and is in no way due to pressure. As a constructive feat it is unique in the animal kingdom both as regards the material, moulding, and ornamentation. The most expert glass-blower cannot rival, far less surpass the extreme minuteness, delicacy, and accuracy of the work. What explanation can be given of the fact that one of the gems of nature's architecture is produced by one of the simplest animals, unless it be that the First Cause works through plant and animal alike from the monad to the man? Similar remarks apply to the marvellous constructions (shells, \&c.) of the Foraminifera, and still lower grades, witnessed in plants.

FIG, 1.-Helmet-shaped empty lorica or protecting covering of animalcule (Codonella galea), showing tesselated structure, resembling hexagonal epithelial, liver, and pigment cells, $\times 200$.

FIG. 2.-Same animalcule extended beyond the orifice of its lorica. n, Nucleus ; cv, contractile vesicle (Haeckel). This animalcule bears a considerable general resemblance to certain jelly fishes (the Author).

FIG. 3.-Empty lorica of Codonella campanella, $\times 200$, shows exquisite symmetry and regular circular markings.

FIG. 4.-Opalina ranarum. Body elliptical and flattened. Shows temporarily assumed parallel disposition of the cilia, $x 100$ (W. Saville Kent).

FIGS. 5 and 7.-Examples of Arcchnidium globosum with tentacles extended (Fig. 5) and retracted (Fig. 7), $n$, Nucleus; cv, contractile vesicle, $\times 1200$. Body nearly spherical, oral cilia thick and tentaculiform, nucleus large and curved. The tentacles when retracted assume a spiral form.

FIG. 6.-Ventral aspect of animalcule (Halterix grandinella), $\times 600$. Body subglobose; springing setæe long, fine, and forming central girdle; the adoral setæ at one point exhibiting spiral involution. Illustrates spiral, concentric, and radiating arrangements (the Author).

FIG. 8.-Abnormal multiple subdivision of animalcule (Didinium nasutum), $\times 200$ (Eberhard). Body barrel-shaped, provided with two wreaths of cilia-anterior and central. Shows transverse segmentation.

FIGs. 9, 10, and 11.-Natatory animalcule (Didinium nasutum) illustrating the seizure and engulfment of a paramecium as delineated by Balbiani, $\times 200$. Body and cilia as in Fig. 8.

FIG. 12.-Didinium nasutum in the act of dividing by transverse fission, $\times 200$ (Balbiani).

FIG. 13.-Adherent zooid (Urocentrum turbo) dividing by transverse fission, $\times 300$ (W. S. Kent).

FIG. 14. -Ailult zooid (Gyrocoris oxyura), $\times 300$ (Tatem). Body bell-shaped, subpyriform; claw-like lail or style; cilia forming posterior marginal fringe very long and fine. Contractile vesicle large.

FIG. 15.-Adult animalcule as figured by Eberhard, $\times 300$. Shows well-marked spiral contour greatly resemlling that of many shells (the Author).

Ficis. 16 and 17.-Lateral and ventral aspects of Trichodina baltica showing denticles of adherent disc as drawn by Quennerstedt, $\times 350$. Body turban-shaped, having ring of suctorial disc toothed on both its outer and inner borders; outer teeth recurved outwards, $\times 350$. Body turban-shaped, having ring of suctorial disc toottred on bothits its on concentric, radiating, and spiral arrangements (Fig. 17).

Figs. 18 and 19.-Adult expanded (Fig. 18) and contracted (Fig. 19) zooids (Zoothamnium curcini), $\times 200 . \quad$ Illustrate the power possessed by zooids of changing shape by centrifugal and centripetal movements (the Author).

FIG. 20.- Extended and contracted zooid (Vorticella cratera) as drawn by D'Udekem, $\times 250$. Body broadly campanulate, inflated, and hemispherical ; peristome bodies flattened. Ciliary disc but slightly elevated; pedicle long and stout and jrovided with spiral 
PLATE LXXII

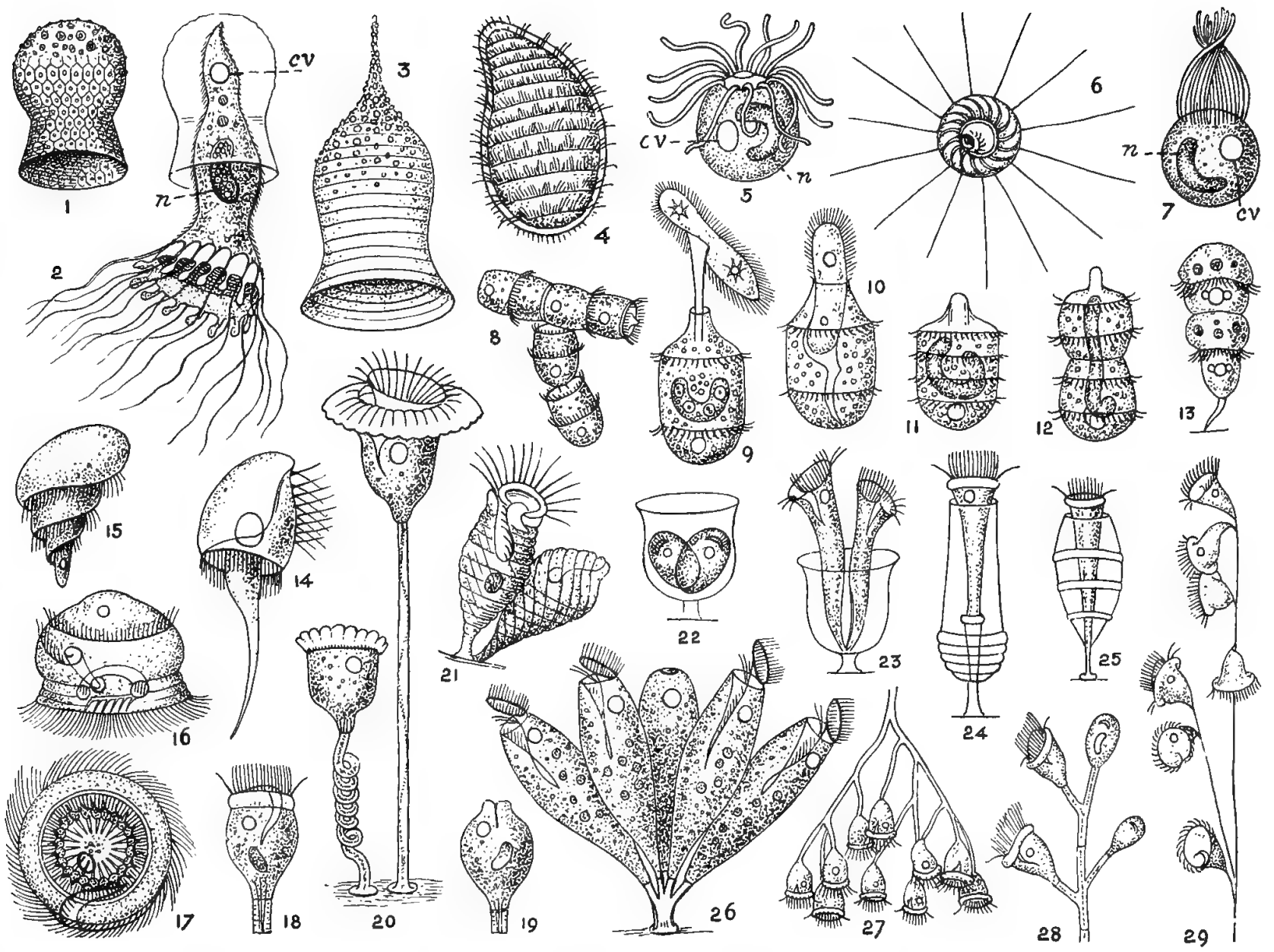

PLATE LXXII (continued)

band which confers spiral movements on it. Illustrates the spiral action of the pedicle and the power of expanding and contracting the body by centrifugal and centripetal movements.

FIG. 21. -Adherent zovid (Scyphidia rugosa) in the expanded and contracted states, $\times 280$ (Dujardin). Body pyriform, attached posterior extremity most attenuate; integument furrowed obliquely and spirally in two directions; pharyngeal cleft prolonged. Shows how spiral markings and structures are affected by centrifugal and centripetal movenents (the Author).

FIG. 22.-Lorica or protecting sheath with two zooids (Cothumia patula) in the contracted condition, $\times 200$ (De Fromentel).

Fig. 23. - Shows the same lorica and the same zooids (Fig. 22) in the extended condition. These figures illustrate in a striking manner the centripetal and centrifugal movements of zooids (the Author).

FIGs, 24 and 25.-Two zooids (Cothurnia cohnii and Cothurnia mpa), $\times 400$ (Cohn and Eichwald), occupying elegantly shaped lorica with aunular transverse markings. At Fig. 24 the lorica is elongate ovate and subcylindrical; the anterior margin being abruptly truncated. At Fig. 25 the lorica is symmetrically ovate and widest in the middle.

FIG. 26.-Colony stock of Opercularia stenostoma, $\times 200$ (D'Udekem). Bodies elongate and pyriform, widest in nilddle and tapering finely towards pedicle, which is small, rigid, and dichotomously divided. Peristome border and ciliary disc very narrow ; the latter bearing an elegant single circlet of cilia. The general appearance is plant-like. The zooids are provided with remarkable ciliated lids resembling those of the trap-door spiders.

FI 1 . 27.-Adult colony stock of zooids (Zoothamnium carcini) in an inverted position, $\times 80$. Illustrates branching distribution of zooids.

FIu. 28. - Terninal branchlet of colony stock of zooids (Carchesium epistylidis) as figured lyy H. E. Forrest, $\times 300 . \quad$ The zooids at the left of the figure are expanded or open; those at the right contracted or closed. Furnishes example of centrifugal and centripetal movements.

Fra, 29. - Termination of branchlet showing unilateral distribution of zooids (Carchesium polypinum), $\times 150 . \quad$ Figs. 28 and 29 jresent a general flower-like appearance. 
PLATE LXXIII

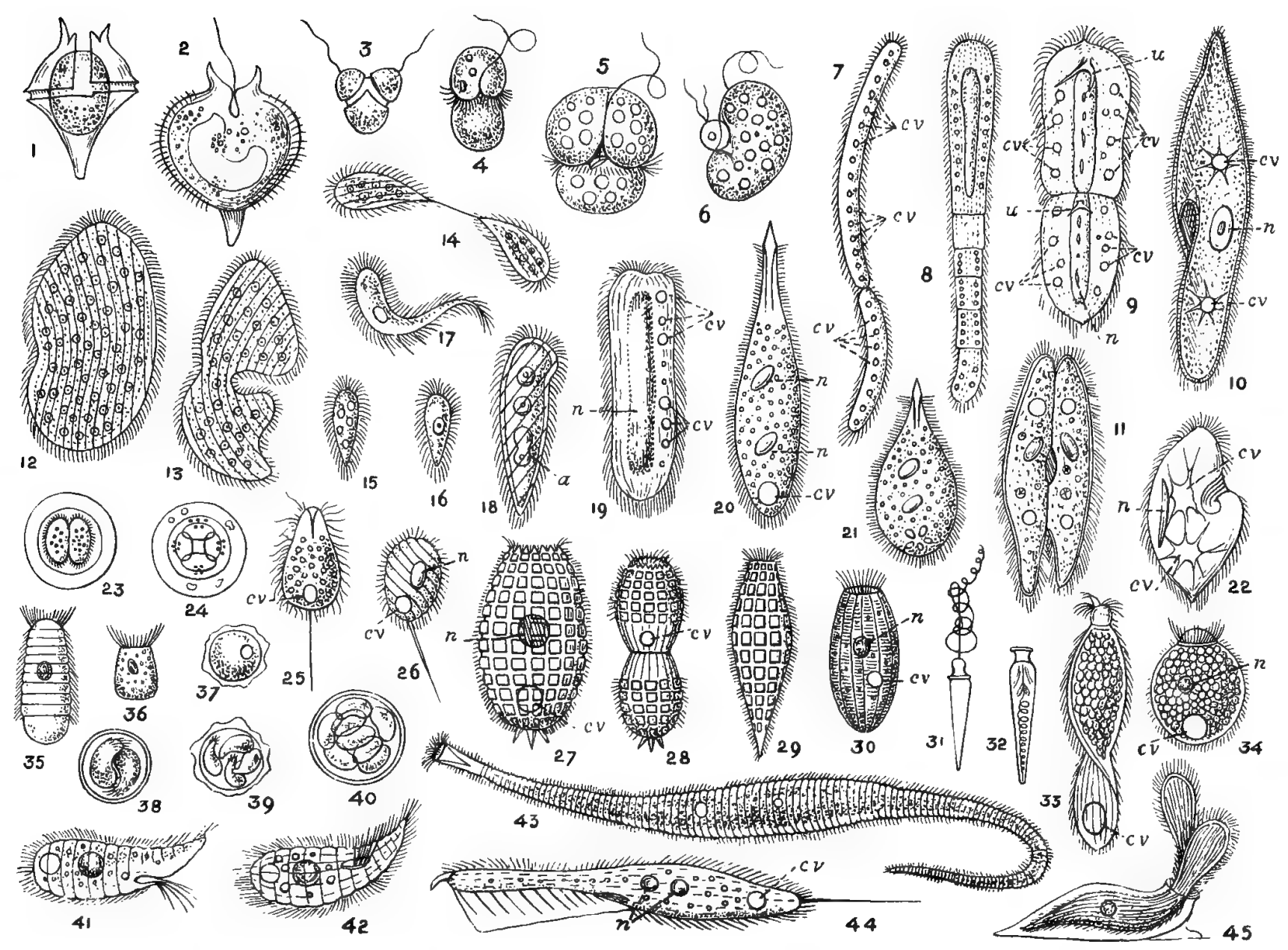

PLA'TE: LXXIII

This plate illustrates the presence of cilia in large numbers in Infusoria. They occur on various parts of the body and have a high significance functionally, as they act as organs of locomotion, produce currents which assist in alimentation, and, in some instances, entangle food. Several of the figures reveal longitudinal and transverse markings (incipient and real segmentation); others indicate spiral structure.

FIG. 1.-Encysted animalcule (Ceratium divergens), $\times 500$.

FrG. 2.-Similar animalcule with carajace cast off.

Firis. 3 and 4.-Animalcule (Gymnodinium lachmanni) dividing ly longitudinal fission, $\times 300$.

FITS. 5 and 6.-Two monads (Gymnodinimm marimum), one of which (Fig. 6) is devouring a small 4 r monad, $\times 600$.

FI $x_{\text {. }}$ 7.-Anoplophrya filum covered with cilia and displaying numerous contractile vesicles $(c r)$, $\times 120$. Shows transverse division of segmentation.

Fic: 8,--Adult animalcule (A noplophrya proliferu), ciliated; affords a good example of transverse division or segmentation, $\times 120$ (Clap. and Lach.).

Fir. 9.-Adult zooid (Hoplitophrya lumbrici), ciliated; dividing by transverse fission. $r$, $c v$, Contractile vesicles; $u$, $u$, horny uncini; $n$, nucleus, $\times 200$ (Stein). Shows trace of internal skeleton.

FIs. 10.-Paramecium aurelia, ciliated ; remarkable for its power's of progression. $n$, Nucleus ; $c v$, cv, contractile vesicles, $\times 200$ (Müiller).

FIG. 11.-Two ciliated zooids conjugating and fusing.

Frg. 12.-Adnlt animalcule (Opalina ranarum), ciliated; showing striation, $\times 100$.

FIfs. 13, 14, 15, 16, 17, and 18.- Show successive phases of division ending in the production of mirute orate zooids with one, two, or more nuclei. At Figs. 15 and 18 are seen phases of zooid re-emerging from the encysted stute; the zooid at Fig. 15 having only one nucleus, that at Fig. 18 having three nuclei, the lower one of which (a) is in the act of subdividing. At Fig. 1' a young zooid with ragged pseudopodium-like lateral extensions is represented (Ernst Zeller and Engelinann).

FIG. 19.-Adult ciliated zooid (Anoplophrya flum), $\times 200 . n$, Nucleus ; $c v, c v$, contractile vesicles (Ray Lankester). 


\section{PLATE LXXIII (continued)}

FIGs. 20 and 21.-Ciliated zooid (Trachelophyllum apiculatum) in the extended and contracted state. $n$, n, Nucleus ; cv, contractile vesicle $\times 200$ (Wrzesniowski). These figures illustrate the power possessed by zooids of changing their shape by centrifugal and centripetal movements; the centrifugal novement elongating the body, the centripetal one shortening it (the Author).

FIG. 22.-Adult ciliated animalcule (Otostoma carteri) showing hand-like nucleus or endoplast $(n$ ), and stellate contractile vesicles $(c v, c v), \times 100$ (Carter).

FIGs, 23 and 24.--Successive phases of suldivision of same animalcule (Fig. 22) into sporular elements (Carter).

Figs. 25 and 26.-Urotricha lagenula and farcta, $\times 300$ and 350. The latter (Fig. 26) shows spiral markings. $n$, Nucleus; $c v$, contractile vesicle (Claparele and Lachmann).

FIts. 27. - Adult ciliated animalcule (Coleps hirtus), $\times 400$. Shows pronounced longitudinal and transverse markings, $\times 400$. $n$, Nucleus; $c v$, contractile vesicle.

FIG. 28. - The same dividing liy transverse fission. $c v$, Contractile vesicle.

Figs. 29 and 30.-Ciliated animalcules (Coleps fusus and Plagiopogon coleps). $n$, Nucleus; cv, contractile vesicle, $\times 350$ and 250 (Clap. and Lach. and Pertr). Show longitudinal aud transverse markings, the fomer most distinct.

FIts. 31 and 32.-Supposed trichocysts with thread-like spiral filament in the extended and retracted states (Polykrikos schwartaii), $\times 200$ (Bütschli).

$\mathrm{FI}_{\mathrm{x}}$ 33. - Ciliated animalcule (Lacrymaria cohnii) illustrating spiral twisting of body. cv, Contractile vesicle, $\times 250(\mathrm{Cohn})$.

FIG. 34.-The same animalcule spherically contracted. $n$, Nucleus; $c r$, contractile vesicle. These figures show with what facility animalcules can change their shape by centripetal and centrifugal movements. They have complete control over every part of their bodies, and their movements are voluntary (the Author).

FTG. 35.-Ciliated adult zooid (Metacystis truncata) showing transverse annulation (incipient segmentation), $\times 400$ (Cohn).

FIG. 36. - Young zooid of same animalcule (Fig. 35) as yet devoid of transverse markings.

FIGs. 37, 38, 39, and 40.-Mucous plases of encystment and sporular mode of multiplication in Colpoda cucullus, $\times 200$ (Stein).

FIGS. 41 and 42.-Anophrys sareophaga. Show longitudinal and transverse markings and segmentation, $\times 400$ (Cohn).

FIG. 43.--Trachelocerca phanicopterus. Shows elongated well-formed body, with fine transverse or annular markings, $\times 300$ (Cohn). Presents worm-like appearance and displays a distinct advance in organisation.

Fig. 44. - Proboscelle verminu, $\times 500$. Shows fin-like swimming arrangement in addition to cilia. $n$, Nucleus; cv, contractile vesicle (W. S. Kent).

FIG. 45.-Folliculina boltoni with flask-shaped protecting covering or lorica, $\times 200$ (W. S. Kent).

\section{PLATE LXXIV}

This plate illustrates the very great variety in size and shape of Infusoria; the presence of longitudinal, transverse, and spiral markings; the prevalence of cilia and setæ for natatory and feeding purposes; the curved spiral form of peristome; the power of changing shape spirally or otherwise; the double spiral arrangements seen in certain immature forms, \&c.

Frg. 1.-Animalcule in an extended condition (Chonic teres), ciliated and free swinming. 't, Contractile vesicle, $\times 200$ (W. S. Kent). Presents slender, worm-like appearance.

FIG. 2.-One of the largest of the animaleules (Amphileptus gigas), ciliated, attenuate variety with contractile vesicles (cv, cv) in linear series along dorsal horder; $t r$, trichocysts, $\times 100$ (Wrzesniowski). Shows well-marked longitudinal markings.

FIG, 3.-Lembus velifer-adult example; cilia comparatively long, $\times 400$ (Cohn). Shows undulating swimming membrane and well-marked transverse markings.

FItr. 4.- Normal zooid (Cyclidium glaucoma); setre very long and fine. $n$, Nucleus ; $v$, contractile vesicle, $\times 600$ (W. S. Kent).

FiG. 5.- Young of above (Fig. 4) as yet devoid of oral membrane. Setre as in Fig. 4.

FIG, 6. Cyclidium citrellus, $\times 300$; setre pronounced (Cohn).

FI(x. 7.-Adult animalcule (Trachelocerca olor). $c r$, $c v$, Contractile vesicles, $\times 300$. Shows swan-like lody and neck covered with fine cilia; the body displaying spiral markings. This is one of the most elegant of the animalcules; the neck when the animal is feeding being arched and curving in all directions.

FI + S. 8, 9, and 10.-Ciliated animalcules (Metopus sigmoides) in the extended and spiral state, $\times 200$ (Stein). Fig. 8 shows the animalcule in the extender state covered with cilia and displaying longitudinal and oblique markings. Fig. 9 shows it extended and slightly twisted and revealing pharynx $(k)$, nucleus $(n)$, and contractile vesicle $(c v)$. Fig. 10 sliows it with the body spirally contorted. The body is very plastic and can readily assume the various shapes indicated. On the contraction or shortening of the l,ody, the peristome with the pharynx, for the time, describes a conplex spiral circuit.

FIGS. 11 and 12.-Animalcules (Dinenympha gracilis) with bodies spirally contorted; the latter (Fig. 12) revealing a row of spore-like lodies, $\times 666$ (Leidy). The bodies in these cases are elongate-fusiform, flattened, band-like, and covered with fine cilia. They are also striated Iongitudinally. They display great activity and power of movement.

FIr. 13. Z Zooid (Spirostomum ambigunm) with its body extended, $\times 150$ (Stein). The body is elongated, cylindrical, and covered with cilia; the nucleis or endoplast $(n)$ braided and cord-like, and the contractile vesicle (cv) much expanded posteriorly.

FIri. 14.-A similar and larcrer zooid with its body spiraliy contorted. $n$, $n$, Braided cord-like endoplast; $c v, c v$, contractile vesicle extending throughout hody and greatly dilated posteriorly; or, oval aperture. This is one of the largest of the infusorial type and can readily be seen with the naked eye. It jossesses great mobility and displays the most graceful spirals. 


\section{PLATE LXXIV}

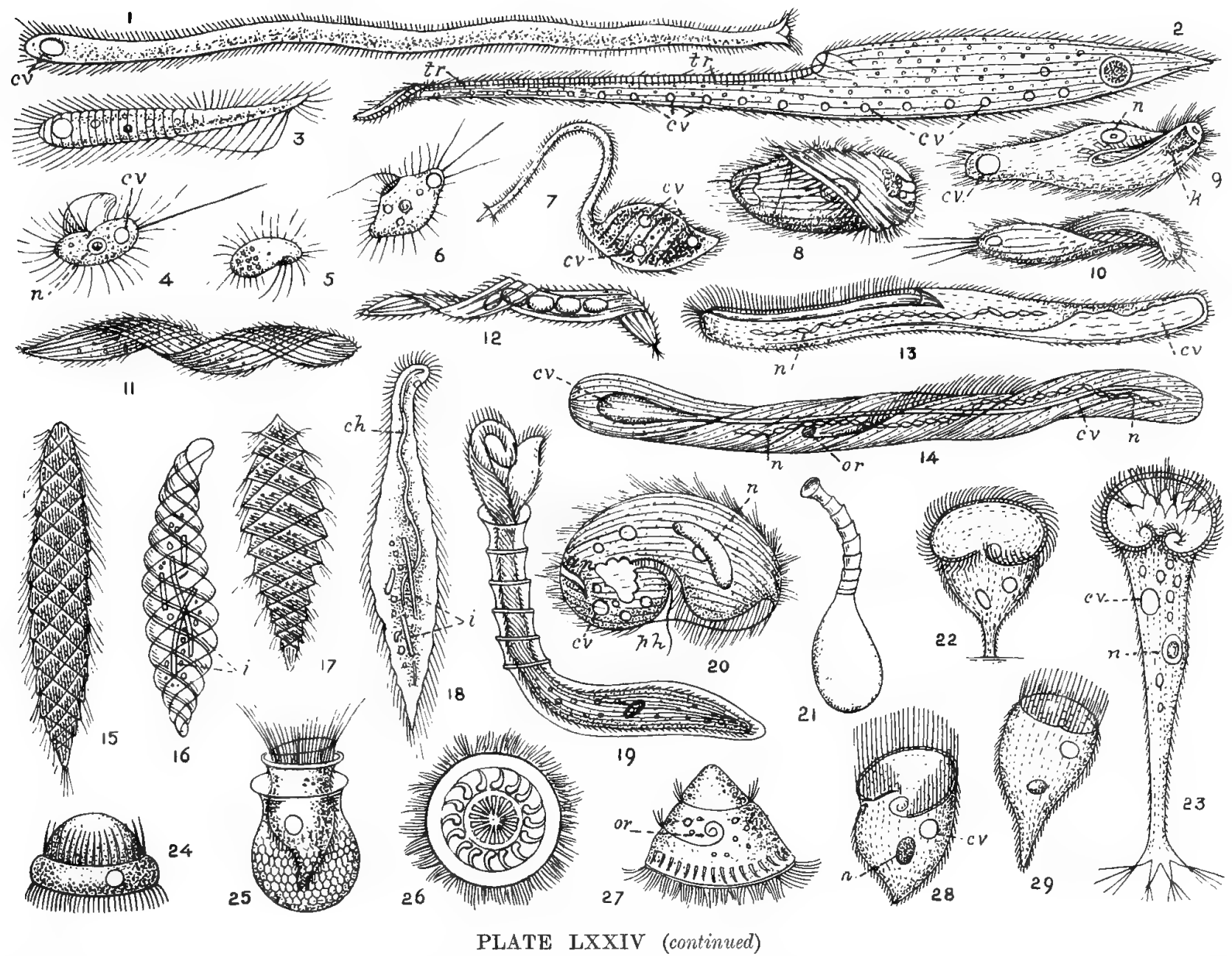

FIGs. 15 and 17.--Supposed immature examples of Trichonymphe agilis, $\times 500$ (Leidy). Bodies elongate ovate, covered with four sets of cilia, the longest cilia being spirally arranged. Show beautiful double right and left-handed spiral markings remarkably resembling those seen in the fir-cone, pine-apple, \&c. Compare with Plate xi., Fig. 1, and Plate xxiii., Fig. 1. The appearance of spiral markings and arrangements in immature simple forms furnishes another proof of the fundamental nature of the spiral in organic beings (the Author).

FIG. 16.-Animalcule (Pyrsonema vertens) also showing double spiral markings. i, Ingested food particles, $\times 666$ (Leidy). The cilia in this case are apparently absent; the body presenting a more or less contorted membranous appearance.

FIG. 18.-Ciliated animalcule with ingested fool particles of wood fibre (i), showing curious undulating cord-like structures (ch), $\times 666$ (Leidy).

FIG. 19.-Animalcule (Folliculina ampulla) with ornamented lorica, $\times 200$ (Stein). Shows spiral and segmented structures; the spirals occurring in neck and peristomal lobes of animalcule; the segmentation or circular markings in the adult sheath or lorica on animalcule.

Fig. 20.-Nyctotherus cordiformis. Body kidney-shaped, ciliated and marked with longitudinal striations. $n$, Nucleus; $c v$, contractile vesicle; $p h$, vestibulum of pharynx; an, anal aperture, $\times 150$ (Stein).

Fig. 21.-Ornamental adult sheath or lorica of animalcule (Folliculina ampulla), $\times 200$ (Stein). Shows elegant flask shaje ; the neck presenting annular rings or segmentations.

FIG. 22.-Attached fully extended animalcule (Stentor igneus), $\times 70$. At Figs. 28 and 29 examples are givell of the same animalcule in the free or swimming condition. $n$, Nucleus; $c v$, contractile vesicle. Show curver, spiral arrangement of the cilia.

FIG. 23.-Stentor pediculatus, $\times 250$. n, Nucleus; $c v$, contractile vesicle (De Fromentel). Body trumpet-shaped when extended, elongate ovate when contracted. Expanded peristome circular, slightly spiral, and covered with cilia. Contains numerous raised papillæ with fine hair-like setæ; the posterior adherent extremity is provided witl six digitiform prolongations and four fine setæ each.

FIGs. 24 and 26.--Lateral and ventral views of Trichodina scorpana, $\times 400$ (Rohin). Body turban-shaped, convex above, concave below, supplied with two wreaths of cilia and having armature of acetabulum. Fig. 25 shows beautiful concentric, curved, and radiating arrangements.

FIG. 25.- Normal zooid with elegant flask-shaped protective covering or lorica (Tintinnus lagenula), $\times 300$ (Clap. and Lach.). The zooid is provided with brush-shaped tuft of cilia.

FIG. 27.-Conically contracted animalcule (Trichodina pediculus) showing oral aperture (or) as drawn by Busch.

FIGs. 28 and 29.-Free-swimming nnimalcules (Stentor igneus). $n$, Nucleus; $c \%$, contractile vesicle, ×70. See 1under Fig. 22 , VOL. I. 


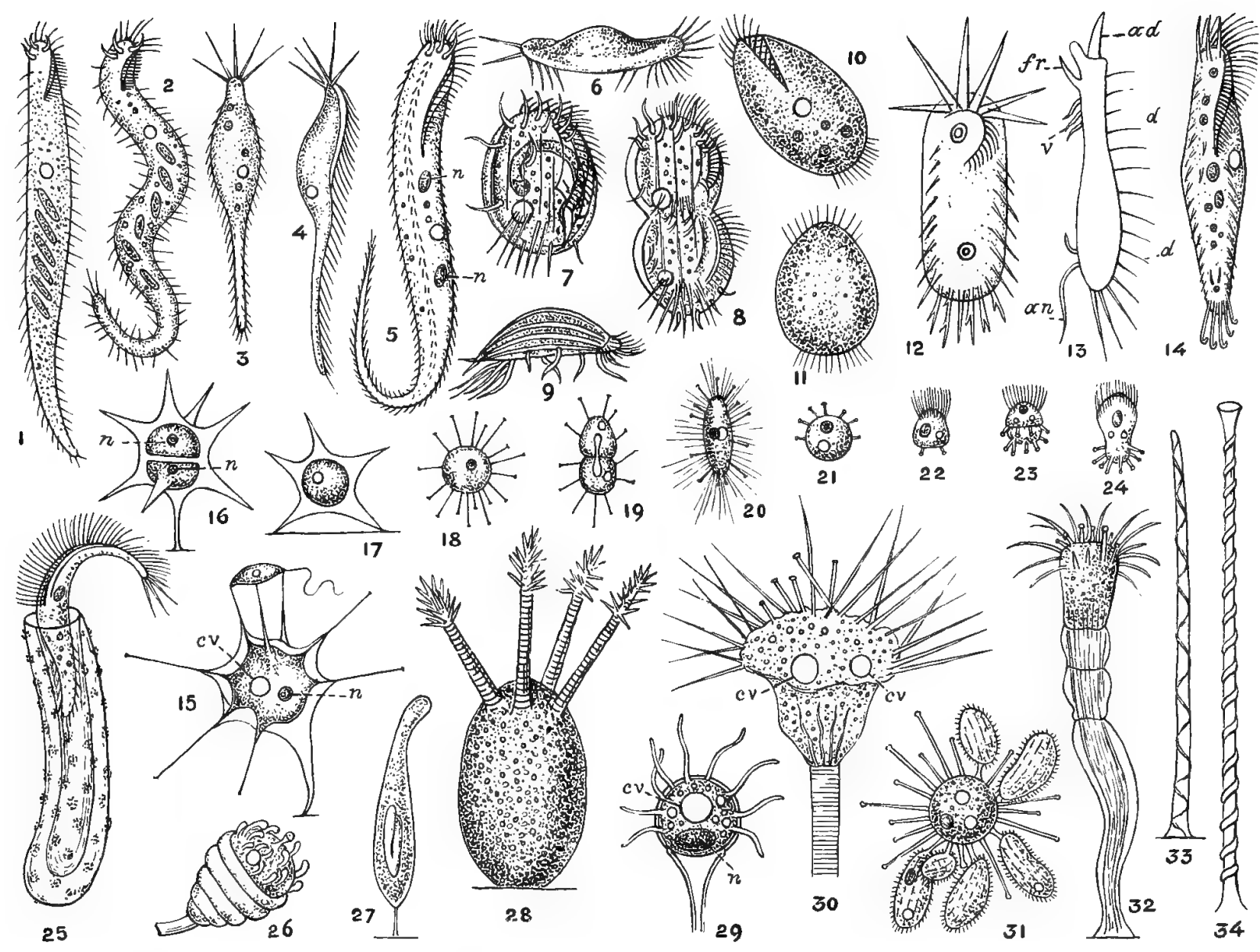

PLATE LXXV

This plate reveals an obvious advance in infusorial structure and function. It displays suctorial and prehensile tentacula; some of the latter exhibiting beautiful spiral formations and spiral movements; it also displays modes of division, modes of feeding and differentiation generally. In studying the lower animal forms depicted in Plates lxvi. to lxxiii. inclusive it is impossible to come to the conclusion that the one is evolved out of the other. Each in its own way is independent, and the most that can be done is to refer the individual to a type or group having structural, functional, and developmental peculiarities. In other words, no gradual transitions, such as evolution takes for granted, can be made out.

FrGs, 1 and 2.-Extended and spirally contracted examples of ciliated zooids (Uroleptus mobilis), $\times 150$ (Engelmann). Body flexible, elongate, linear, cylindrical; peristome very narrow; frontal styles uncinate; ventral and lateral setæ-the latter long and fine; nuclei or endoplasts six in number and rod-like in shape.

FTrs. 3 and 4.-Dorsal and lateral views of Epiclintes radiosa, $\times 300$ (Quennerstedt). Body elongate, anterior portion compressed and bearing at its summit five large straight radiating frontal styles; single marginal row of setæe on anterior and middle portions of body and double row bordering candal portion of body.

Firi. 5.-Ventral aspect of Uroleptus rattulus, $\times 150 . n, n$, Nuclei (Stein). Body persistent in shape, linenr-fusiform, anterior end rounded, tapers from central part of body and ends in long thin tail ; peristome field narrow and extends backward one-fifth of body ; marginal setæe cover body, the ventral setwe forming two rows; endoplasts $(n, n)$ two in number with contructile vesicle between. Shape of body fish-like.

Fir. 6.-Lateral view of Euplotes patella, $\times 200$ (Dujardin).

FIG. 7.- Ventral view of Euplotes charon, $\times 300$.

FIri, 8.-The same dividing by transverie fission, $\times 300$.

Fir: 9.-The same encysted, $\times 300$ (Stein). Figs. 7, 8, and 9 show simple forms of division and development, 


\section{ADVANCE IN LOWER PLANT AND ANIMAL FORMS}

\section{PLATE LXXV (continued)}

FIGs. 10 anı 11.-Extended and contracted zooid (()xytriclut scutellum), $\times 360$ (Colnn). Budy ublong, flattened, pointed anteriorly, rounded posteriorly and highly retractile; peristome-field extending back third of lody; posterior portion of body furnished with transverse row of twelve slender anal styles. Illustrates the centrifugal and centripetal movements seen in the lower animal forme (the Anthor).

FIG. 12.-Actinotricha saltans as figured l,y Colın, $\times 360$. Body elliptical and erpally rounded at both ends, five large styles radiating from anterior border; peristome and adoral eilia produced backwards one-third of body; small styles occur on latero-ventral and posterior portions of body.

FI(t. 13.-Diagrammatic longitudinal section of Orytricha pellionella, $\times 400 . \quad$ ul, Adoral ; fr, frontal ; v, ventral ; an, anal ; and 1 , dorsal cilia or setre (Sterki).

FIG. 14.- Ventral aspect of same as drawn by Stein.

Fig. 15. - Fixed adult animalcule (Atinete strllut inferiorly in short, hollow stalk-like attarhment; its lateral walls at points of exit of tentacles produced outwards in fine radiating tubuli ; contained body stellate; the tentacles, which vary from six to twelve, capitate; contractile vesicle (cv), single; nucleus $(n)$, spheroidal and subcentral. Illustrates the power possessed ly animalcules of seizing and transmitting food into their bodies by tentacles, pseudopodia, \&c. The food is drawn into the body by centripetal movements; it is seized by centrifugal movements. In the one case the sarcode is projected out of the body against the prey; in the other it is drawn into the body by an opposite and counter novement (the Author).

Figs. 16 and 17.--Encysted examples of same with body (Fig. 16) divided into two halves.

FIt. 18. - Normal adult zooid (Spharophrya urostylæ) with suckers, $\times 200$ (Stein).

FIG. 19.--The same dividing by transverse fission; the anterior part provided with suckers and temporary cilia not show11.

FIG. 20.-A similar zooid elongated and with cilia and suckers developed preparatory to subdivision, $\times 200$ (Stein).

FIt. 21.-Spharophya pusilla, $\times 150$ (Claparede and Lachmann). Body minute, spherical, bearing a variable number of short, widely scattered suckers. Nucleus ovate; contractile resicle single.

FIGs. 22, 23, and 24.-Animalcules (Sphærophrya stentorea), $\times 200$ (Steiu). Bodies illoricate, more or less spherical in form, with delicate capitate suckers scattered irregularly throughout the periphery; freely swimming; no fixed pedicle.

FI(i, 25. -Zooid (S'tichotricha secund $\iota$ ) projecting from its mucilaginous sheath, $\times 250$ (Stein). Body very active, lanceolatefusiform, tapers markedly anteriorly and less posteriorly ; peristomal or adotal cilia longest at anterior extremity, diminishing as oral aperture is reached; ventral setæ short, marginal setæ long and slender. Anterior half of body projected from unucilaginous sheath to right in screw-like manner. Animalcule not unfrequently leaves its sheath and swims freely in the water.

FIr. 26.-Adult zooid (Podocyathus diadema) with its tentacles retracted, $\times 300$. Lorica cup-shaped, transplarent, and of rery delicate consistence, rugose or wrinkled transversely; pedicle slender, flexuose; suctorial tentacles few, short, occupying central position; circumjacent prehensile tentacles numerous, extensile to twice the length of diameter of body, their substance finely granular.

FIG. 27.-Verniform zooid (Ophryodendron belgicum), $\times 400$ (Fraipont). Body subcylindrical, seven or eight times as long as broad, attached posteriorly by sessile pedicle.

FIG. 28. - Sessile zooid (Ophryodendron multic(pitatum) bearing four proboscidiform organs with transverse markings, $\times 300(W$. S. Kent). Body ovate, subspherical or pyriform, pedicellate when young, sessile in adult strite, adult zorid supporting as many as four subterminally developed proboscidiform organs, whose distal extrenities bear from thirty to forty cirrhose appendages.

FIG. 29.-Distal region of zooid (Acinetu vorticelloides) showing rudimentary development of the lorica, $\times 300$ (Fraipont). Body subspherical; tentacles developed thronghont exposed peripheral surface, extensile to length equal to diameter of body; lorica rudimentary, consisting of small cup-shaped expansions only of distal extremity of supporting pedicle. Nucleus ( $n$ ), ovate; contractile vesicle $(c v)$, single and of large size.

FIG. 30.-Distal extremity of pedicle bearing animalcule (Hemiophma gemrnipara) with extended tentacles of two orders, suctorial and prehensile; the latter spiral in their nature, $\times 400$ (Hertwig). Body contractile and variable in form, usually cup-shaped, widest anteriorly, narrow where it joins pedicle; tentacles projected in crown-like fashion from entire anterior border, the suctorial ones subcentrally developed, usually five or six in number and short, the prehensile ones being three or four times more numerous and longer. The external surface of the latter are also granular in a spiral direction. Pedicle longitudinally and transversely striate; contractile vesicle $(c v)$ two or nore in number; nucleus rsually scimitar-shaped. Spiral character of prehensile tentacles illustrated at Figs. 33 and 34 .

FIG. 31.-Zooid (Sphærophrya magna) having seized and in the act of devouring by suction half-a-dozen examples of colpode parvifrons, $\times 300$ (Maupas). Body spherical ; tentacles distinctly capitate not exceeding fifty in number; length equal to diameter of body, produced from all parts of periphery. Resemble in function the suctorial arms of the star-fish (the Author).

FIG. 32.-Adult zooid (Hemiophrya truncata), $\times 250$ (Fraipont). Body elliptical or subquadrangular; pedicle massive and irregular, three or four times length of body, same thickness as lower part of body and tapering to point of attachment, its distal end articulate or transversely plicate ; suctorial tentacles develop from centre of distal region of body, the prehensile tentacles (fifty or sixty in number) more slender and prolonger than suctorial ones, round which they form a fringe.

FIG. 33.-Tentacle of zooid (Acinetopsis rara) in the contracted condition and highly magnified, showing externally developed spiral fibrilla, $\times 600$ (Robin). The proboscidiform tentacle is in this case very attenuate, or filiform when extended, its length then equalling five or six times that of the body, exhibiting throughout when contracted an exteriorly developed, very delicate, plicate, spiral fibrilla. The tentacle is structurally and functionally spiral and very remarkable.

FIG. 34.-Tentacle of a normal zooid (Podophrya elongata) in a state of contraction, lighly magnified, and exhibiting a spirally convolute aspect due to the presence of a superficial spirally developed granular crest or film, $\times 800$ (W. S. Kent). The configuration of the tentacles seen in Figs. 33 and 34 is analogous to that witnessed in the spirally disposed fibrilla of the stalk of a vorticella. The of the tentacles seen in Figs. 33 and 34 is analogous to that witnessed in the spirat be seen and studied in order to be even partially realised. 


\section{THE VISIBLE AND INVISIBLE WORLD}

\section{Views of Stewart, Tait, Thomson, Helmholtz, Stokes, Young, Crookes, \&c.}

Professors B. Stewart and P. G. Tait, in their work, "The Unseen Universe," found their main argument on the "Principle of Continuity" in all things. Matter, force, and motion, according to them, pre-suppose pre-existing matter, force, and motion. They, however, take for granted an Invisible Universe and a First Cause or Deity. Thus they state: "The argument is in favour of the production of the visible universe by means of an intelligent agency residing in the Invisible Universe. . . . We are led by it not only to regard the Invisible Universe as having existed before the present one, but the same principle drives us to acknowledge its existence in some form as a universe from all eternity."

It should be noted that if the idea of continuity be carried out logically, the Unseen Universe and the First Cause or Deity should also have had a beginning from a pre-existing Unseen Universe and First Cause or Deity. To say a thing is eternal does not prove continuity in the sense in which that term is employed. "All this (in their opinion) follows from the Principle of Continuity, in virtue of which we make scientific progress in the knowledge of things, and which leads us, whatever state of things we contemplate, to look for its antecedent in some previous state of things also in the Universe. This principle represents the path from the known to the unknown. - That principle of continuity which underlies not only all scientific inquiry but all action of any kind in this world of ours. . We are content to develop from the present recognised condition of things. We take the world as we find it, and are forced by a purely scientific process to recognise the existence of an Unseen Universe. We are likewise led to regard the Unseen as having given birth to the present universe. ... We maintain that the visible universe - that is to say the universe of atoms-must have had its origin in time, and that while the Universe is, in its widest sense, alike eternal and infinite, the universe of atoms certainly cannot have existed from all eternity. ... We believe that an extension of purely scientific logic drives us to receive as quite certain the occurrence of two events which are as incomprehensible as any miracle; these are: the introduction of visible matter and its energy, and of visible living things into the universe. Furthermore, we are led by scientific analogy to regard the agency in virtue of which these two astounding events were brought about as an intelligent agency, an agency whose choice of the time for action is determined by considerations similar in their nature to those which influence a human being when he chooses the proper moment for the accomplishment of his purpose. . . We have assumed that a study of creation leads us up to some conception of God-that we are driven by the faculties which $\mathrm{He}$ has given us to acknowledge the existence of a Paramount Power, and inasmuch as scientific thought leads us to regard the Universe as both infinite and eternal, so we are driven to regard this Power which underlies all phenomena as infinite and eternal also."

One branch of their argument is expressed as follows: "Let us begin by stating at once that we assume, as absolutely self-evident, the existence of a Deity who is the Creator and Upholder of all things. We further look upon the laws of the universe as those laws according to which the beings in the universe are conditioned by the Governor thereof, as regards time, place, and sensation... Nothing whatever lies, or can be even conceived to lie, outside of this sovereign and paramount influence. There is no impression made upon the bodily senses, no thought or other mental operation, which does not take place under conditions imposed by the will of God. . . . In the first place, there is God, the source of power; secondly, there are the Conditions which He imposes; and thirdly, there is the Ego, the being who is thus conditioned. . . As far as we can judge, the visible universethe universe of worlds-is not eternal, while, however, the Invisible Universe, or that which we may for illustration at least associate with the ethereal medium, is necessarily eternal. The visible universe must have had its origin in time, no doubt from a nebulous condition. But in this condition it can hardly have been fit for the reception of life. Life must therefore have been created afterwards. We have thus at least two separate creations, both taking place in time - the one of matter and the other of life. And even if it were possible, which it is not, to get over one of the diffeulties attending this hypothesis, that of creation in time, by regarding the visible universe as eternal; yet even then we must regard matter and life as implying two separate creative acts if we assume the nebulous hypothesis to be true."

As to the origin of life on our globe there are, I may remark, four theories:-

(a) That life is the result of spontaneous generation;

(b) That matter is in some simple sense alive;

(c) That life has been added to matter by a creative act;

(d) That life has been transmitted to our planet from other planets. 
The theory of spontaneous generation of life de nowo $(a)$ has been proved to be untenable by direct experiments frequently repeated.

The theory that matter - all matter-is in some simple sense alive $(b)$ makes demands on the imagination which the theory does not satisfy. "Looking upon the atom as the essential thing in the universe, the various motions of the atom are by this school supposed to be accompanied by a species of consciousness inconceivably simple. Under certain circumstances this eternal and immortal consciousness is supposed to be consistent with that which we call the life of the individual, while under other circumstances these two lives are not consistent with one another. The individual then dies, but nevertheless the simple, immortal lives of the atoms which compose his body remain attached to them as truly as before." 1

The addition of life to matter by a separate act of creation $(c)$ is the theory generally accepted, and most probably the correct one.

The life-transmission hypothesis $(d)$ has been advocated by two of the ablest physicists of modern times, namely Sir William Thomson (Lord Kelvin) and Professor Helmholtz. Curiously enough, these celebrated savants arrived at practically similar conclusions independently and at nearly the same time. If, however, it be granted that the life of our planet has been derived from other planets, the problem of life is not thereby solved: the question then is, Whence came the life which exists in the transmitting planet or planets?

Lord Kelvin attempted to explain the origin of the material universe by the vortex-ring hypothesis, and gravitation by a modification of the hypothesis of ultra-mundane corpuscles. He affirms "that atoms are vortexrings generated out of a perfect fluid filling all space," and supposes "that the ultra-mundane corpuscles are only a finer form of vortices." His belief that the life of our globe was transmitted from other planets by meteors is, in a certain sense, in accordance with the principles of continuity. It is, however, at best only a partial explanation. Helmholtz states his case as under: "If failure attends all our efforts to obtain a generation of organisms from lifeless matter, it seems to me a thoroughly correct procedure to inquire whether there has ever been an origination of life, or whether it is not as old as matter, and whether its germs, borne from one world to another, have not been developed wherever they have found a favourable soil.". 2

Professor Stokes thus speaks of life: "Admitting to the full as highly probable, though not completely demonstrated, the applicability to living beings of the laws which have been ascertained with reference to dead matter, I felt constrained at the same time to admit the existence of a mysterious something lying beyond, a something sui generis which I regard, not as balancing and suspending the ordinary physical laws, but as working with them and through them to the attainment of a designed end. What this something which we call life may be is a profound mystery. . . When from the phenomena of life we pass on to those of mind, we enter a region still more profoundly mysterious. We can readily imagine that we may here be dealing with phenomena altogether transcending those of mere life, in some such way as those of life transcend, as I have endeavoured to infer, those of chemistry and molecular attractions, or as the laws of chemical affinity in their turn transcend those of mere mechanics. Science can be expected to do but little to aid us here, since the instrument of research is itself the object of investigation. It can but enlighten us as to the depths of our ignorance, and lead us to look to a higher aid for that which most nearly concerns our well-being." 3

Professors Stewart and Tait follow a similar line of argument. They say: "Life, whatever be its nature, may be supposed to penetrate into the structural depths of the universe. Its seat is in a region inaccessible to human inquiry, and equally inaccessible, we may well suppose, to the inquiries of the higher created intelligences. Intimations of its presence are no doubt constantly emerging from this region of thick darkness into the objective universe, but when they have reached it they obey the ordinary laws of phenomena, according to which a material effect implies a material antecedent. Notwithstanding all this, life exists just as surely as the Deity exists. . . We have driven the creative operation of the Great First Cause into the durational depths of the universe-into the eternity of the past-but for all that we have not got rid of God. In like manner we have driven the mystery of life into the structural depths of the universe - that region of thick darkness which no created eye is able to

1 Professor O. Lehmann, at a concourse of German physicians and physicists held at Stuttgart, endeavoured to prove that no hard and

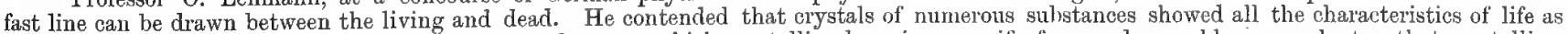

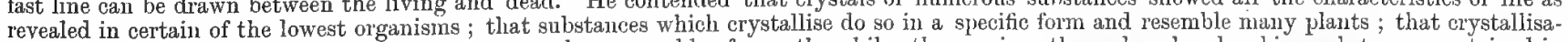

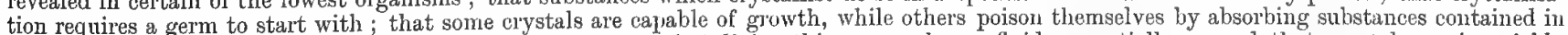

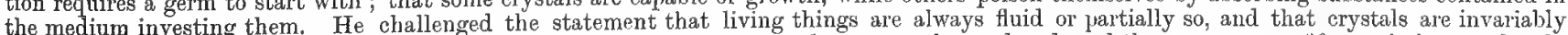

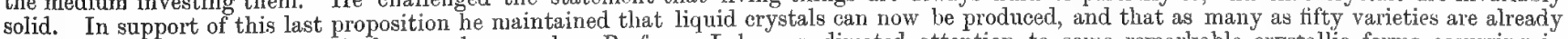

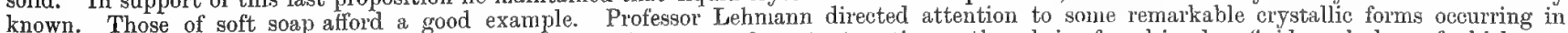

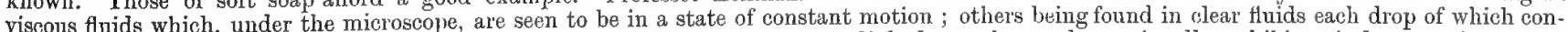

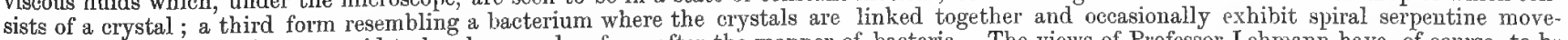

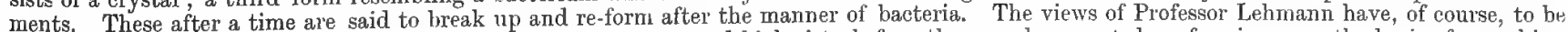

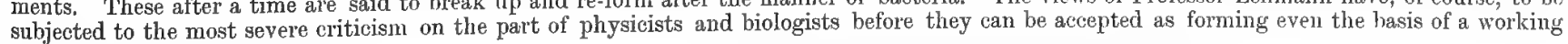
hypothesis.

2 Nature, January 14,1875

${ }^{3}$ British Association Address at Exeter, 1869. 
pierce-but we have not got rid of life." . . "Iife can be produced from life only, and this law would seem to give an indication that the solution of the mystery is not to be found by considering life as merely a species of energy."

Darwin, Wallace, and others have endeavoured to account for life in all its forms as the outcome of evolution, and as apart from separate creations. They assume the existence of a primordial germ, but in so doing they take for granted what requires to be proved, as the primordial germ itself has to be accounted for. "Darwin especially imagines that all the present organisms, including man, may have been derived by the process of natural selection from a single primordial germ. When, however, the backward process has reached this germ, an insuperable difficulty presents itself. How was this germ produced? All really scientific experience tells us that life can be produced from a living antecedent only; what, then, was the antecedent of this germ? Hypotheses have no doubt been started, but we cannot regard them in any other light than as an acknowledgment of a difficulty which cannot be overcome. . If, then, the matter of this present visible universe be not capable of itself, that is to say, in virtue of the forces and qualities with which it has been endowed, of generating life, but if we must look to the Unseen Universe for the origin of life, this would appear to show that the peculiar collocation of matter which accompanies the operations of life is not a mere grouping of particles of the visible universe, but implies likewise some peculiarity in the connection of these with the Unseen Universe. May it not denote, in fact, some peculiarity of structure extending to the Unseen?

"In fine, to go a step further, may not life denote a peculiarity of structure which is handed over not merely from one stage to another-from the Invisible to the visible-but which rises upwards from the very lowest structural depths of the material of the universe, this material being regarded as possessed of an infinitely complex structure?"

The infinitely complex structure here referred to would, if accepted, go far to explain the extraordinary changes which occur in spores, seeds, and eggs when impregnated, whereby each produces only its own kind. These changes are most numerous and most marked in the development of the higher animals, and while the microscope, chemistry, and physics are wholly inadequate to deal with them, there is no room for doubt that living matter, as represented by the union of the male and female elements of plants and animals in reproduction and development, varies infinitely. I directed attention to this point as far back as 1873, when I attempted to refute the then prevailing idea that protoplasm and spores, seeds and eggs, were simple, homogeneous substances and fundamentally identical. ${ }^{1}$

Great strides have been made in physiology and in physics since 1873, and some have gone the length of asserting that the universe as a whole participates in every motion which occurs in even the smallest of atoms.

"The law of gravitation assures us that any displacement which takes place in the very heart of the earth will be felt throughout the universe, and we may even imagine that the same thing will hold true of those molecular motions which accompany thought. For every thought we think is accompanied by a displacement and motion of the particles of the brain, and we may imagine that somehow these motions are propagated throughout the universe. . . 'Mr. Babbage has pointed out ${ }^{2}$ that if we had power to follow and detect the minutest effects of any disturbance, each particle of existing matter must be a register of all that has happened.' .

"We must resort to the Unseen not only for the origin of the molecules of the visible universe, but also for an explanation of the forces which animate these molecules, and not only so, but we are always carried back from one order of the Unseen to another. Now if this be the case-if The UnIverse be constructed with successive orders of this description connected with one another--it is manifest that no event whatever, whether we regard its antecedent or its consequent, can possibly be confined to one order only, but must spread throughout THE Entire Universe."

This intimate blending of matter and force, which includes the molecular operations of the brain and thought, implies a much more intimate union between the organic and inorganic kingdoms, and between mind and matter, than was considered possible a few years ago. The view, however extravagant it may at first sight appear, is corroborated in several particulars. The recent developments in wireless telegraphy conclusively prove that the atoms with their corpuscles or electrons play a leading rôle in everything that pertains to the physical universe, and that they can be made the servants of intelligent, modern man.

The advances recently made in the production and employment of electricity unequivocally point to similar conclusions. While electricity has played and is destined to play a leading part in physics and physiology, its nature and properties are no more understood than are those of the atoms. It is not yet known whether electricity

1 "On the Rolation of [']ants and Anina]s to Inorganic Matter and on the Interaetion of the Yital ancl l'bysical Forers.' (Lancet, 15th Novmulier, 187.).

2 Nintlı Bridgewater Treatise. 
is a fluid or an elastic solid, or a form of motion within, between, or outside ethereal particles. Similarly the exact nature of life and force has yet to be discovered.

Dr. Thomas Young, in his "Lectures on Natural Philosophy," thus describes the possible relations of the seen and unseen: "We see forms of matter, differing in subtility and mobility, under the names of solids, liquids, and gases; above these are the semi-material existences, which produce the phenomena of electricity and magnetism, and either caloric or a universal ether. Higher still, perhaps, are the causes of gravitation, and the immediate agents in attractions of all kinds, which exhibit some phenomena apparently still more remote from all that is compatible with material bodies. And of these different orders of beings, the more refined and immaterial appear to pervade freely the grosser. It seems therefore natural to believe that the analogy may be continued still further, until it rises into existences absolutely immaterial and spiritual."

Fortunately for our race it is not necessary to have a profound knowledge of the substances and forces which so materially contribute to our comfort and enjoyment. At the same time it is our bounden duty to examine with all the energy and ability at our disposal matter and force in all their combinations as they occur in the organic and inorganic kingdoms. Electricity especially promises a rich harvest to the original investigator of the future. It is apparently the silver thread which links the seen to the unseen, the living to the dead, and which pervades all matter, animate and inanimate. It can be generated by living tissues, and discharged, even as the thunderbolt is discharged, by living animals. It can be produced from inanimate substances by friction and otherwise in such quantity and strength as instantly to kill animals in precisely the same way as the lightning itself does. In electricity we have a form of force found in the inorganic and organic kingdoms which is always available. Its presence in nerves, nerve centres, the spinal cord, the brain and the muscles has much significance when it is remembered how intimate the connection between the spinal cord, the brain, nerves, and muscles is. The connection points to a substratum of matter and force pervading dead and living intelligent matter alike. The finer the matter and the more subtle the force, the greater, apparently, is the demand for them in nature, and the wider their applicability. This is especially true of ether. "Regard the ether as we please, there can be no doubt that its properties are of a much higher order in the arcana of nature than those of tangible matter."

Sir William Crookes has played a prominent and distinguished part in discovering and demonstrating the finer properties of matter.

Nearly a quarter of a century ago, as was explained at a meeting of the Royal Society in March 1902, the results of certain famous experiments in highly rarefied tubes led Sir William to assume the existence of an ultra-gaseous condition of matter; and in a memorable lecture at the meeting of the British Association in 1879 he expressed his opinion that "in studying this fourth state of matter we seem at length to have within our grasp, and obedient to our control, the little individual particles which, with good warrant, are supposed to constitute the physical basis of the universe." Every subsequent experiment having for its object the widening and deepening of our knowledge of the nature of phosphorescence, magnetisation, and the heat of the electric arc has helped to strengthen the belief that this "radiant matter" (to use the original name for the "satellites" of Lord Kelvin, the "corpuscles" of Professor J. J. Thomson, and the "electrons" of Dr. Johnstone Stoney) has some of the properties of matter and some of the properties of energy, and that physicists have at last reached that metaphysical borderland where matter and energy are merged in one another. According to Professor J. J. Thomson's measurements the mass of an electron is about the $\frac{1}{00}$ th part of that of the hydrogen atom, and it leaves the negative pole with a velocity about one-half that of light. Therefore, it is easy to see that, when swarms of electrons rush from the negative pole and meet the knots of atoms of gas in a so-called vacuum, their power of producing light and heat must be very great. Sir William Crookes regards the electron as "the unit or atom of electricity," and thinks that a positively-charged chemical atom is one with a surplus, and a negatively-charged atom is one having a deficiency, of electrons. Thus he returns to Franklin's one-fluid theory of electricity.

The electron theory explains an experiment which has long puzzled electricians. It is well known that if a coin be laid on a sensitive plate in absolute darkness and connected with one pole of an induction coil, the developed plate will show an image of the raised parts of the coin. This curious result has generally been explained on the assumption that the electrified stream of air-the so-called "brush discharge" -affects the film just as light does. But Mr. F. Sanford found that even when the coin was embedded in the centre of a block of paraffin three-quarters of an inch thick it could still be photographed by means of the induction coil. In this experiment the coin could not send off electrified air, and the old explanation is out of the question. But since electrons would easily pass from the coin through the paraffin to the sensitive plate, the theory of Sir William Crookes helps us to a satisfactory solution of the puzzle. Sir William has recently (Chemical News, 1906) added another to his many ingenious and bold speculations. It is well known that when the light of an incandescent body, say of the sun, is passed through a spectroscope, the elements present in the glowing body are shown by the lines on the 
picture or spectrum produced. Sir William has been examining the phosphorescent glow of some rare earths, the result of which is to convince him that he has found evidence of new elements. This judgment is founded on the manner in which certain lines are distributed in the phosphorescent spectrum. He writes: "I consider I am justified provisionally, and without undue insistence on what after all is only one of the many tests of newness, in saying that I am in possession of good evidence pointing to the existence of two, if not three, new bodies waiting to be isolated by chemical methods."

It will be seen from what has been stated that matter and force are indestructible; that matter and force (physically speaking) are inseparably united; and that motion (in vortices or otherwise) is a characteristic of matter.

Matter has been defined by Messrs. Balfour Stewart and Tait "as the seat or vehicle of energy-that which is essential to the existence of the known forms of energy, and without which there could be no transformation of energy."

The association of matter with force and with motion is exceedingly interesting and important as bearing upon life, reproduction, growth, and development. Plants and animals are formed from the elements of the universe, and the elements carry into the substance of both a certain amount of the force and movement which inhere in the elements as such. This explains how, under the influence of life, plants and animals assume certain forms and movements, especially spiral forms and movements. If this view be adopted it is seen that life and physical force work in the same directions, and that plants and animals, in building themselves up, at once avail themselves of the matter, force, and movements of the universe. Nor need this view be restricted to spiral forms and movements: it is equally applicable to the formation of crystals, and to all plants and animals which assume crystallic shapes and are more or less symmetrical, of which there are comparatively a very large number. Strictly speaking, living plants and animals are never quite symmetrical. Spiral structures, if single, are lopsided, and if double, they overlap and produce symmetry of a kind. Living forms invariably diverge slightly from absolute symmetry. Pasteur regarded the want of symmetry in living plants and animals as the distinguishing characteristic of life.

\section{$\$ 3$. New Theory of Matter.}

It will be convenient to discuss this all-important subject at this stage, and I cannot do better than quote Professor G. H. Darwin, F.R.S., who gave a lucid and interesting account of it as President of the British. Association in 1905. He states :-

"Until some ten years ago the essential diversity of the chemical elements was accepted by the chemist as an ultimate fact, and indeed the very name of atom, or that which cannot be cut, was given to what was supposed to be the final indivisible portion of matter. The vast edifice of modern chemistry has been built with atomic bricks.

"But within the last few years the electrical researches of Lenard, Röntgen, Becquerel, the Curies, Larmor and Thomson, and of a host of others, have shown that the atom is not indivisible, and a flood of light has been thrown thereby on the ultimate constitution of matter. Amongst all these fertile investigators it seems to me that Thomson stands prominent, because it is principally through him that we are to-day in a better position for picturing the structure of an atom than was ever the case before.

"It has been shown that the atom, previously supposed to be indivisible, really consists of a large number of component parts. By various convergent lines of experiment it has been proved that the simplest of all atoms, namely, that of hydrogen, consists of about 800 separate parts, while the number of parts in the atom of the denser metals must be counted by tens of thousands. These separate parts of the atom have been called corpuscles or electrons, and may be described as particles of negative electricity.

"The corpuscles, being negatively electrified, repel one another just as the hairs of a person's head mutually repel one another when combed with a vulcanite comb. The mechanism is as yet obscure whereby the mutual repulsion of the negative corpuscles is restrained from breaking up the atom, but a positive electrical charge, or something equivalent thereto, must exist in the atom, so as to prevent disruption. The existence in the atom of this community of negative corpuscles is certain, and we know further that they are moving with speeds which may in some cases be comparable to the velocity of light, namely, 200,000 miles a second. But the mechanism whereby they are held together in a group is hypothetical.

"It appears that in general there are definite arrangements of the orbits in which the corpuscles must revolve, if they are to be persistent or stable in their motions. Accordingly we may state that definite numbers are capable of association in stable communities of definite types. 
"To obtain any adequate conception of their size we must betake ourselves to a scheme of threefold magnification. Lord Kelvin has shown that, if a drop of water were magnified to the size of the earth, the molecules of water would be of a size intermediate between that of a cricket-ball and of a marble. Now each molecule contains three atoms, two being of hydrogen and one of oxygen. The corpuscles may stand to the atom in a similar scale of magnitude. Accordingly a threefold magnification would be needed to bring these ultimate parts of the atom within the range of our ordinary scales of measurement. We must return to the intermediate stage of magnification, in which we consider those communities of atoms which form molecules. This is the field of research of the chemist.

"Communities of atoms are called chemical combinations, and we know that they possess every degree of stability. The existence of some is so precarious that the chemist in his laboratory can barely retain them for a moment; others are so stubborn that he can barely break them up. In this case dissociation and re-union into new forms of communities are in incessant and spontaneous progress throughout the world.

"Stability is, further, a property of relationship to surrounding conditions; it denotes adaptation to environment."

In this connection it may be well to state that Professor De Launay, in an article on "The Evolution of Matter," argues that since uranium changes into radium, and radium changes into helium, there is, in general, a spontaneous evolution of matter going on. He mentions that M. Curie was asking himself whether not merely this uranium-radiumhelium group, but all chemical elements, have a certain duration of life, like organised beings, but relatively much longer than that of the radium group.

Matter, as the fundamental substratum of life and being, is invested with quite an extraordinary interest. This interest is enhanced when it is remembered that matter and mind are indissolubly associated in living beings. From the earliest times the nature and composition of matter has engaged the attention of mankind.

Time was when the physical universe was believed to consist of various kinds of ponderable matter combined in various ways and scattered throughout space, subject to the laws of motion, the influence of chemical affinity, temperature, \&c. ; retaining its mass unchanged, and exercising, at all distances, a force of attraction on other material masses according to the great law of gravitation.

There were then certain imponderables, of which heat (phlogiston) was one, two elastic fluids, and corpuscular emanations to constitute light. The all-absorbing subjects of magnetism and electricity were comparatively unknown. Matter was believed to be indestructible, and one of its distinguishing characteristics was that it acted at a distance. No mention was yet made of the law of the conservation and dissipation of energy. Neither had the undulatory theory of light been worked out, nor the existence of ether, as a continuous substance, occupying all space, and which made strain or instantaneous direct action, as opposed to action at a distance, possible, been mooted.

About a century ago Young opened the controversy which established the undulatory theory of light, and necessitated the existence of a continuous medium, that medium being the modern ether. ${ }^{1}$ The universal presence of ether in the universe necessary for the transmission of light not only makes action by strain or at first hand, as opposed to action at a distance, possible, but also accounts for the propagation of sound, the magnetic field, and the electric waves of wireless telegraphy.

Faraday was the first to indicate the true nature of the magnetic field, and to establish the doctrine of strain or immediate action as opposed to gravitation or action at a distance.

Magnetism had been known from the time of Thales (circa 600 B.C.), and it was a common experiment in all physical laboratories to show that pieces of amber or sticks of sealing-wax, if briskly rubbed with a silk handkerchief or chamois skin, attracted light substances such as hairs, small pieces of cork, \&c.

The nature of the attraction was, however, a mystery; the magnetic field was not understood, neither was the relation between magnetism and electricity fully made out. The existence of electric currents, while suspected, had not been demonstrated. Benjamin Franklin by means of his kite and moist string drew lightning from the thunder-cloud, and Galvani and his assistant observed that the muscles of frogs suspended by wires were made to twitch during thunder-storms. There was evidently a connection, a something in common, in all these phenomena. The presence of ether, or a continuous inter-stellar substance occupying all space, furnished the key wherewith to unlock the secrets of the new discoveries.

Matter had long been regarded as indestructible, and the question arose as to whether matter and force were separate and distinct entities or were associated, and, if associated, whether they were always associated or only occasionally. Modern research proved that force inheres in matter, and is exerted by it either as strain (action

1 While the theory of an all-prevailing ether had been broached before Young's day, it may be said to have been established by him and Fresnel.

VOL. I. 
at first hand) or action at a distance (gravitation). This led Helmholtz and others to claim for force the imperishability accorded to matter.

The law of the conservation and dissipation of energy followed as a necessary sequence. It was felt that if matter as represented by the elements and the ether occupied all space, force also occupied all space, and that the changes could be rung on matter and force to an indefinite extent throughout the universe. All this was intelligible, but a school has lately arisen which sets aside and dispenses with every form of gross matter, and deals with what may be regarded as its intangible manifestations. The new doctrine has been ably stated by Mr. Arthur J. Balfour in his admirable address, entitled "Reflections Suggested by the New Theory of Matter," delivered at Cambridge (August 17, 1904) in his capacity as President of the British Association. He says: "To-day there are those who regard gross matter, the matter of every-day experience, as the mere appearance of which electricity is the physical basis; who think that the elementary atom of the chemist, itself far beyond the limits of direct perception, is but a connected system of monads or sub-atoms which are not electrified matter, but are electricity itself; that these systems differ in the number of monads which they contain, in their arrangement, and in their motion relative to each other and to the ether; that on these differences, and on these differences alone, depend the various qualities of what have hitherto been regarded as indivisible and elementary atoms; and that while in most cases these atomic systems may maintain their equilibrium for periods which, compared with such astronomical processes as the cooling of a sun, may seem almost eternal, they are not less obedient to the law of change than the everlasting heavens themselves.

"But if gross matter be a grouping of atoms, and if atoms be systems of electrical monads, what are these electrical monads? It may be that, as Professor Larmor has suggested, they are but a modification of the universal ether, a modification roughly comparable to a knot in a medium which is inextensible, incomprehensible, and continuous. But whether this final unification be accepted or not, it is certain that these monads cannot be considered apart from the ether. It is on their interaction with the ether that their qualities depend; and without the ether an electric theory of matter is impossible. . . A little more than a century ago electricity seemed but a scientific toy. It is now thought by many to constitute the reality of which matter is but the sensible expression. It is but a century ago that the title of an ether to a place among the constituents of the universe was authentically established. It seems possible now that it may be the stuff out of which that universe is wholly built. When the sudden appearance of some new star in the telescopic field gives notice to the astronomer that he, and perhaps in the whole universe he alone, is witnessing the conflagration of a world, the tremendous forces by which this far-off tragedy is being accomplished must surely move his awe. Yet not only would the members of each separate atomic system pursue their relative course unchanged, while the atoms themselves were thus driven violently apart in flaming vapour, but the forces by which such a world is shattered are really negligible compared with those by which each atom of it is held together. Chemical affinity and cohesion are on this theory no more than the slight residual effects of the internal electrical forces which keep the atom in being. Gravitation, though it be the shaping force which concentrates nebulæ into organised systems of suns and satellites, is trifling compared with the attractions and repulsions with which we are familiar between electrically charged bodies; while these again sink into insignificance beside the attractions and repulsions between the electric monads themselves. The irregular molecular movements which constitute heat, on which the very possibility of organic life seems absolutely to hang, and in whose transformations applied science is at present so largely concerned, cannot rival the kinetic energy stored within the molecules themselves. The starry heavens have from time immemorial moved the worship or the wonder of mankind. But if the dust beneath our feet be indeed compounded of innumerable systems, whose elements are ever in the most rapid motion, yet retain through uncounted ages their equilibrium unshaken, we can hardly deny that the marvels we directly see are not more worthy of admiration than those which recent discoveries have enabled us dimly to surmise. . Whether this vehement sentiment in favour of a simple universe has any theoretical justification I will not venture to pronounce. There is no à priori reason that I know of for expecting that the material world should be a modification of a single medium, rather than a composite structure built out of sixty or seventy elementary substances, eternal and eternally different."

The new theory of matter aims at establishing simplicity and unity, and those who advocate it are disinclined to believe in a great number of elements. While it is possible to accept the ether theory and the doctrine of strain, or action at first hand, there is no need to discredit gross matter and action at a distance. The old and new theories of matter are not necessarily opposed to each other. The new view is in no way contrary to older theories of the atomic and molecular theories of matter, but is an extension and explanation of these, and, in the hands of Professor J. J. Thomson, has made, at any rate to physicists, a simplification and rational view of these without introducing the question of physical reality. It is possible to believe in what is seen and felt, and also in what is unseen and intangible. It is also possible to believe in action at a distance, as witnessed in gravitation, and also 
in action at inappreciable distances, as exemplified in atmospheric electric strain, cohesion, adhesion, and whatever more direct and intimate relations are possible. The existence of the finer ether does not destroy the gross matter, neither does the most direct conceivable action destroy action at a distance. The sense organs compel us to believe in the grosser matter, and chemical analysis, and the microscope, with its multiple powers and improved definition. gradually introduce us to finer and finer matter. The limits set to the eye, the telescope, microscope, spectroscope, and the most refined chemical analysis forbid us to deal with ultimate matter, but the experience gained from the grosser matter enables us to realise and appreciate what can be neither seen nor felt.

As already indicated, it was Faraday who first took exception to the doctrine of action at a distance, and on his experiments and views the electric theory of matter was ultimately founded. He, however, failed to account for or explain away the great law of gravitation discovered by Newton.

As pointed out by Mr. A. J. Balfour, "No scientific education is likely to make us, in our unreflective moments, regard the solid earth on which we stand, or the organised bodies with which our terrestrial fate is so intimately bound up, as consisting wholly of electric monads very sparsely scattered through the spaces which these fragments of matter are, by a violent metaphor, described as 'occupying.' The electric theory which we have been considering carries us into a new region altogether. It does not confine itself to accounting for the secondary qualities of matter by the primary ones, or the behaviour of matter in bulk by the behaviour of matter in atoms; it analyses matter, whether molar or molecular, into something which is not matter at all. The atom is now no more than the relatively vast theatre of operations in which minute monads perform their orderly evolutions ; while the monads themselves are not regarded as units of matter, but as units of electricity; so that matter is not merely explained, but is explained away."

The great difficulty in accepting the new theory of matter so well stated by Mr. Balfour consists in its transcandentalism and its tendency to disparage the sense organs as reliable instruments for obtaining accurate knowledge, within limits, of things outside of ourselves.

The trend of philosophy and recent physical speculations, in Mr. Balfour's opinion, is to show that inasmuch as the sense organs, even when artificially aided, cannot adequately deal with matter in its ultimate electrical condition, and inasmuch as they were constructed in a more or less perfect form ages before physical science existed, and even before knowledge and abstract reasoning were desiderata, therefore the senses are not safe guides for acquiring useful and other knowledge. This, it appears to me, is a distorted view of things, and amounts practically to a reductio ad absurdum.

Mr. Balfour virtually adopts the new theory of matter, and, in doing so, throws doubt upon the utility of the sense organs as instruments for acquiring knowledge, especially exact and higher knowledge. In dealing with the sense organs he calls to his aid the theory of "natural selection," than which, as I will show further on, there can be no more unsafe guide. He says: "Sense-perceptions supply the premises from which we draw all our knowledge of the physical world. It is they which tell us that there is a physical world; it is on their authority that we learn its character. But in order of causation they are effects due (in part) to the constitution of our organs of sense. What we see depends not merely on what there is to be seen, but on our eyes. What we hear depends not merely on what there is to hear, but on our ears. Now, eyes and ears, and all the mechanism of perception, have, as we know, been evolved in us and our brute progenitors by the slow operation of natural selection. And what is true of sense-perception is of course also true of the intellectual powers which enable us to erect upon the frail and narrow platform which sense-perception provides the proud fabric of the sciences. It is certain that our powers of sense-perception and of calculation were fully developed ages before they were effectively employed in searching out the secrets of physical reality-for our discoveries in this field are the triumphs but of yesterday. The blind forces of Natural Selection, which so admirably simulate design when they are providing for a present need, possess no power of prevision, and could never, except by accident, have endowed mankind, while in the making, with a physiological or mental outfit adapted to the higher physical investigations. So far as natural science can tell us, every quality of sense or intellect which does not help us to fight, to eat, and to bring up children, is but a by-product of the qualities which do. Our organs of sense-perception were not given us for purposes of research; nor was it to aid us in meting out the heavens or dividing the atom that our powers of calculation and analysis were evolved from the rudimentary instincts of the animal.

"It is presumably due to these circumstances that the beliefs of all mankind about the material surroundings in which it dwells are not only imperfect but fundamentally wrong. It may seem singular that down to, say, five years ago, our race has without exception lived and died in a world of illusions, and that its illusions, or those with which we are alone concerned, have not been about things remote or abstract, things transcendental or divine, but about what men see and handle, about those 'plain matters of fact' among which common sense 
daily moves with its most confident step and most self-satisfied smile. If this conclusion be accepted, its consequences extend to other organs of knowledge besides those of perception. Not merely the senses, but the intellect, must be judged by it; and it is hard to see why evolution, which has so lamentably failed to produce trustworthy instruments for obtaining the raw material of experience, should be credited with a larger measure of success in its provision of the physiological arrangements which condition reason in its endeavours to turn experience to account. . . . Natural science must ever regard knowledge as the product of irrational conditions, for in the last resort it knows no others."

While admiring Mr. Balfour's forensic ability and dialectic skill I wholly disagree with him as to the place to be assigned to the sense organs as instruments for acquiring knowledge useful for the ordinary purposes of life, and also for all kinds of research, even the most abstruse.

As I take it, nothing can be evolved which is not originally designed and potentially involved : ex nitito nitil fit. Organisms of all kinds, and sense organs in particular, are original creations: they are not haphazard, chance productions, as Mr. Darwin and Mr. Balfour believe. Neither are they the outcome of spontaneous generation or evolution. The organs of the simpler and more complex organisms are fundamental-that is, they make their appearance at the same time as the organisms themselves. They are not after-thoughts. On the contrary, they are provided by a First Cause for the well-being, guidance, and perpetuation of plants and animals. The organs are, in every case, means to ends, and the information they give concerning external things, near and remote, is reliable and not illusory.

It is inconceivable that the Creator could or would have provided animals-even the most degraded-with organs expressly constructed (and at the outset) to mislead and deceive, and when the argument is applied to man and tested by facts it simply falls to pieces. Mr. Balfour admits that the sense organs were constructed ages before they were required, and when man "was in the making." This, it appears to me, is a fatal admission to his theory, as it is an unstinted acknowledgment, not of natural selection, but of prescience and design on the part of the Creator.

Natural selection, which is said to work in an accidental, haphazard, blind way, cannot possibly take the place of an intelligent First Cause. Natural selection, moreover, if it really exists, which I greatly doubt, cannot operate upon living things before they are formed, and while yet in the womb of the future.

The subject under discussion is less one of philosophy than of common sense.

If there is one thing more certain than another in the universe it is that plants and animals are graduated -man forming the apex of a pyramid with a very wide base. It is further all but certain that plants and animals are arranged according to types which, while they occasionally approach indefinitely near, do not merge into each other or lose their identity. This explains why gaps of greater or less magnitude are constantly found in the flora and fauna of the globe. Each plant and animal is perfect after a fashion, and each is provided with the means for guiding itself and maintaining its place in nature. The guiding power in the higher animals takes the form of sense organs. In the lower animals it appears as sensitive integument, sensitive hairs, sensitive antennæ, rudimentary eyes, ears, \&c. As showing the special and designed nature of the sense organs in the lower animals, it happens that ever and anon a sense organ is hyper-developed, hence the compound eye of the insect, the far-seeing eye of the eagle, the keen hearing of the stag, the remarkable smelling power of the dog, \&c. Here we have specialisations added to original endowment. Not only special organs of sense, but certain sense organs are elaborated and differentiated to achieve predetermined and definite results in particular directions.

If it be stated that the sense organs were formed countless ages before they were required, it is quite evident that they cannot be the offspring of environment or of any set of external conditions; the light can no longer be regarded as forming the eye, sounding bodies the ear, smelling particles the nose, sapid substances the tongue and palate, and extraneous substances the skin.

The formation of the sense organs in the lower animals before the advent of man, and ages before they were required for the guidance of the animals possessing them, effectually disposes of the claims put forth by the advocates of so-called natural selection. This is one of the strongest possible arguments for design, and puts the Creator and the thing created in their proper places.

If when animals are being created they are furnished with guiding powers in the shape of sense organs or otherwise, and if, further, the organs referred to are formed before they are required, it is clear that the organs are part of the original animals, and absolutely necessary to its existence. This is a wide question, but it is at the root of existence in the animal kingdom. Deprive an animal of its sense organs, or what represents them, and the animal is doomed to death and the race to early extinction. It is impossible to conceive-or, if conceived, to believe - that the sense organs on which so much intelligence and design have been lavished are added (and at 
the outset) to an animal simply to mislead and deceive it. It might very well be asked what is the object of life and of sense organs in animals if it be not self-guidance, self-preservation, the power of reproduction, and the capacity for living in harmony with other animals, with their natural surroundings.

The world is not an illusion. It exists and is composed of a congeries of the most marvellous substances each adapted to the other. Everything about it is carefully thought out. The inorganic and organic kingdoms each play a distinct rôle, but they nevertheless work in unison and according to a general plan. Nothing comes by chance. All nature is co-ordinated and the boundaries of its actions and re-actions are accurately limited and defined. The flora and fauna of the earth are complemental in the widest sense. Each is adapted to the other. The several parts of animals, and especially their sense organs, are necessary to the perfect individual. They are original and fundamental, but not redundant parts, and cannot be removed without inflicting a certain amount of injury on the individual. The sense organs are never "make believes." They can always be relied upon to give an accurate idea of things as they are. The line of argument here adopted shows how wide of the truth are all statements impugning the reliability of the sense organs in man. The subject assumes a graver aspect when we are told that not only do the sense organs habitually deceive us, but that they cannot assist us in our quest after science and the higher learning.

It will be an evil day for science and the higher learning when they are divorced from the sense organs and relegated to the whimsical speculation of the closet philosopher.

I know of nothing more mischievous than the depreciating and belittling of the knowledge supplied by the sense organs, either as regards its accuracy or its amount. Similarly, I know of nothing more illusory and disappointing than placing reliance on the supposed facts furnished by the inner consciousness-facts which the sense organs can alone supply.

There is no breach of continuity in the development of intellect from sense impressions as originally supplied by the skin and the sense organs. The individual who is born devoid of general sensibility and the sense organs cannot deal with even the most ordinary concerns of life. Still less can he grapple with the higher learning and the more advanced problems of science. If this be so it is mere waste of time to discuss the possibility of any one successfully dealing with the more recondite problems of existence, especially if he does not fall back upon the sense organs for his training, experiences, and ultimate intellectual power.

They attempt an impossible task; they seek to build a palace-a great airy structure-without foundations. The sense organs, I venture to assert, furnish the basis of all legitimate speculation regarding the external world; without them there can be no trustworthy training for the acquisition of knowledge in the concrete.

The senses, if not perfect at the outset, can be educated. Nature makes provision for this. The eye can avail itself of the microscope and telescope; the ear can utilise the telegraph, telephone, phonograph, \&c.; and the senses of smelling, tasting, and touching can be sublimated and refined.

The modern microscope, with its increased multiple powers and its greatly improved definition, gradually introduces us to finer and finer matter. The same is true of the spectroscope with its matchless capacity for analysis. This instrument possesses the extraordinary power of dealing with incandescent matter at near and incalculably remote distances, its bands of colour revealing unmistakably the metals present in any particular glowing body, wheresoever situated. The telescope and modern photography also contribute to the analysis of the heavenly bodies in their own peculiar way; nor must modern chemical analysis, with its numerous startling advances and refinements, be overlooked. The several analytic processes have all the one object, namely, the training and perfecting of the sense organs and the revelation of matter in its finer and subtler forms.

The philosophers who ignore the sense organs literally kick away the ladder by which they have mounted.

If there is a limit to the operations of the sense organs so there is a limit to the efforts of self-consciousness and of intellect.

Imagination, however brilliant, can never take the place of fact, and so long as we are composed of matter, and the extraneous universe is material in its nature, it is idle to attempt either to ignore or evade the grosser matter in favour of a subtler matter.

A simple example will illustrate my meaning. We look at a table in the centre of a well-lighted room, and can have no reasonable manner of doubt that the eye informs us correctly of its presence and general appearance. It is a matter of no moment for my present contention whether the table be composed of the grosser matter represented by the time-honoured atoms and molecules or by the ether in vortex motion, electrical corpuscles, electrons, ions, \&c. The same sense organs and the same intellect furnish the knowledge requisite for expiscating both theories: moreover, the two theories are not necessarily exclusive of each other, any more than the sense organs and the brain which they inform and educate are exclusive. 
If the sense organs mislead and deceive, it is because they are in certain instances abnormal or diseased. The eye may give false information by its being colour-blind, by its being astigmatic, by its being myopic or presbyopic, \&c. Similarly the ear may distort facts by its being deaf, by its being exposed to noises in the head or subject to musical spectra, by its being plugged with wax, by the closing or partial closing of the Eustachian tube, by disease of the bones, \&c.

In like manner the nose, mouth, and palate may abrogate their functions because of a severe catarrh in the head, and the sensibility of the skin may be heightened or lowered as the result of certain skin disorders.

These abnormal results must be corrected if the sense organs are to be taken as reliable guides. The normal and abnormal conditions referred to are to be placed in different categories. There is no excuse for mixing the two and constructing a theory on the latter.

The history of the sense organs affords an all-sufficient reply to Mr. Balfour's ingenious speculations.

At the dawn of creation even the simplest organisms were endowed with powers which assured their life, growth, and continuance. At the outset they had no experience to guide them, and, as they could not take care of themselves, they must have been cared for. As time advanced, more complex organisms succeeded the simpler ones; these were provided with organs of various kinds - the higher being furnished with sense organs. The sense organs were connected with special nervous arrangements, and these nervous arrangements culminated in brains of greater or less magnitude and power. The sense organs and the brain and its manifestations (intellectual faculties) became blended and identified; they also interacted; the sense organs became the handmaidens of the brain, which is the most inscrutable and wonderful of living substances. Mind and matter were united to each other. The sense organs were specialised living-structures, and they discharged special functions; the structures preceded the functions. The sense organs were conferred upon animals to guide and not to mislead them. If the senses do not enable us fully to realise the peculiarities of ultimate matter, it is because they are not sufficiently educated. If they do not convey all the knowledge desiderated and which we could wish, it does not follow that the information communicated is not accurate information so far as it goes. To admit defects in the organs of sense is to throw away the weapons by which we hope to conquer ignorance and reach the highest goals in science.

It cannot be doubted that the organs of sense were originally conferred upon the higher animals and man for their guidance and to impart a knowledge of things external. They are the gateways through which everything outside of ourselves enters the great nervous emporium, the brain. A man minus his sense organs would be absolutely helpless; a man with highly developed sense organs is armed at every point, and master of the situation. He knows and can maintain his place in nature.

One of the chief stumbling-blocks in modern zoology and physiology concerns the order of nature. Some there are who assert that the organs-even the organs of sense-are produced by a felt need on the part of the animal; in other words, that function precedes and is the cause of structure. This means that an animal, by willing and voluntary effort, can compel its organs_-organs of sense and otherwise-to be born and grow. Nothing can be more delusive. We have proof to the contrary in our own persons. No individual, however anxious or determined, can, by willing, add to or take from his original structures. These structures make him what he is, independently of himself and in spite of himself.

The history of animals is a history of their organs. The organs are increased as we rise in the scale of being, but, in every instance, they are means to ends; they represent division of labour and increased capacity and accuracy. They represent arrangements which enable us to deal with and interpret extraneous matter in the aggregate and to a great extent in detail. While the organs are forming they are practically of no value; this is specially the case in the embryo and foetus-a circumstance which precludes the operation of natural selection in any form. Natural selection, it is claimed, utilises if it does not produce variations from the utilitarian point of view, to meet demands set up by environment or extraneous conditions. Natural selection, however, as I have already explained, could, allowing it to be a reality, only act upon structures already in existence. Neither evolution nor natural selection could, unaided, produce the organs, and least of all the sense organs.

If we are ever to attain to an accurate and extensive knowledge of things without, and, in certain cases, within, ourselves, it seems to me self-evident that we must cultivate to the utmost, not only our sense organs but also the brain of which they are, strictly speaking, accessories and extensions. No mere process of abstract reasoning will ever satisfactorily explain the riddles of the universe. The cosmos cannot be accounted for by an effort of the inner consciousness; the philosopher, physicist, chemist, physiologist and psychologist must all work together and to a given end if satisfactory progress is to be made in the higher learning and in science. 


\section{INTERACTION BETWEEN MENTAL AND MATERIAL ASPECTS}

\section{$\S 32$. Interaction between the Mental and the Material Aspects of Things.}

This is the title of a paper recently published by Sir Oliver Lodge (Nature, April 3, 1903), and as it has an important bearing on the foregoing section I cannot do better than give a digest of it.

Sir Oliver is an aclnowledged authority in physics, especially electricity, and an interesting writer on the border line which divides the real and tangible from the unseen and spiritual. He sets out by giving definitions of science, faith, prayer, and God. He observes: "All these ambiguous terms are liable to enter into our present discussion, which concerns, I take it, fundamentally the inter-communion and interaction between the divine and the human, chiefly in the regions of volition and of action on the physical world. The influence of the divine on the human has been variously conceived in different ages, and various forms of difficulty have been at different times felt and suggested; but always some sort of analogy between human action and divine action has had perforce to be drawn in order to make the latter in the least intelligible to our conception. The latest form of diffculty is peculiarly deep-seated, and is a natural outcome of an age of physical science. It consists in denying the possibility of guidance or of control, not only on the part of a Deity, but on the part of every one of his creatures. It consists in pressing the laws of physics to what seems their logical and ultimate conclusion, in applying the conservation of energy without ruth or hesitation, and so excluding, as it has seemed, the possibility of free-will action, of guidance, of the self-determined action of mind or living things upon matter altogether. The appearance of control has been considered illusory, and has been replaced by a doctrine of pure mechanism, enveloping living things as well as inorganic nature.

"And those who for any reason have felt disinclined or unable to acquiesce in this exclusion of non-mechanical agencies, whether it be by reason of faith and instinct, or by reason of direct experience and sensation to the contrary, have thought it necessary of late years to seek to undermine the foundations of physics, and to show that its much-vaunted laws rest upon a hollow foundation, that their exactitude is illusory, that the conservation of energy, for instance, has been too rapid an induction, that there may be ways of eluding many physical laws and of avoiding submission to their sovereign sway.

"By this sacrifice it has been thought that the eliminated guidance and control can philosophically be reintroduced.

"This, I gather, may have been the chief motive of an attack on physics led by an American, J. B. Stallo, in a little book called the 'Concepts of Physics,' which has at various times attracted some attention. But the worst of that book was that Stallo was not really familiar with the teachings of the great physicists.

"The armoury pressed into the service of Professor James Ward's attack is of weightier calibre, and his criticism cannot in general be ignored as based upon inadequate acquaintance with the principles under discussion; but still his Gifford lectures raise an antithesis or antagonism between the fundamental laws of mechanics and the possibility of any intervention, whether human or divine.

"If this antagonism is substantial it is serious; for natural philosophers will not be willing to concede fundamental inaccuracy or uncertainty about their recognised and long-established laws of motion, nor will they be prepared to tolerate any, even the least, departure from the law of the conservation of energy. Hence, if guidance and control can be admitted into the scheme by no means short of refuting or modifying those laws, there may be every expectation that the attitude of scientific men will be perennially hostile to the idea of guidance or control, and so to the efficacy of prayer, and to many another practical outcome of religious belief. It becomes therefore an important question to consider whether it is true that life or mind is incompetent to disarrange or interfere with matter at all, except as an automatic part of the machine-or rather, except as an ornamental appendage or dependent accessory of its working parts.

"Now experience-the same kind of experience as gave us our scheme of mechanics-shows us that to all appearance live animals certainly can direct and control mechanical energies to bring about desired and pre-conceived results - for example, the Forth Bridge. Undoubtedly our body is material, and can act on other matter, and its energy is derived from food, like any other self-propelled and fuel-fed mechanism: the question is whether our will or mind or life can direct our body's energy along certain channels to attain desired ends ; or whether direction, as well as amount, of activity is wholly determined by mechanical causes.

"Answers that might be given are :-

"(a) That life is a form of energy, and achieves its results by imparting to matter energy that would not otherwise be in existence, in which case life is a part of the machine, and as truly mechanical as all the rest. I hold that this is false; because the essence of energy is that it can transform itself into other forms, remaining constant in quantity, whereas life does not transmute itself into any form of energy, nor does death affect the sum of energy in any known way. 
"(b) That life is something outside the scheme of mechanics, although it can nevertheless touch or direct material motion, subject always to the laws of energy and all other mechanical laws; supplementing them, but contradicting or traversing them no whit.

“This I hold to be true; but in order to admit its truth we must recognise that triggers can be pulled-force exerted, and energy directed-without any introduction of energy from without; in other words, that the energy of operations automatically going on in any active region of the universe-any region where transformation and transference of energy are continuously occurring, whether life be present or not-that this energy can by means of life be guided along paths that it would not automatically have taken, and can be directed so as to produce effects that would not otherwise have occurred; and this without any break or suspension of the laws of dynamics.

"That is where I part company with Professor James Ward in the second volume of "Naturalism and Agnosticism,' notwithstanding that I feel sure that Mr. A. J. Balfour agrees with him.

"Those who take his view must either throw overboard the possibility of interference or guidance or willed action altogether, which is one alternative, or must assume that the laws of physics are only approximate and incomplete, which is the other alternative-the alternative favoured by Professor James Ward. I wish to argue that neither of these alternatives is necessary, and that there is a third or middle course of proverbial safety.

"On a stagnant and inactive world life would be admittedly powerless; it could only make dry bones stir in such a world if itself were a form of energy; I do not suppose for a moment that it could be incarnated on such a world; it is only potent where inorganic energy is in constant process of transfer and transformation. In other words, life can generate no trace of energy, it can only guide it.

"Guidance is a passive exertion of force without doing work; as a quiescent rail can guide a train to its destination, provided an active engine propels it. If a stone is rolling over a cliff, it is all the same to 'energy" whether it fall on point $A$ or point $B$ of the beach. But at $A$ it shall merely dent the sand, whereas at B it shall strike a detonator and explode a mine. . . John Stuart Mill used to say that our sole power over Nature was to move things; but strictly speaking we cannot do even that; we can only arrange that things shall move each other, and can determine by suitably pre-conceived plans the kind and direction of the motion that shall ensue at a given time and place. Provided always that we include in this category of 'things' our undoubtedly material bodies, muscles and nerves.

"But here is just the puzzle; at what point does will and determination enter into the scheme? Contemplate a brain cell, whence originates a certain nerve-process whereby energy is liberated with some resultant effect; what pulled the detent in that cell which started the impulse? No doubt some chemical process, combination or dissociation, something atomic, occurred; what made it occur just then and in that way?

"I answer, the same sort of pre-arrangement that determined whether the stone from the cliff should fall on point $A$ or point $B$. The same kind of process that determines when and where a trigger shall be pulled so as to secure the anticipated slaughter of a bird. So far as energy is concerned, the explosion and the trigger-pulling are the same identical operations, whether the aim be exact or random. It is vitality which directs; it is physical energy which is directed and controlled both in time and space.

"I lay stress upon a study of the nature and mode of human action of the interfering or guiding kind, because from it we must be led if we are to form any intelligent conception of divine action. True, it might be possible to deny human agency or power and yet to admit the possibility of divine agency, though that would be a nebulous and at least inconclusive procedure; but if we are once constrained to admit the existence and reality of human guidance and control, we cannot deny the possibility of such powers and action to any higher being, nor even to any totality of things of which we are a part.

"The point immediately at issue turns upon the distinction between 'force' and 'energy.' These terms have been so popularly confused that it may be difficult always to discriminate them, but in physics they are absolutely discriminated. A force in motion is a "power'; it does work, and transfers energy from one body to another. But a force at rest-a mere statical stress, like that exerted by a pillar or a watershed-does not work, and alters no energy; yet the one sustains a roof which would otherwise fall, thereby screening a portion of ground. from vegetation; while the other deflects a rain-drop into the Danube or the Rhine.

"It will be said some energy is needed to pull a hair-trigger, to open the throttle-valve of an engine, to press the button which shall shatter a rock. Granted; but the work-concomitants of that energy are all familiar, and equally present whether it be so arranged as to produce any pre-determined effect or not. The opening of the throttle-valve, for instance, demands just the same exertion, and results in just the same imperceptible transformation of fully-accounted-for energy, whether it be used to start a train in accordance with a time-table and the guard's whistle, or whether it be pushed over as by the wind at random.

The shouting of an order to a troop 
demands vocal energy, and produces its due equivalent of sound; but the intelligibility of the order is something superadded, and its result may be to make not sound or heat alone, but history.

"Energy is needed to perform any physical operation, but the energy is independent of the determination or arrangement. Guidance and control are not forms of energy, and their superposition upon the scheme of physics perturbs physical and mechanical laws no whit, though it may profoundly affect the consequences resulting from those same laws. The whole effort of civilisation would be futile if we could not guide the powers of nature. The powers are there, else we should be helpless; but life and mind are outside those powers, and can direct them along an organised course.

"And this same life or mind, as we know it, is accessible to petition, to affection, to pity, to a multitude of non" physical influences; and hence, indirectly, the little plot of physical universe which is now our temporary home has become amenable to truly spiritual control."

Sir Oliver Lodge's chief contentions are :-

(1) That the fundamental laws of physics, complete and accurate as they are, in no way exclude guidance of events by the agency of life or mind or other unknown influence.

(2) That common experience shows that living creatures do exert such guidance, and further, that they are amenable to non-material or spiritual influences from each other.

The testimony of Sir Oliver Lodge in favour of a directing agency in the universe is of the greatest possible importance. Not less important is his belief in life as a guiding power. The merely mechanical or automatic view of the cosmos advocated by Haeckel and others fails, it appears to me, at every turn and in every direction. The time, there is reason to believe, is not far distant when a strong reaction will set in against the fashionable physics of modern times, as against a slow-working mechanical evolution and as against so-called natural selection, in which, as explained, I do not believe. Theories which require unlimited time (untold millions of years) for their realisation, and which rely on accident and chance rather than on a reasoned plan and certainty, can never be other than disappointing when critically examined by the unbiassed inquirer.

\section{MATTER AND FORCE IN THE INORGANIC AND ORGANIC KINGDOMS-THEIR RELATION TO LIFE, AND TO PLANTS AND ANIMALS}

\section{Author's Views regarding Creation, Evolution, Natural Selection, Type, Locomotion, Environment, Geology, \&c.}

Various attempts have been made of late years to reduce all matter to one, and all force to one; the object being to identify organic and inorganic matter, and vital and physical force. It has also been contended that matter and force are inseparable, that matter inevitably exerts force, and that no force can exist as apart from matter. It has further been asserted that matter is eternal and occupies all space, that it had no beginning and will have no end, that it is self-created and self-moving, that it is in a perpetual state of flux, continually entering into new combinations, breaking up and re-forming itself, that it is a fixed quantity and admits of change of shape but of no increase or decrease, that it is indestructible. Similar attempts have been made to unify force. ${ }^{1}$ The transformations occurring in matter and force are said to be incessant and infinite, and these transformations occur in the atoms and molecules and in the tiniest specks of matter, as well as in the nebulæ, stellar bodies, suns, great world spheres, \&c.

It has also been maintained that organic matter is the natural product, under certain circumstances, of inorganic matter, and that plants and animals are the outcome of spontaneous generation, and owe their existence to the operation of purely mechanical, physical, and chemical processes; that, in short, there is no such thing as living matter as apart from the inorganic matter and force of the universe. The idea of a Creator and design in the cosmos is wholly ignored. Matter and force, according to certain scientists, have created themselves, and established their own laws, and these laws are inexorable. The world and all it contains, in their opinion, has been formed and is regulated, not by an intelligent agent or First Cause (a Creator), but by blind chance and accident; the inorganic dead world - giving rise to the organic living world-spontaneous generation, environment, natural selection, and external stimulation being all that are required to produce all known results provided sufficient time be allowed.

1 The indestructibility of matter is comparatively a very old doctrine: the modern view of the indestructibility of force was especially worked out by Robert Mayer (1842) and Hermann Helmholtz (1847).

VOL, I, 


\section{THE ELEMENTS AND THEIR COMBINING WEIGHTS}

The present section will be most fitly terminated by the insertion of a table of "elements with their combining weights." The table has been carefully prepared for me by Dr. John Ferguson, Professor of Chemistry, University, Glasgow, whose kindness I have much pleasure in acknowledging.

The belief in the existence of elements, notwithstanding all that has been written to the contrary, is practically universal. Even such an astute and advanced chemist as Mendeléeff regarded the existence of elements as certain. This famous Russian savant, so late as 1906, in discussing the "Periodic Law," in which some profess to detect a proof of the validity of the assumption of a primordial matter, gave it as his opinion that such a view was untenable. He declined to believe that such an inference was warranted. He saw nothing in the law inconsistent with the idea of the individuality of the elements, holding that until it could be definitely shown that one element could be transformed into another, or that ether and matter were mutually convertible, the elements must be regarded as distinct and separate entities, immutable and unchangeable.

\section{THE ELEMENTS AND THEIR COMBINING WEIGHTS}

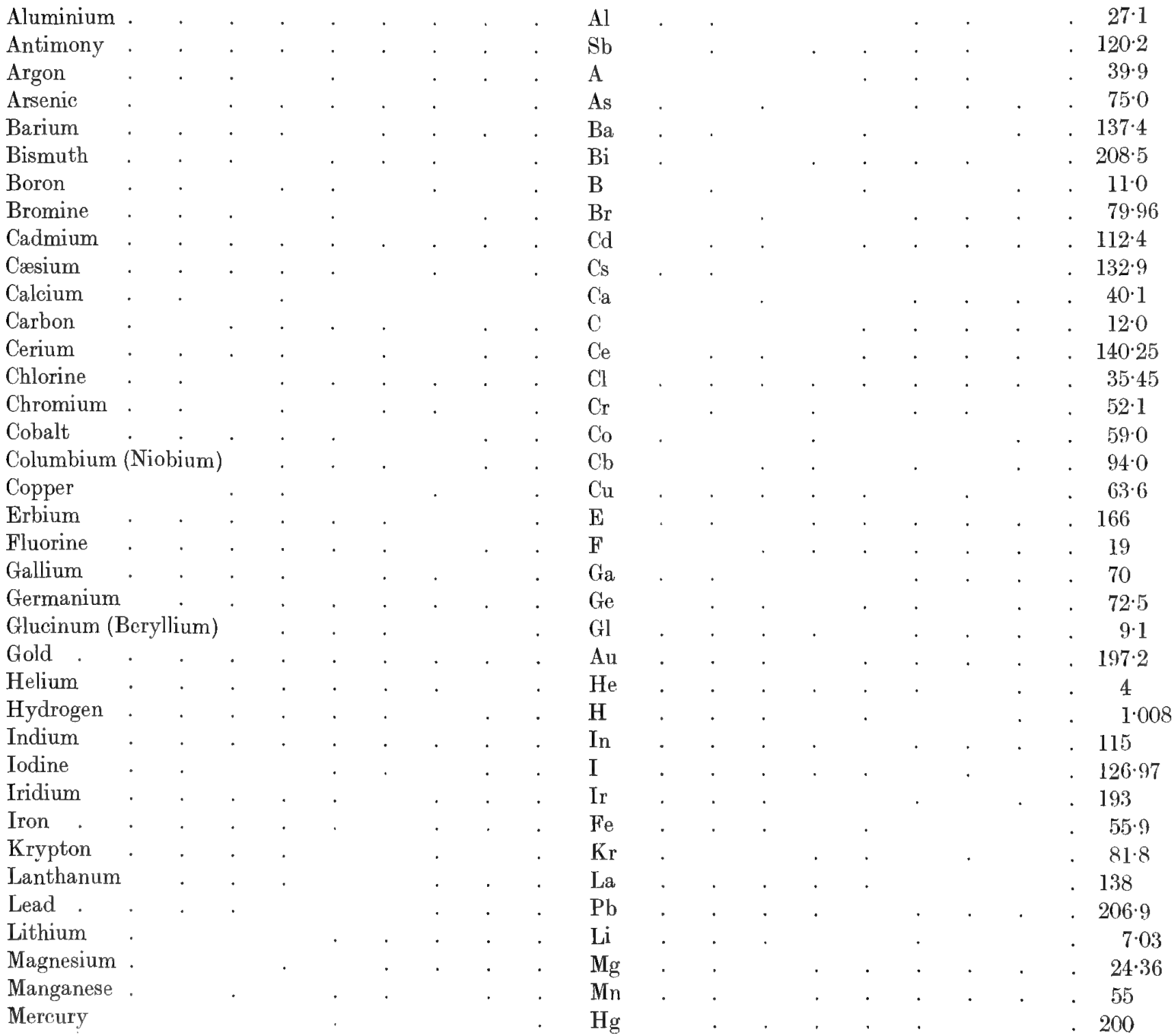




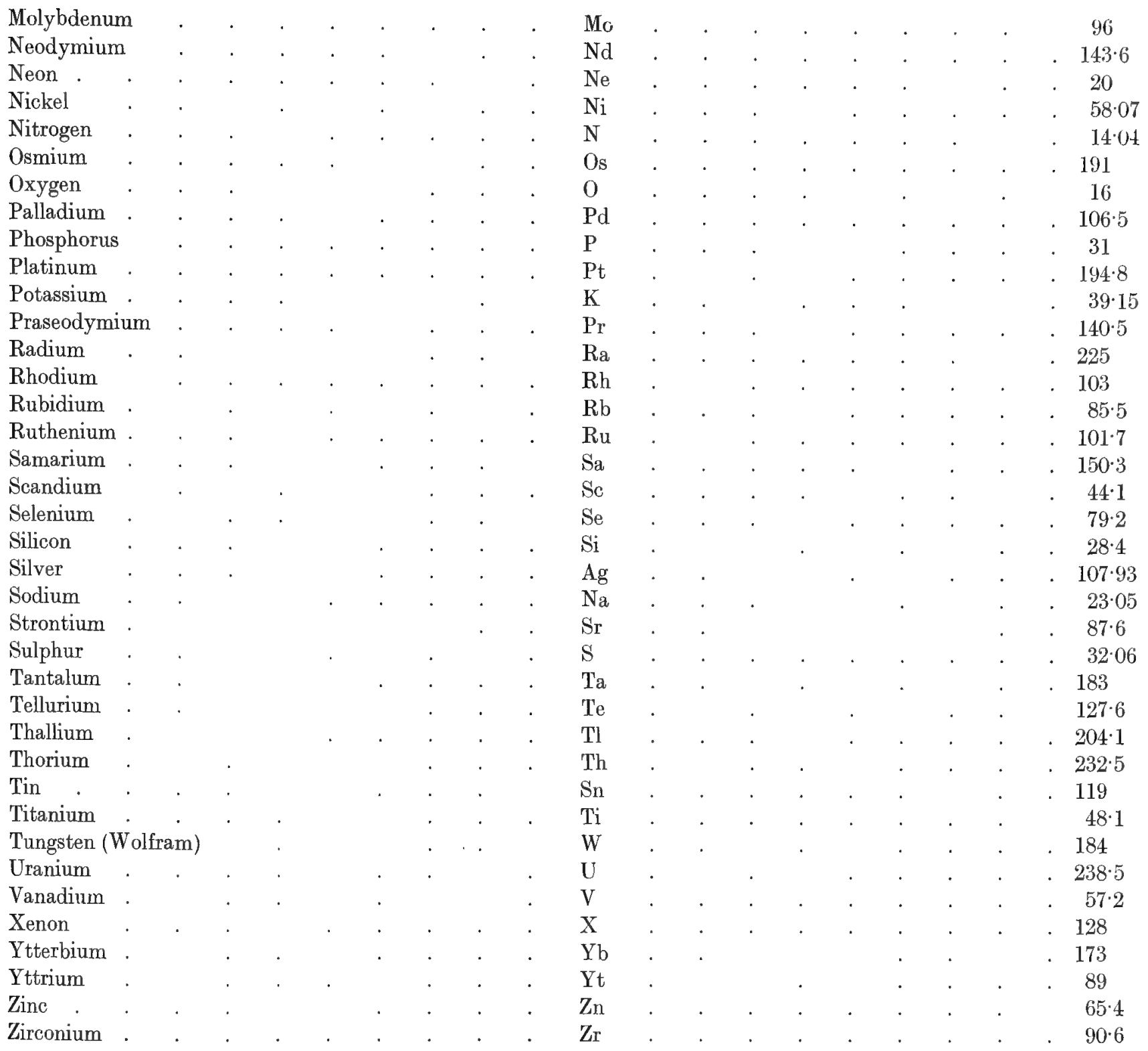

Those who advocate the doctrine that there is only one kind of matter in the universe, and one kind of force, assign to plants and animals the power of modifying and adapting themselves indefinitely (which really means fashioning and in a manner creating themselves); the modifications and adaptations extending over millions of years-periods utterly incomprehensible to even the ablest mathematicians.

Plants and animals are said to select and employ those powers, substances, properties, and qualities in themselves which make for advance and a steady ascent towards perfection, in the absence of a First Cause and design. The power of natural selection is claimed for the simplest and most rudimentary plants and animals, from the monad to the man. Similar powers are claimed for molecules and cells in reproduction and development. Latterly, like claims have been made for inorganic atoms, nebulæ, and all the celestial bodies wheeling in space. In this connection it should be stated that selection implies_nay more, involves --intelligence, for it necessitates the capacity to discriminate, assort, separate, adopt, reject, \&c.

Intelligence is a sine qua non, and the choice lies between intelligence in the atom, the molecule, the speck of protoplasm, the cell, or the tissue, and the Creator Who made all and Who modifies, controls, and adapts all for specific purposes. In order to give coherence to the extraordinary theories propounded by certain investigators as to the omnipotence of matter and force, and the complete absence of a First Cause and of life, the atoms are said to exert likes and dislikes, and to possess what is virtually a mind, and an anima or soul. Intelligence of a kind 
is conceded, but the advanced mechanical school attributes it to the atoms and not to the Deity Who made, energises, and controls the atoms. The school in question transfers to matter the intelligence, or its equivalent, hitherto attributed to the Deity. According to it there is no duality in nature; no Creator and created; no tangible substance and spirit; no free-will; no life after death; no immortality. As there is no getting away from adaptation, which is another word for design, those who deny the existence of a Maker and Ruler of the universe are forced to ascribe a self-forming, and self-moving, and adaptive power to everything the universe contains. They, however (and let this be noted), fail to account for the matter, force, life, and design which they encounter all along the line of their investigations. They assume all these things, and say that matter and force are eternal and indestructible, that life is the outcome of matter, and that design is mere adaptation. What cannot be proved they boldly take for granted. They ridicule faith in the unseen in one direction, and extol it in another direction when it suits their purpose.

\section{§33. Haeckel's Belief in the Omnipotence of Matter.}

Of all those who have written on the potency of matter in its relation to life, force, and cognate subjects, Professor Ernst Haeckel is entitled to take a foremost place. He may be considered the head of the material or matter "cult." I do not agree with his views, but he is entitled to an unbiassed hearing, and I propose to give a somewhat extended account of his conclusions-as far as possible in his own words-in order that the reader may be supplied with the facts necessary to form an independent judgment. Haeckel's peculiar tenets are well known on the continent of Europe, but have not been much studied at home; a circumstance which makes it imperative to consider them somewhat fully in this portion of the work.

Professor Haeckel advocates spontaneous generation, evolution, natural selection, heredity, descent, \&c., in their widest sense. He completely ignores a First Cause and design, and assigns the universe and all it contains to accident and blind chance. He preaches matter pure and simple, and excludes everything which does not centre in matter, and which cannot be explained by it. He regards the highest animals, including the monkeys and man, as the terminal links of one long, unbroken, continuous chain, which extends itself through time and space, beginning with the simplest conceivable living forms (plant and animal), and culminating in the most complex, as represented by man. With him substance is everything, and there is only one living substance which has the power of modifying and adapting itself indefinitely at the bidding of environment, extraneous stimulation, natural selection, accident and other inadequate causes; the simplest plants and animals being, in every instance, the parents or progenitors of the more complex, up to man himself.

In his opinion the most perfect and higher forms are manufactured by endless trifling modifications in the fulness of time out of the rudimentary and lower forms, in one continuous, unbroken series, as apart from a First Cause, design, and types.

The idea of separate creations of types in plants and animals is set aside. With him every living thing runs into every other living thing, from the monad to the molluse, and from the mollusc to the man. All lines of demarcation, all boundaries as between living things, are swept away, and one vast, interminable, inextricable tangle substituted. The principle of a designed, ascending series of plant and animal organisms, with variations within limits, is tabooed. There is no halting-place, no room or opportunity for definitions, classification, or orderly arrangement. No barriers of infertility are recognised.

If, as Professor Haeckel and Mr. Darwin say, species are the result of modification, and are mutable up to a point, then species, according to their own showing, are illusory, inasmuch as fixity cannot logically result from continuous modification, however gradual and however prolonged. A species which is produced by a series of changes cannot consistently become stable or permanent at any period of its history. The reader has to decide as between a chaos of accidental modifications and adaptations in plants and animals extending over illimitable periods, and a designed whole in which plants and animals have, from the first, or at different periods, their limits or boundaries determined. In both cases there is continuity, but in the one the continuity is due to the endless and accidental modification of one substance as apart from a First Cause, design, and life, while in the other the continuity is due to the intelligent modification of various substances with life and a First Cause as dominant factors.

In the former instance, development and heredity apply to the whole organic kingdom, there being no boundaries or natural divisions as between the several plants and animals; in the latter instance, they apply to groups and types of plants and animals where divisions can be recognised and an orderly arrangement more or less satisfactorily established. In the one case, the ascending series consists, so to speak, of one long continuous flight of steps; in the other of several flights of steps with halting places between; that is, points of departure, 


\section{HAECKEL'S BELIEF IN THE OMNIPOTENCE OF MATTER}

and goals, destinations, or points of arrival ; the steps being not only separated from each other, but also arranged in groups which can be individually treated as regards time and space. The ultimate result is substantially the same, but the mode of arriving at that result is wholly different.

Of course, there is no necessity to divide or separate plants and animals into categories, but as one great plan obviously runs through the whole of nature, and design is everywhere apparent, it follows that there must be an orderly sequence with boundaries of a more or less rigid description. These boundaries make classification on a broad basis possible, but the artificial classifications of the makers or rather the manufacturers of species are not thereby endorsed or sanctioned. I find, for example, that living, and even dead matter, lends itself to radiating and concentric arrangements, and that it tends to split up longitudinally and transversely, and to produce fission and segmentation as seen in the branches and stems of plants, and in the limbs, vertebræ, \&c., of animals. This circumstance makes it possible to establish a classification outside all existing classifications, and of a more primitive or fundamental kind. The classifications of botanists, zoologists, and Palæontologists may be very partial and very inaccurate, but the inability adequately to grasp the situation does not seriously impair the great argument for a First Cause and Design, and for the orderly arrangements which have for their object a series of graduated types disposed in a well-marked ascending scale.

Haeckel thus states his case: " Experience has never yet discovered for us a single immaterial substance, a single force which is not dependent on matter, or a single form of energy which is not exerted by material movement, whether it be of mass, or of ether, or of both. ... (1) The universe, or the cosmos, is eternal, infinite, and illimitable. (2) Its substance, with its two attributes (matter and energy), fills infinite space, and is in eternal motion. (3) This motion runs on through infinite time as an unbroken development, with a periodic change from life to death, from evolution to devolution. (4) The innumerable bodies which are scattered about the spacefilling ether all obey the same law of substance; while the rotating masses slowly move towards their destruction and dissolution in one part of space, others are springing into new life and development in other quarters of the universe. (5) Our sun is one of these unnumbered perishable bodies, and our earth is one of the countless transitory planets that encircle them. (6) Our earth has gone through a long process of cooling, before water, in liquid form (the first condition of organic life), could settle thereon. (7) The ensuing biogenetic process, the slow development and transformation of countless organic forms, must have taken many millions of years-considerably over a hundred..$^{2}$ (8) Among the different kinds of animals which arose in the later stages of the biogenetic process on earth, the vertebrates have far outstripped all other competitors in the evolutionary race. (9) The most important branch of the vertebrates, the mammals, were developed later (during the Triassic period) from the lower amphibia and the reptilia. (10) The most perfect and most highly-developed branch of the class mammalia is the order of primates, which first put in an appearance, by development from the lowest prochoriata, at the beginning of the Tertiary period-at least three million years ago. (11) The youngest and most perfect twig of the branch primates is man, who sprang from a series of man-like apes towards the end of the Tertiary period. (12) Consequently, the so-called 'history of the world '- that is, the brief period of a few thousand years, which measures the duration of civilisation-is an evanescently short episode in the long course of organic evolution, just as this, in turn, is merely a small portion of the history of our planetary system; and as our mother-earth is a mere speck in the sunbeam in the illimitable universe, so man himself is but a tiny grain of protoplasm in the perishable framework of organic nature. All the different philosophical tendencies may, from the point of view of modern science, be ranged in two antagonistic groups ; they represent either a dualistic or a monistic interpretation of the cosmos. The former is usually bound up with teleological and idealistic dogmas, the latter with mechanical and realistic theories. Dualism, in the widest sense, breaks up the universe into two entirely distinct substances-the material world and an immaterial God, Who is represented to be its creator, sustainer, and ruler. Monism, on the contrary (likewise taken in its widest sense), recognises one sole substance in the universe, which is at once "God and Nature'; body and spirit (or matter and energy) it holds to be inseparable. The extra-mundane God of dualism leads necessarily to Theism; the intra-mundane God of the monist to Pantheism. . . The whole animal world falls into two essentially different groups, the unicellular primitive animals (Protozoa) and the multicellular animals with complex tissues (Metazoa). The entire organism of the protozoon (the rhizopods or the infusoria) remains throughout life a single simple cell (or occasionally a loose colony of cells without the formation of tissue, a coenobium). The organism of the metazoon, on the contrary, is only unicellular at the commencement, and is subsequently built up of a number of cells which form tissues. The theory of a cell-soul is completely established by an accurate study of the unicellular protozoa, the psychic phenomena of the protistæ form, the bridge which unites the chemical

1 "The Riddle of the Universe," by Professor Ernst Haeckel, M.D., LL.D., D.Sc., \&c. Watts \& Co., London, 1902.

a It will be observed that in $(6)$ and (7) Haeckel tacitly refers the existence of life on the earth to spontaneous generation, a wholly discredited and impossible doctrine. 
processes of inorganic nature with the mental life of the highest animals. For all these phenomena-indeed, for all phenomena both in nature and in the mind-Lamarck takes exclusively mechanical, physical, and chemical activities to be the true efficient causes. Darwin refers them to natural selection. . . All the phenomena of the psychic life are, without exception, bound up with certain material changes in the living substance of the body, the protoplasm. We have given to that part of the protoplasm which seems to be the indispensable substratum of psychic life the name of psychoplasm (the soul-substance in the monistic sense); in other words, we do not attribute any peculiar "essence" to it, but we consider the psyche to be merely a collective idea of all the psychic functions of protoplasm. In this sense the 'soul' is merely a physiological abstraction like 'assimilation' or 'generation." .. In the unicellular protists, the psychoplasm is identified either with the whole of the living protoplasm of the simple cell or with a portion of it. In all cases, in the lowest as well as the highest stages of the psychological hierarchy, a certain chemical composition and a certain physical activity of the psychoplasm are indispensable before the 'soul' can function or act. That is equally true of the elementary psychic function of the plasmatic sensation and movement of the protozoa, and of the complex functions of the sense-organs and the brain in the bigher animals and man. The activity of the psychoplasm, which we call the 'soul,' is always connected with metabolism. . . All living organisms without exception have the faculty of spontaneous movement, in contradistinction to the rigidity and inertia of unorganised substances (for example, crystals); in other words, certain changes of place of the particles occur in the living psychoplasm from internal causes, which have their source in its own chemical composition. . . . At the lowest stage of organisation, in the lowest protists, the stimuli of the outer world (heat, light, electricity, \&c.), cause in the indifferent protoplasm only those indispensable movements of growth and nutrition which are common to all organisms and are absolutely necessary for their preservation. That is also the case in most of the plants. . . The automatic and the reflex movements which we observe everywhere, even in the unicellular protists, seem to be the outcome of inclinations which are inseparably connected with the very idea of life. Even in the plants and lowest animals these inclinations, or tropisns, seem to be the joint outcome of the inclinations of all the combined individual cells. When two cells meet as a result of copulation, or when they are brought into contact through artificial fertilisation! (in the fishes, for instance), they attract each other and become firmly attached. The main cause of this cellular attraction is a chemical sensitive action of the protoplasm, allied to smell or taste, which we call 'erotic chemicotropism '; it may also be correctly (both in the chemical and the romantic sense) termed cellular affinity or 'sexual cell-love.' On a critical study of these different embryonic formations, the evolution of which from each other we can directly observe under the microscope, we arrive, by means of the great law of biogeny, at a series of most important conclusions as to the chief stages in the development of our psychic life. We may distinguish (1) Unicellular protozoa with a simple cell-soul: the infusoria. (2) Multicellular protozoa with a communal soul : the catallacta. (3) The earliest metazoa with an epithelial soul : the platodes. ... The earliest ancestors of man and all other animals were unicellular protozoa. This fundamental hypothesis of rational phylogeny is based, in virtue of the phylogenetic law, on the familiar embryological fact that every man, like every other metazoon (that is, every multicellular organism with tissues), begins his personal existence as a simple cell, the stem-cell (cytula), or the impregnated egg-cell. As this cell has a "soul' from the commencement, so had also the corresponding unicellular, uncestral forms, which were represented in the oldest series of man's ancestors by a number of different protozoa. ... Every living cell has psychic properties, and the psychic life of the multicellular animals and plants is merely the sum-total of the psychic functions of the cells which build up their structure. In the lower groups (in algæ and sponges for instance) all the cells of the body have an equal share in it (or with very slight differences); in the higher groups, in harmony with the law of the 'division of labour,' only a select portion of them are involved - 'the soul-cells.' . . . We find the highest development of the animal cell-soul in the class of ciliata, or ciliated infusoria. The unicellular protozoa give proof of the possession of a highly-developed 'cell-soul,' which is of great interest for a correct decision as to the psyche of our earliest unicellular ancestors. . . The tissue-soul (histopsyche): in all multicellular, tissue-forming plants (metaphyta) and in the lowest, nerveless classes of tissue-forming animals (metazoa) we have to distinguish two different forms of psychic activity-namely (1) the psyche of the individual cells which compose the tissue, and (2) the psyche of the tissue itself, or of the 'cell-state' which is made up of the tissues. This 'tissue-soul' is the higher psychological function which gives physiological individuality to the compound multicellular organism as a true 'cell-commonwealth.' It controls all the separate 'cell-souls' of the social cells-the mutually dependent 'citizens' which constitute the community. . . The plant-soul (phytopsyche) is, in our view, the summary of the entire psychic activity of the tissue-forming multicellular plant (the metaphyton, as distinct from the unicellular protophyton). . . The soul of the nerveless metazoa. Of very special interest for comparative psychology in general, and for the phylogeny of the animal soul in particular, is the psychic activity of those lower metazoa which have tissues, and sometimes differentiated organs, 


\section{HAECKEL'S BELIEF IN THE OMNIPOTENCE OF MATTER}

but no nerves or specific organs of sense. The nerve-soul (neuropsyche); the psychic life of all the higher animals is conducted, as in man, by means of a more or less complicated 'psychic apparatus.' This apparatus is always composed of three chief sections : the organs of sense are responsible for various sensations; the muscles effect the movements; the nerves form the connection between the two by means of a special central organ, the brain or ganglion. The ganglionic cells, or 'psychic-cells,' which compose the central nervous organ, are the most perfect of all organic elements; they not only conduct the commerce between the muscles and the organs of sense, but they also effect the highest performances of the animal soul, the formation of ideas and thoughts, and especially consciousness. The long ancestral history of our 'vertebrate-soul' commences with the formation of the most rudimentary spinal cord in the earliest acrania; slowly and gradually, through a period of many millions of years, it conducts to that marvellous structure of the human brain which seems to entitle the highest primate form to quite an exceptional position in nature. No phenomenon of the life of the soul is so wonderful and so variously interpreted as consciousness. Consciousness is a vital property of every cell. It is found in all organisms, animal or vegetal, but not in lifeless bodies (such as crystals). This opinion is usually associated with the idea that all organisms (as distinguished from inorganic substances) have souls : the three ideas-life, soul, and consciousnessare then taken to be co-extensive. Fechner has endeavoured to prove that the plant has a 'soul,' in the same sense as an animal is said to have one; and many credit the vegetal soul with a consciousness similar to that of the animal soul. In truth, the remarkable stimulated movements of the leaves of the sensitive plants (the mimosa, drosera, and dionaea), the automatic movements of other plants (the clover and wood-sorrel, and especially the Hedysarum), the movements of the 'sleeping plants' (particularly the papilionacea), \&c., are strikingly similar to the movements of the lower animal forms : whoever ascribes consciousness to the latter cannot refuse it to such vegetal forms. The peculiar phenomenon of consciousness is not, as du Bois-Raymond and the dualistic school would have us believe, a completely 'transcendental' problem; it is, as I (Haeckel) showed thirty-three years ago, a physiological problem, and, as such, must be reduced to the phenomena of physics and chemistry. The conception of the soul as a 'substance' is far from clear to many psychologists; sometimes it is regarded as an 'immaterial' entity of a peculiar character in an abstract and idealistic sense, sometimes in a concrete and realistic sense, and sometimes as a confused tertium quid between the two. If we adhere to the monistic idea of substance, we find energy and matter inseparably associated in it. We must distinguish in the 'substance of the soul!' the characteristic psychic energy which is all we perceive (sensation, perception, volition, \&c.), and the psychic matter, which is the indispensable basis of its activity - that is, the living protoplasm. Thus, in the higher animals the 'matter' of the soul is a part of the nervous system; in the lower nerveless animals and plants it is a part of the multicellular protoplasmic body; and in the unicellular protists it is a part of their protoplasmic cell-body. The supreme and all-pervading law of nature, the true and only cosmological law, is, in my opinion (Haeckel), the law of substance; all other known laws of nature are subordinate to it. Under the name of 'law of substance' we embrace two supreme laws of different origin and age-the older is the chemical law of the 'conservation of matter,' and the younger is the physical law of the 'conservation of energy.' Among the various modifications which the fundamental idea of substance has undergone in modern physics, in association with the prevalent atomism, we shall select only two of the most divergent theories for a brief discussion, the kinetic and the pyknotic. Both theories agree that we have succeeded in reducing all the different forces of nature to one common original force; gravity and chemical action, electricity and magnetism, light and heat, \&c., are only different manifestations, forms, or dynamodes, of a single primitive force (prodynamis). This fundamental force is generally conceived as a vibratory motion of the smallest particles of matter-a vibration of atoms. The atoms themselves, according to the usual 'kinetic theory of substance,' are dead, separate particles of matter, which dance to and fro in empty space and act at a distance. The real founder and most distinguished representative of the kinetic theory is Newton, the famous discoverer of the law of gravitation. In his great work, the Philosophiae Naturalis Principia Mathematica (1687), he showed that throughout the universe the same law of attraction controls the unvarying constancy of gravitation; the attraction of two particles being in direct proportion to their mass and in inverse proportion to the square of their distance. This universal force of gravity is at work in the fall of an apple and the tidal wave no less than in the course of the planets round the sun and the movements of all the heavenly bodies. In fundamental opposition to the theory of vibration, or the kinetic theory of substance, we have the modern theory of 'condensation,' or the pyknotic theory of substance. It is most ably established in the suggestive work of J. C. Vogt on "The Nature of Electricity and Magnetism on the Basis of a Simplified Conception of Substance" (1891). Vogt assumes the primitive force of the world, the universal prodynamis, to be, not the vibration or oscillation of particles in empty space, but the condensation of a simple primitive substance, which fills the infinity of space in an unbroken continuity. Its sole inherent mechanical form of activity consists in a tendency to condensation or contraction, which produces infinitesimal centres of condensation; these may change their degree of thickness, 
and, therefore, their volume, but are constant as such. These minute parts of the universal substance, the centres of condensation, which might be called pyknatoms, correspond in general to the ultimate separate atoms of the kinetic theory; they differ, however, very considerably in that they are credited with sensation and inclination (or will-movement of the simplest form), with 'souls,' in a certain sense-in harmony with the old theory of Empedocles of the 'love and hatred of the elements.' Moreover, these atoms with 'souls' do not float in empty space, but in the continuous, extremely attenuated intermediate substance, which represents the uncondensed portion of the primitive matter. By means of certain constellations, centres of perturbation, or systems of 'deformation,' great masses of centres of condensation quickly unite in immense proportions, and so obtain a preponderance over the surrounding masses. By that process the primitive substance, which in its original state of quiescence had the same mean consistency throughout, divides or differentiates into two kinds. The centres of disturbance, which positively exceed the mean consistency in virtue of the pyknosis or condensation, form the ponderable matter of bodies; the finer, intermediate substance, which occupies the space between them, and negatively falls below the mean consistency, forms the ether, or imponderable matter. As a consequence of this division in the mass and ether there ensues a ceaseless struggle between the two antagonistic elements, and this struggle is the source of all physical processes. The positive ponderable matter, the element with the feeling of like or desire, is continually striving to complete the process of condensation, and thus collecting an enormous amount of potential energy; the negative, imponderable matter, on the other hand, offers a perpetual and equal resistance to the further increase of its strain and of the feeling of dislike connected therewith, and thus gathers the utmost amount of actual energy. The two fundamental forms of substance, ponderable matter and ether, are not dead, and only moved by extrinsic force, but they are endowed with sensation and will (though, naturally, of the lowest grade); they experience an inclination for condensation, a dislike of strain; they strive after the one and struggle against the other.

"There is no such thing as empty space; that part of space which is not occupied with ponderable atoms is filled with ether.

"There is no such thing as an action at a distance through perfectly empty space; all action of bodies upon each other is either determined by immediate contact or is effected by the mediation of ether. Every shade of inclination, from complete indifference to the fiercest passion, is exemplified in the chemical relation of the various elements towards each other, just as we find in the psychology of man, and especially in the life of the sexes. This fundamental unity of affinity in the whole of nature, from the simplest chemical process to the most complicated love story, was recognised by the great Greek scientist, Empedocles, in the fifth century B.c., in his theory of 'the love and hatred of the elements.' It receives empirical confirmation from the interesting progress of cellular psychology, the great significance of which we have only learned to appreciate in the last thirty years. On those phenomena we base our conviction that even the atom is not without a rudimentary form of sensation and will, or, as it is better expressed, of feeling (esthesis) and inclination (tropesis) - that is, a universal 'soul' of the simplest character. The same must be said of the molecules which are composed of two or more atoms. Further combinations of different kinds of these molecules give rise to simple and, subsequently, complex chemical compounds, in the activity of which the same phenomena are repeated in a more complicated form. One of the first things to be proved by the law of substance is the basic fact that any natural force can be directly or indirectly converted into any other. Mechanical and chemical energy, sound and heat, light and electricity, are mutually convertible; they seem to be but different modes of one and the same fundamental force or energy. This fundamental principle is now generally recognised in the entire province of physics and chemistry, as far as it applies to inorganic substances.

"It seems to be otherwise with the organic world and its wealth of colour and form. It is, of course, obvious that a great part of the phenomena of life may be immediately traced to mechanical and chemical energy, and to the effects of electricity and light. For other vital processes, however, especially for psychic activity and consciousness, such an interpretation is vigorously contested. Yet the modern science of evolution has achieved the task of constructing a bridge between these two apparently irreconcilable provinces. We are now certain that all the phenomena of organic life are subject to the universal law of substance no less than the phenomena of the inorganic universe. The combination of elements which are peculiar to organisms, and which are responsible for their vital phenomena, are compound protoplasmic substances, of the group of albuminoids. Organic life itself is a chemico-physical process, based on the metabolism (or interchange of material) of these albuminoids. The only element which is capable of building up these compound albuminoids, in combination with other elements (oxygen, hydrogen, nitrogen, and sulphur), is carbon. The peculiar chemico-physical properties of carbon-especially the fluidity and the facility of decomposition of the most elaborate albuminoid compounds of carbon-are the sole and the mechanical causes of the specific phenomena of movement which distinguish organic from inorganic 
substances, and which are called life, in the usual sense of the word. The old idea of spontaneous generation is now taken in many different senses. I (Haeckel) restrict it to the first development of living protoplasm out of inorganic carbonates, and distinguish two phases in this beginning of biogenesis: (1) autogony, or the rise of the simplest protoplasmic substances in a formative fluid, and (2) plasmogony, the differentiation of individual primitive organisms out of these protoplasmic compounds in the form of monera. . . Since Darwin gave us the key to the monistic explanation of organisation in his theory of 'selection' forty years ago, it has become possible for us to trace the splendid variety of orderly tendencies of the organic world to mechanical, natural causes."

\section{§34. Mechanical Views of Kant and Laplace as Interpreted by Haeckel.}

Haeckel continuing adds : "Since Newton (1682) formulated the law of gravitation, and Kant (1755) established 'the constitution and mechanical origin of the entire fabric of the world on Newtonian laws,' and Laplace (1796) provided a mathematical foundation for this law of cosmic mechanism, the whole of the inorganic sciences have become purely mechanical, and at the same time purely atheistic. Astronomy, cosmogony, geology, meteorology, and inorganic physics and chemistry are now absolutely ruled by mechanical laws on a mathematical foundation. The idea of 'design' has wholly disappeared from this vast province of science. The anthropomorphic notion of a deliberate architect and ruler of the world has gone for ever from this field; the eternal, iron laws of "nature' have taken his place.

"But the idea of design has a very great significance and application in the organic world. We do undeniably perceive a purpose in the structure and in the life of an organism. The plant and the animal seem to be controlled by a definite design in the combination of their several parts, just as clearly as we see in the machines which man invents and constructs; as long as life continues the functions of the several organs are directed to definite ends, just as is the operation of the various parts of a machine. Hence it was quite natural that the older naïve study of nature, in explaining the origin and activity of the living being, should postulate a creator who had 'arranged all things with wisdom and understanding,' and had constructed each plant and animal according to the special purpose of its life. The conception of this 'almighty creator of heaven and earth' was usually quite anthropomorphic; he created 'everything after its kind.' As long as the creator seemed to man to be of human shape, to think with his brain, see with his eyes, and fashion with his hand, it was possible to form a definite picture of this 'divine engineer' and his artistic work in the great workshop of creation. This was not so easy when the idea of God became refined, and man saw in his 'invisible God' a creator without organs-a gaseous being. Still more unintelligible did these anthropomorphic ideas become when physiology substituted for the conscious, divine architect 'an unconscious, creative vital force'-a mysterious, purposive, natural force, which differed from the familiar forces of physics and chemistry, and only took these in part, during life, into its service. ...

"Nowhere in the evolution of animals and plants do we find any trace of design, but merely the inevitable outcome of the struggle for existence, the blind controller, instead of the provident God, that effects the changes of organic forms by a mutual action of the laws of heredity and adaptation. And there is no more trace of 'design' in the embryology of the individual plant, animal, or man. Throughout the whole of astronomy, geology, physics, and chemistry there is no question to-day of a 'moral order' or a personal God, whose "hand hath disposed all things in wisdom and understanding.' And the same must be said of the entire field of biology, the whole constitution and history of organic nature, if we set aside the question of man for the moment. Darwin has not only proved by his theory of selection that the orderly processes in the life and structure of animals and plants have arisen by mechanical laws without any preconceived design, but he has shown us 'in the struggle for life' the powerful natural force which has exerted supreme control over the entire course of organic evolution for millions of years. . . The anthropomorphic character of this notion, generally closely connected with belief in a personal God, is quite obvious. Belief in a 'loving Father' who unceasingly guides the destinies of 1,500,000,000 men on our planet, and is attentive at all times to their millions of contradictory prayers and pious wishes, is absolutely impossible; that is at once perceived on laying aside the coloured spectacles of faith, and reflecting rationally on the subject. Since impartial study of the evolution of the world teaches us that there is no definite aim and no special purpose to be traced in it, there seems to be no alternative but to leave everything to blind chance. The development of the universe is a monistic, mechanical process, in which we discover no aim or purpose whatever; what we call design in the organic world is a special result of biological agencies; neither in the evolution of the heavenly bodies nor in that of the crust of our earth do we find any trace of a controlling purpose -all is the result of chance."

The foregaing quotations fairly and fully express the views of Haeckel, and of Darwin as interpreted by VOL. I, 
Haeckel. It is seldom that such astounding assertions have been so dogmatically and unblushingly launched upon the scientific and semi-scientific public. They carry with them no proof, and have nothing whatever to recommend them unless it be the overweening confidence of one who seeks to turn the universe, and all it contains, topsy-turvy, and to obliterate all traces of law and order, design, and a Creator in favour of blind chance and a vain belief in the potency of matter as such. Haeckel's wild speculations are not supported by even a tittle of evidence which is worthy of the name. If his personality and that of Darwin be eliminated there is positively nothing left which would entitle such pernicious views to be tolerated for even a single day. They destroy faith, and cajole and coerce, rather than convince, the reasoning faculty. The conclusions arrived at, everything considered, are lame, halting, and impotent. Theories which substitute chance and so-called natural forces and mechanics for a Creator and Design and for Law and Order, and which require over one hundred millions of years for their verification, are not likely to find favour in the future with thoughtful, educated men. The best intellects will refuse adherence to what are, at best, colossal hypotheses. Moreover, Haeckel contradicts himself. At one part of his writings, as already stated, he says: "But the idea of design has a very great significance and application in the organic world. We do undeniably perceive a purpose in the structure and in the life of an organism. The plant and the animal seem to be controlled by a definite design in the combination of their several parts, just as clearly as we see in the machines which man invents and constructs. . . Nowhere in the evolution of animals and plants do we find any trace of design, but merely the inevitable outcome of the struggle for existence, the blind controller, instead of the provident God, that effects the changes of organic forms by a mutual action of the laws of heredity and adaptation. And there is no more trace of 'design' in the embryology of the individual plant, animal, or man. ... What we call design in the organic world is a special result of biological agencies; neither in the evolution of the heavenly bodies nor in that of the crust of our earth do we find any trace of a controlling purpose-all is the result of chance." If evolution in its extended form has of late years found favour with the multitude and with ordinary unscientific readers, it is because the doctrine has been tricked out to present a new and fashionable appearance, and because it gives a free rein to the imagination. Evolution in the widest sense cannot be proved, and is, to a large extent, unthinkable. The public, in these sensational times, swallow blindly and eagerly what stimulates the palate, especially if what is swallowed does not require to be personally digested and assimilated.

It is important to point out in this connection, that Professor Haeckel and Mr. Darwin, and those who think with them, obscure the issue by the inexact use of language. Thus Haeckel employs the same phraseology when explaining his monistic, mechanical views of life as is employed by the Vitalists and Creationists in describing their dualistic and non-mechanical views. He attributes sensation, consciousness, a soul, likes and dislikes, and other peculiarities of life to atoms and molecules, which, in modern times, have been invariably regarded as dead substances. He revives an old pagan idea. In like manner, Darwin employs the phrase "natural selection" as co-extensive with the phrase "artificial selection," which it certainly is not. Such lax use of terms is at once confusing and misleading. Artificial selection necessitates an intelligent selector outside the thing selected: natural selection, as employed by Darwin and his disciples, means a power inhering in plants and animals (not credited with intelligence) by which they blindly select and perpetuate desirable structures, properties, and qualities in themselves, to the exclusion of others which are not desirable. Plants and animals do not possess the powers claimed for them. We have proofs of this in our own persons. We cannot add to or take from any part of our body. We cannot by wishing, or even by a strong effort of will, become taller or shorter: we cannot grow wings, however much we may desire to fly: we cannot change the colour of our skin or eyes : we cannot grow a third set of teeth when the permanent teeth fail, or a new crop of hair when alopecia sets in: we cannot become beautiful if nature has cast us in a homely mould. In a word, we are absolutely helpless if we attempt to change our bodily parts, even in the slightest degree. We have certainly no power to select and perpetuate what we might consider our better parts to the exclusion and detriment of our inferior parts. If that be so, it follows that plants and animals have even less power in the direction indicated. To the vague language employed by Darwin in the so-called theory of "natural selection" is to be traced the original and growing confusion which dogs it at every step. Of the many who talk of "natural selection" very few have the faintest idea of what it actually means. It is, at best, a mere phrase, and it is, unfortunately for science and truth, a very misleading and mischievous one. Similar remarks are to be made of other catch phrases, such as the "struggle for existence" and "the survival of the fittest." Nature, in normal conditions, furnishes her living things with an abundant supply of foodno struggle being required-and it is the strongest and best which, under ordinary circumstances, perpetuate themselves. 


\section{PROFESSOR HUXLEY'S VIEWS ON EVOLUTION}

\section{§35. Professor Huxley's Views on Evolution, especially in connection with the Reptile, the Bird, and the Horse.}

Professor Huxley approaches the subject of evolution with considerable caution, and discusses it in his "Lectures and Essays" with remarkable adroitness and skill. His utterances are characterised by great frankness and lucidity of statement. He divides his argument in favour of evolution into two parts--one of which may be said to be constructive, the other destructive. The constructive part of the argument is admirably put, and with it I substantially agree. In the destructive part he is less satisfactory and convincing, and with it I find myself ever and anon at issue.

It would occupy too much time and space to state his argument at length. I will therefore content myself with liberal abstracts from it couched, for the most part, in his own incisive language.

His argument, it appears to me, is strongest where he deals with geologic types and with their persistency and permanency in time and space; and weakest where he attempts to set up connecting links, or, as he calls them, sub-groups (intercalary forms), with a view to running together and merging the several types of animals. In the sub-groups, the types themselves virtually disappear, even such distinct types as are represented by the reptile and bird respectively.

These sub-groups, if sufficient time and space be allowed, are said gradually to displace the types, or, what comes to the same thing, they run the types into each other, and so establish a line of descent and consanguinity. In this way he refers the descent of the bird to the reptile and the one-toed horse to a remote five-toed ancestor. If intermediary or intercalary forms be accepted, everything connected with evolution becomes possible. The intermediary groups provide endless connecting links, and when they are absent they are assumed to exist as "missing links," which is a very convenient arrangement for those who support the doctrine of evolution in its entirety. Of course, it is very comforting to all such to be able to say, when an intermediate link is not forthcoming, that it is a "missing link," and will be found sooner or later as science advances.

The destructive part of the argument, as indicated, has for its object to break down all outstanding differences between animals (which is equivalent to the obliteration of types), and to set up a sliding scale which merges, or is calculated to merge, one animal into another. This is an insidious form of argument, and demolishes quietly, but effectively, all distinctions, great and small.

The constructive part of the argument leads in quite another direction, and seeks to set up fundamental distinctions, boundaries, order, and design.

While fully armitting that there are types of plants and animals adapted to special times and to particular localities, and that typical plants and animals in certain cases approach each other indefinitely near, I am not, of those who believe that all plants and all animals merge into each other by insensible gradations. I hold that the fundamental differences between typical plants and animals cannot be got rid of by the introduction of hypothetical intermediary groups, however numerous, and however quickly they follow each other. I hold, moreover, that the theoretical intermediary groups or intercalary forms are assigned a much too important place in classification, and cannot logically be employed as reliable material in tracing the descent of the bird from the reptile or the one-toed horse from an ancient five-toed ancestor.

The intermediary groups in animals (allowing they exist) display, in not a few cases, fewer modifications and adaptations than are found in the limbs and travelling organs, which, whatever the class of the animal, have rigidly to conform to the requirements of land, water, and air transit. In the travelling organs there are what may be designated connecting links in locomotion, but no one thinks on this account of tracing the descent of the bird and bat to the insect, the reptile to the fish, or the mammal to the reptile.

The flying lemur and flying lizard, in the matter of locomotion, connect the land with the air, and the flying fish the water with the air; but the flying lemur and flying lizard are not descended from or connected in any way with the extinct pterodactyls. All that can be said is that both are flying creatures, and in this respect bear a general resemblance to each other. The animals which live in the water nust all conform to the fish type, and display swimming tails, fins, flippers or webbed feet. The animals in question may be birds or mammals belonging to entirely different families - that is, they are not connected by consanguinity and descent. Lastly, the flying things may be insects, birds, or bats, and still further removed from each other as regards descent from a common stock. The structural differences and modifications required in the walking, swimming, and flying organs are much greater in many cases than exist between nearly related animals. I have consequently always felt that slight differences in animals and animal structures are unduly magnified when they are intended to support evolution, much greater differences being glossed over or altogether omitted when they do not favour that doctrine. The great subject of locomotion, being opposed to the popular doctrine, is seldom referred to. 
These remarks have a very special bearing on the descent of the horse, of which so much has been made. This is a one-toed animal, said to be descended from a five-toed ancestor. The premises are inadequate to warrant such a conclusion. The quadrupert, as is well known, has typically five toes. The ox, deer, pig, \&c., however, have only two. Similarly the typical birds have five toes. The ostrich, emu, \&c., have, as a rule, only two, and these birds practically walk and run on one, which is greatly developed. The same is true of the extinct moa. There are, moreover, infinitely greater structural differences between ordinary fore limbs and the flying wings of birds and bats than there are between the one-toed horse and its supposed ancestors with five toes.

From these remarks it will be evident that too much emphasis has been placed on structural modifications in the limbs of the horse as proving descent and evolution, and too little on the structural modifications of the flying wings of birds and mammals as disproving descent and evolution. It is not permitted in science to blow hot and cold, and to strain points in order to buttress untenable positions.

If a special set of structural differences and modifications are quoted in support of a particular theoretical contention, antagonistic differences-provided they exist-should be referred to, or even quoted.

There is warrant for believing that the structural differences in animals and in their travelling organs are due to design and a common plan, and that the animals and their modified limbs are part of the great scheme of nature-the animals and their limbs forming parts of fundamental wholes.

It is not permissible to set aside original endowments by the introduction of sub-groups or intercalary forms or the over-statement of one set of facts and the under-statement or suppression of an equally or more important set.

Professor Huxley, as explained, founds his belief in the doctrine of evolution largely on a consideration of extinct geologic forms, especially those of reptiles, birds, and horses. The birds he is inclined to believe are the descendants of extinct reptiles, and the horse of pristine five-toed mammals. The extinct birds and horses he classifies as intercalary forms or sub-groups, as contra-distinguished from the great groups, including birds and mammals. The sub-groups he regards as connecting links between the great groups. It should, however, be borne in mind that, in a perfect scheme of nature, the intermediary sub-groups may be as important, in a way, as the larger groups; both representing parts of a more or less arbitrary classification. If there are intermediate forms between the extinct bird and reptile, and between the horse and an extinct five-toed mammal, it does not necessarily follow that the birds are evolved by direct descent from the reptiles, or the modern horses from remote five-toed mammals. The so-called intermediate sub-groups and links may be regarded as independent factors, and, as such, are entitled to great consideration. Mere numbers in groups do not seriously affect the question of types. The general plan of creation, as an ascending series, gives practically the same value to groups and sub-groups, and the fact that sub-groups resemble other groups above and below them, neither proves identity nor direct descent.

Huxley, with characteristic candour, admits the extreme persistence in certain cases of various types of plants and animals, and endeavours to explain the absence of change in living forms (as in Egypt) for thousands of years, by saying that the physical conditions in certain countries and during certain periods remain unaltered. He in this way indirectly refers the endless modifications which, according to evolution, occur in living things to externalities, such as climate, environment, extraneous stimulation, irritability, \&c. Of this, however, as I have already shown, we have no distinct proof; the changes which occur in living forms are not due to accidental circumstances. Like other evolutionists, Huxley avails himself of whatever connecting links exist or may come to hand, and at the same time endeavours to show that the geological record, from the nature of things, can never be perfect. He indeed emphasises this point. This is at once an ingenious and clever device. If an advocate of evolution be permitted to employ such geologic forms as exist and which serve his purpose as connecting links to strengthen his argument at one point and to say, when the connecting links fail him, that the geologic record is imperfect, he gains a double advantage: he gets credit for what is, and claims credit for what, there is every reason to believe, is not. The imperfection of the geologic record is elaborated by $\mathrm{M}_{r}$. Darwin in his "Origin of Species" by means of natural selection, and all who write on evolution take refuge in connecting links when possible, and when the supply runs out, they naively turn round and assert that their production is almost certainly merely a question of time as the geologic record becomes more perfect day by day. Another coign of vantage appropriated by evolutionists is that furnished by duration or time. If the changes and modifications said to be effected by evolution cannot be demonstrated, they boldly assert that sufficient time has not elapsed, and some of them (Haeckel, for example) extend the period even to a hundred or more millions of years. In so doing they flout human experience, history, and even the geologic record.

Professor Huxley has stated his views with great perspicuity as follows: 1- "From almost the origin of the 


\section{PROFESSOR HUXLEY'S VIEWS ON EVOLUTION}

discussions about the existing condition of the animal and vegetable worlds and the causes which have determined that condition, an argument has been put forward as an objection to evolution, which we shall have to consider very seriously. It is an argument which was first clearly stated by Cuvier in his criticism of the doctrines propounded by his great contemporary, Jamarck. The French expedition to Egypt had called the attention of learned men to the wonderful store of antiquities in that country, and there had been brought back to France numerous mummified corpses of the animals which the ancient Egyptians revered and preserved, and which, at a reasonable computation, must have lived not less than three or four thousand years before the time at which they were thus brought to light. Cuvier endeavoured to test the hypothesis that animals have undergone gradual and progressive modifications of structure, by comparing the skeletons and such other parts of the mummies as were in a fitting state of preservation, with the corresponding parts of the representatives of the same species now living in Egypt. $\mathrm{He}$ arrived at the conviction that no appreciable change had taken place in these animals in the course of this considerable lapse of time, and the justice of his conclusion is not disputed.

"It is obvious that, if it can be proved that animals have endured, without undergoing any demonstrable change of structure, for so long a period as four thousand years, no form of the hypothesis of evolution which assumes that animals undergo a constant and necessary progressive change can be tenable; unless, indeed, it be further assumed that four thousand years is too short a time for the production of a change sufficiently great to be detected.

"But it is no less plain that if the process of evolution of animals is not independent of surrounding conditions; if it may be indefinitely hastened or retarded by variations in these conditions; or if evolution is simply a process of accommodation to varying conditions; the argument against the hypothesis of exolution based on the unchanged character of the Egyptian fauna is worthless. For the monuments which are coeval with the mummies testify as strongly to the absence of change in the physical geography and the general conditions of the land of Egypt, for the time in question, as the mummies do to the unvarying characters of its living population.

"The progress of research since Cuvier's time has supplied far more striking examples of the long duration of specific forms of life than those which are furnished by the mummified ibises and crocodiles of Egypt. A remarkable case is to be found in the neighbourhood of the Falls of Niagara. In the immediate vicinity of the whirlpool, and again upon Groat Island, in the superficial deposits which cover the surface of the rocky subsoil in those regions, there are found remains of animals in perfect preservation, and among them shells belonging to exactly the same species as those which at present inhabit the still waters of Iake Erie. It is evident, from the structure of the country, that these animal remains were deposited in the beds in which they occur at a time when the lake extended over the region in which they are found. This involves the conclusion that they lived and died before the Falls had cut their way back through the gorge of Niagara. I believe I am speaking within the bounds of prudence if I assume that the Falls of Niagara have not retreated at a greater pace than about a foot a year. Six miles, speaking roughly, are 30,000 feet; 30,000 feet, at a foot a year, gives 30,000 years; and thus we are fairly justified in concluding that no less a period than this has passed since the shell-fish, whose remains are left in the beds to which I have referred, were living creatures.

"But there is still stronger evidence of the long duration of certain types. I have already stated that, as we work our way through the great series of the Tertiary formations, we find many species of animals identical with those which live at the present day, diminishing in numbers, it is true, but still existing, in a certain proportion, in the oldest of the Tertiary rocks. Furthermore, when we examine the rocks of the Cretaceous epoch, we find the remains of some animals which the closest scrutiny cannot show to be, in any important respect, different from those which live at the present time. That is the case with one of the Cretaceous lamp-shells (Terebratula), which has continued to exist unchanged, or with insignificant variations, down to the present day. Such is the case with the Globigerina, the skeletons of which, aggregated together, form a large proportion of our English chalk. Those Globigerine can be traced down to the Globigerinir which live at the surface of the present great oceans, and the remains of which, falling to the bottom of the sea, give rise to a chalky mud. Hence it must be admitted that certain existing species of animals show no distinct sign of modification, or transformation, in the course of a lapse of time as great as that which carries us back to the Cretaceous period; and which, whatever its absolute measure, is certainly vastly greater than thirty thousand years.

"There are groups of species so closely allied together, that it needs the eye of a naturalist to distinguish them one from another. If we disregard the small differences which separate these forns, and consider all the species of such groups as modifications of one type, we shall find that, even among the higher animals, some types have had a marvellous duration. In the chalk, for example, there is found a fish belonging to the highest and the most differentiated group of osseous fishes, which goes by the name of Beryx. But the genus Beryx is represented, at the present day, by very closely allied species which are living in the Pacific and Atlantic Oceans. We may go 
still further back. I have referred to the fact, that the C'arboniferous formations, in Europe and in America, contain the remains of scorpions in an admirable state of preservation, and that those scorpions are hardly distinguishable from such as now live. More than this. At the very bottom of the Silurian series, in beds which are by some authorities referred to the Cambrian formation, where the signs of life begin to fail us-even there, among the few and scanty animal remains which are discoverable, we find species of molluscous animals which are so closely allied to existing forms that, at one time, they were grouped under the same generic name. The same truth is exemplified if we turn to certain great periods of the earth's history-as, for example, the Mesozoic epoch. There are groups of reptiles, such as the Ichthyosauria and the Plesiosauria, which appear shortly after the commencement of this epoch, and they occur in vast numbers. They disappear with the chalk and, throughout the whole of the great series of Mesozoic rocks, they present no such modifications as can safely be considered evidence of progressive modification.

"Facts of this kind are undoubtedly fatal to any form of the doctrine of evolution which postulates the supposition that there is an intrinsic necessity, on the part of animal forms which have once come into existence, to undergo continual modification; and they are as distinctly opposed to any view which involves the belief that such modification as may occur, must take place, at the same rate, in all the different types of animal or vegetable life. There is another order of facts belonging to the class of negative or indifferent evidence. The great group of lizards, which abound in the present world, extends through the whole series of formations as far back as the Permian, or latest Palæozoic, epoch. These Permian lizards differ astonishingly little from the lizards which exist at the present day. Comparing the amount of the differences between them and modern lizards, with the prodigious lapse of time between the Permian epoch and the present age, it may be said that the amount of change is insignificant. If the doctrine of evolution be true, it follows that, however diverse the different groups of animals and of plants may be, they must all, at one time or other, have been connected by gradational forms; so that, from the highest animals, whatever they may be, down to the lowest speck of protoplasmic matter in which life can be manifested, a series of gradations, leading from one end of the series to the other, either exists or has existed. But when we look upon living Nature as it is, we find a totally different state of things. We find that animals and plants fall into groups, the different members of which are pretty closely allied trgether, but which are separated by definite, larger or smaller breaks, from other groups. At the present time, for example, there are numerous forms of non-ruminant pachyderms, or what we may call broadly the pig tribe, and many varieties of ruminants. These latter have their definite characteristics, and the former have their distinguishing peculiarities. But there is nothing that fills up the gap between the ruminants and the pig tribe. Such also is the case in respect of the minor groups of the class of reptiles. In the existing fauna, the group of pig-like animals and the group of ruminants are entirely distinct; but one of the first of Cuvier's discoveries was an animal which he called the Inoplotherium, and which proved to be, in a great many important respects, intermediate in character between the pigs on the one hand, and the ruminants on the other. Another remarkable animal restored by the great French palæontologist, the Palmotherium, similarly tended to connent together animals to all appearance so different as the rhinoceros, the horse, and the tapir. Subsequent research has brought to light multitudes of facts of the same order. Instead of dealing with these examples, which would require a great deal of tedious osteological detail, I take the case of birds and reptiles; groups which, at the present day, are so clearly distinguished from one another that there are perhaps no classes of animals which, in popular apprehension, are more completely separated. Existing birds are covered with feathers; their anterior extremities, specially and peculiarly modified, are converted into wings, by the aid of which most of them are able to fly; they walk upright upon two legs; and these limbs, when they are considered anatomically, present a great number of exceedingly remarkable peculiarities. On the other hand, existing reptiles have no feathers. They may have naked skins, or may be covered with horny scales, or bony plates, or with both. They possess no wings; they neither fly by means of their fore-limbs nor habitually walk upright upon their hind-limbs; and the bones of their legs present no such modifications as we find in birds. As we trace the history of birds back in time, we find their remains, sometimes in great abundance, throughout the whole extent of the Tertiary rocks ; but, so far as our present linowledge goes, the birds of the Tertiary rocks retain the same essential characters as the birds of the present day. Not very long ago no remains of birds had been found below the Tertiary rocks. But, in the course of the last few years, such remains have been discovered in England; though, unfortunately, in so imperfect and fragmentary a condition, that it is impossible to say whether they differed from existing birds in any essential character or not. In America the development of the Cretaceous series of rocks is enormous; the conditions under which the later Cretaceous strata have been deposited are highly favourable to the preservation of organic remains, and the researches carried on by Professor Marsh have rewarded him with the discovery of the forms of birds of which we had hitherto no conception. By his kindness, I am enabled to place before you a restoration of one of these extraordinary birds, every part of which can be 
thoroughly identified by the more or less complete skeletons, in a very perfect state of preservation, which he has discovered. The Hesperornis, which measures between five and six feet in length, is astonishingly like our existing divers or grebes in a great many respects ; so like them indeed that, had a skeleton of Hesperornis been found in a museum without its skull, it probably would have been placed in the same group of birds as the divers and grebes of the present day. But Hesperornis differs from all existing birds, and so far resembles reptiles, in one important particular-it is provided with teeth. But the discovery of an animal which, in every part of its skeleton, closely agrees with existing birds, and yet possesses teeth, shows that there were ancient birds which, in respect of possessing teeth, approached reptiles more nearly than any existing bird does. The same formation has yielded another bird, Ichthyornis, which also possesses teeth; but the teeth are situated in distinct sockets, while those of Hesperornis are not so lodged. The latter also has such very small, almost rudimentary wings, that it must have been chiefly a swimmer and a diver like a penguin; while Ichthyornis has strong wings, and no doubt possessed corresponding powers of flight. The Solenhofen slates have yielded the Archoropteryx, the existence of which was first made known by the finding of a fossil feather, or rather of the impression of one. By-and-by a solitary skeleton was discovered, which is now in the British Museum. The feet are not only altogether bird-like, but have the special characters of the feet of perching birds, while the body had a clothing of true feathers. Nevertheless, in some other respects, Archropteryx is unlike a bird and like a reptile. There is a long tail composed of many vertebræ. The structure of the wing differs in some very remarkable respects from that which it presents in a true bird. In the Archropteryx, the upper-arm bone is like that of a bird, and the two bones of the forearm are more or less like those of a bird, but the fingers are not bound together-they are free, and several, if not all, of them were terminated by strong curved claws, not like such as are sometimes found in birds, but such as reptiles possess; so that, in the Archroopteryx, we have an animal which to a certain extent occupies a midway place between a bird and a reptile. Like the Anoplotherium and the Palrotherium, therefore, Archropteryx tends to fill up the interval between groups which, in the existing world, are widely separated, and to destroy the value of the definitions of zoological groups based upon our knowledge of existing forms. And such cases as these constitute evidence in favour of evolution, in so far as they prove that, in former periods of the world's history, there were animals which overstepped the bounds of existing groups, and tended to merge them into larger assemblages."

Referring to the horse, Mr. Huxley says: "It by no means follows, because the Palrotherium has much in common with the horse, on the one hand, and with the rhinoceros on the other, that it is the intermediate form through which rhinoceroses have passed to become horses, or vice versit; on the contrary, any such supposition would be certainly erroneous. Nor do I think it likely that the transition from the reptile to the bird has been effected by such a form as Irchropteryx. And it is convenient to distinguish these intermediate forms between two groups, which do not represent the actual passage from the one group to the other, as intercalary types, from those linear types which, more or less approximately, indicate the nature of the steps by which the transition from one group to the other was effected.

"I conceive that such linear forms, constituting a series of natural gradations between the reptile and the bird, and enabling us to understand the manner in which the reptilian has been metamorphosed into the bird type, are really to be found among a group of ancient and extinct terrestrial reptiles known as the Ornithoscelida. The remains of these animals occur throughout the series of Mesozoic formations, from the Trias to the Chalk, and there are indications of their existence even in the later Yalæozoic strata.

"Most of these reptiles, at present known, are of great size, some having attained a length of forty feet, or perhaps more. The majority resembled lizards and crocodiles in their general form, and many of them were, like crocodiles, protected by an armour of heavy bony plates. But, in others, the hind-limbs elongate and the forelimbs shorten, until their relative proportions approach those which are observed in the short-winged, flightless, ostrich tribe among birds.

"The skull is relatively light, and in some cases the jaws, though bearing teeth, are beak-like at their extremities, and appear to have been enveloped in a horny sheath. In the part of the vertebral column which lies between the haunch bones and is called the sacrum, a number of vertebræ may unite together into one whole, and in this respect, as in some details of its structure, the sacrum of these reptiles approaches that of birds.

"But it is in the structure of the pelvis and of the hind limb that some of these ancient reptiles present the most remarkable approximation to birds, and clearly indicate the way by which the most specialised and characteristic features of the bird may have been evolved from the corresponding parts in the reptile. In the crocodile, the pelvis is obviously composed of three bones on each side; the ilium, the pubis, and the ischium. In the adult bird there appears to be but one bone on each side. The examination of the pelvis of a chick, however, shows that each half is made up of three bones, which answer to those which remain distinct throughout life in 
the crocodile. There is, therefore, a fundamental identity of plan in the construction of the pelvis of both bird and reptile; though the differences in form, relative size, and direction of the corresponding bones in the two cases are very great.

"But the most striking contrast between the two lies in the bones of the leg and of that part of the foot termed the tarsus, which follows upon the leg. It can be shown by the study of development that the bird's pelvis and hind limb are simply extreme modifications of the same fundamental plan as that upon which these parts are modelled in reptiles.

"On comparing the pelvis and hind limb of the Ornithoscelida with that of the crocodile, on the one side, and that of the bird on the other, it is obvious that it represents a middle term between the two. Taking all these facts together, it is obvious that some of these animals may have walked upon their hind legs, as birds do. In fact there can be no reasonable doubt that one of the smaller forms of the Ornithoscelida, Compsognathus, the almost entire skeleton of which has been discovered in the Solenhofen slates, was a bipedal animal. As Compsognathus walked upon its hind legs, it must have made tracks like those of birds. And as the structure of the limbs of several of the gigantic Ornithoscelida, such as Iguanodon, leads to the conclusion that they also may have constantly, or occasionally, assumed the same attitude, a peculiar interest attaches to the fact that, in the Wealden strata of Fngland, there are to be found gigantic footsteps arranged in order like those of the Brontozoum, and which, there can be no reasonable doubt, were made by some of the Ornithoscelida, the remains of which are found in the same rocks.

"We have had to stretch the definition of the class of birds so as to include birds with teeth and birds with paw-like fore-limbs and long tails. The evidential value of the facts I have brought forward must be neither over nor under-estimated. It is not historical proof of the occurrence of the evolution of birds from reptiles, for we have no safe ground for assuming that true birds had not made their appearance at the commencement of the Mesozoic epoch. There is another series of extinct reptiles which may be said to be intercalary between reptiles and birds, in so far as they combine some of the characters of both these groups; and which, as they possessed the power of flight, may seem, at first sight, to be nearer representatives of the forms by which the transition from the reptile to the bird was effected, than the Ornithoscelida.

"These are the Pterosauria, or Pterodactyls, the remains of which are met with throughout the series of Mesozoic rocks, from the Lias to the Chalk, and some of which attain a great size, their wings having a span of eighteen or twenty feet. These animals, in the form and proportions of the head and neck relatively to the body, and in the fact that the ends of the jaws were often, if not always, more or less extensively ensheathed in horny beaks, remind us of birds. Moreover, their bones contained air cavities, rendering them specifically lighter, as is the case in most birds. The breast-bone was large and keeled, as in most birds and in bats, and the shoulder girdle is strikingly similar to that of ordinary birds. But it seems to me that the special resemblance of pterodactyls to birds ends here, unless I may add the entire absence of teeth which characterises the great pterodactyls (Pteranodon) discovered by Professor Marsh. All other known pterodactyls have teeth lodged in sockets. In the vertebral column and the hind-limbs there are no special resemblances to birds, and when we turn to the wings they are found to be constructed on a totally different principle from those of birds.

"There are four fingers. These four fingers are large, and three of them-those which answer to the thumb and two following fingers-are terminated by claws, while the fourth is enormously prolonged, and converted into a great jointed style. This finger had the office of supporting a web which extended between it and the body. An existing specimen proves that the fingers supported a vast web like that of a bat's wing; in fact, there can be no doubt that this ancient reptile flew after the fashion of a bat.

"Thus, though the pterodactyl is a reptile which has become modified in such a manner as to enable it to fly, and therefore, as might be expected, presents some points of resemblance to other animals which fly; it has, so to speak, gone off the line which leads directly from reptiles to birds, and has become disqualified for the changes which lead to the characteristic organisation of the latter class. The occurrence of historical facts is said to be demonstrated, when the evidence that they happened is of such a character as to render the assumption that they did not happen in the highest degree improbable. Those who have attended to the progress of palæontology are aware that evidence of the character which I have defined has been produced in considerable and continuallyincreasing quantity during the last few years. It is obviously useless to seek for such evidence except in localities in which the physical conditions have been such as to permit of the deposit of an unbroken or but rarely interrupted series of strata through a long period of time; in which the group of animals to be investigated has existed in such abundance as to furnish the requisite supply of remains. It so happens that the case which, at present, most nearly fulfils all these conditions is that of the series of extinct animals which culminates in the horses; by which term I mean to denote not merely the domestic animals with which we are all so well acquainted, 
but their allies, the ass, zebra, quagga, and the like. The horse is in many ways a remarkable animal ; not least so in the fact that it presents us with an example of one of the most perfect pieces of machinery in the living world. The locomotive machinery is resident in its slender fore and hind-limbs; they are flexible and elastic levers, capable of being moved by very powerful muscles; and, in order to supply the engines which work these levers with the force which they expend, the horse is provided with a very perfect apparatus for grinding its food and extracting therefrom the requisite fuel. Without attempting to take you very far into the region of osteological detail, let us turn to the fore-limb. In most quadrupeds, as in ourselves, the fore-arm contains distinct bones called the radius and the ulna. The corresponding region in the horse seems at first to possess but one bone. Careful observation, however, enables us to distinguish in this bone a part which clearly answers to the upper end of the ulna. This is closely united with the chief mass of the bone which represents the radius, and runs out into a slender shaft which may be traced for some distance downwards upon the back of the radius, and then in most cases thins out and vanishes. It takes still more trouble to make sure of what is nevertheless the fact, that a small part of the lower end of the bone of the horse's fore-arm, which is only distinet in a very young foal, is really the lower extremity of the ulna.

"What is commonly called the knee of a horse is its wrist. The 'cannon bone' answers to the middle bone of the five metacarpal bones, which support the palm of the hand in ourselves. The 'pastern,' 'coronary,' and 'coffin' bones of veterinarians answer to the joints of our middle fingers, while the hoof is simply a greatly enlarged and thickened nail. But if what lies below the horse's 'knee" thus corresponds to the middle finger in ourselves, what has become of the other four fingers or digits? We find in the places of the second and fourth digits only two slender splint-like bones, about two-thirds as long as the cannon bone, which gradually taper to their lower ends and bear no finger joints, or, as they are termed, phalanges. Sometimes small bony or gristly nodules are to be found at the bases of these two metacarpal splints, and it is probable that these represent rudiments of the first and fifth toes. Thus, the part of the horse's skeleton which corresponds with that of the human hand contains one overgrown middle digit, and at least two imperfect lateral digits; and these answer, respectively, to the third, the second, and the fourth fingers in man. Corresponding modifications are found in the hind limb. The heel of the horse is the part commonly known as the hock. The hinder cannon bone answers to the middle metatarsal bone of the human foot; the pastern, coronary, and coffin bones to the middle toe bones; the hind hoof to the nail, as in the fore foot. And, as in the fore foot, there are merely two splints to represent the second and the fourth toes. Sometimes a rudiment of a fifth toe appears to be traceable.

"The teeth of a horse are not less peculiar than its limbs. The twelve cutting teeth of a horse are close-set and concentrated in the fore-part of its mouth, like so many adzes or chisels. The grinders or molars are large, and have an extremely complicated structure, being composed of a number of different substances of unequal hardness. The consequence of this is that they wear away at different rates; and, hence, the surface of each grinder is always as uneven as that of a good mill-stone. .. I have now enumerated those characteristic structures of the horse which are of most importance. To any one who is acquainted with the morphology of vertebrated animals, they show that the horse deviates widely from the general structure of mammals; and that the horse type is, in many respects, an extreme modification of the general mammalian plan. The least modified mammals, in fact, have the radius and ulna, the tibia and fibula, distinct and separate. They have five distinct and complete digits on each foot, and no one of these digits is very much larger than the rest. Moreover, in the least modified mammals, the total number of the teeth is very generally forty-four, while in horses, the usual number is forty. . . . The general principles of the hypothesis of evolution lead to the conclusion that the horse must have been derived from some quadruped which possessed five complete digits on each foot; which had the bones of the fore-arm and of the leg complete and separate; and which possessed forty-four teeth. In Europe abundant remains of horses are found in the Quaternary and later Tertiary strata as far as the Pliocene formation. But these horses, which are so common in the cave-deposits and in the gravels of Europe, are in all essential respects like existing horses. And that is true of all the horses of the latter part of the Pliocene epoch. But, in deposits which belong to the earlier Pliocene and later Miocene epochs, and which occur in Britain, in France, in Germany, in Greece, in India, we find animals which are extremely like horses-which, in fact, are so similar to horses, that you may follow descriptions given in works upon the anatomy of the horse upon the skeletons of these animals-but which differ in some important particulars. For example, the structure of their fore and hind limbs is somewhat different. The bones which, in the horse, are represented by two splints, imperfect below, are as long as the middle metacarpal and metatarsal bones; and, attached to the extremity of each, is a digit with three joints of the same general character as those of the middle digit, only very much smaller. These small digits are so disposed that they could have had but very little functional importance, and they must have been rather of the nature of the VOL. I. 
dew-claws, such as are to be found in many ruminant animals. The Hipparion, as the extinct European threetoed horse is called, in fact, presents a foot similar to that of the American Protohippus, except that, in the Hipparion, the smaller digits are situated further back, and are of smaller proportional size, than in the Protohippus.

"The ulna is slightly more distinct than in the horse; and the whole length of it, as a very slender shaft, intimately united with the radius, is completely traceable. The fibula appears to be in the same condition as in the horse. The teeth of the Hipparion are essentially similar to those of the horse, but the pattern of the grinders is in some respects a little more complex, and there is a depression on the face of the skull in front of the orbit, which is not seen in existing horses.

"In the earlier Miocene, and perhaps the later Eocene deposits of some parts of Europe, another extinct animal has been discovered, which Cuvier, who first described some fragments of it, considered to be a Palæotherium. But as further discoveries threw new light upon its structure, it was recognised as a distinct genus, under the name of Anchitherium.

"In its general characters, the skeleton of Anchitherium is very similar to that of the horse. Fach foot possesses three complete toes; while the lateral toes are much larger in proportion to the middle toe than in Hipparion; and doubtless rested on the ground in ordinary locomotion.

"The ulna is complete and quite distinct from the radius, though firmly united with the latter. The fibula seems also to have been complete. There are forty-four teeth. The incisors have no strong pit. The canines seem to have been well developed in both sexes. The first of the seven grinders, which is frequently absent, and, when it does exist, is small in the horse, is a good-sized and permanent tooth, while the grinder which follows it is but little larger than the hinder ones. The Anchitherium, the Hipparion, and the modern horses, constitute a series in which the modifications of structure coincide with the order of chronological occurrence, in the manner in which they must coincide, if the modern horses really are the result of the gradual metamorphosis, in the course of the Tertiary epoch, of a less specialised ancestral form. . When America was first discovered by Europeans, there were no traces of the existence of the horse in any part of that continent. Nevertheless, the investigations of American geologists have proved that the remains of horses occur in the most superficial deposits of both North and South America, just as they do in Europe. Therefore, for some reason or other, the horse must have died out on that continent at some period preceding the discovery of America. Of late years there has been discovered in the Western Territories of America a marvellous accumulation of deposits, which furnishes us with a consecutive series of records of the fauna of the older half of the Tertiary epoch, for which we have no parallel in Europe. The researches of Leidy and others have shown that forms allied to the Hipparion and the Anchitherium are to be found among these remains. They tend to show that we must look to America, rather than to Europe, for the original seat of the equine series; and that the archaic forms and successive modifications of the horse's ancestry are far better preserved there than in Europe. The succession of forms which Professor Marsh has brought together carries us from the top to the bottom of the Tertiaries. Firstly there is the true horse. Next we have the American Pliocene form of the horse. In the conformation of its limbs it presents some very slight deviations from the ordinary horse, and the crowns of the grinding teeth are shorter. Then comes the Protohippus, which represents the European Hipparion, having one large digit and two small ones on each foot, and the general characters of the fore-arm and leg to which I have referred. But it is more valuable than the European Hipparion, for the reason that it is devoid of some of the peculiarities of that form--peculiarities which tend to show that the European Hipparion is rather a member of a collateral branch than a form in the direct line of succession. Next, in the backward order of time, is the Miohippus, which corresponds pretty nearly with the Anchitherium of Europe. It presents three complete toesone large median and two smaller lateral ones; and there is a rudiment of that digit which answers to the little finger of the human hand.

"The Furopean record of the pedigree of the horse stops here; in the American Tertiaries, on the contrary, the series of ancestral equine forms is continued into the Eocene formations. An older Miocene form, termed Mesohippus, has three toes in front, with a large splint-like rudiment representing the little finger; and three toes behind. The radius and ulna, the tibia and the fibula, are distinct, and the short-crowned molar teeth are anchitheroid in pattern.

"But the most important discovery of all is the Orohippus, which comes from the Eocene formation, and is the oldest member of the equine series as yet known. Here we find four complete toes on the front limb, three toes on the hind limb, a well-developed ulna, a well-developed fibula, and short-crowned grinders of simple pattern. The knowledge we now possess justifies us completely in the anticipation, that when the still lower Eocene deposits, and those which belong to the Cretaceous epoch, have yielded up their remains of ancestral equine animals, we shall find, first, a form with four complete toes and a rudiment of the innermost or first digit in front, with probably a 
rudiment of the fifth digit in the hind foot; while, in still older forms, the series of the digits will be more and more complete, until we come to the five-toed animals, in which, if the doctrine of evolution is well founded, the whole series must have taken its origin. The only way of escape, if it be a way of escape, from the conclusions which I have just indicated, is the supposition that all these different equine forms have been created separately at separate epochs of time."

The several points touched upon in the present connection are illustrated in various parts of the work, such as the non-variability and permanency of the human type in Egypt; the ancient extinct reptiles (Dinosaurs, \&c.); the extinct flying reptiles (Pterodactyls); the extinct birds; the supposed ancient extinct horses; and the extinct mammals. Numerous examples of typical modern birds and mammals occur everywhere, and need not be specified.

It is not necessary to discuss the subject of evolution further in this place; suffice it to say that one of its phases, and that perhaps the most important, has not been dealt with. I refer to the teleological aspect of evolution. This lies at the root of the whole matter. The question naturally arises, From what materials, by what means, and for what purposes have the great races of plants and animals been produced? Are they accidental chance products, or are they the work of an intelligent First Cause and deliberate design? I personally advocate the latter view. It appears to me to afford the only possible explanation of the facts. Plants and animals, as already stated, are not automata. They are not self-made, self-adjusting, and self-directing machines. On the contrary, and as has been pointed out, they are created, designed organisms, which have the power of performing (under guidance) a great variety of functions which no mere mechanical contrivance could possibly accomplish. There is, in fact, a great gulf between the mechanical dead machine, which only goes so long as it is wound up from the outside, and the living organism, which goes on practically indefinitely, and winds itself up from within. In the one case everything is haphazard and mechanical. In the other everything is predetermined, and there is an obvious adaptation of means to ends.

If the term evolution is to be regarded as representing gradual progressive advance from lower to higher platforms, it is evident it must be freed of everything which, even indirectly, savours of accident. It can only claim to be the expression of a First Cause and of deliberate design where the end is seen from the beginning, where everything is pre-determined, and where law and order reign supreme. Fvolution, if it means anything, can only mean the unfolding, in strict sequence, of a multitude of facts, none of which are the result of accident. Fvolution, if admitted at all, must mean the orderly unfolding of everything the world contains, and it must rest ultimately on utilitarianism as a base. The greatest good would then be its rallying cry and watchword. The why and the wherefore would be both explained. Everything which exists has been created for a definite purpose, so that the great subject of teleology is vindicated even in its most minute details. Utility is, after all, the touchstone of the universe.

The subject of teleology has been relegated most unfairly and ignorantly to the limbo of neglect, but there are indications that it and design (which go hand in hand) will, before many decades, be reinstated in their places of honour, and bring men's minds back to the fold from which, with great danger to themselves, they have temporarily strayed.

What is here stated will be readily understood from what follows, where I furnish certain details bearing generally on the subject of evolution.

Plants and animals, as I have striven to prove, are never chance products, and the influence of surrounding matter is never sufficient of itself to produce an animal, or any part thereof.

While plants and animals vary within limits they will, as a rule, if left to themselves, and allowed to breed back, revert to their originals. The so-called variations will disappear.

Plants and animals are endowed with original constitutions which are amazingly stable: the permanence of type has never been satisfactorily or sufficiently explained. Professor Huxley himself, while endeavouring to account for the want of change in ancient Egyptian animals for periods of three or four thousand years by saying that the external conditions in Egypt did not vary during the time stated, yet admits that in America (Goat Island and Falls of Niagara) fossil shells are found of exactly the same species as those inhabiting Lake Frie at the present day, and on which no visible change can be detected for a period exceeding, in his opinion, in all probability, thirty thousand years.

Whatever may be said in favour of stability as a result of want of change in climate and surroundings in Egypt cannot possibly apply to America, where the vicissitudes of climate and environment are avowedly very considerable. With stability and permanency in living forms for periods of thirty thousand or more years, it is safe to assert that original constitution and type are factors of the utmost importance in the scheme of creation, and 
cannot be set aside in favour of any theory, not even that of evolution. 'The constitutions of plants and animals must, because of their stability and permanence, be regarded as original endowments, and the variations, of which so much has been made, are, it appears to me, as nothing by comparison. That plants and animals under cultivation and in certain circumstances vary within limits, is admitted, but it is also admitted that they revert to their originals if left to themselves.

Given stable constitutions, it follows that plants and animals do not, of necessity, vary. If they do vary, the degree of variation is not to any great extent determined by any extraneous circumstances, such as environment

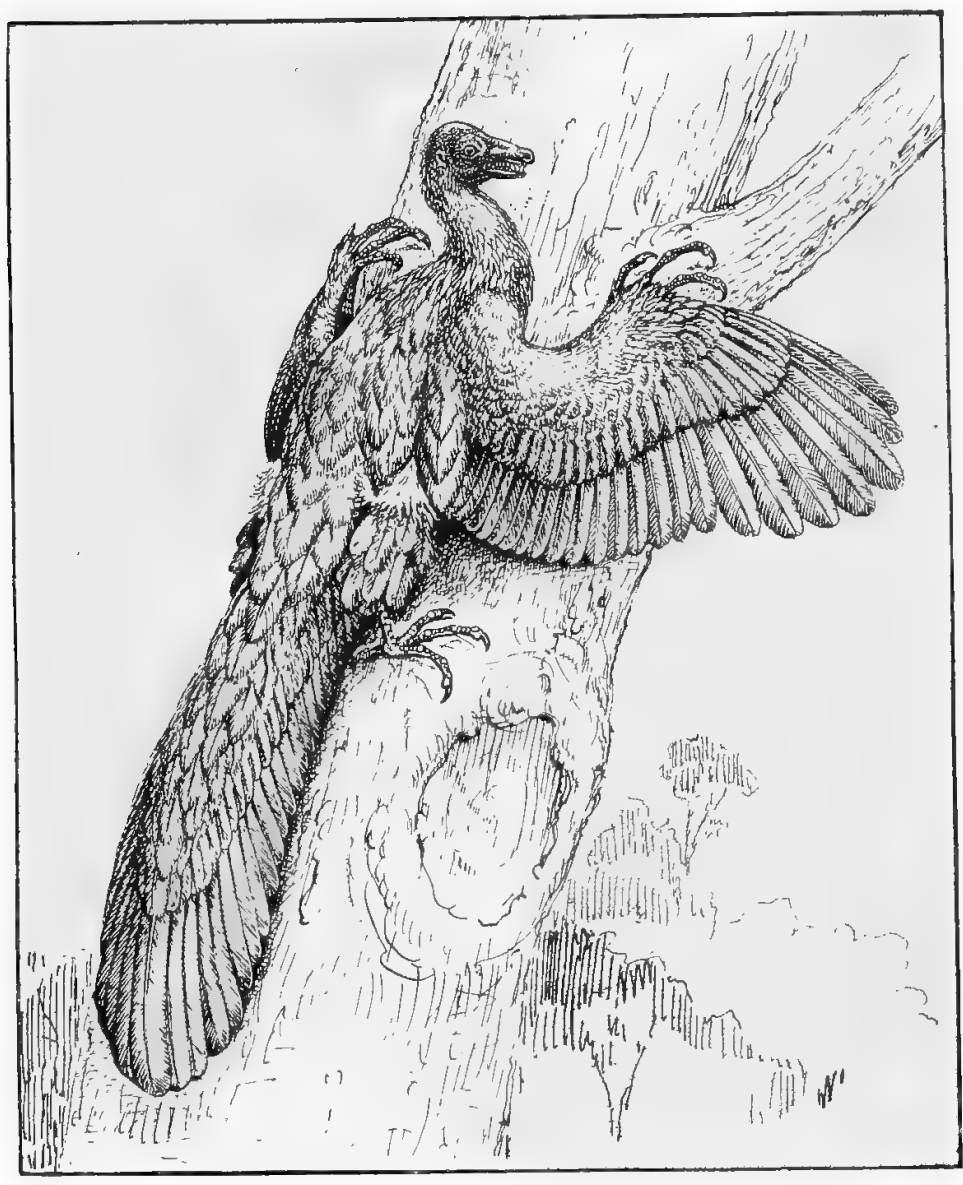

FIG. 36.- Restoration of the Ardieuteryx siemensi, a rare extinct fossil hird. Shows each wing to possess three fingers, each terminating in a hook; a long bony tail with feathers spreading from either side; and the absence of a proper heak, \&c. (Restored according to scale, from measurements taken from a photograph of the fossil specimen, by $\mathrm{C}$. Berjean and the Author.) directions; the trend being limited in such a way as to prevent the mixing up and confusion of types. The result is practically the same. In the one case the First Cause is behind or outside the plant and animal ; in the other it is practically uithin the plant and animal.

It is not possible to get away from a supreme power, and from design, law and order, either in the organic or inorganic kingdoms.

The subject of one great creation confined to a definite period, or several smaller creations embracing various periods, need not be discussed further than to say, that the geologic record would lend itself to support either theory equally well. Time is an element in both forms of creation. When one great creative act is spoken of, the act is not to be regarded as instantaneous : it consists of stages and processes which imply duration. All that is meant is that creation occupied a definite measure of time, and that when the universe and all it contains was made, there was an end of creative activity. The amount of time required is nowhere stated. The six days mentioned in Scripture may mean six thousand years or six millions years. The processes or steps of creation are equally visible in the organic and inorganic kingdoms; in the crust and rocks of the earth, and in the successive geologic flora 
and fauna. It is easy to conceive of one great creative act confined to one period (that period being practically illimitable), but consisting of successive stages. The stages, in this case, represent virtually separate creations. If the element of time be granted, it is equally easy to regard the separate smaller creations as part of one great creation. Creation is essentially a progressive work. It is a continuous adaptation of the organic and inorganic kingdoms to each other in a million different ways throughout an inconceivably great period of time, and these adaptations are unfolded by geology as seen in the rocks and in the fossil flora and fauna imbedded and preserved in them. The ancient flora and fauna are strikingly represented in the geologic period. The types are less numerous than in the modern flora and fauna, but they are equally representative. Successive adaptations of plants and animals to altered circumstances are practically successive creations, provided an intelligent Creator with unlimited power and unlimited time at His disposal are conceded. The real difficulty arises when such a Creator is denied, and when plants and animals are said to be the product of spontaneous generation, and to create and modify themselves indefinitely at the bidding of environment and under the influence of external stimulation.

The descent of the bird from the reptile, and the horse from a five-toed mammal, as argued by Professor Huxley, is by no means proved. The argument hinges chiefly on the modifications in limbs and teeth, particularly the former. Some ancient fossil birds, he avers, possess teeth and elongated tails, and the wings have free digits with claws. The legs and feet of birds are supposed to resemble those of the extinct terrestrial reptile Ornithoscelida, which bear a likeness to those of the modern crocodile. The pelvis of the bird and reptile have also, according to Huxley, some features in common. The resemblances are comparatively few in number, and insignificant when contrasted with the numerous and important differences. The resemblances, such as they are, I venture to assert are due not to evolution, but to original endowment necessitated by the exigencies of their mode of life, occasioned by the nature of the media on and in which they are to live and move.

The old-world fossil bird, sometimes spoken of as the bird-reptile, is commonly known as the Archæopteryx. I furnish a careful restoration of it by C. Berjeau and myseif. The restoration brings into strong relief the more salient features of this most anomalous and interesting form (Fig. 36).

Only a few specimens of the Archropteryx have as yet been discovered. The two most perfect specimens known were found in the fine lithographic stone of Solenhofen in Bavaria. One of these (that first discovered) is preserved in the Natural History Department of the British Museum, London; the other and more perfect specimen of the two (Archropteryx siemensi), which is that restored, has been assigned

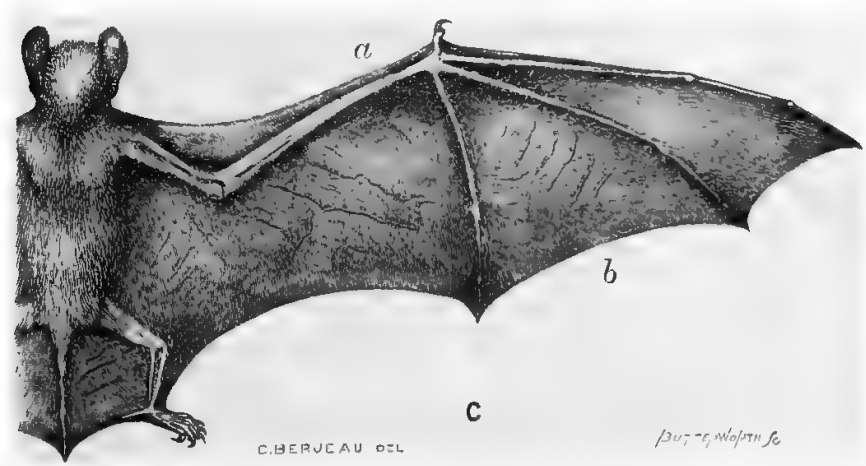

FIG. 38. - Right wing of the bat (I"csprrtitio murinus). Shows how the bones of the arm, leg, and tail all take part in supporting the flying membrane. The first digit of the hand is aborted and terminates in a hook; the remaining three digits being greatly elongated, and tapering to a point to give elasticity to the wing. Drawn by C. Berjean for the Author, from a specimen in his private collection.

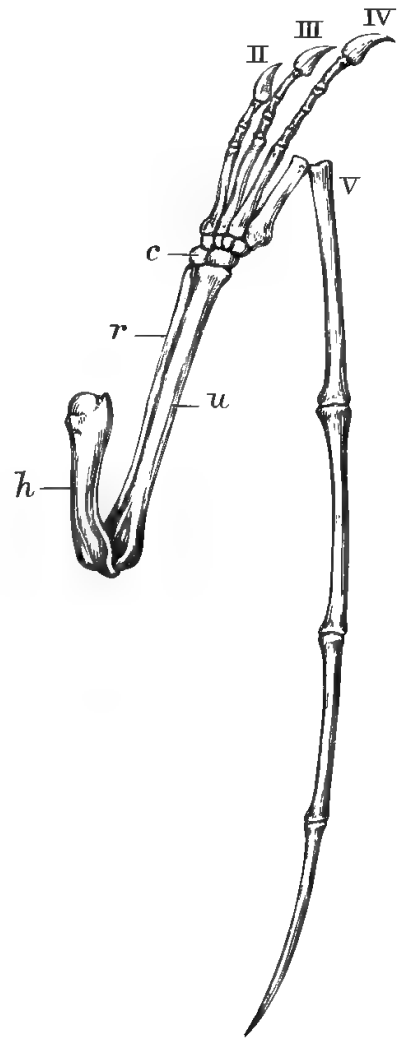

FIG. 37.-Bones of the wing of the Pterodactyl(Scaphognathus crassirostris) or Hying reptile. Shows that the second, third, and fourth digits of the hand are aborted and terminate in claws, and that the fifth digit is thickened and enormously elongated for the purpose of supporting and carrying the flying membrane which constitutes the essential part of the wing as far as flight is concerned (after Nicholflight is concermed
son and Lydeker). a place in the Museum at Berlin. The Archæopteryx was the size of a large pigeon, had a short head, and probably no beak. Its jaws were furnished with small teeth. Both wings had three fingers, each armed with a claw. The legs, which resemble those of living birds, had four toes, also armed with claws. The claws on the fingers and toes were probably employed in clinging to the bark of trees, where the bird found larvæ and other food. The wings, thighs, and body were covered with feathers, and there can be little doubt the bird could fly. The tail was peculiar. It was osseous and much elongated, and consisted of a diminishing series of vertebræ, with feathers arranged on either side of it like a palm leaf.

The proof of the descent of the Archæopteryx from the reptile, it will be seen, is by no means strong or striking, being confined to the presence of small teeth in the jaws, a phenomenally long bony tail, and two wings, each having three fingers terminating in as many claws. 
The modifications and presence of claws in the wings of the Archæopteryx are less remarkable than the modifications met with in the Pterodactyl. The Pterodactyl, or flying reptile, as it is frequently called, had wings much

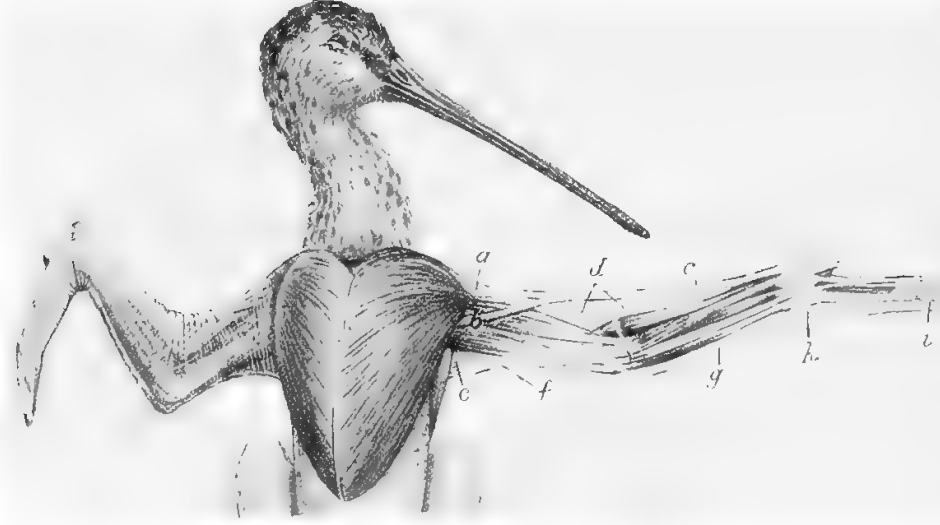

FrG. 39. - Shows ventral aspect of the hard and soft prarts-muscles, tendons, elastic tissues, liganents, and bones-of the wing of the snipe (frallinago rrelestis). In the figure the left wing is flexed and the right wing fully extended. The parts marked $c, g, h, i$ (especially $h, i$ ) show where the wing is flattened ont, solidified, and strengthened to support the roots of the primary and secondary feathers which are the inmediate instruments of flight. The primary and secondary feathers are seen in position at Fig. 40, $d, b$, Muscles connected with the elastic structures of the wing ; $c, d, c, f$, $g, h, i$, nusculo-fibro-elastic tissnes which are mainly concerned in flexing the wing. The great depressor or chest muscles are seen between the wings. (From a dissection and drawing by the Author.)

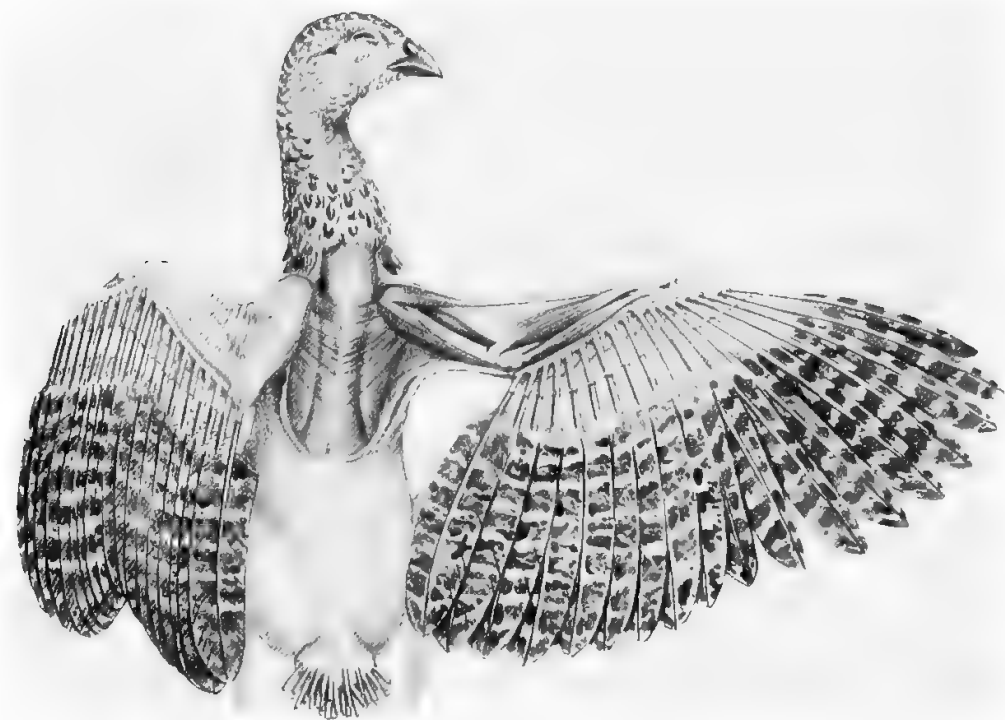

F'Is. 40. - Shows the dorsal aspect of the hard and soft parts of the wing of the pheasant (Phasionus colchicus). In the right wing the roots of the primary and secondary feathers are exposed to show their relation to the fore arm, hand, and greatly moditied phalanges or finger hones. The left wing is fully flexed and ready to make the up stroke. In the left wing the feathers are widely separated, and Iresent a series of vertical knife-like edges through which the air escapes during the uls stroke; in the right wing the feathers are spread ont and firmly pressed together to prevent the escape of air during the rlown stroke. (From a dissection and drawing by the Author.) more modified than those of the Archæopteryx, as shown at Fig. 37.

Similar remarks are to be made of the wings of the modern bat (Fig. 38).

Still more remarkable are the modifications of the wings of existing birds, where the bones of the hands are reduced in number; some being greatly enlarged, and soldered together to provide a flat, osseous support for the roots of the primary and secondary feathers, which play a most important part in all air-flying birds (Fig. 39).

The points which I wish to establish in the present connection are :-

1. That the typical five fingers or toes in the limbs of the majority of vertebrates are not permanent, but a vanishing quantity: the toes of the hind limbs being, in many cases, fewer than the fingers of the fore limbs.

2. That the fore limbs of the elephant and of many fossils have five fingers or digits: that in some of the Dinosaurs (extinct reptiles) there are four digits in the fore limb and only three in the hind limb: that in the extinct moa there are three toes with a rudimentary fourth toe to the hind limbs: that in the modern emu there are three toes to each hind limb: that in the ostrich there are only two toes to each hind limb: that in the camel, ox, and deer there are two digits to each of the limbs: that of the two toes of the ostrich, one is very much longer and stronger than the other: that there is also a slight deviation in this direction in the camel: that in the horse there is only one digit to each limb-. this being very much enlarged and very strong.

3. That the modifications in the digits of the fore limbs of vertebrates are very remarkable, and cannot be accounted for as apart from a First Cause and Design : that in some cases not only are the digits suppressed, but other digits are hyperdeveloped to quite an extraordinary extent: that in the foot of the ostrich, where there are only two digits, the one is dwarfed and the other very greatly enlarged: that in the wing of the pterodactyl there are three digits which are aborted and terminate in hooks - one digit (the fifth) being enormously elongated and hypertrophied: that in the wing of the bird the digits are reduced to three, and that they are run together, solidified, and increased in size to form a flattened, osseous surface which supports the roots of the primary and secondary feathers, the immediate instruments of flight: that in the horse, only one digit remains for each limb; this being phenomenally large and strong.

4. That there are strong grounds for believing that the variations in the number and shape of the digits of 


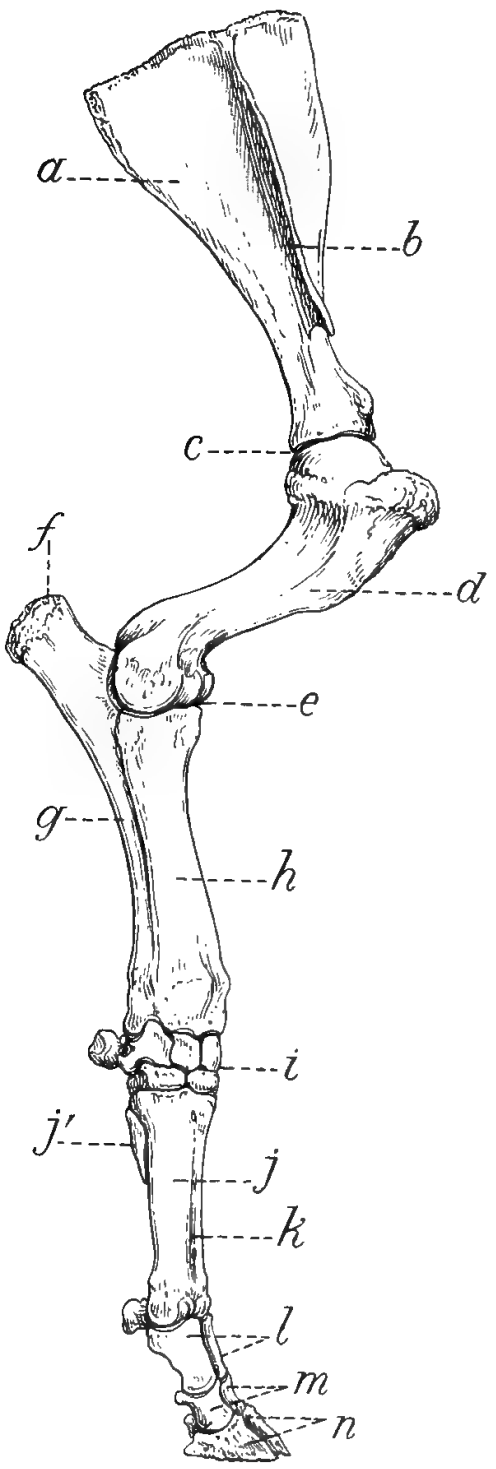

A
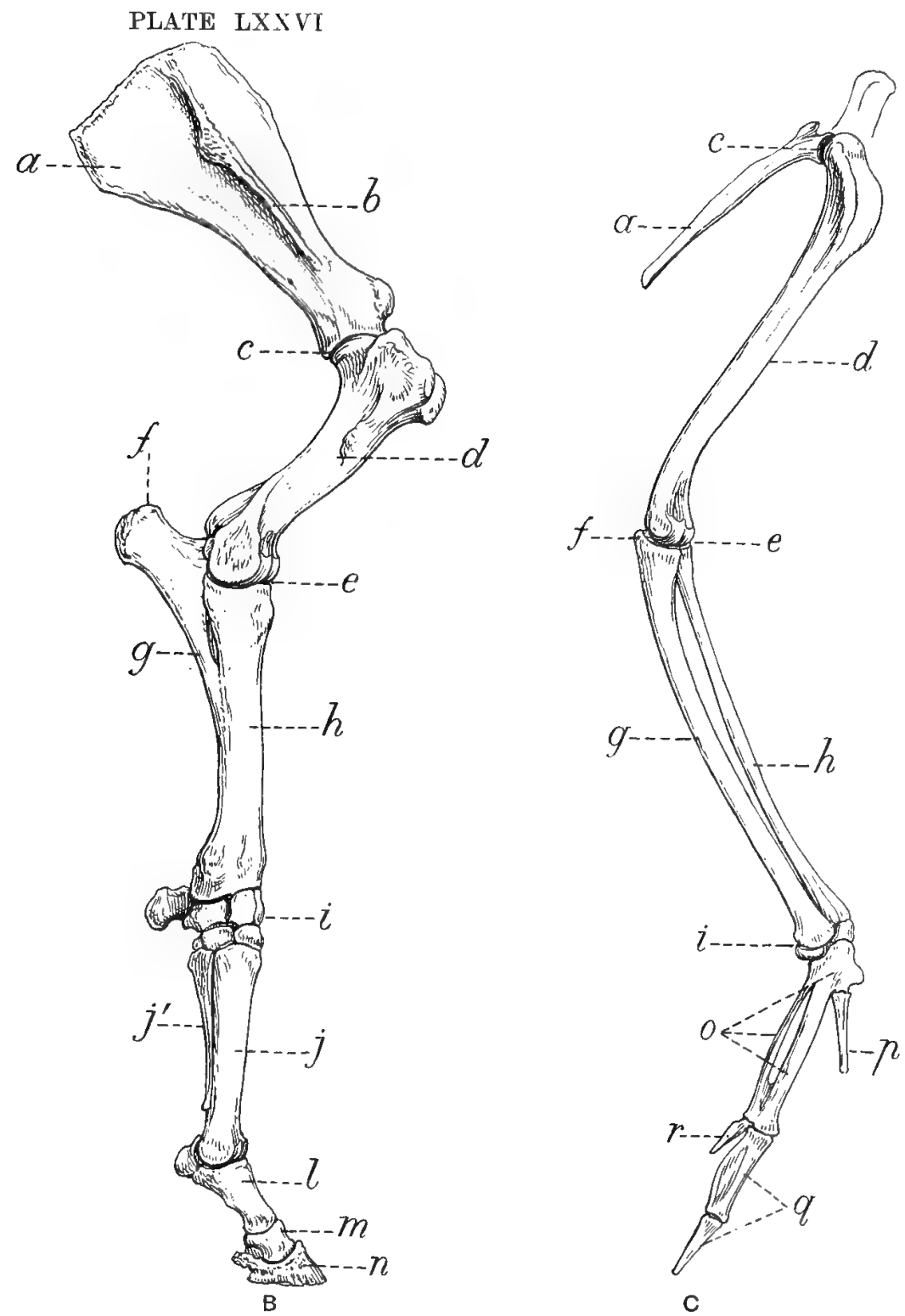

A. Skeleton of the right fore leg of the ox (Bos taurus). $a$, Scapula; $b$, spine of scapula; $c$, scapulo-humeral articulation; $d$, humerus; $e$, humero-radial articulation ; $f$, olecranon; $g$, ulna; $h$, radius ; $i$, carpal bones ; $j$, large metacarpals ; $j^{\prime}$, small metacarpal; $l$, suffraginis; $m$, coronary; $n$, pedal bone $(l, m, n$ form the first, second, and third phalanges). Drawn from nature ly C. Berjeau for the Author.

B. Skeleton of the right fore limb of the horse (Equus caballus). a, Scapula; $b$, spine of scapula ; $c$, scapulo-humeral articulation; $d$, humerus; $e$, humero-radial articulation; $f$, olecranon; $g$, ulna; $h$, radius; $i$, carpal bones; $j$, large metacarpal or cannon bone ; $j^{\prime}$, small metacarpal or splint bone; $l$, suffraginis; $m$, coronary; $n$, pedal bone $(l, m, n$ form the first, second, and third platanges). Drawn from nature by C. Berjeau for the Author.

C. Skeleton of the right wing of the whooper swan (Cygnus musicus). a, Scapula; c scapulo-humeral articulation ; $d$, humerus; $e$, humero-radial articulation; $f$, olecranon; $g$, ulna; $h$, radius; $i$, radio-ulnar-carpal articulation; $o$, carpo-metacarpus; $p$, first digit or thumb; $q$, second digit; $r$, third digit. Drawn from nature by C. Berjeau for the Author.

the limbs of vertebrates are solely connected with the locomotion of the animals, and have nothing to do with descent and consanguinity.

5. That the phalanges (fingers and toes) of vertebrates are original endowments conferred upon animals during their development and at birth, and that they are not after-thoughts or modifications due to externalities, environment, or alteration of plan.

6. That this view is confirmed by the fact that all animals, to whatever order, family, or class they belong, must conform (as far as their travelling organs are concerned) rigidly to the principles which govern progression on the land, in the water, and in the air respectively. 
It is no easy matter to deal satisfactorily with the organs of locomotion as apart from a First Cause, Law, Order, and Design. Given these, there is no difficulty. The subject resolves itself into cause and effect, and the ends attained are always explained by the means employed.

The modifications in the fore arm, \&c., of the ox, horse, and swan are given at Plate lxxvi.

This plate gives comparative views, and shows the modifications, more especially in the fore arm, wrist, hand, and phalanges, of the ox, horse, and wing of the bird. In the ox, the fore arm has two digits armed with two hoofs. In the horse the fore arm has only one digit and one powerful hoof. In the wing of the bird the bones of the hand and fingers are mixed up and soldered together to give additional strength for supporting

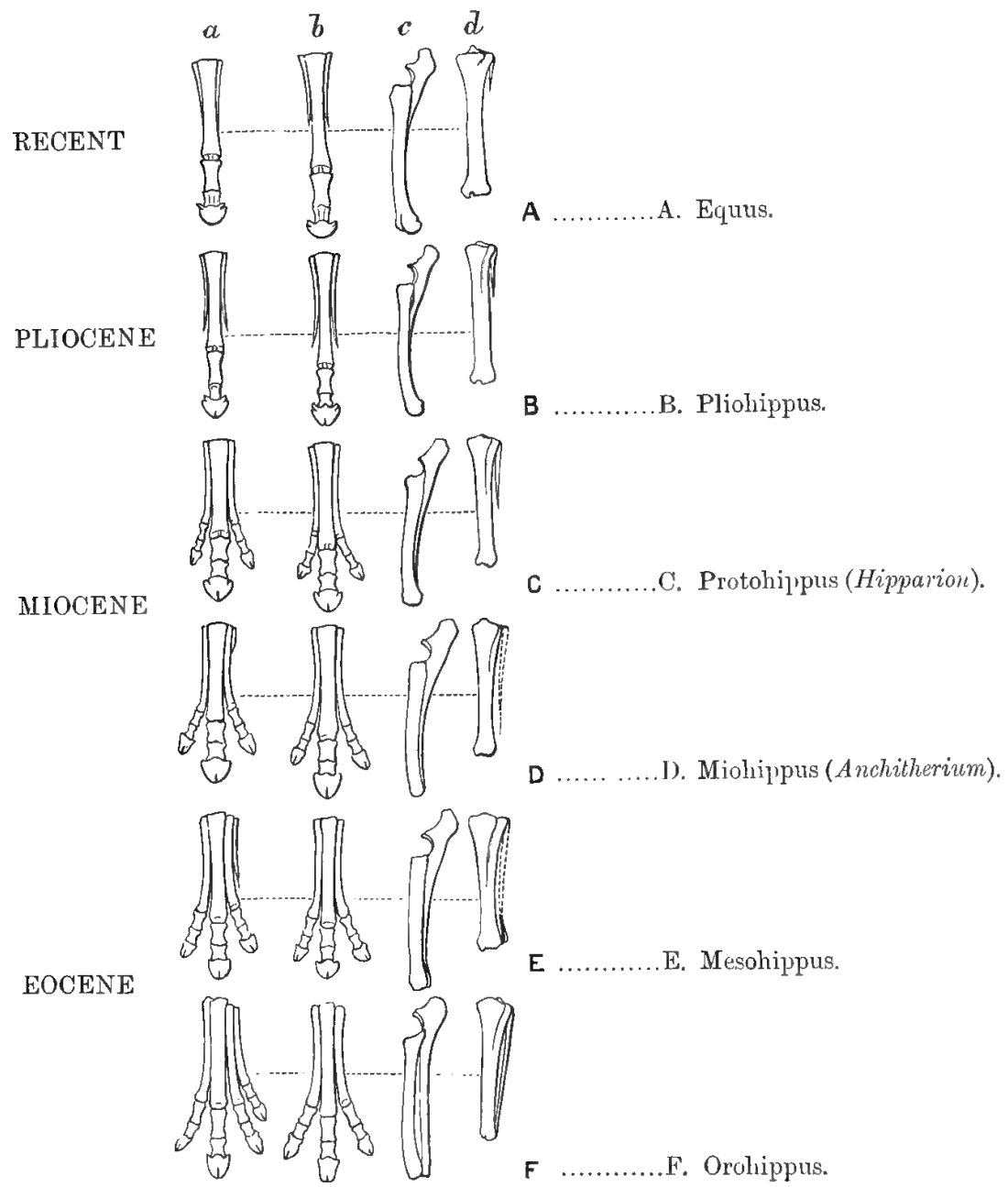

Fig. 41.-In this higure the feet and bone limbs of the horse (Equus caballus) are conpared with those of its supposed ancestors. $a$, Fore foot; $b$, lind foot ; $c$, fore arm ; $d$, leg (after Huxley). the roots of the primary and secondary feathers in flight. The modifications referred to are very striking, and are connected with the locomotion of the several animals. They have nothing to do with heredity and descent, not even in the horse, which is generally regarded as a direct descendant of a five-toed mammal. In the ostrich the foot consists of two digits-the one being very much larger, stronger, and longer than the other. Here again the exigencies of locomotion afford the true explanation of the arrangement. In this quaint bird, which wallss and runs on loose sand, the large digit performs the office of a foot. The extinct moa had three digits and a rudimentary fourth one, and the modern emu has three digits to each foot. In these running birds the dropping of digits is very suggestive and remarkable. The horse and ostrich are the fleetest of existing land animals.

The supposed descent of the horse, according to Huxley and Marsh (especially the latter), from a five-toed mammal is illustrated at Fig. 41.

In Fig. 41 the foot of the horse is traced from past to present time-from the Focene, Mrocene, and Pliocene until we reach the Pleistocene, or recent period. The oldest horses were said to be about the size of a large dog. T'he following is the supposed order of succession: Orohippus (Eocene), with four toes to the fore leg and three toes

to the hind leg; Mesohippus, with three toes and an imperfect fourth toe to the fore leg, and three toes to the hind leg; Miohippus anchitherium (Miocene), with three toes each to fore and hind limbs; Protohippus hipparion, with three toes each to fore and hind limbs, the outer toes becoming thinner and shorter and not quite reaching the ground; Pliohippus (Pliocene), with three toes to each fore and hind limb, the outer toes becoming so thin as to be mere rudiments, and being far removed from the ground; Equus (Recent), with three toes to each limb, but the outer toes so shrunken as to be scarcely recognisable even as vestiges. In these transformations the central toe of each foot is from the first the largest: it gradually hypertrophies: the outer toes becoming meanwhile smaller and smaller.

In the lapse of time, in the opinion of Huxley and Marsh and evolutionists generally, a new animal is formed. There is no warrant for such a conclusion. The dwarfing and virtual suppression of portions of limbs is not confined to the horse. It occurs in several animals, as the Pterodactyl, or flying reptile, and the diving and flying 


\section{THE TRAVELLING ORGANS OF ANIMALS}

bird, to say nothing of similar modifications on a large scale in the vertebrate series, where the toes range from five as a maximum to one as a minimum. Nor must it be forgotten that in certain sea mammals, such as the dugong, manatee, porpoise, and whale, not only the toes, but also the limbs, especially the posterior ones, entirely disappear.

If the dropping of toes proves anything, it proves the existence of type in limbs (as in other parts), where enough remains to show its origin and general relations, and connections of the parts to each other. The dropping has certainly nothing whatever to do with consanguinity and descent, or with evolution.

The object in view in the modification of limbs and other parts of animals is to make the modified parts more perfect for the performance of some special work. The massive single toe of the horse, armed with a strong hoof, is the best possible for dealing with the hard, unyielding earth in walking and running; the wing of the flying bird, with its fingers soldered together and its covering of fleecy elastic feathers, being the best conceivable for dealing with the soft, yielding air in flight. In all this there is the most convincing proof of a First Cause and Design. The instrument is specially created to perform specific work on the most approved mechanical principles-the means and the ends being always found side by side. No effect, great or small, is produced apart from an adequate cause. This argues pre-determination, law, and order in infinite variety. It would be a singular circumstance if the limbs of the horse were modelled in a way no other limbs are. As a matter of fact, the fore limbs of the horse are less modified than the wings of the bird.

It will be seen that a by no means strong case has been made out for evolution and the descent of the modern horse from a five-toed extinct mammal. Perhaps even less can be said when the teeth of the horse and its supposed ancestors form the fulcra of the argument. It was noticed that the crowns of the molar or grinding teeth of the existing horse had a complicated pattern, and immediately palæontologists set about finding similar teeth in mammals, ancient and modern; the mammals in question having toes ranging from five to one. Of course it must not be overlooked that the modern horse and its supposed ancient ancestors are not the only mammals whose molar teeth exhibit complicated patterns. The modifications in the toes and teeth of the horse when the mammalian series is regarded as a whole can scarcely be said to amount even to peculiarities, and assuredly do not justify the outstanding position claimed for them by evolutionists and by those who have a fixed idea that the horse is descended from a five-toed mammal. The supposed relation of kirds to reptiles based on the possession by both of teeth, and peculiarities in the pelvis and fore limbs, is, to a large extent, fanciful, and can scarcely be seriously entertained. The closer the subject is examined the less satisfactory it becomes. A certain amount of detail regarding the configuration, modification and uses, especially of limbs, is necessary at this stage.

\section{THE TRAVELLING ORGANS OF ANIMALS TO BE REGARDED AS ORIGINAL STRUCTURES}

The wings of birds in no respect resemble the fore legs of reptiles as a class. They are structures specially modified to navigate the air. The limbs and the hands and feet of birds vary infinitely, but in every case they are means to ends. Thus there are running, swimming, diving, and flying birds. The ancient dodo had no wings : the same is true of the modern apteryx. Wingless birds can only walk and run. The ostriches and emus have small, short wings useless for flight, and long legs. which make them excellent runners; the penguins have small, flexible, featherless wings, which enable them to dive and to fly in, but not out of, the water-they have also webbed swimming feet. The majority of birds have large wings provided with primary, secondary, and tertiary feathers, by the aid of which they fly in the air. Some birds, such as the ducks, can walk, swim, dive, and fly by turns. The travelling organs of birds are designed and adapted structures. They are in no sense to be regarded as haphazard developments: neither are they to be regarded as evolutions as apart from desigri and a predetermined plan.

Modifications in the travelling organs required for the purposes of progression do not prove that the bird is descended from the reptile. Neither do similar modifications in the horse prove that that animal is descended from a five-toed mammal. There are modifications and peculiarities in the limbs of birds and horses, and there is gradation, but this is all that can be said. As a matter of fact, the modifications in the wings, legs, and feet of birds show as great a variety of structure as is found between birds and reptiles themselves; but the modifications afford proofs of adaptation and design in the travelling organs, and do not support a blind evolution which ignores a First Cause and refers the modifications to chance as apart from Design. Similar remarks are to be made of the limbs and feet of horses. They afford examples of special developments for a purpose : they are not mere purposeless, blind adaptations, as evolutionists would have us believe. In the case of the hands and feet of vOL. I. 
the horse there is a diminution in the number of osseous and other parts, a contraction of the travelling area, and a concentration of power in certain bones, which are greatly enlarged and thickened for a specific purpose (Plate lxxvi., Fig. B). In the case of wings, especially those adapted for flying under water and diving ${ }^{1}$ (Plate l., Fig. 1, J), the same features are to be noted, namely, diminution in the number of the bones of the wing, contraction of the osseous wing-area, and a concentration of power in certain wing bones which act screw-fashion in sub-aquatic flight. Nor does the matter rest here in the case of birds which fly in the air. While the skeleton of the wing of the air-flying bird is being simplified by the fusion of certain of its bones, and its actual osseous area reduced, its covering of feathers, especially the primary, secondary, and tertiary ones, which are the immediate organs of aërial flight, is being much differentiated and greatly amplified to perfect it as an air-flying organ (Fig. 42). All this means design, modification, and adaptation as apart from mere evolution as such. There can be no
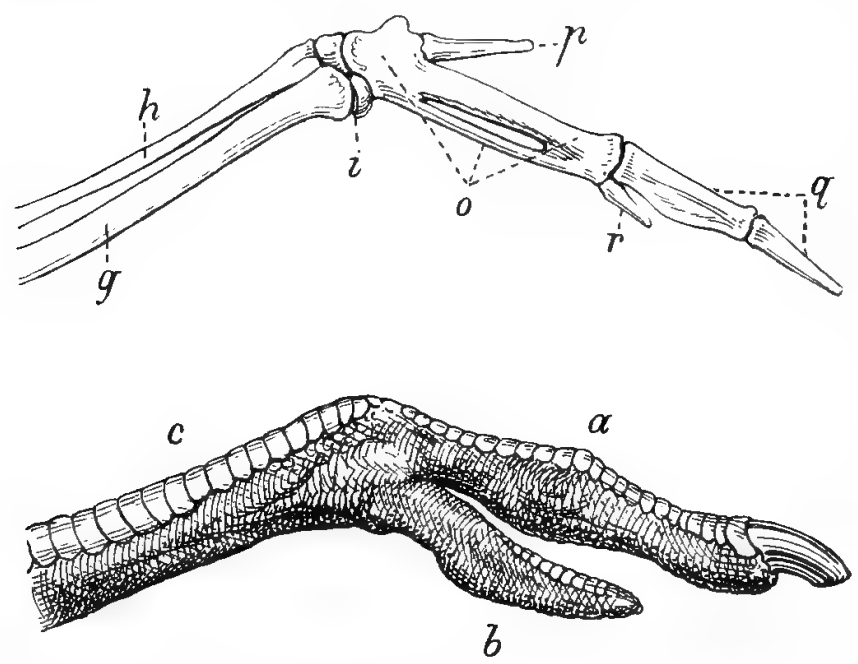

Fig. 42.-Skeleton of the outer portion of the right wing of the whoper swan (Cygnus musicus). Shows that the bones of the hand and phalanges (finger bones) are very greatly modified; certain of the bones being suppressed and others greatly enlarged, strengthened, and soldered together to form at flat osseous platform for supporting the roots of the primary and secondary feathers which are especially engaged in aèrial flight $q$, Ulna ; $h$, radius ; $i$, radio-ulna-carpo articulation ; $u$, carpo-metacarpus : $p$, first digit or thumb ; $q$, second digit ; $i$, thild digit. Drawn from nature by C. Berjean for the present work.

FI: 43.-Portion of the right leg and foot of the ostrich (Stmuthio camelus). The foot of the ostrich displays two toes, and is most interesting. as it forns a transition link between the great extinct moa (Dinornis gigcinteus), which lad four toes, and the modern emu (Dromzus novihollamdit), which has three toes. The dropping of toes in the ostrich tribe is quite as remarkable as the dropling of toes in the horse. "Major toe of the ostrich, which does the principal part of the walking and running; $b$, minor or sulisidiary toe, which takes little part in locomotion; $c$, lower part of leg. Drawn firon nature by C. Berjean for the present work. better examples of rlesign given than are afforded by the wings and the legs and feet of birds as shown at Figs. 42 and 43 . In these figures I have had depicted from nature, and according to scale, the outer portion of the right wing of the whooper swan, an air-flying bird, and the right leg and foot of the ostrich specially modified to walk and run on sand. The wing is a marvel of design. Its bones are twisted and spirally arranged. It is a screw structurally and functionally. It makes a figure-of-8 track in space in stationary, and a spiral, waved track in free flight. The wing feathers (primary, secondary, and tertiary) are beautifully graduated as regards length, direction, and strength. They are elastic, and taper from the roots to the tips. The primary feathers are slightly twisted in the direction of their length, and the primary, secondary, and tertiary feathers are set at various angles, and are geared to each other and overlap in such a way that they open during the up-stroke of the wing and close during its down-stroke. They thus alternately evade and seize the air. The opening of the feathers corresponds with the folding of the wing and the up-stroke, and the closing of the feathers with the extension of the wing and the down-stroke. The arrangements are the most intricate and perfect possible. Shall we be told that the wing, a complicated and inimitable structure largely developed before the bird is hatched out, is a chance product, the outcome of environment and of a groping, eyeless evolution? What is said of the wing of the bird applies equally to the legs and feet. The legs may be long as in running, wading birds, or short as in swimming birds: the feet may possess two, three, or more digits, and they may bear claws as in the birds of prey, or webs as in swimming birds. The legs and feet, like the wings, are modified, and adapted to perform certain functions. They also afford proofs of design, to the exclusion of evolution as a blind factor in the production of specific structures, the functions of which are obvious and lie on the surface. Evolution, it is said, makes for progress, but it has not been shown that the modern horse is a more perfect animal than the five-toed mammal, its supposed ancestor; or that the modifications in the limbs of birds are not as great, or even greater, than those which nccur in the horse. The order of the organic kingdom is one of gradation, and it may be quite a fallacious interpretation of the facts to say that the so-called connecting links are not as important in their way as the great groups themselves. This remark applies to development as a recognised part of gradation. The mere number of individuals in a group, as I have pointed out, does not invalidate the distinction here drawn. From a careful and extended study of the subject I am disposed to believe that the limbs and solid hoofs of the horse tribe are not the outcome of evolution, but are special developments, modifications, and adaptations to ensure strength, and to increase speed on

1 In the case of birds which fly in the air, the artu of the wing is greatly increased hy the utdition of feathers, namely the frimary, secondary, and tertiary feathers; these being chiefly engaged in atirial thight, 


\section{THE TRAVELLING ORGANS OF ANIMALS}

land. The same is true of the limbs and small feet of the ostrich. In the ostrich the digits of each foot are reduced to two ; one digit being mainly employed in running.

Five-toed typical mammals, with heavy, powerful frames, are not so well adapted for runring on hard surfaces as the horses, but they still exist-as witness the elephant. There was no necessity to manufacture the horse from a five-toed mammal. The modified limbs and solid hoofs of the horse are rather to be regarded as a proof of type and of adaptation and design, and, as such, are to be placed in the same category as the swimming tail of the fish and reptile; the swimming feet of the frog, beaver, ornithorhynchus, bird, seal, and walrus; and the flying wings of the insect, bird, and bat. These are all examples of structures adapted to the media the animals are to traverse (Plates l., li., and lii.). They are special creations rather than mere modifications. Environment and externalities cannot produce them: they are constructed in young animals during development and before they are required. They are means to ends, but do not prove consanguinity and descent.

The rehabilitation of so-called missing links, and the modifications of existing structures, especially limb structures, do not afford a safe basis for constructing a chart of consanguinity and descent. The presence of teeth in the heads of a few extinct birds, the pattern of the teeth of the horse, and the decrease or increase in size, and the appearance or disappearance of certain limb bones in the same or other individuals, do not prove evolution, descent, or blood relationship. All these phenomena can be explained outside evolution and descent by reference to the requirements of life and the exigencies of locomotion. That a few extinct birds had teeth does not prove the descent of the bird from the reptile; neither do the suppression of certain limb bones and the greatly increased size of others give a valid clue to the descent of the horse.

As a matter of fact, the changes and modifications introduced into the animal series, because of modifications in their organs of locomotion, are much greater than the data usually relied on for proving the doctrines of evolution and descent. I have, therefore, to express my conviction that evolutionists are not on safe ground when they confine proofs of their views to one or two lines of demonstration; these being by no means the most obvious or convincing.

Professor Huxley regards America as the original home of the horse, rather than Europe, and mentions incidentally that the animal died out on the American continent and had to be re-introduced. If this view be correct, it is difficult to understand how such a contingency could have arisen, considering the vast extent and the resources of the continent occupied. The dying out view is essentially opposed to the theory of environment.

The evolution of the foot of the horse from a five-toed mammal is on a level with the evolution of the wing of the pterodactyl, bird, and bat, from some five-fingered unknown flying ancestor. The wing of the bat (Fig. 38) is composed of an arm, fore arm, wrist and five digits, with an outstretched membrane. The digits are nearly of the same length, and take an equal share in supporting the membrane, and in the function of flight. In the hand and phalanges of the pterodactyl (Fig. 37), as in the foot and phalanges of the horse, several of the digits are dwarfed, one of them (the fourth) being enormously developed, and forming the chief organ of locomotion. Certain of the digits are dwarfed and rudimentary, but no one would, on this account, say that the ancient pterodactyl was evolved from some extinct animal resembling the modern bat, which is a more highly differentiated animal. Similar remarks are to be made of the wing of the bird. The wing of the bird is composed of a humerus (arm bone), a radius and ulna (fore arm bones), modified carpal or wrist bones, and several digits or finger bones run together and fused (Fig. 12). The arm and fore arm bones are independent and separate as in the primates and in ourselves. The wrist and finger bones, on the contrary, are modified almost beyond recognition; certain of them being suppressed, while others are fused and solidified to form one strong compound bone, which bears the great primary feathers, the most essential structures in flight. The very remarkable modifications occurring in the wing of the bird are quite as extraordinary as those occurring in the limb of the horse, but no one would be so rash as to suppose that the bird, because of certain modifications in its wing, was descended from any form of mammal, with distinct wrist bones and five distinct digits. Neither would the bat (a mammal, with five digits in its wing) furnish the ancestor of the pterodactyl (Fig. 37). The dropping of certain bones, and the enlarging and fusing of others, in the limbs and travelling organs of animals afford no proof of evolution in the strict or proper sense. Evolution involves differentiation and increase in the number of parts. It is opposed to devolution, or the decrease and suppression of parts. The increase and decrease of parts here referred to, which occur even in the embryo, while inimical to the doctrine of evolution, supply strong arguments in favour of type, a First Cause, design, and adaptation to given ends.

The modifications required for locomotion can be traced in all animals, however diverse. They are not confined to allied animals, and consequently, and strictly speaking, have nothing to do with evolution; evolution requiring connecting links, and points of resemblance and continuity, in the evolving forms.

The connecting links in locomotion do not support the theory of evolution. Thus the galeopithecus or flying 
squirrel is provided with a flying membrane which extends between its fore and hind limbs; and the flying lizard is provided with a similar membrane, which is supported by its ribs. The galeopithecus, and flying lizard, however, have nothing in common, and no direct connection either with the pterodactyl or with the bird. Similarly, the pectoral fins of the flying fish do not connect that animal with the galeopithecus, flying lizard, pterodactyl, bird, or bat. This modification of limbs for the purposes of progression opens up a wide question quite apart from evolution and descent, and even classification. The dolphins (sea mammals), if the pectoral fins be excepted, have no limbs: the whales have pectoral fins or flippers, but no posterior limbs or the merest vestiges of them. The same is to be said of the Sirenida (the dugong, manatee, rhytina, \&c.), where the flippers bear a general resemblance to limbs. All these animals (mammalian in type) are supplied with swimming tails and fish-like bodies. In the case of the seal, sea-lion, and walrus (likewise mammals), they are furnished with more or less fish-shaped bodies, and modified but well-developed limbs, each of which has five digits. The digits support a swimming membrane, and can be opened and closed, particularly those of the posterior limbs. The digits and swimming membrane of the hind limbs form what is virtually a powerful swimming tail, which, as in the fish, is lashed from side to side, and is the chief organ of propulsion. In the sea-mammals the tail is lashed in a vertical direction. In the flying fish the pectoral fins, which are the homologues of the anterior extremities, are greatly expanded, and have the form of wings, and act as such to a limited extent. The flying fish has, in addition, a swimming tail, and forms a connecting link between progression in the water and the air, just as the galeopithecus and flying lizard form connecting links between progression on the land and in the air. In all these cases, the construction of the limbs (designed and modified, as explained, for particular purposes) affords no proof of evolution. Indeed, and as already stated, the several travelling organs are constructed in the young animals before they are required. As a matter of fact, the animals whose limbs and travelling surfaces are modified and adapted to operate on essentially different media must be arranged in different categories, as they include, or may include, insects, fishes, reptiles, birds, mammals, \&c. That the modifications referred to have nothing to do with descent and evolution as their chief factor, is proved by the changes which occur in the frog during development. In the tadpole state it is provided with a swimming tail adapted for propulsion in water; as it reaches the adult condition its tail rlisappears, and it is furnished with four limbs, each having five digits, with a swimming membrane adapted for water and land transit. The travelling organs are modified to meet the requirements of the water and land respectively, but the water and land and environment do not make the travelling organs; these are designed, specially prepared structures connected with the movements, habits, and breathing of the animal.

The question of limbs as organs of locomotion is a very wide one. The shape of limbs, if it proves anything, proves design as contra-distinguished from evolution. When we find animals of every conceivable kind modified to meet the particular requirements of transit on the land and in the water and air, design of necessity takes precedence of evolution. The transitions of structure and function in the organs of locomotion in animals are very numerous, but they are, in every case, graduated and designed transitions: they are adapted to the land, the water, and the air, or partly the one and partly the other. They are in every case means to ends. They are never chance products.

Small feet suffice for supporting heavy animals on land. The deer, ox, horse, pig, \&c., afford examples. Large feet, often webber, are required for soft or boggy land, as witness the feet of the frog, wading bird, ornithorhynchus, otter, beaver, \&c. Still larger travelling organs are required for the water, as seen in the flippers and feet of the turtle; the feet and swimming tail of the newt and crocodile; the fins and tail of the fish; the webbed hands and feet of the seal, sea-lion, and walrus; the flippers and swimming tail of the dolphins and whales; the fin-like anterior extremities and fish-like tails of the dugong, manatee. rhytina, \&c.

Lastly, very greatly expanded travelling organs are required for transit in the air. These are seen in the extinct flying reptiles and in modern insects, birds, and bats. Then there are, as stated, transition links in the travelling organs, as hetween the land and air on the one hand, and the water and air on the other. The flying lemur, flying squirrel, and flying lizard afford examples of the former, and the flying fish of the latter.

I purposely abstain from an enumeration of the myriad travelling organs in the infusoria and lower animal forms, and in plants. With such an amazing array of designed and adapted travelling structures occurring in innumerable animals, and in not a few plants, it is impossible to come to any other conclusion than that all animals, and all plants with independent movements, are specially designed and constructed organisms as apart from evolution. As environment and externalities do not form the organs of locomotion, neither do they form other organs in plants and animals as a whole.

The subject of the modifications of limbs, and especially those of the horse, is not a fortunate one as proving the truth of the doctrine of evolution. Even in animals so far advanced as the vertebrates, limbs can be dispensed with. The serpent has no limbs, and some of the lizards have the merest traces of them; but both 
perform their several functions perfectly. Limbs, even in the highest animals (man included), are mere buds or outgrowths of the trunk and vertebral column, which are the essential parts of the organism. The hard and soft parts in animals split up transversely as in vertebræ, and longitudinally as in limbs, which terminate in digits and range from one to five or greatly exceed that number, as in the fins of fishes and the flippers of certain extinct animals, such as the Ichthyosaurus, Plesiosaurus, \&c. Illustrations of limbs and limb structures are given at Plates xlvii., xlviii., xlix., and l., which see.

The limbs and travelling organs of animals are the product of a First Cause, and are means to ends. That there is gradation, plan, and adaptation in limbs and travelling organs, as apart from evolution, is proved hy the relation which exists between the travelling organs and surfaces of animals and the media to be traversed. No animal can progress satisfactorily on the land whose travelling surfaces are not adapted to land transit, and the same is to be said of the water and air. The modified travelling organs which connect the land with the water and the water with the air, or each with the other, afford no proof of evolution. The land, water, and air form the three great highways for animal progression, and every animal, to whatever order it belongs, must possess organs of locomotion adapted for one or other. The land, water, and air afford different degrees of support to the travelling organs of animals, and these must be modified to deal with the question of support as well as of propulsion. The more tenuous the medium traversed, the larger must be the travelling organs. No animal is equally well adapted for walking, swimming, and flying, although there are several examples of animals which walk, swim, and fly indiscriminately.

The travelling organs of animals becorne exceedingly interesting when viewed from the teleological standpoint. When so viewed it is all but certain that they are to be regarded as original structures-that is, structures which formed part of the original animals, and which were absolutely necessary to the early as well as to the later and continued existence of the animals. The organs in question were formed even before they were required. They grew and were developed with the other parts of the body. There is no proof that they were made piecemeal, or that they passed through innumerable stages before reaching their present state of perfection.

The travelling organs lead us back to a much disputed subject, namely, were the land animals originally marine animals, or did certain of the land animals take to a marine existence, or were the land and marine animals created separately at the outset? I am wholly in favour of the separate creation view, and of the production of great races and types of animals adapted at the beginning to their several surroundings. I cannot reconcile myself to the production of organs by infinite modifications in infinite time, seeing a creation at first hand was as easy and easier than a creation at any subsequent period, even at a millionth remove.

The Mosaic account of creation is very explicit on this particular point. According to it, not only animals but also plants were created separately and for different regions of the earth's surface. They were also created to live and travel in different media. The several types of plants and animals which exist on the earth at present had their prototypes in the early days of our planet. Fven flying things had a place among the primeval creations. The Mosaic narrative runs as follows: "And God said, Let the earth bring forth grass, the herb yielding seed, and the fruit tree yielding fruit after his kind, whose seed is in itself, upon the earth. . . And God said, Let the waters bring forth abundantly the moving creature that hath life, and fowl that may fly above the earth in the open firmament of heaven. And God created great whales, and every living creature that moveth, which the waters brought forth abundantly, after their kind, and every winged fowl after his kind. . . And God blessed them, saying, Be fruitful and multiply, and fill the waters in the seas, and let fowl multiply in the earth. . . And God said, Let the earth bring forth the living creature after his kind, cattle, and creeping thing, and beast of the earth after his kind: and it was so."

The remarkable feature of creation is its unvarying uniformity and conformity to law and order.

In the case of animals, their general shape and travelling and other organs must satisfy the requirements of the media they are severally called upon to live in. In every case, the animals must be adapted to one or other special set of conditions.

If the land animals take to the water they must have modified or suppressed limbs, a fish shape, and a swimming tail. If the marine animals take to the land they must have a modified or suppressed tail and more or less perfectly developed limbs. The same reasoning applies, within limits, to birds. As the land and water do not create or form the travelling organs, it follows that the animals which move on the land and in the water respectively must be provided with the necessary travelling organs as part of their original outfit and endowment.

\section{\$3. The Travelling Organs in Relation to Environment.}

There is an organic and an inorganic kingdom, and the one is fitted to the other. Both have been made by the same hand, and the activities of both determined. It has not been proved that the representatives of the organic 
kingdom are automata set in motion and regulated by different parts of the inorganic kingdom. The most that can be said is that plants and animals derive their substance and part of their force from the inorganic kingdom. Their forms, their structures, their functions, and their activities generally, are inherent and peculiar to themselves. Plants and animals are living things, designed for specific purposes, and constructed to discharge particular offices. While they are influenced up to a point by environment, they are, in no sense, at its mercy. Environment, be it noted, essentially consists of externalities; conditions of heat and cold, light or its absence, moisture and dryness, state of the earth, the water, and the air, supply of food, crowding, \&c. While environment leads to modifications (within limits) of growth and development it does not alter the constitutions of plants and animals, and produce new individuals. I wish to emphasise this point. Plants and animals slightly modify themselves —or, to be more accurate, are modified by a First Cause - to meet new requirements, but they do not thereby lose their identity. Environment applies all round to certain localities and certain periods. It is more or less a fixed quantity as regards time and space. It is calculated to produce sameness in plants and animals, and certainly would do so if plants and animals were fundamentally alike. It assuredly cannot, of itself, account either for the origin and infinite variety of plants and animals, or the numberless slight modifications which occur in them from time to time. The origin of plants and animals, as well as the modifications and adaptations which characterise them, cannot be referred to it. It is not a chief factor either in the production of plants and animals or in the changes and variations which are known to take place in them.

If, as Professor Huxley believes, the birds resemble reptiles, and the horses certain ancient mammals, the fact, if fact it be, can only be explained by design and intelligent adaptation; and the design and the adaptation must be referred either to an intelligent Designer, or to the living things created and controlled by Him.

Blind, purposeless modification in the principal and subsidiary groups of plants and animals can achieve nothing. The fact that different plants and animals located in a more or less stable environment assume an infinite variety of shapes, and discharge an extraordinary diversity of function, proves to my mind that the main factor in the organic kingdom lies outside environment. To realise what is here stated one has only to remember that the plants and animals on the surface of the globe are legion as regards size, shape, and function, and that the environment, as explained, is more or less a fixed quantity in different localities and at different times. If physical peculiarities and environment were the chief factors in the production and modification of plants and animals, then one would expect to find a certain degree of uniformity which can nowhere be detected. In other words, if the inorganic kingdom and environment in any one locality and at any one period vary little, one naturally inquires, Whence come the numberless plant and animal forms found in that locality and at that period? The great groups and sub-groups of plants and animals, and the individuals composing them, are practically exposed to similar conditions, yet the plant differs from the animal, and both differ infinitely amongst themselves. Plants and animals sometimes appear as mere species of apparently undifferentiated protoplasm, where no traces of structure, in the ordinary sense, can be discovered even with the highest power of the microscope, and sometimes they appear as aggregates of complex tissues, where division of labour is carried to an extreme. The simple and the complex forms occur side by side in the same regions and at the same period of time.

To put it otherwise, the endless diversities in plants and animals cannot be accounted for by mere inorganic changes, however great and however long continued.

This is evident from other considerations. The movements of plants and animals, and the presence of sense organs and organs of locomotion, cannot be referred to inorganic changes and environment. Plants and animals move as apart from irritation and external stimulation. The organs of sense and of locomotion were not made by, but to act upon, extraneous substances. The light does not make the eye, the atmosphere the wing, the water the tail of the fish, or the land the hoof of the horse. Neither is the hand, that most marvellous creation, made by externalities and by use and wont.

There are, as is well known, various kinds of eyes, wings, tails, feet, and hands, but they are, in every instance, intelligently adapted structures. They are fashioned to perform certain functions, and these they discharge unerringly. No better illustration of this can be given than is afforded by the organs of locomotion. Is the surface to be trod hard and unyielding? Small feet are provided. Is the medium to be traversed yielding and treacherous? Large feet with webs or a swimming tail are supplied. Is the intensely mobile and extraordinarily light air to be navigated? Greatly expanded wings are forthcoming. The expansion of the travelling surfaces keeps pace with the tenuity, mobility, and want of supporting power in the media. To this there is no exception. Every kind of animal, be it small or great, be it invertebrate or vertebrate, be it animalcule, mollusc, insect, fish, reptile, bird or mammal, must conform to the inexorable laws of locomotion (see Plates xlix. to liv.).

Environment cannot account for the small feet of the horse, ox, and deer; for the enlarged feet of the frog, ornithorhynchus, and seal; for the expanded tail of the fish, dolphin, and whale; for the comparatively very 


\section{KANT'S AND SPENCER'S VIEWS OF MATTER AND FORCE}

large wings of the insect, flying reptile, bird, and bat. The travelling organs and surfaces of all animals are specially designed, and constructed for specific purposes. They are also (and this is important) constructed in advance and before they are required. The wings would be ineffective for land transit, and the small feet for navigating the air. In like manner, the tail of the fish would be ill adapted for progression either on the land or in the air.

The modifications and changes effected by environment are at best insignificant. They certainly do not produce the various types of plants and animals; neither do they produce the sense organs and organs of locomotion. It is inconceivable that extraneous dead matter could create sense organs and organs of locomotion, however long the time allowed. There is in and behind living things a self-moving, self-modifying, and self-regulating power, which is superior to environment, and which enables them, within limits, to adapt themselves to their surroundings and work out their destinies. Plants and animals have their objectives on which they act, but the objectives, although they slightly influence, do not make the subjectives. There is the thing acting and the thing acted upon; and the thing acting is, for the wisest of purposes, made superior to the thing acted upon. Wherever there are means to ends a distinction must be drawn between the means and the ends.

The subject of vitality crops up here. It is admitted that plants and animals derive their substance and part of their force from the inorganic kingdom, and some aver that there is no such thing as vital force. They strive to show that there is only one kind of matter and only one kind of force, and that both are fixed quantities and indestructible. They seek to identify inorganic and organic matter, and physical and vital force. This generalisation is not warranted by facts. Organic matter can readily be distinguished from inorganic matter, and vital force from physical force. Organic matter lives or has lived; inorganic matter is invariably dead. Vital force can control and alter the shape of inorganic matter; it can also change the direction in which physical force acts; it is superior to both. This superiority implies reserve power, as one power can only be overcome by another which exercises a directive influence or is greater. The question comes to be, Can life control inorganic matter and physical force without trenching upon the modern belief that matter and force are fixed quantities which can neither be increased, diminished, nor destroyed? The vital force, if superior to physical force, must act outside the latter-must, in fact, be an added quantity, of which no cognisance is taken by modern physicists. This view can very well be maintained by those who believe in a First Cause which originated all kinds of matter and all kinds of force. The potter is superior to the clay he manipulates; he is also superior to the potter's wheel which he directs and controls. The distinction between dead and living matter and physical and vital force is wide and deep. Only an all-powerful, all-embracing First Cause can supply a connecting link between them. Granted an all-powerful First Cause, there is no difficulty. Without a First Cause many and insurmountable obstacles present themselves.

The attempt at unification and simplification here referred to has long been extended to matter as matter, organic and inorganic. Inorganic, dead matter is said to consist originally of only one kind, and the same is said of organic living matter. We are asked to believe that all the known elements (some seventy-five in number) ${ }^{1}$ spring from and have a common origin which is homogeneous in its nature, and that all the plant and animal tissues, however numerous and complicated, are the lineal descendants of protoplasm, which is averred to be a simple homogeneous substance resembling the white of egg. The heterogeneous substances in the organic and inorganic kingdoms are said to proceed from the homogeneous ones by condensation, rarefaction, attraction, repulsion, \&c., by infinite permutations in infinite time. The simple substances are held to be the parents or progenitors of the complex ones. All the varieties of chemical substances, all plant and animal tissues, are referred to a single original. Similar remarks are made of force.

\section{\$37. Kant's and Herbert Spencer's Views of Matter and Force.}

Immanuel Kant in his "General Natural History and Theory of the Celestial Bodies" (1775) "pictures to himself the universe as once an infinite expansion of formless and diffused matter. At one point of this he supposes a single centre of attraction set up, and shows how this must result in the development of a prodigious central body, surrounded by systems of solar and planetary worlds in all stages of development. In vivid language he depicts the great world-maelstrom, widening the margins of its prodigious eddy in the slow progress of millions of ages, gradually reclaiming more and more of the molecular waste, and converting chaos into cosmos. But what is gained at the margin is lost in the centre; the attractions of the central systems bring their constituents together, which then, by the heat evolved, are converted once more into molecular chaos. Thus the worlds that are lie

$$
{ }_{1} \text { A list of the elements, so far as at present discovered, is given at } \mathrm{pp} .194 \text { and } 195 .
$$


between the ruins of the worlds that have been and the chaotic materials of the worlds that shall be; and, in spite of all waste and destruction, Cosmos is extending his borders at the expense of Chaos."

The following is Mr. Herbert Spencer's view of this very interesting and difficult subject: "Throughout the universe in general, and in detail, there is an unceasing redistribution of matter and motion. This redistribution constitutes evolution when there is a predominant integration of matter and dissipation of motion, and constitutes dissolution when there is a predominant absorption of motion and disintegration of matter. Evolution is simple when the process of integration, or the formation of a coherent aggregate, proceeds uncomplicated by other processes. Evolution is compound when, along with this primary change from an incoherent to a coherent state, there go on secondary changes due to differences in the circumstances of the different parts of the aggregate.

"These secondary changes constitute a transformation of the homogeneous into the heterogeneous--a transformation which, like the first, is exhibited in the universe as a whole, and in all (or nearly all) its details; in the aggregate of stars and nebulix; in the planetary system; in the earth as an inorganic mass; in each organism, vegetal or animal; in the aggregate of organisms throughout geologic time; in the mind; in society; in all products of social activity.

"The process of integration, acting locally as well as generally, combines with the process of differentiation to render this change not simply from homogeneity to heterogeneity, but from an indefinite homogeneity to a definite heterogeneity; and this trait of increasing definiteness, which accompanies the trait of increasing heterogeneity, is, like it, exhibited in the totality of things, and in all its divisions and subdivisions down to the minutest.

"Along with this redistribution of the matter composing any evolving aggregate there goes on a redistribution of the retained motion of its components in relation to one another; this also becomes, step by step, more definitely heterogeneous. . . . This rhythm of evolution and dissolution, completing itself during short periods in small aggregates, and in the vast aggregates distributed through space completing itself in periods immeasurable by human thought, is, so far as we can see, universal and eternal. . . . All these phenomena, from their great features down to their minutest details, are necessary results of the persistence of force under its forms of matter and motion. Given these as distributed through space, and their quantities being unchangeable, either by increase or decrease, there inevitably result the continuous redistributions distinguishable as evolution and dissolution, as well as all these special traits above enumerated. That which persists unchanging in quantity, but ever changing in form, under these sensible appearances which the universe presents to us, transcends human knowledge and conception-is an unknown and unknowable power, which we are obliged to recognise as without limit in space and without beginning or end in time."

It will be observed that, in Immanuel Kant's and Herbert Spencer's systems of the universe, matter and a power of attraction and condensation and of repulsion and disintegration are taken for granted; this power converting homogeneous masses into heterogeneous masses at one period, and heterogeneous masses into homogeneous masses at another period. The homogeneity and heterogeneity are applied to the inorganic and organic kingdoms alike. Heterogeneity is held to be another term for differentiation, and differentiation applies to the structure of the universe as a whole, and to plants and animals as integral parts of it. Two things are to be considered in this connection. First, can mere attraction and condensation convert strictly homogeneous substances into heterogeneous ones; and second, can mere physical heterogeneity, unaided, produce differentiation and division of labour in plants and animals? This is a subject of the greatest possible importance, for on it hinges the existence or non-existence of a large number of elements in the inorganic kingdom, and a large number of different substances in the organic kingdom, not only in the embryonic but also in the young and adult states. One can readily conceive a highly heated molten mass where the elements of the universe are blended, and apparently form a homogeneous whole. Similarly in reproduction the germ, seed, and egg may consist of apparently homogeneous and identical matter, but (and the but has great significance in the present case) are the elements annihilated by mere superheating, or can elements, if they do not exist in the molten mass, be obtained from it by mere aggregation, con. densation, and cooling? In like manner, are the potentialities and dormant powers of the germ, seed, and egg destroyed by being reduced to a molecular mass, or can the various tissues, soft, hard, and otherwise, of the body be developed from the germ, seed, and egg as apart from latent or actual heterogeneity therein? I do not think they can. I am disposed to believe that much confusion has been introduced into the consideration of the inorganic and organic kingdoms by the lax use of the terms homogeneity, heterogeneity, and differentiation. If a thing be absolutely homogeneous and identical in all its parts and particles it cannot, strictly spealking, be converted by any physical process into a heterogeneous substance. Neither can a heterogeneous substance be converted into a simple homogeneous one. They are the opposites of each other. Mere heterogeneity, on the other hand, does not result in differentiation from the botanical and zoological points of view. To differentiate in biology is to produce different substances, structures, and organs discharging dissimilar functions. This involves life, a First 
Cause, and design. The transition from the inorganic to the organic is not so direct and simple as some philosophers and physicists endeavour to make out. This will be made abundantly clear by a consideration of the exact meanings of the words homogeneous and heterogeneous, and of differentiation in relation to the latter.

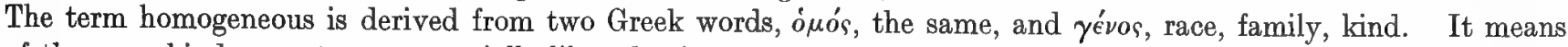
of the same kind or nature; essentially like; having parts of only one kind ; said especially of parts of one whole ; opposed to heterogeneous.

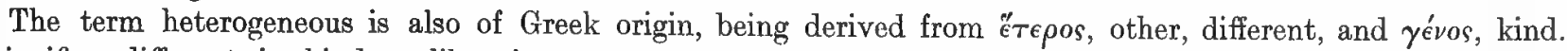
It signifies different in kind, unlike, incongruous; having widely unlike elements or constituents; opposed to homogeneous.

The term differentiation is derived from the Latin differentia, difference. It primarily means the formation of differences. It means secondarily, any change by which something homogeneous is made heterogeneous or like things are made unlike. It also signifies specialisation of structure and function.

The vigorous attempts at generalisations made by Immanuel Kant and Herbert Spencer, supposed to explain everything, in reality explain very little.

Kant presupposes "an infinite expansion of formless and diffused matter" and the setting up of "a single centre of attraction, which gradually reclaims more and more of the molecular waste, and converts chaos into cosmos," and Herbert Spencer takes for granted "an unceasing redistribution of matter and motion," and the conversion of homogeneous substances into heterogeneous ones, and, conversely, of heterogeneous substances into homogeneous ones. Both authors assume the existence of matter and force, and of a directive agency capable of converting formless homogeneous substances into heterogeneous or differentiated substances. The question, Whence come the matter and force and the directive agency has still to be answered.

Kant and Spencer, by assuming that the universe (inorganic and organic) is constructed out of a formless homogeneous mass, virtually ask Nature to make bricks without straw, inasmuch as the heterogeneous materials required for constructing the inorganic and organic kingdoms, and which characterise them, are not forthcoming. They practically ignore modern chemical analysis and the results obtained by the spectroscope, photography, the telescope, and the microscope. If the universe is now composed of a large number of heterogeneous elements, it is difficult to conceive that the elements did not exist in a potential or other form at the beginning. If only one kind of matter is required to construct the inorganic and organic kingdoms, it follows that that matter must either have enormous potentialities, which imply differentiation, or it must, in ultimate composition, be incredibly multiple. Heterogeneous substances, whether organic or inorganic, cannot possibly be produced from absolutely homogeneous substances, whatever the surroundings and whatever the nature of the forces acting upon them. The unlike and the like must be placed in different categories; the one cannot, even by a process of legerdemain, be produced from the other. Either a large number of different substances are required for the production of the different parts of the universe, or the so-called homogeneous substances are complex and compound to an extent not yet dreamt of.

The complexity in question is no doubt traceable to the atoms and molecules composing inorganic and organic matter alike. Everything points to variety, and endless variety, in the atoms and molecules themselves. While we have positively no knowledge of matter and force as ultimates, recent researches go to prove that the differences and differentiations witnessed on all hands in dead inorganic matter and in living organic matter are really present in ultimate matter as such. If the differences which characterise primitive heterogeneous matter cannot be demonstrated, neither can the sameness which characterises primitive homogeneous matter be made out. ${ }^{1}$ The continuity which is claimed for matter in the present day supports the idea of original differences, and the heterogeneous substances in the universe as we know it greatly outnumber the homogeneous ones. If inorganic and organic substances vary, and they do admittedly vary greatly, the primitive or ultimate matter from which both are formed must also vary. Variability in ultimate matter would fully account for the occasionally conflicting results obtained from the employment of chemical analysis, the spectroscope, microscope, telescope, and other means of research. "To chemistry has been entrusted the task of tracking matter back to its simplest forms or form. The result is remarkable so far as present knowledge goes. There are found a considerable number of substances (seventyfive have already been discovered) which have defied all attempts to resolve them into further simplicity. These are also indestructible, passing scathless and invulnerable through every transformation. There is no apparent tendency on their part to lose their peculiar properties or to pass one into the other; although there are a few

1 Lord Kelvin, the greatest of physicists, in an eloquent speech delivered by hin at the University of Glasgow in 1895, on the occasion of his jubilee as Professor of Natural Philosophy, gave utterance to these remarkable words : "I know no more of electric and magnetic force, or of the relation between ether, electricity, and ponderable matter, or of chemical alfinity, than I knew and tried to teach my students of natural philosophy fifty years ago, in my first session as professor."

VOL. I. 
apparently anomalous instances of change of property accompanying change of temperature, as in the case of phosphorus. While, of course, it is open to the theorist to hold that these elements are varieties of some ultimate single substance, there seems no prospect that a proof will ever offer itself." The tendency is for the elements slowly to increase in number as our methods of chemical and other analysis improve.

The discoveries of Sir William Crookes by the aid of vacuum tubes go to show that matter exists in a much more attenuated form than is represented by the elemental atom, and that a new or fourth estate must be assigned to it-that is, a condition in which it is neither solid, liquid, nor gaseous in the ordinary sense. A reference to Sir William Crookes's researches has already been made.

Protoplasm, at one time considered homogeneous and identical, is now known to be compound to quite a remarkable extent. The same is to be said of the elements. As time advances, apparently simple bodies are being resolved into two or more separate bodies. The tendency is not to unify and simplify matter but to split it up indefinitely. The differentiation of matter even in its most initial forms can alone account for the extraordinary powers possessed by the lowest and most rudimentary plants and animals, and for the successive embryological changes and stages through which plants and animals pass during development.

The differentiations which characterise the embryological changes referred to are stereotyped, so to speak, in adult plants and animals.

All the fluids and tissues of plants and animals, in some respects infinitely diverse, cannot possibly be the product of one or two simple substances. This is a matter of common sense no less than of science.

\section{CREATION A PROGRESSIVE WORK}

The consideration of ultimate matter and force raises the whole subject of creation. The most natural view to take of this stupendous question is to regard it as a progressive rather than a finished work. The history of the heavenly bodies, our planet, and plants and animals favours this hypothesis. It can scarcely be doubted that worlds are in process of formation at the present day, and that plants and animals, as a complementary part of these worlds, are being continually produced.

The inorganic and organic kingdoms form one great whole, and are correlated not only in their aggregates but in their details. They are interdependent in the strictest sense, and what is taken from the one is returned to the other, sooner or later. Both kingdoms have a common origin, in the sense that they are the work of the same hand and are controlled and supervised by the same master intellect.

\section{$\S 38$. Scriptural Account of Creation.}

The progressive view of creation is supported by revelation. The account given of creation in the Old Testament is one of progress and separate creative acts. The inorganic kingdom was created first, then came the organic; the plants appearing before the animals, and both appearing according to a more or less ascending scale, culminating in man. All plants and animals were taken from the dust and will be returned to it sooner or later. The scriptural account is briefly as under: "In the beginning God created the heaven and the earth. And the earth was without form, and void; and darkness was upon the face of the deep.... And God said, Let there be light. . . And God divided the light from the darkness. And God called the light Day, and the darkness he called Night. . . And God said, Let there be a firmament in the midst of the waters, and let it divide the waters from the waters. And God made the firmament, and divided the waters which were under the firmament from the waters which were above the firmament. . . . And God called the firmament Heaven. . And God said, Let the waters under the heaven be gathered together unto one place, and let the dry land appear: And God called the dry land Earth; and the gathering together of the waters called he Seas. . . And God said, Let the earth bring forth grass, the herb yielding seed, and the fruit tree yielding fruit after his kind, whose seed is in itself, upon the earth. ... And God said, Let there be lights in the firmament of the heaven to divide the day from the night; and let them be for signs, and for seasons, and for days, and years: And let them be for lights in the firmament of the heaven to give light upon the earth: and it was so. And God made two great lights; the greater light to rule the day, and the lesser light to rule the night: he made the stars also. And God set them in the firmament of the heaven to give light upon the earth, And to rule over the day and over the night, and to divide the light from the darkness... . And God said, Let the waters bring forth abundantly the moving creature that hath life, and fowl that may fly above the earth in the open firmament of heaven. And God created great whales, and every living creature that moveth, which the waters brought forth abundantly, after their kind, and every winged fowl after his kind. .. And God blessed them, saying, Be fruitful, and multiply, and fill the waters in the seas, 
and let fowl multiply in the earth.... And God said, Let the earth bring forth the living creature after his kind, cattle, and creeping thing, and beast of the earth after his kind; and it was so. . . . And God said, Let us make man in our image, after our likeness : and let them have dominion over the fish of the sea, and over the fowl of the air, and over the cattle, and over all the earth, and over every creeping thing that creepeth upon the earth. So God created man in his oun image, in the image of God created he him; male and female created he them. And God blessed them, and God said unto them, Be fruitful, and multiply, and replenish the earth, and subdue it; and have dominion over the fish of the sea, and over the fowl of the air, and over every living thing that moveth upon the earth." 1

"These are the generations of the heavens and of the earth when they were created. . . And every plant of the field before it was in the earth, and every herb of the field before it grew: for the Lord God had not caused it to rain upon the earth, and there was not a man to till the ground. But there went up a mist from the earth and watered the whole face of the ground. And the Lord God formed man of the dust of the ground, and breathed into his nostrils the breath of life; and man became a living soul. . . . And out of the ground the Lord God formed every beast of the field, and every fowl of the air : and brought them unto Adam to see what he would call them : and whatsoever Adam called every living creature, that was the name thereof. [The italics in these passages are mine.l. . And unto Adam he said, Because thou hast hearkened unto the voice of thy wife, and hast eaten of the tree, of which I commanded thee, saying, Thou shalt not eat of it; cursed is the ground for thy sake. ... In the sweat of thy face shalt thou eat bread, till thou return unto the ground; for out of it wast thou taken : for dust thou art, and unto dust shalt thou return." 2

It will be noted that the magnificent scheme of creation quoted above was a designed and pre-determined whole with an adequate cause and an unvarying sequence of events, inorganic and organic. There were no afterthoughts, no patchings, no defects, no want of power in design or execution. The scheme was far-reaching from every point of view, and fully explained the relations of non-living to living things. The universe to begin with was a nebulous waste-it was without form and void. Its matter was first arranged. From being apparently homogeneous it became markedly heterogeneous. Its movements were next seen to. The sun was made to rule the day and the moon the night. Heat and light, the indispensables of life, were added. The heavenly bodies were made to wheel in space according to inexorable laws, and these movements proceed unerringly at the present day. The universe became a moving mass, and its several parts exhibited and continue to exhibit the most extraordinary actions and reactions. Living things appeared on the scene; plant and animal, each after its kind, whose seed is in itself. Not only so, but the plant was created before it was placed in the ground. The same is to be said of animals - even man. "And the I,ord God formed man, and breathed into his nostrils the breath of life." Man, the corner-stone of creation, originally innocent and holy, fell from his high estate through the eating of the fruit of the tree of knowledge, which he was strictly commanded not to do. The scheme of life and death and of rewards and punishments, it will be seen, was established at a very early period of the earth's history. The plant forms the food of animals, and plants and animals the food of man, but plants and animals can only become food in the dead state. Death, from the food point of view, was not therefore the outcome of disobedience and sin. Sin bore its own burdens: "In the sweat of thy face shalt thou eat bread." Punishment was reserved more especially for man and the higher animals endowed with a distinct moral sense.

It may be stated in this connection that disobedience, and punishment as a consequence thereof, manifested themselves soon after man's appearance on the earth. The good and the bad qualities of his nature were not slow to assert themselves. Cain slew his brother Abel, and so became a murderer and an outcast, and other great crimes followed. As it was in the early times so it is now. Good and bad men occupied the same cities, villages, hamlets, homes, and territories. A certain number were prone to wickedness in every form, and deliberately chose the downward course. They were the law-breakers of primeval times, and continue to be so in modern times.

The majority of mankind prefer the good, and strive to attain it directly or indirectly. As the law-breaker cannot become wholly bad, neither can the most exalted members of our race become wholly good. There is a

1 I may here state parenthetically that in order to carny out God's commands regarding the subduing of the earth man must have been provided potentially with the power of directing and controlling matter and force, ind this power he undoubtedly exercises at the been provided potentially with the power of drecting and controlling inate of speech, Adan having named the several animals, in succession. All the forces of nature were and are at his disposal--steam, electricity, gunpowder, dynanite, and a host of powerful agents, only a few of which are known, but which are capable of completely altering the appearance presented ly any part of the earth's surface. This is seen in the universal emplopment by man of fire and water on a large scale ; in the construction of powerful engines; in the hewing down of forests; in the reciamation of lands; in smelting processes for the reduction of the hardest metals; in the luilding of great ships of war; in the making of canals, dams, and docks; in the construction of roads, bridges, and railways; in quarrying and mining operations of unexampled magnitude; in canals, dams, and docks; in the construction of loads, bridges, and railways; in quanessing of waterfalls and waterways; in the laying down of innumerable sulmarine telegraphic cables; in the installation of various systems of telegraphy; in the use of wireless telegraphy; in the employment of the telephone and phonograph, and in endless other ways which need not be enumerated.

2 The Book of Genesis, chapters i., ii., and iii. 
give and take in good and evil as between the most civilised and the most depraved members of the race. Mankind cannot be divided by a hard and fast line, as is commonly done, into three categories - namely, the savage, the semi-savage, and the civilised. The three approach and touch each other at innumerable points in every stage of their history. The fact that men are all inhabiting the same planet at the same time, and are never wholly or widely removed from each other, shows that homo sapiens is originally and fundamentally one, whether of single or multiple origin. Physically, this can scarcely be doubted, and mentally they are never separated by an impassable gulf. The psychologist and alienist can always track the workings of the mind in health and disease, and the more the so-called savage is educated, the more certainly can his mental faculties be traced to a lofty source.

The most depraved nations and individuals have an inkling of good and of a higher power, and as soon as their mental faculties expand and their moral sense asserts itself they recognise the difference between good and evil. They also come to see that punishment is the proper treatment for disobedience, and that a reward is due to the performance of meritorious deeds. The punishment may be death : the reward immortality. But I need not pursue this subject further: my object in referring to it at all is to invite the reader to take a common-sense view of life and death, and of disobedience, sin, punishment, and reward. The scriptural account does not clash with anything here stated, and it is always well, when dealing with abstruse subjects, to look at them from every conceivable point of view. I have to treat the subject from the anatomical, physiological, and psychological side only.

Whatever may be thought of the scriptural account of creation by advanced and hypercritical scientists, there can be no doubt as to the sublimity and force of the language employed, and the great originality and far-reaching scope of the theory advocated. If it be deficient in some minor matters, it is, nevertheless, the most satisfying account of creation to be found in any treatise, ancient or modern. It regards the cosmos as a progressive work; it recognises separate creations; it acknowledges types in plants and animals; it proclaims an ascending series in plants and animals; it says nothing about evolution (in every case the seed of the plant and animal is said to be in itself); it makes for permanence and stability as opposed to variation; it shows that the inorganic and organic kingdoms are complemental, and that plants and animals are also complemental and co-ordinated; it establishes law and order, and is wholly opposed to chance. According to it, everything is foreseen and pre-determined. It advocates in the most uncompromising manner a First Cause and Design; it relegates reproduction to laws which may not be departed from; it indicates a plethora of plants and animals even at the beginning-no time being set apart for the manufacture of the one out of the other. It prepares an inexhaustible supply of food for plants and animals (the fiat is to be fruitful and multiply); it supplies plants and animals with a suitable home in anticipation of their arrival on our sublunary sphere; its one dominant note is permanency in the midst of endless fluctuation; it is absolutely explicit as to every living thing being taken from the inorganic kingdom and returned to that kingdom sooner or later ; it makes no mystery as to the existence of dead and living matter, or of physical and vital force, or of man and intellect as being the crowning or coping-stones of creation. Its language is singularly simple and direct, so that even he who runneth may read. Only the perversely blind can shut his eyes to the astounding truths contained in the opening chapters of Genesis.

The historic account of the great events of creation is sufficiently virile to stand on its own legs, and it should be carefully studied by all those who take an intelligent interest in the great issues of life and death. According to it there is causality, and continuity, and supervision. The several creative acts are attributed to an intelligent and adequate First Cause. The lowest and highest plants and animals are produced with equal facility. There is no imperfection, no blundering, no want of power anywhere observable. Everything comes into being in natural and necessary sequence, and due provision is made for food supplies and all necessary requirements and supervision, so long as life lasts.

The means are, in every instance, forthcoming. The nebulous masses which the modern telescope reveals supply the primitive (not necessarily homogeneous) matter from which spheres of various kinds (planets like our own included) are produced.

The microscope reveals the primitive (not necessarily homogeneous) protoplasm to which plants and animals owe their origin. Given primitive matter (inorganic and organic) as a first instalment of creation, and a directing, controlling agency or power, all known phenomena, past and present, become readily intelligible. The question then, as now, hinges on the nature of the primitive matter. Is it homogeneous and simple, or is it heterogeneous? That the two kinds of primitive matter now referred to are not simple and homogeneous, as many believe, is proved by this, that inorganic matter, as we know it, is infinitely varied, and cannot be produced by physical forces acting for shorter or longer periods on an absolutely homogeneous substance: similarly, organic matter infinitely diverse in its nature cannot be manufactured by vital force out of an absolutely homogeneous protoplasm.

The vital and physical forces--all forces whatever-have free play in the production of the universe, and are 
operative by turns. Gravitation, attraction, repulsion, condensation, rarefaction, cohesion, adhesion, capillarity, osmose, \&c., all take part in building up the inorganic and organic kingdoms. Matter (inorganic and organic) and force (physical and vital) are each and all under law, and the same law; and the actions and reactions which everywhere abound are, in every instance, means to ends. The laws which obtained at the dawn of creation obtain now. Nothing has suddenly leapt into existence. Time or duration is a factor in the production of everything the universe contains.

\section{\$3. Geology as Bearing on Creation.}

The formation of the crust of the earth and of the plants and animals which inhabit it may be said to furnish a history of creation on a small scale. Geology assures us that the surface of our globe is the product of innumerable forces acting for long periods on heterogeneous substances, and that these forces are at work at the present day. They consist of water action (seen in rain, waterspouts, rills, rivers, lakes, and oceans), which produces sediments and strata of various kinds, especially rocks; atmospheric and climatic action, which results in denudation; frost and glacier action, which, with river action, scoops out valleys and transfers huge masses of foreign matters long distances; volcanic action, which begets great upheavals and dislocations of existing horizontal strata and gives rise to new strata; subsidence and elevation of certain areas at different periods (also resulting in dislocation), whereby islands are engulphed or formed, and continents diminished or increased in extent, \&c. These forces, continually at work in the past, as in the present, have produced the conditions which modern physical geography reveals.

The formation of chalk furnishes a striking illustration of natural forces at work on a large scale: "Great light has recently been thrown upon the origin of the unconsolidated white chalk by the deep soundings made in the North Atlantic, previous to laying down, in 1858, the electric telegraph between Ireland and Newfoundland. At depths sometimes exceeding two miles, the mud forming the floor of the ocean was found, by Professor Huxley, to be almost entirely composed (more than nineteen-twentieths of the whole) of minute Rhizopods, or foraminiferous shells of the genus Globigerina, especially the species Globigerina bulloides. The organic bodies next in quantity were the siliceous shells called Polycystinex, and next to them the siliceous skeletons of plants called Diatomacex, and occasionally some siliceous spiculæ of sponges were intermixed. These he supposed to be connected by a mass of living (?) gelatinous matter to which he gave the name of Bathybius, ${ }^{1}$ and which he thought contained abundance of very minute bodies termed Coccoliths and Coccospheres, which have been detected fossil in chalk.

"Sir Leopold MacClintock and Dr. Wallich have ascertained that ninety-five per cent. of the mud of a large part of the North Atlantic consists of Globigerina shells. But Capt. Bullock, R.N., lately brought up from the enormous depth of 16,860 feet a white, viscid, chalky mud, wholly devoid of Globigerinæ. This mud was perfectly homogeneous (?) in composition, and contained no organic remains visible to the naked eye. Mr. Etheridge, however, has ascertained by microscopical examination that it is made up of Coccoliths, Discoliths, and other minute fossils like those of the chalk classed by Huxley as Bathybius, when this term is used in its widest sense. This mud, more than three miles deep, was dredged up in lat. $20^{\circ} 19^{\prime} \mathrm{N}$., long. $4^{\circ} 36^{\prime} \mathrm{E}$., or about midway between Madeira and the Cape of Good Hope.

"The recent deep-sea dredgings in the Atlantic conducted by Dr. Wyville Thomson, Dr. Carpenter, Mr. Gwyn Jeffreys, and others, have shown that on the same white mud there sometimes flourish Mollusca, Crustacea, and Echinoderms, besides abundance of siliceous sponges, forming on the whole a marine fauna bearing a striking resemblance in its general character to that of the ancient chalk."

The progressive formation of strata can at present be seen in the deltas of the Nile, Mississippi, and other large rivers, and in the beds of the several oceans; the upheavals are seen in the occasional and violent action of existing volcanoes; the glacier action is witnessed in Switzerland and other mountainous countries ; denudation from wind, rain, frost, \&c., is constantly going on in hills, mountains, and valleys, and in glacial, water, and tidal courses. The elevation and subsidence of land, though usually a very slow process, is an every-day occurrence. It only becomes sudden and pronounced when due to volcanic action. Sir Charles Lyell remarks: "Such changes have actually" occurred in our own days, and are now in progress, having been accompanied in some cases by violent convulsions, while in others they have proceeded so insensibly as to have been ascertainable only by the most careful scientific observations, made at considerable intervals of time.... In parts of Sweden, and the shores and islands of the Gulf of Bothnia, proofs have been obtained that the land is experiencing, and has experienced for centuries, a slow upheaving movement."

1 The gelatinous supposed living matter met with at the sea hottom, and named Bathybius by Huxley, was found on closer examination to be a chemical product. Huxley's theory on the subject was consequently abandoned by himself. 
It appears, from the observations of Mr. Darwin and others, that very extensive regions of the continent of South America have been undergoing slow and gradual upheaval, by which the level plains of Patagonia,

\section{TABLE OF STRATIFIED ROCKS}

Showing Aproximate Thickness

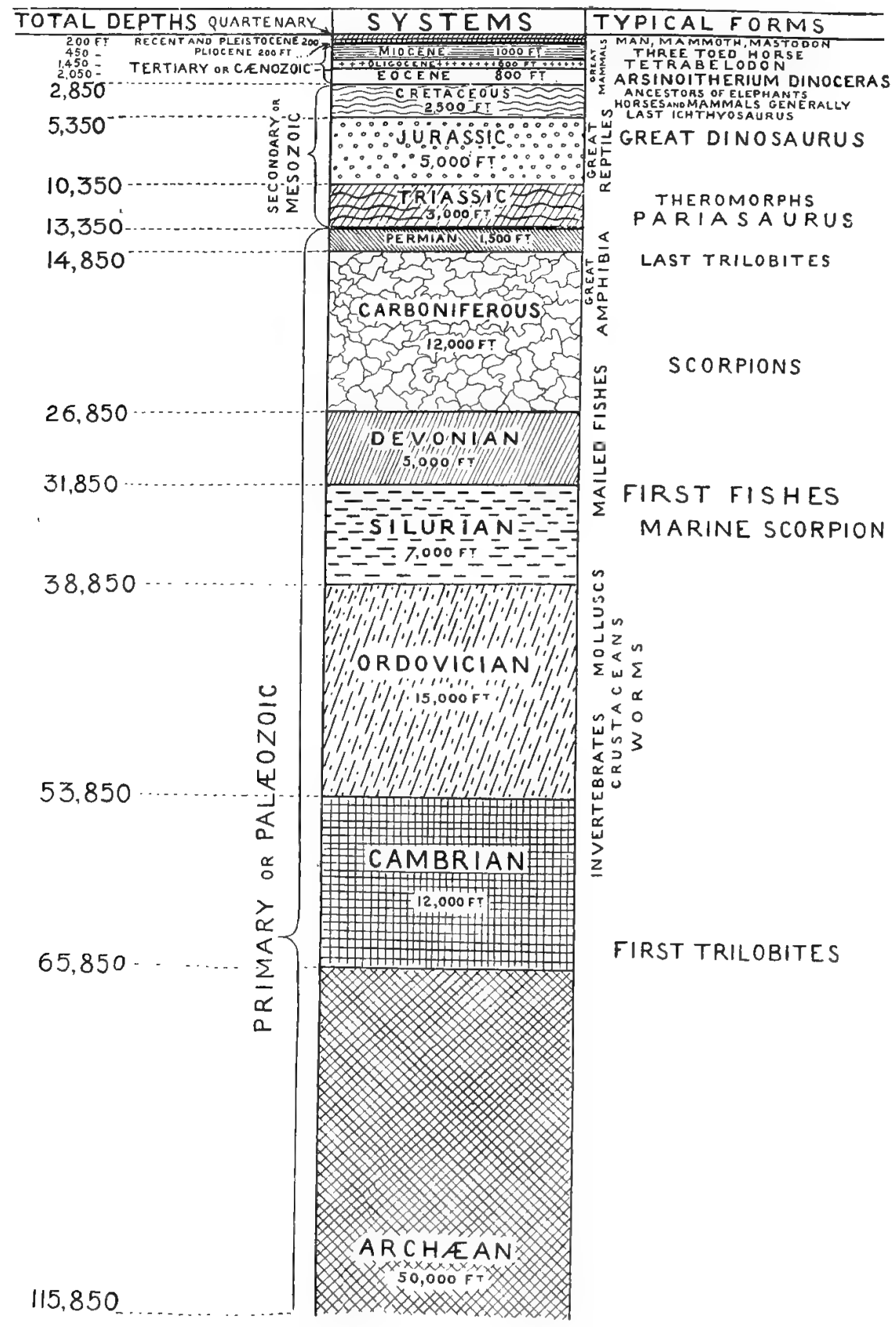

Fif;. 44.- $\Lambda$ tahular view of the strata of the earth's crust, showing the relative thickness of each "system" or group of strata, and the position in which important animal remains have been crumpled, but we can make out their thickness horizontally. In nature they are tilted and other (after Lankester).

covered with recent marine shells, and the Pampas of Buenos Ayres, have been raised above the level of the sea. On the other hand, the gradual sinking of the west coast of Greenland, for the space of more than 600 miles from north to south, during the last four centuries, has been established by the observations of a Danish naturalist, Dr. Pingel. And while these proofs of continental elevation and subsidence, by slow and insensible movements, 


\section{SIMPLE AND COMPLEX PLANTS AND ANIMALS}

have been recently brought to light, the evidence has been daily strengthened of continued changes of level effected by violent convulsions in countries where earthquakes are frequent. There the rocks are rent from time to time, and heaved up or thrown down several feet at once, and disturbed in such a manner as to show how entirely the original position of strata may be modified in the course of centuries.

It is not necessary for me to take up in detail the physical geography of the crust of the earth in geologic and recent times. It will suffice to furnish the reader with a table or chart (Fig. 44 ) in which the several rock strata of the earth's crust are given, in which the comparative thicknesses of the strata are indicated, and in which are located the fossil and other animals in the order of their appearance. Such a table supplies invaluable information in many directions, but unfortunately it conveys no idea of absolute time in relation to comparative time. The standard or unit of time has yet to be discovered. The thickness of the various rock strata affords no reliable clue. If the thickness of one stratum be ten times that of another stratum, one is tempted to conclude that the thicker stratum is ten times the age of the thinner one. This could only be true if the rate of deposit was uniform, which it never is. The rate of deposit of the stratum only partly determines the time taken in its formation: the thickness of the stratum and the time of deposit become comparative questions. The same reasoning applies to everything contained in the strata. While the simpler animals are found deepest in the earth's crust, and were the first to be created, there is nothing to show absolutely how long the several animals existed on the earth.

A study of the table referred to will satisfactorily illustrate my meaning.

The time involved in the various creative acts is inconceivably great, and a practically unknown quantity.

The heavenly bodies, our planet, and its atmosphere had to be prepared before plants and animals could exist. The plants and animals, moreover, had to be specially constructed to meet the exigencies of the earth, and its climate at different periods. Geology shows that the plants and animals of successive periods were specially adapted to the peculiar physical conditions of the period, and were perfect in their day and generation. There were the plants and animals forming the coal-measures; the great conifers and tree ferns ; ${ }^{1}$ the ganoid and old world fishes, the huge reptiles, the monster mammals, \&c., all indicating and chronicling bygone eras.

Not only were the plants and animals as a whole adapted to the earth as a whole at a particular period, but a portion of the plants and animals were adapted to particular parts of the earth at different periods. The earth, its atmosphere, and its climate have changed and are changing; a state of matters which accounts for regions which were once tropical, and contained tropical plants and animals, being now temperate or arctic, and containing corresponding flora and fauna. The distribution of plants and animals on the earth is not an accidental or chance distribution. The organic formations kept and keep pace with the physical changes of our planet; the organic and inorganic kingdoms being, as explained, mutually adapted and correlated. When the continents and climate changed, the plants and animals changed also. "The number of reptilian remains, all apparently of the cretaceous age, is truly surprising; more than ten species of Pliosaurus, one of Dinosaurus, eight of Chelonian, besides other forms, having been recognised. . . The Aix-la-Chapelle (fossil) plants flourished before the rich reptilian fauna of the secondary rocks had ceased to exist. The Ichthyosaurus, Pterodactyl, and Mosasaurus were of coeval date with the oak, the walnut, and the fig." 2

If there is one thing more certain than another it is that the inorganic and organic kingdoms, as we know them, are mutually interdependent, and that they are always and everywhere complementary. The geological record affords conclusive proof that the one is an addition to, and, in a sense, an outcome or extension of the other, and that both are parts of a great whole. At one period of the earth's history neither plant nor animal existed. The earth had to be prepared as a habitation for them, and when a fitting habitation was provided, then, and not till then, did they make their appearance; the plants coming first, and animals afterwards. The plants and animals, moreover, appeared in a certain graduated order; the more simple ones preceding the more complex up to a point which, when reached, enabled the simple and the complex to exist side by side, as at the present day.

\section{$\$$ 40. The Simple and Complex Plants and Animals necessary to each Other.}

While geology points to the production of the lower plant and animal forms before the higher, this fact (if fact it be ${ }^{3}$ does not indicate imperfection or want of power in the Creator. With Him all things are possible. In other words, $\mathrm{He}$ could with equal facility produce a monad or a man. The geologic record of the future may

1 Professor Goppert, after examining the fossil vegetables of the coal-fields of Germany, has detected, in beds of pure coal, remains of plants of every family hitherto known to occur fossil in the carboniferous rocks. Many seams, he remarks, are rich in sigillaria, Lepidodendra, and Stigmarix; the latter in such abundance as to appear to form the bulk of the coal. In some places, almost all the plants were calamites, in others ferns. (Sir Charles Lyell's "Elements of Geology." London, 1871.)

2 Lyell, op. cit. pp. 275,280 .

3 Some scientists are of opinion that the geologic record is so imperfect as not to be reliable. There is, however, the possibility of important fossiliferous strata being destroyed by igneous and other, action at suecessive periods during the formation of the crust of the earth. 
show that many of the lower and higher plants and animals were co-existent at a much earlier period than is generally supposed, or even from the first. The subjects of atmosphere, climate, food, \&c., have here to be considered, and these involve changes necessitating time for their production. Air, water, and soil had to be provided as a necessary pabulum for plants, and plants as a necessary pabulum for animals. Plants and animals live upon each other: generally (but not necessarily) the higher and stronger upon the lower and weaker. Thus the parasites, the most debased of plants and animals, infest the highest plants and animals in large numbers. In a sense the highest plants and animals are as necessary to the lowest as the lowest are to the highest. A completed scheme of creation requires the co-existence at the same time, and in the same place, of the higher and highest and the lower and lowest plants and animals. Man certainly requires the lowest and highest plants and animals as auxiliaries of his existence.

The presence of the simplest and most complex plants and animals in the same place and at the same time goes against the belief that the more complex plants and animals are manufactured directly or indirectly from the more simple and rudimentary ones. It also goes against the idea that plants and animals are capable of indefinite improvement. If this were so, and creation was non-progressive and confined to one period, such is the extreme age of the earth, the stock of rudimentary plants and animals would long ago have been exhausted-only the most perfect plants and animals remaining. As a matter of fact, however, the rudimentary plants and animals existing at the present day are enormously in excess of the complex ones. The rudimentary plants and animals are in reality a necessity. They form the food of the higher ones; a circumstance which is strangely overlooked in discussing the doctrine of evolution. Inorganic and organic matter may be said to prey upon each other. The soil forms the chief food of the plant, and a decomposing plant nourishes the soil. The same is true of plants and animals. The animal devours the plant, and both when dead enrich the soil. Sooner or later plants and animals return to the earth from which, under divine guidance, they originally sprang. Inorganic matter and rudimentary plants and animals are absolute requirements of the higher plants and animals, and the converse.

While plants, as a rule, subsist on inorganic matter, a certain proportion of them (the insectivorous plants) subsist largely on animals. Certain animals (the Herbivora, for example) subsist exclusively on plants. Others again (the Carnivora) live exclusively on animals. Man, as an omnivor, devours all. As a matter of fact, life swarms upon life, and everything largely lives upon every other thing directly or indirectly.

The gradation in plants and animals is practically unlimited, but this circumstance does not necessarily afford irrefragable proof of evolution and descent in the usual acceptation of these terms. It rather points to the inexhaustible resources and infinite constructive skill of the Creator. There are main types and sub-types, a general plan and detailed plans, but all are necessary to a perfect scheme of creation. There is nothing in geology to show that the various substances composing our planet were originally manufactured out of one homogeneous substance : neither is there anything to prove that the great races of plants and animals are the products of a primordial speck of homogeneous protoplasm. All that geology reveals is that certain physical conditions obtained when certain plants and animals lived, and that the physical conditions and the plants and animals changed, up to a point, from time to time in the past as they are doing in the present.

\section{ORDER IN WHICH PLANTS AND ANIMALS APPEARED ON THE EARTH}

In considering the problem of creation in relation to the inorganic and organic kingdoms it is necessary to take into account certain of the physical changes which have occurred and are occurring in inorganic matter, and all the vital changes which have occurred and are occurring in organic matter. It is here that the geologic record becomes so important as an instructor and guide. A reference to the geological chart given above discloses the fact that in the oldest rocks (Primary or Palæozoic) the traces of organic remains are comparatively few; that in the secondary or Mesozoic rocks they are more plentiful; and that in the Tertiary or Cænozoic and Post-Tertiary (which are comparatively recent rocks) they greatly abound.

It is a remarkable circumstance that even in the oldest known rocks organic remains are found. Thus in the Lower Laurentian rocks, in an interstratified bed of limestone 1000 feet thick, what was once thought to be a foraminifer (Eozoon Canadense) has been discovered. Its antiquity is such that the distance of time which separated it from the Upper Cambrian period, or that of the Potsdam sandstone, may, says Sir W. Logan, be equal to the time which elapsed between the Potsdam sandstone and the Nummulitic limestones of the Tertiary period.

The occurrence in the oldest known rocks of organic remains fills the mind with wonder. It shows that life appeared on the earth untold ages ago. Nor is it quite certain that the earliest fossiliferous rocks have yet been discovered. Indeed some are of opinion that these have been destroyed by volcanic and other action. If so, 


\section{PLANTS AND ANIMALS IMPROVABLE UP TO A POINT}

it becomes very difficult, indeed impossible, to say, in the present state of geological science, when and in what order plants and animals made their appearance. "It would," Sir Charles Lyell observes, "be presumptuous to suppose that when a small part only of the globe has been investigated, we are acquainted with the oldest fossiliferous strata in the crust of our planet. Even when these are found, we cannot assume that there never were any antecedent strata containing organic remains, which may have become metamorphic."

As science advances, the tendency is to push life in its simplest and most complex forms further and further back into the abyss of time. The early appearance of life on our globe does not interfere with the order of creation as a progressive work. It concerns the when rather than the how and the why, and enlarges our purview of the time required for the several creative acts. It does not interfere with the limits assigned to the several kinds of plants and animals, or their range and distribution in space. Neither does it affect the infinite gradations in plants and animals, the general plan of creation, the production of types and sub-types, and the improvability of the types and sub-types up to a point, or within prescribed limits. Law and order impose limits or boundaries on plants and animals, as on everything else.

These boundaries apply to the duration and distribution of plants and animals-they live and die in certain places and at certain periods: they apply to the capacities and powers of plants and animals; to their fertility and infertility ; to everything, in fact, which pertains to them.

\section{\$ 4I. Plants and Animals Improvable up to a Point.}

Law and order limit the degree of development of plants and animals, as shown by embryology, and the time required for their reproduction. They also limit the amount of progress possible. To take an extreme example. Man - assuming, as most modern writers do, his savage origin-has gradually emerged from his wild, untutored state : he has laboriously toiled through the stone age, the bronze age, and is now far advanced in the iron age. Still man (as man) has not essentially changed during the historic period, that is, for the last five or six thousand years. His brain and nervous system and intellect have been cultivated, but if he were isolated and left to himself there is reason to believe he would slowly retrogress as he has slowly improved. This, of course, may only show that man is naturally and essentially a social animal. ${ }^{1}$

There is nothing to show that man is capable of indefinite improvement, or that he will, in time, become practically a new being.

If the divine origin of man be assumed, savage man affords a striking example of deterioration. The time occupied in the deteriorating process probably equalled that involved in his civilisation. It is not at all improbable that from a very early period of his history civilised and savage man were contemporaneous: certain tribes being prone to wickedness and backsliding, others to virtue and advancement. The good and evil propensities of mankind are not obliterated in the present day. The history of nations points to progress within limits. The nations of antiquity have all had their rise and fall, and, as far as experience goes, over-civilisation and refinement result in deterioration and disintegration. Over-training and in-breeding, as a rule, impair rather than improve a race.

The late Professor John Goodsir, one of the greatest comparative anatomists and anthropologists of modern times, held firmly to the belief that man was originally an intellectual, moral being. This belief pervades his "Lectures on the Dignity of the Human Body." In Lecture IX., namely, that on "Retrogressive Man," he says : "I believe that man was not originally savage, and that the less civilised races are not undeveloped but degraded forms. Man, in virtue of possessing a spiritual element, stands alone amongst the organised beings of the globe. The existence of this element associates the being possessing it with the spiritual world." 2

The improvable element in plants and animals is traceable to their tissues, especially to the nervous tissues, or what represents them when not differentiated as nerves. The improvable element is essentially intellectual in character. All plants and animals, from the lowest to the highest, have in their substance, or in part of their substance, a guiding, directive principle which enables them to control their actions and to modify and adapt themselves to the circumstances in which they are placed. This guiding. directive principle is not brought into play by irritability and extraneous stimulation. This is equivalent to saying that the First Cause works in and through the plants and animals to given ends: in other words, plants and animals are supervised, and are not at the mercy of environment or of any set of extraneous conditions. They are superior to their surroundings, but they cannot form themselves as wholes or parts of wholes. They are created things, the offspring of progenitors. This view is opposed to the theory of "natural selection," which rejects a Creator or intelligent First Cause, and refers all modifications in plants and animals chiefly to externalities and to chance.

1 Alexander Selkirk, the Scottish castaway and original of Rohinson Crusoe, who lived for several years on an uninhabited desolate island, had, when rescued, nearly lost the power of speech.

2 "The Anatomical Memoirs of John Goodsir, F.R.S., late Professor of Anatomy in the University of Edinburgh," vol. i. p. 276. VoLt. I. 


\section{NATURAL AND ARTIFICIAL SELECTION CONTRASTED AND CONSIDERED}

Natural selection assigns to plants and animals a power to vary, and to discriminate, select, and perpetuate what is best in themselves, to the suppression and exclusion of the less useful or doubtful parts.

While no plant or animal, from the lowest to the highest, can be regarded as a mere automaton mechanically formed, mechanically set in motion, and mechanically kept in motion, so, on the other hand, no plant or animal, from the lowest to the highest, can be credited with the power of selecting and perpetuating the best properties in itself, to the exclusion of the less desirable properties, in the so-called struggle for existence. Plants and animals cannot alter their original constitutions : even man cannot add a cubit to his stature, however much he may desire to do so.

Natural selection, strictly speaking, is the counterpart, or rather the opposite, of artificial selection, but there is no proof that natural selection exists. Artificial selection, as is well known, implies the presence and existence of a selector or discriminator; a judge who detects, assorts, and combines excellences in individuals of different sexes by crossing or inter-breeding. It improves breeds by combining and perpetuating excellences in different individuals. The selector is outside, or apart from, the thing selected. Natural selection, however, claims a power, and a very important one, not recognised in artificial selection; it claims that individual plants and animals, from the lowest to the highest, can select, combine, and perpetuate their own excellences. as apart from sex and crossing, and in the absence of a selector, discriminator, and judge. Natural selection in this higher and more extended sense is a misnomer, and there is absolutely no proof of its existence. If the power to select exists, it must be referred to something outside the plant and animal. It can, as a matter of fact, only be referred to the maker, director, and upholder of the plant and animal.

I am aware that the males of animals in many cases fight for the possession of certain females, and that females prefer certain males, which is a kind of selection; but this is quite a different thing from saying that the males and females select and perpetuate what are regarded as the best qualities or properties in themselves. In reality no such selection is possible. The males and females respectively are to be regarded as aggregates, and they have no power to select and perpetuate certain properties or qualities (essentially details of themselves), as apart from these aggregates. In mating, even in man, the motives in selecting are frequently frivolous and sordid in character, and in no sense calculated to bring out the best qualities of the race. This has only to be stated to be endorsed. One can readily understand how a living plant or animal can be improved by artificial selection, and by the blending of good points in two or more individuals of opposite sexes, aided by suitable pabulum, surroundings, and training; but it is incomprehensible that natural selection can secure the propagation and perpetuation of the best qualities in one and the same individual, and as apart from sex and the conditions referred to. Natural selection cannot be regarded as the main factor in evolution, and evolution itself only obtains in a restricted sense, and as applied to the types and sub-types of plants and animals.

There is no such thing as an unbroken descent of plants and animals from one and the same tiny speck of primitive protoplasm by infinite permutations in practically endless time. All that can be said is that the types and sub-types of plants and animals are capable of improvement within limits. In this sense evolution (if admitted) carries with it the possibility of progress. The best parts in plants may be artificially cultivated, and the best traits in animals artificially developed by training and other means.

In this restricted sense, evolution would account for the education and progress of civilised man in every department of his being; for his intellectual, social, moral, and religious advances. It would account for the modifications and improvements in cultivated plants and in domestic animals. It would account for improvements under favourable conditions, and for retrogression and breeding back under unfavourable conditions, in wild plants and animals. It would lend itself to law and order both as regards progression and retrogression; the rule being advance, with occasional retrogression in particular cases and under peculiar circumstances.

That the scheme of creation, as we know it, is progressive and continuous is proved by the histories of existing plants and animals, by the presence in the crust of the earth of large numbers of fossils, in many cases of rudimentary extinct plants and animals, by the formation of sedimentary, volcanic, and other rocks in ancient and recent times, \&c. The scheme is a well-ordered and supervised one. It completely eliminates the element of chance. It provides for everything that has happened, is happening, and will happen. It is a case of the Creator everywhere in time and space, and of accident and blind chance nowhere. An all-powerful, over-ruling, vigilant ('reator, Supervisor, and Upholder is an absolute necessity in the Cosmos as revealed to us and as we behold it. 


\section{\$ 42. Everything Controlled and under Supervision.}

The view here advanced accords, on the whole, with the account of creation given in the Old Testament. According to that account there was a succession of events, one thing was formed after another: the inorganic first and then the organic; the plant and then the animal in an ascending series, and, last of all, man. The seven, or rather the six days (for the seventh, according to Scripture, was a day of rest), occupied by the creative acts evidently represent six epochs of time; each period being undetermined, and as far as man is concerned, indeterminable.

That the six days of Scripture do not mean six ordinary days of twenty-four hours each, goes without saying, if regard be had to the nature of the heavenly bodies, the slow formation of the crust of the earth, and the production thereon of plants and animals, as revealed by their fossil remains in successive geologic strata.

It is not my province to deal with theological tenets, but it may be stated that the progressive and continuous view of creation set forth in Genesis, which requires an ever-present, originating, controlling, and directing Creator, lends powerful support to the doctrine that all things are pre-arranged and pre-determined. It gives emphasis to the following passages in the New and Old Testament: "Behold the fowls of the air: for they sow not, neither do they reap, nor gather into barns; yet your heavenly Father feedeth them. Are ye not much better than they? Which of you, by taking thought, can add one cubit unto his stature? And why take ye thought for raiment? Consider the lilies of the field, how they grow; they toil not, neither do they spin : And yet I say unto you, That even Solomon in all his glory was not arrayed like one of these. Wherefore, if God so clothe the grass of the field, which to-day is, and to-morrow is cast into the oven, shall he not much more clothe you, 0 ye of little faith?... Are not two sparrows sold for a farthing? And one of them shall not fall on the ground without your Father. But the very hairs of your head are all numbered. Fear ye not therefore, ye are of more value than many sparrows." 1 "Nay but, O man, who art thou that repliest against God ? Shall the thing formed say to him that formed it, Why hast thou made me thus? Hath not the potter power over the clay, of the same lump to make one vessel unto honour, and another unto dishonour?" " "But in a great house there are not only vessels of gold and of silver, but also of wood and of earth : and some to honour, and some to dishonour." 3 "Surely your turning of things upside down shall be esteemed as the potter's clay: for shall the work say of him that made it, He made me not! or shall the thing framed say of him that framed it, He had no understanding ?... Woe unto him that striveth with his Maker! Let the potsherd strive with the potsherds of the earth. Shall the clay say to him that fashioneth it, What makest thou? or thy work, He hath no hands ?" 4

The doctrine of predestination as here recorded directly raises the question of "necessity" and of "free will," but into this I need not enter further than to say that man cannot be granted a free will and a free hand in any other sense than it is granted to all animated created things. He assuredly cannot interfere with the order and progress of creation. He has limits assigned to everything he does, as other animals have. Even plants are hedged in by boundaries which they may not overstep. All things in the ultimate sense are fore-ordained, and foreseen. Anything short of this would mean an end of law and order, and a reign of anarchy and confusion. To those who advocate the doctrine of free will I would respectfully suggest, that to an absolutely omniscient Being, Who foresees the end from the beginning, the element of free will does not necessarily or wholly clash with the idea of responsibility. One may know that a confirmed drunkard will indulge when he has an opportunity; the drunkard, however, is the responsible party. This explanation is satisfactory only up to a point, for it may still be said that what is, is as Crod made it, with all its tendencies and potentialities.

If the Creator be taxed with partiality, favouritism, and unfairness, it may truly be said that as Creator He has a perfect right to do what He pleases with every created thing. He is the Alpha and Omega: His will in everything is supreme and final. No garrulousness on our part is permissible. It is absolutely clear to my mind that the Creator is omniscient and all-powerful, and that He not only made and is making the universe, but that He supervised and continues to supervise all the details of His overwhelmingly stupendous task. The Creator is omnipresent as well as omniscient, and nothing is too great or too little to claim and occupy His all-embracing attention. He is also eternal : He was, and is, and ever shall be. There can be no such thing as a partial Creator. He must be-all powerful and everywhere present in time and space. Law and order must characterise His every act. Every action and reaction bespeaks unmistakable design. The means and ends are never separated. No force and no material are ever wasted. There is no blundering and no confusion when the ultimate purpose is considered and grasped. The tremendous upheavals in the physical universe, and the ravages of war, pestilence,

1 The Gospel of Matthew, chapter vi., verses $26,27,28,29$ and 30 ; chapter x., ver'ses 29,30 , and 31.

2 The Epistle of Paul to the Ronans, chapter ix., verses 20 and 21.

3 The Second Epistle of Paul to Timothy, chapter ii., verse 20.

* The book of the prophecy of Isaiab, chapter xxix.,verse 16 ; chupter xlv., verse 9. 
and famine which occasionally alarm and shock humanity, are part of a great rôle whose objective physiologically is the ultimate well-being of plants and animals, man included. The temptations, trials, and sacrifices incidental to life, even in its highest forms, have a meaning and a value, and it is for us to discover or endeavour to discover the final purpose, and bow to the inevitable.

If we regard the organic part of creation only, it will be seen that death is a necessary part of life, and that suffering in one form or other, in many cases, precedes the highest enjoyment.

\section{§ 43. Plants and Animals subject to Disease.}

Plants and animals are subject to epidemies, which at certain periods carry them off in untold numbers. They are also a prey to bacteria, microbes, and parasites of all kinds. These epidemics, very appalling while they last, generally correct some suspected or known evil. Even disease has a function in removing weaklings and improving the general stock. Certain correctives have to be applied to both plants and animals to keep them in a normal condition. Weeds have to be extirpated among plants, and the fecundity of animals kept within bounds by wholesale slaughter for food and other purposes. Overcrowding in plants and animals invariably results in famine or disease. There is a balance in organic nature which, if disturbed, necessarily results in mischief. It is a mistake to preserve too closely, and to destroy birds and beasts of prey in too great numbers: the grouse, salmon, and other diseases are largely due to overcrowding.

Slight variations as regards locality, soil, and climate are necessary to the well-being of plants and animals. Plants and animals confined within small, circumscribed areas degenerate. ('hange and cross-breeding become sooner or later a necessity; hence the rotating of crops in husbandry, and the introduction of new blood in the production of prize stock.

What holds true of plants and animals also holds true of man. He too is subject to epidemics, and from very similar causes. He suffers from famine; he makes war, and is in turn assailed, decimation occurring on either side; he overcrowds, and pays a heavy penalty in the shape of fevers, consumption, cholera, and other deadly ailments. The marriage of blood relatives, if long persisted in, results in mental and bodily weakness, and, in many cases, early death. The city population is never so robust as that of the country, and the blood of the inhabitants of the country must commingle with that of the inhabitants of the city in order to maintain a fair average of health.

The normal conditions of plants and animals are due to a large number of circumstances over which we have very little control. Many of these are exceedingly baleful in character if individuals only are considered. The baleful character, as a rule, disappears when the good of the race is taken into account. All the so-called evils have their uses in the organic kingdom. They keep plants and animals within bounds, preserve the balance of nature, and maintain a higher standard than would otherwise be possible. Similarly, volcanic eruptions, floods, denudations, glaciers, the elevation and depression of land, extremes of temperature and climate, great winds, \&c., make and keep the earth, as a whole, habitable for plants and animals. If the volcano and other great natural forces produce local havoc, their action is nevertheless beneficent when the finality of things comes to be considered.

\section{THE UNIVERSE AS A WORKING SYSTEM}

In considering the universe as a working system I am forced to fall back upon a Creator or First Cause-an intellectual Designer of means to ends-an Upholder and Controller of everything which is, and without Whom no change can occur either in the tiniest atoms or in the most ponderous bodies known to science.

To the Creator or First Cause is to be attributed the production of all matter, inorganic and organic, and all force, physical, vital, and mental. Everything that is exists by and through the Creator: it is what it is because the Creator made it: the thing does not make itself: nothing happens by accident, and nothing is left to chance. There is no room for spontaneous generation, or for modifications of living things which are not intended and provided for. It is not possible by any supposed laws or formulæ to exclude the Creator from His own universe, and the extraordinary way in which everything hangs together and is inter-dependent and correlated tends to show that all is referrible to one master mind, which is continuously at work. The recurrence of day and night and the seasons, so necessary to the well-being of plants and animals; the arrangements for their activity and repose, and for their proper nourishment and habitation; all testify to unity of plan and harmony as between the Creator and the thing created. Nothing is out of joint. According to this view inorganic and organic matter, and physical, vital, and mental force, have a common origin. They are parts of a great whole, where the parts perform harmonious functions. The parts are in no sense inimical or hostile to each other when the whole plan is considered. While the Creator may, in a sense, and for dialectic purposes, be regarded as distinct from the thing created, He nevertheless pene- 


\section{THE UNIVERSE AS A WORKING SYSTEM}

trates everything, be it matter or mind, to its inmost recesses. He literally and absolutely fills to overflowing everything which is, or can be, from the superabundance of His own being. He works in and through matter, and it is matter, and the manifestations of matter, which appeal more directly to our senses. He, however, also works in and through mind and intellect, and it is through the latter channel we receive our highest conceptions of the universe in its totality and of the Creator and arch-designer of the universe. It is for Him to infuse into matter and force and mind whatever of His own attributes He chooses, but all this is done in such a way as to make it impossible to separate matter from force and force from mind-either the mind of the C'reator, or mind in the ordinary sense (or its equivalent) as manifested in man and the lower living forms. It may be regarded as absolutely certain that the movements of the atoms and heavenly bodies are pre-arranged, and that every living thing, from the highest to the lowest, has its sphere of action defined, and is provided with a full measure of directive or controlling power according to its requirements. It cannot be conceived that the inorganic and organic kingdoms could be left to themselves to proliferate and run into endless variations. The fact that these kingdoms are correlated and interact, at practically innumerable points, renders such an assumption in the highest degree improbable. Correlation and interaction imply natural laws, the limitation of matter and force and mind, and the reduction of variations to a minimum.

The war which has been waged as to the co-existence of an invisible, spiritual, intangible world, and a visible, unspiritual, tangible world, and as to the co-existence of mind and matter as two distinct entities, is a futile war, as it seeks to set up differences and distinctions which do not, strictly speaking, exist. We have no proof that matter (inorganic and organic) is ever separated from spirit or mind, and mind, as we know it, requires matter for its healthy manifestations. If matter and mind cannot be separated it is absurd to speak of independent material and immaterial bodies, and the heated controversies as to materialism and immaterialism are so much wasted breath.

Matter and spirit, and matter and mind cannot, in the present economy, be dissociated without destroying their mutual relations : they are compound wholes which must be considered together.

The inter-blending of matter and spirit, and of matter and mind, in the universe carries in its train law and order, design, and the adaptation of means to ends on a grand scale. The universe, instead of being, as some suppose, a huge conglomerate of chance particles in fierce conflict, is a well-ordered whole, bristling in all directions with intelligent adaptations which command not only the attention, but also the admiration, of the thoughtful observer.

The idea of duration or eternity enters into everything connected with the Creator. With Him there is neither beginning nor end of days. There is continuity and permanence in all He does. There is also progress : what $\mathrm{He}$ does to-day is not undone to-morrow unless of set purpose. He works in specific directions and to given ends, and He assigns limits to all His works : day and night and the seasons return at stated intervals; there is a life period for every plant and animal. Plants and animals come from and are resolved into the elements. The sea is not allowed to swallow up the land, nor the land to usurp the place of the sea. In both cases the dictum, "thus far and no farther," is in full force.

No doubt there are catastrophes in nature, and these apply to the inorganic and organic kingdoms alike. They are of the nature of safety-valves. They have always occurred, and occur ever and anon now. Thus in the inorganic kingdom there are at times destructive storms of wind and rain, great inundations, landslips, volcanic eruptions, \&c., which take part in forming the earth's crust and in enriching geology and palæontology. In the organic kingdom there are epidemics, pestilences, plagues, failures, famines, wars, \&c., which make their appearance at irregular intervals.

These visitations, alarming and unsettling to us, in no way interfere with the mighty progress of events occurring in well-ordered sequence in the universe, and no argument of imperfection of plan, inconsiderateness, or cruelty can be founded on them. The so-called catastrophes, as indicated, have their uses in relieving pressure and clearing the air. They are so trifling in the stately march of events as scarcely to deserve mention. Nature holds on in her sublime course regardless of what, to our limited intelligence, appear minor imperfections and blemishes in her general plan.

If matter and force are eternal and indestructible, they are only so as emanations from the Creator in the sense indicated above. The definitions given of spirit, mind, matter, force, \&c., are, for the most part, quite misleading from the fact that the things defined are not fully comprehended or understood; the part, in not a few instances, being taken for the whole.

It is not permissible to judge the works of the Creator by purely human standards. The accidents and mishaps and pains and penalties which bulk so largely in human affairs have practically no significance when the stupendous concerns of the universe are considered. The destruction of individual plants and animals, and races thereof, are trifling events from the cosmic point of view. The awful avalanche, the dread lightning, the roaring deluge, the howling cyclone, the appalling volcanic eruption, carrying death and destruction in their wake, are 
necessary, under certain circumstances, to establish an equilibrium which is invariably beneficial. The aggregating and marshalling, and the breaking up and distributing of matter goes on incessantly, but the synthetic and analytic processes are all under control and supervision. Is a new molecule to be formed ? The atoms are provided, assorted, and the necessary combining powers conferred. Is a certain cell required? Atoms and molecules of particular linds are forthcoming for its production. Is a tissue, a gland, or a special organ a desideratum? Adequate and special preparations are made which exclude the possibility of failure. All these things are achieved not by accident but by design and forethought.

Is an old star, or sun, or system, to be destroyed and new ones formed? They are broken up and thrown into the seething, nebulous cauldron. From the white, molten, gaseous, formless mass, suddenly a centre of attraction appears which assumes right or left spiral movements, and draws towards itself outlying nebulous matter in ever-widening spiral streams; or conversely, the spiral streams acting from without converge upon a central point and so form a spiral nucleus. That heavenly bodies at times explode, fly off at tangents, and form new combinations is well ascertained. A time arrives when the new sphere is completed and launched into space on its own account.

Is an existing sun waning and wasting? It is fed as regards heat and energy by effete bodies attracted to it, and which, crossing its path and colliding, restore the lost energy and heat in the proportions required.

These cosmic and other operations are all planned. If there were not law, order, and method of the most far-reaching description, catastrophes of the most appalling kind would be incessantly occurring. The fact that they do not occur, or to the most trifling extent, is the most convincing proof we can possibly have that design reigns supreme in the universe.

Until a comparatively recent period the atom was regarded as the smallest conceivable particle. Sir William Crookes, however, showed (circa 1870) that matter in a still more minute state of division than the atom existed, that in fact the atom itself was formed by the coalescence of still more minute particles which pervaded space. He claimed for matter a fourth estate, and maintained that there was a form of matter which could not strictly be classed either as solid, liquid, or gaseous in the ordinary sense; the said matter being ultra or super-divided and separated. The subject has recently (190:3) been taken up by Sir Oliver Lodge. I refer to his researches in passing because of their supposed intimate connection with the ultimate matter of the universe. Sir Oliver remarks that the ultra or super-divided matter consists of fragments of matter, ultra-atomic corpuscles, minute things, very much smaller, very much lighter than atoms-things which appear to be the foundation-stones of which atoms are composed. Professor J. J. Thomson measured the mass of these particles and found that they were of less mass than the atom of hydrogen; whereas the atom of hydrogen had been the lightest body hitherto known. These small corpuscles, also called electrons, were about the one-thousandth of an atom of hydrogen in mass, and he further made this important observation, that whether hydrogen or oxygen or carbonic acid, or any other gas was in Crookes's tube, the particles into which these substances seemed to be broken up by electric action were identical and independent of the nature of the gas in the tube. The speed at which the corpuscles travel was found to be something comparable to that of light-about one-thirtieth or sometimes even one-tenth of the velocity of light. Anything moving with the prodigious speed of several thousand miles per second must have a great amount of energy, and, when stopped by a target, naturally considerable results are produced: their inertia is extremely small, but a body, no matter how small, moving with the speed of light, must have terrible energy. M. Becquerel was the first to discover the radio-active powers of matter. In the researches of Dr. Russell various substances were found to possess this quality of giving out something on their own account. The most important developments were made by Monsieur and Madame Curie in France, who found that polonium possessed the properties of uranium, and that radium in turn possessed the properties of uranium in a most extraordinary degree. The rays given off by these substances are of extraordinary interest; they have marvellous penetrating powers, and are very intense-more intense than the X-rays given by a Röntgen tube. Radium rays will not only penetrate a foot of aluminium or wood, but they will penetrate three-eighths of an inch of lead, and then be as strong as are the rays from uranium. There are three kinds of radiation: (1) particles which are readily stopped by obstacles, absorbable rays; (2) the particles which penetrate obstacles with singularly penetrating power ; and (3) the ordinary X-rays. The X-rays are waves in the ether-not light. The penetrating rays are electrons which are shot off. But the most interesting are the first rays, those which are easily stopped; for these turn out to be atoms of matter shot off with a speed comparable to that of light. Professor Rutherford, now of Montreal, has measured for the first time the speed of these readily stopped, absorbable particles, and also their mass. He shows that they are atoms of matter, and that they are moving with one-tenth of the velocity of light.

The size of the electrons is about one hundred thousandth part of the diameter of an atom, otherwise they would not have sufficient inertia. They are the smallest bodies known. The electrons occupy the atom very effec- 


\section{THE UNIVERSE AS A WORKING SYSTEM}

tively; they are energetic and pushful, though not big. They occupy the atom in the sense that soldiers occupy a country - that is, they will not let anybody else in. The electrons, by the force they exert, make the atom impenetrable; they also give the atom its other properties, and enable it to act chemically. That chemical affinity is electrical force has been known for a long time; it was suspected by Sir Humphry Davy. The relation of the electron to the atom is a matter of the most intense interest. But it is not to be supposed that the electron is stationary in the atom. The electrons are revolving round one another at tremendous speed, so that the atom is a region of intense activity. The electrons are not in the least crowded, although there are a thousand in the hydrogen atom, twenty or thirty thousand in the sodium atom, and one hundred thousand in the mercury atom; for consider how far apart are they in proportion to their size-just as far apart as planets in the solar system are in proportion to their size. The distance of the earth from the sun is to the size of the earth very much as the distance of electrons from each other is to their size in a mercury or platinum atom. The fact is, we come to an atomic astronomy, and the atom is becoming like a solar system, or like nebulæ or Saturn's rings, composed of a number of small particles in a violent state of revolving motion and occupying very little of the whole space with their actual substance. They are so small that collisions are unfrequent; so it is in the solar system and heavens generally - collisions do occur, but seldom, because of the excessively small sizes compared with the distances at which they are spaced out.

Taking any family belonging to a sun, that is, a solar system, it forms something like the same kind of collection as the electrons form in an atom. So when we get in an atom a sort of solar system we begin to question whether there is anything in absolute size after all. It has been suggested that solar systems may be atoms of a still larger universe. These are questions that are too hard. But there appears to be no end to the infinity of the universe, and all that we can say is that the probability is that it is infinite in an infinite number of ways.

The account given above of the electrons of matter opens up an entirely new vista. It shows that the consideration of even the most minute particle of matter indirectly raises the whole subject of the physical universe. Similarly, a consideration of any of the attributes of spirit and mind raises these subjects more or less in their totality: $(a)$ as regards the divine mind; $(b)$ as regards the human mind and its representative in the lower animals; and $(c)$ as regards the effect of both on matter.

I cannot do better than close the present section with the words of Professor J. H. Muirhead, who regards mind and matter, and vital and physical force, from two different standpoints. "If," he observes, "we set out from the existence of mind and matter as two entirely separate substances, there is, it must be admitted, no way in which we can establish continuity or causality between them. On the other hand, if we reverse this assumption, and regard the conception of two worlds, a physical and a mental, as one that grows up within (it is not said created by) our experience, a way seems opened up out of the difficulty. The conservation of energy and momentum, and the determination of their direction by physical antecedents, are from this point of view conceptions which are forced upon us in our endeavour to interpret to ourselves one side or aspect of our experience--that which we call the mechanical. Within the area so describable they are universal, ultimate, admitting of no exception. But the mechanical is only one side of our experience. Besides mechanical energy there is life. The phenomena of life violate no mechanical law, yet open up to us a new aspect of our world, a new form of 'energy.' We may, indeed, try to 'explain' life as only a more complex mechanism, and this has been a common device since the time of Descartes. But the present-day tendency to recognise here a transitio in aliud genus, and to reject (as leading to confusion) the attempt to explain the fuller, more concrete reality by formulæ applicable only to the more abstract, seems to be founded on a truer insight. What holds of the relation of life to mechanism holds also of the relation of mind to life in general."

From the foregoing it becomes more and more apparent that matter, force, life, spirit, and mind are indissolubly associated, and cannot be separated, even temporarily, for the purposes of argument. The one thing above all others to be avoided is the taking of a part for the whole and the endeavouring to establish weighty conclusions on slender and inadequate premises. If the Creator made inorganic and organic matter, He of necessity made force, physical and vital. He also made spirit and mind. The Creator is the fountain and source of everything, material and immaterial, but it is not given to humanity to fully comprehend how force and life are linked to matter, and spirit and mind are associated, and how they interact as between themselves and the universe as a whole. It is quite certain that no mere mechanical solution of the problem will suffice even as a working hypothesis.

\section{§ 44. Consideration of the Terms Irritability, Stimulation, Environment, and Instinct.}

The time has now arrived when these terms should be defined and used in a restricted sense or, what is better, discarded. Their lax employment of late years has done much to discredit biology and physiology as exact sciences, They have introduced confusion where none existed, and have made obscure, phenomena not naturally so. 


\section{§ 45. Irritability.}

This term is erroneously applied to both plants and animals. Plants and animals in a healthy normal condition cannot be said to be irritable. They are sensitive; but this is quite another matter. Irritability, strictly speaking, can only be applied to unhealthy and abnormal plants and animals. The term irritability was first employed to indicate that something in plants and animals which caused them to respond to external stimulation. The term involved a theory, and, to prove the theory, a second theory — namely, that of artificial stimulation-was invented.

\section{§46. Irritability plus Stimulation.}

The theories of irritability and stimulation are necessary to each other. They take for granted that plants and animals require to be jogged into activity by something outside of themselves. They ignore the life, spontaneity, and independence of plants and animals, and regard them as mere automata, which they certainly are not.

To make this matter quite plain, it is only necessary to state that healthy plants and animals can, and do, habitually perform all their normal functions as apart from both irritability and extraneous stimulation.

It is not denied that healthy plants and animals, and parts thereof, respond to electric and other stimulation, such as pricking, cauterisation, the application of mustard, acids, \&c. This fact, however, does not prove that the plants and animals are irritable, and that they can only act in response to stimuli. Healthy plants and animals, as explained, are sensitive, and, as a consequence, shrink from violent treatment. As further explained, they perform all their functions spontaneously and independently, that is, as apart from irritability and stimulation. Moreover, the results obtained by artificial stimulation are not identical with similar results obtained in plants and animals which are not artificially stimulated. The most that can be said is that in certain cases, and under certain circumstances, artificial stimulation produces results akin to, but not identical with, results obtained by natural stimulation, which has its origin in the original endowments and life of the individual. If a muscle be pricked, or a nerve excited by an electric shock, it cannot be shown that the results obtained are identical with those witnessed in natural muscles and nerves not so pricked and excited. In the one case, the stimulation is artificial and from without: in the other case, it is natural and from within. The modus operandi is essentially different. The mechanical treatment of living plants and animals as if they were automatic machines has great attractions for a large number of enthusiastic modern physiologists. They pride themselves on obtaining what they are pleased to designate exact results. They are ambitious to measure, to weigh, to demonstrate, and conscientiously record everything; and they inconsistently, in many cases, resort to the employment of the most delicate, complicated, and expensive instruments, which they use in a clumsy, careless, inexact manner. Their modes of experiment are inexact and crude to a degree. They profess to obtain the secrets of the most sensitive tissues and organs in plants and animals by the employment of every conceivable kind of stimulus, and by hacking them about with knives, scissors, needles, \&c., and torturing them until they are abnormal to an alarming extent, in the vain hope that they are imitating nature. I have seen one of the so-called exact mechanical physiologists constructing a chart of the power exerted by and the movements characteristic of the heart of a frog by partially detaching the organ from the body, by lacerating and displacing its nervous and muscular fibres, and by attaching the latter to a balance furnished with a stile or pen and connected with a recording cylinder. Nothing could possibly be more untrustworthy and incongruous. There was the usual employment of delicate, complicated, exact instruments with the most violent and barbarous, and, I am afraid I must add, ignorant procedure. The heart was deprived of its blood supply, the ganglia of the heart were displaced, torn, and abnormally excited, and, in many cases, destroyed; and the cavities of the heart, especially that of the ventricle, were opened and partly teased out. To crown all, the lacerated, fatally injured, bloodless heart was occasionally jogged into spasmodic activity by the application of electrical stimulation. Here was an imitation of nature's methods with a vengeance; yet the results thus artificially and ruthlessly obtained were duly published and paraded in a scientific journal as an exact physiological research. On another occasion I saw two well-known scientists open the abdomen of a rabbit and literally tear, and maul beyond recognition, the solar plexus with needles to discover the functions of the plexus by direct experiment. These are examples of badly directed experiments and faulty manipulation, but they illustrate the methods in many cases followed by the mechanical sehool. As compared with natural methods, the results obtained by even the most experienced and adroit experimenters are, at the best, doubtful, and to be received with the extreme of caution. It cannot be otherwise. If animal tissues and organs are lacerated or stimulated to any great extent, the circulatory, nervous, muscular, and other systems are abnormally disturberl, and a perfectly normal result, under the circumstances, is impossible. 
To take other examples. Wholesome food administered to a healthy animal produces no discomfort in the stomach or alimentary canal. The feeling engendered is one of comfort and satisfaction, and not of irritation or pain. Administer to the same animal an irritating drug and soon the stomach and bowels are in an agony of pain. In such a case there is the artificial stimulation (the drug) and the consequent irritation or pain produced by the action of the drug. But (and here comes the crux) neither the stimulation nor the irritation are natural, and they do not exist in the normal condition.

The muscular twitching and rigid tetanic condition produced by the continuous administration of strychnine is not normal muscular action; neither is the relaxed, flabby muscular condition produced by the inhalation of chloroform. The strychnine unnaturally exalts the muscular function, and the chloroform unnaturally depresses it. Similarly, the brain may be excited by the drinking of alcohol and other stimulants, or it may be calmed and soothed by the administration of opium and other narcotics. Neither the alcohol nor the opium produces a perfectly healthy brain function. The stimulant, as a matter of fact, produces the irritation and excitement which are otherwise non-existent.

If nature is to be studied satisfactorily she must be watched in her simple, normal manifestations, and not in her abnormal, complex conditions, when she is hacked about and tortured by various external stimuli.

The simplest plants and animals (by preference the transparent ones) yield the best results.

In making these remarks I have no desire to disparage the important results obtained by a judicious use of vivisection. These have been at once interesting, instructive, and useful. Neither do I wish to set aside the valuable results obtained by clinical research in cases of disease; disease acting as a distorted mirror to health. My object is to distinguish between the natural operations and functions of plants and animals, in which neither irritability nor stimulation is present, and the abnormal functions of plants and animals, where irritability and stimulation are artificially induced, or are a consequence of disease.

In the case of cell plants and animals where next to no differentiation obtains, the various functions of feeding, assimilating, excreting, and reproducing are performed in the simplest manner, as apart from irritability and stimulation. The lowest plant and animal forms are sensitive, and act spontaneously in certain directions-that is, they are not controlled to any extent by outside influences. What is true of plants and animals is also true of tissues and organs. The involuntary muscles in animals, on which so much depends-say the cardiac, respiratory, and alimentary muscles - are not, in the normal condition, irritable or jogged into activity by stimuli. On the contrary, they are independent, self-acting structures, whose functions are to take in, retain, and transmit blood, air, and food respectively. The blood does not act as a stimulus to the heart, the air to the lungs, and the food to the alimentary canal. If the blood, air, and food acted as stimuli they could not be retained, as well as taken in and given out at intervals or rhythmically. The secretory and excretory organs also act spontaneously and independently. Secretion and excretion go on so long as the body is in a healthy condition. The muscles, glands, and other structures form part of a living mass, and every part of the mass discharges its own particular function, separately and conjointly, as apart from irritability and extraneous stimulation. The combined action of the mass represents the life and aggregate endowments of the individual. On the spontaneous, independent action of the units depends the integrity and well-being of the plant and animal alike. If plants and animals had to depend for their existence on an elaborate system of artificial stimulation, it goes without saying that mishaps and accidents of the most serious character would continually occur. As a matter of fact, the so-called vegetative functions of animals are, for the wisest of purposes, placed beyond the control of the animals. The involuntary functions of the body are, in a sense, more important than the voluntary ones. They are so, as far as mere life is concerned.

\section{§ 47. Environment.}

The subject of environment is closely associated with the theory of irritability and artificial stimulation. It is held by many that environment furnishes a stimulus which causes plants and animals to modify their constitutions, their organs, and their general shape and appearance throughout the ages, and makes them what they are at the present day. It is even asserted that environment produces the sense organs and the organs of locomotion.

According to this view, environment is a leading factor in the production of every conceivable difference in plants and animals; plants and animals being regarded as living plastic things, which are acted upon by their surroundings nolens volens, or in spite of themselves. Environment is vaunted as superior to plants and animals; these being, at best, mere living automata, with no discriminating or directive power at their disposal. The dead surroundings are supposed to shape and determine the destinies of living plants and animals respectively. The theory is the invention of the mechanical school of physiologists. According to this school externalities form the skin with its sensory nerves; odoriferous substances the sense of smell ; sapid substances that of taste; sound that

VOL. I. 
of hearing; light that of seeing. In like manner the ground is said to form the organs for walking and running ; the waters those for swimming; the air those for flying.

That environment influences plants and animals, up to a point, is readily conceded, but that it makes plants and animals what they are is an opinion which cannot be seriously entertained.

At the outset, plants and animals are endowed with life. They exert directive powers which make them superior to their surroundings. They appropriate and assimilate certain inorganic and organic matters and reject others: they exert vital force, and during their development and life histories they subordinate and guide or override the physical forces, or act in conjunction with them according to circumstances: they act as masters and not as slaves : they adapt themselves to, but are not modified by, their surroundings. There are in plants and animals fundamental and permanent constitutions which are never altered beyond recognition by environment. Plants and animals are originally adapted for their particular habitats : they are created to occupy certain positions in time and space : they are, in no sense, the creatures of circumstances. Plants and animals are to the earth what furniture is to a dwelling. They are parts of a preconcerted whole, and the outcome of design. Plants and animals adapt themselves to altered circumstances in virtue of inherent original powers which environment did not confer, and cannot take away. It is mere perversity to say that plants and animals, or parts thereof, are even indirectly the product of environment. A home had to be provided for plants and animals : that home is represented by the word "environment": but it must be obvious to every one who reflects that the home is not to be confounded with its occupants, still less is the home to be credited with the formation of the occupants or with the changes occurring in them. The universe is divided into two great kingdoms, the inorganic and organic, and the two are complementary and interdependent, and this is all that can be said. Certainly the inorganic kingdom and environment cannot be credited with the direct and unaided production or material modification of the organic kingdom. The two kingdoms are at once independent and interdependent, and their permanency and mutual relations are all provided for in the scheme of the universe as we know it.

If environment or the home for plants and animals was provided before the plants and animals made their appearance, it is only another example of design and prevision. The scheme of creation necessitates environment, but environment plays a distinctly humble and subsidiary rôle. It administers to the life and well-being of plants and animals, but more than this cannot be claimed for it. It certainly does not indefinitely modify the structure and appearance of plants and animals.

While plants and animals, as explained, exercise a discriminating and directive power as regards the matter by which they are surrounded, they have no power to select and perpetuate perfections and suppress imperfections in themselves, as is claimed by those who advocate the doctrine of "natural selection."

The plant and animal cannot prevent, neither can they increase or diminish, the growth of any particular part either during development or during the adult state. The parts forming plants and animals are pre-determined, in the sense that each plant and animal produces only its kind according to types. Neither plants nor animals can, of their own initiative, depart to any extent from their types ; any accidental aberration being, sooner or later, corrected. Plants and animals have no power to develop on lines selected by themselves. They are conditioned and have their prescribed limits; in other words, their activities are confined, both as regards direction and amount. This is proved by the reversion or breeding back of cultivated plants and animals. Plants and animals, if left to themselves, revert to their originals. A distinction is to be drawn between plants in a state of nature and plants and animals artificially selected and deliberately crossed-to perpetuate what are considered good points and eliminate what are regarded as weak points. The cultivator works, in a sense, against nature, and produces, in not a few cases, abnormalities and monstrosities. The artificial breeder obtains his results by fostering development in one direction and repressing it in another. He, in reality, creates an abnormal and artificial state of things. He ilso creates what may be considered a false environment by housing, forcing, manuring, feeding, \&c. The abnormalities referred to are corrected in a state of nature, where there is a marked tendency to equalisation; the strong pairing with the weak, the tall with the short, the fair with the dark, the slender with the obese, and su on, illustrating the adage "extremes are ever neighbours."

Strictly speaking, there is no such thing as "natural selection." Selection implies a selecting power outside the thing selected. In nature no such power exists, if, and when, the Creator is excluded. In the case of artificial selection a selecting power, outside the plant and animal, is always present. The phrase "natural selection" has been introduced as the equivalent of "artificial selection," which it cannot be shown to be, and Mr. Darwin has, it appears to me, unwittingly established a false analogy which is at once pernicious and misleading. To say a plant or animal "naturally selects" and perpetuates perfections in itself, while it suppresses imperfections, is to attribute to the plant and animal the prerogative of the Creator of the plant and animal. The advances made by plants and animals are pre-determined, and form part of the ascending scheme of nature according to types. The 
advance in plants and animals, and the cosmic changes which accompany them, are not the outcome of accident, but of pre-arrangement and design. This ensures uniformity and prevents confusion. While there is a well-marked tendency to advance, progress is at times interrupted. In such cases not only is there no advance but there is deterioration, followed, in not a few instances, by extinction. Here we have the rule and the exception. Plants and animals, permanent to an extraordinary degree, even in their details, at times reach high-water mark, after which they retrogress and partly or wholly disappear. This should not happen according to the doctrine of evolution and "natural selection," where the fittest survive in an unbroken, continuous line ; all plants and animals being derived from a primordial cell and its representatives. There should, according to natural selection and evolution, be no gaps in the flora and fauna, geologically or otherwise. The missing links should be found somewhere. If plants and animals are originally derived from one stem, failure in one line should be made good by another line : the trail should never be lost. The subject of " natural selection" is fully discussed further on.

\section{$\$ 48$. Instinct.}

This term has been variously defined.

According to Webster it is a natural inward impulse; an unconscious, involuntary, or unreasoning prompting to any mode of action, whether bodily or mental, without a distinct appreciation of the end or object to be accumplished: a natural unreasoning impulse by which an animal is guided to the performance of any action, without thought of improvement in the method.

According to Paley it is a propensity prior to experience, and independent of instruction.

According to Whately it is a blind tendency to some mode of action, independent of any consideration, on the part of the agent, of the end to which the action leads.

According to Sir William Hamilton it is an agent which performs blindly and ignorantly a work of intelligence and knowledge.

There is perhaps no word in the English language which has been more vaguely employed and interpreted, and which has introduced more confusion in physiological and psychological problems. Nor is this to be wondered at considering the nature of the definitions given of it. These, for the most part, involve a contradiction in terms. They speak of what are virtually intelligent acts performed blindly, unconsciously, unintentionally, and without previous knowledge, training, and experience. With such a medley of meaning, misapprehension and confusion are inevitable.

It is quite evident that intelligence and consciousness in one form or other, and at one period or other, must be predicated of every instinctive act. Either the animal or its ancestors must be regarded as conscious and intelligent when they arrange and devise means to ends, or the Creator must act directly through them. The so-called instinctive acts are born of intelligence and consciousness, and the instinctive habit is produced by repetition in the individual. In all instinctive acts there is the element of design, but design implies a conscious, intelligent Designer, either within or without the animal which acquires and avails itself of the instinctive habit. There is direct proof in the higher animals that the so-called instinctive acts in the lower animals are produced originally by intellectual conscious efforts frequently repeated. The instinctive or automatic acts in man are so produced.

There are grave difficulties as to the employment of the term instinct in scientific phraseology. It is too inexact to be useful, and it is to be hoped it will soon become obsolete.

In one sense instinct is higher than intelligence, as it acts with greater celerity and with equal certainty; in another sense it is lower, as it is a mere unreasoning prompting in a particular direction without anything to guide it. It is plain that instinct cannot at one and the same time be intelligent and unintelligent, and this is exactly what is erroneously claimed for it. It is obvious that the element of intelligence, past or present, is always present: it is equally obvious that consciousness, past or present, is a factor : finally, it is mixed up with repetition or habit. As generally employed, instinct represents intelligent conscious acts repeated in the individual and in the race until they become (as the result of habit) unintelligent and unconscious - that is, automatic. It really represents acts of unconscious cerebration. Considered from the physiological side it involves the education and training of the nervous system, especially of the brain, in the individual and in the race. Considered from the psychical side it involves the employment of conscious reasoning and the arranging of means to ends, immediate or remote. Intelligence, consciousness, and repetition must all be predicated in instinct, and in this extended sense it is applicable to most of the lower animals and to man. The term is, however, a faulty one, as it includes too much or too little, according as the object is to exalt or depreciate the reasoning powers. In the case of the lower animals, such as the ant, the bee, the spider, bird, \&c., instinct figures as a power superior to reason, where means to ends are secured without consciousness and thought: in the case of the highest animals, instinct has its seat originally in conscious thought, reflection, and repetition. 
It goes without saying that the ant, the bee, the spider, and the bird cannot build their nests and carry on their domestic economy as apart from conscious thought any more than men can build houses and cities and institute governments as apart from conscious intelligent effort. If consciousness and intelligence within limits be denied the ant, the bee, the spider, and the bird, then it is obvious that these attributes must be exercised by the Creator in and through them. The nest of the ant, the honeycomb of the bee, the web of the spider, and the nest of the bird are constructed with surpassing skill. They are, in no sense, chance products. On the contrary, they are paragons of workmanship and design, and the highest resources of mathematics, physics, and mechanics are not unfrequently laid under contribution. Consciousness and intelligence are, though to a less degree, present in their case as they are in man. It is mere evasion to say that an ant, a bee, a spider, and a bird build their nests exclusively by instinct, and that a man builds his houses and cities wholly by conscious intelligent effort. The distinction is altogether artificial. As there is no difference in the nerve substance of the lower and higher animals, and no breach of continuity in the chain which connects the one with the other, it is evident that what holds true of the highest animals also holds true, within limits, of their lower congeners.

I have to express my conviction that conscious reasoning power can be traced in all the lower animals where, in their workings, they provide examples of the adaptation of means to ends. Similar remarks are to be made (but in a modified and lower sense) of plants, where no differentiated nervous system exists. The presence of a nervous system, as we know it, is not necessary to adaptation, and the arranging of means to ends.

The subject of instinct raises the important question of reflex nerve action as it exists in the higher animals and in man. As is well known, the nervous system in man is divided into two parts : the cerebro-spinal part, consisting of the brain and the spinal cord plus the sensory and motor nerves, and the sympathetic part, consisting of a double chain of nerve ganglia extending on either side of the vertebral column plus visceral and other nerve plexuses. These two parts are united to each other at various points; the nervous system in reality being a continuous whole. There is this peculiarity. The voluntary movements of the body are controlled by the cerebro-spinal portion : the involuntary, for the most part, by the sympathetic portion. The modus operandi of the cerebro-spinal system is usually as follows. A sensation due to some external object is transmitted from the peripheral sensory nerves which travels inwards to the ganglia of the spinal cord, and thence to the ganglia of the brain, where it is perceived and interpreted. The brain in turn sends an impulse, which travels outwards by the motor nerves to the muscles which are set in motion. The brain can act independently from within, as apart from sensations or impulses transmitted from without. In other words, the brain, or the mind acting through it, can originate motor impulses similar in some respects to the sensory impulses transmitted by the sensory nerves and the sense organs. In the case of a reflex nerve action the sensation, as a rule, only reaches the ganglia of the spinal cord, where it is perceived and interpreted; a motor impulse being generated and sent on to the muscles by the motor nerves without the knowledge or co-operation of the brain. The reflex act does not involve volition and consciousness in the ordinary sense. It may occur in the decapitated frog and in man where the brain or the upper part of the spinal cord are diseased, and where they are functionally cut off or disassociated from the spinal cord as a whole. In such cases, the reflex act may be largely explained by a nerve habit acquired by constant repetition of acts performed by the entire cerebrospinal system during the life of the individual: the spinal cord and sensory and motor nerves being taught to perform their parts independently of the brain.

The nerve-habit due to the repetition of certain acts becomes after a time automatic, and when once established forms what may be regarded as part of a self-acting machine, which when once set in motion is inclined to go on indefinitely. This is true of nerve reflexes generally, and of the nerve and muscular arrangements of the heart, lungs, alimentary canal, bladder, uterus, \&c.

The nerve-habit in the higher animals is almost invariably associated with a muscular habit, and it is often exceedingly difficult to distinguish between the two. The nerves and muscles, as a rule, act conjointly, but they also, as will be seen further on, act singly and independently. The brain by its motor nerves may send impulses to the muscles of an amputated or paralysed limb. Similarly, the heart may act without its nerves and as apart from the brain. The heart of the chick is composed of a mass of nucleated cells with no trace of either nerves or muscles, yet the organ acts perfectly.

If the sensory nerves be diseased or from any cause paralysed, no sensations reach the brain, and if the motor nerves be similarly circumstanced no motor impulses reach the muscles. The self-acting machine formed by the co-ordinated movements of the nerves and muscles must be intact for the performance of cerebro-spinal voluntary acts. In the case of reflex nerve acts it suffices if a portion of the self-acting machine is unimpaired and in perfect working order.

The point of transcending interest in this connection is the relation of the reflex nerve acts of the higher animals to the nerve acts witnessed in the lower animals having a nervous system (with or without a brain); it being 
customary to regard all the nerve manifestations of the lower and lowest animals as reflex, mechanical, and involuntary, the power of voluntary movement being reserved for the highest animals and man. The effect of this sweeping conclusion in favour of reflex acts is to deprive the lower and lowest animals, wholly or in part, of consciousness, intelligence, and the power of voluntary movement-a state of matters which not only places the lower animals on a very much lower level, but which confines the power of volition and of reasoning to man, the monkeys, and a few of the higher animals. For this there is no warrant. That the majority of the lowest animals move voluntarily and quite apart from reflex action will be readily admitted by all who have made this subject a special study.

\section{INSTINCT AND INTELLIGENCE}

As already stated, intelligence precedes instinct. We have proof of this in our own experience. If voluntary actions are repeated at intervals and frequently, they become instinctive and automatic-that is, they are performed without thought. To learn to walk requires much conscious effort on the part of the child. When the art of walking is acquired it is performed in the absence of all thought and effort. What is true of child movement is true of animal movement as a whole. I shall probably be here told that the lower animal forms have no minds, no reasoning powers. My reply is that they are capable of what are practically voluntary designed movements, and by that I mean movements to given ends. I further reply that the controlled movements witnessed in the lower animals are the outcome of mind, or its equivalent, either in the living thing itself or in the Creator Who made it, and Who regulates and adapts all its movements, be they small or great, simple or complex. It is not conceivable that the innumerable multitudes of living things could exist if they were deprived of volition, or its equivalent, and if they were not cared for. In the great scheme of creation everything is arranged, and contingencies of every possible kind anticipated. To the simplest and most complex plants and animals separate rôles are assigned, and these rôles are performed, in a sense, intelligently. This intelligence in the thing created, or the Creator Who formed and works in it, accounts for the habits and adaptability of plants and animals. It assigns them their place in nature. It explains how plants grow in certain directions, assume given shapes, and produce seed, \&c.; how animals move to and fro in certain well-defined grooves; how they sleep and wake, hibernate, migrate, build nests, store up food, procreate, \&c. If the actions of the lower animals pre-suppose experience and reasoning powers which cannot be conceded to them, then we are forced to fall back upon the inscrutable powers of the Creator. It is beside the question to say the spider spins its web, the bee constructs its comb, the ant its complex dwelling, and the bird its beautiful nest, by instinct; still less can it be said that the bee stores its honey and the ant its food by instinct. These are rational and far-reaching actions. They can only be explained in one of two ways. They are either the result of experience and reason (or its equivalent) in the present race or the ancestors of the spiders, bees, ants, and birds ; or they are due to an intelligent First Cause Who guided the ancestors, and continues to guide the offspring-the descendants of the said spiders, bees, ants, and birds. Instinct in its modern acceptation represents a blind or unpremeditated act, but the spiders' web, the honey-comb, and the ants' and birds' nests are miracles of design and intelligent architecture. No amount of experience in the lower forms of life could, apart from intelligence of some kind, culminate in such marvellous works of art. It has been thought that the accumulation of acquired characters through untold ages, natural selection, and hereditary transmission largely account for the existing state of things, but I would point out that to acquire, select, and transmit characters implies intelligence--intelligence of a high order, and intelligence incessantly at work from the dawn of creation. Moreover, no number of acquired characters, if accidental, would ever produce a progressively improved plant or animal. The acquired characters, to be useful, must be employed according to a given plan by a voluntary intelligent agent. Accidental chance characters can achieve nothing. Progress and improvement imply intellect, and whenever and wherever they occur intellect must be predicated.

It is a mere trifling with language and with facts to talk of instinct as a blind impulse, but one which, nevertheless, attains given ends with the certainty and precision of an unerring, intelligent agent. A thing cannot at one and the same time act blindly and intelligently. The so-called instinctive acts, in every instance, involve intelligence either in the creature or the Creator. The definitions given of instinct necessitate, as I have already shown, a contradiction in terms.

The rule would seem to be, that intelligence is vouchsafed to the animal according as its structure becomes complex, and its powers of independent action are multiplied. The C'reator guides and takes care of creatures which cannot take care of themselves.

It is impossible to disassociate instinct from intelligence and knowledge, and wherever we have clear adaptations of means to ends we are dealing with a planned whole and design as contra-distinguished from chaos and chance. 


\section{$\S 49$. Effect of Cosmic Changes on Plants and Animals.}

Considering the intimate relation which obtains between the organic and inorganic kingdoms-between plants and animals on the one hand, and the elements composing them, and circulating within them, on the other-it follows that plants and animals are more or less influenced by their surroundings and by cosmic changes, such as light and darkness; day and night; the seasons; heat and cold; moisture and dryness; absence or presence of electricity in the atmosphere, \&c.

Plants, on the whole, are more susceptible to cosmic changes than animals, from the fact that their affinities with the inorganic kingdom are closer, and more pronounced. This follows, because they are, with few exceptions, fixed to the earth and dependent, in a great measure, for their food on their immediate surroundings.

The extent to which the cosmic changes referred to influence plants and animals is, in every instance, limited.

The changes in question affect plants and animals most in a state of domestication. Wild plants and animals are comparatively little influenced by them.

That cosmic changes and environment do not essentially alter the forms and functions of plants and animals is proved in two ways :-

(a.) Plants and animals, varieties of which have been obtained by man's selection and fostering care, revert to their originals if left to themselves. 'Thus the several cultivated grains and vegetables, if allowed to run wild, degenerate and breed back. The same may be said of animals: the endless varieties of pigeons, if uncared for, all revert to the blue rock pigeon.

(b.) Animals have not perceptibly changed during the historical period. Thus man has had the same external configuration and traits of character for at least five or six thousand years. Egyptian tombs some five thousand years old display drawings of the five leading races of man as recognised at the present day. They also give representations of a large number of domestic and other animals, which are the same now as they were in the days of the Pharaohs, and in the remote past. ${ }^{1}$

If we go back to geological records, we find foraminifera in all respects similar to those existing at present, which lived at an inconceivably earlier period, and long before the appearance on the earth of fishes, reptiles, birds, and animals.

Sir J. William Dawson, when speaking of the origin of specific types and varieties and the external conditions favourable to their production, and when discussing the question as to "whether the conditions favourable to the appearance of new varieties were also those favourable to the creation of new types or the reverse," says : "In the present state of our knowledge we have no good ground either to limit the number of specific types beyond what a fair study of our material may warrant, or to infer that such primitive types must necessarily have been of low grade, or that progress in varietal forms has always been upward. The occurrence of such an advanced and specialised type as that of Dadoxylon in the Middle Devonian should guard us against these errors. The creative process may have been applicable to the highest as well as to the lowest forms, and subsequent deviations must have included degradation as well as elevation. I can conceive nothing more unreasonable than the statement sometimes made that it is illogical or even absurd to suppose that highly organised beings could have been produced except by derivation from previously existing organisms. This is begging the whole question at issue. . . .

"There is no reason to believe that any specific change has occurred in any plant within the Pleistocene or modern period." "

In an address delivered to the biological section of the British Association, Mr. Carruthers has discussed this

${ }^{I}$ As archaological research is prosecuted, and our knowledge advances, it hecomes nore and more evident that civilisation and art in Egypt are considerably older than was suspected even a few year's ago. It is now helieved that man was in a forward state of civilisation as far back $a s$ 9000 yeus ayn. To Professor Flinders Petrie the discoveries estallishing this fact are mainly due. Many other distinguished investigators have contriluted their quota, among whom may le mentioned 11. Capart, who has written an interesting work on "Primitive Art in Egypt."

II. Capart in his volume shows that Egyltian art is indigenous, and was not greatly alfected by outside inluences. He says that recentlyrevealed earlier strata exhibit a practically continuous series of ornamental ntensils and sculptmred nomments connecting the Neolithic age with. Plaramic times. He ohserves that the flints of Ergyt were of exceptionally fine workmanship, that art is of immemorial antiquity in that ancient land, and that the earliest linds were linked hy continuity of style with the latest. The ohjects of which M. Caprart's work treats are ascrihed to dates lying between 7000 B.C. and 4000 B.C., and the tirst dynasty is supposed to have been found in Petrie's excavations of the small town of Aliyilos.

The following ahstract has reference to a work, "The Egyptians in Eyypt," by Professor Flinders Petrie: "Far back in pre-historic times the savage who wandered over the wild desert mountains of Sinai picked up little scraps of sky-blue stone which pleased his fancy. These were doubtless preserved by being stuck into holes in his weajons and objects of wood, as the Bedawin do now ; and these decorated things were tradcd over into Egypt. The fre-historic man of Egy th demanded more, and a trade in turquoise sprang up, and provided the turquoise beads which were treasured for necklaces in the Nile Valley aboul eight thousand years ago. The primitive workers doubtless extracted the stones from the sandstone rock hy means of the flint-scrapers, such as are found hy hundreds in the old mine-heaps. When Egypt passed into the settled form of unitied government, muder the dynasties, the early kings would not leave this supply of jewels nuclaimed. So in Sinai, as far back as abont 4500 H.C. there are ligures of the Egyptian king smiting the natives, and of the general who headed the expedition. These are the oldest

seulptures known. Several such scenes of trimmpli were carved by later kings, especially those of the pyramid period, as Sencferu, about 4000 n.c." 


\section{EFFECT OF COSMIC CHANGES ON PLANTS AND ANIMALS}

question, and has shown that the earliest vegetable specimens described by Dr. Schweinfurth from the Egyptian tombs present no appearance of change. This fact appears also in the leaves and other organs of plants preserved in the nodules in the Pleistocene clays of the Ottawa, and in specimens of similar age found in various places in Britain and the continent of Europe. ${ }^{1}$

The difficulties attending the ordinary theories of evolution as applied to plants were well set forth by the same able botanist in his Presidential Address to the Geological Association in 1877. This address deserves careful study. One of his illustrations is the ancient willow, Salix polaris, which now lives in the arctic regions, and is found fossil in the Pleistocene beds at Cromer and at Bovey Tracey.

Salix polaris, he states, belongs to a sub-generic group containing twenty-nine species, which are arranged in four sections, that to which $S$. polaris belongs containing six species. Now it is easy to construct a theoretical phylogeny of the derivation of the willows from a supposed ancestral source, but when we take our little $S$. polaris we find that this one twig of our ancestral tree takes us back without change to the Glacial period. The six species would take us still farther, and the sections, sub-genera, and genus at the same rate would require an incalculable amount of past time.

But when we have reached the branch representing the generic form we have made but little progress in the phylogenesis of Salix. The ordinal form, if it ever existed, must necessarily be much older than the period of the Upper Cretaceous rocks - that is, than the period to which the earliest known dicotyledons belong.

All beyond the testimony of our living willows is pure imagination, unsupported by a single fact. So that here, also, the evidence is against evolution, and there is none in favour of it.

Science must indeed always be baffled by questions of ultimate origin, and, however far it may be able to trace the chain of secondary causation and development, must at length find itself in the presence of the great Creative Mind, Who is "before all things and in whom all things consist."

While evolution practically ignores what are virtually æons of time, its supposed proofs are wholly dependent on unlimited time and the endless modifications which unlimited time is believed to beget. The human mind cannot realise or reason about unlimited time and unlimited change, so evolution can neither be proved nor disproved. What proof there is is, however, clearly against evolution, for if it can be shown that during vast periods of time plants and animals have not perceptibly changed, then, $\dot{a}$ priori, we are bound to attribute to plants and animals a very large measure of fixity and permanence : so large a measure as to render evolution next to impossible.

There is another consideration. A large number of plants and animals, as geological records show, have become extinct.

The fact that plants and animals have appeared on, and disappeared from, the earth goes to prove that they are not the product of a process of continued evolution. If they had been, it is reasonable to suppose that the "missing links" would have been reproduced, and made good, by collateral evolutions, which is not the case.

Whatever may be said as to the production of new forms in the past and present, there can be no doubt that large numbers of both plants and animals have disappeared and are disappearing.

Further, geology shows that certain plants and animals have attained a high degree of perfection, and degenerated within limited cycles of time. This is true of the primeval ferns and cuttle-fishes. Moreover, crossing as between the several kinds of plants and animals is in no case unlimited. On the contrary, and especially in animals, barrenness, in the majority of instances, results after the first cross. Thus the horse and ass produce a mule or a hinney, but the mule and hinney are themselves barren.

There is this also to be said. The effect of evolution is to mix up every conceivable kind of plant and animal. This makes for confusion. Separate creations, on the other hand, of genera, and it may be of species, by prescribing limits, lead to law and order.

Evolution and retrogression are diametrically opposed to each other : they are reverse processes : retrogression, however, undoubtedly occurs.

All the parts and organs of plants are developed, not by environment or surroundings, but by inherent vitality and original endowment.

When a part or organ is not used it atrophies, degenerates, and even disappears. Thus in fishes born and bred in the waters of dark caverns, the eyes are reduced in size and are useless as seeing organs. The same holds true of plants and animals which become parasites. The parasitic plant loses its roots, and the parasitic animal its power of locomotion. There is, however, this important difference : while disuse causes the organ or part of it to deteriorate, both as regards structure and function, use cannot make an organ or part of an organ. The so-called vestiges of useful structures so frequently met with in plants and animals are the remains of types and nothing more. They are not genuine organic links which prove either evolution or descent.

$$
1 \text { "Proceedings of the British Association," 1889; "Pleistocene Plants of Canada," Canadian Naturatist, } 1866 .
$$


Whatever view be taken as to the production and continuance of plants and animals on the earth, the necessity for a First Cause or prime mover is abundantly evident.

A wide survey of the subject compels me to believe that whatever modification there is in plants and animals is due not to environment and surroundings, but to original endowment and inherent vital powers, which enable plants and animals to accommodate themselves, within limits, to their surroundings. It is the plants and animals, which, under guidance, modify themselves, and this only up to a certain point. In no case do external stimuli, and environment, produce unlimited modification, or alter materially or permanently the form and functions of either plants or animals. To assert this would be to maintain that the dead surroundings of plants and animals control their activities, while in reality the surroundings are controlled by the plants and animals.

When I say that cosmic changes affect plants and animals, I speak guardedly, and affirm that these changes do not alter permanently the ultimate constitutions of plants and animals as a whole, but only individuals, and within narrow limits.

Thus a fertile soil, rich manure, and a suitable climate, under the direction of man, will increase the stature, and, it may be, slightly alter the form of a plant; the plant reverting to its original type when these conditions are not complied with. The original or fundamental constitution of the plant is not materially altered in either case.

In like manner, the animal, say the horse, may by careful feeding, exercise, and training in a good climate, be improved in size, form, power, and speed. The horse, however, at its best does not differ fundamentally or intrinsically from the pony or under-sized horse. The English race-horse and the Shetland pony are anatomically and physiologically one and the same animal. They are both horses.

Plants grown in the dark, and in cold places, such as a cellar, are feeble, and largely destitute of colour. A potato or an onion grown under these conditions supplies the necessary illustration. The potato and onion, when grown in the dark, do not cease to be what they were and are. On the contrary, when the same plants are grown in the light, and under the genial influence of heat, they are the same as other potatoes and onions.

Similar remarks apply to animals.

Animals habitually born and bred in confinement are never robust, and, after a time, degenerate. Though they have enfeebled constitutions, the constitutions, such as they are, are not materially altered.

Plants, for the most part, do not grow, or grow very little, during the winter. They assume great activity in the spring. Summer finds them at their best. Autumn slowly but surely diminishes their power. Still the seasons do not alter either the constitution or the original endowments of the plant.

A plant grows more vigorously during the day, and when the sun shines, than during the night under stellar or lunar influences. Day and night do not, however, destroy or modify the constitution of the plant, and the same may be said of the animal.

The seasons, the alternations of day and night, \&c., are necessary to the well-being of plants. Plants have necessarily periods of activity and repose. They have to be fed and rested, just as animals have to be fed and rested.

In the case of animals, the influence exerted by cosmic changes can readily be traced. In winter many animals, such as the snake, hedgehog, \&c., hibernate. Animals, as a rule, are active or work during the day, and rest during the night; but the seasons, and day and night, do not alter the idiosyncrasies of animals. Animals require to drink more water in a warm, dry atmosphere than in a moist, cold one. More food is required in a cold than a hot country. Animals are uneasy in a thunderstorm and when lightning is flashing about: still these external conditions (and they are striking and marked) do not in the least interfere with the form and functions of the individual.

Plants and animals are responsive to cosmic influences, up to a point. They are true to, and in sympathy with, their origins. They are of the earth, earthy, and they do not disguise or disown their connection with the elements of the inorganic kingdom. Like true children, they acknowledge the sway of the universal parent, but in so doing they do not give up or lose their identity. They live in harmony, so far as they can, with their surroundings. They are not at war with themselves or with their environment. They are not irritable entities which require for their growth, development, and well-being a ceaseless round of artificial stimulation, such as is supposed to be provided by environment and cosmic surroundings.

The leaves of plants open their stomata and freely imbibe moisture during wet weather. They close them during seasons of drought. The rain and the drought are not the cause of the opening and closing of the stomata. These are not mechanical but vital acts. The opening and closing of the stomata are inexplicable as apart from the life in the leaves of plants. The natural effect of rain and moisture would be to close the stomata by swelling, and of dryness and drought to open them by causing contraction and shrinkage. The daisy (literally day's eye, or the eye of day) closes its petals at night, and opens them in the early morning. The opening and closing movements of plants are not caused by the darkness and light. This follows because plants open and close at all hours 


\section{RHYTHMIC MOVEMENTS IN PLANTS AND ANIMALS}

of the day and night, and a floral clock can be arranged to indicate the hours with nearly as much certainty as the shadow on the sun-dial.

A living clock composed of plants, or parts of plants, opening or closing at particular intervals, is only possible in living structures. Light, heat, cold, moisture, dryness, \&c., are not necessary to the opening and closing movements.

This is proved by the fact that parts of plants open and close at different periods of the day and night; the stomata of the leaves, as stated, opening in damp weather, when we would naturally expect them to close, and closing in dry weather, when we would naturally expect them to open.

The Volvox globator, which is an aquatic plant, opens and closes its vacuoles at stated intervals, as apart from every form of external stimulation. Other plants, when in a vigorous, healthy condition, exhibit rhythmical movements, and notably the Hedysarum (Desmodium gyrans), a native of the East Indies. The leaf in this plant is unequally pinnate, consisting of a larger leaflet at the end of the stalk, and two pairs of leaflets placed laterally. The smaller leaflets come towards and recede from each other with a jerking motion, every three minutes or so. The movements of the heart are certainly not more regular than those of the leaves now referred to. It cannot be the light or heat which produces the movements of the leaflets, for they go on in the dark in a reduced temperature; and as they are most regular when the plant is most healthy, we are not entitled to assume inherent irritability or extraneous stimulation. The movements in the leaflets in some senses foreshadow a heart, and are no doubt connected with the nutrition of the plant. They teach us one very important fact, namely, that living organs and portions thereof can come and go, contract, expand, and perform stated motions at stated intervals, without the presence of nerves, muscles, elastic and other tissues, unless, perchance, these exist in an undifferentiated form, which is by no means improbable. According to Fee, the fluids drawn to the surface of a plant during light are kept in equilibrium by rhythmical evaporation; the rhythmical movements of the leaves being referable to vital changes in the cell contents and vessels.

As a proof that the presence or absence of moisture will not account for all the phenomena witnessed in cells and other structures, it may be stated that in the sensitive plant (Mimosa pudica) there is a swelling at the base of the petiole, the cells of which constitute, as it were, two springs which act in opposite directions ; so that if from any cause the one be paralysed, the other pushes the leaf in the direction of least resistance. The springs, if they may be so called, situated at the base of the petiole are set in motion by the rush of fluid, creating a turgid state of the one set of cells, and an empty state of the other. A kind of rhythmic movement is thus produced. What is it, one naturally inquires, which gorges the one set of cells and empties the other, if it be not a vital power exercised by the plant? The fluid is present to both sets of cells alike. The same fluid certainly cannot stimulate the one set of cells to contract or shorten and the other to enlarge or lengthen; and besides, so far as known, there are no contractile tissues present in the plant. Neither can the presence of moisture act as an irritant, moisture being necessary to the life of the plant, and a normal part of it. The only explanation that can be given is, that the plant lives, and that it sucks in moisture by the one set of cells, and ejects moisture by the other set, just as one part of the heart sucks in blood while another expels it.

\section{RHYTHMIC MOVEMENTS IN PLANTS AND ANIMALS}

\section{$\S 50$. These are Repetitions of Rhythms occurring in External Nature.}

It may, at first sight, seem far-fetched to say that the rhythms in plants and animals are the outcome of rhythms occurring in the inorganic kingdom. As, however, the organic kingdom obtains all its matter, and no inconsiderable portion of its force, from the inorganic kingdom, it need occasion no surprise if rhythms, of a very distinctive and well-marked character, make their appearance in plants and animals.

The term rhythm is derived from the Greek $\dot{\rho} v \mu \hat{u}^{\prime}$. Strictly speaking, it means a measured motion. In a wider sense, it is a dividing into short portions by a regular succession of motions, impulses, sounds, accents, \&c., as applied to music, poetry, the dance, \&c. In its widest sense, it may be regarded as an advancing and receding, a going and coming, a giving and taking, an opening and closing, a lengthening and shortening, \&c.; the repetition at intervals, and the time and manner of the repetition, being accessory to the main idea.

While the element of time enters into our conception of all rhythms, it is necessary to explain that in physiology the time-limit for the recurrence of the movement forming the rhythms varies very considerably. Thus in the vermicular movements of the intestine during digestion, the movements proceed with great regularity and rapidityso much so, that at times the rhythms may be said to run into each other : in the csophagus in swallowing, and in the stomach in discharging chyme, they are considerably slower.

VOL. I. 
In the fotal heart they are 120 or so to the minute; in the adult heart from 60 to 80 to the minute; in the bladder they recur every four hours or thereby; in the rectum every twenty-four hours or thereabouts; in the menses or uterine discharges every month, and in human parturition at the end of nine calendar months.

I propose to divide rhythms primarily into two great classes, namely, Inorganic and Organic.

Under Iroorganic Rhythms I include :-

1. Those great cycles of time during which countries and continents have their climates changed, from excessive heat to excessive cold.

2. The annual revolution of the earth round the sun.

3. The seasons.

4. The rotation of the earth on its axis every twenty-four hours.

5. The alternations of day and night.

6. The waxing and waning of the moon.

7. The rise and fall of the tides, \&c.

Under Organic Rhythmis I include the changes and movements occurring in plants and animals at stated and calculable intervals. They are connected with and more or less intimately dependent upon the inorganic rhythms referred to above.

In Plants the chief rhythms are:-

1. Those connected with reproduction and germination.

2. Those connected with the rise and fall of the sap.

3. Those connected with the production of the leaves, the flower, and the fruit.

4. Those connected with the fall of the leaf.

5. Those connected with the regular movements of different parts of plants, such as occur in leaves and branches, the opening and closing of stomata, the opening and closing of vacuoles or water spaces, respiration, circulation, \&c.

6. Those connected with the movements of cilia in spores, \&c.

7. Those connected with the alternate action and inaction of plants as a whole, as influenced by the seasons, day and night, nutrition and innutrition, \&c.

8. Those connected with the periodic intake and output of solids, semi-solids, gases, \&c. These have a direct bearing on the building up, nutrition, and maintenance of the plant.

In Animals the principal rhythms are :-

1. Those connected with eating and drinking (set meals).

2. Those connected with the organs of alimentation (cesophagus, stomach, bowels, rectum, bladder, \&c.).

3. Those connected with waking and sleeping.

4. Those connected with respiration.

5. Thase connected with the circulation.

6. Those connected with the emotions.

7. Those connected with reproduction.

8. Those connected with locomotion.

Under 1 (Eating and Drinking) fall the rhythms engendered by the desire for food and drink at stated intervals.

Under 2 (Organs of Alimentation) fall the rhythms occurring in : -

(a) The fauces and oesophagus.

(b) The stomach with its sphincters.

(c) The small and large intestines.

(d) The rectum with its sphincters.

(e) The urinary bladder with its sphincter.

Under :3 (Waking and Sleeping), fall the rhythms engendered by alternate activity and repose, hibernation, winking, \&c.

Under 4 (Respiration) fall the rhythms occurring in :-

(a) The nostrils.

(b) The glottis.

(c) The lungs, or parts thereof.

(d) The diaphragm.

(e) The muscles of the thorax, abdomen, \&c.

Under 5 (('irculation) fall the rhythms occurring in :-

(a) The blood-vessels.

(b) The several compartments of the heart. 


\section{RHYTHMIC MOVEMENTS IN PLANTS AND ANIMALS}

Under 6 (Emotions) fall the rhythms which result in sighing, sobbing, crying, laughing, impulses of love, hatred, \&c.

Under 7 (Reproduction) fall the rhythms occurring in :-

(a) Ovulation.

(b) Menstruation.

(c) Coitus.

(d) Parturition, \&c.

Under 8 (Locomotion) fall the rhythms connected with walking, swimming, flying, waltzing, \&c.

The recurring, or give-and-take movements witnessed in the inorganic kingdom, which, as explained, are essentially rhythmic in character, are dependent on physical forces, such as gravitation, attraction, repulsion, cohesion, adhesion, condensation, rarefaction, osmose, chemical affinity, \&c.

Fxamples of rhythms in the inorganic kingdom are to be found in the rise and fall of the tides (give and take of water); the alternation of day and night (give and take of light); the coming and going of seasons (give and take of light and heat); the rise and fall of the barometer (give and take of atmospheric pressure); alternations of damp and dryness (give and take of moisture); the formation and disintegration of clouds (give and take of the sea, inland lakes, the atmosphere, and the land); the appearance and disappearance of frost (give and take of cold and heat); the formation of sound and other waves in the air and water (give and take of atoms in a state of vibration); the production of calms and winds (give and take of heat and cold equilibrating or producing vacua and air currents), \&c. ${ }^{1}$

In order to explain the rhythmic movements which occur in plants and animals, it is necessary to recall and emphasise the fact that all plants and all animals are derived from the inorganic kingdom, and that no element is found in the plant or animal which is not also found in that kingdom.

It is necessary also to explain, as has been already done, that the elements or substances which enter into the composition of plants and animals carry with them certain forces which inhere in them, and which must be reckoned with as factors, and important factors, in living plants and animals.

This follows because it has been shown by Professor Helmholtz and others that not only matter but also force is indestructible.

As it is not possible to destroy matter, neither is it possible to destroy force. The most that can be done is to change the position and form of matter, and to divert the direction of force. There is, it has now been ascertained, a store of matter and of force (physical force) in the universe which can neither be increased nor diminished.

The corollary to all this is, that living plants and animals in the processes of growth and development carry into their bodies, by what are virtually rhythmic movements, a large number of the elements which are found in the inorganic kingdom, and also a not inconsiderable portion of the physical forces of that kingdom, which confer on it its rhythmic and other movements. It was therefore to be expected that the rhythmic movements witnessed in the inorganic kingdom would reappear, in some form or other, in the organic kingdom, and they certainly do. It was also to be expected that the organic rhythmic movements would play quite as definite a rôle as the inorganic rhythmic movements, and this they likewise do. As a matter of fact, the organic rhythmic movements are primary or fundamental movements, and are absolutely necessary, not only to the well-being of plants and animals, but also to their very existence. Without them, plants and animals could neither be formed nor maintained.

The rhythmic movements occurring in plants and animals are spontaneous, independent movements, and neither nerves nor muscles, as they occur in the higher animals, are necessary to their production. It is not possible to explain or get behind the rhythmic movements referred to, and they must be accepted in physics and physiology as ultimate or final. They form parts of a great scheme of movement which pervades all matter, whether living or dead.

The rhythmic movements may, and do, occur in cells and living protoplasmic masses in the vegetable and animal kingdoms where no trace of a nervous system or differentiation of any kind can be detected.

While the cells of plants and animals are endowed with rhythmic movements, not a few of them are provided with cilia or hair-like processes, which, acting first in one direction and then in another, cause them to move in specific directions.

Sir James Paget writes: "Probably the simplest example of rhythmic motions yet known is that detected by Professor Busk ${ }^{2}$ in Volvox globator. At a certain period of the development of this simplest vegetable organism there appear in each zoospore, or in the bands of protoplasm with which the zoospores are connected,

\footnotetext{
1 In this enumeration of inorganic rhythms I do not refer to the manner in which these rhythms are produced. That is a much wider
} question, and would require separate treatment.

2 "Transactions of the Microscopical Society of London," May 21, 1852. 
vacuoles, spaces, or cavities of about $\frac{\pi}{\pi 00}$ of an inch in diameter, which contract with regular rhythm at intervals of from thirty-eight to forty-one seconds, quickly contracting and then more slowly dilating again."

Sir James Paget adds: "The observations of Cohn, ${ }^{1}$ published about a year later than those of Mr. Busk, but independent of them, discovered similar phenomena in Gonium pectorale and in Chlamydomonas, the vacuoles, like water-vesicles, contracting regularly at intervals of forty to forty-five seconds. The contractions and the dilatations occupy equal periods, as do those of our own heart ventricles; and in Gonium he has found this singular fact, that when, as commonly happens, two vacuoles exist in one cell, their rhythms are alike and exactly alternate, each contracting once in about forty seconds, and the contraction of each occurring at exactly mid-distance between two successive contractions of the other. Here, then, we have examples of perfect and even compound rhythmic contractions in vegetable organisms, in which we can have no suspicion of muscular, structure, or nervous, or of stimulus (in any reasonable sense of the term), or, in short, of any of those things which we are prone to regard as the mainsprings of rhythmic action in the heart." 2

The movements in the insectivorous plants are more or less rhythmic and definitely co-ordinated. They are also movements to a given end, and purpose-like, namely, the securing of living flies and small insects as food. Examples of these movements are to be seen in Venus's fly-trap (Diona muscipula) and the sun-dew (Drosera rotundifolia). Arrangements for catching live insects are also met with in the pitcher plants.

In Venus's fly-trap the outer portion of the leaf bifurcates, is orate, slightly saucer-shaped, and provided with serrated edges and six exceedingly sensitive hairs, three on each half of the leaf, which project from the surface, and are consequently in the way of anything moving on the leaf. When any small insect (fly, beetle, \&c.) settles and crawls on the leaf it inevitably comes in contact with the sensitive hairs, and the plant, being informed of the presence of the insect, slowly but surely imprisons, crushes, and destroys it. Nor does the marvel stop here. No sooner is the insect fairly within the grasp of the leaf than a secretion, akin to gastric juice, is poured forth from glands on its surface which enables the plant literally to devour and assimilate the insect. The leaf remains closed until the process of assimilation is completed, when it gradually opens to receive a new victim. Nor is this all. If the plant be tricked by dropping a small piece of cork or some non-edible particle on a leaf, it closes the leaf, but soon again re-opens it; no digestive fluid whatever being exuded. The plant can therefore distinguish between what is edible and what is non-edible. This implies a low form of cognition or its equivalent. The opening and closing of the leaf and the production of a digestive fluid cannot be regarded as, in any sense, haphazard.

When we consider that the spontaneous, definite, co-ordinated movements of Venus's fly-trap are performed in the absence of bones, muscles, nerves, and all those structures which are usually associated with the production of purpose-like movements in animals, they are, to say the least, very remarkable. They, in a sense, widen the horizon of organic movement indefinitely. They show that the potentialities of living matter are practically unlimited. Motion, purpose-like motion, can no longer be regarded as the prerogative of animals. That the movements of the fly-trap are not due to irritation in the ordinary sense is proved by this, that they will close on living, edible, and dead, non-edible particles alike. There is, however, this astonishing difference : the leaves remain closed on edible particles until they are digested and assimilated, whereas they open and extrude non-edible particles.

If the food of the plant acted as an irritant, the plant would naturally get rid of it with all possible haste. This, as explained, it never does. The substances which it will not tolerate are the non-edible ones.

\section{RHYTHMS AND REFLEXES IN PLANTS AND ANIMALS: THEIR NATURE AND USES}

Rhythms in plants and animals may be defined as involuntary movements recurring at stated intervals; the movements being repetitions of each other, and the intervals varying according to circumstances. They are fundamental, inherent, and intimately associated with life in its several phases: indeed life without rhythms would be impossible, as they are, to a large extent, the means by which nutrient materials are added to living bodies and the detritus or waste products carried off. They, in conjunction with certain reflex acts, are responsible for alimentation, respiration, circulation, reproduction, secretion, excretion, and various important functions, the due performance of which is necessary not only to the well-being but also to the existence of the individual. The rhythms make their appearance at the very threshold of life in rudimentary plants and animals as contractile vesicles, and they increase in number and complexity according to the degree of differentiation attained by plants and animals respectively. This is especially the case in animals. Rhythms, in the widest sense, are accountable for most, if not all,

I "Untersnchungen üher die Entwickelungsgeschichte der Mikroskopischen Algen und Pilze." Brestau, 4.to, 1854.

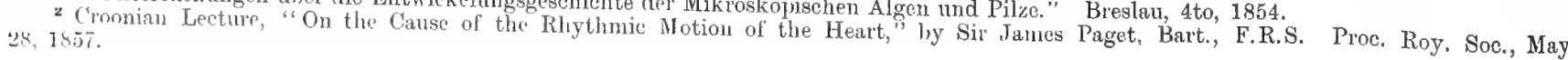




\section{THE MUSCULAR MOVEMENTS}

the involuntary movements occurring in plants and animals. To these are assigned what may be designated the mechanical as opposed to the intellectual functions of living beings. They make sure that the so-called vegetative functions are duly performed, and that the great races of plants and animals do not die out and disappear for want of necessary and proper apparatus to carry on the ordinary business of life.

Rhythms are part of the original equipment of life. They are conferred at the outset by the Creator, and afford irrefutable proof of design. No plant or animal could of itself and unaided assume the rhythmic structures, or carry on the rhythmic functions. It is here that the Master Designer, Director, and Controller asserts Himself. The rhythms are His immediate handiwork, and through them He controls the incomings and outgoings of plants and aninals and the great bulk of their movements. He makes sure that the really essential functions of life shall be duly performed, and in this we have an explanation of the so-called instinctive acts of the lower animals. Strictly speaking, there is no such thing as instinct. All those acts which imply reason and are means to ends, but which are blindly performed, are in reality the work of an intelligent Creator. If a spider and a bee work geometrically in constructing the spider's web and the honeycomb, and the ant and bird build their nests as highlyskilled architects would, it is because the Creator works in and through them. He alone can see the end from the beginning. He provides the rhythms and the intelligence, be it little or great, required by His creatures to fulfil their mission in life. To the spider, the bee, the ant, and the bird He guarantees the precise amount of intelligence for the due discharge of the duties assigned to them. Instinct always implies reason, and there must be a master mind behind animals to make sure that their acts never miscarry.

The rhythms and so-called instinctive acts furnish the key to the situation as far as plants and the lower animals are concerned. To the higher animals, and to man, a greater measure of intelligence has been graciously vouchsafed, and they are free (within limits) to exercise their higher powers; but, even in their case, care is taken that the vegetative functions shall not be neglected. Man, therefore, shares with the lower animals the rhythms, and what I venture to designate the mis-named instinctive acts.

\section{§ 5I. The Muscular Movements, Inherent, Spontaneous, and Independent-Not Caused by Nerve Action.}

Of late years there has been a growing tendency on the part of physiologists to refer the rhythmic movements of the heart and other structures to rhythmic discharges of nerve force from the ganglia situated on and in the substance of the heart and the tissues in which they occur. Sir James Paget attributed them to the nutritive processes going on in the heart, and Dr. Romanes was of opinion that they were due to periods of activity and exhaustion; the active period necessitating a period of inactivity or rest. None of the above explanations are, of themselves, sufficient to explain the phenomenon, and for the following reasons :-

(a) Rhythmic movements occur in organisms where no traces of nerve ganglia can be detected, such as Infusoria, Antherozoids, Spermatozoa; in certain Algæ, Oscillatoriæ, and Diatomaceæ; in the vacuoles of Volvox globator, Chlamydomonas in ciliary action, in the petioles of Hedysarum gyrans, the heart of the chick, \&c.

(b) The processes of nutrition go on in all living structures, but only a portion of these structures exhibit rhythmic movements, at least characteristic rhythmic movements.

(c) The activities of all living structures involve exhaustion and consequent rest, but this fact throws no light upon the rhythmic nature of the activities themselves; it does not explain why the heart, or the compartments thereof, act regularly at intervals, and only at intervals, during the entire life of the individual, while other structures act continuously, but only for given periods.

The illustrious Haller inaugurated $(1760-1766)^{1}$ what proved to be a virulent discussion regarding the nature of muscular movement. Having made numerous experiments and carefully considered the subject, he came to the conclusion that muscle moved independently and spontaneously in virtue of a power which inhered in its substance -in other words, that its power of movement was in no instance traceable to nerve action either in the cerebrospinal, sympathetic, or ganglionic system of nerves. His followers were designated "Hallerians" or "Animists." Those who opposed Haller and were in favour of the intervention of nerves in some shape were known as "Neurologists." I, personally, am wholly in favour of Haller's views ; for facts known to me, and revealed by experiment, have convinced me that certain structures move to given ends where no nerves are present, and what is more, where not even muscular fibres exist. The opening and closing movements of the heart, and the opening and closing movements of the stomach, bladder, rectum, and uterus also make this abundantly clear. It is not possible that the closure of any one of the viscera mentioned can forcibly dilate or open its sphincter. This powerful structure opens of its own accord. The opening and closing movements are rhythmic in character; that is, they are irregular, interrupted movements, and such as no system of nerves could inaugurate or keep going.

1 "Mémoires sur les Parties Sensibles et Irritables." Lausanne, 1760. “Elementa Physiologiæ,” ton. iv. lib. xi. Lausanne, 1766. 
Looking at the subject of rhythm not as an isolated fact in physiology, but as one of a great series of movements universal in their nature and extent, I am disposed to regard it as a primary endowment not confined to any particular living substance, but inhering in all to a greater or less extent, and capable of manifesting itself in plant and animal protoplasm, in rudimentary tissues, and in fully-developed muscular fibres, \&c.

Rhythmic movements, as explained, are not peculiar to the organic kingdom. They occur also in the inorganic kingdom. The recurrence of day and night, the seasons, the rise and fall of the tides, \&c., afford examples of inorganic rhythms. The inorganic rhythms are, so to speak, the harbingers or parents of the organic ones. This follows, because plants and animals derive all the materials which enter into their composition, and not a little of the force which actuates them, from the inorganic kingdom.

The rhythmic movements in plants and animals are essentially give-and-take movements, and, as such, are fundamental ; that is, they are inseparable from life, and form a dominant factor of it; they furnish the means by which life is continued. They are spontaneous original movements which are co-extensive with life. They begin with life and only terminate with death. They are not dependent on anything but themselves; they are not the result of irritation or any form of stimulation, external or internal. They are the media through which, and by which, pabulum and power are supplied to living organisms.

Plants and animals could not exist as apart from rhythmic movements. Plants and animals take from and return to the inorganic kingdom all the materials, gases, fluids, and solids which enter into and circulate within their bodies. The give-and-take movements imply a double power in living structures-a power to take from and return to-a power to appropriate, to circulate, to distribute nutritious juices, and a power to get rid of the effete or waste products produced by vito-chemical and other changes.

This double power of feeding and of transmitting the food through and out of the bodies of plants and animals is a sine qua non of existence. It is the distinctive prerogative of life. Life without rhythmic movements, or their equivalents, in one shape or other, would be impossible. Rhythmic movements are, in a sense, at once the cause and the outcome of life, and they are manifested in the lowest as well as the highest plants and animals. They are, moreover, within limits, the mainspring of life, and are not only inherent in the structures in which they occur, but they are also, for the wisest of purposes, incontrollable by any power existing in plants and animals. It is not possible to discontinue or stop the movements of the heart for more than a few seconds, or the chest for more than a few minutes. These go on day and night, from the cradle to the grave. Rhythmic movements are the outcome of the operation of vital laws which apply to all plants and animals. They are not dependent on the presence even of cells, and they certainly occur in numberless cases where no trace of either muscles or nerves can be detected.

They, moreover, occur in substances and tissues which, in the normal condition, are in no sense irritable. Neither are they naturally caused by, or in any way regulated by, external artificial stimuli. It is mere assumption and abuse of language to say that rhythmic movements are the result either of irritation, excitation, or stimulation.

It is inconceivable that living substances and structures created for the express purpose of taking in food (gaseous, liquid, and solid), circulating the same, and ejecting the waste products, should be dependent for their movements on inherent irritability or stimulation of any kind, especially external stimulation. Living structures move deliberately and to given ends spontaneously, as apart from all kinds of collateral aids. They are prime movers in the most emphatic sense. They set matter in motion, and are not themselves set in motion by matter, unless under abnormal exceptional circumstances.

Rhythmic movements are fundamental and essential to plants and animals. They cannot be separated from them without serious impairment or loss of life. Anything which destroys the respiratory or circulatory movements necessarily results in death: the continuance of rhythms is essential to the continuance of life.

In speaking of rhythms it is important to bear in mind that there are various kinds of rhythms, all more or less necessary to the continuation of life, and therefore fundamental; thus there is the rhythm of feeding, fasting, and defæcating; the rhythm of circulating air and nutritive juices; the rhythm of working and resting; the rhythm of sleeping and waking; the rhythm of hibernating and rousing at long intervals; the rhythm of reproduction, \&c. These several rhythms supply singly, or in the aggregate, the most incontestable evidences of life with which we are acquainted. It is not too much to affirm that their temporary or permanent suspension in every instance results in the dangerous impairment or death of the individual. All living things require food, air, fluids, semi-fluids, and solids: they have to circulate their nutritious juices, to work and rest, to sleep and wake, to reproduce themselves, \&c. The activities involved in obtaining food, circulating nutritious juices, reproduction, \&c., originate in the plant or animal : they express the potentialities of the individual. If one or more fails, death sooner or later supervenes. 
From the foregoing it will be seen that it is a mistake to speak of the rhythmic movements of the heart and chest in the higher animals as isolated phenomena-as something per se.

As a matter of fact, they form part of a great rhythmic system, which, so far as plants and animals are concerned, has for its object the appropriation, incorporation, and circulation of inorganic matters, and the expulsion of its innutritious portions at stated intervals. The rhythms of the organic kingdom are in turn to be regarded as repetitions of the greater rhythms occurring in the inorganic kingdom.

If we grasp the idea that rhythms are necessary to and form part of life, we shall have no difficulty in realising that they are of necessity outside the pale of inherent irritability and extraneous stimulation as known to modern physiology. As rhythmic movements are themselves the origin of the numerous activities of plants and animals it is a waste of time to attempt to prove that they are inaugurated and kept going by outside influences. The rhythmic movements are to be accepted as ultimate factors in physiology, behind which it is impossible to get. They are of the very essence of life, and wherever there is life there is rhythm, in a more or less pronounced form. The addition of matter to, and the subtraction of matter from, plants and animals can only be accomplished by give-and-take, rhythmic movements. Without them there would be universal stasis. Plants and animals could not grow, reach maturity, or reproduce themselves. There would be no circulation of atoms and molecules either of the gases, the fluids, or the solids of plants and animals. Everything would be at a deadlock. The interaction of the vital and physical forces, and of the organic and inorganic kingdoms, would cease and determine, and the whole scheme of the universe, as we know it, would be practically a dead-letter. The universality of rhythms in the organic and inorganic kingdoms invests them with the force of laws. The fact that they are present in the lowest and highest living forms unequivocally proclaims their fundamental and important character. The more I ponder the subject the more strongly am I convinced that the modern theory of irritability and stimulation, as applied to the organs of respiration, circulation, alimentation, \&c., is fallacious, misleading, and mischievous. The time has now come when life must be liberated from thraldom and leading-strings, and especially from trammels which prevent its moving unless when goaded into activity by something outside of itself. Life in the physiology of the future must be given a free rôle, and plants and animals must be allowed opportunities of working out their own destinies according to unalterable laws which dominate the inorganic and organic kingdoms alike. The meddlesome physiology of modern times has complicated and confused the issues of life; has destroyed its oneness, so to speak, by attributing piecemeal functions to organs which act as wholes, designedly and intentionally. This is especially true of muscles, voluntary and involuntary. The involuntary hollow muscles (chest, heart, stomach, rectum, bladder, uterus, \&c.) are spoken of as if they had only the power to close or contract, and similar remarks are made of the voluntary muscles, which are grouped as antagonists and are made to war against each other.

As I have already explained, muscles have a double power (centrifugal and centripetal), whereby they open, elongate, or dilate the one instant, and close, shorten, or contract the next. Nothing short of the double power now claimed will account for the opening and closing movements of the involuntary hollow muscles, and for the elongating and shortening movements of the voluntary muscles as seen in the different parts of the body. While the origins and insertions of voluntary muscles have been described with great care, and the actions of separate muscles indicated, comparatively little has unfortunately been done in describing the grouping and combined action of muscles, which, in some respects, is the most important. Muscles rarely if ever act alone. As a rule, they act in spiral muscular masses and cycles, as their spiral distribution round spiral and other bones, and spiral and other joints, clearly indicates. The muscular masses and cycles are co-ordinated, and work in groups to given ends, and it is this circumstance which makes muscular movements in very young animals so very difficult. All the voluntary movements have to be taught—-that is, the muscles have to be trained to co-operate and act in concert; hence the trouble experienced in learning to walk, swim, skate, \&c., in ourselves ; to walk and fly in the case of birds ; to swim in the case of fishes, \&c. The voluntary muscular systems in man, in the horse, in the fish, seal, walrus, \&ce, are fully discussed further on (see Plate lxxxiii. p. 320 ; Plate Ixxxiv. p. 332).

A glance at these Figures proves beyond doubt that muscles and muscular action cannot be treated separately or in an isolated way, and that to get natural results muscles must be allowed to act according to the laws which govern moving bodies generally : the flexors and extensors must be permitted to act simultaneously and in harmony, and so of the pronators and supinators, abductors and adductors, \&c.; the flexors when they contract or shorten must not be called upon to drag out and forcibly extend and elongate the extensors; the pronators the supinators; the abductors the adductors, \&c. Neither must the extensors, the supinators, and adductors be called upon in turn to drag out and forcibly extend and elongate the flexors, the pronators, and abductors. The flexors and extensors, the pronators and supinators, the abductors and adductors move synchronously, and when the one set contracts or shortens, the corresponding set dilates or elongates, and so muscular energy is conserved to the utmost. The 
so-called antagonistic muscles are not in reality opposed to each other. If this were so, the object in view, namely, the moving of bones and joints, would be to a large extent or wholly defeated. It should be stated that there are three kinds of co-ordination in muscular movements :-

(a) A co-ordination as between the flexors and extensors, the pronators and supinators, and the abductors and adductors respectively;

(b) A co-ordination as between the circumductors and other peculiar muscles ;

(c) A co-ordination as between the muscles of the chest and abdomen, and the muscles constituting the several compartments of the heart, \&c.

When the chest opens, as in inspiration, one set of muscles opens, dilates, or elongates, while another set closes, contracts, or shortens. There is no forcible dragging out of supposed antagonistic muscles in the movements of the chest and abdomen. In like manner, when the auricles of the mammalian heart close, contract, or shorten, the ventricles of the heart open, dilate, or elongate. The movements of the auricles and ventricles occur simultaneously, but the auricles do not by their closure forcibly open the ventricles, neither do the ventricles, when they close, forcibly open the auricles. The movements of the auricles and ventricles, which are centrifugal and centripetal in character, are spontaneous and independent of each other. If it were otherwise, the auricles and ventricles would make war against each other and to no purpose. The movements of the auricles and ventricles are co-ordinated, and the object in view is the transmission of the blood.

What is said of the auricles and ventricles of the heart applies equally to the stomach, rectum, bladder, uterus, and their sphincters. When the bodies of the viscera referred to close or contract, their sphincters open or elongate. Here again the closing and opening movements occur simultaneously and spontaneously. When, on the other hand, the sphincters open or elongate, the bodies of the viscera close or contract. The opening movements, however, do not produce the closing movements, neither do the closing movements produce the opening movements. If this were so, an enormous waste of muscular energy would result. Besides, and as is well known, the muscular walls of the auricles are too thin to force blood (during the systole) into the thick and practically solid ventricles : in the same way, the attenuated walls of the bodies of the other viscera named have no power to force the very narrow passages or outlets of the powerful sphincters. All this is proved alike by observation and experiment. In urinating, it is possible to empty the bladder, and, shortly after, to pass a small quantity of re-accumulated urine. What is passed on the second occasion cannot possibly act either as an irritant or a wedge for opening the sphincter vesicæ. This opens spontaneously when the body of the bladder closes. In nervous individuals, under certain circumstances, such as time or other pressure, urination becomes, for the time, impossible; all efforts at expulsion being rendered futile by the sphincter vesicæ refusing to open spontaneously.

The function of the Iungs, heart, and hollow viscera is to receive, contain, and transmit, at stated intervals, their contents, whether gases, fluids, or solids, but the contents do not act as irritants or stimuli and produce their own expulsion. If they did, the hollow viscera could not possibly act as receivers and retainers, as, under these circumstances, the contents would be ejected before they had time to accumulate or collect. Neither do the contents act from over-distending the hollow viscera, as calls to urinate, defæcate, \&c., if not attended to, pass off. The whole subject of rhythm in muscles requires to be reconsidered from a new standpoint and from a greatly extended purview, and the chances are that the history of muscular action will have to be re-written.

The most remarkable feature about the hollow viscera is not that they expel their contents at regular and irregular intervals, but that they allow the contents to collect within them for longer or shorter periods without producing even a trace of irritation.

This is a view of the subject hitherto altogether overlooked. It is, however, of such paramount importance that I feel called upon to direct attention strongly to it. The hollow viscera are not only transmitting and expelling organs, they are also receiving, collecting, and retaining organs. This one fact destroys of itself the prevailing theory of inherent irritability and artificial stimulation. It also militates against the theory of reflex action as a factor in rhythmic movements.

The subject of muscular action (voluntary and involuntary) is very fully discussed and illustrated in other parts of the work.

There can be little doubt that the spinal cord with its ganglia and complement of sensory and motor nerves has the power, within limits, of acting with or without the brain. This double power may be explained in two ways :-

(a) By the establishing of a nerve-habit in the cord from the frequent repetition of certain acts involving the co-operation of particular sets of ganglia, sensory and motor nerves, muscles, \&c. : and

(b) The separate and independent action of the ganglia and sensory and motor nerves of certain portions of the cord.

In the latter case, the ganglia and sensory and motor nerves of the cord perform virtually the same function 
performed by the brain and its sensory and motor nerves; the only difference being that in the cord the ganglia and its connecting nerves act more or less automatically, whereas in the brain they may and do act voluntarily. Both views are tenable, and there are good grounds for believing that the first arrangement $(a)$ may be traced to the second arrangement $(b)$, where the practically voluntary and independent action of the cord is converted by the force of habit into mechanical and what are known as reflex acts. Reflex acts are in no sense purposeless. This hypothesis of the double action of the cord is greatly strengthened by the fact that the brain is an expansion of the cord, and because the nerve elements in the cord and the brain are essentially the same. When the spinal cord acts independently of the brain, it does so through its ganglia or nerve centres, which perform the functions of little brains, and these I have ventured to designate "brainlets."

Reflex acts are not necessarily confined to the spinal cord. Under certain circumstances they may and do extend to the brain, in which case they are regarded as examples of unconscious cerebration. As it is not possible in a healthy normal animal to separate the spinal cord from the brain, neither is it possible to separate the fundamental functions of the two. This explains how voluntary muscular actions, frequently repeated and at stated intervals, may become involuntary or automatic actions, as happens in the case of walking.

Reflex acts, under ordinary circumstances, can scarcely be said to have an existence as apart from the brain. When an animal is deprived of its head, and co-ordinated and other movements are induced in its limbs through the instrumentality of the cord by artificial stimulation, what one sees are movements stereotyped in the cord due to similar movements occurring originally in the brain, the said movements in the brain being voluntary but rendered involuntary by repetition and habit. Reflex movements derive their value from their original connection with the movements due to the operation of the brain and the nervous system generally. The nervous system in a normal healthy animal acts as a whole. It may, however, under exceptional circumstances act piecemeal or in parts. The brain, for example, is divided into certain areas, which discharge specific functions, and the same is true of the spinal cord up to a point. As the part cannot be taken for the whole, neither can spinal cord reflex acts (which are involuntary and automatic) be regarded as other than secondary to the direct acts of the brain, which are voluntary. The anatomy of the nervous system precludes the separation of the spinal cord from the brain. The nervous system extends to every part of the body, and its branches, plexuses, and ramifications are distributed to all the tissues.

Where there is no breach of continuity anatomically, it would be a mistake to attempt a separation of function, unless in a general way and on particular points.

Reflex acts (in the sense explained above) have important uses. They take a leading part in the performance of all those movements which, whatever they may have been originally, have become automatic or mechanical. They, as it were, supervise all those acts which are beyond the control of the animal, such as the circulation, respiration, alimentation, the vegetative processes, \&c.

In order fully to understand what is meant by a reflex act in animals it is necessary to say a few words regarding the nervous system in its highest form as witnessed in man.

Usually the nervous system in man is regarded as consisting of two parts-the cerebro-spinal and sympathetic systems; the former displaying ganglia, end-bulbs, connecting nerve-fibres, sensory and motor nerves, nerves of special sense, \&c. The latter reveals similar and corresponding nerve elements. The cerebro-spinal system consists of the cerebrum and spinal cord, and these important nerve centres are lodged in bony cavities for protection. The great sympathetic system of nerves consists of a chain of ganglia linked together by a cord of their own, and placed outside the osseous cavities on either side and in front of the spinal column.

A further division is attempted, whereby the nervous system is separated into cerebral nerves, cerebro-spinal nerves, spinal nerves, sensory and motor nerves, nerves of special sense, sympathetic nerves, \&c. Both divisions are convenient, but they are purely arbitrary, and no physiological conclusions of importance can be founded on them.

As the ganglia of the brain, spinal cord, and sympathetic systems have much in common, and are united to each other directly and indirectly by commissural nerve fibres, it follows that neither the cerebral, cerebro-spinal, spinal, nor sympathetic systems are complete of themselves. There is, as a matter of fact, structural continuity which forbids functional differentiation.

The great cerebro-spinal centre which constitutes the chief portion of the nervous system sends its spinal and other nerves to the body in symmetrical pairs. Of these, twelve proceed from the cavity of the cranium through apertures at its base. The next issues from an opening between the occipital and atlas bones. The remaining thirty nerves issue from the sides of the vertebral column, each nerve below the body of a vertebra.

The distribution of the several kinds of nerves enumerated above is very extensive, and, as was to be expected, not a little involved and obscure. Thus the nerves of the sympathetic system are said to send branches to the VOL. I. 
unstriped muscles of the body generally (heart, ${ }^{1}$ blood-vessels, \&c.), and also to the glandular organs. Some viscera, however, such as the heart, lungs, the upper and lower parts of the alimentary canal, \&c., in addition receive branches direct from the cerebro-spinal system; such as are not so supplied being provided by nerves derived originally from the cerebro-spinal nerves through their sympathetic plexuses. The unstriped muscles of the viscera and other parts are also under the influence of fibres derived from the cerebro-spinal nerves.

The account given of the nature and relations of the ganglia and nerve fibres of the cerebro-spinal and sympathetic systems is scarcely less conflicting than that given of the leading divisions of the great nerve centres themselves.

The sympathetic system structurally consists of medullated fibres; the fibres being remarkable for their comparatively very small size. They pass by means of the white rawi communicantes from certain of the cerebro-spinal nerves into the cord and ganglia of the sympathetic. The cerebro-spinal system is composed of white and grey nerve substance in varying proportions.

The trunks of the great sympathetic nerve, as explained, are two in number-one on either side of the vertebral column. They begin at the base of the skull and terminate at the coccyx. The ganglia composing them are united by nerve fibres from above downwards, from below upwards, and transversely. They are also united by nerve filaments with the spinal nerves and their ganglia. Superiorly, they are connected with nerve plexuses which enter the cranial cavity.

It may be stated generally that the cerebro-spinal system of nerves, with its appropriate ganglia and nerve fibres, presides over the striped voluntary muscles and movements of the body, and that the sympathetic system of nerves, with its well-developed ganglia and nerve plexuses, presides over the unstriped involuntary muscles and movements. This is true up to a point, for, as explained, the cerebro-spinal and sympathetic systems in several cases interchange nerve fibres, and both systems supply nerves to the same structures. That the cerebrospinal and sympathetic systems of nerves do exercise a regulating power over the striped and unstriped muscles in the higher animals seems proved by experiment, where the division and stimulation of certain nerves retard or slow the movements of the organs or the parts to which they are distributed; the division and stimulation of other nerves accelerating or quickening the movements. The retarding or slowing nerves are known as inhibitory nerves, those which accelerate and quicken as accelerator nerves.

The famous Sir Charles Bell was the first strongly to direct attention to the existence of separate sensory and motor nerves in animals, and relied for proof on the division and stimulation of the nerves themselves. In this new departure in $1811^{2}$ he was followed by Majendie ${ }^{3}$ in 1822. Other original workers, such as the brothers Weber, Claude Bernard, Brown-Sequard, and Dr. John Reid, soon enrolled themselves under the new banner. Dr. John Reid, one of my predecessors in the Chandos Chair of Anatomy and Medicine in the University of St. Andrews, especially distinguished himself in the new field by a series of papers at once thoughtful and brilliant, the first of which saw the light in $1835 .^{4}$

In dividing and stimulating the nerves in the living animal it is important to bear in mind that shock to the system may largely vitiate the result, and render the experiments more or less futile. This is especially the case where large nerves or branches of large nerves are divided or mutilated. The rule seems to be that the larger the nerves experimented with, the less reliable the results. The shock caused by the experiment largely accounts for what is here stated. Nor must certain fundamental facts be lost sight of. As already explained, plants and the lowest animals are not provided with a nervous system, yet they perform all the important functions of life in its absence. As, however, a nervous system in certain living forms is not required, it would be a mistake to attach too much importance to it when present, and to experiments performed on it.

From the brief account given of the nervous system as a whole, it will readily be inferred that it is no easy matter to deal satisfactorily and conclusively with the reflex acts in animals.

\section{$\S 52$. Nerve Reflexes in Animals-Definitions of Reflex Acts-Subjects connected with Reflex Manifestations, \&c.}

By reflex acts in the higher animals are generally understood acts performed by a portion of the nervous system (mainly the spinal cord), plus ganglia, sensory, and motor nerves, \&c., to the exclusion of the brain. The acts are said to be involuntary and non-intellectual, and yet, in many cases, purposive. They have for their object the

1 The heart, althongh an involuntary muscle, is composed of striated muscle. It forms a connecting link between the involuntary and voluntary muscles.

2 "Idea of a New Anatomy of the Nervous Systen," printed privately in the year mentioned (1811).

3 Original experiment by Majendie published in the Jour. de Physiol. in 1822.

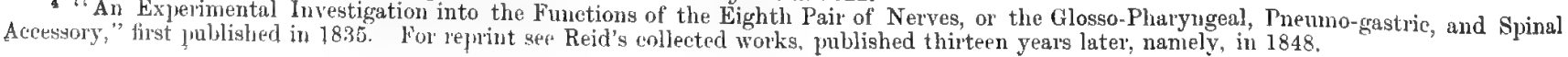


performance of certain important functions intimately connected with the life and necessary to the well-being of the individual. They are, more or less, rhythmic in character, and resemble in some respects, although not identical with, the lower forms of mental acts performed by animals with rudimentary brains. They are supposed to be the outcome of irritability and of external or internal stimulation, but in a healthy organism irritability and stimulation in all their forms are contra-indicated. It is only in the diseased or abnormal animal that irritability and artificial stimulation come into play. A reflex movement is defined by Professors Landois and Stirling ${ }^{1}$ as follows: "By the term reflex movement is meant a movement caused by the stimulation of an afferent (sensory) nerve. The stimulus, on being applied to an afferent nerve, sets up a state of excitement (nervous impulse) in that nerve, which state of excitement is transmitted or conducted in a centripetal direction along the nerve to the centre (spinal cord in this case), where the nerve cells represent the nerve centre; in the centre, the impulse is transferred to the motor, efferent, or centrifugal channel. Three factors therefore are essential for a reflex motor act-a centripetal or afferent fibre, a transferring centre, a centrifugal or efferent fibre; these together constitute a reflex act. In a purely reflex act, all voluntary activity is excluded." The so-called reflex function represents the natural or enforced working of an important part of the nervous system, to which is delegated the co-ordination and supervision of the involuntary movements, and all those operations which, for the wisest of purposes, are removed from the immediate ken and supervision of the individual. It is important to draw a distinction between what I designate natural reflex acts in a healthy body, and induced mechanical reflex acts in a diseased or unhealthy one. That the reflex nerves act spontaneously and purposively seems certain from the following experiment. If a frog be decapitated and a patch of moist mustard be placed, say on the inside of the right thigh, it draws up the left leg and scrapes the mustard off with the left foot. If the mustard be then placed on the inside of the left thigh, it draws up the right leg and scrapes it off with the right foot. This result is due to irritation and stimulation, but as the head of the frog has been removed, it is an abnormal experiment. The experiment, however, is of considerable value, as it shows that the spinal cord, and its ganglia and nerve cells, have an independent power of interpreting sensory impressions and of sending out purposive motor impulses.

The ganglia and nerve cells of the spinal cord hold the same relation to the cord which similar cells in the brain hold to that, the most august of all the organs. It is permissible to assign to the ganglia of the brain and the cord a variety of functions, and even to designate them by special names.

The functions discharged by the brain and spinal cord are ultimately molecular in character, and the ganglia, nerve cells, and nerve fibres, as units of these structures, have an independent and separate existence, and are entitled to the most attentive examination and consideration.

Assuredly all parts of the nervous system can act spontaneously as apart from irritability and stimulation; in other words, the nervous system, like all the other systems of the body, is self-acting. It is this power of spontaneous self-action which distinguishes the living from the dead thing. It is the prerogative of life to use and abuse extraneous dead matter, and to be very little affected by environment. Environment, and the stimuli which it is supposed to supply, neither originate life nor keep it going. Every living organism has the mainsprings of life in itself, and these mainsprings embrace all the systems and orgarss, indeed all the parts of even the most complex beings.

The reflex acts are mainly concerned in the vegetative functions of the body-that is, those functions which are in no way dependent on volitions or efforts of will. They are necessary to life, as they regulate, as explained, the highly important functions of alimentation, respiration, circulation, urination, defæcation, parturition, \&c. They hold an intermediate position between the involuntary rhythmic movements on the one hand, and the voluntary purposive movements on the other.

From one point of view, the involuntary reflex acts are quite as important as the voluntary purposive acts. They depend principally on the spinal cord with its auxiliaries, the ganglia, and the sensory and motor nerves. As, however, the brain is a mere expansion of the spinal cord, and the spinal cord has within it the potentialities of the brain, both as regards substance and function, it follows, that while the reflex acts cannot, strictly speaking, be regarded as voluntary and intellectual, neither can they be regarded as wholly devoid of intention and purpose. They are, in fact, the blind instruments of the Creator, and produce intelligent results. In compound animals, of which man is the best representative, a great many different systems have to be co-ordinated, regulated, and kept going. Thus there is the alimentary system, the respiratory system, the circulatory system, the lymphatic system, the involuntary muscular system, the glandular system, the reproductive system, \&c. These have all to be harmonised and the substances forming them to be duly fed and nourished. This intricate double duty of co-ordinating and feeding is largely handed over to the reflex nerve centres in the spinal cord. It would be impossible personally to superintend the extensive and complicated machinery of life. This, for the wisest of purposes, is placed in higher hands.

$$
1 \text { “A Text-Book of Human Physiology," by Dr. L. Landois and Willian Stirling, M.A., Sc.D., vol, ii, p. } 904 .
$$


A good example of reflex manifestations as bearing on nutrition is supplied by the vaso-motor system of nerves, which regulates the supply of blood in the smaller arteries by alternately narrowing and widening their calibre. This is effected by acting on the muscular coats of the vessels. If the arteries be greatly narrowed and the quantity of blood passing through them reduced, the organ supplied by them is under-fed: if, on the contrary, the arteries are dilated, and the quantity of blood unduly increased, the organ is over-fed.

While the vaso-motor system can and does act independently of the brain, it nevertheless is in sympathy with it and can be influenced by it. Thus in the phenomena of blushing and blanching, the skin is alternately made ruddier or paler than natural. Both results are emotional, and are obtained through the instrumentality of the cerebro-spinal nervous system, especially the brain. An unworthy insinuation or indelicate remark frequently raises a blush, and terror or an appalling piece of intelligence or shock usually induces pallor.

There are constrictor and dilator branches of the vaso-motor system of nerves whose function it is to regulate the amount of blood passing through the smaller arteries and the capillary blood-vessels through which the plasma or liquid portion of the blood oozes and feeds the tissues. As the blood moves very slowly in the capillaries, opportunities are afforded for a reverse oozing of effete matters into the capillaries from without. The outgoing (outflowing nourishing current) and the ingoing (in-flowing waste product current) are osmotic in their nature; the walls of the capillaries being to all intents and purposes osmotic membranes.

As there are constrictor and dilator branches of the vaso-motor nerves for regulating the flow of blood in the smaller arteries and capillaries, so there are two sets of nerves, for quickening and slowing the number of the beats made by the heart in a given time. Similarly, there are two sets of nerves for co-ordinating and regulating the movements of the hollow viscera with sphincters, such as the stomach, rectum, bladder, and uterus. These insure that when the body of the viscus contracts or closes, its sphincter simultaneously and spontaneously opens. Conversely, when the sphincter relaxes or opens, the body of the viscus simultaneously contracts or closes. The diametrically opposite movements witnessed in viscera with sphincters are more or less under the control of the reflex nervous system, which, as explained, acts as apart from irritation and stimulation.

The movements of the viscera in question, though largely, are not wholly, under the influence of the two sets of nerves in question, as the movements, in not a few cases, are more or less voluntary, and, to a certain extent, under the guidance of the brain. They can, under certain circumstances, be temporarily suspended.

The heart, which is to be regarded as the most important of the hollow viscera, is peculiar in this, that it never ceases to perform its opening and closing rhythmic movements, and it is the rarest thing in the world for any one to be able to stop the beating of the heart for even a few seconds. This dangerous power has been practised in only a very few cases, and once with a fatal result.

If the heart once stops beating, it is next to impossible to get it to start beating afresh. Failure of the heart's action is the dread of modern society, and the terror of all those who administer chloroform in surgical operations, especially such operations as involve sudden shock to the nervous system-as, for example, the extraction of teeth, toe-nails, \&c.

What is said of the heart is also true of the rhythmic movements of the chest. If the respiration which is unceasing day and night be once fairly suspended, the chances of reviving it are, as a rule, not great.

In the higher animals, especially man, all the systems are elaborated--division of labour being carried to an extreme. They are also carefully adapted to each other; they are dependent and interdependent; they are finely adjusted and balanced; they are self-acting; they act separately and in concert. In the normal living individual all the systems must be intact and in perfect working order. This is especially true of the nervous system. As regards normal reflex acts the spinal cord, its nerve centres and nerve cells, its sensory or afferent and its motor or efferent nerves, must all be intact and healthy. Disease or mutilation of any of the parts necessarily vitiates or destroys every form of reflex act.

Living compound organisms must be regarded as wholes, and any attempt at isolation generally proves abortive. The part cannot possibly represent the whole. It is for this reason a mistake to speak of the reflex acts as things per se; they are simply nerve manifestations of a kind. They are not wholly dissociated from so-called mental acts traceable to changes occurring in the brain. This follows, because they are the product of nerve matter, which is continuous, and which, as regards the brain and cord, differs less in kind than in degree. All the parts of the nervous system run into each other. The cerebro-spinal and sympathetic systems, ganglia, their nerve cells, sensory and motor nerves, \&c., form one grand whole. It is not possible to have well-defined and entirely isolated nerve function where there is continuity of nerve substance. The vaso-motor nerves, in the phenomena of blushing and blanching, are affected, as stated, by the condition of the brain. Similarly, the action of the heart, and the chest movements, are influenced by mental and emotional states. The heart may be pained, and its movements quickened or slowed, by cerebral conditions. What is known as "breathless excitement" influences the chest 
movements to a marked extent. As the spinal cord expands into the brain proper, so its substance and function reappear in an exalted form in the latter organ. This explains why certain involuntary nerve acts called reflex crop up amongst the volitional or mental acts proper, and how the voluntary acts become involuntary if repeated at intervals and frequently.

Musical performances, which at first are the result of laborious voluntary effort, ultimately become involuntary and automatic. The so-called involuntary movements are the harbingers or forerunners of the volitional or voluntary, and it is very difficult, if not indeed impossible, to draw a hard and fast line between them. It must never be forgotten that what are called automatic, instinctive, involuntary movements in the lower animals are, at the outset, intellectual, and intentional on the part of the Creator.

Reflex action is, in some respects, a very complicated, and extensive subject. Its ramifications involve both the cerebro-spinal and sympathetic systems of nerves. The cerebro-spinal system and ganglia, as explained, preside over the voluntary muscles, and the sympathetic system and ganglia over the involuntary ones. As the reflex nerve centres are connected with both the cerebro-spinal and sympathetic systems of nerves, their actions may be either conscious or unconscious.

Reflex action may be simple or compound, according to the number of parts affected by it. Thus one or several muscles may come under its influence, and so of glands and numerous other structures. A reflex centre may consist of a single ganglion with a nucleus, nucleolus, and a limited number of nerve cells (uni-polar, bi-polar, and multi-polar), or of several ganglia and a large number of nerve cells. In the latter case, the ganglia are connected and have complicated relations, their action being far reaching. Thus in a frog with the brain and medulla oblongata destroyed, tickling of the sole of one foot produces movements in the muscles of that foot and the leg to which the foot belongs. If the tickling be increased, the sensation engendered may pass across to ganglia on the other side of the spinal cord and produce movements of both feet and both legs. If pushed still further, it may produce movements of the superior extremities, and even of the whole body, in which case the frog is convulsed.

The reflex nerve centres are composed chiefly of grey matter; the sensory and motor nerves connected with them consisting of white matter.

The following are a few of the accepted tenets explaining the nature of reflex action according to the modern mechanical school of physiology :-

"Reflex actions may terminate either in movement of a muscle or in secretion of a gland. A reflex movement, of the most simple type, may be thus described : (1) excitation of a sensory or afferent nerve; (2) excitation of an intermediate nervous or reflex centre; and (3) excitation of a motor or efferent nerve which causes the muscular contraction."

"The initial excitation may occur both in nerves of general sensibility and in those of the special senses ; but certain nerves more easily excite reflex actions than others."

"A reflex movement may occur whether we excite a sensory nerve at its commencement or at some point in its course, but in the latter case the action is less intense than in the first."

"The excitability is increased when these centres are severed from communication with psychical centres which preside over voluntary movements."

"Reflex movements may occur in one muscle, or in many muscles or groups of muscles. Thus they may be simple or compound, and when compound there may be contractions of muscles occurring sinultaneously or successively."

"Reflex centres may be so arranged in the body as to constitute a series in which those in the cerebrum govern or control others in the deeper ganglia of the brain, while these in turn have an influence over still lower centres in the spinal cord. This arrangement has been termed the super-position of reflexes."

"It is possible for the same stimulus to produce a reflex action of movement, a reflex action of secretion, and also a conscious perception."

"In compound reflex actions, the initial excitation may occur in psychical centres." 1

Several of the statements here made are more or less contradictory.

According to prevailing views reflex action has its origin or mainspring in irritability and extraneous stimulation. I am wholly opposed to this explanation of reflex action, as neither the irritability nor the stimulation can be detected in a healthy normal animal. These appear only in the diseased or mutilated animal. Irritability, extraneous stimulation, and reflex action are further regarded as the cause of all the involuntary, as contra-distinguished from the voluntary, acts of the body. Here again I am at issue with modern physiologists. The involuntary acts are no more dependent on irritability and stimulation than are the voluntary ones. As a matter of fact the ganglia or nerve centres of the cerebro-spinal and sympathetic systems of nerves, with their afferent and efferent

1 "Outlines of Physiology in its Relations to Man," by John (iray Mekendrick, M.D., F.R.S.E., Professor of Physiology in the University of Glasgow. 
connecting fibres are self-acting, and require nothing outside of themselves but their natural objectives to evoke their peculiar powers. Their operations are, in no sense, automatic or mechanical. They do not require to be jogged into activity by any form of extraneous stimulation. On the contrary, the operations of the nerve centres are due to original endowment. They form part of the original animal. This is proved by the new-born infant sucking as soon as the teat of the mother is placed in its mouth. The reflex acts are inaugurated at birth and continued throughout the entire adult state. A most intimate connection obtains between the ganglia or nerve centres of the cerebro-spinal and sympathetic systems of nerves; their operations in many instances being co-extensive. They form part of one great nervous system, and, in the healthy animal, act together or in conjunction. It is only in the diseased or mutilated animal that they may be said to act separately.

Much confusion unfortunately exists as to the true nature of reflex action. As explained, it is universally referred by physiologists of the present day to irritability in the animal, and to extraneous stimulation of its nerve centres. This, as I have pointed out, does not afford a satisfactory explanation. Reflex action is said to preside more particularly over and produce the involuntary movements of the body, such as the circulation, the respiration, alimentation, the vegetative functions, \&c. The theory of irritability and artificial stimuli, as applied to reflex action, when carefully examined, is found to be misleading and untenable.

I quote recognised examples of reflex action to prove this statement.

1. Sneezing is said to be due to irritation of the Schneiderian membranes acting on certain nerves reflexly. Sneezing, I would observe, is an interrupted or non-continuous act. The irritation of the membranes referred to could not through the instrumentality of a reflex act produce such a movement.

2. Winking of the eyelids.--This is said to be due to the irritation of light falling on the sensory nerves of the conjunctiva, which act reflexly. Winking, like sneezing, is an interrupted rhythmic movement consisting of the alternate closing and opening of the eyelids. The same irritation acting upon reflex nerve centres could not possibly produce the closing and opening movements, which are diametrically opposite in character.

3. Contraction of the iris of the eye is stated to be due to the stimulus of luminous rays of various kinds acting on the optic nerve. In reply I have to observe that the light is always present or constant, and it could not by any nerve arrangement (reflex or otherwise) produce the accasional opening and closing movements referred to. If the light as an irritant produced the opening of the iris, it could not also produce the closing of the iris; these movements in many cases following each other rapidly.

4. Swallowing.--This operation is said to be due to the irritation produced by the presence of a bolus of food in the cesophagus. If this were so, it would result in the closing of the part of the cesophagus in front of the bolus, which is the point first touched. As a matter of fact, the closing takes place behind the bolus - the part of the cesophagus in front of the bolus spontaneously opening at the sarne time. It is quite evident that these opposite movements cannot be due to the presence of the bolus or any form of reflex action caused by irritation and stimulation.

5. Opening and closing of sphincters.-In the stomach, the pyloric valve or sphincter is said to be opened by the forcible contraction or closing of the body of the viscus; the irritant which produces the closing of the stomach, it is affirmed, being the food in conjunction with certain nerves (splanchnic and intestinal). In this connection I may state that no power exerted by the contracting stomach could possibly force the narrow passage of the sphincter. In reality, the sphincter opens or dilates spontaneously when the stomach closes or contracts. The double movement (centripetal and centrifugal in character) is a co-ordinated rhythmic movement, and the one is independent of the other. It is maintained by the mechanical school that the food (plus the nerve centres) is the cause of the closing or contraction of the stomach and of the dilatation or opening of the sphincter. This explanation is wholly unsatisfactory and inadequate. The movements of the stomach and its sphincter are exactly the opposite of each other, and it is, on the face of it, incredible that the same food could produce diametrically opposite movements, especially if those movements follow each other rapidly and are more or less rhythmic in character What is said of the stomach and its sphincter is equally true of the rectum and its sphincters, of the bladder and its sphincter, the uterus and its sphincter, and the hollow viscera generally.

6. When the theory of irritability and stimulation, plus reflex nerve action, is applied to the heart of the reptile, bird, and mammal, it is equally disappointing. If the heart of the mammal be taken as the example, it is said that the interior of the several compartments of the heart is irritable, and that the blood and certain nerve centres form the stimulus which causes the several chambers of the organ to open and close alternately with timeregulated beat, which ceases not night nor day. The objections which $I$ took to the explanations given of the action of the hollow viscera and their sphincters apply with equal and, if possible, greater force to the explanation given of the action of the heart. The mammalian heart consists of four compartments, and is bountifully supplied with nerves and nerve ganglia. The accepted theory is that when the two auricles close or contract they 
forcibly distend or open the two ventricles. The theory further declares that the two ventricles, when they close or contract, forcibly distend or open the two auricles. The forcible distension is said to be due to the blood acting as a fluid wedge. The closing contracting movements are considered vital, the opening distending ones passive. As a matter of fact, both sets of movements are vital. They are, moreover, spontaneous and independent of each other. They are not mechanical movements brought about by the blood and certain reflex acts, in conjunction with irritability and stimulation. Against the mechanical theory it will suffice if I state that the heart contracts and dilates spontaneously by centripetal and centrifugal movements when cut out of the body, deprived of its blood, and placed in an exhausted receiver. I may be permitted to add that the muscular walls of the auricles are so thin and feeble as to be altogether incapable of forcibly opening the ventricles, the walls of which are so thick that they, in many cases, form a solid muscular mass at the beginning of the systole. The remarks now made may with equal cogency be applied to the lymphatic hearts of the frog.

7. The movements of respiration.-These are said to be due to reflex acts caused $(a)$ by the presence of carbonic acid in the air-cells and air-passages ; $(b)$ to the accumulation of carbonic acid in the veins; and $(c)$ to cold acting on the skin, \&c. The difficulties already referred to reappear in a slightly altered form. The respiratory movements are, as is well known, co-ordinated, rhythmic, alternating movements - that is, movements occurring in opposite directions at stated intervals. Now it need scarcely be explained that the presence of carbonic acid in the lungs and veins, and the application of cold to the skin, could not by any possibility account for the give-and-take, opening and closing movements of the chest. The air and carbonic acid in the lungs at any given time vary little, and the latter may, for all practical purposes, be regarded as constant. It could not, by any process known to chemistry, act as an irritant and produce the opening of the chest the one instant, and immediately after act as a non-irritant and produce the closing of the chest. But nothing short of this could, if the mechanical views which prevail are true, produce the alternate opening and closing of the chest as witnessed in respiration.

8. The first respiration of the child.-The first respiration is said to be due to cold acting on the skin and other parts. Here again, cold (a single cause) is assumed to produce double and opposite results. If cold produced inspiration it could not within a second or two produce expiration. The cold is not alternately laid on and cut off, which it would require to be if it were the real cause of the double movements referred to. The cold is present both during the inspiratory and expiratory movements. This fact is, of course, wholly at variance with the theory of irritability and stimulation in their relation to reflex acts.

9. The sense organs.--These are also said to be largely under the influence of reflex nerve centres, but the subject of reflexes need not be further considered here, as I refer to them further on when discussing the nature of respiration.

A consideration of rhythms in plants and animals necessarily involves a consideration of the so-called reflexes in their entirety. Strictly speaking, reflex movements are confined to animals with a nervous system, consisting of a brain, spinal cord, and sensory and motor nerves--the brain, for convenience and the purposes of experiment, being practically eliminated. Their peculiarity consists in their involuntary nature-that is, they occur in animals through the instrumentality of the spinal cord and sensory and motor nerves as apart from, and largely to the exclusion of, the brain. The illustration usually given is the living decapitated frog. It is found that if the frog so mutilated has a hind foot pricked, lacerated, or stimulated in some way, it flexes and draws up the leg of which the foot forms a part: it removes it from the source of irritation. If, further, a little mustard be applied to the inside of one of the thighs of the frog, it scrapes away the offending mustard with the foot of the opposite leg. Here there is a low form of cognition and intelligence which can manifest itself apart from, and independently of, the brain. Similar results can be obtained in the human subject suffering from paralysis of the lower extremities (paraplegia), when, because of disease of the brain or upper part of the spinal cord, the brain, as an integral part of the nervous system, is practically removed. In such cases, the ganglia or nerve centres of the spinal cord and the sensory and motor nerves (their connection with the brain excepted) are intact, and in perfect working order. My explanation of the phenomenon is that the nervous system in certain cases can work voluntarily and reflexly apart from the brain, and that animals with ganglia, and sensory and motor nerves (minus a brain and cord) - say the fiverayed star-fish-can also act voluntarily. This the mechanical school of physiology deny. They insist that the movements of the star-fish are automatic, reflex movements. The mechanical theory of the movements of the starfish is disproved by observation and experiment. The star-fish certainly can and does move voluntarily, as any one may satisfy himself who studies it in its native habitat. Indeed, voluntary movements, of a kind, take place in both plants and animals where neither a nervous system nor a brain can be detected.

While it would be a mistake to deny a low form of cognition and intelligence to the decapitated frog, it would be even a greater mistake to deny the power of voluntary movement to the star-fish. In other words, experiments on the frog such as those referred to do not afford warrant for classing the movements of the star-fish, and of animals 
much lower in the scale of being, as reflex in their nature; that is, movements produced by external stimuli acting on a supposed irritable integument. The movements of the star-fish are certainly spontaneous, voluntary, and purposive: the animal changes from place to place and searches for and secures food, which it ingests, digests, and assimilates. The mutilated frog and the unmutilated star-fish cannot be placed in the same category. The frog whose brain has not been mutilated moves voluntarily and to given ends, and so does the star-fish which never had a brain. Moreover, as already stated, plants and animals having no brain also move voluntarily. Voluntary movements are not confined to animals provided with nervous systems with or without brains. It is necessary on the one hand to guard against placing too high a value on reflex acts when speaking of the lower and lowest animals, and on the other hand against not assigning to them a proper recognition in the higher and highest animals. Reflexes, as a matter of fact, form intermediate links in nervous manifestations. Regarding them as involuntary acts, they have much in common with rhythmic movements, to which they are more or less closely allied. They are intermediate as regards the psychic functions. In this direction they become mixed up with the so-called instinctive acts. They also trench on the voluntary acts. Certain voluntary acts, when often repeated and long continued, become more or less automatic. Reflex acts, so far as they are automatic, are fundamental and vital: they are means to ends, and, as such, afford evidence of design : they are connecting links as between mind and matter, but they do not prove that nerve action may be resolved into mere irritability of the nervous system, or that artificial stimulation is an exciting cause for psychic manifestations. In the reflexes, as in the rhythms, the hand of the Designer can clearly be traced. Both the rhythms and reflexes make for certainty as against accident in lower and higher living things. While it is difficult to assign limits to the rhythms, it is next to impossible to define what constitute reflex acts. The movements of the sensitive and insectivorous plants are both voluntary and reflex after a fashion. Similarly, the movements of the hollow viscera, as a class, display peculiarities which incline to rhythms and reflexes respectively.

The connection between rhythms and the so-called reflex acts are, in many cases, well marked, and a wide survey of the subject inclines me to believe that they are different phases of the same thing. This much is certainthey are fundamental, and occur in both plants and animals. They are not dependent on the presence of a nervous system as we know it, neither are they, strictly speaking, traceable to extraneous stimulation or irritability in the organisms in which they occur. The rhythmic movements of the plant Volvox globator, devoid of nerves, are as perfect, in a way, as those of the heart of the animal richly supplied with nerves. The heart of the chick in its early stages affords an example of an animal structure, devoid of nerves (and even of muscles), but which nevertheless exhibits typical rhythmic movements. This supplies an intermediate or transition link as between the rhythms and reflexes of the plant and animal, and emphasises their fundamental character.

Rhythms and reflexes, there can be little doubt, are primarily inherent vital movements, which are not necessarily dependent on any particular structure, nervous, muscular, or otherwise, nor on any form of irritability or stimulation, and which manifest themselves in young and adult plants and animals alike. They are largely connected with the development, growth, and nutrition of plants and animals, and are, in reality, the chief means by which the elements are taken into and discharged from the bodies of plants and animals in the immature and mature states. They also provide the mechanism and movements by which plants and animals are connected with the outer world-that is, the material universe outside of themselves.

By rhythms and reflexes plants and animals are kept going : they are original endowments by which life asserts itself; by which it incorporates and discharges matter ; and by which it recognises and moves amongst it. Somehow the idea has got abroad, and is almost universally accepted, that the major portion of the movements of plants and animals is due to an inherent irritability of the moving parts, and that an outside stimulus must be applied before movement can occur. Nothing can be further from the truth. All the essential movements in plants and animals are vital movements, and take place independently of either irritability or external stimulation. Plants and animals absorb, assimilate, secrete and excrete, circulate their nutritious juices, and reproduce themselves quite apart from both irritation and stimulation. To hold any other view would be to degrade life, and reduce plants and animals to the condition of machines, which they certainly are not. Plants and animals manage their own affairs irrespective of any outside interference-the Creator excepted. They employ extraneous matter and force in building up their bodies, but they control and direct the matter employed. They select and appropriate certain elements and reject others: they avail themselves of certain physical forces and override and suppress others according to circumstances. Thus, plants grow upwards in spite of the great law of gravitation. They, however, in growing, freely avail themselves of other physical forces, such as attraction, repulsion, cohesion, adhesion, capillarity, and especially osmose. These all play a part in the absorption, assimilation, secretion, excretion, circulation, and respiration in plants. Similar remarks are to be made of animals.

The phenomena of endosmose and exosmose, by which plants and animals largely imbibe nutritive materials 
and throw off waste products are themselves essentially rhythmic in character. They are give-and-take movements in opposite directions, and are comparable to the rhythms of the compartments of the heart, which enable that organ alternately to open and receive blood, and to close and eject it. They are also comparable to all movements occurring in opposite directions, such as the closing and opening of the eyelids, the closing and opening of the chest, the several parts of the alimentary canal, bladder, and so on.

Plants and animals, in virtue of their vital powers, can take in, retain, and eject at discretion the elements on which they live. They can also recognise and take stock of their surroundings; in other words, they can appreciate the matter in their vicinity which forms their environment. This being so, it follows, that certain changes and movements, as between plants and animals on the one hand, and the elements and all parts of the physical universe on the other, must take place. It follows, further, that the changes and movements must, fundamentally, be atomic and molecular in character, and capable of manifesting themselves in all kinds of living substances and tissues, as apart from nerves and muscles, which are mere differentiations. If rhythms and reflexes find their highest expression in nerves and muscles, it must never be forgotten that they do not originate in either, and are traceable to lowly organisms and structures, and even to plant and animal protoplasm.

This is a matter of very considerable importance, because, according to existing views, the highest animals are too sharply defined and too widely separated from the lower animals. The distinctions between plants and animals are also exaggerated. All plants and animals are endowed by nature with movements of some kind. They are also endowed with the power of acting upon the elements which enter into their composition and by which they are surrounded. The movements and powers referred to are necessary to their existence and well-being. If plants and animals cannot absorb, assimilate, retain, transmit, and discharge matter, they languish, pine, and die. In order to live they must be endowed with the power of taking in or receiving, of assimilating and retaining, and of extruding and casting off effete matter. They must be superior to the matter amongst which they are placed. To this there is no exception. This remark applies equally to the plant and animal; to alimentation, respiration, the circulation of nutritious juices, the extrusion of effete matter, \&c. As is well known, the rhythms and reflexes in the higher animals are dominant factors in the respiratory movements of the chest and lungs, in the vermicular movements of the alimentary canal, bladder, and uterus, and in the movements of the several compartments of the heart. They are primarily concerned with the incorporation of matter in the form of air, drink, food, offspring, \&c. They are secondarily concerned in the recognition of matter not to be incorporated; the recognition, in the higher animals, being effected either by the skin, or the sense organs as the instruments and representatives of the brain. The skin and sense organs are specially modified and differentiated for this purpose. The cells and tissues composing them are equal to dealing with many kinds of matter and many kinds of motion-with objects touched, with the qualities thereof, as roughness, smoothness, \&c., with tasting and smelling bodies, with vibrations of various kinds, with the undulations of light and sound, with substances near and remote, with matter in a gaseous, fluid, semi-fluid, and solid form-with, in short, nearly everything in the physical universe. The higher the animal, and the greater the degree of differentiation and division of labour, the greater the power of recognition. It is his superior powers of recognition which places man on a pedestal by himself, and makes him virtually the ruler of the world.

The differentiation and specialisation in the nervous system is proved by the fact that if the optic nerve be divided and the cut end next the brain artificially excited, luminous flashes and the feeling of light are produced in the brain itself. If, again, a motor nerve be divided and the cut end next the muscle stimulated, the muscle to which the nerve is distributed is set in motion. Similar remarks are to be made of the sensory nerves. If, for example, in cases of amputation of the hands and feet, the peripheral ends of the cut sensory nerves become diseased, the patients complain of uneasiness and pain in the fingers or toes of the amputated parts. The nervous system, when intact, feels at every point and moves at every point, and this with or without a brain. It is selfacting in the sense that it originates and sends out motor impulses as well as receives sensory impressions. An animal with a brain, sense organs, and sensory and motor nerves, immediately and directly feels everything it touches. It is the prerogative of the brain to feel to the finger tips and to move to the finger tips. The animal feels throughout its entire cutaneous and mucous surfaces, and moves in all its parts by means of its muscles, voluntary and involuntary. It is the brain which primarily feels and moves in the higher animals; feeling extending to the extremities of the sensory nerves and motion to the extremities of the motor nerves.

The brain and nervous system require no intermediaries for their operations, other than the sensory and motor nerves and the sense organs. Their fundamental function is to feel and move, and this they can do at first hand, as apart from artificial stimulation and irritation.

When an animal elects to feel, it advances part of its body, so as, if possible, to come in contact with the object to be recognised. The eye, so to speak, projects itself into space and searches for things near and remote; the ear VOL. I. 
turns itself in the direction of sounding bodies, and strains to receive on its drum or tympanum the waves of sound; the tongue presses the sapid solids and fluids which form our food and drink against the palate, cheeks, and other parts in tasting; and the nostrils sniff up the minute odoriferous particles floating in the air, from whatever source derived, in the process of smelling. In each case, the animal by its sensory nerves and sense organs performs its work voluntarily, and as apart from stimulation and irritation. When an animal is accidentally touched from without, it is always taken by surprise, and responds at a disadvantage, being off its guard. Herein lies the great difference between my theory of life and action and that of the modern, mechanical school.

I maintain that life is aggressive and masterful in everything it does; that it is self-sustaining, self-acting, self-directed; that it is superior to its surroundings; that it moves spontaneously to given ends; and that it performs its work directly and at first hand, being independent of everything but the First Cause.

The modern physiologists contend that all, or very nearly all, the movements of plants and animals do not originate in the plants and animals themselves, but that they are due to extraneous stimulation and inherent irritability. They attribute to dead matter and stimuli the initiation of the greater portion of the movements and changes occurring in plants and animals, than which nothing could be more unphilosophical. They reverse the order of nature. In so doing, they practically rob life of its prerogatives, and convert plants and animals into automata which are set in motion and regulated from without. No greater delusion can possibly be imagined. Plants and animals cannot be so degraded. The mainspring of action, and the power of regulating action, inhere in the plants and animals themselves. Life as conferred by the great First Cause is, in every instance, the prime mover, and the matter and force of the universe are subject to its sway and under its control.

In rudimentary animals, such as the jelly-fish (Medusa), the brain is absent, the brain function being discharged by the nerves themselves, plus certain nerve cells and substances which enable the animal to feel and move voluntarily. The power of feeling and moving which inheres in the nerves and substances of the medusa does not disappear in the sensory and motor nerves of the higher animals when a brain is superadded. In that case the original or fundamental arrangement is augmented, amplified, and emphasised. In this connection it is well to bear in mind that aninials feel and move voluntarily in the absence of a nervous system. Similar remarks are to be made of the insectivorous plants. Feeling and moving are, so to speak, focussed and made visible by means of the nervous system and the brain.

As already explained, the rhythms and reflexes are not confined to animals. They occur also in plants. Neither do they require for their manifestation nerves and muscles as found in the higher animals.

When nerves and muscles occur, as in the more advanced complex organisms, they are utilised, but the modus operandi is essentially the same. In rhythms and reflexes two things are present, the thing acting and the thing acted upon, and in both cases movement, at longer or shorter intervals, is the distinguishing feature. The movements, at first direct, become, in certain cases, from constant repetition and habit, to a certain extent indirect or automatic. In rhythms and reflexes, a certain amount of time necessarily elapses during the operations ; the moving substances have to get into and out of action. There is, further, a tendency of the moving parts to continue moving, and of the resting parts to contirue resting, as in non-living ordinary matter. The rhythmic and reflex involuntary movements are illustrated by a reference to the voluntary movements. A sensation travels from the skin and sense organs to the brain, and a volition travels from the brain to the muscles. Time is required for both operations. The sensations and volitions are transmitted at a given and measurable speed in opposite directions. In like manner, the living, feeling thing requires a certain time to reconnoitre and recognise the substance with which it comes in contact or touches, and to determine whether it shall move towards or away from it, or remain passive. Living things act more or less deliberately, and the time intervals are increased according to the degree of deliberation. The direct voluntary movements, and the indirect involuntary rhythmic and reflex movements, are or may be rapid, but they are never quite instantaneous. The distinguishing feature of the involuntary movements is the element of repetition. Voluntary movements may or may not be repeated; involuntary rhythmic and reflex movements generally are.

Plants and animals are endowed at the outset with certain powers and propensities which make them superior to their surroundings, which enable them to take in or reject the matter by which they are invested, and which permit them to realise more or less clearly their places in nature. Plants and animals, as a class, are sentient living entities. They are, in no sense, the sport of the elements, and cannot be regarded as mere machines goaded into activity by accident, irritability, or extraneous stimulation.

If a muscle shrinks, contracts, or shortens on being pricked or artificially stimulated, it does not follow that this is its natural or normal mode of action, or that the same result cannot be obtained in the living animal in the absence of stimulation. The heart and the involuntary muscles, as a class, require no stimulation. The voluntary muscles are set in motion by volitions; volitions themselves being, with few exceptions, the outcome of spon- 
taneous intrinsic molecular changes in the brain and nerves, which materially differ from the changes produced by the crude, artificial stimuli of the modern, mechanical school of physiology. Volitions, strictly speaking, are traceable to living, sentient, conscious matter asserting itself directly by means of its nerves, muscles, and other structures placed at its disposal by the First Cause to accomplish certain ends. Volitions are spontaneous, independent acts. They require no prompting, no motive, and least of all any extraneous stimulation or external jogging.

The mind, according to the majority of psychologists, is at liberty to act as it please. Those who espouse the doctrine of "Free Will" maintain that they can do what they like in their own way and at their own time. J'hey are not obliged to act in obedience to certain movements and motives which they can altogether ignore and disregard. The doctrine of "Free Will" has been challenged by those who advocate the doctrine of "Necessity," and who are known as "Necessitarians."

The Necessitarians assert that as everything in the universe is under law and order and conditioned, so the operations of the mind are not free, but are limited and pre-determined, and that intellectual man, in common with everything, organic and inorganic, can only perform a certain rôle. The Necessitarians have certain facts and analogies in their favour, but it cannot be denied that deep in man's nature a something pleads strongly for the recognition and exercise of Free Will.

An attempt has been made to reconcile the opposing doctrines by falling back on the omniscience of the Creator, Who has made everything that is made, and Who knows everything which has happened, is happening, or can happen. To such a being there can be no such thing as "Free Will" and "Necessity" in the sense in which these terms are commonly employed. Free Will and Necessity are human conceptions, and apply to limited intelligences. The mind, while apparently free in the opinion of certain individuals, is nevertheless under restraints which rob it of much of its supposed liberty of action. Man, in exercising his intellect, as he thinks independently, has behind him an inscrutable power which unerringly guides him in spite of himself to do some things and leave others undone. The exercise of his intellect is not wholly under his control. To the prescient mind, the doctrines of Free Will and Necessity are not altogether incompatible. If certain things are fated to occur they will occur, Free Will and Necessity notwithstanding. The theologians have had much to do with bringing the doctrines of Free Will and Necessity into prominence, for, say they, if all our actions are pre-determined we are not responsible agents, and cannot be punished for doing what we could not avoid doing. There is no place for either punishment or reward under such circumstances. On the other hand, if we are free to act as we please, and to do or leave undone certain acts, then and then only does man become a responsible agent. $\mathrm{He}$ is then a fitting subject for both punishment and reward. The subjects of Free Will and Necessity do not, strictly speaking, fall within the scope of the present work, and need not be further discussed.

If a part of a plant or animal dies or is lopped off, by so much is the life of the plant and animal impaired, and its usefulness diminished. It is not permissible, even in the highest animals, to speak of any structure or function exclusively by itself. Every part of a living animal is essential to its integrity and well-being. This is true even of the extra-vascular tissues, epidermis, hairs, nails, horns, hoofs, \&c. The parts of the most highly differentiated plants and animals are all united structurally and functionally, and it is never quite safe to isolate either structure or function. From the beginning it is so. In the embryo of plants and animals there is community of structure and function: development and growth necessitate this. Whatever differentiation occurs later on in the shape of special organs does not destroy the community of structure and function in the widest sense : it merely adds variety. Division of labour in an organism does not alter the fundamental nature of labour. Thus protoplasm and muscle are both endowed with centripetal and centrifugal movements, and plants and animals (without and with nerves) feel. Movement and feeling are fundamental endowments, and extend to both plants and animals; and the fact that movement and feeling are more localised and emphasised in animals than in plants, and in the higher forms of both, does not abrogate the prerogatives common to all. It is on this common ground of structure and function that the lower and higher forms of plants and animals meet, for it cannot be maintained that the higher plants are better adapted for the work they are called upon to perform than the lower ones, or that animals are better equipped for the rôle they have to play than plants. Each living plant and animal is to be regarded as perfect within its sphere; the Designer and the designed cannot be separated from each other, and error cannot consistently be attributed to the Deity or Great First Cause.

In order to keep this inorganic kingdom going, plants and animals are of necessity endowed with the power of moving in whole and in part.

Desmids, and the young of many plants, move freely about in water for the most part by the aid of vibratile processes or cilia, and all plants circulate their nutritious juices. Some plants, moreover, such as the Sensitive and Insectivorous ones, move in parts and to given ends. They also feel. Animals one and all are endowed with the power of moving. This is true of the amoba, and all intermediate animals, up to man. Movements are of 
various kinds. They may be voluntary or involuntary, or partly the one and partly the other. They are voluntary when volition or its representative comes into operation, as when an animal with or without a nervous system, as we know it, throws itself upon food; they are involuntary in the established rhythms and reflexes, as witnessed in the lungs, alimentary canal, and heart; and they are mixed, as in swallowing, defæcation, urination, parturition, \&c. The involuntary rhythms and reflexes are originally the products of protoplasm and rudimentary structures.

They are conferred for the wisest of purposes, namely, for the appropriation and assimilation of food, for growth, reproduction, and the like. It is a significant fact that the involuntary rhythms and so-called reflexes of plants and animals precede and are the parents of what may be designated the voluntary rhythms, as they occur in the voluntary muscles. Curiously enough, the latter, in many cases, after frequent repetition and from the force of habit, merge into the former. There is a give and take as between the voluntary and involuntary muscles and movements. Thus walking, which, at the outset, is a voluntary movement acquired by laborious effort, becomes, after a time, automatic. The same is to be said of musical and other performances where habit and unconscious cerebration are at work.

Of course it may be maintained that rhythms and reflexes, however produced originally, are more or less automatic. This, within limits, is true; but it is important to point out that, at the outset, they are, in every instance, the product of lowly substances and organisms, which act directly or at first hand-that is, as apart from irritation, stimulation, and every form of circumlocation. The rhythms and reflexes, as a matter of fact, under all circumstances, retain much of their spontaneity and power of independent action. Repetition and habit deepen, rather than account for, their original peculiarities. As illustrations of natural normal actions at first hand as compared with unnatural abnormal action at second hand I may mention the following. If a piece of dry bread falls or is suddenly drawn into the fauces, it is at once forcibly ejected. No such result follows if the bread be insalivated and swallowed in the ordinary way. If a strong aperient be administered, the alimentary tract is thrown into violent action, often accompanied with griping pain, and the bowel forcibly and quickly evacuated. No such phenomena occur in natural alimentation and defæcation. If a diuretic be given, the amount of urine is increased and frequent micturition follows. The drug stimulates the secretion and expulsion of urine but causes neither. In like manner, ergot of rye increases but does not cause the rhythmic action of the uterus in parturition. The effect of stimuli is to quicken and increase normal action. Artificial stimuli, if continuously applied, destroy the tolerating, accommodating, and retaining power of the viscera. If by any chance the fauces, alimentary canal, bladder, and uterus be inflamed (and irritable as a consequence) the unnatural results obtained by stimulation are exaggerated.

The movements of living things are to be regarded as the result of inherent endowments : they are means to ends, and the outcome of Design. They are part of the equipment of life, and cannot be traced to irritability of constitution or external stimulation.

Life without rhythms and reflexes could not manifest itself, could not adequately perform its various functions. It is no explanation of these functions to say that the substances and structures which produce them are so formed that a stimulus applied to them causes them to work perfectly; as in the several compartments of the heart, which open and close alternately. The opening and closing movements are diametrically opposed to each other, and no single stimulus could effect the double and opposite result.

The same may be said of light entering the eye, which it is claimed produces the opening and closing of the eyelids in winking. If light be the stimulus which causes the eyelids to close, it cannot possibly at the same time cause them to open. The double movement can only be explained by original endowment.

All plants and animals are fitted by nature to do certain things: they are superior to their surroundings; they take in and retain certain elements and reject others; they act upon their surroundings, near and remote; the surroundings in turn acting upon them to the extent of calling forth new powers, which result in modifications to meet altered circumstances. The modifications, however, are produced by the plants and animals themselves, and are in no sense accidental. They are not the result of mere extraneous stimulation. If plants develop thick rinds and thick leaves in tropical, dry climates, it is to prevent the evaporation of the juices on which their life depends. If animals in the arctic regions develop layers of blubber under the skin and thick furs over it, it is for the purpose of preventing the escape of bodily heat; this being necessary to their comfort and even their existence. The mere presence of heat or cold, as such, cannot be accredited with the production of the thick rinds and leaves of plants, or the blubber and furry coats of animals. These have their origin in the original endowments and powers of plants and animals respectively. Slight changes and modifications in plants and animals, considering the unstable nature of the physical universe which supplies them with food and a home, were inevitable, but these were foreseen and provided for, and plants and animals have, within themselves, the wherewithal to modify all or any of 
their parts, up to a point, and within limits ; they cannot, however, produce new parts. I say within limits, for no satisfactory proof has yet been adduced to show that plants and animals can modify themselves indefinitely, or that they can merge into each other by insensible gradations, and be all traced back to a common protoplasm or parent. Plants and animals cannot alter their original constitutions; they have their boundaries in time and space which they may not outrun and survive. We cannot add a cubit to our stature by willing or wishing. The laws of growth and the amount of growth and modification are regulated and determined in each individual case, a remark which applies even to the amount and length of hair on the head and beard of man himself.

\section{$\S 53$. Rhythmic Muscles-Rhythms not Confined to Involuntary Muscles.}

The rhythmic muscles, as a rule, have their fibres running in two or more directions. In the simplest arrangements they consist of two sets, and run at nearly right angles, as seen in the small and large intestine, in the small arteries, and in the iris of the eye, where they are spoken of as longitudinal and circular. Strictly speaking, the circular fibres consist of two sets of very oblique spiral fibres crossing each other at very acute angles. In the smaller arteries, the circular muscular fibres preponderate. In the respiratory muscles, especially the serrati, the muscular fibres run obliquely and cross each other at obtuse angles. In the œesophagus, stomach, bladder, uterus, and heart, the number of muscular fibres, and the variety of directions pursued by them, are increased. Thus in the cesophagus, five or more sets of muscular fibres having varying directions can readily be made out; while in the stomach, bladder, and ventricles of the higher vertebrate hearts (bird and mammal), the number is increased to seven. The peculiarity of the rhythmic muscles is, that they do not work singly or in sets but collectively and consentaneously. This follows, because while the muscular fibres pursue different directions, and overlap and cross in layers, they are geared to and connected with each other by muscular slips, blood-vessels, nerves, lymphatics, cellular and other tissues.

The different sets of muscular fibres, crossing as they do in every direction and forming beautiful networks, secure the greatest amount of strength with the least possible material. The hollow viscera, as far as their muscular fibres are concerned, are constructed on strictly mathematical principles.

In the case of rhythmic muscles strength and efficiency of movement are the chief desiderata, and these are obtained not by the several sets of muscular fibres acting separately and opposing each other, but by their acting simultaneously and in harmony, and by their performing co-ordinated centripetal and centrifugal movements.

Rhythmic muscles are, in every instance, involuntary, fundamental structures: that is, they work independently of the will, and are necessary to the life and well-being of the individual. They are, in some senses, more important than the voluntary muscles. Their function is to take in, retain, discharge, and circulate the air, fluids, semi-solids, and solids on which, and by which, animals live. Thus the chest muscles, aided by the lungs, take in, retain, and circulate pure and discharge impure air : the œsophagus, stomach, intestine, and rectum take in, retain, and circulate food and drink, and discharge the effete or waste products thereof: the bladder takes in, retains, and discharges urine at stated intervals : the uterus takes in, retains, and discharges, at the full period, the fully matured foetus; and the heart takes in, retains, and discharges, with unfailing regularity and in given quantities, the blood which it is especially designed to propel. The peculiarity of the hollow viscera as a class is, that they retain as well as circulate the substances with which they deal, whether these be gases, liquids, semi-solids, or solids. This distinction is, in some respects, very important, as it shows that the contained substances cannot possibly act as irritants.

The rhythmic muscles (as more than once stated) are endowed with spontaneous, centripetal, and centrifugal movements: in other words, the contents of these muscles do not act as irritants or artificial stimuli and produce the movements in question. This seems quite certain, and for the following reason. If the contents caused the centripetal closing or expelling movements, they could not consistently cause the centrifugal opening movements, which are diametrically opposite movements. Moreover, the structures exhibiting rhythmic movements, as explained, are retaining as well as receiving and discharging structures. The structures in question do not eject their contents the instant they are received. On the contrary, some of them retain their contents for seconds, others for hours or days or months. The ventricles of the adult vertebrate heart, for example, retain their contents only until a full measure of blood is received; the blood being discharged into the lungs and the system from sixty to eighty times per minute. The stomach retains the food for one, or more hours, until it is converted into chyme: the bladder collects and retains the urine from three to six hours or longer : the lower bowel or rectum collects and retains the fæces for twenty-four hours or thereby; and the uterus retains its living contents for periods varying from three weeks to twenty months. 
The following table furnishes some interesting information regarding the periods of gestation in mammals :PERIODS OF UTERO-GESTATION IN ANIMALS

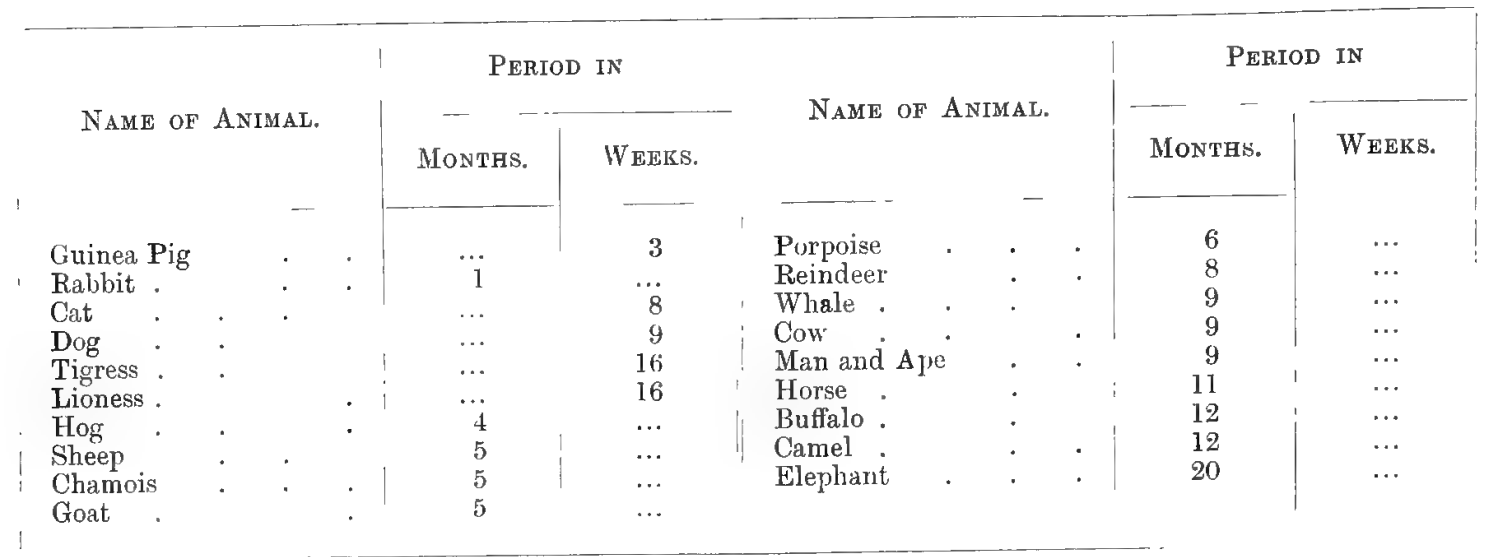

The alimentary canal receives, retains, and passes on the food in relays systematically and at stated intervals; the chest draws the air into the lungs, and keeps it there, for a sufficiently long period to admit of the aëration of the blood, after which it is expelled; the bladder collects and retains the urine for variable periods without discomfort; the uterus receives the ovum, which develops into the embryo and fœtus, and retains them for periods varying from a few weeks up to over a year. Lastly, the several compartments of the heart receive and retain their respective quotients of blood, which are duly discharged either into other compartments or into the great blood-vessels (pulmonary artery and aorta). The several compartments of the heart do not discharge the blood which flows into them the instant it enters. On the contrary, the blood is allowed to collect, and, when a full complement has been received, and not till then, is it discharged. It is, moreover, not discharged until a free space is provided for its reception. All this means prevision and design. When the auricles close and perform their centripetal expelling movements, the ventricles open and perform their centrifugal sucking movements : when the ventricles have drawn full measures of blood into their interior by their centrifugal movements, they close and perform their centripetal expelling movements. The centripetal and centrifugal movements of the auricles and ventricles of the heart are vital in their nature.

The compartments of the heart of the bird and mammal act in pairs, and provide vis a fronte and vis a tergo movements ; the auricles opening, soliciting, and sucking in blood when the ventricles close, discharge, and expel it. The closing movements of the auricles do not occasion the opening movements of the ventricles, or vice versit. The auricles have no power to open the ventricles, or the ventricles the auricles. Each compartment of the heart acts spontaneously and independently, but the movements of the several compartments are co-ordinated, and all act to a given end, namely, the reception, retention, and propulsion of the blood.

In the movements of the heart the elastic properties of the cardiac muscles and the elastic recoil of the great vessels play quite a subordinate part. The muscles of the heart are the prime movers of the blood. The blood cannot cause its own expulsion from one compartment of the heart into another : neither can it cause its own transmission through the arteries, veins, and capillaries. As regards the movements of the heart in the adult, they go on when the organ is cut out of the body, deprived of its blood, and placed in an exhausted receiver-that is, when the heart is deprived of blood and air. This gets rid of inherent irritability and extraneous stimulation as factors in the movements of the heart. That the presence and pressure of blood is not necessary to the movements of the heart is proved by this, that its several compartments npen and close and beat in young animals before they contain blood. Of late years many are inclined to believe that the movements of the heart are due wholly or in part to rhythmic nerve action. This view is negatived by the fact that the heart displays rhythmic movements before nerves are developed in it, and even before it contains muscular fibres. A living muscular fibre, moreover, shortens and elongates when deprived of its nervous supply; all which goes to prove that the movements of the rhythmic muscles are inherent, fundamental, and spontaneous.

While it would be obviously incorrect to deny an inherent power of moving to rhythmic muscles, it would be equally incorrect to say that nerve cells, ganglia, and nerves cannot act rhythmically. The very intimate connection which obtains between muscles and nerves forbids such a conclusion. The force of habit alone, in nerves supplied to rhythmic muscles, would, there is reason to believe, sooner or later, result in rhythmic or interrupted nerve action. 
That nerves influence voluntary and also involuntary muscles is beyond doubt. The voluntary muscles are directly under nerve control. The involuntary rhythmic muscles are likewise, within limits, amenable to nerve action. Thus in the phenomenon known as blushing, a rude remark or suggestion acting on the cerebro-spinal and vaso-motor nervous system causes the arterioles to open or relax, with the result that the capillary bloodvessels become gorged with blood and produce a temporary redness of the skin. Alarm, or fear, or shock, produces an opposite result, by reducing the size of the arterioles and temporarily depleting the capillaries of blood, which induces paleness of the skin. The movements of the heart can also be quickened and slowed by the division and irritation of certain nerves. Notwithstanding the very intimate relation which exists between involuntary muscles and nerves, it is safe to conclude that the rhythmic muscles can, and do, act either with, or without, nerves. The major portion of the nerves going to the involuntary rhythmic muscles are sympathetic ganglionic nerves, and there is every reason to believe that these are endowed with independent rhythmic powers. The sympathetic ganglionic nerves are primary or fundamental, and take precedence of the cerebro-spinal nerves in the order of development. They carry on the vegetative functions of the body independently of, or in conjunction with, the spinal cord. This they do, even in the absence of a brain, as in the anencephalous fœetus. A brain is not necessary to the performance of voluntary movements in certain of the lower animals, as, for example, the medusa and fiverayed star-fish. As I have explained elsewhere, ${ }^{1}$ the involuntary or rhythmic muscles are to be regarded as the forerunners or parents of the voluntary ones: indeed the movements of even the voluntary muscles are essentially rhythmic in character. Sir James Paget endeavoured to explain that the rhythmic action of muscles was due to rhythrnic nutrition, but it is difficult to understand how muscles which are, in a sense, continually in action can be fed at stated intervals; the more especially as the blood or nutritive fluid is constantly circulating through their substance. As the several rhythmic structures referred to are all equally well nourished, it is evident that hypothetical rhythmic nutrition cannot be the real or sole cause of rhythmic movements. In other words, the supply of blood being continuous, it is reasonable to infer that the nourishing process is practically continuous, and not interrupted or rhythmic.

Development and comparative anatomy distinctly point to an inherent rhythmic power in rhythmic structures as apart from nutrition, and even as apart from muscles and nerves and every form of irritability and artificial stimulation. This follows because, as explained, the rhythmic structures are being continuously fed; because the heart opens and closes before it contains blood, muscle, or nerve, and because in plants (in Volvox globator, for example), where neither blood, muscle, nor nerves are present, well-defined rhythmic movements occur.

While rhythmic movements are to be regarded as spontaneous, inherent, and fundamental endowments on which the life and well-being of the individual depends, it is well to bear in mind that in the higher animals, where muscles and nerves are present, the movements can in part be traced to the muscles, and an intimate relation between the muscles and nerves established.

In the higher organisms, the muscles and nerves act together, and both display rhythmic movements or impulses. It is not possible to distinguish with any degree of precision as between the rhythms in muscles and nerves. As differentiation proceeds, and structure is added to structure, the added structures in every instance conform to a general plan and the carrying out of a common object. In other words, added structures do not wage war with pre-existing structures. On the contrary, all the structures, whether developed late or early, work harmoniously together and to a given end. But for design and preconcerted action a compound organism would be impossible. The rise, progress, and completion of the higher animals all proclaim design and a First Cause. The higher animals are to be regarded as completed wholes, the integral parts of which are essential and necessary. For this, if for no other reason, it is never safe to rely on experiments which have for their object the isolation of parts, organs, and systems. In like manner, the division and irritation of nerve structures, and the artificial stimulation of muscles, cannot possibly yield quite normal results. Whatever disturbs the natural balance of an animal inevitably leads to a greater or less degree of abnormal action. Thus the administration of drugs, of chloroform and other anæusthetics, of poisons, \&c., tends to abnormal action; the abnormality being greatly increased where coma and nervous shock are produced.

A violent aperient gives an exaggerated idea of the rhythmic activity of the alimentary canal: an emetic reverses the action of the cesophagus and stomach : strychnine and the injury of certain parts induce tetanus and convulsions : chloroform destroys sensation and renders muscles flaccid : excessive tea drinking produces indigestion and tumultuous action of the heart: excessive stimulation by alcohol results in great general excitement and mental disturbance, and, if long continued, begets coma, collapse, and death. The administration of opium, Indian hemp, \&c., leads to a dreamy condition of the brain and nervous system. The greater the liberty taken with an organism the less reliable are the results obtained by experiment. The common practice of irritating, stimulating, 
cutting, and dividing muscles and nerves and other structures wholesale is never wholly satisfactory. Similarly, the recording of movements, normal and abnormal, by complicated registering apparatus is liable to error. To get quite natural results animals must be studied in their entirety and in their natural condition, and the changes and movements occurring in them recorded, if possible, by instantaneous photography. The simpler animals yield the best results, and amongst them the transparent and semi-transparent animals are the most satisfactory. To obtain normal results, animals must be studied under normal conditions. The extensive mutilation of highly complex, highly sensitive animals, and the registration of movements so produced by graphic methods, have introduced many serious errors into physiology. The methods employed are, everything considered, extremely crude, and the results obtained are, with few exceptions, equally disappointing and misleading. The time, it appears to me, has now arrived for seriously questioning the doings of the mechanical school of physiology.

\section{§54. Respiratory Rhythmic Movements in Animais-New Explanation of these Movements.}

The observations on rhythmic muscles made in the last section (\$ 53) will prepare the reader for what has now to be said regarding the respiratory movements in plants and animals, especially the latter.

The respiratory rhythmic movements in animals are typical of their kind, and in some respects the most important in the body. They begin as soon as the act of parturition is completed and continue as long as life lasts. Any interruption to them for more than a few minutes is almost invariably fatal.

They are vital, fundamental, involuntary, co-ordinated movements - that is, they form part of a living system which, if interfered with to any extent, results in death.

The other great organic rhythms are those of alimentation, the circulation, reproduction, \&c.

These rhythms are coeval with life. The structures which produce them are specially formed during the development and growth of the individual-they are designed and prepared beforehand, and the work to be performed by them is taken up when the proper time arrives. In plants, the fundamental rhythmic movements are carried on in the absence of both muscles and nerves, and it is important to bear this in mind, as one is apt to imagine that muscles and nerves are necessary to the production of rhythmic movements. When muscles and nerves are present the rhythms inhere in the muscles rather than in the nerves and nerve centres; the nerves in the higher animals being necessary to co-ordinate the several systems of rhythms in the several individuals. They cannot be said to occasion them. The nerves, within limits, quicken or slow the rhythms, but that is the most that can be said. ${ }^{1}$

The nerves exercise an influence on the nutrition of the structures in which the rhythms occur. But when all has been said, rhythms in plants and animals are fundamental. They occur, as already stated, in the absence of and as apart from nerves. They are not even referable to nutrition, as the rhythmic structures of plants and animals are not known to feed at certain intervals.

Rhythms in the higher animals are bound up with life, inasmuch as the cessation of any one of the more important of them involves the death of the individual.

They are fundamental, because they are provided for in the constitution and substance of the individual.

The rhythms afford examples of co-ordinated movements, as they are produced by the consentaneous action of a great many parts, and, in the higher animals, by a great many muscular fibres or muscles running in various directions and, in some cases, arranged in several planes. This is especially true of the heart and of the muscles of respiration, where the diaphragm, the thoracic, abdominal, and other muscles are involved. They are involuntary because in the higher animals and in ourselves they go on day and night, waking and sleeping, from the cradle to the grave, without effort on our part. They cannot be controlled (unless for a very brief period) by even the strongestwilled and most resolute individuals. A time comes, and comes quickly, when, if the respiratory movements are suppressed, the want of breath becomes intolerable.

An interesting comparison may be drawn as between the cardiac and respiratory muscles in man. The only point in which the muscular arrangements of the ventricles of the heart differ from those of the chest, abdomen, and diaphragm, is that the muscles of the latter are attached to bones: those of the former having no such attachments and being continuous upon themselves. This really makes no difference; the opening and closing movements of the heart, chest, \&c., being essentially the same.

The chest may be likened to the systematic heart of the mammal, which consists of an auricle and ventricle

${ }^{1}$ It is not usual to sjeak of a rhythmic action in nerves, but there are several circumstances which favour this view. Division of the inferior laryngeal branches of the pneumo-gastric nerves, for example, arrests the movements of the glottis and seriously interferes with respiration. If it
be admitted that interrupted or rhythmic movenents are referable to certain nerve centres and nerves, then it follows that the said centres and
nerves must exert an internittent or rhythming nerves must exert an intermittent or rhythmic action. The origin of rhythms, which I regard as vital and fumdamental in the lowest plant and in the highest animal, is, however, not satisfactorily explained hy this hypothesis. On the whole I incline to the opinion that rhythns are inherent
in living matter - plant and aninal-as apart from nerves. Flyythms certainly oceur in plants and in structures where no nerves exist. 


\section{RESPIRATORY RHYTHMIC MOVEMENTS IN ANIMALS}

with a movable partition (the mitral valve) between them. The thorax may be taken to represent the left auricle, the abdomen the left ventricle, and the diaphragm the mitral valve. The cardiac muscular movements and respiratory movements are rhythmical and alternating; the one cavity opening when the other is closing and vice versa. Thus in the chest the thoracic cavity opens when the abdominal cavity closes, and the converse. These diametrically opposite movements in the thorax and abdomen occur simultaneously. In both the opening and closing movements the diaphragm plays an important part. Thus it descends when the thorax opens, and ascends when it closes. It in this way alternately increases and decreases the capacity of both cavities. The mitral valve of the left ventricle in like manner descends and ascends and alternately increases and diminishes the size of the left auricle and ventricle. The movements of the diaphragm are rhythmic, and accurately adapted to those of the chest and abdomen.

The glottis, situated within the larynx, also takes part in the rhythmic respiratory movements. From this it will be seen that there are four different rhythmic movements connected with respiration in animals, namely, those of the glottis, chest, abdomen, and diaphragm. The respiratory movements, although apparently simple, are highly complex. They have unfortunately never received the attention they deserved. It is therefore fitting that in the present section I should describe the respiratory rhythms somewhat in detail, illustrating what I have to say by carefully selected figures. I consequently, in this connection, give views of the lungs, the larynx, the bronchial tubes, the glottis, the bones and cartilages of the chest, the thoracic and abdominal muscles (esprecially the recti abdominis), the diaphragm, the appearance presented by the body in inspiration and expiration, \&c.

Much of the confusion connected with the respiratory rhythmic movements is traceable to authors failing to grasp the fact that the several rhythms occurring in the glottis, chest, abdomen, and diaphragm are only parts of one great rhythm which may be aptly designated the respiratory rhythm. The confusion is also partly due to anatomists assigning separate movements to individual muscles which can only act in groups or as wholes. As it is not possible to define the action of the muscles in any part of the heart, neither is it possible to define with adequate precision the action of separate muscles in any part of the chest, abdomen, and diaphragm. The chest, abdomen, and diaphragm exercise opening or centrifugal, and closing or centripetal movements precisely analogous to those witnessed in the heart. Lastly, physiologists have never fully realised the inherent nature of the rhythmic respiratory movements, and have referred them directly or indirectly to irritability and extraneous stimulation. They have overlooked the fact that the rhythmic movements are fundamental, spontaneous, and independent-the one movement never causing the other; that the opening centrifugal and the closing centripetal movements are both vital in character, and that elasticity plays quite a subordinate part in respiration. At present there is a prevailing belief that the several compartments of the heart are closed by vital acts; the opening of the compartments being largely due to the elasticity of the muscles which act passively.

Similarly but conversely, the chest is said to be opened by vital movements, the closure being effected passively by the resiliency and elasticity of the muscles and ribs.

Why, one naturally inquires, should the heart be closed by vital movements and the chest opened by them? The contradiction here pointed out demolishes the prevailing theory of muscular action not only in the hollow viscera, but also in the voluntary muscles. The opening and closing of the hollow muscles forming the viscera, and the contraction and relaxation of the voluntary muscles, can only be performed by independent vital acts: mere elasticity cannot be said to influence the action of muscles to any appreciable extent. Up till the present the causes of the rhythmic movements, particularly the respiratory ones, have remained a close secret.

A curious point to be noted in connection with rhythmic movements is this. They occur in the protoplasm of plants and in both kinds of muscles, namely, the pale or unstriated involuntary muscles and the red or striated voluntary muscles of animals.

The explanation is that, strictly speaking, no real distinction (the power of the will over the voluntary muscles excepted) can be drawn between the involuntary and voluntary muscles, either structurally or functionally. The one runs into the other. The voluntary muscle, as I showed in $1872,{ }^{1}$ is a higher development or differentiation of the involuntary muscle, and it can be made to act either with or without the aid of intelligence. A pedestrian can walk either automatically or intelligently, and a clever pianist can perform the most difficult music and keep up a conversation altogether disregarding the movements of the fingers engaged. A lunatic not unfrequently sets up rhythms in his limbs or other parts of his body, and produces a series of grotesque see-saw movements which he continues for hours.

The weaving movements of animals are to be placed in the same category. Some people known to me slowly flex and extend their inferior extremities during sleep. In all cases where the voluntary muscles act involuntarily

1 "The Physiology of the Circulation in Plants, in the Lower Animals, and in Man." Edinburgh Mertical Journal, 1872 , 1873. Also separate volume published 1874 by Macmillan with 250 woodcuts (see pp. 208-214).

VOJ. I. 
or automatically there is a reversion to the primitive type of movement as it occurs in the respiratory, circulatory, alimentary, and reproductive rhythms. Whether movements are voluntary or involuntary is largely a question of habit, repetition, and training, in which the nervous system plays a not unimportant part.

The muscular fibres of the stomach, bladder, and uterus are of the pale unstriated order. The movements of these viscera are strictly involuntary; but (and this is remarkable) the heart, which is a typical involuntary muscle as regards its rhythmic movements, is composed of red striated muscular fibres. In the case of the respiratory muscles they are partly of one kind and partly of the other. Thus the muscular fibres investing the trachea and bronchial tubes are of the pale unstriated sort, whereas those of the diaphragm, the thorax, and the abdomen and other muscles concerned are of the red striated, voluntary kind. The respiratory movements produced partly by pale unstriated and partly by red striated muscles are, however, typical rhythmic movements over which, as explained, we have the slenderest possible control, and only for a very limited period. In other words, rhythmic movements are not peculiar to either kind of muscle; a fact which in itself goes far to break down the arbitrary but convenient distinction between the voluntary and involuntary system of muscles. (The peculiarities of the voluntary and involuntary muscles, and their precise mode of action, are illustrated at Plate lxxxiii., p. 320.)

These preliminary observations are necessary in speaking of the respiratory rhythmic movements, as much ignorance and misapprehension prevails as to their precise nature and the number of the hard and soft parts, especially muscles, which take part in them.

The object of respiration in every instance is the interchange of gases. In plants and in the lowest animal forms that interchange takes place in a very simple way, and without the intervention of any special apparatus. The plant is enveloped in air, and air mingles and circulates with its juices throughout its entire substance. During the day the plant takes in the carbonic acid of the atmosphere and gives out oxygen.

The amoba, which may be taken as an example of the lowest animals, is similarly circumstanced. It is invested by air or air and water, and being molecular and largely structureless the air or the oxygen of the air finds easy passage through its substance. It takes in oxygen and gives out carbonic acid. The same holds true of the skin and mucous linings and all the tissues of the body even in the highest animals. It is only when hearts and a system of blood-vessels containing blood or nutritious juices make their appearance that distinct respiratory organs become necessary. In the higher animals (bird and mammal) the connection between the respiratory organs and the circulation becomes very evident; the lungs being provided with a heart for themselves, known as the right or pulmonic heart, as contra-distinguished from the left or systemic heart-the right heart forcing the blood through the lungs, the left heart forcing it through the system generally.

\section{§55. The Respiratory Organs in Animals and in Man Structurally Considered.}

The respiratory organs are called gills and lungs respectively. They are designated gills in the fish, the water lizards, \&c., and lungs in the reptile, bird, and mammal.

\section{PLATE LXXYII}

Plate lxxvii. illustrates the structure and position of the human lung within the thorax : also the narrow chink or slit in the larynx through which the air passes in respiration, and the position and mode of action of the vocal chords. It also shows the structure of the lung of the fish, menobranchus, and frog.

FiG. 1,-Air lobule of the human lung (Homo sapiens), divided by air cells into numerous separate compartments, Everywhere the air cells are covered with a profusion of delicate cajpillaries, the venous blood within which gives off carbonic acid, which mixes with the air contained within the air cells. By this arrangement the blood is largely depleted of its carbonic acid and has oxygen added to itentering air lobule $(b) ; c, c, c, c$, air cells (after Dalton). gases constitutes the essential part of respiration. $a$, Small bronchial tube

FIG. 2.-Larynx, glottis, trachea, bronchial tubes, and air cells of human lung (after Dalton).

Frg. 3.-Lung of the frog (Rum(u esculent frog is aërated as in man. When the frog is immersed its porous thin skin assists in the process of respiration (alter Dalton).

FIG. 4. Gills of a dipnoid fish (Ceratodus). Affords a good example of the outside or water-breathing lung. $a$, Arcus aortze; $b$, gills containing rows of capillary blood-vessels; $c$, psendo-branchia; $d, d^{\prime}$, two series of gill-rakers belonging to the pseudo-branchia;
$e$, ceratohyal; $f$, glossohyal (after Günther).

FIG. 5.-Head and gills of the Menobranchus, one of the salamanders. are gently waved to and fro in the water to extract more oxygen from it.

FIG. 6.-Photograph of an antero-posterion diaphragm, liver, and stomach in their natural positions; the thorax and abdomen being separated from each other the lungs, heart, 'The viscera completely occupy the thoracic and abdominal cavities, The heart is situated in the middle of the thorax, and shows how 
PLATE LXXIII

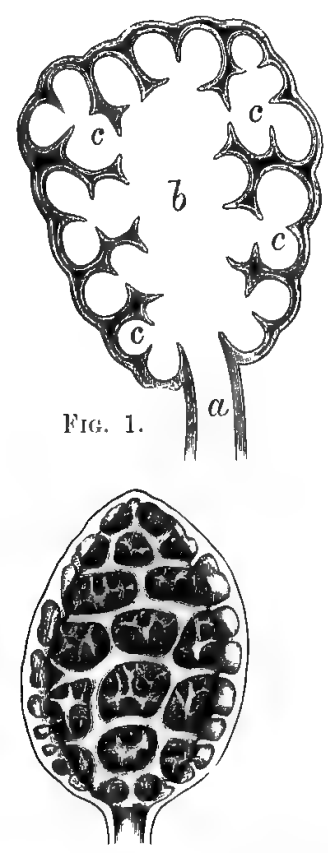

FIi: 3.

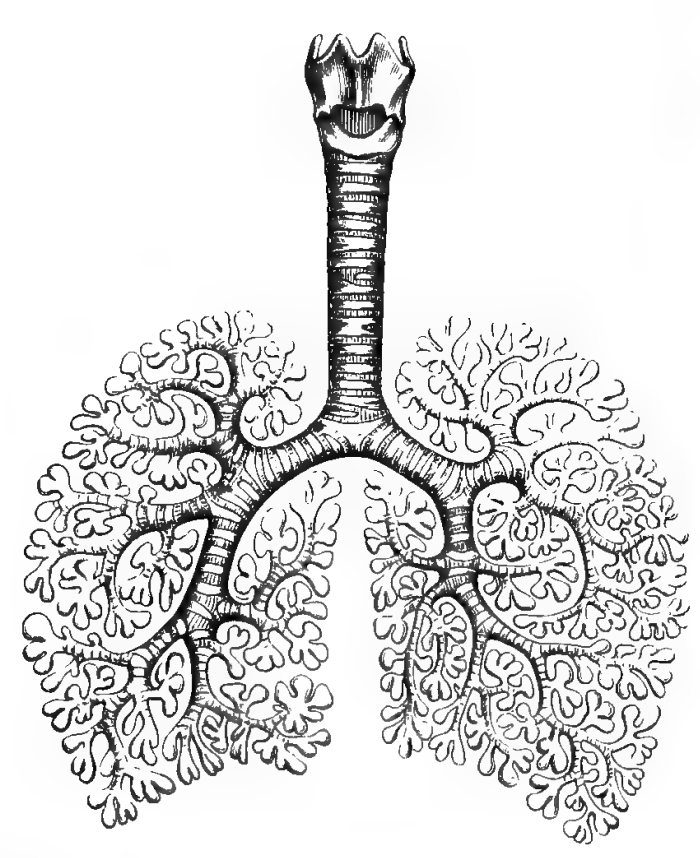

Fig. 2.

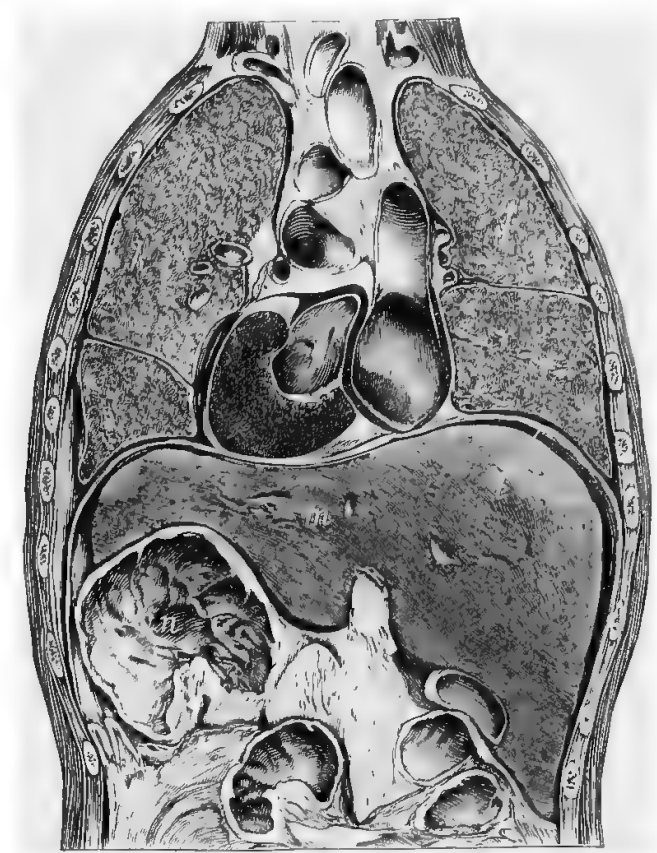

EIG. 6.
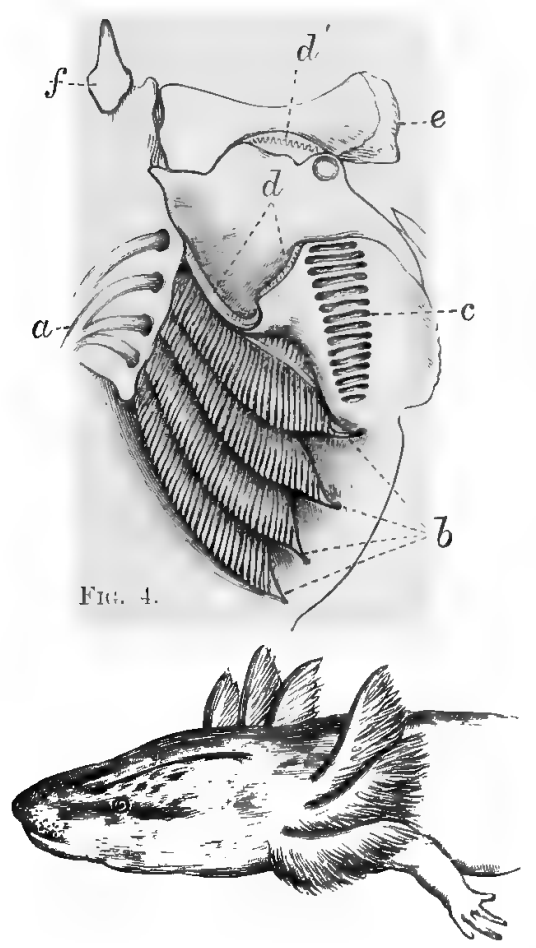

FIG. 5.

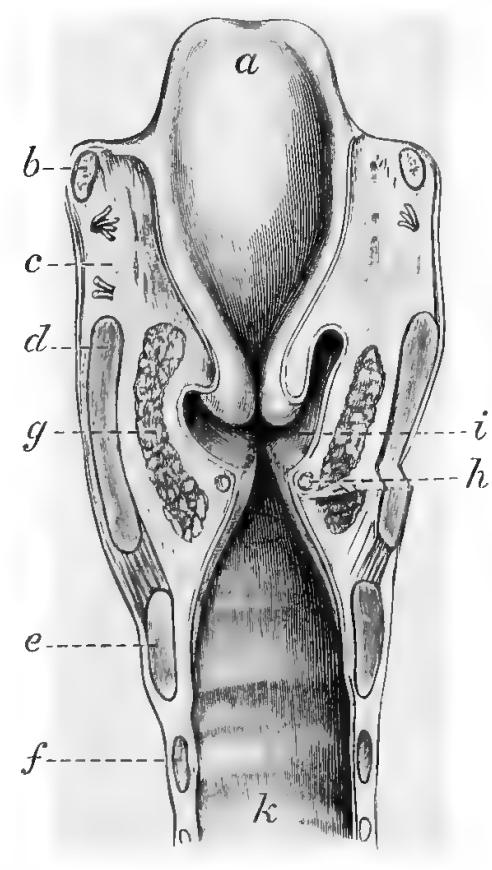

FIG. 7.
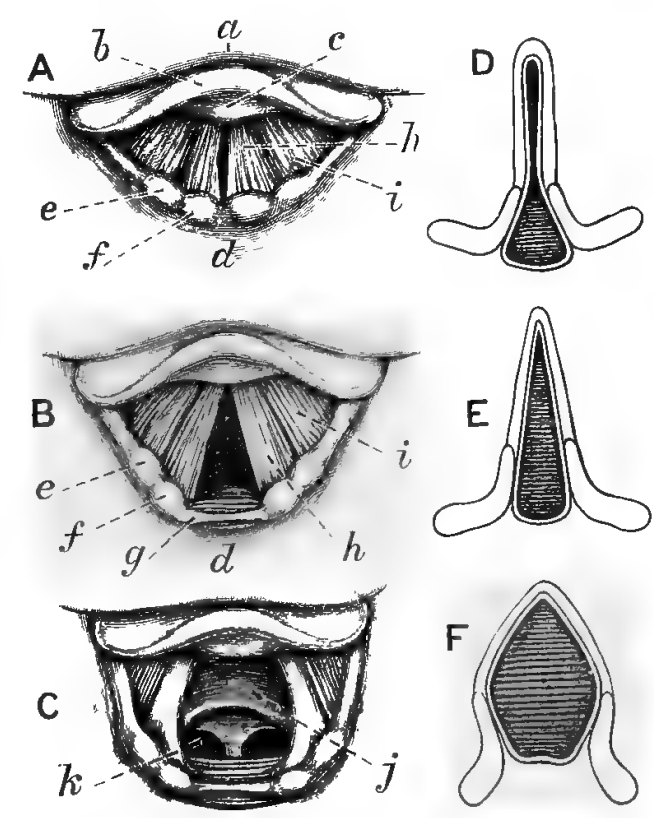

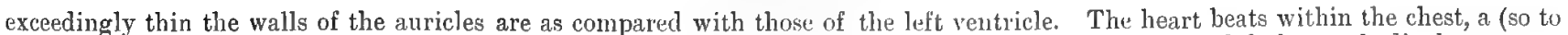

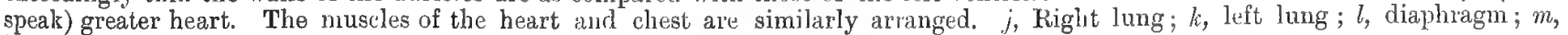
liver; $n$, stomach (the Author, 1873).

Frg. 7.-Anterior hall of section of human larynx neal its middle. Shows the narrow ajerture ol chink (rima giottidis) through

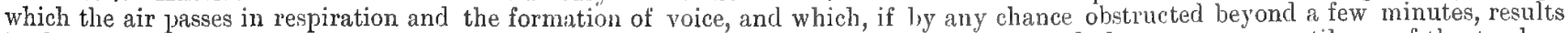

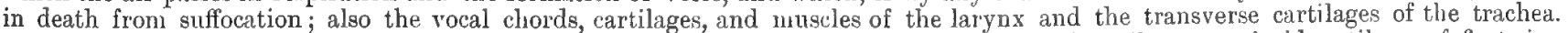

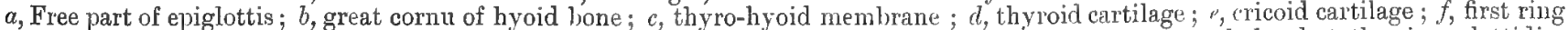

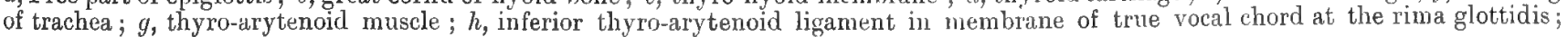




\section{PLATE LXXVII (continued)}

$i$, the ventricle with the false vocal chord above it; $k$, interior of trachea conducting to the lungs. The narrow passage or chink (rima glottidis) through which the air passes during respiration and the formation of the voice is clearly shown (after Thonson).

FIG. 8.-Three laryngoscopic views from life of upjer aperture of larynx, showing vocal chords, glottis, and surrounding parts in different states.

A. Shows the glottis and vocal chords during the enission of a high note in singing. B. In natural inhalation of air. C. In inhaling a verydeep loreath. Diagrams D, E, F, show horizontal sections of glottis, positions of vocal ligaments, and arytenoid cartilages in states corresponding to A, B, C. The same letters apply to the same parts ats in A, B, C (alter Saply).

A, B. $a$, Base of tongue; $b$, upjer free part of epiglottis ; $c$, tubercle or cushion of ditto ; $d$, portion of anterior wall of pharynx behind larynx; $e$, swelling caused by cuneiform cartilage; $f$, ditto corniculum ; $g$, tip of arytenoid cartilages; $h$, inferior or true vocal chords forming lips of rima glottidis or breathing aperture; $i$, superior or false vocal chords with ventricle of larynx between.

C. $j$, anterior wall of receding trachea; $k$, beginning of the two bronchi beyond the bifurcation (after Czermak).

In the fish distinct respiratory rhythmic movements are witnessed. The fish is constantly engaged in apparently swallowing water-this water being made to flow in rhythmic waves over the gills, which consist of a framework on which is arranged a congeries of delicate capillary blood-vessels containing blood. A greater quantity of water, and of the air which is in solution in the water, is thus made to pass over the gills in a given time. As a result more oxygen passes from the air into the blood of the fish, and more carbonic acid out of it than would otherwise be possible.

The menobranchus, one of the water lizards, also displays rhythmic respiratory movements. This curious creature is provided with gills in the shape of six feathery-looking tufted structures, three on each side of the head. These structures are composed of a central portion or midrib with an infinite number of fine capillaries containing blood diverging from it (feather-fashion) on either side. The lizard causes the gills to wave gently backwards and forwards in the water, with the result that a maximum of the oxygen contained in the air in solution in the water is made to pass over the capillaries into the blood and a max.mum of carbonic acid is made to pass out of the blood in the capillaries into the water. The rhythmic movements of the gills, or branchiæ, as they are sometimes called, perform a distinct and useful function.

The frog when developing in the water (tadpole stage) is provided with gills, but when it develops legs and its swimming tail disappears, and it is fitted for a terrestrial existence, its gills are suppressed and true lungs of a simple and primitive type are provided. The frog when it becomes an air-breathing animal develops characteristic respiratory rhythmic movements.

Perhaps the simplest form of lung is that met with in the newt. It consists of a long oval sac which opens by a short single bronchus from a very short trachea. The walls of the sac consist of mucous membrane, epithelium, connective tissue, elastic fibres, pale unstriated nuscular fibres, nerves, blood-vessels, \&c. The blood-vessels which ramify on the sac are so placed that the blood contained in them is aerrated by the oxygen contained in the air, or, as happens occasionally, by the oxygen contained in the air held in solution in water. There is in the newt an arrangement which admits of rhythmic muscular movements in the lung itself.

In the salamanders, which, though air-breathing animals, are aquatic in their habits, the lungs consist of two cylindrical sacs extending nearly the entire length of the body. The air sacs have a smooth internal surface on which may be traced a large number of fine capillary blood-vessels containing blood. The air is forced into the lungs by a swallowing rhythmic movement and discharged at intervals to make room for a fresh supply. By this simple arrangement the oxygen of the atmosphere is transferred to the blood in the capillaries of the lungs, and carbonic acid extracted from it.

The lungs of the frog are more elaborate than those of the newt and salamander because of the rudimentary dissepiments or partitions with which they are supplied, and which enable them to accommodate a comparatively large number of capillary blood-vessels. The honeycomb structure characteristic of higher lungs makes its first appearance in the lungs of the frog. The general structure of the lungs of the frog resembles that of the newt, inasmuch as it contains as an element, pale unstriated muscular fibres capable of conferring independent rhythmic movements on the lungs themselves. The frog, if it takes to the water, must, as is well known, come to the surface ever and anon to breathe, and everybody is familiar with the rhythmic movements of the throat displayed on such occasions.

It is needless to pursue the comparative anatomy of respiration further; suffice it to say that in man there is a pulmonic heart, as contra-distinguished from the systemic heart, an elaborate pair of lungs, and a comparatively very large number of muscles for producing the respiratory movements by which air is taken into and ejected from the chest. (See Plate lxxvii., Figs. 1 to 8.)

The lungs in man are composed of a larynx, a glottis, a trachea, bronchial tubes, air cells, blood-vessels, lymphatics, nerves, muscular fibres of the unstriated type, cilia, glands, epithelium, and a large quantity of elastic tissue.

The walls of the trachea and bronchial tubes are composed of an external membrane consisting of inelastic and elastic tissue and of an internal or mucous membrane. Between the membranes cartilaginous rings occur at 


\section{NEW VIEW OF THE MECHANISM OF RESPIRATION}

intervals. The external membrane, composed largely of inelastic and elastic fibres, is deficient posteriorly. In the space devoid of cartilage two layers of unstriated involuntary muscular fibres occur-a thin, longitudina], external layer and a thick, deeper, transverse or circular layer. The longitudinal layer is confined to the trachea and large bronchi ; the circular layer extending to the small bronchi, even to the air cells.

On the mucous surface of the smaller bronchi cilia in large numbers are found. These, by their waving movements, chiefly in an upward direction, keep the bronchi clear, by sweeping out extraneous substances. They also assist in drawing air into the lungs by creating a counter current. They therefore perform a double function. A certain amount of air always remains in the lungs, and is known as residual air. The air taken into and forced out of the lungs during each inspiration and expiration is called tidal air. The essential part in the respiratory process is the mixing of the carbonic acid of the blood contained within the capillaries of the air cells with the oxygen of the inspired air contained within the air cells themselves.

The mixing of the carbonic acid and oxygen is of the most intimate character, and takes place through the very thin capillary walls of the air cells, which act as osmotic media. It also takes place by the intermingling and transfusion of the gases themselves. In localising the respiratory function in man and in the lower animals it is necessary to bear in mind that the aëration of the blood is not wholly confined to the lungs, but extends to every part of the body where the capillaries and their contained blood, and other structures, are exposed to the action of the air.

In animals the skin, mucous membranes, and the tissues generally all take part in the respiratory process, so that it is at once extensive and complicated. It will be quite correct to say that the body breathes at every pore. Similar remarks are to be made of plants, with this difference, that they give out oxygen and take in carbonic acid. The breathing arrangements of plants and animals are the reverse of each other : the plant exhaling oxygen, which is inhaled by the animal--the latter exhaling carbonic acid, which is inhaled by the plant. A balance is in this way struck as between the gases consumed by the plant and animal respectively. This arrangement affords a striking example of adaptation and of prevision and design.

If an antero-posterior section be made of an adult cadaver in the frozen condition it will be seen that the lungs with the heart and large blood-vessels completely occupy the chest (Plate lxxvii., Fig. 6). The lungs with their investments of pleura have not a cranny unoccupied. The lung pleura and the costal pleura are in contact, but not adherent or united, so that the lungs are free to come and go and to enlarge and diminish with every enlargement or diminution of the chest. The chest forms a vital, air-tight compartment, which is capable of increasing and decreasing in all its diameters. When it increases, it draws out with it the lungs, which in that case are distended to their utmost: when it diminishes, it compresses the lungs to a corresponding extent. The living chest therefore exerts a vis a fronte and a vis a tergo function upon the lungs and upon the air; the object being to bring fresh relays of air and of oxygen to the lungs with a view to aërating the blood and to discharging a corresponding amount of air containing an excess of carbonic acid added to it by the impure venous blood. It will be observed that the lungs exteriorly are protected from atmospheric pressure by the walls of the chest and the diaphragm. They are also protected interiorly because they are inflated with air at birth, which keeps them in a variable state of distension ever after. While air is being constantly drawn into and ejected from the lungs, the lungs, as explained, are never wholly devoid of it. The elastic properties of the lungs assist in keeping up the delicate balance which enables them to follow the ever-varying movements of the chest in respiration.

The walls of the air cells of animals contain a large quantity of fine elastic fibres which ramify and anastomose freely, and are not confined to one air cell ; an arrangement which gives rise to great distensibility and elasticity, properties of considerable importance in keeping the lungs always comfortably filled with air. The presence of pale, unstriated muscular fibres on the larger and smaller bronchi in such plenty suggests rhythmic movements in these tubes. The smaller arteries similarly provided can undoubtedly open and close or partially open and close under nervous guidance, as indicated by the excess of blood in the capillaries in blushing and the absence of blood in them during fear, exposure to cold, \&c.

\section{THE RESPIRATORY MOVEMENTS, ESPECIALLY IN MAN}

\section{$\S 56$. New View of the Mechanism of Respiration-The Muscles of the Chest, Abdomen, and Diaphragm all Involved.}

That blood-vessels have the power of opening and closing is proved by the rhythmic movements occurring in the capillaries of the kidney, in the saphenous veins of the rabbit, in the vessels of the wing of the bat, and in the large vessels at the base of the heart-the aorta and pulmonary artery, which are not supplied with muscular fibres, excepted. 
I am disposed to lay it down as an axiom that whenever pale, unstriated, involuntary muscular fibres occur in quantity, and especially when they assume the annular form, or when they are arranged at right angles and obliquely, rhythmic movements are not only possible but probable.

In order to produce a rhythmic movement all that is required is a series of muscular fibres-striated or nonstriated is immaterial - invested with the double power of shortening and elongating vitally.

The respiratory movements of the higher animals are due to a twofold cause : namely, a want on the part of the system for oxygen, and a desire to get rid of carbonic acid, which is a poisonous, deleterious gas.

The respiratory process is carried on in two ways:-

(a) By means of the skin and mucous surfaces and tissues generally, and

(b) By means of a respiratory apparatus which may take the form of gills or lungs.

The respiratory movements have a wide range as regards frequency, intensity, and duration. Thus in hibernating animals they are reduced to a minimum: the same thing happens in the condition known as trance. The movements are less pronounced in the recumbent than in the sitting, and in the sitting than in the standing positions. They are slower in walking than in running, and in asthma, due to paralysis or partial paralysis of the diaphragm, they are violent; persons so afflicted seizing the backs of chairs or tables to afford fixed points with a view to relieving their breathing.

Respiration is divided into costal and diaphragmatic according as the ribs or the diaphragm take the leading part - the costal being most pronounced in man, the diaphragmatic in woman. There is reason to believe that the use of corsets and tight clothing in modern woman emphasises the diaphragmatic respiration by enfeebling and, to a large extent, destroying the action of the thoracic and abdominal muscles, especially the latter.

The Cheyne-Stokes respiration is a greatly enfeebled form of respiration due to fatty heart and other causes which need not be considered here.

The respiratory movements are spoken of as natural and forced according as they are calm or the opposite. In calm breathing there are usually nine inspirations and nine expirations per minute. In calm as well as in forced breathing not only the muscles of the diaphragm and chest but also those of the abdomen are brought into play. In forced breathing the muscles of the neck, shoulder, root of humerus, back, pelvis, \&c., are involved. The fact that the respiratory movements vary greatly in different individuals at different times and under varying conditions necessitates a certain amount of accommodating power in the muscular arrangements.

In the early foctal condition in man the respiratory system appears as an embranchment or diverticulum of the alimentary system, and we may consider it as a large-branched, specially-modified gland lined with mucous membrane and consisting of a conducting portion and a secreting portion. The lungs being originally a diverticulum from the alimentary canal, it is well not to lose sight of this connection when speaking of the rhythmic movements of the lungs and intestine, and of similar movements occurring in the thorax and abdomen. It is not possible, in my opinion, to separate the rhythmic movements occurring in the chest from those occurring in the abdomen.

As a matter of fact, the thoracic and abdominal cavities open and close alternately; the opening and enlarging of the chest necessitating the closing or diminution of the abdomen and vice versa. This is especially the case in forced respiration. The one movement cannot take place without the other: neither does the one movement cause the other.

The diaphragm by its alternate upward and downward movements plays an important part in the alternate increase and decrease of the thoracic and abdominal cavities. When the diaphragm descends it increases the size of the chest at the expense of the abdomen : when it ascends it increases the size of the abdomen at the expense of the chest.

The muscles of the chest and diaphragm shorten and close when those of the abdomen elongate and open, and the converse. The thoracic and abdominal muscles are co-ordinated for the express purpose of alternately opening and closing the thoracic and abdominal cavities. These cavities are opened and closed by vital muscular movements assisted by the elasticity of the ribs, cartilages, and other structures engaged.

The muscles of the thorax and abdomen are complemental co-ordinated muscles, and the one set closes or shortens when the other set operis or elongates, and vice verse. The diaphragm is also a co-ordinated muscle. It is invested with a double power--that is, it can close or shorten in all its diameters and become flat by a centripetal movement, or it can open or elongate in all its diameters and become arched by a centrifugal movement. The chest, abdominal, and diaphragmatic movements are vital in their nature; the elasticity of the ribs and other structures performing a useful but subordinate function.

The chest, abdominal, and diaphragmatic movements are essentially rhythmic in character. The chest, as indicated, may not inaptly be compared to the left auricle of the mammalian heart; the abdomen to the left ventricle, and the diaphragm to the auriculo-ventricular or mitral valve. 


\section{NEW VIEW OF THE MECHANISM OF RESPIRATION}

That the movements of respiration are due to vital action and very little to elasticity admits of direct proof. They can for short intervals be brought under the influence of the will, in which case inspiration and expiration alike are completely under control-that is, the air is deliberately drawn into the chest and deliberately expelled. In inspiration it is not a case of air entering the chest by atmospheric pressure. On the contrary, the air is sucked in. In expiration it is not a case of mere elasticity and mechanical recoil, as happens in an elastic bag discharging itself independently. The air is deliberately forced out of the chest by muscular effort. In the production of voice it is forced out in very varying quantity, a state of matters which could not possibly be produced by any form of elastic recoil. The mechanism for the production of voice is described under "Phonograph," § 242. There is fundamentally no difference as regards vitality between the inspiratory and expiratory acts. The mechanism by which they are produced is situated partly in the thorax, partly in the abdomen, and partly in the diaphragm.

In order to account for the truly rhythmic character of the chest movements during respiration it is necessary to discard the elasticity hypothesis, according to which the chest is opened by muscles while it is closed mechanically by the elastic properties of the ribs, sternum, and other structures. It is necessary to substitute for the latter a system of muscles which shall close the chest, in a manner not unlike that by which it is opened. In other words it is necessary to provide co-ordinating muscles to those of the chest. 'These muscles are found in the walls of the abdomen, and are as effective in closing the chest as the muscles of the thorax are in opening it. The recti abdominis take a principal share in closing the chest.

In the respiratory movements the muscles of the chest and abdomen co-operate, and in the most natural manner. The muscles of the chest elevate the ribs, those of the abdomen (the transversalis abdominis excepted) depress them. Both sets of muscles act directly and at first hand on the ribs. When the ribs are drawn upwards and outwards, as in inspiration, by the shortening of the chest muscles, the abdominal muscles elongate: when the ribs are drawn downwards and inwards, as in expiration, by the shortening of the abdominal muscles, the thoracic muscles elongate.

The diaphragm closes or shortens in all its diameters and arches upwards during expiration. It has its own. inherent movements, but it is a co-ordinated muscle, and acts in conjunction with the muscles of the chest and abdomen. It exerts a double power, namely, the power of shortening and closing by centripetal movements, and that of dilating and opening by centrifugal movements. In inspiration the diaphragm by its descent and by its becoming flatter compresses and pushes the viscera (especially the stomach and liver) lower into the abdomen. This accounts for the bulging of the abdomen when the chest is expanded.

In expiration the diaphragm by its ascent and by its becoming more arched compresses and presses up the thoracic contents. In the latter movement, the diaphragm receives powerful assistance from the shortening or closing of the transversalis abdominis, which acting on the abdominal contents as a whole presses the liver and stomach into the concavity of the diaphragm, and so aids and assists its movements. The diaphragm dilates and elongates or opens when the transversalis abdominis, and abdominal muscles generally, shorten or close, and vice versid. The diaphragm shortens or closes when the muscles of the chest elongate or open, and the converse. In this way a powerful muscular rhythmic apparatus is provided, sufficient to account for all the respiratory and abdominal movements. The rhythms are muscular throughout, and the ribs and other bones and structures and the elasticity thereof play quite a subordinate part.

The muscles of the thorax, abdomen, and diaphragm form part of a complex rhythmic system which has for its object the intake and output of air, fluids, and solids. The movenents are fundamental, vital, co-ordinated movements, which are absolutely necessary to the life and well-being of the individual. Life cannot be maintained without air, fluids, and solids, and the apparatus dealing with these must be of the most perfect kind; it must work day and night, apart from effort on our part. Of all the vital movements those connected with respiration are in some senses the most important. The exclusion of air from the lungs for more than a few minutes inevitably results in death. Looking at the early connection between the lungs and alimentary canal, and the common rhythmic function discharged by them, it will occasion no surprise if I say, and say deliberately, that not only the muscles of the thorax but also those of the abdomen, as well as those of the diaphragm, are necessary to the due discharge of the functions of respiration.

One has only to study the general disposition and the origins and insertions of the muscles of the thorax, the abdomen, and the diaphragm to be convinced of this. Nearly all these muscles are attached in some way to the ribs, costal cartilages, and sternum; those which are to pull the ribs up being attached for the most part to fixed points above the ribs; those which are to pull the ribs down being attached to fixed points below the ribs.

The respiratory movements proper-and in these I include the rhythmic movements occurring in the nostrils, the glottis, the lungs, the diaphragm, chest, abdomen, and other parts of the body--are at once involved and difficult. 
Notwithstanding the large amount of attention which has been bestowed upon them from early times, the accounts given of them are by no means satisfactory. This arises from three causes :-

(a) The prevailing views as to muscular action.

(b) The high rôle ascribed to elasticity -it being elevated to the position of a prime motor.

(c) The number of muscles employed.

The rhythmic movements of the nostrils are due to the compressors and dilators of the nose. They are as a rule feeble, but there are cases in which they are well marked, and they can always be witnessed in forced respira. tion. They are seen to advantage in the horse and in the sea-mammals, such as the walrus, seal, sea-bear, manatee, porpoise, whale, \&c. During each inspiratory effort, Professor Michael Foster observes, "The nostrils are expanded, probably by the action of the dilatores naris, and thus the entrance of air facilitated. Their return to their previous condition during expiration is effected by the elasticity of the nasal cartilages, assisted perhaps by the compressores naris."

The rhythms of the glottis are due to a complicated system of muscles found on the larynx. Like those of the nostrils, they are faint in ordinary breathing but always perceptible and pronounced in forced breathing. They are more or less mixed up with the action of the vocal chords and the production of voice, and on this account require a separate description.

The rhythms of the lungs are not quite made out, but the presence of large quantities of pale unstriated muscular fibres on the smaller and larger bronchi afford presumptive testimony of their existence. In the interior of the bronchi, ciliary movements which are essentially rhythmic in their nature can readily be made out.

The rhythmic movements occurring in the diaphragm and in the muscles forming the walls of the chest and abdomen practically run into each other, and will be best considered together.

As all the air which passes into and out of the lungs must first find its way through the glottis, its anatomy is no less important than its physiology (Plate lxxvii., Figs. 7 and 8).

In the upper part of the larynx and stretched across its opening are ligamentous bands known as the vocal chords; the two upper or false chords being covered with thick folds of mucous membrane, and the two lower or true chords being covered with folds of very thin mucous membrane. The lower or true alone are concerned in the formation of voice.

Both sets of chords (false and true) are attached to a fixed point between the arytenoid cartilages and posteriorly to the movable arytenoid cartilages. Small muscles, which arise from the thyroid and cricoid and are attached to the arytenoid cartilages, have the power of separating and bringing together the points to which the vocal chords are attached posteriorly so as to open and close the chink or slit between them called the rima glottidis. It is through the rima glottidis that air is admitted into the lungs, and through it all the air expelled from the lungs must pass.

"If," says Professor Austin Flint, " the glottis be exposed in a living animal, certain regular movements are presented, which are synchronous with the acts of respiration. The larynx is opened at each inspiration by the action of the muscles referred to above, so that the air has a free entrance to the trachea. At the termination of the inspiratory act these muscles are relaxed, the vocal chords fall together by their own elasticity, and in expiration the chink of the glottis returns to the condition of a narrow slit. These respiratory movements of the glottis are constant, and are essential to the production of air in proper quantity into the lungs. The expulsion of air from the lungs is rather a passive process, and tends in itself to separate the vocal chords, but inspiration, which is active and more violent, were it not for the movements of the glottis would have a tendency to draw the vocal chords together."

Referring to the diaphragm he says: "From the great increase in the capacity of the chest produced by the action of the diaphragm and its constant and universal action in respiration, it must be regarded as by far the most important and efficient of the muscles of inspiration. Hiccough, sobbing, laughing, and crying are due mainly to the action of the diaphragm, particularly hiccough and sobbing, which are produced by spasmodic contraction of this muscle, generally beyond the control of the will." 1

The diaphragm, which next to the heart is the most remarkable muscle of the body, is deserving of special consideration. It is endowed with characteristic rhythmic movements, which enable it alternately to increase and diminish the thoracic and abdominal cavities. It is a dome-shaped muscle which separates the chest from the abdomen, and its muscular fibres run from its attachments-internal circumference of the lower border of the thorax - to its central tendon in curved lines--the fibres tending to cross at right angles and at every degree of obliquity as in the ventricles of the heart, the stomach, and bladder (Figs. 45 and 46 ). ${ }^{2}$

1 "A Text-Book of Hunan Physiology," by Professor Austin Flint, Bellevue Medical College, New Ynrk, 1. 116, \&c.

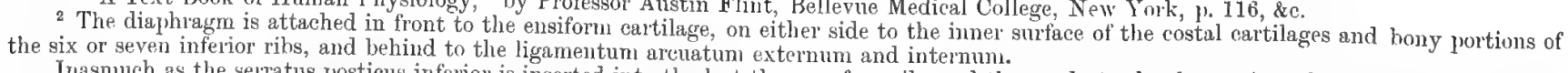

Inasnuch as the serratus posticus inferior is inserted into the last three or four ribs and the ruadratus lumborum into the last rib, it is thought

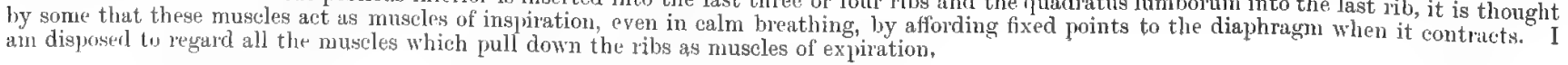


The diaphragm may not inaptly be compared to half a ventricle, half a stomach, or half a bladder. It is moreover endowed with similar movements - these movements being centripetal and centrifugal in character; that is, it can open and close or, in common parlance, relax and contract in virtue of its own inherent powers, independently and as apart from other muscles. When it closes or contracts it enlarges the thoracic cavity at the expense of the abdominal one. When it opens or relaxes it enlarges the abdominal cavity at the expense of the thoracic one. It will be seen from this that both the thoracic and abdominal cavities are engaged in respiration. Further, and as I shall show presently, the muscles forming the chest and the abdomen are likewise both engaged. All the muscles display rhythmic movements. It has been customary hitherto when speaking of calm or normal breathing to

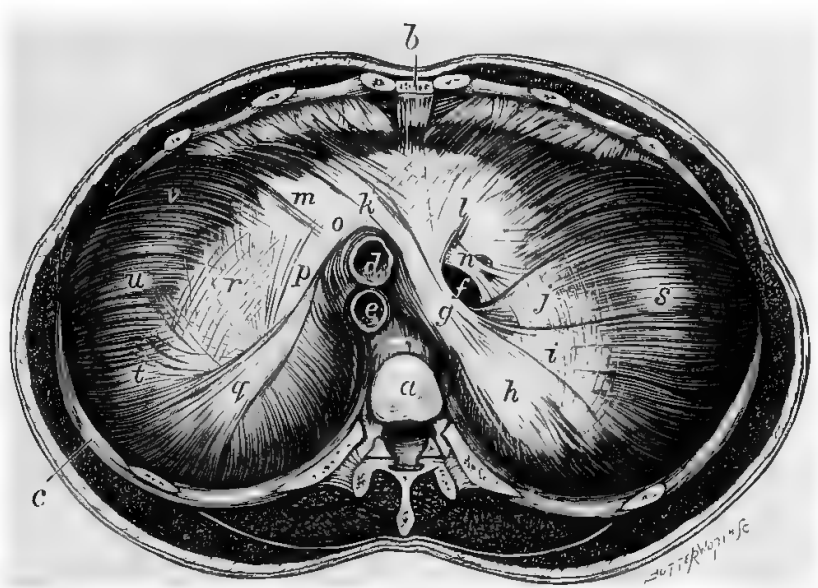

FIG. 45.

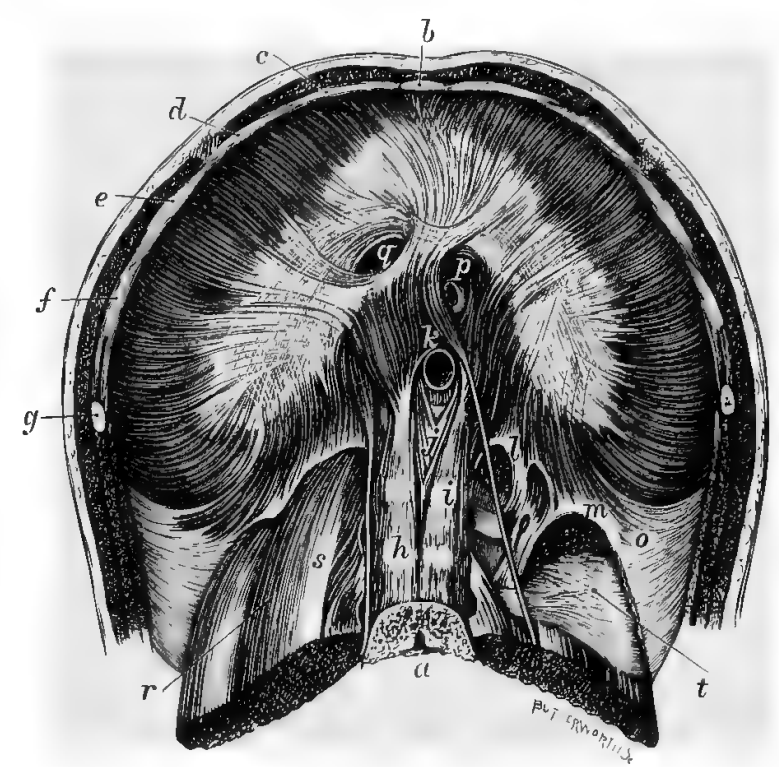

Firi. 46.

FIG. 45.-Muscles of the human diaphragm as seen from above. $a$, Upper surface of the tenth dorsal vertebra ; $b$, section of the lower extremity of the sternum; $c$, segment of the ninth rib; $d$, orifice of the oesoplagus; $e$, section of the aorta; $f$, opening for the vena cava; $g$, middle portion of the oblique fibrous band which separates the opening for the vena cava from the orifices of the cesophagus and the aorta; $h, i, j$, the origins of the fibrous oblique band which forms the thoracic surface of the right leaf of the diaphragm ; $l, l$, triangular expansion of the oblique fibrous band in the anterior middle leaf of the diaphragm; $m$, middle aponeurotic suture of the right and left leaves of the diaphragm blended with the triangular expansion of the oblique fibrous band; $n$, portion of the semi-circular posterior band which forms the external boundary of the vena cava; $0, p, q$, portion of the semi-circular band in front of the osophageal opening on the left leaf of the diaphragm; $r$, aponeurutic bundle of the left leaf of the diaphragm; $s$, the hepatic arch of the diaphragm; $t$, splenic portion of the left half of the diaphragm; $u$, $v$, gastric portion of the left half of the diaphragm (after Bourgery).

Fit. 46.-Muscles of the human diaphragm as seen from below. $a$, Fourth lumbar vertebra sawn through; $b$, tip of xiphoid cartilage ; $c$, seventh costal cartilage; $d$, eighth ditto $; e$, ninth ditto ; $f$, tip of tenth costal cartilage; $q$, tip of eleventh ditto; $h$, expansion of tendon of right pillar (third vertebra) $; i$, expansion of tendon of left pillar (second vertebra); $j$, crossing of the tendinous fibres of the two pillars on the second vertebra; $l$, filsous arch of the aortic opening; $l$, internal fibrous arch; $m$, external ditto.

Left side. - n, Band of insertion of the two arches to the second and third transverse apophyses of the second and third lumbar vertebræ.

Right side -o, Attachment by round liganent to the summit of the twelfth rib; $p$, cesophageal opening between the pillars; $q$, elliptical opening for the passage of the inferior vena cava; $r$, superior extremity of the psoas magnus (right side); s, superior extremity of the psoas parvus (right side); $t$, posterior aponeurosis of the transversalis muscle (after Bourgery).

confine the respiratory movements to the action of the thoracic muscles. I am, however, convinced from a careful and extensive examination of the subject that no respiratory act can ever take place as apart from the co-operation of the abdominal muscles. As a matter of fact, the thoracic and abdominal muscles are correlated and co-ordinated and work together to a common end; that end being the alternate increase and diminution of the thoracic and abdominal cavities.

When the thoracic muscles shorten or contract the abdominal muscles elongate or relax. The thoracic and diaphragmatic muscles shorten or contract synchronously, and by their united movements increase the thorax in all its diameters. The abdominal and diaphragmatic muscles elongate or relax when the thoracic and diaphragmatic ones shorten or contract. All the muscles referred to form part of a complicated rhythmical system. In the correlated co-ordinated movements under consideration the lungs play a not unimportant part.

In order fully to understand the respiratory movements in man it is necessary to regard the muscles of the whole VOL. I. 
trunk (thoracic and abdominal portions) as forming a large oval-shaped cylinder closed at both ends and divided into two by a movable muscular partition-the so-called diaphragm to which allusion has already been made (Fig. 47, A, B).

The muscles forming the cylinder are arranged in straight, curved, slightly oblique, oblique, very oblique, and transverse lines; bones being inserted among them to afford attachment and leverage. The respiratory movements begin and terminate in the muscles, aided by the elasticity of the ribs and other structures.

The straight miscles occur chiefly on the anterior and posterior aspects of the trunk; the oblique, very oblique, and transverse ones are found on all the aspects.

Similar muscular arrangements are met with in the ventricles of the mammalian heart, in the face and neck, in the pharynx and larynx, and in the anterior and posterior extremities. They are repetitions of the primary or typical muscular arrangements which obtain in the hollow viscera.

The straight muscles on the anterior aspect of the body are the rectus sternalis or sternalis brutorum (an occasional muscle) (Quain, p. 243), the rectus abdominis, and the pyramidalis. The last two muscles draw down the sternum, ribs, \&c., in expiration.

The straight muscles on the posterior aspect of the body are the quadratus lumborum and the dorsal muscles, some of which are inserted into the ribs posteriorly. They fix the spinal column and posterior portions of the ribs in forced respiratory movements.

The oblique and very oblique muscles on the anterior aspect of the thorax are the pectoralis major and minor, to which may be added the deltoid. These afford fixed points in difficult breathing.

The oblique and very oblique muscles on the posterior aspect of the body are the trapezius, latissimus dorsi, supra spinatus, infra spinatus, and rhomboidei. These also afford fixed points in difficult breathing.

The oblique muscles on the lateral aspects of the thorax are the external and internal intercostals. These elevate the ribs in inspiration.

The oblique muscles on the lateral aspect of the abdomen are the external and internal oblique muscles. They draw down the ribs in expiration.

The transverse muscles in the thorax and abdomen are the serratus magnus, the serratus anticus and posticus, and the transversalis abdominis. They contract and expand the thorax and abdomen as required.

A combination of all these muscles, as far as the direction and arrangement of their muscular fibres are concerned, is found in the diaphragm. In it, as explained, the muscular fibres run in straight lines, obliquely, very obliquely, and transversely.

Authorities are not agreed as to the number of muscles which take part in the respiratory movements either in calm, normal respiration or in forced, violent respiration.

As a matter of fact, nearly all the muscles of the body may take part in the respiratory movements if they be very violent, as in asthma, severe dyspnnea, \&c. In these cases the muscles of the neck, chest, abdomen, and even the superior and inferior extremities are brought into play. The neck muscles connect the head with the upper part of the thorax and afford fixed points: the muscles attached to the upper part of the humerus (when this is elevated), as the shoulder, scapulæ, and chest muscles, afford additional fixed points, and even the muscles of the lower extremities attached to the pelvis perform a similar rôle.

The number of muscles which take part in the respiratory movements is determined by the violence of the respiratory acts, but even in calm or normal breathing the number is considerably in excess of those usually stated, and I have to express my conviction that in every respiratory effort not only the muscles of the thorax, but also those of the abdomen are engaged; the thoracic and abdominal muscles, as indicated, being co-ordinated and acting together; the thoracic muscles shortening when the abdoninal muscles elongate, and vice versia. The thoracic and abdominal muscles act quite apart from elasticity as a moving force.

This is a new explanation of the respiratory movements, but one to which I attach much importance, as it brings the thoracic and abdominal muscles into line with the muscles of the mammalian heart, which are typical.

It will simplify matters to regard all the muscles with fixed points above the ribs as muscles of inspiration, and those with fixed points below the ribs as muscles of expiration. It may be stated in this connection that there are two large muscles (the recti abdominis) to which hitherto no adequate function has been assigned. The recti abdominis are doubtless muscles of expiration, and when they close or contract they pull the sternum, and the ribs connected with it, downwards and inwards. This they do by a powerful direct pull. The external and internal intercostal muscles are wholly concerned in elevating the ribs, which they do in conjunction with other muscles. By their contraction they are largely instrumental in raising the ribs, and act as muscles of inspiration. The intercostal muscles do not act separately and in opposite directions, as is usually stated. They act simultaneously and in the same direction. Their positions and connections, and the mode in which the ribs are jointer to the vertebral 
column, forbid their acting otherwise. In making this statement I am fully aware of the jointed parallel bar models actuated by oblique elastic bands employed to demonstrate the contrary. The models do not, however, represent the action of the interossei muscles accurately.

When muscles run obliquely and cross each other, the rule is that they act synchronously and produce a common result; in other words, the two sets of muscles do not act against each other, the one set pulling up and the other pulling down and the converse.

I will have occasion to return to the respiratory muscles presently, but it may facilitate matters if, before doing

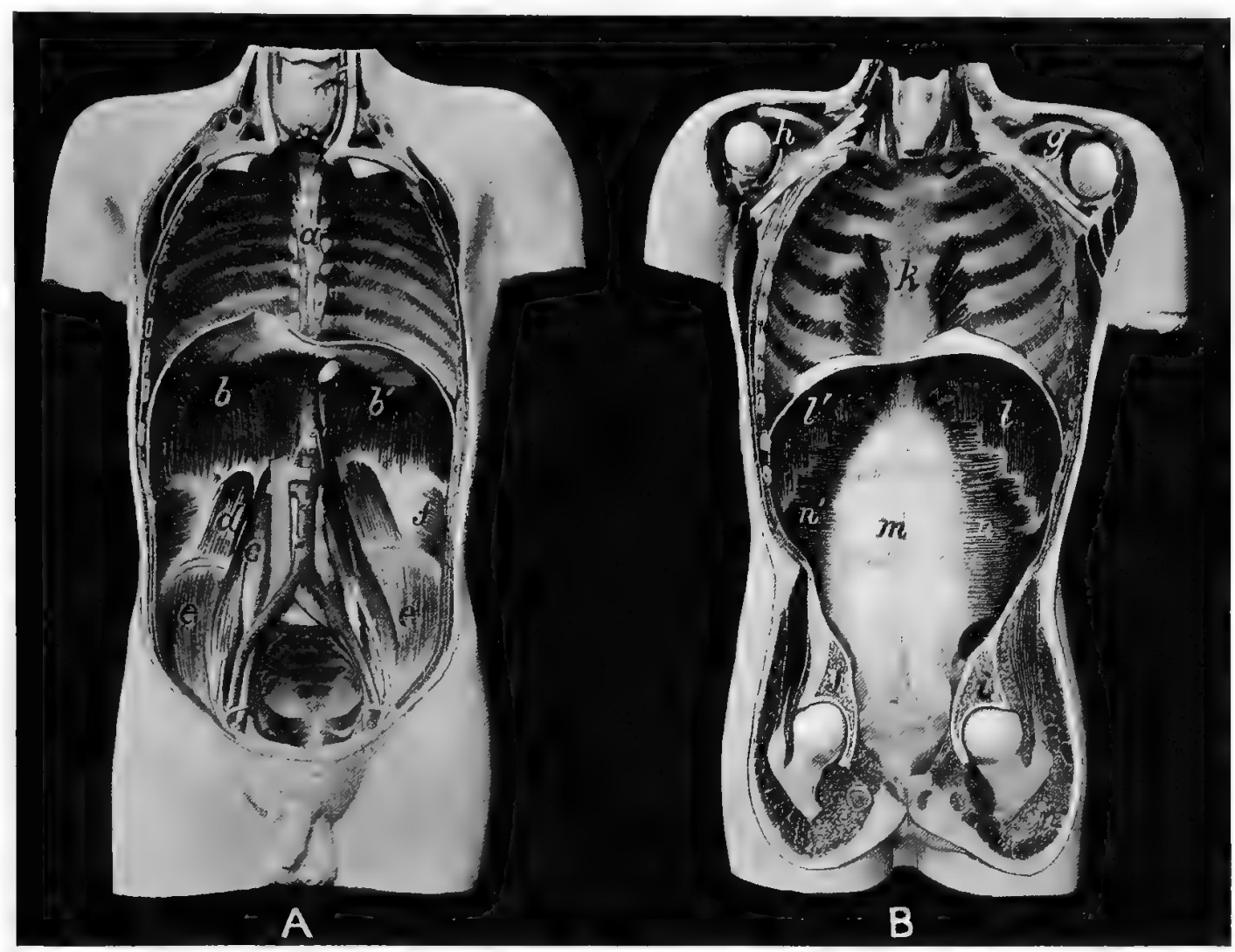

FII: 47.

FIG. 47.-A. Original photograph of the cavities of the thorax and aldomen as seen anteriorly or in front. Shows the rilıs, position of the diaphragm, the great blood-vessels, intercostal and other mulscles. ", Backbone with ribs attached, also intercastal muscles; $b, b^{\prime}$, right and left sides of diaphragm ; $c$, 1sistas magnus and parvus muscles; $d$, quadratus lumborum ; $e, e^{\prime}$, ilincus muscles; $f$, transversalis abdominis muscle (the Author).

B. Original photograph of the cavities of the thorax and abdomen as seen posteriorly or from behind. Shows rounded heads of arms and thigh bones so essential in locomotion, the rilus and intercostal muscles, rectus muscles, and the position of the diaphragm. $g$, Rounded head of right humerus or arm bone; $h$, rounrled head of left arm lone ; $i$, rounded head of right thigh bone; $j$, rounded head of left thigh bone. The arm and leg bones are provided with lall and socket or universal joints, and can loe moved pendulum fashion in any direction in walking and running. $k$, Sternum or breast-bone with sterni muscles, also intercostal muscles; $l, l,{ }^{\prime}$ right and left halves of diaphragm; in, the two recti-abdoninal muscles separated by the linea alba; $n, n^{\prime}$, the two transverse abdominal nusscles (the Author).

so, I give illustrations of the thoracic and abdominal cavities separated by the diaphragm, and also the osseous framework of the chest and abdomen. To these cavities and bones the respiratory muscular arrangements must be ultimately referred (Fig. $47 \mathrm{~A}$ and $\mathrm{B}$ ).

At $A$ and B of Fig. 47 sections of a frozen human cadaver are seen. In both cases the contents of the thorax and abdomen have been removed. At $A$ the cavities are seen from before and at $B$ from behind. In both figures the position and shape of the diaphragm $\left(b b^{\prime}, l l^{\prime}\right)$ are correctly portrayed. The attachments of the diaphragm are also indicated. At B, the recti abdominis covered by their sheath and separated by the linea alba are clearly indicated. At $n n^{\prime}$ the transversales abdominis occur. Other details are given in the descriptions of the figures.

The osseous framework of the chest and abdomen performs a not unimportant part in the great subject of respiration. It acts as a protecting shield or covering, especially to the thorax, and furnishes origins and insertions to the 
respiratory muscles generally, and also to other muscles. It is dome-shaped with the apex of the dome directed upwards, and is compressed in an antero-posterior or lateral direction (Plate lxxvii., Fig. 6, see also Figs. 48 and 49 ). The vertebral column and the ribs forming it are beautifully curved, and both are supplied with cartilages-those of the back-bone being known as inter-vertebral discs, and those of the ribs as costal cartilages. The latter, for the most part, are attached to the ends of the ribs and the sides of the sternum or breast-bone. They give additional elasticity and resiliency to the ribs. The ribs, which are twelve in number on either side of the body, are spiral, most gracefully curved, and so springy and strong that they are sometimes converted into bows for discharging arrows. The roots of the ribs are united to the vertebral column by loose joints which admit of vertical, horizontal, oblique, and rotatory movements, so that they can readily respond to every movement occurring in the respiratory muscles.

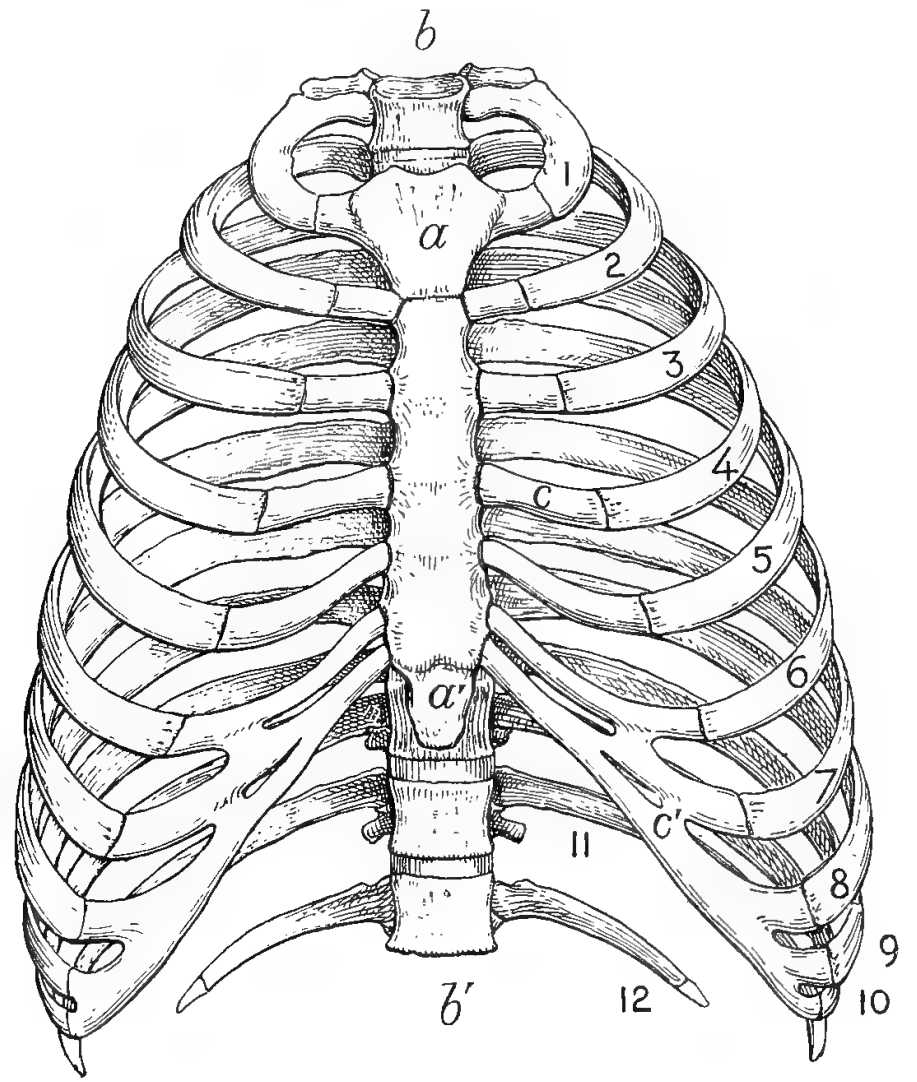

FIF. 48 .

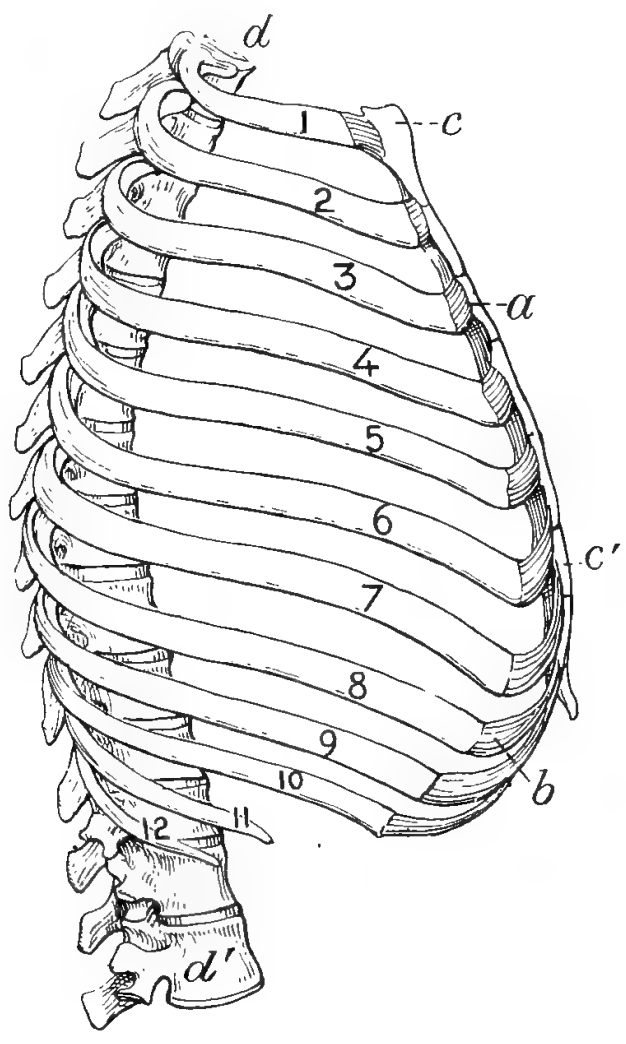

FIG. 49.

FIG. 48.-Anterior view of the skeleton of the thorax and abdomen of the human subject. $u$, $a^{\prime}$, Sternum or breast-bone; $b, b^{\prime}$, vertebral column or back-bone; $c, c^{\prime}$, costal cartilages.

Numbers 1 to 12 indicate the positions and attachments of the twelve ribs (after Holden).

FIG. 49.-Lateral view of the skeleton of the thorax and abdomen of the human subject. $a, b$, Costal cartilages ; $c$ ', $c$, sternum ; $d, d^{\prime}$, vertel,ral column.

Numbers 1 to 12 indicate the positions and attachments of the twelve rils (after Holden).

The ribs are directed obliquely downwards and forwards, and are in the best possible position for being elevated and carried outwards in inspiration, and depressed and carried inwards in expiration by the alternate closing or contraction and opening or relaxing movements of the thoracic and abdominal muscles (Plate lxxviii. Figs. 1, 2, 3, and 4).

Having exposed the cavities of the human chest and abdomen, and given frontal and side views of those portions of the skeleton connected with respiration, we are now in a position to deal with the respiratory muscles themselves.

The diaphragm, as being the chief muscle of respiration, falls first to be considered (Figs. 45 and 46, p. 281). The diaphragm, next to the heart, is the most important muscle in the body. Its muscular fibres run in every direction, in straight, curved, oblique, and transverse lines, in which respect it resembles the heart and hollow viscera generally. It is endowed with universality of motion; its chief movements being in a vertical direction-that is, from above downwards and from below upwards. If the movements of the diaphragm becume irregular, the breathing is at once impaired, and if, by any chance, the muscle becomes paralysed, death inevitably results. Its highly complex 
muscular arrangements are only equalled by its numerous, complicated, and extensive attachments. No muscle in the body has more complex surroundings. It is a purely involuntary muscle, and yet its muscular fibres have the transverse striæ or markings of the voluntary muscles. It is an original fundamental structure: in other words, it is formed in utero before it is called upon to perform any function. It is only after birth, and after the first breath is drawn, that it asserts itself as a working unit of the animal economy. It is a spontaneous, self-acting, rhythmic muscle, and is not dependent on irritability, elasticity, or extraneous stimulation of any kind for its movements. It is not forced into activity by the resiliency of the lungs or ribs: neither is it affected to any extent by pressure exerted on it by the viscera (stomach, liver, intestine, \&c.). While it works in harmony with all the respiratory muscles it is guided by none. It exerts a centripetal and centrifugal power and exercises a pulling and a pushing force. It pulls in inspiration and pushes in expiration; the pull, because of the flaccidity of the muscle, being more marked than the push.

The movements of the diaphragm have been already described.

The deeper muscles of the chest and abdomen (if the recti abdominis and the external oblique abdominal muscles be excepted) show the mechanism of respiration best. What is here stated will be readily understood by a study of Plate lxxviii., and of Figs. 50 and 51, where the thoracic and abdominal muscles are accurately given. It is necessary, however, to be acquainted with both the superficial and deep muscles of the chest and abdomen, as these two kinds of muscles are co-ordinated and act in unison; the chest muscles closing or contracting when the abdominal muscles open or dilate, and vice versit.

The superficial and deep muscles act synchronously or together, so that all the muscles of the chest and all those of the abdomen are in a state of constant motion. In other words, the superficial and deep sets of thoracic and abdominal muscles are at work at the same time. Finally, the chest and abdominal muscles, regarded as wholes or aggregates, are all moving at once. This arrangement provides for the alternate opening and closing of the thorax and abdomen during the entire life of the individual, both in the waking and sleeping states. There are corresponding synchronous movements in the nostrils and glottis, and in the diaphragm.

The respiratory movements are at once extensive and complicated, and have not hitherto been satisfactorily explained. The movements in question are strictly rhythmic in character. They occur in striated muscles, the striated muscles, as a rule, being voluntary muscles. There is thus a departure from use and wont which strongly supports my contention that the involuntary muscles are the precursors and, in a sense, the parents of the voluntary ones, and that the movements of even the voluntary muscles are essentially rhythmic in their nature. ${ }^{1}$

The chest muscles consist of the external and internal intercostals, the pectoralis minor, the serratus magnus, and the pyramidalis sterni. All these muscles are engaged in elevating the ribs. The action of the glottis and diaphragm is not now considered. The abdominal muscles which pull the ribs downwards are the recti abdominis and the external and internal oblique abdominal muscles. The transversales abdominis are powerful expiratory muscles situated beneath the external and internal oblique ones. Their muscular fibres run at right angles to those of the recti abdominis muscles. The recti abdominis muscles are provided with a fibrous sheath derived from the external and internal and transversalis abdominis aponeurosis and display tendinous transverse markings (linex transversæ), which some are disposed to regard as elementary ribs. The points here raised are illustrated at Plate lxxviii. and Figs. 50, 51, 52, and 53.

\section{PLATE LXXVIII}

Plate Ixxviii. displays the thoracic and abdominal muscles engaged in respiration. It shows that the respiratory movements are not due to movements occurring in the chest and diaphragm alone, but to combined co-ordinated and alternate movements, which occur simultaneously in the thoracic and abdominal muscles, and in the diaphragm, respectively. This view of the respiratory muscles and movements, although novel, appears to me to meet all the requirements of the case.

FIG. 1.—a, $a^{\prime}$, External intercostal muscles (human) ; $b, b^{\prime}$, internal intercostal muscles. The external and internal intercostal muscles when they contract ruck up the ribs and force the sternum or breast-bone outwards. $c$, Pectoralis minor; this muscle is a powerful elevator of the ribs. The ribs are pulled down by the contraction of the rectus abdominal muscles aided by the external and intemal oblique muscles. $d, d l^{\prime}$, Serratus magnus muscle; this is an elevator of the ribs. $e$, Subscapularis nuscle; $f$, teres major ; $g$, latissimus dorsi; these three muscles rotate the humerus in the direction of its length. $h$, Biceps muscle; this flexes the forearm on the arm. $i$, Clavicle; $j$, pectoral muscle cut across. The numerals 1 to 8 inclusive indicate the first eight ribs.

FIG. 2.-In this figure the arms are raised. $a, a^{\prime}$, Linea alla or white filorous mesial line; $b, b^{\prime}$, semi-lunar fibrons line; $c, c^{\prime}$, rectus abdominis muscle (of these there are two-one on either side of the white line or linea alba). The rectus abdorninis is covered by its sheath and partly separated by tendinous intersections (linex transversa). The recti muscles play an important part in expiration by

1 Examples of mixed muscles where the movenents are partly voluntary and partly involuntary are found in the fauces. 
PLATE LXXVIII

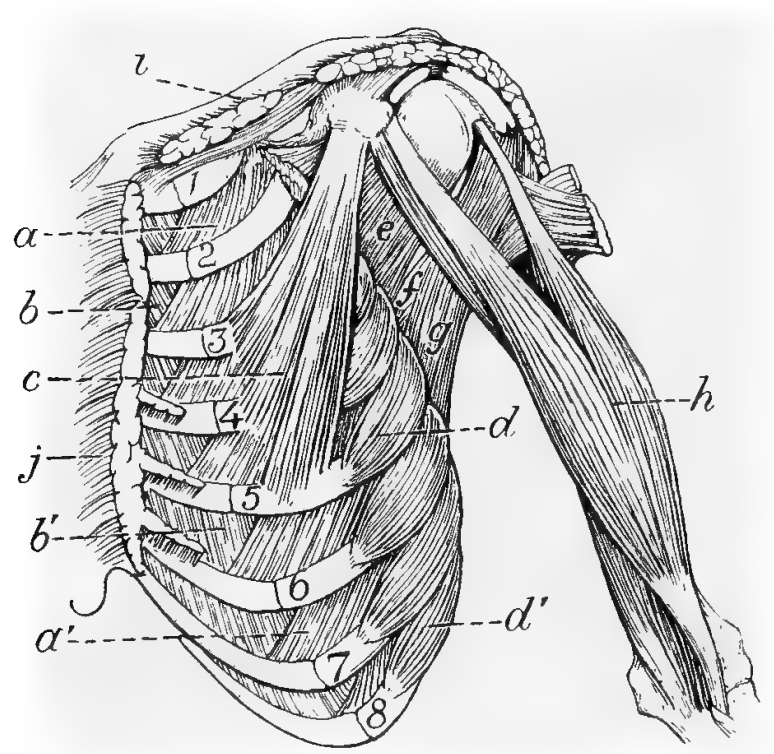

Fis. 1 .

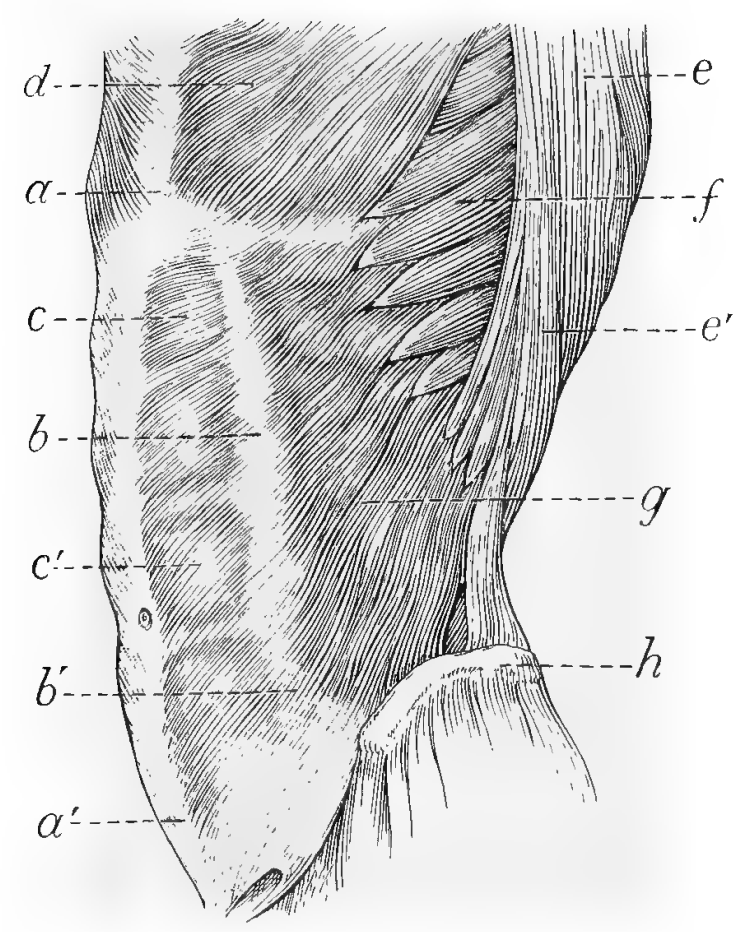

Fig. 2.

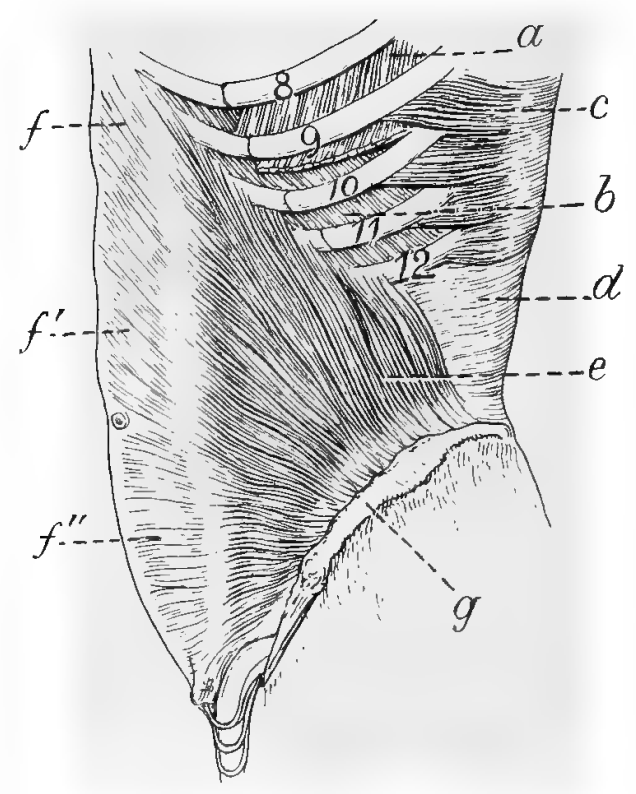

Fit: 3.

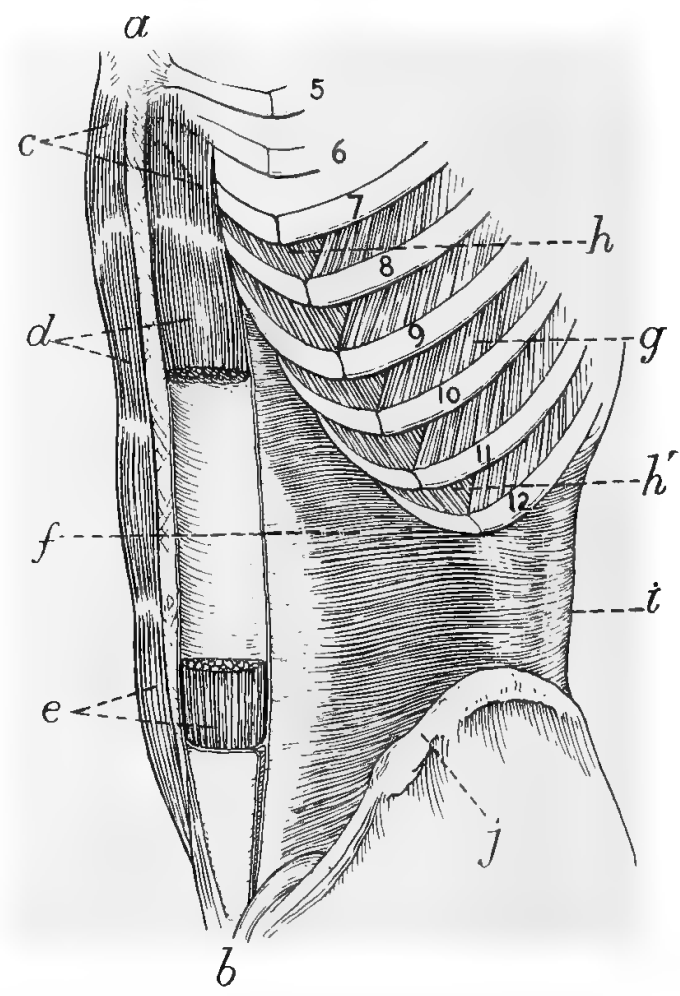

FIG. 4. 


\section{PLATE LXXVIII (continued)}

dragging down the ribs and sternum, and so reducing the size of the thoracic cavity. $d$, Pectoralis major with its muscular fibres drawn up; $e$, latissinus dorsi with its muscular fibres drawn up; $f$, serratus magnus; $g$, obliquis externus; $h$, crest of the ilium.

When the arms are raised and fixed, the pectoral muscles and latissimus dorsi draw up the ribs as in forced inspiration. In this movement the abdominal muscles (external oblique, jnternal oblique, transversalis and rectal abdominal muscles) take part.

FIr. 3.- $a$, External intercostal muscles ; $b$, internal intercostal muscles ; $c$, serratns posticus muscles inferior ; $d$, lumbar fascia; $e$, internal oblique muscle ; $f, f^{\prime} f^{\prime \prime}$, rectus abdominis muscle covered with its shenth ; $g$, wrest of the iliac bone.

The external and internal intercostal muscles cross each other oblinnely, and when they contract take a prominent part with the pectoralis minor muscles and serratus magnus muscles in elevating the rils during inspiration. The ribs are lorcibly dragged downwards during expiration by the contraction of the two recti abdominal muscles aided by the external and internal oblique inuscles. The numerals from 8 to 12 inclusive indicate the positions of the five lower ribs.

FIG, 4,- $a$, Lower portion of sternum ; $b$, symphysis pubis; $c$, the right rectus abdominis muscle, showing the vertical direction of its muscular fibres; $d, e$, the right and left recti abdominis muscles, the latter cut across; $f$, transversalis aldominis. This muscle by its contractions plays an important part in the expulsive efforts of the abdomen in urination, defæcation, and parturition. $g$, External intercostal muscles ; $h, h$, internal intercostal muscles ; $i$, lumbar fascia ; $j$, crest of the ilium.

The numerals from 5 to 12 inclusive indicate the positions of the ribs. The recti and transverse abdominal muscles run at right angles to each other, and by their united contractions, aided by the external and internal oblique muscles, diminish the cavity of the abdomen during expiration in all its diameters. The chest muscles (muscles of inspiration) contract or shorten when the abdominal muscles (muscles of expiration) relax and elongate, and vice versa-the cavity of the thorax being increased when that of the abdomen is diminished, and conversely. The complemental movements referred to are best seen in forced inspiration and expiration.

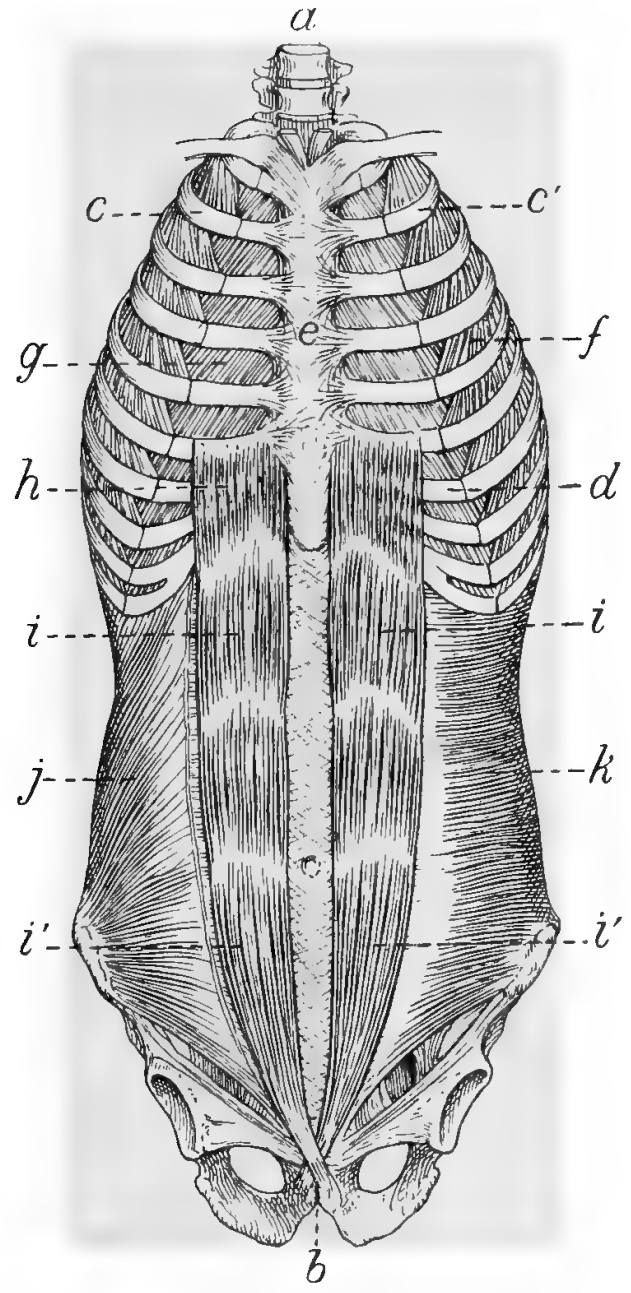

FIG. 50.

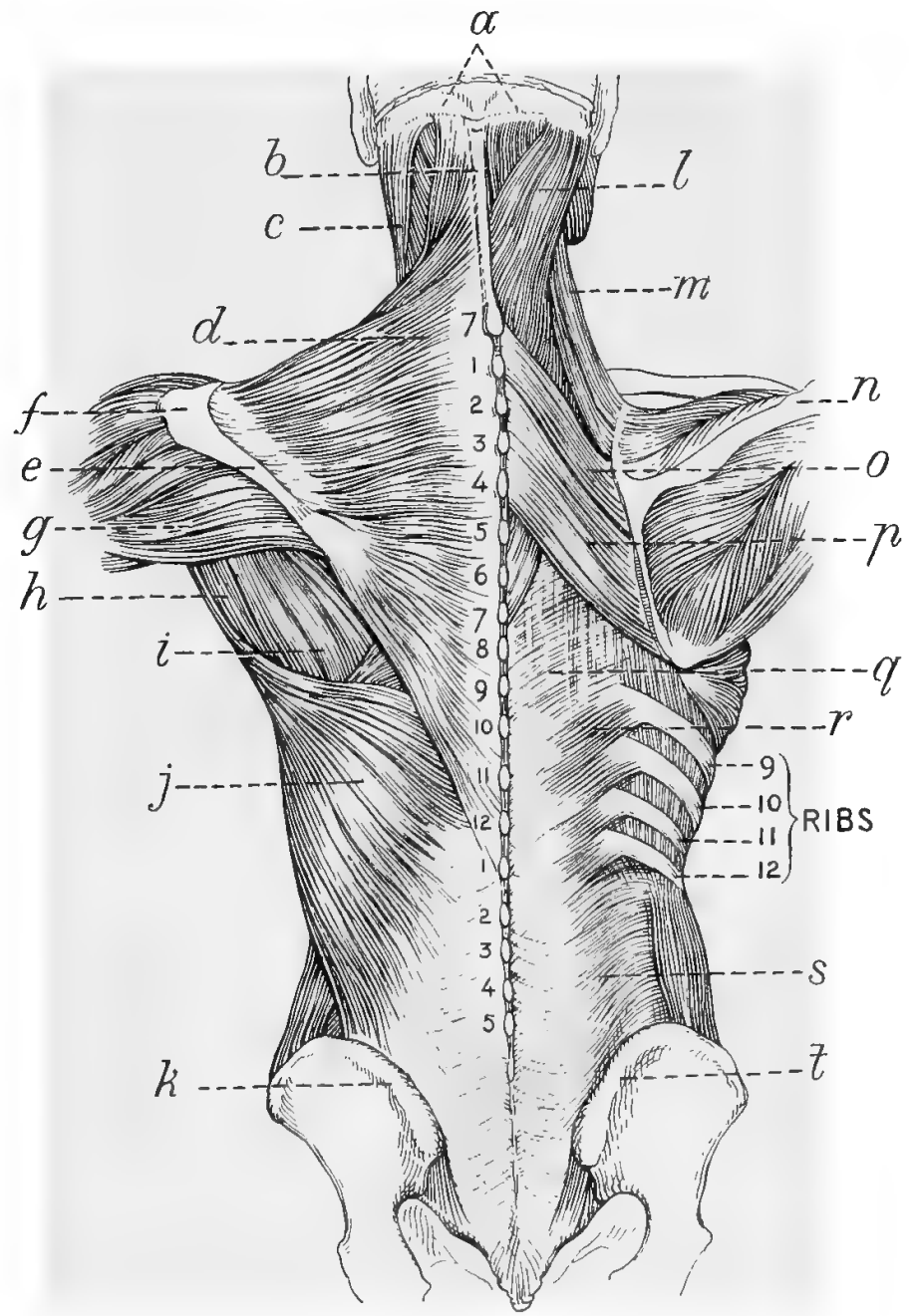

FIs. 51 .

Fig. 50.-Anterior view of the deep muscles of the thorax and abdomen in man (the recti abdominis, which are superficial, excepted). $a$, Spine; $b$, symphysis pubis ; $c, c$, ribs ; $d$, costal cartilages ; $e$, sternum ; $f$, external intercostal museles ; $g$, internal intercostal muscles; $h$, attachment of the rectus abdominis to the cartilages of the ribs; $i, i^{\prime}$, recti abdominis; $j$, the internal oblique abdominis; $k$, transversalis abdominis. 
FIG. 51.-Posterior view of the superficial and certain of the deep muscles of the back in man. On the left side is exposed the first layer ; on the right side, the second layer, and part of the third. a Occipital bone with superior curved line; $b$, ligamentum nuchæ; $c$, sterno-mastoid; $d$, trapezins; $e$, spine of scapula; $f$, acromion process of scapula; $g$, deltoid nuscle; $h$, teres major ; $i$, infra spinatus; $j$, latissimus dorsi ; $k$, crest of the ilium; $l$, splenins capitis et colli ; m, levator anguli scapula; $n$, spine of scapula with supra-spinatus above and infra-spinatus beneath it; $v$, rhomboideus minor; $p$, rhomboideus major; $q$, vertebral aponeurosis; $r$, serratus posticus inferior; $s$, lumhar aponeurosis; $t$, spine of ilium; ninth, tenth, eleventh, and twelfth ribs. The small numerals read from ahove are:-7, spine of seventh cervical vertebra; 1 to 12 , spines of the twelve dorsal vertebræ; 1 to 5 , spines of the five lumbar vertubre. The lowest spines are those of the sacral vertebra and are not numbered.

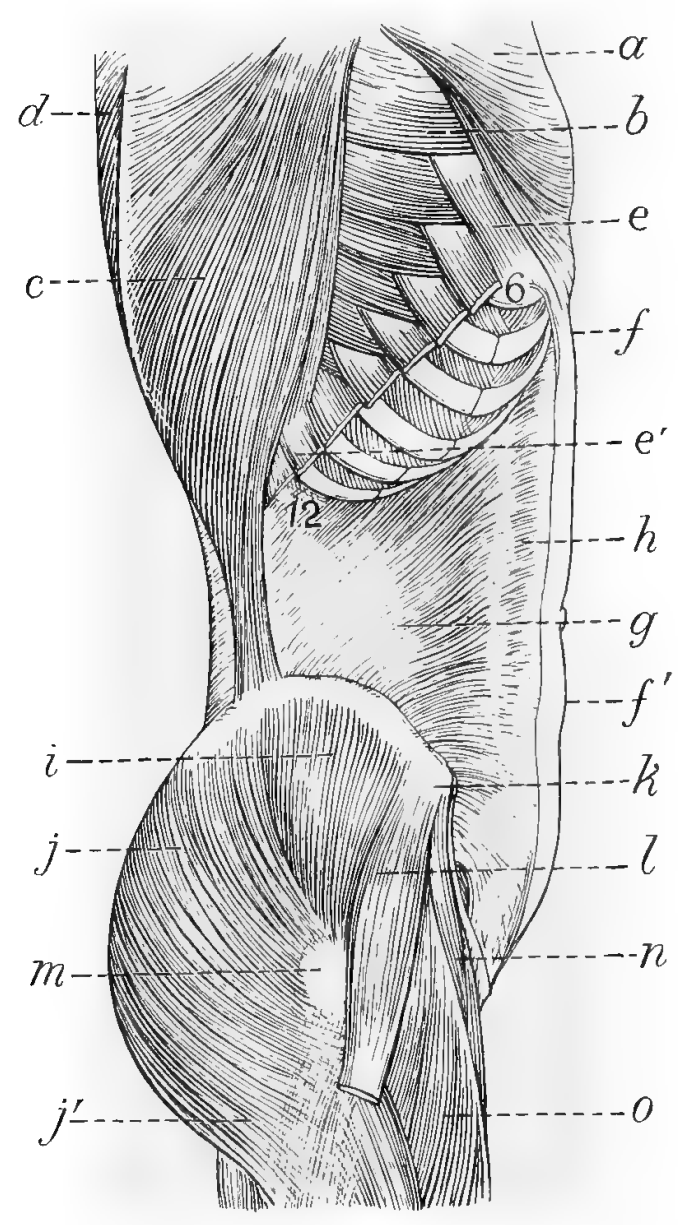

FI 1 , 52.

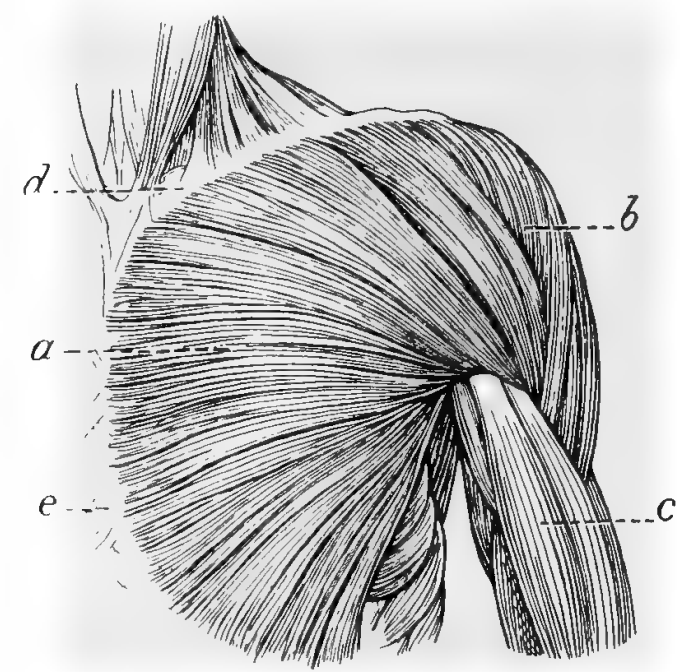

FIr. 53.

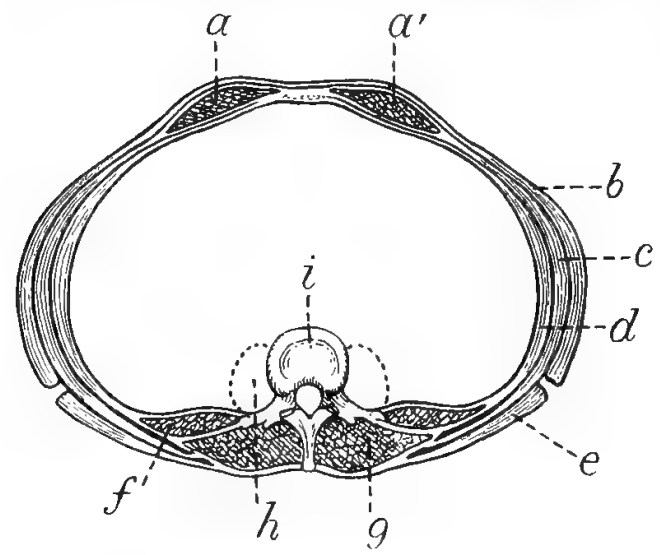

FIG. 54 .

Fr(t. 52.-Lateral view of the muscles of the thorax, alıdomen, and hip (human)-the external oblique inuscle being removed. a, Lower portion of the great pectoral muscle; $b$, lower digitations of the serratus magnus muscle from the fourth to the eighth ribs; $c$, lower costal attachments of the latissimus dorsi muscle; $d$, trapezius muscle-one of the great fan-shaped niuscles of the back; $e, e^{\prime}$, divided attachments of the external oblique muscle left in connection with the ribs; $f, f^{\prime}$, aponeurosis of the external oblique muscle divided in front of the recti muscles; $g$, internal oblique muscle; $h$, line where the internal oblique nuscle separntes to assist in forming the sheath of the recti nuscles; $i$, gluteus medius muscle; $j, j^{\prime}$, gluteus maximus muscle; $k$, anterior superior spinous process of the ilium; $l$, tensor vagina femoris ; $m$, trochanter major ; $n$, spine of pubis ; $o$, rectus fernoris.

The numerals 6 to 12 indicate the ribs connted from above downwards (after Henle).

FIG. 53.- Superficial muscles of the left chest shoulder, and front of the left arm (human), a, 'The great fanl-shaped muscle known as the pectoralis miajor; $b$, the deltoid muscle engaged in raising the arm. The tibres of these two great muscles $(a$ and $b$, like those of the diaphragm, practically run in all directions. Such muscles, for the most part, invest hall and socket or universal joints. Universal joints reupire a universal arrangement of nuscular filres to evoke thuir peculiar powers. Similar arrangements oceur at the hip joints, especially in the deeper muscles. $c$, The biceps muscle which flexes the forenrm on the arm; $d$, the clavicle; $e$, the sternum.

FIG. 54,-Transverse section of the human abdomen in the lumbar region. $a, a^{\prime}$, The two recti abdominis muscles eut across; $b$, ditto, the oblique externus; $c$, the oblique internus; $d$, the trunsversalis abdominis; $e$, the latissimus dorsi; $f$, the quadratus lumborum; $g$, the erector spine; $h$, the psoas; $i$ portion of vertebral column. All these are seen in transverse section. 
According to my researches both the opening and closing movements of the chest are vital acts. The function of respiration is too important for any part of it to be left to irritability, extraneous stimulation, chance, or elasticity. Besides we can, up to a point, breathe voluntarily and prolong the inspiratory and expiratory acts. This one fact, while it effectually disposes of the mechanical theory of respiration, greatly strengthens the belief that the opening and closing of the chest are both vital movements. It, moreover, points significantly to the respiratory rhythmic movements of the chest being fundamental endowments - that is, movements provided for before the animal is born.

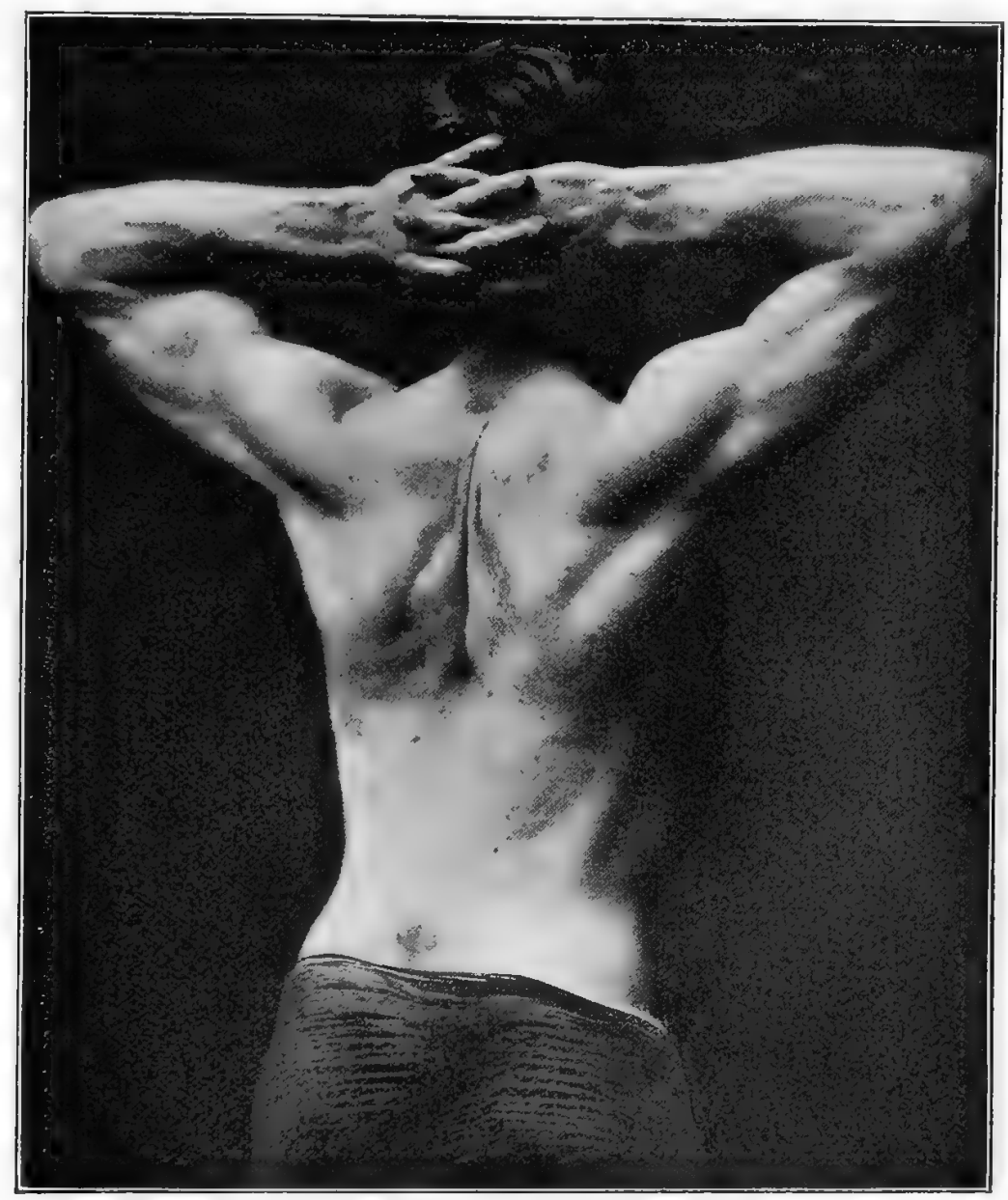

FIIT. 55.

Fig. 55. - Photograph giving a dorsal view of an athlete with the arms raised and the muscles of the arms and scapulæ firmly contructed and thrown into violent action. The muscles of the shoulder joints and the shoulder blades are particularly well seen. 'lhey appear under the skin as great muscular rounded masses. This is especially the case with the deltoid and biceps muscles; the former on the left side assuming a horseshoe-shape on the top of the left shoulder; the latter standing boldly out where the deltoid tapers to a blunt point. The posterior edges of the scapulæ are pressed firmly together, and the various muscles connected with these bones are thrown out into strong relief. The muscles of the forearms are also well defined. Compare with Fig. 51, where the muscles are carefully dissected and the reader can study them at leisure (the Author).

and before either carbonic acid or oxygen are present in the lungs. As a matter of fact, the lungs of the foetus are not distended, and it is only after birth and after the child has drawn its first breath that the mechanism of respiration is brought into play. The first inhalation of air is a vital act, and so are all the opening and closing movements of the chest which follow. That the gases in the lungs do not cause the respiratory rhythmic movements is abundantly evident from the fact that the oxygen and carbonic acid are always present-in other words, are not present and absent at stated intervals, which they would require to be if they produced the intermittent action of respiration. There is no getting over this difficulty if the inherent power of the chest to open and close spontaneously be denied. Moreover, it is the primary and only function of the chest to open and draw in air and to close and expel it. The air can take no part either in its admission to the chest or its expulsion from it. The air holds exactly the same VOL. I. 
relation to the chest which the blood holds to the several compartments of the heart. Neither the air nor the blood act as extraneous stimuli in the ordinary sense.

Plate lxxviii., and Figs. 50 to 54 inclusive, show that the muscles of the human trunk, like those of the ventricles of the mammalian heart, run in every direction, namely, vertically, slightly obliquely, obliquely, and transversely. These remarks apply to the anterior, posterior, and lateral aspects of the trunk. The muscles are also

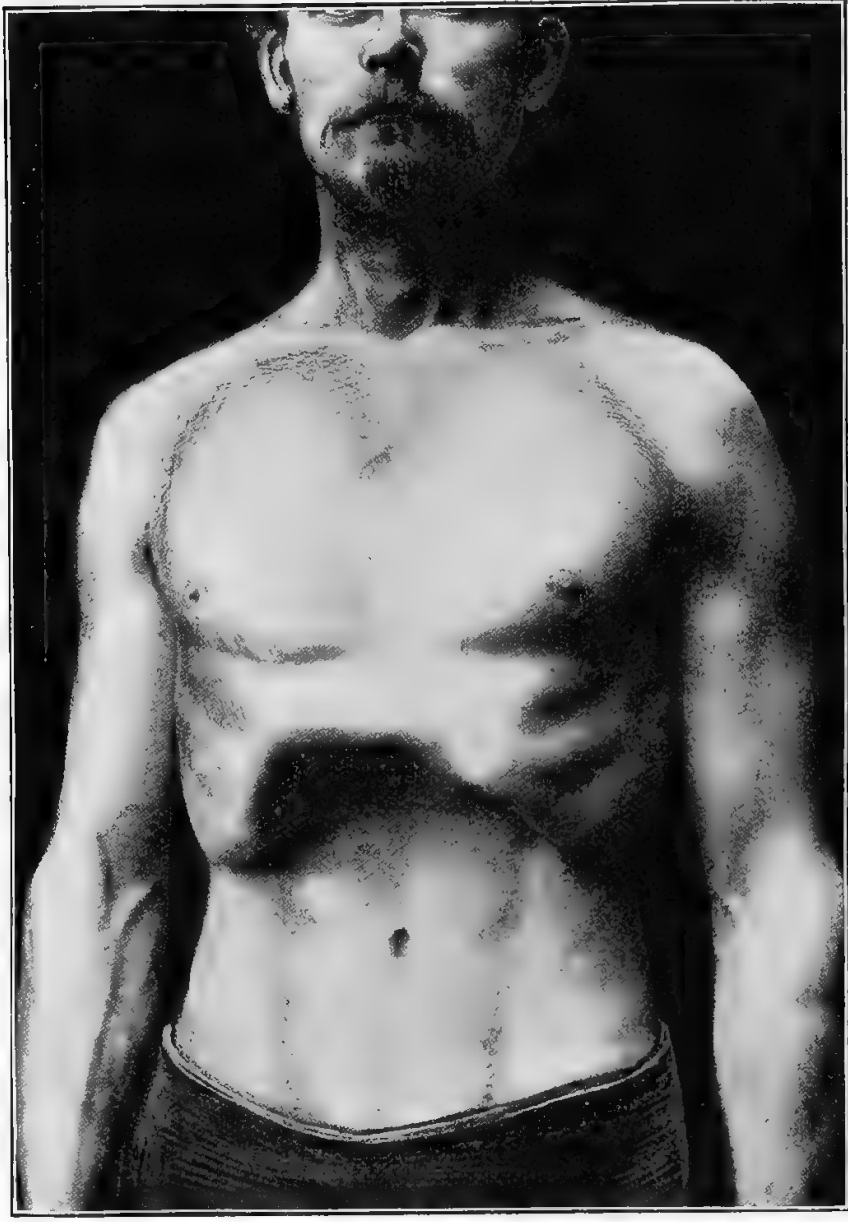

FJG. 56 .

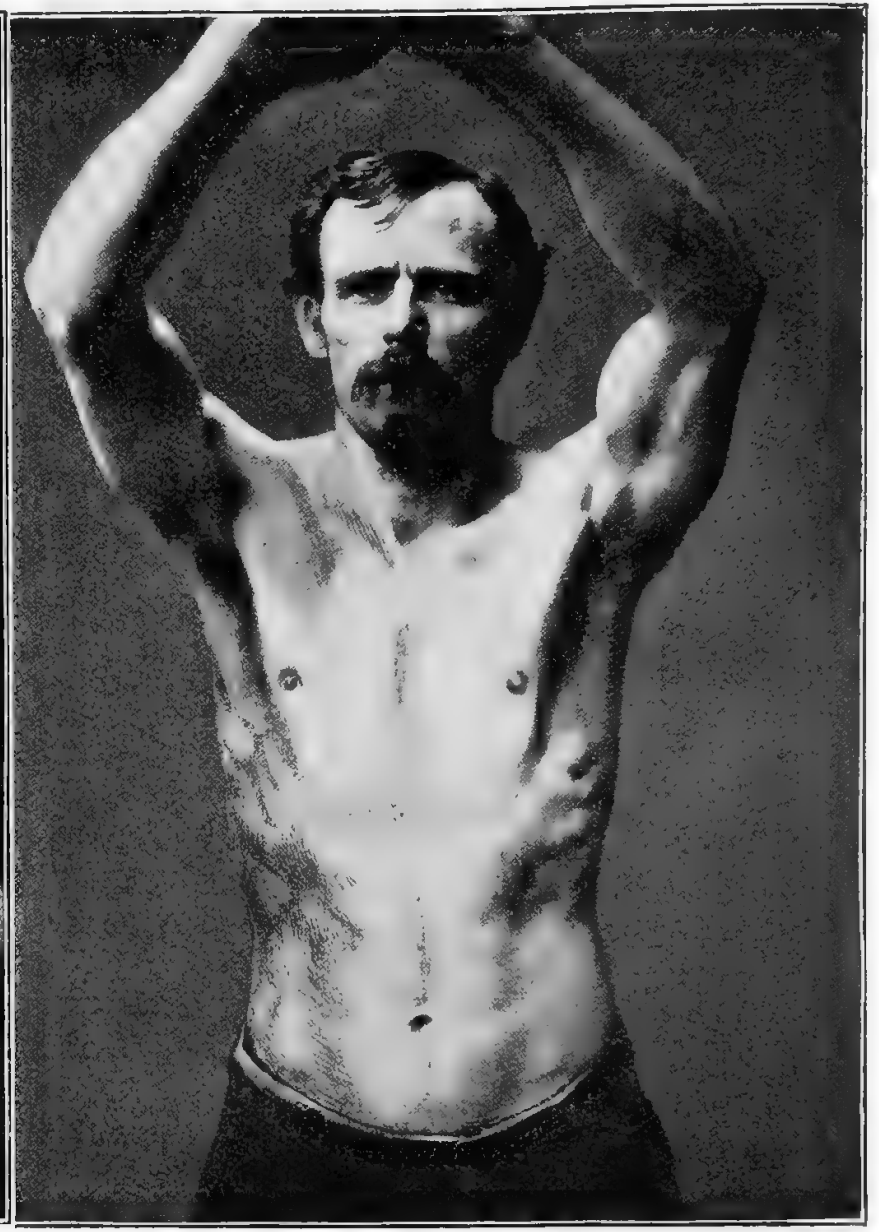

Fir. 57.

FIG. 56.-Photograph of the ventral aspect of an athlete with his arms hamging down holding his breath after having taken a full inspiration. The chest muscles are firmly contracted and the chest is fully expanded in all its diameters; the dome-shaped diaphragm, although concealed, is also contracted and fluttened in a downward direction. The capacity of the chest is increased at the expense of the abdominal cavity. The chest projects and the abdomen recedes. The aldominal viscera are compressed between the under surface of the diaphragn and the upper surface of the pelvis, notwithstanding the relaxed condition of the recti abdominis and of the external, internal, and transversalis abdominis.

The lumpy condition of the muscles of the front of the chest testifies that the lower portion of the pectoralis major, the pectoralis minor, and external and internal intercostal muscles are in a stats of active contruction; whereas the smooth appearance presented lyy the abdominal muscles (especially the recti abdominis) attests an opposite condition. The thoracic muscles are seen relaxed and the abdomiral ones firmly contracted in a complemental photograph (Fig. 57), where a full expiration has been made. The alternate opening and closing centrifugal and centripetal respiratory movements occurring in the chest and alodomen respectively can readily be studied on the living sulject. The rule is, that when the muscles of the chest open or relax, those of the aldomen close or contract, and vice versi (the Author).

FIG. 57.-Photograph of the ventral surface of an athlete with his arms raised who has made a full expiration. This photograph shows exactly the opposite conditions to those seen in the photograph just descrilued (see Fig. 56). In this photogruph the chest muscles are all open or relaxed by centrifugal movements. The muscles of the aldomen, on the contrary, are all closed or contracted by centripetal movements. In this case, the surface of the chest is smooth owing to the muscles being relaxed and flaccid, whereas those of the abdomen are lumpy because of their being firmly closed or contracted. This is especially the case in the recti abdominis muscles, which stand holdly out on either side of the linea alba.

The dialhragm in this photograph is arched upwards into the cavity of the thorax by a centrifugal movement. By these means, the cavity of the chest is diminished and that of the aldomen incleaser. The two photographs under consideration conclusively prove the opening and rlusing consentaneoms moveruents which alternately occur in the thoracic and abdominal muscles during respiration. The throte and alodoninal movenents ure loth vital in their nature (the Author). 


\section{NEW VIEW OF THE MECHANISM OF RESPIRATION}

symmetrically disposed on the several regions of the trunk; an arrangement which secures great accuracy of muscular movement. Fig. 50 shows that the chest and abdomen form a closed, cylindrical, slightly compressed cavity: the cylinders being divided into two by the diaphragm.

The arrangement of the muscles of the trunk is a repetition of that of the ventricles of the heart; the only difference being that in the heart there are no osseous attachments for the muscles, whereas in the trunk there are many. This, however, is a matter of detail, and does not invalidate the important fact that the heart and chest muscles are arranged on a common principle, and that they are invested with centripetal and centrifugal rhythmic movements whereby they can close and open their several cavities in all their diameters. The movements are spontaneous, inherent movements ; the closing movements not causing the opening movements, and vice verso. Both sets of movements are vital in their nature. The cavity of the trunk, divided into two by the diaphragm, constitutes what is practically an enormous heart; the thoracic cavity representing the left auricle, the diaphragm the bicuspid or mitral valve, and the abdominal cavity the left ventricle. It follows that the cardiac muscles supply the pattern or type for the trunk muscles, and that the former pulsate within the latter. The cardiac muscles are the precursors of the trunk muscles.

The muscular arrangements which obtain in the trunk reappear in a well-marked form in the limbs, as is shown further on.

From the foregoing it will readily be inferred that the cardiac and trunk muscles have much in conmon, both structurally and functionally, and that the trunk muscles are largely mixed muscles, that is, they are partly involuntary and partly voluntary. The involuntary nature of the trunk muscles is generally overlooked, but, as a matter of fact, the involuntary muscles are more numerous and important than the voluntary ones.

Fig. 52 affords an admirable example of the typical muscular arrangements which everywhere obtain in the body; the muscles, as stated, running in vertical, slightly oblique, oblique, and transverse directions. It also shows that certain of the muscles (lower digitations of serratus magnus and attachments of external oblique) follow a plicated and interdigitating arrangement, and that others (the latissimus dorsi or great muscle of the back) radiate and spread out so as to assume a characteristic fan-shape. Lastly it shows that the powerful hip muscles (gluteus maximus, medius, minimus, and others) of which the gluteus maximus and medius only are shown in the figure, run in nearly every direction, as is the case in the shoulder muscles (scapular, deltoid, pectoralis muscles, \&c.), and with which they should be compared (see Figs. 51 and 53).

The ball and socket or universal joints, as already indicated, can only be moved satisfactorily by muscles and muscular fibres which run practically in every direction. Universal movements necessitate a universal arrangement of muscles and muscular fibres.

Fig. 52 further shows how certain of the movements of the thorax and abdomen are produced. The external and internal oblique muscles and transverse abdominal ones, aided by the rectal abdominal muscles, when they contract draw down the ribs and diminish the cavities of the thorax and abdomen in a direction from above downwards and so take a prominent part in the movements of expiration. When the thorax, pelvis, and diaphragm are fixed, they powerfully compress the abdominal viscera, and so take a prominent part in defecation, urination, and parturition. The recti abdominal muscles are mainly muscles of expiration. They, however, in conjunction with the transversalis abdominis muscles, which run at right angles to them, exercise a marked influence in diminishing the cavity of the abdomen in its longitudinal and transverse diameters. If to the recti and transverse abdominal muscles be added the oblique ones (external and internal oblique abdominal muscles), which as their names indicate run in oblique directions and cross each other, the most powerful muscular arrangements conceivable for strength and for efficiency in expelling air, fluids, semi-solids, and solids are at once secured. It is the same arrangement which obtains in the ventricles of the mammalian heart, the stomach, the bladder, and the uterus-viscera expressly formed to receive, retain, and expel their contents at given intervals. In these viscera, as has been already shown, the muscular fibres exercise a double power-a centripetal contracting power, and a dilating centrifugal power; and I am disposed to believe that all muscles, voluntary, involuntary, and mixed, exercise the double power referred to. As the viscera in question cannot force the narrow passage of their sphincters, it follows that the sphincters must dilate and open spontaneously when the bodies of the viscera close and contract- the sphincters closing or contracting when the bodies of the viscera open and dilate. The centripetal and centrifugal muscular movements are vital and not vito-mechanical in their nature. In the case of the heart, the auricles contract and close when the ventricles relax and open, and vice vers $\hat{\imath}$.

A similar arrangement is met with in the muscles which alternately increase and diminish the cavities of the chest and abdomen. When the muscles of the abdomen contract and shorten, those of the thorax relax and elongate. When, on the contrary, the muscles of the thorax contract and shorten, those of the abdomen relax and elongate : the two sets of muscles display co-ordinated centripetal and centrifugal rhythmic movements. 
The dome-shaped diaphragm possesses the same powers. When it contracts and flattens, it enlarges the thorax at the expense of the abdomen; the cavity of the thorax being greatest when that of the abdomen is least, and the converse. The obvious conclusion is, that the muscles of the thorax and abdomen and the diaphragm or muscular partition between them have the power of increasing and diminishing the cavities of the chest and abdomen spontaneously and independently.

When the muscles of the thorax and diaphragm contract and shorten they enlarge the cavity of the chest in all its diameters as in inspiration. When, on the other hand, the muscles of the diaphragn relax and those of the abdomen contract they diminish the cavity of the chest in all its diameters as in expiration. The muscles of the abdomen by their contractions assist in diminishing the cavity of the thorax by pulling down the ribs and pushing up such viscera as are placed beneath the diaphragm. The thoracic and abdominal muscular arrangements are necessary to each other in the same way that the auricular and ventricular arrangements of the heart are.

While I venture to claim spontaneous, independent, rhythmic movements for the thoracic, diaphragmatic, and abdominal muscles, I am not unmindful of the fact that the nerves with which these muscles are supplied exercise a certain influence, and that if the ganglion of the tuber annulare of the brain be destroyed the power to breathe is suddenly arrested. This does not prove that the nervous system is to be accredited with the production and maintenance of the rhythmic movements of the chest, diaphragm, and abdomen. The nervous system at best exercises a regulating power. In the case of the heart, the rhythmic movements of the auricles and ventricles continue for some considerable time after the heart is removed from the body and the connection between it and the brain severed. Of course it may be said that the heart contains on its blood-vessels, on its surface, and in its substance large numbers of nerve ganglia, but this only goes to show that the heart, thorax, and abdomen can exercise independent and co-ordinated rhythmic movements as apart from the brain; the movements in question being, for the wisest of purposes, placed beyond our control. It could not well be otherwise. The circulation, the respiration, and the intake, digestion, and output of food and excreta generally are absolutely necessary to life, and, if the several processes had to be superintended by individuals, it goes without saying that life would be a burden and in many ways undesirable.

The relations which obtain between the cavities of the heart and those of the thorax and abdomen are of the closest kind, and the fact that the muscles of the heart act without bones, while those of the thorax and abdomen act in conjunction with bones, makes no difference, for, as I have already shown, the osseous system is, in every case, a mere auxiliary or adjunct of the muscular system. Movement always begins and terminates in muscle or living sarcode of some kind. The bones cannot move of themselves.

Grave discrepancies occur in the descriptions given of the action of the respiratory muscles.

While authorities are not agreed as to the number of muscles which take part in respiration, neither are they agreed as to the functions performed by individual muscles and as regards their so-called active and passive movements. This is especially true of the intercostal muscles. According to Cruveilhier these muscles neither act as elevators nor depressors of the ribs, but only as tensors of the intercostal spaces.

According to Halle the intercostals act together and are elevators of the ribs.

According to Hamberger the external intercostals act as elevutors of the ribs--the internal intercostals as depressors.

According to Hutchinson the external intercostals and the parts of the internal intercostals placed between the costal cartilages elevate the ribs, whereas the lateral portions of the internal intercostals depress the ribs. Hutchinson's view is that commonly accepted at present.

It has been sought to illustrate Hutchinson's theory by the aid of a model consisting of two straight parallel bars of wood representing the ribs, a vertical upright representing the vertebral column, and two oblique elastic bands representing the external and internal intercostal muscles. The parallel bars are jointed at one end to the vertical upright, and the free ends of the hars are furnished with a piece of wood to represent the sternum. When the elastic band which slopes downwards and forwards and represents the external intercostals is put upon the stretch and allowed to recoil it elevates the bars. When the band which slopes downwards and backwards and represents the internal intercostals is similarly treated it depresses the bars.

This ingenious illustration, at first sight very convincing, is, upon careful examination, altogether unsatisfactory, from the fact that the conditions are not identical. The ribs are not straight and parallel structures. On the contrary, they are spiral and variously curved, are set at different angles to the spinal column, are of different sizes, and during their rise and fall rotate upon their axes and within the sockets formed for them by the ribs. Neither do the oblique elastic bands of the model aptly or accurately represent the action of the intercostal muscles. 


\section{NEW VIEW OF THE MECHANISM OF RESPIRATION}

The muscles usually accredited with the production of calm or normal inspiration are-

(a) The diaphragm.

(b) The external and internal intercostals.

(c) The minor pectoral muscles.

(d) The levatores costarum.

(e) The scaleni.

To these I add the following correlated and co-ordinated muscles. The additions are printed in italics.

(f) The recti abdominis. ${ }^{1}$

(g) The pyramidalis.

(h) The triangularis sterni.

(i) The external and internal oblique abdominis.

(j) The transversalis abdominis.

When the muscles which fall under $a, b, c, d, e$ shorten or contract, those which fall under $f, g, h, i, j$ elongate or relax; the two sets being complementary and working harmoniously to increase the capacity of the thorax in all its diameters.

In normal expiration the abdominal muscles shorten or contract; the thoracic muscles, together with the diaphragm, simultaneously elongating or relaxing.

If the abdominal muscles did not elongate or relax when the thoracic muscles shortened or contracted, then the thoracic ones would have forcibly to extend or drag out the abdominal muscles at each inspiratory effort, which is more than they could possibly accomplish. This follows, because the recti abdominis, pyramidalis, triangularis sterni, and the external and internal oblique abdominis muscles directly depress the ribs and are the co-ordinates of the external and internal intercostal muscles, the levatores costarum, and the scaleni, which directly elevate the ribs. The inspiratory and expiratory muscles do not oppose or antagonise each other, as is generally supposed. On the contrary, they work together to produce predetermined results, and they are so placed and so arranged that they do what is required of them as prime movers and at first hand.

Between the thoracic, abdominal, and diaphragmatic muscles there is a constant give and take which brings about by a series of vital rhythmic movements an alternate increase and diminution of the thoracic and abdominal cavities.

According to accepted views, expiration in calm or normal respiration is not produced by muscular action at all. It is said to be due chiefly to elastic recoil. The important function of expiration is left more or less to chance. The elastic recoil mechanical theory of expiration is, on the face of it, erroneous.

The accounts given of the respiratory movements of the nostrils, glottis, and chest are at once conflicting and unsatisfactory. Thus in Quain's "Anatomy" 2 it is stated: "In normal and quiet expiration the diminution of the capacity of the chest is mainly, if not wholly, due to the return of the walls of the chest to the condition of rest, in consequence of their own elastic reaction and of the elasticity and weight of the viscera and other parts displaced by inspiration."

Similar remarks are made of the partial closing of the nostrils and the glottis in expiration.

All this is highly perplexing. If the inspiratory and expiratory acts are co-ordinated and complementary, which there is every reason to believe they are, they have necessarily the same value. It is irrational to assume that inspiration is a vital act produced by the shortening or contracting of living muscles while expiration is a purely mechanical act due to elastic recoil. Moreover, the theory of elastic recoil, if applied all round, is conflicting, inasmuch as it affirms that the several compartments of the heart are closed by vital acts and opened by mechanical ones due to the elastic and other properties of the cardiac muscles, whereas the chest is opened by vital acts and closed mainly or exclusively by elastic ones. Muscle is said to act in a certain way in one place and in another and opposite way in another place.

There are two formidable objections to the elastic recoil theory.

First. There are muscles specially provided for producing the expiratory movements. These, as stated, are the recti abdominis and pyramidalis, the triangularis sterni, the external and internal oblique abdominis, and the transversalis abdominis.

The recti abdominis and pyramidalis, the triangularis sterni. and the external and internal oblique abdominis, when they shorten or contract, directly and at first hand pull down the sternum and ribs, and, aided by the elonga-

1 The abdominal muscles are nsually believed to have no function in calm or normal inspiration, but only in forced or laloured expiration.

"In forced expiration and in efforts of expulsion from the thorax and abdomen all the muscles which tend to dejress the rihs, and those which compress the abdominal cavity, concur in powerful action to enipty the lungs, fix the trunk, and expel the contents of the abdominal viscera" (Quain's "Anatomy," vol. i. 1" 248).

"Seventh edition, vol. i. 1. 249. 
tion or relaxation of the diaphragmatic muscles, diminish the dimensions of the thorax in all its diameters. The abdominal muscles named and the triangularis sterni are as directly connected with the pulling down and lowering of the ribs and sternum as the minor pectorals, intercostals, levatores costarum and scaleni are connected with the rucking up and raising of the ribs and sternum.

Second. If the abdominal muscles and triangularis sterni are not muscles of expiration, then the whole onus of respiration is thrown upon the so-called inspiratory muscles, which are required forcibly to extend the abdominal muscles and to put the ribs and a great many other structures on the stretch to ensure an elastic recoil, which recoil will, unaided and without direction and guidance, produce the important act of expiration. Elasticity. which is a mere mechanical property, is, according to the mechanical school, made to take the place of a first or prime motor.

Expiration is quite as important an act as inspiration. In some senses it is the more important of the two.

The expiratory act has to regulate with extraordinary nicety the amount of air passing through the glottis and the vocal chords in the production of voice, and the marvellous inflexions of which the voice is capable. The regulation of the strong and ever-varying blasts of air required for declamation, intoning, singing, whispering, \&c., could not possibly be left to the haphazard power exercised by elasticity. These air currents, momentarily changing in volume and intensity, demand for their production and management a set of living, co-ordinated muscles with delicately adjusted associated movements. The highly specialised muscles required are found in the larynx, in the thorax, and in the abdomen. It has been sought to explain half the movements of the glottis and vocal chords by elasticity, but I hold strongly that the movements are largely vital throughout. They are too delicate, and require too much fine adjustment to be delegated to any form of elastic recoil.

In the whole animal economy there are no more ticklish functions to be discharged than are performed by the muscles of the glottis, vocal chords, chest, and ahdomen. All the muscles in question are definitely co-ordinated and move with the greatest exactitude. They move, moreover, as highly sensitive organs with a sensory and motor nerve supply. Nothing is left to chance and very little to elasticity.

The statements made by physiologists concerning the movements of the glottis and vocal chords are at once conflicting and confusing. Thus Professor Austin Flint, usually a very careful and exact writer, says of the glottis in connection with voice: "A nearly passive organ, opening widely for the passage of air into the lungs (because the inspiratory act has a tendency to draw its edges together), and entirely passive in expiration, has now become a sort of musical instrument, presenting a slit with borders capable of accurate vibration. The approximation of the posterior extremities of the vocal chords and their tension by the action of certain of the intrinsic nuscles are accomplished just before the vocal effort is actually made. The glottis being thus prepared for the emission of a particular sound the expiratory muscles [it will be observed that the elastic theory of expiration is here discarded] force air through the larynx with the required power. It seems wonderful how a carefully-trained voice can be modulated and varied in all its qualities, including the intensity of vibration, which is so completely under control." [Here again the elastic mechanical theory is set aside.]

He proceeds: "The power of the voice is simply due to the force of the expiratory act, which is regulated chiefly by the antagonistic relations of the diaphragm and the abdominal muscles. From the fact that the diaphragm, as an active inspiratory muscle, is exactly opposed to the muscles which have a tendency to push the abdominal organs, with the diaphragm over them, into the thoracic cavity, and thus to diminish the pulmonary capacity, the expiratory and inspiratory acts may be balanced so nicely that the most delicate vocal vibrations can be produced." I

There is much that is contradictory and misleading in these statements. How, I would inquire, can the glottis be nearly passive in inspiration and wholly passive in expiration? How can the air in phonation be forced through the larynx with the required power by the expiratory muscles if these muscles do not act vitally and as apart from mere elasticity? and how can the vibration of the vocal chords be "completely under control" unless the expiratory muscles and the muscles of the larynx which regulate the vibration are also under control ? How can " the antagonistic relations of the diaphragm and the abdominal muscles" adjust the expiratory act and the power of the voice? Why should the diaphragm be an active inspiratory inuscle, and a passive expiratory one, exactly opposed to the muscles which have a tendency to push the abdominal organs into the thorax? Above all, how can the inspiratory and expiratory acts (seeing the latter put the viscera in motion) "be balanced so nicely that the most delicate vocal vibrations can be produced"?

The confusion and inconsistencies referred to above are the result of an erroneous conception of muscular action and muscular arrangements, and of the part which elasticity is supposed to play in all muscular effort.

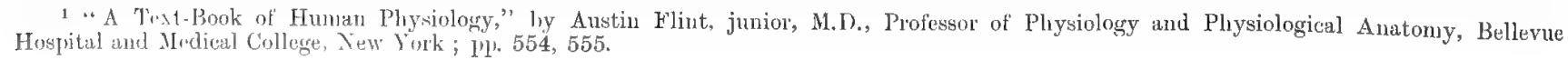




\section{NEW VIEW OF THE MECHANISM OF RESPIRATION}

Professor Flint has given expression to the views of the majority of physiologists of the present day, and is in no way to be held responsible for popular beliefs however incorrect.

For these and other reasons already stated, I hold that the inspiratory and expiratory muscular movements are both vital in their nature, and that the abdominal muscles of expiration are correlated to, and act in concert with, the thoracic muscles of inspiration.

The inspiratory and expiratory movements are produced by spontaneous self-acting muscles; elasticity playing quite a subordinate part in both movements, but being most pronounced in expiration.

As regards the subject of elasticity it may be well to point out that the elastic properties of living muscles and elastic bodies generally, such as steel, caoutchouc, and other springs, are not identical, and give quite different results. The elasticity of muscle acts slowly and is more or less under control-that of mechanical springs acts quickly and is, in no sense, under guidance.

While I do not attach the same importance to elasticity in muscular action as some of my confrìres do, I am far from denying its existence or ignoring its power in animal physics. It certainly does perform a useful function in expiration and in wing and other movements.

The one-sided view of the opening and closing of the chest, whereby the orening is ascribed to vital action inhering in the muscles, and the closing to elastic recoil, is due to the almost universal belief that muscle is endowed with only one power, namely, that of shortening or contracting. A compensating force in that case becomes a necessity, and elasticity is made to do duty in and out of season when a difficulty arises. For reasons already stated, I maintain that muscles are endowed with a double power, namely, a centripetal power of contracting or shortening, and a centrifugal power of relaxing or elongating; these movements being both vital in their nature. An analysis of the movements of muscle (voluntary and involuntary) is given in Sections 54 and 55 of the present work.

According to my views, muscles never oppose and contend against each other as rivals. On the contrary, they work harmoniously together ; elasticity helping them over their dead points and contributing to the continuity of their movements.

Hitherto I have confined my observations to the movements of the respiratory muscles in calm or normal breathing, and before leaving the subject, and in order to emphasise my findings on an avowedly difficult and important matter, I append a table in which not only the muscles engaged are enumerated, but also their attachments set forth. The muscles connected with violent or forced breathing are given further on. They are usually designated extraordinary muscles of respiration.

\section{TABLE OF MUSCLES ENGAGED IN RESPIRATION ACCORDING} TO THE AUTHOR

\section{Inspiratory Muscles Engaged in Ordinary Calm Breathing-}
(a) The external and internal intercostal muscles.
The external intercostal muscles are attached to the outer border's of the ribs; the internal intercostal muscles (sternal portions) to the borders of the costal cartilages.
(b) The pectorales minor muscles. ${ }^{1}$
These muscles are attached to the coracoid process of the scapula and the anterior surfaces and upper margins of the third, fourth, and fifth rilss near the cartilages.
(c) The scaleni muscles (anticus, nedius, ancl posticus).
(d) The twelve leratores costarum muscles.
(e) The diaphrigm, at once inspiratory and ex- piratory.
The scralenus anticus muscle is attached to the third, fourth, and fifth ribs of the "rrvical vertebre and the tubercle of the first rib; the scrlenus medius to the transverse processes of the lower six curviril verterbure ind the upper surface of the first rib; the scalenus posticus to the transverse plocesses of the lower three cervical vertebre and the outer surface of the second rib.
The levatores costarim muscles are attached to the transverse processes of the dorsal vertebre and the ribs bet ween the tuhercles and angles. The diaphragm is attached to the bodies of several of the upper lumbar
vertebre, the liganientum arcuata, the ensiform cartilage, and the carti- lages of the sixth lower rilss. 


\section{Expiratory Muscles ${ }^{1}$ Engaged in Ordinary Calm Breathing}

(f) The recti abdominis muscles.
(g) The external, internal, anil transversalis
abdominis muscles (especially the two
former).

(h) The triangularis sterni.

(i) The serrati muscles.

(j) The sacro-luminalis muscle.

(k) The diaphragm. This muscle takes part in expiration and inspiration.
Attachments.

The rectus abdominis arises by two tendons from the crest of the os pubis and the ligiment covering the symphysis pubis. It is inserted by three portions into the fifth, sixth, and seventh ribs anteriorly.

The exterual oblique muscle is attached to the external surfaces and lower borders of the eight inferior ribs-crest of the ilium, linea alba, and Poupart's ligament. 'The internal oblique muscle is attached to Poupart's ligament, anterior portion of the crest of the ilium and lumbar fascia - the cartilages of the four inferior ribs, the linea alla, the upper crest of the pubis and the pectineal line. The transversalis aldominis is attached to Poupart's ligament, anterior portion of the crest of the ilimm, the lumbar vertebre, the inner surlaces of the cartilages of the six lower ribs-the crest of the pubis, pectineal line, arid linea alba.

The triangularis sterni is attached to the ensiform cartilage, lower border of sternum, and the lower four costal cartilages-the cartilages of the second, third, fourth, and fifth ribs.

The serratus muscle (ilivided into superior, middle, and inferior portions) arises loy eight fleshy digitations from the external borders and upper surfaces of the eight upper ribs and the aponeurosis covering the upper intercostal spaces. It is inserted into the whole length of the inner margin of the posterior border of the scapula.

The sacro-lumbalis is attached to the sternum-the angles of the six lower ribs.

The diaphragm. For attachments of this muscle, see description given under $(e)$.

While the above table illustrates very satisfactorily the number and nature of the muscles engaged in calm normal breathing, it is well to bear in mind that a comparatively large number of extraordinary or auxiliary muscles are brought into action in deep or forced breathing. The movements of the auxiliary muscles are by no means well determined; a circumstance due to the auxiliary muscles, in many cases, acting singly or in small groups, and to their being, in not a few instances, removed to considerable distances from the thorax and abdomen. After all, it is with the ordinary muscles of inspiration and expiration we have to do. It will suffice simply to enumerate the extraordinary or auxiliary muscles of respiration. They are briefly stated as under by Professors Landois and Stirling : ${ }^{2}$

Muscles engaged in Forced Inspiration in the Trunk.-The scaleni, the sterno-cleido-mastoidei, the trapezii, the pectorales minor, the serrati, the rhomboidei, and the extensors of the vertebral column.

Muscles engaged in Forced Expiration in the Trunk. - The abdominal muscles, the intercostal muscles, the triangularis sterni, the serratus posticus inferior, and the quadratus lumborum.

It should be noted that in addition to the respiratory muscles enumerated above, others occur on the nostrils, and on the pharynx and larynx; the latter regulating, within linits, the movements of the vocal chords in the production of voice, and the ingress and egress of air into the lungs.

It only remains to say a few words regarding the causes which produce the muscular rhythmic movements of respiration. Are they due to inherent irritability and stimulation and elastic recoil acting on the tissues involved; or are they the outcome of original endowment stamped on the animal from the beginning and pre-determined to act spontaneously and as apart from elasticity, irritability, and stimulation, and also as apart from nerves, which are rather to be regarded as the regulators than the originators of the movements?

For reasons already stated I am strongly of opinion that the muscular, respiratory, rhythmic movements are spontaneous and independent movements, and that thev are conferred on animals as part of their original equipment, without which the business of life could neither be inaugurated nor continued. One has only to recollect that so long as life endures the demand for breath is incessant day and night, and that the movements of the muscles of respiration are specially adapted to the performance of one great function, namely, the supplying of the lungs with pure air. If the supply of pure air be not forthcoming speedy collapse inevitably follows. To keep the air in the lungs pure there must be no hitch or impediment in the due performance of the breathing process. There must be no waiting for elastic recoil at any stage of the respiratory act.

1 These muscles, as alleady explained, also take part in inspiration.

2 "A Text-Book on Huntan Physiology, including Histology and Microscopical Anatomy," by Dr. L. Landois, Professor of Physiology, University of Greifswald, and Willian Stirling, M.D., Sc.D., Professor of Pliysiology, Owens College, University of Manchester. 


\section{NEW VIEW OF THE MECHANISM OF RESPIRATION}

The inspiratory and expiratory movements are fundamental and vital in their nature. The muscles are living structures both in inspiration and expiration. It is not sufficient to say that the dilatation of the chest is due to vital muscular acts, and that the contraction of the chest is due to passive muscular acts, to elasticity, irritability, and to stimulation. The chest, as already pointed out, is said to be opened up and increased by vital acts and to be contracted and decreased by passive elastic acts. It is further stated that the several compartments of the heart are opened up and dilated by passive elastic and other mechanical acts and closed and contracted by what are undoubtedly vital acts. Both statements cannot possibly be true, and the reader may rest assured that muscle does not act in a certain way in one part of the body and in another and opposite way in another part of the borly. This one fact annihilates the mechanical theory of muscular action.

In taking leave of the muscular respiratory rhythms I wish to explain that I am less concerned with the number of muscles employed in the production of the rhythms than with the rhythms themselves, and the principle which underlies muscular movements as a whole. The points which I am anxious to establish are-

1. That not only are the respiratory movements rhythmic in character, but that they are inherent, fundamental, and co-ordinated.

2. That the movements of the diaphragm, and of the thoracic and abdominal muscles which take part in respiration, are in all respects analogous to those occurring in the heart, stomach, bladder, and uterus.

3 . That the respiratory movements are due to the action of living muscles.

4. That no part of the movements is referable to elasticity alone.

5. That elasticity is a subordinate and not a leading factor in their production.

6. That they are finely adjusted movements, which elasticity by itself could not produce.

7. That they do not primarily owe their existence to nerve initiative.

8. That nerves, under certain circumstances and within limits, regulate them.

9. That they form one of the four great organic rhythms on which life in the higher animals depends; the other three being the rhythms of alimentation, the circulation, and reproduction. The respiratory rhythms are vital, fundamental, spontaneous, and essential to life. The respiratory muscular rhythms cause air, and the oxygen it contains, to circulate within the lungs. The rhythms of the circulation cause pure and impure blood to pervade every part of the economy, so that the tissues are fed and the waste products carried off to the lungs and other parts to be extruded from the system. The rhythms of alimentation bring food, drink, and nutrition generally to the body, and remove from it effete matters in the shape of gases, fluids, and solids; and the rhythms of reproduction, anchored deeply in the constitution of every living being, provide for the continuation of the individual and of the race.

The four great organic rhythms hang together and are mutually interdependent. Indigestion and flatus in the stomach produce palpitation and quicken the movements of the heart; accelerated motions of the heart quicken and disturb the respiratory movements; and an impaired condition of the health dependent upon the abnormal action of the alimentary canal, heart, and lungs, impairs or destroys the sexual appetite.

It needs little reflection to be convinced that a constant supply of atmospheric air, water, and pabulum in a fluid or semi-fluid form is a prime necessity of life, and that the great First Cause has ensured that they shall circulate within every living thing, either with or without specially-designed contrivances for the purpose. The special contrivances in the higher animals are the organic rhythms referred to. The intake and output of air, water, and semifluid food are too important to be left to chance. They are provided for in the economy of all living things; and this one fact shows that a very high place is to be assigned to them in every form of physiological investigation.

There is reason to believe that the movements of both the voluntary and involuntary muscles are fundamentally rhythmic in character, and that the motor nerves distributed to both sets of muscles participate in, although they do not cause, the rhythms.

As already explained, rhythmic movements can and do take place in certain plants where neither muscular fibres nor nerves exist. Similar movements occur in the heart of the chick, composed of nucleated cells, before muscular fibres, nerves, or even blood make their appearance. Rhythmic movements are not, therefore, peculiar to muscle and nerve. In other words, muscular fibres and nerves are not necessary for the production of rhythmic manifestations.

When, however, rhythmic movements occur in structures where both muscular fibres and nerves are present, it follows that, while the rhythms inhere in the muscles, the nerves exercise a guiding or regulating power.

In the higher animals the cerebro-spinal nervous system presides over the voluntary system of muscles-the sympathetic nervous system, aided to a slight extent by the cerebro-spinal system, presiding over the involuntary system of muscles.

The cerebro-spinal system of nerves presides over the voluntary muscles directly, and over the involuntary vol. I. 
muscles indirectly Similarly and conversely, the sympathetic system of nerves distributed to the involuntary system of muscles, in certain cases, influences the cerebro-spinal system of nerves and even the voluntary system of muscles.

While the cerebro-spinal and sympathetic systems of nerves are, in a way, separate and distinct, they are, nevertheless, united structurally. They also co-operate functionally. In like manner, the voluntary and involuntary systems of muscles are separate and distinct up to a point, but they run into each other through the mixed muscles, so that structurally and functionally no hard-and-fast line can be drawn between the cerebro-spinal, the sympathetic, and the voluntary and involuntary muscular systems.

As the voluntary muscles may be regarded as higher developments and differentiations of the involuntary muscles, so may the cerebro-spinal nervous system be regarded as a higher development and differentiation of the sympathetic nervous system.

The two nervous systems merge into each other and the two muscular systems do likewise. With this community of structure and function on the part of the nervous and muscular systems it will excite no surprise when it is stated that the pale, unstriated, involuntary system of muscles, in which are included the heart, ${ }^{1}$ stomach, alimentary canal, bladder, uterus, \&c., and which are characterised by rhythmic movements, have a supply of cerebro-spinal and sympathetic nerves; while the red, striated, voluntary system of muscles, in which are included the diaphragm and the muscles of the chest and abdomen, but which in this case take on rhythmic movements, have a supply of cerebro-spinal nerves alone.

Here we have the cerebro-spinal system of nerves lending itself to rhythmic movements. In other words, we have the highest form of muscle performing rhythmic movements, which, strictly speaking, is the function performed by the lowest form of muscle (involuntary) and the lowest form of nerve (the sympathetic).

This of itself breaks down the distinction between the two kinds of nerve and the two kinds of muscle in question. Nay more, it shows that rhythmic movements are not altogether foreign to the highest form of nerve and the highest form of muscle.

As regards the structure of muscle and of nerve it is a mere question of development and differentiation. The pale, unstriated, involuntary muscle is found in the stomach, intestine, bladder, uterus, lungs, blood-vessels, \&c.; the red striated muscle is found in the heart, the diaphragm, the muscles of the chest, and the muscles of the abdomen - but all the muscles named, striated and unstriated, may and do act occasionally, involuntarily and rhythmically. The heart affords an example of a red striated muscle which moves involuntarily. The muscles of the pharynx and larynx are partly voluntary and partly involuntary, and are known as mixed muscles. The muscles of the limbs consist of red, striated muscles, and, as a rule, act voluntarily; they, however, under certain circumstances, act involuntarily, as in the case of lunatics and, during sleep, in sane persons.

Similarly, what is the sympathetic system of nerves in man suffices for many of the lower animals, which have no brains, but which nevertheless perform what must be regarded as a low form of voluntary movements. The fiverayed star-fish is an example. ${ }^{2}$

If, however, the pale, unstriated, involuntary muscles culminate in the red, striated, voluntary muscles, and the sympathetic system of nerves culminates in the cerebro-spinal, then the following inference may fairly be drawnall muscular movements and all nerve action are, or may be, rhythmic in character.

All the handicrafts are dependent on muscle training. The skilled artificers in every department of labour rely for their deft movements on the highly-trained voluntary muscles of their hands, arms, feet, legs, and other parts of their bodies. The nicety of movement in many cases attained is truly marvellous.

No finer strudy of harmony structurally or functionally can anywhere be witnessed than is presented by a fullydeveloped, highly-trained muscular system. The ease, the grace, the subtlety, the dignity, the power, nay, the majesty of motion, is in some cases transcendent. For the highest manifestations of muscular excellence there must be mind behind to give direction and expression.

As regards the cerebrum or brain, which is the chief portion of the cerebro-spinal nervous system, it is important to point out all that its actions and manifestations are of the interrupted order. Even the brain works and rests at intervals.

It would be easy to multiply indefinitely the many interesting points connected with the rhythms and reflexes in the organic kingdom, and to associate them with protoplasm, with voluntary and involuntary muscles, with nerves or their representatives, with the intake and output of matter in living structures, with a Designer and Regulator of the Universe, with law and order, and endless striking examples of "means to ends," and of cause and effect. It

1 The heart is an exception as regards colour and striation. It affords an example of an involuntary nuscle whose fibres are red and striated. This explanation does not meet the of physe. The star-fish controls its moyements, and of the star-fish to irritability, stimulation, aud reflex action. This explanation does not meet the case. The star-fish controls its movements, and moves in specific directions and to accomplish certain ends.
Its movements are in no sense involuntary or haphazard. 


\section{NEW VIEW OF THE MECHANISM OF RESPIRATION}

is, however, not necessary to elaborate the subject further. It will suffice if I reiterate my statements to the effect that the rhythms and reflexes referred to are the product of fundamental structures, which have their roots deep in the constitution of things, and that they are necessary, in one shape or other, to the very existence of plants and animals in their simplest and most complex forms. The rhythms and reflexes to which allusion is now made are not the offspring of chance, of irritability, stimulation, or environment. They are movements per se, and the more closely they are scanned, the more mysterious and inexplicable do they become unless a First Cause is predicated. There is no getting behind or understanding them unless a First Cause be taken for granted. With a Creator, the whole scheme of original endowment and movement lies open to the gaze of even the most casual observer.

\section{TRANSITION LINKS AS BETWEEN THE PLANT AND ANIMAL}

The breathing of animals has been fully described and illustrated under "The Respiratory Organs in Animals, and especially in Man" (page 274, Section 55, Plates lxxvii. and lxxviii., Figs. 45 to 57 inclusive). The muscular arrangements and movements in animals are described and delineated very fully in Plates lxxxiii., lxxxiv., and lxxxv., Figs. 67 to 75 inclusive. The part played by the muscles in the production of alimentation, respiration, circulation, urination, reproduction, and locomotion are dealt with in detail in different parts of the work.

\section{§57. The Mycetozoa.}

These remarkable organisms form a numerous family, and are pretty well universal as regards distribution, being found in Europe, India, the Cape of Good Hope, Australia, North and South America, \&c. As many as one hundred and seventy-five species are preserved in the Botanical Collection of the British Museum, and, curious to relate, as establishing the cosmopolitan nature of the family, quite a large number of species exhibit precisely the same characters on different parts of the earth's surface.

Great interest attaches to the Mycetozoa physiologically because of their mode of reproduction, their independent amœbic and plasmodium movements, their mode of feeding, their tenacity of life, and their power of subsisting in essentially two different conditions, namely, in a desiccated or dried-up, inactive, or hibernating condition (sclerotium), and in a moist, swarming, streaming, active condition. These several points are illustrated at Figs. 58 and 59.

The dual life of the Mycetozoa, namely, its inactive, resting condition (Fig. 59, A); its power of swarming (Fig. 58, A, B, C); and its aggressive nature in the active or streaming state (Fig. 59, B, C, D, E), are especially outstanding features, and deserve the careful attention of biologists, as they reveal a potentiality, structurally and functionally, which goes far to prove that protoplasm and life are endowed with what are virtually universal powers. The life history of the Mycetozoa certainly shows that reproduction, ingestion, digestion, absorption, assimilation, and movements more or less co-ordinated and definite can be produced in the simplest manner, and with little or no differentiation. It also shows that these processes, in the higher animals up to man himself, are not necessarily dependent on the existence of a nervous system; as a rule, that system controlling but not causing them.

It is important to direct the attention of the reader to this fact, as the trend of late years is to accredit the nervous system, in the higher animals, with every change, physical and mental, which occurs in the animal economy.

It is quite obvious that the movements, rhythmic and otherwise, occurring in plants and a very large number of the lower organisms, are in no way dependent on nerves; these being non-existent, or, at all events, not assuming a definite or palpable form. It is reasonable to assume that the movements connected with alimentation, respiration, circulation, secretion, excretion, \&c., all of which are necessary to the life and well-being of the individual, are pre-arranged, original, inherent, and independent movements. An example will bring out my meaning. The heart of the chick beats regularly while yet a mass of nucleated cells, and before it is provided with muscles and nerves, and even before it contains blood.

To borrow an illustration from the Mycetozoa themselves. The plasmodium of Badhamia utricularis streams out in search of food in fan-shaped, skirmishing order, and covers an area of forty or more square inches; its little advancing fans, two or three hundred in number, springing from a network of branches (Fig. 59, B, C, D, E).

When food, say a portion of Stereum hirsutum, is placed at the root of the network, the little advancing fans and the network itself are simultaneously withdrawn, and the plasmodium, voluntarily as it were, concentrates on the food, which it steadily and slowly devours. The skirmishing order may be maintained for two whole days, while the concentration occurs in five hours.

Here we have what is virtually a voluntary effort put forth by an organism which, according to some, is a 
plant, according to others an animal, and according to a third, a half plant or a half animal. ${ }^{1}$ This raises a question of primary importance in physiology and psychology. Is it necessary to believe that organisms devoid of a nervous system, or, at all events, of a tangible nervous system, are hopelessly ignorant of their own existence, seeing they can move in definite directions and shape their ends? Further, are the lower animal forms (say the five-rayed starfish), which are provided with ganglia and sensory and motor nerves, but no brain, and whose movements are undoubtedly voluntary, devoid of the conscious ego?

The brain of the centipede consists of a slight differentiation and increase of two of a longitudinal chain of ganglia which occupies a central dorsal position in the body of the creature, and similar remarks may be made of the brain even of man. There is in man a striking augmentation of brain substance and brain differentiation, but there is no breach of structural continuity as between the brain and the spinal cord, and no difference in the nature of the nervous matter; it is a mere question of increase, elaboration, and differentiation. There is, in short, not a difference of kind, but only of degree, in nervous matter. The same is to be said of nervous and psychical manifestations in the lowest and highest animals.

If, however, protoplasm can assume every variety of form and movement as apart from a tangible nervous system, it requires no great stretch of the imagination to believe that a nervous system, in the absence of a brain, discharges the essential functions of a brain; the brain being simply a more highly developed and elaborated portion of the nervous system proper. In the lancelet (Amphioxus lanceolatus), the lowest of the "vertebrates," there is a spinal cord but no brain (Goodsir, 1841).

This line of argument militates against the exclusive possession of intellectual faculties and mind by man; but, on the other hand, it accounts for a large number of facts otherwise inexplicable, namely, the voluntary and, it may be, conscious acts of the whole animal series provided with nervous systems, from man downwards. It is well known that the monkey, elephant, dog, horse, and a large number of quadrupeds reason. Similar remarks may be made of birds, and, within limits, of reptiles, fishes, spiders, ants, bees, \&c. In all these cases it is mere assumption to assert that the individual is non-conscious. The words instinct, unconscious cerebration, and reflex action, do not explain the phenomena witnessed.

The fact that the insectivorous plants can, by concerted, co-ordinated movements, catch insects, and digest, absorb, and assimilate them; while the Mycetozoa can advance in search of food and retrace its steps and concentrate on food when found, and digest and assimilate it, have further to be explained. Two explanations can be given: either the Creator works directly upon and through plants and the lower and higher animals, or all these are provided with structures and endowments which, potentially, are equal to all the requirements of life, whether these be physical, mental, or psychical.

The Mycetozoa have been studied to good purpose by Mr. Arthur Lister, who has skilfully summarised our knowledge of these interesting forms. He says :-

"They are characterised by the constant sequence of three main stages in their life history :-

"1. The firm-walled spore gives birth to a swarm-cell.

"2. The swarm-cells coalesce to form a wandering plasmodium.

"3. The plasmodium ultimately concentrates to form either sporangia, enclosing numerous spores (Endosporeæ), or sporophores bearing spores on their outer surface (Exosporeæ).

"Many species are quite common, and are found on old decaying stumps of trees and fallen branches in moist woods and shaded gardens; others inhabit heaps of dried leaves which have lain undisturbed and become soaked with rain. The only stage in which they are conspicuous is that of the sporangia, when they appear as minute objects, some roundish, about the size of small mustard seeds, others rising in clusters of brown columns on black, hair-like stalks, while many take other characteristic forms. The different species display great variety and beauty in the colours they assume, ranging from pure white, golden yellow, bright crimson, and iridescent violet to dark purple and black.

"The various phases in the life history of the group may be described as follows. The swarm-cells emerge from the spores as amoboid bodies; they soon acquire a flagellum at the anterior end, and creep in a linear form with the flagellum extended in advance, or swim in the surrounding water with a dancing motion occasioned by the lashing movement of the flagellum. They possess a single nucleus and a contractile vacuole. To a large extent the swarm: cells of Mycetozoa feed on bacteria, which are caught by pseudopodia projected from the posterior end of the body. The bacteria are conveyed into the body-substance, where they are digested in vacuoles which form round them. There may be one or more digestive vacuoles, each containing several bacteria at one time. The swarm-cells rapidly

${ }_{1} \mathrm{Mr}$. Saville Kent supports the animality view, and traces a connection between the Mycetozoa and the Spongidæ; the resemblance extending to the fission or ancebic stage, to the production in both of spores with Hagella or cilia of sonre kind, to the encysted or resting, hibernating condition, and to the presence in both of rudimentary skeletons in the shape of horn-like elements or keratose, and spicule-like bodies of carbonate
of lime. 


\section{NEW VIEW OF THE MECHANISM OF RESPIRATION}

increase in number by bipartition. When this takes place the flagellum is first withdrawn, and the swarm-cell assumes a globular form; it then elongates, and a constriction occurs at right angles to the long axis. Meanwhile the nucleus is passing through the process of division by karyokinesis, and in the course of a few minutes the two halves of the nuclear plate separate and retreat to the opposite ends of the constricted cell, which now divides into two; each segment soon acquires a flagellum, and resumes the former active state.

"In all cultivations of germinating spores a number of the swarm-cells, after a short time of activity, become encysted in a globular form as microcysts. In this state they may remain dry for several days, but on water being added the cyst-wall is ruptured and the contents creep out and assume again the motile condition. Frequently the entire group of swarm-cells will change to microcysts, and reawaken in the course of two or three days while still immersed in water. A few days after the germination of the spores, the process of bipartition, by which the number of swarm-cells has greatly increased, ceases. The majority now withdraw the flagellum, and adopt true amœboid
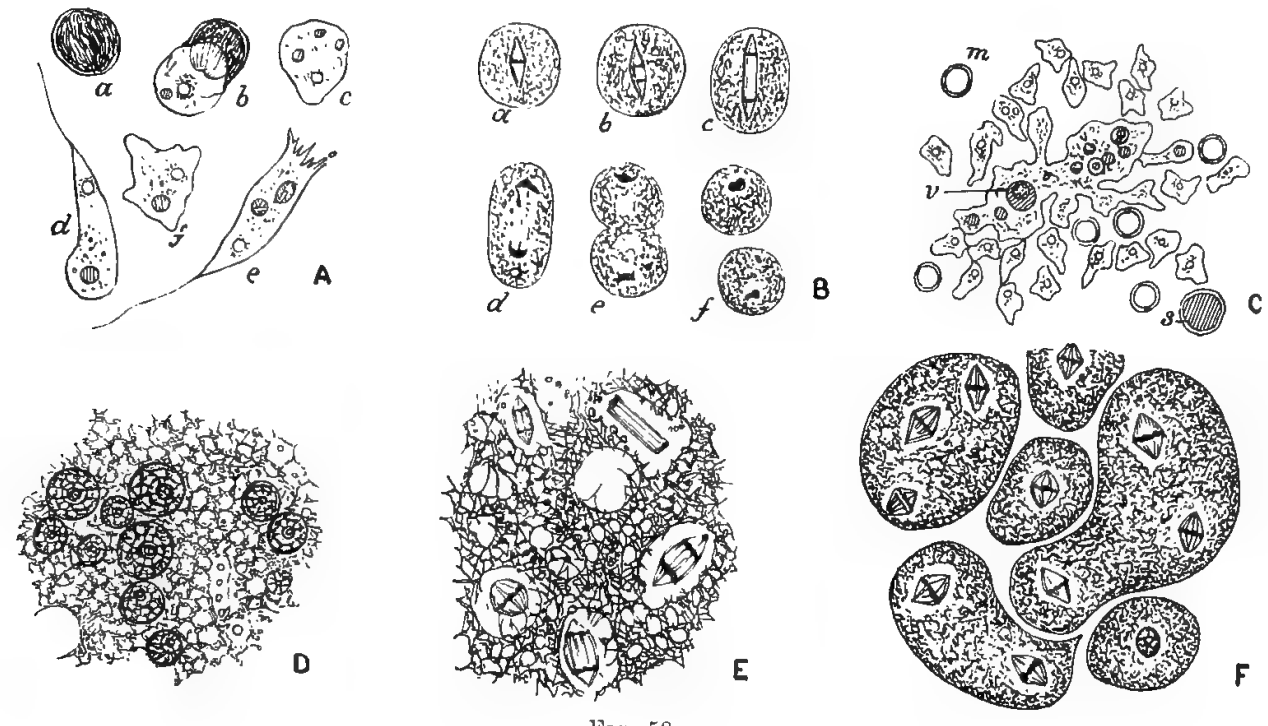

FiG. 58 .

FIG. 58.-Illustrates the history of certain British Mycetozoa.

A. Didymium difforme (Duby). a, Spore; $h$, swarm-cell escaping from the spore-case; $c$, newly hatched swarm-cell containing a nucleus and three vacuoles; $d$, flagellated swarm-cell; $e$, swarm-cell, with two vacuoles containing bacteria and produced at the posterior end into pseudopodia, to one of which a bacterium is attached; $f$, amcoboid swarm-cell. Magnified 450 times.

B. Amaurochcte atra (Rost). a to $f$, Successive stages in bipartition of swarm-cell, accompanied by the division of the nucleus by karyokinesis. Magnified 800 times. Drawn from stained preparations in Canada balsam.

C. Didymium difforme (Duby). Young plasmodium, with attendant amoeboid swarm-cells, some of which have turned into microcysts $(m)$; one microcyst is being digested in a vacuole $(r)$. An empty spore-shell is shown at $s$. Magnified 300 times.

D. Badhamia utricularis (Berk.). Group of nuclei from, actively feeding plasmodium that covered two pilei of A fourteen hours, showing the irregular size of the nuclei and large nucleoli. Stained in picro-carmine and monnted in Canada balsam. Magnified 800 times.

E. Badhamia utricularis (Berk.). Division of nuclei by karyokinesis in the streaming plasmodium. Magnified 800 times. From a preparation stained in safranin, and mounted in Canada balsam.

F. Comatricha obtusatc (Preuss). From a stained preparation of a young sporangium, showing the plasmodium separated into rounded masses about groups of nuclei, which are dividing by karyokinesis; the nuclear division has reached the "spindle stage." The spindles are seen in profile in all cases but one, in which the equatorial plate is seen from one of the poles of the spindle. Magnified 800 times.

movements. These amœboid bodies collect in clusters, and coalesce to form plasmodia, which may be described as masses of naked protoplasm abounding in nuclei and more or less coloured granules. The nuclei at this early stage appear to be those of the individual swarm-cells which have fused together. Careful investigations lead to the conciusion that the vast multiplication of nuclei which takes place during the growth of the plasmodium results from simple division. Apparently one instance only of division by karyokinesis in this stage of the plasmodium has been recorded, and of this observation permanent preparations are preserved. As the plasmodium increases in bulk by the ingestion of nutritive matter and by the union of small plasmodia, it acquires the remarkable streaming movement peculiar to itself. It penetrates the substance of dead wood or spreads over the surface of dead leaves, bark, \&c., in a network of veins, taking a somewhat fan-shaped outline (Fig. 59, B, C, D, E). Through these veins the more fluid matter in the interior streams constantly in a rhythmic flow. The current continues in one 
direction for a certain period, usually a minute and a half, when it stops, and after a moment's pause reverses its course, flowing the opposite way for about the same length of time, but rather longer in the direction in which the mass is advancing. ${ }^{1}$ It is by this means that the plasmodium creeps forward. The object of the onward movement appears to be a search for food. If the yellow plasmodium of Badhamia utricularis, which feeds on woody fungi, is cultivated on Stereum hirsutum, placed on a plate, and covered with a glass shade, it extends itself over the fungus with a turgid advancing border until it has devoured the more delicate hyphæ. Having exhausted the nutriment, it will spread over the plate and the interior of the glass shade. If it is in sufficient quantity, it will, in the course of a couple of days, cover the glass with a net-work of veins over an area of from forty to fifty square inches. A piece of fresh Stereum, soaked in water, may now be inserted beneath the shade, allowing it to come in contact with one of the smallest veins. In a few hours the whole of the plasmodium will have withdrawn from the sides of the shade, and concentrated itself on the fresh food in a dense yellow mass.

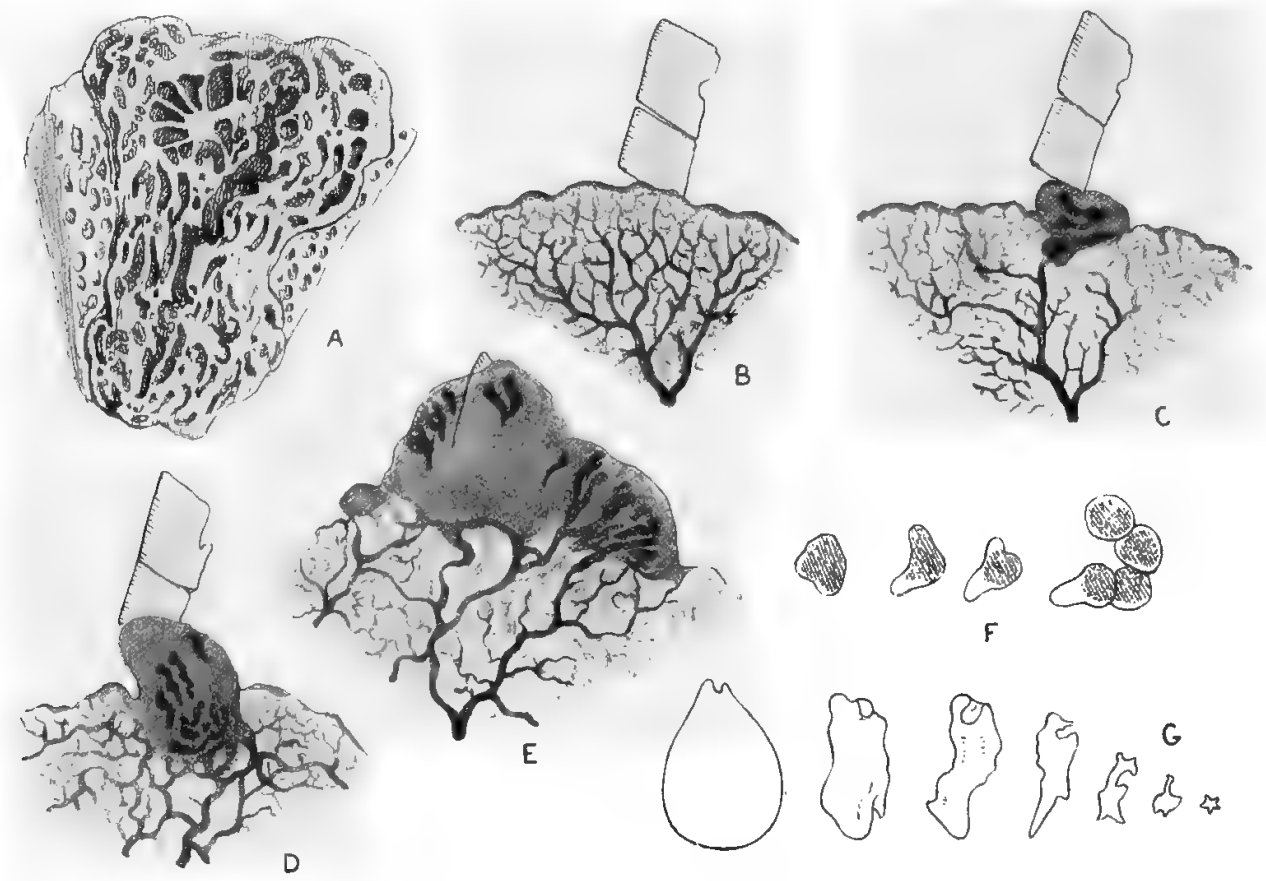

FIG. 59.

FIG. 59.-A. Sclerotium or dry state of Brdhemic utricularis, $\times 4$. Illustrates the resting, inactive condition of the plant.

B to E. Plasmodium of Brelhumiu utriruluris. Shows advance of plasmodium on section of stem of Ayaricus melleus. B, drawn at 12.25 P.M. ; C. at 12.40 P.M. . D, at 1 P.M. ; and E, at 2 P.M., $\times 3 \frac{1}{2}$.

F. Young spores of Bcodhrmit utricularis, showing amceboid movement; the lower spore at right hand of figure changing its shape in the course of a few minutes to that seen at extreme left of figure, $\times 565$. G. Vegetable substance dissolved by plasmodium of Badhamia utriculeris diminishing in size from left to right of figure, taken
at intervals of about a quarter of an hour, $\times 250$ (after Lister).

"The most remarkable activity of plasmodium hitherto witnessed was caused by a supply of Stereum provided in a glass box.

"There was at first, as not unfrequently happens, a shrinking backwards of the margin of the network of plasmodium, as if notice of the presence of a food-supply had been sent off to the more distant parts; then came on a quick stream of plasmodium, and in a quarter of an hour the whole side of the glass box was being covered with astonishing rapidity. The wide meshwork was not sufficient to conduct the abundant supply, and fresh veins started off in all directions, cutting up the broad meshes. At one time the current along them all was so precipitate that it was not possible to follow the course of the particles; they rushed across the field of the microscope at a speed that was truly amazing.

"It was a sight not soon to be forgotten; the marvellous exhibition of such active life in so low an organism was most impressive.

\footnotetext{
${ }^{1}$ These to and fro movements occur also in a considerable number of plants, and in the cil'ulation of the leech, polyp', and other low animal forms.
} 


\section{NEW VIEW OF THE MECHANISM OF RESPIRATION}

"The length of time during which the plasmodium will continue to feed and increase in bulk before changing jnto sporangia differs according to the species, and also to the conditions of its surroundings. Physarum psittacinum, which inhabits the rotten stumps of old trees, appears to pass twelve months in the plasmodium stage; on the other hand, Didymium difforme will go through the several stages from germination of the spores to the formation of the sporangia in a fortnight.

"The plasmodium, if allowed to dry, passes into the sclerotium or resting stage (Fig. 59, A). The sclerotium of Badhamia utricularis is dull orange-red in colour, of horny consistence, and made up of a multitude of thin-walled cysts closely packed together; each cyst being filled with granular protoplasm, among which ten to twenty nuclei are interspersed. On being wetted the sclerotium will revive in the course of a few hours and resume the streaming movement. Preserved in a dry state, it will retain its vitality for three or four years, but it is longer in reviving according to the length of time it has remained in the resting condition.

"Carbonate of lime is usually abundant in the plasmodium and young sporangia of the species comprised in the sub-cohort Calcarines. In most genera of the order Physaracese these granules are withdrawn from the plasma before the spores are formed, and are deposited partly in the sporangium-wall, and partly in vesicular expansions of the capillitium. These expansions take various shapes : they are globose, fusiform, or branched, and are sometimes fused together in the centre of the sporangium, forming a pseudo-columella. In the descriptions of the species they are termed lime-knots. In the Didymiacer the granules in the young sporangia are dissolved at a certain stage, and the salt forms again in crystals on the outside of the wall.

"The sub-class Exospore? is represented by the single genus Ceratiomyxa (Fig. 60, A to H), and is characterised by the numerous white spores being borne on the outside of columnar or branching sporophores. These are delicate, fragile structures, often covering two or three square inches of the dead wood on which they grow. The surface of the sporophore is mapped out into polyhedral areolæ, from the centre of each of which arises a slender stalk bearing a single ellipsoid spore. On placing the ripe spores in water, the membranous spore-wall at once slips off, and the naked contents lie for several hours without apparent change, retaining their ellipsoid form. A constriction then takes place at right angles to the long axis, and before division is completed a second constriction of each half occurs; each of the four lobes thus formed again becomes constricted, and we have eight globular bodies adhering together and exhibiting slow amcboid movement; each of these bodies now produces a flagellum, and the cluster swims away by the aid of the lashing flagella.

"Experiment shows that, whatever may be the digestive principle of plasmodium (possibly a peptonising ferment, as suggested by Krukenberg), it is not confined to any special part of the mass. With starch and the sections of agarics the absorption took place in the streaming interior, while in the case last related it occurred in the hyaloplasm alone; the threads were completely dissolved in the hyaline margin, with the exception of the small fragments referred to, which were kept under constant observation until they were almost ejected by the far-retreating plasmodium." 1

The movements of Badhamia utricularis, especially the advancing movements, afford a striking example of the aggressive nature of life. Living plants and animals continually and habitually project themselves against objects which they incorporate and ultimately assimilate; a due selection of objects being in every case made. The objects approached, incorporated, and assimilated, being for the most part dead, cannot assume an active role. It is, in every instance, the prerogative of the living thing to advance, and it does so, in all cases, as an invader. The plasmodium of Badhamia advances, and in so doing it exerts what is virtually a pushing, elongating, dilating, and centrifugal power: it also recedes, and when falling back it pulls itself together and exerts a pulling, shortening, contracting, and centripetal power. These two movements, which are complemental, are fundamental and indispensable in all living things. They occur in the substance of the amoba, in pseudopodia, and in the voluntary and involuntary muscles; these substances being invested, as I long ago explained, ${ }^{2}$ with the double power of elongating and shortening, and being very largely concerned in seizing and dismissing food and other materials in the economy of the individual; they occur in the respiratory, circulatory, and other rhythmic movements, of which they form

1 "Notes on the Plasmodium of Badhamia utricularis and Brefeldio maxima. (Guide to the British Mycetozoa." London, 1895. See also Annals of Botany for June 1888.

" "On the Physiology of the Circulation in Flants, in the Lower Animals, and in Man," from pages 184 to 243 inchusive. Macmillan \& Co., London, 1874 ; originally published in the Edinburgh Medical Jourual in 1872. 
the distinguishing feature; they are the counterparts of endosmose and exosmose, and of all the give-and-take movements, which are necessary to absorption and assimilation, to secretion and excretion. They are the ways and means which enable plants and animals to take in their natural pabulum, whether solids, liquids, or gases, and to give out the detritus or waste products, which are a consequence of every form of metabolism in living things.

When the mass of plasmodium advances and retires it is permeated by a large number of active, branching streams; the currents of which are, in many cases, extremely rapid. The currents of the larger streams are swifter than those of the smaller ones. When any stream meets with an obstruction which it cannot remove, collateral streams are at once established, which impresses one with the idea that the streams are under control, and can be increased and diminished at pleasure. They apparently can also be quickened and slowed. The collateral streams resemble those which follow the ligature or obstruction of an artery. The appearance presented by the streams and currents in question reminds us of analogous currents witnessed in growing tissues, where branching vessels, the result of cell formation and absorption, appear in suitable positions in definite areas.

Examples of these currents and formations are seen in the developing ovum, chick, bone, \&c.

This is a subject fraught with great interest, as the stream formations of plasmodium are vital and purposive, like the streams of blood and other fluids in young tissues. They are also dendritic in character; the dendritic arrangement not being confined to the organic kingdom, but extending to the inorganic kingdom. As a matter of fact, the dendritic arrangements and formations may be the outcome of either vital or physical force. The dendritic streams of plasmodium, the branching streams of fluids in plants and animals (with or without vessels), and the dendritic flow of nerve impulses in branching nerve cells are essentially vital in their nature; the dendritics of lightning and of frost on window-panes, pavements, \&c., being, on the other hand, purely physical. As has been already explained, the living and dead are inextricably blended, and life can only work through death and dead material. Moreover, and as already stated, when living matter appropriates dead matter it also appropriates, within limits, the force which inheres in the appropriated matter, so that there is a certain degree of "oneness" in the forms and movements of organic and inorganic matter.

This explains, to a large extent, the symmetry of structure which prevails in plants and animals and in many inorganic forms.

The curious and striking phenomena exhibited by the plasmodium of Badhamia utricularis can be most conveniently studied by cultivating portions of the organism in glass cases and beneath bell jars, as under these circumstances the specimens can be examined at all times by the naked eye or by the aid of the microscope. They can also be voluntarily thrown into the inactive, resting or sclerotium stage, or into the advancing, aggressive, active stage.

It is a surprising fact that an organism composed largely of protoplasm with an admixture of cells, nuclei, and nucleoli and no well-defined structure, can exhibit such a variety of movements and discharge such a multiplicity of functions; and, it appears to me, the time has now come when fresh and exhaustive studies of life in its most rudimentary and lowest forms must be made, if life in its highest manifestations is to be intelligently estimated. That masses of living material, large or small, protoplasmic or otherwise, should display amoeboid and rhythmic movements, should wake and sleep or hibernate, should feed and discharge waste products at intervals, should virtually secrete and excrete, should reproduce and perpetuate themselves, and, in a way, discharge all the functions discharged by the highest animals, are matters of primary importance in every form of biological and physiological inquiry.

As a matter of fact, the higher plants and animals are, numerically, exceedingly small as compared with the teeming millions of lower plant and animal forms everywhere met with-animalcules, bacteria, microbes, \&c., \&c. In all of these, living matter and function and the potentialities of both can be witnessed and studied. The amount of life on the earth is incredibly great, but life does not exist as apart from structure and function; differentiation occurring in both until man is reached. Without the potentialities of living matter no progress could be made either as regards structure or function; hence the absolute necessity for studying living matter in its simplest forms and most incipient beginnings.

The difficulties connected with this subject are well-nigh insuperable, alike because of the number and minuteness of the organisms to be investigated. To take one example. Microbes in malaria and other fevers swarm in the blood and actually inhabit the red blood-corpuscles, which corpuscles in man are so minute that they

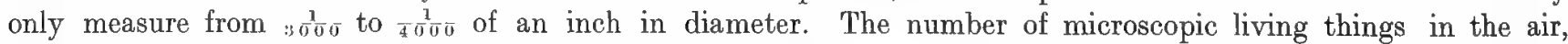
the water, and the soil is legion.

In these the very mainsprings of existence are concealed. In plants and the lower and lowest animals are stored up in a latent or potential form the undefined structures and functions which characterise the highest animals, man included. In the highest animals we are called upon to deal with well-defined, differentiated structures in 
great variety; in the lowest animals it is difficult or impossible to trace differentiation, but it cannot be doubted that it exists.

The fact that the simplest living forms and the ovum are heterogeneous goes far to prove that differentiation of a kind occurs in every department of the organic kingdom, and from the first. Without differentiation, potential or actual, no advance whatever could be made in the development of even the simplest living form, and differentiation, which means division of labour in particular directions and to given ends, goes on through the whole animal series up to man himself. The point here raised is not one of words but of actual fact. In the lowest plants and animals, differentiation of a kind is always present. There is always a sufficient amount for the perfect adaptation of the plant and the animal to their surroundings. There is in them a potential epitome of life. They live, can reproduce themselves, take in food, discharge effete matter, circulate and aërate their nutritious juices, respire, secrete and excrete, \&c. By far the greater number of the functions performed by the highest animals are performed by the lowest. It, however, does not follow that the higher and highest animals are manufactured by a process of evolution out of the lower and lowest. On the contrary, each animal only reproduces its kind. The lowest plants and animals are apparently inextricably mixed up, but this causes no confusion, as law, order, and design everywhere prevail. The means of classification are also assured. As plants and animals only reproduce their kind, so they are arranged according to types and in an ascending series; a state of matters which admits of improvement up to a point, but does not result in the obliteration, sooner or later, of species, genera, and families, which are persistent to an almost incredible extent.

The peculiarities of the lowest plants and animals, in one sense, lie on the surface; in another and wider sense, they are studiously concealed, and require for their expiscation the aid of experience, and an extensive knowledge not only of particular groups but also of the groups as a whole. The wider the knowledge, the safer the deductions. In the borderland occupied by the lowest plants and animals, the intricate path has to be threaded with great care. As explained in the early part of this work, there is a visible and an invisible world-a world of causes and a world of effects. In the visible and invisible, the realms of plants and animals must also be included, as in them cause and effect have free play.

There is nothing in the highest plants and animals which does not have its roots in the apparently undifferentiated protoplasm of the lowest plants and animals. The prevailing view is that in plant and animal protoplasm there is no differentiation, and that the process of building up a plant or an animal begins by a division or splitting up of the so-called homogeneous protoplasm (ovum or otherwise). This cannot be regarded as a full, final, or satisfactory explanation. The question remains: What causes the primary division alluded to ? This is not an accidental process. It occurs at a given period, and always in the same way. The effect is obvious; the cause is, so far, inexplicable. If we were to seek for the cause in an apparently homogeneous impregnated ovum we would have nothing tangible to show. The cause, like other causes, belongs to the invisible world. It is not, however, doubtful that a cause exists. What is said of the original cleavage of the ovum applies to all subsequent cleavages which result in differentiation. It is maintained by the great majority of biologists and physiologists of the present day that the original cleavage is followed by subsequent cleavages of greater or less magnitude; each cleavage inaugurating a progressive series of changes, which result in the development and final completion of the plant and animal respectively. But (and this is the crucial point) none of the subsequent cleavages and changes have any better explanation to give of themselves than had the original cleavage, which, as explained, is altogether inscrutable. Differentiation in plants and animal textures is based on a hypothesis, and that hypothesis fails to explain the most important fact connected with reproduction and the earliest stages of development. It is easy to say that the ovum is homogeneous (I believe it to be heterogeneous), that it spontaneously divides, that a set of primary changes is induced which begets a second set, that the second set begets a third set, and that any changes which occur in it are self-induced.

All this is beside the question. An answer has still to be given to the query, Who or what begins the division and the differentiation? It is denied that the impregnated ovum is a microcosm, but it cannot be shown that one differentiation leads to or produces another. There must be a Power outside or beyond the ovum, and that Power represents design and the very essence of wisdom. It is present at the inception of every plant and animal, and is the major factor in the life history of everything that exists.

Design, and design alone, can satisfactorily explain all that comes under reproduction, development, and life.

It would be difficult to find more illustrative examples of lusty life in a low organism than are furnished by Badhamia utricularis and Brefeldia maxima.

A consideration of these strange plant-animals throws a strong light on the so-called vegetative processes in the higher animals, man included. Their workings and manifestations are all vital in their nature. They are also fundamental and independent. Their movements (rhythmic and amobic) are inherent; their modes of throwing VOL. I. 
themselves upon food, and of seizing, digesting, absorbing, and assimilating it, are in no sense the result of irritation, of stimulation, or of accident. All that can be said is, that they are living entities, and move to given ends; these ends being (within limits) pre-determined. Similar remarks may be made of all plants and animals. They are, to a large extent, superior to their surroundings and environment; they are (when normal) in no instance jogged into activity by what are known as irritability and stimuli ; neither do they owe their existence to a fortuitous aggregation of accidental parts and particles (spontaneous generation), as apart from law, order, design, and preexistent parents.

Examples of low parasitic forms as they occur in various diseases are given at Figs. 61 and 62, and specimens of rudimentary plants and animals are delineated at Plates lxxix., lxxx., lxxxi., and lxxxii.

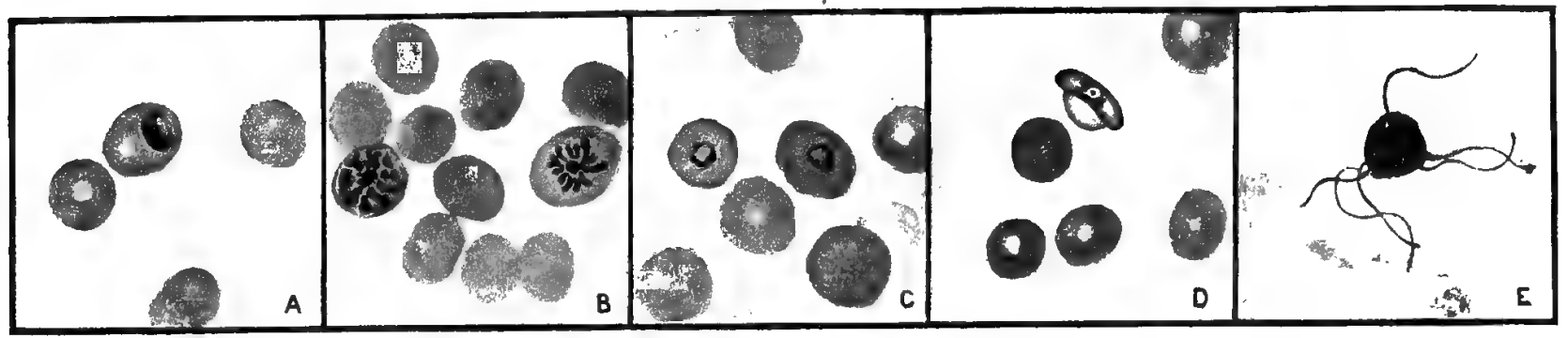

Fif. 61 .

Fig. 61.-Illustrates the parasites seen within the red blood-corpuscles in malarial fever. Magnified 1000 diameters (after Manson).

A. Early intra-corpuscles form of "mild tertian" parasite,

B. Two members of the "rosette" series of the same parasite; that to the right shows the radiate segmentation, that to the left is an earlier stage. In both the pigment is collected in the centre.

C. Two "ring-forms" of the quotidian parasite, within the red blood-corpuscles.

D. Shows a "crescentic body," from a case of malignant tertian fever.

E. A flagellated organism, derived outside the body from a crescentic form.

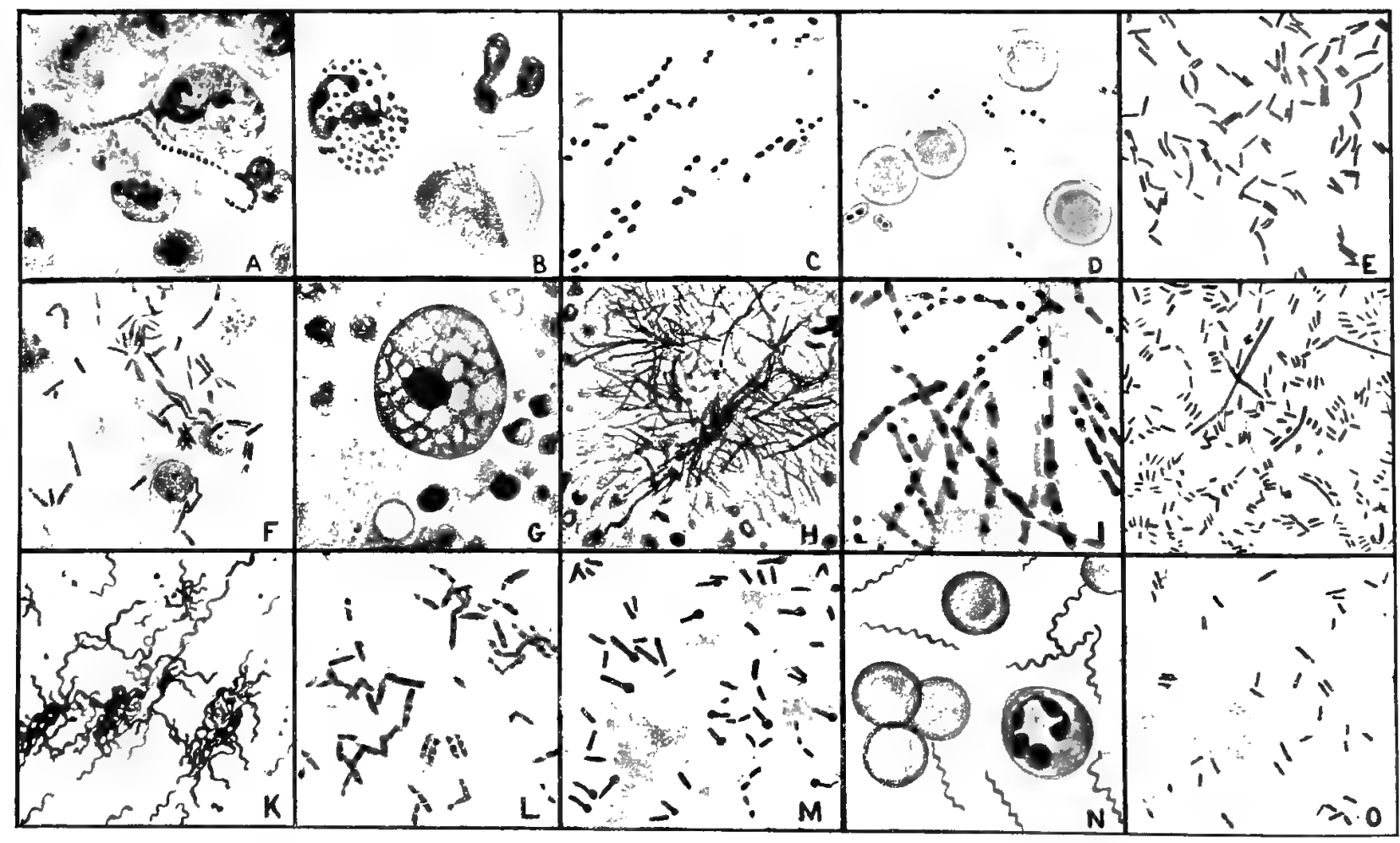

FI $: 62$.

FIG. 62.- Illustrates various forms-dotted, rod-shaped, branching, spiral, \&c, -of bacteria as seen in diseased conditions. Marnified 1000 diameters (after Muir and Ritchie).

A. Streptocroci of acute suppuration. 
FIG. 62 (continued)-

B. Gonococci in gonorrhueal pus-white blood-corpuscles invaded.

C. Pneumococci with capsules arranged in chains as seen in pneumonic sputum.

D. Capsulated pneumococci in blood taken from the heart of a rabbit inoculated with pneumonic sputum.

E. Tubercle bacilli (culture on glycerine agar).

F. Tubercle bacilli from phthisical sputum.

G. Spherical amoeba from the wall of a liver abscess, showing nucleus and vacuolatod protoplasm.

H. Actinomycosis from human liver, displaying a branched felted mass of parasite surrounded by pus.

I. Anthrax bacilli (culture on agar). Contains spores - the darkly-coloured bodies.

J. Typhoid bacilli (culture on agar). Shows filamentous forms.

K. Typhoid bacilli, showing flagella.

L. Diphtheria bacilli (agar culture).

M. Tetanus bacilli, some of which possess spores.

N. Spirilla with red and white blood-corpuscles in relapsing fever (human).

O. Glanders bacilli culture. Shows segmentation of protoplasm.

\section{PLA'TE LXXIX}

Plate lxxix. shows rudimentary plant and animal forms. Illustrates spherical, concentric, radiating, branching, spiral, and segmented arrangements.

FIG. 1.-Sarcina ientriculi. Low form of plant found in the human stomach-magnified $(a)$ 1000, and (b) 250 diameters (Goodsir)

FIG. 2.-Palmella cruenta. a, Patch of jelly with single cells, and dividing and divided paírs, $\times 400 ; b$, similar cells without the gelatinous layer, the smaller granules similar to those seen in the jelly of $a, \times 800 ; c$, cells treated with sulphuric acid and iodine, showing the cellulose coat and granular contents, $\times 800$ (after Griffith and Henfrey).

FIG. 3.-Chlorococcum vulgare. $\alpha$, Group in natural condition, $\times 800 ; b$, an isolated cell, showing granular contents, $\times 1500$; $c$, dividing cells treated with sulphuric acid and iodine, $\times 1500$ (after Grifith and Henfrey).

FIG. 4-A, B, C, Wucor mucedo stages in the formation of a zygospore; D, a sporangium containing spores; E, Oidium lactis; F, Aspergillus glaucus-mycelium (1), gonidiophore (2), and $\mathrm{H}$ bearing spores ; a perithecium (3) and G-the latter containing rudimentary asci; piece of gonidiophore $(\mathbf{J})$ with sterigma $(a)$, and spore $(b) ; \mathbf{K}$, branched gonidiophore of Penicillium glaucum bearing spores; L, M, Sactheromyces cerevisizecells budding; $\mathbf{N}$, the same showing the formation of endospores (after Hansen).

FIG. 5.-Protococe viridis. a, Group of cells, the upper one with eight in a linear series; those to the right $\left(a^{\prime}\right)$, dividing into numerous gonidia; $b$, zoospores set free from the cells by the solution of the cellulose membrane; $c$, an isolated cell dividing, and about to set free its contents as two zoospores; $d$, resting cells with a thick coat and reddish contents; $e$, a zoospore with the cilia cast off ; $f$, zoospore with imperfect or retracted cilia, $\times 400$ (after Griffith and Henfrey).

Fig. 6.-Blood-corpuscles of the cray-fish (Astacus fluviatilis), magnified 1000 diameters. 1 to 8 show the amoboid movements occurring in a single corpusele in a quarter of an hour ; 9 and 10 are corpuscles killed by magenta and having the nucleus deeply stained by the colouring matter; ", nucleus (Huxley).

Fict. 7.-Trichina spiralis, magnified 50 diameters (after Dulton). Encysted worm occasionally found in human muscle, spirally coiled up.

Frg. 8.-Gromia terricola, greatly magnified (after Leidy). Shows an amazing display of sensitive living sarcode streaming and radiating by centrifugal movements from the body of the animal, which is encased in a horny shield. The streams of sarcode (pseudopodia) emerge from apertures in the shield ; they sometimes proceed in straight lines and sometimes branch and form networks. They seize whatever edible particles float in the water in which they are immersed and which come within their reach. These they quickly transfer to the interior of the bódy by centripetal movements. In the whole range of natural history there is no more remarkable example of aggressive, vital, purposive movements. The streaming of Gromia greatly resembles the streaming of the plasmodium of the Mycetozoa. In both cases the movement is an active, aggressive, spontaneous one, essentially rhythmic in character, and consists of an advancing or centrifugal act (extension in space), and a retreating or centripetal act (retraction in space). The centrifugal and centripetal rhythmic movements, in one form or other, may be traced throughout the entire inorganic and organic kingdoms; life without them would be impossible.

The movements in question are seen in endosmose and exosmose, in the formation and disintegration of crystals, in the condensation and rarefaction of nebulr, in cilia, in hollow and solid muscles, in respiration, alimentation, reproduction, circula tion, \&c. (Compare with Fig. 3 of Plate Ixxx.)

FIG. 9.-Amoba diffuens, commonly known as proteus from its continually changing its shape. It affords an example of the lowest animal form. Magnified 400 diameters (after Dallas and Jeftrey Bell). Consists of a granular jelly mass of sarcode with a contractile vesicle and nucleus seen at $\mathrm{D}, \mathrm{J}$, and $\mathrm{G}$, but no other trace of structure. The amoeba possesses the remarkable power of assuming an infinite variety of shapes by rhythmic streaming, as indicated at A, B, C, D, E, F, G. This it does by alternately pushing out and withdrawing finger-like conical processes by centrifugal and centripetal movements, voluntary in character. The creature can slowly advance its whole body, or a portion thereof, in search of food, which, when found, is enveloped as seen at $\mathbf{B}$. That portion of the body in contact with the food forms a temporary stomach. The animal can move to definite ends as in Gromia (Plate lxxix., Fig. 8). It reproduces itself by simple division. The centrifugal and centripetal rhythmic movements have much significance when viewed in connection with similar movements occurring in the sarcous elements of muscles, in the movements of extension and flexion, and in the opening and closing of simple and compound hearts, as fully explained further on. 
PLATE LXXIX

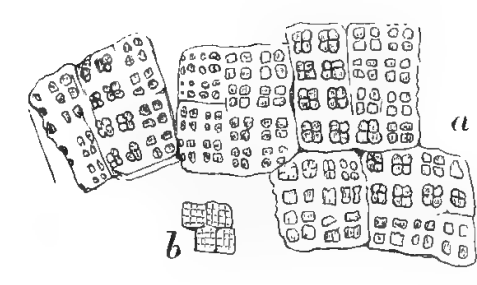

Frg. 1.

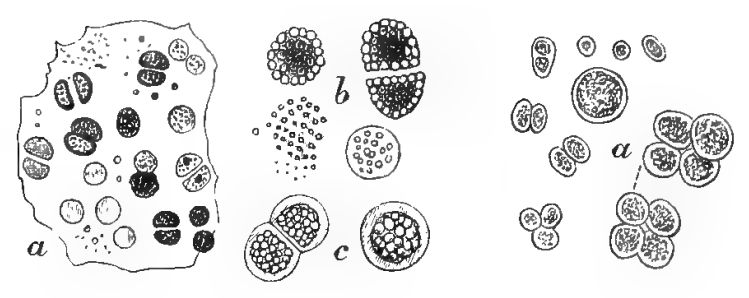

Fig. 2.

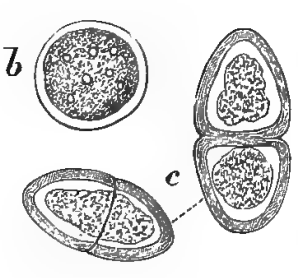

Fig. 3.
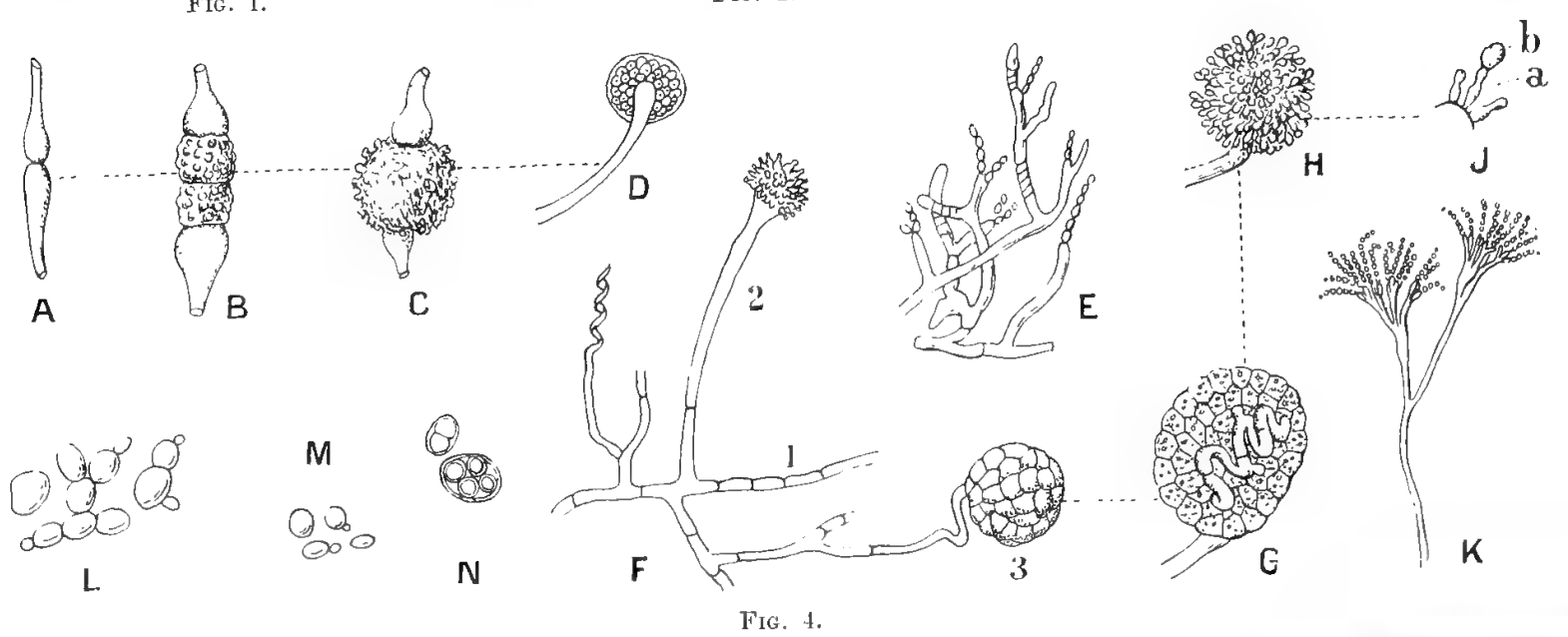

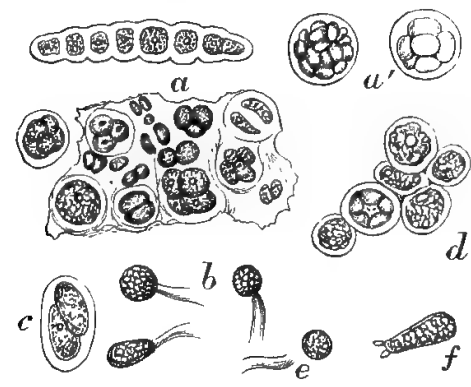

FIG. 5.

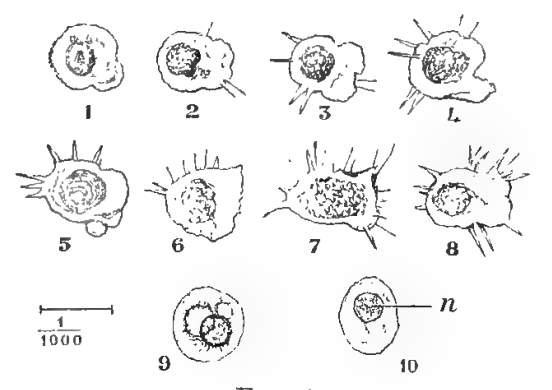

FIG. (i.

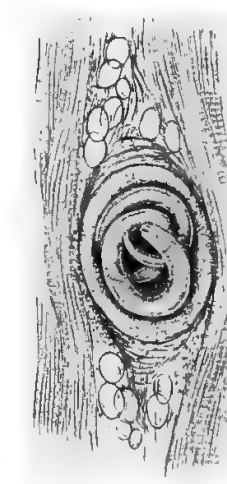

Fig. $\succsim$.

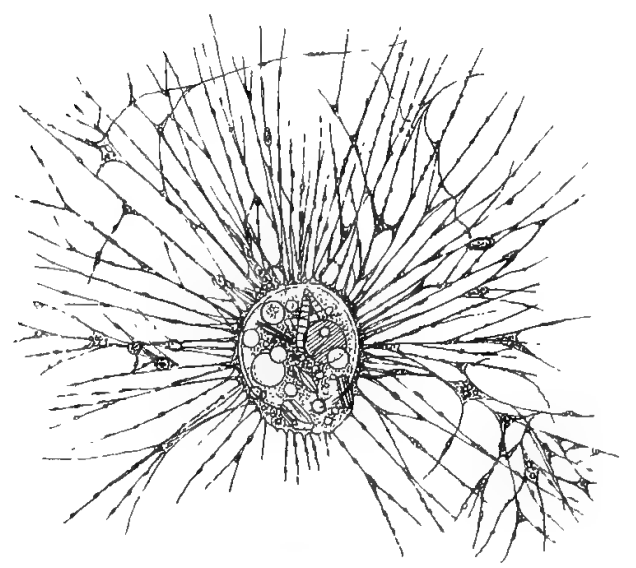

FIG. 8.

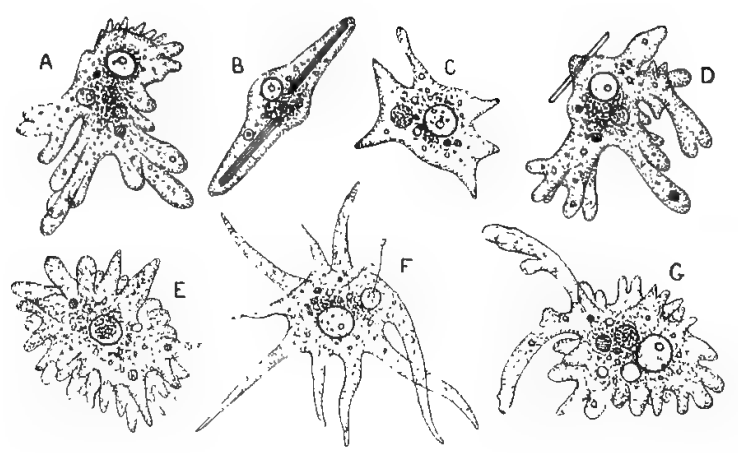

FIG. 9 . 
PLATE LXXX

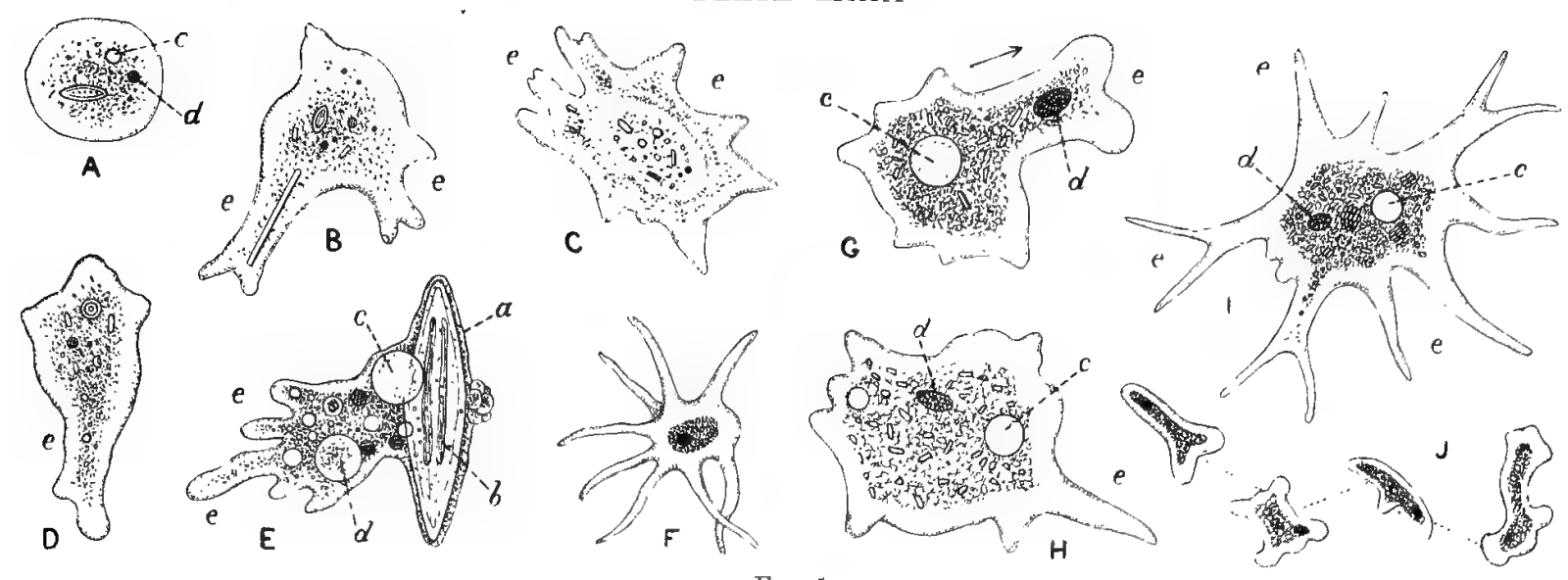

Fir, 1.

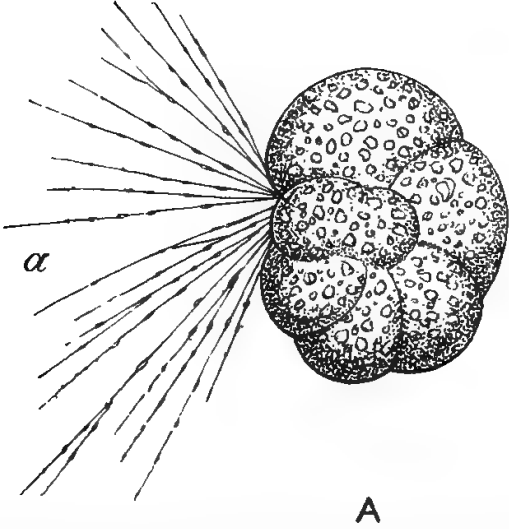

A

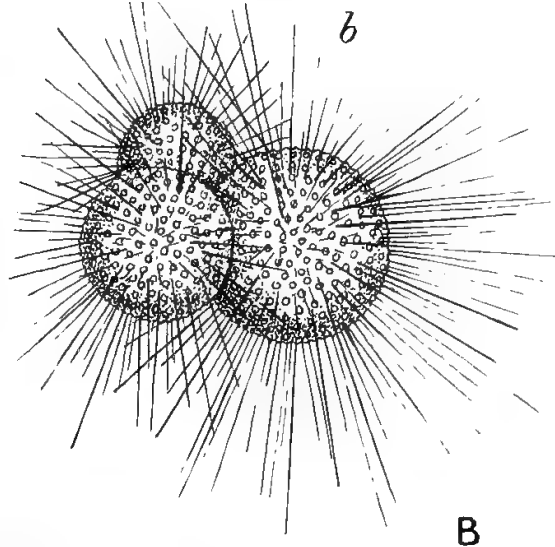

FIษ: 2.

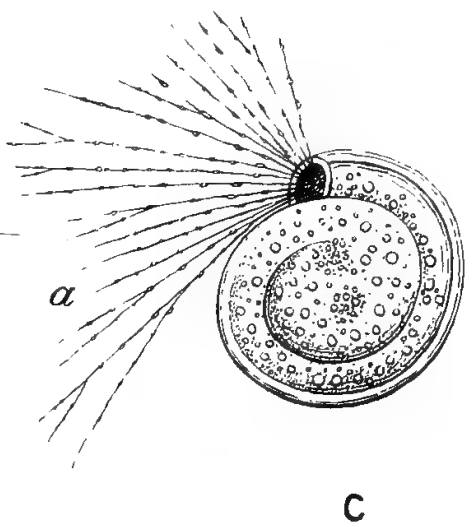

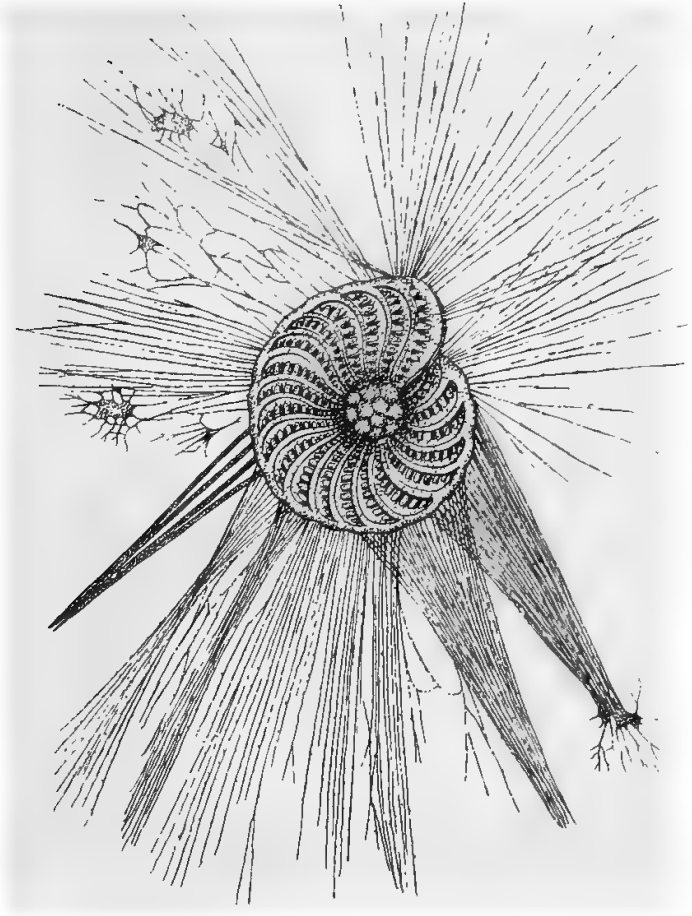

FIG. 3.

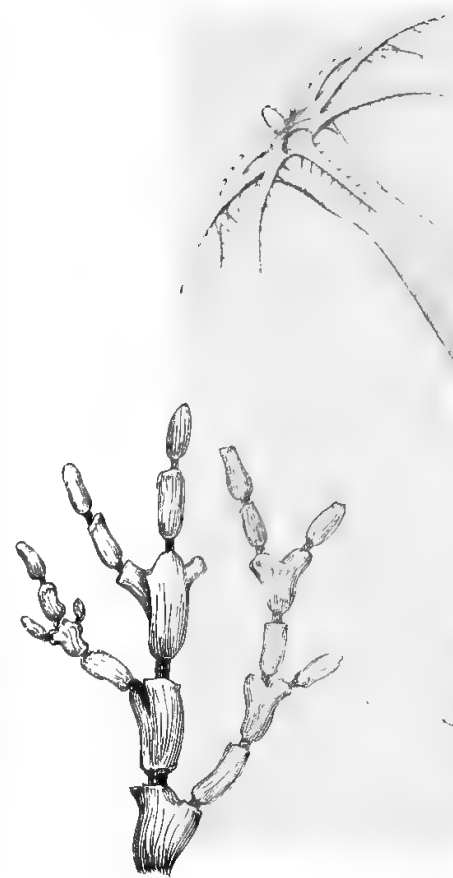

FIG. 4.

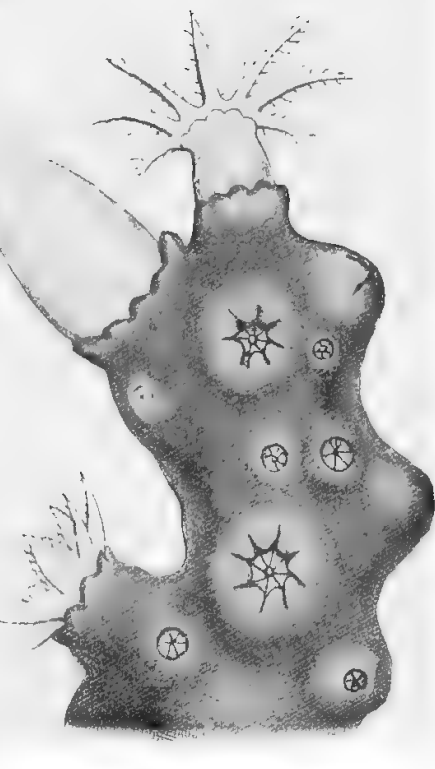

Fig. 5. 


\section{PLATE LXXX}

Plate Ixxx. illustrates radiating, concentric, spherical, branching, spiral, and segmented arrangements in rudimentary animal forms.

FIG. 1.-Amaba protus, as seen under varying conditions of size and shape (A to J), magnified from 400 to 800 diameters (after Masterman, and Marshall and Hurst).

A. Amoba in the encysted state, composed mainly of granular and clear sarcode; $i$, contractile vesicle; $d$, nucleus. It contains an oval-shaped diatom.

B to J. Different shapes assumed by larger and smaller amcebæ. In these figures the contractile vesicle is seen at $c$; the nucleus at $d$, and the finger-like projections at $e$. The projections, sometimes designated pseudopodia, are for the most part conical in shape, as seen at C, D, F, G, H, and I. They are caused by the animal voluntarily forcing a part of its body outwards from the centre centrifugally. They are retracted in an opposite direction or centripetally. They are not due to contractions : if they werc, the projections would not be conical-shaped but constricted where united to the body. In reality, they are produced by an aggressive, streaming, rhythmic movement similar to what occurs in the plasmodium of the Mycetozoa and Gromia already described.

At $\mathbf{E}$, the body $(a)$ of an amoeba is stretched over a large diatom (h).

At $J$, the varying forms assumed by a small amoeba, at intervals of half a minute, are given.

FIG. 2,Foraminifera magnified showing radiating and branched movements in the living sarcode $(", b, c)$. These movements represent lines of force which act in two directions-namely, centrifugally when the sarcode is extended from the mouth of the shell, or through the perforations in the shell into the water to secure food; and centripetally when the sarcode is retracted and carries the food into the body through the mouth of the shell or' the perforations in the shell referred to. These movements are due to vital streaming, as in the Mycetozoa, and are rhythmical in character. They are give and take, cone and go, alternating movements, and are voluntary and of set purpose; the purpose being the securing of nourishment. They are, in no sense, the result of irritability or extraneous stimulation.

A. Nonionina silicea.

B. Globigerina bulloides (var. triloba).

C. Miliolina-young specimen (after Chapman).

Frg. 3.-Polystomella, one of the perforate foraminifera, magnified (after Max Schultze).

Provides beautiful examples of radiating, branched, and reticulated movements in the living sarcode (pseudopodia), by which food is caught and carried into the interior of the body. The sarcode streams through small apertures in the shell of the animal centrifugally and centripetally. It would be difficult to conceive a more delicate and perfect arrangement for securing food when its minute and fragile nature is taken into account. Some of the captured food is seen entangled in the meshes formed by the semifluid sarcode. This foraminifer, like the others already described (Fig. 8, Plate lxxix.), is endowed with voluntary, centrifugal, and centripetal, rhythmic movements whereby it can extend its sensitive living substance into the water in search of food, and retract or withdraw it into the body as soon as the food is secured. The streaming movements of the sarcode can be timed and regulated by the animal with the greatest nicety. The movements cannot be traced either to contractility, irritability, or extraneous stimulation.

FIt. 4.-Part of the axis of Isis hippuris, a form of polyp inhabiting the Indian seas. The axis consists of calcareous joints pieced together by horny rings. Shows segmented, radiating, branched arrangements.

FIG. 5.-Three polyps of red coral in various stages of expansion (after Lacaze-Duthiers). Illustrate radiating, expanding, dilating arrangements, as seen in many flowers, and in the hollow viscera of animals, particularly the heart. The expansion and closing movements are voluntary and time regulated-that is, they occur at certain intervals and in a given order. They are essentially rhythnic in character, and are mexns to ends. The coral feeds when its polyps are expanded, and rests when they are retracted and closed. The expanding or opening centrifugal, and the retracting or closing centripetal, movements are inherent vital movements, and take place as apart from irritability and external stimulation.

\section{PLATE LXXXI}

Plate Ixxxi. shows rudimentary animal forms. Illustrates radiating, concentric, branching, spiral, and segmented arrangements.

FIG. 1.-Red coral "blossoming" (after Lacize-Duthiers). In this case the polyps are more or less fully expanded. They present a stellate, flower-like, symmetrical appearance. They also resemble certain crystals. The coral, as here depicted, is an object of great beauty; the solid substance or skeleton of the coral contrasting finely with its delicate filmy-looking polyps. What is said of Fig. 5 applies also to this figure.

Fí. 2.-Pentacrinoid larva of the rosy feather star (Antedon bifida) - a kind of star-fish resembling in the young condition the beautiful sea lilies (after Herbert Carpenter). The larva is provided with a long flexible stalk formed of calcareous cylinders. In the mature state the stalk is abandoned, and the star-fish creeps freely about the bottom. The body is provided with beautiful slender curled arms, furnished with elegant jointed cirri, by the aid of which and the short ambulacra they secure their food.

FIG. 3.-Obelia-genus Campanulariide (Hydroza) ; reproduces itself by free medusiform zooids.

A. Obelia greatly enlarged (after Parker and Haswell).

B, C. Medusse of Ohelie.

D. The same everted. ", Common body tissue; l, ectoderm; c, endoderm; $d$, stomach ; $e$, generative capsules ; $f$, horny cups ; ! $(\mathrm{C})$, lithocyst ; $h(\mathrm{~A})$, medusa bud; $i(\mathrm{~B}, \mathrm{C}, \mathrm{D})$, manubrium; $j(\mathrm{~A})$, three feeding polyps ; $k$ (C), radial canal ; $l(\mathrm{D})$, tentacle; $m(\mathrm{C})$,
velum.

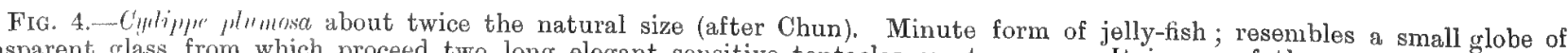
transparent glass, from which proceed two long elegant sensitive tentacles or streamers. It is one of the most beautiful and 
PLATE LXXXI

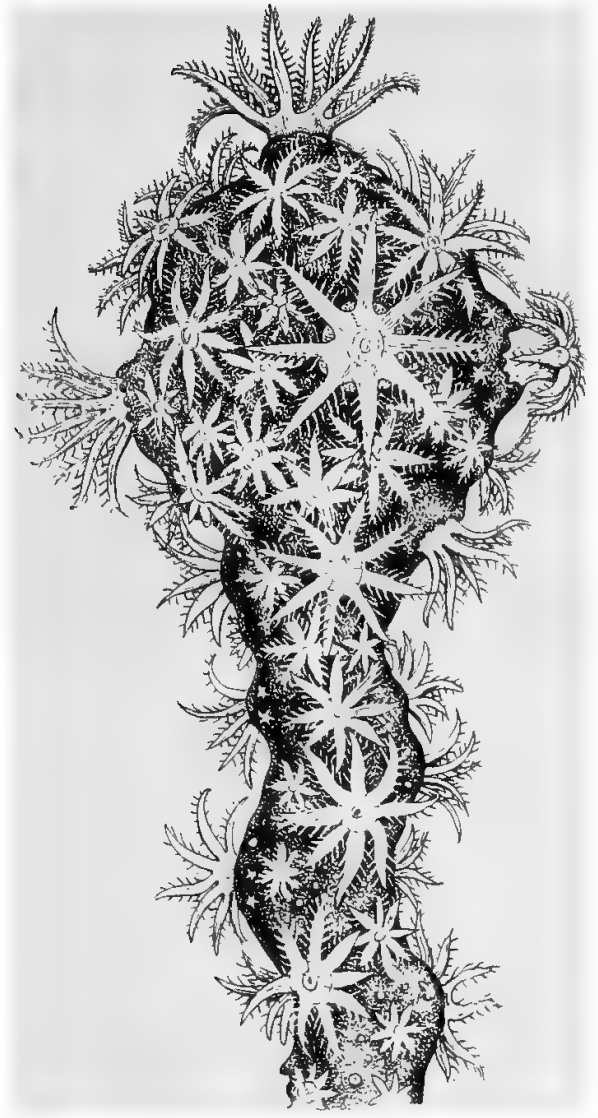

FIg. 1.

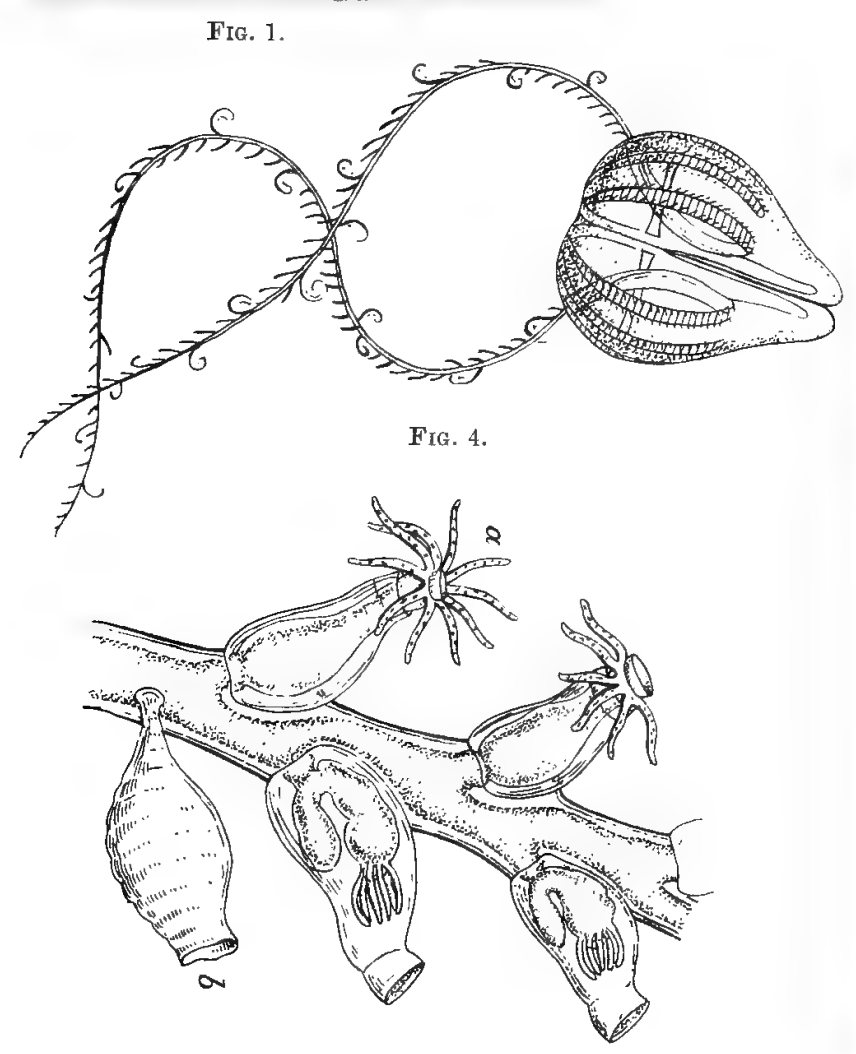

FIG. 5 .

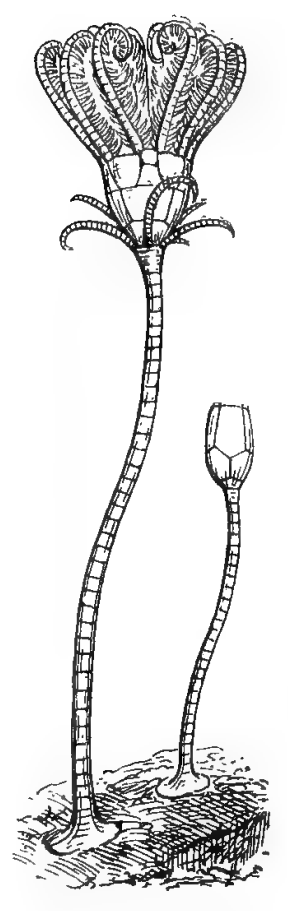

FIG. 2.

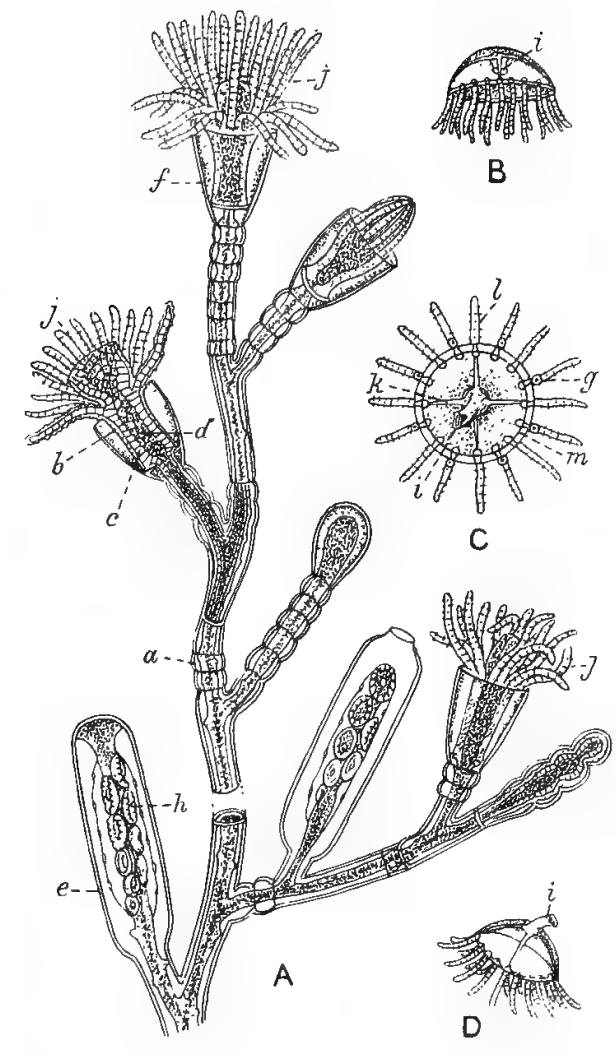

F'JG. 3.

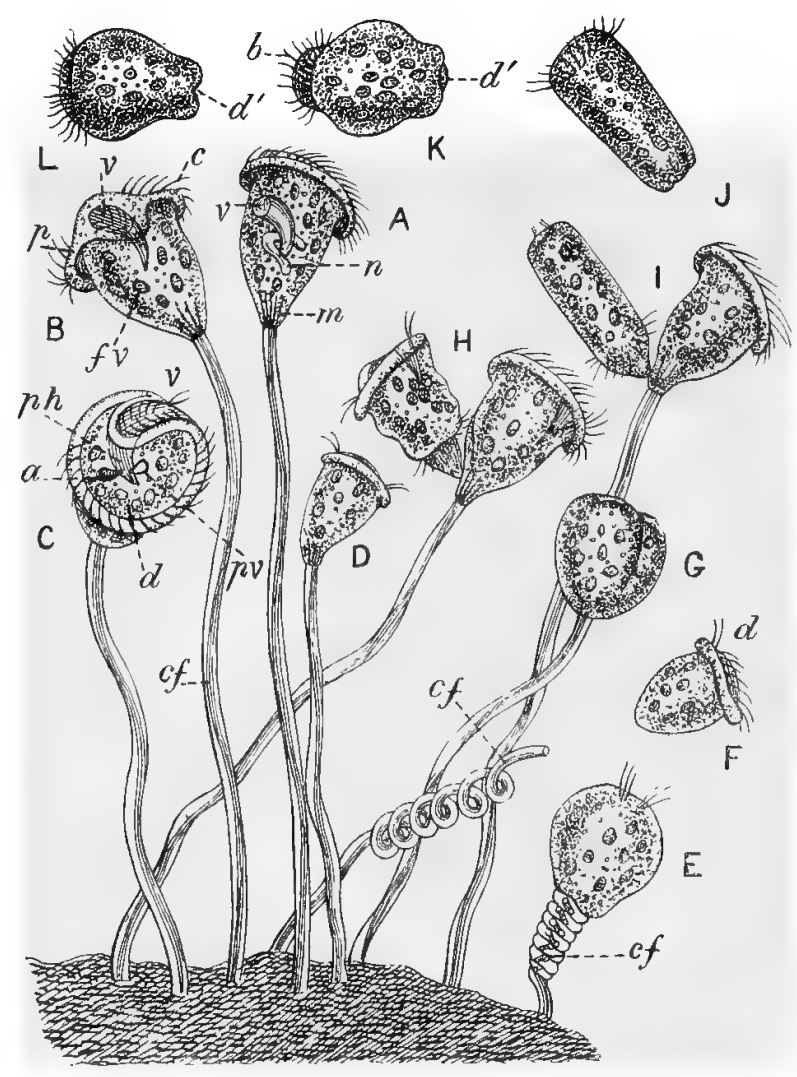

FIG. $b$. 


\section{PLATE LXXXI (continued)}

active little objects found in the sea. It swims by the aid of cilia; its movements being steadied by the two tentacles which bear small branches covered with spirally stalked adhesive cells.

FIG. 5.-Branchlet of Sertularic abietina-magnified (Guide-Book, British Musenm). ", Polyp; l, reproductive capsule. Illustrates radiating, globular, and branched arrangements.

FIG. 6.-A group of Vorticellæ magnified 220 diameters (after Marshall and Hurst).

A, B, C. Show the animal in various positions.

D. A much smaller specimen drawn to the same scale.

E. A specimen made to contract by the action of alcohol.

F. A specimen detached from its stalk and swimming away freely, disc forward.

$G, H, I$. Illustrate three stages of fission.

$\mathrm{J}, \mathrm{K}, \mathrm{L}$. Show the separated individual swimming by means of the aboral circle of cilia-K being slightly contracted, and $\mathrm{L}$ strongly contracted. a, Food-vacuole discharging contents at anus; ", aboral cilia; $c$, cilia of the disc; cf, contractile fibres of the stalk; $d$, disc; $d^{\prime}$, disc retracted; $f v$, food vacuoli; $p h$, pharynx; $p v$, pulsating vacuole ; $p$, peristoma ; $m$, myophan striation; $n$, nucleus ; $v$, vestibule.

The Vorticellæ are remarkable for their beantiful spiral stems and cilia, and the rapidity and grace of their movements. They retract and shorten their stems spirally and suddenly, and elongate them, in similar fashion, slowly. The cilia of the cup-shaped dises are continually in motion, and convey the idea of rotation. The movements of the stems and cilia are voluntary and under perfect control, as any one may readily satisfy himself by careful microscopic examination. The movements are necessary to secure food. They are essentially centrifugal and centripetal, give and take, rhythmic movements.

\section{PLATE LXXXII}

Plate lxxxii. shows red and white blood-corpuscles (human) invaded by disease germs; also rudimentary animal forms. Illustrates spherical, concentric, radiating, branching, segmented, and spiral arrangements.

FIG. 1.-Diseased condition of the red and white blood-corpuscles in malaria in native children on the west coast of Africa, magnified 1250 diameters (after Stephens and Christophers).

A. Young form of gamete in red blood-corpuscle.

B, C, D. Older forms of the same.

E. Macrogamete in red blood-corpuscle.

F. Microgametocyte, ditto.

C, H. Macrogametes showing punctation of red blood-cells in which they are situated.

$\mathbf{I}, \mathrm{J}, \mathrm{K}$. Types of pigmented leucocytes or white blood-cells met with in native children.

FIG. 2.- Hylla - a small fresh-water animal found in ponds, ditches, and also running streams; named for its power of recovering from injury - the mutilated parts becoming perfect animals.

A. Hydra or pond weed, natural size.

B. Section of hydra, greatly magnified. ", Mouth; b, stomach; $r$, ectoderm; $l$, endoderm ; $e, f$, buds ; $g$, ovary ; $h$, spermary ; $i$, thread cells (after Parker and Haswell).

C. Thread cell, highly magnified (after F. E. Schultze).

Fig. 3.-Types of Protozoa-1"ymnomyxu.

A. Vampyrella; B. Mycetozoa; C. Amoeba; D. Arcella; E. Labyrynthula; F. Actinophrys; G. Miliolina; H. Haliomma (after Chapman).

FIG. 4.-Types of Protozoa-C'urtirite.

A. Gregarina ; B. Monas ; C. Noctiluca ; D. Vorticella ; E. Podophrya ; F. Ceratium (after Chapman).

The Protozoa (Figs. 3 and 4) illustrate radiating, concentric, and branching arrangements.

Fici, 5... Trortirell" nehuliferc, magnified 100 diameters (after Griffith and Henfrey). Shows the spiral arrangements of the stem by which the animal cautiously and leisurely advances its ciliated bell-shaped body in search of food, and suddenly retracts the same when surprised or alarmed. The mechanism of the spiral stem is a marvel in its way, and thoroughly under control. FIS. 6.-Joint of stem of Antemularia antennim, magnified (after Allman). a, Polyp; $b$, nematophore; $c$, reproductive cap-
sule. Shows radiating, branched, and segmented arrangements.

\section{$\S 58$. Protoplasmic, Amœbic, Muscular, and other Movements.}

In order to obtain an adequate conception of the movements which occur in plants and animals it is necessary to take a wide survey and to study carefully those seen in rudimentary organic substances and structures, such as spores, spermatozoa, plant and animal protoplasm, pseudopodia, cilia, white blood-corpuscles, the sarcous elements of muscles, the Amœba, Gromia, Paramecium, Zoöids, \&c. The movements of the sarcous elements are especially interesting from their obvious bearing on the respiration, circulation, alimentation, and locomotion in the higher animals.

Motion is one of the characteristics of matter organic and inorganic alike. Of rest, strictly speaking, the universe furnishes no examples. Everything is in motion from the scarcely visible molecule to the great heavenly bodies wheeling freely in space. 
PLATE LXXXII
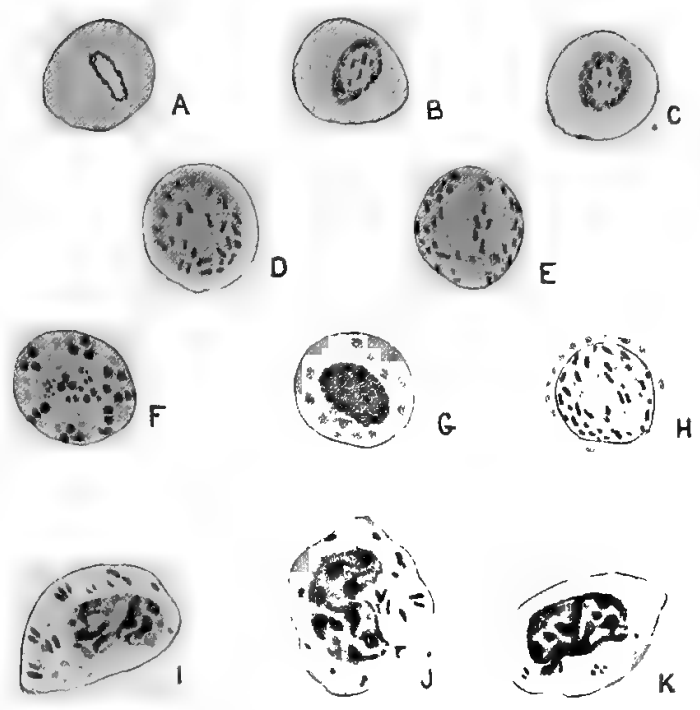

FIG. 1.

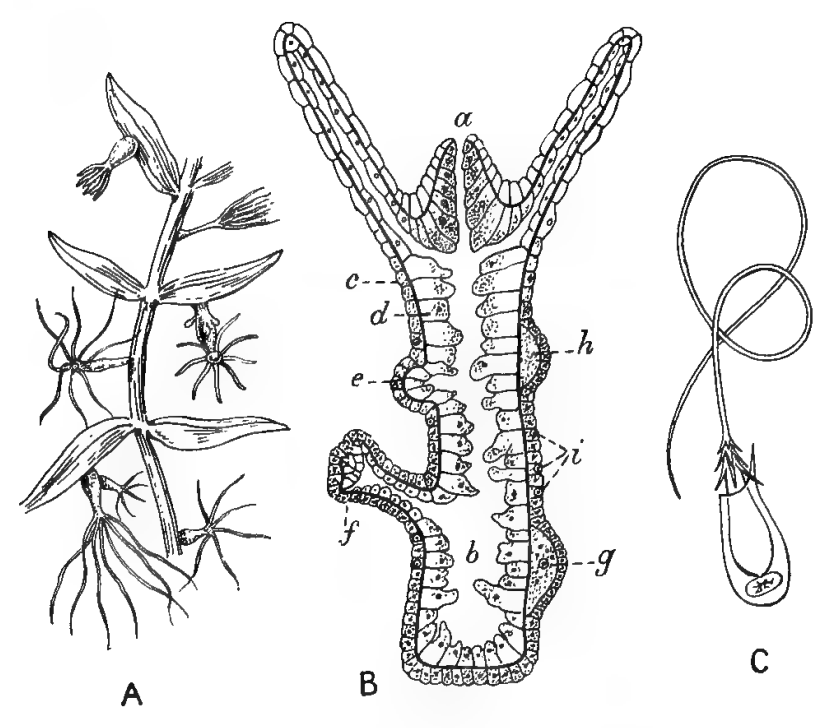

FIG. 2.

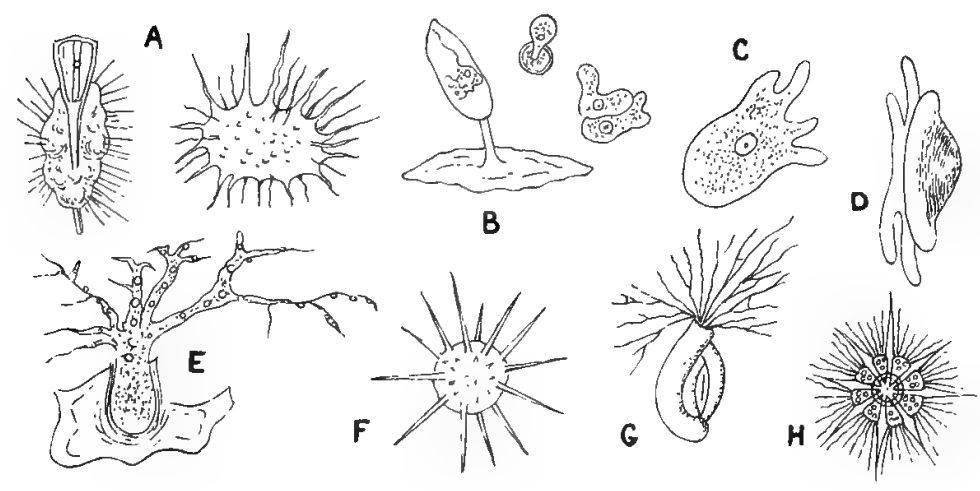

FIG. 3.

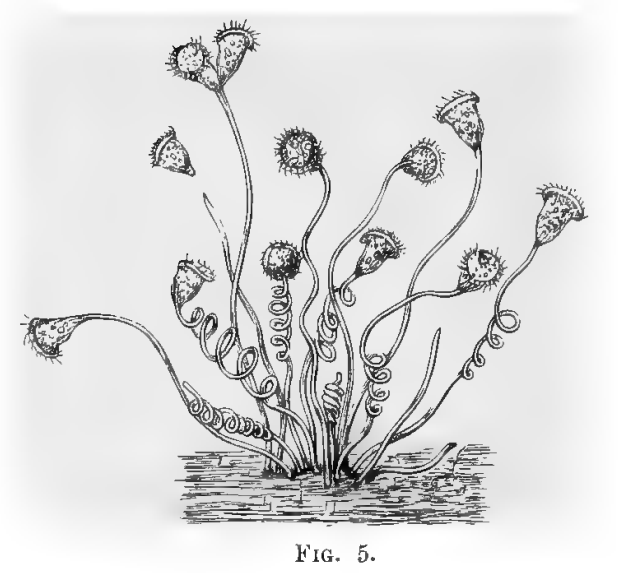

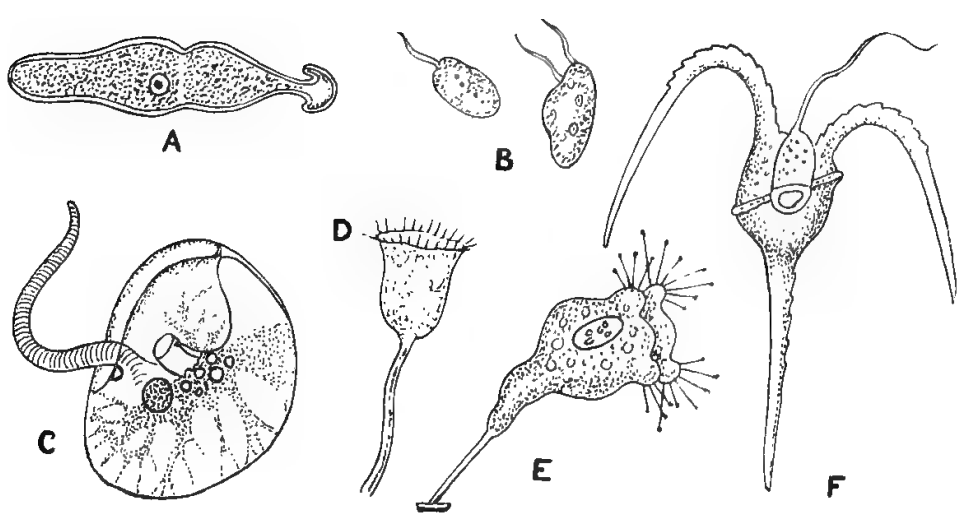

FIG. 4.

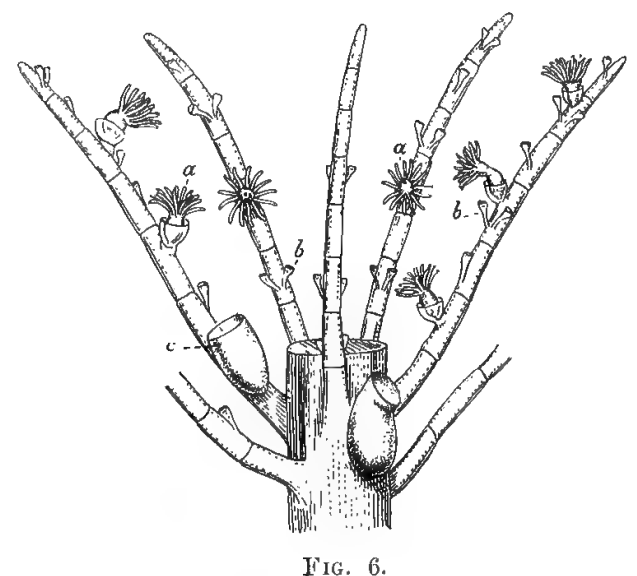

VOL. I. 
Mr. (afterwards Mr. Justice) Grove, in his admirable work on the "Correlation of the Physical Forces," thus puts it: "All matter, as far as we can ascertain, is ever in movement, not merely in masses, as with the planetary spheres, but also molecularly, or throughout its most intimate structure. . . Matter and force are correlates in the strictest sense of the word; the conception of the existence of the one involves the conception of the existence of the other; the quantity of matter, again, and the degree of force, involve conceptions of space and time. . . Motion will directly produce heat; and electricity, being produced by it, will produce magnetism-a force which is always developed by electrical currents at right angles to the direction of those currents. . . . Plücker has recently succeeded in showing that crystalline bodies are definitely affected by magnetism, and take a position in relation to the lines of magnetic force dependent upon their optical axis or axis of symmetry. . . The same principles and mode of reasoning might be applied to the organic as well as the inorganic; and force, animal and vegetable heat, \&c., might, and at some time will, be shown to have similar definite correlations. . . . From Professor Matteucci's experiments it appears that whatever mode of force it be which is propagated along the nervous filaments, this mode of force is definitely affected by currents of electricity."

From the foregoing it will be evident that motion is a characteristic of all bodies, animate and inanimate. It will therefore occasion no surprise if $\mathrm{I}$ lay it down as an axiom that the most rudimentary plants and animals move in all their particles and parts, and that what is true of the lowest plants and animals is equally true of the highest.

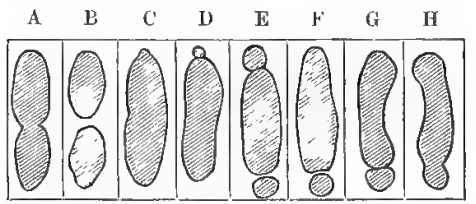

Fig. 63.-Drosera rotundifolia. Diagran of the same cell of a tentacle, showing the various forms ( $A, B, C, D, E, F, G, H)$ successively assumed by the aggregated masses of protoplasm (Darwin).

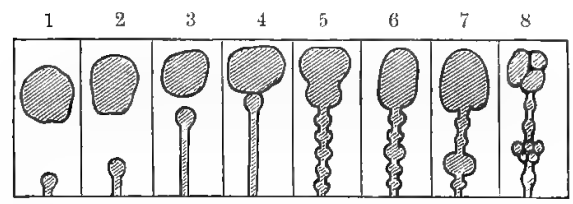

Fig. 64.-Drosera rotundifolia. Diagram of the same cell of a tentacle, showing the various forms $(1,2,3,4,5,6,7,8)$ successively assunied by the aggregated nasses of protoplasm (Darwin).

The microscope becomes a necessity when dealing with minute masses of living matter such as are met with in protoplasm, cells, tissues, low vegetable and animal forms, \&c.

Darwin has described and figured vegetable protoplasm moving and assuming a great variety of shapes. ${ }^{1}$ Thus, when speaking of the aggregation of the cell contents of the glands and tenacles of Drosera rotundifolia, he says: "By whatever cause the process may have been excited, it commences within the glands, and then travels down the tentacles. It can be observed much more distinctly in the upper cells of the pedicels than within the glands, as these are somewhat opaque. The little masses of aggregated matter are of the most diversified shapes, often spherical or oval, sometimes much elongated, or quite irregular, with thread or necklace-like or club-formed projections. They consist of thick, apparently viscid matter, which in the exterior tentacles is of a purplish, and in the short discal tentacles of a greenish, colour. These little masses incessantly change their forms and positions, being never at rest. A single mass will often separate into two, which afterwards reunite. Their movements are rather slow, and resemble those of amobæ or of the white corpuscles of the blood. We may, therefore, conclude that they consist of protoplasm. If their shapes are sketched at intervals of a few minutes, they are invariably seen to have undergone great changes of form; and the same cell has been observed for several hours. Eight rude, though accurate sketches of the same cell, made at intervals of two minutes or three minutes, illustrate some of the simpler and commonest changes (Fig. 63).

"The cell A, when first sketched, included two oval masses of purple protoplasm touching each other. These became separate, as shown at B, and then re-united, as at C. After the next interval a very common appearance I "Insectivorous Plants," by Charles Darwin, M.A., F.R.S., \&c. London, 1875, Ip. 39 to 42. 
was presented as at $\mathrm{D}$, namely, the formation of an extremely minute sphere at one end of an elongated mass. This rapidly increased in size, as shown in $\mathrm{E}$, and was then re-absorbed, as at $\mathrm{F}$, by which time another sphere had been formed at the opposite end."

The cell figured above was from a tentacle of a dark red leaf, which had caught a small moth and was examined by the aid of the microscope under water. Similar movements were witnessed by Darwin in a specimen not immersed. Thus he states: "I placed a fly on a leaf, and when after eighteen hours all the tentacles were well inflected, these were examined without being immersed in water. The cell here represented (Fig. 64) was from this leaf, being sketched eight times in the course of fifteen minutes. These sketches exhibit some of the more remarkable changes which the protoplasm undergoes. At first there was, at the base of the cell 1 , a little mass on a short footstalk, and a larger mass near the upper end, and these seemed quite separate. Nevertheless, they may have been connected by a fine and invisible thread of protoplasm, for on two other occasions, whilst one mass was rapidly increasing, and another in the same cell rapidly decreasing, I was able, by varying the light and using a high power, to detect a connecting thread of extreme tenuity, which evidently served as the channel of communication between the two. On the other hand, such connecting threads are sometimes seen to break, and their extremities then quickly become club-headed. The other sketches in Fig. 64 show the forms successively assumed. Shortly after the purple fluid within the cells has become aggregated, the little masses float about in a colourless or almost colourless fluid, and the layer of white granular protoplasm which flows along the walls can now be seen much more distinctly. The stream flows at an irregular rate, up one wall and down the opposite one, generally at a slower rate across the narrow ends of the elongated cells, and so round and round. But the current sometimes ceases. The movement is often in waves, and their crests sometimes stretch almost across the whole width of the cell, and then sink down again. Small spheres of protoplasm, apparently quite free, are often driven by the current round the cells; and filaments attached to the central mass are swayed to and fro, as if struggling to escape. Altogether, one of these cells, with the ever-changing central masses, and with the layer of protoplasm flowing round the walls, presents a wonderful scene of vital activity."

Professor Julius von Sachs gives an interesting representation of the movements of protoplasm in the middle cell of the hair of a gourd (Fig. 65).

The protoplasm appears as a finely punctuated mass in which are seen larger bodies, especially chlorophyll granules which contain starch, and, in one case, a small crystal of oxalate of lime. The movements of the protoplasm are made more apparent by the presence of the bodies referred to. The streaming of the protoplasm gives rise to a beautiful network, which is at once striking and characteristic.

Darwin, in his account of protoplasmic movements, refers not only to the various shapes assumed by the protoplasm within cells, but also to the gyration of the cell contents.

As regards the former, protoplasm may be said to move and change shape :-

(a) By division and reunion.

(b) By an advance of its molecules in a particular direction.

(c) By a retraction of its molecules in an opposite direction.

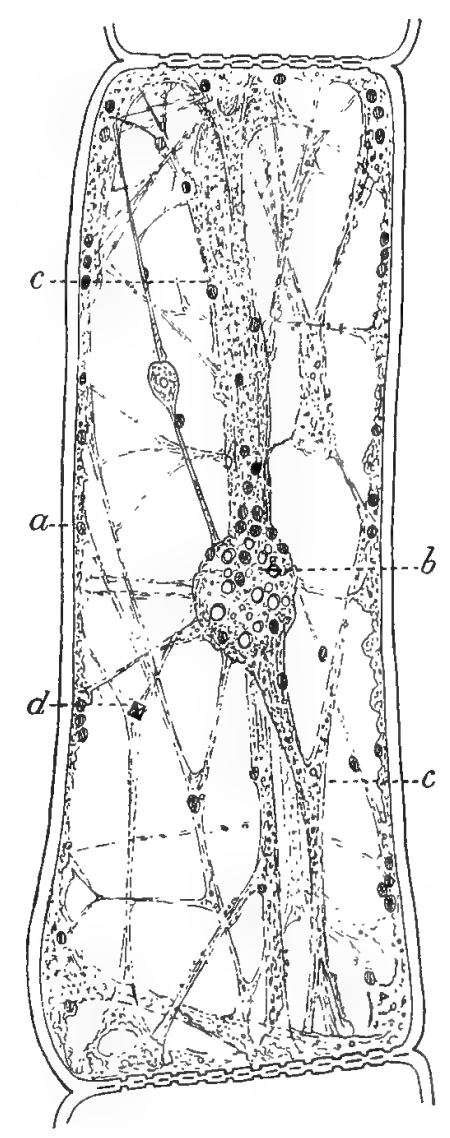

FIG. 65.-Longitndinal section of the middle cell of a hair of the Gourd (from the calyx of the young flowerbud). $u$, Cell-wall; $b$, vacuolated clump enclosing the nucleus of the cell ; $c$, streaming filaments of protoplasm in active movement, and carrying chlorophyll corpuscles (containing starch in their substance); $d$, a crystal being hurried along (after' Sachs).

In the former case it exerts a pushing force, and in the latter a pulling force. Similar powers are possessed by muscles and their sarcous elements.

In these various movements the volume or quantity of protoplasm remains the same. Change of shape does not imply increase or diminution of bulk. The movements of protoplasm are inherent vital movements. They are also independent movements, and are not the result of irritability or external stimulation. Neither are they, to any extent, the product of elasticity. While elasticity is a property peculiar to all bodies, organic and inorganic, it plays quite a subordinate part in vital movements and manifestations-indeed the influence exerted by it is, in the majority of cases, so trifling that it may almost be disregarded. It is the life which inaugurates, regulates, and terminates all movement in plants and animals; the life acting as a prime mover and not delegating its work, to any appreciable extent, to elastic materials and properties which it must evoke and control. 
Elasticity can only come into play when the substance which exhibits it is put upon the stretch or is crushed up, and this stretching out and crushing up in plant and animal movements is, in every instance, due to a vital operation. Elasticity cannot act of itself, and when it is made to act, it acts by recoil and produces a jerky movement; but (and this is the curious thing) when jerky movements are witnessed in plants and animals they are caused not by elasticity, but by life; for example, by the sudden contracting of living cells and hollow viscera, with a view generally to the propulsion of fluids, and by the energetic contraction of muscles, with a view to producing movements in the travelling organs or other parts of the body. The sudden movements referred to are vital movements; movements to a given end and for a purpose. Elasticity, when evoked by life, is always under control, and it is a mistake to assign to it anything but the most subordinate rôlo in plant and animal physics. One has only to watch the movements of protoplasm, of the white blood-corpuscles, of the amoba, the sarcous elements of muscles, \&c., to be convinced of this. It is an error to suppose that vital movements are only manifested by contractions, and by elasticity as a counter-acting force. The vital movements are numerous and various: pushing or elongating movements, pulling or shortening movements, contracting or closing movements, expanding or opening movements, spiral movements, \&c. The pushing, elongating, opening movements are centrifugal in their nature; the shortening, contracting, closing movements being centripetal. The terms centrifugal and centripetal are employed in relation to the central portions of the substance exhibiting the movements. The movements in the living mass, as a rule, spread from and return to a given point; that point, for the most part, corresponding with the position of rest. That living protoplasm can advance and retire as apart from contraction and its supposed counter-acting force of elasticity, is proved by the movements of climbing, sensitive, and insectivorous plants; by the advance of the plasmodium of Badhamia utricularis; by the amœbic movements of the white bloodcorpuscles; by the movements of the amœba, and by the streaming of the pseudopodia of Gromia, and the Foraminifera generally. The plasmodium of Badhamia utricularis when feeding advances as a wedge-shaped mass, no trace of contraction being anywhere observable. The white blood-corpuseles when forcing themselves through the walls of the capillary blood-vessels, as I have satisfied myself by careful microscopic examination, invariably throw out a knuckle or wedge of their substance, the thin end of which perforates the vessels. When the corpuscles have in part forced a passage, they draw or pull the remainder of their substance through the capillary walls, where they assume their original shape. Similarly, the amœba invariably moves by projecting one or more wedge-shaped portions of its substance-the substance streaming towards the apex of the moving wedge or wedges. The amoeba extends its substance in the direction of travel, and, in a sense, pushes itself forward by a centrifugal movement. There is no constriction in the advancing mass to indicate contraction of any kind, and no trace of elasticity can be detected. The advancing mass deliberately-that is, voluntarily-thrusts itself forward in an aggressive manner. The white blood-corpuscles and the amoba exhibit both centrifugal and centripetal movements.

The movements of pseudopodia as seen in Gromia are also centrifugal and centripetal in character; when Gromia is searching for food, it extrudes its protoplasm or body substance voluntarily in continuous streams in more or less straight lines. When prey is caught the protoplasm is voluntarily and suddenly drawn towards and into the central mass. The outward centrifugal movement is a vital, non-elastic, advancing, aggressive movement made in search of food ; the inward, centripetal, retreating, non-aggressive movement (also vital) represents the remaining or complementary half of the capturing act. The centrifugal and centripetal movements are co-ordinated, complemental, and voluntary, and neither movement would be of any use by itself. The movements of the sarcode of Gromia (and of the amoeba) are in all respects analogous to the movements of the white blood-corpuscles and the sarcous elements of muscle, voluntary and involuntary. If a fibrilla of a living, striated, voluntary muscle be examined under the microscope it will be seen that the more or less square, cube-shaped sarcous elements forming it are endowed with a double power, whereby they can elongate first in one direction, and then in another and opposite direction. When all the sarcous elements of the fibrilla elongate in the direction of the length of the fibrilla, the fibrilla is said to relax, in which case it increases in length; when all the sarcous elements elongate transversely to the length of the fibrilla, the fibrilla is said to contract, in which case it decreases in length. The terms relaxation and contraction, when so employed, are at once inaccurate and misleading. Contraction, strictly speaking, implies a diminution in bulk which does not occur when the fibrilla shortens. The sarcous elements are at present (and contrary to observation) accredited with only one power, namely, the power of contracting or shortening the fibrilla in the direction of its length; the power of elongating the fibrilla in the direction of its length being denied. The sarcous elements, according to the mechanical school, are invested with a centripetal, shortening, or closing power, but are said to have no centrifugal, elongating, or opening power. They aver that the closing or contracting power is traceable to the operation of life, but that the dilating or elongating power can only be ascribed to elasticity. This theory of muscular action is alike at variance with the appearances seen under the microscope, and 


\section{PROTOPLASMIC, AMOEBIC, AND OTHER MOVEMENTS}

the phenomena witnessed in moving muscular masses, voluntary and involuntary. In order to give it the semblance of truth, it is necessary to assume that when a limb is voluntarily folded or flexed, the flexor muscles forcibly drag out or extend the corresponding extensor muscles, and that, conversely, when the limb is opened out or extended, the extensor muscles forcibly drag out or extend the corresponding flexor muscles. Similar remarks are to be made of the abductor and adductor, and of the pronator and supinator muscles. According to this theory, the flexor and extensor muscles are constantly at war with each other; they are inconsistently pitted against and opposed to each other-the primary object in view, namely, the movements of the bones to which the muscles are attached and the increase or diminution of the angles made by the bones, and on which voluntary movements mainly depend, being quite lost sight of. As regards the involuntary muscles, the theory is still more unsatisfactory. If the movements of the mammalian heart be taken in illustration, it assumes that the very thin and weak auricles by their closure or contraction forcibly open and dilate the very thick and powerful ventricles. This is an impossible task; the ventricles, at the end of the systole, being a solid muscular mass. As a matter of fact, and as I shall show further on, the ventricles open spontaneously and independently when the auricles close or contract: conversely, the ventricles spontaneously close when the auricles spontaneously open. The two movements are correlated, but the one does not depend upon or cause the other. In proof of this I may state that in the living heart the ventricles open and close even when the auricles, and the blood which they contain, are removed.

The objections to the prevailing theory of muscular action become apparent in cases where two sets of muscles (a longitudinal and a transverse or circular) are arranged at right angles to each other, as in the large and small intestines, in certain arteries, \&c. In such examples, according to the prevailing theory, the longitudinal and circular muscles are opposed to each other and their movements are, of necessity, mutually destructive. This dilemma is avoided by investing both sets of muscles with a double power, namely, a power of contracting, shortening, or closing the one instant, and of relaxing, elongating, or dilating the next. The double power is well seen in the arterioles or smallest arteries with only an imperfect circular layer of muscle. In this case, as is well known, the artery can, in virtue of muscular action alone, be either contracted, narrowed, and elongated, or distended, widened, and shortened. When longitudinal and transverse muscles are arranged at right angles, as in the intestine and larger arteries, they act consentaneously; the sarcous elements of the one set elongating when those of the other set contract, and vice versa. In no other way could the peculiar vermicular movements of the gut and blood-vessels be economically produced. The arrangement here advocated largely dispenses with elasticity as a factor in muscular movements; muscles acting in two directions by the inherent vital powers of their sarcous elements.

These views were advocated by me as far back as $1872,{ }^{1}$ and are fully illustrated by original dissections, photographs, and careful drawings in Plates lxxxiii., lxxxiv., and lxxxy., and also Plates xcvii. to ciii. (seven plates lithographed by Messrs. West Newman, the reference to which must be given further on).

In cases where elasticity forms a factor of movement in animals, elastic structures are, as a rule, provided. Thus, there are elastic inter-vertebral cartilages for the spinal column to diffuse shock; elastic membranes in the wings of insects, birds, and bats to flex and recover the wings after being extended; elastic ribs, cartilages, \&c., to assist in respiration; elastic ligaments to support the strain of heavy parts, as the ligamentum nuchæ supporting the head of the deer-other ligaments restraining and limiting the movements in joints, \&c. In these and other cases special provisions are made for the employment of elastic structures and substances. One of the best examples of elastic structures is afforded by the arteries, especially the aorta, the great blood-vessel of the body, which is subjected to the constantly recurring impulses or shocks generated by the rhythmic contractions of the left ventricle of the heart. It receives the successive discharges of blood from the left ventricle and distributes that fluid to all parts of the body. The aorta is a remarkably elastic structure throughout. It is dilated mechanically by the blood forcibly ejected from the left ventricle of the heart during the systole or closing of the ventricle, and in virtue of its elasticity it recoils and narrows its calibre during the diastole or opening of the ventricle. The aorta by its recoil gives a second or sub-impulse to the blood, and so assists in the circulation and performs a double duty. It receives and diffuses shock and is dilated in the first instance; it is narrowed mechanically by recoil and equalises and assists the flow of blood in the smaller arteries in the second instance. The dilatation of the aorta and the larger arteries of the body with each successive beat of the heart produces the pulse. The pulse is not felt in the smaller and smallest arteries. In these the blood current is largely regulated by the vaso-motor nerves and by special muscles in the arteries themselves-the elastic properties of the vessels being greatly reduced, and mainly confined to the lining membrane of the arteries. In the smaller arteries there are two sets of muscles, a so-called circular set winding round the arteries more or less spirally, and a longitudinal set running in the direction of the 1 "The Plysiology of the Circulation in Plants, in the Lower Animals, and in Mas." 
length of the arteries and at right angles to the circular set. These two sets of muscles act harmoniously and synchronously both in dilating and contracting the calibre of the arteries_elasticity playing, relatively, quite a subordinate part. In the arterioles, or smallest arteries, only the circular set is found. The rule is, that as the muscular elements increase, the elastic elements decrease.

The walls of the smaller arteries are composed of three layers, namely, an inner, middle, and outer layer. The inner layer consists of fusiform epitheloid cells imbedded in a thin basement membrane with their long axes parallel to the axes of the vessels. The cells of the membrane form an elastic layer (tunica intima). The middle layer consists mainly of pale or unstriated muscular fibres arranged circularly and spirally. It contains a small quantity of connective tissue, and is known as the tunica media. The outer layer (tunica extima) consists of connective tissue, the bundles of which are, for the most part, disposed longitudinally. It displays connective tissue corpuscles and a relatively large number of elastic fibres. It will be observed that the elastic elements run, as a rule, in the direction of the length of the arteries, and at right angles to the circular or spiral muscular fibres-an arrangement which readily admits of their being put upon the stretch by the contraction of the arteries. Here the muscular and elastic elements mutually assist each other; the latter giving resiliency and helping the muscles over their dead points. It is, however (and let this be noted), the muscular element which primarily confers the closing and opening movements. The power of muscle to put elastic structures on the stretch is well illustrated

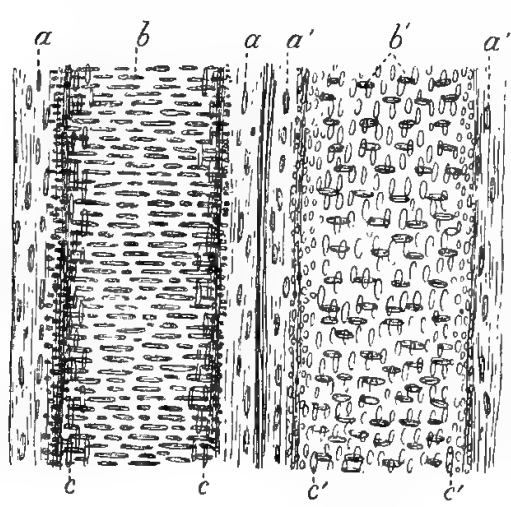

A by the effect produced by the left ventricle of the heart on the aorta. During the systole or closing of the left ventricle the aorta is suddenly dilated. It is not permitted to recoil or contract until the left ventricle withholds its power, which it does during its diastole or opening movement. The left ventricle and the aorta play, as it were, at cross purposes. The ventricle closes, and in closing opens the aorta. The aorta in turn closes by recoil when the ventricle opens. These are co-ordinated complementary movements, and the result is a more rapid, equal, and perfect circulation of the blood. Here, again, the muscular ventricle is the prime mover, and the aorta, which is a passive structure, can only move at second hand, and when distended by the blood forced into it by the left ventricle. The elastic properties of the aorta supplement the movements of the left ventricle. They also confer resiliency on that part of the circulatory apparatus nearest the heart. The resiliency is an important property where shock is to be diffused and recoil

FIG. 66.-A. Small artery. B. Small vein: both treated with acetic acid. $a c a, a^{\prime} \quad a^{\prime}$, Fibrous coats ; $b b^{\prime}$, nuclei of the muscular coat: $c c, c^{\prime} c^{\prime}$, nuclei of the epithelial coat-magnified 300 diameters (after Huxley). encountered, as is the case in
ments are sudden and jerky.

The veins differ from the arteries in being considerably larger and in having much thinner walls; the walls containing, relatively, a larger proportion of white fibrous tissue, and a smaller proportion of yellow elastic tissue.

The structure of the small arteries and veins is given at Fig. 66, where it will be seen the fundamental elements of both are arranged at right angles in a manner analogous to that in which the sarcous elements of muscle are arranged at Plate lxxxiii., Figs. 1, 2, 3, and 4.

This line of argument is greatly strengthened by a reference to the hollow viscera with sphincters, especially the stomach, bladder, rectum, and uterus. The human stomach is provided at its narrow end with a powerful sphincter (the pyloric valve), composed of two sets of strong muscles spirally arranged and crossing obliquely. This valve during the digestion of food is so firmly closed that the thin walls of the stomach cannot open it by even their most vigorous contractions. When the time arrives for the expulsion of part of the digested food (chyme) into the duodenum (or upper part of the small intestine) the sphincter valve opens spontaneously. When the sphincter valve opens the stomach, as a whole, closes or contracts. A double movement is inaugurated. The sphincter opens when the viscus closes, and vice versi- the movements being alternate and rhythmic in character. The contraction or closure of the body of the stomach does not open the sphincter, which, when contracted or closed, forms a solid muscular mass.

The stomach, as my dissections show, is provided with two sphincters-- the pyloric sphincter or valve already referred to, and another which I have designated the ossophageal or cardiac sphincter, from its being situated at the root of the cesophagus, where it enters the stomach, and from its proximity to the heart. That these sphincters act spontaneously and independently, and are not stimulated or set in motion by the food in the stomach, is proved by this, that they are sometimes both open, sometimes both closed, and sometimes one is open and the other is closed. They are both open when no food is in the stomach; the osophageal or cardiac sphincter is open and the pyloric one closed when food is being ingested; the pyloric sphincter is open and the oesophageal or cardiac one closed when 


\section{PROTOPLASMIC, AMOEBIC, AND OTHER MOVEMENTS}

the partly digested food (chyme) is being passed at intervals into the small intestine. The movements of the stomach and its sphincters are co-ordinated, rhythmic wave movements.

The reader will get a good idea of the structure of the oesophagus, duodenum, and human stomach-especially the sphincters of the latter-from careful drawings made by me from my original dissections, preserved in the Royal College of Surgeons of England, London. (Vide Fig. 67, p. 326.)

Arrangements similar to those described in the stomach obtain in the bladder. This viscus is provided with a powerful sphincter at its neck or depending portion. It completely occludes the urinary passage; so much so, that not a drop of urine can escape. When the period for micturition arrives the sphincter vesicæ opens or relaxes of its own accord; the body of the bladder contracts simultaneously and rhythmically, and the urine is expelled. These acts are consentaneous, but they are independent. The one does not cause the other. They are rhythmic wave movements, and are means to ends, and, as such, imply pre-arrangement and design. Neither in the case of the stomach nor the bladder could the practically impervious passages of the sphincters be forced. The same is true of the rectum and uterus in defæcation and parturition. The sphincters ani open when the rectum closes or contracts; the cervix and os uteri open when the uterus contracts. The object of the double movement is to expel the fæces and the fœtus. The contents of the viscera play a merely nominal and passive part in the rhythmic wave movements referred to. They do not cause them. The peculiarity of the hollow viscera (heart, stomach, bladder, rectum, and uterus) is that they are containing and retaining as well as propelling and discharging organs. The double function necessitates the condition of partial rest when the organs are receiving and accommodating their appropriate charges, and active alternating rhythmic movements as between the several parts of the organs when their contents are being expelled. The contents are discharged in each case by the alternate opening and closing rhythmic wave movements.

It should be explained that the involuntary muscles have no origins and insertions, as is the case in the voluntary muscles. They are, however, capable of very powerful action, and display what must be regarded as fundamental centrifugal and centripetal movements. The centripetal and centrifugal movements furnish the types for all muscular action, whether involuntary or voluntary.

The manner in which the involuntary muscles (blood-vessels, heart, stomach, \&c.), the voluntary muscles (flexors, extensors, abductors, adductors, pronators, supinators, \&c.), and the sarcous elements composing both act, will be readily understood by a reference to Plates lxxxiii., Ixxxiv., and lxxxv. which follow.

\section{PLATE LXXXIII}

Plate lxxxiii. shows that voluntary muscular fibres and fibrillæ may be divided longitudinally and transversely and broken up into little squares or cubes (sarcous elements); how the double cleavage gives rise to the markings and striations so well seen under high powers of the microscope; how the sarcous elements have the power of changing shape centripetally and centrifugally in two directions, namely, transversely and longitudinally; how muscles and the parts thereof (sarcous elements) move spontaneously and harmoniously in waves; how the several parts of muscles move simultaneously, one part moving transversely when the other is moving longitudinally, and vice versd; how the several parts alternately change places, there being no such thing as antagonism in muscular action either as regards the sarcous elements of individual muscles, or as regards the so-called flexor and extensor muscles of the body as a whole.

The sarcous elements of the involuntary muscles are less differentiated than those of the voluntary ones. They, however, act in essentially the same way. It is easy to understand how, according to my theory, parts are extended and flexed, how bones and other structures are moved, and how hollow viscera, especially those with sphincters, are opened and closed.

FıG. 1.-A. Elementary voluntary muscular fibres from the leg of a newly-born rabbit after having been expused to the action of alcohol. Shows well-marked longitudinal and transverse cleavage of the muscle, how the muscle breaks up into sarcous elements or little squares and cubes, and how these may be detached as at $a, a^{\prime}, a^{\prime \prime}$ (after Buwman).

B. Sarcous element or cube very greatly enlarged and invested with the double power of vitally changing shape in two directions, namely, horizontally and vertically. The change in shape is effected by combined centripetal and centrifugal movements as indicated by the solid darts $(a, b, c, d)$, and the dotted darts $(e, f, q, h)$. When the sarcous element assumes the shape $\mathrm{C}$ (in the horizontal position), it contracts or shortens (see solid darts ", $c$ ) in a direction from abuve downwards, and relaxes or elongates (see dotted darts $f, h$ ) laterally. When the sarcous element assumes the shape $\mathrm{D}$, it contracts or shortens laterally (see solid darts $b, d)$, and relaxes or elongates vertically (see dotted darts $e, g$ ). The shape of the sarcous elements in the condition of rest is seen at $\mathrm{B}$; the centrifugal movements are indicated at $\mathrm{E}$, and the centripetal ones at $\mathrm{F}$.

When two muscles, say a flexor and extensor, are at work, the sarcous elements of the one are shortening, as at $\mathrm{C}$, while the sarcous elements of the other are elongating, as at 1 (compare with $m, n$ of Fig. 4 ). The sarcous elements can change shape in two directions (see C and D of Fig. 2). The sarcous elements of both muscles move synchronously and harmoniously to a given end, whether that be the flexion or the extension of a part. The flexor and extensor muscles are not pitted against each other or 
PLATE LXXXIII
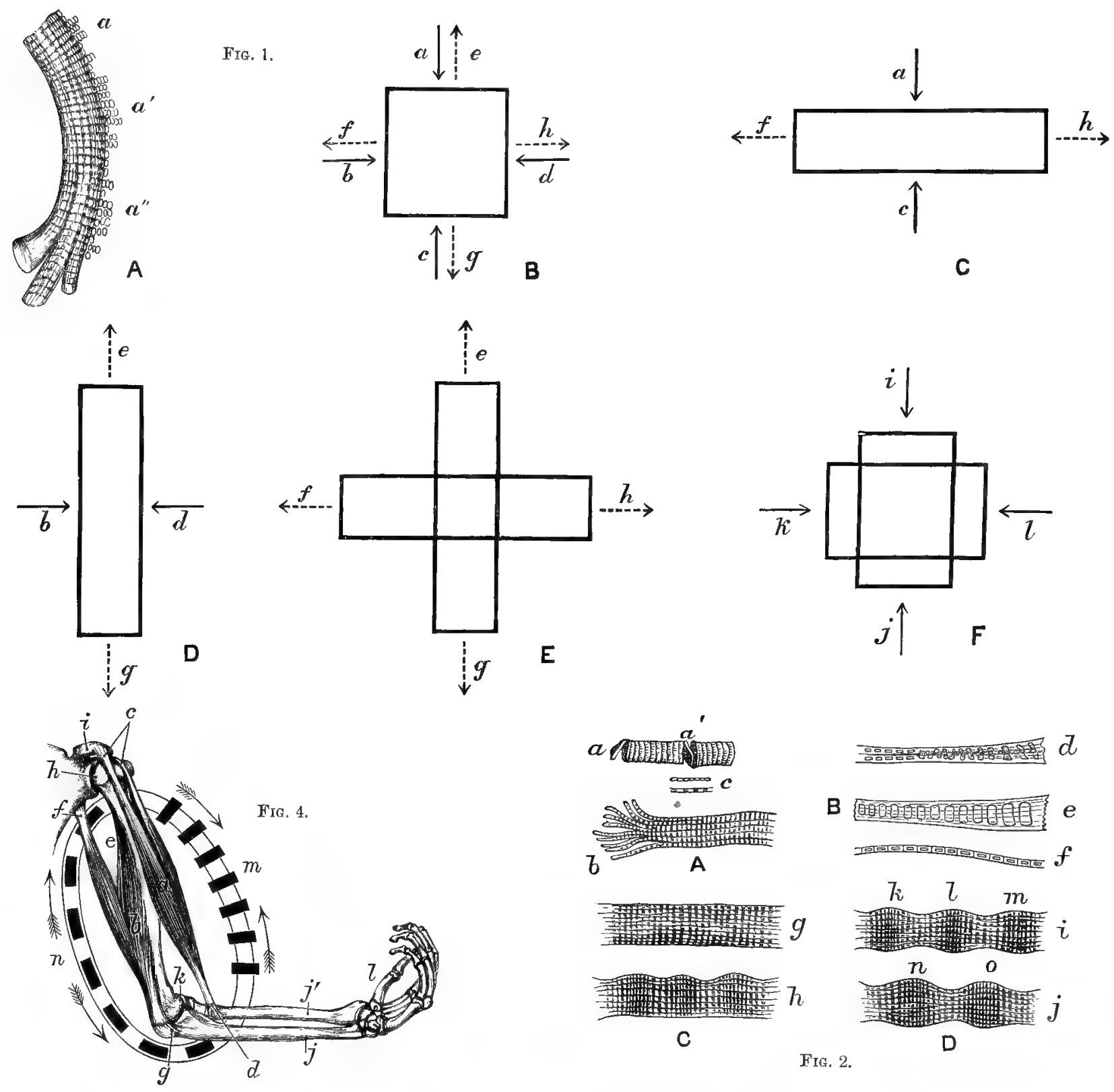

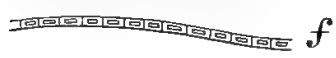
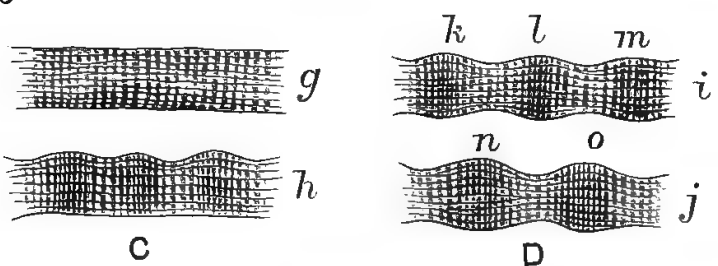

FIG. 2.

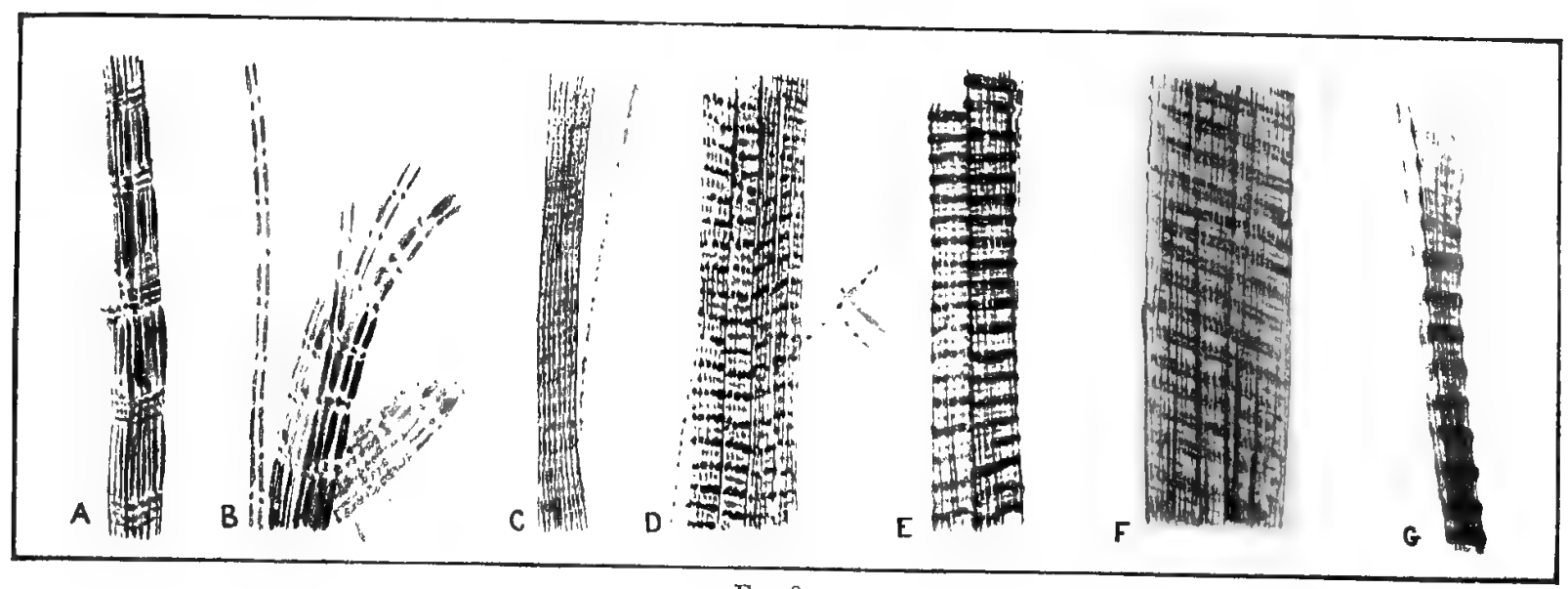

FIG. 3. 


\section{PROTOPLASMIC, AMEBIC, AND OTHER MOVEMENTS}

antagonists in any way. On the contrary, they form muscular cycles which invest bones with joints-one side or half of the cycle always contracting or shortening by a centripetal movement, when the other side or half relaxes or elongates by a centrifugal movement. The muscular cycle formed by the biceps and triceps muscles of the human arm is seen at Fig. 4 ; the arrows ( $m$ ) indicating the contracted or shortened sarcous elemerts of the biceps, and the arrows $(n)$ the relaxed or elongated sarcous elements of the triceps, as witnessed in flexion of the fore-arm. In extension of the fore-arm, the long axes of the sarcous elements reverse and change places $-m$ becoming $n$.

The consentaneous centripetal and centrifugal action of the sarcous elements, while well delineated at Fig. 4, is seen to perfection in the combined movements of the longitudinal and circular muscular fibres of the iris, intestine, and small arteries; the hollow viscera with sphincters; and the co-ordinated independent movements of the auricles and ventricles of the mammalian heart during diastole and systole.

The movernents in question are all typified at $\mathbf{E}$ and $\mathbf{F}$ of Fig. 1; $\mathbf{E}$ representing the centrifugal, elongating, opening-up movement, and $\mathrm{F}$ the centripetal, shortening, closing-up movement (the Author, 1872).

FrG. 2,-A. Fragments of striped elementary fibres, showing transverse cleavage ( $\left(t^{\prime}\right)$ of the sarcous elements. The longitudinal cleavage is seen at $b$. The appearances presented by the separated single fibrillæ are shown at $c$. The sarcous elements of the fibrillæ are, in one case, slightly beaded; in the other they are perfectly rectangular (after Bowman).

B. Muscular fibres of the pig, magnified 720 diameters. $f$, Single fibrils showing quadrangular outlines of the component sarcous elements - their dark central part and bright margin and their lines of junction crossing the light intervals; $d$, $e$, longitudinal segment of fibres consisting of a number of fibrils still connected together; $f$, other smaller collections of fibrils. (From a preparation by Mr. Leatham, after Dr. Sharpey.)

$\mathrm{C}$ and $\mathrm{D}$. Show an elementary muscular fibre of the skate in the uncontracted and contracted states. //, Fibre in the condition of rest; the longitudinal and transverse markings and cleavages being well marked. At $h$ one side of the fibre is contracting or shortening by a wave movement. At $i$, both sides of the fibre are similarly engaged; both sides of the fible being thrown into wave-like swellings $(k, l, m)$. The remarkable thing is that all the parts of the fibre are capable of producing the wave-like swellings; these successively taking each other's places. Thus the constrictions between $l, l$, and $m$ of the letter $i$ become the swellings $n, o$ of the letter $j$ (after Bowman). The power of the fibre to alternately swell out and expand and to retreat and draw in, at exactly the same spots, shows that the sarcous elements are endowed with centrifugal and centripetal movements. As these movements are independent of each other, muscles can alternately shorten and elongate; the rule being, that when the one shorteas the other elongates, and vice versâ. This is the case in voluntary muscles. It is also the case in involuntary muscles with or without sphincters. When no sphincters are present, the involuntary muscles exert their centripetal power in closing or partly closing their cavities. When the cavities are to be opened up and expanded they exert their centrifugal power. The centripetal and centrifugal movements of the voluntary and involuntary muscles are spontaneous and independent, and do not produce each other (the Author, 1872).

FIC. 3. - Shows the structure of voluntary or striped muscular fibres, and the shapes of the sarcous elements in the relaxed or elongated, and in the contracted or shortened conditions.

A. Fibrils of extensor muscle of crab's leg in the extended or elongated state.

B. The same separated; the sarcous elements elongated vertically.

C. Bundles of semi-contracted fibrils from crab's leg-the sarcous elements arranging themselves transversely or horizontally.

D. A further stage of contraction of muscle of crab's leg.

E. Fully contracted or shortened muscle of crab's leg, showing the complete reversal of the long axes of the sarcous elements. (Compare the sarcous elements of this figure with those of Fig. B.)

F, G. Fasciculi of contracted fibrillæ.

In all these figures the longitudinal and transverse cleavages which separate the substance of muscle into sarcous elements are well marked. (From a paper by Rutherford, and as explained by the Author in 1872.):

FIG. 4. - Shows the muscular cycle formed by the biceps or flexor muscle $(a)$ and the triceps or extensor muscle $(b)$ of the human arm, as seen in the flexion of the fore-arm and hand on the arm (the Author, 1872). At $m$ (vide arrows) the centripetal or shortening action of the surcous elements of the biceps in flexion is given, and at $n$ (vide arrows) the centrifugal or elongating action of the sarcous elements of the triceps in flexion is indicated. The long axes of the sarcous elements, $m$ and $n$, are at right angles to each other. In the act of extension of the fore-arm and hand the long axes of the two sets of sarcous elements reverse and change places; thus the sarcous elements $(m)$ of the biceps have their long axes arranged vertically, and those $(n)$ of the triceps transversely or horizontally. The sarcous elements of the flexor and extensor muscles, as already indicated, always arrange themselves at right angles - those of the flexors contracting or shortening when those of the extensors relax or elongate, and vice versit. The flexors do not forcibly drag out the extensors in flexion; neither do the extensors forcibly drag out the flexors in extension. This would be a mere waste of power. The flexors and extensors work consentaneously and harmoniously to produce the acts of flexion and extension respectively. Both are vital acts. There is no jerkiness in either movement, and elasticity plays quite a subordinate part in muscular action. The present figure illustrates how the bones of the extremities form levers, and how they are set in motion by muscular action; the Inuscles acting in cycles, and, for the most part, spirally. $c$, Short and long heads of the biceps or flexor muscle of the arm arising from the coracoid process (i) and upper edge of the glenoid cavity (and ligament) of the scapula; $d$, the insertion of the biceps into the posterior margin of the tuberosity of the radius; $e$, the three heads of the triceps or extensor muscle of the arm arising from the lower part of the glenoid cavity and inferior border of the scapula $(f)$-middle or long head; and from the posterior surface of the humerus-external and internal heads; $g$, the insertion of the triceps into the posterior and upper part of the olecranon of the ulna; $h$, rounded head of the humerus or arm bone; $i$, coracoid process of scapula; $j, j$, ulna and radius; $k$, elbow joint; $l$, bones of hand (the Author, 1872 ).

\section{PLATE LXXXIV}

Plate lxxxiv. illustrates the views of various authors as regards the shape of the sarcous elements of voluntary muscle in contraction and relaxation; how the striation is produced; how there is a transverse and longitudinal cleavage of the sarcous elements--these elements being arranged at right angles to each other; how the transverse and longitudinal arrangements of the sarcous elements reappear in the smaller blood-vessels, in the intestine, and in the iris of the eye; how the transverse and longitudinal sarcous elements work simultaneously and harmoniously, the transverse sarcous elements shortening when the longitudinal sarcous elements elongate, and vice vers $\hat{a}$; how the

vol. I. 
PLATE LXXXIV
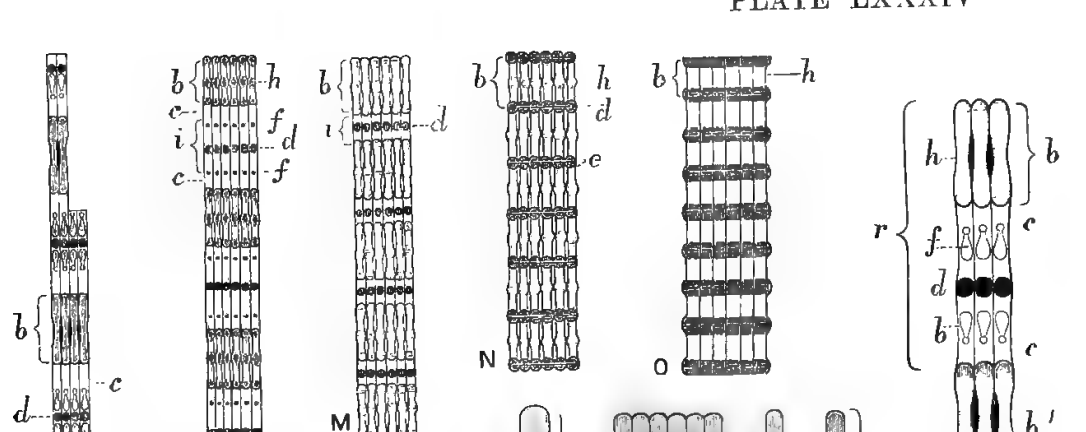

$\mathrm{K}$
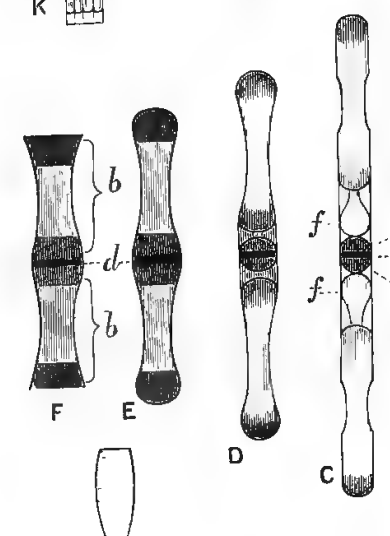

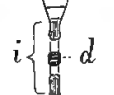
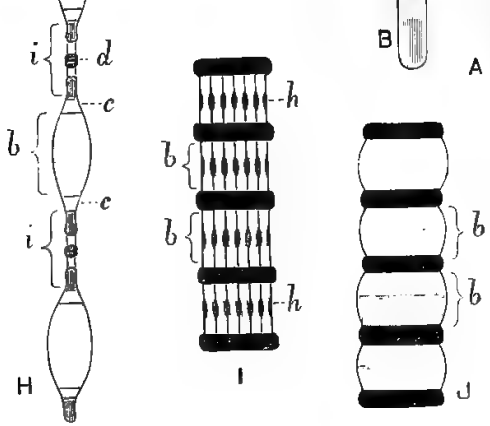

6

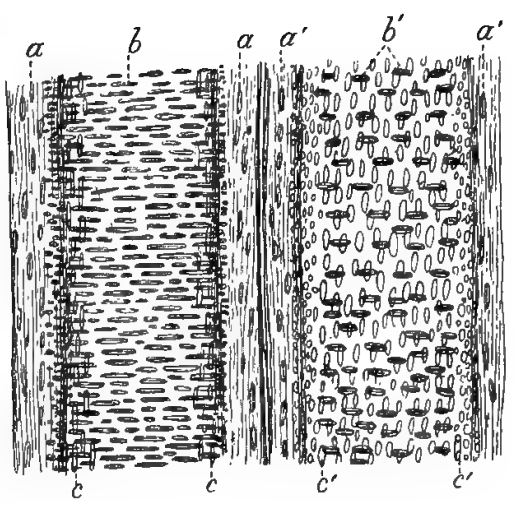

A

$B$

F1G. 2.

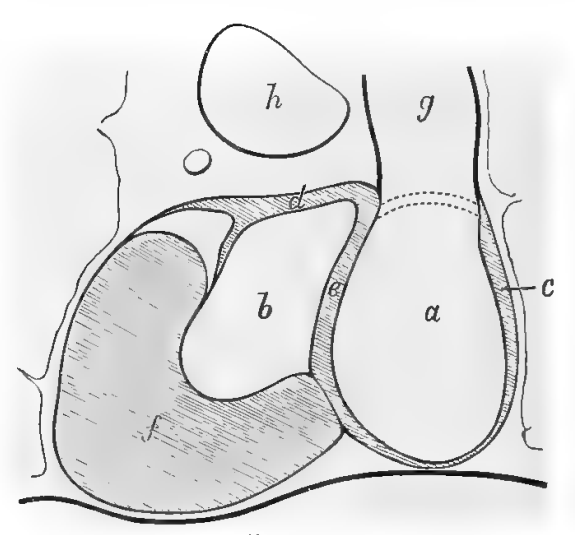

FIG. 4.

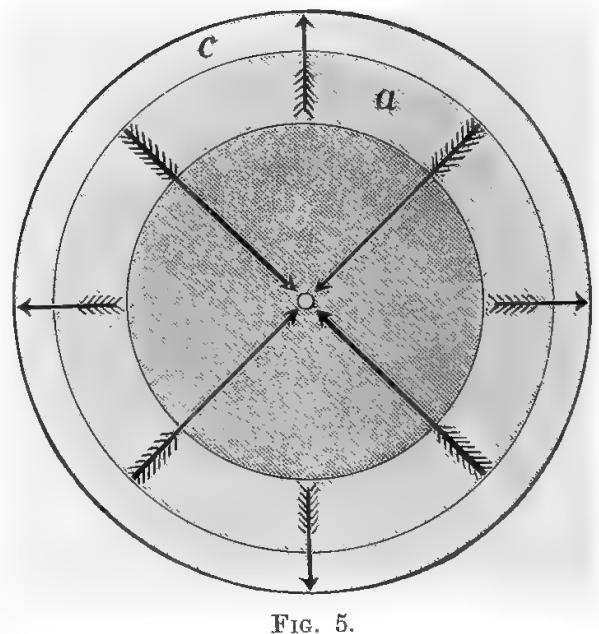

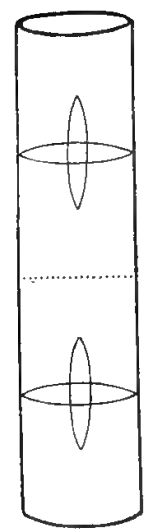

A

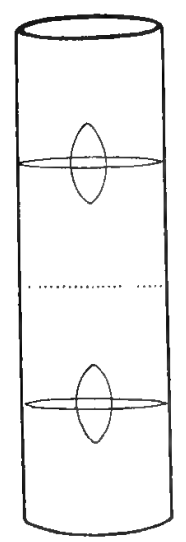

B

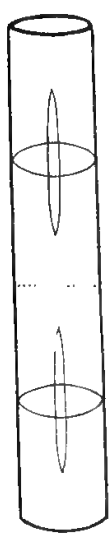

C

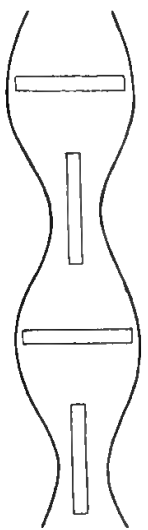

D

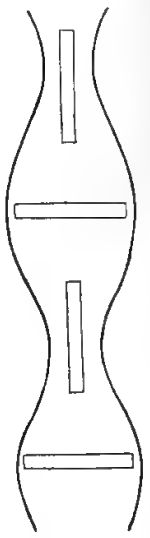

E

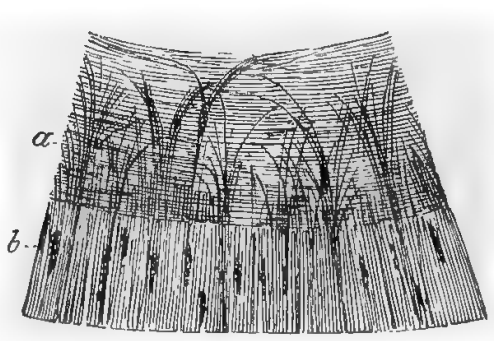

Fig. 6. 


\section{PROTOPLASMIC, AMCEBIC, AND OTHER MOVEMENTS}

several compartments of the mammalian heart open and close spontaneously by centrifugal and centripetal wave movements; how the left ventricle of the heart opens and closes as apart from irritability and stimulation of every kind; how the left auricle is inadequate to forcibly open the left ventricle; how there is a position of rest for the sarcous elements and the muscles of the heart; how the left ventricle opens or dilates when it acts outside or beyond the position of rest, and how it closes or contracts when it works within the position of rest; how these results are obtained by centrifugal and centripetal co-ordinated, independent movements, \&c.

FIG. 1.-Shows the voluntary muscular fibres in various degrees of contraction according to different authors. The italic letters indicate the same things in the figures from $\mathrm{A}$ to ' $\mathrm{T}$ inclusive. Thus a, means hemisphere of chromatin; $h$, Bowman's elements ; c, clear segment proper ; $d$, Dobie's elements; $f$, Flogel's elements ; $h$, Henson's line; i, intermediate segments; $n$, intermediate nodal line.

A, B, C, D, E, F. Semi-schematic representation of portions of fibrils of crab's muscle, showing the appearances of the fibrillar segments in the several stages from complete relaxation (A) to complete contraction (F). The fibrils thicken and shorten as they contract.

G. Three fibrils of crab's leg muscle, showing the successive stages from complete relaxation $(r)$ to complete contraction $(t)$. $b$ to $b^{10}$, show the various appearances of Bowman's elements; $d$ to $d^{8}$, the appearances of Dobie's elements ; $f$ to $f^{2}$, the appearances of Flogel's elements; and c, the clear segments proper.

$\mathrm{H}$. Uncontracted and fully extended fibril of muscle of crab's leg, fixed with absolute alcohol, stained with heliocine, and then treated with acetic acid and glycerine. $b$, Bowman's element swollen; $i$, intermediate segments not swollen; $c$, clear segment, swollen only at end connected with Bowman's element.

I. Contracted muscle prepared as at $\mathrm{H}$, showing appearance of a bundle of fibrils before addition of acetic acid. $b$, Bowman's elements; $h$, Henson's line.

J. The same bundle of fibrils as at I, after addition of acetic acid.

K. Fibrils of muscle of distal segment of pincer claw of lobster uncontracted and extended to full normal length.

$\mathrm{L}, \mathbf{M}, \mathbf{M}, \mathbf{N}, \mathrm{O}$. Show the successive stages from complete relaxation $(l)$ to complete contraction $(0)$ in the muscle of a crab's leg.

$\mathrm{P}, \mathrm{Q}, \mathrm{R}$. Fibrils of muscle of crab's leg uncontracted and extended to full normal length. Flogel's elements seen as granules of chromatin $(f)$. P, is intended to show the effect of shrinkage on clear $(c)$ and intermediate segments $(i)$. Q, shows a fibril unshrunken; and $\mathrm{R}$, a bundle of fibrils. The drawings are nearly double the size of those at $\mathrm{L}, \mathrm{M}, \mathrm{N}, \mathrm{O}$.

S. Flexor muscle of pincer of crab stained with gold chloride. Shows primary bundles of fibrils $(b)$ outlined by longitudinal gold lines, with rounded, deeply-stained particles opposite Dobie's line (d).

T. Optical transverse section of the same bundles (Rutherford).

FiG. 2.- Small artery and vein as seen in juxtaposition, with the sarcous and other elements arranged at right angles to each other. $a, a$, Coats of small artery with sarcous elements arranged longitudinally; $b$, similar elements arranged transversely; $c^{\prime}, c$, the longitudinal and transverse sarcous elements superposed; $a^{\prime}$, $a^{\prime}$, coats of small vein with sarcous and other elements arranged longitudinally; $b^{\prime}$, sarcous and other elements arranged transversely or at right angles; $c^{\prime}$, $c^{\prime}$, the longitudinal and transverse elements superposed (after Huxley).

Fig. 2 illustrates the mechanism by which small blood-vessels (arterioles) are alternately dilated and partially closed-how hollow viscera, such as the heart, stomach, bladder, dc., are opened, and partially or wholly closed; and how the flexor and extensor, the abductor and adductor, and the pronator and supinator muscles are co-ordinated and work harmoniously in pairs (the Author, 1872).

Fig. 3.-A, B, C, D, E. Diagrams by the Author showing that the sarcous elements of the involuntary and voluntary muscles, when in action, are arranged at right angles to each other, and that they are endowed with waved centripetal and centrifugal movements, whereby they reverse and change their shapes when the muscles contract or shorten, and when they relax or elongate. The sarcous elements, when in action, are always arranged in two sets-a vertical or longitudinal, and a horizontal or transverse; and the two sets are co-ordinated and work harmoniously together. In the case of arterioles (small arteries), where there are two layer's of involuntary muscular fibres (a vertical or longitudinal layer, and a horizontal or transverse layer), the sarcous elements, which are spindle-shaped, are arranged symmetrically at right angles as at A. In this figure the vessel is represented at rest, that is, neither dilating or contracting. When the vessel is dilating as at $\mathrm{B}$, the vertical or longitudinal sarcous elements swell out and shorten, while the horizontal or transverse sarcous elements narrow and elongate. When the vessel is closing and reducing its calibre as at $\mathrm{C}$, a reverse movement takes place; the vertical or longitudinal elements narrowing and elongating, and the horizontal or transverse elements swelling out and shortening. The two sets of movements referred to alternately diminish and increase the length of the arteriole.

The sarcous elements in voluntary muscles are square or cube-shaped when at rest. When in action, they form elongated, square-shaped bodies, which are arranged at right angles to each other as at $\mathbf{D}$ and $\mathbf{E}$. The peculiarity of the sarcous elements of the voluntary muscles, as of the involuntary ones, consists in their power of changing shape in two directions; the same sarcaus element elongating transversely or across the muscle the one instant, and longitudinally or in the direction of the length of the muscle the next. This produces an alternating spindle-shaped arrangement of the individual fibrillae of the muscle, and a waved movement of the muscle as a whole.

The sarcous elements of muscles, and the muscles themselves, are never opposed to each other; they always act consentaneously and harmoniously. When one sarcous element, or the muscle to which it belongs, thins and elongates in one direction, its counterpart or fellow thickens and shortens in an opposite direction. There is no such thing as antagonism, in the old sense, in muscular action. The flexors do not forcibly drag out the extensors in flexion; neither do the extensors forcibly drag out the flexors in extension. The same is true of the abductors and adductors, and of the pronators and supinators. When the ventricles of the mammalian heart contract or close, the sarcous elements of the ventricular muscles act simultaneously and exercise their centripetal power; when the ventricles dilate or open, the sarcous elements exercise their centrifugal power. The exercise their centripetal power; when the auricles. The auricles close when the ventricles open, and vice versat, but the movements same is true of the sarcous elements of the auricles. The aur cles close when the ventricles open, and vice versa, but the movements are independent of each other, and the one does not cause the other. The centre bladder, uterus, \&c). In these cases, when the muscles forming the bodies of the viscera are in an active state and contract or close, the muscles forming the sphincters dilate and open. When the viscera are at rest their cavities are open and the sphincters closed.

The power of acting in two directions inheres in both the involuntary and voluntaly muscles, and likewise in their sarcous elements (the Author, 1872). 


\section{PLATE LXXXIV (continued)}

FIG. 4,-Photograph of a frozen section of the human heart where the individual has died during the systole of the organ, that is, when the ventricles were strongly contracted and the ventricular cavities obliterated; the auricles being relaxed and distended to their utmost. a, Cavity of right auricle; $b$, cavity of left auricle; $c$, thickness of wall of right auricle when relaxed; $d, e$, thickness of wall of left auricle when relaxed; $f$, thickness of wall of left ventricle when the ventricle is fully contracted and the ventricular cavity obliterated; $g, h$, great vessels cut into.

Shows the comparative thickness of the walls of the ventricles and auricles during systole, and how it is impossible for the thin auricles to force their contained blood into the ventricles, which, in systole, form solid muscular masses. The obvious inference is that the ventricles open spontaneously when the auricles close. The auricles do not forcibly dilate the ventricles, neither do the ventricles forcibly dilate the auricles. The movements of the auricles and ventricles are co-ordinated but they are independent; the ventricles opening by centrifugal movements when the auricles close by centripetal movements, and vice versî. The ventricles are invested with a double power, whereby they can alternately open and close, and the same is true of the auricles. A similar arrangement obtains in the hollow viscera (stomach, bladder, \&c.) with sphincters.

All the muscular fibres of the ventricles and auricles move simultaneously and harmoniously in the opening and closing movements. The opening centrifugal movements and the closing centripetal movements of the ventricles are shown in Fig. 3 (the Author, 1872).

FrG. 5.-Diagram showing the centripetal and centrifugal movements of the left ventricle during systole, when the ventricular cavity is obliterated, and during diastole, when the ventricular cavity is fully opened, as seen in cross section of the ventricle. The muscular fibres are in a state of activity during both the closing and opening movements. There is, however, a brief interval between the opening and closing movements when the ventricle is at rest. There is also a period of rest for the sarcous elements, which greatly extends the period of the inactivity of the ventricle as a whole. $a$, Zone of rest for the left ventricle; $b$, contracted zone when the left ventrical cavity is obliterated by a strong centripetal novement of the muscular fibres, as indicated by the four long arrows; $c$, relaxed zone when the left ventricular cavity is fully opened up by a more feeble centrifugal movement of the fibres, as indicated by the four short arrows. The centripetal and centrifugal movements are spontaneous and independent of each other. They are also vital in their nature (the Author, 1867).

FIG. 6.- Muscles of the iris of the eye. $a$, Transverse or circular muscular fibres; $b$, longitudinal or radiating muscular fibres. These are arranged at right angles to each other. They are of the plain, unstriped type, and have for their object the increase and diminution of the pupil or aperture in the centre of the iris through which the light passes into the interior of the eye. The transverse or circular fibres have been regarded as forming a sphincter, and the longitudinal radiating fibres a dilator, to the iris; these acting at different times and alternately. The circular and longitudinal fibres are said to be opposed to, and pitted against, each other, after the manner of supposed antagonist muscles. This is an erroneous view. As a matter of fact, the so-called sphincter and dilator muscular fibres are co-ordinated, and act harmoniously and together; both taking part in the opening and closing movements of the iris. The movements of the muscular fibres are centrifugal and centripetal in character, as in small blood-vessels, the hollow viscera, and the voluntary muscles when these produce the movements of flexion and extension, of abduction and adduction, of pronation and supination, \&c.

Some are of opinion that the longitudinal fibres are sparse or non-existent, and that when present they are striated, as in the bird and otter. If this view be adopted it follows that the circular fibres must of thernselves be equal to increasing and diminishing the aperture of the pupil, and that they are consequently endowed with a double power, which enables them to increase or diminish the size of the pupil at pleasure (after Iwanoff).

\section{PLATE IXXXV}

Plate lxxxv. shows how the small arteries are furnished with transverse or circular and longitudinal or straight unstriped muscular fibres arranged in two layers with nuclei interspersed; how the unstriped muscular fibres are ribbon-shaped and flat, and display rod-like nuclei in their interior; how the nuclei reappear in young striped muscle with faint transverse markings; how in the adult heart the transverse markings are pronounced, the involuntary and voluntary muscles having not a little in common, structurally and functionally. In particular the Plate shows how the ventricles of the mammalian heart open and close, the cavities of the ventricles being quite obliterated during the systole; how the muscular fibres of the left ventricle are arranged spirally in two sets, which enter and leave the apex at opposite points, and so produce a symmetrical result, which, curiously enough, finds its exact counterpart in certain shells (Plate xiii., Fig. 1, G) and in double spiral nebulæ (Plate viii.). The last is a most unexpected occurrence, and has much significance, as indicating the prevalence of laws which have symmetry and spiral movements for their objects.

FIG. 1.-A. Small artery consisting of transverse and longitudiual unstriped muscular fibres branching into two smaller ones (b), which split up into capillaries ( $c$ and $d$ ) displaying nuclear elements. Magnified 225 diameters.

B. Nuclei $(", ", n)$ of unstriped involuntary muscles, magnified 350 diameters.

C. Flat, spindle-shaped, involuntary muscles, showing rod-like nuclei $(a, ", a)$; the central specimen being treated with acetic acid. The flat fibres, according to some investigators, display faint traces of transverse and longitudinal cleavage. Magnified 350
diameters.

D, E. Similar fibres with well-marked nuclei $(\alpha, " 1)$.

F. Elementary fibres from pectoral muscle of fuetal calf two and a half months old, showing corpuscles ( $a, a)$, magnified 300 diameters. This preparation affords a link between the involuntary and voluntary muscular fibres structurally and functionally.

G. Elementary fibre from the larva of a dragon fly in an early stage of development, showing central row of corpuscles. Magnified 300 diameters (Bowman).

H. Striped involuntary muscle of the heart. The cardiac muscle is peculiar in this : it is involuntary and yet its substance displays well-marked transverse and longitudinal cleavages. It runs the involuntary and voluntary muscle into each other, and affords proof that both kinds of muscles belong to a common stock, and functionally resemble each other. 
PLA'TE LXXXV

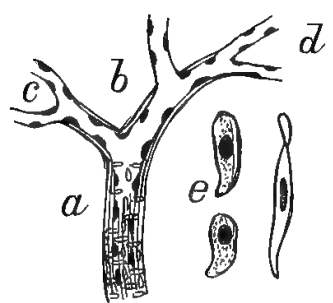

A B

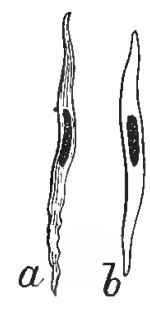

C

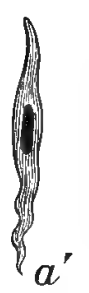

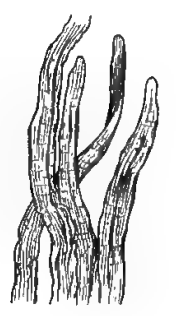

D

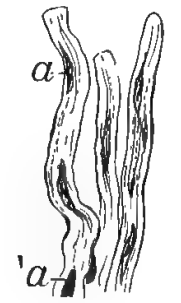

$E$

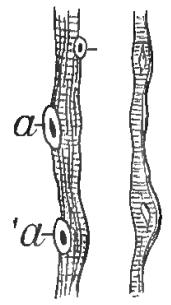

$\mathrm{F}$

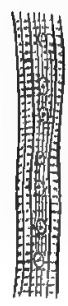

G

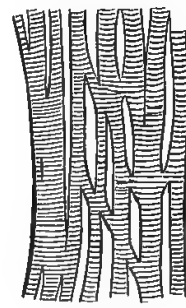

$\mathrm{H}$

FIG. 1.

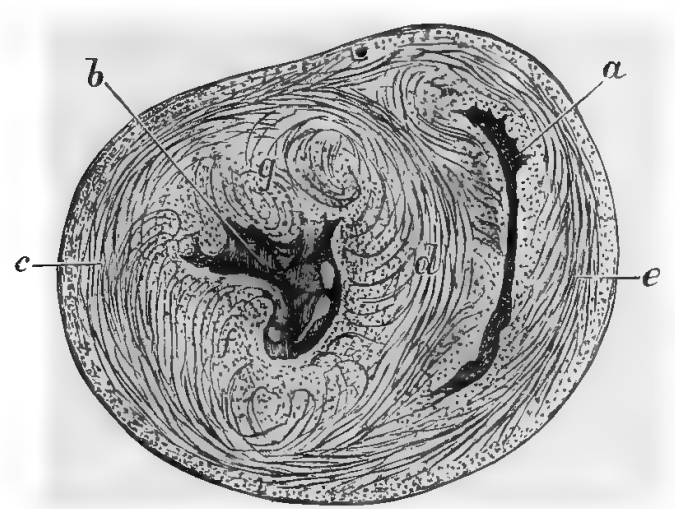

FIG. 2.

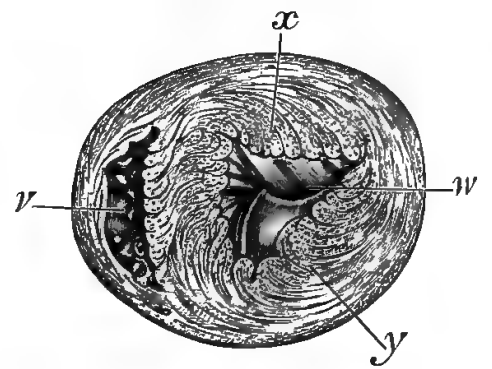

FIG. 6.

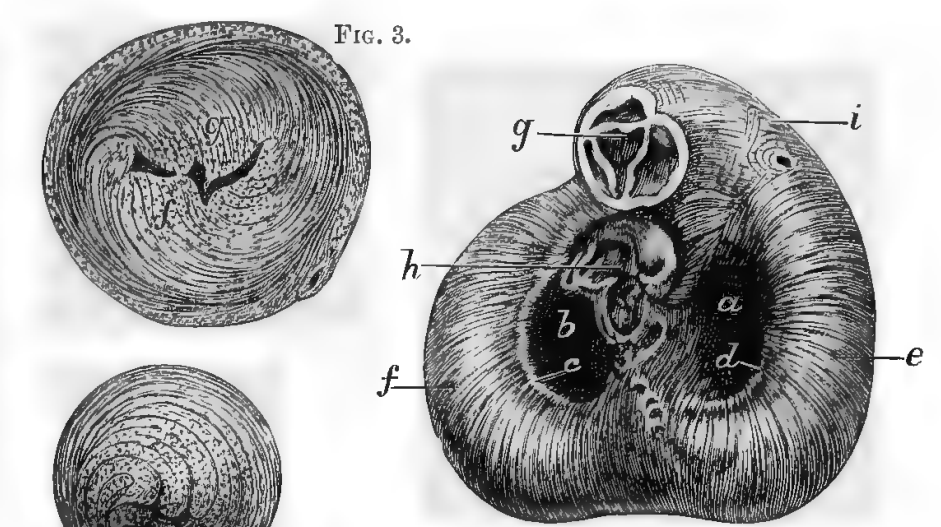

FIG. 5.
FIG. 4.

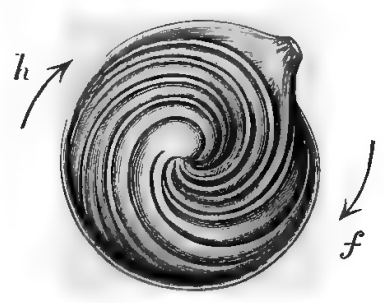

FIG. 7 .

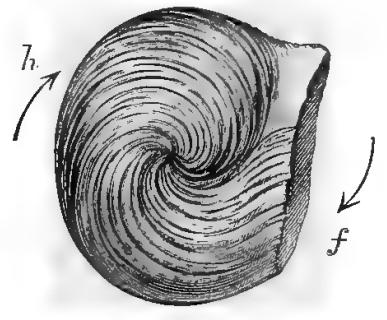

FIG. 8.

\section{PLATE LXXXV (continntr)}

FIG. 2.-Photograph of a transverse section by the Author of the right and left ventricles of the heart of a deer, near the base of the ventricles. Shows that the left ventricular cavity $(b)$ is completely closed by the spiral twining of the musculi papillares ; the right ventricular cavity $(a)$ being all but closed. The walls of the left ventricle and septum $(f, y, d)$ are comparatively very much thicker than those of the right ventricle $(e)$.

FIG. 3.-Photograph of a transverse section by the Author of the left ventricle of the same heart near the apex; the ventricular cavity being all but occluded by the powerful spiral twining action of the musculi papillares $(f, g)$.

FIG. 4.-Photograph of a transverse section by the Author of the same ventricle still nearer the apex; the ventricular cavity being quite obliterated by the strong spiral action of the muscular fibres of the apex.

FIf. 5. - Photograph of a dissection by the Author of the base of the right and left ventricles of the heart of a heifer after removal of the right and left auricles. Shows the ventricular cavities quite open, and the orifices of the aorta and pulmonary artery partly closed by the semi-lunar valves. $a$. Right ventricular cavity, open as in diastole; $b$, left ventricular cavity, ditto; $c$, $d$, tibrous rings from which certain of the superficial muscular fibres of the ventricles arise; the deeper fibres being, for the most part, continuous beneath the rings; $e_{3}$ basal fibres of the right ventricle; $f$, ditto of left ventricle; $g$, orifice of pulmonary artery with remains of semilunar valve; $h$, ditto of aorta; $i$, infundibulum.

FIG. 6.-Photograph of a transverse section by the Author of the apices of the right and left ventricles of the heart of a sheep. The apices $(v, v)$ are open as in diastole. The muscular fibres of the left apex are seen winding spirally out of the interior of the left apex in two sets $(x, y)$. The two sets of fibres $(x, y)$ form the beginnings of the two musculi papillares, which pursue spiral courses within the left ventricular cavity. 


\section{PLATE LXXXV (continued)}

FIG. 7.-Spiral shell with right-handed spiral whorl at the apex. The whorl consists of two parts which twist and plait into each other. It resembles a right-handed spiral nebula (Plate viii., Fig. 4); spiral seeds (Fig. 11, D, E); spiral spermatozoa (Plate xii., Fig. 1, E), \&c. (the Author, 1859, and subsequently).

Frg. 8.-Photograph of a dissection by the Author of the left ventricle of a sheep's heart showing the posterior surface and apex of the left ventricle. The left ventricle of the heart of the mammal is composed of two sets of spiral muscular fibres. $f$, $h$, the two sets of superficial spiral muscular fibres form the first external layer. They are seen involuting or turning into the left apex to make a most exquisite whorl. Compare with Fig. 7 of this Plate. It is these two sets of fibres which are chiefly concerned in the production of the two musculi papillares. The whorl formed by the two sets of spiral muscular fibres of the left ventricle of the heart of the sheep is quite as distinct in the left ventricle of the human heart.
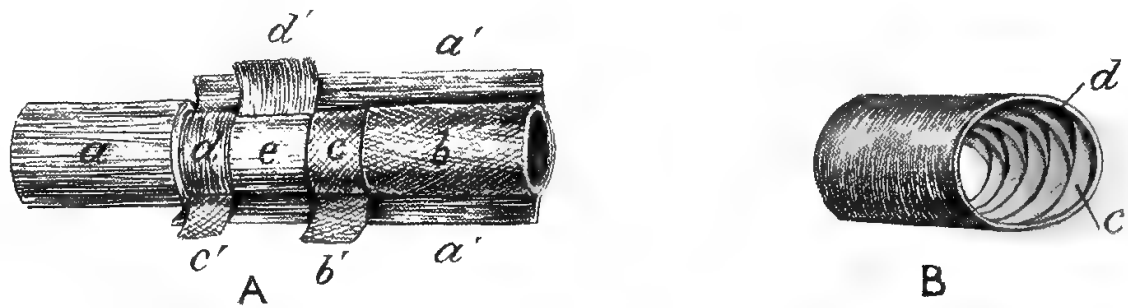

B
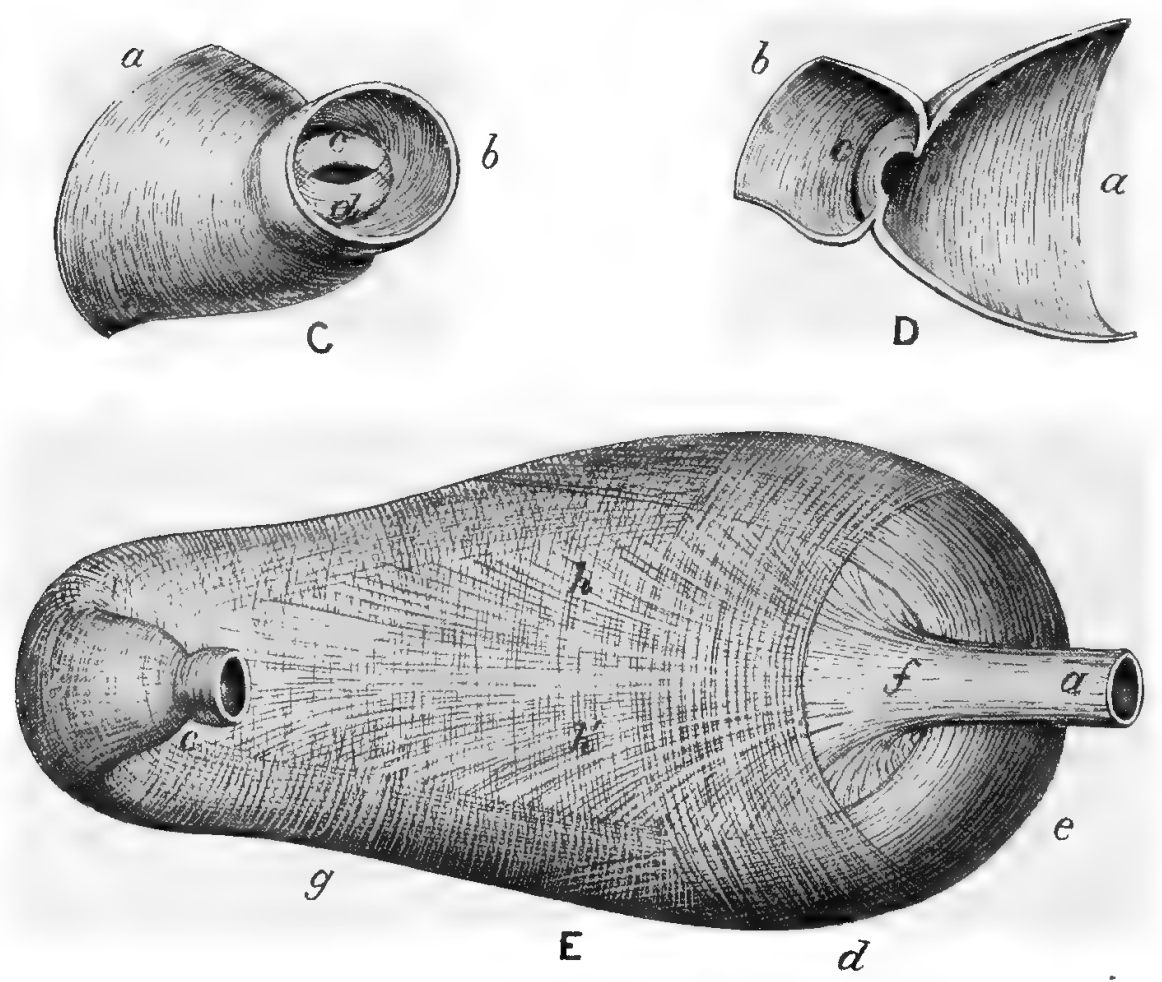

Fig. 67 .

Fic. 67 (A, B, C, D, E).--Represents a series of original dissections and drawings by the Author, showing the distribution of the muscular fibres in the vesophagus, stomach, pyloric valve, and duodenum.

A. Minute dissection of the nuscular fibres of the oesophagus of the horse. In this dissection no fewer than seven layers of muscular fibres can be made out. The distribution is similar to that met with in the left ventricle of the mammalian heart. The tibres are spirally arranged, and consist of three external sets, three internal sets, and a central layer. Proceeding from without inwards the following are encountered-an external longitudinal layer $\left(a, a^{\prime}, a^{\prime}\right)$, an internal ditto $(e)$, a slightly oblique external and a slightly oblique internal layer which cross each other $\left(b, b^{\prime}\right)$, a very oblique external and a very oblique internal layer which also cross $\left(c, c^{\prime}\right)$, and a transverse or central layer $\left(d, d^{\prime}\right)$. This muscular mechanism is endowed with centrifugal and centripetal powers, whereby it opens before and closes behind the bolus in swallowing, with the result that the food is enforced into the stomach. The scheme of the mechanism is indicated at D, E, of Fig. 3, Plate lxxxiv. In vomiting, the swallowing process is reversed; the arrangement working effectively in both cases. One can eat, swallow,
and drink with the body inverted.

The swallowing process is similar to that induced in the teat of the cow in milking. According to prevailing views, the interior of the cesophagus is irritable, and the bolus of food acts as a stimulus which causes the cesophagus to open and close rlternately and so transmit it to the stomach. This cannot be the true explanation. The passage of the bolus into the stomach is a continuous movement, and cannot possibly produce the alternating, interrupted movements which characterise the osophagus. If, moreover, the bolus acted as a stimulant it would cause contraction of the oesophagus in front instead of behind itself. The oesophagus would 


\section{MUSCULAR ACTION (VOLUNTARY AND INVOLUNTARY)}

FIG. 67 (continued).

contract where the bolus first touched it. The rule is, that the oesophagus always contracts behind the bolus ; the part in front of the bolus opening at the same time. In no other way could the bolus be transmitted to the stomach. There is a double power at work in swallowing ; a centripetal, closing, contracting movement behind the bolus, and a centrifugal, opening, dilating movement in front of it. As all parts of the cesophagus are invested with the double power, and the contracting and dilating portions change places, a most efficient steady downward movernent is provided for the downward passage of the bolus.

The movements of the oesophagus in swallowing are the precursors of those occurring in the stomach, sphincters, rectum, heart, bladder, and uterus.

The osophagus is to be regarded as a typical involuntary muscle, and, as such, its movements are worthy of very special study. It combines in itself all the peculiarities of muscular activity. It is not dominated by the will nor by nervous arrancements in any form. It is an independent, self-acting, spontaneous muscle-its primary function being to seize and transmit food whenever presented to it. It forms part of a working system. It is for the head and hands to procure and present the food. When food is presented to the nesophagus, it at once becomes aggressive and seizes it. It does not wait to be jogged into activity by artificial stimulation, which stimulation, as explained, could not produce alternate closing and opening of its several portions. The esophagus is a highly specialised portion of the alimentary canal. It is an original construction, and finds a place in the organisms in which it occurs from the outset. It is a spontaneous and self-acting mechanism. One can swallow air and fluids when no bolus is present. Without an oesophagus and alimentary canal none of the higher animals could exist. ${ }^{1}$ As showing the importance of the oesophagus one has only to realise that its double centripetal and centrifugal powers, which enable it to close or contract at one part while it opens or dilates at another part, foreshadow the movements of the hollow viscera generally, such as the heart, lungs, \&c., and of the hollow viscera with sphincters, such as the stomach, rectum, bladder, and uterus. The osophagus occupies a unique position because of its fundamental nature and its numerous important relations. It is virtually endowed with universality of movement, and a careful study of it forms a fitting introduction to all those structures in which rhythmic movements occur (the Author, 1867).

B. Portion of the human duodedum, or first part of the small intestine, showing the valvulæ conniventes. The valvulæ $(c, d)$ project into the interior of the gut in two segments which interdigitate and overlap. They form part of an obstructive system which has for its object the delaying of food in its downward passage. They have great significance when viewed in connection with sphincters, whose precursors or predecessors they are. Their distinguishing feature is their arrangement in symmetrical pairs, which interdigitate and overlap where they meet. A similar arrangement is seen in the lower portion of the cesophagus of the cat when inverted. The valvulæ are arranged spirally, and not circularly, as is usually stated. They cross where they meet very obliquely, as happens in the muscular fibres of the pyloric valve (see $c, d$ of Fig. O), (the Author, 1867).

C. Pyloric end of the human stomach $(a, b)$, showing the minute or ultimate construction of the pyloric valve $(c, d)$. The valve consists of two sets of muscular fibres which overlap at the angles. They enclose a small elliptical aperture. They are not composed, as is generally believed, of cirular fibres: indeed there are no circular fibres either in the stomach or sphincter. The fibres usually figured and described as circular are the terminal loops of figure-of-8 fibres arranged in a more or less continuous layer. The pyloric valve forms a narrow slit or opening which no power possessed by the muscular walls of the stomach could forcibly open. As a matter of fact it opens or dilates spontaneously when the body of the stomach closes or contracts. This is true of all sphincters. The structure of the cardiac sphincter ( $d$ of Fig. E) gives the key to that of all the other sphincters (the Author, 1867).

D. Vertical section of the pyloric end $(a, b)$ of the human stomach; showing the pyloric valve (c) from the inside. This valve is formed by a duplication or doubling in of the entire walls of the stomach, and is very strong. It projects into the stomach as the valvulæ conniventes project into the duodenum. Only the half of its small aperture is seen. The sphincter, as in C, opens up or dilates spontaneously when the stomach closes or contracts (the Author. 1867).

E. The muscular fibres of the human stomach as dissected by the Author. Seen from the lesser curvature anteriorly. Shows radiating muscular fibres, portions of several layers and the formation of the cardiac sphincter (not hitherto described). $a$, Portion of cesophagus; $f$, root of cesophagus with fibres radiating anteriorly and posteriorly $\left(h, h^{\prime}\right)$ in the direction of the pylorus $(b)$; $c$, sulcus indicating position of the pyloric valve: $d, e$, great muscular bands crossing very obliquely, anteriorly, and posteriorly, and overlapping to form the cardiac sphincter. By the contracting of these bands the root of the oesophagus $(f)$ is readily obliterated. $y$, Portion of the so-called circular layer.

For a more extended account of the stomach and its sphincters, see Plates cii. and ciii.

\section{§59. Muscular Action (Voluntary and Involuntary), as bearing on Locomotion, Respiration, Circula- tion, Alimentation, Urination, Defæcation, and Parturition.}

Normal muscular action is from within and not from without. It is the result of inherent endowment and vitality. It is not caused by artificial external stimulation, and this is where, it appears to me, modern physiology has made a wrong departure. Modern physiology attempts to work muscle from the wrong side, and has to assume that it is irritable or excitable; that artificial stimulation and normal nerve action are one and the same thing; that a large amount of the work performed by muscle is due to elasticity; that muscle is always on the stretch or in a tonic condition-no real interval of repose being ever allowed to it; that flexor muscles when they shorten or contract drag out or forcibly extend and elongate the extensor muscles and vice versa, and so of the pronator and supinator muscles, abductor and adductor muscles, \&c.

That muscle does not require to be goaded into activity by external artificial stimulation is proved by muscular action as a whole, and by the action of the heart in particular. The movements of the heart are inherent, fundamental, and rhythmic in character. In the chick, where the heart is composed of a mass of nucleated cells, they take place in the absence of muscles, nerves, and even blood. No external or internal stimulation is required, and there is no trace of irritability or excitability in the cells forming the heart. The opening and closing movements of the heart are both vital; there is no opportunity for elasticity coming into play, neither is forcible distension possible, seeing

I In cases of constriction or partial obliteration of the oesophagus from any canse the patient can be fed by means of a flexible tube, but this is never satisfactory, and is of course impossible in a state of nature. 
the blood, which is regarded as the distending medium, is absent. Similar remarks are to be made of the adult heart. The heart both in the young and adult condition is a master organ; it is, in a sense, the mainspring of life; its movements, as explained, being fundamental and inherent, and in no way due to caprice or accident. There is no proof that the normal heart is irritable, ${ }^{1}$ or that stimulation of any kind is necessary to its action.

The modern physiologist pricks or applies electricity to a heart or a muscle which is in a state of quiescence, and gets one or more contractions, and straightway concludes that the stimulation is the sole cavse of the movement. He forgets that the heart can and does contract normally without any stimulus, external or internal. This remark applies to all the hollow muscles. It also applies to all the voluntary muscles, as they too are moved from within by the efforts of the will, and as apart from all kinds of external stimulation.

It is true that a muscle can be made to contract by applying an artificial stimalus to it directly, ${ }^{2}$ or to a cut motor nerve attached to it. It does not, however, follow that the artificial stimulation produces exactly the same result as the impulse sent out through an uncut motor nerve to the muscle by a nerve centre. In like manner artificial stimulation, at its proximal end, of a cut sensory nerve produces pain, but no one can say that the kind and degree of pain are identical with that produced by contact with a natural object applied to the uncut sensory nerve at its distal end. At best the results obtained by artificial stimulation and section of the motor and sensory nerves are crude imitations of the normal acts, and consequently to be accepted with caution.

As regards the elasticity of muscle much misconception exists. Elasticity, it is said, only acts when muscles relax, elongate, or dilate; as a matter of fact, the little elasticity there is comes into play also when muscles contract, shorten, or close. This follows, because elasticity, whatever its amount, is a property of the sarcous elements in which muscular movements begin and terminate. The elastic properties of muscle are, as a rule, greatly overstated. When elasticity is to play an important part in living structures, the structures are specially modified for the purpose. Thus in the vorticella a special spiral spring is provided for the retractile or shortening movements of the pedicle or stem. The same is true of the wing of the insect, bird, and bat in flexion. Examples of special elastic tissues are afforded by the inter-vertebral discs or cartilages of the spinal column, the ligamentum nuchæ, yellow elastic tissue, \&c. The large arteries, which contain no muscular fibres, are highly elastic, but the small arteries provided with muscles are very little, if at all, elastic.

Elasticity usually manifests itself by recoil or sudden movements. The sudden movements of muscles, however, do not occur during the elongating or dilating process, when elasticity is said to exert its chief powers, but during the shortening or contractile process, when elasticity is said to be in abeyance. According to prevailing views, the shortening or contractile movements of muscles are vital-the elongating or dilating movements elastic. The elongating movements are said to be mechanical, whereas the shortening, contractile movements are said to be vital. According to my experiments and observations, both movements are vital, elasticity playing quite a subordinate part, but acting to a trifling extent in either case.

But (and this is important) the prevailing theory of muscular action is inconsistent. In the case of the hollow viscera - the heart, stomach, rectum, bladder, uterus, \&c.- the closing, shortening, centripetal movements are said to be vital and sudden; whereas the opening, elongating, centrifugal movements are said to be mechanical and slow.

In the respiratory movements, however, all this is said to be changed. In this case, the opening, elongating, centrifugal movements of the chest are said to be vital and sudden, whereas the closing, shortening, centripetal movements are said to be mechanical and slow. These two positions, it will be observed, are diametrically opposed to each other.

If vital acts are required to close the heart and open the chest, it is abundantly evident that both the closing and opening movements of the heart and chest are vital in their nature, but this is what the theory inconsistently and persistently denies.

There is no difference as to the manner in which the heart and chest are opened and closed. In both, the opening and closing movements are directly due to vital muscular action. The fact that in the chest the muscles act in conjunction with bones and cartilages does not alter the situation. In either case, the movements begin and terminate in the muscles themselves. If, as happens in the chest, the elasticity of the ribs and cartilages is evoked, the muscles are still the prime movers. Bone and cartilage by themselves can neither originate nor perpetuate movement.

1 Chauveau introducerl small edoutchone hags into the hearts of horses without interfering in any way with the normal action of the heart. This experiment is fatal to the theory that the interior of the heart is irritable, and that the blood causes the movements of the heart.

2 If for example, an irritant be applied to a muscle or muscles in the act of elonsating, as in a worm crawling forward, the mussoles by survise, and the forward movemeut is temoraily or muscles in the act of elongating, as in a worm crawling forward, the muscles are taken

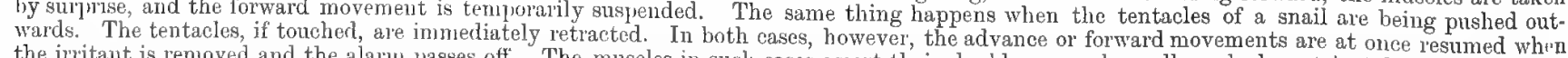
the irritant is removed and the alarm passes off. The muscles in such cases assert their double power ly well-marked centripetal and centrifugal movements. Muscles, or fortions thereof, also withdraw and contract in various parts of the body if the skin covering them he suddenly and
smartly tapped. 


\section{MUSCULAR ACTION (VOLUNTARY AND INVOLUNTARY)}

It cannot be a vital act which closes, and a mechanical one which opens the heart on the one hand; and a vital act which opens, and a mechanical one which closes the chest on the other hand.

A theory which involves such a glaring contradiction, and which relies upon irritability, artificial stimulation, and elasticity as its chief supports cannot be of much account.

The grand fact remains, that muscles in their normal condition move, and move to definite ends, as apart from irritability, and as apart from external stimulation. The blood does not irritate and excite the heart to action, and the air does not irritate and excite the chest to action. The heart is specially formed to receive, contain, and force on the blood at regular intervals by rhythmic movements, and the chest and lungs perform a like function for the air.

The movements of the heart and chest, as already explained, are inherent and fundamental, and they go on so long as life lasts, as apart from irritability and artificial stimulation.

The movements of the heart of the chick, as stated, are not dependent on the presence of either blood, muscles, or nerves. When nerves and ganglia are present they perform the rôle rather of regulating than propelling structures. Similar remarks are, within limits, to be made of the respiratory or chest movements.

The heart and chest are framed on the same general lines, and their movements are fundamentally the same. They even perform a common work, namely, the transmission and aëration of the blood which feeds the tissues. The right ventricle forces the blood into the lungs, and the chest and lungs force air into the blood_- the left ventricle propelling the aërated blood through the system generally. The presence of bones is not necessary to muscular action; the heart acts perfectly in their absence. In the chest, the clavicles, scapulæ, ribs, cartilages, sternum, \&c., supply a scaffolding and protection to the lungs and heart. The chest and heart furnish typical examples of centrifugal and centripetal rhythmic movements. The opening and closing movements of the chest and heart are mainly, if not wholly, muscular. The chest supplies an outer pulsating chamber within which the heart can pulsate safely: as in "Hamlet," there is a play within a play.

Much importance in modern times has been attached to the combination of bones and muscles, and the origin and insertions of the latter have been described with scrupulous exactitude. As stated above, bones are not necessary to co-ordinated and exact muscular action. When they are present, they are, as a rule, the servants and satellites of the muscular system. They are in no instance prime movers. Their main function is one of support; their secondary function is one of leverage.

The prevailing theory of muscular action fails in another and most important respect. It requires, as has been indicated, for its realisation that the flexor muscles, when they shorten or contract, shall forcibly drag out, elongate, or dilate the corresponding extensor muscles; the extensor muscles, when they shorten or contract, forcibly dragging out, elongating, or dilating the corresponding flexor muscles. Here again there is inconsistency. According to the generally accepted theory, the flexor and extensor muscles, the pronators and supinators, the abductors and adductors, \&c., are made to antagonise, oppose, and contend with each other.

The fact that the flexors, pronators, and abductor muscles are arranged on one side of the bones to be moved, while the extensors, supinators, and adductor muscles are arranged on the opposite side, does not convert these muscles into antagonists; in reality, they form muscular cycles within which the bones may be said to vibrate. Muscles are, for the wisest of purposes, disposed in groups, and the groups move simultaneously and in sympathy. The groups are co-ordinated; they are in no way opposed or antagonistic. This is necessary at once for symmetry, harmony, and economy. Moreover, there are muscles which have no antagonists in the sense required by the theory. The diaphragm is an example in point. The diaphragm, which may be compared to half a heart, half a stomach, or half a bladder, elongates or dilates and bulges upwards during expiration, and shortens, contracts, and flattens in a downward direction during inspiration. The dilating upward and the contracting downward movements are equally vital in their nature. The one movement cannot by any possibility produce the other movement. Precisely the same thing occurs in the opening and closing of the several portions of the œsophagus, of the several parts of the stomach and bladder, and of the different compartments of the mammalian heart. The two auricles of the heart close when the two ventricles open; the ventricles closing when the auricles open. This follows, because the auricles are so thin that they lack the power to force blood into the firmly contracted and, for the time being, solid ventricles. In the heart, as in the diaphragm, there is no antagonism whatever. There is, further, no antagonism in the movements of any of the hollow viscera. The stomach, bladder, rectum, uterus, and their sphincters all open and close by spontaneous, rhythmic, vital movements at stated intervals, as apart from antagonism.

Antagonism implies a ruinous waste of power; it, moreover, ignores the object and aim of all muscular action. Nature does not strive against herself. She conserves her energies, and when she puts them forth she does so on the most advantageous mechanical principles. The object of muscular action, in every case, is to perform work; VOL. I, 


\section{DESIGN IN NATURE}

to move bones which form levers, as in the movements of the arms and legs in locomotion or otherwise; to move all parts of the body in the practice of the arts ; to transmit gases, semi-fluids, solid substances, \&c., as in the heart, asophagus, stomach, bladder, rectum, and uterus.

Muscles never neutralise or work against each other. This would altogether defeat the object in view.

All muscular movements are co-ordinated-that is, they are movements to a given end. If the closing or systole of the heart be sudden, and the opening or diastole slow, it is because the blood has to be hurried forward by a vis a tergo movement, and time allowed for it to re-enter the ventricles, which, when they open, exercise a vis a fronte, action.

The deliberate co-ordinated movements of the extremities and other parts of the body are inconsistent with the jerky movements which would be produced by a mechanical forcible dragging out of either the flexor or extensor muscles during the movements of flexion and extension. It is not conceivable that the delicate operations performed by the extremities, hands, feet, and other parts of the body could possibly be effected by muscles (flexors and extensors, pronators and supinators, abductors and adductors) all playing at cross purposes.

The prevailing theory of muscular action fails because it assigns to muscle only one power, namely, the power of shortening or contracting. As a matter of fact, and as $I$ have shown on several occasions in this work and elsewhere, muscle is possessed of a double power, namely, a power by which it elongates or dilates the one instant, and shortens or contracts the next. This double power admits of the most delicate co-ordination; the flexors, pronators, and abductors shortening or contracting when the extensors, supinators, and adductors elongate, and vice versa. In this way no power whatever is lost, and the muscles are under the most perfect restraint and control. There is no violent tugging of muscle against muscle, no muscular warfare; all is harmony and the outcome of design. According to this view, there is also provision for perfect rest to the muscles. Muscles are either acting or at rest. When they act, they act together and at the same time. When they are not acting they are resting. There is no need for muscles being always on the stretch and in the so-called tonic condition.

An appreciable interval of time (the so-called latent period) elapses between the discharge of motor impulses and the movements of muscles, but this interval is common to the flexors and extensors, the pronators and supinators, the abductors and adductors (they form, as explained, co-ordinated groups), so that, when motion occurs in the flexors, pronators, or abductors, it occurs simultaneously in the extensors, supinators, and adductors, and the converse. If muscles are suddenly brought into requisition by slipping, sudden loss of balance, \&c., before a perfect co-ordination occurs, the substance of the muscles is not unfrequently ruptured or torn. The several voluntary muscular movements are associated, co-ordinated movements, and these movements are acquired after much patient and laborious effort and education. The involuntary muscular movements also admit of being trained. They are at once fundamental and intuitive, and are stereotyped by unconscious repetition.

The bones and joints, it may be remarked, are not necessary to locomotion. In the Protozoa or unicellular animals this is effected by an amorphous contractile mass. In the worm, leech, and caterpillar it is effected by imperfect muscular fibres contimuous upon themselves, as in the hollow viscera of Vertebrates. The muscle becomes interrupted in the Crustaceans by the interposition of an external, and in the Vertebrata by the presence of an internal, skeleton. When, therefore, the external and internal skeletons make their appearance, it is to afford the muscular system additional surface and leverage, and to enable it to act with greater precision in a given direction. The skeleton, since it cannot move of itself, is consequently to be regarded as an adjunct or auxiliary of the muscular system. As the muscles are accurately moulded to the bones and to each other, either directly or indirectly (by tendons), and the bones, joints, and muscles move in perfect harmony (the bones being unyielding or rigid), it follows that the osseous system acts as an artificial break or boundary to the muscular one-hence the arbitrary division of muscles into extensors and flexors, pronators and supinators, abductors and adductors, \&c. Instead, however, of dividing the muscles into two opposing, disjointed sets, it would be more intelligible, and, I believe, more philosophical, to regard them as forming continuous muscular circles or cycles bisected by bones, the articular surfaces of which enable the cycles to move the bones with absolute precision in any direction desired. If this plan be adopted, the voluntary system of muscles is readily assimilated to the involuntary, and both are referred to their original, the continuous elementary fibre. This view is favoured by analogy, and by the fact that the muscular system in the higher Vertebrates is in a state of rest (the so-called tonicity of authors), that is, equally balanced or oscillating between two imaginary fixed points, and ready to act, through its extensors and flexors, abductors and adductors, pronators and supinators, with surprising rapidity; the contraction of the extensors on all occasions involving, but not causing, the relaxation of the flexors, and vice versa organised animal may, in this sense, be regarded as a living mass whose parts (hard, soft, and otherwise) are accurately adapted to each other, every part reciprocating with scrupulous exactitude, and rendering it difficult to determine where motion begins and where it terminates, 


\section{MUSCULAR ACTION (VOLUNTARY AND INVOLUNTARY)}

Similar remarks, but in a modified sense, apply to the involuntary hollow muscles, such as the heart, œsophagus, stomach, rectum, bladder, uterus, \&c.

In these there is a distinct double power of opening and closing, these movements being spontaneous and independent of each other.

By ascribing to the sarcous elements of muscles, and the muscles themselves, a double power, namely, a centrifugal and a centripetal power, whereby they can close, contract, and shorten on the one hand, and open, expand, and elongate on the other, all the difficulties connected with muscular action are obviated. The subject is further elucidated by regarding the closing or centripetal and the opening or centrifugal movements as both vital in their nature, as we in this way get rid to a large extent of elasticity as a supposed prominent and necessary factor in muscular movements. By investing the sarcous elements of muscles with the double power in question we assimilate their movements to those witnessed in protoplasm, in white blood-corpuscles, in amœbæ, pseudopodia, \&c. We, in fact, invest muscle with a certain degree of pushing or aggressive power which is fundamental in all vital movements. Animals directly or indirectly advance and throw themselves upon their prey, and the centrifugal advancing power is, in some senses, more important than the centripetal or retreating power. Even plants are aggressive as far as the search for food is concerned. The roots, branches, and leaves continue to advance so long as plants grow. Whenever advance and growth cease, and a condition of stasis sets in, the plant is on the high road to decay. Plants, like animals, possess a centrifugal and a centripetal power; the former enabling the plant to seize the elements required for food, and the latter enabling it to draw in the elements necessary as food into its interior, where they are assimilated.

All cell growth and transformation are the result of atomic and molecular motion, and as cells reproduce themselves endogenously, exogenously, fissiparously, and gemmiparously, the movements are at once extensive and varied.

The elaboration of cells into tissues likewise necessitates movements. Further, the various processes in plants and in animals, such as digestion, assimilation, secretion, excretion, muscular and nerve action, \&c., are the outcome of movements. The living plant and animal, the simplest and the most complex, are fundamentally moving masses of sentient protoplasm, and it is only by studying protoplasm and protoplasmic formations and movements that we can hope to get a real insight into the economy of plants and animals in their entirety.

If we could peer into the interior of a plant or an animal, and our eyes were equal to the occasion, an unparallelled scene of activity would meet our gaze; gases, fluids, semi-solids, and solids all hurrying hither and thither in the most complex and bewildering manner, yet all according to law and order. Indeed all the most wonderful, if not the most beautiful, parts of plants and animals, are concealed by non-transparent tissues and integuments.

Electricians are sanguine enough to believe that at no very distant day a light, sufficiently powerful, will be devised to make all living bodies diaphanous. Should that day ever come, biology and physiology will enter on a new era. Then we shall see face to face, and not through a glass darkly, as at present. The discovery of Röntgen rays is a step in the direction indicated, and already the bones can be photographed within the muscular cycles which actuate them. By these means also foreign substances, which have gained surreptitious entrance into the body, can be detected.

The movements of transparent living matter are of the utmost consequence in biology and physiology, and this fact invests the study of transparent rudimentary plant and animal forms with ever-increasing interest.

The simplest plants known are the unicellular plants, of which the yeast and snow plants are examples. These low rudimentary forms are perfect in their way. They live, grow, and reproduce themselves with as much certainty as the higher forms. They are literally foci of force and movement.

Many of the spores of plants move, some by means of cilia, some by flagella, and some in virtue of their spiral shape. It not unfrequently happens that the young of plants move freely about until they find a suitable habitat, when they cast anchor, develop roots, and fix themselves. Similar remarks are to be made of animals — sponges, for example. The sensitive and insectivorous plants, though fixed, are continuously in motion, and so of many fixed animals.

Plants circulate their nutritious juices; they respire; they secrete and excrete; they grow and reproduce themselves; they move in all their parts. Plants close and open their leaves and the stomata contained in the leaves; they are more active during the day than during the night, and in summer than in winter. The stem grows in length and in breadth; the roots shoot downwards and outwards; the branches upwards and outwards; and the leaves in a great variety of directions. The stems, roots, branches, and leaves often move in a spiral manner. They perform movements of gyration, circumnutation, \&c.

The movements of animal protoplasm are, if possible, more interesting than those of vegetable protoplasm.

Some authors maintain that vegetable and animal protoplasm are not only similar but identical. This 
subject is still sub judice. Kühne showed that vegetable protoplasm placed within the intestine of a cockchafer contracts like a muscle under the influence of electricity.

The movements of the white blood-corpuscles in red-blooded animals are especially deserving of study. In slight local inflammations, such as are produced in the mesentery of a mouse by applying a weak solution of mustard, the corpuscles in question are seen by the aid of the microscope to adhere to the interior of the capillary blood-vessels, and gradually to force themselves through the capillary walls, assuming, as they do so, a great variety of shapes. What generally happens, and what I myself have frequently observed, is this : the corpuscle projects from its substance a wedge-shaped portion, which is forced into the capillary wall; the remainder of the corpuscle streams through the aperture so produced by what appears to be a pushing movement; once outside the capillary vessels the corpuscle assumes its spherical form. The white blood-corpuscles are endowed with independent vital movements. They are also credited with the power of combating and destroying inimical living organisms which invade the blood and engender disease, often of a fatal character.

That living organisms do infest the blood is proved beyond doubt by their being found in certain cases in large numbers in the blood-plasma and within the red and white blood-corpuscles. This happens in malaria and other diseases, as proved by the aid of the microscope. As the red blood-corpuscle in man measures only from the $: \frac{1}{000}$ to the $\frac{1}{4000}$ of an inch in diameter, I leave it to be inferred how very minute the malarial microbes are. As a matter of fact, some of them have a diameter of only the $\frac{1}{2000}$ of an inch or thereby.

The manner in which malaria, plague, sleeping sickness, and other diseases are produced is now well understood. The malarial and other microbes are introduced into the human skin and fluids of the body by the bite of certain mosquitoes, the tsetse and other flies, and float about in the blood-plasma, where certain of them enter the red and white blood-corpuscles, which act as hosts to the invaders. As the red and white blood-globules and the microbes are both in motion, their coming in contact is generally a mere matter of time (Figs. 61 and 62 , page 306 ; Plate lxxxii., Fig. 1, page 313).

Professor Huxley has figured the amœboid movements of the blood-corpuscles of the cray-fish (Plate lxxix., Fig. 6, page 308 ).

It may be useful if at this stage I give a short account of the amoba, paramecium, gromia, and other rudimentary animal forms which supply information of a very important character, not only as regards movement, but also as regards organisation and reproduction.

\section{RUDIMENTARY FORMS IN RELATION TO MOVEMENT, REPRODUCTION, AND LIFE}

\section{$\S 60$. Amœba Proteus.}

The amœba is deserving of very special attention for three things: $(a)$ its simplicity of structure; $(b)$ its universality of function; and (c) its extraordinary power of movement.

It is a rudimentary microscopic organism, varying from the $\frac{1}{30}$ to the $\frac{1}{10 \overline{0}}$ of an inch in diameter, and is found in the mud of streams and ponds. It has no definite shape, and resembles a drop of semi-liquid jelly.

It is a type of the sub-kingdom Protozoa, and consists of a simple cell; it is an example of the phylum Gymnomyxa (naked jelly), its protoplasm being freely exposed to the surrounding water and its whole body performing the functions of ingestion, egestion, assimilation, respiration, reproduction, movement of various kinds, a low form of sensation, \&c. It is representative of the class Rhizopoda, as it throws out blunt pseudopodia or false feet from its substance.

The amœba, designated Amoba proteus from its ever-varying shape, consists of a thin superficial layer of dense clear protoplasm (ectoplasm), and a thinner interior protoplasm (endoplasm). In the latter are found food-vacuoles with or without food; a large contractile vacuole, filled with clear fluid, and a nucleus composed of nucleoplasm. The food-vacuoles occupy various positions; any part of the amoba becoming a temporary stomach. The contractile vacuole is endowed with the extraordinary power of closing and opening by centripetal and centrifugal rhythmic movements, similar to those occurring in highly-differentiated hearts; the vacuole opening slowly and closing or contracting somewhat suddenly. The contracting vacuole communicates by a minute temporary canal with the exterior, by which it ejects its fluid. It is believed to be an excretory organ for the removal of nitrogenous products.

The other matters found in the endoplasm consist of minute granules, small regular crystals, and sand and other grains. The amoba, it will be seen, is very far from being homogeneous in composition, although usually regarded as such.

The general appearance of the amceba is given at Fig. 68 . 
The amoba draws in its pseudopodia and assumes a spherical shape at a temperature of 35' C. If the temperature be raised to $40^{\circ} \mathrm{C}$. it dies. Under unfavourable circumstances, such as drought, the ectoplasm secretes a thin hyaline case, and the amœba becomes encysted and dormant, in which condition it is carried by the wind or other means to various localities.

The amoba is invested with practically universal powers, and furnishes one of the most remarkable examples known of the potentialities residing in rudimentary living matter. Thus any part of its substance may become a stomach and digest its food, and any part of its body may throw off the waste products ; it has, however, neither mouth nor anus. It feeds upon diatoms, desmids, spores of algæ, and other vegetable matter, and occasionally on animal matter, such as portions of rotifers and of Protozoa, notably Arcella.

When the amoeba falls in with a microscopic alga or food particle, the protoplasm flows round it ; the ectoplasm opening to receive it and closing behind it. It enters the endoplasm with a drop of water which constitutes the food-vacuole, and is temporarily included in the body of the amoba. The alga is slowly digested, its insoluble proteids being converted into soluble and diffusible proteids, which pass into the substance of the endoplasm. It

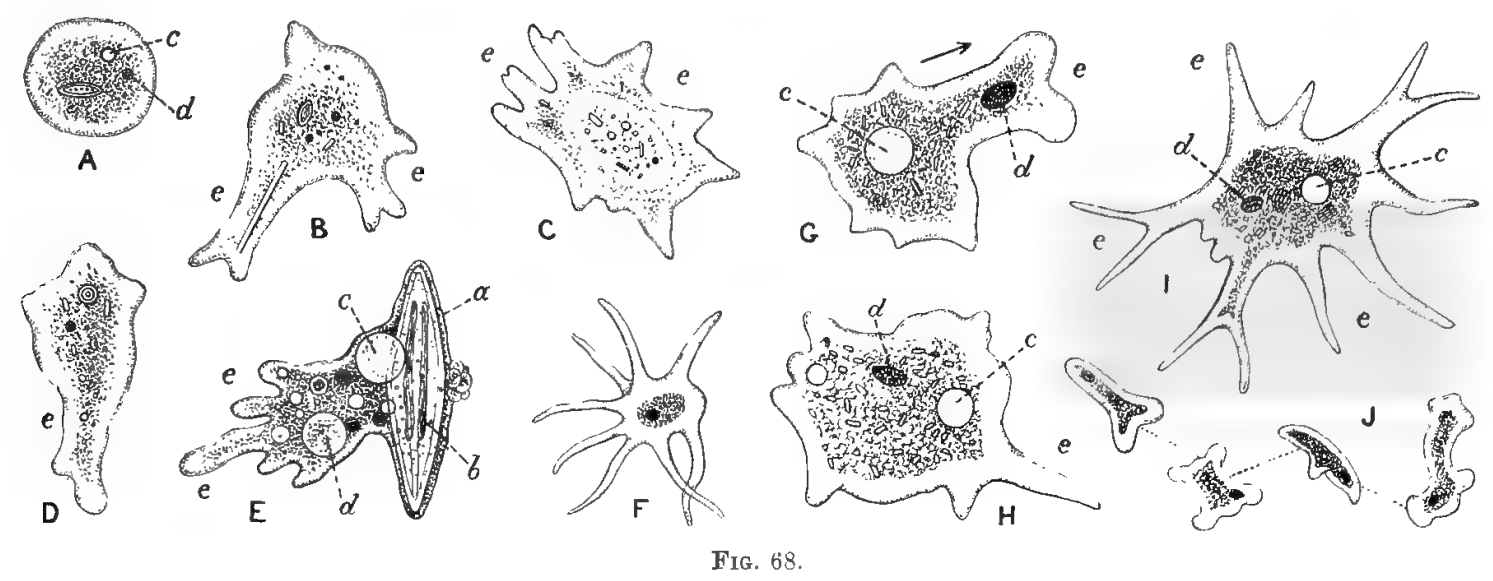

FIG. 68.-Amahn proteus as seen under varying conditious of size and shape (A to J), magnitied from 400 to 800 diameters (after Masterman, and Marshall and Hurst).

A. Amceba in the encysted state, composed mainly of granular and clear surcode; $r$, contructile vesicle; $d$, nucleus. It contains an oval-shaped diatom.

B to J. Different shapes assumed by largex and smaller anwebis. In these figures the contractile vesicle is seen at c, the nucleus at $d$, and the finger-like projections at $e$. The projections, sometimes designated pseudopodia, are for the most part conical.in shape, as seen at C, D, E, F, G, H, and I. They are caused by the animal voluntaluily forging a part of its body outwards from the centre centrifugally. They are retracted in an opposite direction or centripetally. They are not due to contractions; if they were, the projections would not be conical-shaped, but cunstricted where united to the body. In reality, they are produced by an aggressive, streaning, rhythmic movement similar to what occurs in the plasmodium of the Mycetozoa and gromia already described.

At $\mathrm{E}$, the body (a) of an amoba is stretched over a large diatom (li).

At $\mathrm{J}$, the varying forms assumed by a small amoeba, at intervals of half-a-minute, are given.

may be well to state that the proteids are themselves constituents of protoplasm. The amøba, it is believed, cannot digest carbo-hydrates or fats, and does not build up its protoplasm from lower chemical constituents. The indigestible cellulose walls of alga and other intractable materials are extruded from the body. The amœba inhales oxygen and exhales carbonic acid, in which respect it resembles the higher animal forms. It has, however, no branchiæ, gills, or lungs; its whole substance performing this important function.

The reproduction of the amœa is effected in the simplest manner possible. The nucleus, and with it the sarcode of the body, divides into two. In this respect the process resembles the first step in the segmentation of the impregnated ovum of the higher animals. There are, however (so far as known), no male and female elements, and the animal is not hermaphrodite. The original individual becomes two separate entities, each of which, after a short time, becomes as perfect as the parent, and so life is transmitted and continued in the most direct and effective manner. The reproduction of the amœeba is fundamental and thorough-going. It does not require two separate organs or individuals for its consummation. It is thought by some that the amœeba has not only the power of dividing into two by binary fission, but that two amœbæ can come together, coalesce, and conjugate to form one individual. The process of conjugation, while not quite made out in the amoeba, certainly occurs in other low animal forms, such as the Gregarines and Infusoria. The powers inhering in what is practically undifferentiated living matter are at once striking and impressive. They bring us face to face with the eternal verities of matter and 
force, and it takes an effort to realise the amazing potentialities of both. At the very threshold of animal existence, so to speak, we are confronted with tiny microscopic beings which are, in the ordinary sense, structureless, but which nevertheless are endowed with powers and properties which enable them to live amongst matter, to select, assimilate, and incorporate certain portions of it, and to reject other portions; to employ or set aside the forces inhering in the matter; to feed, secrete, and excrete; to respire; to perform the function of reproduction; and to move voluntarily in all their parts and particles to given ends, as happens in the higher animals. No nervous system can be traced in the amoba. It, however, possesses a nervous system potentially.

The movements of the amœba cannot be too carefully studied. In them, it appears to me, are to be found the types, models, and principles of organic movement as a whole. They wonderfully resemble, as already indicated, the movements of the sarcous elements of muscles, on which not only locomotion, but alimentation, respiration, circulation, urination, defæcation, and parturition in the higher animals depend.

If an adult healthy amoba be examined by the aid of the microscope in a good light it will be seen to move wholly or in part. The manner of its movement is peculiar. Its substance flows out in wedge-shaped portions in an aggressive, centrifugal manner. It invades territory, and it envelops food by throwing itself upon or over it. In the advancing, aggressive movement there is no trace of constriction or contraction of any kind in the substance or body of the amoba. The parts thus advanced are, as a rule, numerous; the animal as it were streaming from a central point by means of blunt pseudopods in skirmishing order. When one or more pseudopods encounter a food particle they, with the captured food particle, are withdrawn into the substance and central mass of the animal by retreating, centripetal movements which are the converse of the original aggressive, centrifugal movements. As in the centrifugal movements there was no trace anywhere of constriction or contraction, so in the centripetal movements there is no trace of elasticity. The centripetal and centrifugal movements are spontaneous, rhythmic, vital movements, and are not dependent on either contraction or elasticity; neither are they due to irritability or stimulation of any kind. They are voluntary flow-and-ebb movements, and have equal values. Their function is obvious. They are for the purpose of securing food. When the protoplasm of the amøeba flows as a mass and as a whole in a particular direction, the animal progresses and changes locality. In the movements of the amœba there is no increase or diminution of bulk; the sarcode advancing or retiring in wave-fashion, in good order, and deliberately. The amœba may arrange its substance by an elongating movement of its protoplasm, say from right to left at one time, and at another at right angles by a counter movement, in which case the phenomena witnessed in the sarcous elements of muscles during relaxation and contraction are accurately reproduced. In relaxing and contracting muscles the long axes of their sarcous elements are always arranged at right angles to each other.

\section{§61. Paramecium caudatum.}

The paramecium is a minute, comparatively simple organism which inhabits fresh water and can be seen with the naked eye. It is a type of the sub-kingdom Protozoa, being composed of a single cell with all its activities confined thereto; it is a representative of the Corticata, as it is limited by a cortex and has a definite shape; it belongs to the class Ciliata, cilia forming its means of propulsion.

I append a figure of it (Fig. 69) as enlarged 60 diameters.

The paramecium is an advance on the amœba, having a definite shape, a limiting cortex, a mouth, an imperfect anus, cilia for propulsion and securing food, and the power of causing the food to gyrate within its substance in a given direction. It reproduces itself by a double process of fission and conjugation, whereby two separate individuals complete the reproductive act.

The body of the paramecium is soft, flexible, cylindrical and oval-shaped, with a dorsal and ventral surface. It consists essentially of two parts : $(a)$ an internal semi-fluid protoplasm in which are found a double nucleus, two contractile vacuoles, food-vacuoles and food particles, and, at the periphery, a number of trichocysts with stings; and (b) an external hardened layer of protoplasm designated the cortex, which is covered with a thin hyaline cuticle. In the cortex are found a mouth, an imperfect anus, and numerous apertures, through which protrude a large number of delicate hair-like processes (cilia) and the stings from the trichocysts. The hair-like processes or cilia, which are scattered all over the body and are freely movable, serve at once for propulsion and for securing food; those near the mouth producing artificial water currents in particular directions for the latter purpose.

The possession of cilia constitutes an important departure in the locomotion of the animal, as these hair-like processes are all under control, and can be made to move in rhythmic waves in any direction required, either for the production of food currents or for propulsion. The cilia perform a double function, that is, they can either propel the body as a whole, or simply produce water-currents to collect and bear food to the oral aperture. The ciliary 
movements are spontaneous, voluntary, and very carefully co-ordinated. As a consequence, the animal can dart about and lash into its mouth by the aid of artificial water-currents any food in its vicinity.

The movements of paramecium, as seen under the microscope, are at once striking and interesting. The little creature dashes about at great speed without any apparent means of locomotion; the cilia being so fine as to escape observation when moving quickly. The careering of the paramecium is regulated with the utmost precision; the little creatures avoiding each other and obstacles of every kind with amazing agility. The movements of paramecium are unquestionably under control, and must be regarded as voluntary in character, in which respect they resemble the movements in the higher animals. They are not due to a muscular or nervous system, neither of which are present. Neither are they due to irritability or extraneous stimulation or reflex action of any kind. The existence of direct, spontaneous, voluntary, co-ordinated movements in the paramecium, as apart from nerves, muscles, and other differentiated structures, proves conclusively that rudimentary, undifferentiated, living matter contains in the atoms and molecules of its substance, in a potential form, many, if not all, the peculiarities and powers residing in the most highly organised living matter. This is a subject of great importance, and cannot be too seriously pondered, as it bears directly upon a First Cause, Design, and means to ends.

The mouth of the paramecium is situated on the side of the body in a coneshaped depression or slit (vestibule) at the junction of the posterior and middle third of the ventral surface, and in its immediate vicinity is found the imperfect anus. By the mouth the food, generally microscopic algæ, enters and is made to gyrate within the body (vide darts) in a manner not unlike that by which the food (chyme) is made to travel round the greater and lesser curvatures of the human stomach.

The contractile vacuoles, two in number, possess the remarkable centrifugal and centripetal opening and closing movements described in the amoba. There can be no doubt that these movements are fundamental. This seems proved by the fact that they occur in the most rudimentary and most complex organisms alike. They are connected with the intake and output of extraneous matters, in alimentation, respiration, circulation, secretion, excretion, reproduction, \&c. In all animals, be they small or great, simple or complex, provision must be made for the intake, circulation, assimilation, and output of extraneous matter in a gaseous, liquid, or solid form. Even the simplest cell must be so endowed.

The nuclei are two in number, namely, a large oval-shaped macro-nucleus and a small micro-nucleus. The nuclei are important from a reproductive point of view. When reproduction takes place the nuclei divide; the reproductive process being subsequently completed by a union or conjugation of two separate individuals, thought by some to represent male and female elements.

"During the reproductive process two individuals are in close contact along their ventral surface, their protoplasm becoming continuous through their mouths. as follows:--

"1. In each individual the macro-nucleus breaks up and disintegrates, to be thrown out or absorbed, and the micro-nucleus grows rapidly, and then divides by two rapid divisions into four, two of these pieces being absorbed. Thus by these processes the macro-nucleus and micro-nucleus are now reduced to two fragments of a micro-nucleus. The fragments are each one-fourth part of the original overgrown micro-nucleus.

"2. In each individual one of the parts moves across into the other individual, and fuses with the remaining part of that individual. Sometimes the migrating parts are termed the male pronuclei, and the other two the female pronuclei.

"3. Soon after this, communication between the two individuals becomes interrupted, and they part. In the meanwhile the single-fused nucleus in each divides into two and then into four, so that each individual has then four nuclei.

"4. Two quarters pass to each end of the animal and binary fission takes place. One quarter grows into a macro-nucleus and the other remains a micro-nucleus. The result is a pair of each offspring with a macro-nucleus and a micro-nucleus." 1

The process of conjugation by which the reproductive act is completed, and which requires two separate individuals for its consummation, has an obvious connection with reproduction in the higher animals, so that viewed from the three important points of alimentation, locomotion, and reproduction, the paramecium may be said to

f "Text-book of Zoology," by Arthur T, Masterman, M, A, (Cantah.), D.Sc, (London and St. Andrews), F. R.S.E, 1901, I'L. 91 and 92. 
possess the roots of life as witnessed in many of the more highly differentiated and complex organisms. The potentialities of life receive in paramecium a new and convincing illustration.

Every one who studies the movements of paramecium must be struck with their rapidity, grace, and purposelike character. It is not possible to regard them as haphazard, reflex, or in any way due to irritability, extraneous stimulation, or elasticity. The paramecium is in no sense an automaton.

\section{§ 62. Gromia.}

The gromia, like the amœba and paramecium, is a rudimentary, unicellular, microscopic animal. It belongs to the great group of the Foraminifera, which occurs in incalculable numbers in the ocean, and which has played a most important part in the chalk and limestone formations of the earth's crust from the earliest times. The Forami-

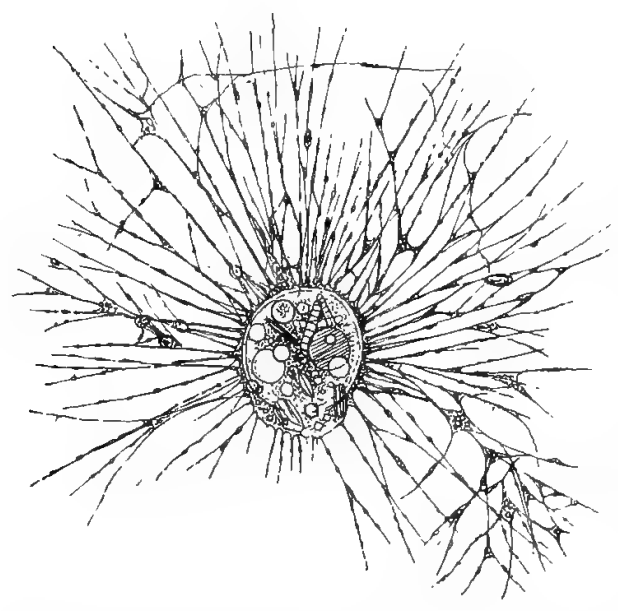

FIG. 70.-Criomia terricolu, greatly magnified (after' Leidy). Shows an amazing display of sensitive living sarcode streaming and radiating hy centrifugal movements. The streaming is reversed by centrijutil movements. In the whole range of natural history there is no more renarkable example of aggressive and regressive, vital, purposive movements. The forward and backward streaming of gromia greatly resembles similar streaming movements in the plasmodium of the Mycetozoa (Fig. 59). In both cases the movements are active, aggressive, and regressive spontaneous movements, essentially rhythmic in character. They consist of an advancing or centrifugal act (extension in space) and a retreating or centripetal act (retraction in space). The movements in question are seen in endosmose and exosmose, in the formation and disintegration of crystals, in the condensation and rarefaction of nebular, in eilia, in hollow and solid museles, in respiration, alimentation, reproduction, circulation, \&e. nifera are remarkable for their minuteness, the extraordinary beauty and variety of their shells, and the delicate colours occasionally displayed by them. Plancus counted as many as 6000 in an ounce of sand from the Adriatic. They are found in millions on the surface and at the bottom of the sea. In the former situation they form a variously-coloured scum, and in the latter they cover thousands of square miles of the ocean bed with a pinkish-white mud, formed chiefly by their skeletons. The shells of the Foraminifera, as a rule, vary from the $\frac{1}{10}$ to the $\frac{1}{20}$ of an inch in diameter. There are, however, genera such as Orbitolites which measure $\frac{7}{1} \overline{0}$ of an inch in diameter. There are also Nummulites with a diameter of $4 \frac{1}{2}$ inches, while the genera Carpenteria and Polytrema measure as much as 5 inches in breadth, and have a thickness of from $1 \frac{1}{2}$ to 2 inches.

"The Foraminifera have in past geological times been among the most active agents in building up the sedimentary rock masses of the earth, by secreting the carbonate of lime from the waters of the ocean. This work is still being carried on by them around the shores of continents, where their accumulated shells go to form, along with the land debris, the terrigenous deposits, and cover large areas of the ocean floor, where, intermixed with coccoliths, they form the well-known Globigerina ooze.

"The organic rocks formed by the agency of Foraminifera are often of very great thickness and extent, and foraminiferal rocks are found inter-stratified with other deposits in almost every geological formation." 1

The most remarkable feature of the Foraminifera is the construction of the shell. This, usually formed of carbonate of lime, is of every conceivable shape; varying from the simple sphere and the plain, nearly straight tube to the most exquisitely curved and ornamented spiral. I give selected examples in considerable numbers in another part of the work (Plates xiii. and xiv.). The marvel, and it is a great and significant one, consists in the fact that a rudimentary animal, low down in the scale of being, consisting of a single cell of sarcode with no visible differentiation other than is provided by a nucleus and contractile vesicle, and with no tissues or organs such as nerves, muscles, glands, \&c., can, nevertheless, produce the most extraordinarily complicated and elaborate shells known, both as regards the variety of the material employed and the perfection and beauty of design of the finished work. In this we have another and striking example of the potentiality of living matter. The marvel can only be explained in one of two ways; either the great First Cause works in and through the apparently simple homogeneous sarcode and directs its atoms and molecules in the direction required; or the sarcode formed by the atoms and molecules is not simple as is generally believed, but differentiated in a way and to an extent of which we have at present no knowledge and of which we can take no cognisance. This follows, because while the sarcode or substance of each animal is apparently identical, the shells, as stated, vary infinitely as regards general design and detail. They also vary as regards material, being sometimes formed of a membrane, sometimes of a chitinous substance, sometimes of carbonate of lime, and sometimes of sand-grains and adventitious matters such as sponge

1 "The Foraminifrit, being an Introduction to the Study of the Protozon," lyy Freslerick Chapman, A.L.S., F. R, M.S., \&c. London, 1902. 
spicules, \&c. Similar variations to those witnessed in shell formation can be traced in the several reproductive processes. The molecular reproductive elements, whether belonging to a simple cellular animal, a hermaphrodite, or a bisexual individual, are, according to popular belief, homogeneous and identical. They are not really so. Each animal only produces its own kind. With the physical conditions of reproduction as regards atmosphere, heat, moisture, \&c., practically the same, the presence of heterogeneous molecules, that is, molecules varying in shape, properties, and powers, and variously assorted and arranged, and with potentialities all their own, becomes a necessity. From homogeneous identical living matter, only one kind of animal could possibly be obtained.

What is true of the molecular basis of reproduction in the aggregate is equally true of the particular sarcode or elementary life stuff of the Foraminifera. To obtain different structures and types even of shells requires peculiarities and special powers in the molecules of the sarcode. There is no logical escape from the belief here expressed.

The following is the account given of gromia by Dr. Carpenter, ${ }^{1}$ who devoted much attention to the subject :"In the Gromix, some forms of which inhabit fresh waters, whilst others are marine, the sarcode body, which is of essentially the same character with that of Lieberkuehnia, is enclosed in a yellowish brown membranous "test"

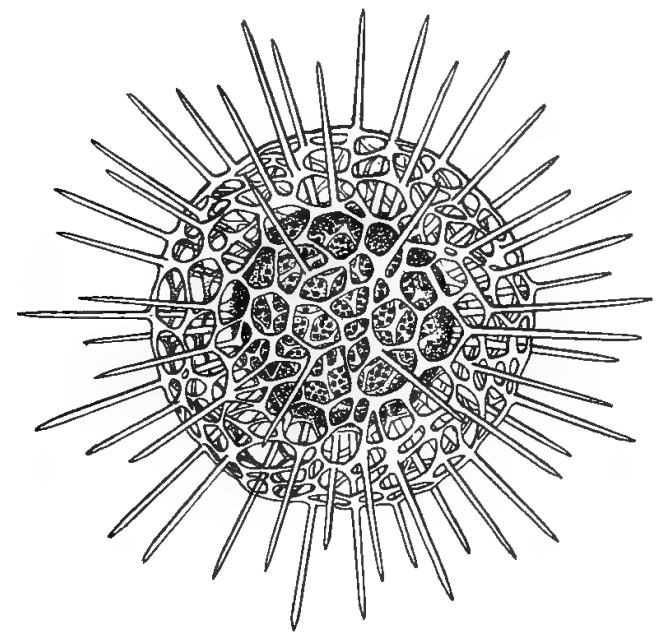

FIs. 71.-Haliomna wyvillii, magnified 200 diameters (after Wyville Thomson).

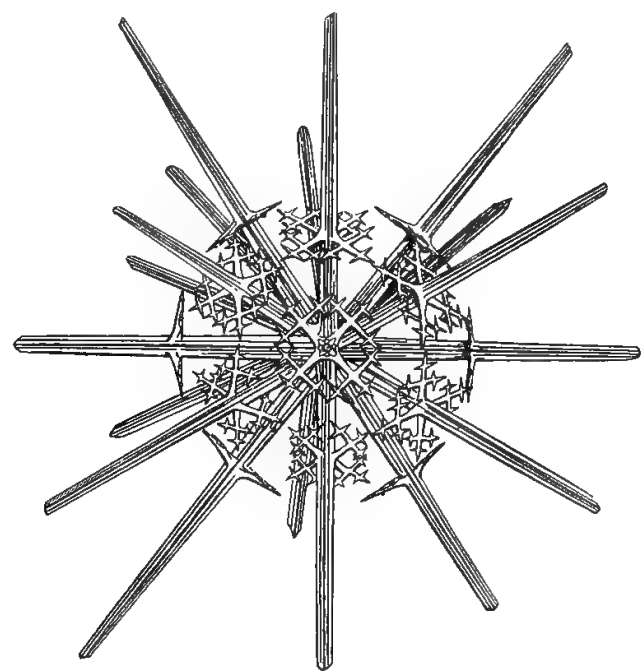

FIG. 72,-Xiphacantha murrayana, magnified 100 diameters. Shows symmetrical, concentric, and radiating arrangement of parts (after Wyville Thomson).

of ovoidal shape, with a single round orifice of moderate size, through which the protoplasmic substance extends itself from the interior through the surrounding medium. When the animal is in a state of rest the whole of this is drawn within the test; and when its activity recommences single fine processes are first put forth, which move about in a groping manner until they find some surface to which they may attach themselves. When this attachment has taken place new sarcode flows into them, so that they speedily increase in size; and they then elongate themselves by sending out finer ramifying processes, which, in diverging from each other, come into contact with those proceeding from other stems, and, by mutual fusion, form a set of inosculations or connecting bridges between the different systems of ramifications, so that the whole becomes a complicated network extending to a distance of six or eight times the length of the body. This network continues to undergo incessant changes, new filaments being put forth in different directions, sometimes from its margin, sometimes from the midst of its ramifications, whilst others are retracted. Not unfrequently it happens that at a spot where two or more filaments meet and fuse together a lamina is formed by the expansion of the viscous protoplasm that flows towards this point; and from such an expansion a new set of thread-like processes is given off, as from the central body."

The general appearance presented by a living gromia is given at Fig. 70 (see also Plate lxxx., Fig. 3, page 309).

The Foraminifera have been divided into two kinds, namely $(a)$ those with one large aperture through which the pseudopodia stream and radiate, and $(b)$ those with perforated shells which give additional outlets for the sarcode in its active state. In both arrangements the sarcode behaves in exactly the same manner ; it exhibits a centrifugal and centripetal rhythmic action: in other words, it flows and streams out of the shell centrifugally in virtually straight lines in a radiating manner; the object being to secure food, fix itself, or change its locality. The streaming of the sarcode in thread-like pseudopodia affords the simplest and best example known of vital force

VOL. I. 
acting at first hand. The streaming is spontaneous and voluntary, and has no connection with contraction or elasticity, with irritability or extraneous stimulation, or with reflexes of any kind. The centrifugal, streaming, radiating, pseudopodic movements, with their concomitant centripetal, retiring, or returning movements, are never haphazard or accidental. They are, in the strictest sense of the term, voluntary, co-ordinated, purpose-like movements ; the object of the double movement being to secure food and convey it into the interior of the body.

As showing common ground between force, growth, and structure, examples are numerous among the Foraminifera and allied groups, where spines and hard permanent substances are developed and arranged in a manner altogether resembling the distribution and arrangement of the soft, streaming, thread-like pseudopodia described. In such cases a symmetrical appearance results. In certain instances an internal skeleton makes its appearance, and when it does, it, for the most part, assumes a concentric, radiating, symmetrical arrangement frequently met with in crystals, plants, and various parts of the higher animals.

The concentric, radiating arrangement referred to is seen in the Radiolaria, the characteristic feature of which is the presence of a membranous "central capsule" which divides the body into two zones, an intra-capsula zone
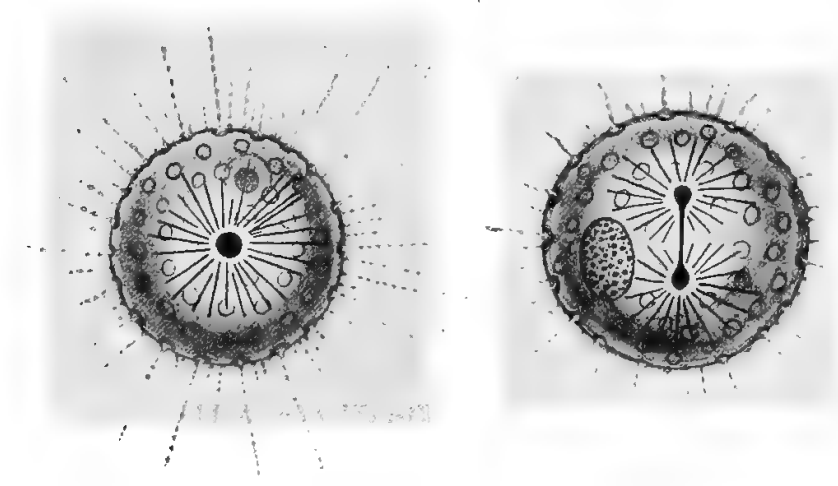

FIG. 73.- Karyokinetic division of the nucleus in Afanthocystis (after Schaudinn).

including the nucleus, and an extra-capsula zone from which the pseudopodia and spines radiate (Figs. 71 and 72).

The body of the Foraminifera consists generally of a transparent sarcode or protoplasm ; the sarcode being occasionally of a brown, pink, or violet tint. The animal is for the most part divided into segments or compartments by septa or divisions in the shell; the sarcode of each compartment being united by slender threads (stolons) of that material. The continuity of the substance of the animal is thus secured.

The Foraminifera, like other Protozoa, possess a nucleus with, in some cases, nucleoli. They also, in not a few cases, possess contractile vacuoles. The nucleus and nucleoli which are immediately connected with reproduction are frequently covered with fine strands of chromatin. The nucleus sometimes divides by binary fission, at other times the nucleoli form a morula-like centre inside the nucleus, the protoplasm outside the nucleus invading it and breaking it up into globules; on other occasions, as shown by Lister in the nucleus of Polystomella crispa, a karyokinetic division of the nucleus takes place similar to what is seen at Fig. 73.

In this figure (73) the radiating arrangement of parts is seen to great advantage, and one cannot help feeling that the lines of force and of growth are in this, as in many other cases, more or less identical.

The Protozoa, of which gromia, paramecium, and amoeba are examples, have been conveniently divided by Messrs. Parker and Haswell ${ }^{1}$ into five classes, which I take the liberty of quoting, the more especially as each class is accompanied by a useful definition :-

"Class 1.-Rhizopoda. Protozoa in which the amœboid form is predominant, the animal always forming pseudopods. Flagella are often present in the young, and occasionally in the adult. Encystation frequently occurs.

"Class 2.-Mycetozoa. Terrestrial Protozoa in which the plasmodial phase is specially characteristic, as is also the formation of large and often complex cysts.

"Class 3.-Mastigophora. Protozoa in which the flagellate form is predominant, although the amoboid and encysted conditions frequently occur.

"Class 4.-Sporozoa. Parasitic Protozoa without organs of locomotion in the adult. Encystation is almost universal, and in the young may be flagellate or amoboid.

"Class 5.-Infusoria. Protozoa which are always ciliated, either throughout life or in the young condition."

The five classes enumerated introduce us to some important distinctions which will be very useful in dealing with other rudimentary forms now to be considered.

\section{§63. The Mycetozoa.}

Perhaps the lowest animal form-if as animal it can be regarded-is supplied by the Mycetozoa. This I have figured and described at length at section 57, Fig. 59, p. 302. It may suffice to say here that it occupies 
the very border line as between the plant and animal. It was long regarded as a plant, and it is only of late years that testimony has accumulated in favour of its being an animal. ${ }^{1}$

Mr. Saville Kent traces a connection between the Mycetozoa and the Spongiidæ; the resemblance extending to the fission or amcbic state, to the production in both of spores with flagella or cilia of some kind, to the encysted or resting, hibernating condition, and to the presence in both of rudimentary skeletons in the shape of horn-like elements or keratose, and spicule-like bodies of carbonate of lime.

\section{§64. Zooids and Monads.}

These are amongst the simplest of living animals. They have separate and combined existences. In other words, they are sometimes found single either in the young or adult condition, and sometimes they are combined to form colonies. The best examples occur in the Infusoria and in the Spongiidæ, which have certain features in common, as proved by the presence in all, in some shape or other, of protoplasm, spores, flagella, cilia, \&c. The protoplasm, spores, flagella, and cilia are also met with in the embryo of the Annelida, Echinodermata, Mollusca, and other invertebrate types.

The presence in incalculable numbers in the air, in streams, lakes, and the ocean of minute rudimentary plant and animal forms is a subject of supreme interest to the reflective mind. There is practically no limit to life, for the most minute living object yet discovered by the aid of the microscope may be resolved into its component parts and beginnings; these being molecular and invisible in their nature. The more rudimentary and minute the plant and animal forms, the more persistent and indestructible in many cases are they. These low types in their germinal or sporular condition can successfully resist temperatures which are fatal to higher organisms. They are proof against boiling water and even higher degrees of heat. So far as extraneous and climatic conditions are concerned they are practically indestructible.

The mere minuteness of an organism does not limit or determine its capacities and powers, and there can be little doubt that unicellular plants and animals are capable of unlimited differentiation in the highest or ultimate sense, that is, in the molecular sense. It is in the atomic and molecular region that the organic meets the inorganic.

It would be a hopeless task to attempt to deal even in a general way with the countless multitudes of infinitely minute and all but invisible rudimentary plant and animal forms. It will be enough to state that they are each and all separate individuals or entities, and reproduce only their own kind. I propose, therefore, to describe very shortly, as representatives of their class, only two of the unicellular organisms, namely, the collared monad (Monosiga gracilis, S. K.) and the vorticella (Vorticella nebulifera).

The monad falls first to be considered. This simple, minute rudimentary animal consists of the following parts :-

(a) An elegant pear-shaped body supported by a slender

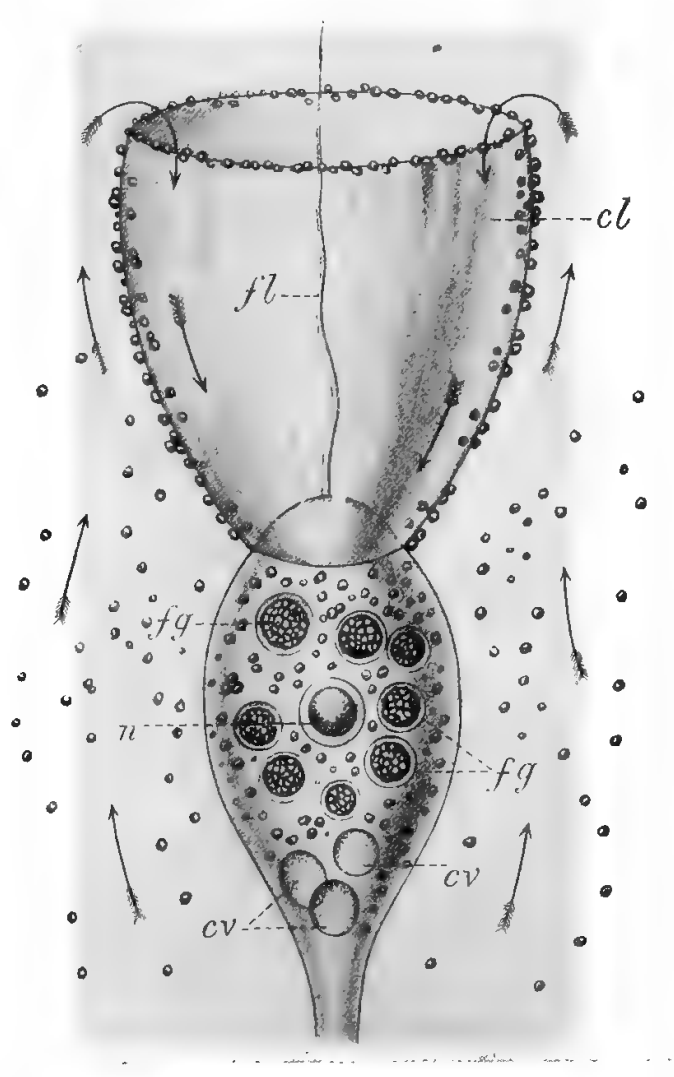

Frg. 74.-Collared monad (LYonosiga yracilis), artiticially fed on carmine and greatly magnified (W. Saville Kent). The animal consists of a peal-shaped body attached to in pedicle or stem (broken off) surmounted by an elegant collar resembling an egg-cup in shape $(c l)$. From the highest portion of the body projects a flagellum or hair-like process $(f l)$, which by its varied movements creates currents in the water, and canses the food particles therein to float into and down the open collar' (see arrows), where they are in a position to be utilised by the body. $f g, f g$, Food granules; $c v, c v$, contractile vesicles; $n$, nucleus. stem, by which it attaches itself to fortuitous objects.

(b) A tapering vibratile hair or flagellum projecting from its upper end.

(c) A delicate funnel-shaped frill or collar fixed to the upper part of the body and investing or surrounding the flagellum.

(d) A nucleus or endoplast.

(e) Several contractile vesicles. 
(f) A large number of food globules and food particles. These are seen to advantage when the animal is artificially fed on carmine, as was done in the case represented by Fig. 74.

All the parts enumerated are of the simplest description. The animal is unicellular and composed of protoplasm, no structure in the ordinary sense being anywhere perceptible. Notwithstanding its extreme simplicity it can live, secure and assimilate food, grow, and reproduce itself. The manner in which it supplies itself with food is the most ingenious and effective possible. It causes its flagellum to perform a rotatory vibratile movement within the funnel-shaped collar in such a manner as to produce in the water in which it lives a series of well-directed currents, which cause the food particles in its vicinity to travel upwards on the outside of its collar and then to turn over and travel downwards on the inside of the collar until they reach the body, where they are ultimately incorporated. Here we have an example of a simple organism performing voluntary co-ordinated movements to a given end, no trace of nerve, muscle, gland, or other structure being present. The movements of the flagellum are evidently controlled, and they are the outcome of arrangement and design as surely as are the movements of the higher animals devised for a similar purpose. Nor are the means of digesting the food so scientifically secured less effective. There is no stomach, nevertheless the food is taken into the protoplasm of the body and digested and assimilated, and the effete or waste products extruded without difficulty. As a matter of fact, every part of the body becomes a stomach pro tem. when food has to be negotiated.

That the monad is a living entity is proved by its life history and by the presence of a nucleus and contractile vesicles in its body; these being entrusted with important functions. The latter (contractile vesicles) pulsate rhythmically, and are endowed with centrifugal and centripetal movements like the heart in the higher animals. The body and collar are endowed with similar movements. All parts of the monad move independently. The nucleus during the reproductive process divides into two; it, with the protoplasm of the body, ultimately becoming two separate individuals. Reproduction in the monad may occur in three ways: $(a)$ by longitudinal division, $(b)$ by transverse division, and $(c)$ by the animal assuming the amœboid and encysted forms prior to the cleavage. It occasionally happens that two monads unite and fuse and become one individual. All the functions of life are satisfactorily discharged by the monad, simple though it be.

\section{§65. Vorticella.}

The vorticella is quite as interesting and instructive as the monad. This is one of the most elegant, active, and wonderful of rudimentary animals. It can just be seen with the naked eye, and occurs both in salt and fresh water. The salt and fresh-water specimens closely resemble each other. The following account applies mainly to the larger fresh water-forms.

A fresh-water vorticella in the expanded condition consists of :-

(a) A cup-shaped body with a spiral stem, by which it fixes itself to plants or other objects, and by which it can advance or withdraw its body at pleasure. Occasionally it dispenses with the stem and swims freely.

(b) A row of cilia attached near a prominent spiral rim (peristome) at the upper, distal, or free end of the body.

(c) A vestibule and pharynx conducting to the ventral surface of the body.

(d) An anus through which fæcal matters are extruded at intervals.

(e) Food-vacuoles which circulate within the endosare or internal fluid protoplasm.

(f) A pulsating vacuole endowed with centripetal and centrifugal movements.

(g) An elongated, curved, rod-like nucleus (Fig. 75).

The vorticella is a protozoon, and the entire animal (the stem included) consists of a single cell, in which respect it resembles the amœba, gromia, and paramecium already described. The body is composed of protoplasm or sarcode, which as in paramecium is divided into an outer, firmer layer (ectosarc) and a central, more fluid mass (endosarc). The stalk, which is supplied with a spiral, contractile fibre or band, is composed of ectosarc alone. The stalk of the vorticella affords a beautiful example of a specially-constructed spiral spring, the movements of which are regulated by the animal. They are very little due to elasticity.

The cilia, which are situated in a spiral groove between the disc and peristome, and also in the vestibule and pharynx, are exceedingly sensitive, and can be moved voluntarily in consecutive order and in specific directions; the object being to produce fluid currents which sweep food into the pharynx and body. The ciliary movements are co-ordinated and spontaneous in a marked degree, and the same is to be said of the spiral movements of the stem. These are perfectly under control, and no more beautiful sight can be witnessed than is afforded by a group of vorticellæ feeding under natural conditions. The cilia are in constant motion, and the body is advanced gently and made to grope about in search of food. If it touches anything suspicious or doubtful it is instantly withdrawn, to be again cautiously and slowly projected. As to the voluntary nature of all the movements of the vorticella there 


\section{GROMIA}

can be no doubt whatever. Not only do the body, stem, and cilia of the vorticella move in a co-ordinated manner, but the several parts of the body move ; each part having the power of contracting centripetally and dilating centrifugally. This power of changing shape in every part is vouchsafed to all, or nearly all, the rudimentary forms. The movements in the vorticella and other rudimentary animals are spontaneous and independent, and are not due to reflex action, irritability, or extraneous stimulation of any kind. Neither are they, in any sense, caused by elasticity.

The pulsating vacuole displays centripetal and centrifugal rhythmic movements similar to those seen in the amœba, paramecium, \&c.

There is no more striking phenomenon in living organisms than is supplied by the throbbing or pulsating movements in question. They appear equally in the simplest and most complex animals, and are, in a sense, coeval with life itself.

The vorticella reproduces itself by longitudinal division. A constriction or dividing line appears at the distal

FIG. 75.-A group of Vorticellæ magnified 220 diameters (after Marshall and Hurst).

A, B, C. Show the animal in various positions.

D. A much smaller specimen drawn to the sume scale.

E. A specimen made to contract by the action of alcohol.

F. A specimen detached from its stalk and swimming away freely, disc forward.

G, H, I. Illustrate three stages of fission.

J, K, L. Show the separated individual swimming by means of the aboral circle of cilia- $\mathbf{K}$ being slightly contracted, and $\mathbf{D}$ strongly contracted. "Food-vacuole discharging contents at anus ; $b$, aboral cilia; $c$, cilia of the disc; of, contractile fibres of the stalk; $d$, disc ; $d^{\prime}$, disc retracted ; $f l$, food vacuoli; $p h$, pharynx ; $\mu$, pulsating vacuole; $\mu$, peristoma; $m$, myophan striation; $n$, nucleus ; $?$, vestibule.

The Vorticellæ are remarkable for their beautiful spiral stems and cilia, and the rapidity and grace of their movements. They retract and shorten their stems spirally and suddenly, and elongate them in similar fashion, slowly, warily, and as if feeling their way. The cilia of the cup-shaped discs are continually in motion, and convey the idea of rotation. The movements of the stems and cilia are voluntary, and under perfect control, as any one may readily satisfy himself by careful microscopic examination. The movements are necessary to secure food. They are essentially centrifugal and centripetal, give-and-take, rhythmic movements.

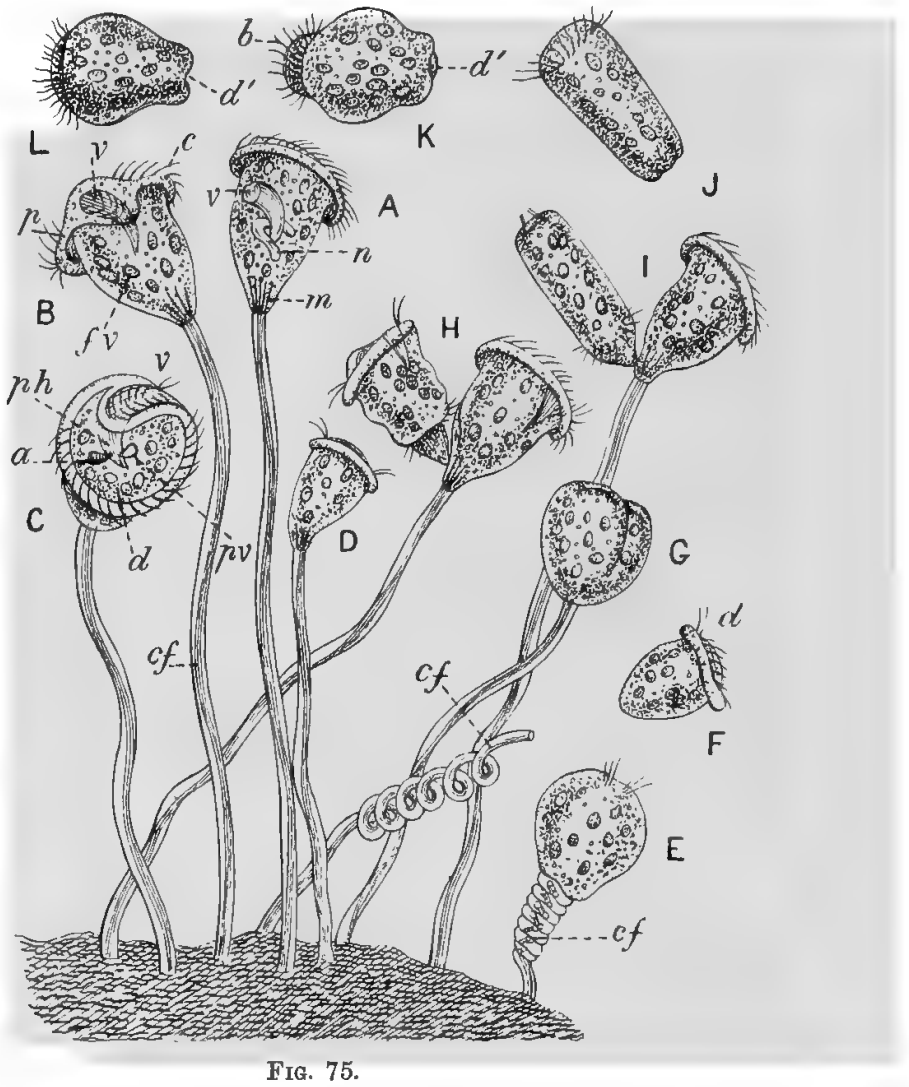

end of the body, and extends downwards in the direction of the stem. The body is thus separated into two, both halves remaining on the stem. The one half resembles the parent and remains on the stem; the other, while still adhering to the stem, develops, near its base, an aboral circle of cilia, by the aid of which, when it has detached itself, it swims away. When a separation is effected the stemless half develops a stalk and fixes itself, the aboral circle of cilia disappearing. The interesting process of fission is completed in from one to two hours. A converse process is occasionally witnessed, namely, the conjugation or union of two vorticellæ. In such cases a small vorticella becomes fused with a larger one, of which it remains a permanent part. Not unfrequently after conjugation, and probably as a result of it, a process of encystment takes place. In this case the disc of the vorticella is retracted, and the cell body becomes rounded and separated from its stalk. Coincident with these changes a cyst is secreted and important changes occur in the nucleus. The nucleus enlarges, elongates, and becomes moniliform, and ultimately breaks up into a number of spores, which are ovoid bodies provided with a circlet of cilia. When the cyst bursts the spores escape and swim freely about. They multiply by fission. Ultimately they fix themselves by their ciliary processes, and develop a disc at the other end of the body. The circlet of cilia then disappears, and a stem resembling that of the parent is formed. This done, each spore becomes a perfect individual.

The movements, the method of feeding, and the modes of reproduction in vorticellæ are all very exact and perfect in their way. They are plainly the outcome of pre-arrangement and design, and this fact, in conjunction with 
similar facts already recorded when describing the amoba, gromia, paramecium, and collared monad, point to a finality in living things; each animal, be it high or low, being perfect in itself-in other words, supreme in its own sphere.

The zooid and monad, in this sense, are as complete as any of the higher animals, although they have neither brain, nerves, muscles, stomachs, lungs, nor hearts.

In the lowest plants and animals, the intelligence of the Creator can be traced. In the higher and highest plants and animals the power of self-direction and government is gradually and in fuller measure transmitted to the individuals themselves, but even in the latter the divine guidance is never wholly withdrawn. Nothing is left to chance in plants and animals, and intelligence reigns supreme throughout the whole organic kingdom. Proof of this is to be found in the fact that millions of bacteria, microbes, and other low forms are incessantly at work preparing the earth, the water, and the air as fit habitations for the more advanced plant and animal forms up to man himself.

The preparation here referred to fixes the boundaries to plants and animals alike; it determines their sphere of action, and the modifications which they may undergo within those spheres so as to secure the most perfect adaptation of means to ends under all possible circumstances.

The whole organic kingdom is conditioned and under control, and boundaries are set to every change which occurs in it. The same remark applies to the inorganic kingdom. All matter, living and dead, is controlled. The universe is an involved, intricate system of action and reaction, but everything is foreseen and provided for. There is no lack of matter or force or of intelligence anywhere. If there were, the inevitable result would be confusion on an unprecedentedly extensive scale. There would be an end to the Law, Order, and Design which everywhere appear. The universe, as we know it, is a conditioned universe, and the Intelligence which created it in the remote past pervades every part of it at the present day. Matter and space are pervaded by intelligence, and the harmony which everywhere obtains is only secured by a rigid adherence to law and order, even in the minutest details. Without a presiding intelligence, everything would assuredly suddenly cease and determine. All this points to gradation and type and finality and law in the ultimate sense.

In order to adequately realise the question of gradation and type in rudimentary plants and animals, and in order to trace successfully the similarity between crystals, plants, and animals, it is necessary to go into the subject in detail and at considerable length, as has been done by the aid of numerous plates and figures, which will be found scattered throughout the entire work. The illustrations cover a wide area, but it is hoped they will supply in a concrete form much valuable information of a not readily available kind.

\section{\$66. Animals specially constructed as Air-breathers and Water-breathers, and for Land, Water, and Air Transit.}

It is all but certain from the geological record and the modern fauna that animals were originally created as air-breathers and water-breathers. It is equally probable that they were primarily endowed with organs of locomotion adapted to the land, the water, and the air respectively. Both these contentions seem proved by the fact that no known animals are at once habitual air-breathers and habitual water-breathers. There is, however, an approach to this state of things. The climbing perch (Anabas scandens) can leave the water for considerable periods and ascend elevations; the goby or walking-fish (Periophthalmus koelreuteri), with its short, well-developed anterior limbs or modified pectorals and tail, hobbles about the shore, when the tide has retired, in search of food the flying-fish takes long leaps in the air at a considerable altitude above the water; the African mud-fish, Lepidosiren (Protopterus annectens), lives for protracted periods in the deposits of rivers and tanks which have become partly or wholly dry.

Wilder noticed that in the mud-fish (Amia calva) the animal, when the water was foul, rose frequently to the surface and took in copious draughts of air. He gave it as his opinion that, on such occasions, an exchange of oxygen and carbonic acid took place, as in the air-breathing lungs of vertebrates.

Ceratodus (C. forsteri Krefft., and C. miolepis Günth.) is found in the fresh waters of Queensland. This fish, known as the Dawson-salmon or Barramunda, is sometimes 6 feet in length, and occasionally weighs 20 lbs. It lives mainly on decayed vegetation, which is found in large quantities in the bottoms of the rivers, and which frequently makes the water turbid and thick. This renders it difficult for the fish to breathe by its gills, which become clogged. It is, therefore, provided with a lung in addition to its gills, so that it can breathe by its gills or lung separately or by both simultaneously. This is a very remarkable circumstance, and must have been foreseen and pre-determined. It affords a very good example of design and of a First Cause. The modification is of the nature of original endowment, that is, a particular structural change specially made to meet an unusual set of conditions. 
The barramunda is credited with the power of going on land, or at least mud-flats. As the fish is heavy, and its pectoral and ventral fins or limbs weak, it is in no way adapted to land transit. It is, however, quite equal to making considerable progress on a marshy soft bottom. While the fish is believed to have the power of leaving the water for short intervals and of breathing by its lung alone, it is more than doubtful if it can survive in a healthy condition for any length of time out of water. The perfect state of its gills favours this belief, and its one lung would be quite inadequate for protracted air-breathing purposes. What in all probability happens is, that the lung is an auxiliary structure, and that the fish breathes habitually by its gills, and employs its lung as an adjunct for taking in great gulps of air when the animal, partly asphyxiated by the gases of the foul water, rises to the surface, which it is constantly in the habit of doing when the conditions of breathing are rendered difficult.

The rising to the surface for the purpose of breathing air also obtains in the sea mammals, where no gills are present, and oxygenation of the blood is effected wholly by the employment of lungs.

One of the most remarkable animals as far as breathing is concerned is the axolotl (Siredon pisciformis). It is provided with six feathery-looking gills, which are arranged on the outside of the body separately, three on each side of the head. The axolotl is a water-breather, and confines its operations exclusively to this medium. Some years since Professor Haeckel ${ }^{1}$ asserted that this quaint creature can convert itself from a water-breather into an air-breather, and he instances some experiments to this effect which he asserts were made at the Zoological Gardens, Paris, where a large number of axolotls are kept and bred. This statement requires to be taken with the extreme of caution, and is as follows: "The water-salamanders, or tritons, which have been artificially made to retain their original gills, are extremely interesting in this respect. The tritons are amphibious animals, nearly akin to frogs, and possess, like the latter, in their youth external organs of respiration-gills-with which they, while living in water, breathe the air dissolved in the water. At a later date a metamorphosis takes place in tritons, as in frogs. They leave the water, lose their gills, and accustom themselves to breathe with their lungs. But if they are prevented from doing this by being kept shut up in a tank, they do not lose their gills. The gills remain, and the watersalamander continues through life in that low stage of development, beyond which its lower relations, the gilled salamanders, or Sozobranchiata, ${ }^{2}$ never pass." It will be observed that the experiments referred to in this quotation are wholly artificial, and of little or no value as far as exact science is concerned. He continues, "Great interest was caused a short time ago, among zoologists, by the axolotl (Siredon pisciformis), a gilled salamander from Mexico, nearly related to the triton; it had already been known for a long time, and been bred on a large scale in the Zoological Gardens in Paris. This animal possessess external gills, like the young salamander, but retains them all its life, like all other Perennibranchiata. This gilled salamander generally remains in the water, with its aquatic organs of respiration, and also propagates itself there. But in the Paris garden, unexpectedly from among hundreds of these animals, a small number crept out of the water on to the dry land, lost their gills, and changed themselves into gill-less salamanders, which cannot be distinguished from a North American genus of tritons (Amblystoma), and breathe only through lungs." (The italics are mine.)

I cannot help feeling that some important error has been committed in this connection. Of course, Haeckel admits the wholly artificial nature of the experiments, but he seems to have mixed up conditions which are essentially separate and distinct, and which are even contradictory in their nature. They are altogether at variance with similar experiments made by myself with young frogs, where the artificial and objectionable features were eliminated. I found that a period arrives in the development of the frog when nature must have fair play and free play. This occurs when the lungs are fully developed and the gills have become useless as water-breathing organs. If at this stage no provision be made for the young frogs leaving the water, resting, and breathing air, they are drowned. It is necessary under the circumstances to erect in the aquarium small, slanting, elevated platforms of stone, brick, earth, or some such material, to permit the young frogs to leave the water and to breathe air at discretion and under favourable conditions. It would defeat the object in view to confine the young frogs exclusively to the water when their arrangements for living in that element have disappeared. We might as well expect the eye to see and the ear to hear when the organs of sight and hearing have, from some cause or other, been destroyed.

It is not permissible to substitute an artificial for a natural process in a growing organism, especially if the artificial arrangements are opposed to nature and bar progress. If an experiment gives an unnatural bias to or stints development, it at once becomes misleading, and little or no importance can be attached to it.

The foregoing affords no proof that the important respiratory processes can be indefinitely modified, and that a water-breathing animal can, within a comparatively short period, be converted into an air-breathing one. Such a possibility would upset the whole scheme of nature, and lead to the dangerous conclusion that all parts of plants and animals are interchangeable, and that the one is directly or indirectly manufactured out of the other.

In this connection it may be useful to state that the sea mammals, such as the whales, porpoises, dugongs, 1 "The History of Creation," vol. i. p. 259 et seq.

2 Ordinarily known as Perennibranchiata. 
manatees, seals, walruses, \&c., afford examples of animals which permanently live in the sea, but which at shorter or longer intervals throughout their entire lives must come to the surface of the water to breathe air.

In reality these animals are true air-breathers. They give no countenance to the theory that they are land animals which have adopted the ocean as their home and are in the act of changing their natures and becoming fishes or fish-like animals. As a matter of fact, the sea mammals occupy a much higher position than fishes in the organic scale. There is nothing in the histories of the sea mammals to show that these most interesting members of the organic kingdom are changing either their natures or their external forms to meet the requirements of natural selection on the one hand, or of evolution on the other.

A certain relation obtains between the breathing habits of animals and their modes of locomotion.

The breathing apparatus and organs of locomotion must be specially provided for during the development and growth of the several classes of animals_- that is, they are original structures, the outcome of design and pre-arrangement, and not chance products developed during their lives by accident, and by the accumulation of trifling fortuitous modifications extending over long periods, as claimed by Darwin in his "Origin of Species by Natural Selection."

The breathing and travelling organs cannot be produced by efforts of will on the part of animals. They are necessities of life, and as such are inborn. Their production is not left to chance, or environment, or any form of irritation or extraneous stimulation.

Animals are not permitted to breathe air and water indiscriminately; neither are they constructed to move on the land, and on and in the water, and the air, with equal facility.

It is not here maintained that one and the same animal may not occasionally walk on the land, swim in the water, and fly in the air. Several insects and birds do this. All that is meant is that animals primarily adapted for land transit are, as a rule, indifferent swimmers and cannot fly, while animals originally adapted for swimming walk badly, or not at all, and cannot fly; animals which are expressly adapted for flying, in the majority of cases, swimming and walking with difficulty.

The walking, swimming, and flying types are well marked; and all animals, be they insects, fishes, reptiles, birds, or quadrupeds, must rigorously conform, as regards their general shape, and the form, size, and structure of their travelling organs, to the laws which regulate locomotion on the land, and on and in the water and the air respectively. In all this there is design. The whole animal is constructed on specific lines to attain certain ends. The travelling arrangements in adult animals are not temporary but permanent, and animals would attempt in vain to perform feats on the land, in the water, or the air, for which they had not structural warrant. Structure invariably precedes function, and until an animal is endowed with the proper travelling organs and surfaces no satisfactory progress can be made along either of the three great highways of nature-namely, the land, the water, and the air. The explanation of these hard and fast arrangements is on the surface. All animals, be they great or small, simple or complex, must be provided with bodies and organs which enable them to pursue, capture, devour, and assimilate their food. Such as are not so provided sooner or later die of inanition. The procuring of food is as essential to animals as the procuring of air, and the plan of construction of all animals is threefold, and such as enables them at once to secure food and air, and to reproduce themselves.

In this connection it is important to point out that no animal can by an effort of will cause its travelling organs to develop in a particular direction ; still less can it originate or inaugurate their growth at any period of its life history. The travelling organs, to whatever class of animals they belong, are original endowments conferred by the Architect of the universe to secure well-defined and desirable objects. They are never chance products; they never grow unbidden, or apart from a pre-conceived plan. On the contrary, they are provided for in the embryo and fœetus. in anticipation of the work to be ultimately performed by them. It is not a case of an animal adapting itself during its life to a particular mode of locomotion; neither is it a case of environment and extraneous stimulation of particular parts of the body originating and ensuring the growth of travelling organs at special points. The travelling organs are part of the original being, and the individual, whatever its position in nature, has no power to increase or diminish their size, to alter their peculiar shapes, or to change the substances composing them. The conditions are rigorous and unalterable, and everything must bend to the inevitable as far as effective progression on the land, on and in the water, and in the air is concerned.

A not uncommon belief is that all living creatures originally proceeded from the water, and that in the course of time they modified themselves in such a manner as enabled them to live and move comfortably on the land, and to fly in the air. There is no proof or even analogy to support this theory. In order for a water-breathing and water-navigating animal to become an air-breathing and air-navigating one, it must develop lungs, and alter its general shape, and that of its travelling organs. These are structural modifications of the first magnitude, and beyond the reach of any animal per se. The animal can only grow along the lines laid down by its parents. If a water-breathing animal be removed from that element and kept on terra firma for a short time it inevitably dies; 
conversely, an air-breathing animal if submerged beyond a certain period invariably drowns. Time is not allowed for a prolonged series of minute accidental modifications such as so-called "natural selection" and evolution require to convert an air-breathing animal into a water-breathing one, and vice vers $\ell$. Moreover, there could be no possible inducement for any animal to undergo the painful suffocating process which such a change would necessitate, especially as the discomfort would have to be endured for ages. If, on the other hand, the changes were sudden the animal would fare no better, as its very existence depends on its structural arrangements for the time being. The only apparent exception to this impossible state of things is when structural changes supervene during development, but these changes cannot be taken into account in this connection, as they are specially provided for, and animals have no power to alter original endowments either in the young or adult condition. Neither can they be altered by environment, irritability, or extraneous stimulation.

It is no reply to what is here stated to say that certain water navigating animals (which are mammals and not fishes) such as the porpoises, whales, seals, \&c., and which breathe air, habitually live in the water and can remain submerged for very long periods. This only means that their original structural arrangements are expressly designed and modified to meet the peculiar requirements of water transit, and air-breathing. In other words, they are provided with fish-shaped bodies, swimming tails, swimming fins, flippers, and feet, and air-breathing lungs. All these animals if kept long enough under water would be drowned. Similar remarks, but in another direction, are to be made of animals which are water-breathers, such as eels, the climbing perch, mud-fish, \&c., which temporarily leave the water and make excursions on the land. They, in turn, if kept too long out of the water, would inevitably perish. Nor is the situation in any degree altered by the fact that certain animals (some insects and the frog for example) begin life as water-breathers with natatory organs, and ultimately become air-breathers with legs adapted for walking, leaping, \&c. In all such cases there must be absolute conformity to the conditions involved in water and air breathing and water and land transit respectively. The air-breather must be provided with lungs or their equivalents; and the water-breathers with gills or branchiæ. To this there is no exception. Tadpoles drown if kept in a smooth glass basin of water with no resting-places above the water during the transition stages, that is, when they are shedding their gills and tail, and before they have acquired lungs and legs. The development of the frog affords a good example of conformity to the air-breathing, swimming, walking, and leaping types. In the tadpole stage the frog is fish-shaped, is provided with a swimming tail, and has no legs. In the adult condition it has no tail, and is provided with four powerful legs which enable it to make considerable progress on land, and also to swim in the water.

It will be noticed that in the development of the frog, spontaneous and independent structural changes take place according to a predetermined plan, and that no modification in function either as regards the nature of the breathing or the kind of locomotion can occur until the necessary structural changes are completed. A change of structure in every instance precedes a change in function.

All this was a priori to be expected. The land, water, and air materially differ from each other, but as they form the abodes, and provide the highways along which animals progress in pursuit of food and other physical advantages, the animals themselves must correspondingly differ. It is absolutely necessary to draw a line of demarcation as between water and air breathing animals, and between animals specially constructed to walk, swim, and fly.

As the land differs from the water, and the water from the air, so must the animals differ from each other according as they are designed to live and move on the land, on and in the water, and in the air. The animals and their travelling organs are specially modified to meet the diverse conditions. They are adapted to their particular work, and there is a co-ordination of parts which prevents accident or mishap in any direction.

The three highways along which animals progress are material in their nature; that is, they are composed of physical particles more or less closely aggregated. The number and arrangement of the particles determine the amount of support afforded to the travelling organs and bodies of animals. Thus in the case of the land, the particles are densely packed together and crowded; the land, as a consequence, supplies what is practically a solid basis of support, and an unyielding fulcrum for the travelling organs of animals. The rule, in land transit, is small feet and heavy bodies. In the case of water, the particles are less numerous and loosely distributed. As a result, the water provides only partial support, and a yielding fulcrum. The rule, in this medium, is bodies of nearly the same specific gravity as water, and large swimming tails. In the case of the air, the particles are comparatively few in number and very widely separated. As a consequence, the air furnishes next to no support, and an exceedingly mobile unreliable fulcrum. The rule in air is light bodies and very greatly expanded wings. It will be observed that the land, the water, and the air provide three different kinds of fulcra on which the travelling organs and surfaces of animals act when they change position. Further, the size of the travelling organs is determined by the density, rigidity, and resistance afforded by the fulcra. There is, therefore, an unalterable and invariable relation as between the fulcra afforded by the three highways along which animals progress, and the size and power of the VOL. I. 
travelling organs possessed by animals in each particular case. Locomotion, primarily and essentially, resolves itself into action and re-action, and it is in every instance a question of power, speed, and support. The quicker the travelling organs are moved the greater the recoil and support obtained from the fulcra on which they act. In flight, if small wings are driven at a very high speed they give as much support as larger wings driven more slowly. If water, which is virtually incompressible, be struck very hard, it affords nearly as much resistance and recoil as a solid. If air, which is very highly compressible, be similarly treated it also furnishes quite an extraordinary amount of recoil and resistance. Flight were otherwise impossible. To fly is equivalent to walking or swimming in the air.

When the niceties of construction involved in water and air breathing, in the size, shape, power, and variety of the travelling organs required for effective land, water, and air transit are taken into account, one is forced to conclude that nothing short of an intelligent First Cause and design will satisfactorily account for the existence of the breathing apparatus and organs of locomotion. The means and the ends are so accurately adapted to each other, and the conditions are so rigorously and uncompromisingly exact, that no other explanation is possible. That the arrangements necessary for water and air breathing, and for land, water, and air transit were brought about by accident, as those who believe in spontaneous generation affirm, or by the accumulation of fortuitous increments extending over vast periods, according to evolution and "natural selection," is in the highest degree improbable-so improbable indeed as not to be worthy of serious consideration. The theories of spontaneous generation, evolution, and natural selection are utterly inadequate to explain such problems, and, if we ignore a First Cause and design, and law and order as the outcome of both, not only is no explanation forthcoming, but the whole subject becomes unthinkable.

A great object lesson is afforded by the breathing arrangements, and by the size, shape, and movements of the travelling organs of animals. These can only be regarded as original, fundamental endowments. They admit of no temporising. An animal cannot at one and the same time be a water and air breather; neither can it progress equally efficiently on the land, on and in the water, and in the air. The physical conditions prevent such a state of things. Little reflection is required to be convinced that in locomotion the cause must be equal to the effect. It works out in a very simple manner. The small feet of land animals when applied to a rigid unyielding fulcrum, such as the earth, at once obtain support and the recoil necessary to forward movement. In like manner, the tails and fins of fishes (especially the tails), when applied by reciprocating movements to the yielding aqueous fulcrum, elicit the recoil necessary to convert them into powerful propellers. Similarly, the comparatively very large wings of the insect, bird, and bat, when made to vibrate rapidly in the air, extract from it the recoil calculated to propel the bodies to which they are attached at high velocities. On the land, support is guaranteed; propulsion only being required. In the water, support is also guaranteed, from the fact that the swimming creature is of nearly the same specific gravity. The fish, consequently, has only to propel itself. In the air, next to no support is afforded, and the wings have at once to support and propel. These are physical conditions which cannot be ignored.

Three things require to be attended to in animal locomotion; namely, $(a)$ the medium to be traversed; (b) the size, shape, and nature of the travelling organs; and $(c)$ the power or force by which the travelling organs are driven. The feet of land animals are generally small, jointed, springy organs; the tails and fins of fishes are expanded, finely tapering, elastic structures of a more or less triangular shape; and the wings of insects, birds, and bats, which are still more expanded, greatly resemble in shape, properties, and functions the caudal and pectoral fins of fishes. The travelling organs are carefully adapted to the work they have to perform. As a matter of fact, they are designed and elaborately constructed organs. The small feet adapted for land transit are not effective as swimming organs, neither are the tails and fins of fishes of any use as flying organs.

There is a general rough resemblance as between land, water, and air as media for the travelling organs of animals, and as between the travelling organs themselves. Dry land for example may shade off into marshy soft land; or water may be solid or semi-solid from freezing; or it may be semi-solid as in snow and sleet; or it may be attenuated as in steam by the administration of air. In all these cases the breathing and locomotory organs must be specially modified. The feet of land animals may become expanded and webbed to deal with marshy soil and water, and the pectoral fins of fishes may increase in size as in the flying fish, and deal with the air after the manner of wings. Land animals may also essay flight by means of expanded integuments, as happens in the flying dragons, flying lemurs, and bats. These are the exceptions to prove the rule. The modifications of the travelling organs are as inexorable, in their way, as the breathing arrangements. Indeed, the whole animal must be designed to play a perfectly distinct rôle as a water or air breather, and as a denizen of the land, the water, or the air respectively. The peculiarities of function must be structurally provided for in advance in the embryo and fotus. Nothing is or can be left to chance. ('hance in such important and vital matters is out of the question.

The foregoing remarks on the travelling organs apply more especially to the higher animals, but they are applicable in principle also to those of the lower and lowest animals. Every animal, even the most rudimentary, is endowed 


\section{A CREATOR AND DESIGNER NECESSARY TO UNIVERSE}

with the power of movement. This movement may occur in protoplasm, in white blood-corpuscles, in cells, in tissues, \&c., but wherever it occurs it is due to prearrangement and design. It is never accidental or haphazard. The movements in each case are co-ordinated to given ends. An amœba, for instance, slowly shoots out and draws in its finger-like processes (pseudopodia). This it does deliberately and of set purpose. The white blood-corpuscle when, because of local inflammation, it forces itself through the wall of a capillary blood-vessel, attacks the vessel as a wedge, the thin end of the wedge always leading. When a voluntary muscle, in response to a volition, shortens or contracts by a centripetal movement, it soon after elongates or relaxes by a centrifugal movement; if two voluntary muscles, say an extensor and a flexor, are in action at the same time, the one (the extensor) shortens or contracts, while the other (the flexor) elongates or relaxes, and vice versâ. Muscles are endowed with the same properties as pseudopodia, that is, the sarcous elements are possessed of a double power, namely, the power of alternately shortening and elongating in the case of long muscles, and of closing and opening in the case of hollow muscles and sphincters.

Cilia also possess this double power. In cilia, no trace of muscles or nerves can be detected, yet they can bend first in one direction and then in another and opposite direction. (ilia, for the most part, are moved voluntarily and to given ends. They can produce food and other currents in the vorticella and various other rudimentary forms. They propel the ova along the Fallopian tubes, and mucus and air along the smaller bronchial tubes, and mucous surfaces generally. They form the organs of locomotion in paramecia and other low animal forms. Structure and differentiation, in the ordinary sense, are not necessary to voluntary movements, but the substances in which the movements occur are equal to the work they are called upon to perform. The substances have, in themselves, the potentiality and the power of movement, and they move methodically and to given ends.

The low moving forms are legion as regards number and variety, but in every case there is adaptation and efficiency. The movements may occur in animals with or without muscles and nerves; with or without feet; with or without an external or internal skeleton; but whenever and wherever they occur due provision is made. The movements, moreover, occur in a certain way and in a certain order. There is no such thing as sprawling, indeterminate movements in nature. Movements in living things are never objectless. To be convinced of this one has only to study the movements and habits of the Infusoria and other rudimentary organisms under the microscope. These are seen to dart about with great alacrity and precision in pursuit of food or other objects. They are seen systematically to avoid each other unless when they attack and seize each other as prey. Their movements are evidently voluntary, and regulated. The mechanism by which the movements are produced is, in many cases, not visible, but it cannot be doubted that the substances and bodies in which they occur are in every case equal to the results obtained. In animals provided with cilia there is no difficulty; the visible means of progression are equal to the result. They can propel the bodies on which they occur in any given direction. What is true of ciliated animals is true of all others. In the creeping animals there is a movable ventral integument provided with rugæ, setæ, or feet, as in the worm and caterpillar. In animals which walk and leap, feet and legs with joints are provided as in insects. The apparatus and mechanism of movement become more apparent as animals become differentiated, and culminate in the beautiful, jointed feet, and limbs of quadrupeds and bipeds; the graduated, elastic tails and fins of fishes and sea mammals; and the expanded, delicately constructed elastic wings of insects, birds, and bats.

My contention is, that the travelling appliances and organs of locomotion are original structures, and are as necessary to the existence and well-being of animals as the breathing apparatus, the circulatory apparatus, the organs of reproduction, \&c. Without the means of movement, visible or invisible, no animal could possibly exist.

\section{$\$ 67$. A Creator, Designer, and Upholder necessary to the Universe as We know it.}

In considering the universe as a whole, or in part, it is necessary to postulate an intelligent Creator or First Cause, a Designer and Adapter, an Upholder and Sustainer. Ex nihilo nihil fit is a trite and almost universally accepted adage. From nothing comes nothing. Those who do not believe in a Creator, who ignore design and the supervision which design implies, are, of course, entitled to ask who or what made the Creator? They are logically entitled to put this question, and, if put, the only satisfactory reply that can be given is that intelligence, law, and order everywhere prevail in the universe; a state of matters which implies, if it does not actually prove, the existence of a Supreme Being Who is at once omnipotent, omnipresent, omniscient, and eternal. If a Creator be not predicated, then matter must be regarded as self-forming, self-moving, self-adapting, and endowed with the power of developing life de novo by a process of spontaneous generation, which is wholly opposed to modern scientific beliefs. As intelligence is excluded from the mechanical view of the formation and working of the universe, it follows that chance takes the place of design, of law, and of order; everything being a law unto itself as apart from co-ordination and co-adaptation. It is the harmony which prevails in the universe and the obvious 
adaptation of means to ends which pleads so eloquently for the existence of an intelligent First Cause and of a Designer and Upholder. Given a Creator or First Cause all else is comparatively easy. It is, of course, impossible for the finite mind either fully to comprehend the infinite mind, or the universe which the infinite mind has produced; nevertheless, a careful analysis and study of our own minds, and a contemplation of the works of our own hands, supply us with important collateral information. As physical, mental, and moral man is a part of the universe, it is permissible to think that he is not wholly alien to the great scheme of creation, and that his thoughts and works are scintillations and reflections, however faint, of those of the great Framer of the Universe. There is, however, the following important difference.

The Creator can produce or annihilate matter and force, whether physical, vital, or mental. To man it is not permitted to create matter or force, still less to annihilate them. All he can do is to change the form of matter and the direction of force. For him matter and force are practically indestructible. For him there is in the universe a store of matter and of energy which cannot be either increased or diminished. That the natural processes which go on in the universe as a working concern are not wholly different from those which mark the activities of man is shown in various ways. In both there is cause and effect: in both there is obvious design: in both there is the adaptation of means to ends: in both there is thought. The element of chance is largely, if not wholly, eliminated: a reign of law is substituted for one of chaos. This is nowhere better seen than in the adaptation of day and night, and the seasons, to the requirements of plants and animals. Day and night and the seasons recur with a regularity which excludes the idea of chance. Similarly, plants and animals repeat themselves, each according to its kind or type, so regularly and persistently that a law of reproduction must be admitted in which chance or accident plays next to no part. In these cases a First Cause, a Designer, and Upholder is a sine qua non. Day and night, the seasons, and the existence of plants and animals on the earth afford examples of forethought and intelligent adaptation of means to ends where co-ordination and co-adaptation and inter-dependence play a conspicuous part. Plants and animals require the alternations of day and night for their periods of activity and repose; they also require the revolving seasons to provide the variations in climate so needful to a healthy tone in both. Plants and animals are largely dependent on cosmic changes, and the organic kingdom is to a considerable extent a product of the inorganic kingdom. In both the organic and inorganic kingdonus a reign of law prevails, which, to the thoughtful observer, appeals powerfully in favour of an intelligent Creator, Designer, and Upholder. In both kingdoms cause and effect can be constantly traced: everything is planned: nothing falls out by accident: the mechanism of the heavens and the mechanism of plants and animals are as obviously the product of an intelligent Creator or First Cause as are a watch, a steam-engine, an electric battery, or a telephone.

There is no getting away from intelligence in either case. The co-ordination of parts and movements can only be the outcome of intelligent forethought and design. Wherever there are examples of means to ends in the works of the Creator and the creature, intelligence and design must be predicated.

As already stated, everything is possible with the Creator. He can make and unmake matter and force at discretion: He can produce or abolish plant and animal life in its infinite varieties: and $\mathrm{He}$ can confer or withhold the extraordinary attributes of mind which culminate in man, but which can be traced as a continuous chain low down in the animal kingdom. While the mental attributes of man are necessarily limited, they are, nevertheless, sufficiently numerous and discriminating to enable him clearly to perceive endless examples of design in the universe as a whole, and in the inorganic and organic kingdoms considered separately.

A question has been frequently put of late years as to whether all matter may not be referred to one primitive substance simple in composition, and whether force is not a product of matter also characterised by simplicity and oneness. ${ }^{1}$

Whatever matter and force may have been at the outset in the hands of the Creator, there can be little doubt that in our times they are not one and indivisible, that is, identical. Matter and force, as we know them, are differentiated, and to this differentiation all the peculiarities in the structure and movements of inorganic substances, and in the structure and movements of plants and animals, are due. It is differentiation which separates one physical body from another, and which sets up distinctions between plants and animals. From

1 Professor Enst Haeckel in his "Riddle of the Universe" expresses himself' in this comnection as follows: "All the partionlan' advances of physics and chemistry yield in theoretical importance to the discovery of the great law which brings them to one common focus, the 'Law of Substance.' As this fundamental cosmic law establishes the pternal persistence of matter and force, their unvarying constancy throughout the entire universe, it has become the jolo-star that guides our Monistic Plilosophy through the mighty labyrinth to a solution of the world-problem. .. The world is nothing else than an eternal evolution of substance. . . The conviction that these two great cosmic theorens, the chemical law of the persistence of matter and the physical law of the persistence of force, are fundamentally one, is of the utmost importance in our monistic system. The two theories are just as intimately united as their objects-matter and force or energy. Indeed, this fundamental unity of the two laws is self-evident to many monistic scientists and philosophers, since they merely relate to two different aspects of one and the sanie object, the cosmus." 
wholly identical substances in the inorganic and organic kingdoms only repetition, as apart from differentiation, can result. There is no universal matter, dead or living, which, being absolutely homogeneous and identical in all its parts and particles, can of itself, and by itself, produce the various substances met with in the inorganic kingdom, and in the several plants and animals found in the organic kingdom. The differentiation of matter and force is fundamental alike in the inorganic and organic kingdoms. The so-called affinities in chemistry favour this belief. We have proof of it also in the histories of the spores, germs, and seeds of plants; in the eggs and ova of animals; and in the peculiar properties of the male and female elements on which reproduction depends. The reproductive elements, whether consisting of germs, seeds, eggs, or ova, self-fertilising or otherwise, vary infinitely as to their ultimate substance, and this variation or differentiation can alone account for the bewildering multitude of plants and animals on the earth. It is not otherwise possible to explain how from one seed proceeds a lichen, from another a fern, from another an oak; or how from one ovum emerges a fish, from another a reptile, from another a bird, and from another a mammal. Neither the microscope nor chemical analysis can detect the differentiation here referred to, but that it exists cannot be doubted when the final results are duly considered. The conditions of reproduction are, in numerous cases, nearly, if not altogether, identical, but the reproductions are as the poles asunder. Mere force, however gentle or violent, cannot convert practically identical matter into anything other than itself. This goes without saying. Heat, light, electricity, and other forms of motion, while within limits convertible, do not destroy the substances on which they act, and which they influence to a greater or less extent. At one time, and indeed till very recently, protoplasm was regarded as a simple, wholly undifferentiated organic substance whose parts and particles were identical in ultimate composition. Protoplasm is now known to be reticulated and to vary considerably in chemical composition. The difficulties involved in the differentiation of plants and animals during development are not overcome by saying that one change begets another, and that one set of conditions inaugurates others in endless succession, until the several plants and animals with their various organs are completed. To get the initial changes and conditions which inaugurate successive developments there must be plurality and heterogeneity of both matter and force. Of simple ultimate matter and force we have no knowledge. All our instruments of research, the microscope, the telescope, chemical analysis, the spectroscope, the polariscope, \&c., reveal not one kind of matter but a great and ever-increasing variety of matter. Already there are over seventy known elements, and these will doubtless be added to as time advances. Within the last few years no fewer than four new gases have been discovered (Argon, Helium, Krypton, and Zeon), and some new metals, notably Radium. ${ }^{1}$ The tendency is not in the direction of simplicity as regards matter and force, but complexity; the complexity being of a very bewildering kind, as manifested more especially in heat, light, magnetism, electricity, wireless and other telegraphy, ether in its several phases, optics, acoustics, Becquerel, Hertz, Röntgen, and other rays, to say nothing of the delicate sensitiveness of the plates employed in terrestrial, celestial, and other photography. The extraordinary powers possessed by radium (one of the new metals) have invested matter and force with untold possibilities and an ever-increasing interest. Radium apparently presents the unique phenomenon of an element breaking up or disintegrating and emitting streams of matter and force, which for intensity are altogether unparalleled.

The radio-active elements and their emanations are opening up what is practically a new field in physics. The. properties of these elements investigated by Plucker, Hittorf, Crookes, Curie, Lodge, Rutherford, Ramsay, and others can be exhibited in the laboratory. By means of a vertical glass vacuum tube 18 inches long and an inch wide, connected at top and bottom with the negative and positive poles of an electric battery, electric sparks (artificial lightning) can be transmitted; these causing the interior of the tube to glow with a beautiful rose-coloured shimmer. Sir William Crookes demonstrated that each particular substance gives out its own special radiance. Thus alumina gives a deep red flare, the sulphate of zinc a rich green, a portion of calc-spar a fine crimson, and so on. Something like a stream of radiance rushes through the glass tube. According to Crookes there proceeds from the negative pole or cathode, a shower of amazingly minute particles, not beams of light, but things, which carry negative electricity. They can even be made to propel a miniature wheel on rails within the tube. When the electric current is turned on, the little wheel travels briskly. The inference is, that whatever the nature of electricity the so-called cathode-rays are things, particles, or corpuscles, substantial entities. As proving the substantial or material nature of the rays they can be deflected or bent by the aid of a magnet applied outside the glass tube. Certain rays cannot be so deflected. In these cases they are regarded as rays of light. This is true of Röntgen's $\mathrm{X}$-rays. As the X-rays cannot be deflected by the magnet they are believed to be not matter, but waves in the ether that fills all space. In this connection M. Henri Becquerel made an important discovery, namely, that the metal

1 Argon was discovered by Lord Rayleigh and Sir William Ramsay jointly in 1894, Helium and Lirypton by Sir Willian Ramsay, Zeon by Sir William Ramsay and Mr. Travers, and Radium by M. and Mne. Curie at a later date. 
uranium, which is derived from pitchblende, prints a picture of itself in the dark on a photographic plate. Uranium is always giving out or exerting this photographic influence. The marvellous new metal, radium, also derived from pitchblende by Mme. Curie, exhibits these radio-active properties to an extraordinary extent. The radio-active elements, as Sir William Ramsay has shown, make the air around them conductors of electricity. He took a tassel of silk threads and rubbed it with fur and so electrified the tassel; then, bringing a minute portion of radium near, the electricity was discharged.

The most wonderful property of radium is its continually giving off a gas or emanation that shines by its own light. From the gas proceed three kinds of rays, namely, A-rays, which can be seen striking against a chemical screen; B, or cathode-rays, which will pass through lead; and G-rays, which are those of Röntgen. When the spectrum of the gas is thrown on a screen, and the gas fades away, Helium is left; a circumstance of the utmost consequence as indicating, according to Ramsay and Soddy, that radium is an element breaking up, and in its dissolution giving out energy. If the heat of radium could be retained it would become incandescent. Radium, uranium, and thorium are all heavy elements and self-destructive on account of their mass. In the opinion of certain astronomers, suns, beyond a certain size, also matter, force, heat, light, \&c., though very intimately related, are not inextricably or hopelessly blended, and can be separated by the employment of sufficiently delicate instruments and tests and exercising due care.

Sir William Crookes, by his experiments and writings, has given a great impetus to the consideration of the finer varieties and ultimate composition of inorganic matter. He claims a fourth condition for it. Matter in the aggregate is to be considered as solid, liquid, gaseous, and ethereal. In detail it is infinitely divisible. There is, so to speak, matter within matter: each kind of matter having specific movements and exercising certain powers such as gravitation, attraction, repulsion, cohesion, adhesion, \&c. The movements of matter are at least of three kinds, namely, wave movements, movements of rotation round an axis, and movements of bodies round each other. It does not suffice nowadays merely to say that atoms form molecules and molecules cells, tissues, and organs; for it has been demonstrated that in the interstices between the atoms and molecules finer matter exists, and that this finer matter (generally called ether) possesses powers of attraction and repulsion, cohesion, and adhesion, \&c., and displays rotatory and wave movements similar to those which obtain in the atoms and molecules themselves. The tendency is to credit the finer masses of matter with analogous properties and powers to those possessed by the larger bodies, even the celestial spheres. Ether has been variously defined. It is regarded by some as imponderable, neither solid, liquid, nor gaseous, not made up of particles (atoms), but continuous and intimately connected with light, radiant heat, magnetism, and electricity.

As it is not possible to unify or simplify matter beyond a certain point, neither is it possible indefinitely to unify or simplify force. Physical, vital, and mental force cannot be regarded as identical and different phases of one and the same thing.

Large and small masses of inorganic matter possess and exert inherent force and act and re-act upon each other according to fixed laws. The inherent force referred to is fitly designated physical force. It is not of a directive or adaptive kind. Large and small living bodies, such as plants and animals, and parts thereof, possess force which exercises a directive power. This has appropriately been designated vital force. Lastly, there are the forces wielded by the Creator, which are directive to an extent which it is next to impossible even partly to realise. These forces are the product of intellect in its highest and most concentrated form. Only in the Creator can force (physical, vital, and mental) be unified, and what is said of force may also be said of matter. The Supreme Being works in and through matter, organic and inorganic, and through physical, vital, and mental force, and no change can occur in matter, be it great or small, near or remote, which is not inaugurated and controlled by the Creator as the First Cause. He is the Alpha and Omega of all things. Without the absolute control of matter, inorganic and organic, and of the physical, vital, and mental forces which energize it, there could be no co-ordination, no co-adaptation, no arrangement of means to ends.

I am well aware that certain Physicists, Chemists, and Physiologists maintain that there is no such thing as vital force, and they ignore the idea of design and a Creator or First Cause. They support the purely mechanical theory of the origin and working of the Universe. They attempt a vast generalisation without being able to explain or prove the particulars. They take everything for granted; theirs is a case of petitio principii, pure and simple. They boldly assert that matter and force are eternal; that force inheres in and is inseparable from matter; that matter is self-forming and self-directing; that matter assumes movement, and, under certain circumstances, life ; that there is no Creator or First Cause; no design; no directive agency; no adaptation of means to ends; no law and order in the ordinary sense. They regard the universe as a huge congeries of stray particles acting in a haphazard or accidental manner. They resent and reject any interference from without, and declare that the world of matter does not require and does not admit of any interference on the part of a Creator or First Cause. 
They boast a mechanical system which literally bristles with law and order, but they curiously and with strange inconsistency exclude the great Lawgiver and Arch-Artificer. The existence of life on the earth is a stumblingblock and pitfall to the mechanical school. They cannot produce life-neither can they explain it. No Physicist, Chemist, or Physiologist, with all the resources of the most admirably equipped modern laboratory at his disposal, has ever produced anything which remotely resembles life even in its simplest forms. The best they can do is to say that they have artificially produced substances resembling starch, albumen, and some other products, which have hitherto been regarded as organic in their nature. They do not-they cannot—claim to have produced a living plant or animal.

As yet no bridge has been devised to directly connect the living with the dead, or to convert physical force into vital force. No doubt inorganic and organic matter and physical and vital force have much in common: the organic proceeds from and returns to the inorganic, and the same elements are found in both. In like manner the elements which build up plants and animals transfer to the plants and animals a proportion of the force which inheres in them. Further, the same laws, up to a point, regulate inorganic matter and physical force and organic matter and vital force: still the subtle and mysterious something, known as life, which cannot be artificially produced or imitated, intervenes and prevents the unification and identification of matter and force so eagerly desired by the mechanical school. That school is obliged to fall back on the theory of spontaneous generation, which has again and again been proved impossible by direct experiments. The mechanical school have to make extraordinary assumptions in support of its theory. They have, among other things, to assign sensation, volition, and a soul to the atom, and souls and memory to the cells and tissues. They have also to attribute a chemical sense-activity and perception to the male and female sexual elements of animals. Thus, according to J. C. Vogt, "The minute parts of the universal substance, the centres of condensation, which might be called pyknatoms, correspond in general to the ultimate separate atoms of the kinetic theory; they differ, however, very considerably in that they are credited with sensation and inclination (or will-movement of the simplest form), with souls, in a certain sense -in harmony with the old theory of Empedocles of the 'love and hatred of the elements.' Moreover, these 'atoms with souls ' do not float in empty space, but in the continuous, extremely attenuated intermediate substance, which represents the uncondensed portion of the primitive matter."

According to Ewald Hering, "Memory is a general property of organised matter." According to Professor Ernst Haeckel, "Unconscious memory is a universal and very important function of all plastidules ; that is, of those hypothetical molecules, or groups of molecules, which Naegeli has called micelle, others bioplasts, and so forth. Only living plastidules, as individual molecules of the active protoplasm, are reproductive, and so gifted with memory; that is the chief difference between the organic and inorganic worlds. . . The elementary memory of the unicellular protist is made up of the molecular memory of the plastidules or micella, of which its living cellbody is constructed. . E Equally interesting examples of the second stage of memory, the unconscious memory of tissues, are found in the heredity of the individual organs of plants and the lower, nerveless animals (sponges, \&c.). ... In the same way we must regard the third stage, the unconscious memory of those animals which have a nervous system, as a reproduction of the corresponding 'unconscious presentations ' which are stored up in certain ganglionic cells. In most of the lower animals all memory is unconscious. . . . Conscious memory, which is the work of certain brain-cells in man and the higher animals, is an 'internal mirroring' of very late development, the highest outcome of the same psychic reproduction of presentations which were mere unconscious processes in the ganglionic cells of our lower animal ancestors."

Professor Haeckel continues: "The theory of a cell-soul is completely established by an accurate study of the unicellular protozoa, and the psychic phenomena of the protistæ form the bridge which unites the chemical processes of inorganic nature with the mental life of the highest animals. . The tissue-soul [he designates] histopsyche. In all multicellular, tissue-forming plants (metaphyta), and in the lowest, nerveless classes of tissue-forming animals (metazoa), we have to distinguish two different forms of psychic activity-namely, (1) the psyche of the individual cells which compose the tissue, and (2) the psyche of the tissue itself, or of the 'cell-state' which is made up of the tissues. This 'tissue-soul' is the higher psychological function which gives physiological individuality to the compound multicellular organism as a true "cell-commonwealth.' It controls all the separate 'cell-souls' of the social cells. . . . The plant-soul [named by him] phytopsyche is [in his opinion] the summary of the entire psychic activity of the tissue-forming, multicellular plant (the metaphyton, as distinct from the unicellular protophyton). . . The soul of the nerveless metazoa [is according to him] of very special interest for comparative psychology in general, and for the phylogeny of the animal soul in particular, it being the psychic activity of those lower metazoa which have tissues, and sometimes differentiated organs, but no nerves or specific organs of sense. ... The nerve-soul [he has designated] neuropsyche. The psychic life of all the higher animals [he avers] is conducted, as in man, by means of a more or less complicated 'psychic apparatus.' This apparatus is always 
composed of three chief sections: the organs of sense are responsible for the various sensations; the muscles effect the movements; the nerves form the connection between the two by means of a special central organ, the brain or ganglion. . The ganglionic cells, or 'psychic-cells,' which compose the central nervous organ, are the most perfect of all organic elements; they not only conduct the commerce between the muscles and the organs of sense, but they also effect the highest performances of the animal soul, the formation of ideas and thoughts, and especially consciousness."

It is not necessary to follow further what many will regard as the extravagancies and absurdities of Professor Haeckel. His theory of life is a bewildering maze of assumption and wild speculation wholly unsupported by evidence, and shows to what extraordinary straits the mechanical school, to which he belongs, is from time to time reduced. That school, by denying a Creator, Designer, and Upholder, is forced to take up an interminable line of impossible positions, which it cannot possibly define, and which it cannot afford to abandon.

Professor Haeckel endeavours to make out that there is no First Cause, no spirit or mind, but only matter ; that matter is eternal and omnipotent, that inorganic or physical force, and organic or vital force, are essentially one, and that all matter and force are referable to what he designates the "law of substance," whatever that may mean.

It may be here stated that Professors Rudolph Virchow and Emil Du Bois-Reymond, two of the foremost experimenters and thinkers of modern time, who originally supported Professor Haeckel's monistic or matter philosophy, ultimately abandoned it in favour of the dualistic philosophy where matter and mind or spirit are regarded as separate entities.

Others scarcely less distinguished who had adopted Haeckel's monistic philosophy also abandoned it on mature consideration. This is true of Wundt, Karl Ernest Baer, Mr. J. G. Romanes, Oscar Hertwig, \&c.

The doctrine of "oneness" is, as has been stated, not borne out by facts in the inorganic and organic kingdoms. It altogether fails to account for life, and for the phenomena connected with sensation, perception, consciousness, and all that is commonly known as intellect or mind. A clear line can still be drawn as between dead and living matter, between physical and vital force, and between insentient, brut matter, and sentient, thinking matter.

Swedenborg divided the universe into two categories: the spiritual or world of causes, and the material or world of effects. Newton in like manner distinguished between the celestial spheres and the Power which made and set them in motion.

Professors Balfour Stewart and P. Guthrie Tait discuss the problem of the Unseen Universe and the endless sequence and succession of events. They proceed on the principle that every existing state and condition was preceded by a pre-existing state or condition. They take a beginning or creation for granted. While it is permissible, in the case of the Creator, to separate the creative spirit or force from the matter it produces, regulates, and directs, it is proper to state that, in the created thing, the matter and force are, as a rule, associated. This is especially true of the living creature, where the matter and force (physical, vital, and mental) act and re-act upon each other; where the vital and intellectual forces are influenced by the condition of the body (as in animals with nervous systems); where concussion of the brain for the time being destroys the mind; or conversely, where the mind being normal powerfully affects the body. As life is super-added to matter and can be withdrawn from it at death, so mind, as the product of matter and life in animals, is liable to temporary or permanent extinction. Granted that mind, as we know it, is always associated with living matter, the question arises when is mind united to the living matter or body? The reply is at impregnation: when the male and female elements meet and fuse to form the impregnated ovum. Mind in its potential form is an attribute of living matter even in its earliest stages, and is transmitted from parents to offspring in unbroken continuity as life itself is. Sensation, consciousness, and mind, or their representatives, in varying degrees may be predicated of all animals and certain plants, notably the insectivorous plants. The coalescence of the male and female sexual elements in animals forms a new being which includes all the physical and mental peculiarities of the parents. The impregnated ovum, consisting as it does of two distinct and different elements, forms a single, but not a simple or undifferentiated cell. There is duality physically and mentally in the impregnated ovum, as the body and brain of both the parents are represented and are present in a potential form. The continuity of life and of the intellectual attributes (be they simple or complex) which collectively constitute mind, run down the ages. Heredity forms the connecting link between past, present, and future generations of living things. Living matter and mind, or what represents them, have always been associated. They are coupled with the doctrine of the indestructibility of matter and force, and conduct not unnaturally to the conception of the immortality of the soul. It is very difficult to draw a hard and fast line as between matter and force and spirit as we know them. As has been explained, matter shades off by fine gradations until we reach what is virtually the intangible ether. The division of matter, there is reason to believe, even goes beyond the ether, so that substance of one kind or other is assumed to occupy all space. The familiar terms 


\section{DIVISION OF LABOUR IN RELATION TO DESIGN}

material and immaterial, because of this shading off and refinement of matter, become blurred, and lose, to a certain extent, their meaning. Of course, the term immaterial, strictly speaking, means something which is separate and distinct from matter, however rarefied and however minutely divided.

There is no such thing as immaterial substance, but immaterial ultimate force or spirit as it exists in the Creator has long been recognised. This ultimate force or spirit manifests itself in the creation, distribution, and direction of matter and force in the universe as a whole. Vital and mental force have also been regarded by the majority of philosophers and psychologists as essentially immaterial. In this connection, it is proper to point out, that in the higher animals and in man, where vital and mental force or mind can be studied and analysed, intellectual endowment has a physical basis; intelligence gradually increases with the growth and differentiation of the nervous system as we rise in the scale of being; the monkeys having less reasoning power than savage man, and savage man than civilised, modern man. The reasoning powers advance pari passu with the development of the nervous system.

As proving the ultimate connection between mind and body, it suffices to say that mind temporarily disappears during sleep, also when the molecules of the brain are suddenly disturbed, as in concussion, also when a poisonous gas is inhaled, also in certain diseases, \&c. The mind returns when the body awakes from sleep, and when it resumes its normal functions. We have no knowledge of mind in the organic kingdom as apart from the body, and this brings us to the border line which divides the material from the immaterial. It has never been shown that the mind of man exists as apart from his body, so that its continuation after death as spirit or soul must be assumed unless immortality be based on the indestructibility of matter and of force. Such a state of things is not only conceivable but highly probable; indeed it may be said to follow as a corollary, if matter and force are regarded as inseparable.

It is beyond the scope of the present work to discuss the mind as a separate entity; but I may venture to point out that the thinking individual never thinks without his body, and that abstract thought affords no proof of the separate existence of mind or of the immortality of the soul.

\section{DEVELOPMENT AND DIVISION OF LABOUR IN RELATION}

\section{TO DESIGN}

Plants and animals are, without exception, derived from cells or portions of cells. Some plants and animals remain cells_that is, they do not divide, differentiate, or advance to anything else. We have examples of cell plants in the snow plant and Sarcina ventriculi, and of cell animals in the zooids, the amoba, and other low forms.

The fact that there are plants and animals which never get beyond the cell stage, while others differentiate into complex beings up to man, where there is a highly-developed nervous, vascular, lymphatic, respiratory, muscular, osseous, and glandular system, shows very plainly that cells fundamentally differ from each other, and that each cell, and system of cells, is originally distinct. In other words, the primordial or primitive cell from which plants and animals are developed is endowed with properties essentially its own, and has limits set to its development, and the direction of its development; each cell and each plant and animal being capable of reproducing their like, but only their like; it being impossible to produce an animal from a plant and vice versâ, or a vertebrate from an invertebrate, or different vertebrates from each other. This means, that a reptile cannot be obtained from a fish, a bird from a reptile, or a mammal from a bird. I am well aware that all this is opposed to natural selection, and evolution in its widest sense, and that Mr. Darwin and others have endeavoured to prove that plants and animals have a common origin, and that even man, with his God-like form and wealth of imagination and intellect, is indirectly and remotely the product of an oyster or some rudimentary, soft-bodied animal with neither brain nor nervous system, and wanting in all those elaborate arrangements which characterise the higher animals. The grand climax in development witnessed in man is said to be reached by an infinity of accidental, trifling modifications in practically unlimited time, but no actual proof of the several modifications and transformations referred to has been, or can be, adduced. Professor Haeckel has done his best to show that mammals closely resemble each other during their developmental stages, and Professor Huxley has given it as his opinion that the birds have been manufactured from the reptiles and are their direct descendants. The views of Mr. Darwin and Professors Haeckel and Huxley are traversed in other portions of this work, and all that need be said here is that, lacking proof, they are inconclusive and unsatisfactory.

It is abundantly evident from the permanency of existing forms that cell plants and animals, and plants and animals as a whole, have limits beyond which they may not stray. It is equally certain that cells and cell plants and animals develop along given lines, and that they have no power either to direct or control their growth. These

vol. I. 
facts admit of only two explanations. Either cells and cell plants and animals develop and differentiate from the first in a haphazard way by accident, or their development and differentiation are predetermined, supervised, and regulated by an intelligent Agent or First Cause. If the thing developing is not the sport of nature, it follows that it must have in itself, in a potential form, all the structural and functional peculiarities and attributes of the future being, whether plant or animal-nay more, that when a nervous system and brain are characteristics, these must also exist in a potential state. In making this statement, the reader is not to infer that the several structures and attributes which constitute the compound plant and animal are all contained in the primordial cell from which plants and animals are developed; only this, that the cells have in themselves, in the shape of original endowments, the powers which enable them to build up from their surroundings, consentaneously or consecutively, the several tissues, structures, and systems which go to form even the most complex plants and animals. To put it otherwise : there is nothing in nature to show that plants and animals are accidental formations at the outset, or that their subsequent development and differentiation are at any period due to chance or natural selection. Plants and animals are certainly not the product of spontaneous generation. The accidental or chance hypothesis of the production, existence, and continuation of plants and animals on the earth would produce endless confusion, and completely overthrow the law and order which are known to exist alike in the organic and inorganic kingdoms.

Much emphasis has been placed by Mr. Darwin and his followers on what they term "accidental modifications" in plants and animals; these being frequently of the most insignificant and trifling description. They ignore the fact, that plants and animals in a state of nature breed true-that is, they revert to their originals even in the most minute details : any accidental departure from the normal standard being, as a rule, corrected very soon after its appearance. They claim that the accidental modifications in plants and animals gradually and steadily accumulate, and, in the fulness of time, produce what are virtually new beings. They, in reality, seek to establish a false analogy as between so-called natural and artificial breeding, and have propounded the theory that plants and animals, in a state of nature, have the power to select and perpetuate whatever is advantageous to themselves and to suppress and obliterate whatever is disadvantageous. This briefly is the famous theory of "The Origin of Species by means of Natural Selection." It is safe to assert that plants and animals have no such powers. The theory takes for granted that plants and animals can dispense with a selector outside of themselves, which they certainly cannot do. The theory of "Natural Selection" is fully discussed further on. In natural and artificial breeding a selector is a sine qua non. In nature the selector is, as a matter of fact, the First Cause which created, controls, and sustains the plant and animal. In the breeding of domestic plants and animals the selector is the individual who perceives and takes advantage of accidental modifications or sports and perpetuates them for his own purposes, not unfrequently to the detriment of the typical or natural parts of plants and animals. He breeds against nature, and produces abnormal or monster plants and animals. He disturbs and destroys the symmetry and balance of nature. That this artificial breeding is an outrage on nature is proved by this : plants and animals when left to themselves and allowed to run wild invariably return to their typical form; the several varieties of pigeons breed back to the blue rock pigeon, and the tender cultivated vegetables revert to their non-succulent, hardy types. Natural selection, as a matter of fact, is wholly based on assumption. As already indicated, no plant or animal can, ipso facto, either develop or suppress any of its parts at discretion.

While plants and animals cannot select and perpetuate what is good in themselves and repress and obliterate what is bad, they do nevertheless exert a certain amount of selecting and rejecting power in special cases. Thus secreting and excreting glands select and reject certain ingredients of the blood: they distinguish between those substances which are to be retained in the system and to be employed in a new form or to be extruded as waste products. The same is true with regard to food. Plants and animals do not feed indiscriminately on everything presented to them. On the contrary, they take kindly to certain articles of diet and refuse others. This power to discriminate between substances possessed by plants and animals, and parts thereof, is in turn referable to original endowment and a First Cause. Plants and animals, when absorbing and assimilating solids, semi-solids, fluids, and gases, can, within limits, select and appropriate or reject, but this is quite another matter from saying that plants and animals make themselves in a haphazard way, apart from design and a general plan, and independently of a Creator. Plants and animals are no doubt influenced up to a point by the food they consume, and may be larger or smaller according as the food is nutritious and abundant or innutritious and scarce. They are also influenced by heat and cold, habitat, \&c. The ultimate nature or constitution of the plant and animal is, however, not altered by food, climate, habitat, \&c. A queen bee (which is considerably larger than the common bee) can be manufactured by a particular food from a common bee grub, and a man and a horse may be larger or smaller physically. The bee, nevertheless, remains a bee, the man a man, and the horse a horse.

There is no escaping from a First Cause, from types, and from design, law, and order, in things organic and inorganic. 


\section{DIVISION OF LABOUR IN RELATION TO DESIGN}

If cells, and cell plants and animals, which supply the simplest examples of living things, characteristically differ from each other from the beginning, and cannot be traced to a common source, what shall we say of the supposed identity of the more complex plants and animals?

The cell, notwithstanding all that has been said to the contrary, is not a simple structure. It is, as a rule, composed of a cell wall, cell contents or protoplasm, a nucleus, and one or more nucleoli. It has a molecular basis, and in the molecules, the differentiation which distinguishes one cell, and one cell plant and animal, and plants and animals as a whole from each other, occurs. The nature and powers of the molecules cannot be determined by the microscope or by chemical analysis, but that the molecules and cells are differentiated from the first is rendered certain by this, that they unerringly reproduce themselves, and only themselves. Cell plants do not produce cell animals or the converse, and the great races of plants and animals characteristically differ from each other as regards their leading features and types.

To say that all plants and animals are originally derived from absolutely homogeneous and identical matter is to ignore numerous important facts.

According to recent researches, molecules, protoplasm, cells, germs, seeds, and eggs vary infinitely. There is no such thing as absolutely homogeneous identical living matter, and in its absence no common basis or startingpoint can be claimed for plants and animals. If such matter really existed, it would avail nothing in the absence of a designer and director outside of itself. Such matter at best could only reproduce itself. A designer and director is absolutely necessary to inaugurate and carry out the divisions and differentiations which characterise the leading types of plants and animals. No progress whatever could be made in the construction of the several kinds of plants and animals as apart from a designer, and distinct differences in the general matter from which the plants and animals proceed. The power to divide and differentiate is an inherent original power conferred by the Creator. Plants and animals are not chance products. Everything about them is due to design and the operation of an intelligent First Cause.

The histories of plants and animals from their earliest beginnings to their latest developments confirm this view.

If there are unicellular plants and animals which never advance beyond the cell stage, but which, nevertheless, perform all the functions of life and reproduce themselves, and only themselves, it is evident that a limit or boundary is assigned them. The irrevocable fiat has gone forth, "thus far and no farther." They have their spheres of usefulness, and to these their activities are confined. The fiat applies not only to all plants and animals, but to all parts of plants and animals, and in this we have an explanation of the so-called vestiges or rudiments, where development is suddenly checked; a part which attains large dimensions in one case becoming dwarfed or disappearing in another. A good example of interrupted growth and stunted development is furnished by the appendix vermiformis in man.

On the higher plants and animals a greater power of differentiation is conferred, and conferred at the outset. It is original differentiating power which makes plants and animals what they are. The amount of differentiating power varies according to types, and according to an ascending scale; but in every instance limits or boundaries are set up. These limits or boundaries are the outcome of design, law, and order, and prevent confusion. Without them the members of the organic kingdom would be mixed up in one vast, inextricable tangle.

The power of differentiating, here contended for, applies not only to cells, cell plants and animals, and plants and animals as a whole, at their earliest beginnings, but also to every stage of their development. In other words, all plants and animals can and do differentiate from the first as molecules, cells, germs, seeds, and eggs: they also differentiate as they develop from the imperfect to the perfect being. The adult forms can always be referred to their primordial masses, whatever these happen to be. As the special molecules, cells, germs, seeds, and eggs cannot be mixed up and confused, so their ultimate products remain distinct, and cannot be mistaken for each other. The special germinal matters, and the final adult forms, whether plants or animals, never lose their identity. On the face of it, rudimentary plants and animals cannot engender de novo a power of differentiating. The power to differentiate is a conferred power, and furnishes the key to the organic kingdom. As the organic kingdom is superior to the inorganic kingdom, so the members of the former are superior to those of the latter. The organic kingdom occupies a higher platform than the inorganic, and takes precedence when the two kingdoms come into contact or opposition. The living plant and animal are superior to their surroundings or environment. They appropriate and reject matter according to their requirements. They play the rôle of masters. They direct and control, and are very little under the influence of extraneous matter. Accidental, dead, extraneous matter and artificial stimulation do not form the several organs and systems of plants and animals. These are all provided for them by nature from the beginning. Neither does extraneous, non-sentient, non-living matter form the plants and animals themselves. 
The histories of plants and animals during development furnish important evidence of the differentiating power inhering in molecules, cells, germs, seeds, and eggs. If all these were originally identical, no differentiation or division of labour could possibly occur : plants and animals would all resemble each other. Instead of this, plants and animals vary infinitely. As the variation cannot be traced to the elements of the inorganic kingdom, it must be referred to the minute, living, primordial masses from which plants and animals originally proceed. These tiny, living masses are potential entities of the most extraordinary kind. They are independent things, each with a course, a career, and a goal. They arrive at the goal at a stated time and by a special route. They cannot alter either the route or the time of arriving at the goal. Plants and animals reach maturity in spite of themselves and by well-defined paths. They have no knowledge of the paths along which they are to travel, or the bourne at which they are ultimately to arrive in their course of development. The several parts of plants and animals are developed sometimes consecutively, and sometimes simultaneously- that is, the growing parts sometimes appear separately and independently, the one after the other, in a given order, and at other times synchronously or together. The parts fit into each other in the completed whole; plants and animals being structurally and functionally perfect from the anatomical and physiological standpoints. The several portions do not produce each other : they form part of a scheme - a designed whole-and are means to ends. They are adapted to each other and to their surroundings.

While the original molecules, cells, germs, seeds, and eggs cannot control the direction of their growth and the degree of their development, neither can the adult plants and animals modify or materially alter the molecules, cells, germs, seeds, and eggs which they, in turn, ultimately produce to perpetuate the types. The laws of reproduction are, in a great measure, stereotyped. There are not only the most perfect arrangements for plants and animals reproducing themselves after their kind, but the tendency and desire to reproduce are universal. The great command, "Increase and multiply," applies to everything which lives.

The relations of the rudimentary and young to the more perfect adult plants and animals are of the most intimate description. In the case of animals, the duties of the parent, as a rule, do not cease with the production of the ova or eggs. The eggs of birds have to be hatched out by the parent, and the ova of mammals have to be carried and developed by the mother until the period of parturition arrives. The ova occupy the ovaries of the mother even before the period of puberty arrives. After birth the offspring has to be carefully tended and nursed for longer or shorter periods. The arrangement in the case of mammals entails the most remarkable changes in the mother before and after the birth of the offspring. The mother supplies from her own blood and being during pregnancy the wherewithal to develop the embryo and foetus, and during the parturient period her mammary glands swell and produce a copious supply of milk in anticipation of the advent of the young animal. The food supply and the progeny are timed to each other.

The amazing transformations which occur in the progeny during pregnancy and after birth are all provided for. Can it be thought for a moment that the various phases of reproduction and development are due to chance?

A watch, which is one of the most ingenious of human contrivances, is a crude and clumsy invention when compared with a mammalian foetus; but what is true of the one is true of the other as regards a designer. It is inconceivable that an impregnated human or other ovum could arrive at maturity in the uterus, and be born and cared for after birth, as apart from design. At every stage of the reproductive process there is evident adaptation even in the most trivial details. The various systems of the fœtus are developed in anticipation of the functions they are to discharge. The heart is prepared to receive blood, the lungs air, the alimentary canal food. The organs are made for, and not by, the materials with which they have to deal. The blood does not cause the movements of the heart, the air those of the lungs and chest, and the food those of the alimentary canal. These movements are due to original endowment, and are necessary to the continuation of life. Similarly, the secreting and excreting glands are formed before they are called upon to discharge their peculiar functions. The salivary and gastric glands, the pancreas, liver, \&c., are ready to digest the food and enrich the blood: the sudoriferous glands and kidneys to discharge effete products: arrangements are made not only for nourishing the offspring after birth, but also for keeping its blood pure. The lungs and skin take a prominent part in the purification. The several systems of the body are necessary to each other, and are all developed before they are required. The digestive system converts the food into chyle, the lymphatic system absorbs and adds it to the blood, the vascular system transmits the blood and feeds the tissues, the respiratory system oxygenates the blood and the tissues, the muscular system moves the several parts of the body, the osseous system gives them strength and leverage, and the nervous system co-ordinates and regulates the entire animal economy. These facts are wholly opposed to the theories of spontaneous generation, natural selection, evolution, irritability, and extraneous stimulation. They are also opposed to the mechanical theory of life. They support my contention of inherent, independent powers in molecules, cells, 


\section{DIVISION OF LABOUR IN RELATION TO DESIGN}

germs, seeds, and eggs, and of original capacity for development in specific directions in every part of every plant and animal existing on the earth.

What is true of the several systems of animals is also true of their sense organs. These are all formed before they are called upon to act. The eye is developed before it is exposed to the light; the ear before it is acted upon by a sounding body; the nostrils before they come into contact with smelling particles; the mouth before it is supplied with sapid substances (food); and the skin before it receives impacts from the outer world. The young animal, if deprived of its sense organs, would, in a great measure, be helpless and very inadequately equipped to cope with the exigencies of life. The sense organs are prepared by nature in advance, and for the express purpose of making the animal superior to its surroundings and master of the situation. The sense organs grow and develop because of powers inhering in the impregnated ovum. They are not caused by externalities: light does not form the eye; sound the ear; odoriferous particles the nose; sapid substances the mouth; and the outer world the skin. Neither are the sense organs the product of voluntary effort on the part of the sentient animals themselves.

The facts revealed by development unequivocally point to a First Cause and design, and it is mere perversion to attribute any one of them to chance. It is also, in my opinion, impossible to account for them by "natural selection " and "evolution," if by this is meant the manufacture of an animal out of a plant or of the several races of animals out of each other. Such a process must be inaugurated, regulated, and supervised by an omnipresent, intelligent Agent. These statements are not met by saying that rudimentary forms develop by accident or by effort, and by increments throughout the ages. There is no proof of this. The several types of animals are provided from the first with the organs (sense and otherwise) which adapt them to their surroundings and their peculiar modes of life. That the sense and other organs of the higher animals are not evolved from the rudimentary sense and other organs of the lower ones is evident from this, that many of the lower forms have certain of their organs more highly developed than are the corresponding organs of the higher animals.

The sense organs are as truly original endowments as the organs of locomotion, whether these consist of suckers, cilia, feet, legs, hands, arms, fins, flippers, propelling tails, or wings. To say that rudimentary animals develop by accident or by voluntary effort during untold ages the organs on which their very existence depends is to reverse the order of nature. Such belief takes for granted a period of probation for the development of the organs during which the animals would be helpless, and, for the most part, starve. Animals live because the sense organs and organs of locomotion enable them to detect and to secure food. Where would a fish be as regards its food supply if deprived of its eyes and of its tail and fins, or a bird if deprived of its keen sight, wings, and legs, or a mammal if deprived of its eyes, ears, feet, legs, hands, and arms?

All animals move freely about in search of food, and their sense organs and organs of locomotion are, in every instance, adapted to their peculiar mode of life. ${ }^{1}$

The electric organ of the electric fish affords a good example of a large special structure which, strictly speaking, is not necessary to the fish as such. It is an original endowment; its function being one of defence and attack. It cannot be accounted for either by "natural selection" or "evolution." The development of such a large heavy structure by increments extending over long intervals and before it was of use would have been an incubus and burden to the fish which it could scarcely have survived. Its development pari passu with the other parts of the fish entails no burden. The structure and function keep abreast of each other, and are mutually explanatory.

The sense and travelling organs of animals are, in the majority of cases, due to infoldings of the skin and outgrowths from the body, but the essentials of the infoldings and outgrowths are in the body itself. It is not conceivable that a fish could voluntarily develop eyes and a swimming tail and fins at discretion, or a bird and bat their eyes, ears, and highly complex and wonderfully differentiated wings, or a quadruped its five senses and beautifully devised, cunningly constructed legs, joints, and feet. Still less is it conceivable that these remarkable structures could be developed by accident from extraneous matter, that is, from the substances with which they come in contact, or the media on which they act. Animals cannot develop their sense and travelling organs by efforts of will, however long continued; neither can they be developed accidentally by environment. The only possible explanation is that they are original creations. As a matter of fact, the sense and travelling organs are necessary to the continuation of life, in the same way that the molecules, cells, germs, seeds, and eggs are necessary to the beginnings of life. The sense organs and organs of locomotion are original endowments, specially provided to enable animals to maintain their places in nature.

It is a remarkable fact that the several orders of animals, and each order separately, conform as regards their travelling organs to the requirements of their physical surroundings. Thus the animals which confine their movements mainly to the land are distinguished by small feet; those which swim have expanded feet, flippers, fins, or

1 In the case of plants and the more stationary animals the food either invests them or is brought to them hy pre-deternined arrangements. The plant and stationary aninal are provided for even in the alsence of a loconotory apluaratus. 
swimming tails; those which fly have greatly expanded wings (Plate 1., Fig. 1, letters $\mathrm{A}$ to $\mathrm{N}$ inclusive). These remarks apply to all animals without distinction: to animals with and without a skeleton, and with and without limbs. The laws of locomotion are inexorable to the extent, that the animals frequenting the land, the water, and the air respectively, must be provided with travelling organs and surfaces adapted to at least one of the three highways of nature. The quadruped cannot swim so well as the fish, and the fish cannot fly so well as the insect, bat, and bird. The animals which excel in walking, swimming, and flying are of necessity provided with special walking, swimming, and flying organs. The sea-mammals, such as the porpoises and whales, are fish-shaped, and supplied with the expanded flippers (modified fins) and tails which characterise fishes. The reptiles which take to the water are furnished with expanded feet, flippers, or a broadly expanded swimming tail ; the fishes which fly are endowed with large, powerful, pectoral fins resembling wings, and the insects, bats, and birds which indulge in aërial locomotion are, in every instance, equipped with greatly expanded pinions. Whatever the nature of the animal, its travelling organs are, and must be, adapted to one or more of the highways of nature.

As further illustrating the special modifications and adaptations manifested in the locomotion of animals it may be stated that animals with small feet, such as the horse, deer, dog, and hare among mammals, and the emu and ostrich among birds, confine their movements almost exclusively to the land: those with expanded feet, such as the otter, platypus, seal, sea-lion, and walrus, restricting their operations largely to the water. The modifications and adaptations are even more marked in the sea-mammals, which have fish-shaped bodies, and can only swim and dive. The same is true of the penguins among birds. These have small rudimentary wings like flippers, and are most expert swimmers and divers, but most awkward, imperfect walkers. The flying fish, because of its greatly expanded pectoral fins, can make considerable excursions in the air, and the flying squirrel, galeopithecus, and flying dragon, which have large integumentary membranes extending between their anterior and posterior extremities on either side of the body, can take long leaps and glide parachute-fashion from elevations, trees, \&c. The bat, the only mammal which now flies, has very large, splendid wings, their area exceeding that of the wings of many birds.

As in the present, so in the past. Time was when the extinct reptile fish, the Ichthyosaurus, swam fish-like by the aid of a swimming tail and by flippers; the extinct reptile bird, the Plesiosaurus, swimming by the aid of flippers alone. The extinct reptiles, the Pterodactyles, were provided with ample wings and flew in the air. The extinct sea-mammals, the Halitherium and Rhytina, were fish-shaped and adopted the fish form of locomotion. The extinct birds, the Dinornis giganteus, the Apteryx, and Dodo, had strong legs; the wings being very rudimentary. They ran but could not fly. The various animals, past and present (whatever their nature) had to be provided with suitable organs of locomotion for the land, the water, or the air. No animal is equally well adapted for walking, swimming, and flying. If it walks and runs well, it, as a rule, swims indifferently: if it swims well, it, for the most part, walks and runs badly: if it flies well, the chances are it neither swims nor walks well. There are, it should be stated, insects and birds which walk, swim, and fly indiscriminately, but they never attain to perfection in all three kinds of locomotion. The eagle, vulture, kite, falcon, and other large birds of prey fly magnificently, but jump and hobble when they attempt to walk : they cannot swim. The albatross, swift, swallow, and bat, while among the best of flyers, can scarcely be said to walk: the albatross only can swim. The penguin flies with great velocity under the water, but cannot fly in the air; neither can it walk with any degree of steadiness. There are other birds, such as the grouse, partridge, and pheasant, which run and fly well but cannot swim. In all this there is design and law and order. If one or more of the three great highways of nature are to be traversed, the mechanical conditions of the highway or highways must be satisfied, and the animal, whatever its nature, must be provided not only with the organs and surfaces requisite, but it must possess the power and the skill to apply the organs and surfaces so as to obtain the reactions necessary for propulsion, or for propulsion and support. Animals could not maintain their places in nature unless they were differentiated and modified as regards the sense and other organs to adapt them to their peculiar modes of life. The developing frog in its tadpole stage illustrates the fish-tail swimuming arrangement. In this particular case there is no time to manufacture a swimming tail according to the plan advocated by Mr. Darwin and the Evolutionists. The accidental trifling modifications, extending over vast periods, required by "natural selection" and "evolution" are wholly absent. The same is true of the young fish. The fish develops from an egg in which there is no trace of a swimming tail. A tail, however, as in the tadpole, is developed in a surprisingly short time and before our eyes. The fish-tail is not an accidental but a pre-determined structure. It is arranged for in the development of the fish from the egg. The young fish grows in two directions —dorsally and ventrally. The dorsal portion includes the back-bone, muscles, nerves, chief blood-vessels, \&c.; the ventral portion the alimentary canal, glandular system, \&c. At first there is no mouth, alimentary canal, or vent; the young fish being provided with a ventral bag of pabulum, which is gradually absorbed as development proceeds. The young fish is not permitted to die of inanition during development, from neglect or the absence of a First Cause and design. At the same time that the bag of pabulum is being absorbed, a mouth and perfect alimentary canal 


\section{DESIGN A PROMINENT FACTOR IN NATURE}

for taking in and dealing with extraneous food, and a swimming tail to enable the young fish to catch it, are being provided. The alimentary canal would be of no use without the swimming tail, and the two are consequently developed together or synchronously. In the developing fish as in the developing frog, the accidental trifling modifications extending over protracted periods claimed for the production of organs and the several kinds of animals are wholly and entirely wanting. The Darwinian theory and evolution break down even in their boasted stronghold of development. If the swimming tail can be developed before our eyes, and while we are, so to speak, watching, there is no need to assume an infinity of accidental trifling modifications extending over practically unlimited time for its production. If the arguments for "natural selection" and "evolution" miscarry in cases of structural development which can be studied from day to day, and which are perfected in a few weeks or months, they ought to be discredited and abandoned. The development of a swimming tail is clearly a predetermined natural process, and has no connection either with "natural selection" or with "evolution." If the swimming thing had to wait for its swimming tail until formed by "natural selection" and "evolution" it would simply be non-existent: it would die of inanition while its swimming tail was in process of formation. There is no proof that the accidental trifling modifications which are said to occur from time to time in plants and animals, and which, according to the theories of "natural selection" and "evolution," are necessary to the production of the organs of plants and animals, and plants and animals themselves, are constant and cumulative. The missing links and gaps in plants and animals in present and past time are wholly opposed to these theories.

\section{$\S 68$. Design a Prominent Factor in Nature.}

Of design it may truly be said circumspice. It everywhere reveals itself to the careful observer. There is an eternal fitness in things - a fitness which has obtained from the earliest times and obtains now. Everywhere there is adaptation to environment. There is nothing out of joint, although to the finite mind, attempting to grasp the infinite, there are many things which are incomprehensible. There are cause and effect, law and order, and continuity which proclaim a great First Cause as potent to-day as it was when the world was first created. There is also a persistent supervision of everything the universe contains as regards inorganic matter and physical force, and as regards organic matter and vital and mental force. Everything is interdependent. The organic kingdom comes from and returns to the inorganic one. Plants live upon the soil, the air, and the moisture and ingredients which these contain. Animals live upon plants and upon each other, and all, sooner or later, are resolved into the elements found in the earth, water, and air respectively. The sun, the great representative of force, heat, and light in our universe, is to be directly or indirectly credited with the movements of the earth and those marvellous cosmic changes which result in day and night, the seasons, the rise and fall of the tides, and various other phenomena on which the life and well-being of plants and animals depend. The elements form the tissues and substances of plants and animals through which they have, so to speak, a right of way. Plants and animals are incessantly taking in and giving out inorganic matter. They are, at no period in their histories, absolutely and identically the same. There is a constant give and take, an endless series of reactions as between living and dead things, which cannot possibly be accounted for by chance, and which necessitate the operation of an all-ruling Providence.

It matters not in what direction we turn. Everywhere there is evidence of law and order in the inorganic and organic kingdoms, and it is the continued adaptation of the one to the other throughout the ages that more than any other thing proves the existence of an Omnipresent God. All physical and all vital phenomena are conditioned: they have their boundaries beyond which they may not go. While the phenomena in question are the outcome of law, a slight degree of variation is permissible to living things which have in themselves a corrective power-a power which enables them to depart from, and return to, their originals. The variation is, in every instance, trifling in amount, and confined as to time and place. It is thus we explain types and varieties in plants and animals.

All research, so far, goes to prove that plants and animals are created according to types, the types being, as a rule, well marked, but, in some cases, shading off and becoming modified, and making it difficult to discover the type. These modifications have been seized upon by Evolutionists as a proof that all plants and animals are the outcome of a continued evolution from lower to higher forms, a conclusion by no means warranted by the facts. In the majority of cases the types can readily be made out, and they are distinguishable alike by the kind and degree of development, and the distribution of the types in time and space.

The geological record shows, in many cases, an advance, maturation, and decline of a type. It also displays gaps in the flora and fauna which, while they are quite consistent with the separate creations of types, and the modifications, within limits, of these types, are inconsistent with, and destructive of, the theory of evolution, which logically requires unbroken continuity. 


\section{DESIGN IN NATURE}

The Evolutionists evade this serious difficulty by saying that while the geological record is admittedly imperfect at present, there are good grounds for believing it will not remain so; some gaps having been partially.filled up of late years. The argument is more ingenious than convincing. Conjecture cannot take the place of positive evidence. It is quite conceivable, and more than probable, that the Deity has placed boundaries to the great leading races of plants and animals. Such an arrangement makes for law and order. Evolution and endless modifications, for the most part, lead to confusion. As in the inorganic kingdom, crystals and other physical objects are built up in the same way and according to rules to which there are no exceptions, so in the organic kingdom, which is a differentiation and outgrowth of the inorganic one brought about by the introduction of life, there are main lines of development which are never departed from to any extent, but which, if departed from, even to a slight extent, can be righted by a power of correction which inheres in the individuals through which the departure takes place. The modifications in such instances are in no case indefinite. We have examples of this power of righting in cultivated vegetables, which if left to themselves, sooner or later return to their originals. The same is true of domestic or cultivated animals. The several varieties of pigeons return to the blue rock pigeon. There are limits, moreover, to cross-fertilisation. The mule and hinny are both barren, and similar remarks apply to crosses in birds. Granting, however, that evolution in the widest sense obtains, it affords no proof of the nonexistence of a First Cause. Evolution beginning in the vastly remote past in a jelly speck of apparently homogeneous, undifferentiated protoplasm, and ending, after infinite permutations in infinite time, in the most highly cultured modern man, would, in some senses, be a more extraordinary creative feat than the formation of types at various periods of the world's history. Evolution does not get rid of the Deity. Neither does it prove that life and the formation of plants and animals are the result of spontaneous generation. No one has ever seen the birth of a new plant or animal, and no chemist or physicist has been able to manufacture either.

Plants and animals are, in every instance, the product of pre-existing germs, seeds, or eggs. It has been again and again shown that if the germs, seeds, and eggs which float in the air are rigorously excluded from organic solutions such as are furnished by chopped hay and water, turnips and water, flesh and water, \&c., the infusions are invariably barren. This was shown by the illustrious Pasteur more than a quarter of a century ago, and the late Professor Tyndall afforded convincing proof in the same direction. M. Pasteur found that if he trapped by long, bent, crooked tubes, or destroyed by great heat, the infinitely minute germs, seeds, and eggs which are suspended in the atmosphere, and prevented their getting into the organic solutions, the solutions invariably remained barren.

Tyndall arrived at the same conclusion by another method. He made two small closed glass chambers with sliding doors, the interior of one of which he smeared with glycerine. These he kept in a still place and away from draughts. After a considerable interval the germs, seeds, and eggs floating in the air in the glycerine chamber were caught (trapped) on the bottom, sides, and top of the chamber. He then cautiously introduced equal portions of the same organic infusion into the two glass chambers respectively, with the result that the portion in the glycerine chamber (the air of which was, so to speak, filtered) invariably remained barren, while that in the nonglycerine chamber sooner or later teemed with life. Other investigators equally competent to deal with the subject obtained similar results by other and ingenious methods devised by themselves.

So far as is known at present there is no such thing as spontaneous generation or a creation of life de novo.

Spontaneous generation is for some a fascinating doctrine. It is delightfully vague, and gives a free rein to the uneducated, and to those who are anxious to evade responsibility by denying the existence of a God, and who prefer to refer the universe, and everything it contains, to accident or blind chance. They say, without the faintest tittle of evidence, that matter created itself, that it assumed motion as in the heavenly spheres, and that it ultimately assumed life as we know it on the earth in living plants and animals. Strangely enough, Professor Tyndall latterly attributed a universal power to matter as apart from life. In like manner Mr. C. Darwin committed himself to evolution and the development of man from lower forms. These great scientists were apparently carried away in their enthusiasm by views which they at first accepted charily and with extreme caution. Where so many outstanding evidences of design present themselves, it is difficult to make a wise selection of proofs. No doubt the most astounding examples of design are revealed by the telescope when directed to the heavens. There we behold what are practically unlimited matter, unlimited space, and unlimited power : great suns and solar systems; untold planets, satellites, and comets; stars which in number exceed the sands of the sea; the Milky Way with its marvellous wealth of nebulæ; and, greatest marvel of all, the vast, ponderous heavenly bodies delicately poised and wheeling in space as lightly as if wholly devoid of substance.

Those stupendous orbs, the sizes and distances of which can only be approximately computed, thread their way among each other with extraordinary exactitude, and, at times, with incredible rapidity-a rapidity so great that mathematicians are at a loss to compute and the mind to grasp it. The heavenly systems, so vast and so various as regards size, shape, and movement, cannot possibly be explained by any concatenation of circumstances into 


\section{DESIGN A PROMINENT FACTOR IN NATURE}

which the element of chance enters. The heavenly bodies are all under law and order, and so great is that law and order that astronomers can calculate and predict unerringly the dates of the eclipses of the sun and moon, the appearance and disappearance of comets, \&c. As science advances and more powerful telescopes are invented, undreamt-of wonders reveal themselves, and the mind, surfeited and in a manner overwhelmed, recoils from the stupendous task of describing and delineating them.

What is true of the telescope is equally true of the microscope. The universe is at once inconceivably great and inconceivably little. There is practically no limit to the division of matter and the number of rudimentary plant and animal forms which the modern microscope reveals. Here, as in the heavens, untold wonders await us. Not only are there endless bacteria ${ }^{1}$ and other low vegetable forms all but invisible with even the highest powers of the microscope, but there are corresponding minute animal forms in incalculable numbers.

The bacteria and low vegetable forms, and the minute rudimentary animal forms, especially the former, possess a truly amazing power of fertility. They are to be counted by millions in even the most confined spaces, and play a leading rôle not only in the fertilisation of the soil and the production of food stuffs, but they are also active agents in putrefaction and in setting up nearly every form of contagious disease. They have thus a high mission to perform; a mission so important that one cannot help regarding them as the emissaries of an all-wise Being Who directs and controls their operations under all circumstances. Microbes and animalcules enter largely into our food and drink in the healthy state, and are accountable for most of our diseases. Here is interdependence of a pronounced kind as between the most rudimentary and despised living forms and man himself. Nothing lives by itself and for itself, and all things are made subservient to each other and for the wisest of purposes. Man with his boasted superiority, mentally and physically, is associated with the lower rudimentary vegetable and animal forms in a way which, to some minds, is not a little humiliating. He, in common with the higher animals, is a host and hostelry for an incalculable number of low living forms which at times inhabit his tissues and revel and multiply in his blood. There are friendly and unfriendly microbes, and the prevalence of the one over the other, in a large number of cases, determines the degree of health and of disease. Truly the issues of life and death hang on very slender threads, and the wonder is not that we are occasionally sick and out of sorts, but that we are ever well.

In addition to the microbes referred to, there are other pests, such as the Trichina spiralis, guinea worm, thread or round worms, tape-worms, bots, \&c. The subject of parasites favours design, inasmuch as certain plants and animals avail themselves of the labours of other plants and animals; the higher forms being made a convenience for the lower forms. It is not conceivable that the lower forms should acquire this mastery as apart from an intelligent Cause giving them the victory.

Very powerful arguments for design are to be drawn from the growth, life history, and reproductive powers of plants and animals.

Before plants and auimals could exist on the earth much and elaborate preparation was necessary. The heavenly bodies had to be created and set in motion. The sea, earth, and atmosphere had to be formed. Plants which directly draw their sustenance from the earth, air, and moisture derived from the sea, lakes, or rivers were first produced. Animals which, for the most part, live upon plants were a later or subsequent creation. Plants only can live on inorganic matter : animals cannot live directly on the elements; their food must be prepared. This important function is discharged by the plants, which store up the elements in their own substance in a form which makes them available and readily assimilable by the animals. The plants which feed upon inorganic matter supply the food of animals.

The relations which obtain between plants and inorganic matter, and between plants and animals, have always existed. They have, however, varied slightly at different times. In the immeasurably remote past, the composition of the atmosphere was not exactly what it is to-day. It contained a larger amount of carbonic acid. The temperature was also higher. These differences induced corresponding differences in plants and animals. The flora and fauna of the early day had to accommodate themselves to existing circumstances. The hot, humid atmosphere surcharged with carbonic acid supplied the conditions for a vigorous race of tree and other ferns and tropical plants, which grew so luxuriantly that they covered large portions of the earth's surface in successive layers ; plants growing upon plants ad infinitum. These huge deposits of plants, due mainly to excess of heat and light, and a moist, peculiar atmosphere, form the coal measures of the present day. Their present position in the bowels of the earth was a mere question of subsidence extending over long periods. They gradually sank and were covered over by cosmic dust, by water deposits, and by agents which are constantly at work in the reduction of rocks and the production of soil.

1 Cohn has divided bacteria into (a) sphero-bacteria, or spherical bacteria; (b) micro-bacteria, or bacteria in the form of short rods, including the genus Bacterium; $(c)$ desmo-bacteria, or bacteria in straight filaments, of which the genus Bacillus is a type; $(d)$ spiro-bacteria, or bacteria in spiral filaments, as the genus Vibrio.

VOL. I. 
That the flora of bygone ages formed the coal measures is abundantly proved by this, that the microscopic structure of coal is absolutely identical with the structure of many plants, and coal when ignited burns exactly as a piece of well-dried timber does. It thus happens that the heat and light stored in the aboriginal tree-ferns and other tropical plants are both available for the use of man at the present day in as great a degree as the modern forest is. There is no destruction of either matter or force. The form of the one and the direction of the other only are changed.

The apparently inexhaustible supplies of petroleum which unbidden flow from the interior of the earth have also a plant origin. This is proved by the fact that spirit can be distilled from petroleum, and even a cheap kind of champagne manufactured from it. It is also proved by the multiplicity of plant products obtained from coal tar, among which may be mentioned the exquisitely beautiful aniline dyes, and numerous delicate and much appreciated flavours.

It is difficult to avoid coming to the conclusion that those stored and hidden vegetable treasures were originally designed for the use of man even though he had not yet made his appearance on the earth. This view would be quite in accordance with other pre-determined arrangements seen in the case of parent and offspring, where provision is made for the development of the fotus in utero, and for its nutrition the moment it is born.

The primary conditions of the earth, atmosphere, and climate referred to affected not only the pristine flora but also the pristine fauna. Both plants and animals in the remote past were different from what they are to-day. The plant life was more luxuriant, and the animals were, as a rule, larger. As already stated, the majority of animals live upon plants. If, however, the plants of the early day were more abundant, more luxuriant, and more juicy and succulent, it follows that the animals, more bountifully and effectively fed and nourished, would increase in size, hence those great and magnificent ante-diluvian mammalian types which include the Megatherium giganteum, the Toxodon, Woolly Rhinoceros, Rhinoceros tichorhinus, Palreotherium, Mammoth, Elephas primigenius, Giant Irish Deer, Cervis giganteus, Dinotherium giganteum, Titanotherium robustum, Elasmotherium, Dinoceras mirabile, Arsinoitherium Zitelli, Helladotherium, Mylodon, Glyptodon, and Diprotodon. Likewise the huge old-world reptiles which are at once the wonder and admiration of the modern savant; such as the Iguanodon bernissartensis, Atlantosaurus, Brontosaurus, Ceteosaurus, Diplodocus, Triceratops, Stegosaurus, Pariasaurus, Dimetrodon, Plesiosaurs, and Ichthyosaurs.

Nor must the great flying reptiles, the Pterodactyles, be omitted in this connection. Reference must also be made to the great wingless bird of New Zealand, the Moa, allied to the ostriches of Africa, the emus and cassowaries of Australia, and the rheas of South America.

To give an idea of the enormous size of some of the extinct animals named, it is only necessary to state that the thigh-bone of the Atlantosaurus is six feet in length, that of a large elephant being only four feet. The Diplodocus carnegii measures eighty feet in length. The giant wingless bird, the Moa (Dinornis maximus), is from twelve to fifteen feet high.

While the majority of the extinct reptiles, birds, and mammals greatly exceeded that of modern times, it must not be inferred that there are no large animals in the present day. On the contrary (so far as is known), the modern whales are much larger than any animals known to palæontology.

The great size of the whales is, in all probability, due to the excess of rich, nutritious food in the ocean, the size and food of animals bearing a certain relation to each other.

One very remarkable fact to be noted in connection with the great extinct animals is, in many cases, the very small size of the brain in comparison with the size of the body. This would seem to indicate that the animals in question were content to vegetate and lead indolent lives. It is just possible that the want of brain power had something to do with their extinction. The peculiarity of the smaller modern animals is their comparative excess of brain. This is especially the case in man, who is admittedly the ruler of the world.

Everything in the universe hangs together. External conditions (within limits) influence and modify races of plants and animals, but the modifications do not destroy the identity of the types, and old-world plants and animals have their representatives in various parts of the world at the present day. Tree-ferns are found in New Zealand, and the Indian and African elephants are the near relatives of the Mastodon.

The changes in the external conditions of plants and animals in pre-historic and present times do not destroy the fundamental relations between plants and inorganic matter on the one hand, and between plants and animals on the other. The plant, whatever its size, shape, and nature, must find its pabulum and substance in the inorganic kingdom; and the animal, be it great or small, must find its nourishment and sustenance in the plant or in some other animal which has lived on the plant. The mere form and size of plants and animals do not disturb or destroy the fundamental relations referred to.

That the several races of plants and animals are, in some measure, dependent upon the quantity of heat, light, 


\section{DESIGN A PROMINENT FACTOR IN NATURE}

and moisture in the localities in which they are found is apparent from this, that in mountainous regions there are belts of vegetation in which particular trees and shrubs flourish; each zone of vegetation characteristically differing from the others. Thus the highest zones produce plants and animals whose homes are in northern climates -. for example, the arctic regions; the lower zones producing such only as are found in warmer climates, tropical, subtropical, or otherwise, in different parts of the earth. When, in mountainous districts, the snow line is reached, only mosses and the meanest and hardiest plants are met with. The giant trees and tall shrubs are found at lower levels, where the climate is milder. The geological record testifies to the same thing in animals. Remains of whales have been dug up in the valley of the Tay in the vicinity of Perth, Scotland; and Mastodons have been exhumed in the valley of the Thames, near London, England. These two facts conclusively go to show that the climate of Great Britain has been at one time arctic in character, and at another, tropical or semi-tropical.

Plants and animals are adapted to their habitats by the Great Designer, but they have also, up to a point, a power of adaptation in themselves. The rhinoceros was originally and is now a typical tropical or semi-tropical smooth-skinned pachyderm. When it migrated northwards, which it did at one period, to a colder climate, it grew hair, and hence the woolly species. The same is true of certain of the elephant tribe, which acted in a precisely similar fashion. The Mastodon had a hairy integument. The negro, who inhabits various parts of Central Africa and who is exposed to excessive heat, has developed a thick, oily skin crowded with dark pigment, woolly hair, and a comparatively thick skull. These changes in the skin and hair of the rhinoceros, the elephant, and the negro are largely due to the operation of external influences. They are also traceable to a power of accommodation inhering in the individuals themselves. Moreover, the changes are little more than skin deep. The negro remains a man, and the woolly rhinoceros and hairy elephants have not parted with their distinguishing characteristics. Environment invokes, rather than causes, slight modifications in plants and animals. If more be conceded, mere externalities are accredited with powers they do not really possess: the externalities usurp the prerogatives of life. In other words, modifications in plants and animals come from within more than from without. Mere environment can achieve nothing. The question in every case comes to be, Can plants and animals modify and accommodate themselves to their surroundings and live, or must they go to the wall and die because of their inability to modify sufficiently? One often hears environment spoken of as the chief factor in the modification of plants and animals, but it is safe to assert that environment never leads. All modifications, be they great or small, begin and terminate in the plants and animals themselves. In order to give undue prominence to the power of environment, those who theorise on this subject assume that plants and animals are provided with irritable constitutions, and external surfaces which are influenced by stimuli supplied by environment. They largely ignore the vital and sensitive powers of plants and animals, and regard plants and animals as mere machines or automata, which they certainly are not.

I have alluded to the power of growth and reproduction in plants and animals, and there is perhaps no subject in the whole range of physiology in which design and the persistent nature of types can be more readily and satisfactorily made out. Growth and reproduction are fundamental in their nature. Like produces like, both in plants and animals. However much germs, seeds, and eggs may resemble each other even under the highest powers of the microscope, they essentially and materially differ. An orchid cannot be produced from the seed of a wallflower, neither can an alligator be produced from the egg of a fish, or a giraffe from the ovum of a lion. The essential differences which meet us at the very beginnings of life must be admitted and faced, whatever the microscope says and whatever ultimate chemical analysis says. The microscope, chemical analysis, and human vision aided and unaided, are incompetent to distinguish and demonstrate the difference, but the adult plants and animals testify to an inherent dissimilarity which makes them what they are, and which has distinguished them from the moment the male and female elements came together and made reproduction possible. Every germ, seed, and egg (and the same is to be said of the male elements where they exist) have characteristics which cannot be ignored or set aside. The division of plants and animals into families, orders, genera, and species depends upon this difference.

Some investigators, bent on simplifying the mysteries and complexities of nature, have ventured to assert that all plants and animals proceed from protoplasm which is absolutely homogeneous and identical in all its parts and particles. This view is d priori unthinkable. The same protoplasm, placed in exactly the same conditions of heat, air, moisture, \&c., would produce only one kind of plant or one kind of animal. It would be impotent and wholly inadequate to produce the leading races of the vegetable and animal kingdom. To have a legitimate distinction, there must be an actual difference, and the obvious inference is, that the great races of plants and animals differ because their male and female elements (which are their most essential parts) differ.

If for the sake of argument it be admitted that the ovum or female element in plants and animals is homogeneous and identical, then it must be conceded that the male element, in whatever form it appears, is characteristically and fundamentally different. From male and female homogeneous absolutely identical elements, as already stated, only one kind of plant or animal could possibly proceed. The beginnings of plants and animals are in every 
instance conditioned. The perfected plant and animal exist potentially in the male and female elements, the conjunction of which produces the new being. There is no such thing as chance in the development of any part of a plant or animal. The end is seen from the beginning, and the atoms, molecules, and soft and hard parts of every organism arrange themselves according to law and order, and according to a fixed plan which is never departed from. Growth cannot, under natural conditions, act by chance or in a haphazard way. Plants and animals in growing follow the lead of their progenitors. Some grow in straight lines and radiate like certain crystals; others grow in curves; others in spirals. As a rule, plants and animals are symmetrical : symmetry of itself implies design. But (and this is the remarkable thing) the symmetrical plants and animals repeat and slavishly copy the general form and peculiarities of their parents.

\section{$\S 69$. Design as seen in the Gradation of Plants and Animals, and in the Arrangements for Walking, Swimming, and Flying.}

Another subject having an obvious bearing on the question of evolution considered from the developmental and morphological point of view is the appearance on the earth, according to the geological record, of the more simple before the more complex plants and animals. While fully recognising the gradual differentiation which characterises the development of man and the higher animals in utero, and the existence of the simple animals at a period anterior to the advent of complex ones, I cannot admit that the higher forms are the lineal descendants of the lower forms in the evolutionary sense. In other words, I am not disposed to regard man as the product of a molluse even if unlimited modification and unlimited time be granted. Such an assumption (and evolution must always remain an assumption) is, it appears to me, derogatory to the stupendous powers inhering in the Omnipresent Framer and Upholder of the universe. Evolution implies an ascent from a lower to a higher type, from an imperfect to a more perfect form, by a continuous process of development. In nature, however, there is not a perfectly uniform advance. On the contrary there is, at times, a deterioration; the plants and animals retrograding. This is the case in many parasites. But apart from advance and retrogression, it cannot for a moment be doubted that the Great First Cause would have had no more difficulty in creating a highly complex organism than a simple one. Evolution is not necessarily a part of the scheme of creation as we know it. All things are possible with the great "I am." Time, space, and matter are equally at His disposal. He commits no mistakes. The Ancient of Days has nothing to learn. His works were as perfect at the dawn of creation as they are now, and no good reason can be assigned why the higher animals, and even man, should not have appeared on the earth in as perfect a condition as we now behold them.

The existence of an Omnipresent Deity is, however, not excluded even by evolution. Nothing can be evolved which is not previously involved, so that evolution in its widest and most philosophic sense requires a First Cause and a pre-existing state of things. Further, the great Designer could with equal facility have furnished the world with its existing and pre-existing races of plants and animals by a series of separate creations according to types, or He could have impressed upon living matter (plant and animal) at the outset those tendencies which would inevitably result in a state of things identical with that which exists now and has existed from all time. In either case creation would be a progressive work. The world and all it contains is possible on either supposition.

The creation, at different periods, of advancing types, and a continuous evolution from lower to higher forms may both be explained by design and a pre-arranged plan. In either case a common idea runs through the whole.

The plan adopted in the construction of plants does not essentially differ from that adopted in the construction of animals, and both more or less closely resemble in their external configuration and general arrangements similar plans met with in the physical universe and in the mineral kingdom. The plans referred to provide the original stellar, dendritic, spiral, circular, and other forms with which botanists, zoologists, anatomists, and physiologists are familiar. Nor will this occasion surprise when it is remembered that plants and animals are the direct product of the mineral kingdom, plus life; the elements which form the bodies of plants and animals being in every instance supplied by the physical universe. Further, the same elements, to a large extent, enter into the bodies of plants and animals alike. If, however, plants and animals are the offspring, so to speak, of the physical universe, it is plain that the organic and inorganic kingdoms must have much in common, and be amenable to the same laws, and so it is in reality. All living things, plants and animals alike, are of the earth earthy, in the sense that the elements which form their bodies come from, and ultimately return to, the physical universe. In this is to be found an explanation of the fact, that crystals and dendrites resemble each other, that plants resemble crystals and dendrites, and that animals resemble plants, crystals, and dendrites respectively.

The symmetry and the repetition of parts which characterise crystals and dendrites reappear in both the vegetable and animal kingdoms. This was to be expected, as the articulata foreshadow the vertebrata, and even the highest 


\section{DESIGN A PROMINENT FACTOR IN NATURE}

animals, man included. All are the outcome of a common design. The universe is to be regarded as a harmonious whole. It is in no sense a patchwork. The inanimate physical part of it supplies the original bodies of plants and animals : it also provides a home and food for them. The inanimate and animate kingdoms cannot be separated from each other even for the shortest interval of time. The two are interdependent in the widest sense. Not only are plants and animals : ndebted to the physical universe for everything they contain at the outset, they are also indebted to it for subsistence throughout their entire lives. The heat, the moisture, the atmosphere, and the pabulum of plants are all physical. Plants form the food of animals, so that even the food of animals is physical at one remove. Heat, moisture, air, and food are necessary to both plants and animals, and both are constantly taking in nutritive materials and giving out waste products. Plants and animals are active and passive by turns : they require rest at stated intervals. Again, the physical universe comes to the rescue: the seasons, and day and night, are provided. Plants and animals, as a rule, rest more or less completely during the night: they also rest in a wider sense during winter. Rest in plants and animals does not mean a complete cessation of the activities of life, but only a damping or lowering down. A large number of plants partially close their leaves at night—in other words, sleep. The same is true of animals. Both plants and animals are more active in summer than in winter, and not a few hibernate.

The juices of plants and animals circulate with less vigour during the night than during the day, and during the winter than during the summer, but the juices are never suppressed or their movements wholly stopped. The juices and movements are both present, and they are slowed and quickened to meet the vicissitudes of the seasons and the alternations of day and night. All this means that the relations which exist between the inorganic dead kingdom and the organic living one are of the most fundamental and intimate description. Cell growth is only possible at certain temperatures, and is prevented by excess of cold or excess of heat. Proofs of design in the inorganic and organic kingdoms, and of the adaptation of the one to the other, everywhere abound. Not only do plants and animals directly proceed from the physical universe, but all their modes of life and habits conform to it. Nay more, their movements (when movements occur) are adapted to it. Here again design and law and order come to the front. The physical universe provides three great highways for animals, namely, the earth, the water, and the air, and all animals which elect to traverse these highways must have their travelling organs and surfaces modified and adapted to one or other. To this there is no exception. The land animals which walk and run must be provided with small feet; the swimming animals with tails, fins, and flippers; and the flying animals (insects, birds, and bats) with wings. The travelling organs of animals furnish a striking proof of design. They are specially modified to meet the requirements of the earth, water, and air respectively; the earth providing an unyielding fulcrum for the feet of animals, the water a yielding incompressible fulcrum for the tails, fins, and flippers of fishes and sea mammals, and the air a still more yielding and highly compressible fulcrum for the wings of insects, birds, and bats.

Wings, of all the travelling organs, are the most delicately and beautifully constructed. They are marvels of design, being at once strong and light, accurately poised, and mobile and elastic in an astonishing degree. They are of three kinds, as seen in the insect, bird, and bat (Plates li., liii., and liv.). In every case they are triangular in shape, slightly twisted upon themselves, and delicately and exquisitely graduated; the thicker parts occurring at the root and along the anterior margin, the thinner and most elastic parts at the tip and along the posterior margin. So inexorable is the law which regulates the make and shape of wings, that some of the winged seedsthose of the plane tree, for example-conform to the prevailing type. The winged fruit of the plane tree might, as far as the structure and shape of its wings are concerned, be mistaken for a large moth.

The wing of the insect is a very dream as regards structure, function, and colour. The dainty, delicate, evervarying tints of the wing of the butterfly; the transcendently beautiful tracery of the wing of the dragon-fly; and the extraordinarily complicated yet simple movements of all wings, baffle description. It is only the entomologist, physiologist, and physicist who can even approximately appreciate their many perfections.

It would be easy to multiply indefinitely examples of design in the organs of locomotion as a whole, but as this subject is treated at considerable length in another part of the work, it is not necessary to discuss it further at present.

The lesson to be learned from the foregoing (and it is an important one) is that the needs of plants and animals as regards food, and the modes and means by which they obtain it, exercise a marked influence on their general form and that of their organs, and must be taken into account in considering types and all kinds of classification.

The simplest plants and animals (cell plants and animals, protista, amœbæ, \&c.) have ew wants, and there is next to no need for differentiation; although even in these their bodies are not formed of absolutely homogeneous materials. A little plain food suffices. As soon, however, as the food becomes compound, varied, scarce, or difficult to procure, distinct organs become a necessity. An alimentary canal or its equivalent, which means a digesting 
apparatus in some form, is called for, and this implies a circulatory and respiratory apparatus to distribute and purify the nutritious juices. Nor does the matter rest here. The food may not be at hand, and may have to be searched for and even caught. Travelling and capturing organs in the shape of protrusions of sarcode, pseudopodia, cilia, flagella, teeth, limbs, fins, flippers, wings, \&c., become imperative. These travelling and capturing organs in turn necessitate (or may necessitate) the presence of bones, muscles, nerves, brain, and other structures capable of producing movements.

The question of food very largely dominates the shape and the degree of differentiation of plants and animals. From the first they are modified to cope with the food on which they are to subsist. Indeed it may safely be asserted that plants and animals are primarily modelled and planned to meet the food requirements. The call for food is incessant, and much time and energy are necessarily devoted to securing and dealing with it. The appetite for food, and the fundamental tendency to reproduction, account for the major portion of the activities of both plants and animals.

Living organisms as a whole, and all parts thereof, must, in the nature of things, possess the power to sustain and reproduce themselves; and in order to discharge these two primary functions they are, in all cases, specially designed and specially constructed. The desire for food takes precedence of that for reproduction, as plants and animals must be well fed and healthy before reproduction can take place. Not only the general shapes of plants and animals, but the shapes of their several organs, and the arrangement of the atoms and molecules forming the organs, are all modified to deal effectively with the subject of food. Plants and animals in the matter of food are aggressive. They lay themselves out for and stretch forth their substance to obtain it. They advance towards the food and are ready to seize it when it is in their vicinity. The food has to be drawn into their substance. The craving for food is peculiar to plants and animals and is in themselves. Food does not produce appetite or act as a stimulus, neither is it to be regarded as an irritant. Hunger in animals occurs equally in the absence and presence of food. The several kinds of food, moreover, have nothing to do with the production of the organs provided to seize and deal with it. These are supplied independently and in advance. Each plant and each animal has its special food and other endowments.

In the insectivorous plants, Venus's fly-trap, sundew, \&c., the leaves are flattened and furnished with highly sensitive hairs, which when touched inaugurate movements capable of catching flies, beetles, ants, \&c. In the collared monad (a low animal form) a pear-shaped body surmounted by a funnel-shaped cavity with a vibratile flagellum is supplied. This flagellum, wielded with extraordinary dexterity and skill, produces water currents which float the food into the interior of the animal. In the paramecium the body is covered with a multitude of swiftly-moving cilia which enable it to dart in any direction it pleases in pursuit of food. In the vorticella the cilia invest the margin of the cup-shaped disc, and by their movements direct the floating particles of food unerringly into its substance. In addition the vorticella is provided with a most exquisite spiral stem, by the aid of which it can instantly project itself against or recede from submerged particles, edible or otherwise. Other animals adopt other modes and assume various shapes in securing food. The Articulata become segmented and develop legs, by means of which they move towards their food. These movements may be slow, as in the caterpillar, or comparatively quick, as in the spider and ant. As we rise in the scale of being, distance and time become elements in the food supply. The larger, complex animals require more food, and in order to obtain it must travel further afield and at a greater pace. Travelling extremities, fins, flippers, and wings must be added to the food-securing apparatus. We have, as a consequence, not only creeping things, of which the caterpillar is a good example, but creatures which can walk, run, and leap on the land; fishes and water-going mammals which can swim in the water; and insects, birds, and bats which can fly in the air. A series of the most extraordinary modifications is introduced by the food requirements. The food question practically determines the anatomy and physiology of plants and animals.

It is scarcely possible to attach too much importance to the food-securing and procreative powers. The food supply, physically speaking, is at the root of the whole matter. Plants and animals, as explained, derive their substance, directly or indirectly, from the inorganic kingdom : plants also derive their food from the same source and at first hand; animals deriving their food at second hand from the plant, or at third hand from other animals. The special forms, arrangements, and laws peculiar to the inorganic kingdom become, by incorporation, the forms, arrangements, and laws of the organic kingdom. These laws are practically inexorable, and so it happens that the elements, and the atoms composing them, both without and within living bodies, have a tendency to act in given directions and to assume characteristic typical shapes. In this we have an explanation of the radiating, concentric, curvilinear, spiral, dendritic, branching, and other arrangements met with in crystals, plants, and animals respectively.

The reproductive function, as stated, is second in importance to the food-securing and digesting, assimilating function: but here again all the peculiarities referred to reappear. Plants and animals resemble each other, and both resemble certain crystals; but plants and animals in the reproductive process simply ring the changes, as each 


\section{DESIGN IN THE REPRODUCTION AND GROWTH OF PLANTS}

produces only its own kind. Plants and animals in reproducing themselves cannot dissociate themselves from the matter of the universe and the forces and laws which govern and compel it, nolens volens, to assume certain distinct forms. A more comprehensive scheme and division of plants and animals than has yet been attempted becomes possible. Hitherto, classifications have hinged largely on slender and, in many cases, trifling points of agreement. This is well seen in the case of animals, where the data for classification consist of the possession or non-possession of a stomach or its equivalent; the number and nature of the teeth; the presence or absence of segmentation, a back-bone and extremities; the possession or non-possession of gills and lungs; the presence or absence of a circulatory apparatus, and a heart with one, two, three, or four cavities; the presence or absence of glands, lymphatics, a muscular, osseous, nervous system and sense organs; the mode of development, placentation, \&c. Now all these peculiarities of structure are directly or indirectly traceable to the food problem.

In the higher animals, a segmented body with muscles, bones, nerves, sense organs, and appendages in the shape of limbs, fins, flippers, and wings is necessary to secure the food; an alimentary canal with teeth, stomach, glands, and a lymphatic system to digest and assimilate it; a circulatory apparatus to distribute it; and a breathing apparatus to aërate the blood it produces. When the animal is built up and in a healthy condition, then, and only then, does the subject of reproduction, placentation, and other peculiarities of development come into play. The food appetite at once precedes and produces the reproductive appetite. All else follows. The amenities and pleasures of life largely depend on the abundance and quality of the food, and on the possession of healthy bodies.

\section{$\S$ 70. Design as Manifested in the Reproduction and Growth of Plants.}

The seed of the plant in growing displays design in a marked degree. When planted under favourable conditions, in a suitable soil, the one part of it (the plumule) ascends into the air and seeks the light and forms the leaves and stem; the other part (the radicle) descends into the earth and seeks the darkness and forms the root. The plumule and radicle never change places or commit mistakes as to their respective positions and functions. ${ }^{1}$ Drop seeds as one may, when germination occurs the leaves and stem always ascend while the roots and rootlets descend. In this a directive agency is displayed either by the seed itself or by a power outside the seed. Clearly the seed can only grow in one particular way, and it displays, in growing, prescience of a kind.

The plant grows upwards by its stem, downwards by its roots, and outwards by its branches and leaves. It spreads itself in space to carry on its vito-chemical processes. If the vertical stem be placed in a horizontal position it will, after a time, curve upwards in a perpendicular direction; if the roots from any chance become exposed, they will re-enter the ground at the first opportunity.

The plant is imbued with what is virtually a sense of direction. It is a living, sentient, responsive thing. Its stem, branches, and leaves invariably grow upwards into the air, where there are light, heat, and moisture ; and the roots as invariably grow downwards into the soil, where there are darkness, gases, moisture, and a certain degree of heat. The plant has either in itself a guiding power which it exercises spontaneously, or it is controlled by an intellectual Power outside itself. The plant is obviously a conditioned thing, free to act only in certain directions and within certain limits. All its parts are controlled to given ends. Its structure is never haphazard. It assumes an infinite variety of the most beautiful forms, and these are, for the most part, symmetrical. In many cases these forms are so exact that they resemble crystals; the parts repeating themselves with admirable precision. In other cases the parts are arranged in the most exquisite spirals, single and simple, double and compound. There is no end to the diversity, but design can, in every instance, be traced. In some instances the stems are straight, in others spiral and twisted, and so of the branches, leaves, flowers, and fruit. On making transverse sections of the parts enumerated, especially the stems and branches, a radiating, concentric arrangement is witnessed similar to what occurs in many crystals. These indicate rays and rings of growth. There is an obvious analogy in the fundamental construction of plants and crystals, and also of animals (Plates xxv., xxvi., and xxviii.). They all grow outwards from centres. These fundamental similarities bespeak design, law, and order.

Plants are constructed to grow in the air, or in the water, or partly the one and partly the other. Some of them are inclined to burrow more in the ground than others. Whatever their mode of life they are all specially adapted. There is no example of a plant performing a function not intended, and for which there is not structural warrant. The climbing plants and the sensitive and insectivorous plants are specially designed and endowed, and discharge their particular functions as apart from so-called irritability, external stimulation, and environment. As living things they are superior to their surroundings; they are, in every instance, masters of the situation.

The plant may vary its structure slightly for a short period and revert to its original; the individual never

1 Roots when exposed to the air and light occasionally develop leaves, and growing branches imbedded in the soil, at times, produce roots. These, however, are abnormal conditions, and do not materially affect the general statement, which holds good when the circumstances are natural. 
really altering its constitution or finally departing from its primal type. In everything the plant does, traces of intelligence and a First Cause may be detected. It is free in one sense, it is not free in another; the controlling, governing power is always behind it. It has been said that plants wander indefinitely from their originals if only sufficient time (thousands or millions of years) be allowed. There is no proof whatever of this statement, and the extraordinary persistence of certain species of plants gives a distinct denial to it. It is also said that plants anticipate their own wants and make their own arrangements as apart from a First Cause. This is assigning to the plant a power not possessed by any living thing. Not even in the highest animals (and we have proof of this in our own persons) can structural wants be satisfied and structural changes induced at will. A man may not add a cubit to his stature. Still less can he cause a hair to grow, or to cease growing, in a particular spot. He has no control over his so-called vegetative functions any more than the plant. All the organs in plants and animals are original endowments : they are created, transmitted products, and neither plant nor animal can, of its own accord, alter its constitution or form to any appreciable extent. As a matter of fact, plants and animals are under supervision, and all their wants, present and prospective, provided for.

Plants develop and grow in certain directions, not as automata, but as individuals and living entities, under guidance and under law and order. The plant is never wholly at the mercy of environment or external stimulation. It is actuated by inherent vital force, and capable (under guidance) of inaugurating independent action. It does not normally act under the influence of irritability as an efficient cause. The movements of climbing plants show this conclusively. It has been generally assumed that climbing plants, whether they climb by rootlets, suckers, hooks, bends, or spirals, must be touched by something before the climbing apparatus can be developed and set in motion. On the contrary the climbing apparatus is developed first. That the tendency and power to climb inheres in the plant, and not in anything outside of it, is shown in the circummutatory movements of certain plants where the stem is spirally twisted and the free or apicial end of the stem makes wide movements of rotation in the air in search of something round which to twine. The twining apparatus is complete before the support is encountered. The latter, therefore, cannot be the cause of the twining and rotatory movements. These are, of necessity, inherent and spontaneous.

In the case of the passion flower (Passiflora alata), the vegetable marrow (Cucurbita Pepo), the melon (Cucumis $M e l o)$, \&c., the tendrils make elaborate preparations for seizing objects, but these preparations are carried through with or without supports. Thus in the passion flower, the tendrils first appear as long, limp, sensitive shoots, quite straight or with a terminal curve. As the tendril grows older its free end becomes spiral either in the air or in connection with something it touches. It not unfrequently curves and twists spirally first in one direction and then in another and opposite direction. This it does in the air as apart from contact and stimulation, as well as when it comes in contact with a support. The spiral and the reversing spiral movements are inherent, and in no sense due to irritability and supposed external stimulation afforded by supports.

Similar remarks are to be made of the tendrils of the vegetable marrow (Cucurbita Pepo). In this case the spiral arrangements are more elaborate. The spirals of the tendrils first appear as small, close, flat spires. These gradually unwind and straighten till they resemble those of the passion flower, which, as explained, first appear as long, tapering, limp, sensitive processes, either straight or slightly curled at the free extremity. In this condition they feel about in the air for something to lay hold of. If they succeed they twine round it and form single or double spirals. These single and double spirals are, however, formed in the air when nothing is touched, so that they are to be regarded as independent formations whose primary object is support. The marvellous thing is that the elaborate spiral mechanism is developed in advance and in anticipation of finding a support. The plant is guided to produce a structure and perform a function calculated to contribute to its well-being. The plant could have no knowledge of the necessity for a support, and the structure and function are equally examples of design and means to ends.

The sensitive and insectivorous plants also display design of a high order. These are dealt with in other parts of the work.

One of the most remarkable features in plants is their mode of fertilisation. In a very large number of cases they are hermaphrodite; the male and female elements being found on the same plant. Under these circumstances contiguity of position and the interposition of air currents, as a rule, secure the necessary contact, blending, and mutual interpenetration of the sexual elements. In other cases, the male and female elements occur on different plants separated occasionally by considerable distances. Under these circumstances a medium, or go-between, becomes indispensable. The air in motion may perform the office of medium; more frequently this important and delicate task is entrusted to the movements of insects and animals of various kinds. In order to attract the insects and animals in question, plants display the most extraordinary lures in the way of colour, form, perfume, nectar, \&c. These lures are the immediate outcome of design, and cannot be referred to natural selection or modifications 


\section{DESIGN IN THE REPRODUCTION AND GROWTH OF PLANTS}

occasioned by prospective requirements. The plant could not foresee the necessity for producing brilliant colours and storing sweet-smelling nectar to attract insects to assist in its fertilisation. The chain of events is too complicated and too far-reaching. The only explanation that can be given is that the arrangements are the work of an intelligent First Cause, Who sees the end from the beginning. It is a mere misuse of language to say that the insect is endowed with instinct, and that instinct and natural selection can account for everything. As a matter of fact they account for nothing. Instinct and natural selection are non-existent in the sense here indicated. They are mere phrases, and a cloak for ignorance.

Granted that instinct and natural selection exist, who, it may be asked, conferred the instinct, and the power to select? Instinct is at once a most unscientific and objectionable term, and should never be employed; but if employed it should be distinctly understood that it is, in every instance, directly or indirectly, the outcome of intelligence; intelligence either in the thing exhibiting it, or in its ancestors, or in the Creator Who made them. Instinct, used in a loose or inexact sense, confuses all the issues of life both in the plant and the animal.

Perhaps no better examples of the Creator's continued presence in His work can be supplied than are afforded by the fertilisation of plants, and the organs and accessories used in this important function. These organs and accessories include the preparation of the male and female elements on the same or on different plants ; the direction and control of air currents ; the employment and guidance of insects and other animals as media for carrying and mixing the generative ingredients, \&c. There is a long sequence and chain of events, and the chain includes inorganic and organic processes, some of them of a most complicated character. Nothing short of the very highest wisdom and power such as that possessed by the Creator can supply an adequate explanation. The harmonious working of so many diverse substances, organic and inorganic, necessitates a knowledge not possessed by any plant or any animal. There is no warrant for asserting that cross-fertilisation, in a state of nature, is due either to instinct or natural selection, or both combined.

How, it may be asked, will instinct or natural selection explain the floating apparatus of the utricularia, the movements and habits of Silene nutans (Nottingham catchfly), or of Marcgravia nepenthoides (a tropical orchid) in their relation to cross-fertilisation?

In the Utricularia an intimate knowledge of hydrostatic and mechanical science is displayed not only in theory but in practice, as the following account by Mr. Brown conclusively proves: " "Small ascidia or sacs are connected with the leaves which, about the time of flowering, are filled with air, and buoy the plant to the surface. The opening of each sac is surrounded by forked hairs composed of four cells, and is closed by a transverse cellular membrane, like the valve of a pump, capable of opening from without inwards, and which resists when it is pressed from within outwards. The physiological action of these ascidia is full of interest. At first they are filled with a somewhat gelatinous liquid, which by its weight assists in retaining the plant at the bottom of the water. Very soon the branching hairs already described, which project into the interior, secrete a gas, which accumulates as the gelatinous substance diminishes. By-and-by, when the vessels are full, the plant gets light and buoyant, and, disengaging its roots from the soil, rises to the surface of the water and flowers. The flowering over, and the fruit mature, the air disappears from the ascidia, the valve allows the water to enter, and again the plant sinks to the bottom, to remain there until spring stimulates its ascidia again into activity."

The adaptation of means to ends displayed by the Utricularia is profoundly impressive. The machinery employed is at once elaborate and complicated, and the plant, or that which directs the operations of the plant, exhibits an acquaintance with natural laws which is truly astounding. It roots and uproots itself at precisely the right moments ; it fills its ascidia with a gelatinous fluid when the plant is to be weighted and sunk, and with gas when it is to be floated and raised to the surface. When the flowering and fruiting which take place at the surface of the water are over, the valves of the ascidia are opened and water admitted, which sinks the plant and prepares it for its winter sojourn at the bottom.

To take another example, observed by Sir John Lubbock in Silene nutans in connection with the visits of moths and fertilisation: "The upper part of its flowering stem is viscid, from which it has derived its local name, the Nottingham catchfly. This prevents the access of ants and other small creeping insects. Each flower lasts three days, or rather three nights. The stamens are ten in number, arranged in two sets, the one set standing in front of the sepals, the other in front of the petals. Like other night flowers, it is white, and open towards evening, when it also becomes extremely fragrant. The first evening, towards dusk, the stamens in front of the sepals grow very rapidly for about two hours, so that they emerge from the flower; the pollen ripens and is exposed by the bursting of the anthers; so the flower remains through the night, very attractive to, and much visited by, moths. Towards three in the morning the scent ceases, the anthers begin to shrivel up and drop off, the filaments turn themselves outwards so as to be out of the way, while the petals, on the contrary, begin to roll themselves up, so that by day- 
light they close the aperture of the flower, and present only their brownish-green outsides to view, which, moreover, are thrown into numerous wrinkles. Thus, by the morning's light the flower has all the appearance of being faded. It has no smell, and the honey is covered over by the petals. So it remains all day. Towards evening, however, everything is changed. The petals unfold themselves, by eight o'clock the flower is as fragrant as before, the second set of stamens have rapidly grown, their anthers are open, and the pollen again exposed. By morning the plant is again asleep, the anthers are shrivelled, the scent has ceased, and the petals roll up as before. The third evening again the same process, but this time it is the pistil which grows, and the long spiral stigma on the third evening takes the position which on the previous two has been occupied by the anthers, and can hardly fail to be dusted by the moths with pollen brought from another flower."

The Nottingham catchfly is remarkable for its nocturnal habits and its delicate adjustments to ensure fertilisation. It produces honey and scent and a fair exterior as lures to certain moths; the upper part of its flowering stem is viscid to prevent the approach of ants and creeping insects; the different parts of the flower mature at various and suitable periods; it is a centre of attraction during the night, but closes up and shrivels during the day; it wakes during the night and sleeps during the day, and comports itself generally as an intelligent agent. It is not possible to conceive of the Nottingham catchfly as the product of natural selection and variation, however gradual and however long continued. The plant in every direction is a crowning example of design. The plant not only develops its several parts to meet certain requirements at certain times, but it adapts itself to the visits of certain moths at night, and excludes other insects whose visits are not desirable.

Dr. Crïger furnishes a highly illustrative example of the mode of fertilisation of a tropical orchid-one of the Coryanthes. "He found that the labellum or under lip of this orchid is hollowed into a great bucket, in which drops of almost pure water continually fall from two secreting horns which stand above it, and when the bucket is half full the water overflows by a spout on one side. The bare part of the labellum stands in the bucket, and is itself hollowed out into a sort of chamber with two lateral entrances; within this chamber are curious fleshy ridges. The most ingenious man, if he had not witnessed what takes place, could never have imagined what purpose all these parts serve. But Dr. Crüger saw crowds of large humble-bees visiting the gigantic flowers of this orchid, not in order to suck nectar, but to gnaw off the ridges within the chamber above the bucket. In doing this they frequently pushed each other into the bucket, and, their wings being thus wetted, they could not fly away, but were compelled to crawl through the passage formed by the spout or overflow. Dr. Crüger saw a continual procession of bees thus crawling out of their involuntary bath. The passage is narrow, and is roofed in by the column; so that a bee, in forcing its way out, first rubs its back against the viscid stigma, then against the viscid glands of the pollen-masses. The pollen-masses are thus glued to the back of the bees which first happen to crawl out through the passage of a lately expanded flower, and are thus carried away. When the bee, thus provided, flies to another flower, or to the same flower a second time, and is pushed by his comrades into the bucket and then crawls out by the passage, the pollen-masses necessarily come first in contact with the viscid stigma and adhere to it, and so the flower is fertilised."

Mr. Belt in his "Naturalist of Nicaragua" shows how the bird may be made to do duty in fertilisation. "A climbing plant (Marcgravia nepenthoides) expands its flowers in a circle, and these hang down like inverted candelabra. From the centre of the floral circle and underneath the flowers there is suspended a number of pitchers, which are full of nectar when the flowers are ripe. The honey attracts insects and the latter attract birds, especially hummingbirds. Before the latter can get at the honey-bearing pitchers, their backs must brush the open flowers out of which the pollen is ready to be shed, and in this manner they convey it from plant to plant and cross the flowers."

In the examples cited of cross-fertilisation, such an amount of ingenuity and adaptation of means to ends is displayed as to leave no reasonable doubt in the mind of any reflective person that intelligence is to be traced either to the plant or to the Creator of the plant. The situation is enhanced when the visits of the moths and birds are taken into account. These visits show clearly enough that the actions of both plants and animals are controlled, and that the Creator maintains His hold upon plants and animals in their various and mutual relations.

Mr. Baildon states the case very fairly in the following words: "The more one reflects upon the phenomena of life, especially of vegetable life, the more is one convinced that they can only be caused and directed either by a consciousness existing in the organism itself and controlling its conduct, or some pervasive consciousness without the organism which ordains for it its actions, either of which hypotheses seems to imply some pre-existing intelligence ; for Nature must be a power even more miraculous than we esteem her, if she be either herself wise without thought and prudent without knowledge, or capable of endowing her productions with a consciousness, wisdom, and foresight of which she herself is innocent." 1

Various other examples of design in plants might be given, but sufficient have been quoted for my present purpose. 1 "The Spirit of Nature," by Henry Bellyse Baildon, B.A. (Cantab.). London, 1880, p1. 161, 162. 


\section{DESIGN IN THE REPRODUCTION AND GROWTH OF ANIMALS}

\section{$\S 7$ I. Design as Manifested in the Reproduction and Growth of Animals.}

Strong arguments for design are furnished by a consideration of the important subjects of reproduction and growth in animals. These require separate and detailed treatment. The peculiarity of reproduction consists in its spontaneous and independent nature; the changes which occur during the process being self-inaugurated and resulting in prospective structures and functions necessary to the adult individual. The self-induced changes and transformations are means to ends, but the causes and the effects are beyond the ken of the parents, and must be referred to the intelligent action of a First Cause. The most simple and complicated changes are equally under guidance, and chains of events, sometimes highly intricate and involved, occur in unvarying sequence, and in such a manner as can only be explained by design. This is very evident in the reproduction of the more highly differentiated animals. In mammals, for example (and what is true of them is relatively true of other living things), the heart which is to propel the blood is formed before blood is admitted into it; the stomach and alimentary canal are formed before food is eaten; the lungs and chest are formed before air is breathed; and the bones and muscles are formed before walking is attempted.

Structure precedes function; but the structures and functions are not determined by the substances with which they have to deal, or on which they are to act: the blood is not the prime mover of the heart; the food does not set up the vermicular movements of the intestine; the atmosphere does not occasion the rhythmic movements of the chest ; the ground does not produce the movements of walking, the water of swimming, or the air of flying.

In living things (plants and animals alike), the structures and organs, be they simple or complex, are formed for a purpose, and they are formed independently, and in anticipation, so to speak, of their life work. The roots and leaves of the plant are pushed out, aggressively as it were, to obtain nutritious saps ; and the feeding appliances of animals are advanced voluntarily to seize, incorporate, and assimilate food.

Plants and animals, and everything outside of them, are related as subject and object; the subject being produced from, but not by, the object. The subject, as a rule, is superior to and dominates the object. The subject as here employed is aggressive and active ; the object being quiescent and passive. The subject must have something outside of itself to work upon; that something being the object. The subject would have no raison d'être as apart from its natural or appropriate object. The subject and object are complemental; the one being made for and adapted to the other. The subject consists of living matter, as seen more especially in animals, where there is usually a slight admixture of sensation, perception, or consciousness. The object, on the other hand, is composed of dead matter such as we behold in the physical universe, where no traces of sensation, perception, or any attribute of mind can be detected.

The subject acts, the object is acted upon. There is a tendency in the present day to reverse this natural order of things, and to refer all the activities of plants and animals to mechanical arrangements as apart from life, and as apart from a First Cause, and design. The mechanical school regards living things as mere automata, and attributes all structure and all function to the play of natural forces and blind chance operating on brut matter. They claim law and order for the universe, while they practically exclude and deny the First Cause ; that is, the directive agency on which everything depends.

Nothing, perhaps, in the wide range of the organic kingdom can convey a more elevated conception of the powers and possibilities of life than the several processes concerned in reproduction. Here there can be no question of innate ability on the part of every living thing to work out its own destiny as apart from every form of environment, irritation, and outside stimulation. Reproduction is essentially the act of life. It is not dependent on any form of external aid or excitation. Given healthy plants and animals and the necessary conditions of normal life, reproduction follows in the natural order of things and of necessity. Whatever may be said of other functions, the function of reproduction is assuredly fundamental and traceable to powers inseparable from the living organism, whether that be simple or complex. It could scarcely be otherwise. If reproduction, which is the most important function of life, were in any way dependent on fortuitous circumstances, there would, in many cases, be a considerable danger of the extinction of dominant races.

In certain plants the organs of generation occur in different individuals, and in these instances winds, insects, \&c., take an indirect part in the reproductive process. It must, however, be noted that, even in these cases, the extraneous aids do not act as stimuli, but only as media of communication. Moreover, the winds, insects, and other aids to reproduction are all under control, and themselves afford evidences of design. The stimulation in every instance comes from the union of the male and female elements. The union in the majority of cases is followed by fertilisation, which is a vital act. It is important to be clear on this fundamental point. Plants and animals have in themselves the wherewithal to initiate and accomplish the reproductive process quite apart from all kinds of outside interference. The reproductive acts begin and terminate in living organisms, and these acts are spontaneous 
and independent in their nature. If, however, plants and animals can unaided reproduce themselves in virtue of inherent vital powers, and discharge the highest functions incidental to life, it follows that they may be credited with the power to discharge the minor functions, among which may be included the process of feeding, growing, secreting, excreting, moving, resting, \&c. In other words, if plants and animals can in the absence of foreign matter, irritation, and external stimulation perform the major and higher functions of life they may be trusted to perform the minor and lower functions.

Reproduction is, in all cases, essentially an atomic, molecular, vital process. It is in the atoms and molecules, or the cells formed by them, that the future being, whether plant or animal, finds its origin and source.

In the higher animals, where there are male and female elements and separate sexual organs, the elements are usually contained in cells. Thus in mammals the ovum and spermatozoon are both cellular in character. In the several orders of plants, reproduction is, for the most part, effected by spores, germs, and seeds which are atomic, molecular, or cellular in character, according to circumstances. It is, however, often effected artificially by detaching and planting or grafting parts of the originals (stems, branches, leaves, \&c.) in suitable localities. Plants may also reproduce themselves by means of roots, runners, bulbs, \&c.

It is in reproduction that heredity makes its most indelible impression. Impregnation and fecundation furnish examples of the subtlest and most permanent combinations of living matter. As we cannot see by the highest powers of the microscope the atoms, and the molecules formed by them, or analyse by the aid of any known process in chemistry those practically invisible and intangible entities, we are forced to accept results, which while they cannot be actually demonstrated, are, nevertheless, clear as inferences, deductions, and matters of reason. If from the coalescence of known molecular male and female elements plants and animals always and invariably identical are produced, it follows that in the male and female elements themselves reside or inhere the potentialities necessary to the production of the plants and animals in question. In other words, if the male and female molecular elements positively refuse to form plants and animals essentially differing from those of which they themselves are component and important parts, then it goes without saying that plants and animals, in every instance, only reproduce themselves.

A fig tree cannot be obtained from a thorn; a mammal from a reptile; or a bird from a fish.

From two wholly dissimilar male and female molecular elements such as those obtained from the several orders of plants and animals no living thing can be produced. Indeed the limits within which fructification and reproduction are possible are exceedingly narrow. A mule and a hinny can be produced by cross breeding as between the horse and the ass, but the mule and hinny are invariably barren.

The reproductive act has features in common in all classes of plants and animals. It is, however, simplest and most easily studied in the lowest forms. Here the new individual is sometimes produced by division and sometimes by budding. What is virtually a protoplasmic mass simply splits into two or throws out a process or bud, which in due time becomes detached from the parent, and discharges on its own account all the functions originally discharged by the parent.

In these cases only one individual and one sexual substance is required for the reproductive process. In other cases one male and one female element are contained in one and the same individual. This is the rule in hermaphrodite plants and animals. In the higher and highest plants and animals, the male and female elements are found in separate individuals. In bisexual plants and animals the male and female elements must be brought into contact either directly or indirectly, and must commingle and interpenetrate before fertilisation and reproduction can take place. Reproduction is undoubtedly an evidence of design and of life. Only living things can reproduce themselves. A crystal forms or may be formed under certain conditions, but it cannot reprodure itself after the manner of a plant or animal.

It may be convenient at this stage to refer briefly to the several kinds of reproduction. The protamoba of Professor Haeckel, one of the simplest of living forms, consists, according to him, of a minute albuminous mass. He does not explain whence came the mass or why it divides. On attaining maturity it splits into two, and in due time each half becomes as perfect as the original whole. This is the simplest possible form of reproduction. It is bisection or multiplication by division. This fundamental form of reproduction in certain cases repeats itself; the two original halves being divided into four, the four into eight, and so on; the parent, as it were, resolving itself into a swarm. This primary form of division is also seen in the cleavage of the impregnated human ovum. Multiplication by division is a vital act, and provision is made for it in the original individual. It is in no sense the result of irritation or oxternal stimulation.

Reproduction by budding is slightly more complex. This is seen to advantage in cell multiplication in plants and animals. In the budding process the parent sets apart and prepares a certain portion of its substance for a separate existence. In due course the separation takes place, and in such a manner that the parent is in no wise 


\section{DESIGN IN THE REPRODUCTION AND GROWTH OF ANIMALS}

injured, although it has contributed a quota of its own living matter to the offspring; that offspring having in turn the power of reproducing itself by germination. Reproduction by budding is well seen in colonies of corals. Like reproduction by division, it is a vital process.

In addition to the foregoing there is reproduction by alternate generations, that is, where two generations are required to form an individual. The medusa known as Cladonema radiatum affords an example. This animal is the offspring of the Stauridium or cross-polype of Dujardin. The Stauridium grows upon a stalk like a polype, and produces rounded gemmules, which gradually assume the appearance and structure of medusæ. In due course they become detached and float away as medusæ. The medusæ reproduce themselves sexually, but from the eggs of the medusæ only stauridia can be developed. There is thus an intermediate stage in the reproduction of medusæ. Here again reproduction is unquestionably a vital act. It begins and terminates in living matter which is not goaded into activity by externalities.

A somewhat similar arrangement obtains in the larvæ of certain dipterous insects (Cecidomyia, Miastor), where maggots produce maggots, and where the first race of maggots is devoured by the second race, which is the only one to survive, and from which the insects referred to are ultimately produced.

In the case of butterflies there is first the egg, then the grub or caterpillar, then the chrysalis, and finally the completed insect. Von Siebold has shown that hibernating fertilised female wasps lay eggs in the spring from which females mainly, with occasional males, are produced. The spring colony of female wasps in turn lays eggs from which males are developed.

In the case of the tapeworm (Tania solium) there are not only alternate generations but two distinct sexual organs and elements in each segment of the mature individual. If the fecundated eggs of the tapeworm be eaten by an animal, say the pig, young tapeworms are developed in the flesh of the pig in the form of cysts, and constitute the so-called measly pork. This is the first stage in the development of the tapeworm. In the first stage the tapeworm consists of a bladder-like cyst (scolex) with the head of the future tapeworm plus four curved hooklets inverted or concealed in the body of the cyst. If the measly pork be eaten by man, or other animal, the cysts contained in it in due time develop into tapeworms in the alimentary canal. This they can readily do, and in the following way. The cysts are introduced into the alimentary canal with the food. Once there the cysts or future tapeworms evert and free their heads armed with the four curved hooklets referred to. By means of the hooklets they fix and anchor themselves in the mucous lining of the alimentary canal, from the contents of which they derive nourishment by absorption. They grow apace in flat, elongated, quadrilateral segments (proglottides), very small and slender in the vicinity of the head, but gradually increasing in size as this is receded from. The segments ultimately measure an inch or more in length and fully a quarter of an inch in breadth; each mature segment containing male and female organs (plus male and female elements), and constituting a separate individual. The adult segments are crowded with impregnated eggs, and when voided by the anus are in a condition to be carried into any animal which is unfortunate enough to give them a lodging and act as a host. This is the second and final stage of development. Two separate animals or hosts are required for the reproduction and continuance of the tapeworm; but it will be observed that in both the first and second stages of reproduction the reproductive acts are spontaneous, vital, and in no way due to irritation or extraneous stimulation. The impregnated eggs become encysted worms in the first host and tapeworms in the second host. The tapeworms take advantage of the two sets of conditions supplied by the two hosts, and profit by both.

In plants and animals where the male and female organs of generation and elements are situated in different individuals, something of the nature of coitus or mutual contact is indispensable. The contact in the case of plants is brought about by winds, insects, and other means. Reproduction by coitus, or its equivalent, can be readily studied in the fish. If spawning salmon be watched it will be seen that after much serious fighting on the part of the males for possession of the females, a male and female salmon pair. They proceed to a convenient spot in the bed of the river, preferably where sand and gravel occur, and scoop out an elongated trough in which the female fish places herself and deposits her spawn. The male fish in turn occupies the trough and deposits his milt over the spawn. In some cases the female turns on her side and the male rushes at her and discharges his milt in passing. The male and female elements commingle, the spawn is fertilised, and the reproductive act completed. It only remains for the parent fish to cover up the fertilised spawn with sand and gravel to protect it from other fish, birds, \&c. In a short time the young salmon are hatched out, and what is most curious, each young fish is provided with a bag of pabulum, from which it obtains nourishment until it can develop a mouth, an alimentary canal, and a vent, when it is in a condition to obtain food from without. The history of reproduction in the fish shows clearly that the entire reproductive act is vital in its nature, and not only vital but voluntary. In some cases, as in the viviparous fishes (shark, skate, blenny, \&c.), the young are produced alive. Here certainly there is no room for irritation or extraneous stimulation. 
In the amphibia, of which the frog (Rana temporaria) is a good example, the conjugation or confluence of the sexes is more intimate. In this case the male frog takes his place on the back of the female, and while she is depositing her spawn he sheds his milt over it. The spawn thus fertilised, aided by heat and moisture, soon develops into tadpoles, which swim about like fishes and are water-breathers. The tadpoles in due course shed their tails and gills or branchiæ, and develop four legs and lungs and become air-breathers. The reproduction of the frog is vital in all its stages. It is in no way dependent for its initiation and accomplishment on external conditions or environment, although these latter aid in the process. The developing frog gradually but surely produces organs which enable it to leave the water and assume a terrestrial existence : the water does not form the branchiæ and swimming tail, neither does the earth form the four legs. All those wonderful transformations, which adapt the animal first for an existence in the water and subsequently for an existence on the land, are spontaneous, vital, and independent. The frog in its various stages of development is superior to its surroundings and environment. All the really important transformations are from within and vital, and are in no sense due to irritability of constitution or external stimulation.

In the reptiles coitus takes place, the females producing eggs which they either hatch out themselves or bury in the sand to be hatched out by the heat of the sun.

Similar remarks are to be made of birds. In this case the eggs are hatched out by the mother or artificially by incubators; the hatching being a mechanical process. The impregnation of the eggs, which is a vital act, takes place at an early stage of their development. The impregnation is in no sense due to irritability and extraneous stimulation. It suffices if the living male and female elements are simply mixed as apart from coitus. The formation and transmission of the eggs down the oviduct are representative vital acts, and furnish the most remarkable illustrations of the powers of life and vital movements known. There is no irritability and no extraneous stimulation anywhere. The egg is formed by vital processes, and the oviduct transmits it from the ovary to the cloaca by vital acts. The oviduct also supplies the egg, during transmission, with its various membranes and coverings, but (and this is the remarkable feature) the developing egg does not, as is generally believed, act as an irritant, and produce its transmission along the oviduct or its expulsion from the cloaca.

The oviduct is expressly formed to receive, contain, and transmit the egg by a series of peristaltic movements in all respects resembling those of the œosophagus, stomach, intestine, bladder, uterus, heart, and chest. The peristaltic movements are inherent and fundamental, and are not dependent on irritability or stimulation of any kind. The bolus does not cause the peristaltic movements of the cesophagus, the food those of the stomach and intestines, the urine those of the bladder, the fotus those of the uterus, the blood those of the heart, the air those of the chest, or the egg those of the oviduct.

If they did cause the movements in question, the structures referred to could not possibly act as receiving and containing structures. The fact that they are receiving and containing, as well as transmitting and expelling structures, shows plainly enough that the peristaltic or rhythmic movements must begin and terminate in the structures themselves. Structures endowed with peristaltic movements have, as a rule, a fundamental and very important vital function to perform. They are necessary to the continuation of life, and occupy a unique position, in so far that they are not subject to extraneous control, or even the control of the will.

The peristaltic structures are to be regarded as special creations devised for the express purpose of carrying on life, and the most important details of life, in its several departments. They invest life with a superior dignity, and make it very little dependent on environment and mere externalities. They make living things superior to dead things. The plant and the animal are on a higher platform than their surroundings; they are living, active factors, and control the inanimate matter which surrounds them. They select and appropriate certain portions of it, and cause it to circulate within their bodies. They reject other portions, and eject at intervals the detritus of that originally selected.

In mammals the reproductive process is in some respects the most complicated. Indeed it is held by some to represent in its several phases all the other forms of reproduction referred to above; the developing mammal, according to them, being in succession a fish, an amphibian, and a reptile, or at all events exhibiting peculiarities which associate it with the several orders of animals named. Thus it has gills or branchial arches and bloodvessels like a fish and amphibian, and a mixed circulation like a reptile.

The male and female organs and elements in mammals are situated in different individuals, and conjunction or coitus in the ordinary sense is the rule. In certain abnormal conditions coitus has been dispensed with, the semen of the male being simply injected into the vagina of the female. The essential act of reproduction in the mammal, as in all other cases, consists in the union and interpenetration of the male and female elements. In the human species it is now known that the spermatozoon or male element actually plants or buries itself in the ovum or female element, and that unless this intimate union and commingling takes place neither impregnation nor conception occurs. 


\section{DESIGN IN THE REPRODUCTION AND GROWTH OF ANIMALS}

The production of the male and female elements in the mammal is an elaborate process, and begins at an early age, even prior to puberty. The testes of the male and the ovaries of the female supply the spermatozoa and ova respectively. The spermatozoa or male elements, when puberty is attained, are always available; the ova or female element being only available monthly or after each menstrual period. The spermatozoa are furnished in incredible numbers, the ova in small numbers, generally one or two, but sometimes three, four, or more. The number of the offspring corresponds with the number of impregnated ova which occupy the uterus. Single births are most common, but twins and triplets are by no means rare.

The manner in which the ova are produced and find their way into the uterus is at once interesting and instructive.

The ovaries, two in number, are situated near the fundus of the uterus, of which they may be said to form appendages, although they are altogether distinct from it. They exist in the female child, and as puberty approaches the ova which they contain begin to ripen. When puberty is attained the ripe ova-one, two, or more-are discharged monthly from the surfaces of the ovaries and caught by the funnel-shaped, fimbriated extremities of the Fallopian tubes in a very special and remarkable way, and conveyed by them to the interior of the uterus. The Fallopian tubes with their fimbriated extremities are not structurally attached to the ovaries, but by a series of vital and most extraordinary movements they sweep over the surface of the ovaries so effectually that they almost invariably succeed in catching the ripe ova on their being discharged. By some refined and totally inexplicable affinity they feel about for the ripe ova, and, having located them, temporarily attach themselves to the portion of the ovaries about to erupt or discharge. The movements of the Fallopian tubes are essentially vital in character. They are spontaneous and independent, and are not caused by any inherent irritability or extraneous stimulation. The Fallopian tubes are special fundamental structures, and perform a most important function in the reproduction of mammals. If they fail to catch and convey the ovum or ova to the uterus, extra-uterine gestation, that is, gestation in the Fallopian tubes, or abdominal cavity, occurs, which sooner or later endangers the life of the mother, and necessitates the performance of the Cæsarian operation or section.

Gestation and development usually occur in the uterus, but, as indicated, they may occur in the Fallopian tubes, or even on the surface of the ovaries, giving rise to Fallopian tube or extra-uterine pregnancy--both requiring operation. Seeing the impregnated ovum or ova can live and develop outside the uterus, and on what are practically living, serous surfaces which merely furnish heat and moisture, it follows that quickened ova are to be regarded as parasites, that is, living things growing upon living things, as happens in mistletoe growing upon apple or other trees. I first directed attention to the parasitic nature of the developing human ova in $1872,{ }^{1}$ and more extended observation and experiment confirm me in this view. The view, I am glad to be able to state, has found general acceptance.

The impregnated human ovum contains in its substance the pabulum required to nourish it until it has found a suitable niche in the mucous lining of the uterus. Once there it adheres by simple apposition. At the outset, and until the shaggy chorion is formed, it is fed by osmotic currents consisting mainly of liquor sanguinis exuded from the blood-vessels of the mother. When the shaggy chorion is formed, the ovum throws out thread-like processes (villi) which increase the osmotic surface. Later, the ovum develops blood-vessels and capillaries ; the latter interdigitating and passing between similar capillaries found in the mucous lining of the uterus of the mother. In this way osmotic exchange is still further increased. The ovum in the later stages of development also obtains nourishment from uterine glandular secretions. The interchange of fluids and gases between the fœetus and the mother is carried on by the placenta, which is composed of a foetal and a material portion; the two portions very closely resembling each other structurally.

The maternal portion of the placenta consists of the thickened mucous lining of the uterus, of glands, lymphatics, nerves, capillary, and other blood-vessels. It at once forms a stomach and a lung for the foetus, as it provides the serum of aerated blood and nutritive gland products. The foetal portion of the placenta consists, for the most part, of connective tissue and of capillary and other blood-vessels. The maternal and foetal portions of the placenta are bounded by epithelium, and as they are merely placed in apposition with fluids and gases on either side the epithelium, a free interchange of nutritive and waste products, fluid and gaseous, takes place as between the parent and offspring. As the foetus is only temporarily grafted on the parent by a loose uterine arrangement, it follows that at the full term the foetus is expelled by rhythmic uterine contractions which induce the so-called labour-pains. When the child is born the fœtal portion of the placenta known as the after-birth is separated from the maternal portion by uterine contractions, and by a vital process resembling that by which the leaf is separated from the branch in autumn.

In healthy natural labours there is next to no laceration of blood-vessels, either maternal or foetal, and conse1 "Physiology of the Cireulation in Plants, in the Lower Animals, and in Man." Edinburgh Leelicel Juetrual, 1872-73. 
quently little or no hæmorrhage. The union of offspring and parent, although, in one sense, of the most intimate possible description, is, in another sense, a loose union; that is, a union of simple apposition, temporary in character, and dissoluble during any period of gestation. Unless, however, the foetus be permitted to ripen during nine calendar months, and the natural period of dehiscence as between the maternal and fœtal portions of the placenta be allowed to supervene, there is apt to be flooding from the rupture of uterine blood-vessels.

During the period of gestation, particularly in the later months, the mammæ increase in size, and produce a rich nutritive fluid (milk), which forms the first and all-sufficient food of the infant.

All the stages through which the impregnated human ovum passes are vital. The impregnated ovum at the outset consists of two separate, living elements. They coalesce and unite to form one living being, having in it the peculiarities and potentialities, physical and mental, of both parents. The impregnated ovum, the fœetus, and the future infant owe their existence to a series of vital changes which are spontaneous and fundamental in character. Similar remarks are to be made of the enlargement of the mammæ and the production of milk in anticipation of the birth of the progeny. These changes are in no way caused by irritability of the substances in which they occur, neither are they due to any form of extraneous stimulation.

In considering the subject of reproduction in the mammal the male and female elements are to be considered together. They each take part in the process and are equally important. The male element (sperm-cell or spermatozoon) is the product of the testes, and the female element (germ-cell or ovum) that of the ovaries.

Both the male and female elements are cellular in character; the ovum being a nucleated cell. The ovum when discharged from the Graafian follicle of the ovary is very tiny, and just visible to the naked eye. It appears as a clear speck, and measures $\tau^{\frac{1}{2}}$ th of an inch in diameter.

The changes witnessed in the maturation and fertilisation of the ovum are of the most interesting and instructive character, and point to an independent and self-directive power in the male and female elements, as well as a conjoint and interdependent power. They also indicate that the male and female elements act directly on the protoplasm of the ovum by causing its molecules to aggregate or separate, to converge or diverge, to coalesce or divide, to involute or evolute, to protrude or recede, \&c. The molecules, when fertilisation is a completed act, are apparently under the joint control of the male and female elements. The male and female elements are evidently foci of force; their function consisting in marshalling and arranging the molecules as development proceeds and new combinations take place. They exercise a formative power, which reveals itself in straight line, curve, circular, spiral, and other formations seen in the growth of plants and animals generally, and in the crystalline and dendritic arrangements met with in the physical universe (Plates lxi., lxii., lxiii., and lxiv. ; see also Plates i., ii., iii., and iv.). At times the male and female elements, especially the former, exercise what is virtually a magnetic power, whereby the molecules are attracted or repelled and arranged in straight lines, curves, ovals, ellipses, spirals, circles, \&c. This circumstance points to a much more intimate union as between organic and inorganic matter, and as between vital and physical force, than is generally suspected. The vital and physical forces work together, and are practically under the same laws. The life leads, but it freely avails itself of the matter and forces of the universe.

The changes above referred to take place in the male and female elements of all animals, man included. They are best seen in the transparent ova of echinoderms and in Ascaris megalocephala, a parasitic thread-worm found in the horse, in which the various changes can be followed in one and the same ovum.

Professor E. v. Beneden gives a most interesting account of the process of fertilisation, and of the division of the nucleus in Ascaris (Plate lxxxviii., page 385). He regards the male and female elements and their chromatin as distinct, even when in union. Prior to their conjunction the male and female pronuclei each contain two short chromatin rods (chromosomes) surrounded by a clear nuclear matrix. The rods in question initiate what is virtually a process of weaving and differentiation. Thus they undergo a series of changes which result in skein and looped$\mathrm{v}$-shaped formations; these being succeeded by new structures which exhibit stellar, radiating arrangements, and ovoid and circular arrangements.

Concurrently with the skein and looped-v-shaped formations two attraction-spheres make their appearance between the pronuclei. The attraction-spheres have central spots or poles, around which their molecules are arranged in a radiating and concentric manner. The poles of the spheres are connected by a chromatin fibre arranged in the form of a spindle. The attraction-spheres, there is reason to believe, are centres of growth, and plav an important part in the formation of the daughter cells which are the first product of the divided ovum.

A very considerable degree of differentiation, it will be seen, occurs in the male and female reproductive elements (pronuclei) and in the attraction-spheres before the fertilised ovum is ready for segmentation and the production of a new being. The several changes referred to negative the idea that either the male or female elements are fundamentally simple, homogeneous structures. On the contrary, they differ from each other before they come into contact. They also undergo several important changes during the fertilising process and before the quickened 


\section{COMPOSITION OF THE HUMAN OVUM}

and altered ovum is in a condition to assume the cleavage necessary to the formation of the blastodermic membrane, which in the higher vertebrates is the starting-point for the embryo and fœtus. The changes which supervene during the fertilising process bear a certain analogy to those occurring in the fœetus itself (Plate xcii., Fig. 1, E, F, G, H, I, page 396 ), so that the scheme of reproduction is, from the first, involved and complex, and the outcome of prearrangement and design.

While the matrices of the two pronuclei appear to blend, their chromatin filaments maintain their identity.

The chromatin flaments form two sets of looped-v-shaped fibres of two each, and as these ultimately divide longitudinally they make two sets of four each. At one stage they flatten and arrange themselves transversely across the ovum, and so participate in, if they do not actually cause, the division of the ovum into two. They subsequently open out transversely, and then at right angles, and in conjunction with radiating, concentric, ovoid, and circular arrangements in the attraction-spheres complete the division of the ovum.

The several points discussed in connection with the maturation, discharge, and fertilisation of the ovum are illustrated at Plate xc., Plate xci., and Plate xcii., Fig. 1, pp. 391, 393, and 396.

\section{$\S 72$. Parts Entering into the Composition of the Human Ovum.}

The human ovum in its entirety consists of the following parts : (1) globular zona pellucida, otherwise called zona radiata ; (2) globular vitelline membrane; (3) globular vitellus or yolk, consisting of protoplasm and nutritive material ; (4) globular germinal vesicle containing globular germinal spot.

The same parts are encountered in the rabbit.

The several parts of the ovum can only be made out by the aid of the microscope. In Plate lxxxvi., page 383, A, the ovum is seen under pressure and is more or less flattened. In B, C, the zona pellucida or radiata, which has considerable resisting power, is purposely burst; the protoplasm and nutritive material forming the vitellus or yolk $(b)$, and the germinal vesicle $(c)$ having escaped. At $\mathrm{D}$, the radiation in the zona pellucida (now known as zona radiata) is indicated $(a)$, as also the vitellus $(b)$, germinal vesicle $(c)$, and germinal spot $(d)$. The radiation of the zona pellucida is believed to contain pores, which permit the passage of nourishment to the ovum and provide entrances to the spermatozoa. The zona pellucida on leaving the Graafian follicle is invested with a clear, granular substance resembling the white of egg, which is supposed to contribute to the nourishment of the ovum, and disappears when the ovum reaches the Fallopian tube. The protoplasm of the ovum on a casual examination is a clear, apparently structureless, substance. On a closer examination, and when re-agents are employed, it is seen to consist of a fine reticulum especially observable near the periphery of the ovum and around the germinal vesicle : in other words, the protoplasm is differentiated. The other ingredients of the ovum (deutoplasm) contribute to its nourishment.

The germinal spot, which measures $\frac{1}{800}$ to $\frac{7}{500}$ of an inch in diameter, consists of a nuclear membrane containing a clear material, with strands of karyoplasm enclosing one or more nucleoli. If only one nucleolus occurs it is known as the germinative spot (macula germinativa). A certain amount of differentiation is also traceable in the germinal vesicle. The old idea, that protoplasm is identical and structureless, may now be regarded as exploded. The amount of nutritive material supplied to the ovum is less in mammals than in fishes, reptiles, and birds, and Professor F. M. Balfour conveniently divided ova into those with $(a)$ accumulation of nutritive or yolk matter at one pole (telolecithal ova-fish, reptile, and bird among vertebrates); (b) those with the accumulation of yolk in the middle ovum (centrolecithal ova-in arthropods); and (c) those with yolk scattered equally and in small amount through the protoplasm of the ovum (alecithal-mammals, amphioxus, echinoderms). This distribution of the nutritive material (proto- and deuto-plasm) determines to a large extent the nature of the segmentation in the several ova.

The development of the ovum after it leaves the ovary is at once intrinsic and extrinsic : intrinsic as regards changes occurring in itself, and extrinsic as regards changes induced in it by the presence of the spermatozoon or male element.

The intrinsic changes consist in the extrusion of certain constituents of the nucleus of the ovum known as the "polar globules": the extrinsic changes consisting in the intrusion into the nucleus of a spermatozoon or spermcell to take the place of the extruded polar globules. The ovum or female element on its part makes room for the spermatozoon or male element, and the latter takes full advantage of the arrangement. The male and female elements come together, and either fuse and merge into each other's substance or, as E. v. Beneden thinks, they maintain their identity, and mutually produce a double or compound cell, which is the starting-point of the new being (Plate lxxxvi., $\mathrm{H}$ to $\mathrm{S}$ inclusive, page 383 ).

The fertilisation of the ovum, it will be seen, is brought about by a union of the most intimate kind; the male 
element actually entering into the female element. Not only does the ovum extrude part of its substance to make room for the male element, but an aperture, in many cases, is provided for the admission of the male element, which aperture is closed soon after this element has effected an entrance.

Further, the male and female elements move towards each other both before and during impregnation. Thus, after coitus and before impregnation, the spermatozoon uses its vibratile, swimming tail on its way through the uterus to the Fallopian tube, and the movements of the cilia of the Fallopian tube assist the ovum in its passage through it; the junction of the male and female elements and impregnation usually taking place in the tube. In the process of impregnation the male and female elements move still more closely together, and carry out in the fullest sense the original purpose of a perfect union. That the male and female elements should be produced in two separate individuals and independently, and after the congress of the sexes should seek each other and travel long distances to effect a union, is at once a fundamental and striking fact in physiology, and plainly proclaims pre-arrangement and design. Reproduction consists of a progressive series of co-ordinated movements and changes in both the male and female elements; the spermatozoon even shedding its vibratile tail when it enters the ovum and the tail is no longer required. The changes to which allusion has been made take place before the division or segmentation of the ovum occurs. There are in reality two sets of changes to be considered, namely, $(a)$ changes occurring during impregnation and before segmentation of the ovum takes place; and $(b)$ changes which occur after segmentation or division of the ovum. It is necessary to say a few words regarding each, as the impregnation and development of the ovum afford some of the best illustrations of design known to anatomy, physiology, and biology.

\section{$\S 73$. Ripening of the Ovum: Formation of Polar Globules.}

Either before or soon after the ovum escapes from the Graafian follicle of the ovary it undergoes a peculiar change, consisting of unequal cell-division or germination, and the extrusion from its vitellus of two minute spherical bodies, the so-called polar globules or directive corpuscles (Plate lxxxvi., G to $\mathrm{N}$, inclusive). The change in question is independent of, but connected with, fertilisation, and is most important, as fertilisation does not occur without it. The cell division and extrusion of polar globules are almost universal in animals. They occur also in plants, and have therefore much significance. The polar globules (directive corpuscles) determine the pole at which the first segmentation will occur in the fertilised ovum. The globules consist of two small portions of the nucleus of the ovum, plus a certain amount of protoplasm. When they are to be extruded the germinal vesicle seeks the surface of the vitellus and undergoes changes indicative of a nucleus about to divide. It also loses its peculiar outline and shape. The vesicle, as a matter of fact, divides into two; the one portion being extruded into the perivitelline space, the other being retained in the vitellus to be extruded subsequently.

The remains of the germinal vesicle, designated the female pronucleus, now leaves the surface of the vitellus and seeks the centre, where it awaits the arrival of the male element (spermatozoon); the latter, when fertilisation takes place, forming the male pronucleus.

The junction of the male and female pronuclei results in the formation of a new nucleus, and ultimately, a new being. It happens occasionally that the ovum receives a spermatozoon before it extrudes its polar globules.

According to Minot "every cell which results from the division of a fertilised ovum is hermaphrodite." He also believed that the descendants of every such cell are also hermaphrodite. He founded his belief on the duality of the fertilised ovum, this containing male and female elements. Weismann in his theory of heredity propounded a somewhat different view. In his opinion " every animal and vegetable cell contains two kinds of living matter, namely, nuclear plasma and nutritive plasma: the former endowed with germinative, directing, and hereditary functions; the latter with the assimilation of food and the more purely physical functions, such as contraction, nerveconduction, secretion, \&c." The nuclear plasma, according to Weismann, controls the functions discharged by the nutritive plasma. Weismann went further and divided the nuclear plasma into germinal plasma and histogenetic plasma. To the former he assigned primitive form and periodicity; to the latter the division, growth, and differentiation of the cell. As fertilisation implies the addition to the ovum of a certain amount of male germinal matter, Weismann assumed that the ovum, prior to fertilisation and development, must get rid of its histogenetic plasma and a proportion of germinal plasma equal in amount to that brought to it by the spermatozoon. This adjustment is arrived at by the extrusion ( $a$ ) of one (histogenetic) polar globule, and (b) of the other (germinal) globule. By this arrangement a certain amount of the primitive or germinal plasma of the original ovum always remains, and in due course transmits the accumulated ancestral peculiarities of both parents. Heredity is essentially molecular in its nature. It is to molecules and the atoms forming them that questions of heredity and affinity must ultimately be referred. Atoms and molecules precede all cell formation and division. 


\section{$\S 74$. Fertilisation of the Ovum.}

The ovum, when expelled from the Graafian follicle of the ovary, is caught by the fimbriated extremity of the Fallopian tube, and transmitted by ciliary action along the tube in the direction of the uterus. If coitus has taken place it may, and generally does, encounter the male element (spermatozoon) in the tube. Less frequently, impregnation occurs in the uterus itself. Impregnation may, as already indicated, occur outside both the tube and the uterus, in which case the impregnated ovum may fall into the abdominal cavity and produce extra-uterine gestation. As a rule only one spermatozoon is required for fertilisation. The changes incidental to fertilisation are best seen in transparent ova such as those of echinoderms and ascaris. In echinoderms one spermatozoon generally may be seen to enter the gelatinous envelope which takes the place of the zona pellucida, and thrusts its head into the outer surface of the ovum, which rises up to meet it (Plate lxxxvi., $O, P, Q, R, S$, page 383 ).

In Ascaris, according to E. v. Beneden, a special aperture in the vitelline membrane (micropyle) is provided for the entrance of the spermatozoon, this always entering at the same part of the ovum (polar disc). The aperture in question is closed soon after the male element has passed in. The relations between the ovum and spermatozoon are in E. v. Beneden's opinion well defined and exact. They are obviously pre-arranged and the outcome of design.

When the head of the spermatozoon has penetrated the ovum it quickly increases in size, and assumes the appearance of a nucleus. It forms the male pronucleus, and is to be distinguished from the female pronucleus formed by the remains of the germinal vesicle. The male pronucleus travels towards the centre of the ovum in the direction of the female pronucleus, and in so doing causes the molecular and granular contents of the ovum to arrange themselves in well-marked and very remarkable radiating and concentric lines. The male pronucleus is evidently a directive force. When it reaches the vicinity of the female pronucleus the latter moves towards it as if by preconcerted arrangement. The male pronucleus at this juncture throws off its vibratile tail, it being no longer required. The male and female pronuclei when they come together both take part in the stellate-concentric arrangement of the contents of the ovum above referred to. They form by their union a new nucleus, capable of forming new cells by division.

Fertilisation is to be regarded as " the conjunction of part of the nucleoplasm of a sperm cell (protozoon) with part of the nucleoplasm of a germ cell (ovum); the result being the production of a complete nucleus endowed with active properties of division and reproduction."

The peculiar structures and properties possessed by the nucleus as seen in the cell of the larva of the salamander are illustrated at Plate 1xxxvii., A to $\mathrm{Q}$ inclusive. In this case the contents of the nucleus when resting are arranged in a network. When active and the nucleus is dividing they pass through a series of remarkable changes. Thus in the first stage of division the chromoplasm is transformed into a skein of closely contorted filaments; in the second stage, the filaments become larger and looser; in the third stage, the filaments become still larger, and present a looped appearance ; in the fourth stage, they are arranged rosette-fashion with a central clear space; in the fifth stage, the filaments converge and present a stellate appearance. The filaments subsequently split up longitudinally, and divide into two sets (metakinesis) to form daughter nuclei. The daughter nuclei ultimately separate, and repeat in their life histories the history of the parent nucleus just given. It will be seen that the nucleus is by no means the simple structure it was once supposed to be. On the contrary, it is highly complex; its activity being correspondingly great.

It would be impossible to exaggerate the importance of atoms, molecules, ova, cells, nuclei, and other rudimentary structures connected with reproduction. As these structures exhibit from the first, and on the very threshold of life, globular, concentric, curved, spiral, radiating, branched, and segmented arrangements, it behoves us to give more than passing attention to living matter in the young and adult states, and to dead matter as a constituent of the inorganic kingdom.

Much and general misunderstanding exists as to the relations between dead and living matter in the universe. It is, for example, not generally recognised that the inorganic and organic kingdoms are made for each other and obey similar laws; that they are co-ordinated and mutually interdependent; that they have much in common, and are not opposed to or destructive of each other; that impassable limits are set to both; that life is administered and kept going by the outside world; that while individuals die off in time and space, the types of the race continue to live and to persist from generation to generation; the stock of life being, as it were, transmitted unimpaired by innumerable ancestors, which have a continuous existence, and only reproduce their own kind.

In the whole range of biology there is no more outstanding feature than is furnished by plant and animal types and by the stability and persistency of these types. Many millions of plants and animals have inhabited and continue to inhabit the earth, but (and this is the peculiarity) they have not become mixed up or lost their identity to any appreciable extent. This is an extraordinary circumstance, and cannot be too deeply pondered. 
That typical plants and animals should remain unchanged for thousands of years simply surpasses belief.

There has always been a necessity for types or independent life centres. In geologic times the flora and fauna differed materially from those of the present day, and it is next to impossible for the geologist and biologist to distinguish between them. The differences cannot be bridged over by any form of modification, however long continued, or by any kind of evolution. The various extinct and living plants and animals do not merge or run into each other. They maintain, and have always done so, a separate existence. The belief in the separate creation of plants and animals in time and space is rapidly gaining ground. It is, however, admitted that modifications more or less extensive are required to meet the exigencies of climate, atmospheric conditions, environment, \&c.

Type and the persistence of type go together.

Mr. William Carruthers has shown that the earliest vegetable specimens described by Dr. Schweinfurth from the Egyptian tombs reveal no trace of change. This is also seen in the unchanged leaves and other organs found in the Pleistocene clays of Ottawa, on the Continent, and in Britain. Mr. Carruthers shows that the ancient willow (Salix polaris), which now lives in the Arctic region, is found in the fossil form in the Pleistocene beds at Cromer and Bovey Tracy.

Still more remarkable examples of the persistency of types are witnessed in animals. Here we are on comparatively safe ground, as plants and animals are figured side by side on the ancient Chaldean and Egyptian monuments. Civilised man, the head of the great vertebrate type, is now known to have occupied Egypt and the neighbouring countries for at least 8000 years; but as there was a civilisation long anterior to this, when flint was the only substance employed in the arts, that is, in the formation of arrow-heads, javelins, spears, knives, scrapers, hatchets, hammers, \&c., the period of semi-civilisation must be very greatly extended. Indeed the antiquity of man may be transferred backwards to a practically indefinite period.

Professor Huxley has furnished proofs at once of the fabulous antiquity of animals and of the marvellous stability and persistency of type. He points out that, "The progress of research has supplied far more striking examples of the long duration of specific forms of life than those which are furnished by the mummified ibises and crocodiles of Egypt. A remarkable case is to be found in the neighbourhood of the Falls of Niagara. In the immediate vicinity of the whirlpool, and again upon Goat Island, in the superficial deposits which cover the surface of the rocky subsoil in those regions, there are found remains of animals in perfect preservation, and among them shells belonging to exactly the same species as those which at present inhabit the still waters of Lake Erie. . . . We are fairly justified in concluding that no less a period than 30,000 years have passed since the shell-fish, whose remains are left in the beds to which I have referred, were living creatures.

"But there is still stronger evidence of the long duration of certain types. I have already stated that, as we work our way through the great series of the Tertiary formations, we find many species of animals identical with those which live at the present day, diminishing in numbers, it is true, but still existing, in a certain proportion, in the oldest of the Tertiary rocks. Furthermore, when we examine the rocks of the Cretaceous epoch, we find the remains of some animals which the closest scrutiny cannot show to be, in any important respect, different from those which live at the present time. That is the case with one of the cretaceous lamp-shells (Terebratula), which has continued to exist unchanged, or with insignificant variations, down to the present day. . . . Hence it must be admitted that certain existing species of animals show no distinct sign of modification, or transformation, in the course of a lapse of time as great as that which carries us back to the Cretaceous period; and which, whatever its absolute measure, is certainly vastly greater than 30,000 years." 1

The marvel of the permanency of plants and animals on the earth is only surpassed by the marvel of their creation. It is difficult to realise that from tiny specks of living matter the interminable races of plants and animals proceed, and that from one speck comes a highly complex form like the bee, and from another a simple animal like the amoba. The living microscopic particles produce with equal facility the smallest and largest plants and animals, as represented by the snow plant and Wellingtonia on the one hand, and by the zooid and leviathan, ancient and modern, on the other. The permanency of tiny living specks, their refusal to coalesce and lose their identity, and their capacity to produce plants and animals "after their kind," argue the possession of wholly exceptional powers. These powers are inherent, and are in no way connected with irritability, extraneous stimulation, environment, or chance. They afford examples of " means to ends," design, spontaneity, and vitality, and become wonderful in proportion to the care bestowed on their examination.

The points of interest in the present connection are briefly these :-

(a) Plants and animals are arranged according to types.

(b) The types are permanent and independent, and do not change into anything different from themselves.

(c) Types are known to persist for very many thousand years.

$$
1 \text { "Lectures and Essays," by Thomas Henry Huxley (Macmillan's series). London, 1904, pages } 22 \text { and } 23 .
$$


(d) They are the product of a First Cause, and are in no way the result of accident.

(e) They are living entities and are not to be confounded with automata in any form.

$(f)$ They are self-acting, and work out their destinies, under guidance, by spontaneous movements.

$(g)$ They occasionally die out, and when they do, their disappearance is due not to mischance but to a predetermined plan. The extinction of certain plants and animals at a given period does not interfere with nature's general plan or with the identity or permanence of plants and animals.

(h) The reproductive elements of plants and animals have a life of their own, and are living from the first. They bring with them into the world the wherewithal to nourish them while they are developing.

(i) There is a life of the individual and a life of the race, and both are persistent, although in varying degrees. The duration of the life of the race greatly exceeds that of the individual, but the one life is inseparably bound up with the other, both having a common source.

(j) Limits have been set to the outward and inward workings of plants and animals, and the same is true of the several parts of the inorganic kingdom.

(h) The inorganic and organic kingdoms are the counterparts of each other, and the two are complementary in the widest sense. They give to and take from each other at every point, both as regards matter and force.

( $l$ ) Any little tendency there is to variation in plants and animals is sooner or later corrected by a well-marked tendency to revert or breed back. This reversion contributes to the perpetuation of types and to the permanency of plants and animals on the earth.

The permanency and non-mixing of plants and animals here insisted on are seen at their birth, in their development, and in their career generally.

While everything in our planet is daily and hourly changing, the great flora and fauna practically remain stable. Stability or permanency, under the circumstances, bespeaks guidance and the operation of forces and laws directly referable to a First Cause. The existence of such a state of things can only be explained by pre-meditation and design.

The most striking feature connected with ova, cells, and nuclei is their ultimate composition. Do they possess a common structure, and have they in their substance at birth or at any period of their life histories, actually or potentially, a something which indicates a general plan according to types and a special descent, whereby each plant and animal reproduces only itself ?

The changes which occur in reproduction are far-reaching. They raise the very important and much-disputed question of the homogeneity and oneness of the ovum, and the identity of the matter forming the atom, molecule, cell, and nucleus in the embryos of plants and animals. Until the advent of the phenomenally high powers of the microscope it was customary to speak of the ovum, cell, and nucleus as simple or differentiated. A cell wall, nucleus, nucleolus, and cell contents or protoplasm were, as a rule, predicated. There were, however, endless disputes as to which of the structures mentioned was the most important. The consensus of opinion was ultimately in favour of protoplasm, which, variously designated and described, was regarded by the majority of biologists as the "physical basis of life," "the life stuff," \&c. This protoplasm was considered simple, homogeneous, and identical throughout its entire substance. It was maintained, with few exceptions, to be wholly undifferentiated. This statement is no longer tenable, as chemical analysis has shown protoplasm to be heterogeneous, and to vary markedly in ultimate composition. It may be accepted as an axiom, to which there is no exception, that differentiation can only occur in heterogeneous substances. No differentiation is possible in absolutely simple bodies. In living plants and animals "like produces like." A simple cell plant or animal can be obtained from an undifferentiated speck of protoplasm, but to get a differentiated organism, a heterogeneous, complex speck of matter must be employed. The theory that everything, inorganic and organic, had a simple origin is a very fascinating and a very old one. It was re-stated and amplified by M. Jean Lamarck in 1801, and received a fresh fillip from the writings of Mr. Charles Darwin in 1859 and subsequently. It largely rejects a First Cause and design, and leans upon chance as a main factor. It is denied by an ever-increasing number of advanced modern workers, and no longer holds the supreme position it did. It is especially discredited by those who believe in life as a separate entity, and who are opposed to spontaneous generation, utilitarianism, and opportunism.

It is now a matter of common knowledge that the ovum and nucleus are complex to begin with, and that they undergo the most extraordinary changes during the process of reproduction. There is in these facts warrant for the belief that no part of the ovum is homogeneous and simple in the ordinary sense, and the same is true of the ovum as a whole in all its stages, from its impregnation until the offspring reaches maturity (Plates lxi. to lxiv., and lxxxvi. to lxxxix. inclusive).

All this can have but one meaning. The generally accepted theory that the ovum is simple, homogeneous, and identical is, when carefully examined, found wanting, and must give way to the pressure of accumulated recent facts. 
Evolution, as indicated, is naturally divided into an ancient and modern form, and comes into operation whenever an attempt is made to obtain complex substances or beings from simple substances by an immeasurable series of slight modifications said to extend over practically unlimited periods. The ancient and modern theories of evolution are both faulty, and fail us at the most critical point, namely, where the simple substance is said to be converted into the complex one, and where differentiation is held first to make its appearance.

There are two phases of development with which the ancient and modern doctrines of evolution are altogether inadequate to deal. They have completely failed to explain the existence of type and the persistence of type. Neither can they account for the general plan and design which everywhere obtain in nature. Those who are opposed to evolution in all its forms not unnaturally inquire, How can plants and animals which vary to quite an extraordinary extent in ultimate composition be produced from an ovum which is, in all its parts and particles, homogeneous and simple, that is, undifferentiated? They say, and say truly, that complex plants and animals can only be produced from compound, heterogeneous substances; in other words, from substances which are themselves directly or indirectly multiple. The compound matter, according to them, is necessary to the production of the compound plant and animal at the outset, and at every stage of its career.

The only question which now remains is as to whether the ovum is a microcosm, that is, a miniature structure where all the parts composing it are present at one and the same time; or a conglomerate, consisting of many parts where only certain of them are in evidence at any particular period; these forming the foundations and scaffolding for the others, which appear in succession at intervals in a given order, until the compound plant or animal is produced. In the one case, the plant and the animal are the outcome of one continuous set of changes or developments ; in the other, of several sets of consecutive changes or developments.

According to the supporters of evolution in its most ancient and widest sense there is one long continuous act of development; simple matter having impressed upon it at the outset certain tendencies which inevitably result in differentiation in a particular direction, and which finally produce the perfected plant and animal. Otherwise stated, the original ovum has in itself certain potentialities which it has no power to resist, and which act in specific directions in the production of plants and animals according to types. Evolutionists seek to explain types for the most part by chance, and as apart from a First Cause, a pre-arranged plan, and design. To all such views, if the Deity be excluded, I am wholly opposed.

These opinions are further discussed in other parts of the work, and the illustrations connected with them have an interest all their own, and should be carefully examined and studied.

\section{PLATE LXXXVI}

Plate lxxxvi. shows various kinds of ova and the changes occurring in them before and after impregnation.

Ovarian ovum of a mammal (A, B, C).

A. Entire ovum seen under pressure. a, Zona pellucida or radiata; 7 , yolk substance and protoplasm; $c$, germinal vesicle with germinal spot.

B. $a$, Zona pellucida burst by pressure; $b$, yolk substance and protoplasm escaped; $c$, germinal vesicle set free.

C. Germinal vesicle with thin coating of yolk substance (after Allan Thomson).

D. Ovum of cat greatly magnified and semi-diagrammatic. a, Zona pellucida or radiata showing radiated structure; $b$, yolk or vitellus invested with delicate membrane; $c$, germinal vesicle; $d$, germinal spot (after E. A. Schäfer.).

E. Diagram of a holoblastic (alecithal) ovum. $b, c$, Germinal vesicle and germinal speck.

F. Ditto of a meroblastic (telolecithal) ovum. In these figures the nutritive part of the yolk appears as clear globules (b). At $\mathrm{F}$, it is seen in excess unless in vicinity of germinal vesicle $(c)$, where the protoplasm abounds (after E. A. Schäfer).

G. Ovum of rabbit from Fallopian tube twelve hours after impregnation. Shows formation of polar globules. a, Zona pellucida with spermatozoa (male elements); $b$, vitellus or yolk; $c$, perivitelline space with spermatozoa; $d$, polar globules extruded from vitellus (after Bischoff).

H. Formation of first polar globule in egg of Ascaris megalocephala. a, Zona pellucida; l, yolk substance and protoplasm ; $c$, germinal vesicle converted into spindle of achromatic fibrils with fibrils radiating into the protoplasm; $d$, eight portions of chromatin; $e$, head of spermatozoon becoming male pronucleus.

I. Shows the eight chromatin particles separated into two sets. a, Zona pellucida; $b$, yolk and protoplasm considerably shrunken; $d$, the two sets of chromatin particles.

J. Shows separation and extrusion into perivitelline space of half of germinal vesicle (four outer chromatin particles) with portion of protoplasm to form polar globule, $a$, Zona pellucida : l, yolk protoplasm; $d$, half of germinal vesicle with four chromatic particles extruded into perivitelline space; $e$, line of division (V. Genuchten).

K. Formation of second polar globule in Ascaris meyalocephala. a, Zona pellucida; $b$, yolk substance and protoplasm; $i$, position occupied by extruded first polar globule; $d$, spindle of uchromatic fibrils with four chromatin particles at equator formed by remaining half of germinal vesicle.

L. " Zona pellucida; $c$, first polar globule; $d$, y-shaped spindle of fibrils with chromatin particles approaching surface of ovum ; $e$, male pronucleus formed from spermatozoon.

M. Extrusion of remaining half of germinal vesicle commenced. a, Zona pellucida ; $c$, first polar globule; $g$, partial extrusion of second polar globule. 

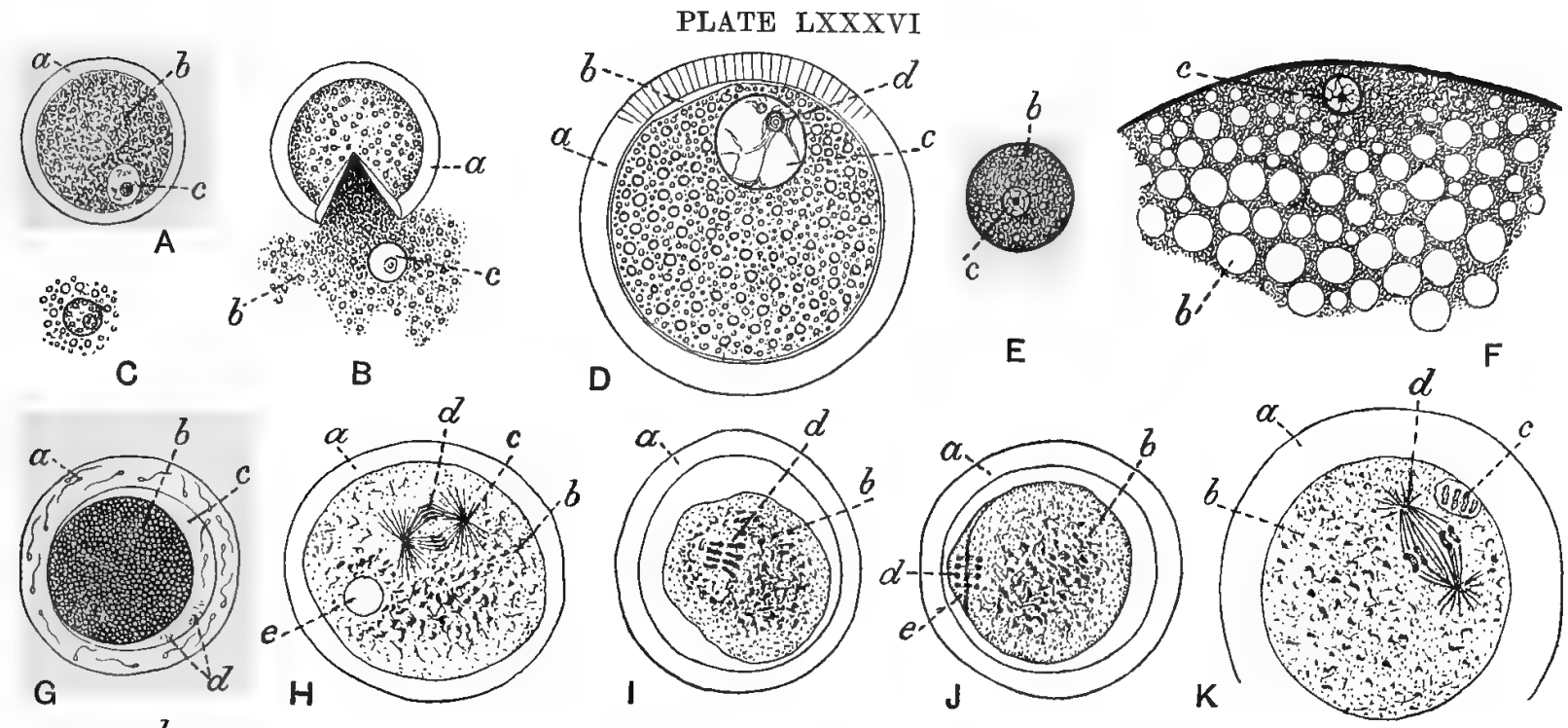

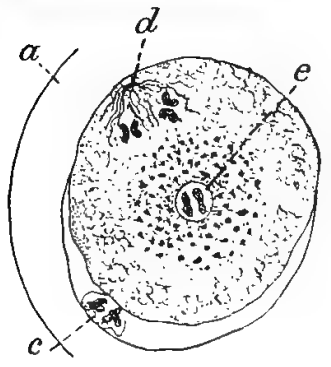

L

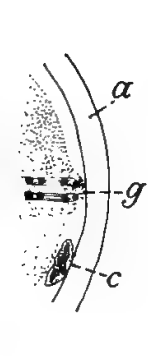

M

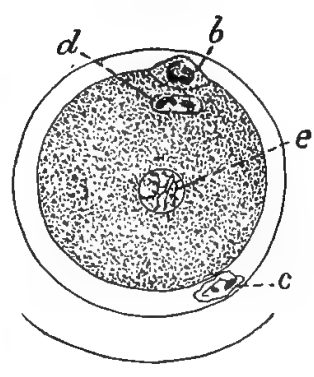

N

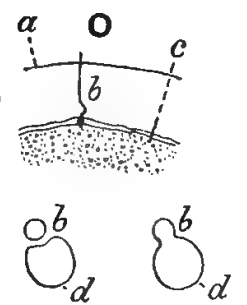

$\mathrm{R}$

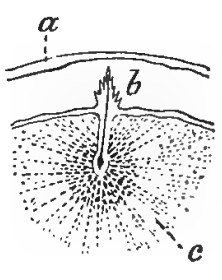

$P$

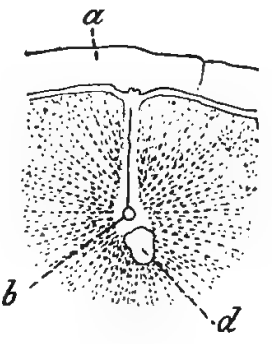

Q

PLATE LXXXVI (continued)

N. Separation of second polar globule (b), with two chromatin particles from the ovum; the other two with remains of germinal vesicle $(d)$ forming the female pronucleus; $e$, male pronucleus; $c$, first polar globule (Carnoy).

$O$. Fertilisation of the ovum of an echinoderm. "Z Zona pellucida; $b$, spermatozoon perforating vitelline membrane; $c$, protoplasm of vitellus.

P. $a$, Zona pellucida ; $b$, entrance of spermatozoon into protoplasm of vitellus $(c)$, with radiating and concentric arrangement of its granules.

Q. $a$, Zona pellucida ; $b$, head of spermatozoon enlarging to form male pronucleus ; $d$, female pronucleus. The radiating and concentric arrangement of protoplasmic yolk granules is well marked in this figure.

$\mathbf{R}, \mathbf{S}$. Blending of the male $(b, b)$ and female $(d, d)$ pronuclei (Selenka).

\section{PLATE LXXXVII}

Plate lxxxvii. illustrates different stages of karyokinesis in the epithelium cells of the larva of the salamander. These cells are hardened in chromic acid and stained with hæmatoxylin. Highly magnified (Flemming).

A. Resting cell, displaying the nuclear network.

B. First stage of division, the chromoplasm being converted into a skein of closely contorted filaments.

C. Second stage of division, filaments increased in size and less closely arranged. (In this and all the other figures, Fig. A excepted, the nuclear matrix is clear.)

D. Filaments slightly more magnified than in the other figures; these showing a looped arrangement, better seen at E, where they are rosette-shaped and enclose a central clear space.

F. Filaments converging towards the centre.

G. Stellate phase or aster.

H. Completion of longitudinal splitting already begun at F and $\mathrm{G}$.

I. Filaments beginning to separate into two groups (metakinesis).

$J$. Further separation into two sets.

K. Separation further advanced.

L. Stellate phase of daughter nuclei (diaster).

M. Commencing convolution of filaments.

N. Filaments more contorted.

O, P. Gradual passage of daughter nuclei into condition of rest as seen at network Q.

Note. -The division of the protoplasm begins at the stage $(\mathbf{M})$ and is rapidly completed at (N). 

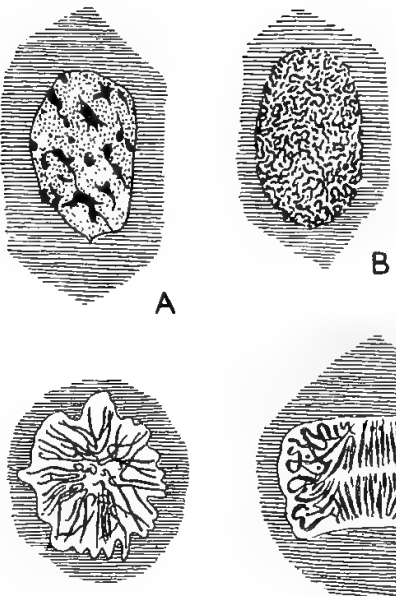

H

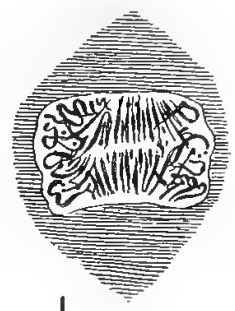

I

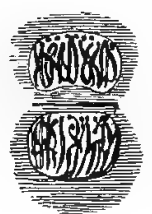

0

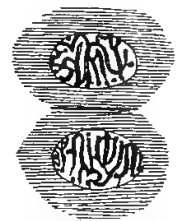

$P$

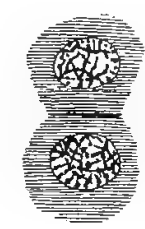

Q

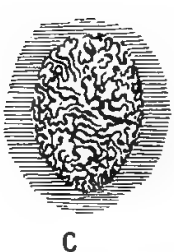

C

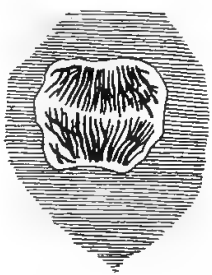

J

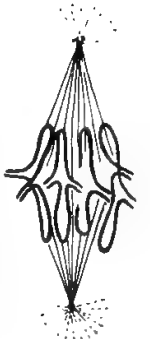

$\mathrm{R}$

PLATE LXXXVII

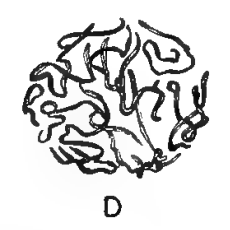

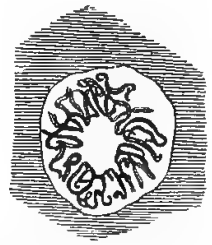

E

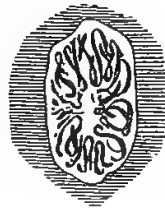

$F$

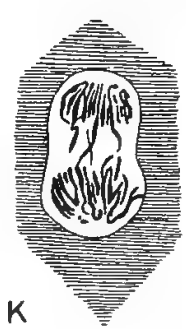

$\mathrm{K}$

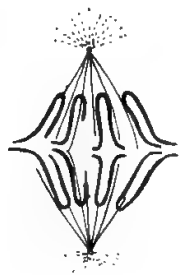

S

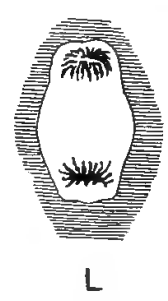

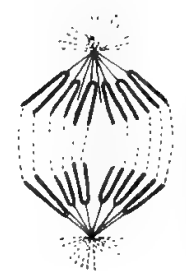

T

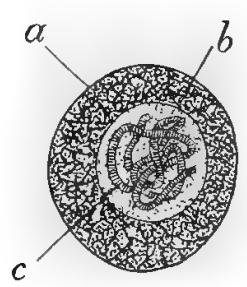

U

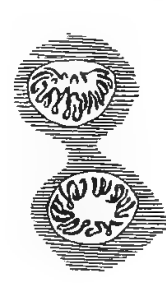

M

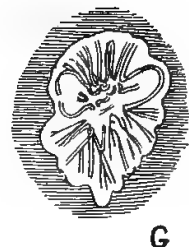

G

PLATE LXXXVII (continued)

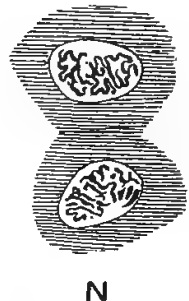

N

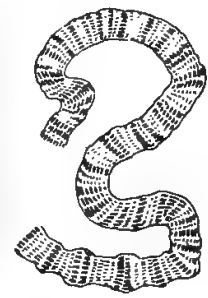

V

(R, S, T.) Stages of metakinesis according to Rabl.

R. The split chromosomes beginning to separate.

S. The separation further advanced.

T. The separated chromosomes passing along the fibres of the achromatic spindle.

U. Gland-cell from a larva of nemocera. $a$, Cell membrane; $b$, protoplasm; $c$, nucleus with convoluted filament.

V. Part of the nuclear filament seen at U greatly magnified (Carnoy).

\section{PLATE LXXXVIII}

Plate lxxxviii. illustrates the formation and conjugation of the pronuclei in Ascaris megalocephala according to E. v. Beneden.

A. $a$, Female pronucleus ; $b$, male pronucleus, each containing two chromatin particles, the male $(b)$ exhibiting skein formation; $c$, one of the polar globules; the second polar globule has been extruded.

B. The chromatin in the female $(a)$ and male $(b)$ pronuclei forming skeins; , polar globule.

C. The skein formation of the female $(a)$ and male $(b)$ pronucleus more pronounced; remains of spermatozoon adhering to $b$. Two attraction-spheres $(d)$ with central particles united by spindle of achromatic fibres now appear ; $c$, polar globule.

D. The female $(a)$ and male $(b)$ pronuclei enlarged and skein formation completed; $c$, polar globule.

E. $a$, Female pronucleus with skein contracted and thickened; $d$, attraction-spheres on one side of ovum connected with periphery by cone of fibres forming polar circle $\left(e, e^{\prime}\right) ; f$, equatorial circle.

F. Female $(a)$ and male $(b)$ pronuclei now in contact; spindle system arranged across their axes. The attraction-spheres $(d, d)$, placed between pronuclei. At G, H, I, and J, these spheres are omitted.

G. Construction of skein and $\mathrm{v}$-shaped formation of chromatin filaments in female $(a)$ and male $(b)$ pronuclei, which now indent each other; $c$, polar globule.

H. V-shaped chromatin filaments more pronounced, and union of female $(a)$ and male $(b)$ pronuclei more intimate; $c$, polar globule.

I. V-shaped chromatin filaments dividing by Jongitudinal cleavage $a$ and $b$, male and female pronuclei ; c, polar globule.

$J$. Highly magnified to show structure of $v$-shaped chromatin filaments $(a, b)$; consist of fine granules of chromatin; c, polar globule.

K. $a, b$, Four chromatin loops in middle of elongated ovum about to divide into two cells; the loops forming a spindle-shaped system of granule with fibrils radiating in the direction of the attraction-spheres $\left(d, d^{\prime}\right)$, which in turn radiate into the protoplasm of the ovum; $c$, polar globule.

L. Shows chromatin filaments $(a, b)$ of conjugated nuclei separating; also system of radiating fibres from attraction-spheres $\left(d, d^{\prime}\right)$; $c, c^{\prime}$, polar circle ; $d, d^{\prime}$, central circle ; $e$, equatorial circle (semi-diagrammatic). 


\section{PLATE LXXXVIII}

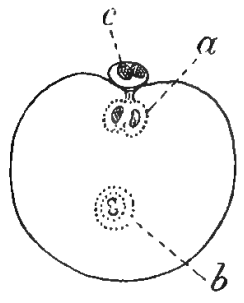

A

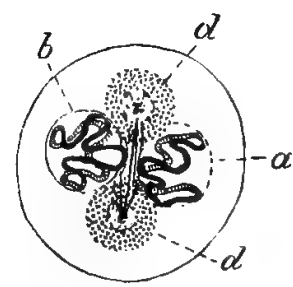

$\boldsymbol{F}$

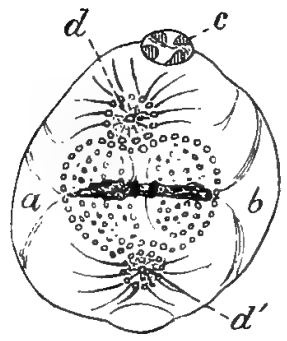

K

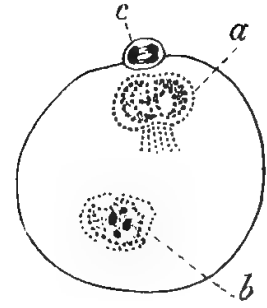

B

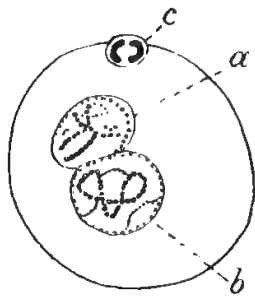

G

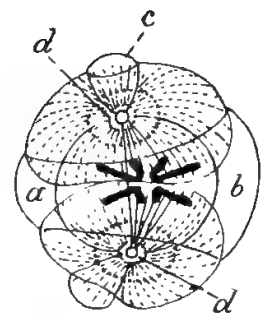

$L$

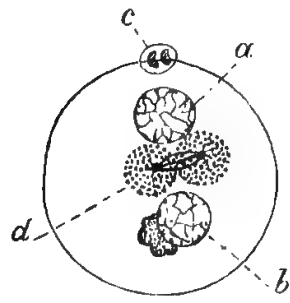

C

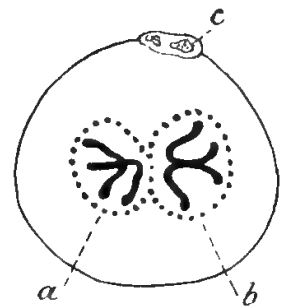

H

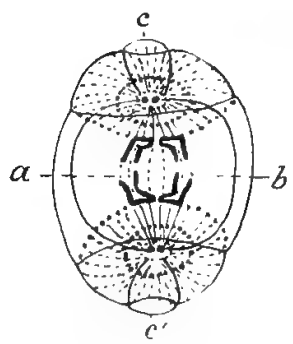

M

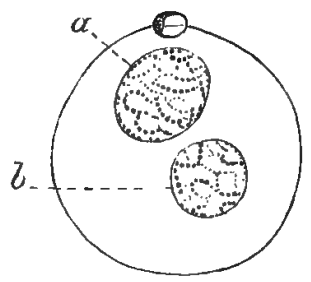

D
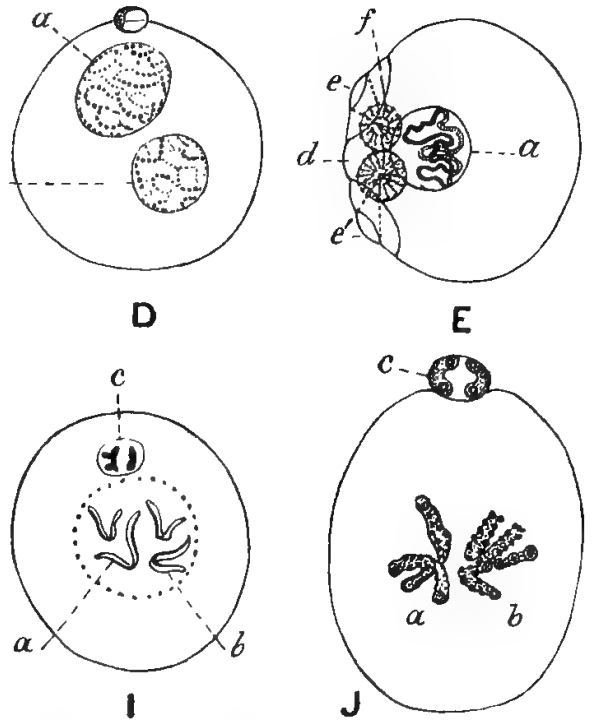

E
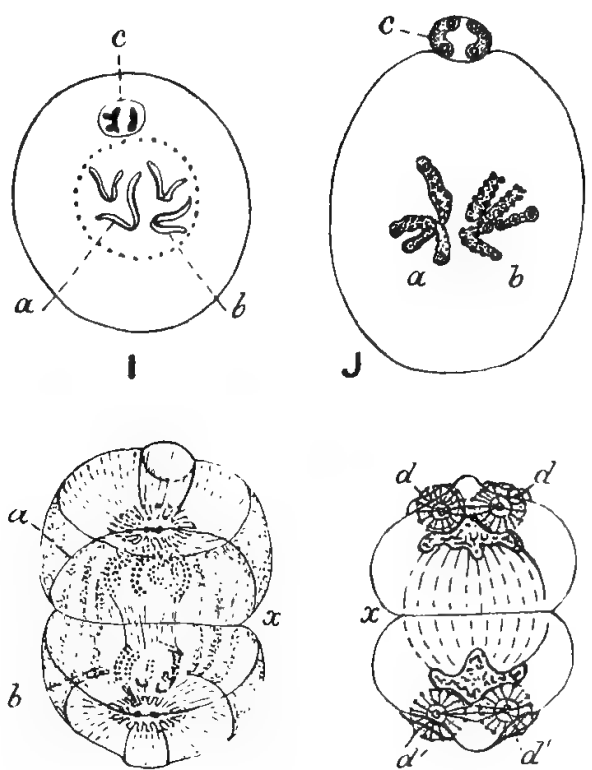

N

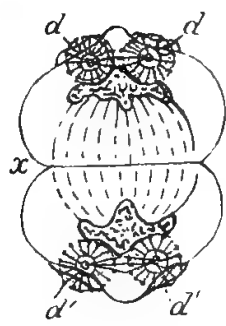

0

PLATE LXXXVIII (continued)

M. Chromatin filaments (", b) more widely separated. The central particle of each attraction-sphere $\left(d\right.$, $\left.d^{\prime}\right)$ has now divided into two.

N. Chromatin filaments $(a, b)$ becoming developed into the skeins of daughter nuclei united by achromatic fibres; protoplasm of ovum dividing into two at $x$.

o. $x$, Division of ovum progressing. The attraction-spheres have now divided into two $\left(d d, d^{\prime} d^{\prime}\right)$, and are joined by achromatic fibres to each other, and to periphery of cell as at D. The daughter nuclei display a chromatin network.

\section{PLATE LXXXIX}

Plate lxxxix.-Dividing nucleus displaying spindle of achromatic fibres with the chromosomes arranged astrally at the equator of the spindle (A). Shows also well-marked radiating and concentric arrangements (Rabl).

A. $a, a^{\prime}$, Central particle; $b, b$, polar radiation attraction-sphere; $d$, chromatin loop cleft longitudinally; $e$, clear area; $f$, cell substance.

B. Dividing nucleus at "aster stage," seen from one of the poles, with the achromatic spindle foreshortened. Shows radiating looped arrangement. $a$, Outline of nucleus; $b$, achromatic spindle with central polar particle ; $c$, chromatin loops.

C. to J. Illustrate the stages in the division of the ovum or egg-cell of a worm. Show radiating, spindle-shaped, concentric arrangements.

C. Ovum before division.

D. Spindle-shaped fibres with chromatic nodules (chromosomes) at the equator of the spindle.

E. Separation of chromosomes into two parts, which travel towards the poles of the spindle and there become transformed into new (daughter) nuclei, the protoplasm meanwhile also separating into two parts, as seen at $\mathbf{F}, \mathrm{G}, \mathrm{H}$.

VỌL. Is 

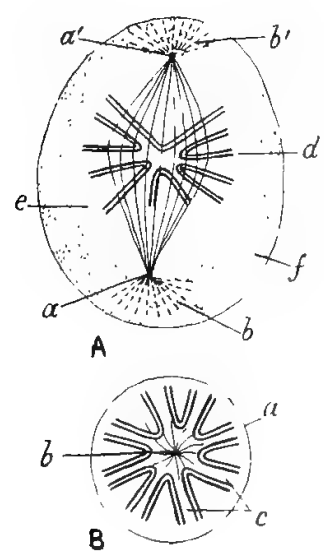
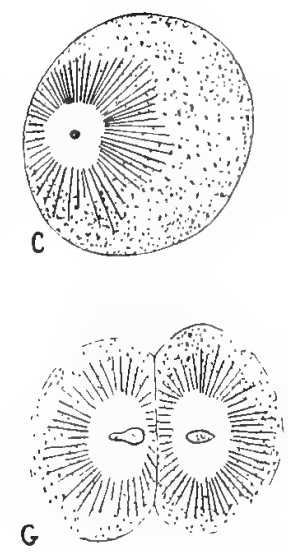

PLATE LXXXIX
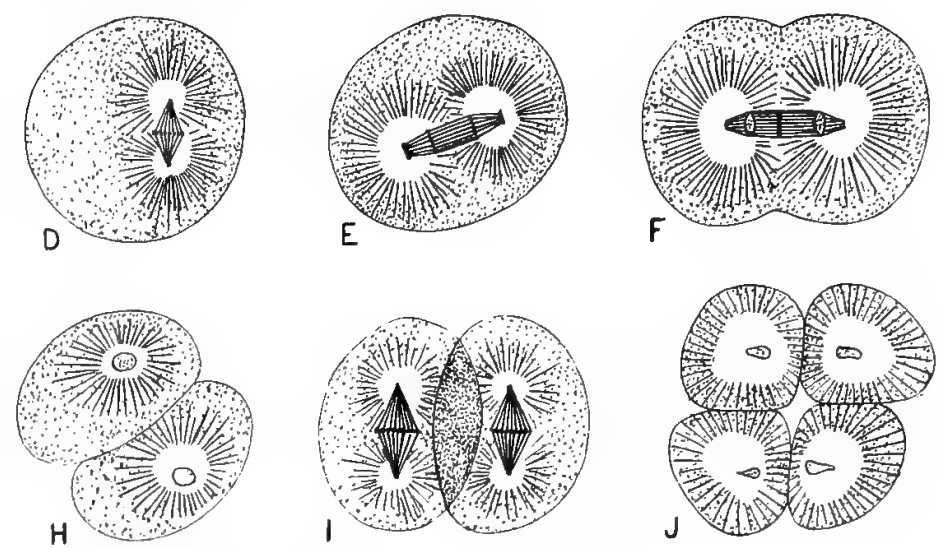

PLATE LXXXIX (continued)

At $\mathbf{F}$ there is thickening of the achromatic fibres in middle of spindle indicating plane of division of cell.

I. Repetition of dividing process with formation of spindles in daughter nuclei.

J. Results of division (Strasburger).

\section{§ 75. Reproduction in the Bird.}

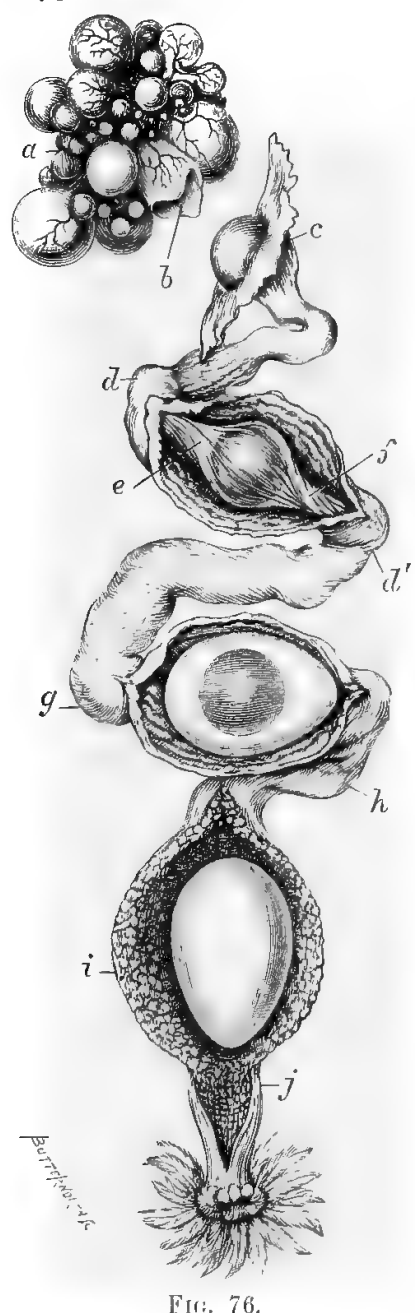

The reproductive process can be readily and conveniently studied in the barn-door fowl. The egg, which represents the female element of the bird, must in every instance be impregnated or quickened by the presence, contact, or actual admixture of the male element. This impregnation, as a rule, occurs at an early stage, when the eggs are being formed. The formation and descent of the quickened egg down the oviduct are among the most interesting and beautiful processes in nature, and afford unmistakable evidences of design (Fig. 76).

FIG. 76.-Shows ovary, oviduct, and eggs of common barn-door fowl. a, Ovary, with eggs at various stages of development; $l$, Graafian follicle from which an egg has been discharged; ', fimbriated extremity of oviduct with egg entering it; $c, d, d$, portion of oviduct supplying egg with moisture and twisted chalaziferous membrane $(e, f) ; d, y, h$, portion of oviduct engaged in providing the egg with a coating of albumen (the white of the egg); $h, i, j$, portion of the oviduct furnishing the three membranes of the egg (two soft and one hard) (after Dalton).

The young egg is formed in the ovary (Fig. 76, $a, b$ ) and consists of a germinal vesicle, a yolk, and a vitelline membrane. At this stage the egg is far from complete. It is dropped into the fimbriated extremity $(c)$ of the oviduct, the mucous lining of which provides it with various coverings and membranes, soft and hard. Thus the upper part of the oviduct $(c, d)$ supplies it with fluid to make it more flexible and yielding; a second part $\left(d, d^{\prime}\right)$ with a gelatinous membranous deposit which forms the chalaziferous membrane $(e, f)$ twisted at either end as if to prevent the escape of the vitellus; a third part $\left(d^{\prime}, g\right)$ provides it with layers of a gelatinous albuminoid substance known as the white of the egg; a fourth part $(g, h)$ furnishes it with three membranous layers (the external, middle, and internal fibrous membranes); a fifth part $(i, j)$ infiltrates the external membrane with lime to produce the hard shell, the internal and middle membranes forming the soft shell. The egg of the fowl, it will be seen, is added to and completed in its passage down the oviduct. The completed egg is shown at Fig. 77. Nor does the marvel stop here. The egg while it is being formed is steadily passed along the oviduct by a series of peristaltic, rhythmic movements akin to what occur in swallowing and in the passage of food along the intestine. At one portion of the oviduct the egg is made to rotate, with the result that the chalaziferous membrane is twisted in opposite directions to form the chalazæ. The oviduct at once completes and transmits the egg prior to its discharge through the cloaca. This it does by providing various secretions and membranes, and by establishing a series of spontaneous, independent, vital movements. The egg does not at any stage of its progress along the oviduct act as an irritant or stimulus either for the production of the secretions or the peristaltic movements. 
The variety of the secretions and movements wholly forbids such an assumption. The secretions and movements are provided by nature for the obvious purpose of completing the egg and transmitting it to its destination outside the body, where it is to be hatched. The secretions and movements are the result of inherent vital action as apart from every form of irritation and stimulation. As a matter of fact the oviduct takes the lead both in the secretions and the movements. The history of the egg, so far, plainly points to adaptation and design. All the parts involved work spontaneously, consentaneously, and harmoniously to a given end; that end being the completion, transmission, and discharge of the egg.

The hatching of the egg is, in a way, as wonderful as its production.

The impregnated egg can be hatched either by the parent bird, or artificially by means of an incubator. Heat, air, a certain amount of moisture, and turning of the egg at intervals are all that is required.

\section{§ 76. Division of the Impregnated Ovum.}

The changes which occur in the egg during the hatching process are of the most complicated and extraordinary character. No better example of the stupendous powers and resources of life can be adduced. The germinal vesicle, surrounded by the yolk and vitelline membrane (the original portions of the egg), begins the great process of differentiation by spontaneously dividing into two parts, then into four, then into eight, sixteen, thirty-two, sixty-four, and so on, until it forms a continuous membrane, the so-called blastodermic layer; the subdivisions assuming a cellular character. The dividing process is still seen in the impregnated human ovum (Fig. $7 \&, \mathrm{D}, \mathrm{E}, \mathrm{F}, \mathrm{G}, \mathrm{H}$ ). Here again we have an example of spontaneous vital action as apart from irritation and stimulation.

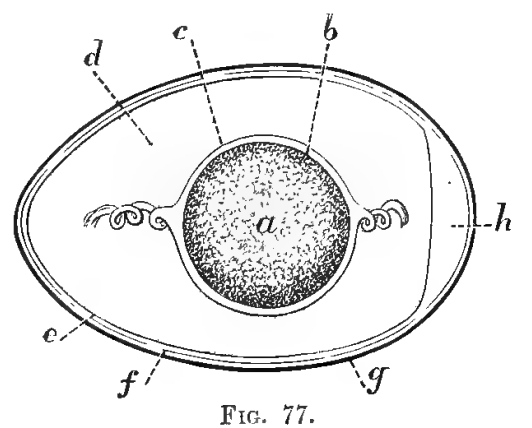

FIG. 77.-Egg of barn-door fowl, consisting of yolk ( 1 ); vitelline membrane (b); chalaziferous membrane (c); albumen (d); internal and middle shell membranes $(e, f)$; calcareous shell $(g)$; and air chamber $(h)$ (after Dalton).

Fig. 78 illustrates the history of the impregnated human ovum.

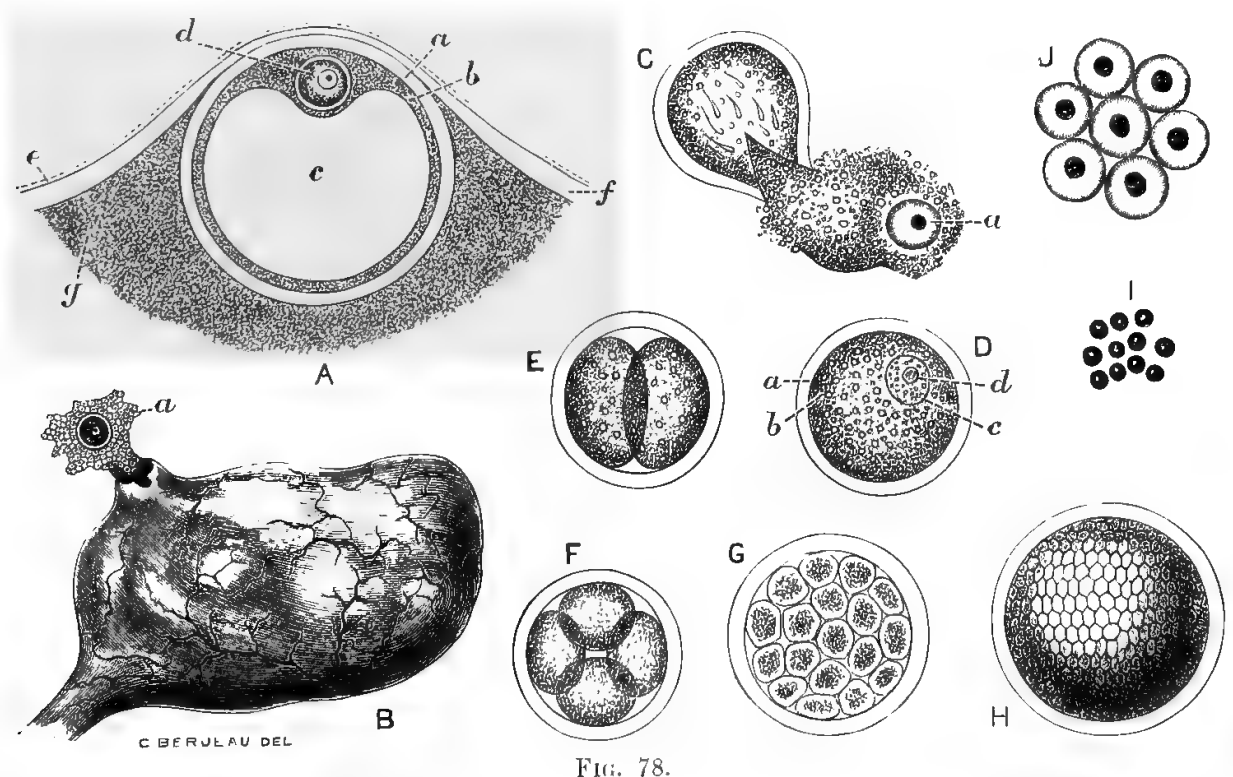

FIG. 78.-A. Portion of human ovary with Gruatian follicle near the period of rupture. Shows the membrane of the vesicle (a); the membrana granulosa $(b)$; the cavity of the follicle $(c)$; the egg $(d)$; the peritoneum $(e)$; the tunici albuginea $(f)$; and the tissue of the ovary $(g)$.

B. Human ovary with Graafian follicle ruptured. Shows ovum discharged with a portion of the membrana granulosa (a).

C. Human ovum ruptured by pressure. Shows escape of vitellus and germinal vesicle with germinal spot $(n)$.

D. Human ovum, magnified eighty-five diameters. Shows membrana pellucida and vitelline membrane $(a)$; vitellus or yolk $(b)$; germinal vesicle $(c)$; germinal spot $(d)$.

E, F, G, H. Four different views of the segmentation of the vitellus.

I. Adult frogs' eggs as they occur in the ovary.

J. The same when they have passed through the oviduct (after Dalton). 


\section{§ 77. Development of the Embryonic Membranes or Layers from which the future Being proceeds.}

The division and subdivision of the ovum inaugurates what is practically a process of weaving; the result being the production of a continuous membrane, known as the blastodermic layer, which in turn admits of division. This layer first divides into two and constitutes the external and internal blastodermic layers. At this stage the egg presents the appearance of a globular sac enveloped in three concentric membranes, namely, the structureless vitelline membrane, the external blastodermic membrane composed of cells, and the internal blastodermic membrane, also composed of cells. The cavity of the egg is occupied by a transparent fluid.

The process of segmentation of the vitellus and the formation of the blastodermic membranes are the most important processes in the development of the egg. By these the vitellus or yolk, composed originally of an albuminous matter, plus oily granules and protoplasm, is converted by a series of marvellous transformations into the several organs and body of the future chick. From the blastodermic membranes all differentiation proceeds. The segmentation of the vitellus and the formation of the blastodermic membranes is essentially the same in all animals, whether the ultimate product be a fish, a reptile, a bird, or a quadruped; the distinguishing features of the several classes of animals being due to the development of accessory structures in particular directions. These, in the human subject, as will be shown presently, are numerous and important.

The two layers of the blastodermic membrane represent the commencement of all the organs of the fotus;
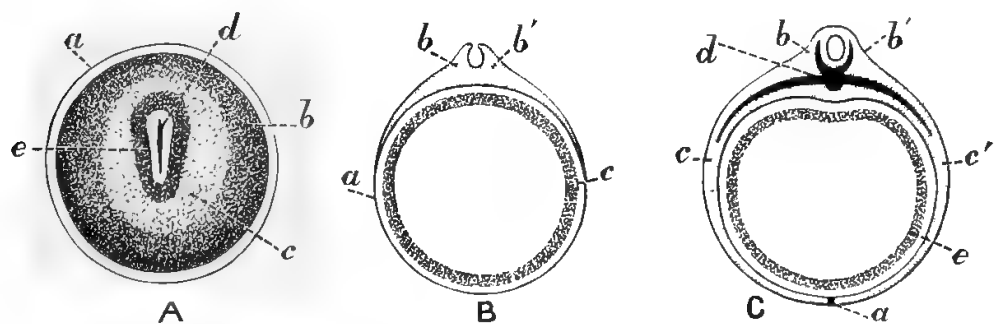

FiG. 79.-A. Inpreguated egg showing first traces of embryo. ", Vitelline nem. brane; $b, c$, vitellus or yolk ; $d$, primitive trace in area pelhucida; $i$, embryonic spot.

B. Transverse section of egrgat an early stage of development. $a$, External layer of blastodermic nemhrane; $b, b^{\prime}$, dorsal plates; $c$, internal layer of blastodermic membrane.

C. A similar section more advanced. " External layer of blastodermic membrane $b, b^{\prime}$, dorsal plates united and enclosing spinal canal ; $c, c^{\prime}$, abdominal plates; $d$, section of spinal column, with lamina and ribs; $e$, internal layer of blastodermic membrane (after Dalton).

cyline are produced, the one containg the brain and spinal cord; the other the intesting tho thes or (Fig. 79, A, B, C).

Of late years the blastoderm has been divided into three layers, namely, an outer, which is the epithelial membrane of the blastodermic vesicle (Rauber's layer); an inner, the primitive entoderm; and an ill-defined middle stratum consisting of somewhat granular cells. The three layers were supposed by $\mathrm{E}$. v. Beneden to form the three permanent layers of the blastoderm; but this view has been disputed by Rauber, Kölliker, Lieberkuhn, and Heape, who maintain that in the rabbit and mole the middle stratum at this stage of development blends with Rauber's layer to form the primitive ectoderm. According to Bonnet there is in the sheep an addition to the middle layer from the peripheral thickened portion of the hypoblast, the cells of which are supposed to form the connective tissue and blood. "The appearance of a middle layer causes the originally bi-laminar blastoderm to be tri-laminar, and its three layers have received the names of ectoderm, mesoderm, and entoderm; or epiblast, mesoblast, and hypoblast."

The following table gives briefly the structures derived from the three layers in question :-

From the epiblast proceed-

(a) The nervous system, consisting of the brain, spinal cord, peripheral nerves and sympathetic nerves.

(b) The epithelial structures of the organs of special sense.

(c) The epidermis and its appendages (hair and nails).

(d) The mammary, sebaceous, and sweat glands, with the muscular fibres of the latter.

(e) The epithelium of the mouth and of the glands opening into it; the enamel of the teeth, \&c.

( $f$ ) The epithelium of the nasal passages, part of the pharynx, and the cavities and glands opening into the nasal passages.

$(g)$ The epithelium of the anus, vagina, and urethra. 


\section{DEVELOPMENT OF EMBRYONIC MEMBRANES OR LAYERS}

From the mesoblast proceed-

(h) The generative and urinary organs (the epithelium of the bladder and urethra excepted).

(i) The voluntary and involuntary muscles (the muscular fibres of the sweat glands excepted).

(j) The vascular and lymphatic systems, serous membranes, and spleen.

(k) The skeleton and connective tissues.

\section{From the hypoblast proceed-}

(l) The epithelium of the alimentary canal and the glands which open into it.

(m) The epithelium of the urinary bladder.

(n) The epithelium of the Eustachian tube, the tympanum, the bronchial tubes, and the air sacs of the lungs.

(o) The epithelium lining the vesicles of the thyroid body, and the epithelial nests of the thymus.

Some investigators, following His, have still further divided the blastoderm. They describe the true mesoderm as consisting of two epithelial lamellæ. According to them the complete blastoderm would consist of four layers ; namely, epiblast, outer or somatic mesoblast, inner or splanchnic mesoblast, and hypoblast.

The separation of the blastoderm into layers is followed by other changes in rapid succession. Very soon bloodvessels and a rudimentary heart, composed of nucleated cells (minus muscular fibres and nerves) make their appearance (Plate xc., Figs. 1, 3, 4, and 5). The heart, as already stated, is formed prior to its containing blood. The organ is made before it is called upon to discharge its peculiar function. The same holds true of other organs-the lungs, for example. The formation of organs before they are called upon to discharge specific functions affords the strongest possible evidence of design. This evidence is strengthened when it is borne in mind that the organs and systems are, in many cases, simultaneously produced. It is not a mere case of successive developments, but of successive and synchronous, continuous developments ; the Master-Architect and Builder carrying out His work in part piecemeal and in part by simultaneous, complicated, combined operations.

The heart is not only made before it contains blood, but it also assumes peristaltic rhythmic movements for propelling the blood when it reaches its cavities. The blood, therefore, is not the cause of the movements of the heart, as is generally believed. As a matter of fact, the heart, before it contains either muscular fibres, nerves, or blood, opens and closes with time-regulated beat, and quite independently. The same holds true of the so-called contractile vesicles in plants and animals. This is due to the circumstance that the heart is a containing as well as a propelling and transmitting organ. All the hollow viscera (stomach, intestine, bladder, uterus, \&c.), even in the adult, are endowed with similar powers. They are all containing as well as propelling organs.

The changes which occur in the heart and vascular system during the process of development and in the transition from the fotal to the adult state are of the most complicated and startling description, and will be adverted to further on when speaking of the placental, foetal, and adult circulations in man.

The developing chick lives upon the albuminous and other materials in its vicinity, plus a little moisture and air. Organ after organ appears in anticipation of the function to be discharged by it. The brain and nerves, alimentary canal, glands, lungs, lymphatics, muscles, bones, feathers, \&c., are all developed in a comparatively short period, and in advance of the peculiar work to be performed by each. The incubating process over, the completed bird emerges from what is practically an empty shell into the outer world, and immediately sets about procuring extraneous food.

If the development of the chick within the egg is marvellous, and a triumph of design infinitely greater than that achieved by the watchmaker when he laboriously constructs a watch, the advent of the fully-fledged chick on terra firma and its instant and eager pursuit of food are, in a sense, still more wonderful. What an extraordinary combination of foresight and power does the development and birth of a chick and its immediate search for food as an independent being imply. All the functions of its multiple existence are at once brought into play: its lungs breathe air, its alimentary canal digests food, its muscular and nervous systems enable it to run here, there, and everywhere, its blood circulates, its glands secrete and excrete, and its brain and sense organs connect it in the most effective way with the outer world, in which it is henceforth to live and move. Shall we be told, or if told, shall we believe, that the fully-developed living chick is the result of accident - a mere matter of spontaneous generation-a product of irritability and artificial stimulation, or forsooth of environment? 
PLATE XC;

Plate xc. illustrates development in the bird, fish, and mammal.

FIG. 1.-A. Egg of fowl at early stage of incubation. Shows unformed body of chick ( $a$ ), with area vasculosa (b) extending on either side and partly covering the vitellus $(c)$; all being bounded by the twisted chalaziferous membrane $\left(d, d^{\prime}\right)$.

B. The same further advanced. Head and body of chick (a) becoming defined; the body being developed by amnion at $b$, and having the umbilical vesicle $(d)$ depending from its under surface. The vascular allantois curves out from the under surface of the body, and spreads above it like an umbrella $(c)$. The allantois acts as a lung to the chick, and the umbilical vesicle as a stomach (after Dalton).

Fit. 2.-Egg of fish, showing the circulation in the vitellus or yolk (Dalton).

FIG. 3.-Young fish, showing formation of umbilical vesicle.

A. Vesicle seen depending as an open sac from ventral surface. Contains the food of the young fish.

B. Umbilical vesicle nore closed and communicating with future alimentary canal.

C. Umbilical vesicle of human embryo at fifth week (after Dalton).

FIG. 4.-A. Impregnated egg of fowl with allantois completed. ", Umbilical vesicle ; h, amnion ; r, allantois. Dalton).

B. The same further advanced ; alimentary canal beginning to make its appearance. ", Umbilical vesicle; $b$, allantois (after

FIG. 5.-The same still further advanced; the alimentary canal appearing as a convoluted tube. a, Pharynx, and proceeding from it the resophagus, stomach, alimentary canal, and bladder; $b$, heart; $r$, mesenteric arturies; $d$, umbilical vesicle and bloodvessels thereof, greatly reduced; $e$, large umbilical arteries passing to placenta $(f$ ) (after Dalton).

FI(:. 6.-Section of human uterus with the impregnated ovum (b) lodged in the mucous membrane (decidua vrru) (after Dalton).

Fif. 7.-Human uterus laid open to show the vagina, os uteri, interior of uterus, right Fallopian tube, dc. The darts indicate the direction of travel of the male and female elements (spermatozoid and ovum) after coitus. "Fundus of uterus ; $h$, right ovary; $r$, fimbriated extremity of the right Fallopian tube which seizes the ovum as it escapes from the ovary; $l, c$, right Fallopian tube laid open - shows route taken by ovum on its way to the uterus (see darts); $f$, os uterus laid open-the dart shows the route taken by the spernatozoon; the route is further indicated by the dart; $g$, the male and female elements meet either in the Fallopian tube, or in the uterns; $h$, portion of vagina ; $i$, os uteri.

Fı(x. 8.-A. Human ovum, about the end of the tirst nonth. ", Umbilical vesicle; l, amnion ; c, shaggy chorion; $d$, amniotic space ; $c$, body of embryo.

B. Human ovum at the end of the third month. ", Remains of shaggy chorion with villi developed to form the foetal portion of the placenta; $b$, chorion devoid of villi (after Dalton).

\section{§ 78. Subjects connected with the Development of the Human Embryo and Fœtus.}

The formation of the human embryo and fotus is, in some respects, more remarkable than that of the chick, inasmuch as the relation between the parent and offspring is much more intimate and of longer duration, and involves a double set of changes, namely, changes in the mother and changes in the progeny. The relations referred to, moreover, do not suddenly cease at parturition as in the case of the chick.

The impregnated human ovum, as happens in the bird, carries with it its own pabulum until it reaches and is, so to speak, grafted on the mucous lining of the uterus. Arrived there it is nourished by a process of osmosis by uterine fluids and gases, namely liquor sanguinis, oxygen, and glandular secretions. The nourishing process is largely mechanical, but in no sense due to irritability or artificial stimulation. Soon a series of the most delicate, involved, and far-reaching changes are inaugurated. The tender embryo is to be protected and housed as well as fed, and the most elaborate preparations are made for both purposes. The os uteri is plugged with mucus to guard against danger from without. Two thin but tough membranes or envelopes (the amnion and chorion) containing fluids are provided, so that the embryo may float daintily and freely, and be protected from any injury which may overtake the parent.

The ovum increases its absorbing surfaces by developing villi or hair-like processes on the chorion or outside membrane, which may very aptly be compared to the roots of a tree (Plate xcii., Fig. 1, F, G, H, I, J, L, page 396). By these processes it, at a later period, establishes a connection with the circulation of the mother by which it is nourished, and by which its own blood, when formed, is arirated (Plate xcv., Figs. 1, 2, 3, and 4, page 407).

The period of gestation in the human female extends over nine calendar months, and during that protracted term a series of the most astounding developments which it is possible to imagine occur. The impregnated ovum, from being a tiny round speck about the size of a small pin head, consisting of a vitelline membrane, vitellus, germinative vesicle, and germinative spot, slowly, but surely, assumes the human form.

In the human female child, long before the age of puberty (indeed at birth), a pair of ovaries crowded with immature ova make their appearance. At puberty certain of the ova or eggs, fully matured, burst in succession 


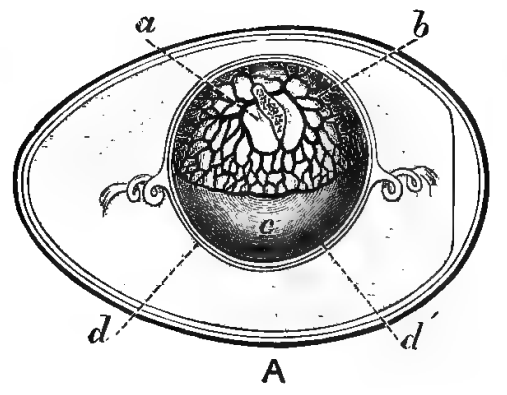

PLATE XO
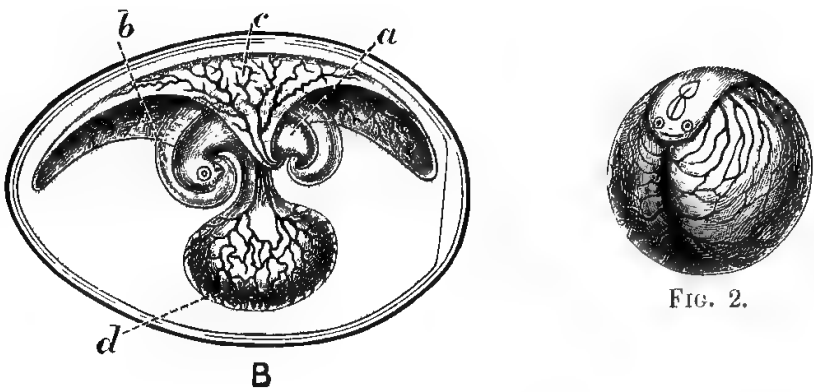

FII. 1.

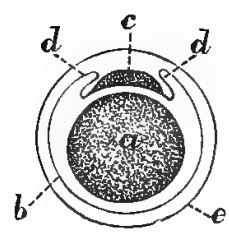

A

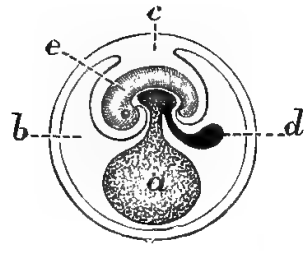

B
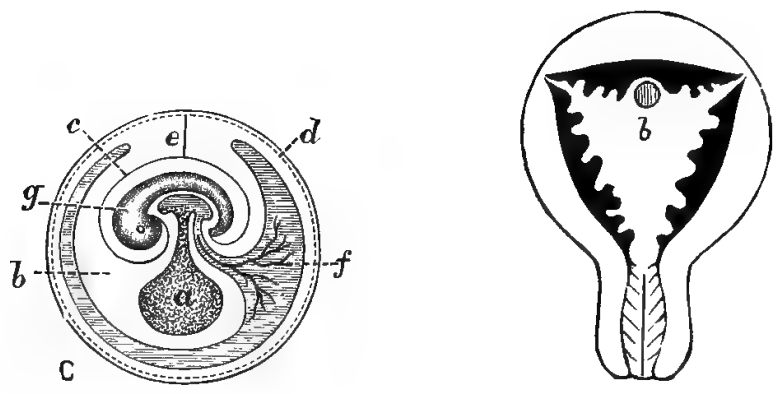

FIG. 6.

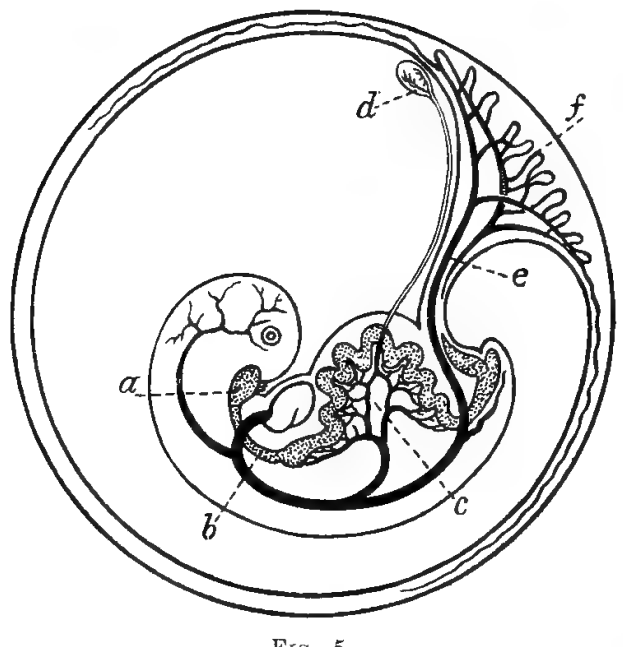

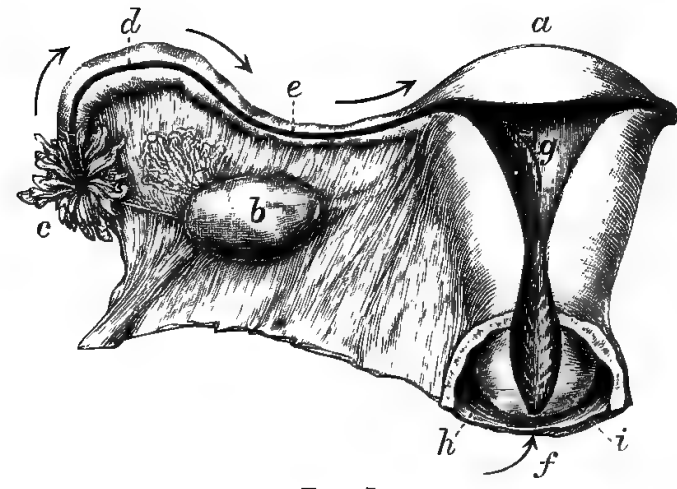

FIG 7
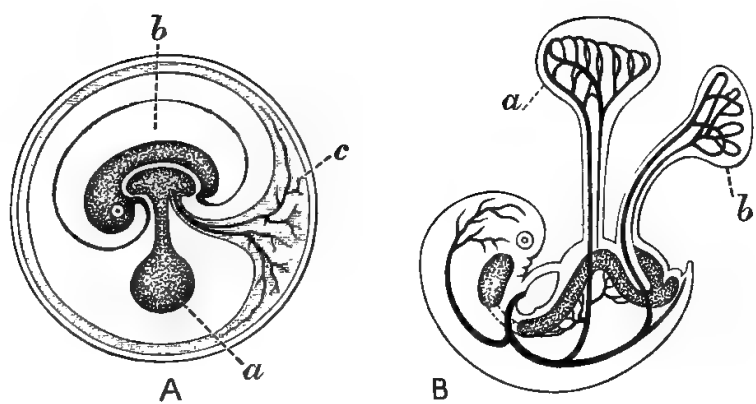

FIG. 4.

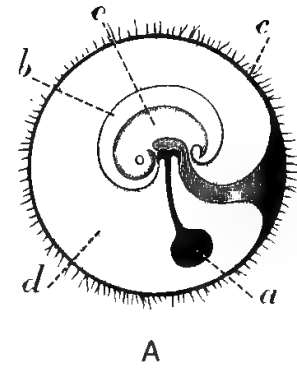

FIG. 8.

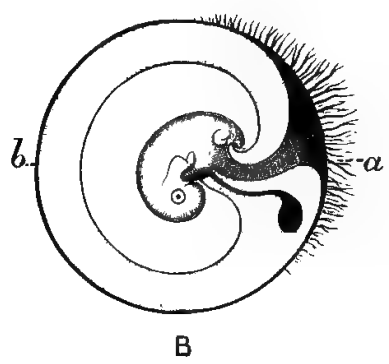


(at or about the monthly periods) the structures which confine them on the surface of the ovaries. The original production of the immature eggs, their ripening, and discharge from the ovaries cannot, even by a stretch of the imagination, be regarded as due to inherent irritability or stimulation of any kind, and still less to environment (Plate xci., Fig. 3, page 393).

The eggs are discharged for the most part singly or in pairs by an accumulation of serous fluid, it occasionally happening that three and even four or more eggs are liberated : a multiplicity of eggs meaning, as a rule, a multiplicity of offspring. The egg or eggs liberated from the surfaces of the ovaries tend to, and occasionally do, fall into the abdominal cavity, in which case the dangerous condition known as extra-uterine pregnancy occurs. In order to prevent such a catastrophe, nature, ever watchful, provides two special tubes called the Fallopian tubes to conduct them to the interior of the uterus. 'These tubes are very remarkable structures, and are armed with very ample powers. They spring from the horns of the uterus, and terminate in free, funnel-shaped, fimbriated extremities ; their function being to seize the mature egg or eggs and transmit them to the interior of the uterus (Plate xc., Fig. 7, page 391).

The manner in which they do this is of transcending interest, and affords one of the best-known examples of co-ordinated, vital action. It also affords a striking instance of design. The free, fimbriated extremities of the Fallopian tubes literally search for and find the ripe egg or eggs on the surface of the ovaries. These they seize as if with hands, and slowly transmit along their interior (largely by ciliary action), coating them in transitu with a layer of albumen which at once affords them nourishment and facilitates their progress. The Fallopian tubes exercise similar functions to those discharged by the oviduct of the bird already described. They perform their movements and provide their peculiar secretion quite apart from irritation or stimulation, as supposed. The egg or eggs, when once fairly lodged in the Fallopian tubes, are in the best possible position for impregnation, and here impregnation generally takes place. As already explained, it happens occasionally that gestation occurs in the tubes, and when it does, the abnormal and critical condition known as Fallopian tube pregnancy is established. At rare intervals the impregnated ovum escapes into the abdominal cavity. Abdominal and Fallopian tube pregnancies are, fortunately, exceedingly rare events. The world-renowned Cæsar was the product of an abdominal pregnancy, and gave his name to the formidable obstetric operation known as the "Crsarian section."

The spermatozoids, ova, uterus, \&c., are figured at Plate xci., page 393.

\section{PLATE XCI}

Plate xci. illustrates the developments of the brain : it also shows various kinds of spermatozoa, an ovum and ovary, and a human uterus (natural size).

Fig. 1.-A. A nerve tube with opening at either end.

B. Expansion and bulging of brain substance at anterior end of nerve tube.

C. Expansion and division of nerve tube into three primary vesicles : I. forebrain ; II. midbrain ; III. hindbrain.

D. Further expansion and subdivisiun into five primary vesicles. $a$, Cerebrum ; $b$, thalamencephalon ; $c$, optic lobes ; $d$, cerebellum; $e$, medulla oblongata. The primary nerve tube is now known as spinal cord $(f)$.

E. Further expansion and differentiation. $a$, Cerebrum ; $b$, pineal body ; $r$, optic lobe ; $d$, cerebellum ; $e$, medulla oblongata ; $f$, spinal cord ; $g$, olfactory lobe; $h$, pituitary body ; $i$, notochord (after Huxley).

FIG. 2.-Spiral spermatozoa of various kinds.

A. Spermatozoon of Triton (Titon cristutus), $\times 450$.

B. Spermatozoa of rabbit (Lepus cuniculus), $\times \mathbf{4 5 0}$.

C. Spermatozoon of field mouse (Arvirola arvalis), $\times 450$.

D. Spermatozoon of wood shrike (Lrenius rufus), $\times 450$.

E. Spermatozoon of gold finch (Fringillu elegans), $\times 450$.

F. Spermatozoon of Perca (Perra fuviatilis), $\times 450$.

G. Spermatozoon of blackbird (T'urdus merula), $\times 450$.

H. Spermatozoon of man (Homo sapiens), $\times 450$.

I. Spermatozoon of frog (Rana temporatio), $\times 450$.

J. Spermatozoon of rat (Mus rattus), $\times 450$.

K. Spermatic cyst of common creeper bird (C'erthiu femitionis), containing a bundle of spermatozoa, $\times 500$.

L. Spermatic cyst of rabbit. "The globules, each of which contains a spermatozoon, $\times 350$; $b$, separate globuli, $\times 500$ (after Griffith and Henfrey).

FIG. 3.-Portion of human ovary with mature and immature ova. a, Mature follicle and ovum, consisting of membrana granulosa, zona pellucida, vitellus, germinal vesicle, and germinal spot; $b$, epithelium ; $c$, stroma; $d$, immature ova ; $e$, immature follicle
(after Fothergill). 
PLATE XCI

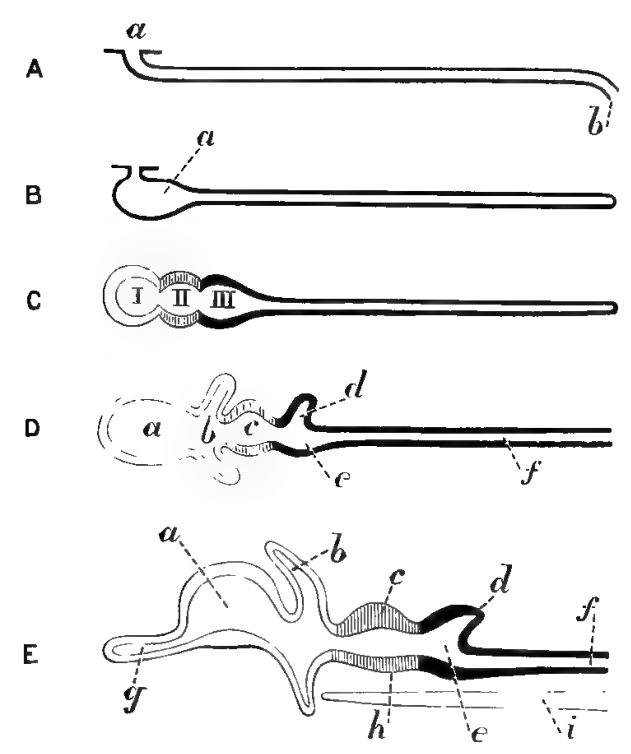

FI $\div 1$.

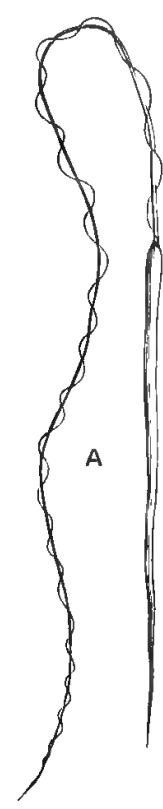

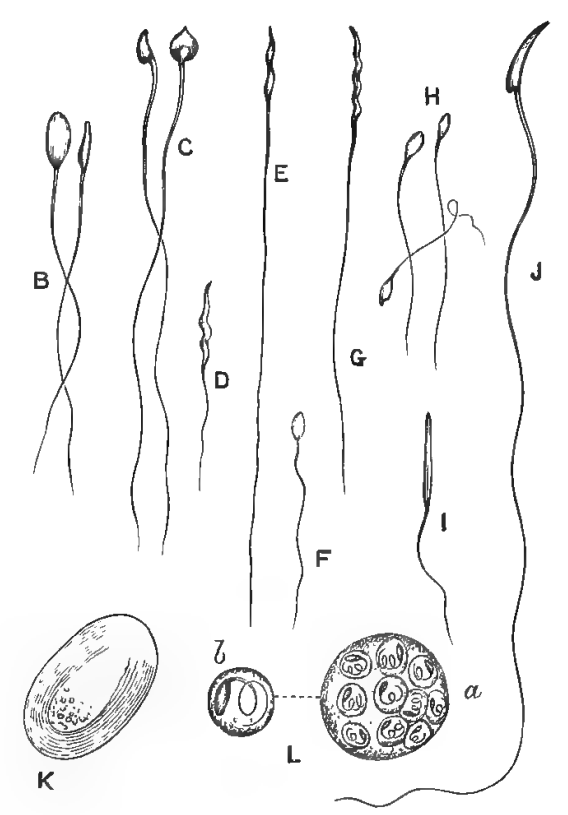

FIG. 2.

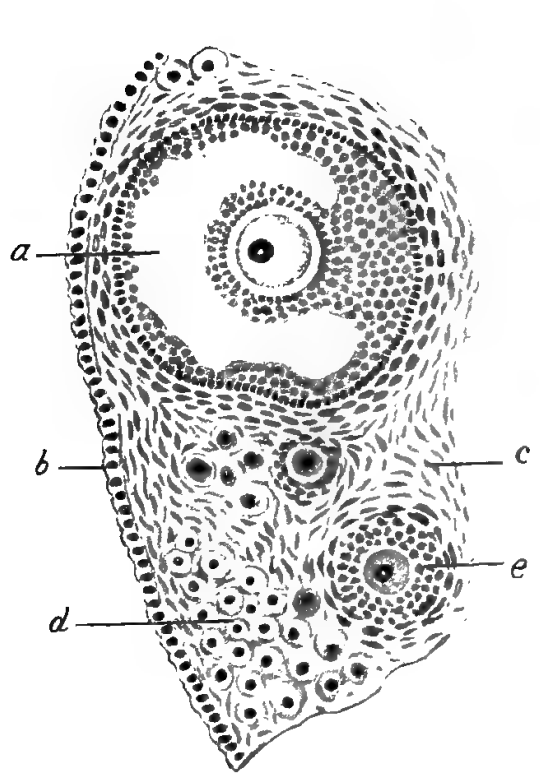

FIG. 3.

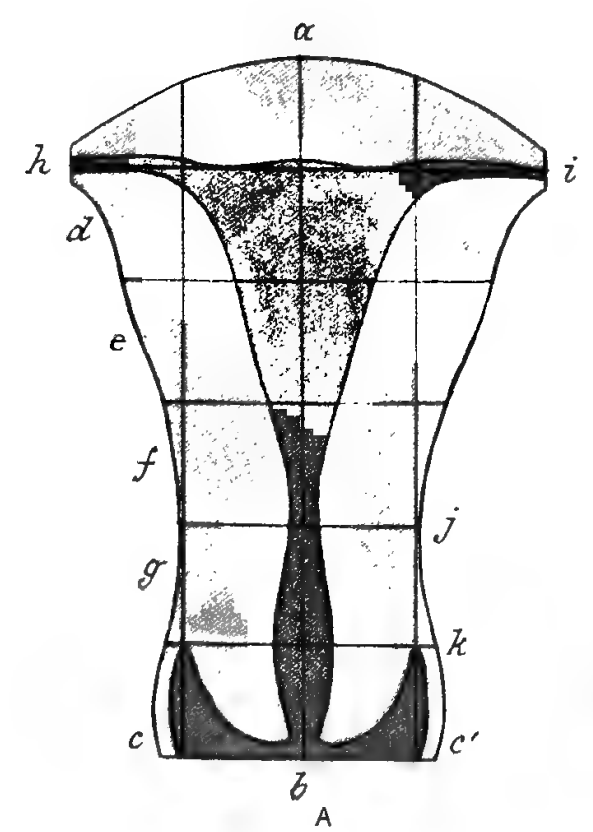

lini. 4.

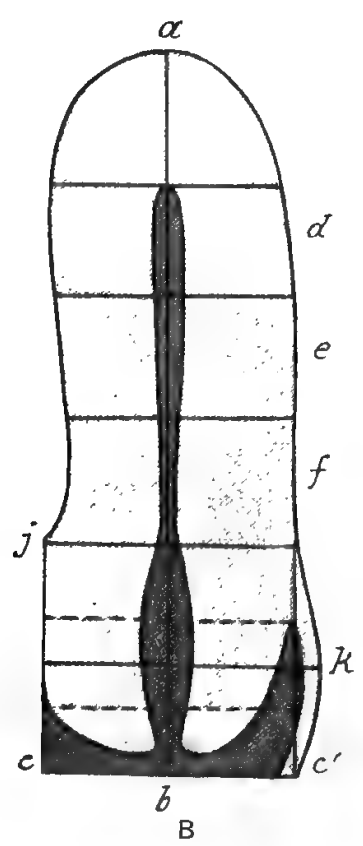

B

PLATE XCI-continued

FIG. 4.-Coronal and sagittal sections of human uterus drawn over half-inch squares to show the natural size of the organ. The same letters are employed in Figs. A and B.

A (Coronal section). a, $h$, Mesial line section of uterus, showing fundus ( $(l)$ and external os uteri $(l) ; c$, $c^{\prime}$, section of upper portion of vagina; $d, e, f, g$, portions into which the uterus may be divided; $h$, $i$, right and left Fallopian tubes entering the triangular-shaped cavity of the uterus; $j$, contraction corresponding with the internal os uteri and separating the triangular cavity of the uterus from the spindle-shaped cervix of the uterus; $k$, line intersecting the spindle-shaped cervix of the uterus.

B (Sagittal section). ",$b$, Mesial line section of uterus, showing fundus ( $(1)$ and external os uteri $(b) ; c$, $c$, section of upper portion of vagina; $d, e, f$, portions into which the uterus may be divided; $j$, constriction corresponding with the internal os uteri; $k$, line intersecting the spindle-shaped cervix of the uterus (after Fothergill).

VOL. I. 


\section{$\S 79$. Career of the Unimpregnated and Impregnated Human Ovum.}

There is the greatest possible difference between the unimpregnated and impregnated ovum. The former, although a living thing, dies sooner or later after reaching the uterus, and is, in due course, discharged with the catamenia. The impregnated ovum, on the other hand, quickened as it were by contact with the male element, embarks upon a new career; upon a series of the most complicated and extraordinary changes with which it is possible for even the professional mind to deal.

Those who endeavour to explain all physiological action by the theory of irritability and artificial stimulation would doubtless argue that the male element (spermatozoon) irritates and excites the female element (ovum). The argument, if employed, would be a mere assumption. The male and female elements are equally alive : they are independent yet interdependent entities, and are absolutely necessary to each other so far as reproduction is concerned. The male element is not more necessary to this act than the female element. They are physiologically of equal value. Their coming together and interpenetration is not due to irritation or extraneous stimulation, as generally understood, but to pre-determined vital arrangements. The male element seeks the female one, and when it finds it, it literally buries itself therein : the lashing, vibratile movements of the spermatozoon cannot be referred to the virtually stationary ovum which, at the outset, is nowhere near. The spermatozoon is endowed with independent movements, and the ovum is moved along by the action of cilia in the Fallopian tube ; it being arranged that the male and female elements shall infallibly meet. The movements of the spermatozoon, the cilia, and the ovum are all independent of each other. They are simply means to ends, and the palpable outcome of design. There is no need for either irritability or stimulation in the case.

In the whole range of science I question if a better example of design, or of adaptation of means to ends, can be found than is supplied by the history of a human ovum before and after impregnation. The impregnated human ovum runs through a whole gamut of changes, some of them as puzzling as they are formidable. It is to be credited with changes in itself, as well as changes in the mother, of the most far-reaching description, and I venture to assert that not one of the numerous changes referred to can be regarded as accidental or the result of irritation or artificial stimulation. The changes are in every instance spontaneous, independent, and vital, and they are all foreseen and provided for from the beginning. The changes are the result of co-ordinated movements in the hard and soft parts, partly in the fotus and partly in the mother; and the changes in the mother keep pace with those in the fœtus, so that when the period of parturition arrives the child is free to breathe pure air and regale itself with maternal milk prepared for it beforehand. This production of a nutrient, all-sustaining fluid, constitutes the final stage of a superlatively interesting series of developmental acts. The babe and its milk are no chance products. They come together at precisely the right time, and afford overwhelming evidence not only of a Designer, but also of an overruling and ever-watchful Providence.

\section{§ 80. Development of the Brain and Vessels of the Body-Placental and Fœtal Circulation-Successive Changes witnessed in the Growing Parts.}

It may interest the reader and serve a good purpose if, at this stage, I give a very brief account of the history of an impregnated human ovum.

The impregnated ovum, when it reaches the interior of the uterus, which it usually does about the eighth day after coitus, is, roughly speaking, about the size of a small pin head, and consists of a vitellus and vitelline membrane ; the germination vesicle and germinative spot (which are prominent parts of the ovum before it leaves the ovary) having disappeared. The ovum when it first reaches the Fallopian tube measures as nearly as may be $i_{2}^{2} \overline{5}$ of an inch, and when it leaves the tube from $\frac{3}{i 0}$ to $\frac{1}{25}$ of an inch.

The living, impregnated ovum attaches itself to the living mucous surface of the interior of the uterus, usually its posterior portion. The union is at first of the most casual description. By-and-by it becomes more intimate, but at best it is temporary in character ; the ovum, the embryo, and the fœetus, as I pointed out in $1872{ }^{1}{ }^{1}$ occupying the position of parasites as far as the parent is concerned. They are living things which derive their sustenance from the mother, and when the period of parturition arrives, the temporary, but withal intimate, union is severed by a natural process akin to the separation of the leaf from a tree in autumn. Neither the original union with, nor final separation from, the parent occasions much difficulty, both being natural processes.

That part of the mucous lining of the uterus which the ovum originally touches, and to which it adheres, is termed the decidua serotina, the parts of the lining in its vicinity the decidua vera, and the parts which are said to curve up over the ovum (a modification which I have never seen demonstrated) the decidua reflexa. When the ovum has comfortably settled itself in the mucous lining of the uterus of the mother, it immediately begins to imbibe

\footnotetext{
1 "The Physiology of the Cinculation in Plants, in the Lower Animals, and in Mun." Edinburgh Medical Journal, $1872-73$.
} 


\section{DEVELOPMENT OF THE BRAIN AND VESSELS OF THE BODY}

nourishment by a vital osmosis; the pabulum being, as indicated, mainly supplied by the liquor sanguinis, by the uterine glands and secretions of the mother. As yet the ovum reveals no internal structure. Very soon the process of segmentation of the vitellus and the formation of the blastodermic and other membranes begin. This process has been already described, and need not be repeated. In the human embryo and foetus various and important accessory structures and fluids are developed and produced. The chief of them are the chorion, amnion, and their special fluids ; the umbilical vesicle, the allantois, the umbilical cord, and the fotal portion of the placenta. They are seen at Plate xcii., Fig. 1, page 396.

\section{PLATE XOII}

Plate xcii. illustrates the development of the impregnated human ovum in its several stages as embryo and foetus ; also the development of the brain, blood-vessels, \&c.

FIG. 1.-A to L inchusive. Represent changes occurring in the impregnated human ovum during gestation (after Crusius).

A. Human ovum two weeks old, natural size.

B. Human ovum two weeks old, twice natural size. ", Ovum embedded in mucous lining of uterus, namely, the decidua vera $(b, b)$, decidua serotina $(c)$, and decidua reflexa $(d) ; e$, villi of chorin.

C. Human embryo three weeks old, half natural size. a, Body of embryo; $b$, decidua ; $c$, amnion ; $d$, chorin ; $e$, villi of chorin ; $f$, umbilical vesicle.

D. The same as C, three times natural size (lateral view). 1, b, c, Anterior, middle, and posterior brain; $d$, eye vesicle; $\ell$, ear vesicle ; $f$, visceral arches; $g$, navel; $h$, primitive segments (vertebral column); $i$, amnion; $j$, allantois; $k$, umbilical vesicle.

E. The same as D, still more magnified (frontal view). a, $b$, Frontal and mid-brain; "l, eye vesicle placed laterally ; $l$, aperture of mouth; $s$, maxillary process; $f$, the four visceral arches. By the third week the embryo measures one-sixth of an inch in length and is covered with villi ; the allantois reaches the chorion ; the primitive circulation is established, the back is curved, and visceral arches appear. Traces of primary brain, eye and ear vesicles are distinguishable. The alimentary canal occurs as a straight tube and the mouth as a five-sided orifice.

F. Human embryo four weeks old, natural size. a, Body of embryo; ", amnion; $d$, chorion with villi $(e)$; $f$, cuvity of chorion ; $g$, navel; $h$, umbilical vesicle.

By the fourth week the embryo measures one-third of an inch in length. The chorion is wholly covered with villi and the umbilical vesicle fully developed. The eyes are seen as two black dots, the superior and lower extremities appearing as buds. The proto-vertebræ (future vertebral column) are also well marked, and terminate in a caudal portion or tail.

G. Human embryo four weeks old, three times natural size. "H, Head with eye, ear, and anterior, middle, and posterior brain ; $f$, visceral arches; $i$, amnion ; $m$, superior and inferior $(n)$ limbs appearing as buds ; $h, h$, rudimentary column ending in a caudal portion; 0 , foetal portion of placenta; $k$, umbilical vesicle.

H. Human uterus with embryo five weeks old (semi-diagrammatic). a, a, ", Walls of uterus ; b, cavity of uterus ; 4 , os uteri; $d$, cervix uteri with plug of mucus to seal the entrance; $e$, decidua vera; $f$, decidua reflexa; $g$, decidua serotina; $h$, villi of chorion. The embryo is seen enlarged at I.

I. Human embryo five weeks old, twice natural size. a, Head with face, eyes, ears assuming shape; $c$, amnion invests embryo ; $m, n$, superior and inferior extremities growing, fingers and toes forming; 0 , foetal portion of placenta with villi composed of fine loops of blood-vessels ; $l$, allantoic vein $; r$, chorion ; $k$, yolk sac and vitellus ; $l$, vitelline stalk.

J. Human fuetus seven weeks old, half natural size. (The term fuetus takes the place of embryo when the parts assume shape.) $r$, Chorion; $s$, villi of chorion; $t$, blood-vessels of umbilical cord spreading on the inside of the chorion; $u$, amnion enveloping fuetus: $v$, body of foetus ; $1 "$, umbilical vesicle.

K. Human foetus eight weeks old. The shape of the body is now clearly indicated. $a, b$, $c$, anterior, middle, and posterior portions of head and brain; $d$, eyelids forming; $e$, ear forming; $m$, superior and inferior $(n$ ) extremities show main divisions of limbs, and the hands and feet fairly formed; $r$, body of uterus assuming shape, the caudal portion diminishing.

L. Human foetus twelve weeks old within the amnion, half natural size. $u$, Amnion with fluid investing the fcetus and guarding it against accident; $i$, chorion ; $o, o$, villi of chorion with fine loops of blood-vessels forming furtal portion of placenta; $x$, body of futus well formed; head, face, eyes, ears, and superior and inferior extremities well defined.

Note.-For placental and fuetal circulation, see Plate xcv., Figs. 1 and 3, p. 407.

FIG. 2.-Plan of section of uterus with fully formed ovum (human). g, Mucous lining or membrane of uterus, opposite placenta (decidua serotina of authors); $h, e$, lining membrane on body of uterus (decidua vera of authors); $d$, lining membrane of foetus (decidua reflexa of authors). This membrane (e) is found on the free surfaces of the chorionic villi (a), being in fact the mucous lining of the chorion. Such parts of it as are not engaged in covering the chorionic villi become thinned away and disappear towards the full term. It may, however, always be found on the free or uterine surface of a normal placenta. a, Chorionic villi constituting fuetal portion of placenta (the maternal portion is formed by capillary vessels found in mucous lining, $/ 1$ ); $/$, chorion, from which the chorionic villi spring ; $f$, amnion ; $i$, unbilical vesicle; $j$, Fallopian tube.

Note-As pregnancy advances the parts marked $e, n, l$ approach each other, and become inculately apposed. The same holds true of the parts marked $b$ and $d$. This apposition and blending of maternal and foetal structures facilitates the exchange (chiefly by osmosis) of nutritive and effete materials between parent and offspring (the Author).

FIG. 3.-Face or front views of various embryos as seen in man (A), in the cat (B), in the sheep (C), and in the bat (D) (after Hackel). These resemble each other, and bespeak a general plan as represented by types and as apart from and independent of evolution.

FIG. 4.-Circulatory systems of the fretus.

A. Truncus arteriosus, with one pair of aurtic arches $\left(a, a^{\prime}\right)$, and dotted lines indicating the future position of the second and third pairs of aortic arches. These are numbered $1,2,3 . \quad b$, Thoracic aorta.

B. Truncus arteriosus with four pairs of aortic arches $\left(a, a^{\prime}\right)$, and indications of the fifth aortic arch. The aortic arches are numbered $1,2,3,4,5 . \quad b$, Thoracic aorta ; 1 , external carotid ; $d$, internal carotid.

C. Truncus arteriosus with three posterior pairs of aortic arches ( $\left(a, a^{\prime}\right)$ from which the permanent vessels of the embryo are developed, with dotted lines showing the position of the two (now) obliterated anterior arches $\left(b, b^{\prime}\right)$. $\quad c^{\prime}$, External carotid; $d^{\text {, in- }}$ ternal carotid ; $e$, thoracic aorta ; $f, f^{\prime}$, vertebral arteries; $g, g^{\prime}$, axillary arteries ; $h$, subclavian arteries. 

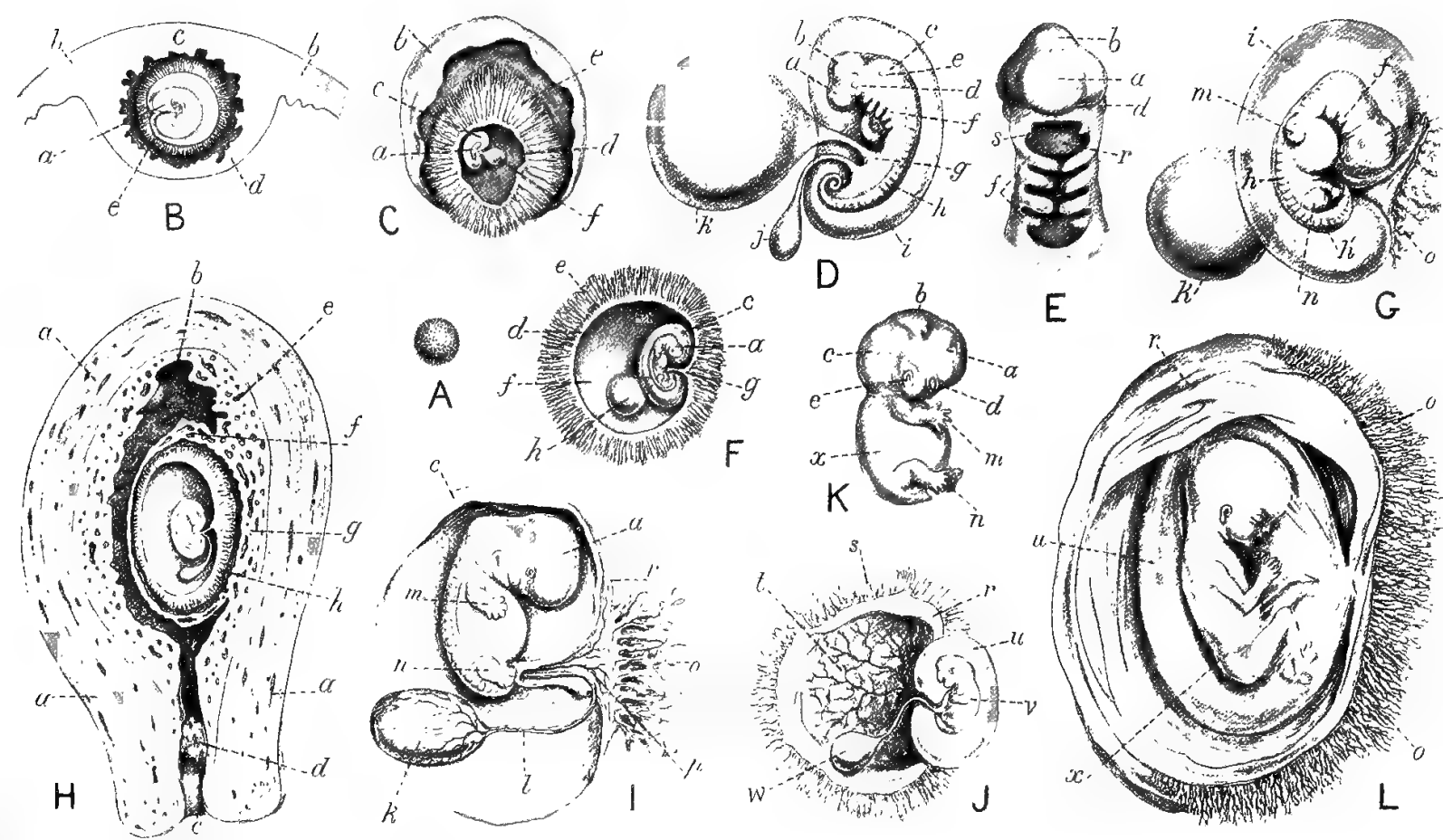

F'TG. 1.
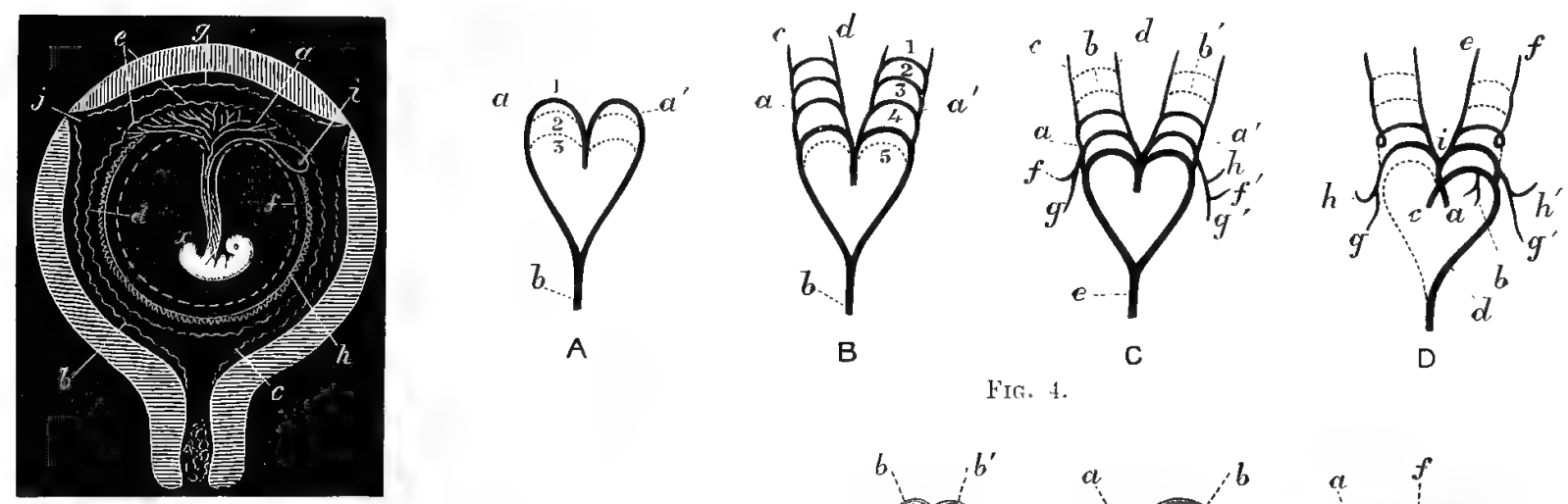

$$
\text { FIT. } 4 .
$$

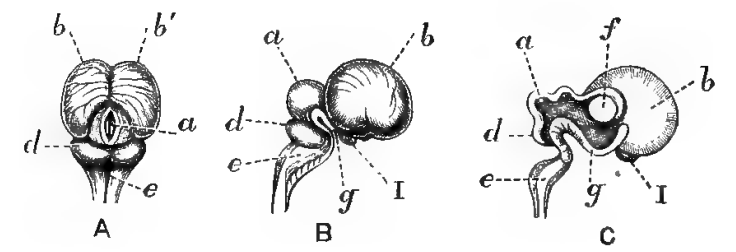

Fit: 5.
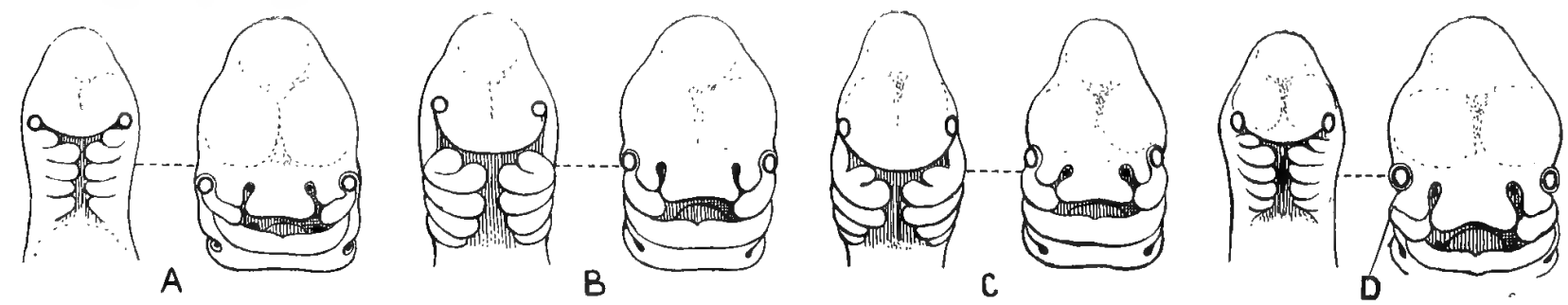

FII. 3. 
PLATE XCII (continued)

D. Permanent arterial trunks in their primitive form, the obliterated portions still shown in dotted lines. ", Aorta; $b$, branches to the lungs; 1 , pulmonary artery ; $d$, root of thoracic aorta ; $c$, internal and external $(f)$ carotids ; $g, l^{\prime}$, axillary arteries ; $h, h^{\prime}$, vertebral arteries; $i$, common carotid artery (after Carpenter).

FIG. 5.-Formation of cerebro-spinal axis. ", Vesicle of the hemispheres; $b$, vesicle of the tubercula quadrigemina; $c$, vesicle of the medulla oblongata (after Dalton).

FIG. 6.-Brain of human embryo at twelfth week.

A. Seen from behind.

B. Side view.

C. Sectional view. The same letters apply to ull the figures. ", Corpord quadrigemina; $b, h^{\prime}$, hemispheres; $d$, cerebellum; $e$, medulla oblongata ; $f$, optic thalanus ; $g$, floor of third ventricle; 1 , olfactory nerve (after Carpenter).

The ovum, as stated, generally reaches the interior of the uterus about the eighth day. At the twelfth or fourteenth day it is a rounded, flattened sac, and measures from three to five millimetres. It is smooth unless at the equatorial zone, where short, unbranched villi appear. At the end of the second week the embryo is discernible, and measures about $\frac{1}{12}$ of an inch. The amnion and allantois also make their appearance at this period. It is not necessary to go into the structure of the several parts forming the embryo and foetus ; suffice it to say, that the amnion is a delicate but tough membranous envelope which invests the embryo and contains a fluid in which the embryo floats ; the umbilical vesicle being the precursor of the umbilical cord or navel string, and the allantois that of the bladder. The chorion (shaggy chorion) is the outer covering or membrane of the embryo, which in its early stage is covered with villi or hair-like processes which play an important part in nourishing the embryo. In the later stages of gestation the chorion only retains part of its villi ; but these are modified and differentiated, and contain a very large number of looped, capillary blood-vessels, which with cells, cellular and other tissue, form the foetal part of the placenta (Plate xcii., Fig. 1, I, page 396). As the looped capillary blood-vessels of the chorion when gestation is well advanced interdigitate with the capillary blood-vessels in the mucous membrane of the uterus (maternal part of placenta), they play a leading rôle in nourishing and a rating the blood of the foetus (Plate xcv., Figs. 1 and 3 , page 407). Of course the aëration of the blood of the foetus is primarily effected in the lungs of the mother. In like manner the nourishment of the foetus is primarily traceable to the operations of the stomach and alimentary canal of the mother. It is, however, through the thin, osmotic walls of the capillary blood-vessels of the fœtal and maternal portions of the placenta that the actual interchange of aerated and specially enriched blood on the part of the mother, and of more or less impoverished, impure blood on the part of the fœtus, takes place. The placenta at once acts as a lung and a stomach to the fotus. The changes which occur in it are vito-mechanical and chemical in their nature. The blood of the foetus parts with carbonic acid and obtains oxygen; a very necessary interchange, as fœtal blood is, at best, a mixed, impure blood. The hearts of both mother and fœtus are at work in carrying pure and impure blood to and from the placenta, and the delicate, thin walls of the innumerable capillary bloodvessels of the maternal and fœetal portions of the placenta act as osmotic media for the constant interchange of fluids and gases alike. All these arrangements are in no way due to irritability of the parts concerned, or to extraneous stimulation of any kind. They are one and all natural and necessary processes, and take part according to original design in the great and pre-arranged scheme of reproduction. The vito-mechanical arrangements, and the complicated and extensive series of reactions, are all necessary to bring about the desired result, and nothing is left to chance.

The placenta is the indirect means of communication between the mother and fotus, and it is through this temporary bond of union that both are influenced. The placenta is an indirect mode of communication, because the blood of the mother in its entirety does not pass to the fœtus or that of the fotus to the mother; the interchange being partial, and confined to the gases and finer fluid parts of the blood in either case. The interchange, as explained, is osmotic in its nature, there being no open vessels either in the maternal or fœetal portions of the placenta.

The impregnated human ovum very soon begins the work of differentiation. At the end of the second week it is about the size of a small pea; the entire ovum measuring about $\frac{1}{4}$ of an inch, and the embryo $\frac{1}{12}$ of an inch. The amnion, allantois, and first rudiments of the embryo appear. The chorion and its villi are also indicated. At the end of the third week the structures referred to are more developed, the embryo as a whole measuring $\frac{1}{6}$ of an inch. At this period (third week) the shaggy chorion (chorion plus villi) is in evidence, and traces of the primary division of the brain, the cephalic visceral arches, the primitive ocular and auditory vesicles, the primary circulation and a straight alimentary canal with a pentagonal-shaped mouth can be traced.

At the end of the fourth week the embryo with its coverings is about the size of a pigeon's egg; the embryo itself measuring $\frac{1}{3}$ of an inch in length. At this period (fourth week) the whole chorion is covered with villi, the umbilical vesicle fully developed, and the body curved upon itself almost in a circle. The segmentation of the trunk is also indicated by visceral arches and by square-shaped proto-vertebræ; the future spinal column terminating in 
what is, practically, a curved tail. The superior and inferior extremities also appear as four buds: the eyes as two black dots.

At the end of the fifth week the body is less curved and the head more erect; the limbs showing signs of differentiation into arm, forearm, and hand in the case of the superior extremity, and into thigh, leg, and foot in the case of the inferior extremity.

Between the fifth and sixth week the embryo measures about 15 millimetres in length, and is contained in a chorionic vesicle about 40 millimetres in diameter. At this period the allantois becomes vascular throughout.

At six weeks the embryo measures about 19 millimetres in length, the rudiments of eyelids are seen, and the concha of the ear is indicated. The umbilical vesicle ceases to be active, and the fingers and toes are separated. The embryo, having assumed a more or less distinct human form, is now called a foetus.

At the end of eight weeks (second month) the foetus measures 25 millimetres in length ( 1 inch); the tail beginning to disappear. At nine weeks or so (beginning of third month) the foetus and its coverings are nearly the size of a hen's egg, the fotus measuring from 25 to 30 millimetres ( 1 to $1 \frac{1}{4}$ inch) : the umbilical cord has lengthened considerably, but as yet its blood-vessels are not twisted round each other. The arm, forearm, and hand, and the thigh, leg, and foot are distinctly mapped out; the tail has also disappeared.

At ten weeks (two and a half months) the fotus measures 32 millimetres and traces of sex appear; the genital tubercle projecting about 1.5 millimetres, and having a knob-like extremity-the future glans penis or clitoris. On the under surface of the tubercle the genital groove bounded by the genital folds (the nymphæ in the female) appears. The genital tubercle has on either side fleshy ridges which in the female form the labia majora and in the male the serotum.

At seventy-five days, or about eleven weeks, the fotus measures 55 millimetres; the eyelids are fairly well formed and the nails and ribs begin to show themselves.

At eighty-four days (towards the end of third month) the foetus measures from 75 to 80 millimetres, or about 3 inches in length. The fotal portion of the placenta is now formed, the chorion having shed more than half its villi : the remaining villi dividing and displaying looped capillary blood-vessels. The umbilical cord is also considerably elongated and twisted, the umbilical vein and the two umbilical arteries twining round each other. The umbilical vesicle and allantois have also disappeared.

At three and a half months the foetus measures $4 \frac{1}{4}$ inches; the placenta and umbilical cord continuing to develop.

Towards the end of the fourth month the fœetus measures about 125 millimetres or 5 inches; the placenta has increased in size and thickness, and the umbilical cord, which now measures 3 inches in length, has been thickened by the addition of Wharton's Jelly.

The chorion and amnion have united, the eyes, ears, nose, and mouth have assumed their proper shape, and the sex can be readily determined.

At four and a half months the foetus measures 6 inches or thereby. The skin has assumed a rosy hue, and hair appears on the scalp and elsewhere.

Towards the end of the fifth month the foetus measures about 8 inches, and weighs a pound or thereabouts. The placenta and umbilical cord are well developed and the foetal circulation is established.

The limbs are shapely, the nails fairly well formed, and the movements of the fotus distinct.

At the end of the sixth month the fotus measures about 12 inches and weighs about a pound and a half. The skin is loose and wrinkled, eyebrows and eyelashes can be detected, and the nails begin to project over the extremities of the fingers and toes.

It is not necessary to follow the development of the foetus further. Enough has been said to show that by a series of successive developments, the child in utero becomes more and more perfect; the developments being traceable to continued growth and differentiation in particular directions; each differentiation plainly carrying out a part of a pre-conceived plan or design. There is also suppression of growing parts.

The only thing that remains in this connection is the segmented character of the fotus, and the placental and fotal circulation (Plate xcii., Fig. 3, page 396; Plate xciii., Figs. 7 and 9, page 403; Plate xciv., Figs. 1 and 3, page 404; Plate xcv., Figs. 1, 2, 3, 5, and 6, page 407).

It is a common belief that the human foetus during gestation passes through the several changes and stages which characterise the adult fish, amphibian, reptile, and bird, to say nothing of all animals lower in the scale of being, even to the protiston, which consists of an amorphous speck of protoplasm. Some go further and assert that the kaleidoscopic changes referred to afford crowning proof of the truth of the doctrine of evolution. I am not of this way of thinking. The doctrine, though $\dot{t}$ priori plausible, is not, in my opinion, tenable. There is nothing known to me in geology, botany, zoology, or physiology which seriously supports it. There are, for example, gaps 
and missing links in the geological record which cannot be bridged over or satisfactorily accounted for; plants and animals are persistent to a practically unlimited extent : species, if produced by natural selection and modification, should never reach a point where further modification is impossible; plants and animals, if cultivated and then left to themselves, invariably breed back to their originals; they deteriorate as well as advance; sterility follows attempts at interbreeding plants and animals in the absence of affinities, and where they do not belong to the same root stock. All the peculiarities attributed to evolution can, it appears to me, be more readily and satisfactorily explained by the existence of progressive, that is, gradually ascending types; each type having prescribed limits. Evolution, strictly speaking, can only apply to development and advance within types. It does not date back to, or account for, the beginnings of life. ${ }^{1}$ Similar remarks are to be made of heredity. This extends only to types and centres of departure, and has a limited range. It is not a continuous advance from the lowest to the highest in either plant or animal. Reproduction, development, and heredity are parts of the same problem, and what is true of the one is true of the other. To be convinced of this, it is only necessary to bear in mind that in the inorganic, as well as in the organic kingdom, each substance plays a distinct rôle, its relations and combinations being conditioned and pre-determined. The elements are separate and distinct; fusion and union being possible only up to a certain point. So in plants and animals. All plants and animals are not originally formed out of exactly the same materials. Even protoplasm (the so-called physical basis of life), which is almost universally regarded as a simple homogeneous substance, identical in all its parts and particles, is composed of oxygen, hydrogen, nitrogen, and carbon (four elements), and to these, in many cases, are to be added varying proportions of sulphur and other elements in small but appreciable quantities. The cells, moreover, which according to the Germans produce protoplasm, in some instances contain glycogen, in others cholesterin, in others protagon, and in others myosin. There is a protoplasm for the several kinds of plants and animals, each producing its own kind; there is even a protoplasm for each of the tissues, nerve, brain, bone, and muscle. Some protoplasm produces fat, some pepsine, and some pigment. As Stricker explains, protoplasm may be fluid, semi-fluid, or firm and resisting. It also varies greatly in shape, being at times club-shaped, bottle-shaped, spindle-shaped, branched, prismatic, and polyhedral. Protoplasm, it will be observed, is not the simple substance it is claimed to be. If, however, it is compound (and this must be admitted), the argument in favour of evolution in the wider sense disappears. That doctrine, as is well known, refers the origin of all plants and animals to protoplasm as seen in the protiston, amoeba, and other low rudimentary forms. Some, as stated, even assert that plants and animals are evolved from dead matter (spontaneous generation). If, as explained, protoplasm is not a simple homogeneous substance, then it follows that plants and animals have not a simple but a complex origin, and the differentiations which characterise them, even up to man, are original endowments; a state of matters which naturally results in types; the types being conditioned, with boundaries in time and space.

In the inorganic and organic kingdoms there is, as has been pointed out, a tendency on the part of the substances composing them to split up longitudinally and transversely, to radiate, to arrange themselves in concentric lines, and to assume spiral and other shapes. These arrangements give rise to typical leading forms which are persistent and permanent. Thus in igneous rocks longitudinal and transverse cleavages are seen (Plate xl., Figs. 1 and 2 ; see also Figs. $3,4,6,8$, and 9 , page 63$)$; in certain crystals radiating and concentric arrangements are met with (Plate i., page 3); in other cases dendritic or branching arrangements occur (Plate ii., page 5; Plate xxxiv., and xxxv., pages 54 and 55); while in others curved and spiral arrangements are encountered (Plates $x$. and xi., pages 24 and 25 ; Plates xiii. and xiv., pages 28 and 29; Plates xviii. and xix., pages 34 and 35). Different kinds of matter under particular conditions assume certain forms, but there is a limit to the forms, and everything bespeaks design, law, and order.

The histories of plants and animals, and especially their reproduction, favour this view. The more rudimentary plants and animals, as has been already fully explained, reproduce themselves by simple division of their substance as apart from sexual organs. They are equal to the performance of every function of life, and are potential living entities from the first. When sexual organs make their appearance the male and female elements frequently occur in the same individual. In other cases they occur in different individuals; a great variety of co-ordinated movements being required to bring the sexual elements (always molecular and cellular in character) into contact. Fructification and development proceed on given lines; the offispring in every case closely resembling the parent or parents. It is not a case of plants and animals proceeding from those immediately below them by unbroken continuity of substance, but of plants and animals created on higher levels according to a common plan; the plants and animals being conditioned and forming types with more or less well-defined boundaries, the possible variations being limited.

1 Extreme evolutionists, who believe in spontaneons generation, regard the organie kingdom as the product of the inorganic kingdom, as apart from a Creator, Designer, and First Cause. 
It is quite true that the more simple plants and animals appeared on the earth before the more complex ones, and that the more complicated structures and organs in both have comparatively simple beginnings. This fact, however, does not prove the doctrine of evolution. If it proves anything it proves that design, law, and order prevail in the universe; that like laws obtain in the inorganic and organic kingdoms; that the simpler forms and combinations lend themselves to the construction of the more complex forms in an ascending series; and that there is continuity of plan in the great races of plants and animals.

Every one conversant with the subject will readily admit that traces of the more simple plants and animals constantly reappear in the higher plants and animals, and this holds true of the several systems and of the organs comprising them. To take an example. The plant breathes by the stomata of its leaves as an animal breathes by its lungs; the plant circulates its nutritious juices pretty much as an animal circulates its blood; the spores of certain plants move freely about like many animals ; and certain animals (sponges, for example), are fixed, as happens in the majority of plants. In all these cases there is a common plan and common arrangements. There is, however, no proof that the animal is derived from the plant, or that community of structure and function in the plant and animal are due to original identity. In like manner the more complex plants and animals, during their development, pass through stages met with in the adult condition of the more simple. In this case, there is community of plan, and also continuity of substance and function, as is well seen in the development of the human embryo and fœtus; which, as stated, have been likened at one time to a fish, at another to an amphibian, at a third to a reptile, and at a fourth to a bird. The conditions of life in utero necessitate these resemblances. The foetus cannot breathe air by its lungs or digest food by its alimentary canal. Special provisions have to be made, and these provisions furnish eloquent testimony of design, as they show that the end was seen from the beginning; the uterine changes being necessary when the completed animal is taken into account.

The development of the mammal bespeaks a general plan or scheme of a progressive character according to types; the higher types including the lower, with which they are in harmony from the beginning. The fact that the mammal during its development gradually dispenses with the arrangements peculiar to the fish, amphibian, reptile, and bird, and advances to something higher, shows that while it has certain things in common with the lower types, it has also other things which they do not individually, or in the aggregate, possess, and which distinguish it from everything lower in the scale of being. According to this view, the organic kingdom consists of a number of types arranged in an ascending series, each having a limited range; the lower approaching towards but not actually merging into the higher, which, as indicated, has peculiarities of its own not found in any of the lower types. This explains the cropping up of vestiges, remnants, or rudiments of lower types in higher types in combination with excellences not found in the lower types, and to which they can never attain.

It is generally maintained that the so-called vestiges, remnants, or rudiments afford proofs of a common origin and of evolution from a pristine stock of undifferentiated protoplasm. It is further averred that the remnants deteriorate from inactivity and disuse, as they are not required in the higher animals. The facts, carefully considered, point rather to the conclusion that all plants and animals are formed on a general plan which repeats itself; that the simpler types precede the more complex; that portions of the simpler types reappear in the higher, and that the structures in both the lower and higher types are largely due to a repetition of parts by division, by budding, by branching, by infolding, by outfolding, by invagination, by evagination, by doubling, by crumpling, \&e. It is quite true that if a part is not exercised, or ceases to be used, it deteriorates and tends to disappear; but the presence of vestiges, or so-called rudiments, is not fully explained either by evolution, heredity, or disuse. To take examples. The blood-vessels connected with the heart in the mammalian foetus are simple and symmetrical, and resemble in their general arrangement that which obtains in the gills of the fish; in other words they consist of five symmetrical sets of loops or arches on either side; each set being capable of carrying blood. A time, however, arrives when the vessels, especially on one side, largely disappear, not, be it noted, from disuse, but because of the exigencies of design. What was symmetrical becomes unsymmetrical, and we have, as a consequence, the twisted aorta and pulmonary artery with their respective ramifications. Here we have a case of suppression of parts and change of form, not because of disuse, but because of general plan. The appendix vermiformis, another vestige, represents a considerable cul-de-sac in the intestine of certain of the lower animals, and is functionally active. In man, it is relatively a very small, narrow cul-de-sac with next to no function. Here again there is dwarfing not because of disuse but because of general plan. If it had been a mere case of dwindling and deterioration occasioned by disuse, the structure would long ago have entirely disappeared. No change, however, has occurred in it for thousands of years; a circumstance which goes far to prove the existence and persistence of type, as apart from evolution. Similar remarks may be made of the presence of hair on the human skin. This is largely confined to particular regions, and is scanty as compared with that found in the integuments of the lower animals. The persistence of hair in diminished quantity and in particular localities in man is, however, no proof either of unbroken descent or 


\section{TRANSITION LINKS IN RELATION TO TYPES}

disuse. It merely bespeaks repetition, type, general plan, and the persistence which general plan necessitates. The appearance and partial or complete disappearance by absorption or otherwise of developing fotal structures afford additional proof in the same direction.

\section{\$ 8r. Transition Links in Relation to Types.}

The lancelet (Amphioxus lanceolatus) (Plate xciv., Fig. 1, page 404) affords an example of a low type which foreshadows all the higher vertebrates. This anomalous rudimentary creature is fish-like in its general appearance, possessed of fins, has a rudimentary nervous system, a tiny brain, a notochord or incipient spinal column, bloodvessels but no heart, an alimentary canal, and a well-defined, symmetrical muscular system. Notwithstanding all this it is a degraded type, and displays little intelligence and little activity. The following is the account given of it and other low fish forms by Dr. Günther: "The lancelet seems to be almost cosmopolitan within the temperate and tropical zones. It inhabits shallow, sandy parts of the coast, and rarely exceeds three inches in length. It belongs to the lowest sub-class of fishes, comprises one form only, and possesses a skeleton of the most primitive type. The vertebral column is represented by a simple chorda dorsalis or notochord only, which extends from one extremity of the fish to the other, and, so far from being expanded into a cranial cavity, it is pointed at its anterior end as well as at its posterior. There is no trace of vertebral segments or ribs." 1

Dr. Günther describes other transitionary types, which may be mentioned in this connection from their obvious bearing on development. "The skeleton of the Cyclostomata (lampreys and sea-hags) shows a considerable advance of development. It consists of a notochord, the anterior pointed end of which is wedged into the base of a cranial capsule, partly membranous, partly cartilaginous. This skull, therefore, is not movable upon the spinal column. No vertebral segmentation can be observed in the notochord, but neural arches are represented by a series of cartilages on each side of the spinal cord. . . The Chondropterygians exhibit a most extraordinary diversity in the development of their vertebral column; almost every degree of ossification, from a notochord without a trace of annular structure to a series of completely ossified vertebræ, being found in this order. Sharks, in which the notochord is persistent, are the Holocephali (if they be reckoned to this order, and the genera Notidanus and Echinorhinus). Among the first, Chimsera monstrosa begins to show traces of segmentation; but they are limited to the outer sheath of the notochord, in which slender sub-ossified rings appear. In Notidanus, membranous septa with a central vacuity cross the substance of the gelatinous notochord. In the other sharks the segmentation is complete, each vertebra having a deep conical excavation in front and behind, with a central canal through which the notochord is continued; but the degree in which the primitive cartilage is replaced by concentric or radiating lamellæ of bone varies greatly in the various genera, and according to the age of the individuals. In the rays all the vertebræ are completely ossified, and the anterior ones confluent into one continuous mass." 2

The rudimentary types referred to above form the natural connecting links in the great vertebrate series, and as such are entitled to great consideration. They, moreover, illustrate successive stages in the development of mammals in utero, which is the subject at present under discussion. In the early human embryo the vertebral column is formed round a simple rod-like structure, the primitive skeletal axis or notochord, which in the majority of vertebrate animals disappears more or less completely during development.

The differentiations in the young or growing frog provide some of the best-known examples of transitional or growing structures (Plate xciii., Figs. 2 and 3, page 403). These are at once striking, interesting, and instructive. The changes which occur in the development of the frog are spontaneous in their nature, and, in no sense, due to irritation, extraneous stimulation, or environment. They are means to ends; the object being to convert a fish-like, water-breathing animal into a land animal with four limbs, which breathes air. The exigencies of the land and water respectively demand the striking structural differences which characterise the tadpole and the frog. But (and this is the important point) the differences are inaugurated and carried out in anticipation of the change from the water to the land: they are not caused by the water and land, the so-called environments. What holds true of the frog holds true of all animals constructed to walk, run, or leap on the land, to swim and dive in the water, and to fly in the air. In all these cases, the organs of locomotion are specially formed in advance, and in anticipation of the special function to be performed. There is no getting away from these facts. The obvious conclusion is, that special external conditions are duly provided for according to a pre-arranged plan, nothing being

1 Dr. Masterman gives a somewhat similar description: "The lancelet is a very small marine organism from one inch to an inch and a half in lencth. It is of an elongated, fish-like shape, flattened laterally, the body being plano-symmetric. It is of a milky-white, semi-transparent appearance, and a number of the organs may be seen through the skin in the living animal. There are no definite external divisions of the body The muscular system is well developed, there is no heart, but the bases of the afferent branchial vessels are contractile. The nervous system lie immediately dorsal to the notochord, and consists of a long tube, the front portion of which forms a small brain and the rest the spinal cord."

2 "An Introduction to the Study of Fishes," by Albert C. L. F, Giunther. M.A., M.D., F.R.S., 1880.

VOL. I. 
left to chance or even to the individual effort of any particular organism either during its developmental or adult condition. In the case of the frog the embryo begins its career as a fish does. It has a ventral bag of pabulum (vitellus), no alimentary canal or mouth, no tail, and no gills (branchiæ). As development proceeds the bag of pabulum disappears and a mouth and alimentary canal are formed, a swimming tail, and gills or water-breathing outside lungs make their appearance. Further on, the tail shrinks and falls off, and four rudimentary limbs are provided instead. The gills or outside lungs adapted for water-breathing also give place to inside or true lungs adapted for air-breathing. These changes, be it remarked, are not due either to the disuse or the inadequacy of the tail and gills, but only to the exigencies of design and final purpose. They are means to ends, and the means are employed to secure the ends in anticipation, and according to pre-arrangement. In the menobranchus (a fish-like animal) the gills are persistent, and so are the limbs (Plate xciii., Fig. 4, page 403).

\section{PLATE XCIII}

Plate xciii. illustrates development and peculiarities in the fish, frog, menobranchus, and other animals.

FIri, 1.- Egg of fish.

A. Egg of fish, showing formation of umbilical vesicle.

B. Young fish with umbilical vesicle.

C. Human embryo, with umbilical vesicle (about the fifth week).

Fig. 2.-. Shows the development of the frog.

A. Diagram of frog's egg, in an early stage of development, as seen in longitudinal section. "Thickened portion of external blastodermic layer, forming body of fa'tus; $b$, anterior extremity of f(xtus ; $c$, posterior extremity ; $d$, internal layer of blastodermic membrane; $e$, cavity of vitellus.

B. Egg of frog, in process of development; tail appearing.

G. Egg of frog, further advanced; eye appearing, tail increasing in size.

D. Tadpole fully developed ; eye more advanced, and mouth and alimentary canal formed.

FIG. 3.-Shows tadpole developing limbs and adult frog. In the first figure the tail is diminished in volume, and rudimentary limbs are appearing. The gills or branchiæ are also in a transition stage; in other words they are changing from outside waterbreathing lungs to inside air-breathing ones. In the second figure the frog is fully developed. It has lost its tail and is provided with four powerful limbs and true air-breathing lungs.

FIG. 4.-Head and gills of Menobranchus. In this curious creature the outside branchim or water-breathing lungs and the limbs are persistent.

FIG. 5.-Gills of a ganoid fish (Ceratollus). Affords a good example of the outside or water-breathing lung. ", Arcus aortre; $b$, gills containing rows of capillary blood-vessels ; $c$, pseudo-branchia; $d$, $d$, two series of gill-rakers belonging to the pseudobranchia ; $e$, ceratohyal ; $f$, glossohyal (after Günther).

FIG. 6.-Diagram of the larva of a calcareous sponge in its gastrula phase. The larva at this stage consists of a simple pouch or stomach with a mouth $(a)$. The walls of the stomach are formed of two layers of cells, an internal and an external; the external cells being elongated and furnished with filaments (cilia), which serve as organs of locomotion. These two layers of cells have been likened to the internal and external layers of the blastodermic membranes in the higher animals (after Haeckel).

FIG. 7.-Ascidian.

A. Early tailed larva.

B. Tailed larva, seen on the right side (altered from Seeliger).

C. Transverse section through the tail of an ascidian larva (after Seeliger).

A. a, Brain ; b, neural tube ; $c$, notochord ; $d$, mesenteron.

B. $a$, Brain, with eye and ear; $b$, nerve cord ; $c$, notochord ; $d$, atriopore ; $e$, intestine ; $f$, remains of caudal intestine ; $g$, neuropore; $h$, papilla ; $i$, laryngeal clefts $; k$, endostyle in wall of pharynx; $j$, heart.

C. $a$, Median fin ; $b$, nerve tube; $c$, notochord ; $d$, muscle; $\rho$, caudal hypoblast. The parts here enumerated foreshadow advancing types; a vertebral column, nervous system, alimentary canal, vascular system, and sense organs being all indicated. The ascidian is to be regarded as intermediate between the molluscoid invertebrates and the vertebrata.

FIG. 8.- $a, b, c, d$, Polar capsules of Mysobolus ellipsoides (after Balbiani). Illustrates spiral, vibratile swimming arrangements in rudimentary structures.

FIf. 9.- Shows a series of embryos exhibiting similarity of structure and development in their primary and secondary stages. The upper row shows the gill arches and the absence of limbs; the lower row shows the partial disappearance of the gill arches and the budding of the linibs.

A. Gill arches and rudimentary limbs in tortoise.

B. Gill arches and rudimentary limbs in fowl.

C. Gill arches and rudimentary limbs in echidna.

D. Gill arches and rudimentary limbs in koala or Anstralian bear.

E. Gill arches and rudimentary limbs in rabbit.

F. Gill arches and rudimentary limbs in pig.

G. Gill arches and rudimentary limbs in deer.

H. Gill arches and rudimentary limbs in cat.

I. Gill arches and rudimentary limbs in monkey.

J. Gill arches and rudimentary limbs in man (after Haeckel).

Note.-The figures A to J of Fig. ", representing stages of development in various animals, and consisting as they do of mere repetitions of parts, indicate a general plan or type. They afford no proof of evolution, or the production of the higher animal furms from the lower ones. The upper and lower rows of figures, taken separately, show repetition as apart from advance. 
PLATE XCIII

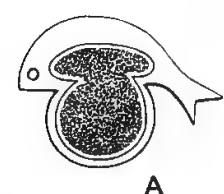

A

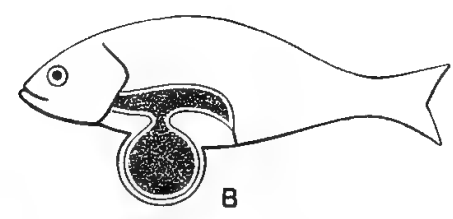

FIG. 1.
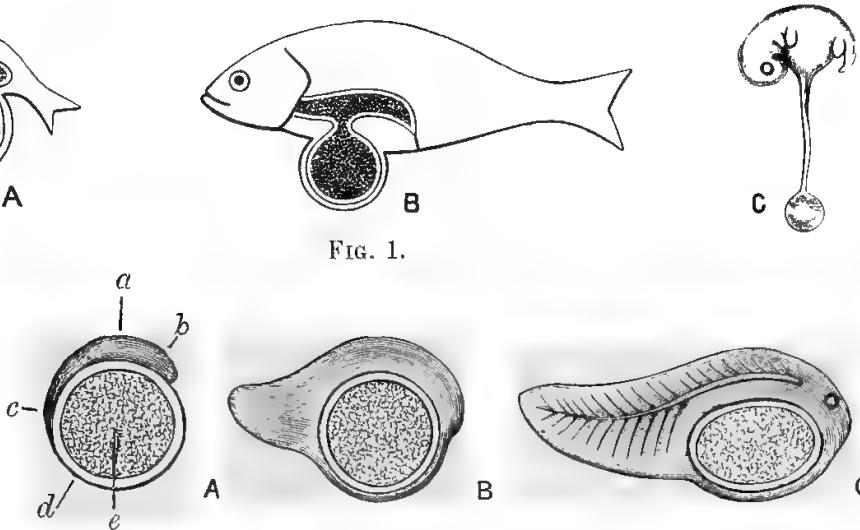

$f-\{$
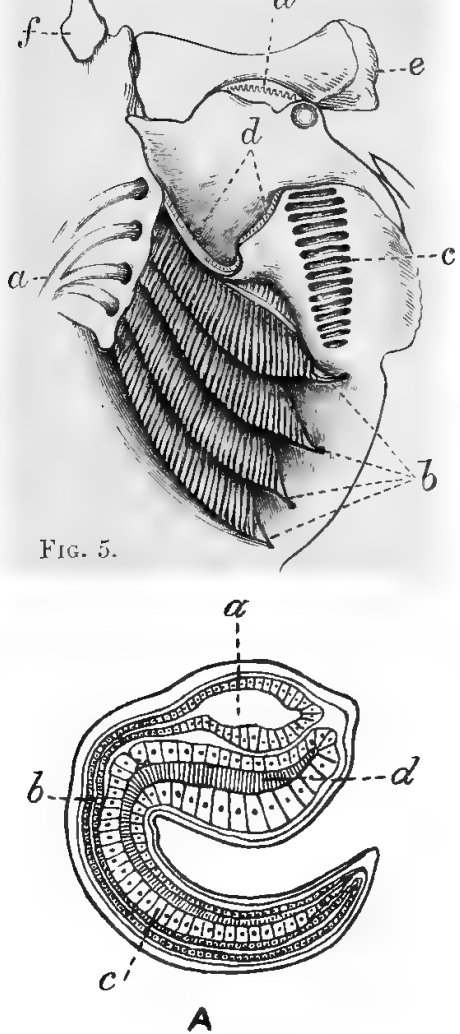

A

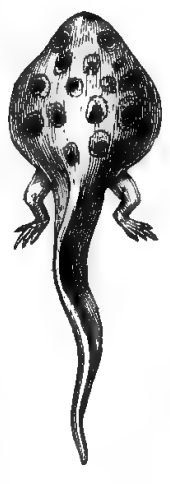

FIG. 2.

B

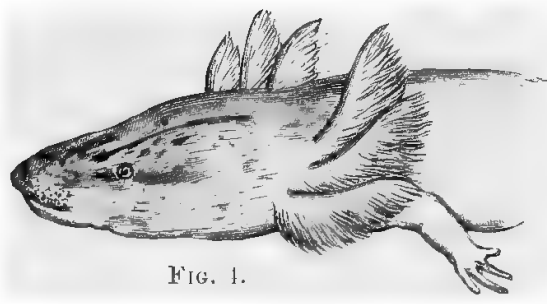

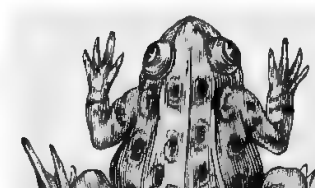

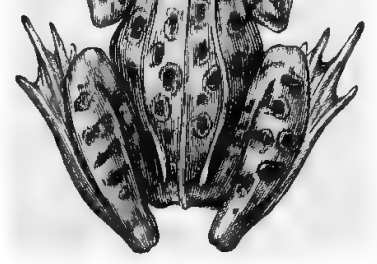

FIเ: :3.

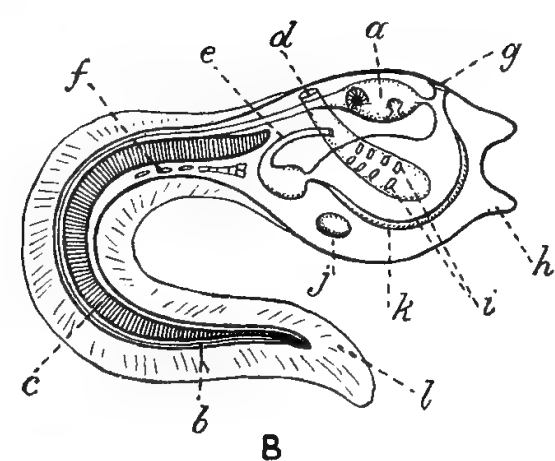

Fig. 7.

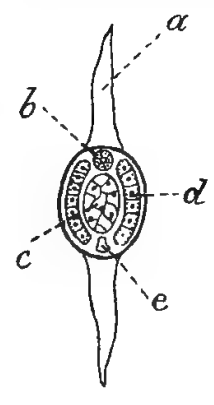

c

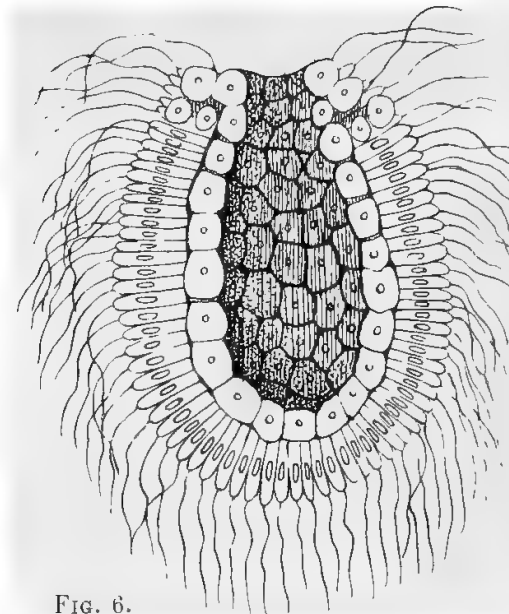

FIg. b.
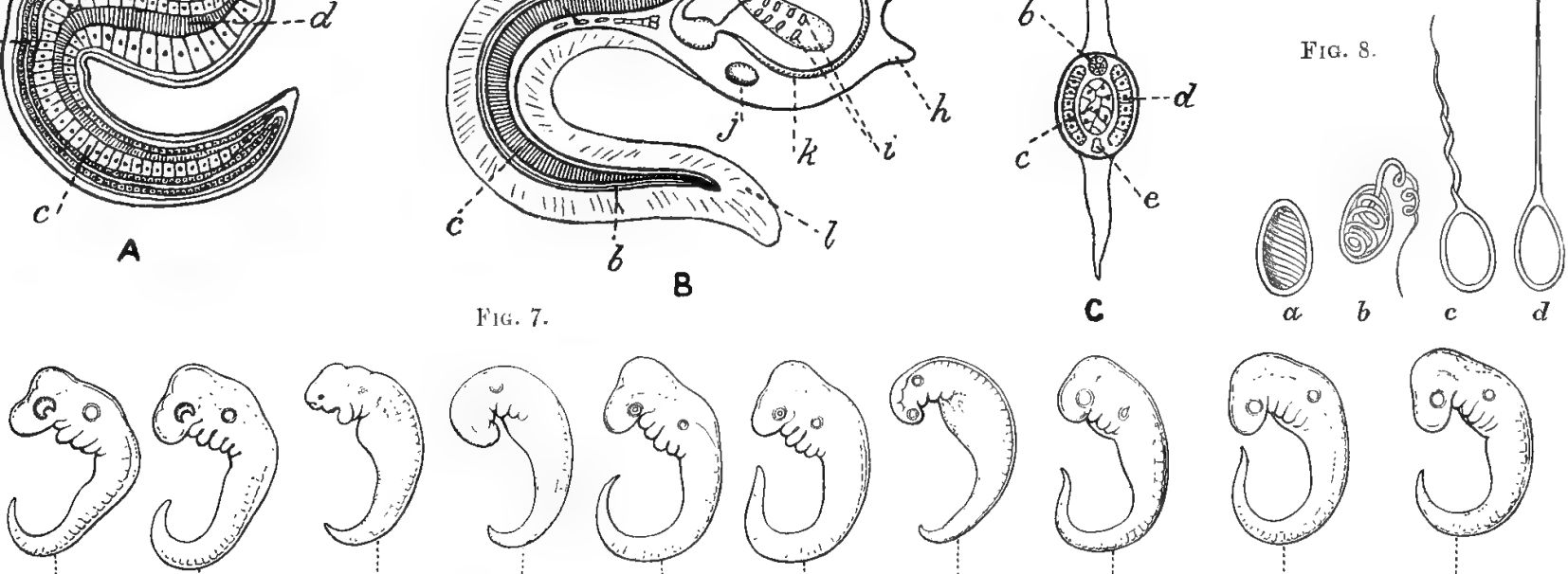

5
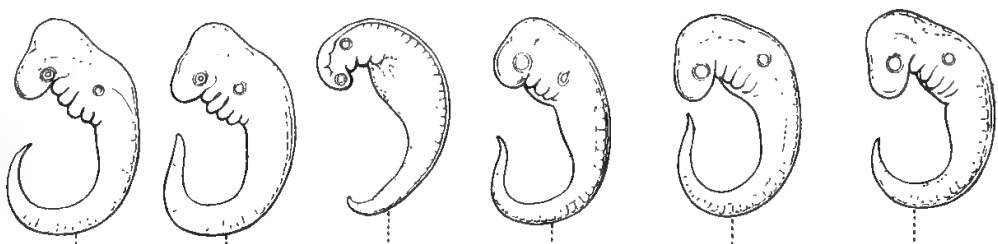

(6) (a)
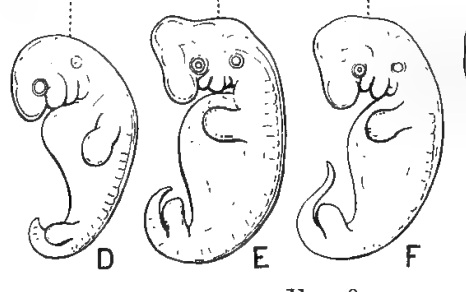

FIG. 9 .
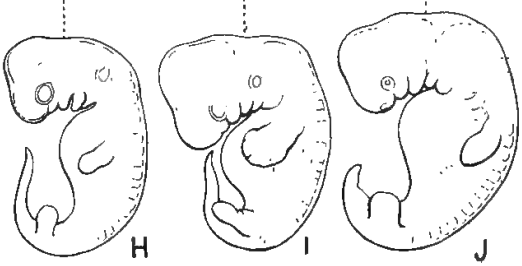


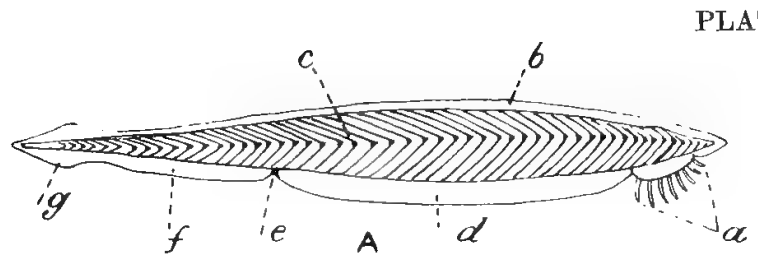

PLATE XCIV

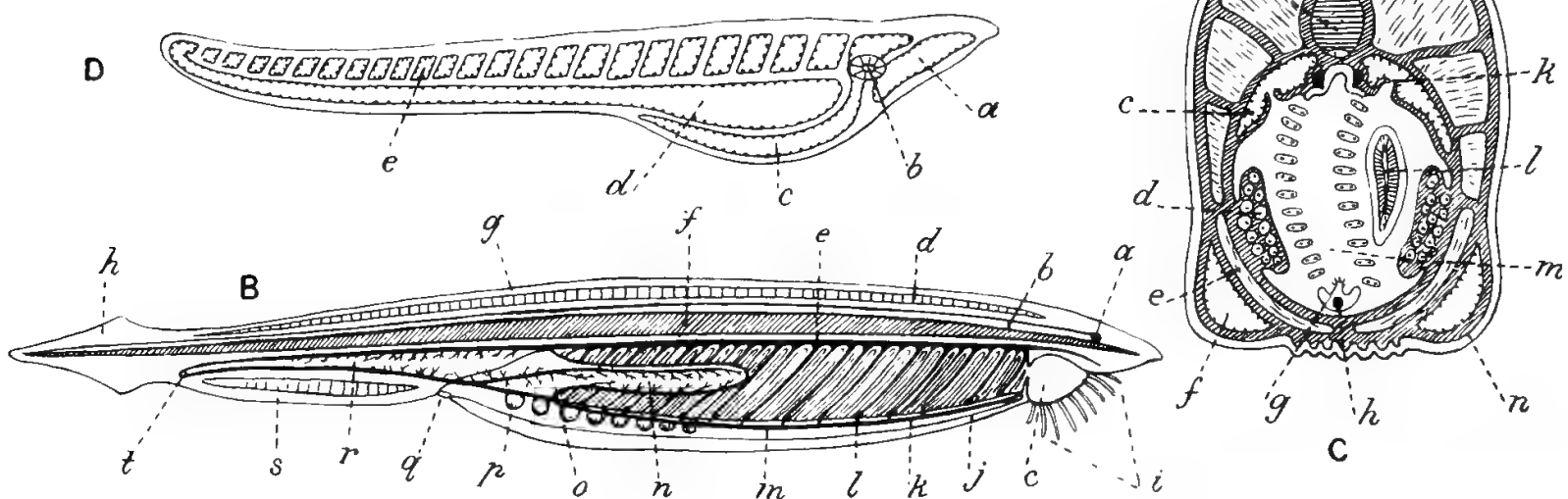

FrG. 1.

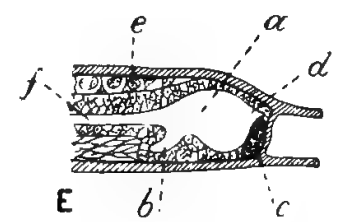

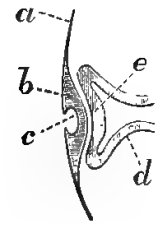

A

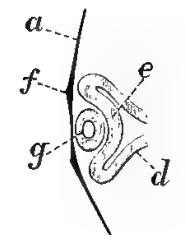

B

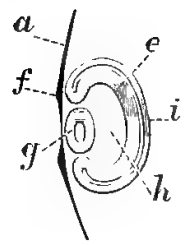

C

FIG. 2.

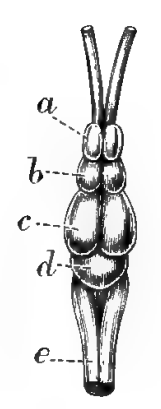

A

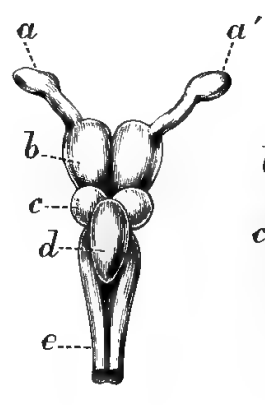

B

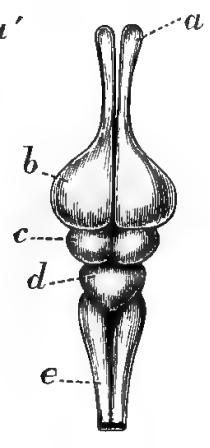

C

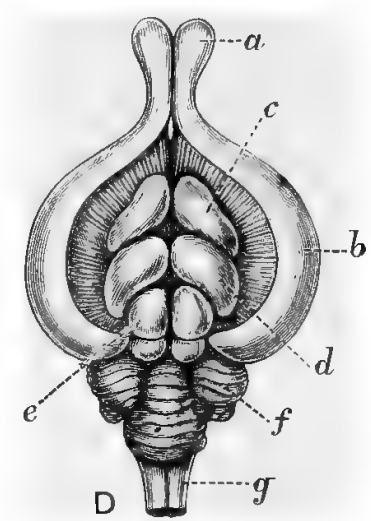

FIG. 3.

\section{PLATE XOIV}

Plate xciv. illustrates the anatomy of the amphioxus, once supposed to form a connecting link between the softbodied animals and the vertebrates; also the development of the eye, and the comparative anatomy of the brain.

Fig. 1.-Amphioxus (Amplioxus lanceolatus).

A. Lateral view of Amphioxus lanceolatus. u, Oral cirri ; b, dorsal fin ; ', myomere muscles; $d$, metapleural fold; $e$, atriopore ; $f$, anal fin ; $g$, tail.

B. View of amphioxus from right side. a, Brain; b, spinal cord; ', buccal cavity; d, dorsal fin (skeleton); ", dorsal aorta ; $f$, notochord; $g$, dorsal fin; $h$, caudal fin; $i$, oral cirri ; $j$, metapleural fold; $l$, branchial artery ; $l$, contractile bulb; $m$, branchial artery ; $n$, liver ; $o$, portal vein ; $p$, gonad ; $q$, atriopore ; $r$, intestine ; $s$, anal fin ; $t$, anus.

C. $a$, Nerve cord ; $b$, notochord ; $c$, peri-visceral coelom ; $d$, gonad ; $e$, ventral muscles ; $f$, metapleural cavity ; $y$, endostyle ; $h$, ventral blood-vessel; $i$, dorsal fin skeleton; $j$, myomeric muscle; $k$, nephridium ; $l$, liver ; $m$, pharynx leading into arterial cavity through clefts ; $n$, metapleural fold.

D. $a$, Right head cavity ; $h$, left head cavity; $r$ collar sae ; $d$, peri-visceral coelom ; $"$, myotomes.

E. Median section of brain of amphioxus. a, Cerebral vesicle; $h$, infundibulum ; ', pigment spot; $d$, unpaired olfactory lobe ; $e$, ganglion cells ; $f$, neural tube (after Masterman).

FIG. 2.-Longitudinal section of the eye of embryo of fowl.

A. From an embryo at about the sixty-fifth hour of incubation.

B. From an embryo a few hours older.

C. From an embryo at the fourth day of incubation. The sume letters apply to the same parts. $a$, Corneal lamina; $b$, lens in Fig. A, still connected with the corneal lamina and possessing a small cavity (c) in its interior; $d$, posterior part of optic vesicle; $\varepsilon$, folded portion of optic vesicle.

In Figs. B and $\mathrm{C}$ the lens $(g)$ is detached but still hollow; $e$, introverted portion of the primitive optic vesicle, subsequently becoming the retina; $d$, posterior part of the optic vesicle, which, according to Remak, probably becomes the choroid coat, ciliary 


\section{CHANGES IN, AND PECULIARITIES OF, THE HUMAN FCETUS}

\section{PLATE XCIV (continued)}

processes, and iris, and in Figs. A and B is still connected with the brain by the hollow optic nerve; $f$, thickening of the corneal lamina around the spot from which the lens $(g)$ has detached itself; $h$, vitreous body (after Carpenter).

FIG. 3.-Brains of various kinds.

A. Brain of cod. $a$, Olfactory lobes; $b$, cerebral hemispheres; $r$, middle brain giving rise to the optic nerves; $l$, cerebellum; $e$, spinal cord.

B. Brain of shark, lettering as in A (after Dallas).

C. Brain of alligator. $a$, Olfactory ganglia; $b$, cerebral hemispheres ; $r$, optic tubercles ; $d$, cerebellum ; $e$, medulla oblongata.

D. Brain of rabbit. $a$, Olfactory ganglia ; $b$, cerebral hemispheres, separated to show corpora striata $(c)$, optic thalami $(d)$, and tubercula quadrigemina $(e) ; f$, cerebellum; $g$, spinal cord (after Dalton). The comparative anatomy of the brain illustrates the repetition of parts with certain additions; the several parts consisting of expansions of the spinal cord.

\section{\$2. Changes in, and Peculiarities of, the Human Fœtus.}

That the fotus during development assumes a great variety of forms every one conversant with the subject will readily admit. The several forms, however, only indicate general plan and advance of type. They do not countenance the belief that the higher plants and animals are actually manufactured from the lower ones by a process of evolution extending over long periods. What the anatomist and physiologist have to deal with is the completed product. It is not for them to show that the completed product has many things in common with the lower plants and animals, but that it possesses properties and attributes which are only found in itself, and which distinguish it from every other living thing.

A general plan for the formation of plants and animals, an upward trend, and types are conceded. What is denied, is unbroken continuity of substance, and the actual production of higher plants and animals out of lower plants and animals by endless modifications in endless time. The two things are essentially different. In the one case, there are differentiation and advance according to a plan and on common lines to a finish, and to a high standard; in the other, there are differentiation and advance also according to a plan and on common lines, but both the differentiation and advance are partial : in other words, the higher plants and animals go all the way-the lower ones only part of the way, and that the least difficult part. It matters not by what route the final goal is reached ; suffice it to say, that only the highest forms can, by any possibility, attain it. It is the amount of differentiation which distinguishes the higher from the lower forms, and it avails notbing to say that man during his fœtal existence presents features found in animals below him in the scale of being. The question really at issue is, not what man has in common with the lower animal, but, what he has which they have not. The principle which underlies mechanics is the same in all machinery, but a distinction is to be drawn between the simple and complex machine.

I have no desire to minimise or underrate the resemblances in the several grades of plants and animals, but I must insist on the differences. I therefore feel it my duty to refer to the former so far as they concern man.

During the early stages of human gestation, when the blastodermic and other membranes and parts are being formed, it would be difficult to distinguish the human embryo from that of a fish, a reptile, or a bird. Then at a later stage the human embryo and foetus display segmentation and a symmetrical repetition of parts met with in the articulata ${ }^{1}$ and in the lower vertebrates. The segmentation in question appears in the visceral arches and in the square-shaped proto-vertebræ (future spinal column). The human foetus also displays a short, rudimentary tail, which is persistent in the majority of quadrupeds. Its limbs appear as buds, and some children are born without limbs; a condition which prevails in fishes and many reptiles. The arrangement of its blood-vessels and circulation is, moreover, peculiar. The distribution of the great vessels connected with the heart resembles that found in the fish, amphioxus, \&c. The vessels in question are arranged in five symmetrical pairs in the region between the thorax and head; certain of the symmetrical vessels ultimately disappear, and are replaced by a non-symmetrical set, consisting of the aorta and pulmonary artery and their branches (Plate xcii., Fig. 4, A, B, C, D, page 396).

The fotal circulation is further a mixed circulation, that is, the blood circulated is partly arterial and partly venous ; a condition which obtains in fishes and reptiles.

The mixed circulation is due to the imperfect formation of the foetal heart; the right and left auricles, which contain more or less perfect venous and arterial blood, communicating with each other by means of the Eustachian valve, with the result that the blood forced by the auricles into the left or systemic ventricles is partly venous and partly arterial. The blood is further mixed, in a minor degree, by means of the pervious ductus arteriosus. The circulation in the fœetus and in the placenta, and other points connected with the development of the blood-vessels. heart, \&c., are illustrated at Plate xcv., page 407.

1 This sub-kingdom of Cuvier has been divided into Arthropoda (Insects, Myriopoda, Malacopoda, Arachnida, Pycnogonida, Crustacea) and Anarthropoda, including the Annulata and allied forms. 


\section{PLATE XCV}

Plate xcv. illustrates the foetal and placental circulations; also the development of the blood-vessels and heart.

Fig. 1.-Circulation in the human foetus (semi-diagrammatic). In this figure the darts show the direction in which the blood flows. The quality of the blood is indicated by the colours of the blood-vessels. Thus the white blood-vessels contain arterial blood, the black vessels venous blood, and the shaded vessels mixed blood. $a$, External surface of placenta; $b$, internal surface of placenta; c, circulation in the head (mixed and venous blood); $d$, circulation in the arm (mixed and venous blood); $e_{2}$ circulation in the leg (mixed and venous blood); $f$, venous circulation in the intestine; $w$, venous circulation in stomach and spleen; $g, g, g$, umbilical vein containing arterial blood from the placenta to the liver $(n)$ and ascending cava $(0) ; h, h$, $h$, umbilical arteries conveying mixed blood from the hypogastric arteries $(j)$ and body of foetus to placenta $(a, b)$; $i$, continuation of umbilical vein (arterial blood); $l$, ductus venosus (arterial blood); $l$, vena portæ, containing venous blood from intestine $(f)$, stomach $(u)$, and spleen ; $m$, descending aorta, dividing (a) into the common iliac arteries and containing mixed blood; 0 , ascending cava, containing venous blood at lower part and mixed blood at upper part; s, ascending cava discharging its mixed blood through the right auricle and Eustachian valve into the left auricle, from which it finds its way into the left ventricle $(r)$ and thence into the ascending, transverse, and descending portions of the aorta, from which it is distributed to the head and neck $(c)$, the arms $(d)$, legs $(e)$, body and viscera $(f, u)$; $y$, descending cava discharging its venous blood into the right auricle and right ventricle $(z)$. From the latter it finds its way into the right and left lungs, which as yet are rudimentary and take no part in aerating the blood; this function being vicariously performed by the maternal portion of the placenta. The blood from the lungs $(n)$ is discharged into the left auricle.

In this figure the blood-vessels (the placenta excepted) terminate in loops formed by the continuation of the arteries with the veins. The capillary blood-vessels are omitted for the sake of simplicity. Drawn by C. Berjeau for the Author.

FIf. 2.-Human umbilical cord composed of one vein (a), and two arteries (b), intertwining to form a symmetrical left-handed spiral. Resembles spiral water-spout (Fig. 5), and the twining stems of the hop (Plate x., Fig. 2, A). Two spirals at least are necessary to produce symmetry, structures composed of one spiral being incomplete or lop-sided. Drawn from injected specimen in the possession of the Author by C. Berjeau.

FIG. 3. - Diagram showing the course of the circulation in the human fcetus (vide arrows). $y^{\prime}, i$, Umbilical vein, conveying arterial blood from the placenta; $h$, umbilical arteries, conveying venous blood to the placenta; $k$, ductus venosus ; $l$, vena portæ; ", vena cava, inferior; $y$, vena cava, superior ; s, Eustachian valve and right auricle ; $q$, right ventricle ; $v$, ductus arteriosus, uniting aorta $(u)$ and pulmonary artery; $t$, left auricle ; 1 , left ventricle; $m$, descending aorta ; $x$, common iliac artery, dividing into external and internal iliac arteries; $j$, hypogastric arteries, continuous with umbilical arteries $\left(h^{\prime}\right)$.

Diagram illustrating the relation existing between the maternal ( $m$ ) and fuetal ( $n$ ) portions of the placenta (human). The maternal and fuetal surfaces are represented as separated from each other by ic certain interval $(m, n)$ to avoid confusion. They are slightly uneven from the projection into them of the maternal and fuetal capillary vessels. During pregnancy the two surfaces accommodate themselves so as to dovetail and fit accurately into each other. In the diagram the vessels represented by solid lines contain arterial blood; those represented by dotted lines venous blood. "I, Mucous lining of uter'us, with nucleated cells on its surface; $b$, sub-epithelial, spheroidal, and fusiform corpuscles, embedded in connective tissue; ", $d$, utricular glands lined with epithelium, and opening on mucous surface of uterus. They pour utricular secretion into utricular space $(m, n)$. This space is mapped off, on the one hand, by the villi and coverings of the maternal portion of the placenta; and on the other, by the villi and coverings of the foetal portion of the placenta. The utricular secretion is necessary to a free osmosis between the maternal and fcetal vessels. $n$, Limiting membr'ane of feetal portion of placentic covered with nucleated cells; $a$, sub-epithelial, spheroidal, and fusiform corpuscles embedded in connective tissue; $i, i$, amnion; $g$, umbilical vein, conveying arterial blood to fretus ; $h$, umbilical arteries, conveying venous blood from foetus. The umbilical arteries and vein break up to form the villi of the fuetal portion of the placenta ; these being directed towards similar villi $(e, f)$, constituting the maternal portion. The maternal and foetal villi are separated from each other by the utricular space $(m, n)$ containing utricular secretion, by two layers of cells, by two membranes, and by a certain proportion of connective tissue, spheroidal, fusiform, and other corpuscles $(a, b)$. Goodsir regarded the placental villus as consisting of a maternal and foetal portion; the maternal portion being composed of a membrane and nueleated cells, and corresponding to $m$ of diagram ; the fuetal portion being composed of a membrane and nucleated cells, and corresponding to $n$ of diagram. Between the maternal and fuetal portions (as he termed them) of a placental villus, he represents a space which corresponds to the space between $n$ and $n$ of diagram. This space he regarded as the cavity of a secreting follicle. Goodsir has, it appears to me, appropriated the lining membrane of the maternal portion of the placenta ( $m$ of diagram) and added it to the limiting or lining membrane of the foetal portion ( $u$ of diagram). Described and drawn by the Author from specinens in 1872.

FIG. 4.-Gravid human uterus about the end of the seventh month. a, ", Foctal portion of placenta; $b$, umbilical cord ; c, body of foetus (after Dalton).

FIF. 5.-Shows the early and adult condition of the arterial system in the mammal.

A. Early state of the arterial system, showing the heart $(a)$, with its two ascending trunks, giving off on each side five cervical arteries which terminate in the vertebral arteries $\left(h, b^{\prime}\right)$. The vertebral arteries unite below the heart to form the aorta $\left(c^{\prime}\right)$.

B. Adult state of the arterial system. $u, "$, Carotid arteries; $b$, $b$, vertebral arteries; $c$, $c$, right and left subclavian arteries ; $d, d^{\prime}$, right and left superior intercostal arteries : $e$, left aortic arch which remaius permanent ; $f$, right aortic arch which disappears (after Dalton).

FIG. 6.- Shows spiral development of human heart.

A. Earliest form of foetal heart. $a$, Venous extremity ; $b$, arterial extremity.

B. Fuetal heart twisted upon itself. $a$, Venous extremity; $b$, arterial extremity.

C. Fotal heart divided into right and left cavities. $a$, Venous extrenity ; b, arterial extrenity ; $c, c$, pulmonary branches.

D. Fuetal heart still further developed. $a$, Aorta ; $b$, pulmonary artery ; $c, c$, pulmonary branches; $d$, ductus arteriosus.

E. Heart of infant, showing disappearance of arterial duct after birth. $a$, Aorta; $b$, pulmonary artery ; $c, c^{\prime}$, pulmonary branches; $d$, ductus arteriosus becoming obliterated (after Dalton).

FIG. 7.- Shows human foetal and adult hearts.

A. Heart of foetus at the end of the sixth month. a, Inferior vena cava; $b$, superior vena cava; cavity of the right auricle, laid open in front; $d$, auricular appendix; $e$, cavity of the right ventricle, also laid open; $f$, Eustachian valve. The arrow placed in the inferior vena cava passes behind the Eustachian valve, crosses the cavity of the right auricle, and passes through the foramen ovale to the left auricle.

B. Indicates the course of the blood through the adult heart (vide darts). $a$, a, Vena cava, superior and inferior; $b$, right ventricle; $r$, pulmonary artery ; $d$, pulmonary vein ; $e$, left ventricle; $f$, aorta (after Dalton). 
CHANGES IN, AND PECULIARITIES OF, THE HUMAN FOETUS 407 PLATE XCV

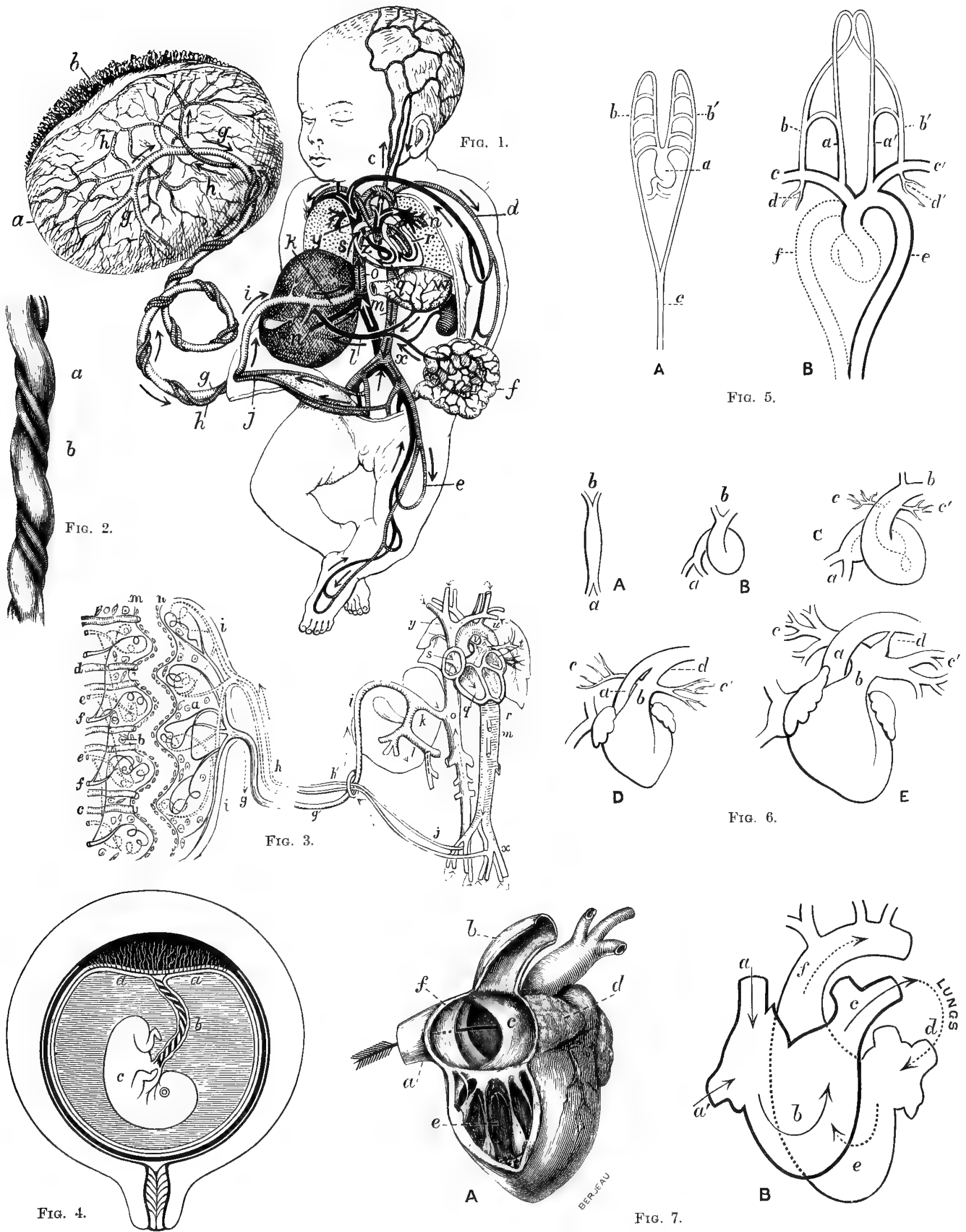


The circulation of mixed blood through the body of the fœetus resembles that of the reptile, where there are two auricles and only one ventricle; the right auricle containing venous blood and the left one arterial blood. As the right and left auricles in the reptile communicate directly with the ventricle, the venous and arterial blood are mixed in the ventricle and a mixed circulation produced. The fœtal circulation resembles that of the fish to the extent that the blood of the fœetus is aërated not by coming in contact with air, but with the oxygen in solution in the blood of the mother contained in the maternal portion of the placenta. The fish and fotus aërate their blood by means of a fluid medium. In the fish the heart consists of a single auricle and ventricle, with two accessory structures, the ductus venosus and the bulbus arteriosus, and the circulation is mixed because a perfect circulation is impossible as apart from a heart with four separate compartments ; the two right compartments being devoted to the venous circulation, and the two left compartments to the arterial circulation. All this does not make the man successively a fish, a reptile, or a bird. It only shows that he is formed upon a common plan, and that he forms one of several types. His distinguishing feature is his completeness.

The mixed circulation of the fotus is converted into a double or perfect circulation soon after birth; the Eustachian valve closing and the ductus arteriosus becoming imperforate. These structural changes are not due to irritability, extraneous stimulation, or environment. The effect of them is to form a heart consisting of four distinct and separate chambers; a right and left auricle, and a right and left ventricle. The right auricle and ventricle receive venous or impure blood from all parts of the system and force it into the lungs, and are, from this circumstance, designated the pulmonic or right heart; the left auricle and ventricle receive arterial or pure blood from the lungs and force it through the entire system, and are termed the systemic or left heart. The left heart, because of the excess of work to be performed by it after birth, becomes the more powerful and typical one.

In the fœtus before birth the placenta equalises the amount of work performed by the right and left ventricles respectively. The history of the formation of the heart affords a pregnant illustration of design.

At first it appears as a simple tube slightly expanded in the middle and with two vessels at either end. After a time it throws itself into a spiral loop which overruns and is free at either end; the free ends being continuous with the blood-vessels which ultimately form the cavæ and pulmonary artery and aorta. Subsequently the original, looped, spiral tube is divided by duplications or partitions into four compartments ; namely, two auricles and two ventricles; the auricles opening into each other by the Eustachian valve, and the pulmonary artery and aorta also opening into each other by the ductus arteriosus (Plate xcv., Figs. 1, 3, 6, and 7, page 407). While these changes are going on, a most intricate arrangement of the muscular fibres forming the auricles and ventricles, especially the latter, is taking place. The arrangement of the muscular fibres in the left ventricle is exceedingly involved and complex-so much so, that for over 200 years it formed the Gordian knot in anatomy. The muscular fibres are arranged in four distinct spiral sets, namely, two external and two internal ; these being continuous with each other and with other fibres at the apex and base of the ventricle. The two external sets wind spirally from the base to the apex and form right-handed spirals. Conversely the two internal sets wind spirally from the apex to the base and form left-handed spirals (Plate xvii., Fig. 4, page 32). The two sets of spiral external fibres involute or turn in at the apex, and the two sets of spiral internal fibres evolute or turn out at the base in such a way that the external and internal fibres become continuous with each other both at the apex and the base of the ventricle. Other deeper and more oblique spiral external and internal fibres, continuous with those already mentioned, occur. It follows, that the several sets of external and internal spiral fibres cross each other at ever-increasing angles until the centre of the muscular wall is reached. As a consequence of this arrangement, and as I explained in $1858,{ }^{1}$ the spiral muscular fibres of the left ventricle may be separated into seven well-marked layers; the layers increasing in thickness from without inwards. Thus if the ventricle be dissected from the outside, the two sets of spiral fibres forming the first layer are found to wind from left to right and from base to apex of the ventricle in a nearly vertical direction; the two sets of fibres forming the second layer winding in a similar direction slightly more obliquely; those of the third layer winding still more obliquely until the fourth or central layer, where the fibres pursue a nearly transverse direction, is reached. When the fourth layer is exposed everything is reversed; the two sets of spiral fibres forming the fifth layer running from right to left and from apex to base very obliquely, the two sets of spiral fibres forming the sixth layer pursuing a similar but less oblique direction ; the spiral fibres forming the seventh or most internal layer also proceeding from right to left in a nearly vertical direction. In virtue of this arrangement, the fibres of the first and seventh layers cross each other spirally at very acute angles, those of the second and sixth layers crossing spirally at more obtuse angles, and those of the third and fifth layers crossing spirally at still more obtuse angles. The fibres of the central layer, as explained, pursue

1 In this year my dissections of the heart, over 100 in number, obtained the Senior Anatomy gold medal of the University of Edinburgh, awarded liy the famous Professor . Tohn (Koodsir, the facile princeps of anatomists. They formed the subject of the Croonian Lecture of the Royal Soriety of London in 1860 ; an abstract of the lecture being published in the Proceedings of the Society in the same year. Later, the lecture, in ull extended form, and very fully illustrated, was assigned a place in the Philosophical Transactions (1864, Part iii.). 


\section{DEVELOPMENT OF BLOOD, \&c., IN MAN AND MAMMALS}

a more or less transverse direction. Two plates of illustrations of the left ventricle of the heart are given further on (Plates xcvii. to xcviii.). It may be stated in passing that the muscular fibres of the stomach, bladder, and uterus are distributed in much the same fashion. These are illustrated by four plates (Plates c. to ciii.). The left ventricle of the heart, which is the chief propelling organ in the circulation of the blood, is symmetrical, and constructed on mathematical principles to secure the greatest possible strength with the least possible material. It is stayed and strutted in every direction, after the manner of a girder bridge, to resist strain at every point. It is expressly designed and expressly built to withstand continuous wear and tear, such as no other organ in the body is exposed to. The heart forces the blood through the system during the entire life of the individual. It rests neither day nor night from the cradle to the grave, and on its efficiency the health and life of every one of us depends. It forms the central engine of all our activities, and any imperfection in it inevitably results in disaster. It is no wonder that so much prevision and care should be expended in its production. Whatever may be said of other animal organs, the heart (and particularly the left ventricle) is no chance product. Its structure and function have only to be examined to convince every one who reflects, that it is a fundamental organ, in large measure necessary to, and coeval with, life; an organ, the foundations of which are laid in the fœetus and perfected soon after birth. It is the great prime mover of the blood and muscles from the physical point, as the brain is the great prime mover from the mental or psychical point. In order to carry on its important work it is endowed with a centripetal and centrifugal power; each compartment having a double independent movement, whereby it alternately opens, expands, and sucks blood into it, and closes, contracts, and forces blood out of it. Each compartment exercises a vis a fronte and a vis a tergo power; the heart forming one of the most perfect and effective forcing-pumps known to modern science.

\section{§ 83. The Development of Blood, Blood-vessels, Nerves, Muscles, Bone, Lungs, Glands, Sense Organs, \&c., in Man and Mammals.}

The manner in which the red and white blood-corpuscles, blood-plasma, blood-vessels, nerves, muscles, bone, glands, sense organs, \&c., are produced in the embryo is exceedingly interesting from a teleological point of view. These several substances and structures, various as regards composition, consistence, and ultimate function, are all being developed at, or nearly at, the same time. The one is not manufactured out of the other. On the contrary, they spring from separate centres, and each grows in its own particular way and independently. They do not crowd or jostle each other, but fit into their own particular niches in the organic edifice as specially-ordered, dressed stones fit into a carefully-planned building. There is no waste either of energy or material, and the completed animal bears the unmistakable impress of an intelligent Designer and Constructor. The production of the several structures referred to is spontaneous, and in no sense due to irritability, extraneous stimulation, or environment. There is division of labour from the first. Each substance is supreme in its own department. The organism does not consist of a vicarious arrangement of substances and of parts. On the contrary, everything is independent and, within limits, interdependent, in the sense that each thing forms a part of the same whole. The structures be they simple or complex, are typical of their kind, just as the several varieties of plants and animals are typical. To take examples: the young blood is formed simultaneously with the blood-vessels which are to contain it; the brain, spinal cord, and nerves are growing at the same time as the muscles they are to control ; and the bones are developing pari passu with the muscles to which they are to afford attachments and leverage, and by which they are to be moved. There is no waiting of any one part of the embryo on any other part; all are up to time, and everything moves with the regularity of clockwork, and precisely as a planned and ordered whole would be expected

to move. "The first red blood-corpuscles are formed very early in embryonic life simultaneously with and in the interior of the first blood-vessels. They are developed in the mesoblast in a circular area which surrounds the part of the blastoderm which is occupied by the developing body of the embryo. The area is known as the vascular area, and the first blood-vessels and blood-corpuscles are, therefore, formed outside the actual body of the embryo. T'hose mesoblastic cells in the vascular area which are concerned with the formation of vessels (angioblasts) become extended into processes of varying length, which grow out from the cells in two or more directions. The cells become united with one another, either directly or by the junction of their processes, so that an irregular network of protoplasmic nucleated corpuscles is thus formed. Meanwhile the nuclei become multiplied, and whilst the greater number remain grouped together in the original cell-bodies or nodes of the network, some are seen in the uniting cords. The nuclei which remain in the nodes accumulate, each one around itself, a small amount of cell-protoplasm. The corpuscles thus formed acquire a reddish colour, and the protoplasmic network in which they lie becomes vacuolated and hollowed out into a system of branched canals enclosing fluid in which the nucleated coloured corpuscles float. As soon as the heart is developed, or even before this happens, the blood begins to move within the vessels of the vascular area. These first-formed red blood-corpuscles are nucleated cells resembling the pale corpuscles

VOL. I. 
except in their colour and in the clearness of their protoplasm, and, like the white corpuscles, they are capable of amoboid movement, and of undergoing multiplication by division. The colourless corpuscles appear to be formed in great number in the embryonic liver as soon as this is developed, as well as in the lymphatic glands, spleen, and thymus gland. It has been supposed that the colourless corpuscles formed in these organs acquire colour, and are converted into nucleated red corpuscles, but there is no direct evidence in favour of this view.

"The primary nucleated red corpuscles are at length succeeded by smaller disc-shaped red corpuscles without nuclei, having all the characters of the blood-dises of the adult. This substitution proceeds gradually, until, long before the end of intra-uterine life, the nucleated red corpuscles have almost entirely vanished from the blood. The disc-shaped red corpuscles are produced in the interior of angioblastic connective tissue cells in the following manner :-A part of the protoplasm of the cell acquires a reddish tinge, and after a time the coloured substance becomes condensed in the form of globules within the cells, varying in size from a minute speck to a spheroid of the diameter of a blood-corpuscle, or even larger; but gradually the size becomes more uniform. Some parts of the embryonic connective tissue, especially where a vascular tissue, such as the fat, is about to be developed, are completely studded with cells like these, occupied by a number of coloured spheroids and forming nests of bloodcorpuscles or minute 'blood-islands.' After a time the cells become elongated and pointed at their ends, and processes grow out to join prolongations of neighbouring blood-vessels or of similar cells. At the same time vacuoles form within them, and becoming enlarged coalesce to form a cavity filled with fluid, in which the reddish globules, which are now becoming disc-shaped, float. Finally the cavity extends through the cell-processes into those of neighbouring cells, and a vascular network is produced, and this becomes eventually united with preexisting blood-vessels, so that the blood-corpuscles which have been formed within the cells in the manner described get into the general circulation." I

The "intracellular" mode of development of the red blood-corpuscles in most animals ceases at or before birth. They are supposed to be developed after birth (in the growing child and adult) from the red marrow of the bones, especially that of the ribs, and from the leucocytic marrow-cells called erythroblasts. This view is, however, disputed, and the erythroblasts, according to Bizzozero, are not developed from the leucocytic marrow-cells, nor from the white corpuscles of the blood, but are corpuscles sui generis, which multiply by karyokinesis, and become gradually transformed in the mammalia, with the disappearance of the nucleus, into the red blood dises.

This view is favoured by Professor Schäfer, who states that the coloured cells observed by him "have almost always been distinctly smaller than the ordinary marrow-cells, often of irregular forms, and sometimes appear to be undergoing division. They are amœboid cells, the protoplasm of which is coloured by hæmoglobin, and they closely resemble the nucleated red blood-corpuscles of the embryo. It appears, therefore, probable that the cells in question are descendants of the embryonic red blood-corpuscles, and that they are transformed into the ordinary blood-dises by the gradual atrophy and disappearance of the nucleus and the moulding of the coloured cell-substance into the shape of the bi-concave red corpuscles." It will be observed that the series of changes which result in the formation of blood and blood-vessels are self-inaugurated, and in no way influenced by irritability, external stimuli, or environment.

The production of young blood is especially interesting in its relation to the capillary blood-vessels ; the blood and the channels through which it is to flow being practically developed at the same time. The simultaneous development is an important feature as indicating design. The blood-vessels and their contents are evidently part of a general scheme.

Similar arrangements are witnessed in the growth of young bone, which may be developed in membrane or in cartilage. In the flat bones of the head (membranous to begin with), such as the frontal and parietal, the membranes are stiffened by the deposition of lime (earthy salts). This lime is not, however, scattered all over the bones. On the contrary, it radiates from an appointed centre stellate-fashion, and spreads towards the periphery of the bones. In like manner the long bones which have their origin in hyaline cartilage have separate centres of ossification; the centres increasing according to age until puberty is reached. Thus in the femur or thigh bone, the first centre of ossification appears in the middle of the shaft about the beginning of the third month after impregnation. In this case the ossification extends up and down the shaft before the other centres appear. Towards the end of fœtal life a second centre appears at the lower portion of the bone and forms part of the knee-joint. A year after birth, a third centre appears at the upper end of the bone and assists in forming the hip joint. During the fourth year a fourth centre appears and forms the knob known as the trochanter major; a fifth centre appearing during the fourteenth or fifteenth year to form the trochanter minor. The thigh bone is thus developed from five centres (the sacrum has as many as thirty-three centres). The shaft or body of the bone is called the "diaphysis" ; the other parts being designated the "epiphyses." In all this there is marked order and strict adherence to a building plan;

1 Quain's "Elenpents of Anatomy," edited by E. A. Schäfer, LL.D., F.R.S., and George Dancer Thune, vol. i. past ii. 1898, pp. 217-219. 


\section{DEVELOPMENT OF BLOOD, \&C., IN MAN AND MAMMALS}

the mode of construction being always the same. The long bones forming the arms and legs (as also those forming the vertebral column) are separated by layers of cartilage, and this circumstance, taken in conjunction with the successive appearance of the centres of ossification, favours the argument for design; certain portions of the bones becoming ossified to support weight, other parts remaining cartilage to prevent concussion or shock. Professor R. Owen states the argument thus: "The young lamb or foal can stand on its four legs as soon as it is born; it lifts its body well above the ground, and quickly begins to run and bound. The shock to the limbs themselves is broken and diminished at this tender age by the division of the supporting long bones, by the interposition of the cushions of cartilage between the diaphyses and epiphyses."

The formation of the vertebral column and its muscles is deserving of special notice because of its central position and segmented nature. It consists in the embryo of symmetrical, square-shaped, cellular and cartilaginous masses, which are virtually repetitions of each other. The vertebræ, as development proceeds, afford a protective osseous canal for the safe lodgment of the spinal cord. They also afford support for the ribs, and attachment for the muscles. The vertebræ and ribs divide the body into what are practically transverse layers. The young muscles connected with the backbone emphasise this arrangement. "The muscles of the trunk are formed from the protovertebræ. These are at first separate masses of mesoblast, the cells of which have at the periphery of the mass a tendency to a radial disposition, whilst towards the centre they are loosely arranged, and may even leave a more or less distinct space unoccupied by cells (proto-vertebral cavity). Whether there be originally a cavity or not in it, the proto-vertebra presently becomes filled up with cells and then forms a fairly compact mass of cells which are most irregularly arranged, but externally (next to the cutaneous epiblast) become regularly disposed into an epithelium-like plate of columnar cells. This is known as the muscle-plate, and in it the original mesoblastic segmentation continues to be exhibited. They do not long remain as a single epithelium-like layer, for the extremities of this layer fold sharply round and become continuous with a cell-stratum, which immediately lines the internal surface of the columnar layer and forms an inner muscle-plate. It is uncertain whether the cells of this inner muscle plate were derived from part of the columnar layer which has folded over, or whether they spring from other cells of the proto-vertebra. Soon after their appearance as a distinct layer of the muscle-plate they begin to elongate in the sagittal (antero-posterior) direction, and it may presently be observed that they are becoming developed into longitudinal groups or segments of muscle-fibres which stretch between the original intervals between the protovertebræ. Although the muscle-plates are originally mainly concerned with the formation of the muscles which move the central skeletal axis, it is probable that all the skeletal muscles both of the trunk and limbs are eventually derived from them. The vertebral column is developed around the notochord, except at the anterior end of that structure, which is imbedded in the basis cranii. It is formed from proto-vertebral mesoblast. The outer part of each proto-vertebra is transformed into a muscle-plate, and thus the original mesoblastic segmentation is maintained. The inner parts of the proto-vertebræ do not, however, remain distinct, but blend with one another on each side of the neural canal to form a longitudinal mass, which extends to the side of and subsequently encloses the notochord, and finally sends dorsal prolongations over the neural canal. The first appearance of the permanent vertebræ is in the form of cartilage, which becomes formed in this mesoblastic investment on either side of the neural canal, nearly opposite the interval between each two muscle-plates, to form the neural arch. This part of the vertebra therefore alternates with the original mesoblastic somites as represented by the muscle-plates. The serial arrangement of the musculature represents phylogenetically the original segmentation of the vertebrate body. The segmentation of the vertebral column, on the other hand, has been arrived at later, and has been carried out in dependence upon the muscular segmentation."

The cartilage makes its appearance on the fourth day in the chick, on the eleventh or twelfth day in the rabbit, and in the fourth or fifth week in man (Kölliker). It is completed by the sixth or seventh week, soon after which ossification begins. To form the inter-vertebral discs, the mesoblast between the bodies of the vertebræ acquires a fibro-cartilaginous character, while at the same time the notochord, which gradually elsewhere becomes reduced in size and eventually disappears, here undergoes enlargement, and its cells form an irregular network in the central inter-vertebral pulp.

The limbs, which ultimately form important auxiliaries of the back bone and body, "arise as outgrowths from the lateral part of the trunk in the thoracie and pelvic regions, on the third day in the chick, and in the third and fourth week in the human embryo. They appear as flattened semi-lunar thickenings of the parietal mesoblast covered by epiblast, budding out from a lateral ridge which is seen in the early embryo near the line of cleavage of the mesoblast and close to the outer margins of the muscle-plates, and several of which subsequently send prolongations into each limb; they are therefore connected with several mesoblastic somites, as is also indicated by their nerve supply." 1

1 Quain's "Elements of Anatomy," edited by E. A. Schäfer', LL.D., F.R.S., and George Dancer Thane, vol. i. part i. 1898, 11. 159-163. 
The pre-arrangement and design so conspicuous in the development of the blood-corpuscles, blood-vessels, muscles, and bones, is emphasised and accentuated in the case of the nervous system. This may be said to form the keystone of the lofty and superlatively beautiful organic arch. The nervous system in man has a cellular origin, and grows like all other parts of the body. It becomes more and more complex as differentiation proceeds. Structurally and functionally it attains to the most exalted position. Originally simple, it ultimately consists of an extraordinary assemblage of nerve cells, ganglia, and nerve fibres. These, in one sense, are independent, in another they are interdependent and inextricably interwoven. Collectively they form the organ of the mind. The several portions of the brain are to be regarded as expansions of the spinal cord, as the several parts of the skull are to be regarded as expansions of the vertebræ forming the vertebral column. The spinal cord and brain are segmented, highly-symmetrical structures, and consist of pairs of ganglia arranged on either side of the mesial line of the body at regular distances. The segmentation is indicated by the beaded outline of the medulla oblongata in the embryo; the medulla being the first expansion of the spinal column. The double chain of ganglia referred to are connected with each other longitudinally and transversely by commissural nerve-fibres. They are also connected with symmetrical sets of sensory and motor nerves which extend to all parts of the body, and by the aid of which, in the adult, they receive messages from the outer world through the skin and sense organs, and despatch commands to the voluntary muscles which they set in motion. The following is the description of the origin of the nervous system as given by Quain: " "The whole of the central nervous system takes origin from the thickened walls of a dorsally situated axial groove, subsequently converted into a canal, which runs forwards in front of tho primitive streak, and the anterior end of which becomes enlarged and converted by constrictions into three successive vesicles, around which the several parts of the brain are formed, and which are known as the primary cerebral vesicles (Plate xci., Fig. 1; Plate xcii., Figs. 5 and 6). The remainder of the neural canal is of nearly uniform diameter, and its walls become converted into the substance of the spinal cord, while the cavity itself becomes eventually the central canal of the cord. The walls of the neural groove are composed of epiblast, and it therefore follows that the whole structure of the central nervous system is laid down in epiblast, and consists in the main of more or less modified epiblastic elements, except where mesoblastic tissues subsequently penetrate into it, conveying blood-vessels into its substance. As was shown by Balfour, the same is in all probability true for all the nerves of the body, cranial and spinal.

"The cord is at first oblong in section, with an angular depression in each side which serves to mark off the situation of the future posterior columns and their corresponding grey matter from the antero-lateral region. These two parts of the lateral neural epiblast may be distinguished as the dorso-lateral (alar) and the ventro-lateral (basal) laminæ; with the former, the afferent nerve fibres become connected, whilst from the latter the efferent fibres take origin (His). In the human embryo of six weeks, they are well marked off from one another, and their respective connections with the posterior and anterior nerve roots are very distinct. The nerve fibres of the white columns are at first entirely non-medullated, and the white substance has a greyish transparent appearance. The medullary sheath is not formed simultaneously in all parts, but appears at different times in different parts corresponding with the tracts of conduction: the last of these tracts to become medullated are the pyramidal tracts.

"The vesicles of the brain, which are at first three in number, become subdivided so as to form five in all, which may be termed in succession from before back, the first, second, third, fourth, and fifth secondary vesicles. Of these five parts the first two, which represent the cerebral and thalamic parts of the future brain (third ventricle), are derived from the first primary vesicle, and the last two, the cerebellar and bulbar parts (fourth ventricle), form the third primary vesicle, while the third, middle, or quadrigeminal part represents the undivided second primary vesicle (Sylvian aqueduct).

"The first and most striking change which occurs in the primary brain is the outgrowth on either side of the first primary vesicle of a hollow protrusion (primary optic vesicle), which becomes developed eventually into optic nerve and retina.

"Subsequently another pair of hollow outgrowths sprout from the fore-brain, and these rapidly extend forwards, laterally, and backwards; they form the vesicles of the cerebral hemispheres.

"The principal parts of the brain appear as thickenings in different parts of the walls of the vesicles. Thus the corpora striata are formed in the floor of the hemisphere vesicles, whilst the principal mass of each hemisphere is formed from the roof and sides (mantle) of those vesicles, and the olfactory lobes are hollow outgrowths from them. The optic thalamus is formed by a thickening of the lateral wall of the second vesicle, the cavity of which comes to be the main part of the third ventricle; the corpora quadrigemina are thickenings in the roof, and the crura cerebri thickenings of the sides and floor of the third vesicle, which becomes the aqueduct of Sylvius; the cerebellum and pons are respectively thickenings of the roof and floor and the crura cerebelli of the sides of the fourth

$$
1 \text { op. cit. p1. 57-63. }
$$




\section{DEVELOPMENT OF BLOOD, \&c., IN MAN AND MAMMALS}

vesicle (anterior part of hind-brain), the cavity of which becomes the anterior (superior) part of the fourth ventricle; and finally, the medulla oblongata is developed as a thickening of the wall of the fifth vesicle, the cavity of which expands from the central canal of the spinal cord to form the calamus scriptorius of the fourth ventricle.

"While these changes are going on in its walls the embryonic brain does not remain straight as at first, with its axis in a line with that of the spinal cord, but undergoes certain flexures, the general result of which is to bend the anterior end towards the ventral surface."

As the nervous system is, in a sense, inoperative in the embryo and fotus it is not necessary to pursue the subject further here. It is, however, fully treated further on, both in its simple and complex condition, as it occurs in the lower animals and in man. It is only necessary to state that the nerve-cells, ganglia, spinal cord, brain, sensory, motor and sympathetic nerves which constitute it, and which furnish an unparalleled display of the highest forms of organic matter, are all prepared in advance of the complicated functions to be discharged by it. The nervous system, more than any other system in the body, bears eloquent testimony to design and a Designer. In no other system is there such extraordinary elaboration; such a magnificent array of well-chosen means to definite and obvious ends. Nor is the labour expended in any sense squandered or wasted, when it is remembered that on the quantity, quality, and integrity of the nervous system all mental and conscious manifestations depend.

Fig. 80.-Nervous systems of the centipede and of man.

A. Centipede (Scolopendra). The nervous system of the centipede consists of a linear series of nearly equal and similar ganglia arranged in pairs, situated upon the median line, along the ventral surface of the alimentary canal. Each pair of ganglia is connected longitudinally and transversely by commissural nerve fibres, and distributes sensory and motor nerves to its own segment or articulation. In the first articulation which forms the head ( $(c)$, the ganglia are larger than elsewhere, and send nerves to the antennæe and to the organs of special sense. This pair is termed the cerebral ganglion, or the "brain." $c$, $c^{\prime}$, Longitudinal commisural nerve fibres, which with their ganglia form what is virtually a spinal column (compare with $c, c^{\prime}$ of Fig. B).

B. Cerebro-spinal system of man (Homo sapiens). a, Cerebrum; b, cerebellum; $c, c^{\prime}$, spinal cord giving off sensory and motor nerves (compare with $c, c^{\prime}$ of Fig. A); $d$, brachial nerves; $e$, sacral nerves (after Dalton).

The nervous systems of the centipede and of man bear a striking general resemblance to each other; allowance being made for the greater development and cumplexity of the latter. The cerebro-spinal nervous system in man is seen on an enlarged scale at Plate exxxii. Fig. 1.

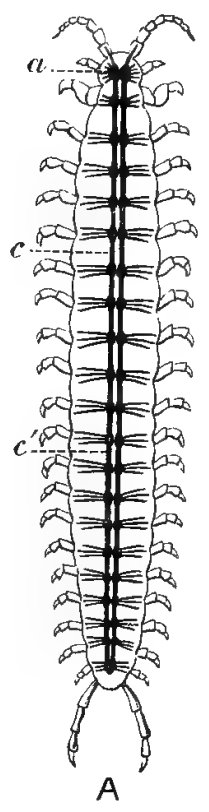

FIG. 80 .

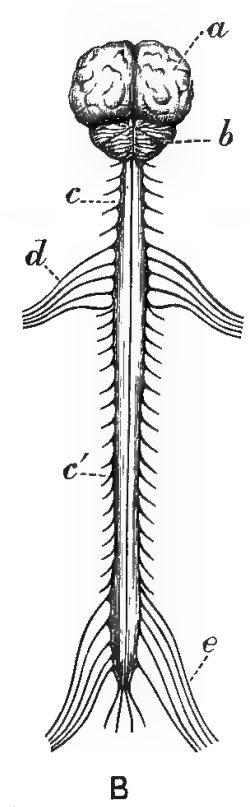

B

The evidence of design so conspicuous in the construction of the heart and brain is equally apparent in the development and construction of the lungs. The heart, the brain, and the lungs may be said to form the tripod of life in the higher animals and in man.

The brain, as already explained, is an expansion and modification of the spinal cord, and literally consists of millions of nerve cells connected directly and indirectly with nerve fibres. It is simple in its inception, and becomes complex in the adult and as the animal rises in the scale of being. Here again there is community of structure and proof of type as between the lower and higher animals. In the centipede, one of the articulata, the nervous system consists of a brain and what is virtually a spinal cord, with sensory and motor nerves given off at regular intervals, as shown at $\mathrm{A}$ of Fig. 80, above. 'The body of the centipede is segmented, that is, divided transversely; the sensory and motor nerves being distributed to the segments. The nervous system is divided longitudinally, the portions representing the quasi-cord and brain being composed of two equal halves. The quasi-cord is composed of a double chain of ganglia united by nerve commissures longitudinally and transversely; the cephalic ganglia being enlarged and approximated to form a rudimentary brain. Here there is longitudinal and transverse division and a repetition of parts indicating general plan and type. In the human brain and spinal cord a precisely similar arrangement of parts obtains, as shown at B of Fig. 80, above.

Thus the human spinal cord and brain are divided longitudinally into two symmetrical halves, and the ganglia are arranged in pairs, or in duplicate both in the cord and brain. The sensory and motor nerves, moreover, are 
given off at regular intervals, indicating a transverse division. Further, the ganglia in the spinal cord and brain are connected together by longitudinal and transverse commissural nerve fibres. The longitudinal and transverse arrangements seen in the nervous system of such a simple animal as the centipede are, it will be seen, repeated with comparatively little variation in the highest members of the animal series. As it is not possible to regard the nervous system of the centipede as the direct or even the indirect progenitor of that of man, we are forced to fall back upon a general plan and types for an explanation.

Similar remarks may be made of the lungs. In the lower animals, say the triton, the lungs are composed of simple sacs displaying a large number of capillary blood-vessels. In the lungs of the frog the sacs are folded in and duplicated and crumpled up to form partitions and loculi. In the human lungs this process is carried to an extreme; the lungs, on section, presenting a honeycombed, porous appearance. In the human embryo the lungs first appear as simple tubular sacs; as development proceeds they are divided into lobes, lobules, and air vesicles; one of the latter resembling in its details the entire lung of the frog. The human lungs are a crowning example of longitudinal, transverse, and oblique cleavage and of infolding, duplicating, and crumpling. It may be noted in passing that the same is true of the cortex of the human brain, and kidneys.

The bronchial tubes, as well as the substance of the lungs, divide and subdivide to quite an extraordinary extent, and present a characteristic tree-like appearance. The object is to provide a comparatively large space in a constricted area; the idea being to obtain a very extensive frame-work for the support of endless capillary blood-vessels; these containing the blood, which is aërated and purified during respiration. The appearance presented by the lung of the frog, the human air vesicle, and the human lung is seen at Plate lxxvii., Figs. 1, 2, and 3 , page 275 .

Other organs besides the brain, heart, and lungs are laboriously built up after undergoing numerous important modifications in utero. This is true of the liver, kidneys, and glands generally. These exercise a powerful influence on the digestion and in keeping the quality of the blood at a high standard. The blood glands proper, and the lungs, contribute to this result. The food enriches the blood and the blood feeds all the tissues, hard and soft, in all parts of the body. Everything hangs together in a living compound organism, as everything hangs together in the universe itself. There is mutual interdependence.

The stomach, liver, kidneys, and glands are simple in the foetus as compared with what they are in the adult, and in the lower animals as compared with man. There are, moreover, numerous points of resemblance between them. These, however, indicate a general plan and types rather than the direct or indirect evolution of the one from the other. Neither plant nor animal can add to or take from their fundamental structures, either during development or in the adult condition.

The fact that all the organs of the body are prepared in advance of the function to be performed by them, and before the offspring is born, furnishes, it appears to me, an unanswerable argument in favour of design, and of means to ends, which neither natural selection nor evolution can even partly explain.

Other weighty arguments in the same direction are to be deduced from the development of the sense organs in man. In the fotal condition the sense organs are all in abeyance; in other words, they are not called upon to perform any function. Nevertheless, they are duly formed in anticipation of the work they will have to discharge after birth. In utero no food reaches the mouth and palate, no odours penetrate the nostrils, no sounds distract the ear, and no light falls upon the eye. As yet no relation is established between what may be called the subjective (the conscious ego) and objective (the outer world); there is no irritability, extraneous stimulation, or environment in the ordinary sense. This fact affords an all-powerful argument against the theory that the sense organs are the creation of externalities. In reality, the sense organs are expressly constructed to deal with and interpret extraneous matter, that is, matter outside the individual; and it is a mere perversion of language and of fact to say that outside substances and environment form any part of the body, and, least of all, of the sense organs. The senses in the child develop pari passu with the intelligence, but the actual structures forming the sense organs are virtually completed before birth. The sense organs are trained and educated after birth as the muscles are, but the organs, as organs, are in actual existence before birth. After birth they form the medium between the individual and the universe, and everything the universe contains. The extreme value of this medium can scarcely be adequately estimated, as it brings the individual directly or indirectly into contact with, at once, the smallest and lightest, and the greatest and heaviest, masses of matter near and remote. It enables him to taste the most minute crystal of sugar, to smell the tiniest particle of musk, and to survey the sun in his mid-day strength, and the mysterious stars in their far-off. jewelled splendour. The sense organs perhaps more than any other organs in the body reveal the hand of the great Designer. They form the veritable connecting-links between matter and mind, and are, from this circumstance and properly, designated the gateways of knowledge. 


\section{DESIGN IN MIGRATION OF BIRDS AND OTHER ANIMALS}

\section{§ 84. Design as Witnessed in the Migration of Birds and Other Animals.}

It is very difficult, if not indeed impossible, fully to explain the phenomenon of migration. No doubt it is largely connected with the food supply and, in the case of birds and fishes, with nidification and spawning. The seasons, the increase of heat or cold, and the velocity or force of the winds are also to be taken into account. The conditions attending migration are complex, and such as cannot be fully appreciated by the animals which migrate. The distances covered extend occasionally to hundreds or thousands of miles, and, in not a few instances, the migrations are made during the night. This is true of large numbers of birds.

Great flights are made by birds in the murky darkness, and fish migrate in shoals into and out of deep water, where their eyes can be of little or no service. Birds and fishes perform their migrations, as it were, without sight, and minus a compass. Their landmarks, if they have any, must be few and far between. As they cannot be credited with a full knowledge of the complicated circumstances which determine their movements, the movements themselves are not a little mysterious.

Birds reared in certain localities migrate, and in due season return to the place of their birth for nesting and other purposes. Young salmon, sea-trout, \&c., make long journeys down the rivers in which they are hatched to the sea, where they remain for stated intervals. They attain maturity in the sea, from which they finally emerge full of spawn. They ascend the parent rivers, and in turn deposit their spawn there, which is hatched out as before. Young salmon and sea trout which have been artificially marked invariably return to the same rivers. They take next to no food in the fresh water before spawning, so that the question of food prior to spawning may be largely eliminated in the migrations of these valuable food fishes. Birds also when rearing their young are indifferent to food.

In considering the subject of migration, one of two things is evident. Either the migrations of animals are arranged for and are pre-determined, or, in the remote past, the migrations must have been performed intelligently. This means that the migrating animals understood what they were about, ${ }^{1}$ and that they were largely guided to their respective destinations by landmarks which are now wholly or in great measure swept away.

It is worse than useless to attempt to explain the phenomenon of migration by the ill-defined and looselyemployed term instinct, which, in this connection, means nothing. Instinct, moreover, as I show elsewhere ( $\$ 48$ of the present work) is, in every instance, preceded by intelligence. To explain migration, intelligence, or its equivalent must be conceded to the parents and to the offspring. If that be withheld, a guiding power has to be substituted, and that guiding power can only be traced to a First Cause. The intelligence of birds and fishes (as we know them) is limited, and inadequate fully to explain all the peculiarities of migration. If it accounted for short journeys made during the day with landmarks as guides it would not account for long journeys made during the night without landmarks. If, again, the ancient progenitors of birds and fishes were wiser than their modern progeny, then birds and fishes have not evolved or become more perfect. On the contrary they have retrogressed. This affords an argument for the creation of types as contra-distinguished from a continuous evolution from lower to higher forms, from the monad to the man. On the whole a First Cause and design furnish the more feasible explanation of the migratory habit.

One can understand how animals pressed by hunger because of the failure of the food supply in certain regions at certain times make short journeys in daylight, but when they make long journeys in the darkness the explanation is by no means on the surface. Animals are no doubt endowed with powers not vouchsafed to man. Eels which are taken out of the water, if left to themselves, immediately make for it. Young turtles hatched in the hot sand make straight for the water even when turned about and placed with the head away from it. Land crabs often make long journeys to moist regions with apparently nothing to guide them, and homing pigeons seldom make a mistake in direction, and frequently travel incredibly long distances. Young ducks reared by hens make for the pond almost as soon as hatched. Chickens scarcely out of the shell begin to peck, and pick up food before they can have any idea of what food means.

Dogs and cats removed by train or boats fifty or more miles distant very frequently return to their original homes, travelling through unknown territory as quickly as if they knew every foot of the way. In like manner bees, however devious their course when leaving the hive, as a rule, return to it in a straight line-hence the phrase "bee-line."

If, however (and here comes the crux of the matter) animals can perform, more or less unerringly, long journeys without apparent landmarks and even in the darkness, it follows that the guiding power is not wholly within themselves, and that in their migrations they are carrying out a design which they can, at best, only partly realise.

1 Many are of opinion that old, intelligent, sterile birds act as leaders in migratory flight, and direct the movements of the younger and less well-informed birds in their protracted journeys. Not a few are of opinion that migration is determined by the velocity of the wind. No migrations are performed where the speed of the wind exceeds from thirty-tive to forty miles an hour. 
The British Association in the year 1880 instituted an extensive inquiry into the migrations of birds in Great Britain and Ireland which was brought to a conclusion in 1903. The committee appointed to carry out the observations put themselves into communication with the numerous lighthouse keepers, some 200 in number, on the British and lrish coasts. For eight years (1880-1887) the numerous observations accumulated; a digest of them being prepared for the Liverpool meeting of the Association in 1896. ${ }^{1}$ This gave in a condensed form the general results of the inquiry in all its aspects, geographical, seasonal, meteorological, \&c. Then followed a series of histories of eight carefully-selected migrant British birds, whose migratory movements and the conditions under which they were made were studied and duly noted. Mr. William Eagle Clarke, in a recent article in Nature (March 31, 1904, p. 516), gives a resumé of the British Association Reports, and adds important observations of his own. I am indebted to him for the following interesting remarks: "Turning now to some of the special results of the inquiry, in the first place it was clearly proved that a considerable proportion of our native-bred song-thrushes, blackbirds, skylarks, starlings, rooks, lapwings, and other species, which are usually regarded as being wholly resident throughout the year, are migratory; indeed, they are as essentially summer visitors to our isles as the swallow and the cuckoo. They leave us before the end of summer for Southern Europe, and are the first harbingers of spring to appear on our shores, arriving during February and early March. ${ }^{2}$

"As regards the geographical aspect of the subject, perhaps the most interesting of the varied movements investigated are those remarkable intermigrations which take place between the south-eastern coast of England and the opposite shores of the Continent by a westerly autumn and easterly spring flight. The birds reach England in the autumn and return to the Continent in spring. Day after day in late September and during October, when the weather is suitable, vast numbers of skylarks, starlings, chaffinches, tree sparrows, rooks, and jackdaws rush across the southern waters of the North Sea, proceeding chiefly due west off the mouth of the Thames (the centre of the stream), south-west off the coast of Kent, north-west off Norfolk, and north-north-west off the Humber. Corresponding return migrations in opposite directions are witnessed in the spring. A noteworthy feature of these movements is that they are performed during the daytime; indeed, they are the main diurnal flights observed on the British coasts.

"During the preparation of the digest and of the various reports," Mr. Clarke observes, "I was so much impressed with the singularity and importance of these movements that I decided to make some further investigations regarding them, and to this end I spent nearly five weeks on the Kentish Knock Light-vessel, situated thirtytwo miles east of the Eissex coast and out of sight of land, during the past autumn (see Ibis, pp. 112-142). I was previously uncertain as to whence came these hosts of migrants; now I am of opinion that they are emigrants from western Central Europe, which, having probably descended the Maas, Rhine, and Scheldt, quit the Dutch coast at the mouths of those rivers en route for winter quarters. Some of them remain during the winter in England, others proceed to Ireland, and others, again, depart from our southern shores for more southern lands. There can be little doubt that many of those which remain in our islands winter in latitudes north of their summer homes.

"Turning next to the meteorological aspect of bird-migration, it has been possible to make a careful comparison between the unique data obtained through the inquiry, and the reports issued by the Meteorological Office, and thus to establish satisfactorily certain relations between migrational and meteorological phenomena. For instance, it has been found that each great arrival on our shores of migrants from North-west Europe in the autumn is correlated with a certain type of pressure distribution which establishes fine weather over the North Sea between Scandinavia and the British Isles. Such conditions, however, though they may prevail at the all-important point of departure, and hence induce migration, do not always extend so far as Britain, and when this is the case the migrants pass into more or less unfavourable weather ere they reach our shores.

"During a month's sojourn in the Eddystone Lighthouse (see Ibis, 1902, pp. 246-269) in the autumn of 1901, I paid special attention to the weather conditions under which the migrants set out to cross the Channel. I found that no movements were witnessed when the weather was in the least degree unfavourable for the passage, and that the wind is undoubtedly the main factor in migration meteorology. The direction of the wind was of no moment, for the birds flitted southwards in winds from all quarters. It was otherwise when its velocity came to be considered, and no movements were performed when this exceeded about twenty-eight miles an hour. At thirty-four miles the few stragglers observed were in distress, and the only birds moving when it exceeded this and approached forty miles were swallows and martins. My subsequent experiences at the Kentish Knock Lightship confirmed these conclusions.

"The supposed influence of the direction of the wind on migratory movements has been the source of much misunderstanding, chiefly because the dependence of the wind upon atmospheric pressure does not appear to have been taken into consideration. We now know that certain types of pressure distribution are favourable for, and

1 Reports of the British Association for 1896 (p1) 451-477).

${ }^{2}$ British Association Reports for the years 1901, 1902, and 1903. 


\section{DESIGN IN THE PRODUCTION AND DISTRIBUTION OF SEEDS}

conducive to migration, and the winds also resulting therefrom have erroneously come to be looked upon as the cause for such movements.

"Finally, the investigation of certain movements, namely, the emigrations, has presented exceptional difficulties, due chiefly to the fact that they are habitually performed under conditions which enshroud them in all but complete obscurity. The reason for this is that, with few exceptions, emigration is undertaken during the hours of darkness, and thus entirely escapes notice at the place of embarkation. It was the object of investigating this phase in the phenomenon of migration that led me to visit the Eddystone, where it was possible to observe these emigrants immediately after their departure from our shores. There I found that at least 90 per cent. of the various emigrants crossed the Channel during the night. Indeed, night movements are undoubtedly the rule when considerable expanses of sea have to be traversed. To this rule the chief exception has already been mentioned: but both at the lighthouse and at the lightship I found that day migration was confined to a few species only."

\section{§85. Design as seen in the Production and Distribution of Seeds.}

The production and distribution of seeds is a wide and fundamental subject. In the simplest plants and animals every part of their substance possesses the properties and powers of the whole, and any part thrown off or broken off is capable of independent existence. Certain plants and animals can be cut into pieces, or portions detached, and yet propagate themselves. Reproduction is effected by simple fission or splitting. It is also effected by budding, where a portion of the original individual is extruded and thrown off at particular periods ; the bud or portion thrown off possessing in a latent form the properties and powers of the parent.

It is only in the higher and highest plants and animals that germs, seeds, and eggs make their appearance.

As plants and animals can have no existence as apart from life conferred, so the higher and highest plants and animals can have no existence, as apart from seeds or ova of some kind. The possession of seeds, or their equivalents, is as fundamental as the possession of life, and each seed produces only its own kind. The seeds never become accidentally mixed up. A fig-tree is never produced from the fruit of a thorn or other tree, and the fish, reptile, bird, and mammal are, in every instance, the product of specific eggs, seeds, or ova of some kind. There are boundaries to seeds as to everything else. The power of producing seeds is an original endowment, and the very essence of life in its higher forms. Life, under all circumstances, is the starting-point of plants and animals, and the power of reproduction by means of germs, seeds, and eggs is necessary to the continuation of the higher life on the earth. Only the healthy and vigorous plants and animals are capable of producing seed. Not only is the seed-producing power conferred, but the most extraordinary precautions are taken for its continuation. Thus, seeds are produced in incalculable numbers, in many cases in millions. As a matter of fact seeds, eggs, and the immature young form a very large portion of the food-supply of animals.

The ova and the countless young fishes which frequent the rivers, lakes, and seas yield a practically unlimited food-supply for the finny tribes generally; berries, nuts, and fruits form the chief sustenance of birds; while seedbearing and other grasses constitute the chief pabulum of the herbivora.

The young of terrestrial animals bulk largely in the food of the carnivora. Man, as an omnivorous animal, eats fish, fowl, and the herbivora indiscriminately. He eats everything (the carnivora excepted), and it is impossible to indicate to what extent he benefits by the arrangement, as he lays not only the young, but the middle-aged, and even the old, under contribution. He draws his food-supply indirectly from spores, germs, seeds, and eggs, which, as explained, are enormously in excess of mere reproductive requirements.

The fruits, seeds, and eggs are, in many cases, delicately flavoured and tempting. They consist of starches, albumen, proteids, \&c., and contain, in many instances, the most delicious juices, as witness those of the apple, pine-apple, lemon, orange, loquat, peach, nectarine, grape, \&c. These no doubt are cultivated fruits, but the same holds true of wild fruits, as the raspberry, bramble, wild strawberry, \&c. The cereals have each their particular flavours and properties. The cultivated fruits, as a rule, furnish a more delicate flavour, but in certain cases the wild flavour excels the cultivated one.

Each individual seed is provided with its own pabulum, on which it subsists until it develops sufficiently to obtain other food. The seed is as dependent on its envelope, or food covering, as the individual is on the seed. The seed is, in a sense, at once the progenitor and support of the individual.

The fact that all seeds are invested with a covering of food affords a crowning proof of design, as it means support to the seed itself and support to the organic kingdom as a whole, directly or indirectly. The exigencies of the seed, the individual, and the race are all provided for. Design is also manifested in the distribution of seeds. They are literally thrown broadcast, by the winds and waters, and by animals of all kinds. Insects take a prominent part in the fructification of plants, and birds and other animals carry seeds to great distances. The inorganic

VOL. I. 
and organic forces both contribute to the dissemination of seeds. These forces are directed and controlled in the operation. Nay more, the seeds, in many instances, are specially constructed to assist in the disseminating and distributing process.

One of the best-known examples of a volant or flying seed is supplied by the goat's-beard.

The goat's-beard (Fig. 81) forms a typical parachute which for elegance of design and efficiency cannot be surpassed. It consists of an exquisite flattened disc slightly depressed in the centre; the free margin being a little turned up. It is supported in every direction by tapering structures, which radiate from a central point at regular intervals (A). Between the radiating structures a most delicate network of vegetable fibres resembling the finest filigree work extends; imparting to the whole a delicate, gauzy appearance which indicates extreme lightness and suggests the idea of flotation or flight. The radiating supporting structures display very graceful double curves;
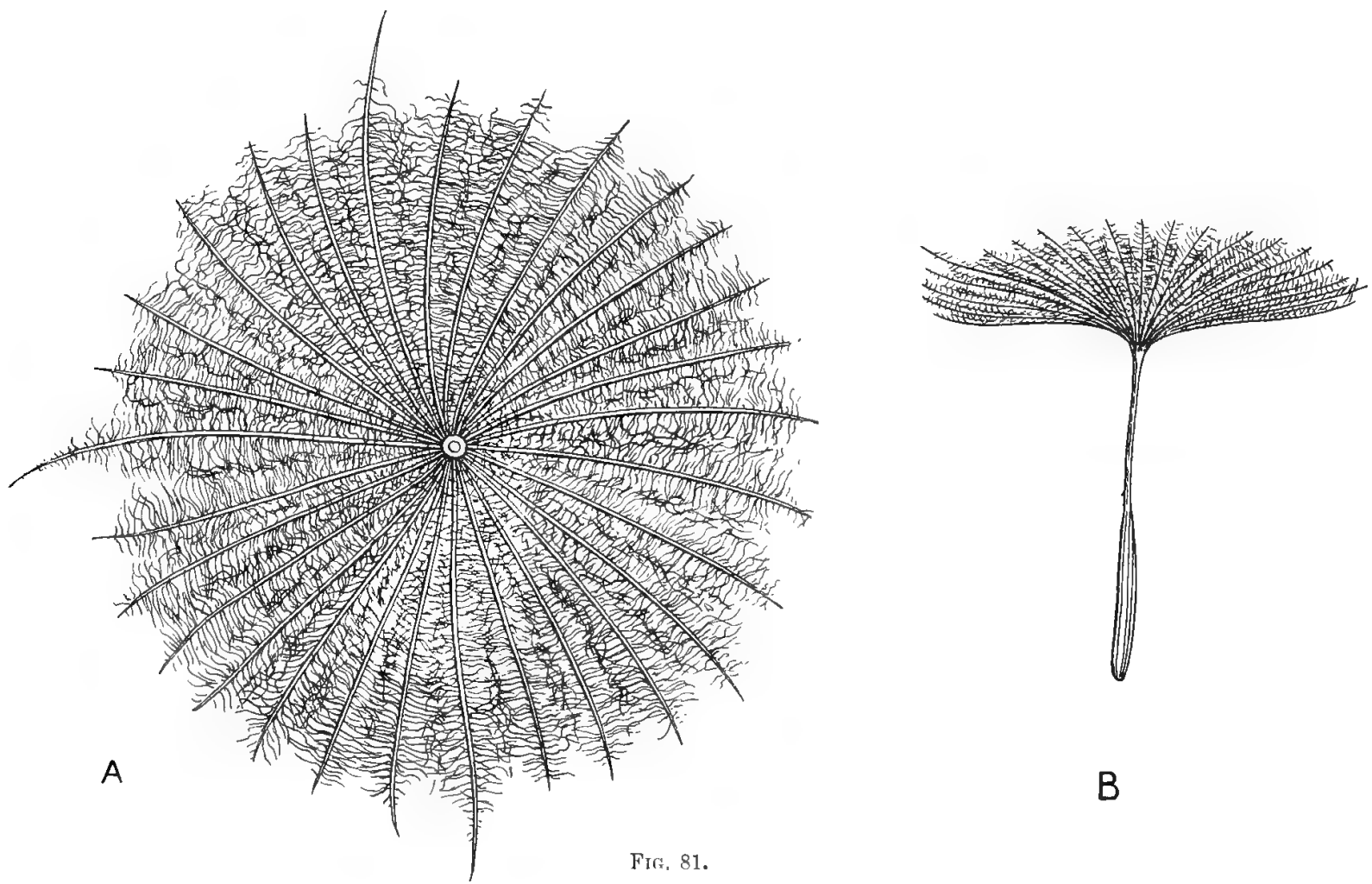

FrG. 81.-The goat's-beard (Truyplogon pratensis), as seen from above (A) and from the side (B).

A. Goat's-beard from above. Shows the radiating supporting structures of the dise with a delicate network between. Enlarged.

B. Goat's-beard from the side or in profile. Illustrates the parachute-nature of the goat's-beard. Natural size.

Drawn with special care from a fresh specimen in the Author's collection by C. Berjeau for the present work.

those nearest the centre of the disc having their convexities directed upwards, those at the periphery or free margins having their convexities turned downwards (B).

Depending from the centre of the disc is a vertical portion or shaft, which may not inaptly be compared to the stick or handle of an umbrella (B). This acts as a balancer, and so ensures the stability of the centre of gravity of the seed whatever the direction of the wind. A more perfect arrangement for the waftage of the seed by the wind to a distance cannot be conceived. As an example of "means to ends" it occupies a unique position, and the annals of botany will be searched in vain for a better example of design and the operation of a First Cause. Here we have an instance of a structure, made in advance for the discharge of a special function. The structure is in the highest degree striking, but the function to be discharged is by no means so obvious. The object of the structure is, no doubt, to provide a surface which shall enable the wind to seize and waft the seed about until it finds a suitable soil for planting itself. The seed is prepared for its flight by gradually becoming lighter as it ripens. The function performed by the seed is passive (if any function can be so designated). The chief function is performed by the actively moving wind. We have a structure exercising, as it were, no function. The structure and the function, however, must be associated if any good is to result from the combination, and this can only be done by enlisting the co-operation of the wind, which is the moving power in the present case. There is a dual arrange- 


\section{DESIGN AS WITNESSED IN WINGED SEEDS}

ment. From the first it is intended that the seed of the goat's-beard shall be wafted about, and it is arranged that the wind shall be the propelling force. The arrangement is not an accidental but a designed one, and it is the employment of a structure in a new and apparently incongruous combination which stamps the act as one of design. The living seed is made the sport of the wind for a definite purpose. The seed cannot move of itself and the wind comes to its aid. The living seed is set in motion by the inanimate but not inactive wind. In other cases, the wind brings seeds from the same or neighbouring trees together. In certain instances insects and birds take part in the flotation and fructifying process.

In the several cases referred to there is an arrangement whereby nature insures that the male and female elements of plants shall be made to meet and interpenetrate each other. The seeds must be able to fly by the aid of the wind; or animals must discharge a function which in the absence of wind they alone can perform. Good examples of floating seeds are furnished by the dandelion and by the soft down of the Scotch and other thistles.

Among trees the ash and plane-tree supply splendid examples of highly-organised, winged seeds. The winged seeds appear to me so important that I describe them separately, and devote a plate to their illustration (Plate xcvi., Figs. 1 and 4 ).

\section{PLATE XCVI}

Plate xcvi. illustrates the remarkable fact that certain winged seeds display many of the structural peculiarities of real wings. Thus the seeds of the ash-tree are twisted upon themselves propeller-fashion as in the wings of insects, birds, and bats; while the seeds of the plane-tree are characterised by having a right and left wing with a body (the seed) between them.

The wings of the plane-tree are triangular in shape, and taper from the body in a direction from within outwards and from before backwards. The nervures or stretchers of the winged seeds also radiate from the body of the seed from within outwards and from before backwards as in true wings.

FIG. I.-The seed of the ash-tree (Fraxinus excelsior). This seed is twisted in the direction of its length like the blade of the ordinary screw propeller employed in steamships. Its margins exhibit the double figure-of-8 curves of the propeller in a marked manner, and show unmistakable evidence of community of structure (as far as outline is concerned) with the wing of the insect, bird, and bat, and with the chief wing feathers (primaries) of the bird. The seed of the ash is a winged seed, and the object of its screw configuration lies on the surface. It is intended to retard the fall of the seed, and so afford it an opportunity of being wafted by the wind to considerable distances where there is room to plant itself. It is most interesting to watch the shedding of the seeds of the ash-tree in the autumn. If there be no breeze they twirl round and round in a leisurely manner, and fall not far from the root of the parent tree. If, however, a stiffish breeze springs up, they are whisked away, in some instances, to quite remarkable distances. The same is true of the seed of the plane-tree, which is the most perfect example of a winged seed known.

Various other examples of winged seeds might be cited, prominent among which are the thistle-down and goat's-beard, the latter forming a most elegant and perfect parachute. $a, b$, Seeds of the ash-tree showing a twisted or spiral contour, the margins of each crossing screw-fashion; $c$, similar seed from another point of viow; $d$, similar seed cut across near its attachment. Drawn from nature by the Author.

FIG. 2.-The little auk (Aptenodytes minor, Linn.). Drawn for the Author from a living specimen by C. Berjeau. In this quaint bird the flying wings are dwarfed and employed as swimming and diving organs, with or without the feet. They are triangular in shape and graduated like other wings; their small size and want of feathers rendering them useless for flying in the air. They are flexible and elastic, but stiffer than ordinary wings; a modification necessitated by the greater density of water as compared with air. They are made to vibrate like other wings, and twist and untwist during their action, and make figure-of-8, screw movements. The flight of the bird under water is astonishingly rapid and graceful, as the Author can testify from careful observation. The moditied wings of the little auk furnish another example of design in flying structures generally in their relation to winged seeds.

FIG. 3.-Wings of the kestrel (Falco tinnunculus), seen from above and from below and behind. The lettering is the same in both cases. $a, b, c$, Posterior margin of the wing, consisting of primary, secondary, and tertiary feathers; $d, k, f$, anterior margin of the wing.

The wing of the kestrel was drawn from the specimen while being held against the light, and shows how the primary, secondary, and tertiary feathers overlap and buttress and support each other in every direction. Each set of feathers has its coverts and subcoverts; the wing being conical in shape from within outwards and before backwards. The lower figure presents a spiral contonr, and shows how it twists and untwists during its action. In certain positions it resembles the blade of an ordinary screw propeller as employed in steamships. Drawn from the natural wing by the Author.

FIG. 4.-The seed of the plane-tree (Acer pseudo-platenus). This seed is remarkable in many ways: (a) as regards its weight; (b) as regards its shape; (c) as regards its venation or nervures. It is a heavy double seed united in the middle, and provided with two very perfect wings, resembling in all respects the wings of insects, birds, and bats (compare with Figs. 2, 5, 6, 7, and 8). The wings have all the characteristics of genuine wings; that is, they are triangular in shape, are elastic, and taper in two directions, namely, from the root in the direction of the tip, and from the anterior margin in the direction of the posterior margin. They have a thick, semi-rigid anterior margin ( $c$ ) and a thin, flexible, posterior margin (c), and the venation or wing-supports curve outwards and backwards as in all the wings described. It would be difficult to produce a more perfect example of design and type in nature than is afforded by this winged seed. It shows that types are not confined to any one division of the organic kingdom, and that the First Cause employs the same or similar means to produce like results. Drawn from nature by the Author, for the present work.

FrG. 5.-The bat (Vespertilio murinus). In the bat, the peculiarities of the wing of the insect and bird are repeated. Thus, the 


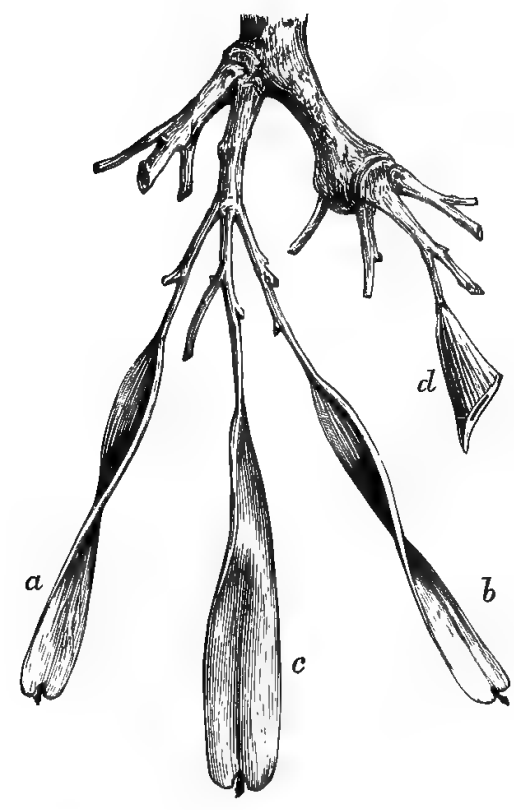

Fig. 1.

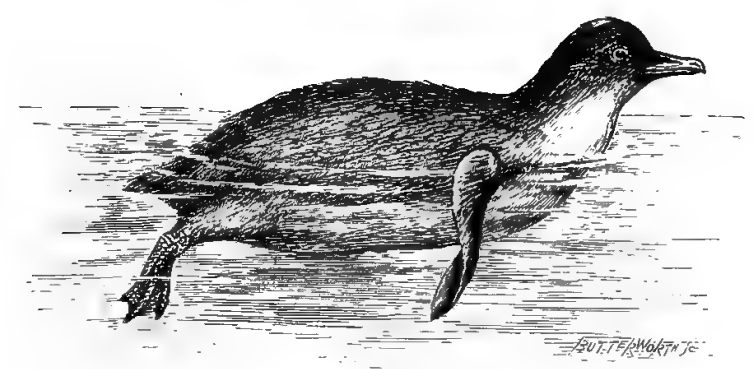

FIG. 2.
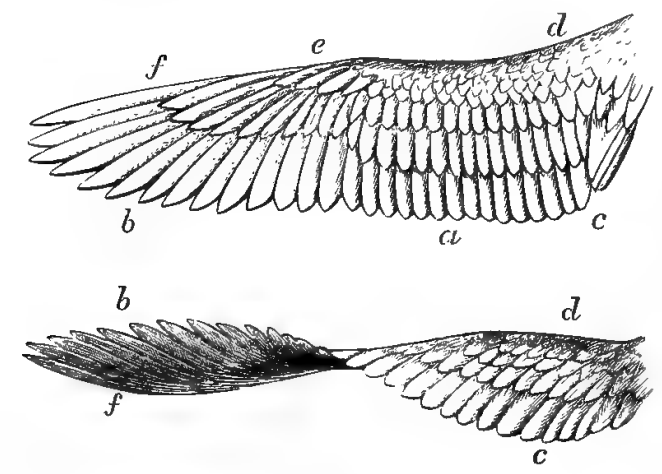

FIG. 3.

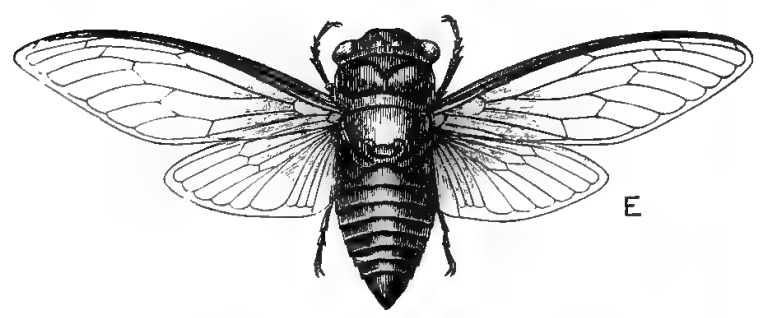

FIG, 7.

\section{PLATE XOVI}
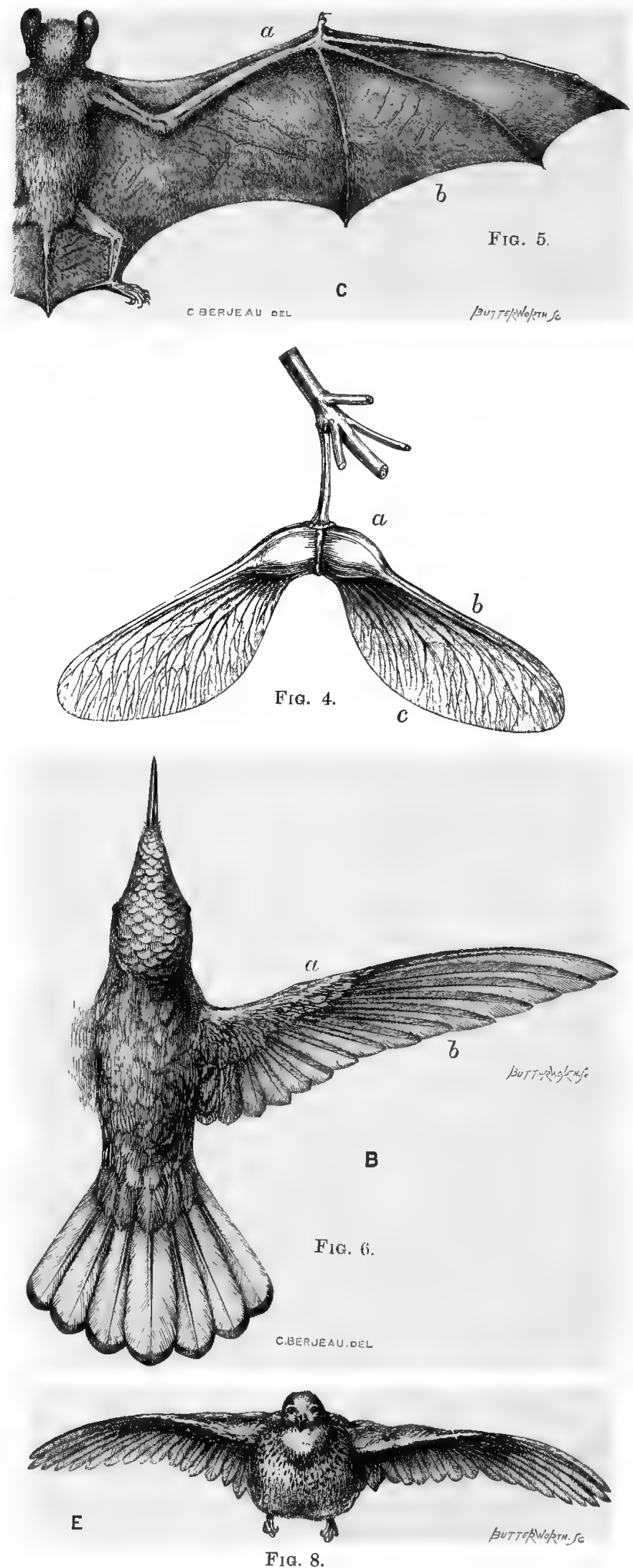


\section{PLATE XCVI (continued)}

wing is triangular in shape, concavo-convex, and elastic throughout. It tapers from the root towards the tip, and from the anterior towards the posterior margin. It is thickest, strongest, and most rigid at the root and along the anterior margin (a), and thinnest, weakest, and most elastic along the posterior margin $(b)$. It presents a continuous membrane to the air during both the up and down strokes, as in the insect; it, however, partially closes or folds during the up stroke and fully expands during the down stroke, as in the bird. The wing consists of muscles, bones, and a continuous elastic membrane which is supported by the bones of the arm, fore-arm, and hand, and by the bones of the thigh, leg, and tail. It is a most elegantly constructed, serviceable wing; the bats being splendid flyers. Drawn for the present work by C. Berjeau, from a specimen in the Author's collection.

FIG. 6.-The humming-bird (Amarilia fuscocaudata). Displays a most dainty, exquisitely-formed, concavo-convex wing, composed of bones, muscles, and feathers, the latter radiating in an outward and backward direction. The feathers which take part in flight are divided into primary or rowing feathers (tip of wing), secondaries (middle portion of wing), and tertiaries (root of wing). The wing resembles in all respects that of the insect, with this difference, that the primary, secondary, and tertiary feathers open up and separate during flexion and the up stroke, and close during extension and the down stroke. The wing of the insect, and also that of the bat, presents a continuous, unbroken surface during both the up and down strokes. a, Semi rigid anterior margin tapering from root to tip of wing ; $b$, posterior, thin, elastic, yielding margin of wing formed by the free ends of the primary, secondary, and tertiary feathers. Drawn for the present w ork by C. Berjeau from a specimen in the Author's private collection.

FIG. 7.-The cicada (Cicada septemderim). This is one of the best flying insects; its body being compact and finely formed, and its wings, which are four in number, being ample, long, and somewhat narrow. The body and wings are carefully adapted to each other, and the spectator is impressed with the idea of fitness in the general arrangements. The wings are beautifully transparent, and the venation nervures or supports of the wings radiate in an outward and backward direction, and are connected by transverse nervures which produce an open network. The nervures forming the anterior margins of the front wings are finely graduated, and taper from the root in the direction of the tips of the wings. The wings of the cicada may be regarded as typical wing structures. It is a curious circumstance that the winged seed of the plane-tree greatly resembles them, both as regards general contour and venation. Drawn for the present work by C. Berjeau, from a specimen in the Author's collection.

Fig. 8. - The red-legged partridge (Perdrix, rrbra), with the wings fully extended as in rapid flight. Shows the deeply concave form of the wings, how the primary and secondary feathers overlap and support each other during extension, and how the anterior or thick margins of the wings are directed upwards as in the kite. The wings in the partridge are wielded with immense velocity and power, and this is necessary because of their small size when compared with the great size and weight of the body. From a photograph taken by the Author in 1867, and drawn with great care by C. Berjeau during the same year.

It may appear far-fetched, but there is a more or less direct connection between the distribution of seeds and the migration of animals, especially of birds. This connection is most perceptible in the case of well-formed winged seeds, and such as are furnished with appliances which permit the wind to lay hold of them when falling and to carry them to considerable distances from where they were originally grown. Nature has provided quite a large number of plants with a distinct mode of dissemination.

The most outstanding examples of winged seeds, as indicated, are provided by the ash-tree and plane-tree, both of which structurally greatly resemble certain wings. Thus the seed of the ash is twisted upon itself in the direction of its length, propeller-fashion; while the seed of the plane-tree is scarcely distinguishable from any of the volant wings.

The seeds of the ash and plane-tree, especially the latter, reveal a most unlooked-for resemblance between wings which are intended to vibrate and sustain volant animals in the air, and wings which are not intended to flutter, but only to act as parachutes and delay the rapidity of the fall of the flying bodies. In the one case, the flight is active and is due to inherent muscular powers; in the other, it is passive and is traceable to the action of the wind - this causing the winged seeds to gyrate in their descent and to be carried, in some instances, long distances before they reach the ground.

The resemblances between winged seeds and real wings afford another outstanding example of type, and design as revealed by type, which is everywhere met with in the organic kingdom. Type, which in one sense is another name for general plan, while it finds its most striking examples in the organic kingdom, is not confined to that kingdom. On the contrary, it goes further and deeper. It includes the inorganic kingdom also. This is seen in the structure of crystals, dendrites, basaltic rocks, \&c. In the organic kingdom the types or examples of a general plan are increased in number, but they all tell the same tale, namely, the story of premeditation and design.

The winged seeds are to be regarded as the homologues or precursors of true wings.

As indicating the persistence of type or general plan it should be stated that there were wing structures in early geologic times, and that several of the extinct reptiles (Pterodactyls) flew, as proved by their fossil remains. The winged type of structures is very ancient, and the fact that it has persisted to the present day shows very clearly that it is no chance product, but rather a part, and a leading part, of the great subject of locomotion, on which the majority of animals, and not a few plants, depend for their food-supply. 
Printed by Ballantyne, Hanson \& Co.

Edinburgh \& London 


. 


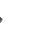



